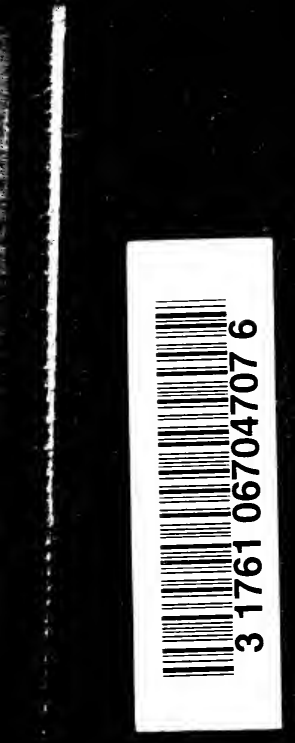




$$
\begin{aligned}
& \text { Ex 整 Librí } \\
& \text { Pskar Aloty }
\end{aligned}
$$




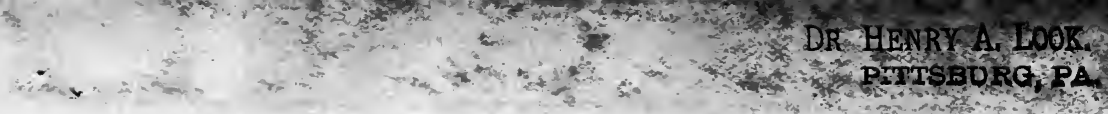

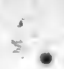

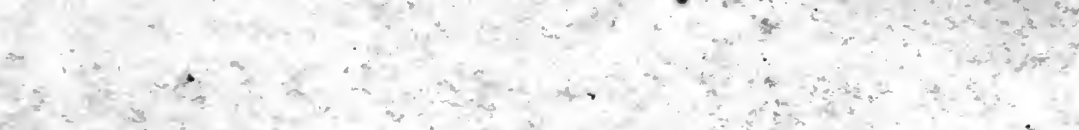

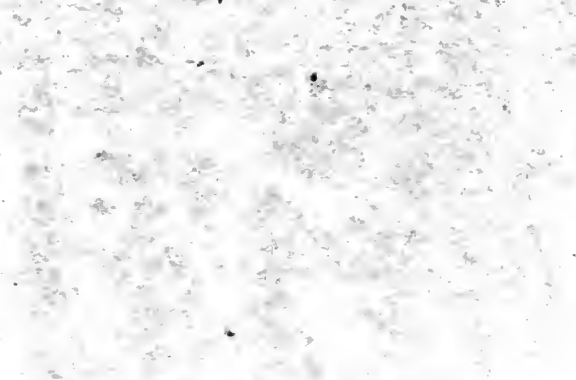

$2-\therefore-20$

$=$
$=$

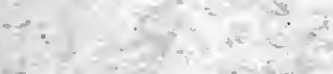

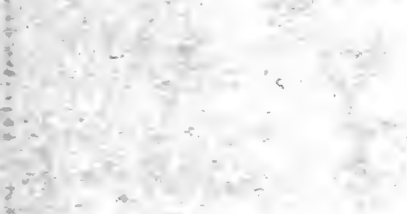

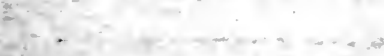
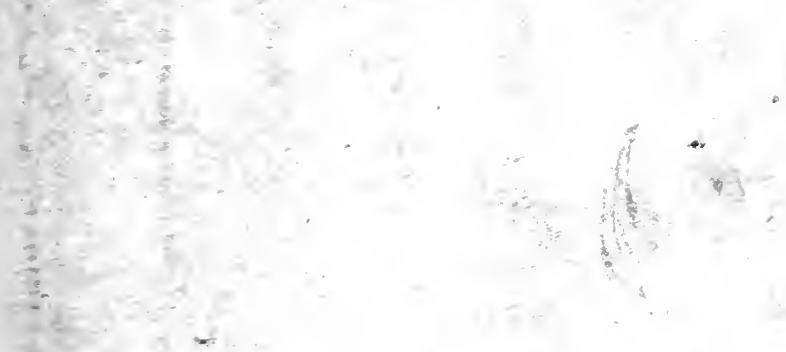

$+$.

is 
Digitized by the Internet Archive in 2007 with funding from Microsoft Corporation 




\title{
A SYSTEM OF
}

\section{GENITO-URINARY DISEASES SYPHILOLOGY AND DERMATOLOGY}

\author{
BY VARIOUS AUTHORS
}

\author{
ED́ITED BY
}

PRINCE A. MORROW, A. M., M. D.

CLINICAL PROFESSOR OF GENITO-URINARY DISEASES FORMERLY LECTURER ON DERMATOLOGY IN THE UNIVERSITY OF THE CITY OF NEW YORK SURGEON TO CHARITY HOSPITAL, ETC.

WITH ILLUSTRATIONS

IN THREE VOLLHES

VOL. I

GENITO-URINARY DISEASES

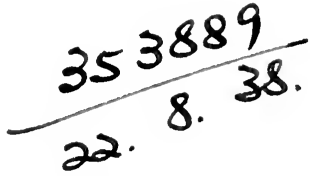

$\mathrm{NE} \dot{\mathrm{W}}$ Y O R K

D. A P P L ETON A N C COM PAN Y 
Copyright, 1893

BY D. APPLETON AND COMPANY.

Electrotyped and Printed

at the Appleton Press, U.S. A. 


\section{P R E F A C E.}

THe genius of modern medical literature is clearly in the direction of division of labor and associated effort. The marked favor with which the numerons "Systems" and "Cyclopædias" which have appeared in recent years have been received by the profession would seem to show that the composite treatise represents the ideal method of bookmaking. In fact, co-operation is the essential condition of thoroughness and completeness in a work covering a wide range of subjects.

The field of research in every department of medicine has grown so large that it is hardly possible for any one individnal to carefully sift from the mass of new material accumulated by the great body of workers the facts and opinions which represent a distinct advance in our knowledge, and have a definite and permanent value.

Especially is this true of the three associated departments of general medicine and surgery embraced in this System. The evolutionary requirements of these specialties demand a new and standard work which shall embody the numerous and important additions made to our knowledge of the subjects they embrace, and at the same time be sufficiently comprehensive to serve as a compendium of reference.

The editor has sought to attain this object by enlisting the co-operation of distinguished specialists, each of whom has been selected for his special fitness to write on the subject assigned, and which has been, as far as practicable, the subject of his choice. Especial effort was made, by clearly defining the ground each article was to cover, to avoid overlapping of subjects and useless repetition, and at the same time secure an organic unison of the completed work, thus making it, as nearly as possible, as coherent and connected as if written by one individual.

It was impressed upon each contributor that, while his article was to be written from his own independent standpoint, the work was designed to be, first of all, thoroughly practical, and adapted to the wants of the 
general practitioner as well as the specialist. With this object in view it was recommended that the subjects of diagnosis and treatment should be presented fully and in a clear and practical light.

The authors are responsible for the views expressed in their respective articles, and to them is due the credit for whatever value the work may possess.

The editor assumes the responsibility of the general plan and arrangement of the work and the articles bearing his name.

In Volume I it will be observed that new material not ordinarily found in text-books on genito-urinary diseases, but of great practical interest and value, has been introduced; such as the chapters on Functional Disorders of Micturition and their Relation to Various Morbid States, the Diagnostic Significance of Pathological Modifications of the Urine, Urine-analysis, Uro-genital Tuberculosis, etc. In addition, the complete monographs on Endoscopy and Cystoscopy contain elaborate and exhaustive presentations of the latest knowledge respecting these valuable aids to diagnosis and treatment.

The idea of grouping Genito-urinary Diseases, Syphilis, and Skin Diseases as proper subjects of associated study had its origin in this country, and it seems eminently fitting that the fruitful results of this idea should be presented by American writers.

It was found necessary, in order to compress the volume within reasonable limits, to omit the extensive and very complete bibliographies which accompanied many of the articles.

It is due to the publishers to state that they have shown a commendable liberality in having executed in the best and most artistic manner the numerous illustrations which form an attractive feature of the work, and serve so valuable a purpose in the elucidation of the text.

New YoRk, December, 1892. 


\section{ALPHABETICAL LIST OF CONTRIBUTORS.}

\section{VOLUME I.}

SAMUEL ALEXANDER, M. D. CHARLES W. ALIEN, M. D. JOSEPH A. ANDREWS, M. D. WILLIAM T. BELFIELD, M. D. JAMES BELL, M. D.

GEORGE E. BREWER, M. D. F. TILDEN BROWN, M. D. JOSEPH D. BRYANT, M. D. JOHN P. BRYSON, M. D. EDWIN C. BURNETT, M. D. ARTIIUR 'I. CABOT, M. D. ANDREW F. CURRIER, M. D. JOHN A. FORDYCE, M. D. GEORGE RYERSON FOWLER, M. D. EUGENE FULLER, M. D. RAMON GUITERAS, M. D.
FRANK HARTLEY, M. D. EDWARD L. KEYES, M. D. S. LUSTGARTEN, M. D. HERMANN G. KLOTZ, M. D. WILEY MEYER, M. D. PRINCE A. MORROW, M. D. WILLIAM K. OTIS, M. D. ALEXANDER W. STEIN, M. D. LEWIS A. STIMSON, M. D. PAUL THORNDIKE, M. D. JAMES P. TUTTLE, M. D. W. W. VAN ARSDALE, M. D. FRANCIS S. WATSON, M. D. J. WILLIAM WHITE, M. D. GEORGE WOOLSEY, M. D. JOHN A. WYETH, M. D. 



\section{O N T E N T S.}

\section{Anatomy and Physiology of the Genito-Urinary Organg Pag BY GEORGE WOOLSEY, M. D. \\ Professor of Anatomy and Clinical Surgery in the Medical Department of the University of the City of New York; Surgeon to Bellevue Hospital, etc.}

I. The kidneys

II. The ureters

III. The urinary bladder

IV. The prostate gland

V. The penis

VI. The urethra

VII. The male perinæum

VIII. The scrotum

IX. The testicles

Spermatic cord .

Diseases of the Penis

By RAMON GUITERAS, M. D.

Surgeon to the City Hospital, New York, Venereal Department; Physician to the Skin Department, University Dispensary.

Abnormities of the penis

Injuries to the penis

Wounds of the penis

Fracture of the penis .

Dislocation of the penis

Cutaneous affections

Lymphatic affections of the penis .

Erysipelas of the penis

Gangrene of the penis .

Tumors of the penis

Elephantiasis of the penis

Epithelioma of the penis .

Methods of amputation

Morbid conditions of the prepuce

Phimosis

Circumcision

Paraphimosis

Diseases of the glans and prepuce .

Balanitis and balano-posthitis

Diabetic balano-posthitis

Herpes progenitalis

Verrucæ 
Diseases of the corpora cavernosa . . . .

Acute inflammations . . . . . . . . . . . . 73

Chronic inflammation . $. \quad . \quad . \quad . \quad . \quad . \quad . \quad . \quad . \quad . \quad 74$

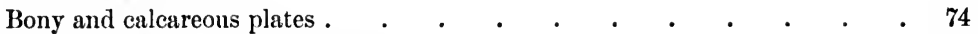

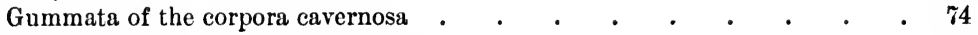

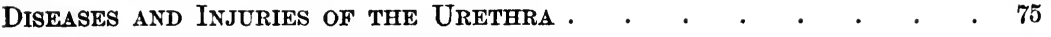

By F. TILDEN BROWN, M. D., New York.

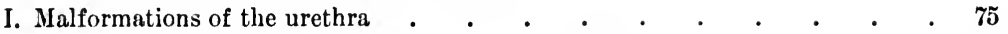

Absence and complete obliteration of the urethra . $\quad . \quad$. $\quad . \quad$. 75

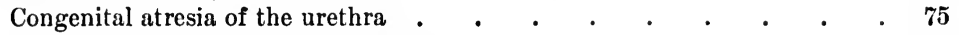

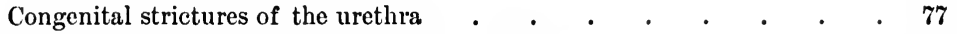

Congenital diverticula of the urethra or urinary pouches $\quad$ - $\quad$ - $\quad . \quad$ 79

Hypospadias . . . . . . . . . . . . 81

Balanic or glandular hypospadias $\quad . \quad$. $\quad . \quad . \quad . \quad . \quad . \quad . \quad 84$

Penile hypospadias . . . . . . . . . . . . . 85

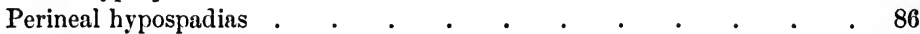

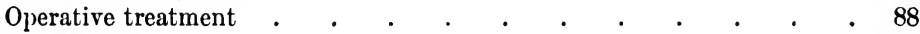

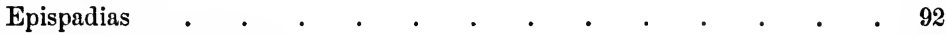

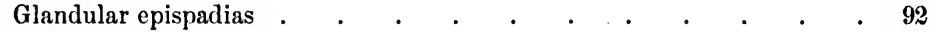

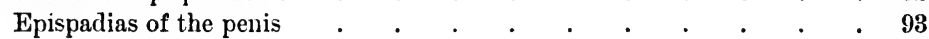

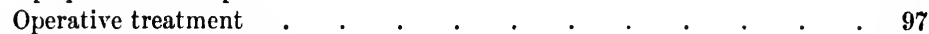

II. Urethral and periurethral abscess . . . . . . . . . . 103

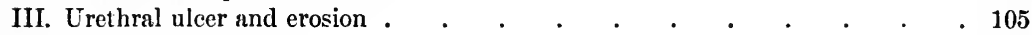

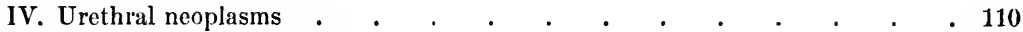

Urethral initial syphilis . . . . . . . . . . . . 110

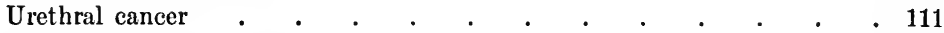

Urethral vegetations and polypi . . . . . . . . . . . . 113

V. Wounds and lacerations of the urethra inflicted from without $\quad$. $\quad . \quad$. 114

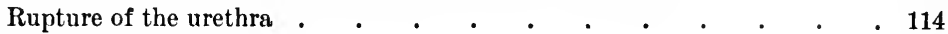

VI. Wounds and lacerations of the urethra inflicted from within . . . $\quad$ - 126

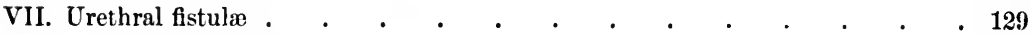

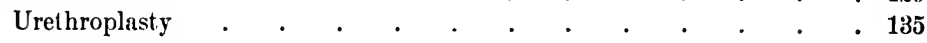

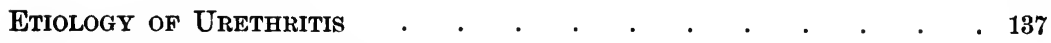

By S. LUSTgarten, M. D.,

Dermatologist to Mount Sinai Dispensary, New York ; formerly " Privat-Docent" on Skin and Venereal Diseases, Imperial Royal University, Vienna.

A. Infectious urethritis .

$\cdot \quad \cdot \quad \cdot \quad \cdot \quad \cdot 137$

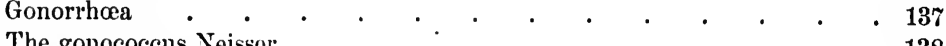

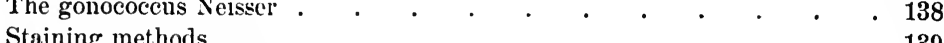

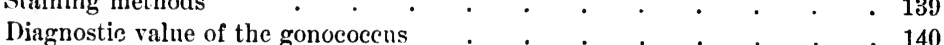

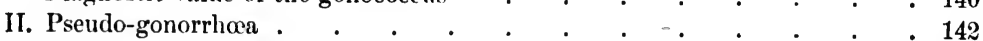

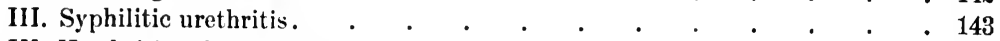

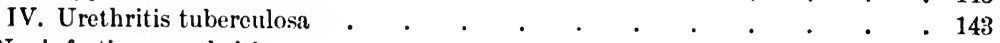

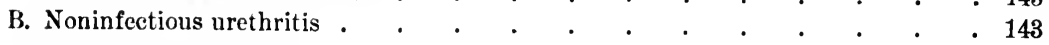

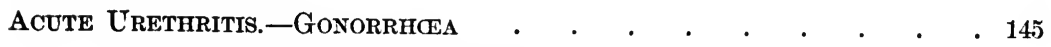

By GEORGE EMERSON BREWER, M. D.,

Assistant Demonstrator of Anatomy, College of Physicians and Surgeons,

Varietics . New York.

Clinical history 
Treatment

Complications of urethritis

Posterior urethritis

Epididymitis

Periurethral inflammation.

Folliculitis

Cowperitis

Prostatitis

Vesiculitis

Cystitis .

Pyelitis .

Balanitis

Phimosis

Paraphimosis

Lymphangitis and adenitis .

Bx WILIIAM K. OTIS, M. D., New York.

Local treatment

Injection

Suppositorics

Endoscopic treatment

Drainage .

General treatment

ENDOSCOPY

By HERMANN G. KLOTZ, M. D.,

Consulting Surgeon to the German Hospital, New York.

Development of the endoscope

Endoscopic armamentarium .

Method of examination

The endoscopic picture

Normal appearance of the urethra

Pathological appearances

Endoscopic diagnosis

Endoscopic treatment

GonorRhoEal OpHThalmia

By JOSEPH A. ANDREWS, M. D.,

Ophthalmic Surgeon to the Charity Hospital, New York.

Symptoms

Treatment

Ophthalmia neonatorum.

\section{GonorRhoeal RheumatisM}

By FRANK HARTLEY, M. D.,

Surgeon to the New York Hospital.

I. Acute monarticular gonorrhœal rheumatism . . . . . . . 243

II. Chronic monarticular gonorrhœal rheumatism, hydrarthrosis, gonocele . $\quad$. 243

III. Polyarticular acute gonorrhœal rheumatism . . . . . . . . 244

IV. Polyarticular subacte gonorrhœal rheumatism . . . . . . . . . 244

V. Polyarticular chronic gonorrhœal rheumatism . . . . . . . . 244 
Gonorrhcea of the Rectum, Nose, Mouth, Ear, Umblicus, aNd Axilla 249

By JAMES P. TUTTLE, M. D.,

Professor of Diseases of the Rectum, New York Polyclinic.

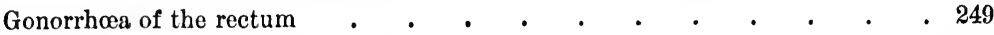

Mucous membranes susceptible to gonorrhœa . $\quad . \quad$. $\quad$. $\quad$. $\quad 250$

Mucous membranes refractory to gonorrhœa . . . . . . . . . 250

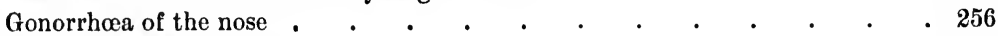

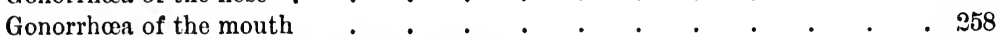

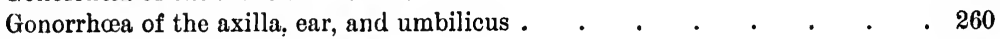

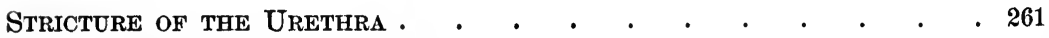

BY J. WILliaM WHITE, M. D.,

Professor of Clinical Surgery in the University of Pennsylvania ; Surgeon to the University and German Hospitals, Philadelphia.

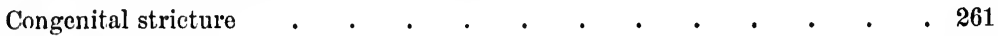

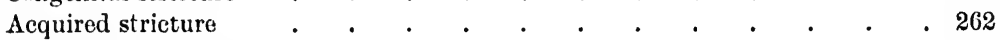

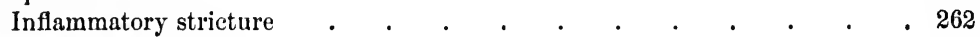

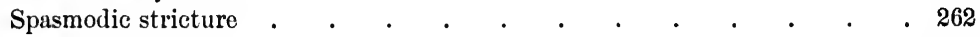

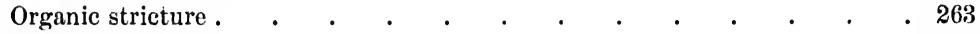

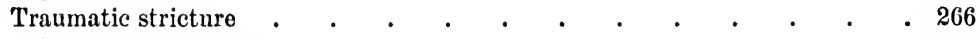

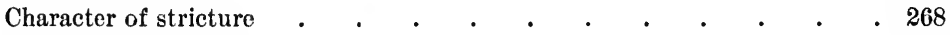

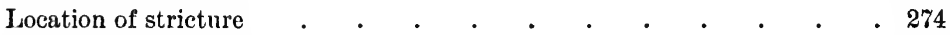

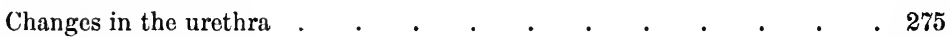

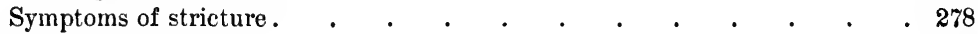

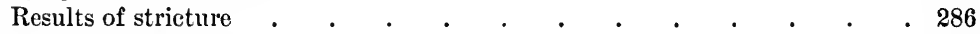

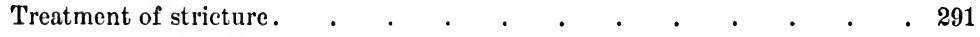

1. Gradual dilatation . . . . . . . . . . . . . . . 291

Catheterisin . . . . . . . . . . . . . . . 294

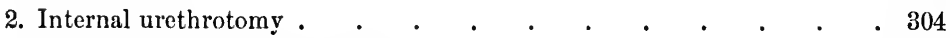

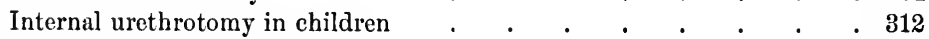

3. External urethrotomy . . . . . . . . . . . 313

Combined internal and external urethrotomy $\quad$. $\quad . \quad$. $\quad . \quad$. 317

Perineal section . . . . . . . . . . . . . . . 319

Retrograde catheterization . . . . . . . . . . . . 323

Drainage after external nrethrotomy and perineal section . . . . . 323

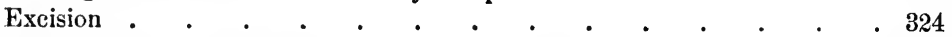

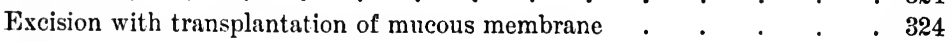

Electrolysis . . . . . . . . . . . . . . . . . . 325

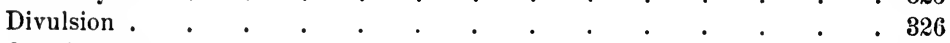

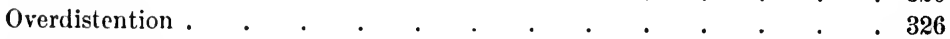

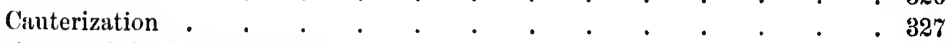

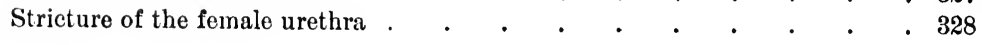

Diseases of the Prostate

BY W. T. BELFIELD, M. D., Chicago, Ill.
. . . . . . . . . . . . . . . . 331

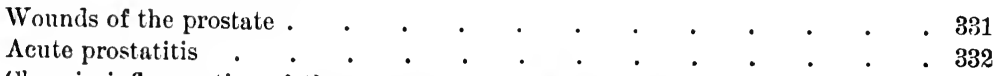

Chronic inflammation of the prostate and appendages, including chronic pros-

tatitis, prostatorrhœa, prostatic abscess, and pelvic abscess . . . . 333

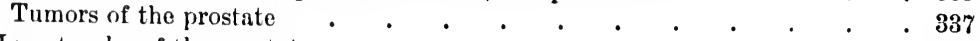

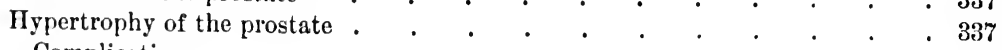

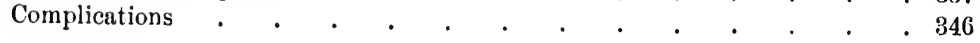




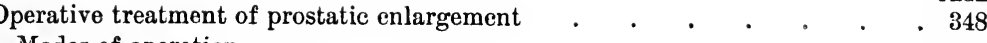

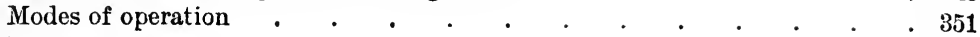

Prostatectomy . . . . . . . . . . . . . . . . $\quad . \quad . \quad 354$

Hypertrophy of the prostatic sphincter . $\quad . \quad . \quad . \quad . \quad . \quad . \quad . \quad . \quad . \quad 357$

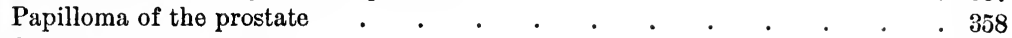

Cancer of the prostate . . . . . . . . . . . . . . . . . . 358

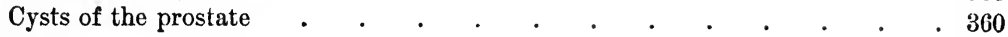

The Functional Disorders of Micturition . . . . . . . . 361

BY JOSEPH D. BRYANT, M. D.,

Professor of Anatomy and Clinical Surgery and Associate Professor of Orthopedic Surgery, Bellevue Hospital Medical College, Surgeon to Bellevue and St. Vincent's Hospitals, New York.

Physiology of urination . . . . . . . . . . . . . . 361

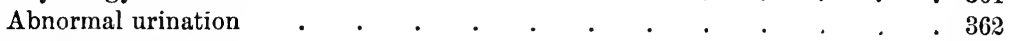

Retention of urine . . . . . . . . . . . . . . . . 364

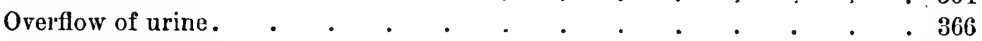

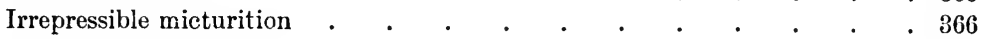

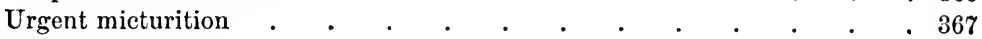

Difficult micturition . . . . . . . . . . . . . . . . 370

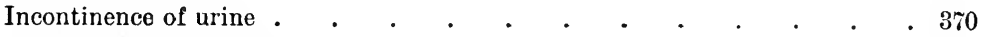

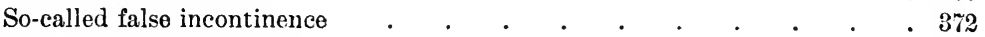

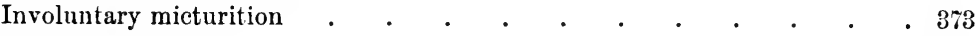

Painful micturition . . . . . . . . . . . . . . . 374

Diagnostic signifieance of painful urination . . . . . . . . . . . $\quad . \quad 374$

Diagnostic significance of force, size, form, and direction of the stream . 381

\section{Diagnostic Significance of Pathological Modifications in the Urine}

(including the most Practical Methods of Urine Analysis) . . 385

By EUGENE FULLER, M. D., New York.

1. General characteristics of the urine . . . . . . . . . 385

2. Chemical constituents of the urine subdivided as follows: . . . . . 385

a. The normal chemical constituents . . . . . . . . . 390

$b$. Those that are normal only when present in very small amounts, abnormal

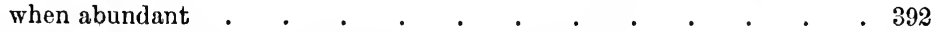

c. Products of chemical decomposition of normal ingredients . . . . . 395

$d$. The abnormal chemical constituents . . . . . . . . . 395

Albumin . . . . . . . . . . . . . . . 396

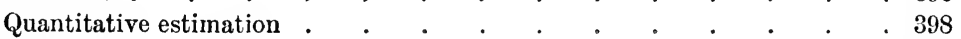

Sugar . . . . . . . . . . . . . . . . . 401

Fermentation test . . . . . . . . . . . . . 402

3. Organized sediments . . . . . . . . . . . . . . 404

Casts . . . . . . . . . . . . . . . 408

Pus . . . . . . . . . . . . . . . . . 410

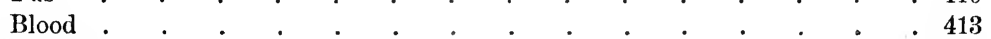

4. Miscellany . . . . . . . . . . . . . . . . 416

Bacilli . . . . . . . . . . . . . . . 416

URINARY Fever. . . . . . . . . . . . . . . . 419

By J. A. FORDYCE, M. D.,

Lecturer on Dermatology, New York Polyclinic; Surgeon to the City Hospital, New York.

Acute urethral fever

Chronic urinary fever 
Etiology • • • • • • • • • • • • • . . 426

Pathogenesis . . . . . . . . . . . . . . . . . . 428

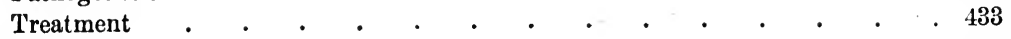

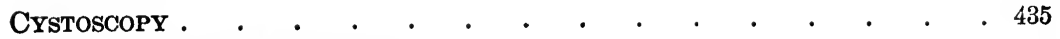

BY WILLY MEYER, M. D.,

Surgeon to the German Hospital and the Skin and Cancer Hospital, New York.

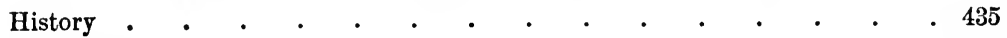

Instruments . . . . . . . . . . . . . . . 438

The batteries . $. \quad . \quad . \quad . \quad . \quad . \quad . \quad . \quad . \quad . \quad .453$

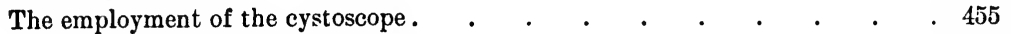

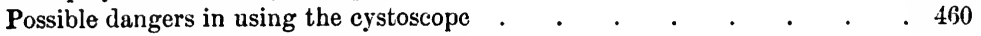

Rules for performing cystoscopy . . . . . . . . . . . $\quad$. 463

Cystoscopic appearance of the healthy bladder ..$\quad$. $\quad . \quad$. $\quad . \quad 4470$

Cystoscopic appearance of the diseased bladder . $\quad . \quad$. $\quad . \quad$. . . $\quad$. 473

Cystoscopy with reference to kidney diseases . . . . . . . . . . . . . 483

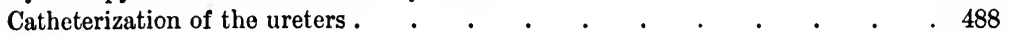

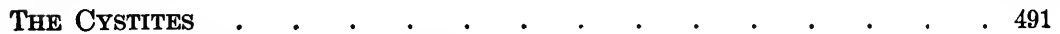

Bx SAMUEL ALEXANDER, M. D.,

Professor of Genito-Urinary Surgery, Dermatology, and Syphilology, Bellevue Hospital Medical College; Surgeon to Bellevue Hospital.

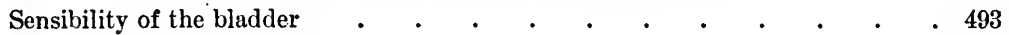

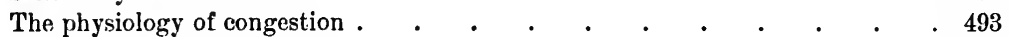

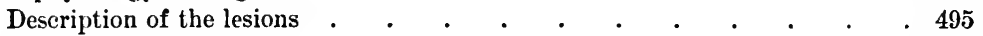

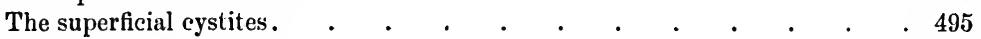

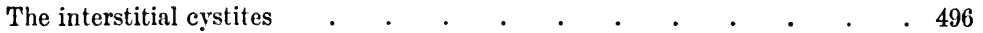

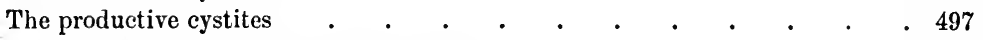

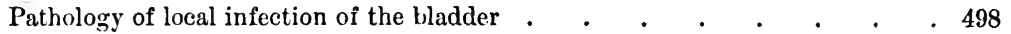

Conditions which favor local infection of the bladder ..$\quad$. $\quad . \quad$. $\quad$. 502

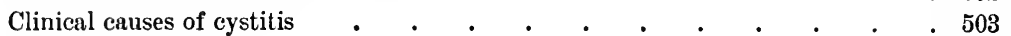

Symptomatology . . . . . . . . . . . . . . . 507

General and operative treatment . . . . . . . . . . . . 515

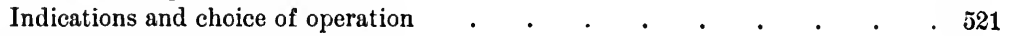

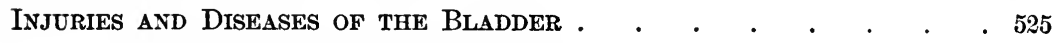

By GEORGE RYERSON FOWLER, M. D.,

Surgeon to St. Mary's Hospital and the Methodist Episcopal Hospital, Brooklyn, N. Y.

I. Wounds of the bladder .

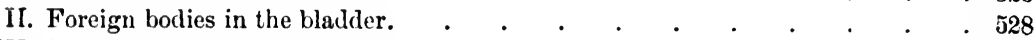

III. Malformations and malpositions of the bladder . . . . . . . . . . 532

Absence of the bladder • . . . . . . . . . . . . . . 532

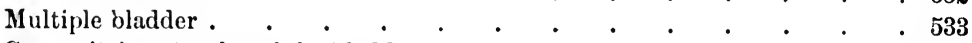

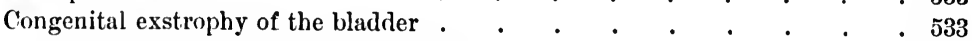

Congenital defects of the posterior bladder wall $\quad . \quad$. $\quad . \quad$. $\quad . \quad$. 537

Patent urachus . . . . . . . . . . . . . . . . 537

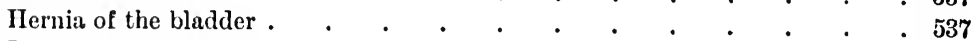

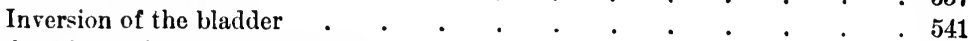

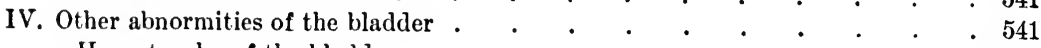

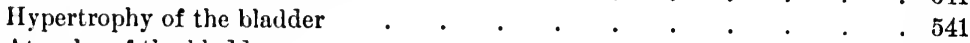

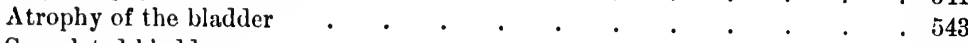

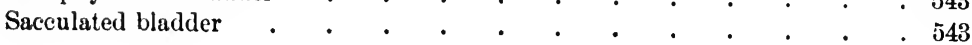


Suprapubic vesical puncture in obstructive disease at the resical neck . .545

$\cdot \cdot \cdot \cdot \cdot 546$

RUPTURE OF THE Bladder . . . . . . . . . . . . 553

BY ALEXANDER W. STEIN, M. D.,

Location . Surgeon to the Charity Hospital, New York.

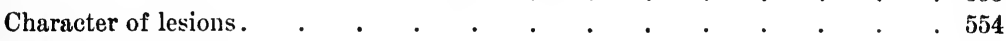

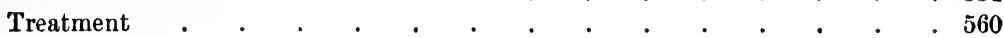

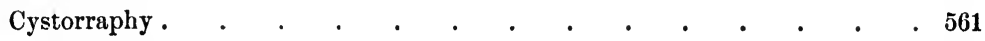

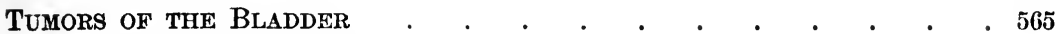

By FRANCIS SEDGWICK WATSON, M. D.,

Assistant Visiting Surgeon, Boston City Hospital ; Instructor in the Surgery of the Genito-Urinary Organs, Harvard Medical School ; Assistant in Clinical Surgery, Harvard Medical School.

Pathologs and etiology of bladder tumors . . . . . . . . . . 565

Papilloma . . . . . . . . . . . . . . . . 567

Myxoma (Polyp) . . . . . . . . . . . . . . . . . 570

Myoma . . . . . . . . . . . . . . . . .

Cysts . . . . . . . . . . . . . . . . . . . 571

Carcinoma . . . . . . . . . . . . . . . . . . . . 571

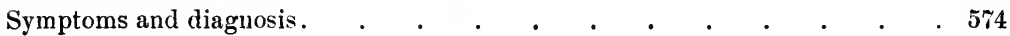

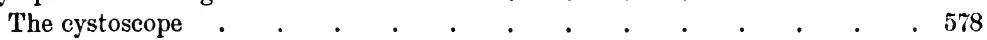

Palliative treatment $. \quad . \quad . \quad . \quad . \quad . \quad . \quad . \quad . \quad . \quad . \quad .587$

Operative treatment of benign growths . . . . . . . . . . 588

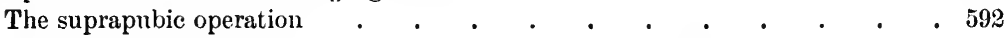

A new means to assist in the removal of intravesical growths through a supra-

pubic cystotomy . . . . . . . . . . . . . . . . 598

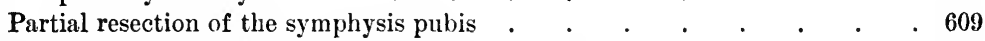

Operative treatment of bladder tumors in the female . . . . . . . . 610

Ultimate results of operation for the removal of benign growths . . . . 611

Treatment of malignant growths . . . . . . . . . . . . 613

Drainage for relief in malignant disease . . . . . . . . . . 613

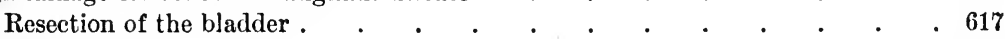

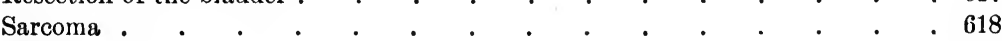

Extirpation of the bladder in the female $\quad$. $\quad$. $\quad$. $\quad$. . . . 618

Stone in the Bladder, Prostate, Urethra, ANd Ureters . . . 621

BY ARTHUR T. CABOT, M. D.,

Surgeon to the Massachusetts General Hospital ; Lecturer on Genito-Urinary Surgery, Harvard Medical School.

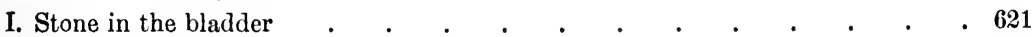

Definition . . . . . . . . . . . . . . . 621

Chemical and physical characteristics. . . . . . . . . . 621

Color . . . . . . . . . . . . . . . . . . 628

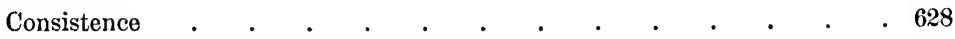

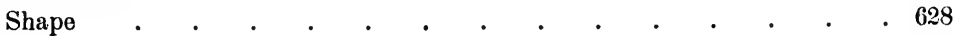

Number . . . . . . . . . . . . . . . 628

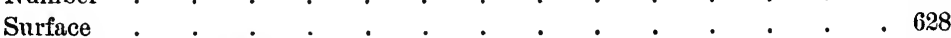

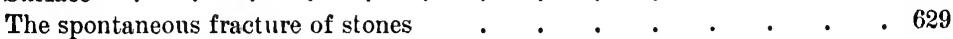

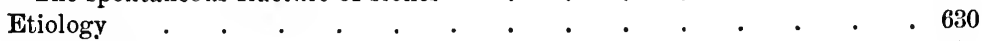

Heredity . . . . . . . . . . . . . . 630 
Diet and habit

Diathesis

Alkaline fermentation

Symptomatology

Diagnosis

The operation of sounding

Exoloration with litholapaxy-pump

Examination with the cystoscope

Digital exploration of the bladder .

Exploration through suprapubic openings

Preventive treatment .

Solvent treatment of stone.

The operative treatment of stone

History

Choice of operation

Interference with the functions of the parts

Description of the different operations .

Completeness of cure following the different methods of operating

Selection of operation .

Complications which may modify our choice of operation.

Preparation for operation

1. Lithotrity

History and development .

2. Litholapaxy.

The operation

Complications which may arise during litholapaxy

Complications after operation

Treatment after litholapaxy

3. Lithotomy

Perineal-lithotomy

Anatomical consideration of the various incisions

Lateral lithotomy

Bilateral lithotomy

Median lithotomy

Medio-bilateral lithotomy

Accidents and complications during operation

Complications which may arise subsequently

Wound complications .

Bilateral operation

Median operation .

4. Perineal lithotrity

5. Suprapubic lithotomy

The after-treatment of the suprapubic wound $\quad . \quad$. . . . . . 729

Accidents and complications in suprapubic lithotomy . . . . . . 731

Stone in the female bladder.

Operative treatment.

Vaginal lithotomy

II. Prostatic calculi

III. Stone in the urethra

IV. Stone in the ureter .

Syinptomatology

Diagnosis

Treatment 
By LEWIS A. STIMSON, M. D.,

Professor of Surgery in the University of the City of New York ; Attending Surgeon to the New York, Bellevue, and Chambers Street Hospitals.

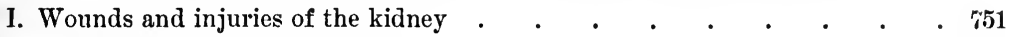

Traumatisms of the kidney . . . . . . . . . . . . 751

Subcutaneous injuries of the kidney . . . . . . . . . . . . $\quad$. 752

Gunshot-wounds . . . . . . . . . . . . 752

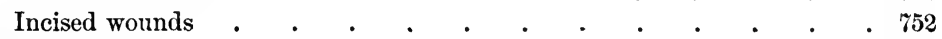

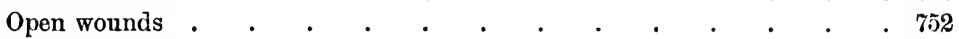

Subcutaneous lacerations and ruptures . . . . . . . . . . . . 5757

II. Nephrolithiasis-gravel-kidney stone . . . . . . . . . 764

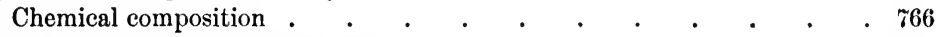

Nephrolithotomy ; nephrectomy . . . . . . . . . . . 781

III. Pyelitis, pyelonephritis, pyonephrosis, nephritis, tuberculosis . . . . 781

A. Pyelitis, pyelonephritis, tuberculosis . $\quad$. $\quad . \quad$. $\quad . \quad$. $\quad . \quad$. 781

B. Primary suppurative nephritis-gangrene of kidney . . . . . . 794

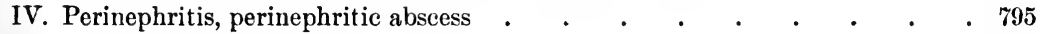

1. Primary perinephritis. $a$, Traumatic; $b$, Spontaneous . . . . . 796

2. Secondary perinephritis, $a$, Due to a general cause (infectious perinephritis); $b$, Due to a local cause (perinephritis by extension) . . . 796

v. Renal and perinephritic fistulæ . . . . . . . . . . 803

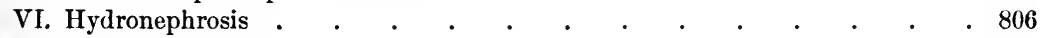

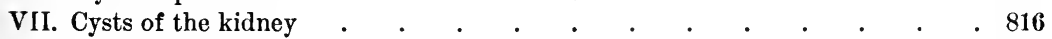

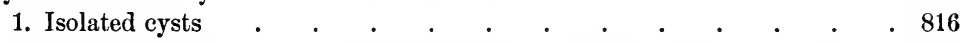

2. Conglomerate cysts, or cystic degeneration, or large polycystic kidney $\quad$. 819

3. Hydatid cysts . . . . . . . . . . . . 820

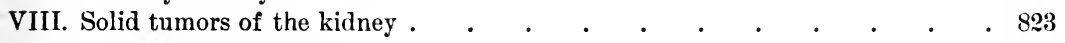

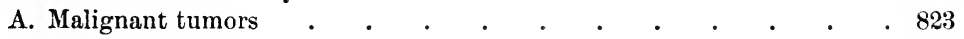

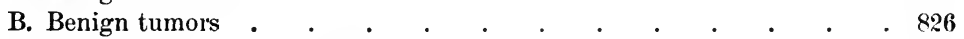

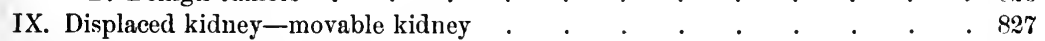

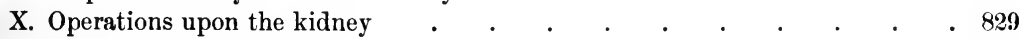

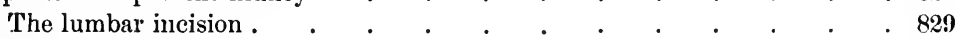

The longitudinal incision . . . . . . . . . . . . 830

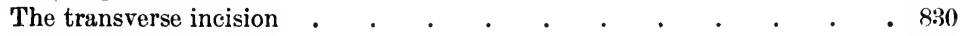

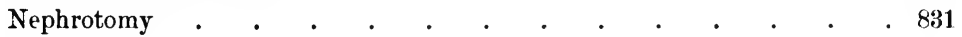

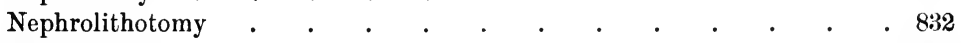

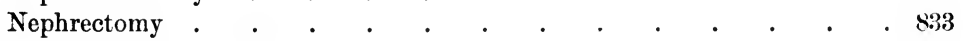

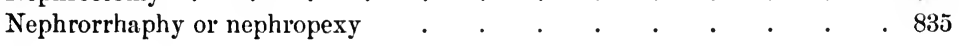

TURerculosis Uro-genitalis . . . . . . . . . . . . . . 837

BY JOHN P. BRYSON, M. D.,

Professor of Genito-Urinary Surgery, St. Louis Medicai College, Washington University.

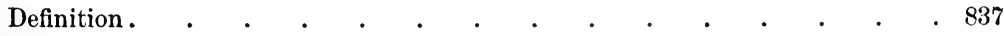

Varieties . . . . . . . . . . . . . . . . . . 838

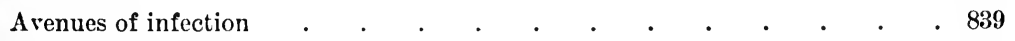

Heredity . . . . . . . . . . . . . . . 848

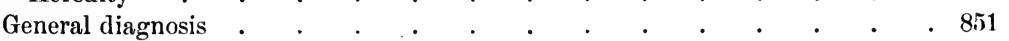

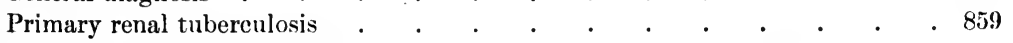

Differential diagnosis of primary renal tubercle in early stage and renal stone

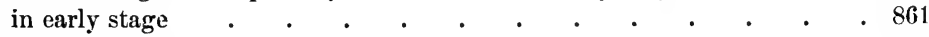

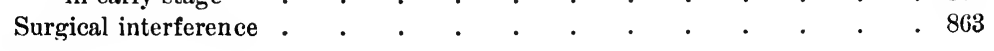


Tuberculosis of the bladder Symptoms and diagnosis Cystoscopy

Tuberculosis of the seminal vesicles

Tuberculosis of the prostate

Symptoms and diagnosis

Tuberculosis of the urethra

Tuberculosis of the testis and cord.

Diseases of The Scrotum

By CHARles W. ALleN, M. D.,

Surgeon to the City Hospital, Genito-Urinary Department; Attending Physician to Bellevue Hospital, Out-door Patients, Genito-Urinary Department ; Clinical Assistant to the Chair of Genito. Urinary and Venereal Diseases, University Medical College.

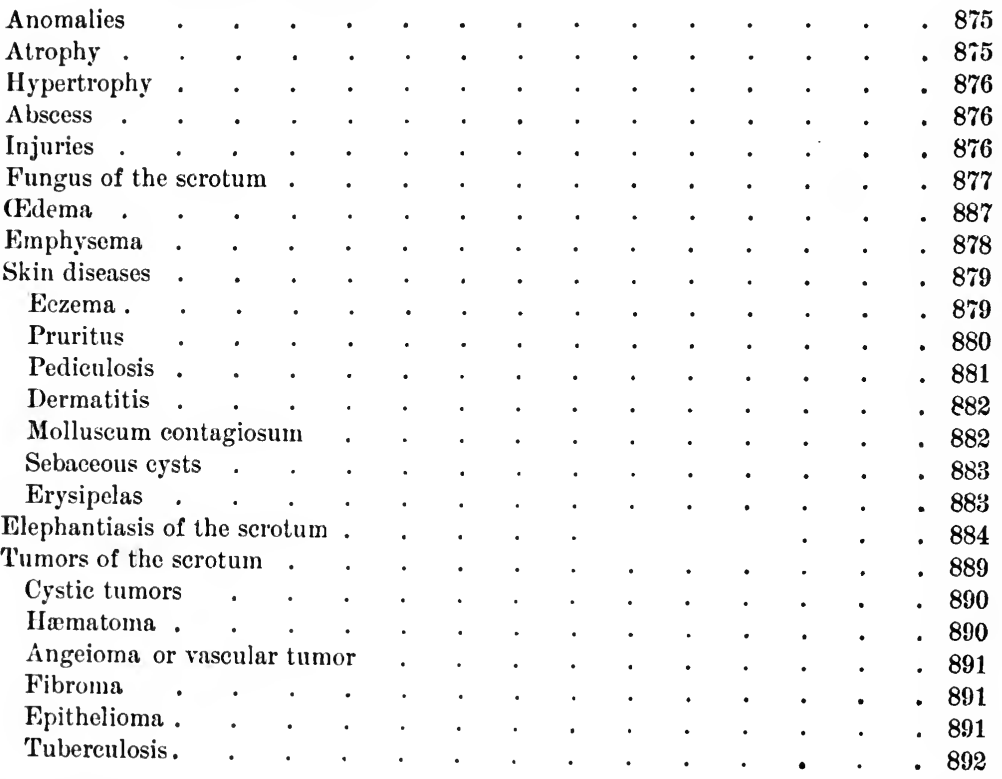

Diseases of the Testicle . . . . . . . . . . . . . . 894

By JAMES BELL, M. D.,

Associate Professor of Clinical Surgery, McGill University; Surgeon to the Montreal General Hospital.

Hæmatocele

Hamatocele of the cord

Free bodies in the tunica vaginalis

Anomalies of the testicle.

Absence of the testicle.

Supernumerary testicles

Iypertrophy of the testicle .

Atrophy of the testicle

Seat of the testicle

Misplaced testicles 


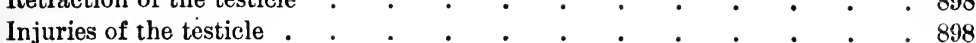

Diseases of the Testicle . . . . . . . . . . . . . . 899

BY EDWIN C. BURNETT, M. D.,

Chief of Clinics of Skin and Venereal Diseases, St. Louis Mcdical College.

Acute orchitis .

Epididymitis

Etiology

Chronic orchitis

Etiology

Chronic epididymitis

Syphilitic sarcocele .

Fungus of the testicle

Diseases of the Testicle

By JOHN P. BRYSON, M. D.,

Professor of Genito-Urinary Surgery, St. Louis Medical College, Washington University.

Cystoma testis .

Dermoid cysts of the testis and scrotum . . . . . . . . . . 930

Solid tumors of the testicle . . . . . . . . . . . . . . 931

Enchondroma . . . . . . . . . . . . . . . . . 931

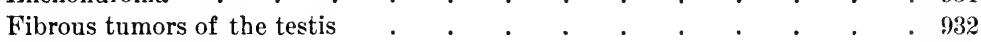

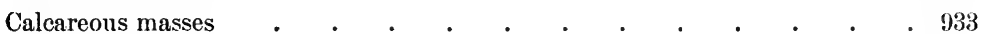

Carcinoma of the testis . . . . . . . . . . . . . . . 933

Sarcoma of the testis . . . . . . . . . . . . . . 935

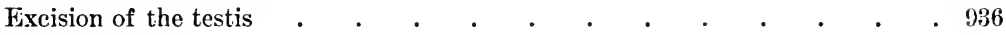

Irritable testis . . . . . . . . . . . . . . . . . 938

Hydrocele AND SPERMatocele . . . . . . . . . . . 941

By JOHN A. WYETH, M. D.,

Professor of Surgery, New York Polyclinic; Visiting Surgeon to Mount Sinai Hospital, New York; and

W. W. VAN ARSDALE, M. D.,

Adjunct Professor of Surgery, New York Polyclinic; Assistant Surgeon, New York Cancer Hospital.

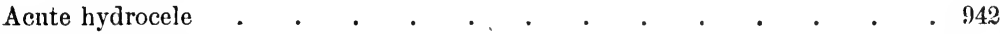

Chronic hydrocele . . . . . . . . . . . . . . . . 945

Periorchitis prolifera . . . . . . . . . . . . . . 948

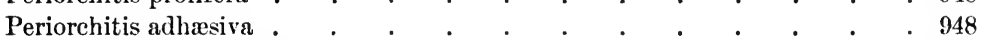

Periorchitis hæmorrhagica . $. \quad . \quad . \quad . \quad . \quad . \quad . \quad . \quad . \quad .949$

Hydrocele communicans . . . . . . . . . . . . . 957

Hydrocele of the spermatic cord . . . . . . . . . . . 958

Diffuse hydrocele of the cord . . . . . . . . . . . . 961

Bilocular hydrocele . . . . . . . . . . . . . . 962

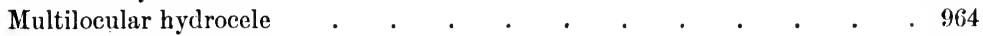

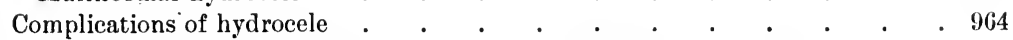

Differential diagnosis of the various forms of hydrocele and their complications

Spermatocele 
VARICOCELE .

BY EDWARD L. KEYES, M. D.,

Consulting Surgeon to Bellevue and Charity Hospitals, New York.

Symptoms

Diagnosis .

Treatment

The operation .

Ablation of the scrotum .

Diseases of the Seminal Vesicles

By PAUI, THORNDIKE, M. D.,

Surgeon to Out-patients, Carney Hospital, Boston; Surgeon to Genito-Urinary Department, Boston Dispensary.

Anatomy .

Physiology

Injuries .

Fistulx.

Tumors .

Malignant disease

Sarcoma

Cysts

Spermatic colic

Inflamination

'Tuberculosis

Radical operation for the removal of a tubercular seminal vesicle . . 993

Functional Disorders of the Male Sexual Organs . . . . . 99 \%

By PRINCE A. MORROW, M. D.,

Clinical Professor of Genito-Urinary Diseases, University of the City of New York; Surgeon to Charity Hospital, New York.

I. Spermatorrhœa

Nocturnal pollutions . . . . . . . . . . . . . . . . . . 998

Diurnal pollutions

False spermatorrhœa .

Pathological significance

Masturbation

Sexual excess

Continence

Course of spermatorrhœn .

Local effects

Constitutional effects

Treatment

II. Impotence

Physiology of erection.

Organic impotence

Psychical impotence

Relative impotence

Irritable impotence

Paralytic inpotence

Treatinent

III. Sterility

Physiological characters of the semen

Pathological modifications . 
Aspermia

Relative aspermia

Temporary aspermia

Oligospermia

Oligozoöspermia

Azoöspermia

Chromospermia

Hæmatospermia

Treatment

Gonorrhoea in the Female .

BY ANDREW F. CURRIER, M. D.,

Assistant Gynæcologist to Skin and Cancer Hospital; Attending Gynæcologist, Bellevue Hospital, Out-door Patient Department.

\begin{tabular}{|c|c|c|c|c|c|c|c|c|}
\hline History & . & . & . & . & . & & & 1039 \\
\hline Variety of forms & . & . & . & . & . & . & - & 1044 \\
\hline 1. Vulvitis . & . & . & . & . & . & . & . & 1048 \\
\hline 2. Bartholinitis & . & . & . & . & . & . & . & 1050 \\
\hline 3. Vaginitis . & & . & . & . & . & . & . & 1050 \\
\hline Senile vagini & & - & . & . & . & . & . & 1052 \\
\hline 4. Urethritis & . & . & . & . & . & . & . & 1053 \\
\hline 5. Endometritis & . & . & . & . & . & . & . & 1054 \\
\hline 6. Salpingitis . & . & . & . & . & . & . & . & 1056 \\
\hline 7. Ovaritis. & . & . & . & . & . & . & . & 1058 \\
\hline 8. Peritonitis & . & . & . & . & . & . & . & 1058 \\
\hline Complications . & . & . & . & . & . & . & . & 1059 \\
\hline Condylomata & & . & . & . & . & . & • & 1059 \\
\hline Enlarged inguina & al gle & ands & . & . & . & . & . & 1060 \\
\hline Gonorrhœa of no & se an & $\mathrm{d} \mathrm{me}$ & outh & . & . & . & . & 1060 \\
\hline Gonorrhœa of an & uns an & ad rec & ctum & . & . & . & . & 1060 \\
\hline Gonorrhœal arthr & & . & . & . & . & . & . & 1060 \\
\hline Cystitis. . & & & . & & & & & 1060 \\
\hline Syphilis and char & neroi & & & . & . & . & & 1060 \\
\hline Relation of gonor & rrhœ & a to $s$ & sterilit & & & . & & 1061. \\
\hline Treatment . & . & . & . & . & . & . & & 1062 \\
\hline
\end{tabular}





\title{
LIST OF ILLUSTRATIONS.
}

\author{
Chromo-Lithographs, etTC. \\ PLATE \\ I.-Normal micro-organisms and gonococci of male urethra (5 figures). \\ II.-Gonorrhœal ophthalmia in adult and in new-born infant (2 figures). \\ III.-Endoscopic pictures (24 figures). \\ IV.-Cystoscopic pictures (8 figures). \\ V.-Multiple villous papilloma of the bladder. \\ VI.-Mucous polypi of the bladder from a young girl. \\ VII.-Cancer of the bladder. \\ VIII.-Elephantiasis of the scrotum.
}

\section{Half-Tone Picteres, etC.}

TIGORE

\section{Anatomy and Physiology of the Genito-Urinary Organs.}

1. Frontal section through the kidney, pelvis, and calyces

2. Relations of viscera to kidneys (anterior view). . . . . . . . . . 3

3. Díagram showing relation of the viscera to the parietes (posterior view) . $\quad$ - 5

4. Diagram showing the direction, form, and relations of the kidneys . . . 6

5. Horizontal seetion of body between second and third lumbar vertebre . . . 8

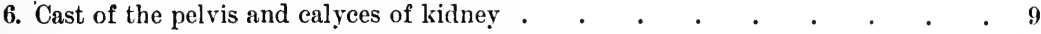

7. Lower part of the male bladder, with the beginning of the urethra $\quad$. . . 14

8. Median section of a frozen male subject . . . . . . . . . . 15

9. Diagram showing relations of the posterior and inferior regions of the bladder in man . . . . . . . . . . . . . . . . 17

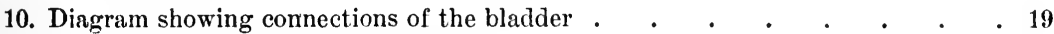

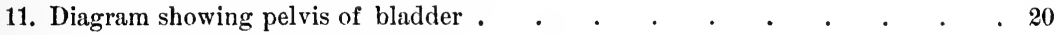

12. Median section of the lower part of the bladder, with the prostate and beginning of urethra . . . . . . . . . . . . . . . 22

13. Cross-section of the prostate in a series from base to apex . . . . . 24

14. A healthy prostate from a man aged thirty-five years, showing internal meatus and ejaculatory ducts . . . . . . . . . . . . . 25

15. Diagram of urethra in natural condition . . . . . . . . . . . 26

16. Scheme designed to show the direction of urethra, and its relations with the

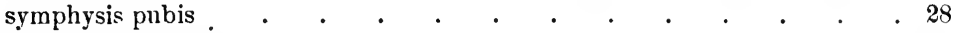

17. Cross-section of the penis . . . . . . . . . . . . . . . 28

18. Cross-section through the cervix and glans penis . . . . . . . . 28

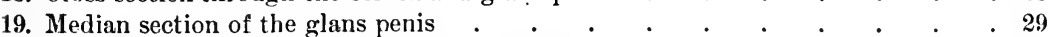

20. Diagram showing end of the penis from below. . . . . . . . 30 


\section{FIGURE}

21. Diagram of oblique coronal section through the pelvis .

22. Sagittal section through bladder and rectum .

23. Diagram of slightly oblique coronal section of pelvis _ . . . . . . . . 33

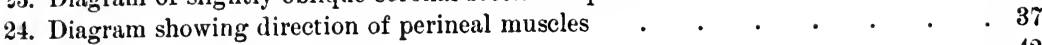

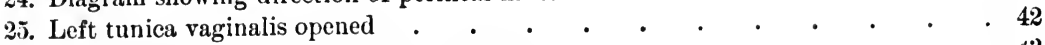

26. Injected testis, epididymis, and vas deferens . . . . . . . . . . . . 43

\section{Diseases of tile Penis.}

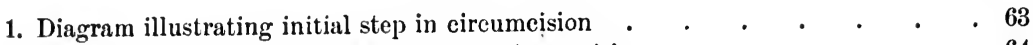

2. Diagrain showing position of stitches in circumcision $\quad . \quad$. $\quad . \quad$. $\quad . \quad$. 64

3. Clamp employed in eircumcision $\quad$.

4, 5. Slitting corona in eircumcision $\quad . \quad$ •

6. Diagram illustrating method of redueing glans penis in paraphimosis . $\quad . \quad$. 66

\section{Diseases and InJuries of the Urethra.}

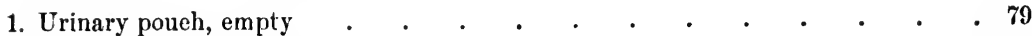

2. Urinary pouch, distended .

3. Glandular segment of urethra in a case of urethral diverticulum . . . . $\quad 80$

4. Glandular or balanic hypospadias . . . . . . . . . . . . . 84

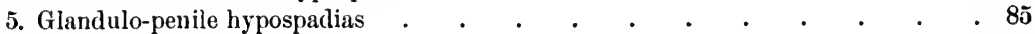

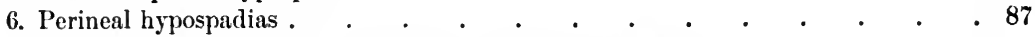

7. Duplay's operation for hypospadias; straightening of penis . . . . . . $\quad$ - 89

8. Duplay's operation for hypospadias; formation of glandular meatus . . . 90

9. Duplay's operation for hypospadias; formation of new urethral canal . . . . 90

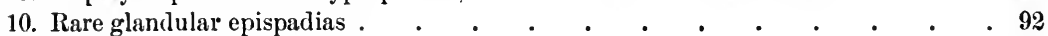

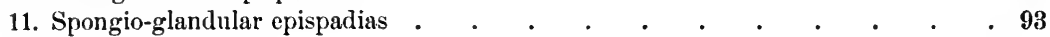

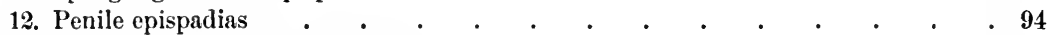

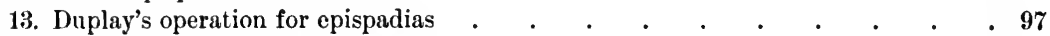

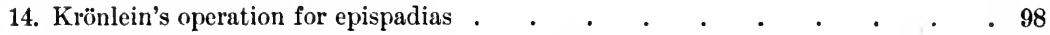

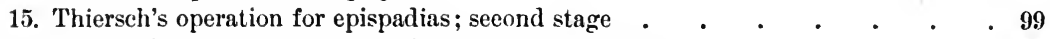

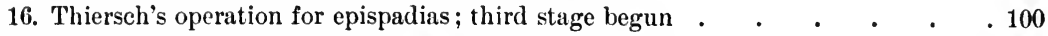

17. Thiersch's operation for epispadias : third stage eompleted . . . . . $\quad$. 100

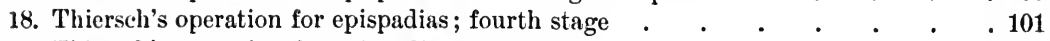

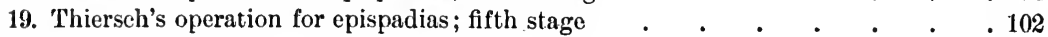

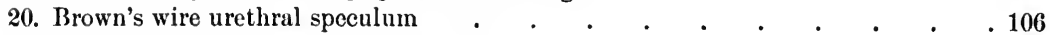

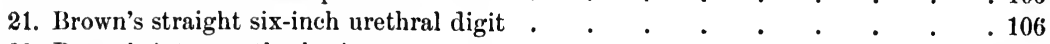

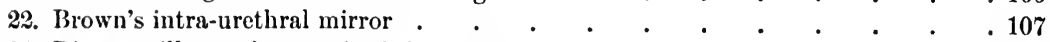

23. Diagram illustrating urethral illumination by electric light condenser . . $\quad$. 107

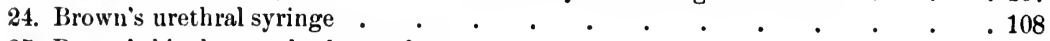

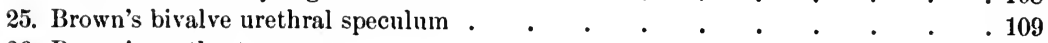

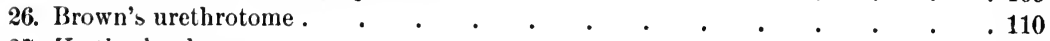

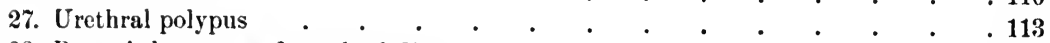

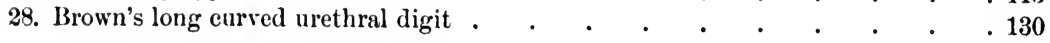

\section{ACUTE URETHRITIS.}

1. Diagram showing apparatus for retrojection of hot water through urethra . . 161

2, 3. Diagrams showing apparatus for continnous irrigation of urethra . $\quad . \quad$. 162

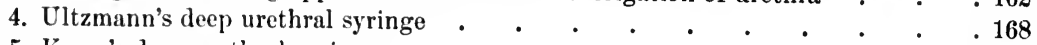

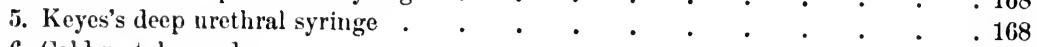

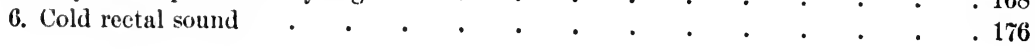




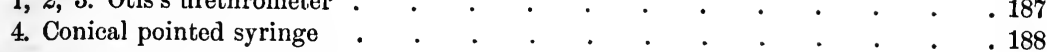

5. Otis's electro-urethroscope.

1, 2, 3, 4, 5. Endoscopic tubes

ENDOSCOPY.

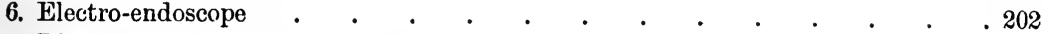

7. Diagram showing instrument for removing urethral polypi . . . . . . . 221

\section{Gonorrheal Ophthalmia.}

1. Diagram showing gonococci in conjunctival discharge . . . . . . . . 223

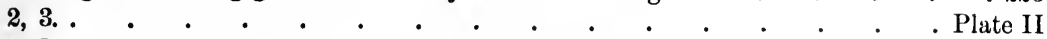

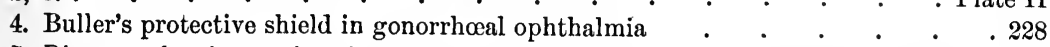

5. Diagram showing patient in position for irrigation in gonorrhœal ophthalmia $\quad .229$

6. Pus basin . . . . . . . . . . . . . . . . . . . 229

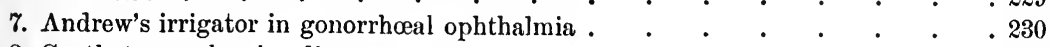

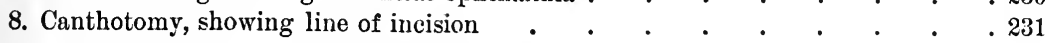

Gonorrhea of the Rectum, Nose, Mouth, Ear, Umbilicus, and Axilla.

1. Tuttle's rectal irrigator

Stricture of the Urethra.

1. Linear stricture

2. Annular stricture

3. Tortuous stricture

4. Bougie à boule .

5. Whalebone filiform bougies

6. Tunneled catheter

7. Thompson's conical steel sounds

8. Flexible bougies

9. Nercier catheter

10. False passages in urethral catheterism

11. Blunt tenotome .

12. Maisonneuve's urethrotome

13. Maisonneuve's modified urethrotome

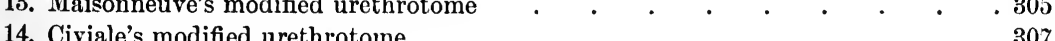

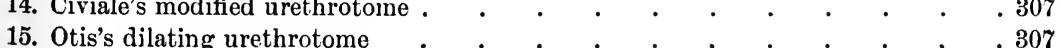

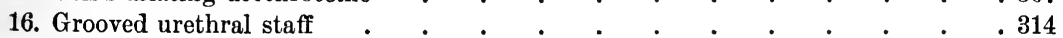

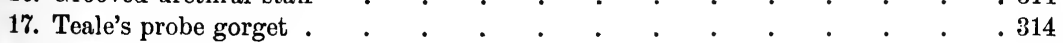

18. Hooked staff

Diagnostic Significance of Pathological Modifications in the Urine.

1. Fermentation saccharometer

\section{Urinary Fever.}

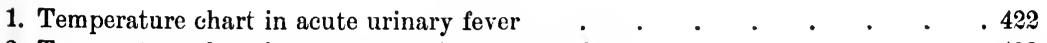

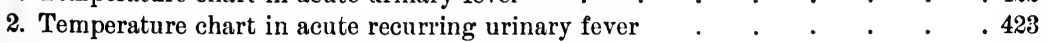

3. Temperature chart in chronic urinary fever . . . . . . . . . 425

4. Temperature chart in non-febrile urinary cachexia . . . . . . . . . 4425

5. Temperature chart in combined renal infection by micrococci and the bacterium

pyrogenes . . . . . . . . . . . . . . 432 


\section{Cystoscopy.}

PAGE

1. Light-conductor for illuminating the internal cavities of the body $\quad$. . . . 435

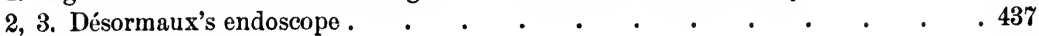

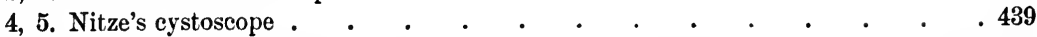

6, 7, 8, 9, 10, 11. The modern Nitze cystoscope and its varieties . . . . 441-443

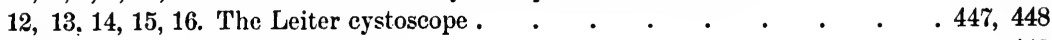

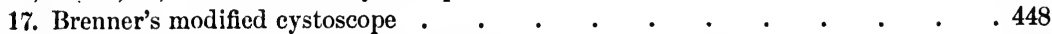

18, 19. Boisseau Du Rocher's modification of the Nitze cystoscope $\quad$. . . . . 450

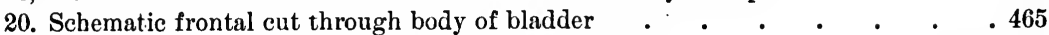

21, 22. Diagrams showing funnel of cystoscope in bladder $\quad . \quad$ • $\quad . \quad$. $\quad .466,467$

$23,24,25$. Diagram illustrating introduction of cystoscope in the bladder . . 468-470

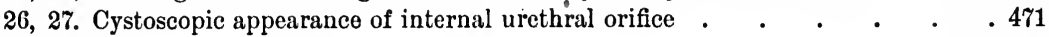

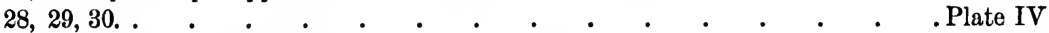

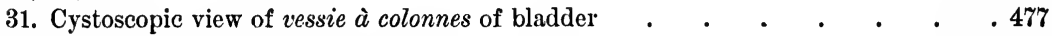

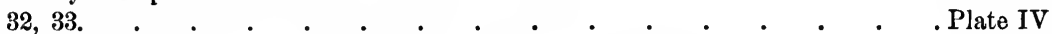

34, 35. Cystoscopic view showing foreign body in the bladder . . . . . . 478

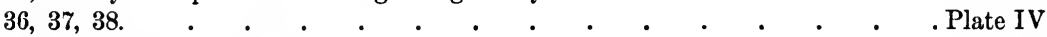

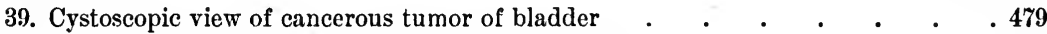

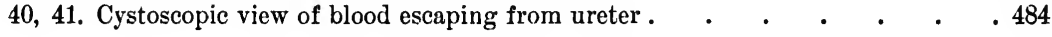

INJURIES AND DISEASES OF THE BLADDER.

1. Mercier's duplicator . . . . . . . . . . . . 530

$2,3,4,5$. Diagrams showing the crossed suture and its method of application in hernia of the bladder . . . . . . . . . . . . . . . 538,539

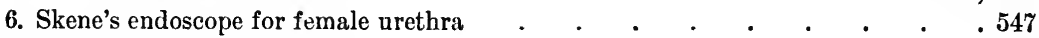

7. Reginald Harrison's trocar.

Rupture of the Bladder.

1. Diagram showing method of placing sutures in rupture of bladder.$\quad .562$

TuMORS OF THE BLADDER.

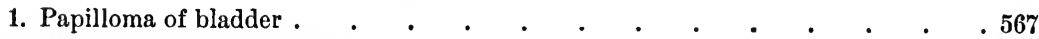

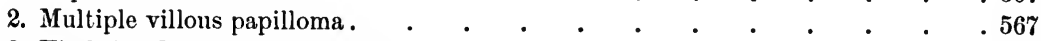

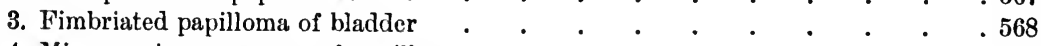

4. Microscopic appearance of papillomatous growth . . . . . . . $\quad$. 569

5. Transitional type of bladder tumor . . . . . . . . . . . 570

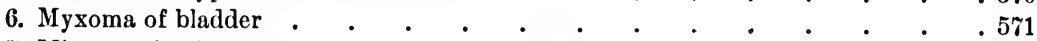

7. Microscopic view of carcinomatous growth of bladder . . . . . . . 572

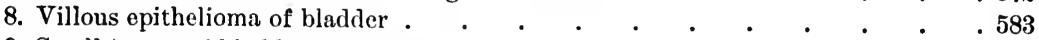

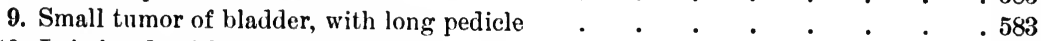

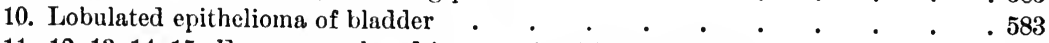

$11,12,13,14,15$. Forceps employed in remoring bladder growths $\quad . \quad$. $\quad . \quad 589$

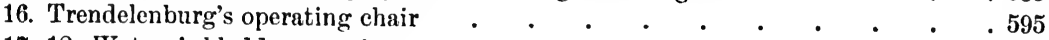

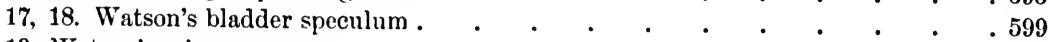

19. Watson's scissors cautery .

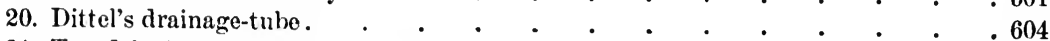

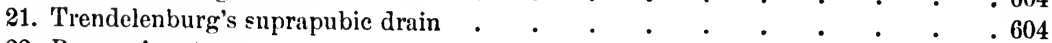

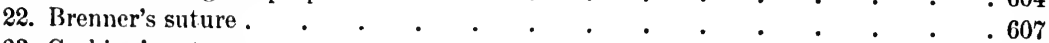

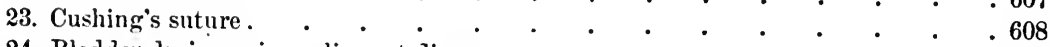

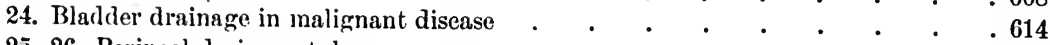

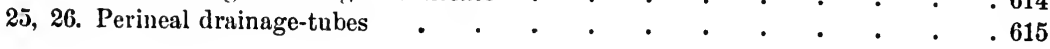




\section{Stone in THE BIADDER.}

FIGURE

1. Stone in bladder having a nucleus of soda, a middle layer of oxalate of lime, and an outside layer of earthy phosphates

2. Uric-acid stone

3. A stone composed of earthy phosphates

4. A stone composed of oxalate of lime and uric acid, showing the manner in which the several nuclei may coalesce and form one stone . $\quad . \quad$. $\quad . \quad$. 625

5. Highly magnified section of oxalate-of-lime stone $\quad$. . . . . . . . . . . 626

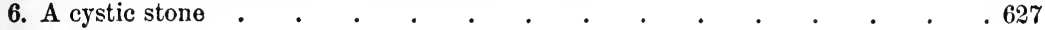

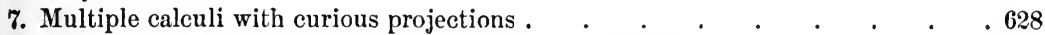

8. Fragments of stone spontaneously fractured in bladder . $\quad . \quad$. $\quad$. $\quad$. $\quad$. $\quad$. 629

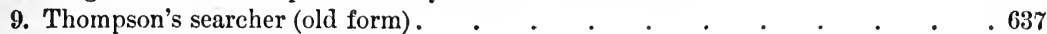

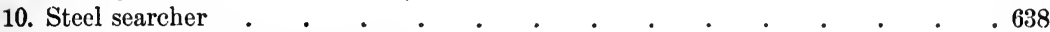

11. Thompson's searcher (latest form) .

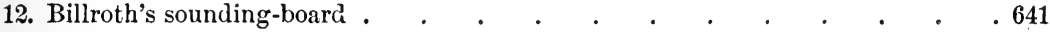

13. Andrew's searcher, with a tube to convey the sound to the ear $\quad . \quad$. $\quad . \quad 642$

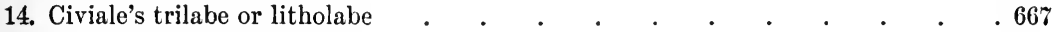

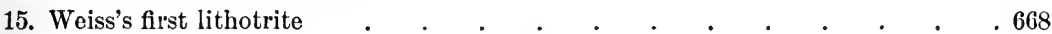

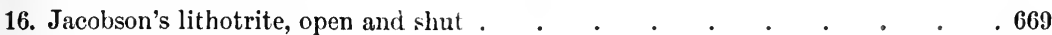

17. Heurteloup's percuteur . . . . . . . . . . . . . . . . 669

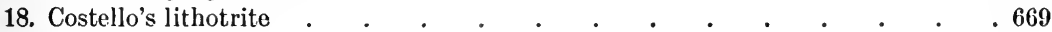

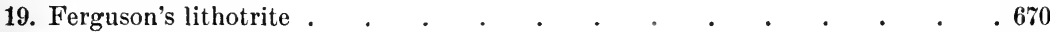

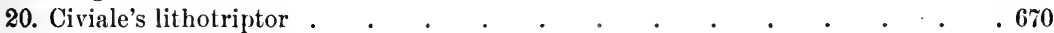

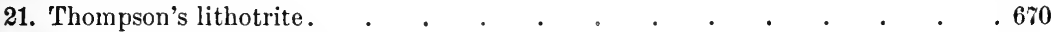

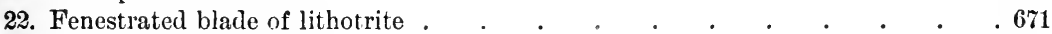

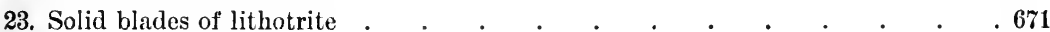

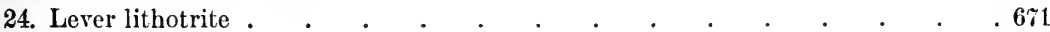

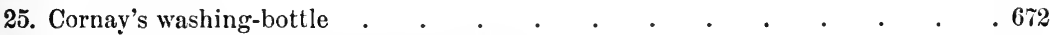

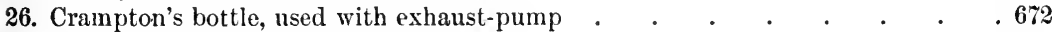

27. Nélaton's evacuating apparatus . . . . . . . . . . . . . . . . 672

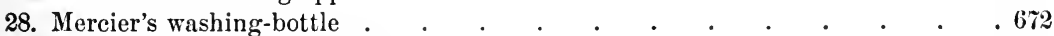

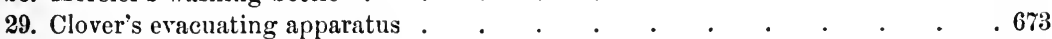

30. Blades of Bigelow's lithotrite, open and shut . . . . . . . . . . . . . . . . 674

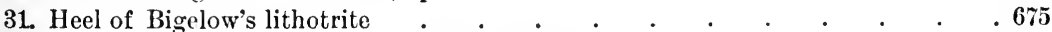

32. Diagrain showing urethra with blade of lithotrite within it . . . . . . . 675

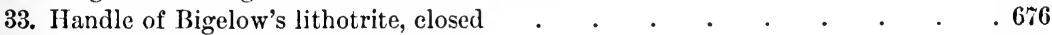

34. Handle of Bigelow's lithotrite, open .

35. Bigelow's fenestrated blades . . . . . . . . . . . . . . . 676

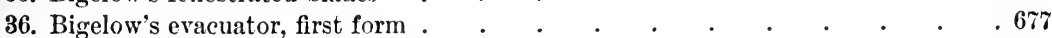

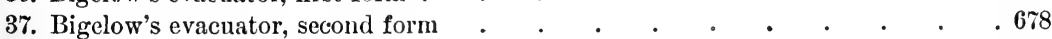

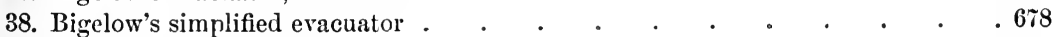

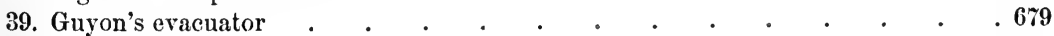

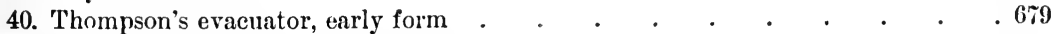

41. Thompson's evacuator, latest form $\quad$.

42. Thompson's evacuator, detail of receiver . $. \quad . \quad . \quad . \quad . \quad . \quad . \quad .680$

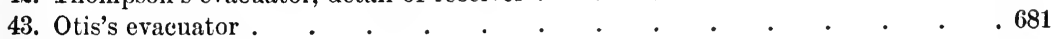

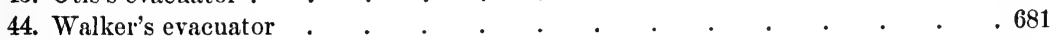

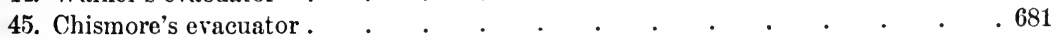

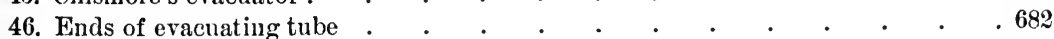

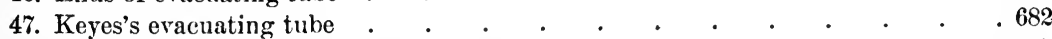

48. Cast of bladder with an instrument depressing the floor . $\quad . \quad$. . . . . . $\quad .685$

49. Diagram showing manner of holding the lithotrite when in search of fragments . 686 


\section{FIQURE}

50. Straight tube with the end turned up to facilitate introduction.$\quad .689$

51. Showing manner of holding the bulb . . . . . 690

52. Chismore's evacuating lithotrite . . . . . 691

53-62. Instruments required in performing lithotomy . . . . . . 703, 704

63. Gouley's lithoclast . . . . . . . . . . . . 705

64. Artery pressure forceps . . . . . . . . 705

65. Chemise cannula $. \quad . \quad . \quad . \quad . \quad . \quad . \quad . \quad . \quad . \quad . \quad .705$

66. Cannula with chemise closed . . . . . . . . . . . . . . . . . 705

67. Browne's air-tampon and cannula . . . . . . . . . . . . . 706

68. Rubber apron to be put under hips . . . . . . . . . . . . 707

69. Bar to hold the legs . . . . . . . . . . . . . 707

70. Patient in lithotomy position with the staff correctly held . . . . . 708

71. Anklets and wristlets. .708

72. Outline sketch of encysted stone . . . . . . . . . . . . 716

73. Dupuytren's double lithotome caché. . . . . . . . . . . 719

74. Dolbeau's dilator . . . . . . . . . . . . . . . . . . 721

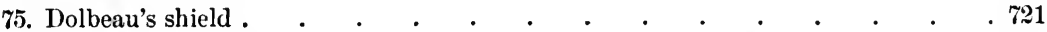

76. Dolbeau's lithoclast . . . . . . . . . . . . . . . 721

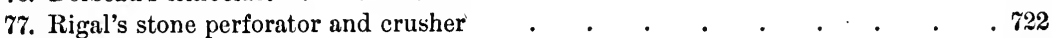

78. Sonde-à-dard . . . . . . . . . . . . 724

$79,80,81,82$. Frozen sections, showing bladder and urethra . . . . . . 725

83. Rubber bag for distention of the rectum . . . . . . . . . . . . . . . . . . . .

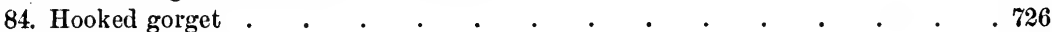

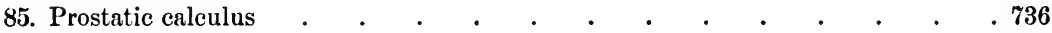

86. Alligator forceps . . . . . . . . . . . . . . . . . . 738

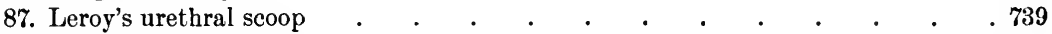

88. Reliquet's urethral stone-crusher . . . . . . . . . . . . . 739

Surgical Diseases of tue Kidney.

1. Section of the kidney with a shot perforation from before backward . . . 753

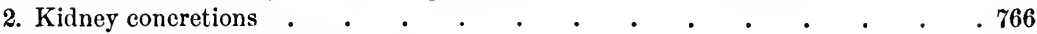

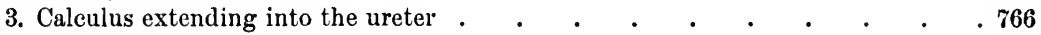

4. Disorganized kidney containing calculi . . . . . . . . . . . 767

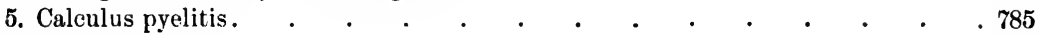

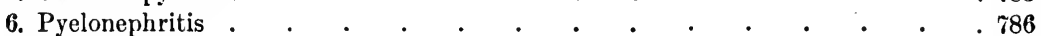

7. Perinephritis . . . . . . . . . . . . . . . . . . . 801

8. Hydronephrosis, first stage . . . . . . . . . . . . 809

9. Hydronephrosis, second stage . . . . . . . . . . . . . . . 510

10. Hydronephrotic right kidney, posterior view . . . . . . . . 812

11. Hydronephrotic right kidney, opened at its outer edge . . . . . . $\quad .813$

12. Isolated eyst of cortex of kidney . . . . . • . . . . . . 817

13. Vesicular cysts of the mucosa of the pelvis and ureter . $\quad . \quad$. $\quad . \quad$. 818

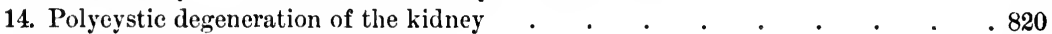

15. Cystic degeneration of the kidney . . . . . . . . . . . . 822

16. Cancer of the kidney . . . . . . . . . . . . . . . . . . . . . .

\section{Hydrocele and Spermatocele.}

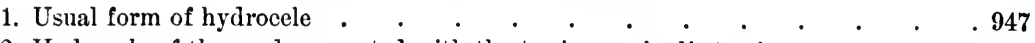

2. Hydrocele of the cord connected with the tunica vaginalis testis . . . . 959

3. Hydrocele of the cord connected with the peritoneal cavity . . . . . . . 959

4. Encysted hydrocele of the cord . . . . . . . . . . . . . 959

5. Iydrocele of the tunica vaginalis testis . . . . . . . . . . . . . . .

6. Bilocular hydrocele . . . . . . . . . . . . . . . . . 
VARICOCELE.

IMGURE

Diagram showing operation for varicocele

. Modified Reverdin straight needle .

Diseases of the Seminal Vesicles.

1. Diagram showing seminal vesicles . . . . . . . . . . . 984

2. Diagram showing prostate; vas deferens; seminal vesicles; bladder; rectum . 993

Functional Disorders of the Male Sexual Organs.

1. Spermatozoa in last drops of urine with alkaline reaction . . . . . $\quad$. 1030

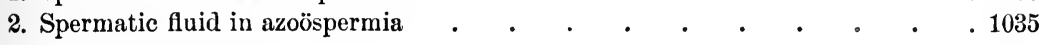





\title{
ANATOMY AND PHYSIOLOGY \\ OF THE GENITO-URINARY ORGANS.
}

\author{
By GEORGE WOOLSEY, M. D.
}

Although a correct knowledge of the anatomy of the genito-urinary organs is essential for accurate diagnosis and intelligent treatment of these parts, it has seemed best to the writer to confine the description in the following pages mainly to those points in the anatomy of the genitourinary organs which may have some practical application in the succeeding chapters ; consequently, it may be noticed that many minute details have been intentionally omitted.

\section{THE KIDNEYS.}

The KIDNEYs are situated deeply, one in either lumbar region, behind or ontside of the peritonæum.

Form.-The form of the kidneys is that of an oval, flattened from before backward, thus presenting flattened convex anterior and posterior surfaces, a convex outer border and a shorter concave inner border, deeply notched in the middle by the hilum, thus giving the kidney its peculiar shape like a bean, and known as reniform. The hilum is placed at the posterior part of the middle of the anterior edge, and therefore the pelvis is best reached along the posterior surface. The upper and lower ends are convex and broader than the middle, and the upper end is broader than the lower. The left kidney is longer and also narrower than the right.

Color.-The color of the kidneys is a brownish red, with deeper purplish shading in the medulla.

Size.-The average kidney measures four and a half inches long, two and a half inches broad, by one and a quarter to one and a half inch thick. The average weight is about four and a half ounces in the male, and somewhat less in the female. The figures as to size and weight are subject to rather wide variations between two healthy subjects, or between the two kidneys of the same subject.

Surfaces.-The anterior surface is convex and flattened, and looks forward, outward, and slightly upward, owing to the fact that the upper 
ends are nearer together. The posterior surface looks in the opposite direction, and is somewhat more flattened by the muscular mass on which it lies. The long convex outer border looks outward, backward, and slightly upward; the shorter concave inner border looks in the opposite

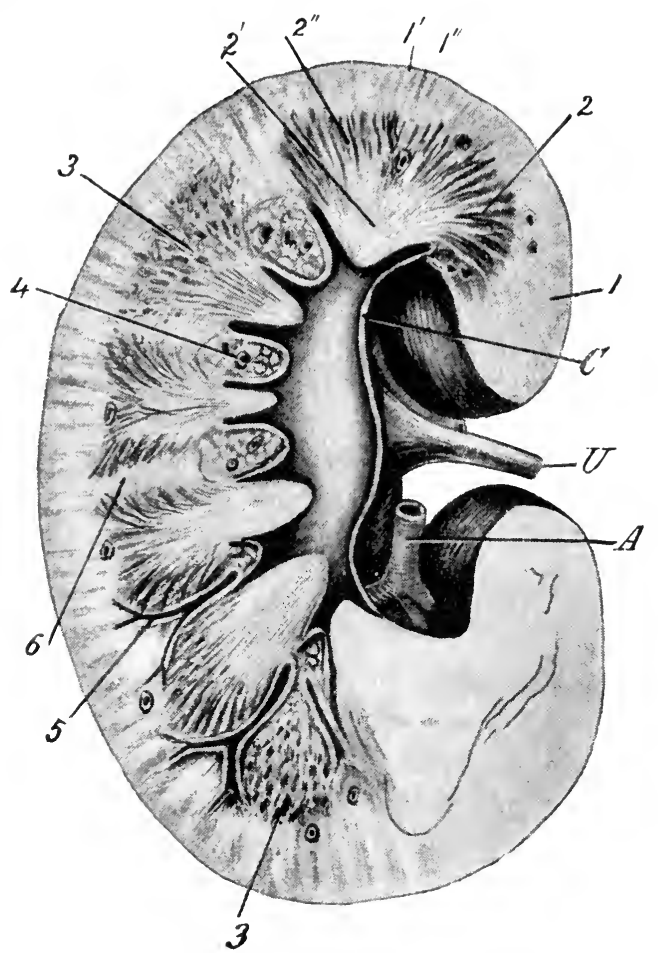

Fig. 1.-Frontal seetion through the kidney, pelvis, and ealyces. $A$, branch of the renal artery; $U$, ureter; $C$, ealyx; 1 , eortex; $2^{\prime}$, medulla; $2^{\prime \prime}$, boundary zone; 4 , fat of sinus of kidney ; 5 , arterial branehes. (Ilenle.)

direction. The anterior border of the left kidney looks slightly more forward than that of the right.

The thicker, wider, rounded upper ends lie nearer one another, as has been mentioned, than the lower by the space of a quarter to half an inch; the upper ends also lie slightly more posteriorly.

Position.-The normal kidneys can not be distinctly felt, but are most accessible at the outer edge of the erector spinæ muscle below the last rib.

As to bony points, the kidney corresponds to the last dorsal and upper two and a half lumbar vertebræ. The upper end lies between the eleventh and twelfth ribs, or between the eleventh and twelfth dorsal vertebræ, and corresponds to the lower part of the tip of the spine of the eleventh dorsal vertebra. 
The lower end is nearly opposite the lower part of the spine of the third lumbar vertebra and the middle of the third lumbar vertebra, or a vertebra higher than the crest of the ilium, above which, or the umbilicus the left kidney lies about two inches, the right kidney about one and a half to one and a quarter inch.

The hilum lies two inches from the middle line and between the first and second lumbar spines. The inner border lies nearly parallel to and about one inch from the spines of the lower two dorsal and upper three lumbar vertebræ. This border inclines slightly outward as it passes downward.

The outer border is two to two and a half inches external to the line of the inner border.

The upper end of the right kidney is lower than that of the left, owing to the position of the liver on the right side, but the lower end of the left

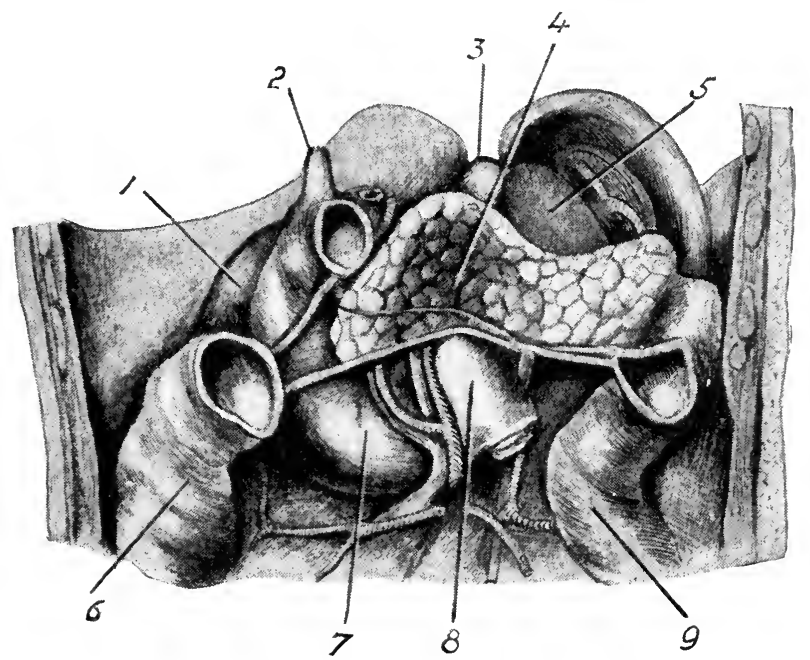

FIG. 2.-Relations of viscera (anterior view). 1, right kidney ; 5 , left kidney ; 2 and 3 , suprarenal capsules; 4 , pancreas; 6 and 9 , ascending and descending colon; 7 , duodenum; 8 , its junction with the jejunum. (Quain.)

kidney may reach as low as its fellow, owing to its greater length; the right, however, is usually lower.

The position of the kidneys under the liver and spleen makes enlargement of those organs cause displacement downward of the kidneys. Thus the hilum may be so lowered as to be opposite the fourth lumbar vertebra.

A pleuritic exudate may cause a similar displacement.

In the upright position the kidneys descend to a distinctly lower position than they occupy in the reclining position, and they descend still 
lower on deep inspiration. These changes affect the right kidney rather more than the left. As the last rib is rudimentary, or is more like a transverse process, so as not to reach the border of the erector spinæ, in a considerable number of cases, it is always well to count the ribs before operating, in order to determine the position of the kidney.

The kidneys are separated from the vertebræ, on either side of which they lie, by the muscles in relation to them.

They lie almost equally on the diaphragm and the quadratus lumborum, the latter covered by the anterior layer of lumbar fascia ; and, to a less extent, on the psoas magnus, which lies more especially in relation to the inner border of the kidney. It slightly overlaps the quadratus lumborum laterally, and here comes in contact with the posterior fascia of the transversalis muscle. The outer border of the quadratus corresponds to the middle of the crest of the ilium, at its attachment to that bone; but, as the muscle passes obliquely upward and inward at a point midway between the last rib and the iliac crest, the outer border of the muscle lies about an inch posterior or internal to a line drawn vertically up from this point of its attachment. On the surface of the body anteriorly, if a line be drawn vertically up from the middle of Poupart's ligament, one third of the kidney lies to the outer side of this line, the remaining two thirds to the inner or median side; and we have already seen that they do not quite reach, inferiorly, a horizontal line drawn through the umbilicus. Posteriorly its position, as indicated above, is one inch from the spines of the vertebræ, and between the spines of the eleventh dorsal and third lumbar vertebræ.

The position of the kidneys between the crest of the ilium and the lower ribs is such, that when the body is bent forwards the kidneys lie where the body is most sharply bent, and in this position they may sometimes be squeezed between the two bony margins.

Misplacements.-One, or, less often, both kidneys may be misplaced, the left more often than the right, and when it is misplaced it is often misshapen. It may be misplaced so as to lie over the sacro-iliac synchondrosis, the promontory of the sacrum, in the iliac fossa or in the pelvis.

Relations.-The kidney is in immediate relation to areolar tissue, called tunica adiposa, which envelops it, and is found most abundantly along the inner and outer borders, and more abundantly posteriorly than anteriorly. As it is largely owing to this investing tunica adiposa that the kidney retains its position, its presence and amount are matters of practical importance.

Thus, when this tissue, at one time abundant, is absorbed, owing to emaciation, a movable kidney is made possible. The amount of this tunica adiposa varies more or less with the fatness or leanness of the indi- 
vidual, and may mislead one as to the size of the kidney. It is through this tissue that perinephritic abscesses extend, and inflammation in it may lead to adhesion with the fibrous capsule of the kidney, making it hard to expose the organ.

The relation of the kidneys to viscera varies more or less on the two sides; the relation to muscles is practically the same on both sides. $A n$ -

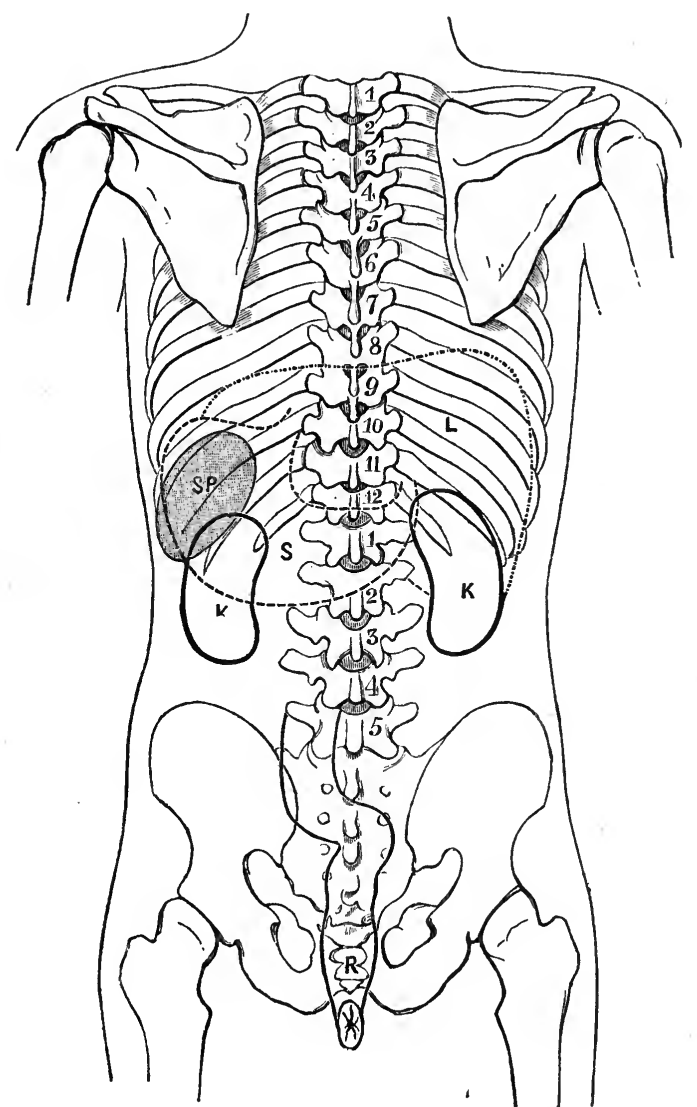

Fig. 3.-Diagram showing relation of the viscera to the parietes (posterior view). S, stomach. $\mathrm{L}$, liver; K, kidney; SP, spleen; R, rectum. (Treves.)

teriorly the right kidney has at its upper part the right lobe of the liver; lower down, and in front of the inner border, the second part of the duodenum; and crossing the lower end obliquely from below upward, and from without inward, is the ascending colon. The colon passes farther upward in front of its inner aspect, where we find the commencement of the transverse colon. 
The left kidney las in front of its upper end the fundus of the stomach separated by the lesser omentum; below this comes the pancreas, and descending vertically along the front of the outer margin is the descending colon. The left kidney has the spleen in contact with the upper two thirds of its outer border; while in many eases the right kidney has the head of the pancreas, embraced by the duodenum, in contact with the inner margin, though this relation is not usually spoken of. The muscles directly behind the kidneys have already been mentioned, in defining the

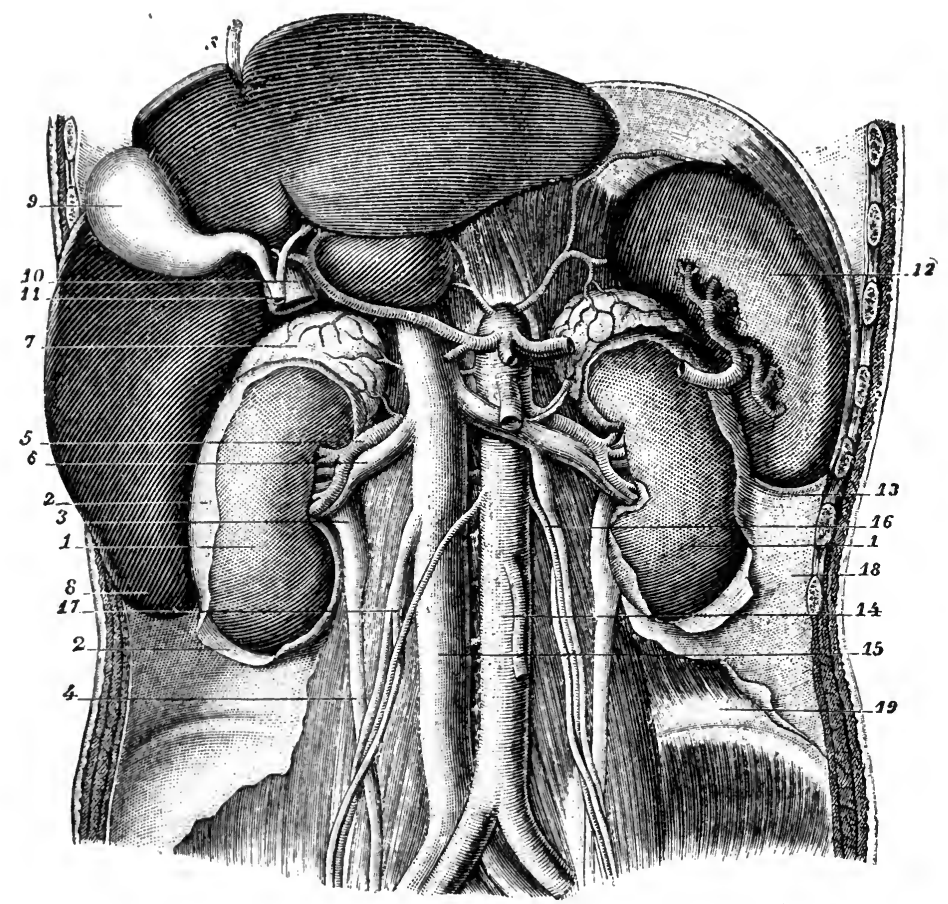

FIG. 4.-Situation, direetion, form, relations of the kidneys. 1, 1, the two kidneys; 2, 2, fibrous eapsule; 3 , pelvis; 4 , ureter; 5 , renal artery ; 6 , renal vein; 7 , suprarenal capsule $; 8,8$, the liver lifted up; 9, gall bladder; 12 , spleen; 14 , abdominal aorta; 15 , inferior vena cava; 16 , left spermatic artery and vein. (Sappey.)

position of the organ. Posterior to the quadratus lumborum, and separated from it by the middle layer of the lumbar fascia, lies the erector spinæ muscle, forming the principal part of the muscular mass on either side of the spine. The outer edge of the erector spinæ lies internal to that of the quadratus lumborum, so that in operating through the lumbar regions the outer margin of both muscles form important landmarks. Internally both kidneys are in relation to the artery, vein, and ureter, which pass in or out at the hilum. 
Relation to Peritonæum.-The peritonæum is in relation only with that portion of the anterior surface of the kidney which is not covered by viscera free of peritonæum. In over fifty per cent of cases the ascending and descending colons have no meso-colon, and are in direct contact with the kidney. Four fifths of the anterior surface of the right kidney and but two thirds of the left are covered by peritonæum; the rest of the surface is separated from peritonæum by the other viscera in contact. The peritonæum is readily stripped from the kidney. The distance between the lateral edge of the quadratus lumborum and the parietal peritonæum external to the kidney is less on the left side by at least one centimeter. The peritonæum helps to hold the kidneys in place, and in cases of movable kidney it is usually more or less stretched, to allow the movement to take place. In the congenital variety, or "floating kidney," there is a complete peritoneal covering and a meso-nephron, the excursion of the kidney being only limited by the length of its pedicle.

Relation to Pleura.-That the lower cul-de-sac of the pleura lies behind the upper end of the kidney, may be inferred from their relative positions; for the lower limit of the pleura normally extends from the lower end of the last dorsal vertebra almost horizontally outward to the lower margin of the eleventh rib, and even with a normally developed twelfth rib the pleura may reach below its lower or inner border. With a rudimentary twelfth rib the pleura extends some distance below the last or eleventh rib, as the line of the pleura is the same as above. The lower end of the diaphragm is indicated, as we reach it by a lumbar incision from behind, by the line of the last dorsal nerve. Besides this nerve, we find, crossing the posterior surface of the kidney obliquely from above downward and outward, branches of the first lumbar artery and the ilio-hypogastric and ilioinguinal nerves.

Structure.-The fibrous capsule of the kidney is a firm, thin, fibroelastic membrane, smooth externally, and on being stripped off from the kidney, which is easily done with a healthy organ, the surface of the kidney and the inner surface of the capsule appear quite smooth, though the two are connected by a delicate reticulum of fibrons tissue and capillary vessels, continuous with the fibrous framework of the kidney. In certain diseases of the kidney accompanied by cirrhosis this reticulum is hypertrophied and the capsule is removed with difficulty, leaving a rough surface. The elastic fibers in the capsule give it the power of adapting itself to the size of the organ, varying according to the vascular tension. Under the capsule is a partial layer of plain muscle-fibers. By splitting the kidney longitudinally from its inner to its onter border, the notch on the inner border, known as the hilum, is found to lead into the substance of the organ, and there expand into an inclosed cavity known as the sinus. 
The capsule, closely investing the tissue of the hilum, here passes in and lines the sinus of the kidney. Here around the bases of the papillæ it becomes continuous with the strong fibrous coat of the calyces. The solid substance of the organ is made up of cortical and medullary substance, the latter composed of from twelve to twenty pyramidal-shaped segments whose apices project into the sinus. They are separated from one another laterally by cortical substances dipping down between, and indicating the development of the kidney from as many segments as there are pyramids. This segmentation shows on the surface in the fœtal kidney, and sometimes persists as a lobulation through life. The cortical substance, besides passing between and separating the pyramids, and here reaching the sinus, as the columnæ Bertini, forms a thin superficial shell from one sixth to one fourth of an inch thick, and lying immediately under the capsule.

The cortex is of a lightish red-brown color, soft in comparison to the medulla. The latter is much darker and purplish, except at the apices,

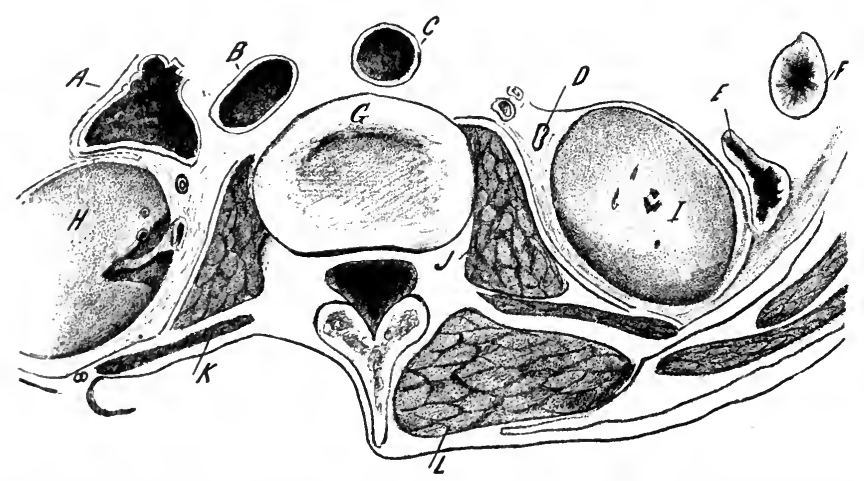

FIg. 5. - Horizontal seetion of body between second and third lumbar vertebra (surface of upper seetion). $I$, right kidney ; $I$, left kidney ; $D$, ureter. (Annals of Surgery.)

where the color is lighter, and it is distinctly striated owing to the diverging but nearly parallel tubules and blood-vessels. At the boundary between cortex and medulla bundles of tubules pass into the cortex, separated from other bundles, by cortical substance, and giving the appearance of rays radiating toward the surface and known as the medullary rays.

Excretory Apparatus. - On tracing up the ureter, we find that it begins to enlarge opposite the lower end of the kidney, and expands into a funnel-shaped dilatation, called the pelvis, which passes into the sinus through the hilum. Within the sinus the pelvis divides into three, or sometimes two, primary branches, which subdivide into a number of short, truncated pouches called calyces. The widened mouths of these calyces receive one or more papillæ. Consequently there are more papillæ than calyces, as 
one calyx may correspond to as many as three papillæ. The calyces are too narrow to admit the finger for exploration. The outer fibrous coat of these calyces is continuous around the bases of the papillæ with the fibrous capsule of the kidney which, as we saw, passed in and lined the sinus. The circular muscular fibers of the ureter, pelvis, and calyces, end as a circular muscle around the base of the papillæ, while the longitudinal fibers are lost near the mouth of the calyx. The epithelium of the internal or mucous coat is reflected over the summit of the papillæ where it becomes continuous with the lining of the uriniferous tubules.

The sinus of the kidney is further occupied by the renal artery and vein and their primary branches, numerous lymphatics, and nerves; all imbedded in a quantity of connective tissue and fat, the fat separating and imbedding the other structures.

These structures, with the pelvis, form the so-called pedicle of the kidney, which assists in holding the kidney in position, and which must be ligated in the operation of nephrectomy.

As the various structures pass in or

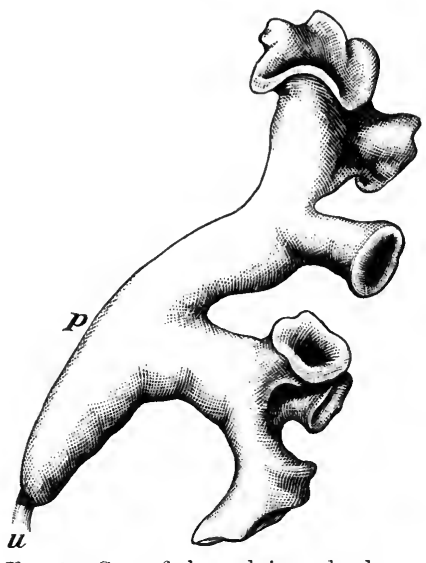

Fig. 6.-Cast of the pelvis and calyees. $u$, ureter; $p$, pelvis. (Henle.) out at the hilum of the kidney they bear the following relations to one another: From before backward, vein, artery, and pelvis lie in the order named. From above downward, the pelvis has the lower position, the artery the upper. The vein is thus situated where it is least exposed to pressure.

Minute Structure.-If a papilla of a fresh kidney is squeezed, a little urine appears from many fine orifices on the surface. These are seen, with the aid of a lens, near the apex of the papilla, and are often found in large numbers at the bottom of a foveola, or shallow depression near the summit; or, more often, there are a number of small foveolæ over the surface.

These minute openings are the mouths of the uriniferous tubules, which, as we trace them toward the cortex, bifurcate repeatedly at acute angles. The number of tubules thus constantly increases toward the bases of the pyramids.

These tubules, known as collecting tubes, pass up through the boundary zone, between medulla and cortex, into the medullary rays, thence laterally into the cortical substance as a junctional tubule, beyond which is the second convoluted tubule, and then the irregular tubule. The tubule 
enters a medullary ray and, after passing down into the medulla, turns in a loop, known as "Henle's looped tubule," and passes up again into a medullary ray and out into the cortex as a spiral tubule, which is followed by the first convoluted tubule. This is separated by a narrowed neck from a dilatation, which is mostly occupied by a vascular, convoluted, capillary tuft, known as a Malpighian tuft. The tubules are lined by epithelium varying in different parts of the tubule. This lining is continued in the dilatation as a flattened layer which covers also the surface of the vascular tuft.

Blood-vessels. - The vessels are very large for the size of the kidneys. The renal artery, as large as the brachial, is given off from the aorta a little below the superior mesenteric artery, and passes nearly horizontally under the vena cava on the right side, to reach the hilum of the kidney, before entering which it divides into four or five branches. These pass in the cellular fatty tissue between the calyces, and, dividing and subdividing, reach the cortical substance on the surface of the sinus between the pyramids. In this cortical substance the arteries pass toward the surface until they reach the boundary zone between the cortex and medulla, where they turn to the side and form partial arches between the cortex and medulla.

From this arch smaller branches pass between the medullary rays toward the surface, and branches of these again break up into the capillary tufts above mentioned. Before joining the complete venous arches between the cortex and medulla these capillaries form an efferent vein, smaller than the afferent artery, as some of the fluid of the blood transudes through the Malpighian tuft. This efferent vein breaks up into a capillary network surrounding the various tubules.

The veins follow much the same course as the arteries, and, collecting into four or five trunks, pass out of the hilum, soon uniting into one, which, passing in front of the aorta on the left side, enters the inferior vena cava, receiving in its course the spermatic vein.

Nerves.-Both cerebro-spinal and sympathetic filaments enter the kidney accompanying the artery. They come from the renal plexus and lesser splanchnic nerve.

Lymphatics are numerous, and comprise a superficial set in or beneath the fibrous capsule of the kidney, communicating with a deeper set which accompany the blood-vessels.

The various structures of the kidney, thus briefly described, are bound together and supported by interstitial connective tissue, continuous by a fine reticulum, with the fibrous capsule as already mentioned.

Irregularities of the Renal Vessels. - This occurs in the arteries in over forty per cent of all cases, and may concern the origin, number, place of entrance into the kidney, branches to neighboring parts, etc. The num- 
ber of arteries is more often increased than decreased, though a common origin of the two from the aorta has been observed. The origin of multiple arteries may be the aorta, suprarenal, lumbar, middle sacral, colica dextra, right hepatic, or one of the iliac arteries.

The usual place of entrance is at the hilum, but they may enter the kidney at either end or on either surface.

The neighboring parts which may receive branches from the kidney are the diaphragm, colon, pancreas, liver (right lobe), or testis.

The veins may also vary in number, places of emergence, etc.

Abnormities.-The two kidneys may be more or less completely fused together. The lowest degree is represented by the horseshoe kidney, where the two are joined at their lower parts across the median line by connective tissue or kidney substance. From this we may have all degrees of union up to complete fusion, along the inner borders, into a disk in the median line, with one or two pelves. Horseshoe kidneys occur once in 1,600 cases, single-fused kidneys once in 8,000. If a horseshoe kidney is joined by connective tissue, it offers no bar to operation. Entire absence of one kidney or extreme atrophy occurs as often as once in 4,000 cases. On the other hand, there may be three kidneys.

\section{PHYSIOLOGY.}

The physiology of the kidney, briefly stated, is as follows: In the Malpighian tufts by a process of filtration are separated most of the water and inorganic salts of the urine. The amount of the filtration depends upon the blood-pressure in the kidney. The urea and like bodies, with more or less water, etc., are derived by a true secreting action of the cells of the convoluted tubules. These separate elements mix in the tubules, and are propelled by the vis a tergo of the drops of fluid behind them. The circular muscle fibers around the bases of the pyramids may by their contraction have a milking action on the urine in the tubules of the pyramids. The drops of urine are collected in the calyces, and pass on to the pelvis and into the ureter.

\section{THE URETERS.}

The expanded pelvis passing out through the lower part of the hilum gradually tapers funnel-like, and becomes cylindrical opposite the lower end of the kidney. Thence, under the name of the ureter, it continues downward to the termination at the base of the bladder. The length is about fourteen to sixteen inches and the caliber about that of a large goose-quill, though often dilated especially at the lower end. The lumen varies; it is narrowest at its lower opening, and in its passage through the bladder wall it is narrower than above. 
Course.-The course of the ureters is downward, converging slightly, to the brim of the pelvis near the sacro-iliac synchondrosis. Thence it passes downward, forward, and inward in the posterior false ligament of the bladder in a curve like that of the posterior pelvic wall, and lying nearly parallel to the lateral edges of the sacrum. It lies behind the peritonæum, and as it approaches the pelvis it is found in the angle between the bodies of the vertebræ and the psoas muscle.

Relations.-In this course the ureter is firmly adherent to the peritonæum at a point near the attachment of the latter to the spine, one half to one inch from this point on the left side, and a little more on the right side, where the ureter is displaced outward by the vena cava.

Consequently, we find in a section between the second and third lumbar vertebræ that the right ureter is four centimetres from the margin of the parietal peritonæum external to the outer border of the kidney; the left is six centimetres distant. The ureter lies on the psoas magnus muscle, crossing it and the genito-crural nerve obliquely, and only loosely connected with the muscle. It is crossed on either side in front by the spermatic or ovarian vessels. On the right side it lies external to and close to the inferior vena cava. Near the brim of the pelvis it commonly crosses on the left side the common iliac vessels behind the sigmoid flexure; on the right side the external iliac vessels behind the termination of the ileum.

In the posterior false ligament of the bladder the ureter lies near the attached margin of this fold and at the side of the fundus of the bladder. It is placed below the line of the obliterated hypogastric artery, and, in the male, the vas deferens arches over it here, between it and the bladder, and thus comes to lie internal to it. It enters the wall of the bladder .about two inches from the opposite ureter, and passes for three quarters of an inch obliquely through the bladder wall, so that the two inner slit-like oblique openings are about one and a quarter to one and a half inch apart, and the same distance from the urethral orifice of the bladder.

In its passage through the bladder wall it lies mostly between the muscular and mucous coats, and the obliquity of its course through the bladder gives it a valve-like action.

In the female, the ureters pass on either side of, and three quarters of an inch from, the uterus and fundus of the vagina to reach the base of the bladder. For the last two inches or more they run in the broad ligament in close relation to the upper part of the vault of the vagina, through which they may be reached.

Structure.-The wall of the ureters is composed of three layers : 1 , an outer fibrous layer containing some elastic fibers; 2 , a middle layer of unstriped muscle-fiber consisting of an internal circular and an external longitudinal layer, with sometimes a circular layer outside the longitudi- 
nal ; 3 , internally a mucous membrane continuous above with the epithelium of the tubules of the kidney below, with that of the bladder, which it closely resembles. A few mucous glands are sometimes found scattered throughout the ureter, pelvis, and calyces, and in some cases small blunt papillæ are found in the lower part of the ureters when they also occur in the bladder. In the passage of the ureters through the muscular layer of the bladder wall its own muscle-fibers become directed in two ways : 1 , transversely to join corresponding fibers of the other ureter : 2 , obliquely downward to the floor of the urethra, where it meets those from the opposite ureter. The structure of the walls of the pelvis and calyces is identical with that of the ureter. The ureters may sometimes be dilated so as to be as large as the small intestine, but this is due to the accumulation of urine in them, and not to reflux from the bladder. Two malformations of the ureters have been found as causes of hydronephrosis : 1 , a kind of congenital valve in the very commencement of the ureter; and, 2, an origin of the ureter from the upper instead of the lower end of the pelvis, so that the pelvis fills before it is emptied, and in the full condition may press upon the valve-like opening of the ureter and close it. three.

As abnormities, there may be but a single ureter, or as many as

\section{PHYSIOLOGY.}

The urine flows through the ureters partly by the action of gravity in the erect position, but more especially by means of the peristaltic contraction of the muscular layer in its walls. These contractions occur somewhat irregularly. Thus, I have seen, through the cystoscope, the end of a contraction as shown by the opening of the slit-like orifice into the bladder as follows: After the first contraction there was another in four to five seconds, and then, after an interval of sixteen to twenty seconds, two more at intervals of four to five seconds, etc. Others have described the contraction as occurring once every three quarters of a minute or so. Reflux is prevented by the oblique passage of the ureters through the bladder wall.

\section{THE URINARY BLADDER.}

The bladder serves as a reservoir for the urine, and has an average capacity of about a pint, or 400 to 500 c. c., but may hold as much as 1,000 c. c. under normal conditions.

In cases of distention due to retention, the bladder has contained as much as 3,000 to 4,000 c. c. of urine, while some contracted bladders have a capacity of no more than 10 to $20 \mathrm{c}$. c. The bladder of the male is somewhat more capacious than that of the female. The different parts of the bladder are designated from their position; thus, the summit 
or apex refers to the upper and more narrow end; the fundus or base to the broader lower end, resting on the perinæum in the male, the vagina in the female.

The term fundus has been limited to that part of the lower half between the orifice and the fold of peritonæum, and not covered by peritonæum. There is no anatomical neck.

Further, we distinguish an anterior surface which looks also downward, a superior surface looking also somewhat backward, an inferior surface or base looking also backward, and two lateral surfaces.

The size, shape, and position of the bladder vary with its degree of distention. The shape of the empty bladder is much disputed, but either

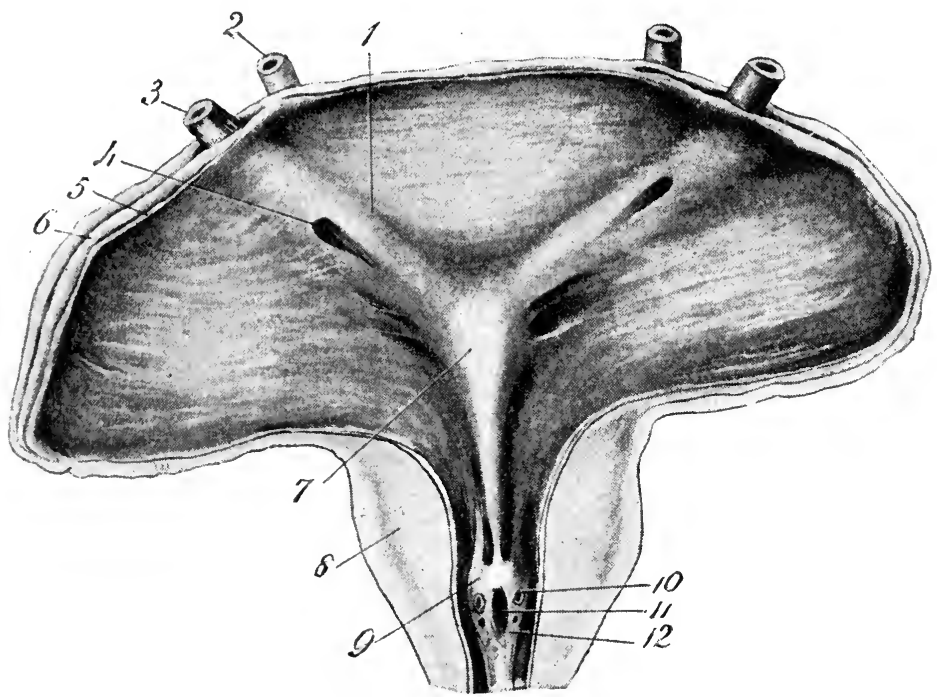

Fig 7.-Lower part of the male bladder, with the beginning of the urethra. Exposed by ineising the anterior wall and laying it open. 3, ureter; 4, opening of the ureter; 2 , vas deferens; 9 , eollieulus seminalis; 7, Bell's musele; 8 , seetion of prostate; 10 , orifice of the common ejaeulatory duet; 11, opening of utriele; 12, mouths of prostatie gland-duets; 1 , Mereier's band. (Henle.)

of two forms may occur: first, oval, like a small lemon, firm, flattened anteriorly, convex superiorly toward the abdomen, and the bladder cavity with the urethra forms a curved slit on sagittal section; second, this is larger and softer, triangular on section, the superior wall is concave toward the abdomen, and fits into the concavity of the inferior wall. On sagittal section the bladder with the urethra forms a $\mathrm{Y}$-shaped figure, the angle of the $\mathrm{Y}$ corresponding to the concavity named. This has been called a diastolic empty bladder, in distinction to the former or systolic one. When moderately distended it has various forms, according to the condition of the surrounding viscera-rounded, flattened, or transversely 
oval-but it still lies within the pelvic cavity. If completely distended it is oval, and its summit lies above the pelvis and against the anterior abdominal wall. The anterior wall is flattened, resting against the symphysis, while the convexity of the posterior wall is increased so that it may lie higher than the apex, which has curved more and more forward. When fully distended the bladder is often flattened from above down-

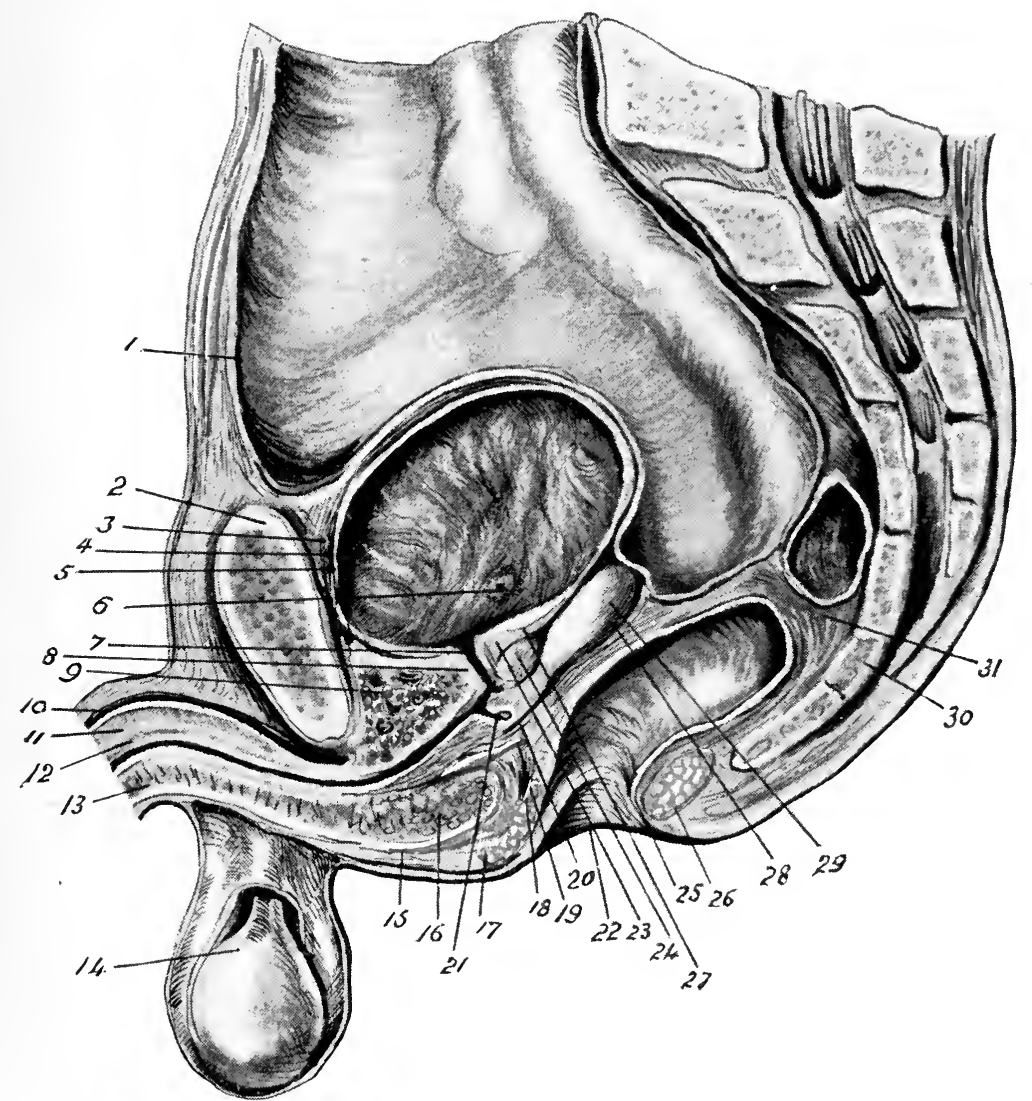

FIG. 8.-Median section of a frozen male subject. The small intestine is removed, 1, peritonæum; 6 , opening of the ureters; 8 , internal sphineter vesiea; 9, external sphineter, with the compressor urethre nuscle; 10 , dorsal vein of the penis; 15 , bulbo-cavernosus musele; 16 , bulb of the urethra; 17 , sphineter ani ; 21, utriele; 24, "midule portion" of prostate; 29, seminal vesieles. (Henle.)

ward, and bulged laterally. The base depresses the perinæum. When completely distended the bladder does not lie in the median line, but deviates slightly to the right, dne partly to the rectum on the left side, and partly to the greater size of the right half of the bladder.

The distended bladder may even reach as high as the diaphragm. 
Position.-In the erect position the orifice of the bladder is the most dependent part, and lies in a horizontal line drawn throngh a point a little below the middle of the symphysis pubis, and it lies one and a quarter inch behind the latter. The long axis of the distended bladder is directed nearly horizontally from the coccyx to the upper margin of the symphysis. It is within the pelvie cavity, except when distended. It lies on the rectum or vagina below, against the anterior pelvic wall in front, and in contact with the rectum, small intestines, and uterus behind:

Relations. - The base of the bladder rests on the front of the second part of the rectum, separated from it and at the same time connected with it by the recto-vesical fascia. That part of the bladder firmly connected with the rectum corresponds to the trigonum on the internal surface, and here the bladder may be punctured from the rectum, about one and a half inch above the angle in the anterior wall of the latter. Above the base of the trigonum the bladder and rectum are separated by the rectovesical fold of the peritonæum. In the female the bladder and rectum are separated by the vagina and cervix uteri, the two latter having the same relation to the bladder as does the rectum in the male. Lying between the rectum and bladder in the male, bounding the sides of the triangular space where the two are firmly bound together, and converging toward the orifice of the bladder, are the seminal vesicles, with the vasa deferentia internal to them. These are held to the inferior surface of the bladder by the process of recto-vesical fascia separating bladder and rectum. Behind the base of the attached area of the bladder the vasa deferentia curve upward, and then forward and outward, on to the lateral surfaces of the bladder, passing between the bladder wall and the lower ends of the ureters, which enter the bladder just behind the posterior angles of this attached area. The base of the bladder further rests on the anterior part of the pelvic floor, or levator ani muscle, forming the upper or abdominal end of the "perineal body." This is depressed in the distention of the bladder, and is therefore held up by the rectal bag in suprapubic operations.

The antero-inferior or pubic surface is separated from the symphysis and body of the pubes, the pelvic fascia, and, during distention, from the anterior abdominal wall, by loose areolar tissue, at the bottom of which, near the bladder, is often found a network of veins.

Relation to Peritonæum.-The peritonæum covers the whole of the superior surface from the apex down. It covers the lateral surfaces as low as the line of the obliterated hypogastric arteries, thus covering a part of the vas deferens lying on this surface. It is prolonged on to the base of the bladder as far as the upper end of the seminal vesicles in the male, and the base of the trigonum in both sexes; thence it passes on to the rectum in the male and the nterus in the female, forming the recto- 
resical and utero-vesical pouches, respectively. This pouch separates the bladder from the rectum in the male and the uterus in the female, and is usually filled with convolutions of the smaller intestine. The lower end of this pouch in the male is about one inch from the base of the prostate or three inches from the anus; in the female the fundus is free of peritonæum higher up, so that the utero-vesical pouch is not so deep as the recto-vesical. Normally the peritonæum lines the anterior abdominal wall down as far as the upper end of the symphysis pubis, from which it passes onto the summit and superior surface of the bladder. In

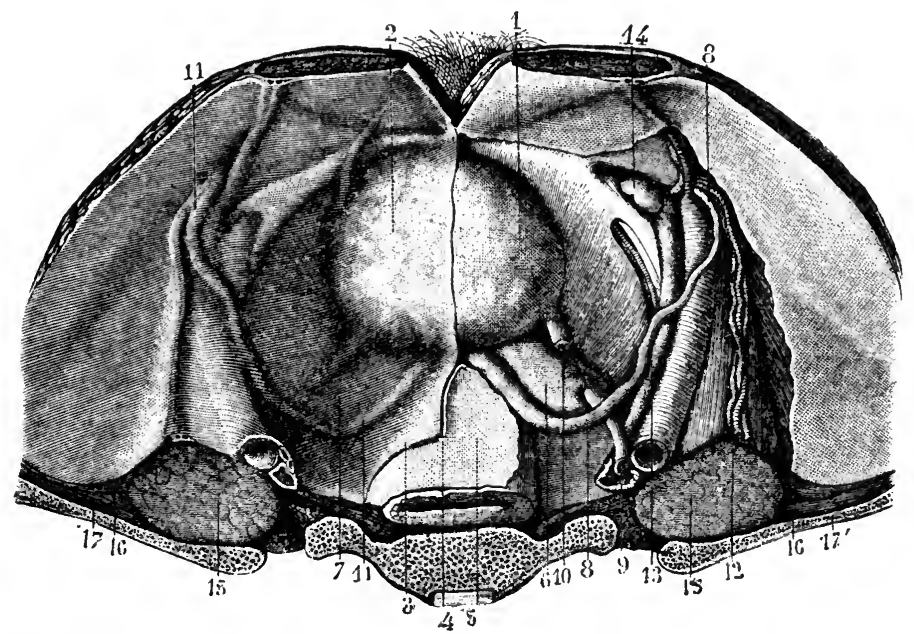

Fig. 9.-Relations of the posterior and inferior regions of the bladder in man. 1, right half of posterior surface of bladder; 2 , left half covered with peritonæum; 3 , semicireular fold which this membrane forms when empty; 4 , median section of this fold; 6 , right seminal vesiele; 8 , seminal duet; 9 , ureter; 11,11 , left ureter, eovered by peritonæum; 12, spermatic vein and artery; 13, external iliac artery and vein. (Sappey.)

distention of the bladder, as its apex rises above the symphysis, in apposition with the anterior abdominal wall, the peritonæum is raised from the symphysis and forms a cul-de-sac between the upper end of the antero-inferior bladder wall and the wall of the abdomen. As the bladder rises into the abdomen the lower half of the distance between the apex of the bladder and the symphysis pubis is free of peritonæum. Thus, if the apex be half-way between the pubes and the umbilicus, the lower limit of the peritonæum is at least two inches above the symphysis. It is through this space, between the symphysis pubis and the lower end of the peritoneal cul-de-sac, that puncture or suprapubic incision of the bladder is performed, without wounding the peritonæum. The bladder wall proper is here separated from the abdominal wall by loose areolar tissue throughout a triangular space, with the base downward (cavum Retzii). 
In this areolar tissue, inflammation, suppuration, or extravasation readily spreads. In some cases the peritonæum is found firmly attached to the symphysis pubis at its upper border, or even lower, and when this is the case it would be incised by a suprapubic incision, unless first dissected off and raised.

The female bladder, as compared with that of the male, is less capacions. Its neck lies a little nearer the symphysis, on a line with the lower border of the symphysis, and is very distensible, as there is no prostate. It is nearly as broad as it is long, and the peritonæum does not come down as far on to the base, which is of less extent.

The opening of the ureters is only three centimetres from the cervix uteri.

The bladder in the child lies largely above the pelvis in the abdominal cavity, and yet its anterior surface is uncovered by peritonæum, so that it is easily accessible in front. It is pyriform or egg-shaped, the vertical axis being greater than in the adult, and it has but little fundus. At birth the urethral orifice lies on a level with the upper end of the symphysis, and the peritonæum reaches the urethral orifice postero-inferiorly, the prostate being minute. The bladder wall is exceedingly thin.

Connections or Fastenings of the Bladder.-The bladder, though fairly firmly fixed, is found in inguinal, femoral, and vaginal herniæ. Above it is freely movable, but it is held in place below by ligaments of two kinds, true and false, and by its connections to neighboring parts. Thus it is fixed to the walls of the pelvis by the prostate, it is strongly joined by connective tissue to the rectum or vagina, and is less strongly held by the ureters, urachus, obliterated hypogastric arteries, and by numerous blood-vessels.

The false ligaments are folds of peritonæum as it is reflected from the bladder on to neighboring parts. Five folds or ligaments are found. One is median from the summit of the bladder to the umbilicus, triangular in outline, the sides of the triangle representing the obliterated hypogastric arteries. The course of the urachus, contained in this fold, bisects the triangle in passing to its apex. Posteriorly on either side a fold passes from the sides of the rectum, or uterus, and pelvic wall to the sides of the back of the bladder, known as the posterior false ligaments, in the bases of which lie the ureters. Between the two posterior false ligaments lies the recto or utero-vesical cul-de-sac.

The lateral fulse ligaments pass from the lateral pelvic wall to the sides of the bladder, but are not distinctly marked off from the posterior ligaments except by the line of the obliterated hypogastric artery. Under the fold of the lateral false ligaments pass the vas deferens and the vesical artery.

The true ligaments consist of reflections of pelvic fascia. The reflec- 
tion on either side, passing to the side of the base of the bladder, is known as the lateral true ligament. The anterior true ligament, also known as the pubo-prostatic, passes from the back of the pubes at the level of the lower border of the symphysis pubis to the front and sides of the bladder just above the prostate, which the ligament embraces. Between these two

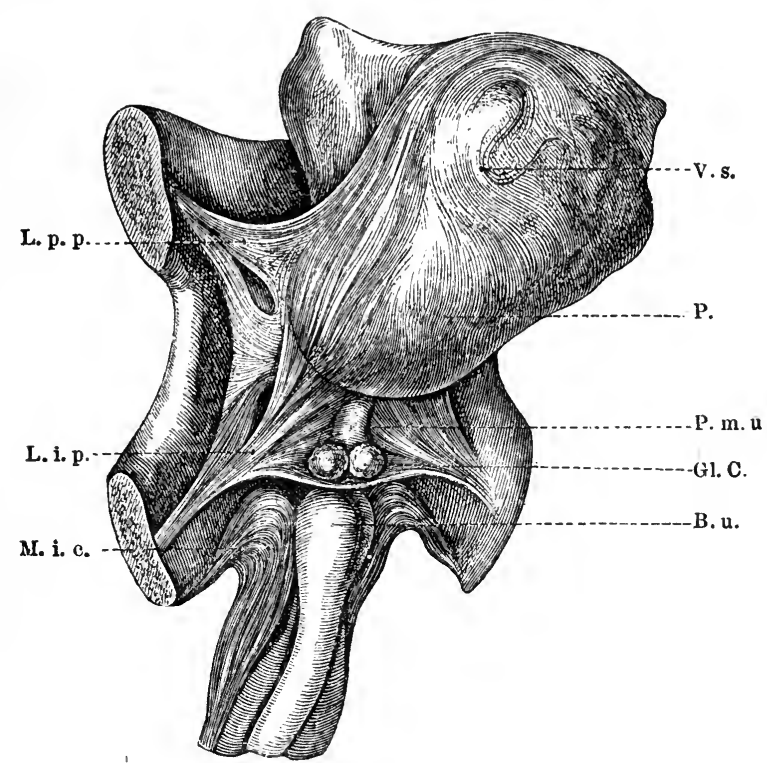

Fig. 10.-L.p.p., pubo-prostatic ligament; L.i.p., ischio-prostatic ligament; M. i. c., ischio-cavernosus muscle; V.s., seminal vesicles; P., prostate; P.m. u., membranous urethra; Gl. C., Cowper's glands; B. u., bulb of the urethra. (Dittel.)

anterior ligaments is a depression filled with loose connective tissue and fat, at the bottom of which lies a plexus of veins, in front of the prostate, connected with the dorsal vein of the penis.

The urachus itself might be considered a true ligament, as it consists of fibrous tissue with muscle-fibers prolonged from the bladder on to its lower part.

The fotal tubular condition of the urachus may persist, usually in the form of an interrupted cavity lined by an epithelium like that of the bladder. This may open at the umbilicus, and discharge a mucous secretion, or, in rare cases, urine may pass through the whole length and escape at the umbilicus.

Interior of the Bladder.-On opening the bladder, we find its interior lined by a smooth mucous membrane loosely connected with the other coats and thus thrown into rugæ when the viscus is empty. Furthermore, in some cases a network of muscular bands projects slightly into the blad- 
der, the mucous membrane dipping down into the meshes of the network and forming the sacculated bladder. Corresponding to these saccules the bladder wall is thin, so that it may give way, forming one or more larger sacculi, in which important pathological changes may occur. At the base of the bladder there is a triangular area, the trigonum, where the mucous membrane shows no rugæ, as it is closely adherent to the parts beneath. This triangular area corresponds to that part of the base which is firmly adherent to the anterior rectal wall, and which is bounded on the sides externally by the vesiculæ seminales. The trigonum is very small in young subjects. Internally the three openings into the bladder are found at the angles of this trigonum. At the apex in front is the orifice

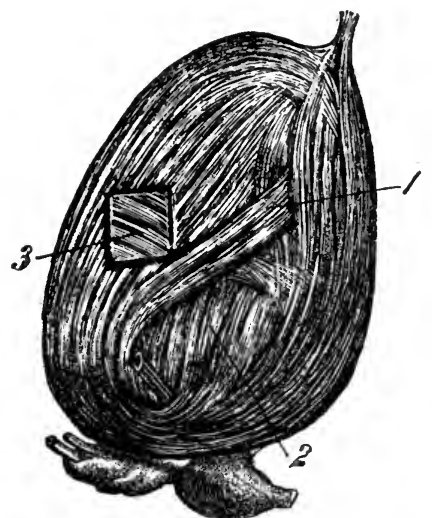

Fig. 11.-1,2, lateral oblique and straight fibers; 3 , middle eireular fibers. into the urethra, and at the angles of the base we have the oblique slits of the openings of the ureters, about one and a quarter to one and a half inch apart, and the same distance from the orifice.

Joining the openings of the ureters is a transverse curved ridge, and passing from the opening of both ureters is another slight ridge, which ends in a minute median projection, on the base of the bladder at the orifice, known as the uvula vesica, often giving the orifice a semicircular shape on cross-section.

The ridges mentioned above are due to the prolongation of the muscle-fibers of the wall of the ureters, and are known, the posterior as Mercier's bar, the anterior pair as Bell's muscle. Behind Mercier's bar there may be found a depressed pouch, especially in old age. The trigonum, more often in old age, may be elevated quite a little above the surrounding surface of the bladder.

Structure.-The partial covering of the peritonæum has already been sufficiently described. The outer of the three remaining coats is the muscular, composed of unstriped muscular fibers arranged in three layers more or less well marked. The outer or longitudinal layer is found especially on the superior and antero-inferior surfaces, passing from the "neck" up toward the summit, where some fibers are continued into the urachus, while at the lower end they are continued into the prostate or vagina, and, through the anterior true ligaments, to the anterior pelvic wall (musculi pubovesicales). Laterally these fibers are more or less oblique. The anterior wall is the thickest, owing to the thickness of its muscular coat, and is especially prone to hypertrophy. In the next deeper layer the fibers are more or less circular, especially in the lower part, where 
they are somewhat aggregated, so as to be formerly called the sphincter vesica. They do not act as a sphincter, however, but merely to expel the last drops of urine, as shown by Henle and others. The most internal fibers form an incomplete layer of obliquely arranged reticulating fibers, which give the reticulated appearance to the interior of the bladder, especially when they are hypertrophied.

Internal to the muscular coat is a well-marked layer of areolar tissue, mixed with elastic fibers. This coat binds the muscular and mucous coats loosely together, more intimately connected with the latter, and allowing it to move freely on the former and to be thrown into rugæ when the bladder is empty. In the trigonum the muscular and mucous coats are closely bound together.

The mucous membrane lining the bladder is smooth, pinkish, and lax, to accommodate itself to the varying size of the bladder. It is lined by stratified epithelium of about three layers of cells, whose form varies with the fullness of the bladder, being flattened when the bladder is full, more or less cubical and oval when it is empty, and forming but a single layer when it is largely distended. Over the trigonum the mucous membrane does not stretch, so that the epithelium here does not vary in form. In the neighborhood of the orifice and fundus numerous very small racemose mucous glands may be found, and in some bladders one finds closely gathered, small, blunt papillæ, as well as in the lower end of the ureters, which probably stand in relation to the later appearance of papilloma.

Blood-vessels. - The arteries are the superior and inferior vesical from the internal iliac, the former through the pervions part of the hypogastric. The uterine arteries in the female also send branches to the bladder. The base-which includes the trigonum-and the "neck" are the most vascular portions. The veins, forming a plexus around the base and neck of the bladder, where they communicate with the prostatic plexus, empty finally into the internal iliac veins. The venous plexus may form varicosities, which project into the interior of the bladder at its lower part.

Nerves.-The nerves come partly from the hypogastric plexus of the sympathetic and partly from the third and fourth sacral nerves, branches of the cerebro-spinal system. The mucous membrane of the trigonum and that lining the neck and lower part of the fundus of the bladder are very sensitive, being well supplied with sensory nerves, which are scanty elsewhere. The sensitiveness of the above-named parts is well seen when diseased.

Development.-The bladder, as well as the prostatic and membranous portions of the urethra in the male and the entire urethra in the female, is developed from the lower part of the intra-abdominal portion of the allantoic vesicle, the upper part of the same forming the urachus. In 
early fœtal development the bladder vesicle connects with the cloaca through that part which afterward forms the trigonum, and this opening may persist between bladder and rectum in cases of mal-development.

\section{THE PROSTATE GLAND.}

Although its very existence has been questioned, and clearly defined marks are wanting to indicate with precision its anatomical boundaries in all directions, yet it is enough of an entity, anatomically and pathologically, to deserve a description as such. It is a firm, partly glandular, partly muscular body, containing the upper portion of the urethra and adjoining the "neck" of the bladder. The shape is that of a short, truncated cone, compressed in front and behind, or like that of a chestnut, the base projecting farther upward behind than in front. The surfaces are anterior and posterior, and both are convex and flattened, the anterior surface looking also somewhat upward and the posterior downward. The

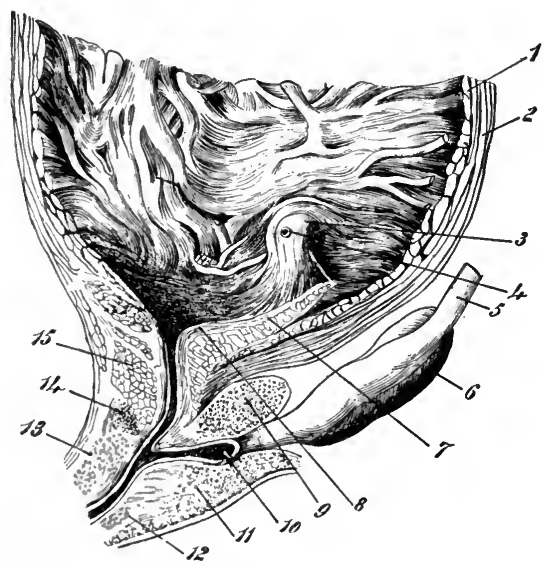

Fig. 12.-Median section of the lower part of the bladder, with the prostate, and beginning of the urethra. 1, inner; 2, outer layer of museletibers; 3 , musele-fibers of ureter; 4 , opening of ureters; 7,12 , sphineter vesiea internus and sphincter prostatie externus ; 10 , utriele; 5 , vas deferens; 6 , vesieulie seminales. anterior or pubic surface is described as having a median longitudinal furrow corresponding to the course of the urethra; but this is not usually present here, though it does exist on the posterior or rectal surface as a shallow groove. Further, the course of the ejaculatory ducts is often indieated at the upper part of this surface by two slight lines. The base, looking upward and slightly backward, shows a funnel-shaped depression for the neck of the bladder, at the bottom of which is the urethral orifice, one quarter to one third nearer the anterior than the posterior sur. face. One quarter to one third of an inch farther back is another funnel-shaped depression, where the ejaculatory ducts penetrate the "middle portion." The apex is somewhat flattened as it rests on the inner layer of the deep perineal fascia. The lateral borders are prominent and
rounded.

Size. - The transverse diameter is one fifth to one sixth greater than the antero-posterior, averaging one and three quarters inch, while the diameter from base to apex measures one and a quarter to one and a 
half inch, and the thickness is but three quarters of an inch. The greatest distance between the prostatic urethra and the capsule of the gland in an average normal prostate is seven eighths of an inch. This is the measurement in the oblique diameter, outward and backward, which is the direction of the incision in lateral lithotomy.

The average normal wEIGHT is between four and a half to four and three quarters drachms, though both the size and weight may be very much increased in hypertrophy of the prostate.

Position.- The prostate is situated between the "neck" of the bladder above and the upper layer of the triangular ligament below. It rests on the rectum behind, and in front it lies just behind and below the lower border of the symphysis pubis, half an inch intervening in the erect position.

Relations and Connections.-The prostate is quite firmly fixed in the pelvis to neighboring parts, thongh it yields somewhat to a full bladder or rectum. It is joined to the bladder by a direct continuity of the longitudinal and circular fibers of that viscus, but especially of the circular fibers.

The passage through it of the urethra also fixes it to the bladder above and the deep layer of the perineal fascia below. It rests on the anterior wall of the rectum at the lower part of its middle portion, just where it bends to end in the third or anal portion, and at this point, the bend in the anterior rectal wall, it is easily palpated by the finger in the rectum. It is connected with and separated from the rectum by its outer capsular covering of recto-vesical fascia. Above and behind the common ejaculatory ducts enter the posterior part of the base of the prostate.

The sides of the prostate are embraced by the anterior true ligaments of the bladder, or pubo-prostatic ligaments, as they pass back from the pubes to the sides and front of the "neck" of the bladder. They leave between them a cellular interval, at the bottom of which lie the apex of the prostate and a part of the prostatic plexus of veins. Farther below and behind the most anterior part of the levator ani, known as the levatores prostatæ, passes downward and backward, the most anterior fibers, proceeding beneath the apex to the central tendinous point of the perinæum. 'The more posterior fibers are attached along the lateral borders of the prostate. The onter capsule of the prostate by its connections also serves to hold the gland in position. In CHILdRen the prostate is quite minute, and is more vertical, rounded, and softer than in adults, and it does not increase much in size until after puberty.

Structure.-The prostate has a donble sheath or capsule. The external is firm and fibrous, and consists of a reflection of recto-vesical fascia, which is continuous with the deep layer of the deep perineal fascia at the apex of the gland. The inner or proper capsule, though thin, is firm, and 
is not continuous with any other fascia, but is composed of muscular, connective, and elastic-tissue fibers continuous with that in the

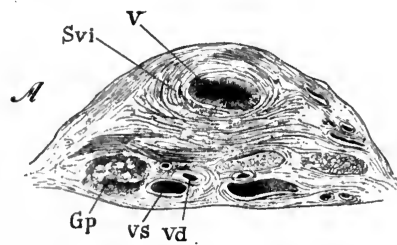
stroma of the gland. It can not be readily removed from the gland. Between these two coverings is situated the prostatic plexus of veins, continuous with the dorsal vein of the penis, and especially developed at the sides of the prostate.

Lobes. - The prostate is usually de-
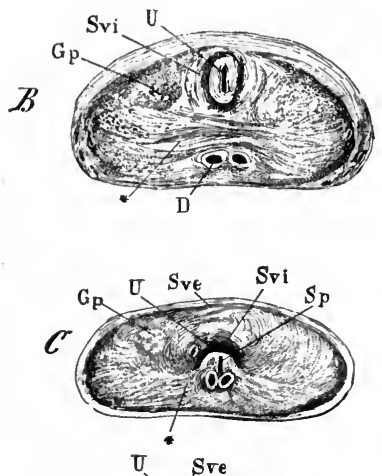
scribed as composed of three lobes. Up to the fifth month of fotal life the prostate consists of two laterally placed lobes. These two lobes are joined in the median line along their anterior edges by the so-called anterior commissure, which is narrow, and contains no glandular tissue.

Posteriorly the two lobes are similarly joined below by the posterior commissure, but above the two lobes diverge behind, leaving a pyramidalshaped space behind the upper end of the urethra and "neck" of the bladder, which is filled by tissue rich in glands. This tissue represents the socalled third or middle lobe, and, though it is subject to pathological changes by itself, its anatomical features do not justify the term lobe, but it is better to call it the "middle portion," as do the French.

This rounded pyramidal " middle portion" is penetrated by the two common ejaculatory ducts. It lies behind the verumontanum, and it is prone to hypertrophy.

When the so-called middle lobe is present it is a morbid growth, causing obstruction to micturition, and is seen projecting between the openings of the urethra and ejaculatory ducts.

The course of the urethra through the prostate is from the base a little anterior to its middle, to the apex. In its course from base to apex, 
the distance of the urethra from the anterior and posterior surfaces is about equal, though it is generally described as lying nearer the posterior surface, and it sometimes does so to a slight extent. Very rarely the urethra grooves the surface of the prostate instead of tunneling through it.

The course of the common ejaculatory ducts is downward and forward for the distance of half an inch. They enter the base of the prostate in the "middle portion" in a funnel-shaped depression one quarter to one third of an inch posterior to the urethral opening; they then pass side by side, separated by a thin stratum of tissue, until they reach the prostatic utricle in the walls of which they lie, and open one on either side of the opening of the utricle in the floor of the prostatic urethra.

The Muscular Tissue of the Prostate.--Just external to the mucous membrane is a thin layer of longitudinal pale muscle-fibers, continuous above with the fibers of the innermost layer of the bladder, and continued more or less throughout the entire urethra. External to these fibers is a thicker but unevenly distributed layer of circular fibers continuous above with the circular fibers of the bladder, and continued below as far as the bulbous portion of the urethra. These circular fibers, surrounding the urethra, are grouped together at the base of the prostate and here form the internal sphincter of the bladder or prostate. In the middle of the prostate they form a thinner layer, but are again somewhat aggregated toward the apex of the gland, where they are mingled with striped muscle-

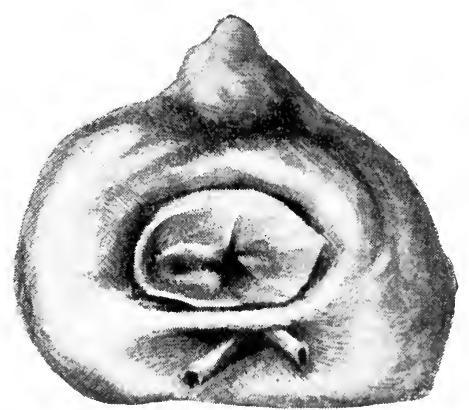

FIG. 14.-A healthy prostate from a man aged thirty-five years, with its posterior or rectal surface downward-the internal meatus being seen above, and the ejaculatory ducts in their depression below. (Thompson.) fibers to form what is sometimes called the external sphincter of the prostate. The striped muscle-fibers of this "sphincter" are first found as we pass from above downward, only in front of the urethra, lying transversely; then in a crescent in front, and still lower down, they encircle the urethra. Both the circular and longitudinal fibers contain elastic and connective-tissue fibers. Throughout the rest of the prostate musele-fibers occur abundantly, mixed with fibrous and elastic tissue to form the stroma of the gland, supporting the glandular elements and continuous with the muscle-fibers in the proper sheath of the gland. These fibers radiate more or less from the line of the urethra. The glandular portion of the prostate occurs most abundantly in the lateral lobes and in the portion behind the urethra, especially the "middle purtion." The glands resemble, though not exactly, racemose 
glands. They appear yellowish on section, and occur in "lobules," forty or fifty in number. Their ducts open on the floor of the prostatic urethra, especially in the sinus prostaticus, by twenty to twenty-four openings. Concretions are very commonly found in the prostatic glands.

Vessels and Nerves. - The prostate is supplied by branches of the inferior vesical, together with smaller branches from the internal pudic and middle hæmorrhoidal arteries. The accessory pudic artery, when present, passes along the anterior surface of the prostate, before perforating the deep layer of the triangular ligament, and gives branches to the gland. The prostatic plexus of veins, communicating with those at the base of the bladder, the hæmorrhoidal and the dorsal vein of the penis, empties into the internal iliac vein. Phleboliths occur frequently in these veins. The lymphatics accompany the veins.

The nerves are derived from the prostatic plexus, a continuation downward of the hypogastric plexus, and comprise both medullated and nonmedullated fibers. The nerves to the penis send filament to the prostate in passing.

\section{THE PENIS.}

The penis is composed of three cylindrical masses of erectile tissue, the two corpora cavernosa and the corpus spongiosum, joined together by fibrous tissue into a three-sided body, which is covered with skin and contains the spongy portion of the urethra. It is attached at its root to the symphysis pubis, pubic arch, and triangular ligament of the perinæum

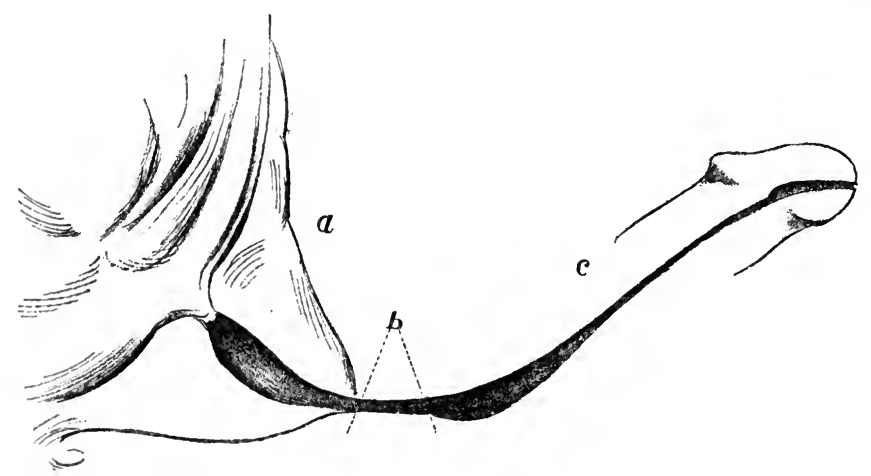

FiG. 15.-Diagram of urethra in natural condition. $a, b, c$, representing the prostatic membranous and spongy portions respectively. (Thompson.)

by means of the suspensory ligament, the crura of the corpora cavernosa, and the bulb of the corpus spongiosum, respectively. In front it ends in a more or less conical expansion, the glans penis, continuous with the corpus spongiosum. 
The body of the penis has three sides and three rounded borders. The glans is conical, flattened from above downward, and has a longer dorsal than ventral surface. It has, a little below the center of its rounded extremity, a vertical slit, the meatus or external orifice of the urethra, from the lower end of which passes a narrow fibrous cord, the frænum præputii, to be attached at its other end to the prepuce. The base of the glans is expanded, and projects above the level of the body of the penis. This is known as the corona, and is obliquely placed, extending farther toward

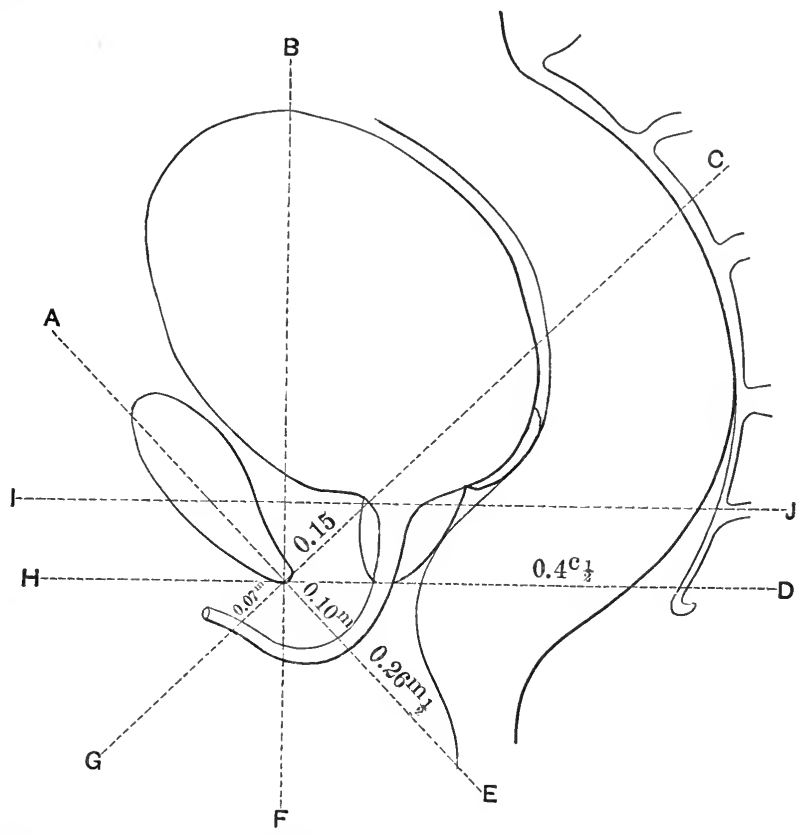

FIG. 16.-Scheme designed to show the direction of the urethra, and its relation with the symphysis pubis. (Sappey.)

the root of the penis above than below, where it is interrupted in the middle line by the frænum. Behind the corona is the constriction called the cervix.

Structure.-The corpora cavernosa are attached one to each side of the pubic arch, where they are expanded and then taper off, as we trace them backward on to the ischium, and are known here as the crura. The crura are invested by the ischio-cavernosi or erectores penis muscles. They converge in front of the symphysis and come to lie side by side, blended together for their anterior three fourths and flattened on their median aspects. From their point of union forward they are closely bound together by a common bluish, elastic, shiny sheath, in which muscular 
and elastic fibers are numerous. Each corpus cavernosum is further provided with a separate similar sheath of its own, forming a median partition between them. This median septum is not complete in the anterior part, where there occur numerous vertical slit-like openings where the two corpora cavernosa communicate with one another, giving the name
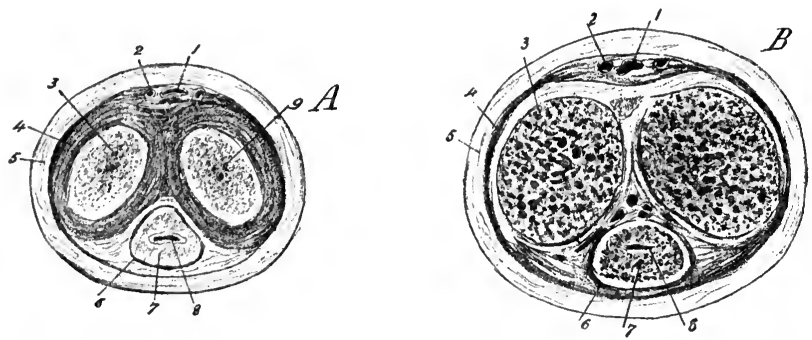

FIg. 17.-Cross-section of the penis. $A$, in flaceid, $B$, in erect eondition; 1 , dorsal vein of penis; 2 dorsal artery of penis; 3 , network of the eorpus eavernosus; 5 , skin of the penis $; 7$, network of the eorpus spongiosum; 8 , urethra. (IIenle.)

of septum pectiniforme to what remains of the septum. At the point where the two corpora cavernosa diverge from one another their fibrous sheath is reflected on to the inferior pubic angle to form a continuous structure with the anterior layer of the triangular ligament. The elasticity of the coverings of the corpora cavernosa readily allows of change in size, as in erection of the penis. The corpora cavernosa are composed entirely of erectile tissue, the trabeculæ of which are continuous with their sheath, and are composed of the same fibrous, elastic, and muscular elements. The corpora cavernosa end anteriorly in a blunt conical ex-
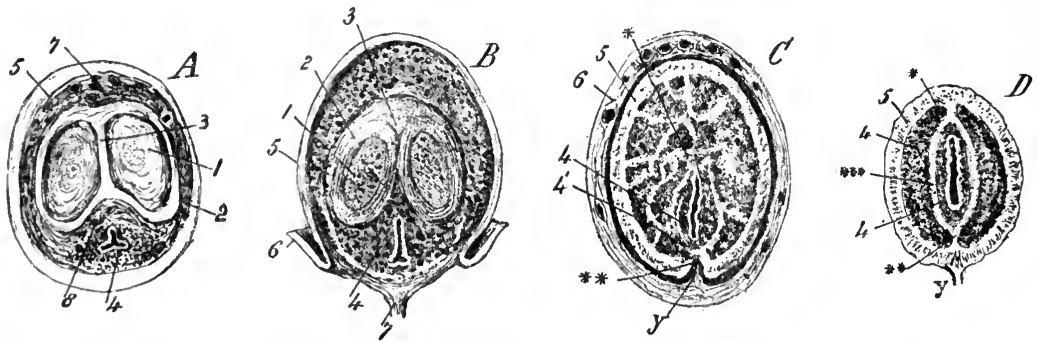

Fig. 18.-Cross-seetion through the cervix and glans penis. $A$, through the eervix; $B$, through the posterior border of the glans; $C$, through the middle of the same; $D$, just behind the meatus; 1 , eorpus cavernosum; 2, sheath of same; 3, septum between the same; 4, eorpus spongiosum; 5 , skin of glans; 6 , prepuee; 7 , dorsal veins; $*$, eonnective tissue eonnecting frænum with sheath of corpus spongiosum; $y$, frænum. (IIenle.)

tremity, which is received into the base of the glans penis. In the groove on the upper surface between the two corpora cavernosa lies the dorsal vein of the penis, while the groove on their under surface lodges the corpus spongiosum. 
The corpus spongiosum is a single median mass of erectile tissue lodged below and between the two corpora cavernosa and containing the spongy portion of the urethra. It begins posteriorly by a rounded expansion, the bulb, which is closely applied to the front of the triangular ligament, behind the point of union of the corpora cavernosa, and is situated mostly below the urethra. The bulb is closely invested by the bulbcavernosus or accelerator urinæ muscle. It presents, on its under surface, the indications of a division into two lateral halves, the indications con sisting of a slight median furrow superficially, and internally an incomplete fibrous septum. The corpus spongiosum ends in an anterior expansion-the glans, already described. Throughout its course it is closely bound to the sheath of the corpora cavernosa, as its bulb is to the triangular ligament, which is continuous with that sheath. The urethra, passing through the upper part of the bulb, lies in the center of the body of the corpus spongiosum and in the lower part of the glans. Iike the corpora cavernosa, it consists of erectile tissue, though its venous spaces are smaller and its fibrous sheath is thinner and more elastic. The elasticity of the corpus spongiosum is lost in chordee, so that it acts like the string of a bow in erection of the penis. The fascia penis is a compact fibrous and elastic layer, investing the structures already described, attached firmly around the cervix, continuous behind with the -superficial perineal fascia and in front with the suspensory ligament of the

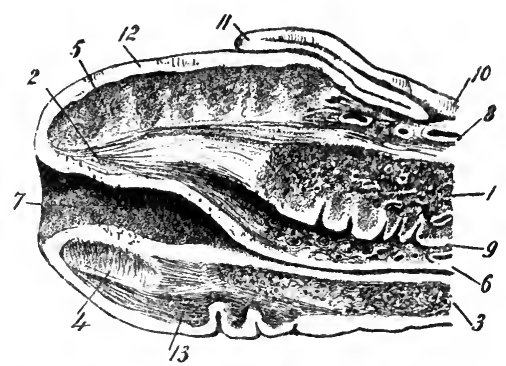

Fig. 19.-Median seetion of the glans penis, ete. 1, eorpus eavernosum: 3, corpus spongiosum ; 5 , substance of glans; 7 , fossa navieularis; 8, venous network of dorsum; 10, skin; 11, prepuee; 12, skin of glans; 13, frænum. (Henle.) penis. This ligament or thick band of fibro-elastic tissue, triangular in form, connects the anterior part of the symphysis pubis with the two corpora cavernosa at their angle of junction. Its anterior border is free.

Outside of the fascia penis is a loose layer of areolar tissue loosely connecting the skin and fascia, and allowing very free movement of the former. It is owing to the laxity of this tissue, which extends to the end of the prepuce, that very large œedematous or inflammatory swellings so rapidly occur in the penis. For the same reason also the skin of the penis can readily be drawn down over the organ-a fact to be remembered in circumcision, otherwise the entire skin of the penis may be removed. The skin is thin, very distensible and movable, free of fat and also of hairs in its anterior two thirds. Beneath, in the middle line, there is the indication of a median raphé continuous with that of the scrotum.

At the level of the cervix the skin is doubled into a loose fold of 
varying length, the prepuce, the internal layer of which is firmly attached around the cervix, and thence is continued over the glans. The skin on the inmer surface of the prepuce, and that covering the glans, approaches the type of a mucous membrane, being red, moist, and thin.

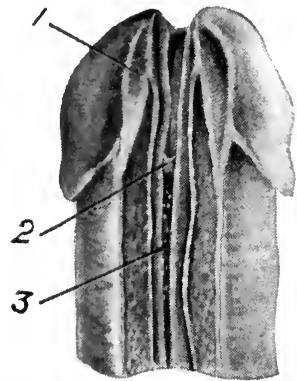

Fig. 20.-End of the penis from below, the urethra opened in the middle line below. 2 , valvula fossæ navicularis; 3, laeunæ. (Henle.)

Around the cervix are gathered numerous sebaceous glands, called glandula odoriferce, from the peculiar odor of their secretion; elsewhere in the anterior two thirds of the penis sebaceous glands are almost or entirely wanting. Over the glans there is no subcutaneous connective tissue, but the skin is intimately connected to the spongy tissue beneath. Thus, a chancre here has little if any induration, owing to lack of tissue to become infiltrated; while a typical indurated chancre may occur at the corona, where the subcutaneous or submucous tissue is abundant and lax. In the skin over the glans occur many large vascular and nervous papillæ. Beneath the skin of the penis, and connected with it, is a thin layer of muscular fibers, continuous with the dartos of the scrotum. It forms a partial sphincter at the end of the prepuce.

Vessels and Nerves.-The arteries of the penis are numerous, and comprise the following branches of the internal pudic, the artery of the bulb, the artery of the corpus cavernosa, and the dorsal artery of the penis, as well as branches from the external pudic arteries. The veins accompany the arteries, and most of them communicate with the prostatic plexus. It is rather remarkable that the dorsal vein of the penis is single, while there are two arteries of that name. This vein lies beneath the fascia penis, as well as its arteries, and perforates the triangular ligament half an inch below the subpubic angle.

The nerves of the penis include the dorsal nerve of the penis, a branch of the pudic nerve, and branches of the prostatic plexus of the sympathetic, the latter distributed to the erectile bodies.

The lymphatics are numerous in the skin and subcutancous tissues of the prepuce and glans, and pass chiefly into that set of inguinal glands situated most internally.

Development and Abnormities. - The penis is developed from two lateral halves which fuse together along their median surfaces. The urethra is at one time but a groove on their under surface, the edges of which grow together inferiorly from the proximal to the distal extremity, to form the urethral canal.

Abnormities occur from failure to unite along the upper or lower borders of the median surface, resulting in epispadias and hypospodias, 
respectively, of which the latter is by far the more common. It occurs in all degrees, from a slight lengthening of the meatus below to a complete failure to unite along the entire spongy urethra. All degrees of approach to hermaphroditism are caused by the complete or partial failure of the bilaterally formed external genitals to unite mesially.

The end of the prepuce may be congenitally narrow, causing congenital phimosis. The opening may be as small as a pin-point or only slightly narrowed, just preventing its retraction. This condition hinders the development of the glans, and is apt to set up adhesions between the prepuce and glans; hence the advisability of early operation.

\section{THE URETHRA.}

The male urethra extends from the urethral orifice of the bladder through the prostate, the two layers of the deep perineal fascia, and the length of the corpus spongiosum, to end at the meatus at the extremity of the glans penis. Corresponding to the parts through which it passes, it has been divided, for convenience of description, into three parts, which have received the following names in order from above downward-prostatic, membranous, and spongy or penile portions.

The urethra can not be regarded, strictly speaking, as a canal, but rather as a closed valve, the walls of which are usually in close apposition, and which is only open for a few seconds at a time to transmit fluids.

The length of the urethra averages eight and a half inches in the dead subject, and seven and a half to seven and three quarters inches in the living. The condition of the penis may alter the length temporarily, and a hypertrophied prostate may lengthen the urethra.

To give the caliber of the urethra in absolute figures is impracticable. Its relative dilatability is more important. This varies in the different parts of the urethra. The meatus is the narrowest and least dilatable part, averaging about a quarter of an inch in length, and admitting a 24 (French) sound. Next comes a spindle-shaped dilatation, the fossa navicularis, contained in the glans penis. The caliber again narrows, and continues uniform until we reach the bulb, where it is dilated, pouchlike, along the floor. This part is about the same in caliber as the prostatic portion, and even more dilatable. The membranous portion is, next to the meatus, the narrowest part of the urethra. Its caliber is uniform, and it is much more distensible than is the meatus. In the prostatic portion again we have a spindle-shaped dilatation, narrowing to the upper end, where we have the orifice of the bladder. This portion is also very dilatable.

We have therefore from end to end a narrow portion alternating with a dilated portion. Any instrument which can pass the meatus should also 
pass the rest of a normal uretlira. In the order of dilatability the different parts are arranged as follows: The meatus is least dilatable, next the mem-

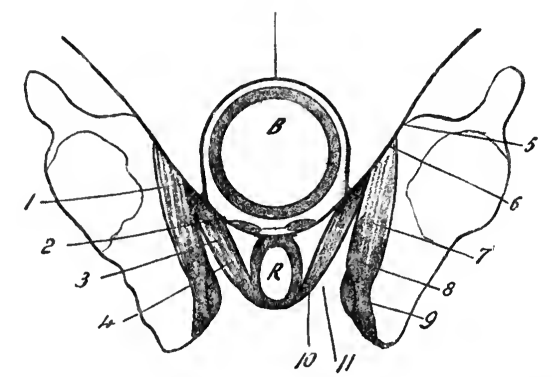

FIg. 21.-Diagram of oblique coronal section through the pelvis, showing the cut outline of the fascia. $B$, bladder; $R$, rectum ; 1 , white line; 2 , rectovesical fuscia; 3 , rectal fascia; 4 , levator ani ; 5 , united iliac pelvic fuscia; 6 , pelvic fascia; 7 , obturator internus; 8, obturator fascia ; 9, Alcock's canal; 10 , anal fascia; 11, ischio-rectal fascia. (MeAllister.)

branous portion, the spongy portion, the prostatic portion, and, lastly, the most dilatable is the bulbous portion. The following figures indicate the relative rather than the absolute caliber of the canal, and are given both in fractions of an inch and in the number of the sound (French scale), which can pass without giving pain: Meatus, three tenths of an inch, 24 sound; spongy portion, six tenths of an inch, 28-30 sound; bulb, seven tenths of an inch, 32 sound ; membranous portion, five tenths of an inch, 26,27 sound; prostatic portion, seven tenths of an inch, 30-32 sound; orifice of bladder, five to six tenths of an inch. According to Otis, there is a constant relation between the caliber of the urethra and the circumference of the penis.

The Direction of the Urethra. -In the anterior three quarters or so of the spongy portion the parts inclosing the urethra are very movable, so that this part of the urethra can not be said to have any fixed direction, except that given it by gravity, hence the name (pars pendula sen mobilis). Posteriorly the corpora cavernosa become attached to the pubic rami, and the corpus spongiosum to the triangular ligament, hence the posterior two inches or so of the spongy portion, or that part lying behind the angle of junc-

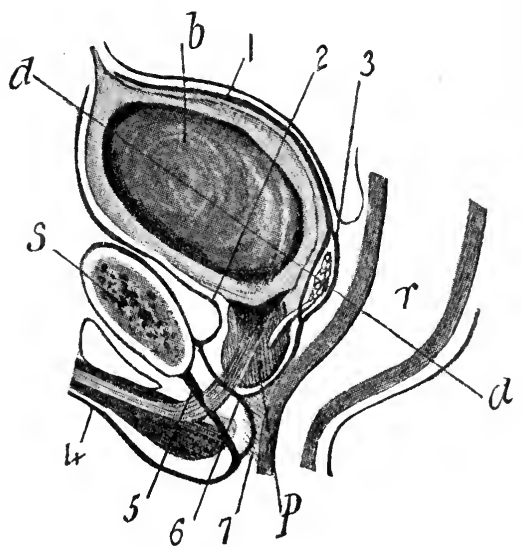

FIG. 22.--Sagittal section through bladder and rectum. $r$, rectum; $b$, bladder; $p$, prostate; 1 , superior vesical fascia; 2 , superior true ligament; 3 , fascial sheath for vesiculæ seminales; 4, Colles'; 5, anterior layer; 6, suprapubic fuscia; 7 , perineal body. (IIcAlister.) tion of the corpora cavernosa, is more or less fixed (pars immobilis, etc.). Here the canal begins to curve beneath the symphysis, and in the membranous portion ascends (in the erect position of the body), with a slight curve, convex upward and for- 
ward, which is continued through the prostatic portion. The curve begins about one and a half inch anterior to the bulb, and forms the arc of a circle about three and a quarter inches in diameter, the chord of the arc averaging two and three quarters inches. In spare, small men the curve is sharper, and also in children, on account of the high position of the bladder in the latter. In large, stout men, on the other hand, the curve is flatter. The lowest portion of the curve of the urethra, in the erect position, is that in contact with the anterior layer of the triangular ligament, or the anterior part of the membranous portion and the posterior part of the bulbous portion. Various morbid conditions may interfere with the direction and curve of the urethra.

Prostatic Portion. - Its course through the prostate, averaging one and a quarter inch in length, has already been described. As above mentioned, it presents a large, spindle-shaped dilatation about its center, being narrower above and below. The upper boundary of this portion is the uvula vesicce, passing from which we may trace on the floor of the urethra a slight ridge as a whitish band. Farther downward it rises into a narrow median ridge about one eighth of an inch high and eight to nine lines long, and then sinks down again, to be continued as a single or double white line along the floor of the urethra into the membranous and bulbous portions. This is variously called the verumontanum, caput gallinaginis, criste urethro and colliculus

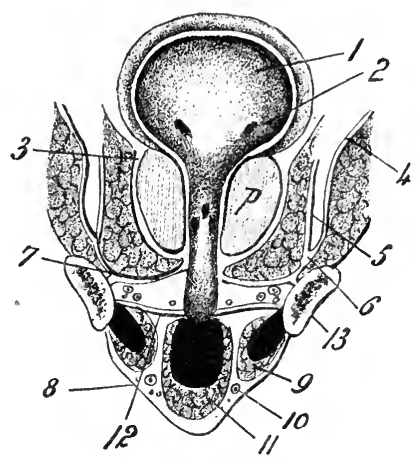

FIG. 23.-Diagram of slightly oblique coronal section of pelvis through the bladder, prostate, and urethra - the erectile bodies are darkly shaded. 1, bladder; 2, ureter; 3 , capsule of prostate; 4 , obturator faseia; 5 , anal fascia; 6 , forward continuation of these united; 8 , deep layer of superficial perineal faseia of isehio-cavernosus; 10 , long perineal artery; 11, bulboeavernosus. (MeAllister.)

seminalis. The longitudinal grooves on either side, called the prostatic sinus, are somewhat depressed, owing to the dilatation of the urethra at this point, and the floor is perforated by the orifices of the prostatic ducts. The prostatic sinus, even when not unusually deep, may impede the passage of a catheter. About the summit of the verumontanum is the opening of the utricle, in the lateral margins of which are placed the mouths of the two common ejaculatory ducts. The utricle, utems masculinus, etc., corresponding to the uterus in the female, run backward and slightly upward in the "middle portion," and in the course of the ejaculatory ducts which lie on either side in its walls. It measures two and a half to four lines deep, and two to three lines in width. The verumontanum is composed in part of erectile tissue, and contains many of the longitudinal muscle-fibers of the wall of the urethra. On cross-section the shape of 
the prostatic urethra is somewhat triangular, with its apex forward. The mucous membrane of this portion lies in delicate longitudinal plaits ; it is of a pale, pinkish-yellow color, and is lined by cylindrical epithelium. With the longitudinal muscle-fibers just external to the mucous membrane is mingled more or less erectile tissue.

The Membranous Portion, or that part situated between the two layers of the triangular ligament, measures but one half to three quarters of an inch in length. This measurement is rather less along the floor of this portion where the bulb encroaches to some extent, though the projection of the latter on the triangular ligament is rather more away from the urethra than along it. It lies beneath the pubic arch, passing through the two layers of the triangular ligament about an inch below the subpubic angle. Its inferior convex surface is separated by a short interval from the third portion of the rectum, and looks toward the surface of the perinæum. Its mucous membrane, which is redder than that of the prostatic portion, is surrounded by an internal longitudinal and an external circular layer of muscle-tibers. The circular fibers are continued forward from the prostate, and with the longitudinal is mingled some erectile tissue continued back from the bulb. External to these layers the compressor urethræ, a voluntary muscle, surrounds the urethra, the fibers passing mostly transversely in front and behind the wrethra. In the same compartment, between the two layers of the triangular ligament and supported on the anterior layer, lie the two Cowper's glands, one on either side and below the urethra. They are compound racemose glands, about the size of a pea, and of a yellowish color. Their excretory ducts run forward just beneath the urethra, to open on the floor of the bulbous portion by two small oblique openings. The ducts are one to one and a half inch long.

Spongy Portion.-This portion, six to six and a half inches in length, begins posteriorly in a dilatation-the bulb, or sinus of the bulb, as that part contained within the bulb is called. The dilatation occurs almost entirely on the floor and sides of the canal, so that the floor of this part may be one to three lines below the opening into the membranous portion through the triangular ligament This and the membranous portion are on the lowest level of the curve of the urethra, in the erect position. The layer of fascia separating them forms the dividing line between the "anterior" and the "posterior" urethra. This distinction is an important one, on anatomical, embryological, pathological, and therapentic grounds. The "posterior" urethra or muscular portion is active in micturition, less active in coition, while the "anterior" or erectile portion is passive in micturition, active in coition. The "posterior" is developed from the uro-genital sinus, the "anterior" from the surface.

At the anterior end of the spongy portion is another dilatation, the 
fossa navicularis, occurring largely on the roof of the canal, and contained within the glans. The meatus is a firm vertical slit, while a cross-section of the spongy portion back of the glans shows a transverse slit.

The mucous membrane of this portion of the urethra has a cylindrical epithelinm, except at the anterior extremity, where it approaches the squamous type and is deeper in color. External to the mucous membrane is a single layer of longitudinal muscle-fibers.

Throughout the mucous membrane of the urethra we find small racemose mucous glands and follicles, the glands of Littré. Besides these there are the larger lacunce, whose openings, like those of the glands of Littré, point downward toward the meatus. There is one larger recess, the lacuna magna, situated on the upper surface of the fossa navicularis, one inch from the meatus and about one fifth of an inch deep. In this recess small bougies, etc., may readily be caught. The valvula fossce navicularis is a fold of membrane often found about half an inch from the meatus on the inferior surface. This may be the site of an occasional congenital narrowing of the urethra.

\section{PHYSIOLOGY OF THE BLADDER AND URETHRA.}

Briefly stated, the physiology of the bladder consists in the study of its two functions of holding and voiding urine.

I. The urine is held in the bladder by means of the sphincters. There is no sphincter in the bladder itself, but they are placed along the upper portion of the urethra, one in the upper end of the prostate, superior or internal sphincter vesicce seu prostatce, one in the lower end of the prostate, inferior sphincter prostatce, and one around the membranous urethra, the external sphincter or compressor urethræ muscle. The upper of these three sphincters is involuntary, the middle partly so, and the external wholly voluntary.

In old age the sphincters are weaker, so that old men can hold less urine, and must therefore urinate more often.

If the sphincters are paralzed the urine dribbles, and spastic contraction may cause retention of urine.

II. Micturition consists of three factors: 1 , the detrusors or the entire muscular coat of the bladder; 2 , abdominal pressure aided by elevation and contraction of the diaphragm of the pelvis; 3 , hydrostatic pressure. Besides these factors we must have a relaxation of the sphincters. As urine collects and distends the bladder, the internal pressure finally reaches such a point that the "internal sphincter" yields, and a few drops enter the prostatic urethra, causing a desire to micturate. This may be resisted for some time by the lower sphincters, by the action of the will, or they may be relaxed by the same action, when the expulsive forces mentioned empty the bladder. The last drops in the bladder are expelled by 
the circular fibers near the orifice. The urethra is only open during the passage of urine, the last drops of which are expelled by the muscular tissue in its wall, aided by the accelerator urinæ, which helps to empty the bulb, and may hasten the flow of urine contained in it. Between the acts of micturition the sphincters remain closed, those composed of involuntary muscle-fibers continuously, those composed of voluntary muscle-tissue when irritated. Thus the passage of an instrument or the injection of a fluid into the anterior urethra receives such a check at the external sphincter or compressor urethræ muscle that a fluid can not pass, but flows back and is voided at the meatus. If the injecting catheter is passed behind this point, however, the action of the sphincters above does not prevent the passage of the fluid into the bladder.

\section{THE MALE PERIN EUM,}

The boundaries of the perinæum proper are the rami of the pubes and ischium, and a line drawn between the two ischial tuberosities. These boundaries can be felt from the surface. The perinæum thus bounded has the form of an equilateral triangle, of which the sides measure three and a half inches. From the apex to the center of the base the measurement is one and a half to one and three quarters inch. The base is not a straight line between the ends of the side lines, but inclines forward in the middle toward the apex, corresponding to the course of the transversus perinei muscle. The depth of the perinæum, or distance from the skin to the pelvic floor, varies with the amount of subcutaneous fat, and averages two to three inches in the posterior part, while the anterior part is less than one inch in depth.

The skin of the perinæum is thin, quickly showing ecchymoses, and has in the median line a raphé continued forward on to the scrotum and penis and backward to the anus.

Beneath the skin is the superficial perineal fascia, made up of two layers. The external layer contains the subcutaneous fat, and is continuous with similar tissue on either side and behind. The deep layer is firm and membranous. Behind, it passes around the posterior border of the transversus perinei muscle, where it joins the posterior end of the triangular ligament, and becomes continuous with the anal fascia lining the under surface of the levator ani muscle. At the sides it is firmly attached to the bony rami. In front it is continuous with the dartos of the scrotum and the fascia of the penis.

On account of its connections there is but one outlet for fluids, etc., beneath it, and that is between the spines of the pubes on either side. The course of urine and other extravasations beneath it is therefore into the scrotum and penis or on to the abdomen. 
From this layer of fascia onward we have an aponeurotic layer alternating with a layer of muscles etc., so that the latter are inclosed on two sides by fascia, the other two sides being formed by the rami of the pubic arch. From the superficial perineal fascia there is an incomplete median septum partly dividing the space into two lateral halves. In the exact median line of the perinæum there is therefore but little danger of hæmorrhage, as even the bulb has a partial median septum, and apart from the

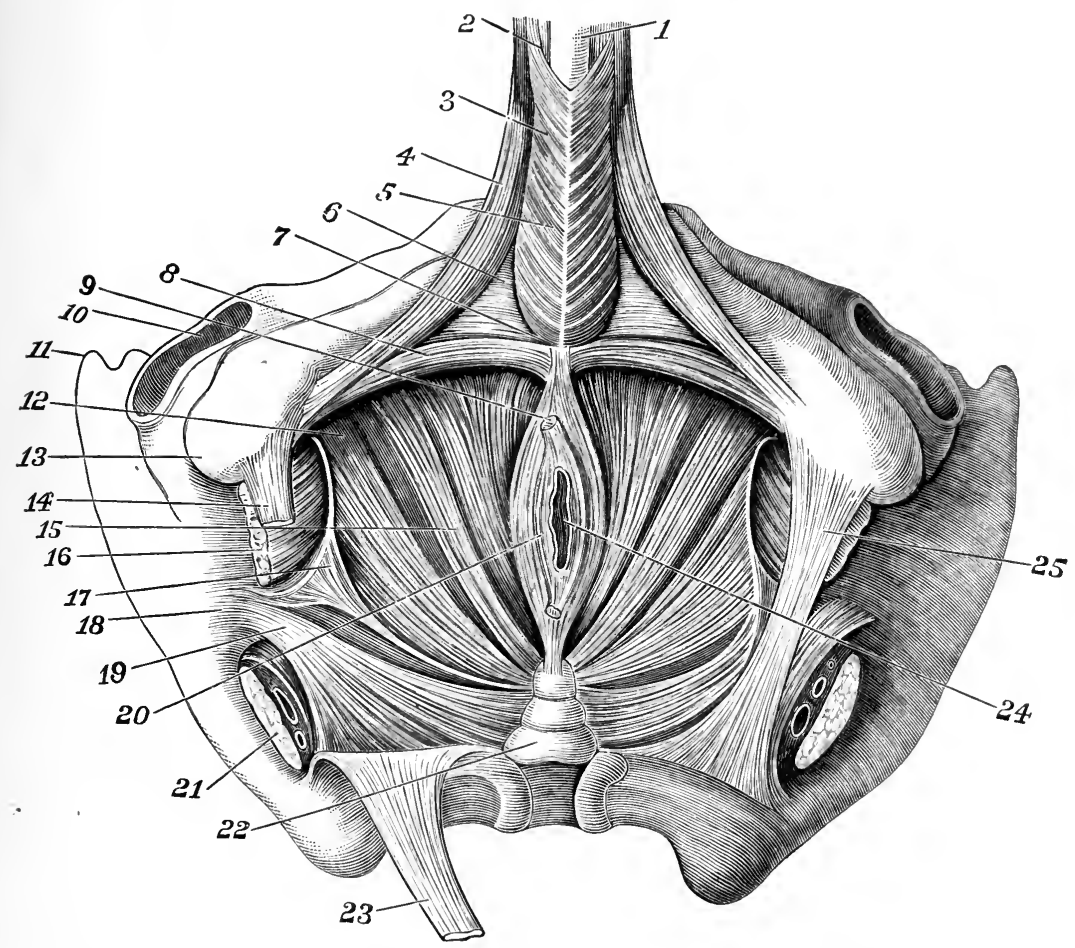

Fig. 24.-Dissection of perineal muscles. 13, tuber isehii ; 14, great sacro-sciatie ligament; 18, spine of the ischium; 17 , obturator faseia; 2 , corpus eavernosum ; 1 , corpus spongiosum ; 16, obturator internus muscle: 3 , bulbo-cavernosus muscle; 4 , ischi-cavernosus muscle; 8 , transversus perinei musele; 15 , levator ani ; 7 , median; 5 , transverse septum of the perineal muscles; 6 , anterior layer of the deep perineal faseia. (Henle.)

bulb there is no danger of hæmorrhage here. The next layer consists of the bulb of the urethra and its spongy portion, as far as the scrotum, three pairs of muscles, vessels, and nerves.

The bulb and corpus spongiosum are median in position.

The bulbo-cavernosus or accelerator urinæ muscle surrounds the bulb at its posterior end. Farther forward it passes around the sides of the bulb to become attached to the triangular ligament; still farther forward it passes around to meet in front of the corpus spongiosum; and the most 
anterior fibers join the anterior fibers of the erectores penis muscles and pass in front of the corpora cavernosa, anterior to the dorsal vein of the penis. It is formed of two lateral halves united by a median fibrous raphé. The erectores penis muscles embrace the crus penis of either side. The superficial transversus perinei muscle passes forward as well as inward from the ischial tuberosities to join with the bulbo-cavernosi and sphincter ani muscles at the so-called central tendinous point of the perinæum, one inch or more in front of the center of the anus. The three muscles thus briefly described may form almost a continuous muscular layer (uro-genital diaphragm), or they may leave a considerable space between them in which is seen the next layer. With the transversus perinei muscles pass the branches of the internal pudic artery and nerve of the same name as the muscle; they are unimportant branches, however, and are divided by the incision in lateral lithotomy with impunity. The deep perineal fascia or triangular ligament consists of two layers, a superficial and a deep. They are attached respectively to the anterior and posterior margins of the internal borders of the pelvic rami as far back as the tuber ischii. Here the two layers are united, and are joined to the deep layer of the superficial fascia as it passes back behind the transversus perinei muscles to become continuous with the anal fascia. In front the two layers are separated, the greatest distance between the two varying from one half to three quarters of an inch. The anterior layer is continuous with the sheath of the corpora cavernosa, and to it the bulb of the corpus spongiosum is firmly adherent. The posterior layer is continuous with the pelvic fascia above the levator ani muscle intervening between the two below, and with it the sheath of the prostate is continuous. The depth of the triangular ligament from the subpubic angle to its base is one and a half to one and three quarters inch. One inch below the subpubic angle the triangular ligament is perforated by the urethra, that part of the urethra between the two layers being the membranous portion. The dorsal vein, arteries, and nerves of the penis perforate the anterior layer about half an inch below the angle of the pubes.

Between the two layers of the triangular ligament we have an osseoaponeurotic compartment occupied by the membranous urethra, the compressor urethræ muscle, Cowper's glands, and the artery of the bulb. The compressor wrethræe muscle has sometimes been described as consisting of two portions, a deep transversus perinei and a circular external sphincter muscle. This division is an artificial one; the fibers pass both more or less circularly around the urethra, and also transversely in front and behind or above and below the urethra. Laterally this muscle is attached to the borders of the pubic rami between the layers of the triangular ligament. It lies on a line more or less anterior to the superficial transversus perinei muscles. Its function is as a voluntary sphincter vesicæ, which may also 
be excited by reflex action. The membranous urethra and Cowper's glands have already been described, the latter lying on either side of the median line, below the urethra, closely applied to the deep surface of the anterior layer of the triangular ligament.

The artery of the bulb passes inward, in this compartment, from the internal pudic artery to enter the bulb, after penetratiug the anterior layer of the triangular ligament. In its course it lies about one and a half inch from the anterior margin of the anus, so that the incision in lateral lithotomy should not begin in front of this point, for fear of wounding this artery.

The posterior layer of the deep perineal fascia is continuous above with the pelvic fascia; lower down it has behind it another muscular layer, the anterior part of the levator ani muscle. On this layer rests the apex of the prostate, the sheath of which, formed by the recto-vesical fascia, is continuous with it. On the deep surface of the levator ani muscle lies the recto-vesical fascia separating the muscle from the sub-peritoneal connective tissue and the peritonæum.

Along the outer border of this space runs the main trunk of the deep perineal artery and nerve. At the tuber ischii the artery lies at a depth of one to one and a half inch from the surface of the tuberosity.

\section{THE SCROTUM.}

The scrotum invests the testes and lower part of the spermatic cord in the form of a double pouch. Its great mobility affords an admirable protection to the testes. A superficial division into two lateral halves by a median raphé indicates its embryological formation. The scrotum is made up of a number of layers, but practically there are but two layers, separated by loose connective tissue.

The skin is fine, thin, and transparent, thus showing an ecchymosis beneath it very quickly. It is elastic, allowing of great distention, as in large herniæ, etc., and also redundant, so that much may be removed without being missed. It is dark in color, and furnished with scattered, flattened hairs and sebaceous follicles whose secretion has a peculiar odor. It is thrown into ruga, which are a sign of health. These rugre are caused by the contraction of the next layer beneath.

The dartos is a reddish-brown, muscular layer continuous with the superficial fascia of the groin, perinæum, and penis, and differing from the latter by being entirely free from fat. The skin forms but a single pouch, a division into two parts being only indicated on the surface by the median raphé, which is continued forward on to the penis and backward on to the perinæum as far as the anus. The dartos, however, forms two separate pouches for the two testes. These two pouches are joined 
together along the median line forming the so-called septum scroti, adherent to the raphe and extending to the root of the penis. The contraction of the dartos is slow and peristaltic, and it alters the shape and size of the scrotum. When the scrotum is incised, the contraction of the dartos is liable to invert the edges of the skin, with which it is firmly adherent. In fact, these two layers form but a single one, especially in the lower half of the scrotum. In the upper half of the scrotum the two layers may be somewhat separated by a subcutaneous connective tissue, continuous with that of the abdomen, in which the swelling due to cedema or elephantiasis may occur.

The layer formed by the skin and dartos is separated from and connected with the layer beneath by a cellular layer in which occur effusions of blood after contusion of the scrotum. This coupling layer is regarded by some as the INTERColduMNar or SPERMatic fascia derived from the tendon of the external oblique muscle of the abdomen at the external ring. Others speak of the spermatic fascia as firm, and closely connected with the next deeper layer.

The cremaster consists of scattered loops of striped muscle-fibers connected together by a fibrous membrane. Above, it is continuous with the lower border of the internal oblique muscle, or at least its attachments are similar to those of its conjoined tendon. This layer is known as the cremasteric fascia, and by its contraction the testicle is raised and the cord is shortened. The size of the cremaster varies with the weight it has to suspend. The cremasteric reflex is due to contraction of this muscle.

The infundibuliform fascia closely connects the cremasteric fascia external to it to the tunica vaginalis internal to it into a second or internal composite layer formed by the skin and dartos. This fascia is continuous above with the fascia transversalis, and is connected to the tunica vaginalis by a delicate areolar tissue continuous with the subperitoneal connective tissue. Astley Cooper named this the fascia propria. This layer closely invests the cord, and within it, among the loose areolar tissue in front of the cord, there can sometimes be traced from the internal ring to the upper end of the tunica vaginalis a fine fibrous cord. This is the remains of fœta! processus vaginalis peritonei, which connected the peritoneal cavity with that of the tunica vaginalis. Below, the infundibuliform fascia is adherent to the back of the testis over a small area where the serous covering of the latter is wanting.

The tunica vaginalis forms a closed serous sac whose opposite walls are in contact. It is described in two parts, a parietal and a visceral. The visceral layer closely invests the testis except along its posterior border. Here it is reflected on to the epididymis lying along the outer side of this border. At the upper and lower ends of the epididymis the 
serous membrane passes quite directly between it and the testis, but between these two points it forms a pouch (digital fossa) between the two. The fold which forms the pouch has been called the meso-testis. This layer invests the epididymis, except along its posterior border and over a small area at the two ends of its internal surface. The parietal layer closely invests the inner surface of the infundibuliform fascia, and forms a cul-de-sac, extending half an inch or so above the level of the testis and on either side of it at the beginning of the cord. At the lower end of the posterior superior border of the testis, where the visceral serous covering is reflected on to the parietal, the tunica albuginea of the testis comes in contact with and is adherent to the fibrous infundibuliform fascia. This anchors, as it were, the testis to the inferior and posterior part of the tunica vaginalis, and this is its position when the serous cavity is filled with fluid, except in those cases of inversio testis when it has a reverse position.

The blood-vessels and ducts are connected with that part of the testis where the visceral layer of its serous covering is reflected off-i. e., along the posterior border.

Vessels and Nerves.-The blood-supply of the two layers of the scrotum is more or less independent as is that of the testis. The external layer is supplied by branches of the external pudic and the superficial branch of the internal pudic. The deeper layer is supplied by the cremasteric artery and the artery of the vas deferens, leaving the spermatic artery and some branches of the artery of the vas deferens to supply the testis. The veins accompany the arteries. The nerves which supply the scrotum come from various sources, including the ilio-inguinal and iliohypogastric, the superficial perineal branch of the internal pudic nerve, and the inferior pudendal, a branch of the small sciatic. These supply mostly the skin and dartos. The cremaster muscle is supplied by the spermatic branch of the genito-crural nerve. Irritation of the cutaneous branch of this latter nerve causes a reflex contraction of the muscle known as cremasteric reflex.

The lymphatics of the scrotum pass to the inguinal glands. Although the blood and nerve supply of the scrotum is abundant, its vitality is not great, so that it sloughs from inflammation or pressure ; therefore it is advisable to be careful in strapping.

The scrotum is, furthermore, one of the first parts to show cedema, and the most frequent seat of elephantiasis. 


\section{THE TESTICLES.}

The testes are suspended in the scrotal pouch by means of the cord, and their attachment to the tunica vaginalis, at unequal heights, that of the left side being usually lower than the right.

Shape.-In shape they are ovoid, compressed somewhat laterally. They thus present two surfaces, internal and external; two borders, anterior and posterior; and two extremities, superior and inferior.

As to size, they average one and a half inch long, one and a quarter inch from front to back, and about an inch transversely. The left is

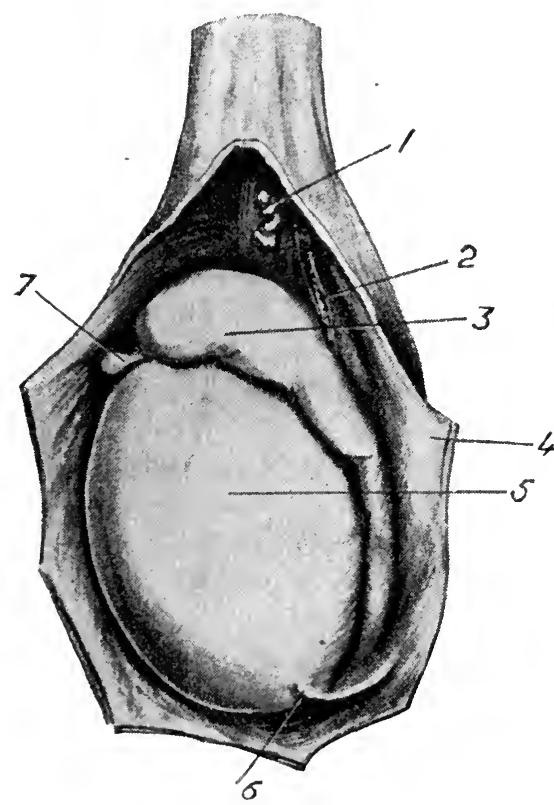

Fio. 25.-Left tunica vaginalis opened, showing testis, epididymis, ete., from outer side. 1, organ of Giraldès; 2, vas deferens; 3, epididymis; 4,6 , tunica vaginalis; 5 , testicle; 7 , hydatid of Morgagni. (Quain.) often a little the larger of the two, the average wEIGHT varying from three quarters to one ounce.

Position. - The testis is suspended obliquely, so that its upper end points somewhat obliquely ontward and forward as well as upward. Thus, the posterior border looks also upward and slightly inward, the anterior border in the opposite direction; and the outer surface is inclined somewhat downward and outward, the inner surface in the opposite direction. The testis is normally fixed to the posterior and inferior part of the tunica vaginalis, and retains this position when the latter is filled with fluid. In the cases of so-called inversion of the testis its position is directly opposite, in the front and lower part of the scrotum, as if it were rotated on its vertical axis through one hundred and eighty degrees. The testis may also be retained in the abdominal cavity, or in the inguinal canal in its passage into the scrotum. On the other hand, it may pass through the scrotum into the perinæum, by the continued traction of the gubernaculum, or it may pass through the femoral instead of the inguinal canal.

The lower position of the one testis, usmally the left, prevents compression of the two together when walking, etc. 
The consistence of the testes is everywhere equal, so that any hardness is pathological.

The Epididymis. - This is made up of convolutions of the first part of the excretory duct of the testis, is attached to the posterior border of the latter, and rests on its outer surface, anterior to this border. Its enlarged upper end, called the head or globus major, projects slightly forward as it rises a little above the upper end of the testis. The body, or middle portion, rests against the back of the onter surface of the testis by its concave internal surface. The convex outer surface and the anterior border are free. Along its posterior border we find the line of reflection of its serous covering, which connects it with the testis and the two with the parietal layer of the tunica vaginalis. The lower end, smaller and more pointed than the upper, is known as the tail or globus minor, and ends in the beginning of the vas deferens.

The epididymis is directly connected to the testis at the upper and lower ends of its concave internal surface, and between these points by a reflection of tunica vaginalis from their posterior borders, which forms a pouch (digital pouch) between the two bodies.

The hydatids of Morgagni are found at the front of the globus major, and more to its outer side. They are pedunculated, covered by tunica vaginalis, and consist of connective tissue and vessels. One or more may occur, but one is regularly present, pyriform in shape, and lies between the globus major and the testis. They represent remains of the ducts of Müller.

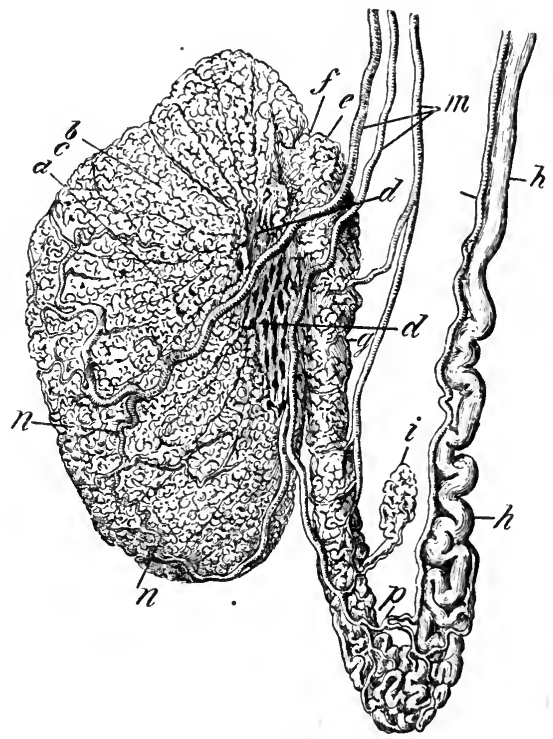

FIG. 26.-Injected testis, epididymis, and vas deferens. $a$, body of testiele; $b$, lobules; $c$, vasit reeta $; d$, rete vasculosum; $e$, vasa efferentia; $f$, coni vaseulosi; $g$, epididymis; $h$, vas deferens; $i$, vas aberrans; $m$, branehes of spermatic artery : $n$, ramification in the testis; $p$, union of the vas deferens artery with a twig of spermatic artery. (Quain.)

Structure.-The outer or serous coat of the testis and epididymis forms a complete covering for each, except along their posterior borders, where the visceral tunica vaginalis is reflected off to become continuous with the parietal layer. At the upper and lower ends of the internal surface of the body of the epididymis it is also connected directly with the testis. Along the posterior, free border of the testis, above, we have the ducts 
passing out which go to form the epididymis ; just below this the bloodvessels enter the gland; and at its lower end the tunica albuginea of the testis is firmly connected with the infundibuliform, or fascia propria, of the scrotum.

The tunica albuginea of the testis is a firm, thick, white layer of fibrous tissue covering the testis beneath its serous coat. It is owing to the firm, unyielding character of this coat that in inflammation of the testis the swelling occurs so slowly, but is accompanied by so much pain; and also that hernia testis may occur when this coat is once perforated. Along the posterior border of the testis the tunica albuginea projects into the gland as a vertical, median, fibrous septum, mediastinum testis or corpus IIighmori, broader above than below. From this corpus Highmori numerous incomplete fibrous septa radiate out to the inner surface of the tunica albuginea, dividing the organ into three hundred to four hundred conical compartments. A vascular network, supported and connected by areolar tissue, lines the inner surface of the tunica albuginea and the fibrons septa, and has been called the tunica vasculosa.

The glandular substance of the testis consists of as many conical lobes as there are compartments (three hundred to four hundred). In each lobe there are two or more convoluted tubuli seminiferi. These tubules when straightened out are two feet or more long; they present small bulgings near their commencement, and frequently communicate with one another laterally at their distal ends. They number between eight liundred and nine hundred, and measure $\frac{1}{100}$ to $\frac{1}{50}$ of an inch in diameter. As the tubuli seminiferi approach the corpus Highmori they unite into a smaller number of tubules, which, becoming straighter, are for a short distance close to the mediastinum, called tubuli recti. These split up into a network of channels in the mediastinum, known as the rete vasculosum testis, from the upper and back part of which the secretion of the testis is conveyed away by the vasa efferentia. These, twelve to twenty in number, perforate the tunica albuginea along the upper part of the posterior border of the gland, and, becoming convoluted, pass upward as a number of cone-shaped bodies, which open above into the single tube of the epididymis at intervals of about three inches. These cone-shaped masses of the efferent tubes, known as coni vasculosi, form a portion of the head of the epididymis.

The single cancl of the epididymis is very much convoluted, so that on being unraveled it is found to measure twenty feet or more in length. Its convolutions, which are grouped more or less into lobules separated by imperfect septa, pass from the globus major to the globus minor of the cpididymis, and are there continued into the vas deferens. The canal of the epididymis decreases in size toward the globus minor, but increases again before becoming the vas deferens. Connected with the lower part 
of the epididymis is generally found the vas aberrans, a narrow, blind, convoluted tube, two to fourteen inches long, often dilated at its distal end, and sometimes branched. It resembles the vas deferens in structure, and may be connected with the Wolffian duct of the fœetus in origin.

The walls of the tubuli seminiferi in the testis are composed of several layers of cells, the inner forming the basement membrane, being alone complete. This basement membrane is continued in the tubuli recti, but the rete vasculosum testis has no proper wall. The wall of the vasa efferentia and epididymis contain some muscle-fibers, and become thicker as we approach the vas deferens.

The mucous membrane of the tubuli seminiferi comprise several layers of epithelium, of which the outer alone is regular and complete. In the internal or nutritive layer lie imbedded the heads of the spermatozoa. In the tubuli recti the mucous membrane consists of a single layer of cubical or flattened cells continuous with the outer complete layer of the tubuli seminiferi and with the single layer of flattened cells in the rete vasculosum testis. In the vasa efferentia and the epididymis the columnar cells are ciliated, the movement of the cilia being toward the vas deferens. In the globus minor, near the vas deferens, the cilia disappear.

Vessels and Nerves. - The spermatic artery supplies the testis and epididymis, together with a few small anastomosing branches of the artery of the vas deferens. Their point of entrance is along the posterior border, at which point also the several branches of the spermatic vein pass out, and unite together into a plexus, the pampiniform plexus. The lymphatics, beginning as intercommunicating spaces between the tubuli seminiferi, accompany the spermatic vessels and terminate in the lumbar glands. The nerves are derived from the sympathetic, and come mostly from the aortic plexus along the spermatic vessels, forming the spermatic plexus. A few filaments come from the hypogastric plexus along the artery of the vas deferens.

Spermatic Cord.-This consists of the following structures, which are grouped together by delicate areolar tissue in their passage from the internal abdominal ring to the upper end of the testis. They have the same coverings as the testis while they are contained in the scrotum, except for the tunica vaginalis, which is often found represented by a fibrous cord among the other structures of the cord. Above, the dartos covering is replaced by superficial fascia, subcutaneous connective tissue intervening between it and the skin. The spermatic fascia extends only to the external abdominal ring, to be there continued as the aponeurosis of the external oblique muscle. The internal oblique muscle also takes the place of the cremaster layer in the inguinal canal. Briefly described, the cord in the inguinal canal is bounded in front by the aponeurosis of the external oblique muscle, and to a slight extent, externally, by the muscle- 
fibers which form the conjoined tendon; behind, by the transversalis fascia, and below or internally by the conjoined tendon; below, by the union of the aponeurosis of the external oblique with the transversalis fascia; and above, by the conjoined tendon and its muscle.

The structures forming the cord are as follows: (1) vas deferens; $(2,3)$ the spermatic artery, and the artery of the vas deferens; (4) the spermatic plexus of veins; (5) the sympathetic nerve-fibers; (6) lymphatics. The cremaster muscle, its artery, and the genito-crural nerve belong properly to the coverings of the cord.

The spermatic plexus of veins (or pampiniform plexus) is divided into two groups: (1) a larger group, placed anteriorly with the spermatic artery; (2) a smaller group, with the artery of the vas deferens lying more posteriorly. It is the former only of these two groups which is to be included by the ligature in operating for varicocele. Above, the veins form two or three trunks, which passing into the abdomen unite to form a single trunk, which empties into the vena cava on the right side, the renal vein on the left.

The following are some of the anatomical reasons for the frequency of varicosities in the spermatic veins: their length, dependent position, vertical course, large size as compared with the arteries, few and imperfect valves, and tortuosity. Further, they lack external support, being in a loose tissue, and are exposed to pressure in the inguinal canal.

The vas deferens continues the canal of the epididymis, and ascends on the inner side of the epididymis, from which and the posterior aspect of the testis it is separated by the spermatic vessels. It then forms one of the elements of the spermatic cord, and ascends almost vertically to the external inguinal ring, lying behind the other structures of the cord, except in inversion of the testis, when it lies in front.

From the external ring it passes upward and outward to the inner margin of the internal inguinal ring, where in turning inward it forms a crescentic margin in the infundibuliform fascia; it then turns sharply inward and downward into the pelvis, across the external iliac vessels, turning around the outer and upper aspect of the deep epigastric artery. It lies beneath the lateral false ligament of the bladder, where it crosses the obliterated hypogastric artery. Having reached the side of the bladder, it arches backward and downward over the ureter to reach the base of the bladder to the inner side of the ureter. Here it lies to the inner side of and parallel to the seminal vesieles, and finally joins with the duct of the latter, near the base of the prostate, to form the common ejaculatory duct. The course of the latter through the prostate has already been described.

At its commencement the vas deferens is rather tortuous; it then becomes straight, and of nearly uniform caliber, but at the base of the 
bladder near its termination it is dilated into a large sacculated ampulla, resembling a seminal vesicle, and then narrows again before joining with the latter. It measures nearly two feet in length, and one tenth of an inch in its average diameter. The walls of the vas deferens are dense and thick, so that it ean readily be detected by its firm, cord-like sensation when pinched. The wall consists of a thick external fibrous coat; a middle muscular coat, the inner fibers of which are circular, the outer longitudinal ; and an internal mucous coat. The muscular coat is thick and yellowish, and near the epididymis there is an additional thin longitudinal layer. The mucous membrane shows three or four longitudinal ridges. The epithelium is columnar, and not ciliated. In the ampulla occur tubular mucous glands, and in many other respects this part resembles the seminal vesicles.

(For the anatomy of the seminal vesicles, see the article on Tur Seminal Vesicles.) 


\section{DISEASES OF THE PENIS.}

By RAMON GUITERAS, M. D.

\section{ABNORMITIES OF THE PENIS.}

Absence of the Penis is rare. Vinogrodoff reported the case of a boy seven years of age in whom there was no penis, although the scrotum and testicles were normal. In some cases neither penis nor scrotum are present. In other eases the penis and anus are absent, as in one case reported by Agnew, where a man passed fæces from his mouth for a period of forty years. Absence of penis may be due to accident, self-mutilation, wounds followed by sloughing, phagedenic diseases, etc. A case came under my observation at the Charity Hospital, some years ago, of a man thirty-five years of age, who had been suffering from a chancroidal ulceration of the glans penis, evidently phagedenic. He had used a wash prescribed for him by an apothecary. The ulceration, however, continued to extend, and had eaten away lis penis up to the pubes, when he decided to enter the hospital, fearing that it might ulcerate into the bladder.

In still other instances the absence of the penis is due to operation, as in the case of eunuchs. There are two classes of eunuchs, the complete and the ineomplete. The complete, employed in the harems of the East, are castrated when small, either by amputation or by tying a ligature about the genitalia close to the pubes and then placing them in sand-holes until gangrene and slough of the genitals follow. This is a very dangerous procedure, and the majority of the children die from its effects.

The incomplete or simply castrated eunuchs were principally among the Italians, where they were formerly seen as soprano singers. The operation is still performed among certain sects in parts of Russia and Roumania.

Rudimentary Penis.-Instances of this abnormity are often seen. Dr. Joseph Jones, of New Orleans, reported a ease in which the penis was but an inch in length, the testicles and scrotum being absent. In other cases where the penis is absent and the scrotum present this defect is generally due to an imperfect fusion of the septum between the anterior or uro-genital and the posterior or anal portion, thus producing a broken or bridged eondition of the cloacal wall. This not only aceounts for the 
absence of the penis from the nonappearance of the genital eminence, but also for the displacement of the urethra, so that its orifice is in the rectum or some fold in the skin.

Concealed Penis.-This is occasionally observed in infants, either beneath the skin folds of a cleft scrotum or buried beneath the fat of the mons. In these cases careful examination will reveal it as a small, firm cylinder.

The operative measure consists of an incision made through the overlying tissues, thus liberating the organ-flaps sufficient to cover it being taken from the adjoining skin.

Multiple Penis.-Such cases are rare. They may be situated either beside one another, over one another, or separated from one another. The functions of urination and copulation are generally intact. The case reported by Ernest Hart (Lancet, 1866) was a very interesting one. A strong, healthy man had a withered thigh, leg, and foot between his thighs. In front of this was a scrotum, and on either side testicles and a penis, all in thorough working order.

Imperfect or Deformed Penis.-The two principal varieties of imperfect and deformed penis are those connected with hermaphroditismus and hypo- and epispadias. True hermaphrodism-i. e., when one side has the male germinal gland, the testicle, and the other the ovaryare rarely if ever seen, the eases which come principally to our notice being those of the spurious variety, which are very puzzling at birth, in that the organs belonging to both sexes seem to be present. For instance, a male with a cleft scrotum, undescended testicles, and a diminutive, hypospadic penis, with the urethral opening in the perinæum, closely resembles a female with elongated clitoris and an atresic vagina.

The following cases, one of true the other of pseudo hermaphrodism, illustrate the characteristics of the two conditions:

Case of Lukomsky.-Patient thirty years of age; penis two inches long, without urethra, below which was a scrotum with two testicles. Below the scrotum was a normal vulva, with a labia majora, minora, and clitoris, beneath which was discovered the urethral opening. The vagina was about three inches in length, with a normal uterus and cervix. The patient had never menstruated. In having coitus with a female, a whitish fluid was ejaculated through the vaginal slit.

Boudareff's Case.-Patient thirty-five years of age; genital slit about one and one fourth inch. Labia majora contained oval bodies resembling testicles, with spermatic cords running from them into the pelvis. Labia minora small, resembling continuation of prepuce of clitoris. Clitoris was two and a half inches long and two inches in circumference. It contained no urethra. The urethral orifice was at the upper angle of the vaginal opening, and admitted two fingers, through which the patient probably had intercourse. The vagina was shallow, measuring a little over an inch, and ending in a rudimentary uterus. Patient had never menstruated. 
From the numerous cases of true and pseudo hermaphroditismus quoted by different authors, certain conclusions may be drawn :

1. That the greater part of these are cases of hypospadic males.

2. That the predominating sex should be carefully searched for and decided upon by physiological as well as anatomical observations; that in so doing the two principal signs to be considered are, the presence of menstrual fluid, or a discharge from some point of a whitish fluid containing spermatozoa.

3. That too much importance should not be attaclied in adult life to their male or female characteristics and sexual affinities, as these are governed to a great extent by the sex according to which they were brought up.

Treatment.-Careful examination having been made and the predominating sex decided upon, surgical interference should at once be instituted to correct, if possible, by plastic work the existing state of affairs, and to favor the healthy development of the parts by freeing them from any tissue tending to ind nce the deformity by its interference; also, to cover over or remove any crevices or tissues which are of no other use than to disguise or deform the true sex. In some cases, where it is uncertain which functions will predominate, it is better to wait and perform simple temporary operations until further developments take place.

Malformation and congenital defects of the sexual organs are generally accompanied by imbecility, idiocy, and epilepsy. Bourneville and Sollier, from a careful study of a large number of these cases, reported that of 223 under thirteen years of age, 164 were idiots, while 59 were idiotic and epileptic as well. Of 505 cases over thirteen years of age, 172 were idiotic and 333 were affected with epilepsy. The cases were principally those of phimosis, hypospadias, atrophy of testicles, ectopy of testicles, and bell-clapper glans.

Undersized Penis is a condition occasionally found where, although the organ is perfectly formed, it is not large enough to permit of satisfactory connection. In such cases the organ may be enlarged by the use of various forms of apparatus which have been invented for this purpose, the object being to increase the amount of blood in the part, and thus favor hypertrophy.

Oversized Penis is a condition which often causes a great deal of trouble to the possessor. First, because, if he leads a dissipated life, he is in constant danger of having its tissues torn, and therefore infected through coitus; and, secondly, because he is liable to injure the organs of those with whom he may cohabitate. 


\section{INJURIES TO THE PENIS.}

Wounds of the Penis.-The classification is the same as for wounds of other parts.

Incised wounds are generally made with knife or razor by jealous women, or by monomaniacs for the purpose of self-mutilation. They are often irregular on account of the tough, trabecular nature of the tissue and the extreme mobility of the part.

Lacerated wounds are not common; they are most often due to the organ being caught in machinery.

Punctured wounds are also rare, except in time of war, from sword and bayonet thrusts.

Contused wounds are caused by blows, kicks, jams, and similar accidents. They are often serious, and are liable to be complicated with fracture of the penis or rupture of the urethra.

Strangulation is generally classified with this condition. It has heen observed in boys suffering from incontinence of urine or seminal emissions, from tying a string around the organ as a preventive. If the resulting strangulation is not relieved, the string may cut through the urethra, or gangrene and slongh of the penis may result. Oftentimes after the string has been removed a band of ulceration remains, which may give rise to a cicatrix, interfering with the function of the organ. Strangulation may result from other causes. Mr. Potts reported the case of a man who came to him with his organ greatly swollen and discolored, the glans being quite black. About two thirds back on the organ was a ring deeply imbedded, which had been slipped over it by some woman a few hours before. This was filed off by passing a director beneath it.

A peculiar case was observed at the Chambers Street Hospital some years ago. A man had, for some unaccountable reason, put his penis in a bottle. Strangulation ensued, and he entered the hospital some hours afterward wearing the bottle. The part of the penis within the bottle was very much swollen and congested, and the organ was liberated with great difficulty.

Gunshot-wounds of the penis are rare, occurring usually in time of war. The urethra may be injured, or a part or the whole of the penis may be carried away.

Treatment.-The three dangers to be guarded against are hæmorrhage, urinary infiltration, and curvature as the results of a wound of the corpora cavernosa. In all cases wash, cleanse, and thoronghly remove any débris. Ligate severed vessels, and approximate and sew up incised wounds with silk or wire. If the urethra is cut, its edges should be brought together and a catheter retained. The subject of urinary infiltration is treated 
elsewhere. From all these wounds cicatrices causing curvature, as in cases of fracture, may occur, the curve depending on the site of the wound.

Fracture of the Penis is generally due to injuries received when the organ is in a state of erection, by "missing the mark," as Bryant aptly terms it, when in coitus the penis strikes the outer wall of the vulva or the pubes; or it may occur in relieving a chordee by a blow- "breaking the chordee," as it is termed. In this manner a traumatic stricture may be caused. Fracture of the penis is followed by pain and induration in one or both of the corpora cavernosa. Later an atrophied condition at this point, which interferes with a complete turgescence of that side, may result in lateral curvature.

Fracture of the penis may be complicated with rupture of the urethra, in which ease there is often an abundant urethral hæmorrhage. Fracture of the corpus spongiosum may occur as well, though this is rare. Bryant mentions the case of a boy nineteen years of age who was bitten by a horse, entirely severing the corpus spongiosum, which prolapsed from the urethra as a fleshy mass. This was of no special inconvenience, the urine escaping by its side.

Treatment.-Rest in bed should be enforced, applications of cold water or solution of lead and opium ; pass and retain gum-elastic eatheter. If œedema and swelling are great and slough threatens, full incisions may be resorted to. The organ should be kept elevated.

Dislocation of the Penis.-Malinouski reports a case in an adult where the penis was dislocated into the scrotum by mechanical violence and remained there for four months. It was then liberated and reclad with its own integument patched ont with scrotal. The power of coitus quickly returned.

J. B. Field reports a case where the penis and both testicles were dislocated up under the abdominal integument above Poupart's ligament.

A. P. Hill reported the case of a boy who was caught in a hay-rake, lacerating his scrotum and perinæum, and dislocating his penis upward under the integument of the linea alba. This was not discovered until retention of urine set in. The sheath of the penis was then split up and the organ fished down into place.

\section{CUTANEOUS AFFECTIONS.}

Cutaneous affections of the penis may be classified as acute and chronic. The acute embrace eczema, erysipelas, scabies, and herpes; the chronic include lupus, tuberculosis, epithelioma, and verrucæ. Of these, herpes, epithelioma, and verrucæ will be considered elsewhere. There are other affections, acute and chronic, which, though involving the skin, will for the sake of classification be considered later, as lymphangitis, adenoma, elephantiasis, etc. 
Eczema.-Eczema of the penis may be either of the erythematous or vesicular form. Where scrotum and penis are both affected it is apt to be chronic and very rebellious, and is often accompanied by phimosis and enlargement of the inguinal glands. It is generally found in men between the ages of thirty and fifty, who are of a catarrhal, gouty, or rheumatic diathesis. Its favorite seats are the prepuce near the margin and the base of the penis. Eczema of the prepuce is generally subacute, and occasioned by the rubbing of the organ against the underclothing or other ill-fitting garments. When accompanied by an inflamed and infiltrated base and enlarged glands in the groin, it often causes considerable anxiety to the patient, especially if it occurs soon after a suspicious coitus, as he is very apt to fear that he has the initial lesion of syphilis. Eczema may appear on the inner layer of the prepuce instead of the outer. Eczema of the base of the penis is generally chronic and slightly infiltrated. It may cause very little inconvenience for months or years.

Treatment.-The acute variety is best treated by powders. The patient should be directed to wash the surface twice a day with hot water, and afterward to sprinkle on a powder consisting of boracic acid, bismuth, and lycopodium or starch. In some cases, after sprinkling with the powder, I lay over it a soft piece of sheet lint covered with vaseline. Eczema in this location, as elsewhere, is often due to faulty excretion of the kidneys or an accumulation of excrementitions matters in the blood in individuals of a gouty or rheumatic diathesis. Considerable assistance may be derived from the use of acetate of potash, grs. xv, t. i. d. Inflamed or enlarged inguinal glands should be painted with iodine.

In the chronic variety, such as occurs abont the base of the penis, ointments seem to be most efficacious; and as it is necessary in these cases to resort to mild stimulation, good results are often obtained by adding oil of cade, one half drachm to one drachm to the ounce of zine oxide, Lassar, or diachylon ointment. If much moisture exist, dust on the powder above mentioned.

Scabies.-Scabies is the cutaneous affection which causes perhaps more alarm than any other acute nonvenereal lesion found on the penis. The lesions may be either papular or pustular. A patient with one or two papules or pustules on the organ, enlarged glands in the groin, and an eruption on the body, may believe that he has syphilis, especially if he happened to have a sore throat, a cold, or malaria at the same time. Careful examination, however, would at once show the difference. The eruption of scalies itches, and is acute and irritable. The presence of burrows is an important diagnostic sign, the most typical of which are found on the penis and between the fingers. The lesions of this disease on the penis at times resemble moist specific papules or mucous patches, for which they are often mistaken. 
Treatment.-Wash with soap and water, and apply the following ointment: Carbolic acid, ten grains ; oil cadini, one half drachm ; ung. sulph., one ounce. Scabies of the rest of the body should be treated in the same way every night for five nights, and the patient should be directed to put on fresh linen every morning.

Lupus. - Lupus of the penis is a rare affection. The erythematous variety is differentiated from epithelioma, with which it is very often confounded, by the duration and slowness of the disease, the characteristic scaling, and the presence of cicatricial tissue in the places that have already been invaded. Lupus vulgaris differs also in duration, being a disease beginning during boyhood or youth, while epithelioma is a disease of old age. Lupus advances slowly, leaving cicatrices behind it; while epithelioma advances more rapidly, quickly tending to glandular involvement and ulceration.

Tuberculosis of the Penis is an exceedingly rare disease. Kraske has recently reported a case of this kind in a man forty-nine years of age, who had tubercular-looking nlcers on his glans. Amputation of the organ was performed, and a microscopical examination confirmed the diagnosis, both typical giant cells and tubercle bacilli being found. The grow.th was more marked deep in the substance of the organ, thus showing that the infection was not a surface one.

\section{LYMPHATIC AFFECTIONS OF 'THE PENIS.}

The various lymphatic and suppurative inflammations of the penis, lymphangitis, lymphitis, abscesses, phlegmon, erysipelas, etc., are closely allied, in that the infecting material is introdnced through the lymphatic channels and the inflammation begins around them.

Lymphangitis. - In one variety of lympliangitis a hard cord appears on the dorsum of the penis, the result of acute inflammation, as gonorrhœa. This afterward becomes reddened, painful, and tender, and is followed by enlargement of the inguinal glands.

There is another form of lymphangitis, in which the lymphatics on the side of the penis become very much enlarged, and often the whole organ is swollen, red, and inflamed. When the lymphatic inflammation becomes so great that suppuration takes place, the pus is generally discharged through the prepuce, coming out from its inner side.

Still another variety of lymphangitis, known as virulent lymphitis, occurs generally in connection with chancroids, in which case the virus sets up an inflammation in the lymphatics of the penis before they reach their terminal ganglia.

Treatment.-Lymphangitis of the penis should be treated during the acute stage by lead and opium wash, hot or cold water, and elevation of the organ. If suppuration threatens, poultice; if cellulitis develop, poul- 
tice, open, and treat as cellulitis elsewhere. In cases of lymphitis, with virulent abscesses, open and treat as buboes.

Abscess of the Penis may develop, as elsewhere on the body, with no apparent cause. I remember the case of an Italian, possessing an unusually large organ, on the left side near the middle of which was an abscess one or two inches in diameter and one half inch in height. The man was otherwise in perfect health, and not suffering from any venereal disease.

Treatment.-Poultice until fluctuation takes place, then open and dress antiseptically.

Cellular Inflammation of the Organ, or Penitis, is of rare occurrence, and usually complicates some other existing disease. It is generally diffuse, and occupies the lower part of the organ, which is very much swollen, reddened, and infiltrated, with more or less marked fluctuation. There may be a considerable elevation of temperature.

Treatment.-Poultice; quinine tonics; open when fluctuation takes place, and treat antiseptically.

Periurethral Phlegmon is a little abscess occurring in the cellular tissue beneath the urethra. It may develop anywhere along the penile portion of the urethra, although the most frequent seats are over the fossa navicularis and at the peno-scrotal angle. It is generally due to a very acute urethritis, or to neglect or over-indulgence during an attack. The phlegmons vary in volume from the size of a pea to that of a walnut; the larger ones usually occur about the peno-scrotal angle. They develop rapidly, and break, if not lanced, leaving a deep hole behind; occasionally they undergo resolution.

Treatment.-Poultice until fluctuation is observed, then open and wash with antiseptic solution; after this treat with astringent powder, iodoform, or aristol; or by keeping on a piece of cotton soaked with lotio nigra, aromatic wine, or 1-to-2,000 bichloride solntion over the opening. Cauterize the base every second or third day until it looks healthy.

Folliculitis.-This is ordinarily a chronic process. In cases of chronic urethritis one of the follicles of Morgagni becomes inflamed, and its lining membrane hypertrophies, thus occlnding the duct. The secretion of the follicle, and perhaps some pus, accumulate in this sac, which continues to grow slowly like a sebaceous cyst. This may remain indolent for a time, when, on account of some irritation, it will inflame, discharge its contents, and leave a fistulons opening behind.

Treatment.-Open, and treat antiseptically.

Erysipelas of the Penis may occur hele as elsewhere, either primarily in the organ or secondarily throngh extension from the surrounding tissues. This generally terminates in resolution, but it may result in suppuration or even in gangrene. 
Treatment.-Lead and opium wash locally every two hours. Tineture of chloride of iron and some saline diuretic internally. If it goes on to suppuration or gangrene, treat accordingly.

Gangrene of the Penis is a rare condition, depending upon some mechanical constriction, a condition of phimosis or paraphimosis, phagedenic ulceration of the organ, or urinary extravasation. Again, it may follow the exanthematous fevers, especially small-pox and typhoid fever, or it may be due to poisoning by ergot. There are swelling and discoloration of the organ, followed by diminished sensibility ; a line of demarcation is formed, and a sulcus behind this, through which urine escapes.

Treatment.-Good diet. Charcoal poultices till part sloughs off; then treat as an open sore.

\section{TUMORS OF THE PENIS.}

These may be either malignant or nonmalignant.

The nonmalignant tumors are cystic (mucous or sebaceous), adenoma, fibroids, vascular tumors, and elephantiasis.

Mucous Cystic Tumors are rare; they may occur anywhere on the balano-preputial mncous membrane. Sebaceons cysts may occur in any region where sebaceous glands are present.

They should be treated like wens in other localities.

Vascular Tumors are of three kinds-capillary, as nævi; venous, as angiomata ; and arterial, as erectile. Of these, the most common variety is the venous or the varices, found on the dorsum, consisting of large, soft masses of tortuous veins. They have been reported of such a size as to interfere with the proper erection of the organ.

Treatment.-Excision, and treatment by means of the galvanic current, produce the best results. Parona reports three cases of varix of the dorsal vein cured by injections of half a gramme of saturated solution of chloral hydrate.

Capillary and arterial tumors are exceedingly rare.

Fibroid Tumors usually occur upon the body of the penis, either on the side or on the dorsum. They are probably due to a plastic effusion into the trabecular structure of the corpora cavernosa, and a fibroid development as a result.

Marcus Beck reports the case of a man fifty-three years of age, healthy and robust, who had on the left side of the penis, below the glans, a tumor about the size of a hen's egg, irregular and nodular in shape. Its base almost surrounded the penis. It was very hard, and free from pain. The lower part of the tumor was in an ulcerated condition. Thirteen years before, a warty growth was discovered on the inner side of the prepuce near the base of the glans. In two years this had reached the size of a walnut. Circumcision was then performed, removing the growth. Two years later a tumor developed in the cicatrix. This grew slowly until six months ago, when it began to develop rapidly, interfering 
with coitus, though not with micturition. Amputation of the penis was performed. The growth was found to be continuous with the corpus spongiosum and the corpora cavernosa, evidently arising from the latter. Microscopically it showed a delicate, white, fibrous structure.

Elephantiasis of the penis may occur alone, but it is generally associated with that of the scrotum. In this case it may assume a large size, or it may become completely hidden in the enlarged serotum, only a small hole remaining for urinating, etc. After an operation, however, the penis recovers its former activity. The literature on this subject is exceedingly limited. I have seen but one case, that of R. W. Taylor, of New York city. It occurred in the prepuce of an old soldier, as a result of a former wound of lymphaties on the dorsum of the penis near its base, received in the late war. The growth of the prepuce progressed slowly, and when the patient presented himself was about the size of a duck's egg. Circumcision was performed, giving very good results, the integument of the body of the penis being very slightly involved.

M. Trippier, in the Gazette Médicale of 1836, described the ease of a young man suffering with elephantiasis of the penis, which had been growing for thirteen years. At date the organ was nine inches in length and seven in cireumference. This case was occasioned by a carbuncle, which had interfered with the lymphatic circulation of the organ.

Adenoma of the penis is rare, the following being the only case which has ever come under my observation:

Man, thirty-four years of age. No venereal history other than that of a urethritis eighteen years ago. Two years previously he noticed that his prepuce was swollen and oedematous; the inflamed condition became gradually less diffuse and more nodular. The skin of the prepuce and body of the penis eontained several small subcutaneous tumors about the size of a pea or marble, and also eysts containing a thick, mucilaginous fluid. The inguinal glands were enlarged.

Horny Growth of the penis is comparatively rare. An interesting case was reported by J. H. Brinton, in the Medical News-that of a farmer, sixty-two years of age. The horny growth sprang from the base of the glans and body of the penis. It was one and seven eighths inehes in length, and one and three eighths in eircumference at its base. There was also a plate of horny tissue eneircling the end of the glans penis, surrounding and narrowing the meatus. The frænum was gone. The horn was striated like an exaggerated thumb-nail, and when dry of a smooth, polished appearance.

Malignant Tumors of the Penis.-Cancer and sarcoma are most common.

Scirrhous cancer occasionally occurs, but is very rare; the more common form is epithelioma. 
Epithelioma of the penis usually develops as a arty growth, most frequently situated on the inner side of the prepuce near its margin, or near, or at the sulcus. This causes very little pain or annoyance, as it is at first superficial and its growth slow. Soon, however, sufficient infiltration occurs to make the retracting of the prepuce difficult, and finally complete or partial phimosis exists, and yet the patient has no disagree. able symptoms, excepting perhaps a few vague pains in this locality. A little later, however, an offensive ichorous discharge takes place from beneath the prepuce; painful erections occur at night, followed by a bloody tinge to the discharge, with an area of induration beneath the prepuce. The disease occurs in men of middle or past middle age, perhaps cachectic, but often strong and lusty; the inguinal giands may or may not be enlarged. Epithelioma spreads slowly, involves the lymphatics slowly, and infects the general system still more slowly. In the further progress of the case ulceration takes place, the lymphatics become infiltrated, enlarged glands appear in the groins, and amputation is imperative. If this growth is examined microscopically, it will be found to consist of epithelial cells, flattened and crowded together, with a tendency to arrange themselves in nests, and situated in the interstices of a network of fibrous tissue.

Treatment.-The operative procedure depends very much upon the stage of development when the patient first seeks advice. There are four operations to choose between, depending on the situation, duration, and development of complications of the disease, viz., circumcision, curetting, excision, and amputation. There is no disease of this organ where the variety of operation depends as much upon the surgeon's individual judgment as it does in this one. If the lesion is situated on the prepuce near its margin, of short duration and no complication of the lymphatics, circumcision shonld be performed. If situated near the balano-preputial fold, on either the prepuce or the glans, uncomplicated, it may be either curetted or excised. A good method is first to curette thoroughly and cauterize the base; then, if there be a recurrence, excise. If after this a return takes place, amputate. If ulceration has begun and is at all extensive, amputate immediately.

A question arises as to the importance to be attached to glandular enlargements. Almost any lesion of the penis in an irritated condition may give rise to enlarged inguinal glands. The enlarged glands in a case of this kind may be due to irritation or inflammation, or, on the other hand, to direct cancerous infiltration. If dne to the first, they will subside after removal of the growth. If due to cancerous infiltration, they should be removed at once.

Methods of amputation are as follows:

1. Ligature by a wire or string, to be tightened every day until 
slough of parts takes place. This is an old, crude way, and is not advisable.

2. Ecraseur. This is a method formerly advocated in France, but with few supporters in this country.

3. Galvano-cautery. This mode of amputation is a very good one, as it controls hæmorrhage at the same time. It should be performed slowly, with catheter inserted in the urethra.

4. Cutting operation. In amputation with the knife, it must be remembered that the skin and urethra shonld be cut longer than the shaft of the penis. This prevents a shrinking back of the urethra, and allows enough loose skin from the shaft to cover the bleeding corpora cavernosa. A good plan is to sew skin and urethra together after the operation. Make a sweeping incision through the organ one half inch in front of the place where we intend to finally amputate, then a dorsal and belly incision in the median line, and dissect up skin-flaps for one half inch. Next secure corpora cavernosa on either side by forceps or ligature, and amputate at the point to which the flaps have been dissected back. We now have urethra and corpus spongiosum left one half inch in length. We amputate the corpus spongiosum, having dissected it from the urethra, which we split up in the median line superiorly and inferiorly and stitch to the skin-flaps on either side, then bringing urethra together again with a stitch above and below. There is no necessity for cutting the urethra one half inch longer than the corpora cavernosa, as one fourth inch will be sufficient.

H. Keller, of St. Louis, advocates uniting by deeply lying transverse sutures both sides of the corpora cavernosa. In this way the two cut surfaces are in apposition, and the sheath of both corpora cavernosa are in contact in front and behind. Then follows a second row of stitches, drawing together the external skin of the penis over the sutured stump. Oozing is thus controlled.

Horteloup, in performing amputation for epithelioma, always removes the inguinal glands on both sides.

Sarcoma of the penis is rare and generally rapidly fatal. It may develop on glans or corpora cavernosa.

Treatment.-Immediate operation.

\section{MORBID CONDITIONS OF THE PREPUCE.}

Phimosis is an inability to draw the prepuce back over the glans; and paraphimosis is an inability to draw the prepuce forward over the glans when once it has been drawn back. Phimosis is often congenital; paraphimosis is always pathological.

Phimosis is of two kinds, congenital and accidental.

Congenital Phimosis causes a great deal of irritation in childhood, and 
may give rise to serious nervous as well as local troubles. Retention of urine may be due to this cause. As the individual grows older, other complications may develop, such as balanitis, constant itching, pain, inordinate excitability of the organ, frequent erections, erotic dreams, seminal emissions, vesical tenesmus, incontinence of urine, general lassitude, and imperfect development of the testicles and penis. The nervous symptoms which may follow later in life are coxalgia, moral and physical depression, convulsions, epileptiform or other, chorea, inco-ordinate muscular movements, including those of speech, sterility, paresis, hyperæsthesia or a mental condition resembling hysteria or hypochondriasis.

In some cases the whole or a part of the mucous membrane lining the prepuce may be adherent to that of the glans. In still other cases preputial calculi, hard calcareous deposits, may take place in this adherent prepuce, or in the interspaces between the adhesion of the glans to the prepuce. These are easily removed when the prepuce is liberated. In congenital phimosis the organ looks pointed or wedge-shaped, and there is generally abundant loose integument about its end even when the prepuce is adherent. On retracting this tissue, the orifice of the prepuce can be seen as a little, round, tight ring about one eighth inch in diameter, and surrounded by mucous tissue. In the center of this little ring will be seen a vertical slit-the urethra. The redundant tissue resembling mucous membrane surrounding this ring becomes inverted when the pressure of the fingers is removed, and forms a trough, through which the urine escapes.

Treatment.-Circumcision is the treatment for congenital phimosis.

Accidental Phimosis.-This term is applied to the condition when retraction of the prepuce is prevented by inflammation of the balanopreputial fold or swelling of the glans, as in balanitis, or from the presence of new growths or ulcerative lesions, as verrucx, chancre, or chancroid.

The treatment of accidental phimosis may be expectant or operative.

Expectant treatment consists in the injection of some astringent, disinfectant, soothing, or cleansing solution beneath the prepuce, to reduce the inflammation; after the subsidence of which, operative procedure may be resorted to. This applies principally to acute accidental phimosis, and that condition of subacute or chronic sluggish thickening following the initial lesion of syphilis.

Operative procedure consists either in circumcision, the dorsal incision, the dorsal excision of the $V$-shaped piece, or the lateral incisions. The treatment depends also a great deal upon the cause and the acuteness of the attack, and here the physician must rely in a great measure upon the history. Verrucæ or venereal warts are mentioned as the cause of accidental phimosis; they are generally of long standing, coming on slowly, 
the acute inflammation being due to some sudden source of irritation. In a case of this kind the physician should inject subpreputially a solution of bichloride 1-10,000 to a $1-5,000$, solution of permanganate of potash $1-500$ to $1-100$, solution of sulphate of zine 1-100, or carbolic acid 1-100. $A$ solution of borax gr. $x x$, glycerin $3 j$, and rose-water $z j$, is a nice preparation and of great efficacy in these cases. When the inflammation subsides and the phimosis is reduced the vegetations may be removed. If the inflammation subsides, but on account of hypertrophy of the verruce the phimosis is not reduced, make Taylor's lateral incisions, which will give us antero-posterior tlaps, and treat accordingly.

In phimosis due to concealed ulcers the prepnce is very much inflamed, swollen, and cedematous. The discharge of pus from the preputial trough is abundant-so much so that in some cases the dripping is almost constant. This pus is often of so rich a color that one might imagine, were it not for the history, that there was an acute attack of urethritis to deal with instead of concealed ulceration. This condition is often difficult to diagnosticate, and must be treated more or less blindly. One thing must be remembered, that however bad the case may appear, or however imperative operative procedure may seem, a few days of quiet, regular life, with a tonic, if the patient's system is below par, and frequent subpreputial irrigations of warm water, followed by astringent injections, will in ninety-five per cent of the eases stop or diminish the amount of the discharge, allow the retraction of the prepuce as easily as before, and permit the regular treatment, as in eases of ordinary superficial ulcerations. Very often the condition may be aided by immersing the organ for fifteen minutes every two hours in warm water, or by enveloping it in soft eloths saturated with lotio plumbi et opii, and keeping it elevated.

If no treatment is pursned, the following may result: First, slough of the prepuce or glans; second, ulceration throngh prepuce; or, third, uleeration through prepuce, with protrusion of the glans. The first of these conditions is rare. It is due to too much pressure, which should be relieved by preputial incisions. The second. is due to neglect, or the ulcer having taken on a phagedenic form. The third may be caused by localized sloughing or ulceration through the dorsum of the prepuce. The protruding glans, beeoming caught in this hole, is pressed upon by the sides, and an cedematous condition takes place. This may be relieved or reduced by elevation, and constant applications of lotio plumbi et opii, or hot-water immersions. When the inflammation and ulceration have subsided, trim off the prepuce at a level with the proximal border of the ulceration.

Phimosis due to simple balanitis and posthitis is not so acute or severe, and more amenable to treatment. Any of the injections first mentioned would probably reduce the inflammation and allow retraction 
of the prepuce. In cases of chronic accidental phimosis due to syphilitic or other cicatrices circumcision is called for.

Circumcision was in vogue long before the Christian era, not only among the Jews but among various races on the different continents. It has always been a custom among the Egyptians, Arabians, Turks, Persians, East Indians, North and South American Indians, certain tribes in Africa, Australians, and other South Sea islanders. Among different races it was customary to perform it at different ages-e. g., among the Jews on the eighth day; among the Egyptians from the sixth to the tenth year; among the Arabians when they were about to enter puberty. In olden times the methods of procedure were very crude ; the prepuce was generally chopped off with a piece of shell or a sharpened stone.

As the science of surgery has advanced, other and more delicate operations have succeeded these crude methods, and many instruments, clamps, etc., fastened by springs and catches, have been devised to assist the various operative procedures.

In the blades on either side of certain of these clamps are spaces two to three inches in length and wide enough to admit a needle. In others are little holes one fourth of an inch apart and opposite to one another, through which needles may be passed. With an instrument of this kind the quickest method of circumcision can be performed. The prepuce consists of two layers, an upper or integumentary and an inner or mucous, the space between which is filled with cellular tissue. These two layers are attached in front by a margin in common; behind, one is continuous with the skin of the shaft of the penis, abdomen, scrotum, etc., while the other or mucous membrane is made fast behind the corona, and from there ex-

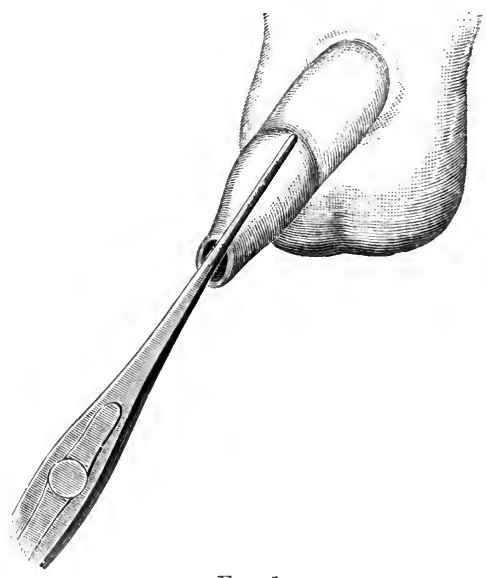

Fig. 1. tends up over the glans to the meatus.

The following are a few easy and rapid methods of performing circumcision : 1. Draw the prepuce forward by means of forceps, inserting inner blade up beyond corona and catching mucous membrane firmly as in Fig. 1; put on a clamp, having first determined the line along which the circumcision is to be made; fasten the clamp, pass four stitches through the slit, as in Fig. 2, or eyes, as in Fig. 3, in the blades of the clamp, with scalpel cut off the prepuce above the elamp, retract the prepuce, draw up the stitehes in the middle and eut them in two, thus mak- 
ing four stitches on each side, or eight in all. If the preputial mucous membrane is adherent, or too tight, slit it up in the median line over the dorsum; tie the sutures on either side, and dress accordingly. This is a

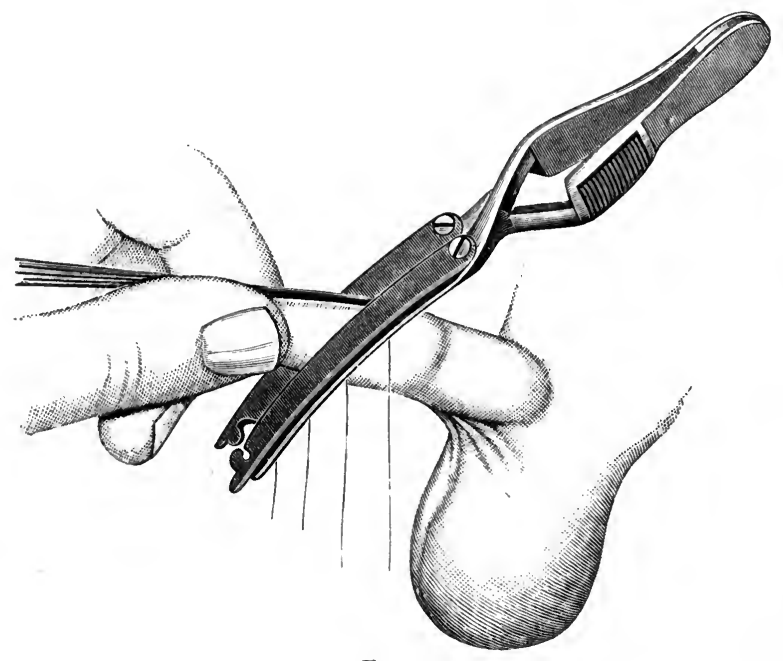

FIG. 2.

quick and simple method, but in order to clear the glans an inch or more of redundant mucous membrane must be left, which detracts from its appearance and leaves a fold of tissue. 2. Catch the preputial margin on either side of the median line of the dorsum with forceps, and draw the prepuce forward; with scissors cut up to the corona through both layers, as in Fig. 4 ; then, taking forceps No. 1 in the left hand, cut around over the corona to the frænum, as in Fig. 5 ; then, holding forceps No. 2, cut around on the other side to the frænum. Sew the two layers together on either side by continuous suture. By this method is generally obtained a good result, although occasionally the two sides

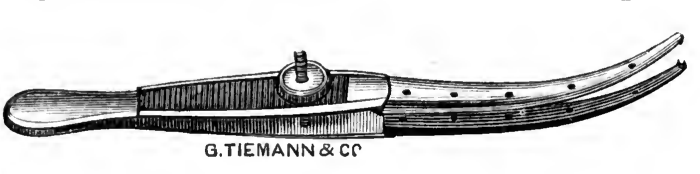

Fig. 3. are cut unevenly. 3. Before describing my favorite method of performing this operation, which gives the best results, I may say that circumcision can in nearly every case be performed under the influence of cocaine, if properly employed. Ether is unwarranted and uncalled for. It requires some time-from ten to thirty minutes-to get the patient under the effects of cocaine. I have never seen a case where the parts could not be cocainized, and that, too, generally by surface applications and subpreputial injections, without resorting to subcutaneous in- 
jections into the cellular tissue. I use a ten per cent solution of cocaine, injected subpreputially, and poured on a thin film of absorbent cotton on the outside of the prepuce. Before tha operation I mark the proposed line of incision on the organ as it hangs in a flaccid state, allowing for retraction or shrinkage of the skin : beginning at the end I wind the penis with a narrow bandage to the base, where I ligate ; patients are more easily cocainized after the penis has been wound than before, probably because less of the cocaine is carried away into the circulation, and therefore comes more readily in contact with the local nerve filaments. I now adjust my clamp (usually Fisher's, Fig. 3), and cut off the prepuce with a scalpel; I then remove the clamp, allowing the skin to retract, after which I trim the mucous membrane around one fourth inch from the corona, and sew the two cut surfaces together. The first stitch should be a dorsal one in the median line, followed by three lateral stitches on either side between this and the frænum. These sutures should be

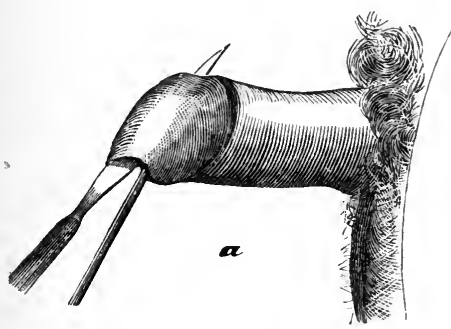

Fig. 4.

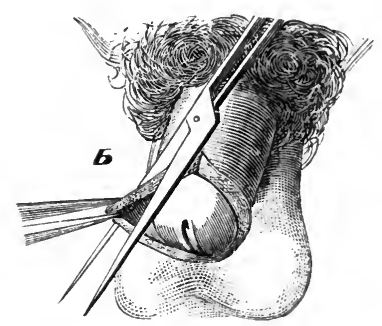

Fig. 5 .

either of catgut or fine silk, and, if of the latter, should be taken ont in two or three days. Dressing, iodoform ganze. Bandage a piece of oiled silk over the glans, with an aperture through which the urine can be passed without soiling the dressing. Put the patient to bed, with a cradle over the hips; give bromide of potash in large doses, to prevent erections; it generally requires sixty grains, given in fifteen-grain doses, to keep the parts quiet. In this way union by first intention is generally obtained, and a clean-cut surface results.

Palmer, of Lonisville, Ky., advocates the dorsal incision in the infant in preference to circumcision, arguing that it is less trouble to the surgeon and the infant at the time of operation, answers all the therapentic indications, and that atrophy of the unnsed cover takes place, insuring him in adult life as well finished an organ as if circumcision had been performed. This seems a very rational procedure.

Paraphimosis. - This term is applied to the condition where the prepuce becomes strangulated behind the glans; it may be either reducible or irreducible. It occurs generally in cases of gonorrhoea, chan- 
croid, or chancre, although it may result from nonvenereal causes, even from coitus. A recent condition of this kind is easily reducible, but after a few days a plastic infiltration takes place into the strangulated tissue, adhesions form, and reduction is impossible unless the restricting bands are cut, and even then it is effected with difficulty. The appearance of the organ in this condition is enlarged, the glans swollen, red, and tense. Over and behind the sulcus is a brawny swelling, in some places white, in others pink or red, densely infiltrated, which in old cases has a hard and cartilaginous feel, especially when due to the initial lesion of syphilis. Below this the tissues are inflamed and œdematous, and behind the organ presents a wrinkled appearance. If no treatment is given, ulceration will probably take place through the constricting preputial orifice, thus relieving the strangulation. It may in rare cases result in gangrene, involving the skin and glans, or it may even slough through into the urethra.

Treatment.-First drive the blood out of the organ by squeezing it with the hand or winding it with a narrow bandage, either in toto or simply the glans, in order to reduce it sufficiently to push it through the preputial orifice. If this does not answer, bathe the organ in cold water and elevate it. If much cedema is present, scarification may be practiced. In order to reduce it, grease the glans well with sweet oil, working some of it under the preputial constriction, encircle the body of

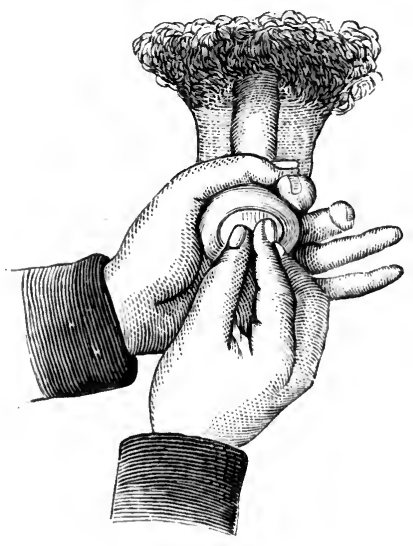

Fig. 6 . the penis with the thumb and forefinger of the left hand, as in Fig. 6, grasp the glans between the thumb, index, and middle fingers of the right hand, and squeeze until bloodless; then proceed to work the finger-nails under the preputial orifice or constriction, all the time squeezing the glans, and continue pressure until the fingers as a wedge have pnshed themselves through the preputial constriction, with the glans penis between them. Various writers have advocated certain instruments to assist in this reduction by inserting them under the constriction and prying it up, as by hairpins, directors, etc. Forceps have also been made especially to compress the glans so that the prepuce may slip over. Various local applications have also been advocated to assist in reducing this condition, as belladonna ointment, ete.

When the paraphimosis is irreducible, hot water is of the greatest assistance in promoting absorption of the infiltration. I direct the patient to hold the organ in water as hot as he can stand every two hours for 
ten or fifteen minutes, and then keep it elevated. If any dangerous complications threaten, such as gangrene or extensive ulceration, the constricting bands should be cut at once. To do this, take a bistoury and insert it under the preputial orifice, with the blade flat, and, having brought it into the median line, cut upward, thus severing the constriction. After an old paraphimosis which has been accompanied by a good deal of infiltration has been reduced, a thickened mass of hypertrophied tissue often remains behind. This can be removed, as suggested by Mauriac, by making two elliptical incisions on either side of the hypertrophied tissue, cuttir out the mass and then bringing the edges of the wound together and uniting with sutures. Dress antiseptically.

\section{DISEASES OF THE GLANS AND PREPUCE.}

Balanitis and Balano-Posthitis. - From the urethra to the margin of the prepuce we have a mucous membrane which is drawn comparatively tight over the glans, is quite adherent to the tissues behind the corona, and is then loosely reflected along the prepuce, to which it acts as an inside lining, extending as far as its margin. This is the balano-preputial mucous membrane; and an inflammation of the part covering the glans is called balanitis, while that of the part covering the preputial fold is called posthitis. When the inflammation affects the whole membrane, it is called balano-posthitis. It is most commonly found in persons having a badly fitting prepuce-too long, too tight, with a tight orifice, or a short frænum. The contributing causes are various subpreputial pathological conditions, such as chancres, chancroids, vegetations, herpes, syphilis, etc.

The exciting causes are carelessness in regard to cleansing the parts, thus allowing an accumulation and decomposition of the sebaceous matter; too frequent coitus with women suffering with uterine troubles complicated with leucorrhceal discharges, pus from urethritis, or ulcerating lesions situated in this locality. Another cause is diabetes, on account of the decomposition of the urine with which the parts are so frequently bathed.

Simple Balanitis.-The surface of the glans presents a bright red or pinkish color, having a soft, moist, spongy appearance, and covered in places with a white, creamy coating. When posthitis occurs as well, and more pressure exists, it has a more mottled appearance, due to the macerated epithelium, which is wanting in places. In this case swelling and redness of the prepuce near the margin take place. Sometimes the balanitis is complicated by a vesicular eruption over the glans, which gives rise to erosions when their rupture takes place. These erosions occasionally develop into ulcerations.

Secondary Balanitis is due to the presence of vegetations, herpes, chancre, or chancroids, both on account of their irritating discharges and 
the interference in circulation caused by their pressure. Ordinarily this condition can be easily detected by drawing back the prepuce, only the phimotic cases are puzzling. In these cases there are very profuse discharge, great redness, swelling, and œdema.

Specific Balanitis is of two kinds: 1. Infecting balano-posthitis. In these cases there is no primary ulceration, but an infiltration of the glans and prepuce, imparting a general feeling of induration on pressure. The inflammation is subacute in character, and the surface varies in color from a pinkish white to a brawny red; erosions are frequently seen, but deep ulcerations are rare. This form of initial lesion comes on so insidiously that frequently the patient does not call upon his physician until constitutional symptoms appear. The character of the lesion and the enlarged glans in the groins and elsewhere are sufficient diagnosis. 2. Secondary syphilis may give rise to macular or papular eruptions of the balano-preputial membrane, and in uncleanly patients not only is the inflammation of the membrane general, but the lesions become excoriated, and may take on a form of superficial or even deep ulceration. The papular efflorescences may become hypertrophied, or may develop into a condylomatous condition - the condylomata lata. In other cases a diffuse induration may take place, resulting in a permanent contraction of the prepuce. These local manifestations may occur either in conjunction with or independent of the secondary eruption on the body.

Chronic Balano-Posthitis is generally found in middle-aged men. It comes on subacutely, with an occasional slight exacerbation. The balanopreputial membrane is stiffened, loses its elasticity, and has $a_{\text {a tough, }}$ leathery feel. The color of the glans is paler, a bluish white, and is occasionally covered with excoriated patches. It causes a great deal of inconvenience to the patient on account of the constant irritation, and the want of freedom and elasticity in the prepuce during coitus. It is a condition which is accompanied by great epithelial hyperplasia; if not attended to, it may result in a serious complication as epithelioma.

Treatment.-Simple balanitis can generally be cured by washing twice a day with warm water, dusting on a little "A. B. C. powder"--i. e., acid. boracic, bismuth subnit., calomel āā._-and then placing a thin film of absorbent cotton between the glans and the prepuce. If a lotion is preferred, lotio plumbi et opii or lotio nigra may be used, every three or four hours. In applying lotions the mistake should not be made of using too bulky wads of cotton. Take a piece of cotton between the fingers, pull it out into a thin film, and spread it over the glans; then pour the lotion over this, when it will flatten to the shape of the parts. If erosions are present, they should be touched with a nitrate-of-silver solution $(3 \mathrm{j}-3 \mathrm{j})$. The best method of using silver solution on erosions, ulcers, etc., is by means of an ordinary wooden toothpick, with a thin film 
of cotton twisted around the end. The end of this should be moistened with the solution and applied lightly to the lesion.

In cases of secondary balanitis, if the prepuce can be retracted, remove the cause-i. e., vegetations, chancroids, etc.-by applying some mildly astringent powder or lotion to the inflammed membrane. In cases of specific balanitis, acute or chronic, use "A. B. C." powder; calomel ; lotio nigra; sol. bichloride $1-5,000$ to $1-2,000$; on a film of cotton, having cleaned the parts thoroughly. If erosions are present they should be touched with a silver solution. Give antisyphilitic treatment, and if the patient is cachectic, tonics. In cases of chronic balanoposthitis, circumcision should be resorted to at once. In fact, in all cases of recurring balanitis, which is always due to a badly fitting prepuce, circumcision should be performed.

Diabetic Balano-Posthitis. - This affection occasionally occurs in connection with diabetes. It is a very rare complication, however. Durand Fardel, in speaking of his observations in diabetics, stated that he did not see it once in three hundred and forty-four cases. This trouble begins with the usual symptoms of balano-posthitis-i. e., itching and burning of the glans and prepuce and redness of the mucous membrane covering them, together with a profuse and generally foul pustular discharge. The exuded smegma sticks to the mucous membrane, forming a membranous-like coating. An artificial eczema of the prepuce is now set up, at first subacute in character, accompanied by a redness and thickening of the skin. Little ulcerations appear around its margin as a result of erosions of the mucous membrane and ruptured vesicles. These are at first round, soft, and flat, but later increase in size and assume an irregular form. Fissures and exuberant grauulations, very vascular and tender, may also occur. The prepuce becomes still more indurated from the plastic infiltration into its tissues and around the base of the ulcerations; thus causing a condition of phimosis, which is so common in these cases that the disease is often known by the name of diabetic phimosis. In some cases where this condition is not relieved the redness and infiltration become more marked, and a gangrenous dermatitis develops, followed by a slough, more or less extensive, of the prepuce and perhaps some of the adjoining tissues. In the preputial secretion in this disease a vegetable parasite is found, called by Friedreich the Aspergillus, consisting, as is usual in this class of parasite, of the mycelium and spores. The spores are round or oval, and usually occur either singly or in pairs. The mycelii are either short and single or long and branched, and sometimes contain spores imbedded in them. They are considered pathognomonic of diabetic balano-posthitis and eczema, and are never found in ordinary smegma. This preputial condition often occurs when there is no other sign of diabetes, and occasionally is the first symptom to point to it. As it has been proved that it may oceur 
in cases where there is a minimum amount of sugar in the urine, it is always wise, in suspicious cases of balano-posthitis or eczema in this region, to examine carefully the secretion from the parts as well as the urine.

Treatment. - The greatest danger to be feared is that of a split in the prepuce, which may be followed by gangrene, and might prove fatal. Thorough cleanliness, as by irrigations with antiseptic solutions, especially of carbolic and boric acid, after each urination, should be insisted upon, to be followed by a dusting powder of bismuth, borax, and lycopodii, āā. It is also advisable to have the patient urinate through a broken test-tube, so that no urine will touch the tissues. The patient should of course be placed upon an antidiabetic diet. Often the inflammation is so great that antiphlogistic treatment, by keeping the patient quiet in bed with cloths soaked in lotio plumbi et opii wrapped about the organ, has to be resorted to for a few days. Occasionally gangrene threatens, and in these cases it is advisable to operate immediately on its first appearance and with the most rigid antiseptic precautions. The object of this operation is to relieve pressure, which can be done either by a dorsal incision or by removing a $\mathrm{V}$-shaped piece from the dorsal aspect of the prepuce. If a slough occurs, the parts should be dressed with charcoal and iodoform āā.

Herpes Progenitalis is a condition characterized by the appearance of one or more vesicles at some point along the balano-preputial membrane, the most frequent locality being the sulcus behind the corona. This is generally an ephemeral condition, and occasionally a recurring one. It may be simple, the vesicles breaking and drying up in a few days, or they may take on an ulcerated form closely resembling chancroids. Herpes zoster may also occur in this region, as elsewhere.

Simple Herpes appears as one or a cluster of vesicles, surrounded by a little areola. When these break, an eroded surface is left, as that of a cold sore. When these take on an ulcerated form or are situated on the membrane behind the corona, they often have a punched-out appearance, with round or oval clean-cut edges. When they coalesce they do not lose their characteristic outline, but unite in a polycyclic form. They are covered with a little serum, which, when the lesion is near the skin, forms a yellow crust.

Neuralgic Herpes occurs in persons subject to neuralgias. The attack is preceded and accompanied by itching, burning, and painful neuralgic symptoms in the sciatic regions, in the penis, or throughont the whole genito-urinary tract. Painfyl micturition, and even strangury, are frequent accompaniments. It is evidently closely allied to herpes zoster.

Ulcerating Herpes occurs generally in the cachectic, uncleanly, or in persons who have so tight a prepuce that the lesions are torn whenever it is retracted, thus keeping up a constant irritation. They are very difficult to diagnosticate from chancroids. 
Recurrent Herpes is often accompanied by balanitis. The lesions are generally situated in the sulcus, and as quickly as one or two are healed others appear; they sometimes continue to recur for weeks. They are generally found associated with a tight prepuce.

Etiology.-Herpes generally occurs in men of a catarrhal diathesis, having a tendency to gout and rheumatism, or in neurotic individuals. The predisposing causes are a tight or badly fitting prepuce, stricture, or some localized inflammatory condition along the genito-urinary tract; also balanitis, urethritis, chancre, and chancroids. As exciting causes may be mentioned exacerbations of any of the localized inflammations aloug the genito-urinary tract; excessive venery; contact with acrid secretions, as the menstrual discharge; or irritation, by badly fitting garments.

Diagnosis.-Herpes is often difficult to diagnosticate from chancroid, chancre, and mucous patches. Herpes differs from chancroid in the vesicular manner of its appearance; in its grouping; in having less inflammation, less discharge, and a more regular outline; in the history of exposure to contagion; in the lymphatic enlargements, herpes being accompanied by little enlargement of the inguinal glands, even in the ulcerated type, chancroid being usually accompanied by enlargement, tenderness, and frequently suppurating buboes. Chancroids are auto-inoculable, herpes usually not. Chancroids are accompanied by a feeling of soreness, herpes by a sensation of itching or burning.

-Herpes very often resembles that variety of chancre which appears as an erosion. The differential diagnosis will be based upon the history, duration, and method of appearance, herpes generally occurring in groups, chancre singly; the base of the chancre being indurated and stiff, that of herpes soft'and pliable; outline of edges-the lesions of herpes being often sharply cut, as though punched ont, without much elevation of sides, those of chancre usually elevated and indurated ; variety of colorherpetic lesions being usually pinkish or light red, those of syphilis darkred or coppery ; the appearance of the surface-that of herpetic erosions being moist and fresh, that of the chancre smooth and glistening. On squeezing the lesions, a serous fluid will exude from a herpetic; from a chancre nothing. The greatest similarity to herpes is shown by the multiple herpetiform chancre. Fomrnier states that the principal difference is in the contom of their respective margins. In chancre the edges are regularly or irregularly rounded or oval, while in herpes they are polycyclic, simply round vesicles fused together.

Herpetic lesions of the penis are differentiated from mucous patches by the history of the case; by their vesicular appearance; by their form, lesions of herpes usually being acuminate, those of mucous papules flat; by their surface, that of vesicles being covered with serum, that of mucous 
patches with macerated epithelium. In the female, when situated on the integument about the vulva, the resemblance is most striking.

Treatment. - Cleanliness, and the use of astringent and soothing powders or lotions, give the best results. If the prepuce is ill-fitting, or the patient is subject to recurrent attacks, circumcise. As a powder, use any bland one-e. g., one consisting of borax, bismuth, and calomel āā. Very often powders keep the parts too dry and accumulate in thick chunks, thus tending to keep up the irritation and to favor the stretching and tearing of the membrane when the prepuce is retracted, and in this way hindering the healing. In such a case a lotion should be substituted. Lotio nigra is exceptionally good in recurrent cases which go on to ulceration, and will often prevent the small vesicles from developing, drying them up almost immediately. When erosions or ulcers develop, they should be touched with a nitrate-of-silver solution every second or third day until cured. If the patient is debilitated, give nerve tonics. Herpes frequently occurs in men who are fat, flabby, soft, and easily overcome by slight exertion; also in the gouty or rheumatic. If due to any trouble along the genito-urinary tract, this should be rectified. If there is much inflammation and pain, as in the neuralgic type, use lotio plumbi et opii.

Verrucæ.-Venereal warts are characterized by hypertrophy of the papillæ of the skin, and an increase in the connective tissue and vascular supply. They are either soft or hard, depending upon their seat. On mucous membranes they are soft, on the integument they are hard. The favorite seat for the moist variety is the balano-preputial membrane, especially the sulcus behind the corona. The hard generally occur on the integument, between the scrotum and thigh. They may be single and pedunculated, at other times sessile, and form cauliflower masses of large size. These sometimes grow to such dimensions subpreputially as to cause not only phimosis, but also ulceration and sloughing of the prepuce. In color they vary from a pinkish white to a deep red. They secrete a pungent, viscid fluid having a disgusting odor.

Etiology.-They generally occur in young adults who are uncleanly, those who are suffering from urethritis, or in those whose parts are unusually moist.

Diagnosis.-Verrucæ acuminatæ can only be confounded with the condylomata lata of syphilis and epithelioma. Verrucæ acuminatæ develop slowly; the condylomata lata appear as flat papules in a syphilitic subject, and later become roughened, moist, and vegetating. Verrucæ differ from epithelioma in that they appear in younger individuals, and are of a much more rapid growth.

Treatment. - There are various methods, such as cauterizing with acetic or nitric acid; curetting and cauterizing the base, or applying 
persulphate of iron; cutting them off with scissors; tying sutures about the base in pedunculated cases; the thermocautery, etc. My method of treatment is as follows: Wash the parts thoroughly with hot water, bathe the vegetations with a ten-per-cent solution of cocaine, then paint them superficially with a drachm-to-the-ounce solution of bichloride of mercury in collodion every day or two, and dust on a powder composed of oxide of zinc, tannin, and calomel, āā. The bichloride exerts both an astringent and a caustic action; the collodion, contracting, holds down the already reduced papules; while the powder tends to keep the parts dry. This application should be made by the surgeon himself, and the patient should be instructed to wash the parts twice a day, and to dust on the powder. He should also be instructed to keep the prepuce drawn over the glans after these applications, otherwise paraphimosis might occur. If the solution is applied carelessly, it will run down between the growths and set up a severe acute inflammation and perhaps a slough.

\section{DISEASES OF THE CORPORA CAVERNOSA.}

Acute Inflammations are rare, and generally result from fracture or wounds of the penis while the organ is in a state of turgescence. A phlebitis is set up and a plastic infiltration takes place into the tissue, causing a little pain and tenderness on pressure, with a slight redundancy. This only inconveniences the patient when erection takes place, giving some pain and producing a curvature of that side, the exuded material preventing a comple turgescence of the corpora cavernosa at that point. The inflammation may occur on one or both sides. "In the course of an attack of gonorrhœa the inflammation may extend to the corpora cavernosa and produce an effusion of plastic lymph, which will obliterate the cavities of the bodies and interfere with complete distention in the state of erection of the organ" (Taylor). "The same effect may be produced by small apoplectic deposits in the substance of the corpora cavernosa, the cicatrization of which always entails a deposit of a certain quantity of plastic tissue" (Robert). This likewise causes a curvature toward the affected side, and in exceptional cases, where both sides are affected, erection may take place posteriorly to the trouble, while a portion of the organ in front remains flaccid. Abscess of the corpora cavernosa occasionally but, rarely, occurs.

Treatment. - When the disease of the corpora cavernosa is acute, and pain and swelling are present, some antiphlogistic remedy, as lotio plumbi et opii, may be applied locally, and camphor, bromide of potassium, or lupulin given internally to prevent erections until the inflammatory stage has passed over, after which alteratives, iodide of potassium or mercurials, both externally and internally, should be employed. In case of abscess of the corpora cavernosa, poultice and treat as abscess elsewhere. 
Chronic Inflammations are more common, especially the variety characterized by the development of thin, fibrous growths or plates. These may occur on one side, on the dorsum, or they may be multiple. They vary in size from a pumpkin-seed to that of a lima bean. They are hard, firm, with a well-defined border, and possess a feeling of elasticity. They are generally sensitive on pressure, and a dull pain may exist. They usually occur in middle-aged men, and most often in those who have had former attacks of urethritis, and cause a curvature toward the affected side. H. V. Miller, of Atlanta, reports a case where two were present on the organ, one on the dorsum about an inch from the pubes, the other on the side about an inch from the glans, causing a double curvature on erection. Johnson reported a case where the fibrous wall of the corpora cavernosa was irregularly indurated, while thickening and consolidation invaded the erectile tissue within. The man complained of lancinating pains in the penis and scrotum. Erections were painful, during which the organ gradually assumed a spiral twist, like a pig's tail or a vermiform appendix. This was on account of the multiplicity of the lesions.

Treatment. - The treatment consists of tincture of iodine externally and iodides internally ; later, frictions of hydrarg. externally, with iodides internally. Some favorable results have been reported from the use of ointments which have as a base lanolin or placement, their greater efficacy depending upon the fact that they cause the medicines to penetrate through the tissues much more readily.

Bony and Calcareous Plates may occur in the corpora cavernosa. Dr. William Porter reported a case in the Medical Record some years ago of a man aged sixty-three, who had a small tumor on the right side of the organ attached to the fibrous tissue of the corpora cavernosa. This was cut down on to and shelled out. It proved to be a bony mass or plate six centimetres long by three centimetres wide. It contained lacunæ and canaliculi. These bony growths often develop in the furrow between the two corpora cavernosa, to which they are attached.

In the Museum of Pathological Anatomy in Vienna is a plaster cast of a penis with a bony growth in the dorsum between the corpora cavernosa which bifurcated about the middle of the organ.

Gummata of the Corpora Cavernosa. - Specific lesions are not as frequent in the cavernous bodies as they are in some other parts of the organ, the most frequent seat being the furrow between them. They may be single or multiple. They appear as nodes in the substance of these bodies, and may cause curvatures of the penis. They may remain indolent for a long time, then break down and ulcerate, or, under specific treatment, undergo resolution. The treatment is the same as for gummata elsewhere. 


\section{DISEASES AND INJURIES OF THE URETHRA.}

By F. TILDEN BROWN, M. D.

\section{MALFORMATIONS OF THE URETHRA.}

\section{ABSENCE AND COMPLETE OBLITERATION OF THE URETHRA.}

THis deformity is due to union and adhesion of the urethral halves, and their transformation into a fibrous cord.

A certain amount of urine is secreted during intra-uterine life into the bladder. The distended viscus presses upon the umbilical arteries, and seriously interferes with the fœtal circulation. Hence these fœtuses rarely survive the eighth month of intra-uterine life. Yet Kaufmann has collected seven cases of complete absence of the urethra in which the child lived after birth.

Evacuation of the urine being impossible by the natural passages, one of these things happens: The urachus may reopen and the urine be discharged at the navel; a vesico-rectal perforation may ensue; or the child, surviving until birth, comes into the world with an enormously distended bladder. A number of these latter cases have been operated on with good results.

The symptoms, diagnosis, and treatment are the same as those following complete congenital atresia of the urethra.

\section{CONGENITAL ATRESIA OF THE URETHRA.}

This occurs from the fusion of the opposing walls of the urethra. The meatus urinarius or its vicinity is its usual seat. Most often they occur in conjunction with hypospadias, but a certain number of uncomplicated cases are recorded.

Atresia of the Meatus Urinarius.-Here the mucous membrane passes unbroken over the head of the glans, and there is nothing to indicate the site of the meatus. It arises from the same process that causes the "epithelial adhesion" of the mucosæ of the glans and prepuce that is so frequently seen. But few cases of the condition are on record. As a rule, the membrane is very delicate. Voillemier records a case in which it protruded at each effort of the child to urinate, and seemed ready to burst. This explains the apparent anomaly of its infrequent record. The 
pent-up urine ruptures it either in utero or immediately after birth, and spontaneous cure takes place.

Diagnosis.-Immediately after birth the child draws attention to itself if anything is wrong. Examination of the penis shows absence of a urethral orifice, the penis is enlarged and swollen, the urethra forms a tense cord on the under surface of the organ, and in extreme cases the membrane may project forward on account of the urinary pressure.

Treatment.-An ordinary small sound or probe can be forced through the membrane. If it be tough, an incision will suffice.

Atresia of the Glandular Urethra.-Here the meatus urinarius is usually present, either in apparently normal form or as a furrow lined with mucous membrane, though cases occur in which the glandular urethra is imperforate, together with atresia of the meatus. Most frequently the meatus is well formed, and ends in a cul-de-sac at a distance of a few millimetres. The obstruction is more resistant and less evident than in the foregoing variety, and here we meet the more serious consequences of urinary retention, dilatation of bladder and ureters, and hydronephrosis.

The diagnosis is made from the absence of urination, the distention of the bladder and the patent portion of the urethra, and the distress of the child.

Treatment consists first in an attempt to reach the urethra by puncture at the meatus, if any indication of it is present; if not, at the apex of the glans. With a fine trocar an attempt is made to reach the distended urethra, and the immediate flow of urine and disappearance of the symptoms show its success. Systematic dilatation with small metal sounds must then be undertaken.

In case the attempt to reach the urethra is not successful, other means must be resorted to. Whitehead punctured the urethra from below, forming an artificial fistula.

Atresia of the Deep Urethra.-This may occur anywhere in the urethral canal; but it is commonest in the pars cavernosa, where it occurs either alone or in conjunction with atresia of the glandular canal. There is frequently also deformity of the rectum, atresiæ or fistulous communications with the bladder. The membranous urethra is less commonly affected, and the prostatic urethra least of all.

Complete retention of urine is the immediate consequence of this condition. The bladder and the ureters are distended, and hydronephrosis occurs. The foetus usually snceumbs at the seventh month, or even earlier, from the pressure of the distended viscus on the vessels, and two thirds of the cases recorded were born dead. The more anterior the occlusion, the greater is the chance of its natural relief by the formation of a urethral perforation or a hypospadias. In some cases the testicles have been 
found undescended, the enlarged bladder forming an obstacle to their passage. The same distended viscus has formed an obstacle to delivery, and has had to be punctured before that operation could be effected.

Spontaneous fistula formation has relieved some cases, and at least twice the urachus has become patent from the urinary pressure, and the secretion has been voided from the umbilicus. In a few other cases that have been born alive operative procedure has been successful.

The diagnosis rests on the absence of urination, the recognition of the vesical tumor in the abdomen, the distress and vomiting of the child, and, finally, the failure to introduce a catheter.

Prognosis is only fair ; operations have not always succeded in finding the end of the canal, and a number of these patients have died almost immediately.

Treatment.-Our first resource is catheterization, which may be successful in cases where the occluded segment of urethra is of small extent, an ordinary catheter or buttoned probe being employed. If this does not succeed, a sharp trocar or a bistoury must be used, and the anterior end of the posterior urethral segment found, or an artificial passage made into the bladder. This must be subsequently kept open by the frequent passage of a probe or catheter. Ebert and Rose employed it in their cases.

It would probably be an improvement on the above operation to enter the bulbous or membranous urethra by means of a perineal incision, and then to attempt to reach the glans from behind forward. The free perineal opening would obviate the dangers of infiltration of urine incident to the method of Ebert and Rose, besides giving immediate relief to the retention.

\section{CONGENITAL STRICTURES OF THE URETHRA.}

These may occur at the meatus or at any lower point in the urethral canal.

Congenital Stricture of the Meatus Urinarius is usually not revealed until catheterism is attempted. It is frequently accompanied by congenital phimosis. There are cases, however, in which the stenosis is so great that only a fine probe can be introduced, and this naturally interferes greatly with micturition. Kocher operated twice on cases in which he could only introduce No. 9 French. One of these patients was forty-seven years old.

The symptoms are usually confined to difficulty of urination; ischuria, inefficient erection and consequent impotence, and various reflex disturbances, have also been noted.

Treatment.-Repeated catheterism is sometimes effective. Incision is certainly quicker and more direct. It should be done downward, toward 
the frænum. A strip of iodoform gauze is placed in the wound, and a short, straight conical sound of full size must be passed every second day. Contraction always occurs to a certain extent, and it is well to cut to several sizes above the one which the meatus is desired to retain. Bleeding is frequently persistent and troublesome, though never dangerous; it may be controlled by introducing into the urethra a section of full-sized rubber bougie and bandaging the penis over it. In some cases it is well to unite the cut edgres of the urethral and glandular mucous membrane with sutures.

Congenital Stricture of the Deeper Urethra.-Of this there are a number of recorded cases; but the fact of their having existed from birth is in most cases not susceptible of proof. A stricture may be acquired at any age.

The symptoms consist of a history of slow micturition from birth, with a stream that may be as thin as a fine knitting-needle, swellings of the abdomen in the vesical region, attacks of colicky pain due to retention of urine, and examination will show a marked stenosis.

Treatment consists of dilatation with sounds, or internal urethrotomy.

But besides these stenoses of the ordinary kind, valve-shaped strictures occur in various portions of the urethra. Thus, the valve of the fossa navicularis may be abnormally developed, or, rather, the early fœtal septum between the glandular and penile urethra may be abnormally persistent. The partial septum looks forward, and hence is obstructive rather to catheterism than to micturition, the obstacle being met with about three quarters of an inch from the meatus. It is readily seen with the speculum, and sometimes, if the meatus is very large, with the unaided eye.

Valvular folds may occasionally form strictures in the prostatic urethra. Several are normally present in the region of the ejaculatory ducts, and as their concavity is directed backward, they readily form obstructions to the urinary flow if of larger than average size. Totenatschen has recorded the post-mortem findings in three such cases. The anterior urethra was normal, but that behind the fold was markedly dilated. The bladder was not much dilated, but its wall was greatly thickened from muscular hypertrophy; the ureters were dilated, and hydronephrosis and atrophy of the kidneys were present. Such patients usually die, soon after birth, from renal embarrassment due to the urinary obstacle.

Of the diagnosis and treatment of stenoses caused by these posterior folds we can say but little. They have not yet been recognized during life. It is well to bear them in mind, however, if in the newborn infant there is retention of urine, and yet catheterization can be effected. Such a condition may then be surmised, and proved later, by the use of a small endoscopic tube of seventeen millimetres circumference. When thus 
located they may be severed by a dilating knife passed down the urethral speculum.

\section{CONGENITAL DIVERTICULA OF THE URETHRA OR URINARY POUCHES.}

These are very rare malformations. In all the cases recorded they have been situated on the under surface of the penile urethra. They were of large size, especially when distended with urine, and extended from just behind the glans to the peno-scrotal fold (Figs. 1 and 2). Hüter operated upon two cases in which the pouch, when distended, was as large as a pigeon's egg; in others it has been much larger, measuring in circumference ten and a half inches, and containing a pound of urine.

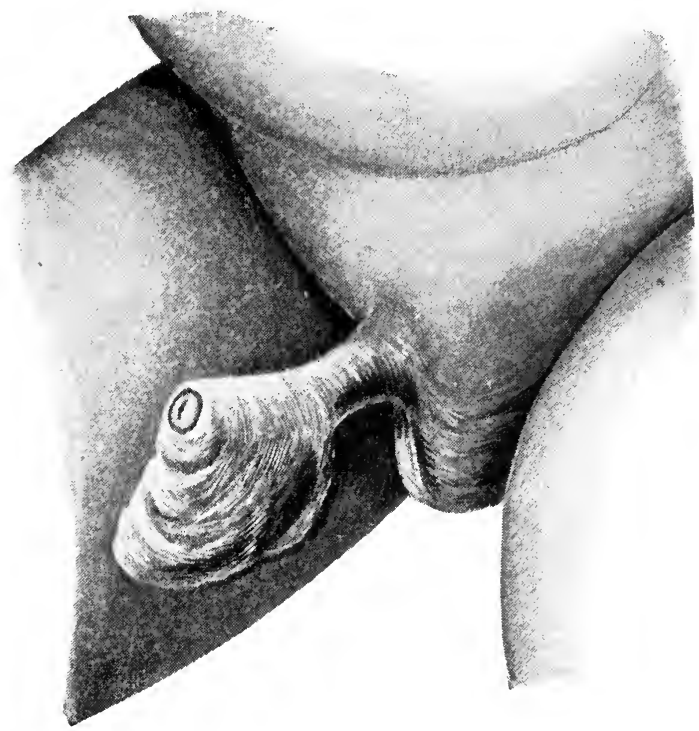

FIG. 1.-Urinary pouch, empty.

The walls of these pouches are thin, sometimes remarkably so, and they are clothed with normal mucous membrane. Sometimes the tenuity of the sac is so marked that the connective-tissue bundles, spread apart by the continuous pressure, can be distinctly seen to be continuous with the bundles of the normal urethra behind. In all cases the diverticula occur at the expense of the lower urethral wall; the upper wall is normal, as is also the dermal covering.

As regards the origin of these peculiar malformations but little is known. Anterior obstructions to the urine flow have not always been noticed; in every case catheterization has been easy. A few observers (Hendriksz, Bökai, Hüter) have found valve-shaped backward prolonga- 
tions of the glandular segment of the urethra (Fig. 3), which undoubtedly acted as valves when the sac was filled with urine; but in most cases none such were present, and they can not be regarded as the absolute cause of the condition in question. Kaufmann's hypothesis is the most probable.

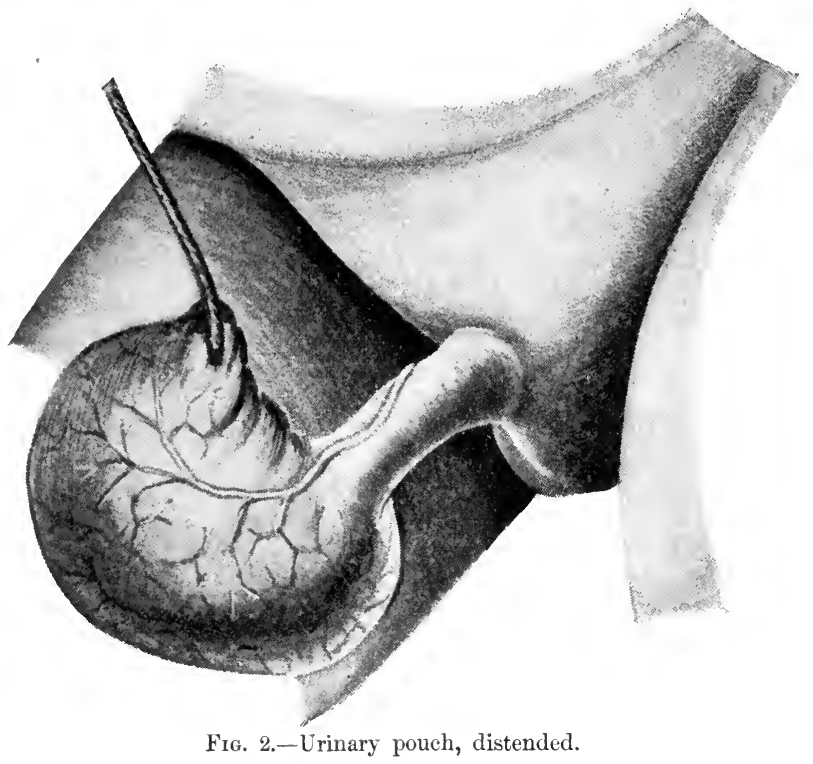

He regards it as due to a disturbance of the exact apposition of the glandular and penile portions of the urethral canal at a period of intrauterine life when urine is already flowing from the bladder. These two portions of the canal, being developed separately, and from opposite directions, any failure as regards the exact time or place of coalescence would cause the urine to accumulate in the anterior blind end of the pos-

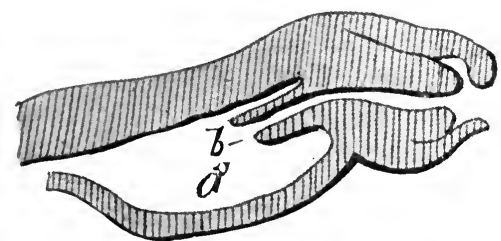

Fig. 3.-Valve-shaped backward prolongations of the glandular segment of the urethra in a ease of urethral divertieulum. terior segment. The lower urethral wall, being the weakest, is naturally the one that gives way, and thus the pouch is formed. When, finally, the growth of the parts or the pressure of the urine does make the canal patent, the distended sac remains with urine in it. Hence it does not tend to disappear, but rather to increase in size.

The diagnosis of these congenital diverticula or dilatations is made from the very apparent alteration in the shape of the penis and the disturbance of urination. The tumor, when collapsed, is soft and fluctu- 
ating; when distended, it is tense and resistant; it can be emptied by pressure, it is translucent, and never shows any signs of inflammatory reaction. In about half the cases there has been permanent incontinence of urine; in Langier's case the patient urinated normally, while in those of Hendriksz and Lotzbeck the entire bladder contents flowed into the pouch when the patient passed water, whence it had to be manually expressed. A diverticulum can be readily catheterized, and the instrument can be plainly felt in the empty sac. In only one case is there any note of ammoniacal decomposition of the urine in the sac.

One case only, that of Bökai's, ended fatally ; the autopsy showed the usual lesions that result from urinary obstruction, viz., vesical and ureteral dilatation and hypertrophy, cystitis, and purulent nephritis.

Treatment is operative, and has always been successful. The lower wall of the pouch is split on a staff. A long segment is then cut off from each wall of the sac, leaving just enough when united to form a tube of the normal urethral caliber. Valvular folds of the mucous membrane are to be severed when present. The mucous membrane and skin can then be separately united. Any small fistulous opening that may be left closes readily under cauterization.

The fatal case above mentioned shows that this malformation is liable to cause all the troubles so familiar in cases of obstruction of the urinary passages; hence, all cases should be operated upon.

\section{HYPOSPADIAS.}

Hypospadias is that congenital condition in which the urine is not evacuated from the normal point at the extremity of the glans penis, but escapes through an abnormal opening on the lower surface of the penis at a variable distance from the tip of the glans. Our exact knowledge of the condition dates from the memoir of Prof. Bonisson in 1861, and Duplay, Pitha, and Bardeleben have made the condition the subject of exhaustive studies.

Most authorities recognize three distinct varieties or grades of the affection. First, balanic or glandular hypospadias, in which the urethral opening is either in the glans or immediately behind it. Second, penile hypospadias, in which the opening is somewhere on the under surface of the urethra, between the glans and the peno-scrotal fold. Third, perineal, in which the scrotum is split, and the urine escapes through an opening between its folds or in the perinæum. Three additional grades include the mixed forms of hypospadias: 1. Glandulopenile. 2. Peno-scrotal. 3. Perineo-scrotal.

Hypospadias is not only the commonest of all urethral malformations, but at the same time one of the most frequent of the entire body. Bouisson says it occurs once in 300 cases of sexual disease. Chaussier found 
among 23,293 newborn at the Maternité of Paris, 132 malformations, two of which were hypospadias. This is entirely too low a proportion. The French military surgeon Rennes found ten hypospadiases in 3,000 recruits - a proportion of one in 300 males. The statisties vary much, but of its frequency there can be no doubt.

Etiology.-The causes of hypospadias are obscure. Certain observations would tend to show its heredity, and the existence of some influence that determines its appearance in several members of one family. Bryant records three cases in which both father and son were affected. The older theory, that it is due to a rupture of the urethra, has been questioned, and it is more generally regarded as referable to an arrest of development. The urethra is primarily a groove, open below, which grows forward out of the sinus urogenitalis in conjunction with the corpora cavernosa; it gradually closes from behind forward. Incomplete closure to any varying degree of this primitive urinary groove would therefore cause hypospadias; in hypospadias perinealis, the urethra's entire length; in hypospadias penis, its anterior half; and in hypospadias glandis, its anterior extremity, remaining incompletely formed.

But this simple developmental theory by no means explains all the cases that we meet. Hypospadiases show the greatest possible varieties of form, and are very difficult to classify, which would hardly be the case were their origin so simple. The glandular portion of the urethra is not developed in the manner the theory supposes, and cases of balanic hypospadias, the commonest of all, conjoined as it often is with imperforate meatus, can not be so accounted for. Then there are cases in which the urethra is apparently normally developed, and there are one or more congenital urinary fistulæ on its under surface, perhaps with distinctly cicatricial tissne in their calloused edges. In fact, most cases do show special changes in and about the misplaced meatus. This orifice is smaller than natural; the skin around it is smooth and shining, pigmented and cicatricial. Besides which there are often bends and twists of the penis, adhesions of that organ to the scrotum, anomalies of the prepuce, asymmetry of the raphé and the frrenum. Some better explanation is evidently necessary.

In certain cases there can be no doubt of the fact that rupture of the urethra occurs during fœetal life. Wiener has shown us that the secretion of urine is active during fœtal life; the bladder is repeatedly filled and emptied. If there is any fault in the proper fusion of the glandular and penile portions of the urethra, the urine will tend to force its way through the proximal urethral walls. Some hypospadial orifices are so manifestly cicatricial that there can be no doubt at all of their originating in this manner.

Upon this fact, and upon his own observations of cases and museum 
specimens, Kaufmann has based his theory of the formation of the meatus which seems to fit all eases, including normal and abnormal ones. His idea is that cases of hypospadias arise when there is a mechanical obstruction to the functional activity of the urethra after the urinary secretion has been established in the fœtus, with consequent retention and final rupture of the urethra. This rupture occurs oftenest, of course, in the direction of least resistance, which is the septum between the external invagination which forms the glandular segment of the urethra and the deeper canal. But if that section is absent, or if it is backward in its development, the urine forces an opening somewhere else.

Thus the glandular urethra may be entirely absent, or it may be present in whole or in part, and end in a blind sac. In some cases the two urethral segments have almost come together; a thin valve-like partition, perhaps perforated, being all that remains, yet at the time that the first urine accumulations occurred the obstruction must have been much more resistant, since the hypospadic perforation has occurred in preference to the normal one.

The point of rupture of the penile urethra is oftenest situated at its extreme anterior end, just posterior to the glans. The under surface of the prepuce is invariably absent, and usually also the lower half of the glandular urethra which is thus represented by a groove. This is probably caused in the same way as is the urethral opening; the urine infiltrates the periurethral tissues and forces its way out.

Hypospadias of the penile portion of the urethra admits of a similar explanation. The same obstruction between the glandular and penile urethra has been present, but for mechanical reasons perforation has not been effected through the anterior end of the penile segment. Hence ensues dilatation of the urethra, with rupture of the tube, infiltration of urine, and a destruction of tissue varying in extent and locality in different cases. The urethral pouches, which we considered above, help to bear out this theory of the mechanical origin of hypospadias. The two conditions are intimately related, it being only the final establishment of the communication between the two urethral segments that prevents the rupture of the pouch and the formation of a penile hypospadias. Congenital urethral pouches are rare, because in most cases of obstruction rupture does occur and hypospadias results.

The cicatricial tissue frequently found associated with hypospadias and the various cicatricial contractions are also best accounted for by Kaufmann's hypothesis; as are torsion and flexion of the penis, adhesions to the scrotum, and fissures of the same. Even in the lightest degree of glandular hypospadias the glans is often drawn down at an angle with the shaft of the penis; in higher grades the entire organ is usually flexed; and the extensive loss of skin and subcutaneous tissue with 
cicatricial contraction sufficiently accounts for it. When the urethral dilatation is great, the scrotal skin is necessarily implicated, the urinary infiltration is greater, and the loss of substance and contraction is proportionate. Adhesion of the penis to the scrotum is unknown save in cases of hypospadias, and is to be explained by the same infiltration and cicatricial contraction. In this way also occurs the defective penile development observed in the worst cases of perineal hypospadias.

Balanic or Glandular Hypospadias (Fig. 4) is that form in which the opening of the urethra is situated on the under surface of the glans, in the place of the absent frænum. The prepuce is deformed in every case. On the dorsum of the glans it is well developed, and forms a thick fold that projeets beyond its anterior extremity; thence its two lateral folds run backward and downward, getting thinner until they become continuous with the margins of the urethral opening. The meatus is usually at the spot where under normal eircumstanees the anterior extremity of the frænum is attached to the glans penis, or it may be situated a little farther back in the retro-glandular sulcus. In the newborn it is often merely a punetiform opening, which can be recognized only during micturition; in older children it will admit a fine probe. It always remains, however, extremely small. The skin and mucous membrane at the orifice is frequently distinctly cicatricial. The opening is usually single; but two, and even three, openings have been observed, the supernumerary ones either communicating with the primary orifice, or ending in blind sacs.

The details of form vary much in different cases. Thus, the glans may be imperforate and the glandular urethra be entirely absent (illustration, F. A. v. Ammon, Die angeborene Chirurgisch. Krankh. d. Menschens, Berlin, 1842, Table xviii); or there may be a more or less perfect meatus in the usual situation, ending in a blind sac of varying length (Forster, Atlas, Fig. vii).

In most cases of balanic hypospadias a deep furrow extends from the normal position of the meatus to the urinary opening. Its lateral walls are continuous with the remains of the prepuce. The remains of 
the fœtal septum between the glandular and penile urethra are sometimes persistent as a crescentic fold; it being the same structure occasionally observed under normal conditions, and known as the valve of the fossa navicularis.

A change in shape of the penis is observed in nearly all adult eases of balanic hypospadias. This change generally consists in a downward deflection of the glans from the direct penile axis. The amount of deflection, which varies in the different cases, is usually small; but it may be marked, and in the worst cases the glans may even point backward, the urethral orifice being situated in the angle between the base of the glans and the apex of the penis itself. Union of the penis. with the scrotum is also

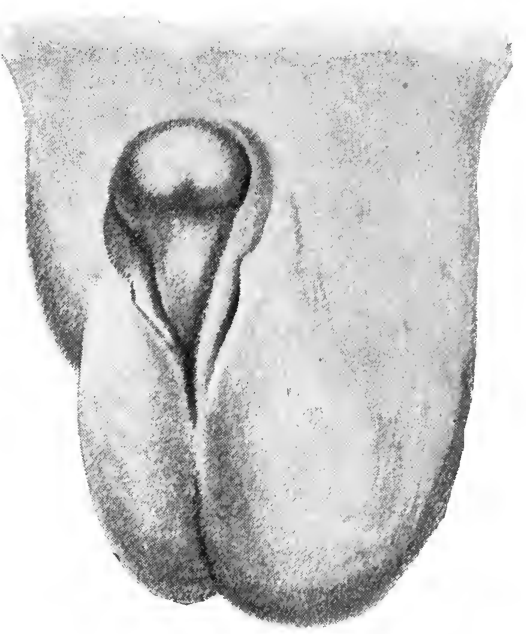

FIG. 5.-Glandulo-penile hypospadias. sometimes seen. Two distinct forms of this deformity have been observed. In the lesser form a thin membrane unites the raphés of penis and scrotum together, interfering moderately with erection. In the other variety the skin covering the penis is continuous on both sides directly with the scrotal integument; the glans is the only free portion of the organ, and the urinary opening lies completely concealed in the angle formed by scrotum and glans.

Torsion of the penis is present in a certain number of cases of balanic hypospadias. In Verneuil's case the dorsal surface of the penis looked toward the scrotum, and its urethral surface was turned forward and to the left. The urethra itself had been correspondingly twisted from right to left and from before backward; it was twisted spirally around the left corpus cavernosum. Finally, a livid condition of the glans, the scrotum, or the prepuce-or of all of them-may coexist with glandular hypospadias. These organs then have more or less resemblance to the vulva, and form one of the species of false hermaphroditism.

Penile Hypospadias.-Here the urinary opening is situated somewhere between the base of the glans and the peno-scrotal fold. It is usually small, and may be covered with a fold of skin; its shape is oval, with its large diameter in the axis of the penis. Its edges are sharp, smooth, and shining; sometimes they are distinctly callonsed and cicatricial. As a canal, the urethra in front of the hypospadic opening is almost always absent, but a deep groove is frequently present, provided with more 
or less elevated erectile margins. Sometimes a hard ridge alone marks the course of the normal urethra to the apex of the glans. It is usually six to eight millimetres broad in the adult.

Certain concomitant abnormalities are frequent and important. Thus, the scrotum may be distinctly divided into two halves by a deep median furrow apparently cicatricial, which runs from the urethral opening to the lower border of the scrotum. The scrotum, otherwise well developed, ascends as a thick, wrinkled mass on each side of the penis, and is continuous with the skin covering its upper surface. The deep scrotal furrow permits the under surface of the arch of the pubes to be distinctly felt, with the urethra winding under it. The corpora cavernosa are so separated that no bulb of the urethra is apparent, the introduced catheter seems to lie quite subcutaneously in the perinæum, and the prostate shows a marked thinning at its center.

The mucous membrane of the deeper urethra is continued directly forward on the floor of this groove. The openings of Littre's glands are usually dilated. Marked narrowings and even partial obliterations of the anterior visible urethral groove have been recorded by various observers. Sabatier (Voillemier, Maladies de l'urèthre, Paris, 1868, page 551) records a unique case, in which every indication of the anterior urethra was absent.

On the other hand, in some cases the urinary canal is fairly perfect anterior to the opening, though the meatus in these cases is usually imperforate. Arnaud, Lacroix, and Lippert have recorded cases of this kind.

The penis in these cases is almost invariably smaller and shorter than normal, both in children and adults, the cavernous tissue being deficient. The penis is curved downward-a deformity which is especially noticeable during erection, which causes the penis to be pulled down and backward. Retraction of the corpora cavernosa, as well as what remains of the urethra, is an efficient cause of it.

Torsion of the penis has been observed, of which De St. Germain gives a remarkable example (Chirurgié orthopédique, page 428).

Perineal Hypospadias (Fig. 6) is the rarest of all kinds of this malformation. The deformity is greater and more complicated than in the other two varieties. The scrotum is completely divided, and the urinary opening is situated in the depths of this furrow-some three or four centimetres in front of the anus. It is generally in the shape of a little slit, elongated vertically, and edged by two muco-cutaneous folds, which unite behind and suggest the introitus of a small vagina.

The urethral segment anterior to the urinary opening shows the same variety of conditions as in penile hypospadias. Most often it is present as a more or less shallow groove running along the scrotal cleft and the under surface of the penis. Sometimes it is represented merely by a 
fibrous cord occupying the same position. In a case recorded by Marestin, the urethra was perfect anterior to the hypospadic opening, but the meatus was closed by a membrane. Or, again, the meatus may be normal, but it ends in a blind sac; the segment intervening between the posterior end of the sac and the abnormal urinary opening being represented by a groove or fibrous cord, as above described.

The penis itself is usually atrophied, and is more or less firmly applied to the scrotal fissure, concealing the urinary opening. The organ is shorter than normal, especially on its inferior surface, though the glans is often well developed. It is strongly incurvated, due, according to Bouisson, not to tension of the muco-cutaneous urethral ridge or furrow, that extends from the hypospadic opening to the glans, but to arrest of development of the corpora cavernosa, even the septum of which is sometimes found strongly contracted.

Marked cases of perineal hypospadias, where the cleft between the lateral halves of the scro-

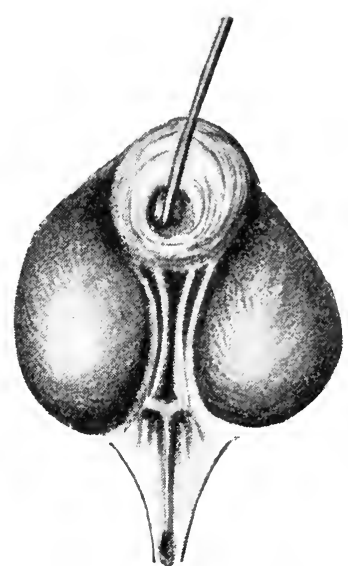

FIG. 6,-Perineal hypospadias. tum, lined with a pale, rosy mucous membrane, was deep, and the atrophy and retraction of the penis were great, have caused considerable difficulty in the determination of sex. Particularly was this the case when the testicles were atrophic or undescended.

Symptoms. - These consist in the first place, of course, of the changes appreciable to the examining eye and tonch. They are sufficiently evident from the detailed description given above. But there are other symptoms referable to derangements of function that demand attention. They occur in connection with micturition, copulation, and fecundation.

Disturbances of urination are common. In glandular hypospadias the function is normal, and even in penile hypospadias it ean be well accomplished if the patient lifts up the penis so as to completely uncover the opening. But in perineal hypospadias the abnormal position of the urinary opening causes much trouble. The membranous and prostatic portions of the urethra being well developed, the urine is projected with force; but the stream, breaking against the incurvated penis, is scattered in all directions; so that patients affected with perineal hypospadias are compelled to urinate in the sitting position, like women, or they soil their garments. Other urinary troubles are present in some cases. Persistent urinous odor and eczema are annoying complications.

In balanic and penile hypospadias copulation is not much interfered 
with, though fecundation may be entirely abrogated; this will certainly be the case if the urinary opening is situated so far back as to lie outside the vagina during copulation. In hypospadias perinæi the facultas coeundi is usually absent. The abnormal downward curve of the penis, and its adhesion to the scrotum, causes the glans to be carried during erection backward and downward into the fissure of the scrotum.

Diagnosis. - Save in the newborn, the diagnosis of hypospadias is easy. Mere inspection suffices. The act of urination will ordinarily confirm the diagnosis. In infants just born, however, the hypospadic opening may be so very small that it is overlooked. Rauchfuss has reported a case in which the atresia of the meatus led to a diagnosis of urinary obstruction, leading to the formation of an artificial urethral canal with the trocar, subsequent examination, however, showed that a hypospadias was present. Percussion of the bladder should enable us to avoid such mistakes. Traumatic splitting of the penis may be confounded with a hypospadias. Chopart recounts a case in which an individual split his urethra gradually from meatus to scrotum; and a traumatic fistula may simulate a hypospadias.

The determination of sex in infants affected with perineal hypospadias is sometimes difficult, especially if the testicles have not descended. The two scrotal halves may look very like the labia, and the small and adherent penis like an abnormally large clitoris. Abdominal palpation, combined with rectal and vesical exploration and sounding, will, however, in all cases reveal the absence of the uterus, while the presence of the prostate gland will determine the diagnosis.

Prognosis varies with the severity of the case and condition and age of the patient. Surgical procedures will restore to almost all these individuals both external symmetry and satisfactory genital and urinary functions.

Treatment.-To relieve the urinary retention that is dependent on the narrowness of the abnormal orifice is usually easy. In children it often suffices to dilate the opening with fine sounds. In older individuals this is not enough. Bökai recommends the circular ablation of the skin around the borders of the opening, the median splitting of the mucous membrane, and the uniting of the cut edges of the skin and mucous membrane by sutures. Kaufmann recommends the splitting of the urethral mucous membrane longitudinally, and the insertion of a small triangular flap of the external skin between its edges.

Bouisson was the first to practically treat the hypospadias itself as a deformity. He showed the necessity of a preliminary straightening of the incurvation of the penis before attempting to make a new canal; for this incurvation obstructs the passage of urine and semen, and interferes more or less completely with coitus. That being done, the forma- 
tion of a new urethral canal may be attempted with hope of better success.

If the hypospadias assumes the shape of a congenital urinary fistula immediately behind the glans, freshening of the edges, and an exact suturing, usually suffices. Any abnormally developed valve of the fossa navicularis must be ablated at its base. If there is membranous closure of the external meatus, it must be incised. If the balanic urethra is normal, but ends in a blind sac, the missing urethral segment must be reproduced by Duplay's method (Archives générales de médecine, Mai et Juin, 1874), and then united to the glandular and penile urethral segments.

If the glans is entirely imperforate, a new canal must be made with the trocar, means being then employed to keep it patent. Dieffenbach has given the following instructions for this operation: "The penis being put on the stretch and drawn as far away as possible from the body, the trocar is pushed through the glans along the under portion of the organ from its apex to the anterior end of the penile urethra. The cannula is allowed to remain in place after the trocar is withdrawn, being later exchanged for a sound, until the canal has cicatrized." Nevertheless the tendency of the new canal to close is very marked.

Gouley's process is similar, but in addition he takes out a wedge-shaped transverse section from the dorsum of the penis, so that the resulting cicatrix shall tend to straighten the organ.

Duplay's method for hypospadias is the most complete. He divides the entire process into three successive stages:

1. Straightening the penis, and formation of a glandular meatus (Figs. 7 and 8$)$.

2. Formation of a new urethral canal from the extremity of the glans to the neighborhood of the hypospadic opening (Fig. 9).

3. Junction of the two portions of the canal.

1. First Stage (Fig. 7). - This may be omitted in those cases in which the penile incurvation is very slight. A free transverse incision or incisions must be made into the middle of

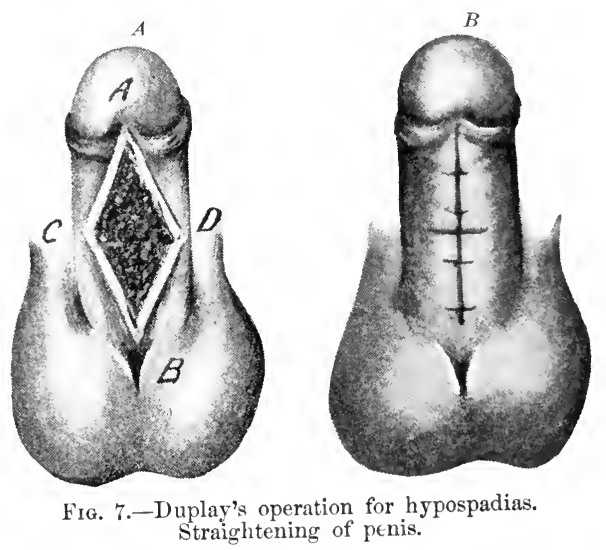
the ridge which unites the glans to the hypospadic opening, as deeply as may be necessary to secure straightening. The fibrous sheath may have 
to be divided, and even the corpora-cavernosa deeply incised; both may be done with impunity. During cicatrization the penis should be kept extended by means of adhesive plaster, or, better, by lateral splints and dorsal sutures.

It is well to delay the second stage of the operation until it is certain that incurvation will not recur. After six or eight months this will be assured.

2. Second Stage (Fig. 8).-Duplay insists upon the necessity of the new canal being as much as possible surrounded by erectile tissue, especially in

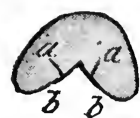

Fig. 8.-Duplay's operation for hypospadias. Formation of glandular meatus.

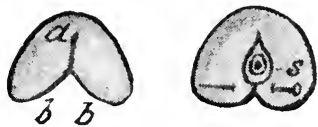

(0) $s$ its glandular portion. The meatus must be situated in the glans. If the semen escapes from a flaccid canal below it, ejaculation will be imperfect, and the chances of fecundation must be limited.

The edges of the glandular furrow are to be freshened, and if it is deep enough they may be united over a sound or staff. If it is not deep enough, a single median longitudinal or two lateral and parallel incisions are to be made, the sound is laid in them, and the canal is enclosed by suture over it. Primary union is almost always obtained. Some opera-

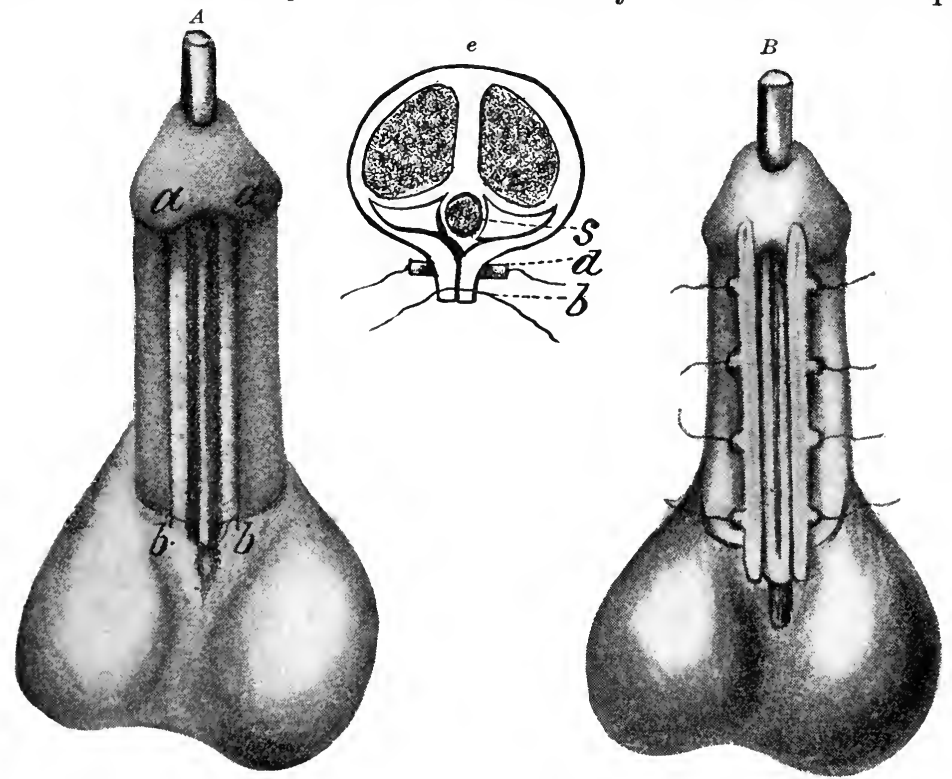

Fia. 9.-Duplay's operation for hypospadias. Formation of new urethral canal.

tors prefer to combine this first part of the second stage with the straightening operation, and time may undoubtedly be gained by so doing. 
To form the new penile canal the following method is employed : The penis is held up, and on each side of the median line is made a longitudinal incision reaching from the base of the glans to within a quarter of an inch of the hypospadic opening. These incisions are parallel one with the other, and not less than a quarter of an inch apart. The internal lip of each incision is then to be slightly dissected up. Each external lip must now be freely dissected, so as to be able to draw to the median line the skin of the lateral parts of the penis. Then the cutaneous surface of the inner flaps is turned toward the cavity of the canal; the raw surfaces are turned outward, and are covered by the raw surfaces of the external flaps. The canal thus formed is only half cutaneous, but this suffices to prevent cicatricial contraction.

Now the displaced flaps are to be united in the median line by modified quilled suture (Fig. 9). A fine single silver wire is to be used, in order to avoid the tracts and large openings of the ordinary wire, the sutures being about a quarter of an inch apart. The ends of each wire are passed through holes made in small leaden tubes; then these lateral tubes are evenly approximated and there fastened with perforated shot. A few points of interrupted superficial suture may be employed to complete the canal externally. Contact is exact, and erection can be resisted.

Third Stage.-Another interval of time is necessary, to be sure that the new canal shows no tendency to contraction, and then we can proceed to join the two portions of the urethra by obliterating the hypospadic fistula. The circumference of the opening must be freely vivified for half an inch all round, and a catheter having been introduced by the new canal into the bladder, the same quilled single-wire suture as described above is to be employed to close the defect that remains.

As regards the management of the urine, Duplay recommends the employment of a retained catheter left constantly open, so that the urine may escape as fast as secreted. After two or three days the patient is allowed to urinate without assistance, being placed in the knee-elbow position to facilitate the process.

As regards the age at which the operations are best undertaken, the first stage should be done early - at the fourth year-and the second in the next year or so, so that the relatively normal penis may participate in the development natural to the period. The last stage should be postponed until puberty, since a certain amount of intelligence on the patient's part will greatly help the surgeon. Nevertheless it may be done in a shorter time. Lücke has cured a case, aged nineteen years, in one year, and Duplay one twenty-one years old in eighteen months. 


\section{EPISPADIAS.}

Epispadias is a malformation characterized by a more or less extensive division of the upper wall of the urethra, through which the urine escapes. Congenital vesical fissures, often found combined with epispadias, belong to the section on deformities of the bladder, and will not be considered here.

Various degrees of the deformity are met with. In glandular epispadias the urinary opening is situated just behind the glans penis; in penile epispadias it is situated more posteriorly, on the dorsum of the organ or just in front of the symphysis.

Epispadias is of extremely rare occurrence compared with hypospadias. The commonest form of epispadias is that of the deeper urethra in conjunction with ectopia of the bladder; less common is epispadias of the penis, and least common of all is epispadias of the glans. Exactly the reverse of hypospadias in relation to position.

Glandular Epispadias (Fig. 10).- The rarity of this malformation is shown by the fact that but three cases of its existence are recorded. The first was observed by Adelmann and drawn by Von Ammon (F. A.J. von Ammon, Die angeborene Chir. Krankh. d. Menschen, Berlin, 1842,

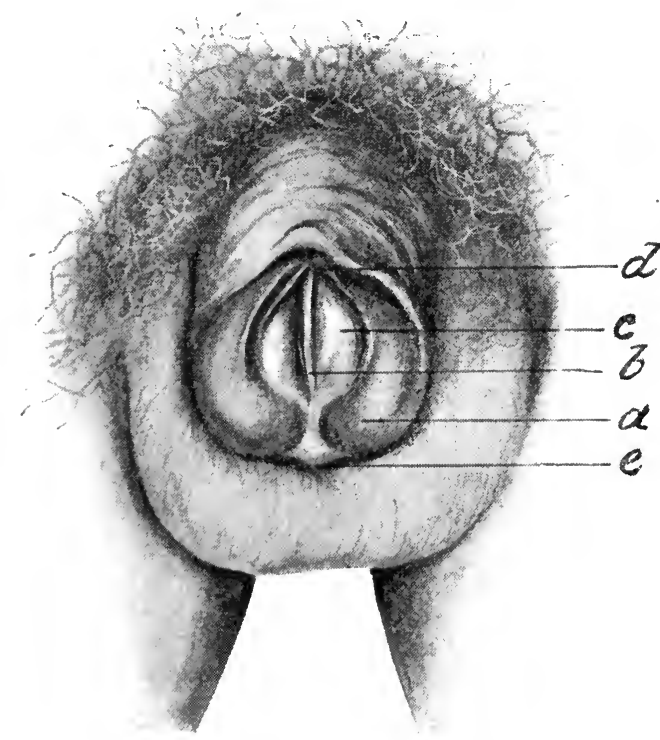

FIt. 10.-Rare glandular epispadias. Tab. xviii, p. 85). The second and third are those of Marchal de Calvi and Dollinger, of which complete descriptions are given by Kaufmann (loc. cit., page 40). These cases show us two distinct forms of penile epispadias. In the rarer form the entire body of the glans is divided into two halves, only united at their lower margins by the floor of the urethra. More commonly the glans is entirely solid, being furrowed upon its upper surface by a urethral trough but a few lines deep.

The other changes in glandular epispadias are not very marked. The penis is somewhat shorter, and, as it were, more voluminous and spread out than normally. It is fairly well formed 
externally. It is worthy of note that the prepuce never seems to be involved.

Epispadias of the Penis (Fig. 11).-Here the urinary opening is on the dorsum of the penis, and most commonly immediately in front of the symphysis. Only a single case, that of Foncher's (Fig. 11), is partial in the sense of the French authors, the opening being situated on the middle of the dorsum penis, and all the other relations being normal; it is the spongo-glandular epispadias of Duplay (Fig. 11). In the usual type (Fig. 12) the massive prepuce hangs as a triangular fold upon the normal scrotum. The frænum is well developed, and is attached to the lower and anterior surface of the glans, which lies in front of the pubis. The upper surface of the glans is in contact with the protruding abdominal skin, which is continuous laterally with the scrotum, there being apparently a depression in the abdominal skin for the reception of the glans.

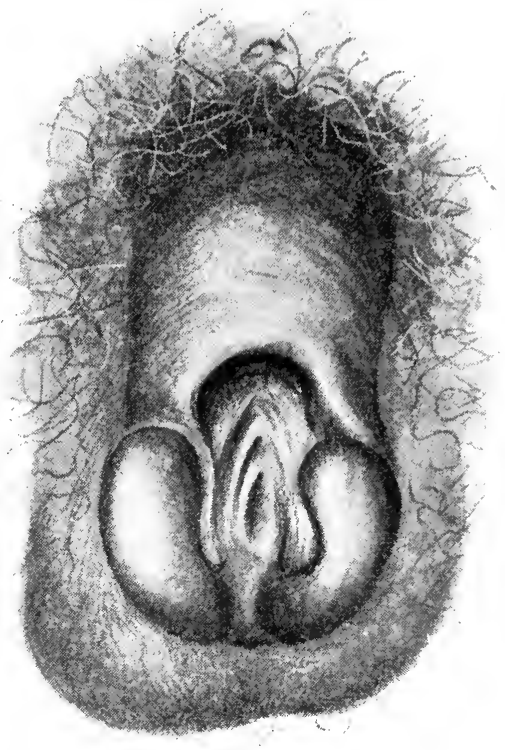

Fra. 11.--Spongo-glandular epispadias. Rare form of penile epispadias.

When the glans is dragged downward and forward the true relation of the parts is seen (Fig. 12). The penis is rudimentary, being one to two inches long. It is curved upward, and almost always twisted. Its upper surface is occupied by a broad and not very deep groove, beginning at the glans and running to the root of the penis. This groove may ex: tend to the apex of the glans, or may end half an inch behind it. In some cases no urethral furrow has been present at all. Both in penis and glans the urinary furrow is lined with mucous membrane, and the pin-sized openings of the mucous follicles are very apparent in it. The edges of the urinary furrow are usually rounded, and the mucous membrane merges gradually into the surrounding skin. In the cases of Larrey and Guyon, the urinary furrow was a deep and narrow cleft, with a bridge over it just behind the glans.

Just in front of the symphysis the urethral groove ends in a deep, funnel-shaped opening, covered in part by a fold of the abdominal wall. Above this fold is the aforementioned depression in which the glans 
penis rests; it is lined with a mucous-membrarelike reddish, shining tissue. Palpation reveals the fact that the symphysis is absent, the hori-

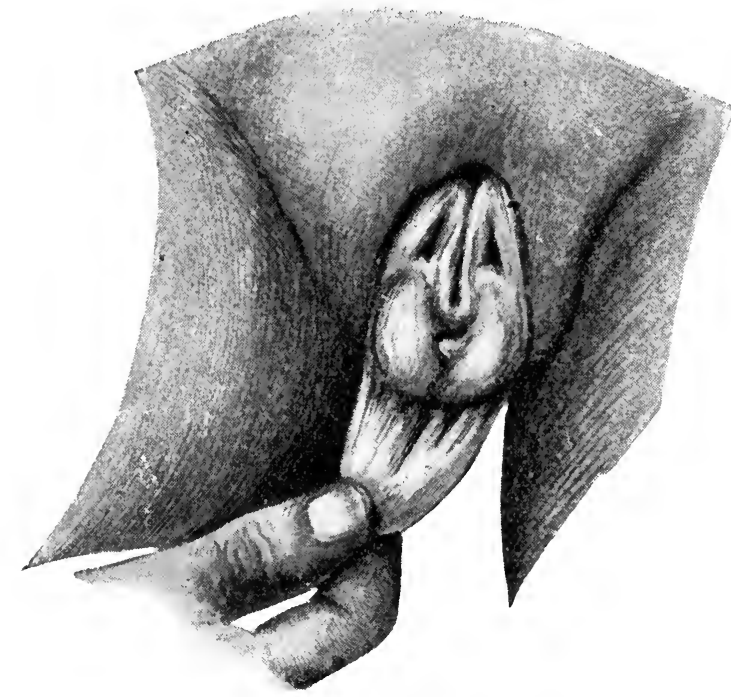

Fia. 12.-Penile epispadias. Commonest form.

zontal rami of the pubes are half an inch to an inch apart, and between their ends lies the depression for the glans.

The segment of normal urethra that remains is usually very patent, so that in some cases the index-finger could be readily carried into the bladder.

As regards the rest of the genitourinary organs, we have but few data. In the records of the four autopsies that have been collected the bladder and the ureters were found unchanged. The prostate gland has been found absent by Breschet and by Thiersch. Diastasis of the pubis has been found in about half the cases. The corpora cavernosa were well developed in their posterior portions, but of the corpus spongiosum only a piece an inch in size was present, and the bulb was smaller than normal. No erectile tissue was found at all in the anterior portion of the penis. In fact, the relations of the erectile tissues have varied much in the different cases.

Etiology.-Much that has been said in regard to the etiology of hypospadias holds good here. In fact, it is epispadias that gives the chief support to the etiological theory advanced for both conditionsviz., that both are due to a rupture of the urethra in consequence of urinary retention by the absence or retarded formation of the glandular urethra.

The usual theory of epispadias is similar to that which explains hypospadias as due to incomplete or faulty development of the parts affected. Thus, Thiersch regarded the malformation as due to a pelvic closure and division of the cloaca, which was faulty as regards time. Under normal circumstances pelvic closure occurs first, so that the corpora cavernosa developing in connection with the rami of the pubes are already agglutinated to the sexual buds before the sinus urogenitalis is pushed forward 
by the developing perinæum. But if the cloacal division occurs before pelvic closure the two halves of the corpora cavernosa are not united; the urogenital sinus is pushed forward between them, and they unite below instead of above it.

This hypothesis evidently depends for its premise upon the absence of the symphysis, and, unfortunately for it, the symphysis is not always absent.

On the other hand, a number of facts undoubtedly favor the rupture hypothesis, and are quite inexplicable on any theory of defective derelopment. Absence or imperviousness of the urethra leads to accumulation of urine in the bladder and dilatation of that organ; a rupture occurs at a time when the abdominal envelopes are not yet quite perfect; and this rupture may affect the entire urinary sac from the navel down, or only its lower part. So we get epispadias with exstrophy of the bladder, or simple epispadias. Thus, Thiersch has proved, both in the dead and the living subject, that the ureters are much dilated in all these cases, he even succeeding in passing a No. 6 English catheter throtgh the ureters into the pelvis of the kidney. Scar tissue also is found all around the bladder opening "as from an unsuccessful plastic operation"-distinct evidences of rupture and cicatrization.

But the most incontestable proof of the correctness of this so-called mechanical theory of the origin of epispadias is found in the cases of intra-uterine healing of exstrophy of the bladder and of epispadias, of which the best case is that of Küster. Here, in a boy one year and seven months old, an unmistakable scar stretched from the umbilicus on to the dorsum of the penis.

In fact, it may well be that the separation of the pubic rami is the direct effect of the same urinary retention that causes rupture of the vesical.and urethral walls. Thus, an early rupture of the urethra would give us a simple epispadias with normal symphysis. Whereas, if this early rupture does not occur, the symphysis separates; if the urethra now ruptures, we get simple epispadias with absence of symphysis. If both bladder and urethra rupture, we get exstrophy of the bladder and epispadias.

What, then, are the causes of this urinary retention, and what are the factors that decide whether it shall rupture on its lower or upper surface; whether hypospadias or epispadias shall result, the glandular segment of the urethra being imperfect or absent? In the great majority of cases, as we have seen, this occurs on the lower border. It is not possible for us to say precisely why in a few cases it selects the upper surface. Evidently the place of least resistance will give way. It may be that the formation of the glans is abnormal and belated; it is possible that the position of the fotus may be such, or the cord may be so located, as to 
put abnormal pressure on the lower surface of the penis to prevent hypospadias and induce an epispadias.

Such a dorsal perforation is by no means so simple a matter as the ventral one; the urine has to force its way, first upward at the posterior border of the glans, then upward and forward through the glans itself. The furrow on the upper surface of the glans is not the divided urethra; it is due to the perforation. If the glandular urethra is present at all (which is rarely the case), it is much deeper, as Dollinger's case shows.

The non-union of the symphysis is explained by the condition of the bladder. Thiersch is of opinion that the mere presence of a distended bladder will prevent union of the rami. In any case the amount of force is not great. The shortening of the penis depends on the deformity of the symphysis and the division of the corpora cavernosa, as well as from the cicatricial contraction that follows the rupture of the urethra and the infiltration and breaking down of the surrounding tissues.

Epispadias probably occurs at the time of the first accumulation of urine in the bladdet, as does hypospadias. The adherents of the developmental theory fixed its occurrence at the fourth to the sixth week, when the pelvic girdle is formed and the cloaca divides. It probably occurs much later, though its precise date can not be fixed.

Symptoms.-These include the results of examination, which are fully given above, and certain functional disturbances of varying degree. When epispadias is incomplete, these latter symptoms are not marked. The most important of these is incontinence of urine. It was not present in the three cases of glandular epispadias that have been recorded, as well as in a number of cases of complete deformity. The stream, however, is imperfect even in those patients who can hold their urine; it is divided and scattering, soils the clothing, wets the neighboring parts, and leads to excoriations, etc. But in most cases there is permanent incontinence, and, this added to urinous odor, constitutes the most serious symptom of the deformity. Some of these individuals can retain their urine for a considerable period of time when lying down, but change of positian, coughing, or anything that increases the abdominal pressure, will cause it to exude. The explanation of the incontinence is somewhat obscure, but since the bladder itself is normal there must be some defect of the closing apparatus. Where the symphysis and prostate are absent its occurrence is readily understood. In other cases Trendelenburg has shown it to be due to abnormal width of the urethra, so that the sphincter can not work completely. The incontinence got better when the urethra was narrowed.

The sexual function of the penis is fair, in spite of its misshapenness. Erection and ejaculation occur, but copulation is imperfect and fecundation impossible. 
The separation of the pubic bones is readily recognized by palpation. It is a source of great annoyance to the patient, since it enlarges the epispadic opening and still further interferes with the sphincter. Exstrophy of the bladder occurs with epispadias, but it is a distinct malformation, and will be considered elsewhere.

Prognosis is favorable. The incontinence usually improves after a closed urethral canal has been formed, so that the patient can hold his water for from one to two hours. If incontinence remains after operation, this can usually be controlled by wearing an apparatus to close the new urethra.

Treatment.-The operations for the relief of epispadias are among the triumphs of modern surgery, not only relieving the deformity, but
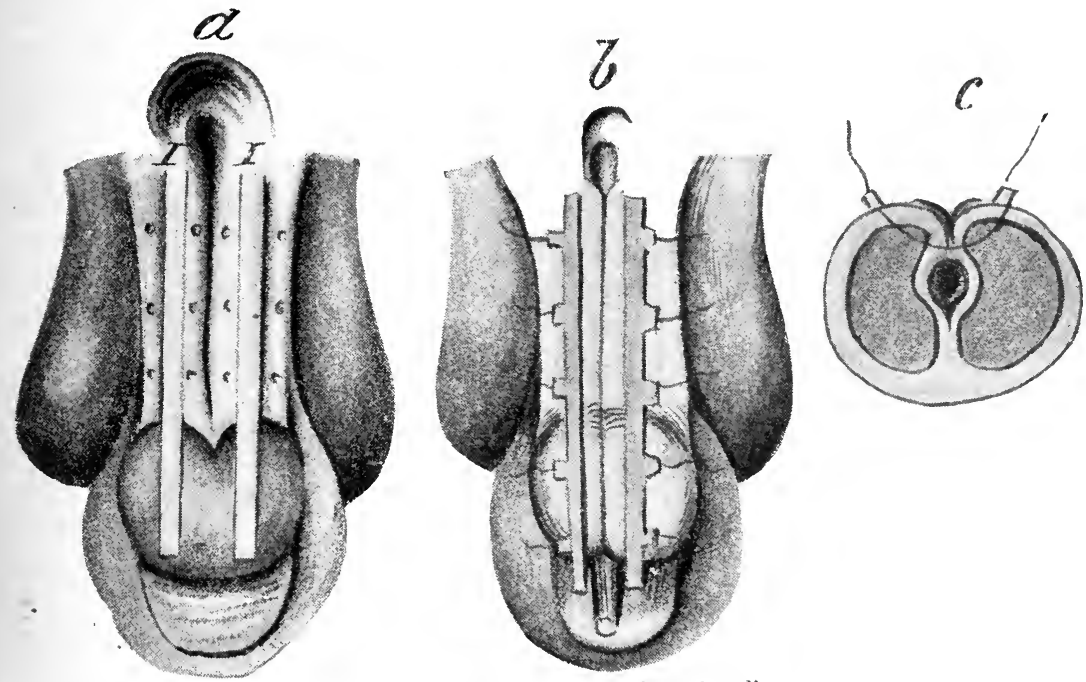

FIG. 13.-Duplay's operation for epispadias.

restoring an almost perfect symmetry to the parts. Two methods have been most successful:

1. Median union of the edges of the urinary groove (operation of Dieffenbach and Duplay).

2. Plastic closure of the urinary canal by the formation of flaps (method of Nélaton, Dolbeau, and Thiersch).

I. Duplay divides his operation into three stages:

1. Straightening of the penis.

2. Formation of a new canal from the extremity of the glans to the neighborhood of the epispadic opening.

3. Junction of the two portions of the canal.

1. It is often difficult to straighten out the penis, owing to defective 
development of the corpora cavernosa. Single or multiple incisions are made into those bodies in the neighborhood of the pubes. The process is the same as that used by Duplay for hypospadias, but done on the dorsum of the penis. Subsequent development of the penis helps the straightening.

2. An attempt is now made to form a new canal almost exclusively from the corpora cavernosa and the corpora spongiosum. The septum of the corpora cavernosa in epispadias is fibrous and thin; by depressing it from above downward the corpora cavernosa are approximated, and a groove is formed in which the sound may be laid. It can be deepened

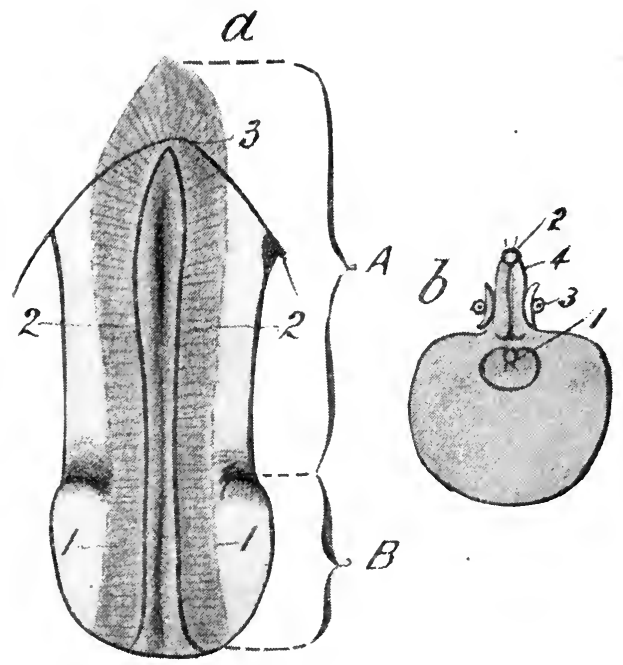

Fı. 14.-Krönlein's operation for epispadias. by incision if too shallow. Then a strip on each side of the penis about a quarter of an inch in breadth is vivified, running from the extremity of the glans to the neighborhood of the epispadic opening (Fig. 13, a). Apposition is secured by the same single-wire quilled suture as was used in the hypospadic operation (Fig. 13, b). A permanent catheter is now passed into the epispadic opening, to draw off the urine as fast as secreted, and prevent its bathing the wounded surfaces.

In most epispadiases the prepuce is very large, and forms, especially in its lower portion, a cutaneous mass which is disfiguring, and may hinder coitus. This exuberant prepuce is to be transplanted to the dorsal surface of the penis. The upper surfaces of the corpora cavernosa are suitably vivified; a large button-hole is made in the thickness of the prepuce at the base of the glans, into which the head of the penis is passed; the two folds of the prepuce, having been dissected free, are applied to the dorsal surface of the organ. This fulfills two important indications : it removes the redundant prepuce, and the thin, rosy, mucosa-like skin of the dorsum of the penis is replaced by normal integument.

3. The epispadic opening is now a fistula, furrowing deeply under the pubis. Its edges are to be freely vivified and then approximated by shotted suture. More than one operation for this purpose may be necessary. 
Krönlein modifies Duplay's operation by employing two instead of three stages (Fig. 14).

Plastic operations for the relief of epispadias date from Nélaton, in 1852 ; his operation was afterward modified by Dolbeau. It consisted essentially in building up a new canal by means of flaps taken from the abdominal wall and scrotum. But Nélaton's autoplasty did not alter the faulty shape of the penis, nor was the urinary control sufficient. The process invented by Thiersch has entirely displaced it. Thiersch's operation for epispadias is divided into five stages:

1. Making a Preparatory Perineo-vesical Fistula.-The left indexfinger or a male catheter having been introduced into the bladder, the bladder neck is pushed toward the perineal raphé as much as possible, and an incision made through the raphé in front of the anus. An elastic or metallic tube is put in, and a fistula is produced. Thus the urine is entirely diverted from the subsequent field of operation.

2. Change of the Glandular Furrow into a Tube, with its Mouth at the Apex of the Glans (Fig. 15).-On each side of and parallel to the glandular furrow an incision is made. The two cuts converge at an acute
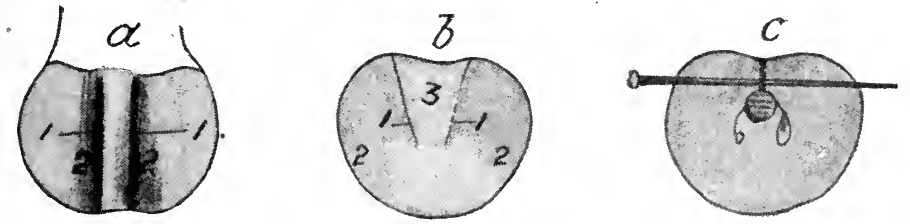

Frg. 15.-Thiersch's operation for epispadias. Second stage.

angle, and should go two thirds through the thickness of the glans, dividing that organ into two lateral flaps and a middle wedge-shaped piece. A narrow strip upon each side of the incisions is now vivified, and the two lateral flaps are pulled together over the central depressed segment. The vivified surfaces are united by two or three stitches, and the new canal gets its epithelial covering from the central sunken wedge (Fig. 15).

3. Transformation of the Penile Furrow into a Tube (Fig. 16).Two thick, long, and rectangular flaps are cut on the dorsum of the penis on each side of the urinary furrow. Both look the same way, the attached margin of the right one being along the right border of the dorsum of the penis, while the attached margin of the left one runs parallel and near to the left margin of the urinary groove. This last flap is now turned over, so that its epidermal surface faces the groove, and its raw surface is external. It is temporarily fixed in this position by three or four sutures, to the free ends of each of which a separate needle is attached. The right flap is then drawn over the left one, so that the two raw surfaces coincide, and the entire denuded tissue is covered. Before, however, the lips of 
the right flap are fixed in their new position, both ends of the sutures put in the left flap must be passed out through the base of the right flap and

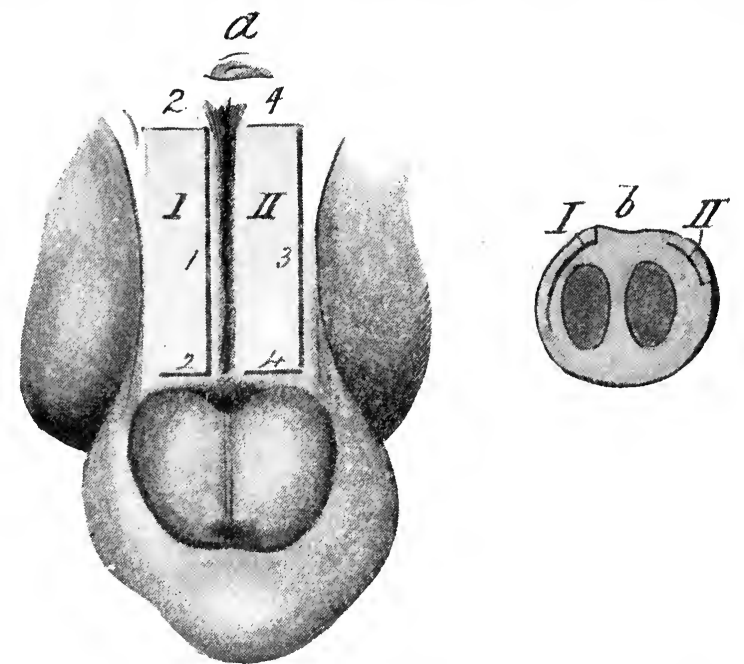

Frg. 16.-Thiersch's operation for epispadias. Third stage begun.

tied; then the left margin of the covering flap must be fixed by sutures. In this way we obtain a tube completely clothed with epidermis, and the entire surface is covered with skin (Fig. 17). Care must be taken that
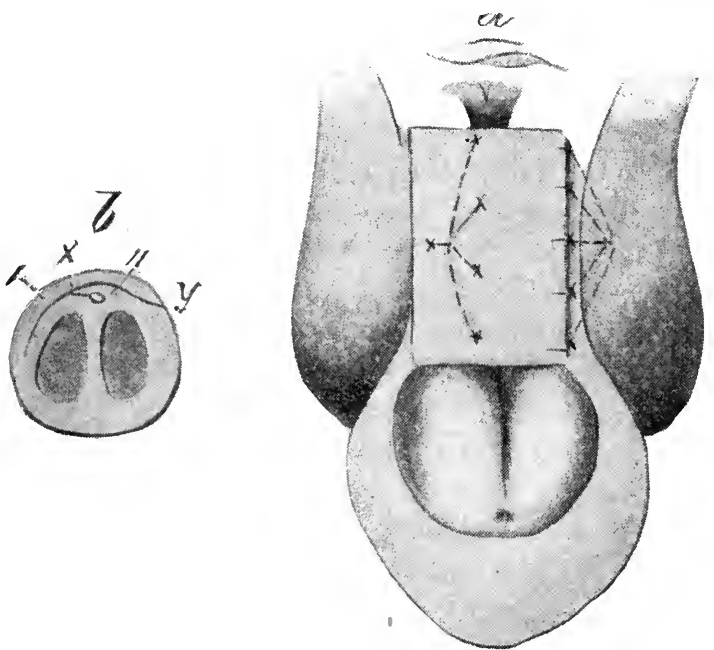

Fra. 17.-Thiersch's operation for epispadias. Third stage completed.

the flaps are as thin as possible, and they must be very freely loosened from their bases, to prevent all possible tension. 
4. Uniting of the Glandular and Penile Portions of the New Urethra (Fig. 18). - The opening between the two segments is to be closed by the anterior half of the prepuce. A hole is cut through its entire thickness large enough to admit the glans, which is thrust through it. The cleft between the glandular and the penile urethra is freshened, and the two leaves of the prepuce, being somewhat separated, are sutured to it and to the vivified lower border of the glans.

5. Closure of the Funnel (Fig. 19).- Two flaps are cut from the abdominal skin above and to one side of the orifice. The first flap at the left side has the form of a right-angled triangle, whose base covers half of the upper margin of the opening. It is so turned down
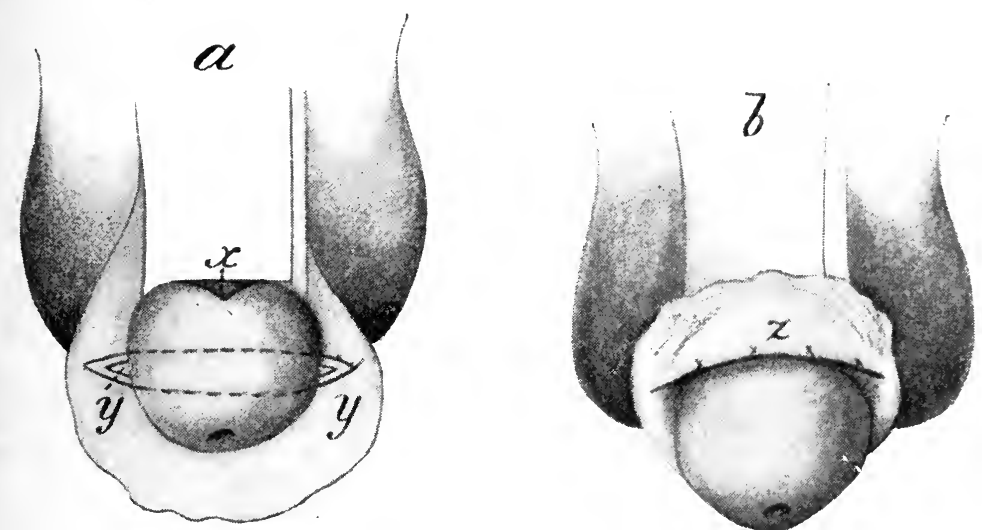

Fra. 18.-Thiersch's operation for epispadias. Fourth stage.

(Fig. $19 A$ ) that the dermal surface looks into the funnel, and its free margin. is then united to the vivified edge of the new covering of the penis. The second flap forms a long quadrilateral (Fig. 19 B), with its base in the region of the right inguinal canal. Then it is pulled down until the raw surfaces of both flaps coincide, and are fixed with sutures. The denuded places in the abdominal skin are allowed to heal by granulation.

The perineal fistula heals of itself - and the treatment is ended.

Thiersch calculates that the perineal fistula takes fourteen days to be established; the closure of the glandular groove, fourteen days; the closing of the penile groove, twenty-one days; the transplantation of the foreskin, fourteen days; the closure of the funnel and subsequent operations, forty-two days-altogether about four months.

The results are excellent in every respect, and Billroth characterizes the operation as one of the most brilliant in the entire range of plastic surgery. 
There occur cases, however, in which the skin of the penis is not sufficient to form a new urethra. For these cases Lossen has modified Thiersch's operation. He proceeds as above described until he comes to the formation of the second dorsal flap, for which purpose he takes integument from the right side of the scrotum. Then a prepuce is formed, after Thiersch. A left scrotal flap has to be taken to close the urinary funnel.

As regards our selection of operative procedure, it will depend upon the case. Median union of the urinary furrow is simplest and best where it will suffice, as it sometimes will in cases where there is but little penile
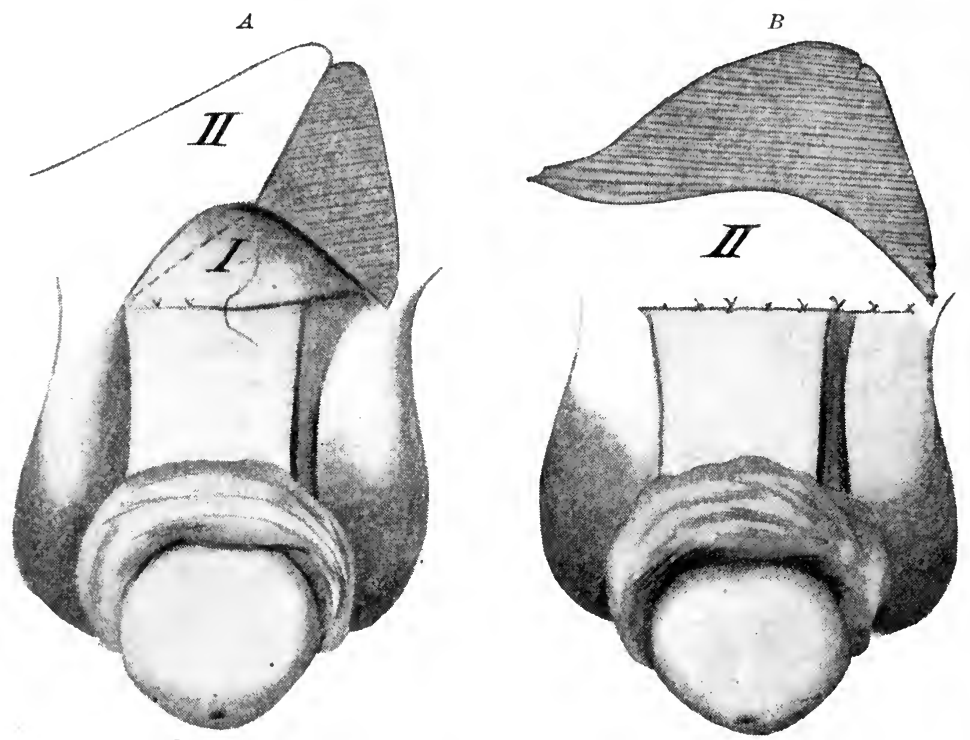

Fra. 19.-Thiersch's operation for epispadias. Fifth stage.

deformity. For other cases we can choose between the methods of Dieffenbach, Duplay, and of Thiersch. Nevertheless, the latter will undoubtedly become the chief method, both on account of its certain and brilliant results and its almost universal applicability.

In Thiersch's operation a triple row of stitches and a double covering protect the new canal; so that when, as frequently occurs, some of the superficial stitches tear out, the parts are still kept in position. The avoidance of a permanent catheter is also of great importance; its presence frequently spoils the most carefully executed plastic operation. 


\section{URETHRAL AND PERI-URETHRAL ABSCESS.}

Collections of pus occur in the peri-urethral connective tissue, and may grow to a considerable size. Perforation of the urethra from any canse permits the urine to escape from the canal, whence it slowly makes its way into the surrounding tissues. Here it acts as an intense irritant, provoking inflammation, suppuration, and even gangrene of the invaded tissues. From such causes we meet with abscess in an acute and chronic form. The microbic infections of these punctured wounds, whether by the penetrating body, or by entrance into the wound, from its urethral side, of any of the pathogenic micro-organisms which are known to be normally present in this canal are to be thought of as more direct cansative agents than the urine, which in the latter cases can only, as the possible carrier, be looked upon as the indirect cause. Urine free from micro-organisms, ptomaines, and leucomaines is inadequate for abscess formation.

Acute Urinary Abscess.-A stricture of the urethra leading to rupture is the common precedent of this condition. The course of the pus and the resulting damage vary in accordance with the location of the urethral lesion. If the rupture is in front of the triangular ligament, as is most often the case, the pus collects in the anterior perineal region, spreading to the sides of the anus and scrotum, even reaching the under surface of the penis, which may be half buried in the infiltrated mass, and is tender and resistant to the touch. If, on the other hand, the rupture is in the membranous urethra, behind the triangular ligament, the pus collects in the prostatic fossa. Its forward advance is hindered by the triangular ligament, and it makes its way into the ischio-rectal fossa, and points near the anus. The course and complications of these deep urethral abscesses are very similar to abscess of the prostate and abscess of the seminal vesicles, which are described elsewhere.

Only in rare cases does such an abscess break into the urethra, pus flowing from the meatus in the intervals of micturition and appearing in larger quantities when the tumor is squeezed. Spontaneous cure then occurs, the tumor diminishing gradually in size, and the small indurated lump left behind gradually disappearing. Almost always the abscess opens externally; pus and urine infiltrate the cellular tissue, and troublesome fistula and grave disorders result.

Symptoms. - Slight chills, irregular fever, tension in the perinæum, and difficulty of micturition are the indefinite symptoms that mark the advent of pus in the cellular peri-urethral tissue anterior to the triangular ligament. The pulse is rapid and irritable, the skin parched, the tongue dry. In the anterior perineal region is found a rounded, hard, painless 
tumor, extending perhaps so far as to encroach on the scrotal and anal districts. If the abscess is behind the triangular ligament, the general symptoms are the same; but no distinct tumor is appreciable in the anterior perinæum; only the skin covering the ischio-rectal region is indurated and brawny, and rectal examination will often reveal fluctuation, especially unmistakable if it has spread posteriorly-that is, over the sacrum and coceyx.

Diagnosis. - If a patient who is the subject of stricture presents general symptoms - of sudden advent-as described above, a careful examination of the perinæum should at once be made. The local symptoms may not be pronounced enough to attract the patient's attention; or he may state that the induration or tumor has been present for weeks, and can not be the cause of his trouble. A perineal abscess, not communicating with the urethra, may be mistaken for a urinary abscess; or, conversely, an apparently simple perineal abscess may, a few days after opening, show by the flowing of urine from the wound that it originated in the urethra. Luckily, the treatment is the same in both cases.

Treatment.- The first step is the free incision of the tumor, practiced even before fluctuation is perceived. The pus is very deep-seated; sometimes two or more inches of tissue must be divided before it is reached. If not given an external vent early, the pus, being limited bythe perineal fascia, will pass backward, and cause mischief around the neck of the bladder and in the cellular tissue of the pelvis.

Many anthorities are satisfied to open the perineal abscess, leaving the stricture to be treated later by dilatation or otherwise; but Bryant regards this as temporizing, and advises the division of the stricture at the same time. A grooved staff is passed through the urethra down to the abscess, if it can not be passed into the bladder; the tissues are then freely divided through the perinæum. If the orifice of the vesical end of the urethra can be detected, a large catheter should be passed and left in. In any case the stricture has been divided, the pus is freely evacuated, danger of extravasation of urine is prevented, and a free passage for the kidney excretion is provided.

Chronic Urinary Abscess. - Urine infiltrated into the peri-urethral connective tissue is so irritating that it almost always causes an acute abscess; chronic urinary abscesses are rare. They occur in the pendulous urethra as well as in the perinæum. In the perinæum they occur as small, rounded, hard tumors, adherent to the urethra, and covered with normal skin. In the pendulous urethra they may be multiple, and form little hard nodules adherent to the urethra. Both perineal and penile chronic abscesses may at any moment become acute; or they may long remain quiescent, and even ultimately undergo absorption and obliteration.

Symptoms are hardly present. They are often so painless as to 
escape the notice of the patient. Examination then alone reveals their presence.

Diagnosis is ordinarily free from difficulty. However, a peri-urethral abscess may be mistaken for a urinary abscess, and vice versa; but since the treatment in both cases is the same the mistake is not serious.

Treatment.-Tincture of iodine may be applied externally over the chronic perineal urinary abscesses; it is not always advisable to open them. The penile ones can also be left alone, save when annoying; in that case they may be evacuated, and the resulting fistula treated secundum artem. Where the urethra will permit of the introduction of the wire speculum, it has recently been my practice to expose by it the urethral opening of these small blind abscesses, when by external pressure the contents, even if very trifling, can be seen to exude into the urethra. Now with a fine blunt bent silver tip (Fig. 24, B) a few drops of hydrogen peroxide are forced by syringe into the cavity. On evacuating this again by pressure a minim or two of five-per-cent argentum nitrate solution is introduced. Fine as is this syringe-tip, it is not always easy to introduce it into the very minute fistulous opening in the urethra. A fine, probe-pointed bent knife may then be used to open the abscess more freely. on the urethral surface, after which cleansing as described is easily done. By this procedure all danger of urethral fistula is avoided.

\section{URETHRAL ULCER AND EROSION.}

Ulceration, more commonly erosion, of the urethra occurs as a complication of specific urethritis. It is generally a sequela rather than an accompaniment of the acute stage of gonorrhœea. Whether the gonococcus by deeper invasion than usual, or a mixed infection, or personal neglect, is most often responsible for the occurrence of these lesions, has not yet been determined. Rough and septic foreign bodies introduced into the urethra, the continued contact of urethral calculi, herpes of the urethra, may all serve as causative agents. Urethral chancroid, although presenting much the same anatomical appearances, and amenable, but more slowly, to the same treatment, has, I believe, a distinct etiology. I purposely omit consideration of the slight inflammatory erosion often existing at some one point on the proximal side of a stricture.

The most common locality for all these lesions is within two and a half inches of the meatus. I have found them more commonly on the upper rather than the lower segment of the urethra. With the urethral wire speculum (Fig. 20) they are readily seen and treated. They vary from a pin-head to a pencil-head in size; they may be single or multiple. The appearance of these lesions is different, when viewed at the extrem- 
ity of a cylindrical Klotz tube, from their appearance as seen through the lateral bars of the wire speculum. This difference is due to the mechanieal pressure of the speculum; and by reason of it the true hyperæmia
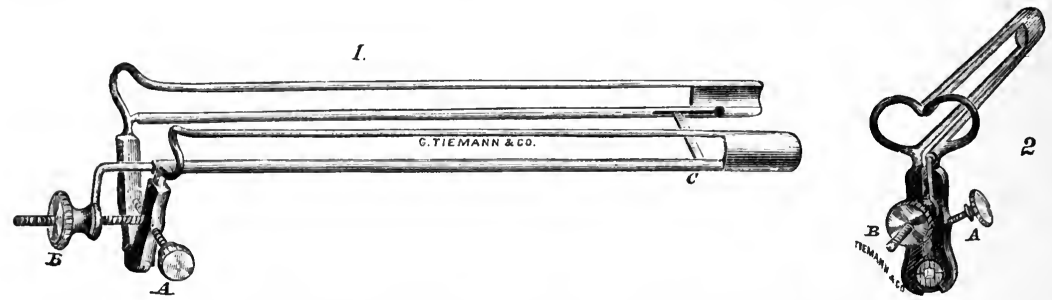

FIG. 20 (1).-The author's wire urethral speeulum, open. $C$, the lever for spreading the vesical end, which is governed by the right-angled rod attached to the traveling screw-button, $B ; A$, the screw which spreads the base or meatus part of the speculum. (2.) The author's wire urethral speculum, closed.

of the area surrounding the ulcer or erosion is less striking, but a much greater field is shown, and the details of the individual lesions are better exposed for observation and treatment.

Erosion of the urethra shows an absence of the smooth, pinkish-yellow, normal mucous membrane, and a faint cellular infiltration about its margin, attended by increase of color. When such a lesion is exposed to sight and its surface touched with a blunt instrument, the extreme sensitiveness of the lesion becomes manifest.

Ulcer of the urethra shows a complete loss of the mucosa; its edges are precipitous or in part overhanging, so that a right-angled probe, or the urethral digit (Fig. 21), will be perceptibly caught as it is drawn

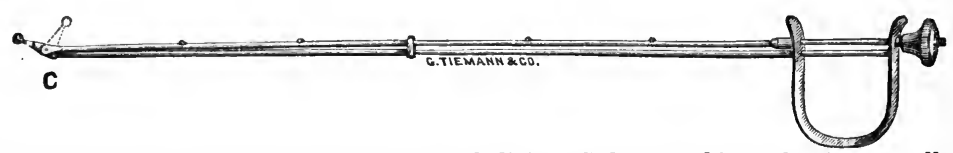

FIG. 21.-The author's straight six-inch urethral digit. $C$, the movable probe-tip eontrolled by $\dot{\mathrm{U}}$-spring and thumb-serew at handle. Inch-marks are indieated along the upper rod.

across the lesion. The edges are clean cut or slightly irregular, considerably infiltrated, and consequently elevated not alone above the floor of the ulcer but higher than the surrounding mucous membrane. The floor of the ulcer is covered with a greyish-white muco-purulent secretion, which on being removed shows an uneven granulating or pitting surface. A secondary inflammatory areola often surrounds the hyperæmic borders of the ulcer. The most positive subjective symptom of urethral erosion, or ulcer particularly, is a sense of pricking pain in the urethra, wholly independent of the act of urination. Urination may elicit the same discomfort momentarily, but it is the sudden striking sensation which is felt in the intervals which will prompt us to look successfully for such a cause. Occasionally quite remote reflex pains are experienced in the 
groin and down the leg. At times these lesions cause functional disturbances of the bladder and muscles of the deep urethra, akin to the reflexes not infrequently excited by anterior strictures of large caliber.

In some cases an appreciable enlargement of the inguinal glands will be noticed.

A trifling but positive muco-purulent secretion from these lesions will, if looked for, be found in each glass of urine passed.

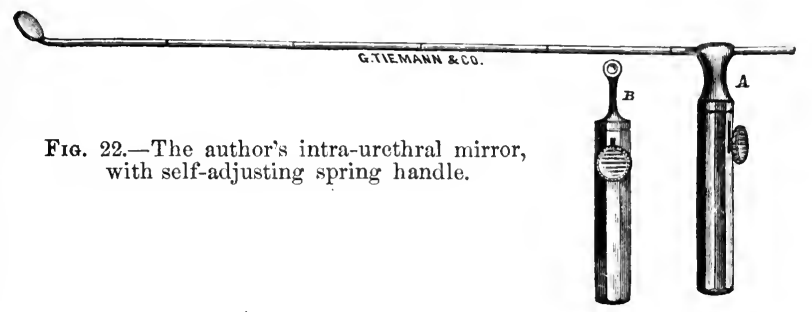

When a bulbous instrument, without giving distinct evidence of stricture at some one point, does at this particular point cause a good deal of sharp pain, an erosion or ulcer of recent formation may be suspected.

The urethral digit will help to verify the diagnosis, but it is only

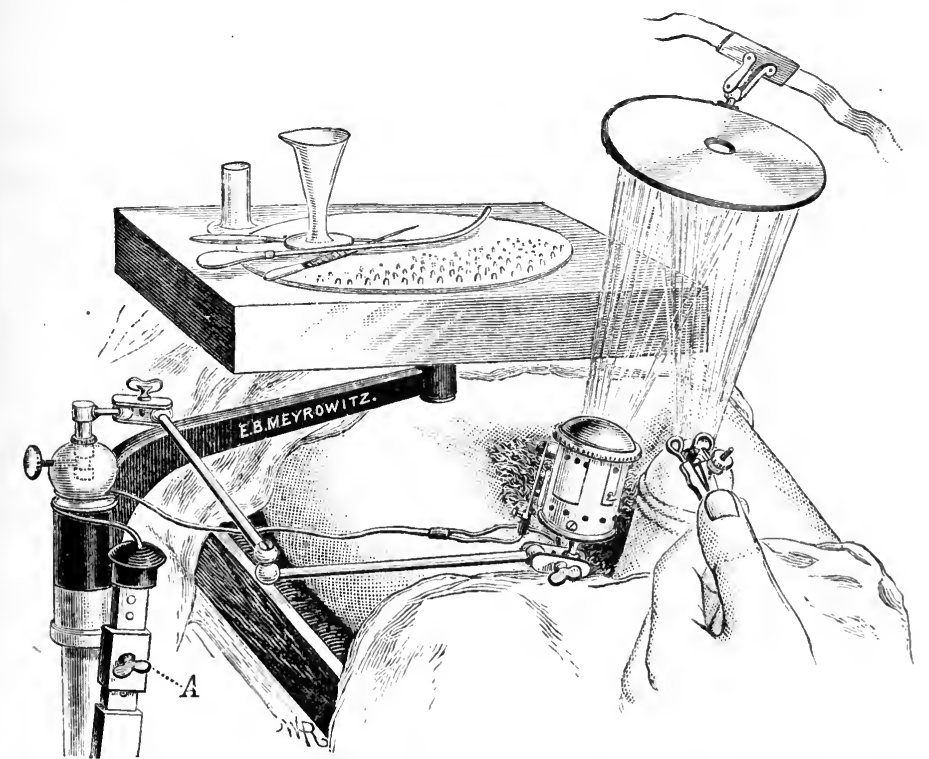

Fig. 23.-The author's method of urethral illumination by electric-light condenser parallel with penis. Head mirror is shown reflecting the light through the speculum into urethra.

by the use of instruments (Fig. 23) which afford an ocular demonstration of the lesions that the disease can be positively diagnosed. 
The intra-urethral mirror (Fig. 22), illuminated by reflected light from the head mirror, will very clearly reveal the details of these lesions as they are exposed by the speculum; but the mere presence of an ulcer with its truncated cone summit and its inflamed borders can be readily determined without the aid of the intra-urethral mirror, particularly if it is not deeper than two inches.

Treatment.-Only by resorting to the use of instruments which will expose these lesions to the eye can the treatment, as well as the diagnosis, be made satisfactory. To effect this, I prefer my wire speculum, already mentioned.

If the patient is nervous, or if the parts are particularly sensitive, a preliminary injection of fifteen to thirty minims of four-per-cent cocaine solution will be of aid. The speculum is introduced closed (Fig. 20, 2), and gradually opened at both ends until a favorable exposure of the ulcer is obtained. Now the floor and walls of the ulcer are to be cleansed with a drop or two of hydrogen peroxide, best applied by a syringe with a long, delicate silver tip (Fig. 24), so bent where it comes off the syringe that hand and syringe do not interfere with the line of vision. The part

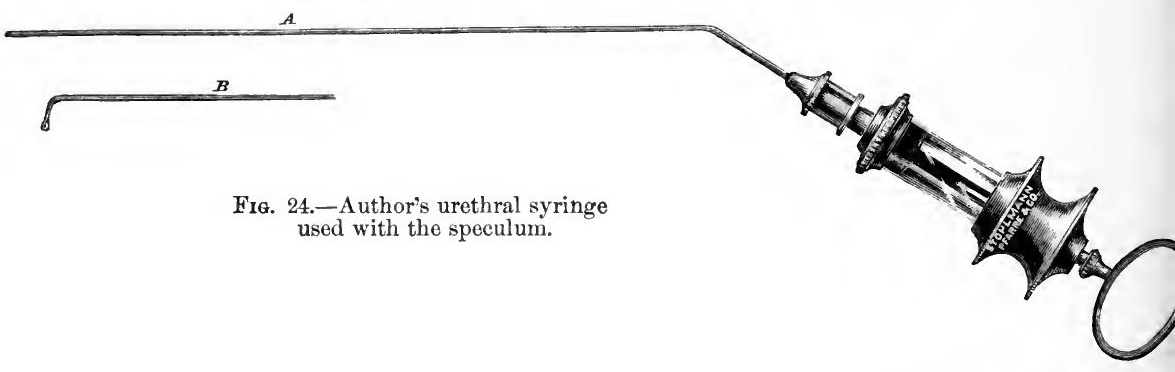

is then wiped with a small cotton swab. Finally, an application of silver nitrate solution, one half to ten per cent strength, is made by a similar syringe-tip wrapped with cotton. The dilatation of the urethra effected by the speculum during such application every fourth or sixth day is an important adjunct to the treatment in promoting absorption of the inflammatory infiltration, thereby lessening the amount of cicatricial contraction which normally attends the healing process.

If such an ulcer of the urethra has existed for several months and has escaped treatment, its appearance will differ from that of the acute ulcer already described, and the treatment must be different. It will now show as an indurated ulcer, the inflammatory process being almost limited to the areola at a little distance from the infiltrated whitish edges and walls of the ulcer, while its base will be affected to a lesser degree by this same whitish infiltration. Tension upon this immediate area of the urethra by the dilating arms of the wire speculum will reveal a marked impair- 
ment of the normal elasticity and the patency of the mucous membrane, consequently an examination by bulbous instruments would now certainly detect a slight but appreciable loss of normal caliber.

At this stage of the ulcer topical applications are of no practical value, for the indolent character of the nutrition processes calls for more energetic treatment.

A certain degree of cicatricial coarctation already exists, and a complete healing of the ulcer by a continuance of Nature's method will be

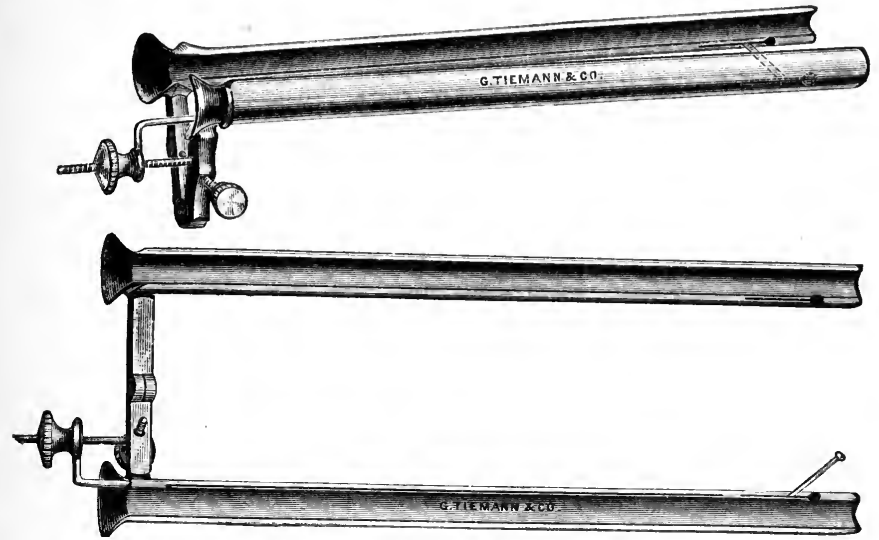

Fig. 25.-The author's bivalve urethral speculum. The figure shows the full extent to which all these specula open for easy eleansing.

effected at the greater expense of the urethra by stricture. Gradual dilatation of the part by use of the sound or the bivalve speculum (Fig. 25 ) will in the majority of cases be effective, but at so great an expenditure of time that it is not to be recommended.

A thorough linear incision through the hard infiltrated walls and floor of such an ulcer is the only proper procedure. To do this, the speculum is absolutely necessary. It puts the tissues upon the proper degree of tension to facilitate cutting, acting at the same time by its lateral pressure as a hæmostatic; and so by ocular guidance a second incision can be made should the first be insufficient. To do this a small knife, shaped like the gum-lance with long handle, is requisite; but, in order to interfere as little as possible with the line of vision, and to guard against cutting any but the tissue intended, as well as to secure uniform but firm vertical pressure, I recommend my diverging urethrotome (Fig. 26), which has been devised for special use in operations which the urethral speculum renders possible-such as annular strictures of medium and large caliber.

For the past three years I have used this or a modified form of tome for making incisions through the speculum under occular guidance. There is every reason to believe that many of the operations which have 
been and are still being done under tactile direction alone will in the future be relegated to the more delicate sense of sight.

The chronic urethral ulcer which has had this initial treatment of linear incision should then be subjected to gradual dilatation with the

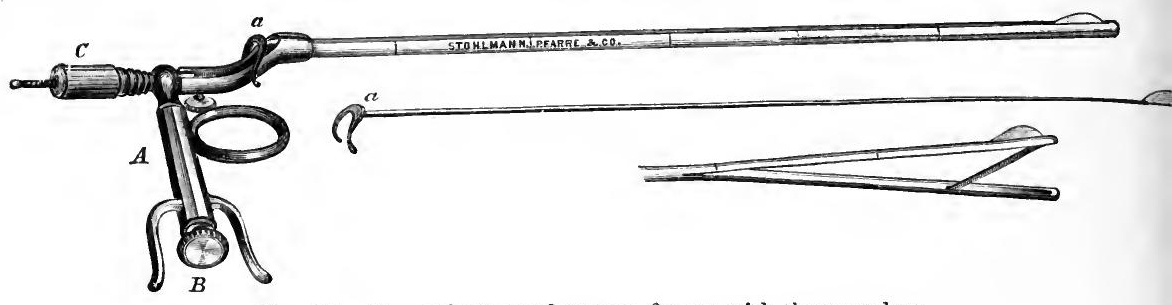

Fig. 26.-The author's urethrotome, for use with the speculum.

speculum, and local application as already described. While speaking of urethral ulcer, although without possession of the data to substantiate the theory, I infer, as elsewhere stated, that a neglected lesion of this kind, occurring in a subject of advanced years and of impaired constitution, may retrograde from its normal tendency to heal as a cicatricial stricture into that rare disease, primary epithelial cancer.

\section{URETHRAL NEOPLASMS.}

\section{URETHRAL INITIAL SYPHILIS.}

Chancre of the uretlira is commoner than is generally supposed, and is undoubtedly the starting-point of many cases of syphilis of unknown origin. It is rare indeed that such an initial lesion occurs at a distance greater than one inch from the meatus. The most common locality for a true intra-urethral chancre is at some point between the extreme limits of the meatus and the fossa navicularis; those occurring at the deeper part of this short portion of the tube are rarer than the more superficial ones.

The earliest subjective symptom of urethral chancre is ordinarily a slight thin discharge, faintly blood-stained, rarely quite purulent. When the last exposure has long antedated (four to eight weeks) this manifestation, it becomes of the utmost importance to positively ascertain the source and nature of this discharge. At this time the patient may also have noticed a moderate impediment to, or, what is more likely, an unusual lateral divergence in, the urinary stream. If this is the case, palpation of the glans penis and the subjacent corpora spongiosum will certainly reveal an induration. The degree of infiltration may be great enough to cause a tumefaction of the overlying parts appreciable to the eye. Again, the lesion may be so small as to make the evidence of palpation doubtful. Under any circumstance, visual examination of the 
urethra is positively demanded, for within certain limitations peri-urethral abscess, urethral ulcer, and urethral chancre have symptoms much in common.

A good demonstration of these lesions is afforded by the urethral speculum.

When such a lesion is felt to be in the anterior urethra, a short twoinch instrument will be selected for use. It can be so turned that the lesion will protrude between the wire arms of the speculum.

The protean types of initial lesions, as they appear in the external genitals, may be reproduced within the urethra. However, I have seen but two varieties, six cases in all ; five of these presented varying types of the smooth, shining, tense, papular syphilide, with a proportionately slight central destruction and depression. The remaining exceptional case, just within a large meatus, on the roof presented a considerable plaque or area of whitish, slightly friable, greatly thickened mucous membrane, with no erosion or destructive central tendency. The external induration felt by transverse palpation of the upper half of the glans penis was marked. Inguinal adenitis and the subsequent cutaneous manifestations must be depended upon to confirm the diagnosis. But the early discovery of a suspicious lesion within the urethra may prove to be of greatest importance to the patient.

In exceptional cases local applications are desirable, but constitutional treatment will best be deferred until the first appearance of unmistakable secondary manifestations.

\section{URETHRAL CANCER.}

Carcinoma of the urethra is usually secondary to cancer of the penis, the prostate, or the bladder. Primary cancer of the urethra is extremely rare. Kaufmann has been able to collect five reliable recorded cases only, in four of which an exact post-mortem and microscopic diagnosis was made. They are those of Schustler, Thiersch, Guyon, Trzebicky, and Guiard.

In all four cases the urethral cancer was found situated in the perineal portion of that tube. In Schustler and in Guiard's cases the perineal swelling simulated an abscess, and led to incision. The carcinoma had evidently undergone colloid degeneration and softening, and masses of detritus had collected in the inclosing walls. In Thiersch and Trzebicky's cases the opening in the perinæum had occurred before the patient reached the hospital. In Thiersch's case the entire urethra had been destroyed; the lower border of the symphysis was exposed, and had already been invaded by the neoplasm. The edge of the bone was so incrusted with urinary salts that, by the catheter, it felt as if an encysted stone were present. 
In the cases that were examined post mortem the mucous membrane of the bladder was found thickened and reddened, and the viscus contained purulent urine. The prostate was enlarged, and the mucous membrane of the prostatic and the anterior half of the cavernous urethra strongly injected. A large, irregular, and ulcerated excavation of the urethra, extended into the corpora cavernosa, varying much in size. The walls of the cavity were lined with hard, irregular masses of tissue of a whitish surface when cut, and exuding a plentiful milky fluid on pressure. Abundant pus, mingled with urine, and of a most unpleasant odor, was present in the cavity. In some cases metastatic carcinoma was found in other parts of the body, in others it was not present. The microscope in every case revealed a carcinoma of the epithelial type.

The origin of urethral cancer is from the urethral mucous membrane, and Thiersch has drawn attention to the possibility of its originating in the region of a stricture. Poncet and Paget record cases in which the carcinoma seems to have originated from the margins of an old fistula. A theory has newly been advocated by Pietrzikowski, namely, that the growth originates from the epithelium of the excretory ducts of Cowper's glands. But in the few observed cases of cancer of these glands the urethra has not been directly implicated, and Pietrzikowski's theory lacks post-mortem confirmation. I incline toward the belief that chronic urethral ulcer, when the lesion has failed to heal by cicatrix, following gonorrhœa in old and debilitated subjects, is the most probable cause of primary urethral carcinoma.

Symptoms.-The first symptom noticed is the interference with urination. Dysuria is present in most cases, anuria in some. Catheterization is difficult in all and impossible in some cases. The beak of the instrument seems to get into a large cavity, and to be stopped there. There may or may not be an abscess-like swelling in the perinæum. Pain is usually present, and is referred to the groin. Stricture, fistulæ, and other urethral lesions are either found or the patient gives a history of having had them in the past. The endoscope has been shown by Grünfeld to be useful for the detection of fungating urethral growths of cancerous origin.

Treatment.-Little may be said under this head, since the cases of urethral carcinoma so far recorded have only been recognized so late that there was no question of any radical treatment. It is to be hoped that the more extended use of endoscopy will enable us in the future to recognize and remove the disease at an earlier stage. Most of the cases have ended very soon after the making of the diagnosis-metastases on the lungs and pleuræ hastening the fatal result in Guiard's case.

Nevertheless, the general verdict is in favor of its being a thoroughly 
local process. In most cases not only were metastases absent, but even the inguinal lymphatic glands were not involved.

In a single case only, that of Trzebicky, the penis was amputated; the patient died four months later from cancer of the inguinal glands.

\section{URETHRAL VEGETATIONS AND POLYPI.}

These rare abnormalities have been endowed with a variety of names, being known as caruncles, polypi, vegetations, fungosities, papillomata, granulations, etc. Our knowledge of their microscopic structure is still too imperfect to allow us to differentiate the varieties that undoubtedly occur, and it is well for us to group them all as fleshy excrescences, carunculæ, or polypi.

They appear as intra-urethral vegetations, similar to the commoner growths found upon the glans and sheath of the penis. Thompson saw
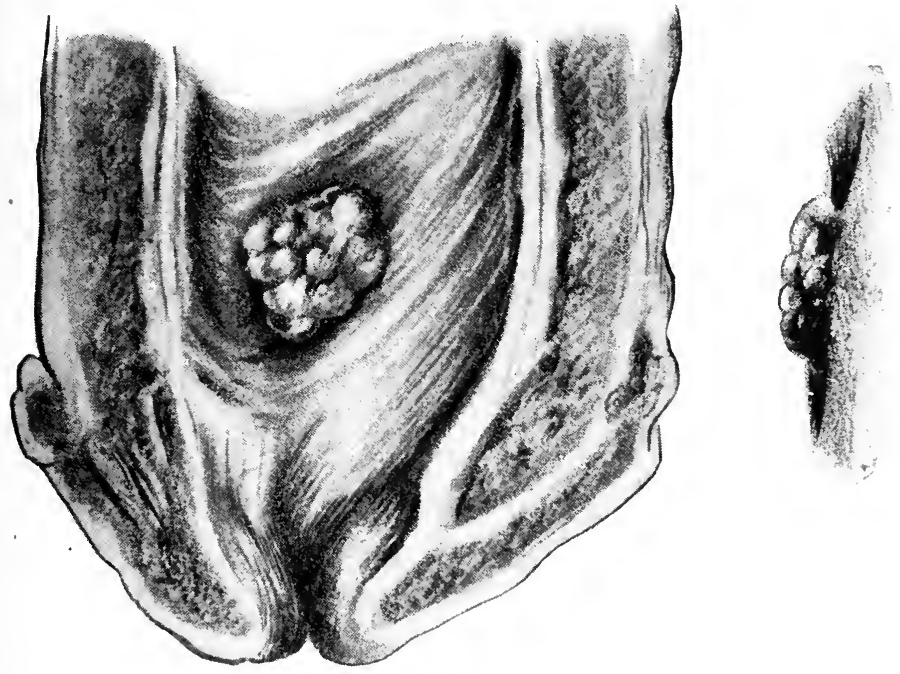

Fig. 27.-Urethral polypus.

one at the junction of the prostatic and membranous urethra, forming a pedunculated tumor nine lines long and three or four broad-a true polypus. Their frequent seat is behind strictured portions of the canal.

The symptoms caused by these growths are few. Rarely do they cause inconvenience or trouble. A slight amount of discharge and some obstruction to the flow of urine are to be noted. Their recognition is possible, of course, only through the endoscope, where they appear as rounded, smooth, or raspberry-like masses, projecting into the lumen of the instrument (Fig. 27). Grünfeld and Oberländer have given us excellent illustrations of the picture thus presented. 
Grünfeld has found that these vegetations may occur anywhere in the canal, and that they may attain a considerable size. Tarnowsky has seen them in connection with urethritis granulosa. They vary in size from a small grain to a cherry.

Treatment is simple and satisfactory. The growths must be torn off by means of the long urethral forceps, the polypus snare, or the polypus scissors. Tedschenko has recently devised an instrument that he calls an endoscopic carunculatome, which is probably very effective when the urethra and the polyp happen to exactly fit the instrument.

I have never seen any of these intra-urethral neoplasms large enough to designate by the name of polypus, but three- or four-millimetre-long papillomatous hypertrophies, with ends of small pin-head size, I have frequently met with. I have found that they were best exposed by the wire speculum, and, as they project between its bars into the open urethral space, they present a perfect opportunity to touch them with glacial acetic acid, under which they rapidly shrink and disappear.

Should the surgeon meet one of these rare polyps of pea-size or larger, its easy removal would be assured by exposing it with the urethral wire speculum, when any of a variety of cutting or seizing instruments would accomplish the extirpation. The point of detachment will also be well exposed by the speculum, for the application of cautery or hæmostatics, if necessary.

\section{WOUNDS AND LACERATIONS OF THE URETHRA INFLICTED FROM WITHOUT.}

\section{RUPTURE OF THE URETHRA.}

Wounds and lacerations of the urethra, coming from without, may be caused in various ways. Wounds proper may be inflicted by any cutting or piercing instrument, by needles, knives, glass, etc. Contused wounds or lacerations of the urethra, with no solution of continuity of the adjacent cutaneous parts, are usually due to direct external violence by a blunt resisting object.

1. Punctured and Incised Wounds of the Urethra are not common, that organ being well protected by the pubes, perinæum, and corpora cavernosa. It can rarely happen that the urethra is wounded without concomitant wounding of other structures.

Punctured wounds are usually not serious. In fact, they frequently heal spontaneously. There may be a small ecchymosis under the skin at the external point of penetration, and a slight hæmorrhage from the meatus. They need no treatment.

It is very different, however, with incised wounds. Their gravity varies with their location and their extent. They are almost always 
complicated with wounds of the perinæum or the corpora cavernosa, and will therefore receive more extended consideration under that heading.

Nevertheless, incised wounds of the male genitals receive a new significance when the urethra is included in the traumatism, since the opening is usually sufficient to allow the urine to escape and to bathe the raw surfaces, though infiltration rarely occurs. We have learned, indeed, that normal fresh urine is not hurtful, and that its presence does not complicate wound-healing. Certain sequelæ are, however, apt to occur : fistula may supervene if the edges of the mucosæ unite with the edges of the skin, instead of with each other; stricture may be caused by the contraction of the cicatrix of a transverse cut.

Longitudinal incised wounds of the urethra heal promptly, without exception, whether closed by suture or not. Transverse incised wounds generally heal spontaneously, but they invariably leave a stricture.

Treatment.-Kaufmann's experiments speak in favor of immediate union of the edges of the wound in transverse incisions of the urethra. The first step is necessarily the introduction of a permanent catheter, to be retained not more than three days. The sutures are accurately applied over the catheter.

When, as is sometimes the case, it is impossible to pass the catheter into the posterior urethral segment, it is proper to follow Reybard's example, and suture the wound notwithstanding. The patient must then be watched with great care, to see if the urinary passage is patent. At the first sign of infiltration the sutures must be removed. If the case is complicated with a solution of continuity of other penile structures, they must be sutured in accordance with the rules laid down under that heading.

Perineal urethrotomy may become a necessary adjunct in severe cases.

2. Lacerated and Contused Wounds of the Urethra.-Lacerated wounds coming from without are rare. They occur almost solely in conjunction with fracture of the corpora cavernosa during erection, either from a sudden bending of the penis or during violent coitus. Violent pain, hæmorrhage, and retention of urine follow; catheterism is frequently impossible. Urinary infiltration is almost certain to occur, with gangrene and loss of substance of the skin, the deeper parts, and the urethra. The patient may die of sepsis. Skinner (Phil. Med. Times, 1880 , page 350 ) cured a case by multiple incision and permanent catheterism, and Dittel one in whom he incised the perinæum on the eleventh day after the accident, and sutured the separated urethral ends.

Rupture of the Urethra from external causes is, of course, simply a lacerated and contused wound of the urethra that destroys its continuity in part or completely.

Contused wounds of the urethra occur from the effect of blunt vio- 
lence which compresses the canal forcibly against the solid and bony parts. They differ much in cause and mechanism in different cases. Terrillon's thesis (Terrillon, Des ruptures de l'urèthre, Paris, 1878) is the completest modern résumé of the subject.

Bryant has observed contusion and rupture of the urethra 19 times in 1,077 cases of affections of the uro-genital organs, being 1.7 per cent of all cases of the kind. If traumatic strictures be included, the number reaches 54, or five per cent. Nevertheless, urethral lesions of this kind are not very common. From the eleventh to the twentieth year is the most frequent period at which these traumatisms occur.

Etiology.-Most often a fall astride of some object is the cause of the accident. The pendulous urethra is rarely affected, its situation and mobility being such as to protect it. Voillemier recites the case of a man whose pendulous urethra was ruptured by the kick of a horse, and Ballard one of a man who was knocked down by a heavy wagon; in both cases the penis was lacerated against the pubis. In one case the dependent penis was caught and squeezed, and the urethra ruptured, when the patient was shutting a drawer. The accident is more frequent when the penis is in the erect condition. Then, the attempt to break a chordee may cause a lesion of the anterior urethra. The effort to accomplish coitus under certain conditions may also give rise to its occurrence, as Demarquay says, either in consequence of a violent attempt at intromission, or when, during coitus, a false movement produces a rough flexion of the penis against the pubes or perinæum of the female.

Lacerations and ruptures of the perineal urethra are more common, and are most frequently caused by a fall upon the perinæum. This occurs over railings, spars, etc. Perineal blows also, though more rarely, occasion it, such as kicks - especially when the thighs are separated, the body inclined forward, and the perinæum protruded. Again, fractures of the pelvis in which the pubis is involved are a fertile source of contusion and rupture of the urethra. The fixed membranous urethra is the part most frequently injured by the fragments of bone. Even a simple dislocation of the symphysis, causing overriding of the bones, may so drag upon the membranous urethra as to rupture it. The dislocation may only be momentary, the bones returning to their normal position, and yet a urethral rupture by traction has occurred.

The mechanism of these perineal urethral ruptures has received much stndy. The older authorities believed that they were caused by a direct crushing of the urethra against the lower border of the pubic arch, or of its bulbous portion against the anterior border of the pubis. Cras, in an important memoir, claimed that the urethra, being fixed by the triangular ligament an inch below the lower border of the pubis, could not be crushed by it, though it might be exceptionally compressed against the 
face of the pubis, which is placed at an angle of only thirty degrees with the horizon. The contusing force is rarely exactly median; it usually forces the urethra aside laterally, and squeezes it against the descending ramus of the pubis, on which there is a fairly sharp ridge. The firm border of the transverse ligament, a concave fibrous bridle half an inch deep, which extends between the descending rami of the pubis, has been accepted by Ollier and Poncet as the efficient factor. Terrillon, basing his conclusions upon experiments with the cadaver, finds that falling astride of a narrow body capable of being included in the subpubic angle, causes rupture of the urethra by crushing it against one of the ischio-pubic rami. If the body that is fallen on completely fills the pubic arch, the urethra is crushed against the anterior face of the pubes.

Pathological Anatomy.-The increased frequency of early operative interference has given us of late years opportunity to observe the condition of the parts immediately after the injury. To this has been added the results of experimentation upon animals and upon the cadaver. It seems certain that laceration and rupture of the urethra may occur in at least three different forms or degrees.

The first is what is designated by Reybard as interstitial laceration. Here the spongy tissue only is involved, the internal mucosa and the external fibrous coat escaping entirely. The spongy tissue of the urethra seems to be especially friable, and such lacerations and partial ruptures are produced by influences that would not affect other tissues. Reybard believes this to be due to the fact that the trabeculæ of the spongy tissue form the walls of the vacuoles containing blood, and this incompressible fluid, when subjected to sudden contusion, bursts its walls. Hence arises a blood-pocket, which can not extend externally on account of the incompressible fibrous sheath, and which therefore projects into the eavity of the urethra, narrowing its lumen.

In other cases there is, besides this interstitial rupture of the spongy body, a rupture of the mucosa and submucosa. The sanguineous interstitial pocket then communicates with the urethra, blood passes out from the urethra, and urine enters the lacerated cavity. The rupture of the mucosa is usually incomplete, since sufficient violence to completely rupture it usually causes the third degree of laceration, or entire rupture.

In still other cases, all three structures-the mucosa, the spongy tissue, and the fibrous envelope-are ruptured, and the cavity of the urethra communicates with the tissues of the perinæum. A part only of the circumference of the urethra may be affected, forming an incomplete rupture. The walls of the canal are usually extensively lacerated, but in almost all cases there remains a bridge of sound tissue connecting the upper parts of the two fragments. This bridge is of great importance for catheteri- 
zation and operation. The edges of the urethra retract, and leave a cavity filled with blood-clots. When the rupture is complete, and the entire urethra is transversely divided, the retraction is more marked, and there may be a space of half an inch between the two ends. In a recent rupture this cavity is filled with clots; later, it contains a mixture of pus, urine, and blood. The torn ends of the mucous membrane curl up and wrinkle; the lacerated spongy body is irregular and filamentous.

Such complete ruptures occur oftenest in the membranous urethra, where the walls are thinnest and the surrounding structures most feeble. In the penile urethra it is much rarer.

The exact seat of the laceration or rupture in the perineal urethra has been much discussed. All the later authorities-Guyon, Cras, and Terrillon-agree that the bulbous region is most commonly involved. Ruptures in the prostatic region do not occur; those of the membranous portion are rare except with fractures or dislocation of the pelvis. Ruptures of the pendulous urethra are exceptional.

The recognition of the extent and exact seat of the laceration during life is, however, a matter of much difficulty.

Even with the most extensive concomitant lesions of the perineal organs the skin usually escapes. A few cases only are on record where the external integument was broken through. Fracture of the pubis may be present and may be primary; in other cases the urethral lesion precedes the fracture. The urethral lesion may communicate, directly or indirectly, with the fractured bone, when there will be danger of osteomyelitis, or the fracture may be simple. The tissue of the corpora cavernosa is frequently lacerated, but their tough fibrous envelope preserves them from serious damage, in most cases. Sometimes these corpora are torn away from their ischio-pubic attachments, and the subpubic ligament is frequently ruptured. More or less blood is always effused.

Symptoms. - Spontaneous pain is usually slight compared with the extent of the damage done. Shock, however, may be quite severe at first. Still, if the bones have been splintered or chipped, there may be marked pain. Pain on pressure is constant, and affects the entire injured area. Moving the patient causes pain, located in the perinæum, and radiating to the vesical neck and the glans. Increase of pain means supervention of a complication-phlegmonous inflammation of the injured part, or urinary infiltration.

Most important are the symptoms referable to the evacuation of the urine. In about one quarter of the cases the patient can still urinate, though in most of these retention comes on in a few hours or days. In three fourths of the cases there is absolute retention. In a very few cases there is a temporary retention, with subsequent re-establishment of micturition. The retention is easily understood in the cases of 
total rupture. The separation and dislocation of the urethral ends, and the accumulation of coagula between them, obviously render urination impossible. In the lesser degrees of contusion, intra-urethral hæmorrhage may likewise block the passage, or a blood collection outside the canal may so compress it as to render it impervious. An injury to the transversus perinei muscle may also have some effect. A very small proportion of the lightest cases show no disturbance of micturition at all; for in the cases where retention comes on some time after the accident it is due to compression from recurrent hæmorrhage, or inflammatory swelling from infection of the injured tissues. Finally, temporary spasm of the membranous urethra may cause retention in the mildest cases.

Urethral hæmorrhage is an important symptom of the injury. Sometimes it is so small that only a few drops of blood, or a coagulum, appears at the meatus; but usually there is a spontaneous hæmorrhage amounting to a couple of ounces or so, and appearing in drops at the meatus, during the first few hours after the injury. Serious hæmorrhage is rarely seen; yet Paul cites a case in which the hæmorrhage lasted for six days. But the intensity of the hæmorrhage bears no relation to the extent of the injury. In injuries to the perineal urethra there may be much blood effused that does not find its way to the meatus. It collects at the site of the injury, causing the primary swelling, while a coagulum may block up the urethra and prevent the blood appearing externally. The first urine evacuated with the catheter is usually mixed with blood which has trickled into the bladder. The presence of blood, then, is a symptom of much importance, whether found at the meatus or in the urine, and shows conclusively the presence of a solution of continuity of the urethral wall.

The swelling is at first limited to the perineal region, and appears as a rounded tumor situated hetween the scrotum and the anus, and as large as or larger than a hen's egg. The skin covering it is normal and elastic, though ecchymotic patches may appear. The swelling is due to hæmorrhage, and will gradually increase as long as that lasts. Limited by the superficial fascia, it may spread on to scrotum and penis. In the worst cases the blood tumor can be felt per rectum, and occasionally pressure upon the tumor will cause blood to exude from the meatus. Rupture in the penile region is followed by a swelling which is generally slight, in the form of a more or less regular collar surrounding the canal.

Course, and Complication.-In the mildest contusions of the urethra the effused blood canses a retention that is readily relieved by the catheter. As the blood is absorbed micturition becomes free, and, like any other subcutaneous contusion, the part usually returns to the 
normal state. Sometimes, however, the sanguineous tumor becomes inflamed, suppurates, and forms an abscess which opens either into the urethra or through the skin, or both ways.

But when the injury to the urethral wall has been greater the complications of urinary retention and wound infection render the accident much more dangerous, and especially is this the case when the rupture has been complete. When the patient attempts to micturate, the urine flows into the contused perineal tissues and spreads thence through the loose meshes of the connective tissue, and infiltration of urine ensues. Where the injury has caused a solution of continuity of the perineal skin this is much less likely to occur, since an outlet is provided for the renal secretion. We need not here describe the course of infiltration of urine. Suffice it to say that the urine brings with it something which irritates the already damaged perineal tissues-they inflame, and suppurate; the skin sloughs. Fistulæ are thus formed.

Not less serious complications arise from the fact that the urethral wound, in spite of its subcutaneous nature, is usually infected. The source of this infection may be micro-organisms conveyed by urine from the bladder, or the pathogenic micro-organisms known to exist in even the normal urethra. But if auto-infection does not happen, septic germs are very prone to reach these lesions from without by means of catheters and other urethral instruments.

This infection spreads almost inevitably to the bruised perineal tissues in connection with the wound, and results in perineal abscess. This is a serious and very common complication of urethral contused and lacerated wounds, and is usually of a peculiarly malignant nature. The intact skin prevents the escape of the noxious materials, and the urine in contact with the bruised tissues increases the danger; for, while quite fresh and healthy urine may be aseptic, decomposition sets in so readily in this fluid that its presence constitutes one of the chief dangers of the condition. Urinary infiltration is also very liable to occur, and septic general infection leading to a fatal result is but too frequent. Cavernitis, phlebitis of the pelvic bones, and pyæmia are occasionally seen.

The most favorable termination of the average case, after the spontaneous or artificial opening of the mass, is the expulsion of the dead tissues and inflammatory material by suppuration and slow healing with cicatricial formation. Spontaneous cure has been very rarely observed, and that only in the lightest cases.

As regards the opening in the urethra, such cicatrix formation is of extreme importance. The extent of the original injury by no means corresponds to the ultimate defect. The bruised margins of the urethral orifice become necrotic and slough away, and the resultant scar may be so extensive as to seriously compromise or entirely close the urethral lumen. 
Hence cicatricial stricture, and that of the worst form, is an extremely common sequel to these injuries.

Complete rupture of the urethra leads to extensive granulation between the divided ends, and the formation of a circular retractile scar, giving rise to one of the most serious of strictures, followed by fistula-formation and other troubles.

Diagnosis. - When a patient has sustained a perineal injury, the possibility of a urethral lesion must always be kept in mind. If blood appears at the meatus the diagnosis is almost certain. If none is there, pressure on the perinæum may cause its appearance. In the entire absence of meatal hæmorrhage the patient should be made to urinate, the perineal swelling being watched while he does so. If the tumor increases during the act, and the patient experiences severe pain, the urethra is probably injured, and all attempts at voluntary micturition should be stopped at once. If the tumor is not increased by urination, and if the urine contains no blood, nothing more than a urethral contusion is present. But retention of urine often accompanies a simple contusion, as we have seen, and here the possibility of catheterization with the absence of hæmorrhage will clear the diagnosis.

When a lesion of the urethra has been thus demonstrated, a well-oiled and disinfected catheter is to be introduced. It may reach the bladder, or it may strike the symphysis and only reach the bladder after careful manipulation; or the bladder may not be entered at all. When catheterization is easy, a small contusion only is usually present. But in some very bad cases there is no difficulty in reaching the bladder, even when subsequent events show that the urethra is completely ruptured. In a recent rupture a number of fibers of submucous connective tissue may unite the torn ends of the canal, and these serve as a guide to the catheter; later, however, when infection has occurred, these, as well as the ends of the urethra and the neighboring tissues, become gangrenous and disappear.

In case the catheter passes ont through a urethral wound into the neighboring tissues, we should at once try to get an accurate idea of the extent and limits of the damaged tissues, using the instrument as a probe.

Inflammation of the injured perineal tissmes is recognized by the pain and fever, the local tension and redness of the skin, the neighboring œdema, etc. The various complications are to be recognized by their appropriate symptoms, elsewhere considered.

Prognosis. - Kaufmann places the mortality of all urethral contused and lacerated wounds, with rupture, at fourteen per cent. In the lightest cases, where there is interstitial rupture, or simple fraying of the mucosa, the prognosis is good; the symptoms often disappear, and the case progresses rapidly to recovery. In severer cases, where the urethral 
walls are torn through and the spongy tissue is involved in the wound, the prognosis is graver; complications are apt to ensue. In the worst cases, where the rupture is complete, the effusion of blood and the laceration of the tissues alnost inevitably cause infiltration of urine-a most serious affair. The immediate prognosis is grave, and the ultimate one also serious, since cicatricial stricture with all its consequences inevitably supervenes.

The cases of all degrees of severity which are accompanied by primary retention are more favorable than those in which the patient can micturate, since they demand and receive immediate medical care-i.e., catheterization-the very thing to prevent that most dangerous of the complications of the condition, infiltration of urine. Cases in which the urine can still be spontaneously evacuated are frequently treated expectantly for some time, and eventuate badly. Contusions and ruptures of the urethra caused by pelvic fractures have been found to be the most unfavorable of all.

Treatment. - A broad distinction must be made between the cases of very slight gravity and the more severe ones, since it would in certain cases be absurd to proceed at once to operative measures. When the symptoms point to simple contusion or to interstitial rupture, temporizing measures are allowable. Pressure and cold applied to the perinæum, and the use of a flexible catheter to overcome the retention, are all that is necessary. But the surgeon must watch the patient closely, and on the first sign of the existence of more serious lesions proceed to take vigorous measures.

In but few cases that come under medical care will the above mode of treatment be answerable. The indications, however, for immediate interference are usually peremptory.

The hæmorrhage is rarely sufficient to demand immediate attention; cold and pressure will soon keep it within bounds. But if retention of urine sets in, we receive a positive indication for interference within a very few hours after the accident. In that case catheterization must at once be resorted to. If it be successful, it is the custom of some surgeons to leave the instrument in situ, and thus to drain the bladder. The results of this method of getting rid of the urine have not been very brilliant. Duplay advocates it in some cases; but Kanfmann rejects it, and reports a mortality of thirteen per cent in those cases in which the catheter was retained; for in more than half the cases in which this plan of treatment was inaugurated it had to be abandoned on account of complicationsabscesses and infiltration.

Permanent catheterization is probably the commonest plan of treatment to-day. It provides for urinary outflow and puts the urethra at rest. But it also directly carries the infective agent to the wounded 
tissue; for, as we have seen, it is practically impossible to make the catheter an aseptic instrument. It also hinders the outflow of effete material through the urethra, thus closing up the only avenne for its escape. But the most conclusive arguments against its use are the results obtained. Urinary infiltration and septicæmia, the worst complications that we have to fear, are not hindered by it. The results are bad, and as a general plan of treatment permanent catheterization is to be absolutely rejected. Occasional catheterization is proper, of course, and is to be used in all cases of retention in which it is practical; and in simple contusion of the urethra with retention-cases a degree severer only than those in which the expectant treatment is allowable-catheterization must be regularly repeated until the blood effusion that presses upon the urethra is entirely absorbed. Meanwhile a most vigilant watch must be kept upon the perineal tumor and on the temperature. Inflammatory disturbance around the tumor and increase of bodily heat being indications for immediate interference.

Puncture of the bladder has been often done in those cases in which catheterization proves impossible; and with a perfectly aseptic needle the operation is harmless, and may well be employed when, from any cause, ordinary catheterization is impossible. But it is only a temporary measure; though it may be repeated a number of times, it does not constitute a treatment for the lesion. It may be preferable to use aspiration of the bladder from the beginning, instead of catheterization-it is possibly less harmful; but the statistics of the French surgeons, and especially those of its advocate, Mallière, do not show that it is less liable to complications than is the retained catheter. Lately some have adopted the plan of permitting the cannula to remain in situ in the punctured bladder, or have replaced it with a permanent soft catheter. But the mortality under hypogastric puncture has been over nineteen per cent, perineal abscesses have been frequent, and the results obtained can not compare with those obtained by perineal section.

Perineal section-or, rather, immediate external urethrotomy-fulfills all the indications for the injury. It gives an external and convenient opening for the torn and bruised area of tissue, allows drainage for the effused blood, the purulent secretions and the urine, and presents an opportunity to treat the urethral wound directly. The objection, that by immediate perineal section we convert a subcutaneous into an open wound, is a fallacious one. These traumata are not subcutaneous, although the perineal skin may be unbroken; the mrethra affords an open door for the advent of disease. As a matter of fact, they are open wounds under good conditions for infection, and bad ones for antisepsis and drainage. The sole rational treatment consists in free opening, remoral of necrotic tissue, and drainage; and it has to be done, finally, in a large number of cases in which other methods are first tried. Of course, if done later, it 
is difficult to recognize the urethra in the midst of the sodden, inflamed tissues; it is comparatively easy if done early. Thus we get an opportunity to do what is necessary for the ruptured organ itself.

Statistics fully bear out the correctness of these hypothetical considerations. We have seen that the mortality in cases treated by vesical puncture was 19 per cent, and in those treated by permanent catheterization, 13.6 per cent; in those in which the early perineal incision has been done it has been only 8.75 per cent. Perineal incision is not to be reserved for the worst cases; it is to be applied to all save those of the very least degree.

The operation is a simple one: The patient is laid on his back in the lithotomy position; the perinæum is shaved and thoroughly cleansed. Local anæsthesia may suffice for adults. The perinæum is incised in the median line, the cut beginning about two inches in front of the anus, and reaching nearly to its margin. First the skin, and then the superficial fascia, are incised. If the injury has affected the bulb of the urethra, the blood effusion will be reached under the superficial fascia; if the membranous portion is injured, the incision must be deepened posteriorly and the membrana propria perinei freely divided. The clots are now turned out and the cavity thoroughly washed; necrotic fascia is to be cut off; open vessels are to be tied; while compression will stop the oozing, and hot sponges or the thermo-cautery end any hæmorrhage that there may be from cavernous tissue.

The catheter is then passed, and the course of the urethra in the wound carefully followed. When the urethra is continuous, it is easy to detect the amount and nature of the injury it has sustained. If it is severed, the torn distal end must be smoothed off with scissors and the proximal end searched for. This is almost always a difficult and apparently in some cases an impossible task. The free proximal end of the urethra forms a movable round cord, bleeding freely. If the patient can be induced to pass a few drops of urine, the urethra will soon be apparent; if the patient is narcotized, pressure upon the bladder will cause a few drops to exude. In the later stages, when a large abscess has formed and the entire necrotic mass is of a dirty, greenish gray, the difficulties are increased. As soon as the proximal urethra is found, a catheter is passed into the bladder and the viscus is emptied.

If the urethral wound is a partial rupture, we can expect spontaneous healing after the perineal opening has been made; the continuity of the mrethra being preserved, there will be no special difficulties in the subsequent catheterism; but if the rupture has been complete, the two stumps of the urethra are united by a mass of cicatricial tissue, which forms a stricture of the most intractable and resilient character. König has recently got good results by the total excision of such strictures, followed by 
the suturing together of the ends of the tube. Suture is to be recommended also, when feasible, in this class of cases of complete ruptured urethra that we are considering. Even partial success in obtaining union by suture will give us much better results than we could otherwise obtain. When there is extensive contusion and laceration of the urethra it is, of course, impossible to suture the ends unless they will permit of some trimming.

The perineal wound is to be washed, powdered over with iodoform, and its edges anointed with boric-acid ointment; it is then stuffed with Lister ganze retained by a T-bandage. The patient urinates for the first few days through the perineal wound, then through the urethra or catheter. The parts must be thoroughly irrigated with boro-salicylic water, and a new bandage applied after each micturition. Defecation must be prevented as much as possible for the first few days by light feeding and opium. On the eighth day a catheter or sound should be introduced, and as large an instrument as possible passed every few days thereafter. The perineal wound usually takes three to six weeks to heal.

If the rupture is a complete one and occurs in the bulbous urethra or anterior to it, the same operation of suturing the severed ends of the urethra may be advantageously supplemented by perineal urethrotomy reaching the bladder for urinary drainage through the membranous urethra. Under these circumstances an aseptic rubber tube can be left in the anterior urethra to act as an internal splint or support to the sutured wound. The opening in the membranous urethra will both minimize the danger of urinous contamination of the traumatic urethral lesion and systemic infection. If the rupture is posterior to the bulb, and so extensive as to jeopardize the chances of successful suturing unless the urine be deflected from its normal channel, suprapubic cystotomy for direct drainage or for ureteral catheterization may be done. The guide for the cystotomy in this case is the tilted-up point of a sound passed after the severed proximal end of the urethra has been found by perineal incision.

Cases which are still further advanced, in which septic infection has occurred, are to be similarly treated by incision and disinfection. Permanent irrigation, or particularly the permanent bath, is to be recommended in these cases. The other complications, urinary infiltration and cystitis, are considered at length elsewhere. 


\section{WOUNDS AND LACERATIONS OF THE URETHRA INFLICTED FROM WITHIN.}

The urethral canal may be injured by foreign bodies introduced into the canal by the patient himself; by the use of faulty urethral instruments; by excessive urethrotomy, resulting in cicatricial curvature; and by urethral calculi, to be considered elsewhere.

1. Injuries caused by the Introduction of Foreign Bodies. -Needles and pins, and less commonly hairpins, are among the most frequent foreign bodies thus met with. Pipe stems, slate pencils, metal chains, a child's tooth, chewing-gum, and shoe strings are some of the other objects too numerous to mention. The satisfaction of erotic impulses is the usual cause of their introduction. They are most often found at the period when sexual feelings are awakening, at the thirteenth to the fifteenth year, and next most often in elderly individuals, who seek by these means to arouse their lost desires.

Objects thus introduced purposely or accidentally show a marked tendency to work their way into the deeper portions of the urethra and to reach the bladder, where they frequently form the nucleus of stone. Opinions have varied as to the cause of this phenomenon. Motions of the organ in various directions tend to the backward displacement of an elongated object in the penile urethra. In the membranous and bulbar urethra muscular forces come into play. The power of these forces is quite considerable.

Elongated foreign bodies, if thin, are not displaced by the stream of urine. Larger bodies may occasionally be expelled by micturition, or they may completely close the wrethral canal.

Symptoms. - These depend upon the diminution or closure of the urethral canal, and the irritation of the urethral walls caused by the foreign body. Burning and pricking pains in the canal, often radiating to the bladder and glans, and intensified by urination or attempts at it, are the consequences of the mechanical irritation. Urination is difficult or impossible, in accordance with an obstruction that is partial or complete.

As a rule, foreign bodies in the urethra cause such discomfort that they are speedily extracted. If, however, they remain for any length of time in situ, they undergo changes similar to those undergone by foreign bodies in the bladder-they become incrusted with lime salts. This leads to a gradual change of shape and increase of size; the urethra is finally perforated by pressure, and the foreign body lies in a kind of pocket communicating with its cavity. Cases have been seen in which sloughing has occurred, and the foreign body has been thus expelled. 
Diagnosis. - The history, the location of the foreign body, its size and hardness, are the factors that render the diagnosis easy or difficult. In the penile urethra external palpation is usually efficacious, especially if the foreign body is hard. In the membranous and prostatic urethræ rectal palpation will often help us, even the projection of a lengthy foreign body into the bladder itself being perceptible by this means. Sounds and catheters are to be employed as little as possible for diagnostic purposes, since, no matter how carefully employed, they always tend to push the foreign body still farther from the meatus. Now that visual examination of the urethra has been perfected, the detection of any foreign body is easy. The introduction of a Klotz tube or Brown's speculum will at once reveal the nature and position of the object.

Prognosis is good. The foreign bodies can almost always be readily extracted, nor have any untoward after-results been observed. But a single fatal case is on record (Henry). The patient was seventy-eight years old. The foreign body was extracted, but a false passage was made, and the patient died of septic infection. Foreign bodies have remained in the urethra for months and even years, and in very old persons, yet they have always been successfully extracted when surgical aid was invoked.

Treatment.- The nearer the meatus the object lies, and the less projecting angles and irregularity of form it has, the easier will be its extraction.

Many of them can be removed by any of the urethral forceps, of which that of Mathieu is the best. The articulated curette of D'Etiolles is effective.

Needles, etc., can usually be readily removed by the forceps. A needle, and even a pin, can be forced through the urethral wall until its shaft can be seized externally. It is now under control, and its head can be readily grasped by the urethral forceps, and the pin withdrawn through the meatus. The large, straight, open-end lithotrity tube has repeatedly been used with success in the removal of foreign bodies from the urethra, the finger being passed into the rectum for ocelusion of the membranous urethra; the urethra is now slightly distended with water through the tube, when quick aspiration with the bulb will suck up the object sought.

Larger foreign bodies, or those whose shape or fixation render the above inethod of extraction inapplicable, must be removed by urethral incision. The operation is the same as for urethral calculi, and will be described under that heading.

The employment of any of the previously mentioned grasping instruments will be greatly facilitated by first introducing the urethral speculum, which by a full dilatation of its tips will spread the mucous 
membrane away from the object, should it be large, and permit a better insertion of the forceps blades between the urethral walls and the object. It is to be presumed that the speculum, with its independent base and tip control, would alone be adequate for the extraction of many foreign bodies; by it the canal can be spread to its fullest extent. When this is seen to be sufficient and well adjusted to the foreign body, simultaneous pressure on the object, through the external covering of the urethra and the speculum, would force the object into the instrument's wire arms, when, by relaxing the spread of its tips, it would act as a forceps for withdrawal of the object.

2. Injuries caused by Faulty Urethral Instruments are essentially either contused or incised wounds inflicted from within. They do not materially differ from similar injuries coming from without, save that injuries to the external soft parts are absent.

Longitudinal incised wounds universally heal promptly. Even when unsutured, and untreated, this is the rule.

Transverse incised wounds may also heal spontaneously, but they rarely do so without causing a ring-formed stricture of the urethra.

Contused wounds cansed by instruments do not differ in their course from ordinary contused wounds as treated above.

The treatment in the most serious of these cases consists of the introduction of a retained catheter.

3. Injuries caused by Excessive Urethrotomy, resulting in Cicatricial Curvature.-Curvature of the penis has occurred in a considerable number of cases. When more than one stricture is divided, and the incision is made deep enough to show hæmorrhagic extravasation into the scrotal and penile connective tissue, this serious complication of curvature is to be apprehended. Otis mentions three such cases, but says they caused no trouble, being apparent only during erection, and passing away in all cases in from two months to a year. I have seen several cases which, after reaching a certain stage of improvement, promised to remain during life.

These curvatures are due to a cavernitis of chronic course, with considerable exudation into the meshes of the cavernous tissue. If this exudation is unilateral, the organ in erection will be bent toward the affected side. This may be so excessive as to render coitus impossible.

Much can be done by treatment for these subjects of chronic cavernitis. Moist warmth is efficacious, as is also the envelopment of the penis in rubber tissue, which has the effect of a continuous bath. Massage, with inunctions of iodide of potash, mercury, and iodoform ointment, will help resorption. Dittel claims by these means to have always improved and frequently cured such cases. 


\section{URETHRAL FISTULF.}

A urethral fistula is an abnormal opening in the urethra through which the urine escapes partially or wholly during micturition. Occasionally they are congenital, being developed during intra-uterine life, and follow a mechanism exactly similar to those that occur later.

Urethral fistulæ may open externally either into the rectum or on to the skin. This dermal opening may be either on the penis or in the perinæum. We may therefore divide urethral fistulæ into three classes:

1. Urethro-rectal fistulæ.

2. Urethro-perineal (scrotal) fistulæ.

3. Urethro-penile fistulæ.

1. Urethro-rectal Fistulæ are much the least frequent form of the malady. The urethral orifice is most frequently seated in the prostatic urethra, less frequently in the membranons portion of the canal. It is usually single and narrow, and is always situated at a higher level than is the rectal opening; hence, while urine flows freely into the rectum, fecal matter does not usually ascend to the urethra. The fistulous tract is narrow and tortuous; its walls are indurated and calloused. The rectal opening can usually be seen through the speculum as a small opening in the midst of a reddish rosette of the mucous membrane. Occasionally the rectal opening is large, and there is a true defect of the recto-urethral septum.

Etiology.-Lithotomy operations, the lateral and median, have sometimes opened both the rectum and the urethra; incisions through the prostate, if made too extensively, have been the cause. Urethral foreign bodies have perforated the urethro-rectal wall and left a fistula; rectal foreign bodies have done the same. Prostatic abscesses and prostatic calculi, causing ulceration, have also occasioned it. Cancerous and tubercular degeneration of prostate or rectum has not rarely been an efficient agent. The extreme rarity of deep membranous traunatic stricture renders stricture an unusual cause of urethro-rectal fistula; but a coexisting stricture in any part of the canal will, after the establishment of fistula by some other means, become an important factor in angmenting the trouble, and an obstacle, while it exists, to any of the methods of cure.

Symptoms. - The chief of these is the intermittent passage of urine from the external orifice of the fistula. This only occurs during micturition, and the point is an important one to distinguish the condition from vesical or uretal fistula. The amount of urine that passes through the abnormal opening varies much. If the urethra is free and the fistula small, it may only be a few drops; if the urethra is much occluded, most of the urine may be passed per rectum. The urine flows from the fistula 
during micturition, but it may in exceptional cases be accumulated and retained for a time in the rectum. Flatus not uncommonly escapes by the meatus, and sometimes, though rarely and with much pain, solid fecal matters pass out per urethram. Semen has been known to pass into the rectum in a marked case of the kind.

Examination per rectum will frequently reveal the posterior opening to the touch alone. With a bivalve or Sims rectal speculum and a probe it can always be found, and is usually surrounded by hard, callous folds. A metallic sound having been introduced into the urethra, a probe is passed into the fistulous opening as found per rectum, and the internal orifice of the fistula is decided by their meeting. In place of the sound the endoscopic tube or urethral speculum offers great advantages when the urethra will admit them.

Diagnosis follows from the above. A vesical fistula is distinguished from a urethral fistula by the fact that in the former case the urine escapes continuously from the abnormal orifice, and not alone during micturition.

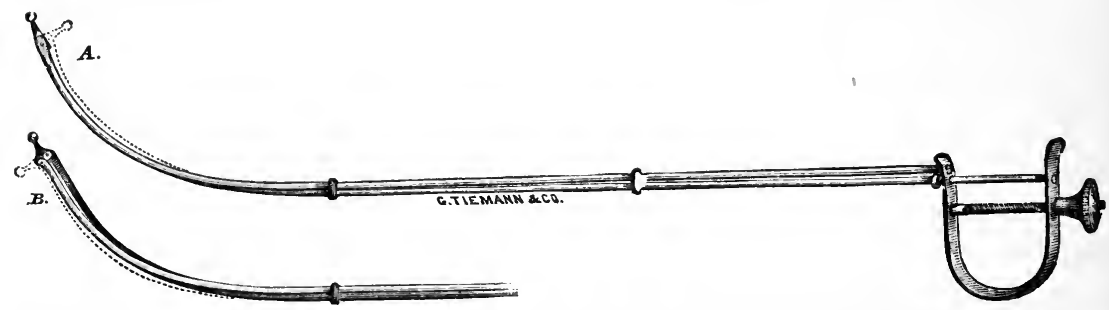

Fic. 28.-The author's long curved urethral digit. $A$, movable probe-tip for upper segment of posterior urethra; $B$, movable probe-tip for under segment of posterior urethra.

Injecting colored liquids through a eatheter into the bladder will also help to a decision. The curved urethral digit (Fig. 28) will be of service in detecting the locality of the urethral opening. When permissible, visual examination of the urethra is valuable.

Prognosis.-This depends largely upon the cause. Those due to tuberculous and eancerous processes or extensive suppuration and other grave conditions are usually ineurable. Traumatic fistulæ are of better prognosis, and have been known to close spontaneously in a few cases.

Urethro-rectal fistulæ are grave also in regard to the conditions that they cause. The irritation of the rectal and vesieal mucous membrane leads to inflammatory conditions of a chronic nature in both organs. The patient's general health may be gravely involved.

Treatment.-If there be a strieture of the urethra, it must be removed by the usual means as an essential preliminary to all treatment of the urethro-rectal fistula itself. Our next object is to protect the fistnlous canal from the irritation of urine, frees, etc. Regular catheterization will dispose of the urine, but it is less easy to control the fæces and 
gas. Duplay advises the use of an cosophageal tube, or of a silver cannula provided with an apron, to close the fistulous tract during the intervals of defecation.

As regards the fistulous tract itself, an attempt may be made to close it by caustics applied to its rectal opening-chemical caustics, or the electro- or thermo-cautery. Only very narrow fistulæ are suitable for such treatment, and only in rare cases does it succeed.

Another and better recourse is the operative procedure, which, however, affords but a fair prognosis. The difficulties inherent to the operation are very great, and the necessary contact of the wounded surfaces with urine, fæces, and gas render healing slow and imperfect.

A modified Sir Astley Cooper's operation is the simplest and probably the best. A grooved sound having been passed to the bladder, a median perineal incision is made through the membranous urethra. The point of the probe, which has been previously passed from the rectal opening through the fistula to the urethra, is now felt for. The urethral orifice of the fistula thus found is freely cut down, so as to be continuous with the existing perineal incision. If old and much calloused, this portion of the fistula should be curetted. Now an incision continuous with that in the perinæum is made, so as to bisect the remaining fistulous tract at some point in the recto-vesical septum, the presence of the probe still acting as the objective point. Additional curetting in both directions is to be done when the probe is reached. The bottom of this wound is firmly packed with iodoform gauze, also leading some of the same gauze into the groove caused by slitting the urethral orifice of the fistula. A full-sized drainagetube, or short, soft catheter, is led through the perineal wound into the bladder and there retained.

$\because$ To secure cleanliness and favor repair of the fistula, frequent dressings will at first be requisite.

If this operation should fail in curing wholly, it will probably succeed in effecting a closure of either the urethral or the rectal half of the fistula. If the latter should remain, it can, as a secondary step, be cured by the ordinary cutting operation for complete anal or rectal fistula.

The success of this operation may be enhanced by the use of a fine platinum probe in place of the silver one, making use of it when reached by dissection in the mid-perineal region for an electric-cautery arc, by seizing it here with an insulated clamp electrode, then making a similar connection by the opposite pole with the platinum probe as it protrudes in the urethra. Having cauterized this half of the fistulous tract, the urethral electrode is conveyed to the rectum, for seizure of the platinum wire as it there protrudes, thus completing the cauterization.

In very exceptional cases this same operation of Cooper's may be made more promising by a simultaneous suprapubic cystotomy, to permit 
of two or three days' permanent catheterization of the ureters, leading the distal ends of the catheters out through either the suprapubic or perineal wound-preferably the latter. It is probable that catheterization of the ureters will shortly become a feasible procedure to effect through the perineal wound alone. Advanced cancerous or tubercular disease of the rectum or prostate, of course, counterindicate all operative procedure.

2. Urethro-perineal Fistulæ.-These are the commonest of the urethral fistulæ. Often they are multiple, as when they arise from extensive urinary infiltration. Or a fistula single at first may give rise to others; a small quantity of urine remains behind in the tortuous tract, inflammation arises, small abscesses form, and secondary sinuses appear.

The urethral orifice of these fistulæ may be large and funnel-shaped or narrow and slit-like. It is usually single, though it may be double or even multiple. On the cutaneous surface, on the other hand, there are nsually a number of orifices scattered over scrotum, perinæum, and the root of the penis. Instances occur in which the pus travels far, and openings of sinuses are found in the groin, the thigh, just above the knee, the buttocks, and the lumbar and the hypogastric regions. The sinus itself varies much in length, but is almost always irregular, with pus and urine pockets and blind cul-de-sacs at various places. Its walls are usually lined with a smooth, red, and shining membrane.

Profound alterations of the soft parts occur sooner or later in all these cases; and even when the normal caliber of the urethra has been restored, and the urine follows its natural channel, the fistula may persist, and cause continually further trouble. Thus, there is always marked induration along these tracts if they have existed for any time, palpable as a thick, fibrous cord, or even as distinct nodular masses. Calculi are not infrequently found in them, originating perhaps from the bladder or prostate, but caught in the fistulæ, and increasing there by the continuous deposition of urinary salts. In some cases these salts incrust the walls themselves.

Etiology.-Large abscesses, extensive urinary infiltrations-such as follow ruptures of the urethra and perineal contusions-are the common cause of these fistulæ; while lithotomy, external urethrotomy, and cleancut wounds are rarely followed by them. Glandular and peri-urethral abscesses, such as occur in simple or virulent urethritis, and in which the abscess empties both externally and into the urethra, may originate them.

But the commonest of all causes is the presence of a traumatic or inflammatory stricture, which may readily engender an abscess, a urinary pouch, infiltration of urine, and even immediate rupture of the urethra. Urinary fistulæ are the necessary sequelæ of such accidents.

Symptoms.-More or less urine flows through the abnormal openings during micturition; sometimes almost all of it does so. A muco- 
purulent fluid exudes from the urethra in the intervals of micturition, its quantity depending on the extent and number of the tracts, the diverticulæ, etc. The spermatic fluid usually escapes partly by the normal and partly by the abnormal opening. The appearance of the external orifices has already been described. The hard, fibrous cord surrounding the fistula is sometimes very apparent, especially in those running through the loose scrotal tissues.

Diagnosis. - Occasionally so little urine escapes through the abnormal openings, and that so mixed with muco-pus, that it is difficult to decide if the exuding liquid is urine at all. If the urinous odor does not decide it, we can compress the penis at the moment of micturition, so as to force the urine through the fistulous tracts.

Combined eatheterization of urethra and probing of the fistula may be necessary to complete the diagnosis. If the sinus is not too tortuous, we can thus gain fairly exact ideas of its direction, lengtl, and dimensions. Colored liquids may be injected into the tracts or urethra in cases of doubt. The straight urethral digit (Fig. 21) and endoscopy are aids in the diagnosis.

Prognosis.-This depends much on the location and the nature of the fistula, and its cause. The original disease, usually a stricture, must be taken into account. The formation of new abscesses in the penis and sorotum, the appearance of urinary infiltration, and of erythema of the skin, also influence it. In general the prognosis is doubtful.

Treatment.-Futile attempts to cure these fistulæ are usually made, when they are first treated, with various injections of silver, iodine, and hydrogen peroxide, as well as the thermo and electric cautery, scarifications with barbed probes, etc. Ineffective as these are as initial resources, they all become valuable aids when used as auxiliaries to the prime requirement-namely, restoration of the urethra to its normal caliber. Some operators are satisfied to attempt this by internal urethrotomy and the retained catheter. In the exceptionally simple cases this may suffice, but even here it is preferable, and in the severer cases it is necessary, to supplement internal by external urethrotomy, thereby gaining free vesical evacuation without contamination of the fistulous tracts, and with greatly reduced danger of systemic infection following the internal urethrotomy.

3. Urethro-penile Fistulæ.-These are not as common as some of the other varieties. The fistulous tract is always straight and short, being rarely more than half an inch long. Sometimes it is so short that the urethral and integumental openings seem blended into one, the tissues being much thinned. They are almost always single, and have no diverticulæ. The internal orifice is frequently funnel-shaped; the external orifice is lined with a thin, smooth membrane. When the fistula is at 
the level of the glans, and especially at that of the fossa navicularis, there have been seen a number of minute orifices instead of a single larger one. Indurations and callosities are not frequently met with. The size of these fistulæ is very variable; from those that will hardly admit a bristle to those measuring a half or three quarters of an inch in diameter.

Etiology is much the same as in the foregoing varieties. Both internal and external traumatisms are frequent causes-simple and contused wounds. Infibulation, and the habit some ignorant women have of attempting to prevent their infants wetting the bed by tying up the penis, cause some cases. Strictures may cause penile as well as deeper fistulæ. Peri-urethral abscesses are common causes, and a number have been effected by phagedenic chancres and chancroids.

Symptoms. - There are disturbances in micturition and ejaculation, both urine and spermatic fluid flowing wholly or in part by of the abnormal opening. If the fistula is so small that there is doubt as to its existence, compression of the glans at the moment of micturition will reveal the escape of the smallest amount of urine.

Diagnosis may be completed by conjoined urethral and external probing.

Prognosis is good. While these urethro-penile fistulæ are less dangerons than the deeper forms-in that there is much less liability to complieations on the part of kidney and bladder-they are frequently obstinate. Some of the smallest resist all those means of treatment so long as only the trivial operations of stimulation and catheterization are resorted to.

Treatment. - As in the other forms of urethral fistulæ, if stricture be the cause or only a coexisting complication, the urethra must first be restored to its normal caliber; then, if the fistulous tract be a small one, its canterization by the electric loop, as described in considering the treatment of urethro-rectal fistula, will be most valuable. Uniform evacuation of the bladder at stated intervals must then be practiced for at least four days. If the fistula be large and short, after severing its infiltrated wrethral margins and cutting any existing stricture on the urethral roof, the wire speculum should be introduced and opened so as to well expose the fistula. Then its edges can be freshened, either from the cutaneous or mucous side, or both, and if not resulting in too great an encroachment upon the urethral caiber, the freshened edges of the fistula may now be bronght together by a separate linear row of sutures for both mucous and cutaneous surfaces-the presence of the wire speculum rendering it possible to work by sight within as well as without the urethra. In such a case regular catheterization may suffice, but a perineal urethrotomy will render snccess much more certain. 
When the fistulous opening is too large to permit of closing by the operation of urethrorraphy as just described, some one of the various plastic operations must be resorted to.

4. Urethroplasty.-The chief forms of this operation are three:

1. By eutting and displacing the neighboring portions of skin.

2. Formation of a bridge of skin, by loosening of the subcutaneous connective tissue.

3. Formation of a skin flap from the nearest general integument, and transplanting it by two stages. The foreskin, scrotum, or loins may be used.

The best and most praetieal operations are as follows:

If the fistula is near the serotum, the skin of that organ had better be used. A thick metallic eatheter or the urethral speculum is passed, to fix and to make tense the urethral walls and the edges of the fistula. The calloused edges of this latter are now so cut away that a quadrilateral opening remains (Figs. in Dittel, Deutsche Chir., 49, p. 241 et seq.). The scrotal skin is now divided parallel to the posterior edge of the defect, and one to one and a half ineh behind it. The bridge of skin loosened from the subcutaneous tissue, and its anterior borders united to the anterior borders of the defect. When union has taken place, the posterior margins of bridge and defect are freshened and united.

In the flap-transplantation operation the fistula is given the form of an elongated oval by freshening its edges. A flap of slightly larger dimensions is made of scrotal skin (Dittel, loc. cit., p. 241). This is twisted or turned, as in the rhinoplastic operations, and united to the defect. After that has united, the base is adjusted and the posterior fistula is closed.

If the fistula is near the middle of the penis, a metal catheter or urethral speculum is introdueed, and the edges of the defect are freshened. A bridge is then made, as described above, the skin being taken from where it is most available. The sutures ean be removed in forty-eight hours. Periodic and eareful catheterism or perineal urethrotomy is necessary.

In the operation by lateral sliding flaps, the edges of the fistula are freshened into an elongated oval; then a suitable flap is made parallel to the side of the fistula; only three sides are cut, and the attached flap is dissected up from its base; then a narrower flap of the same length is entirely removed at the opposite side of the fistula, and the wounded surfaces adjusted by suture.

In the operation by longitudinal sliding flaps the defect is given the form of a transverse oval. A transverse bridge is made in the skin nearest the fistula; on the other side of the defect a smaller denudation is made; then the denuded portions are sutured, and when union is assured the posterior flap-wall and the posterior edge of the fistula are to be united in a similar fashion. 


\section{EXPLANATION OF FIGURES ON PLATE I.}

Figs. 1, 2, 3, micro-organisms of the normal urethra. Fig. 1, $a$ and $b$, bacilli resembling tubercle bacilli. Fig. 2, hyaline epithelial cell containing streptococcus. Fig. 3, epithelial cell containing pseudo-gonococcus. Fig. 4, pure culture of the latter on Agar. Fig. 5, gonococci from gonorrhœal pus : $.1: 1000$ diameters. 


\section{PLATE I}

FIG. I.
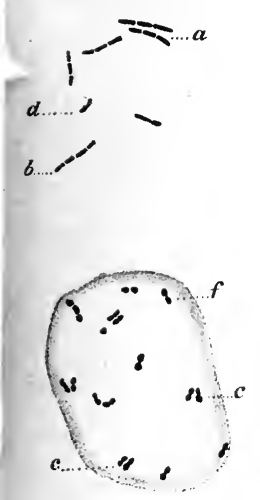

F/G.2.

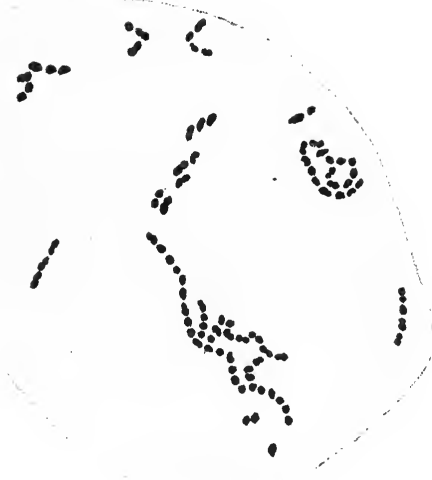

FIG. 5.

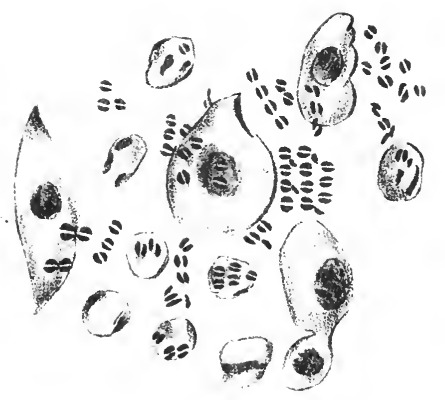

FIG.3.

FIG. 4.
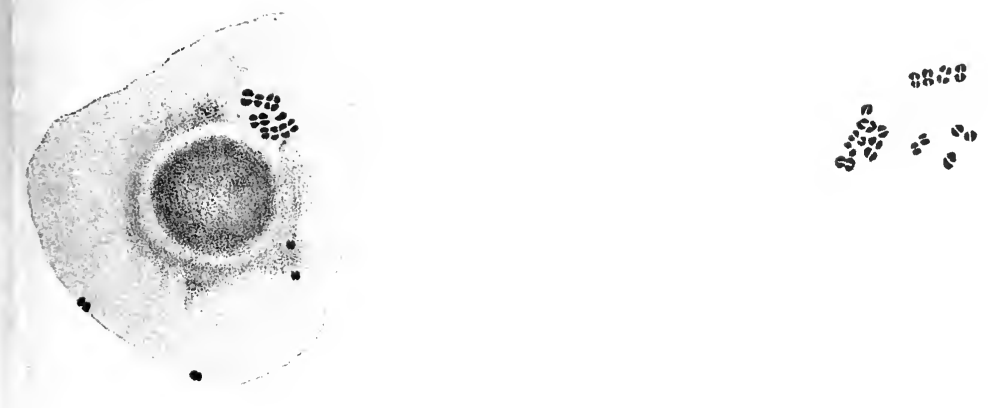

Normal microorganisms and gonococci of male urethra. 



\section{ETIOLOGY OF URETHRITIS.}

By S. LUSTGARTEN, M. D.

WHEN we speak of urethritis, it is understood that a number of etiological factors may enter into its production. From a practical point, however, the gonorrhœal form is that which demands the closest consideration. In the present incomplete state of our knowledge we deem it best to divide the subject into the two chief classes-infectious and noninfectious urethritis.

\section{A. INFECTIOUS URETHRITIS.}

Gonorrhœa.-Without attempting to give a complete review of the history or even literature of the question, we have to remember that especially through Ricord's anthority the fact has been established that gonorrhœa is a disease entirely distinct from syphilis-the "identists" theory, which had so long held sway in consequence of Hunter's misinterpreted experiment upon himself, having been abandoned. Ricord's teachings brought about a controversy as to whether gonorrhœe is simply a catarrhal process or a specific disease possessing contagious properties. The adherents of the catarrhal view, the so-called "avirulists," claimed that only through its irritative action, and not through its contagious qualities, gonorrhœeal pus reproduces the disease when brought into contact with healthy mucous membranes. In support of this belief, instances of gonorrhœa were cited which had developed in the male through contact with leucorrhoeal and menstrual discharges, as well as after a variety of mechanical and irritative causes. This school found many followers in this country, which had given birth to its famous founder.

Ricord's opponents, on the other hand, maintained that a secretion of a mucous membrane which does not contain the contagion of gonorrhœa can never cause gonorrhœe. These men argued and proved that bougies covered with pus from abscesses and other sources, when introduced into the healthy urethra do not occasion a true gonorrhœa ; and that cohabitation with women at the time of menstruation, or with those suffering from leucorrhœa or cancer of the uterus, do not of necessity bring abont catarrh of theurethra; the distinction between catarrh and true gonorrhœa lying in the fact that the former has no period of incubation, is readily 
and rapidly cured, and shows no tendency to attack adjacent organs. It is needless to say that the "virulists" steadily gained ground in the profession, and it was not long before diligent search began to be made for the specific cause of the disease. Various micro-organisms supposed to possess infectious qualities have been discovered by different observers, since, first in 1837, a harmless infusorium, trichomonas vaginalis, was accused, just as were Salisbury's threadlike germs, which he found in the pus of gonorrhœa, 1868.

Neisser, by his discovery of the gonococcus in 1879 , finally put an end to many fruitless and often simply hypothetical discussions, and put a new phase upon the question more in keeping with modern scientific achievements.

The Gonococcus Neisser.-To discover the presence of the gonococcus, staining methods must be employed, and this is readily accomplished by means of the various aniline dyes. Then, with a high magnifying power, the relatively large micro-organism, measuring as it does from 0.8 to 1.6 micro-millimetres in length and 0.6 to 0.8 micromillimetres in width, is seen either occupying the pus and epithelial cells, or lying free between these cells. The single coccus is kidney-shaped, or has somewhat the appearance of a coffee bean. They occur usually in pairs, lying close together, with their flattened surfaces looking toward each other. Multiplication takes place by each coccus splitting in two, so that groups form, which show in their grouping some resemblance to sarcina. These groups at times so completely fill up the pus-cells, that they seem to have occasioned a rupture, allowing the cocci to escape. After many unsuccessful attempts at cultivating the gonococcus, in which others had failed, Leistikow-Löffler, Krause, and finally Bumm, succeeded, the latter by employing serum of the blood of the human placenta as a culture medium. The cultures grow very slowly, and new culture-tubes must be inoculated quickly because of the tendency of the coccus to die in a few days. Bumm also was the first to give the final proof of the pathogenic rôle of these micro-organisms, by producing an acute gonorrhœal urethritis in a healthy female urethra by inoculation with first a second and subsequently a twentieth generation of the pure culture. Later on Von Schrötter and Winkler reported successful cultures on plover-egg albumen, although they did not carry out inoculation experiments. Aufuso has also succeeded in reproducing the disease from a twelfth generation of a culture made from the serum of a hydrarthritis. Quite recently E. Wertheim found that gonococcus are easily cultivated and grow rapidly upon culture plates according to Koch's method, made with one part of human blood serum and one or two parts of peptone agar or gelatin solution. By this method the gonococcus grows so rapidly that within twenty-four hours the cocci show a very extensive develop- 
ment, and form very fine transparent colonies. This observer materially strengthened the results of the preceding investigators in reproducing the disease with these pure cultures in five paralytic subjects. Wertheim's investigations have been confirmed by Gebhard.

The gonococcus has been found in the secretions of the various mucous membranes, which are now well known to be capable of taking on gonorrhœal infection, such as the urethra, vagina, uterus, conjunctiva, rectum, and even the mouth and nose in a few rare instances, besides, although not constantly, in Bartholinitis, pyosalpinx, periurethral abscess, periurethral follieulitis, as well as in joint affections. It has, however, yet to be determined by future investigations what percentage of the above-named complications, as well as of such others as Cowperitis, cystitis, pyelitis, prostatitis, buboes, perimetritis, etc., is due to the gonococcus alone or to other or mixed infections. Bumm, in his well-known work on the gonococcus, has made the assertion that only cylinder epithelium is a suitable medium for the invasion and development of the gonococcus; but more recent investigations have not substantiated this claim, since, for example, in paraurethral folliculitis the gonococci have been observed by Touton to penetrate the flat epithelium and invade the deeper strata of the tissue; and since Dinkler had claimed their penetrating qualities in ophthalmia gonorrhoica.

It is interesting and important from a medico-legal point that stains of gozorrhœal discharge on linen or underwear will permit the demonstration of gonococci even after several weeks, if the dried secretion is scraped off after being macerated and subjected to the staining process. This was first pointed out by Charles W. Allen in 1887, and quite recently confirmed by Kratter.

Staining Methods. - The gonococcus, as previously intimated, is readily stained with almost all basic aniline dyes, like fuchsine, methyl violet, gentian violet, methyl blue, Victoria blue, etc., and are with equal facility decolorized by alcohol, acids, or Gram's method. The latter has been claimed by Roux to be of practical importance for differentiation, since all other diplococci which resemble the gonococci retained the color when treated by the Gram method, while the gonococci lose it readily. The writer does not agree with these views, and will speak further on this question, so important and still so difficult of solution, in the following section.

An easy and quick way of staining is the following one: A small drop of pus or secretion * is spread in a very thin film on a cover-glass or slide

* In case too scanty a discharge is present, Neisser recommends an irritating injection either of sublimate 1 to 10,000 to 20.000 , or nitrate of silver 1 to 2,000 , which in the course of a few hours will set up a sufficient discharge. 
with the aid of a platinum-wire loop. It is allowed to become dry on the air. The specimen is then passed three times, rather slowly, through the flame of an alcohol lamp or a Bunsen burner, with the right side turned upward. Then the preparation is covered with the staining fluid by means of a glass rod, and allowed to remain so for one or two minutes, after which time it is washed off with a jet of cold water, and examined either in water or, after carefully drying, in Xylol-Canada balsam. The staining fluid is better prepared fresh each time by adding to a watchglassful of distilled water with a pipette a small quantity of a concentrated alcoholic solution of methylene blue or Victoria blue, so as to obtain a dark-blue liquid.

Fig. 5 represents a preparation stained in this way with Victoria blue. It shows epithelial cells and lymphoid cells containing gonococci, as well as gonococci free in the field. The epithelial cells are here seen in such number as they are only found at the beginning and in the declining and chronic stage of the gonorrhœeal process, while at the height of an acute attack the lymphoid cells being the almost exclusive forms present.

Gram's method, which may be used in dubious cases, is more complicated. To fresh aniline water (aniline oil, shaken. well with water and filtered through a moistened filter-paper) a concentrated alcoholic . solution of gentian violet is added, drop by drop, up to the point of saturation-i. e., until the liquid loses its transparency. The cover-glass, prepared as above, is allowed to float on this solution for ten minutes. It is then washed with water, and placed for five minutes in a solution of iodine 1 part, iodide of potassium 2 parts, distilled water 300 parts, and from there put in absolute alcohol, where it remains until no more color is extracted. After a renewed washing the preparation is subjected for half a minute to a second process of staining in a weak (light brown) watery solution of Bismarck-brown or vesuvin, washed again with water, and examined as before in water or Canada-balsam. If a preparation treated in this manner shows blue diplococci, one is sure that they are not gonococei; but in case of brown diplococci no absolute certainty is reached. For the bacterioscopic examination of gonorrhœal or urethral discharges a good microscope with Abbé's condenser and highly magnifying lenses is needed. Dry objective lenses are not to be recommended, except Zeiss's new apochromatic systems, while a one-twelfth-inch homogeneous immersion lens and an ocular No. 2 and No. 3 will answer the purpose very well.

Diagnostic Value of the Gonococcus.-The characteristics of a gonorrhœal discharge are (1) the presence of gonococci; (2) the habitat of these in lymphoid and epithelial cells ; (3) their staining qualities, espeeially their becoming decolorized when subjected to Gram's method. These three points, which especially Neisser has emphasized and many 
authors have accepted, no doubt hold good for every true gonorrhœal affection. The question now arises whether we are always entitled, when we find the stated conditions, to diagnosticate gonorrhœa a virulent and contagious disease, or whether not, under certain circumstances, harmless diplococci are apt to lead us astray. It hardly needs to be pointed out of what practical and forensic consequence this question is. Without wanting to go too much into details, our own researches (Lustgarten and Mannaberg, Vierteljahresschrift f. Derm. u. Syphilis, 1887), which have been confirmed in their essential particulars by Legrain, Poney, and others, have shown that the normal male urethra is inhabited by a variety of micro-organisms, as Figs. 1, 2, 3 on Plate I show. Although most of these bacteria are harmless parasites, and will lead into error only a careless and inexperienced worker, still three of them deserve special attention, and these are (1) a pyogenic coccus, the staphylococcus aureus, whose presence explains to a certain extent the occurrence of acute inflammatory processes in and around the urethra without an infection from outside; (2) a bacillus which shares with the tubercle bacillus the morphological and the usually applied tinctorial qualities, Fig. $1, a, b$ (probably identical with the smegma bacillus), and whose frequent presence in the normal urine must be borne in mind to prevent a too hasty diagnosis of genito-urinary tuberculosis; and (3) one or several species of diplococci which resemble completely Neisser's gonococcus in shape and tinctorial qualities, especially in being decolorized by Gram's method. Only the last-named ones have any bearing upon the present question. They are furthermore found in epithelial cells (Fig. 3) and in lymphoid cells, which can not be distinguished from true pus-cells, although the latter can not of course be expected in the normal urethra. But as these "pseudo-gonococci" exist, they may, for instance, in a traumatic urethritis, be included by pus-cells, and thus simulate true gonorrhœea. (That would explain M. von Zeissl's observations in artificial urethritis.) We must, therefore, with all due respect to his great merits, contradict Neisser's assertions of the pathognomonic value of gonococci. Even a pupil of Neisser, Steinschneider, had to concede, in response to our publication, the possibility of error, which he estimates at five per cent, but which we would judge to be far higher without venturing to give any exact figures. Other careful observers, among whom are Fürbringer and Keyes, take also a reserved stand on this question.

A distinction in principle between a gonorrhoic discharge and the secretion of the normal urethra (or certain nongonorrhoic discharges) seems to consist only in the fact that in the first cases the gonococci are to be found in larger number and in greater masses, and are not accompanied by so many banal micro-organisms.

But in an individual case, especially in that of a very chronic gleet, 
the differentiation may be very difficult, and indeed almost impossible, for the decision of the question whether a gonorrhoic and consequently contagious affection or a simple catarrh exists; and aside from the microscopical examination we may have to rely on the clinical features and our own clinical experience.

The same uncertainty applies to pus obtained from the vagina, since first Bumm and many others after him stated the normal presence there of several diplococci species resembling gonococci.

To sum up the well-established facts concerning the gonococcus according to the present state of knowledge, we arrive at the following conclusions :

1. The gonococcus Neisser produces gonorrhœea.

2. If an acute or purulent secretion contains gonococci with their characteristics, as described above, the affection is produced by gonococci. That does not of necessity apply to vaginal discharges of adults, as likewise reserve is indicated in the (often epidemic) vulvo-vaginitis in children, about the true gonorrhoic character of which doubts still may be entertained.

3. If in a very chronic torpid urethritis with scanty, whitish, or grayish discharge,* diplococci with all the characteristics of gonococci are found, it does not prove that the affection is of gonorrhoic nature and consequently contagious ; especially not, if these diplococci are only to be found in comparatively small number and accompanied by a variety of other micro-organisms. This uncertainty is much to be regretted, as the question of the contagiousness of such cases turns up almost daily in the practice of the specialist and of the general practitioner as well, and as an absolutely reliable way of ascertaining the contagiousness would lessen considerably the professional responsibility. As far as personal experience goes, I am inclined to believe that many of the cases of (3) are indeed not contagious, and that the wives of such men remain healthy and prolific.

II. Pseudo-gonorrhœa.-Under this name Bockhart has described fourteen cases of urethritis, resembling in their symptoms an average gonorrhœa, for which he blames a very small diplococcus and an ovoid streptococcus. These micro-organisms could be cultivated and were inoculable in producing an acute urethritis. His statements have not been confirmed as yet. Under the name of pseudo-gonorrhœea we might as well in general include all kinds of infectious urethritis which can not be otherwise classified, as, for instance, one case reported by Neisser and one by Finger, probably produced by bacilli. To judge by the seanty re-

\footnotetext{
* Cases which, in the opinion of eminent clinicians, and in accordance with frequent experience, are no longer virulent.
} 
ports, the cases belonging to this class must be very rare. It may be that we have to count among them the urethral discharges observed sometimes in acute febrile diseases, like typhoid fever, and which may be produced by the respective viruses of those diseases, in analogy with the exanthemata on the skin and other mucous membranes. Such occurrences, however, have a greater theoretical than practical interest and need further study.

III. Syphilitic Urethritis. - So have been called slight and indolent urethral discharges, seen by many observers (Bassereau, Bumstead, Taylor, etc.) during the secondary stage of syphilis, and which, no doubt, are the consequences of roseola or mucous patches in the urethra, as Grünfeld has demonstrated by endoscopic examination. Although of little consequence to the bearer, they deserve attention, as they may be the source of contagion, even when all externally visible symptoms of syphilis are wanting.

IV. Urethritis Tuberculosa.-Tuberculosis of the urethra, leading to tubercular ulceration, may in rare instances be a partial symptom of a more or less generalized uro-genital tuberculosis, or in even far rarer cases start primarily in the urethra. Primary lupus of the latter has, as far as I know, never been recorded. Quite recently Schuchardt reported a new and surprising form, the primary tubercular catarrh of the urethra and bladder, about which further communications have to be looked forward to before a decided opinion can be reached. In all these forms the clinical diagnosis will have to be corroborated by the demonstration of tubercle bacilli in the urethral discharge. Particular care has to be taken, and special methods have to be resorted to,* to carry this out in an irreproachable manner, and to prevent confusion with the smegma-bacilli, the common parasites of the normal urethra and urine.

\section{B. NONINFECTIOUS URETHRITIS.}

Urethral discharges, which we are at present justified in believing noninfectious, are observed under a variety of different etiological conditions. They are of comparatively little importance, and this in proportion to the causes from which they arise. These discharges have no period of incubation, and show a marked tendency to rapid cure if the cause is removed. For practical purposes it will suffice to simply enumerate, as causative factors, general conditions, traumatism, and chemical irritation. Under general conditions may be mentioned chronic bronchitis, chronic constipation, gout, oxyuris vermicularis, the excessive use of new beer and new cider, and possibly the presence of herpes in

* Boiling the preparation in a two-per-cent alcoholic solution of caustic potash before staining. 
the urethra, etc.; under traumatism, the use of instruments, stone in the urethra, the introduction of foreign bodies, masturbation, excessive coitus, etc.; under chemical, the use of irritating injections, the internal administration of cantharides, and possibly the cohabitation with women during their menstrual period, and those suffering from leucorrhœa. Further investigations may show that the latter cases may be more properly ranged among the infectious forms. 


\section{ACUTE URETHRITIS.-GONORRHCEA.}

\section{By GEORGE EMERSON BREWER, M. D.}

THE writer desires, at the outset, to enter a plea for the more general use of the etymologically correct term urethritis, as descriptive of the condition about to be considered, rather than the term gonorrhcea, which implies a pathological process known for more than two hundred years to be of incorrect application. Although it would be quite impossible to exclude from our vocabulary all the derivatives of this unfortunately incorrect term without creating confusion, still, in the pages that follow, use will be made as far as possible of terms which correspond to a more modern and a more correct nomenclature.

Definition.-An inflammation of the urethral mueous membrane, characterized by the presence of a purulent discharge and painful micturition.

History.-Regarding the history of this disease, it is only necessary to say that it is a disease of great antiquity, having been mentioned in the early medical writings of Hippocrates and Celsus. It was described with great accuracy by many writers during the middle ages, who distinctly recognized its symptoms and contagiousness. By these early observers it was generally regarded as a disease of the secretory apparatus, resulting in an abnormally large and more or less constant flow of semen from the external urethral orifice. Hence the name gonorrhoea.

Great confusion was created toward the end of the fifteenth century by the appearanee throughout Europe of syphilis, chiefly from the fact of its being communicated, like urethritis, by sexual eontact. For more than two hundred years these diseases were regarded by the majority of medical writers as due to one and the same virus. This view, however, was combated by Cockburn, Hales, Ellis, and many others. In 1767 John Hunter thought to establish the non-identity of the two diseases by inoculating himself with the pus derived from an individual during an attack of an acute urethritis. Unfortunately, however, the patient from whom the pus was secured was at the same time suffering from an unrecognized syphilis, and the resulting chanere and other manifestations of the latter disease which followed, retarded the further progress of knowledge upon this point for nearly half a century. The reaction from this 
view began with the publication by Benjamin Bell, in 1793, of an exhaustive argument founded upon a large number of observations which seemed to disprove the theory adopted by the majority of authorities as a result of Hunter's experiment. His position was subsequently strengthened by the careful observations of Hernandez, published in 1811. The subject was finally cleared of its obscurity by the masterly thesis of Ricord, who demonstrated, from an analysis of over six hundred carefully observed cases, the non-identity of syphilis and gonorrhœea.

Varieties.-The urethra, in common with other organs and tissues of the body, may be the seat of several distinct varieties of inflammation. Until quite recently writers upon this subject contented themselves by describing only two forms of the disease. In view of modern investigation, and our increased knowledge regarding the causes of inflammation in general, this classification must be somewhat enlarged. Clinically, ever since the disease has been recognized, it has been observed that some cases present symptoms of great severity, while others pursue a comparatively mild course. The duration of the former is seldom under six or eight weeks, and often prolonged indefinitely even under the most careful treatment, while the latter frequently disappears spontaneously in a few days.

It has been for some time the generally accepted belief among the members of the medical profession that, in the severe cases, the inflammation is caused by contact with a specific virus contained in the purulent secretions from the diseased organs of a person similarly affected, but that the mild cases may arise from the irritating vaginal secretions occurring during menstruation or an attack of leucorrhoea ; or they may be the result of an irritation of a sensitive point in a previously diseased urethra, caused by prolonged sexual excitement and the effects of alcohol. There are still other cases, though of very rare occurrence, where an attack of urethritis arises without contagion and in persons whose urethral mucous membrane has never been the seat of inflammatory disease. A certain number of these patients present in their urethræ characteristic lesions of tuberculosis, while others show, mainly by the effects of treatment, the inflammation to be the local manifestation of rheumatism or gout.

Urethritis may also be occasioned by traumatism and by the presence of lesions of syphilis, chancroid, and possibly herpes.

The question as to whether a perfectly healthy man by cohabiting with a perfectly healthy woman can by any amount of physical exertion, sexual excitement, or alcoholic stimulation, "give himself the clap," as Ricord expresses it, is far from being settled in the affirmative. Although I am aware that the great majority of writers upon this subject during the last half-century have declared that to be a fact, still, in the light of our increased knowledge regarding the behavior of gonorrhœal disease in 
women, the question is still an open one. That a careful examination of the vagina and external genitals of woman fails to reveal the presence of disease, by no means proves that the subject of such examination is free from disease, for Noeggerath and many other authorities upon diseases of women have demonstrated that the cervix uteri, Fallopian tubes, and even the body of the womb itself, may be the seat of a grave gonorrhoal inflammation, without the slightest evidence being revealed by the most careful examination of the external genitals and vagina. Noeggerath goes so far as to say that nine tenths of all pelvic diseases peculiar to women may be traced to gonorrhœa.

It is a well-known fact also that the gonorrhœal infection in women almost invariably begins upon the mucous membrane of the urethra, and, indeed, may never spread to the vagina or cervix. The passage of urine just before an examination would in this case remove all evidence of disease, especially if the inflammation had passed the acute stage, leaving no tumefaction or redness of the urethral orifice to excite suspicion. Furthermore, in a chronic inflammation of the vulvo-vaginal glands, it is well known that a secretion can often be pressed out which is muco-purulent in character, containing gonococci in abundance. In view of these facts, and many others, the statement by Fournier that "a man gives himself the clap oftener than he receives it" must be accepted with a certain amount of reserve.

In this connection it may be stated, in further confirmation of this view, that during the years 1887 and 1888 the writer, while in charge of the genito-urinary room of the out-patient department of Roosevelt Hospital, had an opportunity of investigating a large number of these supposed cases of strain, or attacks of acute urethritis, acquired by individuals while cohabiting with presumably healthy females. In a considerable number of instances also in private practice an opportunity has been afforded of continuing these investigations. Although the number of such cases is not large, and the opportunities for thorough examination of both individuals afforded in only a small percentage of these, still, during the past six years twelve or fifteen such cases have been thoroughly investigated, with the result that in no instance have the acute inflammatory symptoms been accounted for in any manner other than by direct contagion, by contact with the gonococci-bearing secretions from the mucous membrane of some portion of the genito-urinary tract in the female, or by an exacerbation of an uncured urethritis in the male.

Etiology.-In 1879, Neisser, after a large number of observations, pointed out that the pus from the severer forms of urethritis and conjunctivitis differed from other varieties of pus by the presence in the former of a peculiar micro-organism which he called' the gonococcus, and that in no other pus was this organism found. This statement has been 
confirmed by a large number of observers since that time, so that to-day it may be safely stated that no one who has investigated the subject will undertake to deny the constant presence of this organism in these affections. Proof as to the exact etiological significance of this observation, however, was not furnished until several years after its discovery. This delay was due in a great measure to the difficulty in producing pure cultures of the organism, to the existence of large numbers of other organisms bearing a marked morphological resemblance to the gonococeus, and to the fact that inoculation experiments were necessarily applied only to the human urethra, animals, so far as is known, enjoying an immunity from this disease. As a result, however, of the carefully conducted experiments of Bockhart, Bokai, Bumm, Roux, and others, the gonococcus has finally been isolated, successfully cultivated, and the disease produced with all its characteristic symptoms by the inoculation of a pure culture, as far removed as the twentieth generation, upon healthy urethral mucous membrane. The relationship between the gonococcus and the severer urethral inflammations, therefore, seems to be established.

In an article published by Bockhart, in 1886, some interesting experiments are reported regarding the etiology of the non-specific, or, as he terms them, the pseudo-gonorrhceal inflammations. His observations are based upon fifteen eases of mild urethritis, in the secretions of which no gonococci were found. Investigation with other methods of coloring revealed the presence of two organisms differing wholly from the gonococcus, and from each other in staining reaction and appearance. These were also found in the vaginal secretions, alkaline in reaction, occurring in women as the result of menstruation, leucorrhœa, and cancerous disease of the cervix. The relation of these organisms to the milder forms of urethritis was subsequently rendered probable by successful culture, and inoculation experiments which resulted in the production, in healthy individuals, of a mild urethritis, corresponding in symptoms, course, and duration with the case from which the original cnlture was made. As in the writer's experience, in not more than one, or at most two, per cent of the cases of urethritis is it necessary to search for a cause other than the gonococcus to account for the symptoms, the term urethritis, when used in the following pages, unless otherwise defined, may be considered as referring to the usual form of the disease-that is, specific or gonorrhœal urethritis.

Infection.-The number of instances in which acute urethritis is acquired by means other than sexual intercourse is extremely small. Cases have been reported by Jullien, Horand, Winslow, and others, where the disease has been acquired and transmitted through the practice of unnatural sexual intercourse. Cases have undoubtedly been observed also where infection has taken place through mediate contagion, one such having 
fallen under the writer's observation. Given the case of a healthy man who has sexual relations with a female whose genitals are contaminated by the secretions from a gonorrhœal infection, and the chances are largely in favor of his acquiring the disease, in spite of the many precautionary measures which he may subsequently take. That he may occasionally escape, will not be questioned, and in this connection it might be advisable to consider briefly the conditions which favor infection and those which diminish its probability.

In his recently published work on this subject, Finger states that the development of gonococci is favored if the culture medium or tissue upon which they are deposited is alkaline in reaction. Ordinarily the mucous membrane of the urethra is bathed in a fluid the reaction of which is distinctly acid. Under conditions of strong sexual excitement, however, the glands and follicles of the urethra pour out an abundant alkaline secretion which neutralizes the previous acidity of the canal. Conditions, therefore, which heighten sexual excitement would tend to increase the probability of infection by insuring an abundant flow of alkaline mucus. In this manner may be explained the well-known effects of alcoholic stimulants, long-continued and frequently repeated sexual acts. A large urethral orifice is said also to favor infection by allowing easy ingress of the infecting material.

Regarding the conditions which diminish the probability of infection only two need be mentioned. The first and most important of these is immediate urination after the coitus; the second, prompt attention to cleanliness. The former, probably, acts more by restoring the acid reaction of the canal than by any mechanical removal of pathogenic organisms.

Incubation.-By this is understood the time which elapses between the contact of the virus and the first symptoms indicating an inflammatory reaction on the part of the mucous membrane. During this period the few pathogenic organisms which find their way into the urethral canal develop and multiply, until they are present in sufficient numbers to cause by their presence alone, or by fluids or gases elaborated in the process of their development, sufficient irritation to give rise to the reactionary symptoms which constitute the first evidences of the disease. The duration of this period is generally from one to fourteen days. Cases are occasionally encountered which exceed this latter limit. In the great majority of cases, however, the first symptoms are noticed on the third day; in at least two thirds of the cases, during the first week. The excessive use of alcohol, and long-continued erotic excitement, may shorten this period by supplying the conditions which favor a rapid development of the organisms. In cases of doubtful origin the observation of a distinct period of incubation is of value, as excluding those inflammations of the urethra due to the mechanical or chemical injury, as these have no 
period of incubation, the reaction appearing almost immediately after the injury.

Clinical History.-About three days after exposure the patient experiences an itching sensation in and about the external urethral orifice. There is also a slight burning during and immediately after the passage of urine. The meatus is red, slightly swollen, and sensitive to the touch. An increased amount of mucus is present. These symptoms gradually increase in severity: the itching is replaced by a sense of heat and soreness; the lips of the external orifice become thickened, everted, œdematous, and highly congested; the pain, or ardor urino, is increased, and the mucous secretion becomes more abundant and assumes a distinctly purulent character. If the case is untreated, this progressive increase in the severity of the symptoms continues until the acme is reached. This is usually from the twelfth to the fifteenth day. The patient now presents all the characteristic appearances of an acute inflammation; the glans penis is intensely red, and often excoriated; the prepuce is oedematous; the whole organ hot, and sensitive to the touch, and in a constant state of partial erection. The discharge is very abundant, thick, creamy-yellow, or greenish in color. Each urination is accompanied by most excruciating pain; the stream is small, twisted, divided, or scattering, owing to a diminution in the calibre of the urethra from the swelling of the mucous membrane. At this stage there is, in nearly every instance, marked sexual irritability. Erections occur, chiefly at night, with great frequency, and are often prolonged for several hours. Owing to the inflamed and infiltrated condition of the mucous membrane its elasticity is greatly diminished, which prevents its partaking in the general expansion of the tissues during an erection. As a result of this, the organ becomes curved npon itself, the concavity looking downward. This alteration in the form of the organ during erection, or chordee, may vary greatly in extent, from a slight bending of the glans to a curve approximating a right angle. This condition frequently gives rise to great pain, often rendering the nights wholly sleepless. Seminal emissions at this period are of frequent occurrence, and add greatly to the general discomfort of the patient. Under unhygienic surroundings this condition may continue for many days and weeks with but slight, if any, abatement in the severity of the symptoms. Generally, however, improvement begins by the twentieth day, and the sufferings of the patient are gradually lessened, until there is no soreness, pain on micturition, chordee, or sexual disturbance. The discharge, however, may continue for some time after the acute inflammatory symptoms have disappeared. This, however, finally diminishes in quantity, becomes progressively less purulent and more mucous in character, and finally ceases, as it began, by a small, transparent drep.

The above description applies only to an uncomplicated case, where 
the inflammation does not extend beyond the bulbo-membranous junction. The duration of such a ease, if untreated, is extremely variable, probably never under four weeks; generally from eight to ten, and often for an indefinitely protracted period. Absolute rest, the avoidance of stimulating food and drinks, and freedom from sexual excitement, will often not only diminish greatly the severity of the symptoms, but shorten to a marked degree the duration of the disease. On the other hand, inordinate physical exertion, indulgence in alcoholic and venereal excesses, will often, in spite of the most careful treatment, prolong the disease indefinitely, increase the severity of individual symptoms, and render the patient liable to grave complications. If the inflammatory process extends beyond the compressor urethræ muscle, a number of complications, such as posterior urethritis, epididymitis, cystitis, prostatitis, etc., may occur, and greatly prolong the duration of the disease. These, however, will be considered in separate sections.

Pathology.-From the careful observations of Bumm and others, it is probable that the inflammatory process is occasioned and takes place after the following manner: A small amount of purulent material containing gonococci is deposited during the sexual act upon the mucous membrane of the fossa navicularis. These living organisms penetrate the epithelial covering of the mucous membrane, and, finding their way through and between the superficial cells, eventually reach the upper stratum of the sub-epithelial connective tissue. The irritation caused by this microbian invasion results in an acute hyperæmia of the part, with dilatation of the capillary vessels and exudation of serum. There is also an increased glandular secretion with epithelial exfoliation. Later, an abundant transudation of leucocytes takes place from the dilated capillary vessels. These, during their outward passage through the epithelial layer, absorb and carry with them large numbers of gonococci. If the wrethra is examined at this time, the mucous membrane will be found to be thickened, deep red in color, and covered with an abundant muco-purulent or purulent secretion. The orifices of the mucous follicles appear as deeply injected, slightly elevated spots. Epithelial erosions and sometimes areas of genuine ulceration are present. The process begins in the fossa navicularis and gradually extends downward, reaching the bulbo-membranous junction about the twentietl day. When the wandering leucocytes have succeeded in removing the micro-organisms from the sub-epithelial tissue, and the gonococci-invaded epithelial cells have been washed away, the symptoms begin to subside. The hyperæmia diminishes, the sub-epithelial round-cell infiltration is absorbed, the erosions and ulcerations receive a new cellular covering, and the disease is at an end. Resolution takes place more slowly in the glands, which often continue to secrete pus long after the process has ceased in other portions of the mucous membrane. 
Differential Diagnosis. - When the physician is called upon to assume the care of a patient who presents a discharge from the urethra, the first question to be decided is, whether or not the inflammation is of gonorrhoal origin. This can usually be determined by any one who is familiar with the use of the microscope. If the urethral discharge contains gonococci, we have to do with a case of true gonorrhœal urethritis. If, on the other hand, after thoroughly examining three or four slides, no gonococei are found, gonorrhœa may be excluded. Of the several conditions which may be mistaken for acute specific urethritis, the one which in its initial symptoms resembles it most closely is acute non-specific urethritis. In this instance we have to do with an inflammation of the urethra, due to contact with the irritating secretions of a simple leucorrhœa, menstrual fluid, cancerous disease, or pelvic abscess. The symptoms of this condition, which have been described by Bockhart and Aubert, may vary from a slight irritation of the meatus, with an increased flow of mucus, to a urethritis, which, for a short time at least, may be compared in severity to an ordinary gonorrhoea. There is usually a distinct though variable period of incubation. The acme is reached in from two to six days, and the duration of the disease is seldom over twelve. The differential diagnosis can only be established by a microscopic examination of the secretion. A urethritis due to mechanical injury or chemical action may be differentiated by the absence of any period of incubation, by the sudden and often violent beginning and the equally rapid subsidence of the symptoms, and also by the history of the case, which, as a rule, clearly points to its origin. Chancroidal ulcers and the initial lesion of syphilis, when situated just within the meatus, may furnish a secretion which has been mistaken for urethritis. It is, however, hardly necessary to enumerate the differential points, as any one familiar with these conditions can scarcely fall into error if an ordinarily careful examination be made. A mucous or muco-purulent discharge from the urethra is occasionally present during the early secondary stage of syphilis, presumably due to the presence of mucous patches in the deeper portions of the canal. This may lead one into error in supposing that he is dealing with the first stage of a gonorrhœea. Observation for two or three days will, in most cases, suffice to establish the diagnosis. All of the above conditions are rare, and require but a passing notice.

The symptoms which most resemble an acute gonorrhœal infection are those due to an acute exacerbation of a chronic urethritis. It may be safely stated that, of all the cases of supposed acute urethritis that a physician is called upon to treat, in persons who have previously suffered from the disease, at least one half are cases not of a fresh infection, but of an awakening of an ancient and uncured urethritis which may have been latent for many months, and even years. Except for the fact that 
these cases show little or no period of incubation, and that the severity and duration of the acute symptoms are generally reduced, they differ in no way from the primary attack. From a therapeutic standpoint their difference is unimportant; from a social or medico-legal point of view, however, the decision of the physician may be of the greatest moment, especially when the honor of a member of the patient's family is at stake. The following facts should always be borne in mind in determining such a question :

First, that cessation of the discharge by no means indicates a complete recovery from the disease.

Second, that cases of chronic urethritis may exist where the inflammation is limited to one or more granular patches or small areas of chronic inflammation in the bulb or deeper portions of the urethra, the only evidence of disease remaining being the presence in the urine of small, thread-like bodies or tripper faden, regarding which mention will be made later.

Third, that the disease may remain latent in this manner for months and years, but under favoring conditions may again become active, and furnish a secretion which may contain gonococci in abundance.

Fourth, that this awakening of the latent disease may be occasioned by ill-health, the passage of urethral instruments, the excessive use of alcohol, and prolonged sexual indulgence.

Prognosis. - Among a certain class in the community this disease is regarded as an affection of but little or no importance; in fact, "no more to be dreaded than a cold in the head"; and, from the careless and indifferent manner with which it is treated by a large number of the medical profession, it would seem that this opinion regarding its innocuousness was not wholly confined to the laity. The quicker the spread of such ideas is checked, the better will it be for the reputation of the medical profession and for the welfare of mankind in general.

It is only under very exceptional circumstances that this disease threatens the life of the patient. Under unfavorable hygienic surroundings, and in subjects greatly reduced through dissipation and disease, the inflammation may become so acute as to result in local gangrene, septic infection, and death. A fatal termination not infrequently results from the immediate or remote effects of some of the complications of this disease, as acute suppurative prostatitis, purulent arthritis, stricture, and pyelo-nephritis. In general, however, the prognosis regarding life may be said to be good.

Regarding the probable duration of an uncomplicated case of acute urethritis modern authorities differ greatly. Ricord says, "Une chaudepisse commence, Dien le sait quand elle finira." Keyes places the average duration at from four to six weeks. Prof. Otis was accustomed to tell 
his class that no discharge which recovered under four weeks by any treatment was of gonorrhœal origin. These views were, however, somewhat modified during the last two years of his teaching. In a paper published in 1887, entitled Some Observations upon the Modern Treatment of Urethritis, the writer states, as a result of some experiments with bichloride of mercury, that "in uncomplicated cases of acute specific urethritis recovery may be expected within two weeks"; and during the five years which followed the publication of that paper, in a large number of cases, permanent cessation of discharge has been known to have taken place within fourteen days from the beginning of treatment. The following may be quoted from a paper on the subject, giving statistics from observations during five years upon the treatment of this disease with bichloride of mercury, which was read before the Genito-Urinary Section of the New York Academy of Medicine in June, 1891, and which will fairly represent the writer's present views npon the subject:

"These statistics I believe to be as conscientionsly prepared and as accurate as any others gathered in the same manner from the same class of cases suffering from this disease, yet I believe them to be absolutely worthless from a scientific point of view, for even my comparatively limited experience in the treatment of urethral disease has tanght me that the simple cessation of discharge by no means indicates a cure of the disease; and I am prepared to say, without the slightest hesitation, that it is my belief that, had a careful and thorough examination been made in each instance at the time when I reported the cessation of all discharge, not one case of my three series of cases would fail to show the unmistakable evidences of an uncured urethritis, which might under favoring conditions again become active and furnish a secretion which would be contagious.

"Regarding the length of time necessary to effect a complete and perfect cure by this or any other method of treatment, I must confess myself wholly unable to present a rule which would be even approximately correct, as in this disease, perhaps more than in any other, success depends upon so many conditions which are intimately associated with the temperament, personal habits, and general health of the patient."

Treatment.-Up to the present time the treatment of this disease has been mainly empirical. The reason for this probably lies in the fact that until quite recently but little was definitely known regarding its correct pathology.

As the investigations of Bamm and others have shown that the gonococci very quickly penetrate the epithelial covering of the mucons membrane, and collect in great numbers in the lymph-spaces of the upper connective-tissue layer, the logical conclusion is inevitable that the ideal scientific treatment must consist in measures which would insure the 
presence in these lymph-spaces of a fluid containing an antiseptic agent capable of destroying these organisms, or at least of inhibiting their further development. This, for obvious reasons, could only be brought about by employing remedies which act through the circulating fluids of the economy, as substances present in the excreted urine, and those injected into the urethra, must necessarily fail to reach the deeply imbedded bacteria.

Clinical experience, however, has shown that certain antiseptic fluids, when injected into the urethra, do exert a marked action on the disease, both in subduing the acuteness of the symptoms and limiting its duration.

If our modern ideas regarding the pathology are correct, this can only be explained by the theory advanced by Halsted, which is that, although large numbers of gonococci undoubtedly do penetrate the tissues and become deeply imbedded in the connective tissue, the development of these organisms takes place at or near the surface of the mucous membrane, and consequently within reach of injected fluids.

- In perhaps no other diseased condition have the resources of the pharmacopœia been so thoroughly tested as in the treatment of urethritis. As it would be a useless expenditure of time to enter into an exhaustive consideration of the many methods of treating this disease which have been suggested or employed in the past, only those procedures will be described which experience has proved to be of positive value. Before inaugurating any plan of treatment a few general directions should be laid down by the physician, the observance of which by the patient will greatly add to his comfort and the chances of ultimate success.

The use of alcohol should be absolutely prohibited. In the opinion of the writer, a large proportion of the cases which resist treatment may be accounted for by the fact that the physician is not sufficiently explicit in his directions in this regard. Even the moderate use of alcoholic drinks is deleterious. The only instances in which it should be allowed are in cases where from its long-continued use grave nervous disturbances would follow its sudden withdrawal.

Sexual excitement should be rigorously avoided, as the physiological congestion always present under this condition adds to the existing inflammation, and favors its extension to other portions of the genitourinary tract.

Inordinate physical exertion must also be guarded against during the acute stage, as the hyperæmia induced by bodily exercise exerts the same untoward influence as that resulting from alcohol and sexual indulgence.

Rest in bed during the first four or five days of the inflammation, especially when combined with constant applications of hot water, affords the most effective means at our command of controlling the painful 
symptoms of the disease and of diminishing its severity. In the great majority of cases, however, this is impracticable, and measures must be employed which will not necessitate absolute repose or confinement to the house. The pain which accompanies micturition, although probably due to a distention of the inflamed canal by the jet of urine rather than to the acidity of the fluid, as formerly supposed, seems to be modified often by diuretics and alkalies. The drinking of a large amount of pure water often fulfills this indication better than the employment of any other agent. Strong lemonade is more agreeable, and when taken in a sufficiently large amount will act as a diuretic and alkali, the citric acid, in common with all vegetable acids, being converted into alkaline carbonates in the economy. The relief of dysuria may often be afforded by immersing the penis in hot water during micturition. The injection also of a four-per-cent solution of cocaine into the urethra four or five minutes previous to urination will in most cases completely do away with the pain.

The Expectant Plan of Treatment.-The expectant plan of treatment, advocated by Fournier and other French surgeons, needs but a passing notice. Their plan is to defer all treatment, save hygienic, until the stage of decline, from four to six weeks from the beginning of the disease. As any acute inflammation of the urethra is liable to result in granular patches, local ulceration, and sub-epithelial deposits of lymph, which may, and generally do go on to the formation of stricture if allowed to remain unchecked for a month or six weeks, and as it is well known that the probability of all inflammatory complications is greatly increased by each day that the original source of infection remains unchecked, to adopt the French plan would seem to lay ourselves open to a charge of malpractice, especially as we have means at our control to arrest the inflammation, if not cure the disease in from two to four weeks.

Internal Treatment.-It has long been the hope of those interested in the treatment of urethral inflammations that some remedy would eventually be discovered which, when taken into the economy, would, either by its action through the blood or the urine, in destroying or at least inhibiting the irritating action of the pathogenic organisms, cure the disease. This hope has not up to the present time been fully realized.

Certain well-known drugs, as the alkaline diuretics, cubebs, copaiba, and sandal-wood oil, undonbtedly exert, when judiciously employed, a marked effect in subduing the painful and disagreeable symptoms of the disease, and occupy, therefore, a well-deserved place in the therapeutics of urethritis. That they limit the duration of the inflammation, has not been demonstrated ; they should therefore be regarded as auxiliary measures, to be used when necessary in connection with such local applications as experience has shown to exert a positive influence in cutting short the 
inflammation. The action of these remedies is doubtless through the agency of the urine rather than the blood; and the therapeutic effect is the result of contact of the urethral mucous membrane during micturition with the substances themselves or the products of their decomposition contained in the excreted urine. That this is true of copaiba at least, is evidenced by the experiments of Ricord and Roquette, who prescribed this agent to patients suffering from urethritis, and who at the same time had urethral fistulæ! As a result of these experiments, it was found, during the internal administration of this drug, that that portion of the urethra behind the fistula would show marked improvement, while the portion anterior to the fistulous opening, through which no urine passed, remained unchanged. Subsequently, injections were made into the anterior portion of the canal of a portion of the urine passed by the patient while under the influence of the balsam; this produced the same amelioration of the inflammatory symptoms which was noticed in the posterior portion. Some years ago, a number of experiments were undertaken by the writer at the outdoor department of one of our large hospitals to test the efficacy of the different drugs in the treatment of this disease. Several cases of acute specific urethritis were treated by each of these remedies, and compared with control experiments upon patients who received no treatment at all save some simple placebo. As a result of these observations, it was found that simple diuretics exerted apparently no influence either upon the severity of the symptoms or the duration of the disease; that alkalies in some instances seemed to lessen the pain during urination, but showed no tendency to diminish the discharge or otherwise modify the course of the disease. Cubebs, when given in sufficient doses (a teaspoonful of the powder every two hours), would often stop an abundant purulent discharge in from twenty-four to thirtysix hours, and so diminish the other painful symptoms as to render a previously suffering patient comparatively comfortalle. The symptoms, however, would reappear in proportion to the degree to which the dose was diminished; and when discontinued entirely, even after several weeks' use, the disease would still appear and pursue much the same course as if untreated from the first. The continued use of this drug in large doses would frequently produce grave gastric disturbance, necessitating its withdrawal. Copaiba was found to be less useful than cubebs, chiefly for the reason that it produced more general digestive disturbance. When borne in large doses its action was practically the same as that of cubebs. Complete anorexia, severe vomiting, diarrhœa, and cutaneous erythemata were the common accompaniments of its exhibition. Sandal-wood oil was slightly less efficacious than the other two drugs, but had the marked advantage that it created much less gastric irritation. It was the only agent that could be used in sufficient doses to markedly modify the symp- 
toms for a sufficient length of time to be of any practical value. Epigastric and lumbar pain, with anorexia and vomiting, may occasionally occur as a result of its use, and, rarely, a macular erythema. The following résumé of the agents employed, their preparation and doses, may be of value :

Pure water taken in quantities of from two to four quarts a day proved most useful as a diuretic agent. Potassium acetate, when given in doses of fifteen to thirty grains three or four times a day, largely diluted, acted as a reliable diuretic. If the dose was so increased that the patient absorbed from one to two ounces of the salt in twenty-four hours, the effect was to render the urine alkaline in reaction. Potassium citrate prodnced much the same result, but had to be given in somewhat larger doses. Sodium bicarbonate acted as a reliable alkali, in doses of twenty or thirty grains, frequently repeated. The most satisfactory preparation of cubebs was the freshly made powder; of copaiba, the oleo-resin in capsules of ten minims each. Of the sandal-wood oil preparations, the one found most useful was the "Santal midy capsule" made by Fougera $\&$ Co., Paris ; these should be given in doses of two to four capsules after each meal.

The following prescription, known in hospitals and dispensaries as the "Lafayette mixture," is found to be useful:

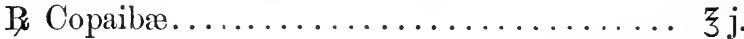

Liq. potassii................... $3 \mathrm{ij}$.

Ext. glyeyrrhizæ.................

Spt. ætheris comp...............

Syr. acaciæ.....................

Ol. Gaulth .................. M.

Sig. 3 ss. t. i. d.

Several other drugs closely allied to the balsams above described have been advised and more or less extensively used in the treatment of this disease. Among these may be mentioned gurjun balsam, which was brought to the notice of the profession by Vidal in 1877. This drug is similar in action to copaiba, and is said to possess the advantage of exciting much less nausea and digestive disturbance. The dose is from one half to one drachm daily, preferably taken in mucilage of acacia or some sirupy fluid.

Kava-kava was at one time used to a considerable extent as a substitute for cubebs. It is employed in the form of an infusion, or in pills made from the extract, the dose of the latter being one to two grains three or four times a day.

Oil of turpentine undoubtedly exerts a marked effect upon inflammations of the mncons membrane of the urinary passages, but its exhibition is attended with such marked discomfort to the patient that its use has been practically abandoned. 
Local Treatment.-The local treatment of this disease has engaged the attention of medical men for a considerable period, and has been steadily growing in favor.

When one considers the fact that urethritis is a purely local disease, limited during the first few days at least to the anterior portion of the urethral mucous membrane, it is not surprising that many have entertained the hope, and with a considerable degree of reason, that some local procedure should be found which would arrest the inflammatory process and destroy the poison which produced it. In no other local inflammatory condition have efforts proved so uniformly unsuccessful. The reason of this possibly lies in the fact, which has previously been mentioned, that the micro-organisms have already developed to a large extent, penetrated the epithelial layer, and formed colonies in the lymph-spaces and connective-tissue layers of the mucous membrane long before the inflammatory reaction has made its appearance, and that any local measures which do not entirely destroy the mucous membrane itself fail to exert their influence upon the deeper-seated organisms.

Several so-called abortive methods have been suggested, and employed with varying degrees of success. These will be considered first.

Nitrate of Silver.-The injection of a strong solution of nitrate of silver (ten to thirty grains to the ounce) into the urethra immediately upon the first appearance of the symptoms of this disease, has been practiced by many eminent genito-urinary surgeons and with a certain degree of success. The great pain caused by its employment, however, and the uncertainty of result, combined with the wide prevalence of erroneous ideas regarding its probable causative influence in the production of stricture and other grave complications, have led to its practical abandonment by most surgeons. Quite recently an article has appeared in the London Lancet by Mr. C. E. Cotes, describing a method of employing nitrate of silver, which has proved in his hands most successful. The anterior urethra is thoroughly cleansed of all pus by urination, and subsequent swabbing with cotton by means of the endoscope. A solution of nitrate of silver, ten grains to the ounce, is then applied through the endoscopic tube to the entire anterior portion of the urethra by means of a cotton applicator. An extra amount of this fluid is employed upon the anterior third of the canal. The patient is then instructed to remain in bed for from forty-eight to seventy-two hours. The intense inflammatory reaction which follows this application subsides in three or four days, and no further treatment is required. The author reports forty cases successfully treated by this method, and if equally happy results can be secured by others the treatment of this disease will be relieved of many of its embarrassments.

Guiteras's Method.-In a paper read before the Genito-Urinary Section 
of the New York Academy of Medicine in June, 1892, Dr. Ramon Guiteras suggests a new method of treatment by the use of solutions of nitrate of silver. His plan is to make one injection, every second day, of a solution of nitrate of silver, beginning with the strength of two grains to the ounce, and increasing by two grains at each application, until the strength of twenty grains is reached. In the interval which elapses between the treatments the patient simply keeps the urethra thoroughly washed out with a mild solution of zinc acetate, boric acid, or peroxide of hydrogen. He reported several cases of apparent cure in from two to fourteen days.

Dr. F. Tilden Brown has also reported a case which yielded to this method in five days. The plan promises well, but has not as yet had a sufficient trial to demonstrate its real value.

Thiery's Method.-In an article upon the treatment of urethritis, by Dr. Paul Thiéry, which appeared in Le Progrès Médical, March 3, 1887, attention is called to a method of employing iodoform in the urethra, by which he succeeded in aborting six cases in from five to twenty-five days. His method was by the simple injection of a mixture of iodoform powder in sweet almond-oil or glycerin. This application was made once daily, after urination, and the fluid retained in the canal for fifteen or twenty minutes. These injections, it is claimed, produce little or no immediate discomfort to the patient, and relieve to a marked degree the acutely painful symptoms of the active stage of the disease.

Several other agents have been employed with a view to aborting the disease, among which may be mentioned bichloride of mercury, permanganate of potassium, and peroxide of hydrogen; and although experience has not shown that any agent at present at our command has the power in all cases to abort the inflammatory process, it is not unreasonable to hope that such an agent may be discovered in the future. Certain it is that several remedies are known to have the power to markedly modify, diminish, and prevent the extension of the inflammation, limiting thereby to a considerable degree the duration of the disease and producing a marked amelioration in the disagreeable symptoms.

Space will not permit even a partial review of the many drugs which have been employed in the local treatment of this disease. Moreover, such an enumeration would be unprofitable, as in nearly every instance previous to 1887 , when reported cures were published by this or that agent, no attempt was made to distinguish between a genuine fresh infection of an acute urethritis and the other less troublesome forms of the disease; and brilliant results have frequently been reported with drugs and chemical agents which are known to all familiar with the subject to be practically inert in the acute and severer forms of the disease. 
Heat and Cold.-These agents have been used with marked effect, the former for the hyperæmia and cedema so often present in the early stage, the latter in controlling the erections and chordee, which render the nights so much to be dreaded. Hot sitz-baths frequently repeated, soaking the penis in a cup of lot water, and the more or less constant application of hot fomentations, will be found of the greatest value. The sexual hyperæsthesia is best controlled by the cold-water coil of Otis or by the employment of an ice-bag to the perinæum.

The application of heat directly to the urethral mucous membrane by means of the retrojection of hot water was first brought to the notice of the profession by its inventor, Dr. H. Holbrook Curtis. His apparatus consists of a tin pail, beneath which is fastened a platform for an alcohol-lamp or Bunsen burner (Fig. 1). The pail is connected by means of a long rubber tube with a No. 18 F. soft-rubber catheter. The whole is suspended from the ceiling by means of a cord or pulley, and can be raised or lowered at will. The patient, after having first passed his water, is seated on the edge of a chair, over a large-sized slop-jar or pail. The catheter, well oiled, is passed carefully into the urethra about five inches, and the current of water started. This is at first about the temperature of the body, but is gradually raised until it is as hot as the

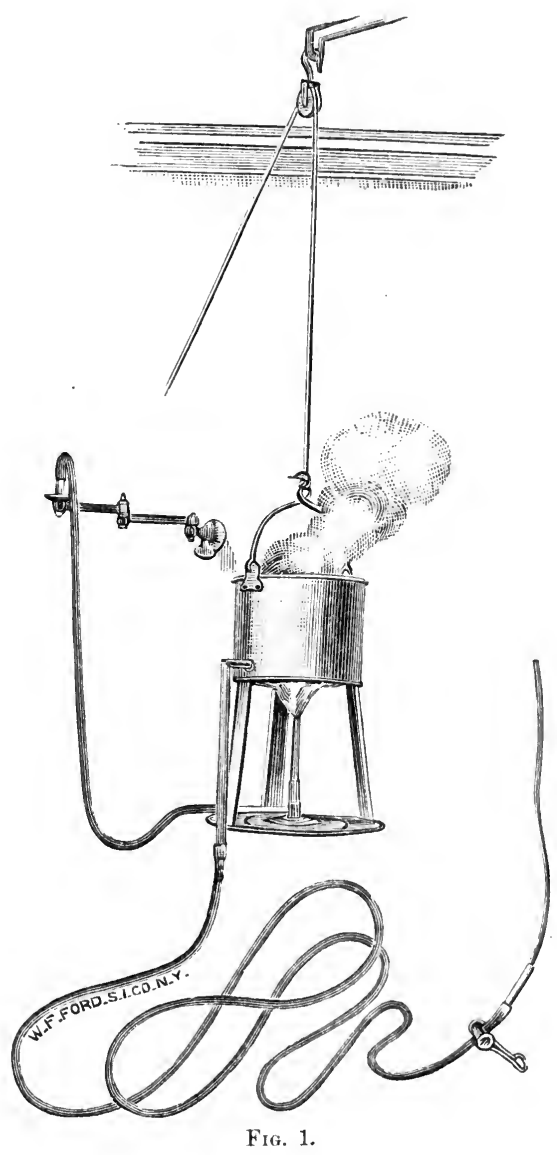
patient can bear. About two quarts are passed through the urethra at each sitting. This should be repeated two or three times each day. This insures thorough irrigation of the canal and the prolonged action of heat upon the mucous membrane. As the result of this method of treatment, the patient experiences at once marked relief from the pain and other inflammatory symptoms. Unless, however, the fluid employed con- 
tains some astringent or antiseptic agent, the effect on the discharge or the ultimate duration of the disease is not marked.

In hot retrojection, however, we have perhaps the best method of applying medicated solutions to the urethral mucous membrane. The advantages are that it thoroughly cleanses the canal, and brings the fluid in contact with every portion of the mucous membrane (a result rarely accomplished by any other procedure). It produces but slight irritation, and is followed by a feeling of comfort to the patient.

The other methods of local application are by the Halsted plan of continuous irrigation, by simple injection, and by the use of ointments and soluble bougies. The method of continuous irrigation was first suggested by Dr. W. S. Halsted, and is as follows: An elevated reservoir containing the fluid is connected by

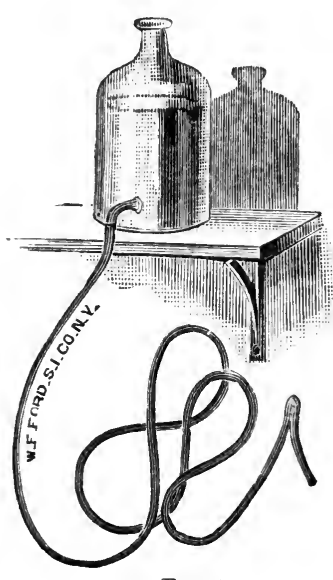

Fig. 2. means of a rubber tube with a glass or gutta-percha nozzle (Figs. 2 and 3). The patient is instructed to pass his urine, and then, standing in front of a sink or basin, the nozzle of the irrigator is firmly pressed against the urethral orifice. The current is so di-

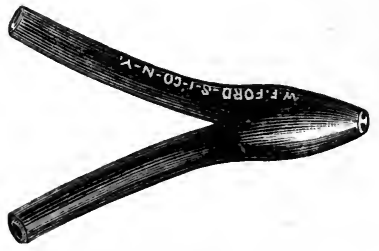

FIG. 3.

rected that the stream of fluid enters in the line of the canal, and not obliquely against the urethral walls. When this is accomplished, all that portion of the urethra anterior to the bulbo-membranous junction can be felt to be completely distended or "ballooned out." Sufficient outflow is now permitted to keep the fluid in motion while the urethra remains distended. From one to two quarts of fluid should be allowed to pass through the urethra at each irrigation. The ordinary method of applying astringents or antiseptic agents to the urethra is by means of solutions injected into the canal. To properly inject the anterior urethra, two things are necessary - a suitable syringe, and a certain amount of dexterity on the part of the patient. Regarding the former, an ordinary glass or hard-rubber syringe having a conical nozzle, an even barrel, and well-fitting piston, should be chosen. The capacity should be from two to three drachms only. The most satisfactory instrument of this 
kind is one having a hard-rubber barrel and soft-rubber tip. The patient should always urinate just before injecting, to clear the canal of pus. The nozzle of the syringe should then be introduced within the meatus as far as it will go, and the lips firmly pressed with the thumb and forefinger, laterally against its nozzle. The direction should be in the axis of the canal, and the fluid slowly introduced by pressure on the piston. If this is properly done, not one drop of fluid will escape until the canal is completely distended. The syringe should then be removed and the lips of the meatus compressed. The fluid should be retained for at least one minute and then allowed to escape.

The plan of introducing medicated ointments into the urethra was employed during some experiments by Dr. Halsted several years ago, but without demonstrating any advantage.

Soluble bougies have been extensively used by some surgeons in the treatment of this disease. In the writer's experience, however, the irritation produced by their presence so frequently resulted in posterior urethritis and other complications that they were discarded.

In the choice of methods of making local applications to the uretlra, the physician must necessarily be governed by circumstances. If possible, one of the irrigating methods should be employed. Equally good results are often effected, however, by the judicious use of the syringe in patients capable of appreciating and carrying out the instructions regarding its proper use. A large number of individuals, especially in dispensary practice, seem incapable of acquiring sufficient skill in the use of this instrument to insure contact of the fluid with the entire anterior urethra. No patient should be given a syringe to use until he has demonstrated, in the presence of his medical adviser, his ability to distend the canal properly.

Alkalies.-The local use of solutions of bicarbonate of sodium and other alkalies has been advocated by a number of distinguished surgeons. Theoretically, the local use of alkalies would favor rather than retard the inflammatory process, and a number of experiments by the writer with these agents show that they at least exert no favorable influence on the disease.

Astringents.-Pegarding the use of simple astringents, it may be said that whereas experience has shown them to be of practically no valne in the acute stage of a specific urethritis, their employment in the declining stage is attended often by the inost happy results. In this condition the mineral astringents will be found to be of greater value than the vegetable agents. Of these, the acetate of zinc is probably the best. A solution of this should be injected from four to six times daily, in strength varying from two to twelve grains to the ounce. A milder solution should be used at first, the strength being increased as the discharge becomes more watery. 
Several other salts enjoy the reputation of being equally efficient, such as the sulphate and the sulpho-carbolate of zine, the chloride of zinc, alum, acetate of lead, and the permanganate of potassium. The addition of an aqueous preparation of opium or tannin to the astringent solution seems often to exert a salutary influence. The following prescriptions have been quite extensively used:

B Zinci sulphatis,

Plumbi acetatis............... āā

Aquæ rosæ.....................

gr. $\operatorname{xxx}$.

z vj. M. (Ricord.)

B Zinci sulphatis................

gr. $x v$.

Plumbi acetatis............... gr. xxx.

Ext. krameriæ fl.,

Tinct. opii................ āā 3 iij.

Aquæ.................... (Taylor.)

B Ext. hydrastis fl............... $\xi \mathrm{j}$.

Aquæ..................... ad $\xi$ vj. M.

A consideration of the use of these drugs in detail belongs rather to the chapter on chronic urethritis than in this connection.

Bichloride of Mercury. - The systematic use of bichloride of mercury in the treatment of urethritis was not undertaken until the original experiments of Halsted at the Roosevelt Hospital in 1884. He found that the local employment of a large amount of a very dilute solution of this agent proved far more useful than that of a small amount of a more concentrated solution. His method consisted in thoroughly irrigating the urethral canal by means of his irrigator, described above, with a solution of bichloride varying in strength from 1 to 100,000 to 1 to 10,000 . This was done twice daily, the patient receiving no other treatment. The results of these experiments of Halsted were reported by Dr. S. O. Vanderpoel in the Medical Record for March 27, 1886. He cites the histories of six patients, all with undoubted attacks of acute specific urethritis, cured in from ten to fourteen days. The success which attended the use of this agent seemed so far in advance of that following any procedure previously employed, that experiments were at once undertaken by a number of observers with a view of determining the accuracy of Halsted's observations. During a series of experiments by the writer, a thorongh trial was made of bichloride of mercury, by the method of irrigation suggested by Halsted, by the Holbrook-Curtis method of hot retrojection, and by the use of the simple piston syringe. Over three hundred cases of acute specific urethritis were treated, and the results carefully recorded. In a paper read before the Genito-Urinary Section of the New York Academy of Medicine, in June, 1891, these results are analyzed, and statisties given regarding the length of 
time necessary to effect a cessation of the discharge, and also regarding the frequency of complications. At the close of that paper it was stated that it was the writer's firm belief that "the judicious use of bichloride of mercury in cases of acute gonorrhoal urethritis is attended with better results in subduing the painful and disagreeable features of the disease than that of any other agent; that recovery is more rapid and permanent, and that the frequency of inflammatory complications is greatly reduced."

In the preceding pages an effort has been made to describe briefly the methods of treatment which the most recent experience of those interested in the subject has shown to be of the greatest value, and which, therefore, are at the present time most generally employed. In estimating the comparative value of the different methods, the writer has been guided by personally conducted experiments rather than by the opinions of others.

As a result of these observations, the following plan has been found to be most useful in the majority of cases:

As soon as a patient with an uncomplicated attack of acute specific urethritis presents himself for treatment, he is given a cathartic, and instructed to religiously abstain from alcoholic drinks, highly seasoned food, sexnal excitement, and excessive bodily exercise. If the disease is in its initial stage, and the inflammatory symptoms still unimportant, a hot retrojection of bichloride of mercury $(1$ to 30,000$)$ is employed twice daily. If this plan is impracticable, he is instrueted in the proper use of the syringe, and advised to use the same solution twice daily, taking ten injections in the morning and ten at night, to imitate as nearly as possible the method of continuous irrigation. If the inflammatory symptoms are severe, and the bichloride causes marked pain, the local treatment is suspended for two or three days, and oil of sandal-wood given in full doses. When the inflammatory symptoms have sufficiently subsided, the bichloride is again resumed. Under this treatment the painful symptoms rapidly disappear, the secretion undergoes a change from an abundant, thick, purulent discharge to a thin, watery fluid. This is daily examined for gonococci, and when these are absent, or when the discharge has been watery for three or four days, the bichloride is suspended, and a soothing injection of sulpho-carbolate of zinc, or preferably bismuth suspended in glycerin and water, is employed. This should be given at first after each urination, and the frequency then gradually reduced until it is taken only at bedtime. In uncomplicated cases, where these directions are successfully carried ont, the discharge generally ceases in from six to twelve days. The bismuth, however, should be continued once or twice daily for two or three weeks, as its suspension is likely to be followed by a return of the discharge. Any indiscretion, or departure from the hygienic regulations during this treatment, is also likely to again light up 
the inflammation. After the discharge has ceased, it may be necessary to employ sounds, local application of nitrate of silver by means of the endoscope, and general tonic medication, to complete the cure; for, although the patient may have no visible discharge, and suffers no inconvenience, the disease is still far from well. The two-glass urine test (to be described later, in the chapter on posterior urethritis) should frequently be employed, and a microscopic examination made of the tripper faden. As long as these contain gonococci, or sink to the bottom of the glass, as they invariably do when made up largely of pus, the patient should remain under observation. He can be pronounced well, and not likely to experience a return of the symptoms, only when-

1. There is no visible discharge.

2. When the tripper faden are ahsent, or rise to the surface of the fluid, and contain no gonococci ; and-

3. When neither stricture nor prostatitis exists.

\section{COMPLICATIONS OF URETHRITIS.}

Posterior Urethritis.-Of all the complications which arise in a case of acute gonorrhœa, posterior urethritis is by far the most frequent, occurring, according to different authorities, in from sixteen to eighty per cent of all cases. By posterior urethritis is understood an extension of the inflammatory process to that portion of the urethral mucous membrane between the bulbo-membranous junction and the bladder. Under ordinary circumstances, in a simple case of acute urethritis, the disease confines itself to the anterior urethra. The reason of this is, not, as has been generally supposed, that the compressor urethræ muscle offers a mechanical barrier to its progress, but rather that the membranous urethra, by being much less vascular than the other portions of the canal, and almost devoid of glands and follicles, offers a much less favorable soil for the development of pathogenic organisms, and in that way acts as an effective bar to its further progress. This extension, however, may be favored by many factors, such as a diminished power of resistance on the part of the tissues as a result of tuberculosis, syphilis, or other debilitating diseases, or a favoring congestion of the parts due to inordinate physical exertion, the abuse of alcohol, and sexual excitement. It may also be produced by direct infection as the result of the passage of urethral instruments. Unless caused in this manner, it rarely appears before the end of the third week, but may occur at any time after this period. This extension of the inflammation to the posterior urethra is important, not only on account of the painful symptoms to which it may give rise, but also on account of the fact that, by greatly increasing the extent of the disease, it renders complete recovery much more uncertain and remote; and by its proximity to other important organs greatly enhances the 
probability of a further extension of the inflammation to the bladder, seminal vesicles, and testicles.

Symptoms.-This complication may occur with no appreciable subjective symptoms. Generally, however, there is a sense of heat, heaviness, and discomfort in the perineal region, with an increased frequency of urination. If the inflammation is more acute, the calls to urinate may occur every few minutes, and are accompanied by great urgency and painful bladder tenesmus, with radiating pains and free hæmorrhage at the close of the act. In a particularly severe case under the writer's observation, the urine was passed by involuntary spasmodic effort every two or three minutes, accompanied by the most excruciating pain and marked hæmorrhage. A membranous cast of the prostatic urethra and several smaller croupous shreds were passed during the height of the inflammation. Abnormities on the part of the sexual function are of frequent occurrence in this condition, especially when the inflammation involves the caput gallinaginis. There is heightened reflex. sexual irritability, as evidenced by frequent and prolonged erections, especially at night, accompanied often by involuntary seminal emissions.

Physical Signs.-Rectal examination reveals, in some instances, marked tenderness over the membranous and prostatic urethra, without enlargement of the gland. The only strictly reliable information regarding the presence or absence of a posterior urethritis, however, is to be gained by the careful employment of the two-glass urine test. This test is based upon the fact that the pus resulting from an inflammation of the mucous membrane of the anterior urethra makes its escape by flowing outward through the meatus, progress in the opposite direction being prevented by the action of the compressor urethræ muscle. On the other hand, pus generated in that portion of the urethra behind the cut-off muscle flows backward into the bladder. If, therefore, the patient passes his urine in two glasses, that containing the first portion passed represents a sample of the urine collected in the bladder, plus the washings from the urethra; the second portion, on the other hand, represents only that contained in the bladder unmixed with urethral pus. In the absence of suppurative disease of the bladder or kidneys, pus in the second glass always means a posterior urethritis.

In this connection several facts may be mentioned which will be of value in determining the amount of inflammatory disturbance present. In mild inflammations of the posterior urethra, when but little pus is secreted, if the urine be passed frequently, that in the second glass may remain clear; if, however, an examination be made after a long retention, or in the morning immediately after a night's sleep, the second glass will always show the presence of pus. This occasional presence of a clear specimen (after short retention) is of value in excluding cystitis. 
Treatment.-In mild cases, where the posterior urethritis is evidenced only by an examination of the urine, no subjective symptoms being present, the only indication for treatment is to increase the outward flow of urine, and render it sterile by the internal use of boric acid or salol in doses of five to ten grains of each four or five times a day. The best diuretic for such cases is the daily consumption of from two to four quarts of plain water. If slight frequency and tenesmus exist, the patient should abstain

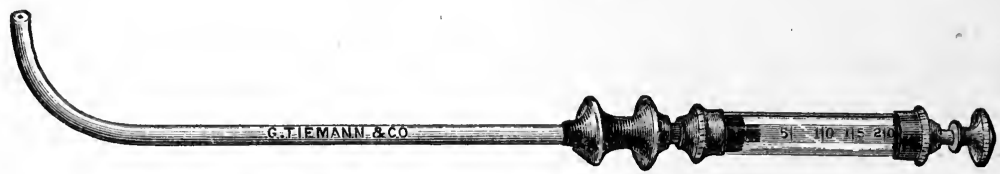

FIG. 4.

from active exercise and remain quietly at home. He should take, in addition to the diuretic agent, small doses of opium or hyoscyamus to control the urgent symptoms. In the severer cases absolute rest in bed must be enforced. Diuretics, and opium in the form of suppositories, should be given until the pain and frequency of the urination have been controlled. In addition to these palliative measures, an application of nitrate of silver should be made direct to the inflamed area every second or third day. For this a solution of from five to ten grains to the ounce of water should be employed. This should be deposited on the inflamed area by an Ultzmann deep wrethral syringe (Fig. 4), or, better still, Keyes's modification of Ultzmann's instrument (Fig. 5). The method of procedure is as follows:

The patient should lie upon his back in bed or on a table. The anterior urethra should be washed out, to remove irritating secretions. The

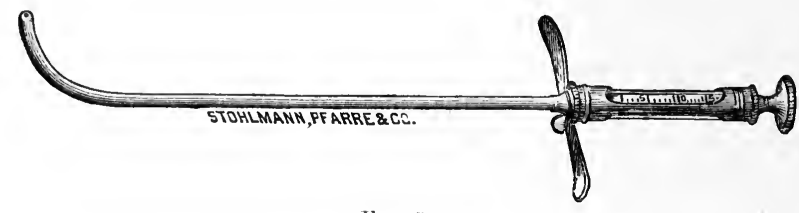

Fig. i.

syringe should be carefully introduced, until the point of the beak lies just behind the compressor urethræ muscle and within the prostatic urethra; that, in ordinary cases, is when the shaft of the barrel is at an angle of $45^{\circ}$ with the horizon. To facilitate its introduction, the barrel should be anointed with a small amount of glycerin, as oily substances protect the mucous membrane from the action of the fluid. From five to ten minims of the fluid should be slowly injected. Before removing 
the syringe, especially where concentrated solutions are employed, it is well to withdraw slightly the piston, which prevents the dripping of the solution while the syringe is being removed. In this way the fluid is prevented from leaking into the anterior urethra. The object of making this application before the patient empties his bladder is, that the action of the nitrate of silver can be quickly neutralized by the passage of urine if it prove too irritating. The results which follow the judicious use of this agent are often strikingly prompt and satisfactory. There is usually at first an increased amount of pain and tenesmus. This should be controlled by local applications of heat and the use of opium, or morphine suppositories. In from six to twenty-four hours after the application there is generally experienced a marked relief from the distressing symptoms. As a general rule, the second injection should not be made until the irritation produced by the first has fully subsided. The strength of the solution should be increased at each application by from two to five grains to the ounce. The relief is usually more prompt in the severer cases. It is a recognized fact that cases of posterior urethritis yield to treatment much more readily if attacked at once, whereas, if allowed to continue until subacute or chronic, they become often the most obstinate and discouraging of patients. The reason for this lies in the fact that the prostatic urethra is so richly supplied with glands and follicles, each of which, with its surrounding network of capillaries, forms a miniature incubator for the development of gonococci. When the disease is allowed sufficient time to penetrate these, it is to a great extent beyond the reach of local treatment, and proves often most rebellious.

In dealing with a case of posterior urethritis, it should be borne in mind that a further extension of the inflammation usually results from the slightest exciting cause. Special emphasis should therefore be placed upon all directions regarding the maintenance of absolute rest, and the avoidance of stimulants and sexual excitement.

Epididymitis. - This complication comes next in point of frequency. Statistics vary largely regarding its occurrence, depending presumably upon the class of cases observed and the amount of eare employed in their treatment. Gouley states that it occurs in about thirty per cent of all cases of acute urethritis. The majority of modern authorities, however, place it at about sixteen per cent. In three hundred and two acute cases treated by the writer with bichloride of mercury, epididymitis occurred in but six instances, or about two per cent of the patients. In three hundred and fifty-two cases treated by other methods, this complication appeared in sixteen per cent. As in the case of cystitis and prostatitis the presenee of a posterior urethritis enhances greatly the probability of its oceurrence. In fact, it may be serionsly questioned if this complication 
ever occurs without a previous extension of the inflammatory process to the posterior urethra.

The infection may take place directly by an extension of the inflammation along the mucous membrane of the ejaculatory ducts, seminal vesicles, and vasa deferentia, or indirectly through the lymphatics. In the former instance we have involvement of the spermatic cord, preceding by several hours or days the inflammation of the epididymis. In the latter the symptoms are first noticed in the epididymis, and indeed may remain wholly limited to that locality.

This complication occurs with most frequency in the third and fourth week of the disease, rarely before the fourteenth day, but after that period it may occur at any time while the urethritis is present. The predisposing cause of the epididymitis is a posterior urethritis. The prophylactic treatment consists in the employment of measures calcnlated to shorten the duration of the original inflammation and prevent its extension to the posterior urethra, strict adherence to hygienic regulations mentioned in the chapter on treatment, and the wearing of a well-made suspensory bandage.

Symptoms.-The inflammation may be ushered in by a chill, followed by a high fever. This is usually accompanied by a more or less severe pain in the region of the testicle. Exceptionally, the pain is at first noticed in the region of the inguinal canal and upper part of the spermatic cord. In these cases the cord can be felt to be considerably enlarged, hot, and tender, before any change can be felt in the tissues of the epididymis. The swelling of the affected parts takes place rapidly, and frequently within a few hours from the first symptom, the affected half of the scrotum will be enlarged to twice or three times its normal size, very hot, and exquisitely tender. Examination reveals the presence of a large, indurated mass nearly surrounding the testicle, composed of the infiltrated tissues of the epididymis. In the severer cases this may be somewhat obscured by the presence of a certain amount of fluid in the cavity of the tunica vaginalis. Under ordinary circumstances, if untreated, this condition may remain stationary for several days ; the pain then gradually subsides, and the redness and sensitiveness disappear. The induration is but slowly absorbed, from six to ten weeks often being required to effect its disappearance. Not infrequently a small nodule may remain at the head or tail of the epididymis for many years, which may cause a complete obstruction to the outward flow of semen. Instances have been known where the inflammation was so severe as to result in local necrosis, gangrene, and suppuration. In general, however, the prognosis regarding life and ultimate resolution is good.

Treatment. - The indications to be met in a case of acute epididymitis are, first, to relieve the hyperæmia and resulting pain; and, second, to 
promote absorption of the inflammatory products. The former is best accomplished by absolute. rest in bed, supporting the inflamed organ by some mechanical appliance, and the application of heat by means of fomentations and poultices. The flaxseed-meal poultice is the one generally employed, and when properly made, and applied with sufficient frequency, it affords prompt relief. The tobacco poultice has long enjoyed the reputation of being particularly serviceable in this condition, and is prepared by mixing a handful of fine-cut tobacco in half a pint of boiling water, and then adding a sufficient quantity of ground flaxseed to give it the required consistence. The advantage of this over the flaxseed poultice is due to the alleged narcotizing effect of the nicotine on the peripheral nerves. In a large number of eases, however, this method of treatment is, for obvious reasons, impracticable, and strenuous efforts have been put forth by surgeons for many years to discover, if possible, some means of relieving the pain and extreme tenderness without necessitating the patient's remaining in bed. The best method which has been suggested, up to the present time, is that by means of the actual cautery, first employed by Dr. W. S. Halsted. His method consists in lightly touching the surface of the skin overlying the affected organ with a white-hot cautery point. The operation requires only a few seconds, and, if skillfully performed, is but moderately painful. A dressing of iodoform-ointment is then applied, and held in place by means of a suspensory bandage. Instant relief from pain frequently follows the application of this treatment, and the patient is, as a rule, able to be up and walk about in comparative comfort. In a report entitled Genito-Urinary Notes, published by the writer in July, 1888, in the Journal of Cutaneous and Genito-Urinary Diseases, forty six cases of acute epididymitis are reported which were treated by this method. In two instances only were the patients obliged to remain in bed after the first application, and in one of these the real cause of the enforced rest was a coexisting cystitis. Prompt relief from pain may also be had by removing the fluid collected in the sac of the tunica vaginalis by means of an aspirating needle. Other forms of counter-irritation, such as the application of a strong solution of nitrate of silver (forty to sixty grains to the ounce) and the ethereal tincture of iodine, have been employed, but these are inferior to the cautery, and often give rise to extensive sloughing of the epidermis. To promote absorption of the inflammatory induration, measures should be undertaken which insure the application of heat, moisture, mild counterirritation, compression, and suspension. These indications are perhaps best fulfilled by the use of the dry poultice, first suggested by Langlebert, a report of which was furnished by the writer to the American Association of Genito-Urinary Surgeons in September, 1891, from which the following description is quoted: "The dressing eonsists in a moderately 
thick layer of cotton-wool well applied over the inflamed testicle. This is covered by a layer of rubber protective tissue so fashioned that it completely incloses the diseased organ, with its edges extended on to the healthy skin of the scrotum in a manner to partly overlap but not entirely inclose the healthy side. This is secured by a snugly applied gauze bandage, and the whole held in place by a suspensory." This dressing, by retaining heat and preventing the evaporation of the moisture abundantly supplied by the sweat-glands, possesses all the advantages of an ordinary flaxseed poultice, and in addition exerts moderate compression, insures suspension, and allows the patient to be up and about.

This procedure has been extensively employed by the writer in hospital and private practice, and with the most gratifying results.

Strapping the testicle is a useful procedure, and, when properly applied, often promotes rapid absorption of the products of inflammation. To be of any value, it must exert compression and completely encircle the inflamed organ. To accomplish this the scrotum should be shaved, the healthy testicle pushed upward out of the way, and a circular band of pure rubber plaster placed above the organ in such a manner, and with sufficient tension, to prevent the testicle being drawn upward through the plaster ring. Alternate longitudinal and circular straps should then be applied until the whole is inclosed. This should be removed and reapplied as often as it becomes loosened.

Inunctions of mercurial ointment seem in some instances to hasten absorption. The advantages, however, are not sufficient to overcome the danger of constitutional disturbance which may follow its employment. For the pathological anatomy and further consideration of this subject the reader is referred to the chapter on the Diseases of the Testicle.

Peri-urethral Inflammation (Folliculitis, Cowperitis, Prostatitis).-An extension of the inflammation from the surface of the urethral mucous membrane to the glands and follicles emptying into the canal, and to the peri-urethral connective tissue, may constitute a complication of acute urethritis which is more or less serious, according to the location of the gland and the severity of the inflammation. This may take place anywhere along the course of the canal, but the usual sites of the inflammatory departure are at the fossa navicularis, at the bulb, or in the prostatic portion, for the reason that the glands are more abundantly located in these regions. If an inflammation, mild in character, extends along the membranous lining of a mucous follicle, or the duct of a racemose gland, the only result is often a localized point of induration and tenderness, and an exudation of pus from the gland into the urethra, which may continue long after the original urethritis has subsided. If the inflammation is more severe in character, the orifice of the duct or follicle may be occluded by swelling, and, as a result of the retention of the secretion, a 
cyst may be formed which often attains the size many times that of the original gland, and which may finally rupture into the canal or externally, or gradually become absorbed and disappear. In more severe cases the inflammation may extend beyond the limits of the gland, and a peri-glandular cellulitis result, which in turn may extend rapidly along the tissues of the spongy or cavernous bodies of the penis, giving rise to extensive abscess, sloughing, and subsequent cicatricial contraction. An infiltration of urine not infrequently occurs when a peri-urethral abscess opens into the canal, especially when this opening exists behind a stricture. This may result in extensive destruction of tissue or a permanent urinary fistula.

Simple folliculitis frequently occurs in the minnte glands situated in the neighborhood of the frænum. These are superficially located, and empty into the fossa navicularis. When inflamed, they appear as rounded, hard nodules on one or both sides of the frænum, corresponding in size to a small pea, tender to the touch, and covered by reddened mucous membrane. Softening takes place in three or four days usually, and a spontaneous external opening is established. Exceptionally, the opening may occur within the urethra.

Treatment.-The treatment of this condition consists in injecting the minute sac with a drop of pure carbolic acid or a concentrated solution of nitrate of silver. This is best accomplished by means of an ordinary hypodermic syringe and probe-pointed needle. One such application is usually sufficient to bring about a cure. If untreated, this small sac may continue to suppurate indefinitely, offering a constant source of urethral reinfection.

When the inflamed follicles are situated posteriorly to the fossa navicularis they develop somewhat more slowly, and more frequently undergo resolution. The patient complains of localized pain in the neighborhood of the urethral canal. The pain is of a dull, throbbing character at first, which becomes burning and often lancinating after the passage of urine or during an erection. Examination reveals the presence of a hard, rounded, tender nodule on the superior or inferior surface of the urethra. This increases in size, slowly at first, and may retain its rounded contour. In some instances it may attain the size of a large pea or cherry. Suppuration frequently occurs, and spontaneous rupture of the sac externally, no connection with the urethra, as a rule, existing. Occasionally the inflammatory process undergoes resolution, and the cyst remains for months and years as a rounded, elastic, painless nodule, which gradually disappears without symptoms. In the graver cases the inflammation extends rapidly beyond the limits of the gland or follicle, and results in a diffuse cellulitis, indicated by severe throbbing pain, and a tender, irregular induration, with œdema, chills, fever, and general malaise. In the milder cases, where the inflammation is confined to the gland, the treatment should consist in 
rest, hot applications, and the inunction of an ointment of the oleate of mercury or white-precipitate. If suppuration occurs, the pus should be evacuated, as soon as detected, by a free incision. The cavity should be thoronghly cleansed with peroxide of hydrogen, and packed with aseptic gauze.

Cowperitis.-This is a name used to designate an infrequent complication of urethritis, namely, the involvement of one or both glands of Méry, located in the perineal region on either side of the raphé emptying into the bulbous urethra. The process of infection is exactly the same as in the folliculitis described above, the difference being only in the size of the gland. This complication occurs usually after the second week of the disease, and is characterized by pain in the perineal region, usually at first appreciated by the patient while sitting. Upon examination, a round, painful nodule about the size of a hazel-nut is felt midway between the scrotal junction and anal orifice on either side of the raphé. This rapidly increases in size, and may extend beyond the median line, and lose its rounded outline. In severe cases retention of urine may be occasioned by its pressure on the urethral canal. Suppuration is usually announced by the occurrence of chills, fever, and softening of the indurated mass. If untreated, rupture usually takes place externally, occasionally internally, very rarely in both directions, resulting in perineal fistula.

The treatment should consist in rest, hot applications, early incision, careful drainage, and thorough asepsis.

Prostatitis. - An inflammation of the prostate gland occurs in the same manner as the peri-urethral inflammations already described. It is, however, far more important than the others, for the reason that, on account of its intimate connection with that portion of the nervous and muscular system presiding over the sexual function, and the expulsion of the urine, its inflammatory involvement is accompanied by grave disturbances of these functions, and also for the reason that its location beyond the reach of direct remedial measures is such also as to render extensive suppuration and burrowing of pus most dangerous. Prostatitis rarely occurs before the end of the third week of the disease, unless the pus is carried directly to the gland by the passage of instruments or great carelessness in the use of injections. Two varieties of inflammation may occur in this gland as the result of gonorrhoal infection-the follicular and the diffuse. In the former, one or more follicles are involved; this gives rise to moderate pain and uneasiness in the perineal region, accompanied by frequent micturition, tenesmus, and sexual hyperæsthesia. These symptoms differ from those produced by a posterior urethritis only in the increased amount of pain in the perineal region, and the marked muscular spasm following urination. It is, however, impossible to arrive at a diagnosis of follicular prostatitis, except as a result of 
rectal examination. This also may be negative; generally, however, some small points of induration may be detected in one or the other lobe of the gland. There is also well-marked tenderness on pressure.

In diffuse prostatitis the symptoms and physical signs are far more characteristic. In addition to the evidence of a posterior urethritis, perineal pain, frequent urination, and tenesmus, the patient experiences a sense of fullness and pressure in the rectum, with painful defecation, and, in severe cases, the passage of small, ribbon-shaped stools. Retention of urine may occur from pressure on the urethra. A rectal examination reveals the presence of an enlarged prostate, which may attain the size of a large orange and completely occlude the rectum. This is hard, hot, pulsating, and tender. Occasionally the prostate is found to be greatly enlarged, without subjective symptoms ; generally, however, there are marked perineal weight and pain. The latter may become severe, and of a throbbing, boring character, extending along the urethra and into the thighs and testicles. Fever is usually present, and its degree furnishes a valuable criterion upon which to base an opinion regarding the prognosis. If the fever does not rise above $101^{\circ}$ Fahr., and is unaccompanied by chills, sweating, and prostration, resolution withont suppuration will probably occur. Indeed, resolution is the rule in diffuse prostatitis when the patient can have the advantages of skillful treatment. In these instances the symptoms disappear rapidly after the acme is reached, and the glandular enlargement progressively decreases until the examining finger fails to detect anything abnormal. When suppuration occurs, the fact is usually announced by a marked chill, high fever, and a considerable diminution in the perineal pain and tension. Fluctuation can occasionally be felt throngh the rectum. If untreated, rupture of the abscess takes place, most frequently into the urethra, occasionally into the rectum, very rarely through the tissues of the perinæum. In severer cases, where the inflammation extends beyond the capsule of the prostate and involves the periprostatic connective tissue, extensive suppuration and burrowing of pus may take place between the layers of the pelvic fascia within the pelvis, resulting in septic absorption and death. Not infrequently the abscess opens in two directions, as into the urethra and rectum, or urethra and perinæum, giving rise to fistnlæ, which, if unrevealed, renders the life of the patient wretched beyond description.

Treatment. - When the diagnosis of acute prostatitis is made out, absolute rest in bed should be insisted upon. A calomel purge at the beginning often seems to exert a favorable influence over the course of the disease, although it may temporarily increase the rectal tenesmus. Leeches to the perinæum, followed by hot sitz-baths and hot rectal enemata, repeated three or four times a day, constitute the best treatment. Sufficient opium, in the form of suppositories, should be given to afford relief to the vesi- 
cal irritation and tenesmus. The application of cold direct to the prostate through the rectum has found favor with some German surgeons. This is best accomplished by means of the cold rectal sound (Fig. 6), by which a stream of iced water is kept continually flowing through the hollow metal bulb introduced into the rectum.

If suppuration is inevitable, the pus is best evacuated by means of a perineal incision and subsequent drainage. In cases where spontaneous rupture occurs into the urethra the course is generally favorable, the abscess cavity rapidly filling with granulations. The healthy urine seems

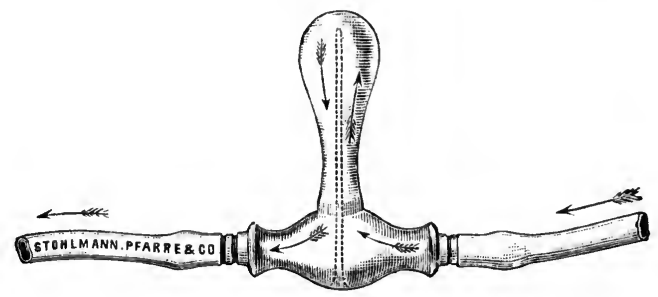

Fig. 6.

to exert in such cases no unfavorable influence. When the opening occurs into the rectum, however, the prognosis is not as favorable, as the introduction of intestinal gas and fecal matter into the cavity of the abscess tends to prevent healthy granulation, and may give rise to an indefinite continuation of the suppuration.

Impotence may result from acute prostatitis, from destruction of the seminal vesicles and their ducts by suppuration, or as a result of cicatricial contraction. Although chronic prostatitis frequently follows the acute follicular form of the disease, it can hardly be considered as a complication of acute urethritis. For its consideration, therefore, the reader is referred to the section on Diseases of the Prostate.

Vesiculitis. - An inflaimmation of the seminal vesicles and their excretory ducts. This occasionally occurs as a complication of acute urethritis. It is evidenced by a sense of weight, burning pain, and throbbing in the region of the vesical neck and rectum, extending to the scrotum, increased by defecation, and is accompanied by marked sexual hyperæsthesia, priapism, and frequent pollutions. The examining finger in the rectum detects one or both inflamed seminal vesicles as elongated, fusiform bodies on the posterior wall of the bladder just above the prostate. These are-hot, and tender to the touch. This condition is usually accompanied by the symptoms of posterior urethritis. As a result of the heightened sexual appetite, frequent involuntary seminal emissions take place, accompanied by a throbbing, burning pain, and excessive voluptuous sensation. The fluid ejaculated is yellow or brownish in color, and upon 
microscopic examination is found to be composed of pus, blood, and dead spermatozoa. Under favorable hygienie and therapeutic conditions the acute symptoms of this disease subside in from four to six days, and ultimate recovery may take place. Not infrequently, however, it becomes chronic, and may prove most rebellious to treatment. The symptoms of the chronic condition are milder in character, and are chiefly those of sexual neurasthenia, combined with those of a chronic posterior urethritis. It is to be differentiated from the latter, and from chronic prostatitis, by an examination of the fluid which may be pressed out by the finger in the rectum. This is best accomplished in the following manner: The patient is directed to pass a small amount of urine in a glass reservoir, after which the seminal vesicles are emptied by the method just described, and the remainder of the urine passed in two portions, and each preserved in a separate glass jar. The first specimen represents the washings from the urethra, the second the expressed contents of the seminal vesicles, and the third the urine as it is contained in the bladder. In chronic vesiculitis the second specimen should contain a larger amount of pus than the third, and, in addition, a number of dead spermatozoa. The proportionate amount of pus in the first specimen is determined by the amount of urethritis present, and is not of value in the diagnosis of vesiculitis. Temporary impotence as a result of resiculitis is common, and due to the admixture of pus with the seminal fluid, which inhibits if not destroys the vitality of the spermatic elements.

Treatment.-In acute eases, rest in bed, opium suppositories, hot enemata, with sitz-baths, and saline laxatives. The psychrophore has been employed by Finger with success. Preparations of bromide of sodium with digitalis and camphor may often be found useful in subduing the sexual hyperæsthesia. To be of value, however, they must be given in large doses. The treatment of chronie vesiculitis is largely hygienic, combined with measures addressed to the usually present chronic urethritis. Cod-liver oil, iron, sea-bathing, and a change of climate will often be found more useful than local measures.

Cystitis. - The frequency of cystitis as a eomplication of acute urethritis has been greatly exaggerated. This is due to the fact that until quite recently the occurrence of frequent micturition, vesical tenesmus, and pus in the urine was thought to be pathognomonic of inflammation of the bladder, whereas we now know that these symptoms may indieate, and in fact generally do indicate, only an inflammation of the posterior urethra. It is a well-known fact that a very considerable amount of cystitis may exist without the occurrence of either frequent or painful micturition, provided the inflammation is limited to portions of the mucous membrane other than the immediate vicinity of the internal urethral orifice. 
The subjective symptoms of this condition are somewhat misleading, often nothing more than a dull pain and sense of weight in the neighborhood of the symphysis pubis being present. There is tenderness in the hypogastric region, with moderate fever. Chills may occur in severe cases, with high fever and general malaise. In cystitis the urine soon becomes neutral or alkaline in reaction from the decomposition of urea. This, as Roosing has shown, is due to the presence of micro-organisms. The free alkali formed by this decomposition, by its action on the pus, forms a substance resembling mucin, which invariably collects at the bottom of the vesical reservoir, is expelled with the last portion of urine passed, and is pathognomonic of intravesical inflammation. In recent cases of cystitis, especially when the bladder is frequently emptied, this material may be absent.

Finger describes three conditions usually regarded as cystitis: First, posterior urethritis; second, posterior urethritis with moderate cystitis in the immediate vicinity of the urethral orifice; third, cystitis without posterior urethritis. These conditions can only be differentiated by a three-glass urine test. If the patient urinates in three glasses, the following facts will be observed regarding the gross appearance of the specimens :

\begin{tabular}{|c|c|c|c|}
\hline Location. & First specimen. & Second specimen. & Third specimen. \\
\hline Posterior urethritis. & $\begin{array}{l}\text { Acid urine; tripper fa- } \\
\text { den; free pus. }\end{array}$ & Free pus. & Free pus. \\
\hline $\begin{array}{l}\text { Posterior urethritis } \\
\text { with eystitis. } \\
\text { Cystitis. }\end{array}$ & $\begin{array}{l}\text { Urine acid or neutral ; } \\
\text { larger amount of free } \\
\text { pus; tripper faden. } \\
\text { Urine neutral or alka- } \\
\text { line; free pus. }\end{array}$ & Free pus. & $\begin{array}{l}\text { Free pus, with moderate } \\
\text { amount of mucoid } \\
\text { material. } \\
\text { Free pus, and large } \\
\text { amount of mucoid } \\
\text { material. }\end{array}$ \\
\hline
\end{tabular}

These tests are to a certain extent vitiated by the presence of hæmaturia or pus from the pelvis of the kidney. In the absence of these conditions, however, they may be relied upon as furnishing the most valuable data upon which to base a diagnosis.

For a review of the microscopic appearances of the urinary sediment, the reader is referred to the article on Urinary Analysis.

Pathology.-In cystitis due to gonorrhœal infection the inflammatory appearances are most marked in and about the urethral orifice. The appearance of the mucous membrane varies, from a slight thickening with moderate injection of the vessels to an intensely red, œdematous tissue covered with pus or fibrinous exudation. Hremorrhages, erosions, and occasionally deep ulcerations, may be present in the severer forms of the disease. The prognosis in cases of acute cystitis is usually good, when the patient can enjoy the advantages of rational treatment. If the dis- 
ease is neglected, the condition soon becomes chronic and very rebellions to treatment. Occasionally the inflammatory process extends beneath the mucous membrane, and invades the connective tissue and muscular layer of the viseus (a condition described as chronic parenchymatous cystitis), with greatly thickened walls and marked diminution in capacity. This is practically incurable, and will be described further in the article on Cystitis.

Treatment.-As in other inflammatory conditions of the genito-urinary tract, rest occupies the first position in the successful treatment of cystitis. Diuretics are of decided value in increasing the secretion of urine and preventing decomposition by frequent emptying of the bladder. The oil of sandal-wood in full doses seems often to exert a happy influence upon the severity of the symptoms, and in connection with other local measures may be of positive value. Sufficient opium, in the form of suppositories, should be given to relieve the pain and bladder tenesmus, if present. After the acuteness of the attack has subsided, the gentle washing out of the bladder by means of a fountain syringe and soft-rubber catheter with a dilute solution of salt in sterilized water, or, better, a two-per-cent solution of salicylic acid, serves to remove the pus and mucoid material. This should be followed by a mild solution of nitrate of silver from 1 to 5,000 to 1 to 1,000 . The internal use of boric acid, in doses of five to ten grains three or four times a day, with the view of sterilizing the urine and preventing the development of bacteria, has been extensively used of late, especially in France, and often with gratifying results.

Pyelitis. - Inflammation of the mucous membrane lining the pelvis and calices of the kidney. This occasionally occurs as a complication of gonorrhœal inflammation when the latter has infected the bladder. The occurrence of this complication is favored by conditions which impede the outward flow of urine, as urethral stricture, prostatic enlargement, and pressure upon the ureters, by unfavorable hygienic surroundings, a vitiated constitution, and the abuse of alcohol.

Pathology. - In the acute form of the disease the mucous membrane appears deeply injected and thickened, and the cavity of the renal pelvis contains a mixture of urine, blood, and pus. In the severer forms minute hæmorrhages may be present, and occasionally a fibrinous pseudo-membrane. The redness extends often into the ureter.

Symptoms.-The symptoms of this disease are pain in the lumbar region, extending downward to the bladder, scrotum, and thighs, increased by exereise; frequent micturition, reflex in character, simulating posterior urethritis; and a moderate amount of fever with chills and profuse perspiration, when the secretions are for any reason retained. In mild cases any or all of these symptoms may be wanting. The physical examination is usually negative, or shows at most only slight tenderness over the kidney 
region. The examination of the urine furnishes the only reliable evidence of the disease. The usual characteristics of a pyelitic urine are the increased amount, pale color, cloudy appearance, acid reaction; when allowed to settle, the pus forms a thick, compact, creamy mass at the bottom of the glass. Albumen is present in a greater amount than is sufficient to account for the pus. The microscope reveals, in addition to an abundance of pus, an occasional hyaline or granular cast; in severe cases, blood may also be present in the sediment. The question as to whether the presence of any characteristic epithelial cell may be said to indicate positively the renal origin of the pyuria in a given case, is far from being definitely settled.

Prognosis.-In uncomplicated cases of acute pyelitis of gonorrhœal origin recovery may be expected, although the presence of pus in the urine may continue for months or years. When marked obstruction to the outflow of urine exists, or a chronic inflammation of the lower urinary passages, the outlook is more grave, as the long continuance of pyelitis invariably leads to pathological changes in the parenchyma of the kidney and resulting nutritive disturbances.

Treatment.-This is mainly hygienic, and consists in removing as far as possible the cause which led to its occurrence, providing absolute rest in bed and the strict avoidance of stimulating food and drink. If the pain be severe, marked relief can often be afforded by the external application of heat in the form of fomentations and poultices, or by the employment of dry cups over the lumbar region. Diuretics are of positive value, and should be used freely. The best agent to be employed is pure water taken in large amounts. Salol, boric acid, and the balsams have been used by many, and may exert at times a happy influence over the course of the inflammation. Beyond this little can be done.

Balanitis. - An inflammation of the mucous membrane covering the glans penis. This occurs frequently during the course of an acute urethritis. As it is a generally recognized fact that the gonococcus develops only under exceptional circumstances upon pavement epithelium, the balanitis is probably the result of a primary irritation and erosion from the caustic action of the gonorrhœal pus, and later an infection by means of some other pathogenic organism. The conditions which favor the occurrence of this complication are uncleanliness and the presence of a long, tight prepuce.

The mucous covering of the glans will be found to be intensely red, and covered with an abundant secretion of foul-smelling pus. Superficial erosions may be seen, especially over the corona and in the sulcus immediately behind. The subjective symptoms are a sense of heat, soreness, and occasionally throbbing pain. With this is usually associated a certain amount of sexual hyperæsthesia. 
Treatment.-Prophylactic; circumcision and cleanliness. During an attack ; frequent bathing, constant exposure of the glans, applications of hot water, and lead and opium wash; later, iodol or some other unirritating dusting-powder.

Phimosis.-By this is understood an abnormal narrowing of the preputial orifice, preventing complete retraction of the foreskin. This condition may be congenital or acquired. As a complication of acute urethritis, we have to do only with the acquired variety, which is oceasioned by an inflammatory odema of the skin and connective tissue.

As a result of this inability to retract the prepuce, the secretions from the urethra collect between the glans and inner layer of the foreskin, and give rise to balanitis, with erosions and often ulcerations of the mucous membrane, adding greatly to the discomfort of the patient.

Treatment.-This consists in cleanliness, and the application of heat to reduce the œdema. The former is best accomplished by the injection into the preputial sac of a saturated solution of boric acid, or some other unirritating antiseptic fluid, by means of a long, pointed glass syringe; the latter, by soaking the penis in hot water five or six times during the day. After the preputial sac has been thoroughly cleansed of pus, an injection of black-wash should be made and allowed to remain.

Paraphimosis is the name applied to that condition produced by the forcible retraction of a phimoticor infiltrated prepuce. The constricting ring which forms the narrow orifice of the prepuce compresses the superficial veins which should convey the blood from the glans penis. As a result of this pressure, the glans rapidly swells and becomes œedematous and livid. In rare instances, where the swelling is extensive and the constriction unrelieved, gangrene may result. Generally, however, the constricting band sloughs, relieving in a measure the strangulation of the parts, and the swelling gradually subsides.

Treatment.-This should consist in hot applications, gentle compression to reduce the size of the glans, and final reduction after the manner described under Diseases of the Penis.

Lymphangitis and Adenitis. - In severe cases of urethritis the lymphatics and neighboring glands may become involved. The former are more frequently affected than the latter. In simple lymphangitis the swollen vessels can usually be felt as hard cords generally on the dorsum of the penis. In rare instances the lymphatic cord can be traced direct to the inguinal glands. If the inflammation is severe, the integument overlying the inflamed lymphatic canal is reddened, hot, and tender, and may be accompanied by œdema of the skin and connective tissue. One or more inguinal glands may become enlarged and tender. There is, however, but slight tendency to suppuration, and when this does occur it is in all probability due to a mixed infection. 
Treatment.-The only measures required for this condition are rest in bed, hot fomentations, and measures directed against the original urethritis.

Various skin eruptions, such as purpura hæmorrhagica, erythema multiforme, and urticaria, have been observed during the course of an aente urethral inflammation; also endoearditis, iritis, and other diseases: but as there is no reason to suppose that the association is other than accidental, they will not be included among the true complications of this disease.

Gonorrhœal rheumatism and gonorrhoal ophthalmia will be considered in separate sections. 


\section{CHRONIC GONORRHEA OR GLEET.}

BY WILLIAM K. OTIS, M. D.

Etiology.-The continuance of a urethral discharge for a period longer than two months from the inception of the disease is inevitably due to the presence of some pathological condition in addition to the original cause of the inflammation, thus preventing the natural tendency to spontaneous cure. These pathological changes consist in isolated granular patches on the urethral mucous membrane, which may occur at any point in the urethral tract, and which fail to heal because of plastic infiltration of the submueous tissue, eausing more or less coarctation of the canal at these points. These infiltrations, according to Mr. Reginald Harrison and others, are the result of an attempt on the part of Nature to prevent the leakage of urine through mucous membrane which has been denuded of its protecting epithelium by a superficial inflammation; but the more probable view, and the one most generally held, is that the inflammatory action extends deeply into the urethral and even the periurethral tissues, causing the deposit of plastie material. This finally results in a true cicatrix, similar in all respects to that following a burn of the skin or of other tracts lined with mucous membrane, not subject to contact with irritating fluids.

The role which the gonococeus plays in the production of these infiltrations is not well understood, but in all probability it penetrates the deeper tişsues and is the direct cause of the inflammatory deposit which subsequently becomes organized cicatricial tissue. Coarctations may have existed in many cases long before the discharge which they prolong, and were originally due to morbid conditions of the urine in early childhood -e.g., an excess of uric acid, which at the time may have passed unnoticed.

In a small minority of eases of chronic gonorrhera no stricture will be found to be present, the discharge being prolonged by either the involvement of the sinuses and glands of the urethra (which are much more numerous and of greater depth than is generally supposed), by the presence of neoplasms, or by the general condition of the patient; but, as a rule, these cases yield readily to treatment, which, at all events, is identical with that pursued in other eases after all stricture lias been removed. 
Diagnosis. - In the diagnosis of ehronic urethritis it is exceedingly important that the location of the diseased areas should be carefully determined. It is well to follow the classification of Ultzmann, who divides the urethra into two portions, the anterior and the posterior. The anterior urethra is that portion situated in front of the compressor urethræ muscle; the posterior, including the prostatic and membranous, is the portion situated between the compressor urethræ muscle and the internal sphincter of the bladder.

If the anterior urethra alone is invaded, and the patient be required to urinate half the urine into one glass and the remainder in a second glass, only the first half will appear cloudy, while the second half will be perfectly clear. If, on the contrary, the posterior urethra is the seat of the disease, the discharge, unable to overcome the pressure of the strong compressor urethræ muscle in front, flows backward into the bladder, and its entire contents will be clouded. In cases where the secretion is scanty, the anterior urethra may be washed out with a solution of salicylic acid, one sixteenth of one per cent, through a soft-rubber catheter passed down to the bulbo-membranous junction. Several ounces of the fluid should be injected by means of a hand syringe, allowing the solution to escape alongside of the catheter, thus completely cleansing the entire anterior urethra.

If now the patient urinates in two portions, and the first portion is cloudy, while the second is clear, the inflammation is situated in the posterior urethra. Should the seminal vesicles be suspected, the urine should be passed into three separate glasses; the first will contain the washings of the urethra, including the neck of the bladder; the finger should then be passed into the rectum and pressure brought upon the seminal vesicles on both sides; the second third of the urine being now passed into a clean glass, immediately followed by the passage of the remaining urine into a third glass. If the intermediate portion is more opaque than the other two, the seminal vesicles are undoubtedly affected.

The discharge itself may be exceedingly profuse, or it may be so scanty as to remain unnoticed by the patient, appearing only as discharge in the form of a single drop in the morning (goutte militaire), or it may only appear in the form of threads in the urine. These threads (Tripperfäden) consist of an amount of purulent secretion exuding from the granular patches, too small to appear as discharge, which, being washed out by the stream of urine, are rolled up in the form of threads and appear as such floating in the urine. They consist of transparent, cylindrical masses, in which numerous pus-corpuscles and a few urethral epithelial cells are imbedded. The more compact such fiber seems, the more pus-cells it contains. If, on the other hand, it is transparent, it consists for the most part of urethral epithelimm, with but little pus. 
The more the epithelium predominates in such a thread the nearer the process is to a cure. Those threads which are principally composed of epithelium have a tendency to float, while those composed chiefly of pus sink rapidly to the bottom.

The attempt has been made to localize the seat of the inflammation by the gross appearance of these threads, the thick, short threads and those which frequently assume the form of a polliwog being supposed to come from the deep urethra, the thinner and longer threads from the anterior to the triangular ligament. This method is so misleading, however, as to be practically without value, and it is only when we discover spermatozoa entangled in the thread that we can definitely conclude that it is formed in the prostatic urethra.

Treatment.- It may be said that the sooner treatment is begun after the disease has arrived at the subacute stage the better. At this time the infiltrations, not having had time to become organized into cicatricial tissue, consist of plastic material alone, and frequently yield to simple dilatation without the necessity of resorting to a cutting operation.

Local Treatment.-The first step in the treatment of a plastic deposit in the subepithelial tissues is to prevent the abnormal development of epithelial structures. The superficial erosions and granulations of the urethral mucous membrane in chronic gonorrhoea are due, as has been already shown, to the implication of the deeper layers, and therefore it is certainly not logical to attempt a cure without directing the treatment to this, the underlying cause of the continuance of the discharge. Pressure is a most serviceable agent in these cases, and is best exerted by the passage of full-sized sounds, which should be allowed to remain in the urethra for three or four minutes each day. As it is manifestly impossible for a solid sound to exert pressure on a urethra in which, as is so frequently the case, the meatus is the smallest portion, it will often be necessary to divide this up to the normal caliber of the canal behind it, cutting on the inferior surface with a blunt-pointed, straight bistourythis for the reason, chiefly, that incisions superiorly in the glans recontract quickly, or, if they do not, absorption of the tissue of the glans may occur in the line of the incision, if at all extensive, which may result possibly in a distinct cleft. The enlargement of the orifice should never be made by a sudden, quick stroke of the knife, as it is sometimes the custom of surgeons, but under cocaine or ether, very deliberately, and by repeated careful incisions, always downward, and exactly in the median line, until by repeated trials with the bulbous sound the precise size required is attained. Should the frænum be in the way, if broad and flat, the incision may be carried through its center as far as its base; if narrow, it should be first snipped away to the same extent. When the meatus is situated too low to allow sufficient room, the surgeon must content him- 
self with an inferior incision that shall not encroach upon the true floor of the canal, and will have to use the urethrometer or its equivalent, instead of the sound, for keeping the sundered ends of the strictures from uniting until healing of the incision is complete. The importance of this operation where the meatus is small can not be overestimated, discharges which have resisted treatment for many years frequently recovering spontaneously after its performance.

No matter in what portion of the canal the disease may be located, the first indication for successful treatment is the removal of all mechanical obstacles to a cure. The urethra should be carefully examined by means of the urethrometer and bulbous bougies, and all coarctations, no matter how slight, carefully noted. As a thorough examination of this kind is somewhat painful, it is well to inject half a drachm of a fourper-cent solution of cocaine by means of an Ultzmann or other syringe, lubricated with glycerin; then, after a few moments, the urethrometer is introduced, closed to the bulbo-membranous junction, and slowly dilated to the size previously estimated, after the rule of Otis* (Figs. $1,2,3)$, and drawn gently forward until it is stopped by the presence of some contraction. The size and extent of this having been noted, it is again dilated and drawn forward until it is again stopped. In this way a perfect map of the urethra is obtained. The bulbous bougies are exceedingly useful in cases in which the lower strictures are smaller than the meatus, or where the meatus has been divided, giving a somewhat more clean-cut impression than does the urethrometer.

All pathological narrowings should now be removed, preferably by dilating urethrotomy, although in recent cases simple dilatation will often prove sufficient.

Injection.-The mechanical obstacle having been removed, we may proceed to the treatment of the diseased mucous membrane with some chance of success. If the disease exists in the pendulous urethra alone, it may occasionally be cured by means of injections with the ordinary pointed urethral syringe (Fig. 4), together with the passage of a full-size straight sound on alternate days. The syringe should have a capacity of not more than two drachms, and the injection should never be sufficiently

* Otis, Stricture of the Male Urethra, p. 89.

Circumference midway of the Flaccid Penis.

3 inches,

$3 \frac{1}{4}$ inches,

$3 \frac{1}{2}$ inches,

$3 \frac{8}{4}$ inches,

4 inches, $4 \frac{1}{4}$ to $4 \frac{1}{2}$ inches, or 105 to 112 millimetres. of Urethra.

30 millimetres, or more. 32 millimetres, or more. 34 millimetres, or more. 36 millimetres, or more. 38 millimetres, or more. 40 millimetres, or more. 
strong to cause more than passing irritation. These injections may consist of any astringent, vegetable or mineral, but should always contain an antiseptic element.

When, however, the disease has extended into the bulbous portion of the urethra, it will be necessary to use some other means of medication. A soft-rubber catheter (14 French) with two lateral openings may be passed in as far as the bulbous portion of the urethra, allowing the medicated solution from an irrigator to flow slowly along the sides of the catheter, thus washing out the entire anterior urethra from the compressor urethræ muscle.

The involvement of the posterior urethra can not be regarded as a complication, but rather as a phase of the disease, which is occasionally, though rarely, absent. According to Letzel and Trzeinski, 92.5 of all cases became posterior and chronic. In fifty cases observed by Heisler, twenty per cent became posterior in the first week, seventeen at the end of the second week, seven at the end of the third week, and ten at the end of the fourth week.

It is desirable in the treatment of chronic gonorrhœa, where, as is usually the case, the entire urethra is more or less involved, that a definite system of treatment should be decided upon, varying somewhat in each case according to the tolerance of the patient. The system devised by Ultzmann is convenient, and is usually effective. It consists in the application of various solutions to the membranous and prostatic urethra, and to the mucous membrane of the bladder. For this purpose he uses either his own silver catheter with slit openings, or an ordinary stiff catheter (Coudé, 15 French) having two lateral openings. This is anointed with glycerin and introduced just beyond the compressor urethræ muscle. A hard-rubber syringe of three or four ounces capacity, filled with a medicated solution, is then attached by means of a short piece of soft-rubber tubing to the end of the catheter, and the fluid gently injected into the membranous urethra, whence, easily overcoming the resistance of the weak internal sphincter vesicæ, it flows into the bladder. This is repeated until the bladder

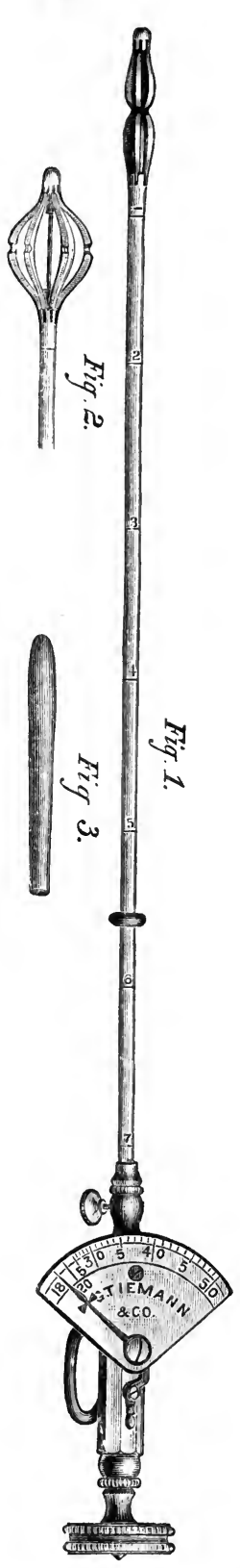

Fies. 1, 2, and 3.-Otis's urethrometer. 
is completely full, when the patient immediately empties it through the urethra. The end of the catheter is known to be in the neck of the bladder by the fluid not flowing out

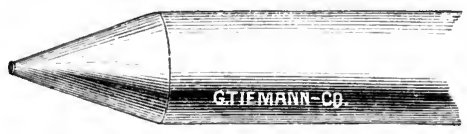

Fı, 4.-Conical-pointed syringe. alongside of it through the urethra, or, when the syringe is removed, through the catheter itself. It is well to begin with a mild solution, gradually increasing the strength each day, and combining this treatment with the use of the solid sound.

A favorite method of Dr. Ultzmann was to begin with a solution consisting of zinci sulphas, alum. crud., acidum carbolicum, āā 1 part; water, 500 parts; on the first day diluting this with water three times; on the second, twice; and on the third, once; then using it full strength. If this is well borne, it is changed for a solution of permanganate of potash, 1 to 2,000 , increasing to 1 to 1,000 , when a 1-to-2,000 solution of argentic nitrate is substituted and gradually carried up to 1 to 1,000 . The solution should be warm. At about the end of the second week, in place of the irrigation on every third day, an instillation or "etching" of a few drops of a five-per-cent solution of argentic nitrate is made into the neck of the bladder by means of the drop catheter or syringe. This consists of a capillary-tubed catheter with a straight shaft of rather abrupt curve, to which is attached an ordinary hypodermic syringe. The desired amount is drawn into the syringe, and the catheter introduced until the end has passed the membranous portion, which is indicated when the long axis of the catheter is $45^{\circ}$ from the vertical, showing that the triangular ligament has been passed. If the application has been properly made, none of the injected fluid will return, and a desire to urinate will be felt soon after the application. This treatment is especially effective in cases of irritability of the vesical neck. Applications should be repeated only after a period of two or three days, or until after the effects of previous applications have passed entirely away. The strength of the solution may be increased up to as high, in some cases, as sixty grains to the ounce, though in others it will be found necessary to begin with a mild solution, even as low as one or two per cent.

Guyon, who formerly used a method very similar to that of Ultzmann, has recently introduced a plan of treatment consisting in the use of solutions of the bichloride of mercury, beginning with a mild solution1 to 30,000 , or even 40,000 , and gradually carrying it up to 1 to 20,000 . He fills the bladder in the same way, allowing the patient to empty it through the natural route, thus washing out the entire urethra.

After a time it will be noticed that this treatment causes so much irritation that little benefit results, when the irrigations are immediately stopped, and instillations, by means of the drop catheter. of a solution of 
the bichloride of 1 to 3,000 , gradually increasing up to as high as 1 to 1,000 , are used. Usually these "etchings" are somewhat painful, the pain not eoming on as rapidly as when the nitrate of silver is used, but lasting somewhat longer, and being much more severe. This treatment will often be found exceedingly serviceable in those cases which have resisted attempts at a cure by means of the nitrate of silver, but in other cases it has proved to be much less efficient than the silver solutions.

The method introduced by Neisser consists in the injection into the bladder of a 1-to-8,000 solution of the nitrate of silver. The eatheter is passed into the bladder and slowly withdrawn, the fluid being injected until the catheter has passed the compressor urethræ muscle. The solution first flows back into the bladder, and then forward, alongside the catheter. The first day a solution of the nitrate of silver, 1 to 8,000, may be used, the patient retaining for two hours the portion injected into the bladder. This is repeated daily, and in a few days the discharge usually ceases, leaving only the threads, which as a rule disappear in a few weeks. Toward the end of the treatment it is only necessary to make the injection every two or three days. Some patients are unable to bear even so weak a solution as 1 to 8,000 , or the irritation caused by the injection and the passage of the catheter is so great that the daily injection is not advisable. In these cases it will be necessary for the surgeon to use considerable judgment, neither pushing the injection to such a strength as to cause the patient an inordinate amount of pain, nor so much irritation of the urethral mucous membrane as to produce itself the very condition which he is endeavoring to cure.

Suppositories.-When the posterior urethra alone is involved, a favorite method of treatment by many surgeons is the introduction of suppositories by means of the porte-remède of Dittel. This consists of an ordinary silver catheter, nine and a half inches in length, open at the distal end, but with a well-fitting obturator with a flexible stem. The obturator in place, the instrument is introduced until the end lies in the posterior urethra, when the obturator is withdrawn and a medicated pencil of cocoa-butter inserted into the proximal end of the instrument and pushed down into the prostatic urethra by reinserting the obturator. These suppositories may be simply astringent, or they may be made to act even as a caustic.

Another method of treating the urethra in these cases is by the introduction of a sound smeared with some form of medicated ointment, either the ordinary smooth sound or one in which depressions have been made in order better to carry the medicament.

Endoscopic Treatment.-Owing largely to the efforts of Grünfeld and Oberländer, and to the introduction of new instruments, one of the most satisfactory methods at our disposal for treating the conditions met with 
in chronic urethritis is by the use of the endoscope. By this means the mucous membrane can be carefully examined, and isolated spots touched with very much stronger solutions than could be used by any other method. The advantages of this are manifest. The most advantageous form of urethroscope is that which will admit of ready access of instruments through the tube, and in which the illumination is sufficient to detect the smallest lesions of the urethral mucous membrane.

In most forms of endoscopy in which reflected light is used, either there is no room for the introduction of instruments, or there is so much extraneous light that after a little time the eye becomes dazzled and tired, very much as one feels after looking at snow. In order to overcome these difficulties, I have constructed a urethroscope, consisting of a small metal cylinder one and a half inch long by one inch in diameter,

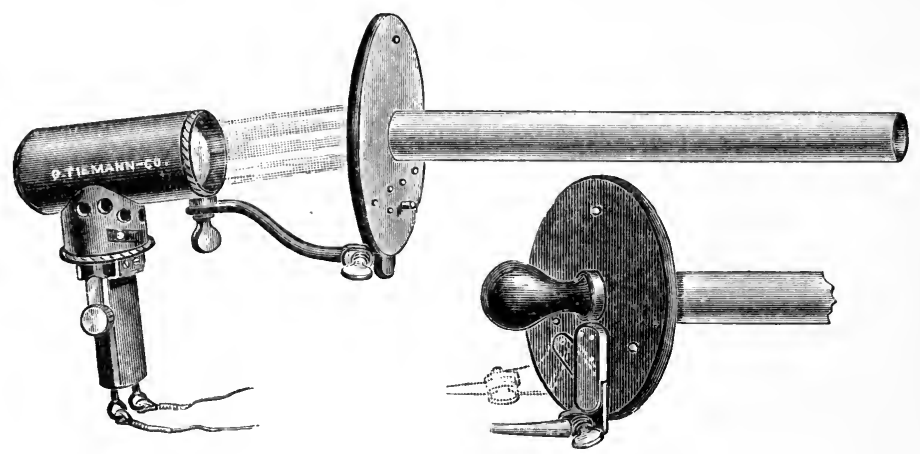

FIG. 5.-Otis's electro-urethroscope.

one end of which is open. Within this cylinder, one quarter of an inch distant from the open end, is a miniature incandescent electric lamp, placed directly behind a convex lens. The electric connections with the lamp are placed in a piece of hard rubber one inch long, which serves as a handle to the instrument, a cut-off being so arranged that the lamp is under control and can be turned on or off, or renewed at pleasure. A stout wire one and a quarter inch in length connects the instrument with the top of the plate of the Klotz's urethroscopic tube by means of a small, flat foot at its distal end, at the outer extremity of which is a smooth pin. This pin fits in a hole in the top plate, and on revolving the instrument a quarter circle the foot swings under a shoulder attached to the plate and is securely fastened. This joint is firm and easy of manipulation, readily allowing the illuminator to be attached or removed at any time during the examination. Hinged joints, swinging in opposite directions at each end of the wire, allow the instrument to be turned in any direction. The rays of light passing through the lens are thrown down the tube, and the illuminated field is easily observed by an eye 
looking over the top of the cylinder. The advantages of the instrument are the exclusion of all extraneous light, a very much more ready access to the urethral field both to the eye and for instrumental applications, and greatly increased illumination.

The instrument is also very light and compact, being much smaller and only one seventh the weight of the Leiter instrument. The eye may be placed much nearer the mucous membrane to be examined. Its great simplicity of construction should insure a moderate cost.

The tube being passed down to the bulbo-membranous junction, the obturator is withdrawn and the instrument applied. Granular spots are easily detected under the strong illumination, and with a little practice the openings of the glands can also be seen.

In making applications, the strong solutions only are of value. Sixty grains of the nitrate of silver on a cotton swab, pure iodine, or even lunar caustic, or the electric actual cantery, may be used. As it is rather difficult to remove the cotton from the end of the applicator after it has been used, the little sticks employed by florists to attach flowers are very serviceable as applicators, and may be thrown away after using once.

Drainage.-In certain cases the invasion of the posterior urethra is attended with chronic tenesmus and frequency of urination, the patient rarely being able to retain more than an ounce or two of urine at a time, and is obliged to micturate every hour, or even more often. He has to wear a urinal, or to constantly remain near some place where he can relieve himself, and, from pain and attendant want of sleep, loses flesh and strength, and becomes so nervous and irritable that life is scarcely bearable either for himself or his friends.

In these cases, which are often mistaken for tuberculosis, local treatment seems to aggravate rather than alleviate the condition, the irritation caused by the introduction of instruments being greater than any benefit derived from the medicated solution. The bladder requires a complete rest, and that for a considerable period, at least several weeks. To establish this the most efficient means is by drainage through the perinæum. The patient having been etherized and placed in the lithotomy position, the perinæum shaved and rendered thoroughly aseptic, a grooved staff is passed into the bladder and firmly held in the median line by an assistant, the bow of the staff being pressed outward toward the operator, who sits in front, and with the left forefinger in the rectum feels for the apex of the prostate. An incision is now made through the skin and superficial structures, about one inch in length, its lower end being about an inch above the margin of the anus. A long, straight bistoury is then passed along the finger into the groove of the staff and the urethra divided for a little over a quarter of an inch. A director is then passed through the incision along the groove in the staff into the bladder and 
the staff removed. The right forefinger may now be inserted and the interior of the bladder thoroughly explored, which is greatly aided by pressure with the other hand above the symphysis. The attendant overstretching of the vesical neck is in all probability exceedingly beneficial, acting by temporarily paralyzing the sphincter, as is done in the case of the sphincter ani in the operation for fissure. A large rubber catheter is now passed through the wound, extending into the bladder cavity only just so far as to drain it, and is held in place by a silk stitch passing through the edges of the wound and the catheter. The patient being put to bed, the end of the tube is placed in a bottle and the bladder allowed to drain freely. At the end of two or three days the stitch is removed and the tube thoroughly cleansed. The bladder should be washed daily through the tube, such solutions being used as cause no undue amount of pain. The tube should be frequently cleansed from any deposit of calcareous material. The relief from this operation is usually instantaneous, and the bladder should be allowed to drain as long as possible, at least for a period of several weeks.

The duration of the contagious period of the discharge is a question of great importance, and one which is not at all easy to decide. It has been made doubly important of late by gynæcologists, who have pointed out that many uterine, tubal, and ovarian diseases are due to contagion from this source. Though we are unable always to detect the gonococci in the discharge or in the Tripperfäden, it is quite evident that they lie hidden in the crypts and intercellular spaces of the urethral mucous membrane, ready to bloom forth upon any exciting cause, such as overindulgence in stimulants or sexual intercourse. Thus we often find patients who have once contracted gonorrhwea continually complaining of their misfortune in the frequency with which they contract new attacks from sources which they considered unimpeachable, whereas in reality it is simply a rekindling of the old malady. While it is probable that the contagious element is not present when Tripperfäden alone exist, yet at any time these cases may present a discharge which is contagious. No patient should be allowed to marry within a year after the total disappearance of all discharge, until all stricture has been removed from his urethra, and until the Tripperfäden consist of epithelium alone. In regard to this last, however, even normal nrethræ which have never been subjected to the invasion of the gonococcus occasionally throw off Tripperfäden containing white cells, so that it will be necessary for the practitioner to use judgment as to the nature of the Tripperfäden in each case.

The great majority of men having gonorrhœa recover entirely, at least so far as any infections element is concerned. They marry, and their wives remain perfectly healthy, even though the husbands continue 
to pass urine containing threads composed largely of white cells, and while uncured gonorrhœe is undoubtedly responsible for a certain amount of ovarian and tubal disease, the probabilities are that this factor has been overestimated.

General Treatment.-In regard to the general treatment of cases of chronic gonorrhœe, it is not necessary to restrict the patient nearly as closely as when the disease is acute. Avoidance of excessive indulgence in alcoholics, particularly champagne and beer, care of the general health, moderate exercise, avoiding horseback and bicycle riding, or too violent exercise, and avoidance of sexual intercourse, are advantageous in these cases, but, after all, it is through local treatment that we must expect to effect a cure.

Finally, in those unfortunate eases which are also the subjects of tubercular infection, the tubercle bacillus and the gonococcus appearing side by side in the discharge, the prognosis is exceedingly grave. In these cases local treatment, as a rule, is worse than useless-in fact, is often harmful, although in some cases emulsions of iodoform may prove of service. It has been suggested by Belfield that the trichloride of iodine, beginning with a solution of one quarter of one per cent, and increasing to one per cent, might be of service; but should these remedies fail, a seavoyage, or life in some salubrious climate, is all that can be suggested to aid these unfortunate sufferers. 


\section{EXPLANATION OF FIGURES ON PLATE II.}

1 , Normal urethra, near fossa navicularis ; 2 , normal urethra, anterior portion of pendulous part; 3 , normal urethra, posterior portion of pendulous part; 4 , normal urethra, central portion, parietal position of tube; 5 , normal urethra, lacunæ Morgagni, one enlarged, parietal position; 6 , normal urethra, bulging in of mucous membrane (caliber of tube smaller than that of urethra); 7, normal urethra, membranous portion; 8 , normal urethra, anterior portion of colliculus seminalis protruding between lateral folds; 9, normal urethra, colliculus seminalis, with aperture of uterus masculinus; 10 , colliculus seminalis enlarged and hyperæmic; 11, membranous portion (new formation of blood-vessels), hyperæmic condition in shape of stripes; 12 , diffuse hyperæmia and oedema of mucous membrane (pendulous portion); 13, patch of granular condition; 14, 15, later stages of granular condition, with infiltration of subepithelial tissue; 16, epithelauflagerungen (new formation of epithelial layers); 17, 18, patch of granular condition with lacunæ Morgagni, before and after local treatment; 19, polyp of membranous portion; 20, papillary excrescences (vegetations) of pendulous portion; 21, 22, large polypus (central position) (Grünfeld); 23, cicatricial stricture (Grünfeld); 24, old cicatricial stricture after dilatation (Grünfeld). 
PLATE $\|$.
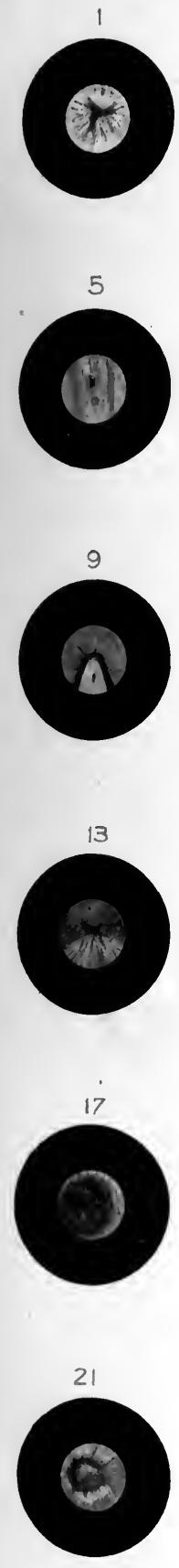
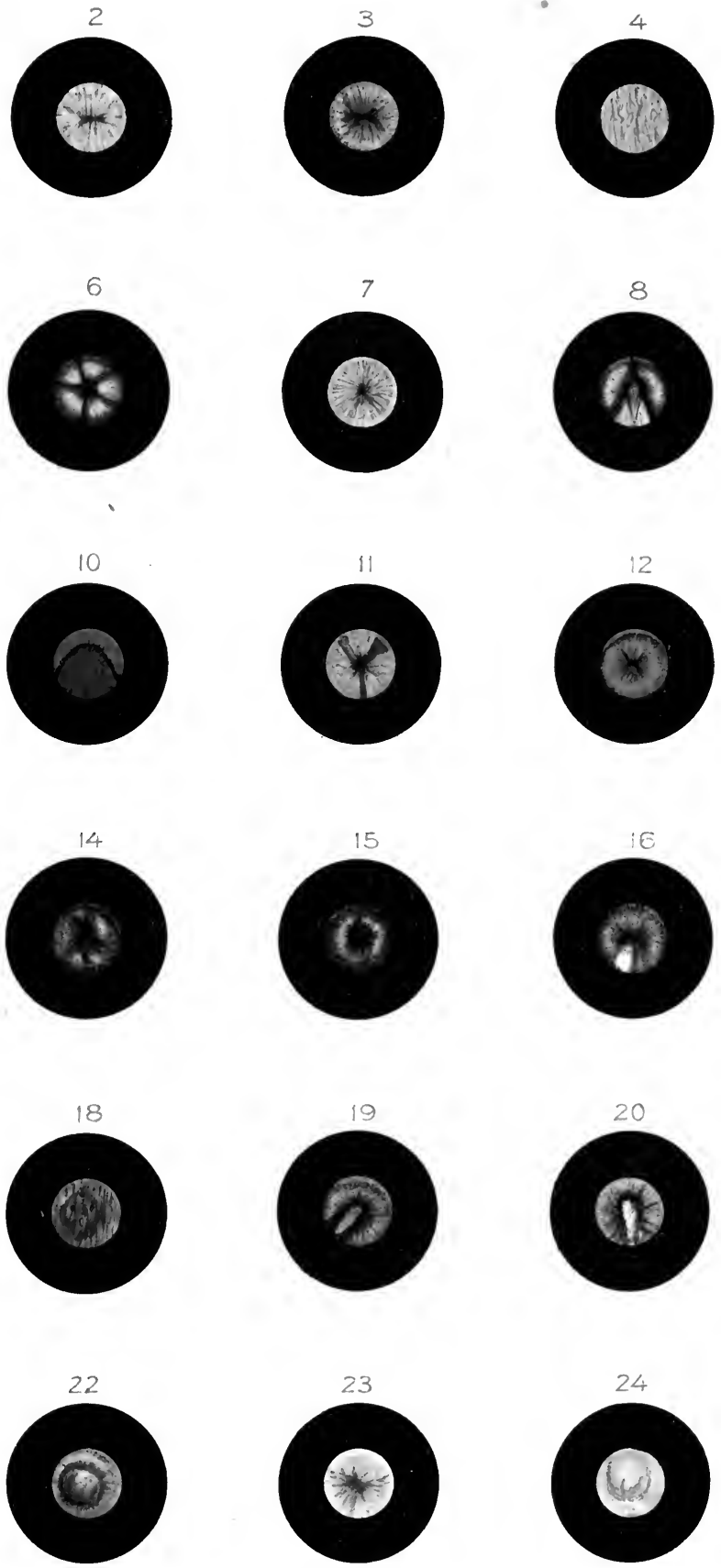

$$
\text { ENDOSCOPIC PICTURES. }
$$





\title{
ENDOSCOPY.
}

\author{
By HERMANN G. KLOTZ, M. D.
}

THE urethra is not directly accessible to examination by the human eye. It is true, by pressing the orifice between two fingers in a perpendicular direction the lips of the meatus will separate sufficiently to allow the inspection of a portion of the fossa navicularis, or, by extending the meatus by means of the blades of a forceps, the mucous membrane may be seen to the depth of nearly one inch. But to look beyond that distance some contrivance is needed ; first, to hold asunder the walls of the urethra, which naturally are in close contact; and, second, to illuminate the cavity thus formed sufficiently to render it distinctly visible at a certain distance. The instruments which have been constructed for this purpose are called endoscopes, and the art and practice of examining the inside of the urethra by means of such instruments, endoscopy. These words are derived from the Greek ěv $\delta o v$, within, inside of, and $\sigma \kappa o \pi \epsilon \hat{\imath} \nu$, to view, and simply mean inspecting the interior. By analogy with the terms used for the visual examination of other interior organs of the human body, as the eye, the larynx, the urinary bladder, viz., ophthalmoscopy, laryngoscopy, cystoscopy, etc., it would be more correct-as, in fact, is frequently done-to use the terms "urethroscopy" and "urethroscope." But since Désormeaux made known his instrument under the name of the endoscope, the general term has almost universally been applied to the examination of the urethra alone, and by an endoscope we understand, as a rule, an instrument for the examination of the urethra. The conditions for the examination of the urethra being somewhat similar to those of the vagina, only much less favorable for illumination on account of the narrowness and length of the urethra, most of the apparatus intended for the purpose of distending its walls follow the shape of vaginal specula, the simple cylindrical speculum being the prototype for most of the tubes introduced into the urethra, while a number of bivalve specula have been devised at different times. But the problem of lighting the cavity of the urethra presented much greater difficulties, and led to the construction of a number of complicated and impractical apparatus, their authors endeavoring to unite in one the different parts necessary for the purpose. 
Development of the Endoscope.-The first instrument which actually stood the test of practice was that of A. J. Désormeaux, of Paris, presented in 1853 to the Academy of Medicine. In 1865 he published the practical results obtained with it in his book on The Endoscope and its Applications to the Diagnosis and Treatment of the Affections of the Urethra and of the Bladder. Désormeaux's instrument was still far from perfect, and the numerous surgeons all over the civilized world who took interest in the subject at once set to work to improve upon it by applying better sources of light, gradually separating the light itself from the instrument proper, and reducing the latter to more simple forms. The decided innovation, to use for illumination the common laryngeal mirror fixed to a headband or handle as a reflector, without any connection with the tube proper, was made in 1862 by August Haken, a practitioner of Riga. This method was soon adopted by other surgeons, particularly by Joseph Gruenfeld, of Vienna, who, by a number of publications beginning in 1874, has probably done more than any one else to demonstrate to the profession in general the comparative simplicity and the practical value of endoscopy. Gruenfeld has given an almost complete history of the development of the endoscope, with a description of the older instruments, in a paper published in the Wiener med. Jahrbuch, 1879. The literature of endoscopy has already assumed considerable proportions. An almost complete list up to 1880 is found in Gruenfeld's book, Die Endoscopie der Harnröhre und Blase, which appeared in 1887 as part of the Deutsche Chirurgie, published by F. Encke. It is the first complete treatise on the subject. The list of publications has been continued as far down as 1888, by E. Burckhardt, of Basel, in his monograph, Endoscopie und endoscopische Therapie der Krankheites der Harnröhre u. Blase, published in 1889; a book full of practical instruction and advice, illustrated by numerous histories of cases and by beautifully executed plates. These are the only books treating entirely on the subject of endoscopy. Among the large number of papers published in periodicals, those of Oberländer, of Dresden, occupy a prominent position, although more of interest to experienced practitioners with the endoscope.

Changes and improvements in the shape and material of the urethral tubes have been devised at different times without material change of the principle of construction. The great progress in the application of the electric light within the last few years has again almost revolutionized the principles of the endoscope, and has furnished us with a number of valuable inventions. There is, therefore, no want of practical instruments ; we are rather confronted by an embarras de richesse in trying to recommend any one particularly to those who wish to employ endoscopy.

The least complicated instrumentarium is required for Gruenfeld's method, with the light and the reflector separated from the urethral tubes 
or sounds, which deservedly form the most important part thereof, and almost entirely usurp the name of the endoscope. From the different instruments recommended by Gruenfeld only one is really necessary. This, the simple endoscope, is a straight cylindrical metallic or hard-rubber

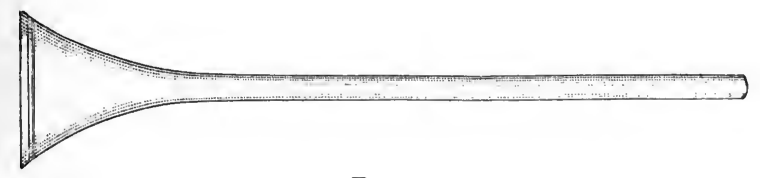

FIG. 1.

tube, cut rectangularly at its visceral end, with a well-rounded and smooth edge; the ocular end widens into a short funnel, the edge of which is rough or indented to afford a firm grip for the finger of the observer. A conductor or obturator, intended to close the visceral end of the tube during its introduction, is formed either of a solid rubber plug, or of a strong wire rod bearing an olive or acorn shaped or cylindrical bulb. It must be well rounded at the top, and well fitted into the end of the tube, so as to bring the shape of the closed instrument as nearly as possible to that of a simple metallic sound or catheter. Gruenfeld's tubes vary from four to thirteen centimetres in length, and from Nos. 14 to 24 of the French scale in width, and are blackened on the inside. An important modification of Gruenfeld's simple endoscope was effected by Steurer, of New York. When the endoscope is introduced to its full length into the urethra, the gradually widening funnel of the ocular end enters the meatus and unduly distends the same, often causing discomfort and pain to the patient. To obviate this, Steurer places a disk between the funnel and the tube proper, by which the glans penis, without stretching of the

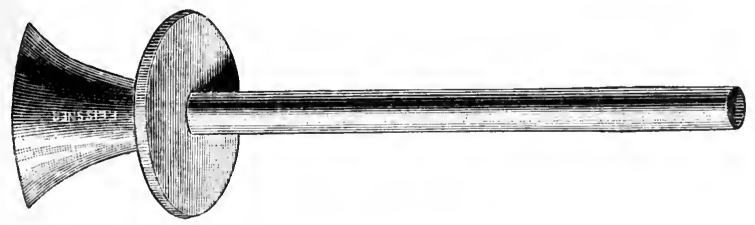

FIG. 2.

meatus, may be shoved back over the tube. Thereby the length of the urethra is reduced considerably, and the deeper portions can be reached with a tube much shorter than the urethra itself. In 1886, after several years' experience with the same, I published a modification of Gruenfeld's, or rather Steurer's, endoscope devised by myself, which since has become well known in the United States as Klotz's endoscope. The funnel-shaped part of the ocular end of Gruenfeld's tube has been eritirely dispensed with. Ostensibly intended to facilitate catching of the light-rays, it in- 
creases the distance between the eye of the observer and the object by almost one inch, without offering any advantage for increasing the quantity or intensity of the light by deflecting any of the rays, as only nearly parallel rays can reach the end of the tube, eight to thirteen centimetres long.

The tube itself is made of coin silver, in preference to cannulas made of brass or other coarser metals, or of hard rubber, because silver can be

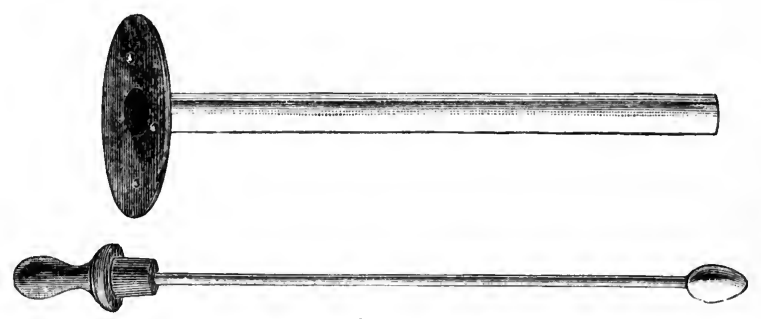

FIG. 3.

worked into much thinner tubes, which, in consequence of the wider bore, furnish a wider field of view for the same size of tube. Besides, silver tubes are much less affected by the chemical action of caustic and astringent solutions, and can be kept much more easily clean and smoothly polished than Gruenfeld's blackened tubes. Auspitz had already been taught by experience that the dissipated reflexes, dreaded so much by others, do not increase in quantity if the inner surface of the tube is always kept clean and smooth, while on the other side in blackened tubes the intensity of the light is greatly impaired by a considerable absorption of light-rays. To avoid annoying reflexes from the surface of the disk, an unpolished hard-rubber plate, one millimetre and a half thick, is riveted to the metallic disk. This is clean, less liable to be injured by chemicals, and insures a tirm and comfortable grip, particularly during exploration of the deeper portions of the urethra, wherein the tube has to be held securely and firmly, and to be moved cautiously against the powerful resistance of the muscles. The disk has a diameter of four centimetres, somewhat larger than Steurer's, to prevent overlapping of abundant prepuces. This very light and simple endoscope is extensively used in the United States, and has been recommended by Keyes and Gerster, of New York ; Newell and Allen, of Boston; Eversole, of St. Louis ; and more recently by S. Alexander and William K. Otis, of New York.

Tubes made of hard rubber have been applied by Weinberg, and later by Schuetz, of Frankfort, who adopted Steurer's disk. They are of very light weight, clean, and can be introduced and advanced without a conductor. To make them strong enough, however, their walls must be of considerable thickness, and the dark inside absorbs much light. Glass 
tubes, which had been used already by Buttles, of New York, in 1867, have more recently been described by Posner, of Berlin, after the model of Ferguson's vaginal specula, lined with silver and covered with black varnish. They undoubtedly have the advantage of cleanliness, resistance to chemicals, and of excellent light. With Unna, who had previously used glass tubes himself, I consider them too dangerous, on account of the liability to break, particularly if introduced into the deeper portions of the urethra. All tubular endoscopes expose to view at the visceral opening only a small portion of the mucous membrane at one time. By their forward or backward movement gradually other parts come into view, and thus the entire lining may be inspected from the deeper portions to the orifice. To afford inspection of a more continuous strip of the mucous membrane, some bivalved dilating specula have been constructed, which can be distended either at the visceral end alone or in their entire

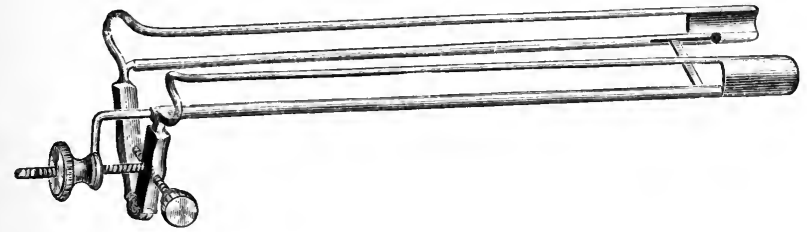

Fig. 4.

length. In 1879 Auspitz placed a dilating speculum before the profession. Meyer, of Berlin (1886), Tuttle (1887), and Tilden Brown, of New York (1888, modified in 1889 and 1891), have described dilatable specula composed of strong wires joined by a metal ring or band at the visceral end. By different screw attachments, or by means of a lever, these specula can be separated independently at the ocular as well as at the vesical end, thus affording equal dilatation for the whole length of the urethra. These dilating instruments are undoubtedly useful for examination of the an-

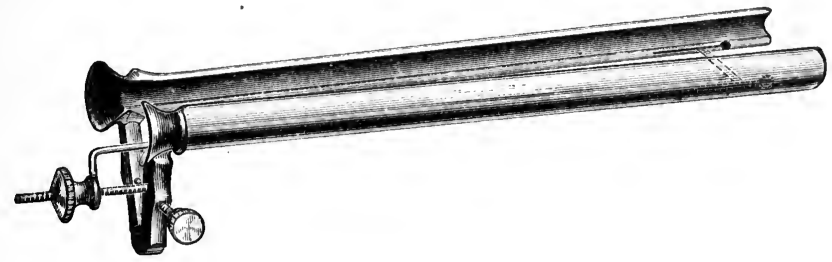

Fig. 5.

terior portion of the urethra. For longer distances the perspective sidelong view of an extended stretch of mucous membrane does not give distinct pictures. Besides, the mucous membrane will bulge into the field of view through the intervals between the wire bars and obstruct the 
view. The pressure of the branches is sure to affect the appearance of the mucous membrane by rendering it more or less anæmic, while the danger of injuring the mucous membrane by catching it between the branches in closing and removing the instrument is certainly a possibility. The substitution of solid bars for the wire rods, introduced by Brown in his more recent modification, may dispose of the bulging in of the folds. It seems that, while not preferable for common use, the dilating endoscopes may prove of particular value for the isolation of papillary and other growths, and for the demonstration of strictures of wide caliber-a question yet to be decided by further experience.

Von Antal's aëro-urethroscope, and a later similar device by F. Heuel, of New York, purpose to distend the urethral walls withont a tube by compression of air within the canal, thereby affording an unobstructed continuous exposure of the urethral surface. The impossibility of using these instruments without an assistant, the influence of the pressure of the air on the blood-vessels of the mucous membrane which is rendered more or less anæmic, the restriction of its use to the anterior portion, and the preclusion of the use of any application for cleaning and treating the mucous membrane, deprive this instrument of every claim for general practical use.

With all these endoscopic tubes or sounds the light is thrown into the urethra by a reflector, either attached to a handle or more commonly to a head bandage, so as to leave the right hand of the observer entirely free. A concave mirror of focus length of ten inches, generally used for illumination of the larynx, answers the purpose very well. It affords entire liberty in the selection of the light itself. By far the best light is direct sunlight, and it should always be employed when it can be secured, particularly in the beginning of endoscopic study. A plain mirror attached to the head-band is best used for its reflection, as the concave mirror develops considerable heating effects. Under sunlight the differences in color appear most natural. The configuration of the surface, and dilated and newly formed blood-vessels, can be more readily distinguished than by any other mode of illumination. Sunlight reflected by white clouds, and, under favorable conditions, even diffuse daylight, afford sufficient illumination; but as direct sunlight is unfortunately not always at our disposal, some artificial light is therefore indispensable. Any good kerosene or gas lamp, with argand burner; either alone or in connection with Toboldt's or other light-intensifying apparatus, will answer more or less well. Auer's and Weissbach's incandescent gas-burners with their almost perfectly white light, and, finally, the Edison electric lamp, are particularly serviceable. In the diaphotoscope of Schuetz, of Frankfort, the electric light is employed in a very ingenious manner; it is highly praised by Burckhardt, who says he has of late used it almost exclusively. 
The electric lamp, in the shape of a perforated disk, is placed directly in front of a perforated concave mirror, and is protected by a condensing lens with a dull ground surface; the whole apparatus, inclosed in a metallic case in such a manner that heating is avoided as far as possible, is placed directly in front of the eye, either by means of a handle or a headbandage, so that the light is placed almost in the eye of the observer. The other instruments for which the electric light has been brought into service, and which naturally recommend themselves to those who enjoy the service of a battery, or can connect the electric lamp with the current from the street furnished by the electric-light companies, are of different construction. A very interesting instrument is Oberländer's electro-urethroscope, recently improved by Kollmann, of Leipsic. Its characteristic feature is the placing of an illuminated platinum-wire loop at the vesical end of the tube in the immediate vicinity of the mucous membrane. The necessity of cooling the tube by a current of cold water, the reduction of the lumen of the tube by the staff bearing the light and the cooling apparatus, the difficulties of making any applications to the diseased portions during illumination, the necessary attention to battery and water-supply, render the instrument, even in its improved shape, rather complicated, although the intensity of the light is certainly very great and the views obtained are very distinct.

Other inventors have united the tube, reflector, and the light into one single piece, returning to the principle of Désormeaux. The best-known representative of this class is Leiter's electro-endoscope (1887). It is recommended as a most serviceable instrument by Finger. A small Edison lamp is placed on top of a handle, through which the wires run, inside of a cover and in front of the concave, slightly inclined mirror. This throws parallel rays of light into the tube to the visceral end. The observer looks from behind and over the mirror into the wrethra through a metallic ring into which lenses for correction or magnifying may be inserted. The ocular end of the tube, being unobstructed, is open for the introduction of tampons, brushes, and other instruments-a great advantage over Désormeaux's instrument, and over the electro-urethroscope of Nyrop (1886), which otherwise shows a construction similar to Leiter's. The principal objections to Leiter's instrument are its size and weight. For observation only, it is convenient enough if both hands are at your service, the left hand fixing the penis and the right one directing the instrument by the handle; but when the right hand is needed for cleaning the field of view, or for therapentic manipulations, it is impossible for the left one to support the penis and the tube with the weight of the optic apparatus attached, and at the same time to effect exact, tender, and subtile handling of the tube which is required for successful examination. 
W. K. Otis, of New York, and after him S. Alexander and F. Tilden Brown, reduced Leiter's optic apparatus to a mere skeleton and its weight to one sixth of the original, which with aluminium as material might still be reduced. A small concave mirror is connected by a stout

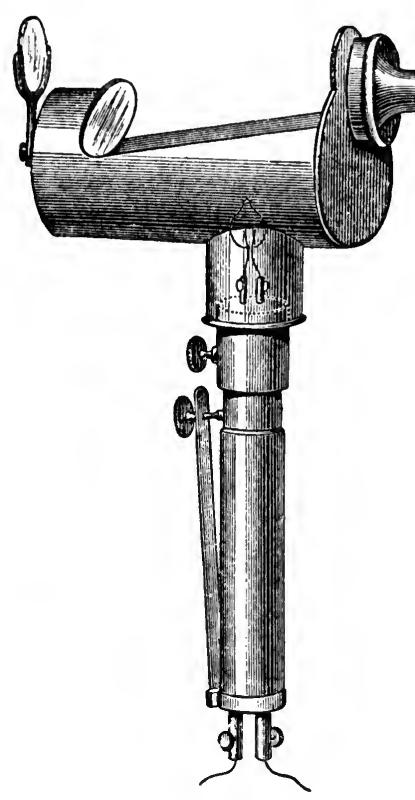

FIG. 6.

wire about two inches in length with the proximal end of the urethral tube. Midway on this wire is attached a small Edison electric lamp, a semicircular screen being placed immediately behind the mirror to cut off extraneous rays of light. It is now attached to the rubber disk of Klotz's endoscope, either by a screw (Alexander) or by a simple attachment (Otis) which allows of rapid change of the endoscopic tube. At the distal end of the wire is placed a small flat foot, at the outer extremity of which is a smooth pin. The pin fits into a hole of the disk, and on revolving the instrument a quarter of a circle the foot swings under a shoulder riveted to the plate and securely fastened.

Brown has connected the same apparatus with his bivalve speculum. In this reduced form the electro-endoscope certainly recommends itself as extremely simple and practical. It must be acknowledged that it is an advantage of the instruments in which tube, reflector, and light are joined in one piece, that the illumination and possibility of inspection of a certain region are assured at once as soon as the instrument is introduced, and will remain so regardless of changes in the position of the observer or of the patient. With the head-mirror the focus of light-rays has first to be directed into the tube and on the desired spot of the urethra. A precise position of the head is therefore exacted, and the accommodation of the same to the changes in the position of the object as well as the observer. Sufficient dexterity in directing the light, unless previously gained from laryngological studies, is, however, easily acquired by practice, and if once acquired affords ample compensation by enabling the observer, by a slight movement of the head, to change the intensity of the light and to focus the rays much more nicely on important points so as to bring out the differences in the surface of the mu- 
cons membrane. This can not be effected with an instrument in which the distance between the light and the visceral end of the endoscopic tube is a fixed one, and tubes of uniform length, therefore, have to be employed, while with the mirror, endoscopes with any desired length can be used. F. T. Brown has tried to eliminate from Otis's endoscope this disadvantage by making the speculum movable along the bar which bears the electric lamp. Perhaps it would be more advantageous to make the lamp itself slide upon the horizontal bar which connects the mirror and the tube.

Endoscopic Armamentarium.-Whatever method of endoscopic examination may be selected, and whichever illuminating apparatus, it will be necessary to have several urethral tubes of different caliber, and, whenever practicable, of different lengths. It is obvious that in general, cateris paribus, the intensity of the light and the distinctness of the picture received by the eye of the observer will increase in proportion with the width and decrease with the length of the tube. As a rule, therefore, the largest caliber that passes the meatus onght to be employed, and the shortest tube that will reach to the spot which it is intended to examine. While there is really little difference in the length of the urethra, the length of the pendulous portion of the penis, and the development of the connective-tissue framework of the same vary considerably in different individuals. In most cases an instrument four inches in length will be sufficient to reach the membranous portion by shoving back the penis with the disk, but for demonstration of the prostatic portion instruments of five or five and a half inches are preferable. In particular cases I have had constructed tubes six and a half inches long, to get a view near the external oritice of the bladder. Tubes under No. 23, French scale, even if only two and a half to three inches long, are of very little use. To those who have had some experience with endoscopy, they may occasionally be of good service for reconnoitering the forward portions of the urethra, and to prove the absence of certain conditions, like warts, ulcers, etc.: but to get an insight into the condition of the mucous lining and of the submucous tissue, it requires tubes from 24 , French, upward, increasing in length with the caliber. A set of endoscopic tubes-No. 23, 3 inches long; No. 24, $4 \frac{1}{2}$ inches long; No. 26, $4 \frac{1}{2}$ to $5 \frac{1}{2}$ inches long; No. 28, $5 \frac{1}{2}$ inches long; No. 30, 4 to $5 \frac{1}{2}$ inches long ; and perhaps one No. 32, $5 \frac{1}{2}$ inches long-will probably be sufficient for all practical purposes.

Besides, a number of auxiliary instruments will be needed for the successful application of the endoscope to diagnosis and treatment-a number of tampon-holders, better not fewer than twenty, for cleaning the surface of the mucous membrane of adherent grease, pus, mucus, blood, or other substances, and for applying drugs in solution or in pow- 
der form. Pieces of cylindrical wood such as used in preparing matches, cut into convenient length, are sufficiently rough to hold tufts or wads of absorbent cotton after a few turns around their axis. They may be armed with tampons at both ends and thrown away after use. Strong wire rods of galvanized wire, brass, or German silver, bent at one end into a ring to serve as a handle, and filed sufficiently rough or bearing a number of turns of a shallow screw, are equally serviceable, and can be used again after removal of the soiled tampons. The tufts or tampons themselves ought to be of different sizes for the different sizes of tubes and according to the extent of the spot to which they are to be applied. The plain rods are preferable to more complicated pincer-like instruments, to which a cotton tuft is fastened by a ring. Occasionally the tampons get loose from the rods and remain in the tube, but usually can be removed without difficulty by the rough end of the wire, or, better still, by a wire drawn out into a fine corkscrew. For the application of medicine, the cotton tampons are preferable to pencils or hair-brushes, because they are always clean, absorb sufficient fluid to communicate it to that portion of the mucous membrane with which they are brought into contact, but do not allow of the falling of a drop on the mucous surface, which would be liable to prolong the effect of the drug longer than is desirable, or to carry it to portions for which its application was not intended and might do harm. Besides, brushes and pencils have to be cleaned at every application, are liable to spoil solutions of metallic salts into which they are inserted, and themselves become easily brittle, hard, or sticky. Powders can easily be carried to any desired spot by the same cotton tampons, but an insufflator, or a tube for blowing finely powdered drugs on the desired spots, may be employed, as recommended by Gruenfeld and Burckhardt. Naturally, almost any apparatus for the insufflation of powders or for the spraying of fluid drugs may be adapted to the needs of the endoscope. Solid caustics, like nitrate of silver, sulphate of copper, etc., are applied by means of porte-caustics, which differ only by their more slender and longer stem from those used for ordinary purposes. A fine curette, ring or spoon shaped, and several probes of different caliber, are, if not absolutely necessary, still very desirable additions to the endoscopic armamentarium. Several minute knives of different shape, pointed, curved, and blunt-pointed; scissors, straight and curved, and forceps, may be required occasionally, as well as a wire snare for the removal of new growths.

Burckhardt has largely used the galvano-cantery and devised differentshaped electrodes - a fine-pointed cautery for scarification, one with a flattened point, and a broad, knife-shaped one. It need hardly be mentioned that all the instruments just enumerated must have handles of sufficient length to pass through the longest of the endoscopic tubes, de- 
manding a length for the entire instrument of from seven to eight inches. It is further desirable, but not absolutely necessary, that the proximal end of all the instruments be bent under a right or obtuse angle, so as to allow of the inspection of the urethra during application. For the cotton tampons it is entirely superfluous.

Special tables for endoscopic examination have been designed by Finger and others, but any surgical chair or table that can be raised to a height of thirty-eight or forty inches will be serviceable. The individual to be examined assumes a reclining position. The upper part of the body may be slightly elevated, the buttocks drawn down as far as possible toward the edge of the seat, the knees and feet well separated so as to allow sufficient room for the observer, the feet resting on some support either connected with the table or on a small chair, or on the front round of a common cane-seat chair occupied by the observer. In this way it is possible to go over the entire length of the urethra without change of position ; only, to lower the ocular end sufficiently to reach the deepest portions of the urethra, it occasionally becomes necessary to relinquish the seat and to kneel in front of the chair or table. If the observer begins the examination while standing, he has to change his position on reaching the membranous and prostatic portions. When a reflector is used, the light is placed on one side of the patient, generally the right, on a small table, which also affords a place for cotton tampons and other instruments, unless a shelf on the chair is provided for. Where the light is connected with the tube greater freedom is allowed for the position. It has already been mentioned incidentally that, as a rule, the examination of the urethra is performed backward from the distal end of the organ; with hard-rubber tubes, and, to a certain extent, with silver tubes it is possible to pass inward from the orifice and inspect the interior while progressing; but in most cases it is easier to introduce the tube with an obturator to the deepest portion which it is desired to inspect, and, after removal of the same, to view that portion which presents itself in front of the straight visceral end of the instrument. During gradual withdrawal the tube will pass every point of the mucous lining, and so bring into view, bit by bit, the entire interior surface.

Method of Examination. - To proceed with the endoscopic examination: Having put your patient, light, medicines, and instruments in the right position, the proper size of endoscopic tube selected, this has to be thinly covered in its entire length with some greasy substancevaseline, with or without boric acid, or some other unirritating disinfectant, being preferable to glycerin. Now the left hand takes hold of the penis, brings it to angle of about $90^{\circ}$ with the abdomen, grasping the glans between the thumb and index-finger and opening the lips of the meatus, while with the right hand, the thumb pressed against the top of the 
handle of the obturator, the second and third finger resting on the edge of the disk, passes the point between the lips, and, feeling its way along the upper wall of the urethra, moves it gently downward until the disk comes in contact with the glans or resistance is felt. In the first case, under somewhat increased pressure, the disk pushes the penis until the end meets with resistance. The bulb of the obturator is now touching the bulbous portion. If it is not intended to inspect more than the anterior urethra, the left hand takes hold of the disk between the thumb and index-finger while the third and fourth fingers support the penis between them; the right hand removes the obturator. If, however, it is intended to examine the entire urethra, the ocular end of the tube is now gradually lowered to a horizontal line, and while being gently pushed on, and then more or less below the horizontal line, until the end of the tube glides on toward the bladder-as a rule, the most painful part of the operation. While the resistance of the external sphincter is rarely very strong, in my experience that of the internal sphincter is very firm, so that even with a sufficiently long instrument it requires a great effort to pass into the bladder. The difficulties in passing beneath the arch of the symphysis vary considerably in different individuals, owing to the difference in the insertion and length of the ligamentum suspensorium penis. Sometimes it is hardly necessary to lower the ocular end of the tube below the horizontal line; sometimes it must be turned almost into a vertical line. In very few instances I found it impossible, even with a small tube, to enter beyond the membranous portion. The end of the tube always turned against the upper wall and was caught by the symphysis, while curved sounds would easily pass around the obstacle. The deepest possible point being reached, the left hand, which now has to contend with all the force of the muscular apparatus which brings strong efforts into play to throw out the instrument, takes firm hold of the disk, the obturator is now removed, and the examination proper begins.

Usually the field of view is covered by some fluid-a mixture of vaseline, mucus, and other secretions of the urethra and of its glands-and has to be cleaned by gentle touch with a dry cotton tampon. This cleaning process has to be continued more or less through the entire urethra. Small quantities of blood can easily be wiped off, but whenever a more serious hæmorrhage takes place the examination has to be given up, at least for the portion of the urethra where it occurs. After inspection, a slight retrograde movement of the tube commences to bring other portions before the visceral end. This movement has to be directed as far as possible in the axis of the urethra, and therefore has to follow the natural curve of the deeper urethra through the horizontal line until the ocular end has again turned upward. As long as this presses against the glans penis and shortens the urethra, it is sufficient to take hold of the 
disk or the proximal end of the urethral tube alone; but as soon as the tube passes out of the urethra it is necessary to take hold of the penis itself with the last three fingers, to prevent too rapid motion and the slipping off of the penis from the tube. The thumb and index-finger then gradually pass from the disk to the tube itself, until the visceral end glides ont of the meatus. During observation alone the right hand may assume the leading out of the tube, thereby assuring greater accuracy and steadiness, while the left one supports the penis and takes control of the tube only when the right one is engaged in cleaning the surface or in other manipulations. Meanwhile the light from the reflector has been directed through the tube on the exposed portions of the surface, and the observer has had a passing view of every part of the interior.

The Endoscopic Picture.-The pictures which present themselves to the eye vary considerably according to the different portions of the urethra. Around the edge of the tube the folds of the mucous membrane, into which it is formed during the natural condition of rest, will be fully extended into a smooth ring. Owing to the natural elasticity, the walls of the urethra have the tendency to collapse again into their former position, and will converge as soon as relieved from the distending pressure of the tube. The surface, therefore, assumes the shape of an inverted cone or of a funnel; at a short distance from the edge of the tube the natural folds of the mucous membrane begin to appear again, and, gradually increasing in height, run together like the folds of a paper filter, reducing the lumen of the urethra into a point or a short line which has been called the central figure (Centralfigur) by Gruenfeld. The form of the funnel largely depends upon the degree of pressure with which the tube is held against the urethral wall. If pressed firmly forward the mucons membrane will bulge into the lumen of the tube. During moderate pressure it will rest flat in front of the vesical opening, and on diminishing the pressure, or on gentle withdrawal, the surface will recede from the edge of the tube and form a funnel or cone. The more exactly the urethral tube is adapted to the size of the urethra, the smoother will be the surface of the funnel and the more slowly will the folds appear, while the mucous membrane will bulge into larger and wider folds whenever the tube fits loosely in the urethral lumen. The natural condition of the surrounding tissue will likewise influence the shape of the funnel, as well as certain configurations of the urethra itself.

Appearance of the Urethra.-The healthy mucous membrane shows a more or less pale pink or red color, quite often longitudinal stripes of lighter shade, and a moist, glistening surface, giving the appearance of a soft, dull, silky tissue, greatly resembling the mucous membrane of the oral cavity. The moist and smooth surface reflects the light thrown upon it in manifold shape, every irregularity becoming apparent through breaks 
or altered shape of the reflexes. Gruenfeld has attached great significance to these reflexes by studying them minutely, and drawing from them conclusions as to the condition of the mucous membrane. Indeed, in reading his book one becomes impressed with the idea that it is impossible to obtain a direct view of the objects, but only indirect knowledge of their condition by observation and interpretation of the reflexes. With narrow blackened tubes, which Gruenfeld persists in using, this is indeed the case, but with sufficiently large and polished tubes and good lights, as Posner has bluntly pointed out, we can simply and directly see the condition of the surface. Therefore, instead of studying the reflexes, they are rather to be avoided, and when they appear the position of the tube is to be changed a little, whereupon they will disappear. In the central position of the endoscopic tube, that is following strictly the axis of the urethra, these reflexes occur more frequently. While this method is more favorable for summary exploration, and is essential for demonstration of the changes in the elasticity of the mucous and submucous tissue, for the study of the surface of the membrane and the lacunæ Morgagni, irregularities of the epithelial covering, etc., the eccentrical position of the vesical end of the tube is much more preferable. By turning the proximal end to the right or left, above or below, the distal end will cover a portion of the interior of the mucous lining, and present it perfectly flat and level to the eye. By withdrawing the tube in slight spiral movements, the entire inside may be gradually unfolded and pass before the eye like a panorama, and allow of the closest observation of every irregularity.

Beginning now at the inner end of the urethra, at the prostatic portion beyond the colliculus seminalis, the mucous membrane forms a very flat cone of decidedly red color, molded into a number of sharply defined low folds which radiate toward the somewhat eccentrically placed lumen of the internal orifice. On starting the outward movement the flat cone or funnel begins to be slightly deeper, while the folds flatten and the color gets paler. Further on, sometimes gradually, sometimes quite suddenly, a more or less flat, rounded protuberance appears above the lower edge of the tube, somewhat paler, as a rule, than the surrounding portions, and with decided longitudinal stripes. This gradually gains in width and height until the lumen of the urethra appears semilunar. This is the colliculus seminalis. On further advance the protuberance ends abruptly, or gradually thins out into a small pointed ridge (caput gallinaginis), which sometimes can be followed as far as the bulbus. In the center of the colliculus quite often a small longitudinal slit or indentation can be distinctly seen, covered with clear, mucus-like fluid, leading into the socalled uterus masculinus. Where the colliculus appears more broad and ribbon-like, it passes gradually into the lateral portions of the mucous mem- 
brane, where it is more elevated and narrow, deep furrows are formed on both sides with a somewhat darker red, uneven surface. It is not possible to distinguish with any certainty the openings of the ductus ejaculatorii, or prostatic-gland ducts, which are located there, and sometimes manifest their presence by the sudden flooding of the field of view with a watery fluid. It is not always possible to keep the tube strictly in the axis of the urethra; it may then happen that only one side of the colliculus with the adjoining groove is seen, or that the tube passes through one of these lateral grooves, and the colliculus is turned toward one side and does not come into view at all. Altogether there reigns great individual variety in the configuration of this extremely complicated region, which with our present knowledge of the pathological conditions may render it very difficult to draw the line between normal and diseased conditions. It has happened that the colliculus was mistaken for a polyp. Occasionally two rounded folds of mucous membrane of a dark-red color bulge in from both sides like curtains over the whitish point of the caput gallinaginis, forming a triangular lumen or central figure.

Reaching the membranous portion, the mucous membrane now assumes a somewhat lighter color; the funnel becomes regular; radial folds unite in one point or into a short vertical slit. The transit from the membranous portion into the bulbus may be very gradual, withont a remarkable change of the color or of the shape of the folds. More often, however, the funnel appears less deep, and divides into several large, somewhat bulging folds. According to Finger, the progress into the bulbous portion is marked by a sudden jerk, the funnel disappears, and two large folds of mucous membrane cover the end of the tube. This is indeed occasionally observed in cases where the bulbus really forms a bag-like dilatation of the urethra. This formation, which is often depicted in anatomical demonstrations of the urethra, does not really exist in the larger number of cases. In the cavernous portion the color of the mucous membrane is of lighter pink, often with fine white stripes. It presents itself as a regular funnel or cone, divided into several more or less wide folds, and uniting in a horizontal slit as a central figure, which is often divided at both ends by broader lateral folds into two small vertical slits. The natural shape of the lumen of the urethra explains the horizontal figure which is maintained until near the glans. It then changes into a triangular form, to become a broad vertical slit between the rigid walls of the so-called fossa navicularis. As with the bulbus, it is often found that the assumed widening of the urethra within the glans does not really exist, but that the appearance of a cavity is solely due to the fact that the rigid urethral walls do not collapse like in the deeper portions. The color of the mucous membrane gradually assumes a more whitish tint, which within the glans turns into a yellowish or bluish one. The surface is generally 
smooth. Along the upper aspect the lacunæ Morgagni may become visible as very fine indentations, as if made with a pin, or very shallow impressions with a sharp, smooth edge. Dark-red lacunæ surrounded by a distinct red ring, as described by Finger and Burckhardt, I can not acknowledge to be a normal condition. In the bulbous and cavernous portion the endoscopic examination will hardly eause any bleeding in the normal condition, but in the membranous and prostatic portion even the most careful handling of the instrument may cause slight hæmorrhages, even in the absence of pathological changes. As a rule, very slight lesions heal without any difficulty, and do not cause any evil consequences.

It is obvious that if the urethra in its normal state can thus be inspected with the endoseope, the same can be done under pathological conditions, and that we are enabled to gain certain knowledge of the nature and seat of the pathological changes. It has been claimed that such knowledge can be obtained by other means, and that therefore the endoscope is not necessary for diagnosis. It is true that a great deal of knowledge can be acquired by examination of the urethra through the sense of tonch, but not even the subtle dexterity of the most experienced surgeon's hand will unravel some conditions, and doubts and errors will occur. The difficulties which present themselves to the effective treatment and definite cure of some affections of the urethra, particularly to the suppression of chronic discharges, and the frequency of these ailments, bear ample testimony to the inefficiency of these means. Many patients can be found who have had their chronic gonorrhœea treated by internal medicines, by the usual injections administered by themselves, by deep injections made by the surgeon, by irrigation, by sounds, and even by an abundant urethrotomy, and who still have a mucous or purulent discharge. In such cases the presence of numerous filaments in the urine makes the existence of some pathological condition almost a certainty, and the desire to look into the urethra and find the cause of the continnous discharge is but natural, and is justified by the fact that in the greater number of cases pathological conditions are easily found. However, in subjecting patients to endoscopic examination great precautions have to be taken. During the stage of acute, and even of subacute inflammation, the endoscope onght not to be applied.

Gruenfeld and other authors have described the appearance of the urethra in its different stages of acute gonorrhœal urethritis, but neither have any practical results been obtained, nor can the introduction of the endoscope in such conditions be considered harmless. Exceptionally an inspection may be justified through a tube of small caliber, where the presence of an ulcer or of a new growth is suspected; but as a rule no endoscopic examination onght to be made until the symptoms and exami- 
nation by other means make it probable or certain that the pathological condition does not extend over the entire length of the mucous lining, but is restricted to some more or less circumscribed portions.

The advice of Finger, to abstain from instrumental examination as long as there exists a copious mucous clondiness of the urine in connection with the gonorrhœeal filaments, may safely be followed. Tarnowsky's rule, adopted by Finger, not to examine a patient with the endoscope until by repeated introduction of metallic sounds it has become somewhat accustomed to the passage of instruments, seems to go too far.

Burckhardt advises beginning the first examination by the introduction of an elastic bougie-a-boule, to be followed by a big metallic sound. It is certainly necessary, unless the history of the patient reveals that sounds of a certain size have been passed within a certain time, to ascertain the caliber and sensitiveness of the patient's urethra by such means. But unless delay is, for some reason or other, out of the question, it is preferable not to let the endoscopic examination follow immediately, but to defer it for one or several days.

If the meatus does not admit easily a tube of at least No. 23, French scale, it must be enlarged in the usual manner. It has been advised by several authors to experiment on the cadaver before trying to use the endoscope on the living. Aside from the impracticability of this rule, the appearance of the mucous membrane on the cadaver differs so much from that on the living that comparison between the two is hardly of any value, and the difficulties met with in the introduction of instruments into the living urethra are entirely different from those on the dead. It must be distinctly understood, however, that nobody ought to undertake to use the endoscope until he is thoroughly acquainted with the anatomy of the urethra, and possesses some experience in the introduction of solid sounds and catheters on the living. The difficulties connected with the use of the straight instrument, against those with the curved one, have often been overestimated. The hand that knows how to tenderly guide the sound rather than push it will have no great difficulty with the straight endoscopic tubes. The first introduction of the endoscope is usually somewhat painful. The use of cocaine ought to be avoided, unless the sensitiveness of the patient makes it absolutely indispensable, on account of the contraction of blood-vessels and the consequent change in the color of the mucous membrane, at least under the influence of stronger solutions. A five-per-cent solution does not cause material alteration, and is sufficiently strong to make the procedure bearable. In subsequent sittings cocaine may be used. Most patients, however, become used to the endoscope quite readily, and will not need it.

While it is the rule to use the largest possible caliber of endoscopic tubes, it may be more judicions to be content with a smaller number dur- 
ing the first examination, so as to make the first experience with the somewhat startling procedure as easy as possible. For the same reason it has been advised, and correctly so, not to extend the first sitting beyond the bulbous or the membranous portion, and to be satisfied with a more cursory and superficial inspection, leaving a more minute exploration to the future.

Opinions are divided on the advisability of having the bladder emptied a short time or immediately hefore the examination. The retention of the urine, aside from the danger of its suddenly entering the tube, undoubtedly increases the discomfort of the patient during the examination and afterward, because he will be obliged to pass it soon after the operation, when the urethra is always more or less sensitive. The advantage of locating spots on which filaments and other secretions are formed is in reality often frustrated by the removal of the filaments by the rim of the tube during introduction. Where therapeutic measures are intended, it is particularly advisable to avoid early micturition after the operation in order to allow of the longer undisturbed action of the drugs on the mucous membrane. Besides, the cleaning of the surface by the stream of urine renders the removal of the secretions by means of cotton tampons more or less unnecessary.

The rules and conditions for the examination of the urethra in persons affected with disease are exactly the same as for the normal urethra, but the mucous membrane will present to the eye modifications from the normal condition in great variety and in numerous combinations, so that one meets with great difficulty in trying to arrange them in definite groups. Unfortunately, our knowledge of the histological conditions of the urethral tissue has been and is very incomplete, but recently Neelsen and Finger, by a number of methodical examinations of cadavers, have studied the changes of the tissue of the urethra. Although the lack of opportunity to examine the same portions during life and after death leaves some doubt about the interpretation of certain conditions, it must also be stated that the extent and the nature of the lesions found in chronic gonorrhœe are by no means always proportionate to the intensity of the preceding acute inflammation. Sometimes considerable alterations are found where at no time more than a very slight discharge had existed, and but insignificant impairment is found after a rather longcontinued profuse running.

In general, endoscopy has proved the erroneousness of some doctrines which have been proclaimed over and over in text-books. The regions pointed out spontaneously by patients as sensitive or particularly tender on the touch of a bougie-a-aboule are by no means always the seat of pathological conditions, but more frequently are found in healthy condition. Nor is it confirmed that the posterior parts of the pendulous 
urethra, the bulbous and membranous portions, are usually or even more frequently affected in chronic urethritis, as has been maintained.

It appears, on the contrary, that in by far the greater number of cases the anterior and central portions are the regions principally affected; that sometimes the two inches nearest the meatus alone are diseased. Among one hundred cases, I found the seat of the morbid changes to be the prostatic and membranous portion alone in five cases ; the prostatic and spongy alone in two; the prostatic, membranous, and spongy in twenty-two; the membranous and spongy alone in twenty-six ; and the spongy portion alone in forty-five cases. The prostatic portion was affected in twenty-nine, the membranous in fifty-three, the spongy in ninety-five cases, the bulbous especially in forty-four cases. This agrees very well with Finger's examinations on the cadaver, who, out of thirty-one cases, found the pendulous portion affected in twenty-two. Out of twenty-four cases relating to the pendulous or membranous urethra only, in seventeen the spongy portion alone was the seat of localized changes.

Pathological Appearances.-The impairment of the normal condition of the urethra may pertain to the color of the mucous membrane, to the character of its surface, and to the volume and elasticity of the entire urethral wall, which determines the shape of the funnel and of the folds in the endoscopic pictures. An cedematous, succulent condition of the mucous membrane is characterized by a reduction of the lumen of the funnel in every direction. It seems flat and narrow; the edge of the tube forms a sharp impression, which disappears slowly on removal of the instrument. In a very high degree of swelling the funnel is entirely obliterated, and the mucous membrane bulges into the tube itself. In all such cases the movements of the tube are followed rapidly by changes in the folds as in a kaleidoscope. In other cases this transformation proceeds more slowly. The receding edge of the tube does not leave an impression; the folds form more sluggishly, owing to the firmer swelling or infiltration of the mucous membrane. It, however, retains its elasticity, and does not offer any obstruction to the tube. Again, we find that the wall clings more tightly to the tube, and on its withdrawal does not collapse immediately nor fold itself, but forms a deep, sharply pointed funnel with rather patulous infundibulum and rigid sides. This sclerotic condition may be found limited in extent or affecting almost the entire urethra, showing either a perfectly smooth but almost white, tendon-like appearance, or more or less natural red color, which, however, on pressure gives way to a whitish, ivory-like shade. This diffuse hardening of the entire urethra, which has been described occasionally but without attracting much attention, must not be confounded with stricture. It ought to be designated by a distinct name, like diffuse sclerosis, or otherwise. This different configuration is due to 
the different degree of infiltration which, according to Finger, may be confined to the subepithelial layer, or may reach deeper into the mucous membrane, into the submucous and cellular tissue, and even into the cavernous tissue, in the latter case leading to retraction and stricture. Sometimes the infiltration does not affect the entire circumference of the urethra. The fumnel, and with it the central figure, appear irregular, occasionally one fold dropping before the lumen of the tube quite suddenly like a curtain. It is impossible, however, to follow up all these details.

Higher degrees of infiltration, which cause the condition generally described as stricture, render the introduction of a tube impossible. It may, however, be pushed as far as the obstruction, and furnish a view of the configuration of the stricture. Sometimes it has become possible, by exposing the front of so-called impermeable strictures, to pass a whalebone filiform bougie into the very small, often eccentrically situated lumen through the stricture, and, by introducing a tunneled catheter over the bongie, to open the stricture to further dilating treatment. In strictures of wide caliber the endoscope will furnish valuable information according to the extent and the degree of hardening of the tissue; in other cases the endoscope may be the means of demonstrating the spasmodic nature of strictures by the absence of all organic alterations of the seat of the suppossed stricture.

In the face of the often-made assertion that chronic urethritis inevitably tends to the production of infiltration of the submucons tissue, I must state that, in quite a number of cases of very long standing gonorrhøeal discharge, extending to eight and ten years, I have observed over and over again normal folds and the normal softness of the mucous membrane. Much more conspicuous than the changes of elasticity are those of the color of the mucous membrane. The natural pink may give way to all shades, from the pure glistening white of a tendon to deep scarlet, to darker shades of bluish and brownish red, or to slate color. These different tints may extend continuously over a large area, or may be restricted to spots or patches, or not rarely to radiating or longitudinal stripes. In considering the diagnostic value of these changes of the color, account has to be taken of individual differences, distant psychical or nervous reflexes, and of the immediate mechanical effect of the instrument. Sometimes the dull luster of the healthy mucous membrane gives way to a more perfect glistening smoothness of œdematous tissue. At other times it appears more shiny, like satin, or even as if varnished. More often, however, the surface loses its soft smoothness and appears rough, like the inside of undressed kid or thin leather. These conditions, which appear in great variety, seem to depend on pathological changes, and very often on the new formation of networks of blood-vessels, which, particularly in reflected sunlight, can be clearly distinguished. 
In other cases the surface appears like velvet, with minute pointed eminences; frequently alterations appear in the shape of more or less white longitudinal stripes, as a rule representing the ridges between folds. Sometimes the normal, sometimes the morbid portions are prominent, giving the appearance of smooth or uneven ridges. Again, the longitudinal or radiating prominences slightly project, with a perfectly smooth epithelial cover, apparently circumscribed subepithelial infiltration. Of more frequent occurrence are more or less circumscribed and disseminated granular patches. The dark-red, uneven surface is broken by elevations of the size of a rape or millet seed, or smaller, which are placed more or less closely together, leaving between them depressions of a deeper, more brownish tinge, and reflecting the light in the shape of numerous points. It is not unlikely, from Finger's observations, that the granular projections are due to considerably dilated blood-vessels rather than to the mucous glands or a papillary construction of the mucous membrane. The great tendency of granular patches to bleed on being touched even lightly would then be easily explained. Granulations have been described by all authors from the earliest days of endoscopy as almost regularly associated with chronic urethritis. From my own experience I can not share this view. The conditions which strictly answer to the so-called granular state are not of so common occurrence. While observing them in almost every part of the urethra, I have found granulations quite frequently associated with affections of the lacunæ Morgagni, which I have found in not fewer than sixty-three out of one hundred cases.

Sometimes on the first examination, sometimes after the swelling of the mucous membrane has been somewhat reduced by treatment, do these lacunæ appear as more or less sharply defined round or oval pits or depressions of the size of a small pin-head to that of a hemp-seed, and quite often covered by a drop of sticky mucous fluid. The edges now appear rough, as if eroded, now smooth and sharply cut; often their proximal end is covered by a semilunar fold or duplication of the epithelium of the mucous membrane; again they appear as slightly elevated craters. The lacunæ are most numerously distributed along the upper aspect of the central portion of the urethra, extending frequently to the lateral corners, which are formed by the meeting of the upper and lower walls of the urethra during natural rest, and sometimes on the lower surface. A particularly well-developed lacuna magna with a sharp semilunar edge is frequently found within or near the fossa navicularis. It is not unusual to observe sharply cut nicks or notches within the perfectly smooth mucous membrane. On extending them by lateral position of the tube and sufficient pressure, these notches may be developed and reveal at the bottom widened lacunæ. Finger's pathological investigations confirm the 
frequent participation and the important part assumed by the lacunæ Morgagni in chronic inflammation of the urethra.

In the lateral corners of the pendulous portions well-defined patches of irregular shape and size, of dark-red or brownish color, and of a dry, finely corroded surface, are not infrequently observed, which, on being touched with Lugol's solution, assume a dark-brown or almost black tint, which makes them vividly contrast with the surrounding normal and smooth portions of the mucous membrane which are not affected by the application of the iodine. Whether this reaction is due to the formation of amyloid tissue, or to changes in the epithelium from cylindrical to pavement cells, I am unable to say.

The occurrence of almost transparent sago-like grains or corns, imbedded in the intumescent mucous membrane, as in trachoma, has been mentioned by several authors. I can confirm the existence of such forms from one single but well-observed case. Circumscribed, mostly slightly elevated, perfectly white, and rather firm patches, rarely exceeding one third of an inch in length, are occasionally seen in different portions of the urethra. Gruenfeld has described them as the formation of new layers of epithelium (Epithelauflagerung), which opinion is borne out by Finger. A change of the nature of the epithelium is most likely connected with the thickening. Sometimes small, deepened white spots are noticed, which look somewhat like scars. It is necessary to mention here scars formed by internal urethrotomy, appearing almost always along the upper aspect of the urethra as perfectly white, more or less deep and wide furrows, extending only over a short space, but occasionally over the entire length of the pendulons urethra. Occasionally, two or three months after the operation, these furrows were found yet unhealed at the bottom. Even if perfectly smoothly healed, deep persisting furrows or scars may remain the seat of the formation of filaments.

Loss of substance is much less frequently found than new formation or thickening. More generally the defects do not extend deeper than the epithelial covering. Such spots appear somewhat depressed, yellowish, without luster, and are very sensitive to the touch. They were found mostly in persons of very nervous habits. Small, well-defined spots, not larger than hemp-seed, and forming a group, with a red surface and deeper colored edge, have been occasionally observed, closely resembling groups of herpes on other mucous membranes. Deeper defects of the mucous membrane, and ulcers bearing the characters of so-called chancroid, have been described. They usually are found near the meatus, where the initial lesions of syphilis are likely to occur.

Better observed are the tumors of the urethra, which occur either as warts or as mucous polypi. The former are frequently found directly within the meatus, often in company with the common venereal warts 
around the frenum and sulcus coronarius. Less often they are found in larger numbers extending into deeper portions, mostly grouped to one or a few larger conglomerates, or studding the surface as numerous disseminated smaller excrescences of most grotesque shapes. They may attain the size of a pea, with a broader base, or consist of single elongated papillæ with slender stems. They come into view, rather suddenly, rising above the edge of the tube as soon as relieved from its pressure, and on further withdrawal new groups come into view. They are generally of very light color, the extreme papillary prominence almost white or transparent, and are easily recognized from their irregular shape. As the mucous membrane normally shows a papillary structure only for a short distance from the meatus, it is difficult to explain their occurrence in the deeper portions, as far as the membranous urethra.

The larger smooth polypi, which are generally found in the deeper urethra, as far as the prostatic portion, likewise appear suddenly before the end of the tube as smooth rounded bodies of bluish color, somewhat transparent on the circumference, according to their size, covering more or less the entire field, or protruding from the edge. On touching them with a probe they are found to be soft and generally movable, and can be passed around, but do not bleed easily. Smaller ones may be brought into view in their totality. Larger ones may require several movements of the tube to demonstrate their volume. It is always necessary, by different manipulations from side to side, to bring the entire polyp within the lumen of the tube, so that its insertion may be determined upon. They may have a comparatively thin stem or grow from a broader base. In the membranous portion I have repeatedly found new growth of decidedly more fibrous or even of elastic structure. They were always elongated, some very small ones sharply pointed. A larger one, nearly one inch in length, protruded from the membranous portion into the bulbous, and, like the others, grew from the lower surface. It is probable that they owe their elongated shape and forward direction to compression by the muscles of the membranous portion, and to the force of the outstreaming urine.

The different pathological conditions just described may vary greatly as to distribution over the different portions of the urethra. Although they all may occur in different regions, certain forms show a decided preference for certain localities, like the enlargement of the lacunæ Morgagni, papillary excrescences, etc. Granular patches may be found all over. The prostatic portion may exhibit some peculiarities owing to its complicated formation, the changes pertaining principally to the colliculus seminalis. A great tendency to more or less profuse bleeding makes it often very difficult to judge of the true condition of these parts. The colliculus may be found considerably enlarged, often occupying the entire 
lumen of the tube, of a decidedly darker red color, without the smooth striped surface. On account of the succulence of the tissue, the opening of the sinus prostaticus can not be distinguished. According to Burckhardt, who has studied the pathological changes of the prostatic portion more than any one else, in cases of spermatorrhœa the opening of the sinus was more patulous, admitting a probe to a considerable depth, while the colliculus was enlarged, but showed no loss of elasticity.

In the face of such great variety of symptoms, the temptation to arrange them into separate groups and to differentiate several species of chronic urethritis is very great, and, indeed, since Désormeaux has been attempted by almost every author. Oberländer, more than any one else, has endeavored to create a systematic division and subdivision of the various forms of chronic urethritis, founded on their pathological alteration. However, the different conditions are found side by side at the same time in the same urethra, and at the same localities at different times, so that they really represent only various phases and various stages of development of the same process. It is therefore not advisable to complicate matters by the adoption of separate forms and names-certainly not as long as many questions are still awaiting solution from further microscopic investigations.

Endoscopic Diagnosis. - The value of endoscopy for diagnosis in the first line rests on the actual demonstration of the exact seat and character of numerous pathological conditions of the urethra which can not be detected during life by any other means, and which, in fact, were almost or entirely unknown before the introduction of the endoscope. On the other side, the examination enables us to definitely exclude the presence of some or any diseased condition at all, which may be of great importance in guiding investigation into the more remote portions of the genito-urinary tract. It may therefore safely be asserted that the endoscope is essential and indispensable in arriving at a certain diagnosis in chronic diseases of the urethra, and often alone affords the means of adopting a rational and successful plan of treatment.

While naturally demanding a certain technical skill and some practice, like every instrumental examination in the human body, endoscopy does not offer any difficulties which can not be conquered by industrious and energetic efforts. Therefore the diagnosis of chronic diseases of the urethra ought not to be considered complete without an endoscopic examination, certainly not by those who claim to make these diseases a special study.

The diagnostic facilities which it offers by no means exhaust the practical value of the endoscope. On the contrary, by making diseased portions of the urethra accessible not only to the eye but also at the same time to the hand of the surgeon, it affords a means for effective and 
direct local treatment, often in cases where other means have utterly failed. The very nature of some lesions described above immediately invites the application of drugs or operative interference which have proved effective in the treatment of affections of the mucous membrane of other organs.

That the scope of endoscopic treatment is a limited one is readily conceded; equally so that in many cases it will be useful only in connection with other treatment. Still, there remain occasions enough where it alone effects a final cure. In general, endoscopic treatment will be restricted to superficial alterations of the epithelium, subepithelial tissne, the mucous membrane proper, and to the glandular apparatus; while more deeply seated processes, new formation of connective tissue, and sclerosis, will remain unaffected. Strictures, therefore, and all cases with decided loss of elasticity, offer no field for endoscopic treatment unless associated with superficial lesions, except occasionally for the possibility to make a narrow stricture accessible to explorative bougies.

Endoscopic Treatment. - The treatment itself consists in the first line in the application of drugs in solution or in powder by means of cotton tampons or of a spray, or directly of the substance itself. Nitrate of silver is the most useful of these drugs, and is applied in solution of from one to twenty per cent, in which strength it does not develop really caustic or destructive effect, but only, according to the strength, increases the natural process of exfoliation and proliferation of the epithelium; while a fifty-per-cent solution, like the crystallized salt itself, really produces a more or less deep eschar.

Next to the silver nitrate the preparations of iodine are most valuable. Lugol's solution (iodine 1, iodide of potash 2, water 12) alone, or with equal parts of pure carbolic acid, bichloride of mercury in one half or one per cent alcoholic solution, sulphate of copper in three, five, ten, or twenty-five per cent solution and in crystals, liquor aquæ plumbi subacetatis, tannin as the glycerol of tannin, or ten-per-cent alcoholic solution, ten and fifty per cent solutions of chromic acid, liquor ferri perchloridi, powders of iodoform and of dermatol, resorcin, ten and twenty-five per cent solutions in glycerin, and trichloracetic acid in ten and twenty per cent solutions, complete the list of drugs. Ichthyol and salicylic acid probably deserve to be tried.

The immediate effect of all these medicines can be watched by the eye. In most instances the surface of the mucous membrane becomes dry, thereby bringing out more prominently every irregularity of the surface. Nitrate of silver leaves more or less white patches, the same as trichloracetic acid. Bichloride solutions likewise produce a dry, pale surface, while glycerin does not change the appearance of the mucous membrane, and apparently renders the applications less painful. 
The sensations produced by these applications vary greatly according to the individual susceptibility, but as a rule the posterior portion, including the prostatic and membranous, are much less sensitive to the chemical irritation. The more the meatus is approached, the stronger the expressions of pain. Even the application of mild substances produces, as a rule, a more or less acute inflammation, which generally becomes manifest by an increase of secretion within the first twenty-four or thirty-six hours, or sometimes after that period, and then gradually and spontaneously disappears again. If it does not show any diminution two days after its appearance, one or two injections of a mild solution of sulphate of zine or some similar salt are usually sufficient to reduce it to its former insignificant state. After the application of stronger solutions of copper, of corrosive sublimate, and tincture of iodine, the secretion generally becomes more copious and more watery; after nitrate of silver and carbolized iodine, more purulent. During the period of exacerbation the urethra is generally somewhat sensitive, particularly during micturition. It is therefore absolutely necessary to advise the patient beforehand of the probability of the occurrence of pain and increased discharge.

If even slight hæmorrhage occur during examination, it is equally advisable to inform the patient that he may find a little blood in his clothes, or rather on the pad of cotton which is usually placed in front of the meatus, for nothing is more liable to frighten patients than the unexpected appearance of blood. As a rule, the patient is to be enjoined to delay urinating as long as possible. Beyond the sequelæ just described, and occasionally a more continued renewal of copious discharge, where a sufficiently chronic condition had not yet been attained, I can not say that I have met with any particular accidents which would justify me to consider endoscopic treatment more dangerous than other methods of treatment. Epididymitis, particularly, occurs but rarely, certainly less often than during the use of sounds.

The length of the interval between the single endoscopic sittings has to depend partly on the sensitiveness of the patient, the intensity of the reaction, and on the strength of the application. Even mild treatment ought not to be repeated oftener than every three or four days, but with the stronger solutions not less than five to seven days ought to be allowed to pass, and as soon as improvement becomes more manifest, the intervals ought to be increased to ten days or two weeks.

It is impossible to definitely state in which conditions the one or the other drug, the milder or the stronger solutions, are to be preferred. At the first application it is safest to apply a solution of nitrate of silver not stronger than two and a half per cent, with or without iodoform or dermatol, which is not liable to irritate much. The conditions of the mucous membrane, together with the character of the secretion, will determine to 
a certain extent the selection of milder or stronger remedies. In more diffuse hyperæmia and swelling, with mucous discharge, the milder solutions of nitrate of silver and sulphate of copper and trichloracetic acid will be serviceable ; in more pronounced shrinking of the tissne, iodine or stronger copper solution. Granulations demand tincture of iodine, ten to twenty per cent solutions of nitrate of silver, and sulphate of copper, and particularly in connection with widened lacunæ, carbolized iodine and sulphate of copper in crystals. As long as gonococci are present, Burckhardt strongly recommends the use of one-per-cent alcoholic solution of corrosive sublimate, the good effects of which I am able to confirm. The brown, dry patches of the lateral folds are best influenced by iodine, under which they gradually assume a lighter hue and smoother surface. The real caustics are applied only to small excrescences which can not well be reached with instruments.

Erosions are the least tractable conditions. I have seen the best effects from a very mild solution of silver, even less than one per cent, and of iodoform. The white patches of thickened epithelium become less prominent under applications of tincture of iodine, and particularly of chromic acid, but it seems doubtful whether they really demand any treatment, as it is not apparent that they cause trouble.

The changes in the prostatic urethra, particularly the swelling of the colliculus, require as a rule the strongest applications. With Burckhardt, I have sometimes found the only effective remedy to be the galvano-calltery. Polypi and large warts require removal by surgical means. Only for smaller excrescences caustics are sufficient. Gruenfeld has constructed quite a number of instruments for this purpose. The instrument which I devised in 1887 has been found useful by several surgeons. It consists

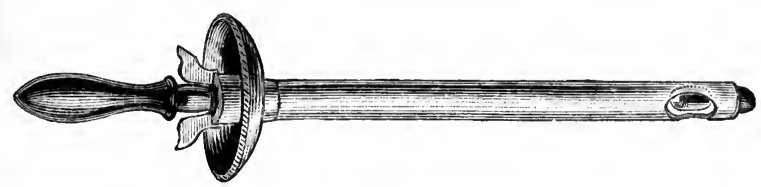

Fig. 7.

of two concentric metallic tubes, the inner one revolving readily within the outer. At the same point near the visceral end of the tubes there is in each an elliptic window. The instrument is introduced so that the polyp projects into the lumen between the two openings. Revolution of the interior tube (the edges of whose windows are sharpened) then cuts off the tumor at its base. The sharp curette can also be used for the removal of smaller growths. The most convenient way, however, for the removal of all growths is probably by the different shapes of the galvanocautery devised by Burckhardt. 
It is remarkable how much changed the urethra often will appear after the first application of some mild solution. The mucous membrane gradually assumes a more natural pale color, the surface becomes smoother, the lacunæ. Morgagni show sharp, well-defined edges and become more and more shallow, differing less in color from the surroundings. The improvement of these organs seems to have a decided relation to the number of filaments in the urine.

With the increasing strength of the epithelial covering the tendency to bleeding gradually subsides. Hæmorrhagic tendency is best treated by applications of resorcin, iodine, or perchloride of iron diluted with glycerin. Altogether, however, the results of endoscopic treatment are quite variable. Sometimes you will be surprised by the rapidity with which the mucous membrane assumes the normal aspect and the symptoms cease; and on other occasions the patience of everybody concerned will be tried to the utmost. It is therefore advisable to abstain from any assurance with regard to the probable duration of the treatment. Whenever, after a reasonable time and after judicious changes in the selection of remedies, apparently no progress is made, it becomes necessary to abandon endoscopic applications, at least for a time, and resort to some other mode of treatment, occasionally even to some internal remedy. Often enough a cure may then be effected by a method which had been tried in vain before, but which will prove beneficial now under conditions altered by the endoscopic treatment. The concomitant use of a mild injection, at least at the beginning of the treatment, is generally advisable. By gradually omitting the same while the patient is allowed to resume his former habits of life, the danger of relapse is greatly diminished.

The effects of endoscopic treatment are but rarely very brilliant and magic-like. As a rule, a cure will be obtained only after a number of applications and after patient work; but, considering the meager success of other means, and the deplorable condition of many patients, a method which certainly effects a cure in a limited number of cases in which other means have failed, deserves a permanent place in the therapeutics of chronic urethral diseases, which after a long trial it has now fairly won. 


\section{GONORRHCEAL OPHTHALMIA.}

Syn.: Gonorrheal Conjunctivitis.

\section{By JOSEPH A. ANDREWS, M. D.}

GoNoRrhGal ophthalmia is an acute purulent inflammation of the conjunctiva, caused by the transfer of the discharge from an acute or chronic gonorrhœal inflammation of the genitals either by the patient himself conveying the poison from his genital organs on his fingers, or linen, towel, etc., either consciously or during sleep, or from the transfer of the poison from an eye similarly affected.

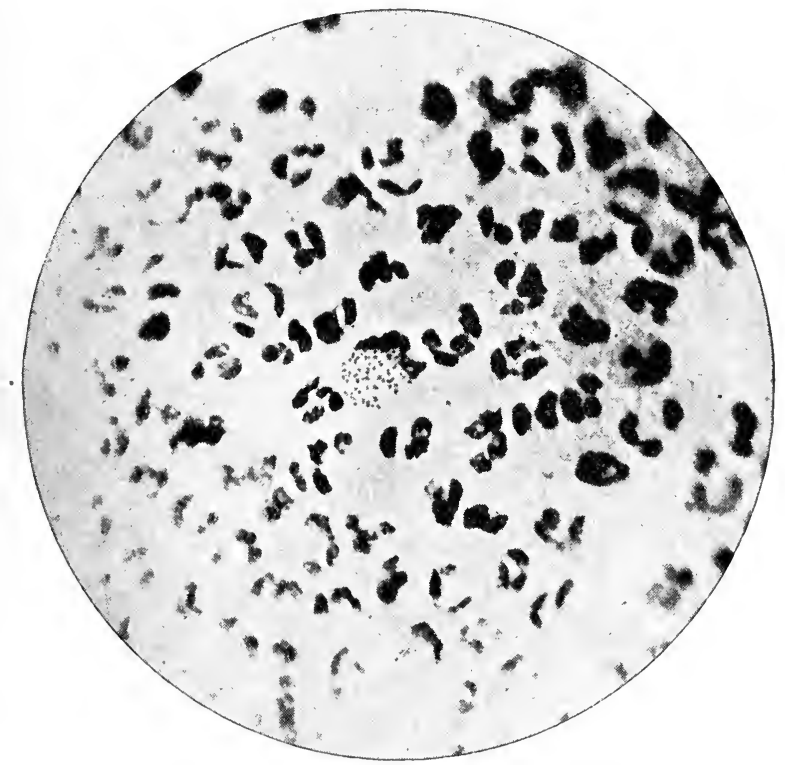

FIG. 1.-Gonococei in conjunctival discharge. Stained according to the method of Schütze. The pus-cells and their nuclei are brought out by the double-stain with safronin. Only one puscell shows the gonococi in this field, but the picture is more satisfactory than the others in which several pus-cells contained them. Zeiss apochromatic objective $2.0 \mathrm{~mm}$., numerieal aperture $1 \cdot 2^{\circ}$ and projective evepiece No, 2. The amplification, about 500 diameters, revealed a very satisfactory microphotograph of this diplococcus, but the reproduction process gave a too faint impression; the pieture was, thereforc, enlarged about one half in the reproduetion. Greater amplification than 500 diameters loses in sharpness what it gains in extent. Dr. E. K. Dunham, Direetor of the Carnegie Laboratory, this eity, kindly made the microphotograph from which this illustration has been produced. 
The investigations of Bumm, and quite recently those of Wertheim (Centralblatt für Gynäkologie, 1891, No. 24, p. 484), who cultivated the gonococeus on human blood-serum, show that a specific germ, the gonococcus, first described by Neisser, of Breslau, in 1879, is the active agent of this disease. The gonococcus almost always occurs in the form of diplococci. In stained preparations and with the highest power-i. e., homogeneous oil-immersion and Abbé's condenser-this coccus is seen as an elongated body, with a furrow in its middle, dividing it into two halves. This is the appearance of the coccus undergoing division.

The gonococci in the secretion lie chiefly in the protoplasm of the cells in small, irregular heaps. It may readily be stained by Schütze's method * (München med. Wochenschrift, 1889, No. 14).

In the following table I have endeavored to show the relative frequency with which the gonococeus is found in active and chronic gonorrhœa:

\begin{tabular}{|c|c|c|}
\hline 1 & $\begin{array}{l}\text { Number of } \\
\text { cases } \\
\text { examined. }\end{array}$ & \begin{tabular}{|l} 
Number of \\
cases in which \\
gonococcus \\
was found.
\end{tabular} \\
\hline 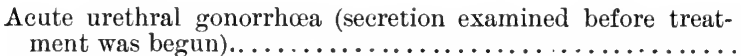 & \multirow[t]{2}{*}{17} & \multirow[t]{2}{*}{17} \\
\hline $\begin{array}{l}\text { Chronic urethral discharge following gonorrhoea (all these } \\
\text { patients had been treated, but were not under treatment } \\
\text { when the secretion was examined). }\end{array}$ & & \\
\hline $\begin{array}{l}\text { Purulent ophthalmia in adults (typical clinical picture of } \\
\text { gonorrhoal conjunctivitis; all of these patients, excepting }\end{array}$ & 144 & 108 \\
\hline 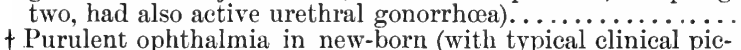 & 17 & 72 \\
\hline ture of gonorrhœeal conjunctivitis) $\ldots \ldots \ldots \ldots \ldots \ldots \ldots$ & 122 & 122 \\
\hline 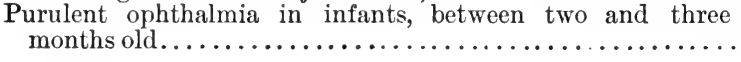 & & 3 \\
\hline Total............... & 364 & 322 \\
\hline
\end{tabular}

Purulent ophthalmia does undoubtedly occur quite independently of gonorrhœa ; but in such cases the clinical picture of gonorrhœal ophthal-

* The carefully prepared cover-glass preparation is placed from five to ten minutes in a cold filtered saturated solution of methylene blue, prepared with a five-per-cent watery solution of carbolic acid. Next wash cover-glass in water and place it for a moment (long enough to count one, two, three, slowly) in a solution of acetic acid ( 5 gtt. acetic acid to 20 c. c. distilled water), and at once again thoroughly wash cover-glass in pure water. The gonococei remain distinctly blue.

† Since the publication of the above table (New York Med. Jour., June 21, 1890, p. 682) the writer has examined the secretion from the vagina of the mothers of eighty-eight infants, in which ophthalmia developed from fifty to seventy-two hours after birth. The gonococcus was found in two only of these cases in the mother and child-the one infant being infected at birth and the other six days after birth through carelessness on the part of the mother. The typical clinical picture of gonorrhœal conjunctivitis was absent in eighty-six cases; nevertheless, one eye was lost in five infants and both eyes in one infant, the disease being non-gonorrhœal. 
mia is absent, and the disease has not, in my experience, been of the severe type which is nearly always seen in gonorrhœal ophthalmia. Gonorrhœal pus contains, in addition to the specific diplococci, other bacteria, some of which look very similar to the gonococcus; and when such diplococci, morphologically identical with the gonococci, are found outside of pus-cells or in suspected fluid which does not contain pus, it is impossible to fix their character until we have resorted to a staining method by which we now believe we are able to differentiate them from all others which they resemble morphologically. The Gram-Roux* method is the best known at present for this purpose.

Period of Incubation.-It is generally impossible to fix the exact moment at which the poison of gonorrhœa has been brought in contact with the eye. Bumm, in his successful inoculation of the urethra with a pure culture of the gonococcus, stated that, for two days following the inoculation, no phenomena were observed. On the third day there was burning in passing urine. However, objective signs of infection may have preceded this subjective symptom for a short time. Moreover, gonorrhœal pus is more virulent than the pure culture of the gonococcus. This fact would seem to be established by the observation that investigators have not always succeeded in reproducing the disease with cultures of the gonococcus; whereas gonorrhœal pus, placed on a healthy human

* Gram-Roux method: A drop of the suspected fluid pus, etc., is placed in the middle of a clean cover-glass; a second cover-glass is laid upon this, and the two are pressed gently together; the cover-glasses are separated by sliding one over the other, not pulling them apart. The cover-glass is now passed two or three times slowly through the flame of a spirit-lamp to coagulate the albumin. The staining is now done by immersing the specimens for twenty minutes in an aniline, gentian, or methyl-violet solution, which should always be freshly prepared: add a few drops of pure aniline-oil placed in a testtube three quarters filled with distilled water and thoroughly shaken. The emulsion is now filtered, and to the clear aniline water is added drop by drop a concentrated solution of methyl-violet (methyl-violet $2 \cdot 25$ parts, distilled water 100 parts) until a slight cloud appears. Excess of color is removed from the cover-glass by washing in a stream of water. The specimen is next immersed for five minutes in the iodine-iodide-of-potassium solution (iodine, 1 part; iodide of potassium, 2 parts ; distilled water, 300 parts). After this, the washing is done as before. This procedure fixes the color on the micro-organisms in general. The specimen is now examined while still wet with an oil-immersion lens. If microorganisms resembling the gonococcus are found, we proceed to test by decolorizing with alcohol. Gram's liquid does not fix the color on the gonococci; alcohol removes the color from them. The specimen is treated with absolute alcohol until the color is as completely removed as possible. It will be now found that all gonococci have disappeared, while all the other organisms which may have been present will be distinctly risible. If, after having been decolorized in alcohol, the specimen be again lightly stained with a solution of Bismarck brown (care must be taken not to overstain; a few drops of the Bismarck-brown solution are allowed to remain on the preparation for six to ten seconds), the diplococci which disappeared under the action of alcohol will again be seen, but of a lighter color than other diplococci (not gonococci) which may have been present in the specimen, and which still retain the original stain. 
conjunctiva, reproduces the disease with the precision of a physical experiment. We may expect to find signs of infection within forty-eight hours after contact of the poison. These signs at the outset will be those of simple catarrhal conjunctivitis-i. e., hyperæmia of the conjunctiva and lachrymation.*

Symptoms. - The first signs of infection are hyperæmia of the con. junctiva, increased lachrymation, and a sensation of irritation, as if a foreign body-i. e., dust-were lodged beneath the lids. This condition is rapidly intensified, and the pronounced picture of the disease is reached on the second or third day. At this time the eyelids are intensely red and glistening, painful to the touch, and so much swollen and œdematous that the patient is generally unable to open the eye. On separating the lids, a copious watery discharge, $\nmid$ with flakes of mucus, pus, and epithelium suspended in it, escape. The conjunctiva is of a deep red; its surface is smooth and glistening, or, in the worst cases, there is a plastic infiltration into the conjunctiva, especially noticeable in the swollen conjunctiva of the globe. The retrotarsal fold of the conjunctiva may also be much swollen and come into view when the lids are separated. When the conjunctiva of the globe is much swollen (chemosis), it forms an annular wall around the cornea (see colored plate, Fig. 2, right eye). The local temperature is increased, and the pain in the eye and frontal region is very severe.

The bodily temperature even in the severe cases does not usually run high, but the loss of sleep and the consciousness of the danger to the sight are so demoralizing in many instances as to make the patient feel like a very sick person. The disease progresses very rapidly, and the discharge, undiminished, becomes more turbid, and finally pure pus. The pus wells out from between the lids, and flows over the cheeks, where it dries in scabs, excoriating the skin beneath. If now the lids be separated, the pus will be seen covering the surface of the cornea, and collected in the hollow formed by the swollen conjunctiva surrounding it, presenting an appearance which might excite the suspicion that the cornea is

* These are the signs for which we must especially be on the lookout in the felloweye, which, if unaffected, will be covered with the protective shield. But we must not confound the slight injection of the conjunctiva in such cases (which may be due to the heat, etc., occasioned by the protective shield) with true infection. A quiet gonorrhœal conjunctivitis (hyperæmia, increased lachrymation, and photophobia) which has been observed in gonorrhœal rheumatism, may puzzle us in a given ease of active, urethral gonorrhœa with rheumatism, but such severe purulent conjunctivitis does not develop in these rare eases, and the treatment must always be of the mildest kind.

t The attendant should be careful of his own eyes in separating the lids in this stage, as fluid in considerable quantity is sometimes pent up in consequence of the edges of the lids being glued together, and when the lids are separated the fluid may be projected for some distance. 
involved in a suppurative process. This suspicion is removed or confirmed by washing away the discharge. The ocular conjunctiva, if swollen, is soft, or in the severe cases firm, in consequence of the serous effusion into the conjunctival and subconjunctival tissue being replaced by a plastic effusion. The great danger now is the implication of the cornea. The swelling and pressure of the eyelids, the chemosis, compress the vessels supplying the cornea and thus impair its nutrition, the evidence of which will be a hazy appearance of the cornea. But it is the irritating noxious character of the discharge which produces ulceration of the cornea. Although cloudiness of the cornea is an unfavorable symptom, it frequently disappears under faithful attention to the antiphlogistic treatment-cold and irrigation. Ulceration of the cornea is always a very serious complication; the earlier in the history of the disease it occurs, the graver the consequences. The ulceration (see colored plate, Fig. 2, right eye) shows itself as a small loss of surface of the cornea at its center or periphery. The ulceration spreads in surface and depth, until, unless arrested by treatment, it involves a more or less considerable extent of the cornea, leading to perforation and its consequences, prolapse of iris, staphyloma of the cornea, and, in the worst cases, to purulent panophthalmitis.

But these terrible consequences are not inevitable, even where the cornea is involved to a considerable extent in ulceration, for we are often able, by appropriate treatment and constant vigilance, to rescue the eye, and give the patient useful vision by the performance of an iridectomy after all signs of irritation have disappeared. But this is the darkest side of this terrible disease. In a large proportion of the cases we may succeed in directing the disease through a favorable course. Then there will follow a gradual diminution of the swelling and secretion, and recovery in about six weeks. In other cases in which the cornea escapes injury, the conjunctiva takes on a condition of chronic inflammation, which does not usually endanger the cornea, and yields to treatment.

Anatomy.-We are indebted to Bumm for the anatomical changes in gonorrhœal conjunctivitis. Bumm's investigations were almost exclusively of preparations of gonorrhœal conjunctivitis in the new-born. These inquiries showed that the gonococcus penetrated the cylindrical epithelium and extended principally in the intercellular tissue, leaving the parts covered with pavement epithelium intact. Toulon, Dinkler, Jadassohn, and others have disproved this latter statement, by showing that the gonococci penetrate into the upper layers of the subepithelial tissues and lie there mostly free. The invasion by this bacterium gives rise, on the one hand, to exfoliation of the epithelial layer ; on the other hand, in the conjunctival tissue, to the migration of white blood-cells, infiltration with round cells, and suppuration. The chief multiplication of the gonococci takes place in the secretion and in the epithelium. Bumm surmises that the 
gonococei in the subepithelial tissue die rapidly, because the conditions there are not favorable to their further development.

Influence of Age.-Recently, Spicer (The Royal London Ophthalmic Hospital Reports, December, 1891, pp. 211-247) has shown statistically, in his eminently practical paper, that there is a marked difference in the resistance of the tissues to the destroying action of the poison of gonorrhœa in the different periods of life. The cornea suffers least in early adult life. In every case in which the age was over thirty-eight years, the eye was lost. However, in this connection we should take into account the character of the inflammation. The severer forms of the disease, in our experience, are as destructive in early adult life as later.

Prognosis. - The issue in every case will depend on the duration of the disease before the application of treatment, the character of the inflammation, the severity of the inflamma-

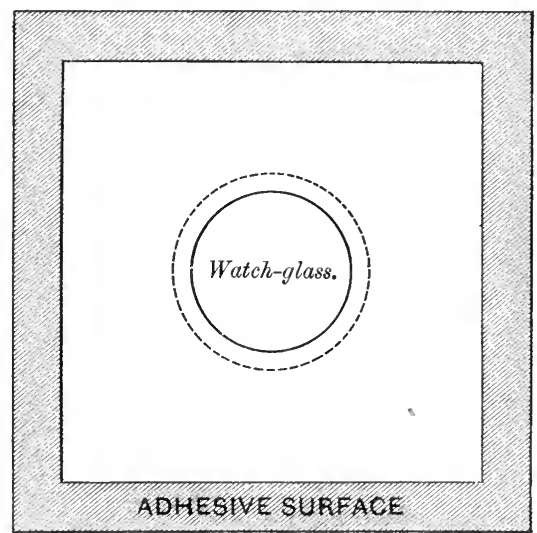

FIG. 4.-Buller's protective shield (reduced one tion, and, further, upon the condition of the eye previous to infection. An eye which is the subject of a pre-existent chronic inflammation of the conjunctiva-i. e., trachoma with pannus *-will be less seriously affected by the infection with gonorrhœa than when the conjunctiva is normal.

Treatment. - The patient must be put to bed. Two nurses, one for the day and one for the night, are indispensable. If one eye only is affected, the healthy eye must be protected. The best protective covering is Buller's shield (Fig. 4). This shield consists of two pieces of rubber adhesive plaster, one four inches the other four and a half inches square, between which, in a hole in the center, an old-fashioned deep watch-glass is fastened. The adhesive plaster is fixed by its free edge to the nose, forehead, and cheek, leaving only the lower and outer angle a little open for purposes of ventilation. This shield enables the patient to see, and the surgeon to inspect the eye, without removing the dressing.

The eye must be kept as free from discharge (day and night) as possible by frequent washing, the frequency of the washing to be regulated by the amount, of the discharge ; in other words, the discharge must be washed

* Previous to the introduction of jequirity, trachoma with pannus was in aggravated cases treated by inoculating the eye with gonorrhœal pus. 
away as soon as it forms. In the interval of washing, iced cloths must be kept applied (day and night) to the eyelids. The cold is best applied by means of pledgets of linen, two inches square, kept cold on a block of ice at the bedside, and laid on the affected eye, changing the linen before it becomes warm. These pieces of linen must never be reapplied to the block of ice, but be burned at once. The eye should be washed with a solution of boric acid (three per cent), or bichloride of mercury ( 1 to 10,000 or 1 to 20,000$)$; the boric-acid solution is preferable. The solution of

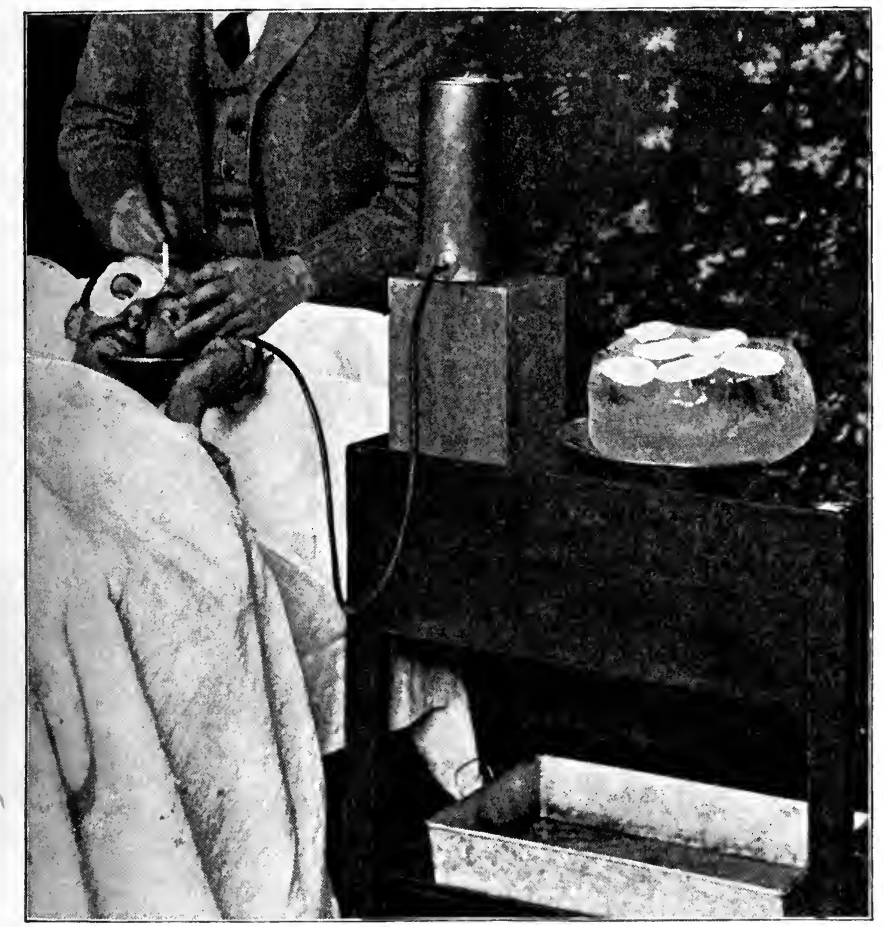

Fig. 5.-Patient in position for irrigation. Pus-basin held by patient under affected eye. Buller's protective shield over right cye. Pieces of linen on block of ice. Pan under table to receive soiled iced-cloths.

boric acid should be prepared with boiled water from the kettle, to insure its being clean. The solution of bichloride of mercury should be prepared with distilled water. The solution should be used cold.

Fig. 5 represents the method employed at the Charity Hospital for applying cold and irrigating the eye. The right eye has been protected by Buller's shield; the head is inclined toward the left, and a pus-basin held by the patient himself just beneath the eye receives the discharge when the eye is irrigated. The fountain syringe, with rubber tubing attached, 
is placed just high enough above the head of the bed to give the flow sufficient force to play in a gentle stream on as much of the conjunctiva

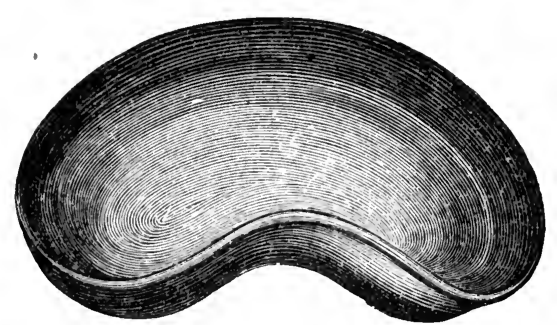

Fig. 6.-Pus-basin. as can be exposed by gently separating the lids.

Some year's ago I devised an irrigator * which I have found useful in many cases. It must be inserted with great care and gentleness beneath the upper lid, to avoid injury to the cornea and giving pain to the patient, for the lids are generally extremely sensitive. When beneath the lid, and the solution is flowing through it, it should be held slightly away from the eyeball. Only a carefully trained nurse should be intrusted with this instrument. A glass tube a quarter of an inch in diameter and two inches long is a useful substitute for this irrigator. The lids being separated with the thumb and index-finger and the upper lid slightly tilted away from the eyeball, the point of this glass tube is held close to the eye (without touching it), and the stream directed up into the conjunctival cul-de$s a c$, and the discharge in this part washed out as thoroughly as possible. It is our custom at the Charity Hospital, in washing out the eye, to allow the stream to play on the conjunctival surfaces for one, two, or three ininutes at a time when the discharge is profuse. If the swelling of the lids is very conspicuous, and it as well as the chemosis shows no disposition to subside under treatment, the outer commissure may be cut to the bottom of the conjunctival cul-de-sac. This is best done with a pair of strong, sharp scissors that will cut to the points. The lids being separated + with the index-finger and thumb (as shown in Fig. 8), thus putting the skin slightly on the stretch, and enabling the surgeon to make a straight cut, one blade of the scissors is inserted between the eyeball and the lid, and the point well down to the bottom of the

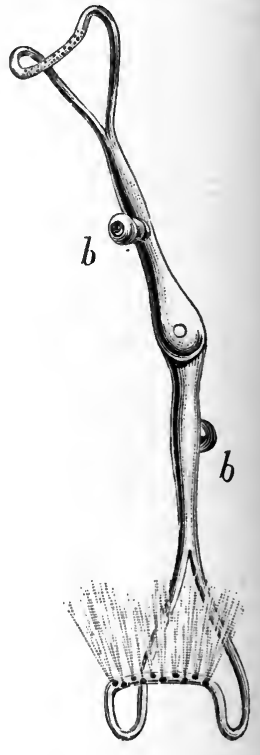

FIG. 7.-Author's irrigator. Consists of a hollow folding lid-elevator of large and small size. Tubing of fountain - syringe is attached at $b$.

* New York Med. Jour., 1885. This irrigator is simply a modification of the foldinglid elevator. one extremity smaller than the other, the whole being hollow, with fine holes at the extremities for the passage of the fluid.

+ The fingers should not touch the eyeball, but be applied to the upper and lower borders of the orbit, respectively, and the lids separated by rolling the skin over the bone. 
cul-de-sac. The blades are now quickly and firmly brought together, making the cut through the skin and conjunctiva, along the line of incision shown at $\mathrm{A}$ in Fig. 8.

\section{Indications for the Application} of Nitrate of Silver.*-In my hands, nitrate of silver has been more useful than any other remedial agent in the treatment of gonorrhœal conjunctivitis. But nitrate of silver is never depended upon to the exclusion of the most exacting cleanliness and attention to the uninterrupted application of cold. The silver assists, nothing more; and unless we employ it at any other time than when it is indicated by the condition of the conjunctiva, it will do harm, even in the weakest solutions.

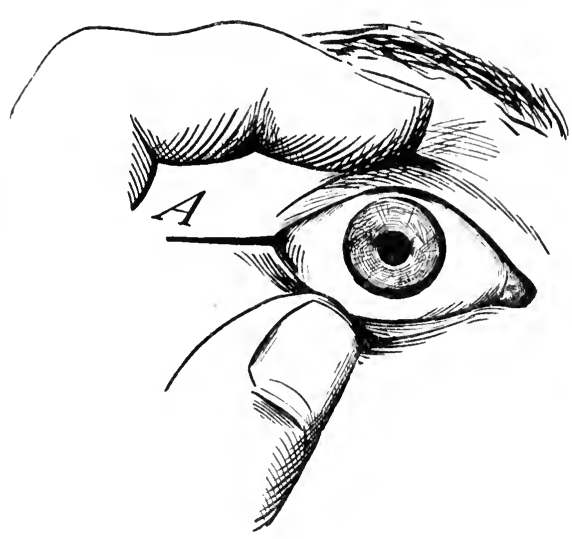

Fig. 8.-Canthotomy. A, Line of incision with scissors.

* Nitrate of silver has been selected, because we consider it the safest germicide to employ in gonorrhœal conjunctivitis. But its value as a germicide is limited. When we have thoroughly tested the germicidal value of a given agent in the laboratory, as shown by its behavior in pure cultures of the pathogenic bacteria, it does not follow that we are therefore prepared to go forth fully armed for the therapeutical contest. Such experiments have impressed upon us the great importance of cleanliness in the treatment of wounds. They teach us that everything that touches a healthy wound must be free from living pathogenic or pyogenic bacteria. But we are not so clearly informed concerning the safest means to adopt to destroy these bacteria when they are already present in the tissues. There is in the body some power of adjustment of forces by which disease is limited or disposed of ; and as we now associate pathogenic bacteria with the cause of disease, and as gonorrhœa, like some other diseases caused by bacteria, is known to be selflimited, and will be eliminated spontaneously, we must assume that the tissues contain a bacteria-destroying agency. The ideal germicide, therefore, would be such a one as would, while destroying pathogenic bacteria, at the same time, instead of irritating the tissues, aid them in their inherent quality of resisting the incursions of the mischievous bacteria. We do not yet possess such a germicide. It is evidently not nitrate of silver. The gonococcus, as has been said, invades chiefly the pus-cells and epithelium. The twoper-cent solution of nitrate of silver exfoliates the epithelium, and destroys the infective properties of the pus in less time and with less harm to the tissues than the stronger solutions, or any other known agent. It is these facts which led the writer to recommend (New York Med. Jour., 1886) the frequent instillation of a two-per-cent solution of nitrate of silver. Sixty years ago Mackenzie (his Text-Book) treated puruleut ophthalmia by washing the eyes with a solution of bichloride of mercury $(1$ part in 4,000$)$ three or four times daily, and brushed a solution of nitrate of silver (4 grains to the ounce of water) over the conjunctiva every six or eight hours. Therefore, in one respect the treatment of purulent ophthalmia with repeated instillations of a two-per-cent solution of silver is not a novelty. But Mackenzie had, of course, no thought of using silver for its germicidai properties; and indeed this solution was laid aside for stronger ones, until the mitigated or solid stick of 
Most text-books * on ophthalmology oppose the application of nitrate of silver or any caustic in the active stage of gonorrhœal conjunctivitis. It has been my experience that the instillation of two or three drops of a two-per-cent solution of nitrate of silver in the early stage of the disease has enabled me, during several years, to save many eyes. The course of the disease has certainly, in many instances, been rendered less severe. The solution which $I$ use is never stronger than two per cent. In the early stage of the disease this solution should be applied once only in twentyfour hours, and then by the surgeon himself.

In connection with the application of silver to the conjunctiva, the following facts should be borne in mind: The eschar produced by the silver is thrown off much more quickly, and with less irritation, from the conjunctiva of the lid than from that of the eyeball. The more hyperæmic and succulent the conjunctival membrane, the more rapidly will the eschar produced by the silver be cast off, and the less time will be occupied in the regeneration of the epithelium. In other words, the shorter will be the two periods following the cauterization; i.e., the period of irritation-the casting off of the eschar-and the period of regeneration of the epithelium. If we bear these simple facts in mind, they will furnish us with the indications for withholding or repeating the applications of the nitrate of silver.

The plan of treatment employed at the Charity Hospital is to instill $†$ two or three drops of a two-per-cent solution of silver immediately after the eye has been freed of discharge by washing, the ice-cloths being applied at once after this application. The cold applications here shorten the period of reaction. If the discharge be not profuse, we do not repeat the instillation on that day; should, however, the discharge increase, the swelling of the conjunctiva persist, and the conjunctiva bear the caustic well, then we repeat the application of silver, say, two or three times in twenty-four hours. Our object here is to control the purulent secretion; but we must also always consider the cornea, and the behavior of the conjunctiva toward the caustic. The application of silver must not be repeated as long as the eschar is present.

Occasionally cases are observed in which, at the end of the first week,

silver had supplanted them. Some of our text-books on diseases of the eye still advise the use of the solid stick of nitrate of silver in purulent ophthalmia, but this recommendation is one of those things which have gained credence from having been transmitted from book to book. The solid stick of nitrate of silver should never be used in the treatment of purulent ophthalmia.

* Schmidt-Rimpler advises the use of a weak solution of silver in the active stage of the disease; but he, in common with other authors of text-books, omits to give the indications and contra-indications for the use of silver.

f Or the silver solution is applied to the conjunctiva on absorbent cotton wrapped about the end of a match. 
the disease has assumed such a mild type that we are tempted to believe that all the danger is over. We must not be deceived by these appearances, but continue our vigilance just as before. Pcrforation of the cornea and loss of the eye may occur when the disease seems far advanced toward recovery. We must therefore be on the lookout for acute exacerbations, and remember that in any such case the cornea is exposed to new danger.

Should the cornea become involved, sulphate of atropine (gr. $\mathrm{ij}-\xi_{\mathrm{j}} \mathrm{j}$ ) is instilled three times a day; or sulphate of eserine (gr. $\frac{1}{8}-z^{3} \mathrm{j}$ ) may be substituted in ulceration of the cornea. When the cornea is involved the silver is never instilled into the eye. If deemed advisable to use it at all in such complications, it should be brushed over the conjunctiva of the everted lid.

The two-per-cent solution of silver is preferred, because the weaker solutions have generally been inefficient, and the stronger ones no more efficient, but attended with greater risk. Should the disease be complicated with a diphtheritic process, the nitrate of silver should not be used; and when the conjunctiva is covered with a membranous deposit, we had better withhold its use. The silver in such cases will not penetrate the deposit, but there is danger that the solution may flow into the crater formed by the swollen conjunctiva around the cornea, and, lying there, injure the cornea by removing its epithelial layer, thereby exposing it to infection.

I have abandoned the practice which I recommended four years ago (New York Medical Journal, October 31, 1885), of flooding the surface of the eyeball with vaseline in all cases of this disease. We found that nurses trusted too much to the ointment, and neglected the washing of the eye. We have found the vaseline-oil (poured between the lids frequently and freely) of benefit in those cases of the disease in which the conjunctiva of the eyeball is firm (plastic infiltration); also in all cases after the more acute inflammatory symptoms have subsided, and the conjunctiva ceased to secrete profusely. The duration of gonorrhceal conjunctivitis is from four to six weeks.

Preparations of zinc, carbolic acid, bichloride of mercury, iodoform, peroxide of hydrogen, have absolutely no serious claim t.pon our consideration in connection with the treatment of this disease, by which statement we mean that "they have been weighed in the balance and (by comparison) found wanting."

The nitrate of silver, in our hands, has proved itself to be the safest and best remedial agent in the treatment of gonorrhœal conjunctivitis; but it is one which requires caution in its application, and full appreciation of the fact that the chief reliance must be placed in the uninterrupted application of cold, and thorough washing of the eye with an un- 
irritating aseptic fluid, a saturated solution of boric acid being given the preference.

\section{OPHTHALMIA NEONATORUM.}

\section{Syn.: Blennorrhoea Neonatorum.}

This disease may result from direct infection from the mother during the birth of the child. When the disease occurs within forty-eight to seventy-two hours after birth, it is most probably in this way. It is, however, more frequently communicated after birth, through the agency of the fingers of the nurse or the mother, or in the first washing after birth, when any infectious discharge which may adhere to the infant's body is mixed with the water of the bath, and in washing the face is introduced between the eyelids. The child may be infected by the mother through her fingers or cloths, which may be soiled with the discharge from her genitals. It is not necessary that the mother's vaginal discharge shall be gonorrhœal; other abnormal vaginal discharges may give rise to purulent conjunctivitis in the new-born and be quite as disastrous to the sight as is gonorrhœal conjunctivitis. In every instance of purulent ophthalmia in the new-born presenting the clinical picture of gonorrhœal conjunctivitis in which I have examined the discharge, I have found the gonococcus. But I am disposed to the belief, based on those microscopic examinations of the vaginal discharge of the mothers and the discharge from their infants' eyes which I have made during the past three years, that ophthalmia neonatorum is, in the majority of instances, not gonorrhœal.

If the blennorrhœa in the new-born is gonorrhœal, it presents, as a rule, the same clinical picture and follows the same course as in the adult -i. e., there are the intense swelling of the lids and profuse purulent discharge. The cornea is also in great danger. As a rule, when originating at birth, it attacks both eyes.

Prophylaxis.-If the mother have a pathological vaginal discharge, thorough and judicious cleansing of her vagina should be practiced before the birth of the child; and when the child of such a mother is born, its eyes should be immediately washed with a saturated solution of boric acid, and two drops of a two-per-cent solution of nitrate of silver (Crede's method) be dropped between the lids of each eye, and iced cloths kept applied to the eyes for about three hours to limit the reaction. The child and mother should not alone be kept from other children, but the mother should be told of the contagious character of the disease, and warned against wiping her child with any towels, sponges, or cloths that may have been used on her own person.

Treatment.-The treatment is essentially the same as that for adults with gonorrhœal conjunctivitis. The discharge must be washed away 
(with fountain syringe *) as soon as it forms, and iced cloths applied uninterruptedly to the lids day and night. The surface of the lids (especially the edges) should be smeared with vaseline. The external commissure may be cut to the bottom of the conjunctival cul-de-sac, if the swelling and pressure of the lids upon the eyeball seem to indicate such a procedure. The same rules for the application of nitrate of silver apply here as in the case of the adult.

When the clinical picture is that of gonorrhœal conjunctivitis, especially when we do not find the gonococcus in the discharge, we should be extremely careful in the use of nitrate of silver. We should bear this fact in mind, that while the purulent conjunctivitis in the new-born which is not gonorrhœic may still be quite severe and endanger the eye, it often runs a favorable course under the simple antiphlogistic treatment. It is, therefore, in this class of cases, better to wait than to begin the use of silver, until the discharge is decidedly purulent, trusting to the antiphlogistic treatment of cold and irrigation, rather than run the risk of converting, by the use of silver, what might have been a mild process into one of those inflammatory conditions with a great inclination to associate itself with destructive corneal processes, which is frequently known to follow the too hasty and improper use of caustics. We should, therefore, feel our way, and postpone the use of silver until the disease shows a disposition not to yield to antiphlogistic treatment. In this latter event a solution of nitrate of silver, one half to one per cent, may be brushed over the everted upper lid, but not repeated again for twentyfour hours.

In the class of cases in which there is a membranous deposit on the conjunctiva, no silver of any strength should be used. The silver, in such cases, will not penetrate the membranous deposit, therefore it is useless; but if it should come in contact with the cornea, it will exfoliate its epithelium and expose it to infection, which it might have escaped if no caustic had been employed. In this latter class of cases I have seen the best results follow the flooding of the eye with as much vaseline-oil as will be retained between the lids. The washing of the eye and application of cold $\uparrow$ must, of course, receive the same exacting attention as when the vaseline is not employed.

* The solution should flow in a gentle stream. The ordinary syringe should not be used, as there is danger with it of splashing the discharge into the nurse's eye.

+ Where the disease is not gonorrhœic and it is very mild, as shown by the absence of heat and swelling of the lids, it is better not to employ the iced cloths. 
EXPLANATIONS OF FIGURES ON PLATE III.

Fig. 2, gonorrhœal ophthalmia in an adult (life-size). Right eye (discharge washed away) shows conjunctiva of globe much swollen (chemosis), forming an annular wall around the cornea, with ulceration of the cornea. Left eye shows swelling and discoloration of the eyelids, with profuse purulent discharge. Fig. 3, gonorrhœal ophthalmia in new born, infant seven days old (life-size), shows characteristic inflammatory swelling and purulent discharge. 


\section{PLATE III.}

\section{$F / G .2$}
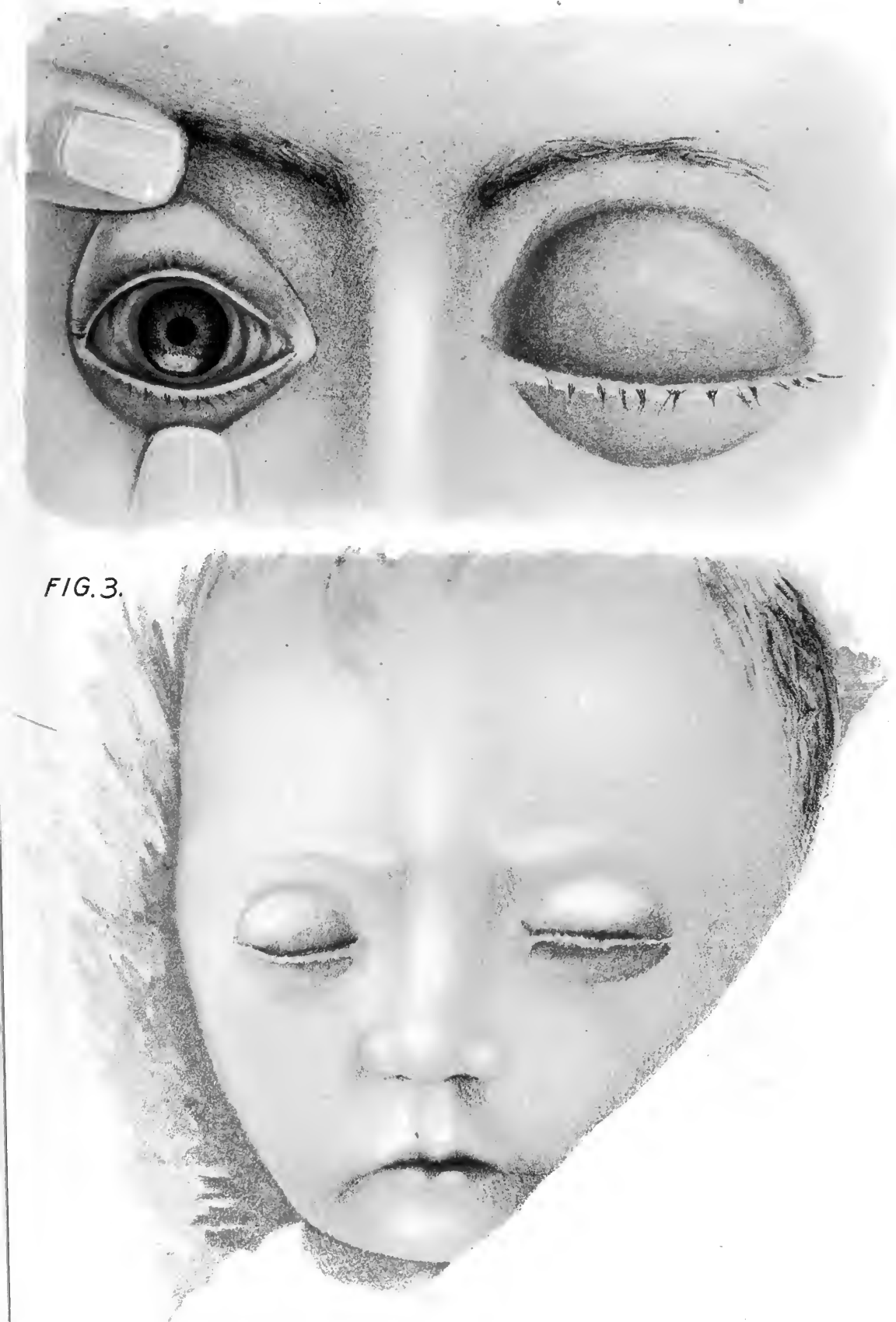

GONORRHOEAL OPHTHALMIA FIG.?. IN AN ADULT. 



\title{
GONORRHEAL RHEUMATISM.
}

\author{
BY FRANK HARTLEY, M. D.
}

Definition.-As the result of a blennorrhœal urethritis, commonly and less frequently of a blennorrhœal ophthalmia or vulvitis, certain affections are observed to occur, which, when existing in the joints, bursæ, sheaths of the tendons, the eye, muscles, nerves, and fibrous tissues, are designated as blennorrhœal rheumatism.

Etiology.-In reference to its etiology we believe it to be an infectious process-a wound infection in which the follicular ulcers (so-called gonorrhœal ulcers) in the posterior urethra are for the most part the points of origin of the infection.

Such a position leads us to a belief in an existing gonohæmia or pyohæmia ; but in either case we must make our belief subject to the three necessary indications of the proof of any micro-organic origin of the disease.

These conditions are, that one and the same form of micro-organism be always found in a given disease, that it be easily recognized morphologically, by chemical relations and reaction to coloring matters, and that the disease be artificially produced in a healthy individual by inoculation with pure cultures.

Neisser, Bokai, Bockhart, Welander, and others have carried out these conditions in so far as concerns the urethral inflammation, but in the complications of a gonorrhea we lack positive proof of the third condition.

Since Bumm demonstrated the truth of the discovery of Neisser the complications of this disorder have been subjected to renewed speculation and study. Very early in the history of the gonococcus as the cause of a specific urethritis, the conclusion was that this micro-organism must be held as the direct cause of its complications.

Brieger, Ehrlich, and Haslund, after a careful study of the subject, denied the presence of the gonococcus in the joint effusions following gonorrhœea of the urethra or vagina.

Petrone, Kammerer, Hall, myself, and others have found a diplococcus in the joint effusions resembling the gonococcus morphologically and in its reaction to coloring matters, but have not produced the neces- 
sary proof obtained from cultures, nor demonstrated the presence of the gonococcus in the blood or lymph.

Until cultures and inoculations have been made which will produce the complications of a gonorrhœa in a healthy individual, we must remain content to side with Bumm, Baumgarten, Neisser, Bockhart, Haslund, and Gerhardt, who regard the complications as the result of a mixed infection.

Before the invasion of the gonococcus the healthy urethra contains many micro-organisms, harmless as well as pathogenic. Four varieties of the diplococcus besides the gonococcus have been met with in the urethra of man. Two of these varieties, as the gonococcus, decolorize by Gram's method, but are infrequent in their occurrence. A diplococcus staphylococcus has also been found in blennorrhœal orchitis, subacute prostatitis, and in the normal urethra of children and old adolescents, presenting the same reaction to nutritive media, upon animals, to chemicals, and to Gram's method. It acts identically with the gonococcus, and causes suppuration in the tissues in which it exists. So far as we know, however, no one of these varieties has been subjected to the three crucial tests establishing a proof of their causative action.

These micro-organisms, resembling the gonococcus morphologically, are not able to penetrate the mucous membrane of the urethra until the gonococcus has attacked it and made of it a "locus resistentiæ minoris." Gonorrhœa is a purely local affection, which can not develop except upon cylindrical epithelium; and all inflammatory processes which are added to it in other tissues than those covered by cylindrical epithelium are the result of secondary infections.

Such cases as those reported by Dr. Ely (New York Medical Record, 1889) are very instructive in this particular:

The case was one of a man twenty years old, who died in coma, with a temperature $105.8^{\circ}$, pulse 130 , and a purulent discharge from the urethra, within a few hours of his admission into the Roosevelt Hospital. The autopsy revealed old pericardial adhesions, fresh vegetations upon the mitral valve, and a hyperæmic liver. The spleen was large and soft. The kidneys contained small infarctions and embolic foci. In the vegetations upon the mitral valve, in the liver, spleen, kidneys, and urethra, staphylococci, streptococci, and bacilli were found. In the urethra a diplococcus resembling the gonococcus was also found.

Nine cases are now recorded, and in some-Besançon's, Lancereaux's, and Ely's-micro-organisms resembling pyogenic bacteria have been found both in the urethra and in the internal organs. These cases seem to establish a series of progressive steps from urethritis to malignant endocarditis and pyæmia. The interpretation of these phenomena taking place at a distance from the primary focus is rendered difficult by the fact that the gonococcus has not yet been found in the blood. Moreover, 
cultures and inoculations in the urethra have not been followed by a rheumatism, with the discovery of the gonococcus in the secondary foci, and its cultivation and inoculation into a healthy urethra with the development of a gonorrhœal urethritis. Until such experiments have been fully carried out, we prefer to believe that gonorrhœeal rheumatism is a mixed infection, with the urethra as the primary focus in most cases.

The earlier authors, who recognized a connection between a gonorrhøa of the urethra and the rheumatism, spoke of the rheumatism as a true and direct gonorrhœal disease. They considered it in the same category with epididymitis and prostatitis, and ascribed the causation to an injury to the mucous membrane of the urethra in the same manner as when provoked by catheterismus. Such writers were Selle (1781), Hunter (1786), Ricord (1836), Foncart (1846), Brandes (1854), and Fournier (1866). In opposition to these, other authors, among whom may be mentioned Guyon (1836) and Thiry (1856), believed in no genetic connection between urethritis and rheumatism, and insisted that the gonorrhoia simply brought forth a latent diathesis in the individual. Guerin (1864), and Lasegue (1876) maintained that gonorrhœa was nothing more than a general disease sui genris, with a long period of incubation.

Lewin (1878) was the principal exponent of the vasomotor theory, and believed that complications considered as rheumatism were reflex in character, as the result of the inflamed urethra. The discovery of the gonococcus led to the theory of a wound infection and to the production of two theories. Of these theories, one, based upon the discovery of the gonococcus in the exudates of secondary foci, considered the rheumatism a true gonorrhœal affection; the other classed the rheumatism as an accidental and secondary affection, brought about by the presence of the puscoccus in the urethra, and, in order to enter the blood or lymph channels, they considered it necessary that the epithelium be destroyed or altered in its nutrition. This destruction or alteration in the nutrition of the epithelium is brought about by the gonococcus. According to this latter theory, gonorrhœal rheumatism has no specific quality, since it is not impossible for a simple catarrh or a trauma to give rise to the same result. Guyon and Janet (1889), and Fürbringer (1890), have classed the rheumatism as a ptomaine-poisoning, and the direct result of the gonococcus invasion.

Such are the various theories that have been presented; and as none have been thoroughly proved, we prefer, in the light of the present facts, to consider gonorrhœal rheumatism as a mixed infection.

Seele and Swediaur, in 1781, were the first to observe articular affections in gonorrhœal subjects, but it was reserved for Hunter, Cooper, Brodie, Bonnet, Diday, and Fournier to establish the fact that this was 
not a mere coincidence, but involved a relation of cause and effect-i. e., certain persons having gonorrhœa acquired a peculiar form of rheumatism which existed with every successive attack of gonorrhœa.

Subsequent investigations have shown that the structures involved were much larger than were at first described, and to-day the lesions may be said to involve the joints, sheaths of tendons, muscles, bursæ about the joints, and fibrous tissues. Occasionally the peri- and endocardium, the nerves, and meninges of the brain and cord, are attacked.

We understand that the acute variety of inflammation of the joints includes four distinct pathological conditions, each one of which has its distinctive name as follows : Serous synovitis, sero-fibrinous synovitis, seropurulent synovitis, and purulent synovitis.

The chronic variety is represented by the word hydrarthrosis, the pathological condition of which is a chronic serous synovitis or a papillary synovitis, and in rarer instances by an ankylosing arthritis or an arthritis deformans. Although the number of antopsies in gonorrhœal rheumatism are not few, yet we are not able to obtain from them the pathological conditions existing in the milder varieties, since it is only in the very severest cases that the result is fatal. In that variety which approaches the serous inflammations, all we have are the records of a few cases where the fluid has been withdrawn from the joint. The exudate was in these cases straw-colored or lightly tinged with blood, and consisted of large epithelioid, white blood-cells, and albumen. In this serofibrinous variety the exudate has consisted in large amount of fibrin in addition to epithelioid and white blood-cells. In the sero-purulent variety the fluid has been mucoid in character, light green in color, the sediment of which consisted largely of pus-cells, most of which showed a fatty metamorphosis. In two cases punctured by Laboulbene, the fluid was of a deep yellowish color, consisting of a sticky alkaline fluid containing no mucine, but pus-cells and fibrin. In the suppurative variety, Fournier,

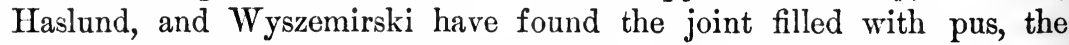
articular cartilages eroded, and the bones bare, with the periosteum separated from the shaft of the articulating bones for a long distance from the joint.

The outcome of these varieties of acute gonorrhœal rheumatism is either a complete restitutio in integrum, which is the case with the serous variety as a rule, and less frequently in the sero-fibrinous and sero-purulent, or a restriction in the motion of the joint, commonly seen in the purulent variety, and less frequently in the sero-fibrinous and sero-purulent varieties. As the result of constantly recurring synovites, hydrops (or chronic serous synovitis), synovitis papillaris, or arthritis deformans, are not uncommon. Statistics on this subject show that in 35 per cent of the cases (106 in 299, Bornemann) a complete resolution takes place. In 65 per cent of 
the cases (193 in 299) the motion was restricted or completely lost. In the polyarticular variety, and less frequently in the monarticular variety, where several attacks have occurred, the pathological condition remaining is a papillary synovitis, arthritis deformans, and a chronic serous synovitis. In the chronic serous synovitis the restriction to motion is very slight; such may be the case also with a papillary synovitis. In the arthritis deformans, however, owing to the osteitis rarificans in the earlier stages and the deformities induced by the softness of the bones, the restriction to motion in the joints is more marked. Some of these cases of the chronic gonorrhœal rheumatism which are polyarticular in character have been attended with a marked amyotrophic condition in the muscles in the neighborhood of the joints, and in rare instances in groups of muscles in no way connected with the joint involved.

Situation.-The frequency with which the various joints are involved is, according to Nolen, who has collected 308 cases, as follows: Kneejoint, 86 ; ankle, 52 ; shoulder, 29 ; hand, 26 ; hip, 15 ; fingers and toes, 17.

According to Fournier, two thirds of the cases occur in the knee, one fourth in the ankle, and one fifth in the fingers and toes. Four cases have been described in the vertebral joints. According to Finger, who has collected 375 cases from various authors: Knee-joint, 136 ; tibio-tarsal, 59 ; wrist-joint, 43 ; phalangeal, 35 ; elbow, 25 ; shoulder, 24 ; hip, 18; temporo-maxillary, 14 ; metatarsal, 7 ; sacro-iliac, 4 ; sternoclavicular, 4 ; chondrocostal, 2 ; intervertebral, 2 ; peroneo-tibial, 1 ; cricoarytenoid, 1.

Of 348 cases collected by Jullien, 205 were polyarticular ( $58 \frac{9}{10}$ per cent), and 143 were monarticular ( $41_{10}^{\frac{1}{10}}$ per cent). According to others, $19 \frac{1}{2}$ per cent of the cases are distinctly monarticular, $12 \frac{3}{4}$ per cent are distinctly polyarticular, and in $67 \frac{3}{4}$ per cent more than one joint is involved; though the disease is not distinctly polyarticular.

Date and Development.-The time for the occurrence of the rheumatism is rarely before the fifth day. In the majority of cases ( 75 per cent) it occurs during the first month. In 25 per cent of the cases it occurs during the second and third months in equal proportion. We are to remember that gonorrhœal rheumatism is not generally a complication of the recent but of the older cases.

Duration.-The duration of the joint lesions in 299 cases collected by Bornemann was on the average sixty-eight days, so that it is fair to infer that the duration will be about two months.

Frequency.-The frequency of gonorrhœal rheumatism as a complication is small. Gresolle gives it as $2 \frac{8}{10}$ per cent. Besnier and Jullien give it a frequency of two per cent.

It occurs more frequently in men than in women or children, and as 
a complication not only of the urethra, but of the vulva, vagina, and conjunctiva. Lucas describes two cases following gonorrhœa of the conjunctiva in the newborn; Deutschmann, following an ophthalmo-blennorrhœa; and myself in five cases of vulvo-vaginitis in children.

The joint lesions occur most frequently as a serous, sero-fibrinous, and sero-purulent exudation. In rarer instances a purulent exudation is present. This exudate is well marked, and fluctuation, especially in the knee, is easily appreciated if it be of the serous variety.

Course and Results.-According to the character of the exudate the synovial membrane and superjacent tissues are infiltrated, tense, and reddened. All the local symptoms of an inflammatory nature involving the parasynovial and cutaneous tissues are more marked in the sero-purulent and purulent than in the serous variety. Though the three varietiesserons, sero-fibrinous, and sero-purulent-show a marked tendency to resolution in the milder cases, this is not the case in the severer forms. The sero-purulent, unless mild in character or aborted by treatment, leads to a superficial destruction of the joint surfaces, and anchylosis or perforation.

Such results of the sero-purulent variety have been described by Duplay and Bruns. In the sero-fibrinous variety an anchylosis from the sealing together of the joint surfaces is also to be feared. Ollier and Gosselin consider this variety as not uncommon. Nicaise has reported fourteen such cases. The purulent variety, though rare, leads to perforation of the joint and subsequent ankylosis. The general tendency of all varieties is to become chronic and to remain as a hydrops, an ankylosis, papillary synovitis, or an arthritis deformans. This is especially the case where recurrences are frequent with every new infection.

In general, the attack begins acutely with pain, heat, and a febrile movement of $100^{\circ}$ to $102^{\circ}$. This is, however, only present at the beginning of the attack. One joint is sometimes involved. Commonly several joints are affected, and it is the rule for them to be involved one after the other.

The varieties of gonorrhœal joint lesions have been described under the following forms:

1. Blennorrhœal arthralgia.

2. Hydrarthrosis.

3. Polyarticular subacute arthritis.

4. Suppurative arthritis.

5. Plastic monarticular arthritis.

6. Blennorrhœal arthritis deformans.

We prefer, however, to adopt a nomenclature which is more in accordance with the terms used in other diseases, viz.: 


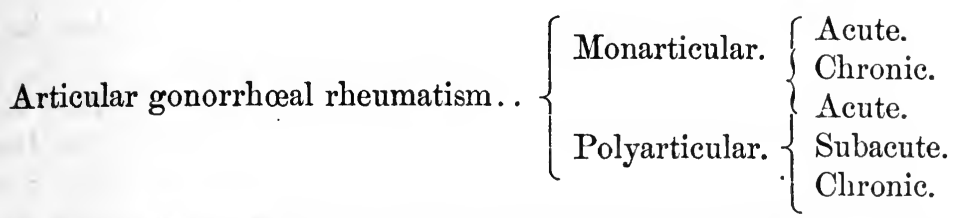

I. Acute Monarticular Gonorrhœal Rheumatism.-This is the most frequent variety. It begins in an acute manner, or is preceded by malaise and pain in several joints. Following rapidly upon this is a swelling of one of the larger joints, generally the knee. The pain is sometimes severe and sometimes slight. The exudation and tension within the joint are marked, and fluctuation distinct. The fever reaches $99.5^{\circ}$ to $102^{\circ}$, and continues for some days. It is more marked in the sero-purulent than in the serous and sero-fibrinous varieties, and is attended with greater local manifestations of tension, pain, œdema, and redness of the skin. In the milder varieties of the serous, sero-fibrinous, and sero-purulent exudations resolution takes place in several weeks, provided complete rest be maintained and no increase in the urethritis occurs. Constant recurrences in the joint lead to the development of a hydrops, a papillary synovitis, or a condition pathologically resembling arthritis deformans.

A rare result of the acute variety is suppuration. This is made known by the chills and fever, together with the increased swelling, œdema, and redness of the joint. The pain usually changes to a throbbing, pulsating variety. Finally, the capsule is involved, and pus, escaping into the sheaths of the tendons and intermuscular spaces, perforates the skin. The result of this form is either recovery, with ankylosis, or pyæmia.

II. Chronic Monarticular Gonorrhœal Rheumatism, Hydrarthrosis, Gonocele.-The chronic form develops either as a primary affection or is an outgrowth of the acute variety after many recurrences. In the former instance a gradual increase in size of the joint takes place, which is discovered by the patient quite accidentally. The joint is painless upon pressure, and only when the fluid is in large amount is the function of the joint interfered with. In extreme cases the ligaments become loose and stretched, and an abnormal mobility exists. In milder cases only the extremes of flexion and extension are hindered. Although at times resolution takes place, it generally persists for months, with many relapses. This constant tendency to relapses results finally in producing a form of papillary synovitis or deforming inflammatory changes in the bones and synovial membrane similar in character to an arthritis deformans.

Constitutional symptoms and local manifestations beyond functional disturbances are occasionally present. It is the rule not to have subject- 
ive symptoms. The variety is usually monarticular. It exists in the knee, ankle, and elbow.

III. Polyarticular Acute Gonorrhœal Rheumatism.-It develops rapidly, and is distinctly polyarticular at the onset. As the disease progresses it confines itself to a single joint, or to two or three joints. When two joints are involved they are frequently the same joints in opposite sides of the body. The number of joints involved is never so great as in articular rheumatism, nor do we find, as in articular rheumatism, a number of joints simultaneously involved. It is the rule in gonorrhœal rheumatism to have the joints involved consecutively.

The fever is usually moderate, subsiding within a few days, while the local lesion continues. During the remission a new synovitis often takes place with a return of the fever. This repetition may occur two, three, or four times. The effusion within the joint is more marked, and the swelling of the tissues about the joint is less distinctly evident than in articular rheumatism. This is strikingly the case except where the sheaths about the joint are involved.

The want of proportion between the constitutional reaction and the local lesions is diagnostic. When a single joint is involved the constitutional reaction is often entirely wanting. When several joints are involved they become so consecutively, and the constitutional reaction is only moderate. When, however, the sero-fibrinous or sero-purulent variety of inflammation is present in an attack, the constitutional reaction is increased in proportion to the degree of involvement. The joint lesions are more stationary than in articular rheumatism, where the shifting character of the lesions is diagnostic.

Resolution is more tardy than in articular rheumatism, and a secondary hydrarthrosis, rare in articular rheumatism, is common in gonorrhœal rheumatism. The excessive acid perspiration, as well as the peri-, endocardial, and pleuritic manifestations, are not so frequent as in articular rheumatism. It is characteristic of this variety to find in the successive attacks the involvement of the bursæ, sheath of tendons, the iris, or conjunctiva.

IV. Polyarticular Subacute Gonorrhœal Rheumatism.Next to the acute this is the most frequent variety. The fever is not high $\left(101^{\circ}\right)$, and the local symptoms are slight.

V. Polyarticular Chronic Gonorrhœal Rheumatism is either an outgrowth of the acute or subacute variety, or it develops as a chronic process from the beginning. It is frequently followed by hydrarthrosisi. e., a chronic serous synovitis-and less frequently by a papillary synovitis, or arthritis deformans in several joints.

Pathological Coincidences.-Besides the joints, other organic structures are involved in the secondary affections following gonorrhœa, 
and among these the involvement of the spinal cord should not be overlooked. Such instances have been dwelt upon by Stanley, Hume, Fournier, Hayem, and Parmentier.

The two latter give six cases in which not only a rheumatism involved the joints, but in which affection of the spinal cord was noted. The disturbances seen resulted in an alteration of sensibility-as neuralgia, hyperæsthesia, and anæsthesia ; or disturbances in motive power, as cramps and paresis-in addition, increased reflexes at the knee and atrophy of the muscles of the lower extremity, with hydrops of the joints.

Fournier agrees with Hayem, and describes a case exhibiting deformities in the joints of the fingers, increased reflexes, twitching of the lower extremities, and atrophy of the muscles of the forearm. Of the pathology of these cases we know absolutely nothing.

The involvement of the nerves has been dwelt upon by Fraenkel and Loeb, who consider it a rare complication to polyarticular rheumatism. Fournier has found the sciatic nerve involved in five out of thirty-nine cases, Scarenzio in two cases. Contagne has seen two cases of crural and inguino-scrotal neuralgia, and Gutherz has seen one case in which the internal pudendal nerve was the seat of the neuralgia.

The involvement of the bursæ in gonorrhœal rheumatism can not be called a rare complication, as it is seen quite often in a moderate number of cases. The bursæ in front of the tendo-Achillis, and that beneath the os calcis, are the more frequently attacked. The bursa beneath the tuber ischii and the bicepital bursa are not so frequently attacked, although their involvement is by no means rare. Terillon has recorded two cases in which the psoas bursa was involved. This is certainly a rare seat. Pathologically the bursæ suffer the same changes as the joints.

The sheaths of tendons are commonly involved with the joints, though they may be affected alone. It is by no means infrequently seen that the sheaths of the extensors of the hand, the dorsal flexors of the toes, and the flexor pollicis, are involved with or without an accompanying inflammation in the joints over which they pass. Less frequently we find the sheaths of the biceps brachii and tendo-Achillis affected. Pathologically the changes in the sheaths are the same as in the joints.

In the serous variety the long swelling corresponding to the shape of the sheath, without redness and much pain, is characteristic. It is especially characteristic for this variety to resolve completely or to pass into a chronic condition of hydrops. In the sero-fibrinous variety the symptoms of pain and crepitation, with only slight tumefaction and redness, are diagnostic. This is the variety in which we expect to find adhesions of the tendon to the sheath, and a consequent inability to perform certain motions with ease. In the sero-purulent variety, the tumefaction, œdema, and redness are the marked symptoms, and in this variety, 
if any result other than resolution occurs, it is an adhesion between the sheath and the tendon, or suppuration.

The muscles of the neck, especially the deltoid and those of the eye, are sometimes the seat of pain, which is present principally upon use of the muscles. The pathology of this condition is not known. Amyotrophia occurring in the course of gonorrhœal rheumatism is localized to the muscles close to. the joint involved, and less frequently to those at a distance from it. Sometimes it exists in a general form. When localized to the muscles moving the joint involved, the consensus of opinion favors as a cause a reflex mechanism, rather than a myositis, or a terminal neuritis of the articular nerves. When general, it has been at times observed to exist together with a variety of spinal lesions; and Urdy, in 1878, Fournier, Hayem, Parmentier, Tibrier, and Strümpell class the amyotrophia as a symptom of the spinal involvement.

The cutaneous phenomena were first described by Seele in 1781, and subsequently have been studied particularly by Jullien, Phillips, Petrone, Mesnet, and Kuppel. They appear under the forms of urticaria, purpura, erythema nodosum, and multiforme alone; or in conjunction with joint inflammations. They are angioneuroses induced by the urethritis through the intervention of the vasomotor nerves.

The involvement of the eye in gonorrhœal rheumatism is generally associated with the polyarticular variety. It may precede or follow the development of the rheumatism. It may be the only evidence of the rheumatism, or be present and alternate with the joint disturbance. Aquo-capsulitis, an inflammation involving principally the membrane of Descemet, is the most common form. In this variety the cornea is transparent, more prominent than usual, and the conjunctiva is injected.

The fluid in the anterior chamber is cloudy, with at times a slight flocculent deposit upon the posterior surface of the cornea. Photophobia is mild or absent. The iris is not generally affected, and there is little or no pain. If the iris be involved (iritis) there is a radiate pericorneal infection, a contracted or deformed pupil, an abolition of the movement of the pupil, an effusion of lymph in the pupil, and a plastic deposit in the anterior chamber. These symptoms are more marked in the gonorrhœal than in the ordinary iritis. Photophobia, and ocular and periorbital pain, are well marked in iritis.

Fournier describes a conjunctival form, which appears as a simple conjunctivitis uniformly distributed over or marked at certain points of the conjunctiva. The secretion is scanty and muco-purulent.

These three varieties are rarely monocular; one eye is usually involved after the other.

They run a rapid course, subsiding in a few days. In rare instances they last for weeks, and are then very prone to relapses. 
Of these three forms the conjunctivitis is the least harmful. Aquocapsulitis is not grave, though troublesome. Iritis is alone liable to be attended with adhesions.interfering with perfect vision.

The involvement of the endocardium and pericardium was first men. tioned by Brandes in 1854. At present about forty cases are known where endocarditis and pericarditis, or both, existed. It is a rare complication, and is present with the rheumatism in other structures, or alone with the gonorrhœa. It is especially observed where a rheumatism complicates the urethritis. In eleven out of thirteen cases collected by Morel this was the case. The symptomatology does not differ from the endocarditis due to articular rheumatism.

In the fibrous tissues, the juncture of the fibro-cartilage of the nose and superior maxilla, the insertion of the ano-coccygeal raphe to the coccyx and the spinous process of the three lower lumbar vertebre have been described as being especially sensitive to pressure for months (seven to eight) after the cure of a gonorrhœal rheumatism. In addition to the above, the calcaneum upon its lateral and inferior surfaces have been observed by Jacquet in 1892 as enlarged and painful in two out of twelve cases. He ascribes it to an osteitis or an inflammatory process involving the calcaneum and the ligaments, and gives to this latter form the name of "pied blennorrhagique."

Diagnosis.-The diagnosis of gonorrhœal rheumatism implies the recognition of three conditions :

1. The existence of a gonorrhœa or a previous gonorrhœal affection, with persisting ulceration in the urethra, etc.

2. The presence of inflammatory lesions at a distance from the local lesion, which are most probably metastatic in character.

3. The objective local symptoms and course of the part involved, as well as the character of the constitutional reaction.

The rheumatism usually occurs during the first month ( 75 per cent). Less frequently it occurs during the second and third. months (25 per cent).

It is customary to have certain joints involved, as the knee, ankle, shoulder, and wrist, together with the tendous sheaths overlying them.

These joints are involved one after the other, and the joint inflammation does not present the shifting tendency so characteristic of articular rheumatism.

From articular rheumatism it may be distinguished by the greater effusion within the joints and the slighter involvement of the parasynovial tissue, except in the sero-purulent variety. Resolution is tardy in gonorrhœal rheumatism, and hydrarthrosis is rare in articular rhenmatism, but common in gonorrhœal rheumatism. The lack of proportion between the constitutional reaction and the local lesion is diagnostic. The fever 
is commonly more moderate than in articular rheumatism, and it subsides within a few days, while the local lesion continues.

The excessive and acid perspiration, the endocardial, pericardial, and

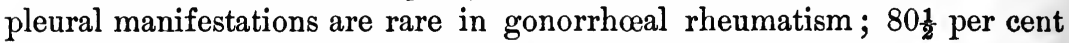
of the cases of gonorrhœal rheumatism are combined with an involvement of the synovial sheaths of tendons, bursæ, iris, or conjunctiva. This combination is more or less diagnostic. There is a combination of symptoms and lesions occurring in syphilis which may be easily mistaken for a gonorrhœal rheumatism. I refer to that class of lesions reported by Schüster, in which, following urethral infection, one or more joints become involved, together with an iritis, endocarditis, and pericarditis, osteitis of calcaneum, os sacrum, tuberositas tibiæ, and sternum. This complex of lesions in which the joints, bones, and iris are affected renders the diagnosis of syphilis quite probable, and the mercurial treatment in the form of inunctions cures or ameliorates these complications.

Treatment.-There is scarcely any treatment which can be called effective for gonorrhœal rheumatisms. Quinine internally in the acute stage, and in the later stages corrosive sublimate and rest to the joint, have been much used. Salicylic acid, cold, and fixation of the joint are often used.

For the pain, antipyrine, $\cdot 75$ to 1 gramme twice daily, has been especially good in some cases for the pain.

Salol internally is now recognized as having a real but feeble action in the acute cases.

Locally, compression, massage, and cold have proved to be of use. Physiological rest to the joint or joints is important. When these measures are not successful in alleviating the local lesions, withdrawal of the fluid within the joint, with irrigation of the joint with solutions of 1 to 40 carbolic acid, or 1 to 1,000 or 1 to 5,000 of corrosive sublimate, are generally snceessful. In that variety in which the pathological changes resemble an arthritis deformans resection of the joint or joints may be performed in order to secure anchylosis in a better position. This, however, is best delayed until the osteitis rarificans has run its course and is in the stage of sclerosis.

The urethra is to be treated in all cases where a lesion still exists.

The best prophylaxis against the rheumatism is the rapid cure of the urethritis. 
GONORRHEA OF THE RECTUM, NOSE, MOUTI, EAR, UMBILICUS, AND AXILLA.

\author{
By JAMES P. TUTTLE, M. D.
}

\title{
GONORRHOEA OF THE RECTUM.
}

THE rarity of gonorrhœa of the rectum in this country may be appreciated in some degree when we hear from men of such large experience as Drs. Bumstead and Van Buren that they have never seen a case. Keyes in his latest work does not even mention it; Dr. J. W. White devotes a few lines to it only to encircle its existence with doubt; Dr. Otis mentions it but to pass it by ; and Dr. Kelsey, with his large experience in rectal disorders, declares as late as $\mathbf{1 8 9 0}$ that he has never seen it.

The experience and opinion of such eminent men as these and others equally celebrated have caused many in the profession to question the existence of the disease, and until the discovery of the gonococeus by Neisser the negative side of the proposition could have been maintained; but since that day its existence can no more be doubted than that of urethral blennorrhagia.

The diagnostic and pathogenetic importance of this microcoecus is questioned by some, and will be discussed in another portion of this book. It suffices here to say that the gonococcus is not found in the discharges from any of the ordinary forms of proctitis. It has never been demonstrated in any instance where there was not confession or presumptive evidence of exposure to the virus of gonorrhœa either through antoinfection, pederasty, or sodomy. If the disease, then, be not specific in its cause it certainly is in its product, and by a tree's fruit shall you know it. The tree which produces apples is an apple-tree, and the disease which produces gonococci is gonorrhœea.

Imbued with this doubt, Bonnière in 1874 made some interesting experiments upon the different mucous membranes of the body with regard to their susceptibility to the gonorrhœal virus. Taking a patient with gonorrhœal ophthalmia and urethritis, he smeared the pus discharged from these infected regions upon the mucous membranes of the nose and anus. The nose showed no evidence of the disease, but on the second day evidences of infection were seen about the anus, and on the fifth day a muco-purulent discharge from the part was seen, with itching, burning, 
and considerable pain upon defecation. He then injected the pus into the rectum through a hollow tube, but with negative results. After a very few experiments, in which microscopic examination was notably neglected, he formulated the following table and conclusions :

\section{Mucous Membranes susceptible to Gonorrlecea.}

Prepuce.

Urethra, especially the fossa navicularis and prostatic portion.

Excretory canals of glands of Littré. Conjunctiva.

Anus.

Mouth.

Prostatic utricle.

Vulva.

Vagina.

Inferior portion of the cervical eanal.

\section{Mucous Membranes refractory to} Gonorrheca.

Rectum.

Pituitary membrane.

Lachrymal canal.

Body of uterus.

Ejaculatory canals.

Seminal vesicles.

Deferent canal.

Prostatic canals.

Bladder.

Excretory canals of the glands of Cowper and Bartholini.

Conclusions.-All the mucous membranes susceptible to gonorrhøea are covered with pavement epithelium, or are supplied with papillæ and a superficial sub-epithelial network of lymphatic vessels. The mucous membranes refractory to gonorrhœa are covered with cylindrical epithelium and have a superficial sub-epithelial network of veins.

These experiments are very interesting and point apparently to some important facts. They have been referred to and quoted by all authors arguing upon the negative side of the question since Bonnière published them; but they are too meager to draw conclusions from at all, and the inferences which he assumes have only a comparative value. Admitting this assumption, that the mucous membrane of the rectum is refractory to gonorrhœal virus, nevertheless it does become infected and the symptoms are unmistakable.

The first account we have of this disease is said to be by Hecker, a Prussian physician, in his Maladies vénériennes, published in 1788. He there mentions a purulent discharge from the rectum which he had often observed in men and women, always traceable to unnatural coition. A German physician of the same name, writing in 1802, in a work on Tripper, speaks of gonorrhœa from hæmorrhoids. Goslin says, "I have seen one case of undoubted gonorrhœa of the rectum, but I have not seen stricture develop from it." Billroth says, "A very acute eatarrh with profuse secretion results from the contagium of gonorrhœe to the mucous membrane of the rectum. It occurs in women most frequently from the inflowing 
of the secretion from the vagina, but in men through direct infection in consequence of passive pederasty."

Rollett says he has frequently seen it, and reports the very interesting case of a man who, being habitually constipated, was in the habit of introducing his finger into the rectun to assist in defecation. Being attacked with gonorrhœal urethritis, he carried some of the pus upon his finger into the rectum and set up a virulent gonorrhcea of that organ.

Allingham says he has seen three undoubted cases of gonorrhœa of the rectum, but, considering it a well-established fact, does not give a minute description thereof. Thiéry reports a case with sodomic history, infundibuliform anus, erased mucous folds, relaxed sphincters, profuse purulent discharge, red and swollen mucous membrane, excoriated and ulcerated in patches.

Dr. Winslow reports two cases, in an institution for boys near Baltimore, which were the source of endemic gonorrhcal urethritis in the institution. Most of the invalids confessed to pederasty, and the disease was finally traced to a boy whose urethra had become infected while on leave of absence, and who had practiced active pederasty on one of the smaller boys upon his return to the school.

Martineau reports two undoubted cases of the disease, one a sodomist and the other a pederast, and at the same time quotes a case of Tardieu's.

Bernard and Tardieu say that they have frequently seen the disease in boys and adults who were always the victims of pederasty. L. Jullien, in the last edition of his work, Sur les maladies vénériennes, gives an accurate and full description of the disease up to that date.

These writers, however, based their opinions upon subjective and circumstantial evidence. Microscopic examination bore no part in their diagnostic methods. As soon, then, as the discovery of Neisser was made known, all these observations went for naught until they should be verified by the discovery of the gonococcus in a certain number of cases. This has been done; the micrococcus has been identified beyond question in anal and rectal discharges, and that, too, in cases the symptoms of which coincided as accurately as possible with those of the cases reported as cases of rectal gonorrhœa by the observers quoted above. Thus are the judgments of our predecessors confirmed by the methods of precision in medicine at the present day, and the cloud which once rested over them is dissipated forever by the microscopic demonstration of the identity of the discharges in urethral and rectal gonorrhoea.

Neisser has observed two cases of rectal gonorrhcea in married women. Their husbands were examined, and found to have the disease in their urethras. Microscopic examination of the secretions showed gonococci similar in quantity and quality to those in their husbands. 
Bumm quotes a ease observed by M. Wolf in the Würzburg Clinic for Syphilis. The woman suffered from a sanguino-purulent discharge from the rectum, which she said had begun a few days after sodomic intercourse. The rectal mucous membrane was swollen, deeply reddened, painful, and inclined to bleed. The discharge was shown by the microscope to contain numerous gonococci. There was no coexisting gonorrhœa of the genital organs.

Merk is reported to have obserred a case in Landschut in 1888, but no satisfactory microscopic examination was made, and altogether the case appears more like tubercular ulceration than gonorrhœa of the rectum.

The most thoroughly observed case so far, however, is that of Dr. Matterstock, of the Würzburg Clinic for Syphilis, and reported by Dr. F. Frisch.

A girl, seventeen years of age, fifteen days after her last coitus, complained of pain and burning in the rectum, unbearable at defecation. She confessed to haring frequently practiced sodomy. Examination showed a funnel-shaped anus, "the parts around were deeply reddened and there was a partial loss of epithelium." The swelling about the parts left only a small opening in the anus, through which dribbled a thick, greenish-yellow secretion. Microscopic examination showed squamous and cylindrical epithelial cells and abundant pus-corpuscles, many of which contained typical gonococei. Now and then a pus-cell appeared in the field which was literally stuffed full of gonococci, so that the nucleus instead of presenting the usual irregular oval shape was more crescent-shaped. Many round lumps of gonococci were seen in the neighborhood of these pus-corpuscles. A similar exhibition of gonococci was found in the discharges from the genitalia. The patient developed pulmonary tuberculosis and died from this disease before the gonorrhea was cured.

Upon post-mortem examination of the part, the large polynuclear round cells were seen stuffed full of typical Neisserian gonococci. Here and there was seen the swarming of the gonococei as mentioned by Neisser. The tubes of the glands and Erhlich's rectal cells were also found to contain gonococci.

This is one of three cases reported with tubercular complications, and all of them have been obstinate in yielding to treatment, a fact which entirely corresponds with my experience in urethral gonorrhœa.

Add to these the three which I have myself reported and I believe we will have prored the diagnosis and opinions of those who, laboring before the discorery of Neisser, yet recognized gonorrhœe of the rectum and treated it as such.

Etiology.-The cause of this disease is the direct infection of the 
mucous membrane of the rectum or anus by the gonorrhœal virus. This may be brought about by the extension of the disease from the vulva to the anus, and thence to the rectum, in women; by careless handling of the parts affected with the disease and thus conveying its germs to the rectum, as reported by Rollett; or, as is most frequently the case, it may be produced by unnatural coitus, the active party being affected with the disease. I believe the disease is due to local inoculation with the virus, and have no faith in the metastatic theory.

Symptoms.-The first symptoms of gonorrhœa of the rectum are itching, tingling, and a sense of heat which may appear at any time from twenty-four hours to ten days after exposure to the disease. The heat soon grows to a burning, the itching into pain, defecation becomes agonizing, and a dull, heavy aching in the sacral region appears, if the attack is severe. At this time, from the fifth to the seventh day, the patient will probably have some constitutional disturbance, the pulse being rapid, the temperature elevated, and general malaise will supervene. The pain incident to defecation causes that function to be neglected, and the tongue soon becomes coated, the appetite disappears, and the patient shows signs of serious illness. After two or three days a discharge appears, at first thin and milky-white, but later thick, greenish or brownish-yellow, and very abundant in quantity. The appearance of the anus will depend on the habits of the patient. If a passive pederast or sodomist, the anus will be more or less infundibuliform, its folds obliterated, the sphincters relaxed, and the mucous membrane swollen, hot, and shining. There will be no hæmorrhoids, but probably numerous little fissures, and occasionally a submucous fistula If the patient be not giren to these practices, the anus will be swollen and pouting; the mucous folds abont its border will be inflamed, enlarged, and œdematous; at their bases, between two folds, the muco-cutaneous tissue will be bathed in pus, excoriated or ulcerated, thus forming anal fissures which are very painful. The disease may not, indeed does not, usually extend above the internal sphincter. When it does, the mucous membrane of the rectum becomes swollen, brightly red, extremely painful, and bleeds easily upon touch; the whole is bathed in a profuse secretion of thick muco-pus which dribbles from the anus, imperfectly closed on account of the swollen folds. Here and there, there are patches of excoriated or truly ulcerated surface. The disease, especially if confined to the anus, is easily cured by simple cleanliness; but if it has invaded the rectum, it is less amenable to treatment, and may become chronic : there will then be points of chronic ulceration and cicatricial healing on the mucous membrane.

Erythema, ulcerations, condylomata, fissures, and eren fistula may be found about the borders of the anus, and sometimes the disease is so severe as to produce dysuria. "The scars and ulcers seen in the chronic 
form are difficult to distinguish from syphilitic proctitis as well as from tubercular forms of rectal disease."

The symptoms, which in the majority of cases are not severe, may pass away rapidly under the ordinary attention to hygiene and cleanliness. Indeed, I believe the large majority of them are never seen by the profession, owing to this fact. But in careless individuals, and in those cases where the rectum is invaded above the external sphincter it may continue indefinitely, if left untreated.

Diagnosis. - The diagnosis of this disease is not often difficult in women, thanks to the usual coincidence of gonorrhœea of the genitalia; but in men it is sometimes far from simple. I have seen one case in a man in which the discharge was so slight, the inflammation so insignificant, and the general symptoms so indefinite, that I was unable to distinguish the case from catarrhal proctitis until I had made an examination of the pus, and found gonococci therein. The existence of gonorrhoeal disease elsewhere, the history of pederasty or sodomy, are necessary to the positive diagnosis. Of course, if gonococci, typical in character, number, and arrangement, be found in the discharges, all doubt is put at rest.

The method of collecting the pus for these examinations in order to distinguish between the anal and rectal forms of the disease is important. The anus is wiped off as gently and as thoroughly as possible with absorbent cotton, and then washed with a solution of salicylic and boric acids-Thiersch's solution. A cylindrical speculum such as my own or O'Neil's is then introduced closed, and the sliding bar on the upper side withdrawn, the patient lying on the left side. The specimen is then taken from the surface of the rectum, and not from the discharge which flows down into the speculum, lest by any chance some of the secretion from the anus should have been carried up on the end of the speculum. Several specimens are then examined, to corroborate one arother, and to avoid as far as possible the error of mistaking disease of the anus for that of the rectum. The solutions used for staining in these cases are gentian violet and ammoniated carmine, and the results are practically the same with both.

But gonococci may not be found, and still the case be gonorrhœa. Blake and Shuldham tell us that, "when gonorrhœa has reached the chronic stage we may fail to find the diplococci or true gonococci, but encounter instead pseudo-cocci, staphylococci, streptococci, or tubercle bacilli." We should therefore not stake our diagnosis upon finding these bacilli, but look upon them as corroborative evidence of an opinion formed otherwise. The history of the case, then, the appearance of the anus, exceedingly relaxed or spasmodic according as the patient is or is not a sodomist, the amount of the discharge, which is usually profuse, 
the pain upon defecation or examination, the fissures between the anal folds, the absence of tubercular or nephritic complications, which both produce ulceration in the rectum similar to gonorrhœa, and the presence of the gonococci free or in the pus-corpuscles, will establish our diagnosis of the disease.

Intertrigo is liable to be confounded with gonorrhœea of the anus, but the history of the case, the presence of gonorrhœa elsewhere, and the detection of gonococci, will easily distinguish them.

Prognosis. - The disease tends toward a mild course, and rapid recovery in otherwise healthy individuals. If, however, there be a tubercular diathesis, local manifestations of this disease are likely to occur during the attack of rectal gonorrhœa, and so far as I have been able to learn, this complication is nearly if not always fatal.

Treatment.-Anal gonorrhœe, if the parts are kept clean and protected from chafing one another, will disappear spontaneously in the majority of eases. When the disease has progressed to the inflammatory, excoriated, or ulcerated stage, or when the rectum has been involved, active and energetic measures are requisite. In the first stage the parts should be bathed gently two or three times a day with a cold saturated solution of boracic acid. Some prefer solutions of bichloride of mercury, but I have not found them so efficacious. Lime-water acts very well as a bathing solution. After the parts have been dried with a soft cloth, a powder of oxide of zine and calomel, equal parts, should be freely applied, and the buttocks separated by a fold of patent lint or plain absorbent gauze. Jullien advises the use of tannic acid in place of the above powder, but it is too irritating, and does not hasten the cure.

If the peri-anal cutaneous surface is inclined to take on an erythematous irritation, keeping it thoroughly greased with vaseline will do much to check it. In case of ulceration, either upon the muco-cutaneous surface or in the cracks between the anal folds, the application of pure carbolic acid or a concentrated solution of nitrate of silver should be made before applying the powder. This treatment, together with gradual dilatation of the sphincter, is applicable to the cases in which fissure exists; but if fistula have occurred, it should be treated at once so that it can not act as a hiding-place for the micrococeci, from which they can break forth and reinfect the parts, after they have been virtually cured. As these fistulæ are usually of the sub-tegumentary variety, involving only the mucous and muco-cutaneous tissues, they may be effectually cured by laying them open and cauterizing them under cocaine. Condylomata may be snipped off with the scissors, but a better method is to tonch them a few times with a fifty-per-cent solution of mono-chloracetic acid, and apply the powder of zinc and calomel. 
When the disease has invaded the rectum proper, the regulation of the bowels, and the thorough irrigation of the reetum with appropriate solutions, will be all that is necessary. I have found the saturated solution of boracic acid to be perfectly satisfactory. The method of using this as an irrigation, and not as a simple injection or enema, is much more effectual. I am in the habit of using my rectal irrigator (Fig. 1). by which the rectum can be distended, and all the cracks and folds of the organ thus reached and acted upon. It also has the advantage that it can be used in bed without soiling the elothing, or inconveniencing the patient, a feature not particularly necessary in these cases, but of great moment in other diseases of the rectum. One to three quarts of this solution should be used at each sitting, and a cure may be confidently ex-

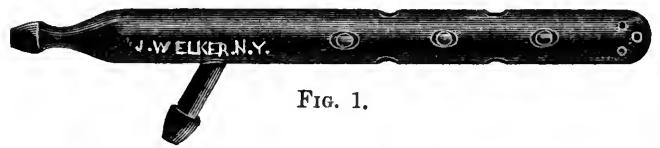

pected in seven to ten days. I have used the mild solutions of biehloride of mercury, but they did not act so pleasantly upon the patient or result in so prompt a cure.

If the mucous membrane of the rectum be ulcerated from the gonorrhceal inflammation, applications of strong solutions (50 to 150 grs. per 3 j.) of nitrate of silver, or equal parts of carbolic acid and tincture of iodine, may be applied to the ulcer after the irrigation has washed away the muco-pus covering the parts. A regular, well-formed stool is desired once or twice a day, in order that the rectum may not become loaded and irritated with either hard or fluid fecal matter. To this end I have found nothing so effective as the following pill:

Aloin, resinæ podophylli, āā gr. $\frac{1}{8}$; extracti belladonnæ, gr. $\frac{1}{4}$; extracti colocynthis eo., gr. $\mathrm{j}$; fiant in pil. No. 1. Sig.: One or two at bedtime.

The irrigation and treatment should not be discontinued until eight or ten days after the discharge has ceased, for the micrococci are liable to beeome concealed in Leiberkuhn's follicles, and the discharge begin anew after several days. Care and patience are necessary to treat these eases thoroughly; and the class in which the disease is likely to be met with offer us little to hope for, either in fees or gratitude.

\section{GONORRHEA OF THE NOSE.}

This disease, although its existence was recorded in the last century, has received no thorough study in recent years. Bonnière, reasoning from one experiment and the character of the epithelium of the organ, includes the pituitary membrane among those refractory to the gonor- 
rhœal virus. The experiment is insufficient, and the reasoning from analogy will not bear the burden of the conclusion, for the epithelial arrangement about the nares is almost identical with that about the anus, and we have shown beyond question the existence of the disease there. However, the rarity of the disease and the absence of the demonstration of the gonococei in the discharges justify the doubt of its existence. Andrew Duncan has reported the case of a young man who, suffering from a severe gonorrhœa, was obliged to use his handkerchief for a short time as a protection against soiling his shirt. After obtaining other cloths he imprudently put it into his pocket, and, without being aware of the consequences, used it for wiping his nose. The result was a disease of the inside of the nose and an affection of the mucous glands there, in every respect resembling the gonorrhœa of the urethra. Hecker says: "If the contagious material should penetrate accidentally into the nose or ear it will occasion a discharge from these parts. This gonorrhœa, which one may call auricular or nasal, according to its seat, is soon eured by cleanliness alone." He does not, however, describe any case or mention having seen them. A. M. Edwards has reported the case of a woman laboring under a severe ulcerative catarrh of the mucous membrane of the nose. She claimed to have used, some six months previously, a handkerchief which had been used by her son, who was at the time suffering from gonorrhœa. It is not stated how soon after the use of the handkerchief the discharge had begun, but the writer infers that the disease was due to the contagium conveyed through the handkerchief.

Already the theory of metastasis, advanced by St. Ives, had been applied to the nose, mouth, and ear; and Jullien quotes the case reported by Forccades as due to this cause and eured by reproducing the urethral catarrh. In the light of modern research there is absolutely no foundation for such a theory.

Lefferts describes gonorrhœal coryza as "aggravated, purulent, catarrhal inflammation arising in children from infection through the mother at birth, and in adults from the direct conveyance of the virus to the Schneiderian membrane by the fingers, soiled pocket-handkerchiefs, etc." He does not, however, cite any cases or refer to any as authenticated.

Stoerk describes chronic blennorrhœa of the nose as occurring among the Polish Jews in Galicia, Poland, Wallachia, and Bessarabia, a people living in poverty and filth, and who attach little importance to personal cleanliness. In the first stage there is at profuse secretion of greenish-yellow pus and the "vascular injection and succulence met with in acute catarrh" or coryza are absent. The disease is prone to extend downward, involving the pharynx, larynx, and vocal cords. Induration and matting together of the soft tissues occur in the disease, but the cartiiages and bones of the nose and throat are never involved. 
Kolbe reported two similar cases, and one communicated to him privately by Dr. Herring, that of a medical student who suffered from a chronic nasal blennorrhœa occurring during an attack of urethral gonorrhœea. Dr. Kolbe does not say positively that his cases were due to gonorrhœal virus, but that he believes such to be more than likely the case, for, says he, "I have seen with my own eyes a patient suffering from a profuse leucorrhœa, preparing for an examination, use her pocket-handkerchief to cleanse both her nose and her vagina, and who can draw the line between leucorrhœa and blennorrhagia?" In the light of the positive knowledge of the present day, it would have been a simple matter for Kolbe to decide whether this disease was gonorrhœal or not. Its history, course, and subsequent results are certainly in the line of gonorrhœal affections, and I have hoped and looked in vain for some report of the accurate microscopic examination of these cases which would prove the origin, cause, and pathology. Until such has been made, we can only surmise and believe in the existence of the disease on clinical symptoms, if at all. If gonorrhœal ophthalmia be once established, I can not see any reason why the virus should not travel down the lachrymal ducts and thus infect the pituitary membrane, or why the virus is not more likely to be carried by handkerchiefs and cloths from the eye to the nose than from the urethra to the eye. While I have not seen such cases, I believe that they are not only possible but probable in the class of patients described by Kolbe and Stoerk, and we will yet see the gonococcus demonstrated in these cases.

\section{GONORRHEA OF THE MOUTH.}

The arrangement of the epithelium of the mouth being so similar to that of the urethra, and the knowledge of the practices of Sapphism, would lead us to expect more frequent cases of the disease in this organ than in the nose. Hölder, after stating that the affection is due to direct infection of the mouth by gonorrhœal pus, either through contact with the genital organs or transmission by the hands, goes on to quote a case from Petrasie, of Kiel, which seems incontrovertible.

Vidal quotes from Baumes the case of a man who, having kissed the vulva of a woman, was attacked with buccal blennorrhagia.

More recently, Cutler has reported a case with such positive history and evidences of gonococei that it deserves special notice. Rizat and Fournier have reported cases of this disease; and Delefosse and Horand have reported cases of urethral gonorrhœa contracted $a b$ ore.

M. Dohrn has reported five cases in children in which the presence of the gonococci were demonstrated by the microscope and verified by culture. The existence of the disease is therefore no longer in question.

Etiology.-Menard has reported four cases of what he terms 
"gonorrhœal ulcero-membranous stomatitis." The patients had all suffered from gonorrhœa of the genital organs and complications, such as orchitis, epididymitis, and arthritis. In accordance with the teachings of Bouchard and Capitan, he holds that the disease is due to the profound infection of the system with gonorrhœal virus; that it always succeeds other complications of gonorrhwea; that it is accompanied by constitutional disturbances; and that it is a systemic not a local disease.

While there is no proof that the coccus may not circulate in the lymph or blood, thus migrate to remote organs, and there finding a congenial soil reproduce the original disease, its presence in these fluids must be demonstrated beyond a doubt before we can accept such a theory. Moreover, even then if confined to the mouth it would still be a local disease, and would not alter our firm conviction that gonorrhœa wherever found is the result of direct inoculation of the part by the gonococcus, regardless of the mode of its conveyance thither. In the new-born it results from inoculation throngh the mother at birth; in adults from the vile practices of Sapphism, or careless and uncleanly habits during attacks of gonorrhœa of the genitals.

Symptoms. - The first indication of the disease appears very soon after exposure to contagion, usually within twenty-four hours. The first sensation is that of a raw, dry feeling in the mouth, accompanied by a disagreeable taste to the saliva. This is followed by a sense of burning, the mucous membrane becomes red, the gums swell, become soft, and recede from the teeth. On the third to the fifth day herpetic sores break out on the lips; the tongue and buccal surfaces as far back as the fauces become swollen, painful upon motion, and bleed easily. Here and there will be seen patches of a grayish-yellow pseudo-membrane, which being detached leave excoriated or ulcerated surfaces. An abundant mucopurulent secretion exudes from the parts and dribbles from the imperfectly closed lips. Deglutition is very painful, the appetite is lost, the pulse rapid, and temperature elevated. The disease does not appear to extend beyond the faucial arch, but the sublingual, parotid, and lymphatic glands about the part usually swell and become painful. So far there is no history of these glands suppurating, but it is altogether within the range of possibilities.

Diagnosis.-The only disease with which this affection is likely to be confounded is stomatitis in its various forms. In the new-born stomatitis develops at a later date than buccal gonorrhcea, the inflammation is less severe, and there is no purulent secretion from the mouth. In adults the history of the case, the absence of exposure to contagions stomatitis, the swelling of the glands, and the presence of gonococci in the pus and membranes, will distinguish bnceal gonorrhea from any other disease.

Treatment.-The treatment of this disease is based upon the same 
principles as that of urethral gonorrhœa. Antiseptic washes, such as solutions of boracic acid, fifty per cent, carbolic acid, two and a half to five per cent, or bichloride of mercury, one to two thousand, are used frequently during the day. Together with these, astringent applications, such as the saturated solution of alum, tannate of glycerin, or nitrate of silver, ten to twenty-five per cent, are made, and serve to reduce the inflammation and restore the power of deglutition. Chlorate of potash and many other remedies have been tried, but the results do not encourage their use. Under the above treatment the disease will usually succumb in seven to fourteen days, but the treatment should be continued for some days longer to insure its eradication.

\section{GONORRHEA OF THE AXILLA, EAR, AND UMBILICUS.}

Of blennorrhagias in these anomalous situations little need be said. They have not been verified by the modern methods of research, and literature does not furnish us with sufficient authenticated cases of the disease in these parts to establish its undoubted occurrence.

\section{BIBLIOGRAPHY.}

Hecker. Verhand. d. phys. med. Gesell. zu Würzburg, vol. xxv, p. 168.

Billioth. System of Surgery, Billroth and Pitha's, vol. viii, part ii, p. 69 .

Rollett. Dict. Encyc. d. Sc. Med., art. Rectum.

Allingham. Diseases of the Rectum and Anus, ed. 1871, p. $23 \%$.

Thiéry. Presse méd. Belge, 1882, p. 201.

Dr. Winslow. Phila. Med. News, vol. xlix, p. 180.

Martineau. Leçon deform. vulvaries et anal., vol. clviii et seq.

L. Jullien. Malad. vénériennes, 1879, pp. 209, 213.

Neisser. Verhand. d. Deutsch. Dermatol. Gesell., Prag, 1889, p. $13 \%$.

Dr. E. Frisch. Verhand. d. phys. med. Gesell. zu Würzburg, 1892, vol. xxv, p. 167 .

Tuttle. N. Y. Medical Journal, vol. xlv, p. 379.

Blake and Shuldham. Med. Press and Circ., 1890, p. $65 \%$.

Andrew Duncan. Medical Cases selected from the Records of the Public Dispensary at Edinburgh, 1784, p. 269.

Hecker. Traité des differentes espèces de gonorrhée, 1787.

A. M. Edwards. London Lancet, $185 \%$, vol. i, p. 342.

St. Ives. A New Treatise on the Diseases of the Eyes, London, 1741, p. 168.

Lefferts. Internat. Encyc. Surgery, vol. v, p. 374.

Stoerk. Krankheiten des Kehlkopfs, Stuttgart, 1880, p. 161.

Kolbe. Wien. medicin. Wochenschrift, 1878, p. 535.

Hölder. Lehrbuch der venerischen Krankheiten, Stuttgart, 1851, p. 288.

Vidal. Malad. vénériennes, 1885, p. 188.

Dr. Cutler. N. Y. Medical Journal, 1888, vol. xlviii, p. 521.

Rizat. Jour. mal. cut. et syphil., 1889-'90, i, p. 305.

Delefosse. Lexon. méd., 1885, p. 279.

Horand. Lyon médicale, 1885, p. 279.

M. Dohrn. Mercred. méd., July 15, 1891.

Menard. Ann. d. derm. et syph., 1889, p. 680. 


\title{
STRICTURE OF THE URETHRA.
}

\author{
By J. WILliaM White, M. D.
}

\section{CONGENITAL STRICTURE.}

StRicture of the male urethra may be congenital or acquired. Congenital stricture is rare, and is usually confined to the meatus or to its immediate vicinity. The cases of atresia of the meatus sometimes reported as congenital are often the result of infantile balano-posthitis caused by a long and adherent prepuce with a narrow orifice. In the cases said to have been observed by Nélaton (Delfau, Maladies des voies urinaires, Paris, 1880, page 241), the contractions were situated in three patients in the penile urethra and in one at the bulb. The six eases included by Thompson in his table of two hundred and twenty cases (Stricture of the Urethra, page 224) were only presumptively of congenital origin. An interesting case is reported (Jameson, Epitome of Practical Medicine, April, 1887, page 189) in which three congenital contractions were said to exist in a boy of ten, plainly perceptible to the touch externally, and necessitating operation.

I am not familiar with any similar cases of undoubted authenticity.

Narrowing of the meatus, reducing it almost to pin-point size, may exist from birth, giving rise to no appreciable symptoms, and often no surgical opinion is procured in reference to it until an attack of urethritis during adult life obliges the patient to seek advice. Such cases are frequent enough to make one doubt the accuracy of these definitions of stricture which include all encroachments upon the average normal urethral caliber and ignore the associated pathological conditions. Such a congenital stricture should be operated upon without hesitancy when any urinary symptoms appear, or when it interferes with the proper exploration or treatment of the urethra or bladder; but the tendency of late years has been rather to overrate the clinical significance and importance of narrowings of the meatus and to divide the latter with unnecessary frequency and freedom. An artificial balanic hypospadias is often thus produced, which leaves the patient with a diminished power of ejection of both urine and semen. 


\section{ACQUIRED STRICTURE.}

Definition. - Acquired stricture includes all other forms than those above mentioned, and may be defined as an abnormal lessening of the caliber or dilatability of the urethral canal associated with changes in the mucous, submucous, or muscular structures constituting its walls * (An American Text-Book of Surgery, page 882). This definition includes the following chief varieties of stricture: (1) Inflammatory; (2) spasmodic; (3) organic- $a$, of large caliber ; $b$, of small caliber.

Inflammatory Stricture.-The term inflammatory stricture is objectionable, as tending to confuse a passing condition and one merely symptomatic of ordinary processes of inflammation with a definite and permanent lesion of much more importance.

The existence of inflammatory stricture has been denied by eminent authorities, who assert that without congestion of the prostate, spasm of the circular muscular fibers of the urethra, or pre-existent organic stricture, no swelling of the mucous membrane alone is competent to give rise to retention of urine. While I believe this to be true, it can not be disputed that occasionally in cases of acute anterior urethritis, with no suspicion of previous stricture and with the prostate unaffected, we have great diminution in the size of the stream of urine, manifestly from the unnatural approximation of the swollen urethral walls. Although the condition is almost always of short duration, and never goes on to retention, it is often the first step in the formation of organic stricture. The treatment is that appropriate to the form of urethritis in question. If, as has been recently suggested (Culver and Hayden, page 88), the term inflammatory stricture were dropped and we spoke merely of the actual condition present in such cases-viz., an acute catarrhal inflammation with recent soft exudation into the mucous and submucous tissues-our nomenclature would be simplified and improved.

Spasmodic Stricture is the result of a contraction of the circular muscular fibers of the urethra, either the unstriped or the compressor urethræ. This is usually reflex, but is sometimes the result of psychical causes, as shame, fear, anger, etc. In the former variety, which is of most surgical importance, it usually depends upon reflex irritation transmitted from some point of irritation in the urethra itself, as from an organic stricture or a patch of granular urethritis in the penile urethra or near the buli). More rarely it arises from reflex irritation from a greater distance. The retention of urine which often follows surgical operations, especially those upon the anus and rectum, is probably as often

* Sir Charles Bell defined it as a loss of elasticity, which would exclude both the spasmodic and inflammatory varieties. 
the result of vesical inhibition as of urethral spasm, although it is usually attributed to the latter. Schatz (Centralblatt für Gynäkologie, June 16, 1858) has, however, called attention to a method of relieving the retention of urine which sometimes follows parturition, which consists in introducing into the bladder an instrument like a glove-stretcher, and dilating the sphincter vesicx and the urethra so that the tip of the little finger could be inserted. He found this almost invariably gave permanent relief. As the condition is similar to the retention above spoken of, it offers an argument by analogy in favor of the theory of spasm.

Spasmodic stricture is also often thought to depend upon a narrowing of the meatus, and large numbers of cases have been reported in which more or less persistent spasm was attributed to this cause and relieved by meatotomy. It must be remembered, however, that spasmodic stricture is particularly apt to occur in nervous, excitable, irritable young men, allied in type to hysterical women, and that in such patients any marked mental impression often causes a disappearance of existing symptoms. Davenport (Keyes, Genito-urinary Diseases, page 101) records a case in which all the symptoms of deep urethral obstruction existed for ten years, and were relieved immediately and permanently by a single catheterization, a result which would doubtless have been attributed to a meatotomy if that operation had been required as a preliminary.

I was at one time an earnest believer in the frequency of urethral spasm as a result of a small meatus, or a large anterior stricture, and I still believe in its possibility. I have seen and reported (Philadelphia Medical Times, 1877) cases which seem to admit of no other interpretation, but I am now satisfied that many cases, in which I then attributed the improvement in my patients to the meatotomy or urethrotomy which was performed, would have been equally benefited by the passage of a goodsized sound through the deep urethra. If the meatus or the anterior contraction is too small to admit such a sound, a cutting operation is, I think, clearly indicated in the presence of otherwise inexplicable urinary symptoms, such as frequent micturition, " urinary stammering," urethrismus, etc., but I certainly do not see among my patients to-day the same proportion of cases which in my judgment require cutting operations as I did some years ago. Keyes (Annual of Medical Sciences, 1888) has arrived at the same conclusion, as have many other genito-urinary surgeons. Guyon (Leçons cliniques, Paris, 1881) says that he has never detected membranous spasm in simple relative atresia of the meatus, but does not deny its possibility.

Organic Stricture may occur in persons of any age, but is most frequent between twenty and forty-five. Women are not entirely exempt. McIntosh and Carter have given extensive statistics, derived from the records of the United States Marine Hospital Service, to show that gon- 
orrhœal strictures of the urethra occur much less frequently in negroes than in white men in proportion to the number of cases of gonorrhœa; they conclude that in negroes the ratio is about one stricture to twentythree gonorrhœas; in white men about one in eight. These observations have not been repeated, so far as I know.

The great majority of such strictures result from a precedent urethritis or from traumatism. The former is by far the more common cause. It acts in a manner easily understood, and is more potent in direct proportion to its duration. Its intensity is of some but of minor importance. Of 164 cases due to urethritis, 90 were reported to have been of long continuance. Inflammation of the urethra is peculiarly apt to run into a chronic form for several reasons. The canal affords periodical passage to a secretion, the urine, which is especially liable, by reason of changes in its constitution, to become an actual irritant; it is exposed, at times of erection, to intense congestion of all its vessels, and the converse is also true, a congested or irritated spot along the urethra predisposing to erection; gravitation, the proportionately excessive supply of blood to the region, and the absence of extravascular resistance due to the loose character of the spongy tissue, all favor the persistence of any congestion left after a first attack of urethritis ; the condition of approximation of mucous surfaces, as of the urethral walls during the intervals of micturition, is here, as elsewhere, unfavorable to the disappearance of granular or injected areas, or other traces of inflammation. The tendency of the gonococcus to establish itself in the deeper layers of the mucous lining, and to multiply there where it is comparatively inaccessible, is another cause of the frequent occurrence of the chronic forms of urethral inflammation.

In chronic granular urethritis the urethral epithelium becomes so damaged at one or more spots, as a consequence of the prolonged inflammation, that it permits the escape of minute quantities of urine into the tissue comprising and surrounding the urethra. Harrison has well summarized (Lettsomian Lectures, 1888) the resulting changes: To prevent urine soaking farther into the tissue, inflammatory exudation is excited, and barriers of lymph, which ultimately become organized, are thrown out opposite the places where the leakages take place. Thus, splints of plastic tissue are formed, corresponding with the spot or spots where the epithelium has been so damaged by persisting inflammation as to cease to discharge its normal function. In this strengthening of the urethra we recognize, in the first instance, a conservative action; eventually, however, as in other compensating processes, certain inconveniences follow which constitute, as it were, an independent disease. In addition to the careful observations which have been made relative to the pathology of gleet and the changes that are induced by chronic inflammation in the 
epithelial lining of the urethra by Dr. Oberländer, of Dresden, there are other considerations which seem to indicate that an excessive form of plastic exudation in the tissues around the urethra is probably excited by the interstitial leakage or exosmosis of some of the constituents of the urine through the walls of the canal.

Among those mentioned by Harrison are: 1. That though the mucous membrane is the tissue chiefly involved in the primary inflammation, it is, as a rule, only secondarily implicated in the stricture-forming process. In many instances it will be found after death that the dimensions of the mucous.membrane are not permanently altered, and that it is possible to split a stricture without necessarily damaging the lining membrane of the canal. 2. That the plastic exudation which makes up a stricture differs from other exudations provoked in other parts of the body by inflammation in the degree of its density and tendency to contract. 3 . The character of the cicatrix which is formed in connection with ruptures and lacerations of the urethra unmistakably shows the effect produced in the healing process of a recent wound, which is constantly submitted to the action of more or less pent-up urine. Here we have a cicatrix which of all strictures is the most resisting and contractile.

Further, the form in which stricture tissue is deposited, and ultimately exercises contractile pressure on the urethral passage, is strongly suggestive that in the first instance it served the purpose of strengthening the wall of the canal, and thus preventing the further leakage of some of the constituents of the urine from taking place at points where the epithelial coat had been more or less permanently damaged. Most strictures are the result of organized lymph which has been deposited in the submucous tissue in an irregular form. An annular stricture is comparatively rare, except when due to traumatic causes-such, for instance, as an injury to the whole caliber of the urethra.

Oberländer, whose views have been alluded to above, thinks that he has demonstrated the existence of two chief forms of chronic urethritis: 1. That in which the infiltration of the mucons membrane is diffuse and superficial and the glandular elements are not involved. 2. That in which the glands of Littré are markedly affected. He describes several subdivisions of this variety, giving to the form which ends in extensive cicatricial bundles projecting high above the level of the mucous membrane the name urethritis glandularis stringens, as it is this condition which, according to him, results in stricture.*

Neelson has confirmed these views by a long series of antopsies, which show that the glandular affection is extremely persistent and easily recog-

* See an excellent résumé of Oberländer's views by Fordyce, Jour. of Cut. and Gen.Urin. Dis., January, 1889, p. 19. 
nizable even when cadaveric maceration has destroyed the evidence of change in the mucosa and of epithelial proliferation.

Bryson, in an admirable paper (Journal of Cutaneous and Genito-urinary Diseases, June, 1889) calls attention to the fact that a definition which makes of stricture a mere mechanical narrowing of the canal includes conditions which no one regards as stricture in the true sense of that term, such as polypi, warty growths, periurethral tumors or abscesses, etc. He thinks that the definite characteristic of stricture is its persistent tendency to become more dense and to contract toward the axis of the affected canal. This excludes narrowings, the essential difference being that in the latter condition we have overlying healthy mucous membrane. He suggests the name of "chronic contracting periurethritis" as accurately describing the "stricture disease," in which we have the changed condition of the mucosa as the essential though passive factor and the urine leakage as the active element. Further evidence in confirmation of this view is furnished by what Mr. Buxton Browne calls " catheter stricture," a form of stricture not usually mentioned in text-books, but which in actual practice causes a great deal of trouble, and even danger. It occurs in elderly men obliged to use the catheter constantly, in many cases appearing to be due to want of care in the selection and use of the catheter. About five or five and a half inches down the urethra the walls of that canal appear to be in a state of spasmodic contraction and inflammation, the mucous membrane loses its polish, and doubtless a certain amount of inflammatory deposit takes place. This condition occasionally occurs in cases where, previous to the introduction to catheter life, lithotrity has been performed for vesical calculus, and the urethra has been accidentally lacerated; while in other cases it has followed the prolonged employment of an India-rubber catheter, which, as the result of frequent use, had become eminently adapted for rubbing the epithelium off the walls of any narrow portion of the urethra and irritating them into a state of contraction.

As a rule, the processes of urine leakage and of stricture formation extend over a considerable time. Thompson found that, in 164 cases, 71 occurred within a year, 63 in from three to eight years, 20 in from eight to twenty-five years, and only 10 soon after the subsidence of the urethritis.

Guyon, in 83 cases, found that 24 appeared in from one to two years, 14 in from two to four years, 29 in from four to ten years, and 16 in from ten to fifteen years or later.

Traumatic Stricture follows blows upon or injuries to the perinæum, most frequently falls astride of a bar, a fence, or some resistant body ; it may occur from fracture of the pelvis or from laceration of the urethra; it sometimes follows injuries or twists of the penis received during coitus, 
or the so-called "fracture of the penis"-a subcutaneous rupture of the erectile tissue from violence while the organ is erect; occasionally it results from the reprehensible practice of suddenly and violently straightening the penis during chordee-“breaking a chordee." Instrumentation in the normal urethra, if rough, clumsy, or unusually prolonged, as in some cases of litholapaxy, may be followed by contraction of the canal. Foreign bodies of all sorts arrested in the urethra, and ulceration from any cause, as chancre or chancroid, are competent causes of stricture, among which must also be included some of the methods of treating previous contractions, as by incisions, excision, cauterization, electrolysis, etc. The widespread popular belief that injections are frequently the cause of stricture is unfounded. If they are very irritant, or so strong as to act as cauterants, they may be ; but as ordinarily used, by lessening the intensity of the inflammation and coating and protecting the urethra, they serve to diminish the exfoliation of epithelium, and thus lessen the leakage of urine, which, as we have seen, is the chief cause of organic stricture.

Prolonged erection, excessive coition, and masturbation have been invoked as competent causes of stricture, more especially by those ardent believers in stricture of large caliber who find them in nearly every urethra, and often in the absence of what Guyon calls a "urethral history," which he considers an essential in the diagnosis of stricture-i. e., the history of gonorrhœa or of some one of the sources of irritation above enumerated. While the possibility of stricture from such a cause as masturbation can scarcely be denied on theoretical grounds, its occurrence is, as a matter of fact, of the greatest rarity. This is shown by the comparative infrequency of strictures among adult males of any community as compared with the number who have at some period of their lives been addicted to the habit of masturbation, and still more forcibly by the history of persons in prisons, asylums, and hospitals for the insane, who have been known to practice the habit to excess over long periods. Some years ago, while acting as medical officer to the Eastern Penitentiary, a prison containing about 1,000 convicts, and conducted on the principle of separate confinement, I began some observations upon the urethræ of those prisoners who were known to be persistent masturbators. I abandoned the investigation on account of the impossibility of eliminating other causes of disease, particularly gonorrhœa ; but it was perfectly clear that among that class of prisoners there was not an unusual number of coarctations; and I do not think masturbation a sufficiently active or efficient cause for their frequent production. If it were a cause, the effects would surely in a certain proportion of cases be more serious and more noticeable; if it were capable in nearly every case, as has been claimed (Gross, Medical and Surgical Reporter, May 5, 1877), of produc- 
ing one stricture of large caliber, it would sometimes be followed by several strictures of smaller caliber. The institution alluded to is one which affords exceptional advantages for the study and observation of onanism and its effects, and the subject was one in which I was specially interested; but I do not remember, during my experience of three years, ever to have seen in a confirmed masturbator retention or incontinence of urine, frequent or difficult micturition, or any of the more marked symptoms which would usually accompany stricture of the urethra. Neither have I learned that those physicians who have charge of the insane, and who see large numbers of onanists, have ever made similar observations.

If stricture is no more common in such pa-

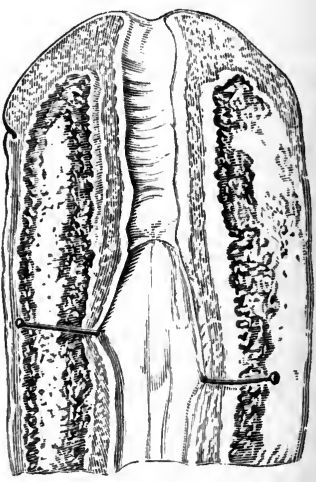

FIG. 1.-(Voillemier.) tients than among their associates, it may safely be assumed that, at least as ordinarily practiced, masturbation can only be productive of organic stricture with such rarity as to make it scarcely worth considering as an etiological factor. The dictum of Sir James Paget may still be said to

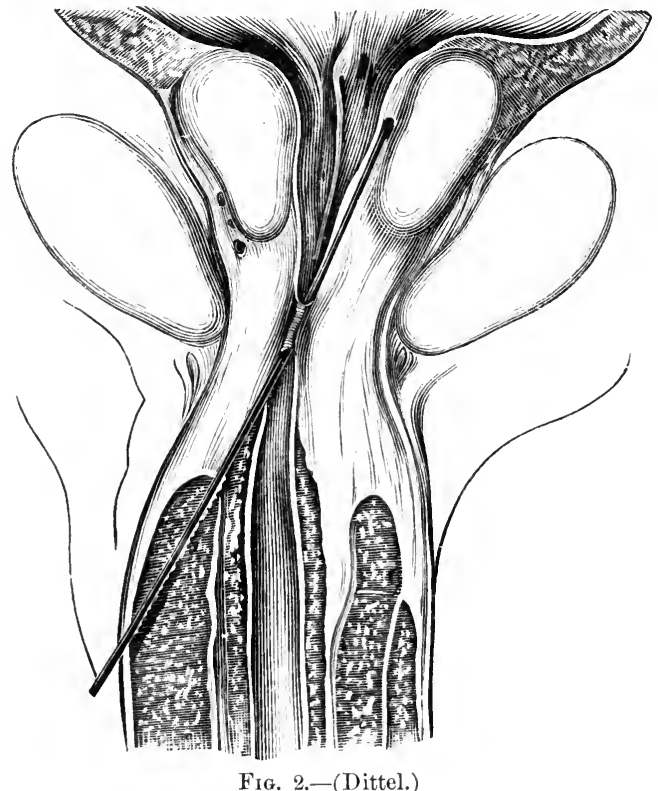
express the view of the profession, namely, that "masturbation does neither more nor less harm than sexual intercourse, practiced with the same frequency, in the same conditions of general health, age, and circumstances" (Clinical Lectures and Essays).

Character of Stricture. - The pathological condition varies from an induration and thickening of the mucous membrane with connective-tissue proliferation occurring in its depth to the formation of a dense mass of cicatricial tissue occupying the submucous region and extending into the meshes of the corpus spongiosum. The strictured portion of the urethra varies greatly in extent from a mere cordlike band, the so-called linear 
stricture (Fig. 1), to one slightly broader (annular stricture) (Fig. 2), and from that to a contraction which may involve as much as two or three inches of the canal, converting it into a devious, irregular channel. This has been called the tortuous stricture (Fig. 3). Many classifications have been adopted, but this answers very well for practical purposes. They have also been divided (Keyes) into-1, soft or recent, including those in which the lesions are superficial and involve chiefly the mucous membrane-this subdivision embraces the strictures of large caliber and the cases of chronic urethritis resembling stricture or constituting its first stage; 2, cicatricial, usually traumatic; and, 3, inodular, always gonorrhœal, and characterized by a lumpy, irregular, ill-defined mass of new fibrous tissue, often cartilaginous in consistence. A peculiar form of contraction of the meatus, of which I have seen several examples, consists in a diffuse induration of the mucous membrane, the hardness extending outward on the glans and for some distance inward. The indurated tissue was scarlike in appearance and semi-cartilaginous in consistency. In one of my cases the condition was associated with a tightly adherent prepuce in a man past middle age. In two cases reported by Greig Smith (Bristol Med.Chir. Jour., September, 1884, page 154) the same condition existed. It appears to be a form of scleroderma. Local

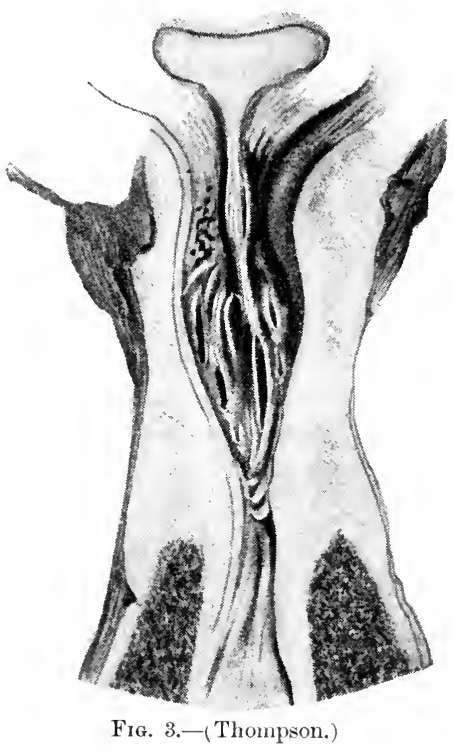
treatment was of little avail, but in my cases there was much spontaneous improvement after a considerable lapse of time.

Strictures are further divided into irritable, when they are readily inflamed and bleed easily upon the touch of an instrument, and resilient, when they are elastic and contractile, returning with great rapidity to their former size after being dilated. These conditions depend upon the relative vascularity and density of the cicatricial tissue constituting the stricture.

They are also divided into those of large caliber and those of small caliber.

For many years the greatest differences of opinion in regard to urethral stricture have centered upon the existence or non-existence, the pathological importance and the treatment, of strictures of large caliber. They have been asserted, on the one hand, to be, by reason of the frequency of 
their occurrence and of the failure to recognize them, the most important of all strictures; while, on the other hand, a recent excellent little manual (Culver and Hayden, page 88) gives them no place in the classification of strictures, but includes the conditions heretofore described as strictures of large caliber under the caption "Chronic Anterior Urethritis."

That every urethral coarctation following on urethritis must at some time have been a stricture of large caliber is self-evident, but just when such a stricture becomes an active pathological factor, and is able to give rise to symptoms, is an unsettled point. Indeed, it is not probable that it ever can be definitely determined in a mathematical sense. The idea that any particular fixed caliber represents the normal condition of the urethra has long been abandoned, the observed variations of that canal being such that no special dimensions can be assigned to it as representing the precise dividing line between health and disease. The old method of regarding the size of the meatus as an indication of the normal caliber of the canal behind it, although it still has the weighty indorsement of Guyon, is also unquestionably fallacious, it having been conclusively shown that no more definite relation exists between them than between any other mucous canal and its corresponding outlet - the mouth and the osophagus, for instance, or the anus and the sigmoid flexure. That there is a certain correspondence between the size of the urethra and that of the flaccid penis is true, the caliber of the one increasing with the circumference of the other, but that this ratio is present in any absolutely unvarying manner has not yet been demonstrated. At the most, the size of the penis may be said to furnish a general indication of the urethral dimensions, but one which is approximate merely. On the other hand, it has been shown that there are usually certain normal variations even in the spongy portion, and that it is impossible with any of the means at our command to distinguish between these natural irregularities and coarctations of equal caliber due to incipient stricture. I have myself always emphasized the occasional pathological importance of even a moderate infringement upon the caliber of the urethra (International Encyclopædia of Surgery, vol. ii, page 353), and have elsewhere summarized (Annales des maladies des org. gen.-urin., Paris, November, 1888) the theoretical arguments in favor of regarding the group of symptoms which includes gleet, frequent urination, dribbling after micturition, and lumbar and hypogastric pain, as usually indicative of some degree of urethral coarctation. Those arguments were in the main as follows :

Habit is a powerful agent in facilitating and controlling the functions of animal life. Illustrations of this fact in other systems than the genitourinary are frequent and familiar. The evacuation of the bowels at certain hours of the day, and the difficulties resulting from inattention to their requirements; the easy and unnoticed digestion of food taken at 
proper intervals, and the anorexia and dyspepsia following irregularity in this respect; the imperceptible and uniform action of the heart under ordinary circumstances; its tumultuous and uneven palpitation when its work is suddenly increased; the many apparently trivial agencies which are notably sufficient to interfere with the mental processes when accustomed routine is broken into-all these are a few of the unlimited examples that might be adduced to show the force of habit in influencing the mode of performance of such functions.

To take up the case in question, it is safe to say that, in a healthy male adult, a certain equilibrium has been established and maintained between the usual efforts and powers of the bladder as an expulsive organ, and a certain average amount of resistance which must be overcome before it can empty itself. At the age of twenty-one years this adjustment of force depends upon a large number of previous distinct acts of micturition-about thirty thousand, if an average of four daily be taken. This balance between the force of expulsion and its work can not with impunity be disturbed, and even a slight interference with the caliber of the urethra may exceptionally tend to produce such disturbance.

Apart from the proclivity to muscular spasm in the neighborhood of and behind every stricture, this interference with the action of the bladder arises from the encroachment of the new deposit upon the urethral caliber. It is a law of hydrostatics that a degree of friction proportionate to the amount and velocity of the current and the size of the tube takes place between the walls of the latter and the liquid; if the tube be narrowed at any one place, the friction is increased at that point, and, to avoid a diminution in velocity, the propulsive force behind the liquid must also be correspondingly increased. The pain excited by the very simple experiment of moderately compressing the urethra during micturition will serve to illustrate the bearing of this fact, as does also the exceedingly slight amount of prostatic enlargement which often suffices to bring on vesical troubles.

Still another point may be mentioned. The act of micturition is one requiring for its perfectly normal performance, first, relaxation of certain muscles to secure patency of the urinary channel and, next, the thorough and complete contraction of those muscles to produce entire evacuation of the contents of the passage, which would otherwise leave it guttatim. The latter portion of this act is accomplished by the contraction of the circular fibers which surround the urethra, and which, during the intervals of urination in a healthy condition, serve to bring and retain its walls in close apposition. The submucous deposit, which increases the friction of the stream of urine at any point, also interferes with the accurate closure of the canal by those muscles whose action is impeded, and whose structure itself is in part often invaded, and, as a consequence, we 
have an imperfect emptying of the urethra at the end of urination. Finally, if, in addition, we recall the intimate nervous connection of the urethra with all the viscera of the abdomen and pelvis, and with the walls of those cavities, we are in a position to sum up the mutual relation of the pathological and subjective phenomena as follows:

The increased friction and resistance resulting from even a slight fibrous periurethral deposit may disturb the normal relations of the bladder, and, by rendering it irritable, bring on one of the common symptoms of stricture, frequent micturition. The imperfect closure of the tube, the muscular action of which at the point of deposit is materially interfered with, causes the equally imperfect expulsion of the last drops of urine, and produces another equally characteristic symptom, dribbling at the end of micturition. The retention and decomposition of these last drops, together with the abnormal friction between the stream of urine and the urethral walls, give rise to a subacute inflammation of the mucous membrane, accompanied with a catarrhal or muco-purulent discharge, constituting the condition of gleet; by reflex irritation transmitted from the area of inflammation, pains in remote organs and situations are developed, notably in the lumbar and hypogastric regions.

This relation of causes and effects has been in the main accepted as correct by the profession for many years. Probably no one denies that in certain strictures in which the urethral caliber is markedly diminished the connection between the pathological changes and the observed indications is about as has been stated. The differences of opinion which now exist are chiefly as to the amount of urethral contraction which is sufficient to produce noticeable effects, and here the argument must rest upon clinical observations supported by the results of autopsies.

Those surgeons who follow the teachings of Dr. Otis, of New York, whose valuable work has added greatly to our knowledge of the subject in question, accept his scale of relation of the caliber of the urethra to the circumference of the flaccid penis, any interference with which they regard as evidence of the existence of stricture. The figures which Dr. Otis gives, while they doubtless represent accurately the distensibility of the male urethra, do not by any means represent what can fairly be called its normal caliber, and fail altogether to recognize the fact that there are points of physiological narrowing, notably along the course of the pendulous urethra, where some of his disciples find the greatest number of contractions. Sir Everard Home, Du Camp, Reybard, and others long ago demonstrated the variations in size and dilatability of the different portions of the urethra; and Civiale observed that in the middle of the spongy urethra there is a notable diminution of elasticity. Weir and Sands, of New York, confirmed by casts of the urethra these older observations, and there can now be no doubt of the existence 
of such physiological points of contraction, or of diminished distensibility.

Delpet has recently.(Ann. des mal. des org. gen.-urin., March, 1892, page 196) made a series of elaborate experiments to determine this and other points in the anatomy of the normal urethra. He found almost invariably a narrowing in the penile urethra at a point three to ten centimetres (one to three inches) from the meatus, at which region tears of the urethral wall from the passage of large instruments occurred with the greatest frequency. The next least dilatable and least resistant region was in the vicinity of the "prepubic angle." In but one instance was there any laceration in the bulbous urethra.

Guyon has, perhaps better than any one else, emphasized (Leçons cliniques, Paris, 1881, page 680) the distinction between the true caliber of the urethra and its distensibility. He believes that the normal caliber can be determined only approximatively, as the urethra is a canal only when traversed by a liquid or an instrument, and in the intervals lies collapsed in longitudinal folds and with its walls in contact. The true caliber is therefore that which separates the walls without stretching them. Sappey has endeavored to ascertain this, and asserts that, exclusive of the meatus externus, the average normal urethral circumference varies at parts from fifteen to eighteen millimetres, and that therefore a sound of No. 15 caliber (French) can be passed without calling into play the dilatability of the canal. Guyon states that his own clinical observations agree with these experimental results, but he also instituted a series of experiments by passing in thirty-seven instances on the cadaver catheters, varying from about No. 31 to No. 34 (French). In thirty instances lacerations were produced; in half of them, in the anterior or middle portion of the penile urethra ; they were almost exclusively on its inferior wall. He, as well as the other authorities mentioned, recognize the fact that the urethra is of varying caliber and has points of normal constriction.

Otis, however, in effect assumes that the urethra should be a tube of uniform caliber, at least anterior to the triangular ligament. The instrument which he has devised, the urethrometer, when used under the guidance of Otis's table, will detect apparent strictures in the majority of normal urethras, and is to-day only employed by practical surgeons in exceptional cases. A very ingenious instrument, the "urethrograph," has also been invented, and is said to be capable of giving an exact drawing of all the irregularities of the urethra; but, as Keyes says, while it seems an ideal mechanical device, its creation is to be deplored, since it "will tend to increase the already too great cutting of that patient canal, the urethra." Dr. Otis's views and teaching both as to diagnosis and treatment have been of great value as showing the distensibility of the 
normal urethra, as thus piving the way for the introduction of the large instruments employed in litholapaxy, as bringing out the full pathological value of true strictures, however slight, and in other particulars adding exactness to urethral surgery. It is not against their use but against their abuse that I desire to protest, and I shall have occasion to recur to the subject when considering the treatment of stricture (see page 310). We may admit at present for purposes of classification, that, exclusive of cases that are best described as examples of chronic urethritis, there are others in which the superficial inflammation has largely disappeared, and in which the periurethral or submucous deposit has begun to contract and to diminish the lumen of the canal, such contraction still admitting fairly good-sized instruments with ease. Such cases are entitled to the designation of stricture of large caliber, which appears to me accurately to describe them.

I have found it convenient, both in teaching and in practice, to make an arbitrary division of all organic strictures into those which will only admit instruments of less than fifteen millimetres in circumference and those which will easily permit larger instruments to pass, calling the former strictures of small caliber and limiting to the latter the term strictures of large caliber.

Location of Stricture.-The situation of stricture varies, but there can be no doubt that the great majority are to be found in the bulbo-membranous region, which includes a space from about one inch in front of the anterior layer of the triangular ligament to the prostato-membranous junction. The next most frequent seat is in the first two and a half inches of the urethra. The frequency of stricture in these regions is due to the fact that they are exceptionally vascular, and that chronic urethritis is especially apt to become localized at those points. Gravitation in both regions favors chronic congestion and may possibly of itself explain the clinical fact. The smallest number are found in the middle of the spongy urethra. These remarks apply to the form of stricture produced by urethritis. Traumatic stricture usually affects the membranous urethra.

The chief differences of opinion in regard to location will be found, when analyzed, to originate in an essential difference in the manner of approaching the subject. Those surgeons who very properly demand evidence of some organic change before admitting the existence of stricture, and base their views on the examination of existing specimens in museums, differ greatly from those who depend upon the urethrameter, and who believe in Otis's teaching of an almost unvarying relation between the caliber of the urethra and the size of the penis.

Thus we find that, while in 320 specimens examined by Sir Henry Thompson he found the stricture in 216, or sixty-seven per cent, in the 
bulbo-membranous region in 54, or seventeen per cent, within two and a half inches of the meatus, and in 51, or only sixteen per cent, in the intermediate spongy portion; Otis describes 258 strictures under his care as having been situated-115 in the first inch and a quarter, 129 between one and a quarter and five and a half inches from the meatus, and only 14 between five and a quarter and seven and a quarter-i. e., in the region of the bulb and the membranous urethra. It is scarcely to be doubted that many of these "strictures" were points of physiological narrowing.

Stricture of the prostatic region is practically unknown.

Guyon* divides the urethra into six regions: 1 . The navicular region, which corresponds to that part of the urethra surrounded by the glans penis, and extending from the meatus externus to the corona glandis. 2. The penile region proper, reaching from the termination of the navicular region to the entrance of the urethra into the scrotum. 3. The scrotal region, including, of course, all that part of the urethra contained within the limits of the scrotum. 4. The perineo-bulbar region, which extends from the posterior limit of the scrotal sac to the passage of the urethra under the pubic arch. 5. The membranous region. 6. The prostatic region. He finds gonorrhoal strictures most frequent and narrowest at the bulb. He adds that there are often, in addition, one just behind the navicular fossa and one at the peno-scrotal junction. In the navicular region cicatricial strictures from local and minor injuries are most common, as they are in the perineo-bulbar region from deep contusions and lacerations. In the scrotal and penile regions strictures are least frequently found.

Traumatic strictures are nearly always single, and may be due to various causes. They occur in the mid-penile portions of the urethra after rupture of a chordee; at the root of the penis when caused by "false movements" in coition; at the perineal or perineo-bulbar portions of the urethra when following falls on the perinæum; in the membranous portion after pelvic fracture, which Guyon thinks is the only traumatism that causes stricture in that region; at the meatus or fossa navicularis when the stricture is of chancrous origin or is due to the irritation of foreign bodies.

Changes in the Urethra.-The urethra behind a stricture undergoes certain progressive changes. It at first becomes dilated and thinned; the walls atrophy; it is deeply congested, the change of color in the surface at this point being often the most conspicuous feature in a strictured urethra laid open after death. As the stricture grows smaller the changes

* Leçons cliniques. See an excellent abstract by Dr. William Mastin (The Annals of Surgery, August, 1882). 
in the mucous membrane become more marked. The increasing pressure canses a corresponding increase in the pouching or dilatation, the retained urine decomposing sets up a superficial inflammation, erosion of the surface occurs, it is denuded of its epithelial layer, actual ulceration follows, and, as the loss of substance becomes greater, larger and larger quantities of urine escape into the spongy tissue, especially toward the floor of the canal, and abscesses followed by urinary fistula result. Delpet (op. cit.) has called attention to the fact that the mucous membrane of the urethra is in immediate contact with the erectile tissue of the spongy body, and that the lacunæ of Morgagni penetrate into the meshes of that structure. Stilling (Archiv für klin. Chirurgie, vol. xv, page 22) has also noted the intimate relation between the muscle and connective-tissue fibrils of the corpus spongiosum and the urethral mucous membrane, and thinks it explains many phenomena otherwise inexplicable, as the occurrence of spasm in the spongy urethra, the dribbling of urine in cases with exceedingly slight obstruction, etc. When the urine first reaches the surface of the body by the newly formed fistula the latter has soft, yielding walls, but these gradually become dense and indurated, undergoing the same pathological changes as did the original strictured region. Bryson has well described (op. cit., page 285) the subsequent changes: At first, when the fistula has formed and has soft walls, there is no great obstruction offered by it, and all the urine passes through by the new channel; but, however wide the fistulous opening may be, some urine comes in contact with the posterior edge of the stricture, perhaps remains constantly in contact with it, and thus there is the necessary condition for a continuation of the periuretbral contraction of the stricture band, and especially of its posterior edge. This answers to this stimulation just as the stricture did originally, and tightly seals the urethral outlet of the pouch, forcing the urine to pass by the new way. It takes time for hard walls to form about the fistula, and more time for them to contract to such a degree as to offer great resistance to the urine. When they do, we have what seems a battle between the urethral narrowing and the obstructive efforts on the part of the fistulous opening, and the periurethral bands at the site of stricture hold their own even when they are only a line or two in breadth, for they are often seen to keep the urethra tightly closed while forcing the formation of one, two, several new fistulous outlets, which latter have at first soft and yielding walls, which offer a minimum of resistance.

There is sometimes seen in front of the stricture under these circumstances a second pouch or infundibulum (Dict. des sci. med., article Urethra), which is, however, of a paler color and does not present the same indications of chronic inflammation. It is due to the atrophy and disappearance of the anterior portion of the coarctation, which, not being 
exposed to the continual irritation of the urine, has undergone retrograde metamorphosis and absorption. All urethræ do not undergo this precise series of changes in the presence of strictures, nor do the latter always contract with the same degree of rapidity. The differences depend on factors which can not be accurately determined or even estimated: the more or less irritant qualities of the urine, the amount of vital resistance of the tissue, the degree of compensatory hypertrophy of the bladder, etc. Occasionally a stricture may remain stationary for long periods, but as a rule the tendency is toward continuous contraction.

The opening of a stricture is only exceptionally found in the center of the urethra; much more commonly it is near the roof, as the bulk of the stricture formation is more apt to take place on the floor of the canal, and by encroaching upon its caliber from that direction to force the aperture out of its normal position and in an upward direction. It may, however, be found at any point of the circumference of the urethra.

The consistence of strictures varies with their age and with the greater or less amount of fibrous and elastic tissue which they contain. Their distensibility varies inversely with the consistence, as does usually their elasticity. The latter quality is undesirable from a therapentic standpoint, as when elastic tissue is in excess we have the so-called "resilient" stricture, which may permit readily of the passage of instruments, but will return almost instantly to its original caliber.

Section of a stricture of the annular or tortuous variety shows a more or less imperfect ring of new inflammatory tissue whose limits taper down gradually; this tissue is hard, yellowish-white near the lumen and darker on the outside, where reddish islets are seen, the result of hæmorrhagic infarcts, which form a focus for new inflammatory changes. The surrounding tissues, as the mucous membrane itself, the spongy, and even the cavernous bodies, may be involved. These changes are always greater on the floor. In the latter situation, according to Brissand and Segond, is found a hard, dense, fibrous, triangular mass, with its summit running up to the mucous membrane and its wide base resting on the albugineous coat of the spongy body, occupying on an average about one fifth of the circumference of the urethra. On the roof is a narrower strip of the spongy body changed to fibrillar elastic elements. This does not involve the whole of the thickness of the spongy body. The two lateral walls are unchanged, their tissue gradually running into the elastic elements above and the fibrous stratum below.

Although Oberländer has shown that the inflammatory process practically begins in the glandular recesses, and these are most abundant on the roof, yet the floor presents the greatest changes, from the fact that the gonorrhœal process is always more active there.

Complete obliteration of the urethra is extremely rare, and it is doubt- 
ful if it ever happens except in the traumatic forms of stricture following extensive laceration or complete separation of the urethra.

Tadriatte (Dict. des sciences méd.) has collected nineteen cases of complete obliteration; fifteen of these were at the bulb, one at the suspensory ligament, two in the penile portion, and one three inches behind the meatus.

The last four cases followed bullet-wounds. The obliteration was usually about one to two centimetres in length, with fistulæ behind; in one case only, that of Peso, a fistulous tract around the cicatricial tissue, maintained a passage for the urine; it was probably the result of a false passage.

Symptoms of Stricture.-The phenomena produced by stricture vary, of course, with the degree and character of the coarctation. They may be described, however, most conveniently by dividing them into, first, subjective symptoms - those recognizable by the patient; and, second, objective symptoms-those elicited by surgical exploration.

The subjective symptoms may be considered in the order of their frequency, certain of these being common to all strictures, others usually confined to very narrow or tight stricture.

a. Inquiry should be made for the so-called "urethral history" (Guyon). This should disclose a precedent urethritis, usually of a date at least one or two years earlier, often having been of long duration; or a trauma to the urethra, perinæum, or pelvis; or a urethral chancre; or some of the causes mentioned on page 267 , as competent to produce a stricture.

b. Alterations in Micturition.-1. Frequency.-This arises first from the change in relation between the expulsive force required of the bladder and the accustomed demands upon it; then from extension of inflammation backward by continuity until the vesical neck is involved; often from the production of a genuine cystitis; later from atony with retention, there being often an unsuspected amount of residual urine present in cases of stricture (Fenwick, Journal of Cutaneous and Genito-urinary Diseases, November, 1888, page 414). In these cases the frequency is worse by day, as in stone; not by night, as in prostatic disease.

2. Change in the Character of the Stream, which may be double, flat, gimlet-shaped, or spray-like, and in tight strictures becomes much reduced in size. This symptom is of but little value, as both the shape and size of the stream depend more on the shape and size of the meatus than upon any condition posterior to it. If the meatus is of good caliber, urethral narrowing may remain unnoticed for some time, as a compensatory hypertrophy of the detrusor urinæ occurs which overcomes the obstruction.

3. Diminution of Expulsive Power.-This is a late symptom, and 
is only developed when vesical atony has succeeded to the hypertrophy just described.

4. Dribbling after Urination depends upon the retention behind the stricture of some drops of urine, which escape by gravity after the act of micturition is complete. It is not infrequently a very early symptom, dependent on irregular action of the circular muscle fibers of the urethra. The dribbling, which is called the "incontinence of retention," the overflow from a distended bladder, is a very late symptom, following retention and usually associated with a high degree of atony. The incontinence of stricture is to be diagnosticated from the incontinence of prostatic hypertrophy by the fact that it is at first worse in the daytime, and only becomes nocturnal later. The reverse is the case in prostatic incontinence. The mechanism of incontinence of urethral origin is simple. The dilatation of the urethra behind the stricture having extended to the neck of the bladder, the urinary reservoir becomes in shape a funnel, the bladder representing the base, the neck situated at the point of stricture. The patient being in the erect position, the weight of the column of urine comes directly on the stricture, which permits it to filter through drop by drop. In dorsal decubitus, on the other hand, the bladder fills up and retains its contents until the changes in it and in the urethra are very far advanced. In the prostatic patient it is possible that the physiological congestion of the lumbar cord produced by the recumbent posture makes urination more frequent at night and during the early morning hours. It lessens as the day goes on, and it is only later when the bladder becomes confirmed in irritability that diurnal frequency follows.

5. Ardor Urince is extremely variable, but is not apt to be marked unless there is a considerable degree of prostato-cystitis present.

6. Retention of Urine may occur early and suddenly from an acute increase of the congestion of the mucous membrane of the strictured region, or it may be a late symptom and dependent on the great obstruction offered by the stricture. In either case it is apt to be precipitated by fatigue or cold, or by alcoholic or sexual excess.

7. Vesical Tenesmus is usually constant during the entire act of micturition; that of prostatic hypertrophy is most violent at the beginning, and grows less as the water begins to flow; that of eystitis is most severe at the end of the act.

c. Urethral Discharge.-Opinions vary as to the constancy of this symptom in its relation to stricture. On the one hand, they are said to have an invariable and reciprocal relation to each other (gleet being the "signal" which stricture throws out in evidence of its presence); on the other, it is said (Dict. des sciences méd.) to have occurred only four times in sixty-one cases of stricture (Janin). According to my own experience, about fifty per cent of strictures are accompanied by a gleety discharge 
from the meatus, and a large majority of the other fifty per cent show mucous and epithelial shreds and pus-corpuscles in the urine. I believe with Desnos (op. cit.), however, that many of Otis's cases of "gleet with stricture of large caliber" are merely cases of chronic urethritis.

d. Interference with Coition.-The physiological congestion is increased; after the orgasm, semen is retained behind the stricture, and great pain may be occasioned by the distention of the urethra at that point; the semen dribbles away slowly for a long time after the subsidence of the erection; ejaculation, when it takes place at all, is often premature; erection is apt to be imperfect or to subside before the completion of the act.

e. Constitutional Symptoms.-These are late, and depend upon the vesical and renal changes with the concomitant alteration in the urine. They are therefore usually a combination of uræmic and sapræmic or septicæmic phenomena. There is a red, glazed tongue, with anorexia, dyspepsia, constipation, etc. The dryness of the tongue extends to the walls of the pharynx, making deglutition painful— "buccal dysphagia" of Guyon. An irregular febrile movement supervenes; the face becomes pinched and yellow, the eyes sunken, the general strength fails, and the patient, after rapid emaciation and profound prostration, dies comatose.

Objective Symptoms of Stricture.-In describing the methods to be employed in the detection of urethral stricture it will be necessary to add somewhat to the statements already made in reference to the anatomy and physiology of the canal. Guyon (Mastin, loc supra cit.) believes that it would be of advantage so to divide the urethra that the location of a contraction at any one point of the canal can be designated at once in an easily expressed and clearly comprehensible manner, or, in a word, that the urethra be examined in its entire length by regions and not by centimetres. He has therefore divided it into the six regions already described. These regional divisions, he declares, are amply justified by clinical experience and certain distinguishing features, since in connection with the navicular portion is to be remembered the care to be exercised in the employment of fine instruments, presenting them to the inferior and not to the superior wall, as is the rule in the other regions, the arresting of extraneous bodies, and the frequency of cicatricial strictures. The penile is the portion which is the most movable, the most superficial, and the least fixed; contractions which are observed in it present the clearest pathological characters; fistulæ occurring here require absolutely special operative measures for their closure. In the scrotal portion, which is deeper and more fixed, stricture is comparatively rare; foreign bodies are seldom arrested; and when it becomes necessary to open it, for instance during the swelling and thickening attending abscesses or urinary infiltration, when it is difficult to trace by the finger an 
instrument introduced into the canal, the median line is the most convenient guide. The perineo-bulbar portion is really fixed and deep; it is the seat of election for blenorrhagic contractions, and here, too, traumatic strictures from deep contusions and lacerations of the urethra are more frequently encountered; again, it is toward this region that the bistoury must be directed in deep urinary infiltration and in practicing external urethrotomy without a guide (perineal section) when it should be opened in the median line exclusively.

Guyon further continues his admirable description by examining the so-called urethral curves. The superior wall has alone anything like a fixed curve or approaches the arc of a circle. The inferior wall, on the contrary, is only a broken line. This inferior surface is composed of three portions, of which the incurvations are unlike, and which are separated by two bends or angles, the form and situation of which are equally far from being fixed. The first portion of this broken curve is formed by that part of the prostate just above the openings of the ejaculatory ducts; the second is represented by the sus-montanale portion of the prostate, more or less developed, and by the entire membranous division, and varies greatly as to length and form; and the remainder is formed by the bulbar portion, which presents more uniformity in its disposition; hence, at the union of these three curved lines are found two species of bends or dilatations, which are the cause of the change of direction of the contiguous parts. Guyon directs attention to the fact that the inferior wall is extensible, soft, and depressible, and is subject to variations of both form and length; hence, the important point to be borne in mind by the surgeon is, that in catheterism he should either follow exactly in the curve of the superior wall, or modify the direction of the urethra; and to determine the adaptability of the canal to instruments, anatomy must be depended upon to indicate to us the normal direction of the urethra and the limit of modification which it will bear withont sustaining a lesion; and here it is to be especially remembered that the first part of the urethral curve-that extending from the suspensory ligament to the entrance into the membranous division, is, above all the rest, susceptible of modification and change of direction. The urethra has no lateral flexions, presenting neither bends nor flexuosities, but is exactly parallel with the median line. Nothing, however, is easier than to produce them in the entire spongy region, particularly in the bulbar portion.

It should never be forgotten, in making examinations for stricture, or indeed in any urethral instrumentation, that the elasticity or extensibility of the urethra resides for the most part in the spongy portion, as is clearly demonstrated by erection; and this elasticity belongs in the greatest degree to the inferior wall, which permits of easy distention or elongation, and changes its dimensions and form with notable facility; 
while the superior wall yields with much more reluctance, and offers a certain resistance to all agents tending to depress or elongate it. This difference increases with age, and obtains especially in senile urethræ.

The surgical interest in all this, in its relation to the treatment of stricture, is evident, since the extensibility of the inferior wall is brought into play even by a moderate force, and the surgeon can not count on its resistance. It glides before an instrument, and can not serve to guide it; it can not be incised with any accuracy or precision; it lacerates or ruptures when surprised by distention, and it yields rapidly and easily to a mechanical pressure testing its extensibility. It should be noted, too, that this elongation of the canal is chiefly at the expense of the anterior urethra. Again, the spongy portion does not yield equally in all its parts, since it has been shown that of the different regions the perineo-bulbar is the most distensible. The inferior wall of the urethra can then be considered as normally longer than the superior surface. The term "surgical wall" proposed for the upper wall by Guyon would seem to be merited because it offers the shortest route to the bladder; it is the most regular and constant as to form and direction; presents the smoothest and firmest surface; is the less capable of gliding before an instrument or being modified by mechanical pressure; offers the greatest assistance to rupture and penetration; is less intimately connected with important structures, and is the least vascular of the two walls. As to the caliber and distensibility of the urethra enough has already been said; but it should not be forgotten that there are two relatively constricted points, the internal and external meatus, and three dilatations, the fossa navicularis, the bulbar cul-de-sac, and the prostatic depression, the last two dilatations presenting numerous individual volieties; and in this comnection it is important to remark that all three of these dilatations are excavated at the expense of the inferior wall of the canal. The urethral curve only remaining regular in the superior wall, it results that the more pronounced the curve the more accentuated are the bulbar and prostatic depressions ; and as a certain degree of lengthening of the urethra always corresponds to the greatest curve--since these are both produced by bulbar and prostatic augmentation of volumeone can reasonably conclude that urethræ of the greatest curves. present at the same time the greatest length. With a knowledge of these facts the instrumental exploration of the urethra becomes a matter of much accuracy and precision.

The most valuable urethral instruments for the purpose of diagnosis are the so-called bougies à boule (Fig. 4). They may be made of metal, with slender stems, having small expanded ends or handles, upon which the number of the instruments may be marked; this should represent in millimetres the circumference of the shoulder of the bulb. More satis- 
factory instruments are, in my opinion, the flexible gum bougies a boule. The shoulder of the acorn-shaped bulb should join the shaft at almost a right angle, and not with the obtuse angle often found in improperly

FIG. 4.

shaped instruments. The size selected for exploration should be determined approximately in the manner already mentioned, by noting the circumference of the flaccid penis. Otis's scale is as follows:

Circumference of penis 3 inches; caliber of urethra 30 inches.

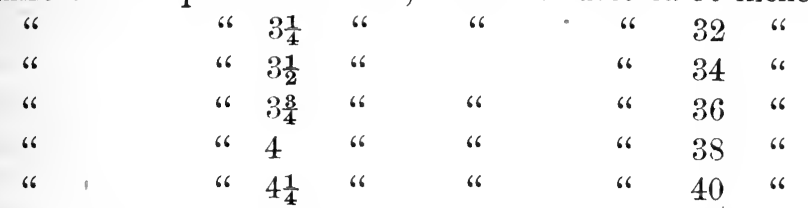

The scale which I have adopted for use, and which differs from that published by Dr. Otis in giving a lower grade of numbers, is as follows:

A penis three inches in circumference at the middle of the spongy portion indicates a urethra which should normally admit an instrument of about twenty-six to twenty-eight millimetres in size; when it is three and a quarter inches, the urethra should have a caliber of from twentyeight to thirty millimetres; three and a half inches, thirty-two to thirty four millimetres; four inches, thirty-four to thirty-six millimetres, beyond which size it is seldom necessary to go.

If the meatus be too small to admit of the introduction of a bulbous bougie of the required size, it should be enlarged. The penis should then be grasped just behind the corona and held gently between the thumb and finger of the left hand, the foreskin, if redundant, having been retracted. The dorsum of the penis should face the abdominal wall. The bougie, well oiled, should then be passed gently into the bladder. If arrested, the point on the shaft corresponding to the meatus should be marked, the distance from that to the bulb representing the position of the anterior face of the stricture; or the region in which it occurs should be carefully noted. If that instrument, or a smaller size, passes through, it should then, after a moment's delay, be withdrawn, and if during its outward passage any contraction is found other than that at the triangular ligament, it is probably due to stricture; though spasm, which often relaxes after a few seconds, or shifts its position in the canal slightly, as measured from the meatus, may give rise to errors in diagnosis. It can not always with certainty be recognized. It is almost never found except at the membranous urethra. It occurs in many urinary diseases, and especially in cystitis and tuberculosis of these organs, although the membranous portion is very rarely affected in tubereular 
cases by the disease itself. It is occasionally seen in cases of atresia of the meatus, or of phimosis, and in spinal cases, or in neuropathic and impressionable patients.

It should be remembered that gonorrhœal cases almost invariably give some point of induration or roughening at least of the urethra just anterior to the bulb. When this region is passed, if stricture exists there, the bougie a boule is released suddenly; spasm will often still hold the instrument firmly ; on returning there is no resistance at this point in spasm, while in stricture there is the same obstruction and the same sudden release at the anterior end of the obstruction.

Pain as a diagnostic symptom is unreliable, but is usually greater in spasm. Metallic instruments used with care almost always pass a spasm. Uncomplicated stricture may be difficult to distinguish from stricture with spasm-i. e., stricture of the anterior portion with symptomatic spasm of the membranous portion.

By the smaller scale I have adopted the probability of mistaking physiological narrowing for stricture is greatly diminished; and I believe that cases which present definite symptoms, such as gleety discharge, frequent urination, dribbling at the end of micturition, etc., and in which such an examination discloses a distinct contraction, may with propriety be considered as cases of organic stricture. This is not unduly conservative, but represents fairly, I think, the views of the large majority of modern surgeons, and especially of those who have any claim from special study or experience to speak with authority on the subject of genitourinary diseases. They will scarcely pass unchallenged by the practitioners who find, and cut, such a remarkable number of strictures in the penile urethra. It is, however, impossible, in my opinion, to read their statements as to the number of cases of this kind which they have cut and the number of strictures which they find in individual cases, and to recall at the same time thre almost entire absence of post-mortem evidence in support of their views, without being led to question either their surgical judgment or their statistics, or both.

As to the diagnosis of stricture of large caliber, even the bougie à boule may be misleading, if used in the deep urethra, on account of the normal points of obstruction to both its introduction and withdrawal which are there met with, while the urethrometer is similarly misleading in the pendulous urethra, especially if its revelations are interpreted according to the unnecessarily large standard of Otis. In the latter region the normal variations account satisfactorily to my mind for that proportion of the statements of a few writers and would-be teachers on this subject, which may charitably be put down to self-deception. In the deep urethra it has been necessary for them to account for their frequent discovery of strictures, even in cases without an active etiological factor, by 
attributing them to masturbation, sexual excess, etc. I have already expressed my opinion as to this point, and I may add that some years ago I endeavored, I believe successfully, to demonstrate that the "deep-seated stricture usually of large caliber found at the subpubic curvature and its vicinity," and described as " an essential lesion of masturbation" (Gross, op. cit.), was in reality the point of normal resistance to the withdrawal of bulbous bougies offered by the posterior layer of the triangular ligament.

The prostatic urethra being at once more movable and more dilatable than the membranous portion, the bulb slips smoothly along it until the point is reached at which this layer of fascia closely embraces the posterior part of the membranous urethra and the outer surface of the prostate. Here, for obvious reasons, it is arrested, and it is at this moment that the deceptive sensation which may be considered indicative of the existence of organic stricture is communicated to the hand. A series of observations and dissections upon the cadaver confirmed me in this view, which was originally purely theoretical, and, moreover, eliminated the possibility of the resistance being due to a spasm of the compressor urethræ muscle which surrounds the canal at this point, arrest of the instrument occurring just as invariably after death as before.

Having in a number of cases carefully brought the bulb closely up to the point of resistance, an assistant held it in position while I exposed it by a dissection of the deep urethra. I always found the shoulder of the bulb in the exact locality of the deep layer of the fascia, the edge of which would often be felt tense and cordlike over the upper wall of the urethra. A division on either side of its attachment to the ramus of the ischium, or to the pubes beneath the crura penis, would then cause an immediate disappearance of the resistance, and the bulb could be drawn outward smoothly and uninterruptedly. If the handle of the instrument were depressed during its withdrawal, the edge of the prostate became a cause of obstruction; and it may act thus to a greater or less degree in all cases.

The recognition of stricture of small caliber is a matter of no difficulty. In their presence it is well to use a medium-sized instrument, Nos. 16 to 18 (French) for purposes of exploration. When it is passed to the anterior surface of the stricture the region occupied by the latter may be easily noted, and its exact caliber determined by using successively smaller instruments. Multiple strictures may be recognized and measured in the same manner if they can be passed at all. The advantages of using a rather large instrument at first is that it eliminates the element of spasm of the membranous urethra, which will often, after a little gentle pressure, allow the blunt, rounded point of a medium-sized bongie to pass, although it would contract firmly and persistently before the point of a fine instrument. By using progressively smaller instruments also, the stricture can be more accurately measured as to both caliber and situation. Some- 
times, when no bouyie à boule will pass, a steel sound several sizes larger will do so with ease. The information it conveys is not so accurate, but is sufficiently so when the stricture is one of small caliber. The chief source of difficulty in diagnosis lies in the possibility of mistaking deep stricture for hypertrophy of the prostate, or vice versa. The distinction must be made by the history of the case, the age of the patient, the character of the symptoms, and the sensations communicated to the instrument in the urethra and to the finger in the rectum. It must be remembered that the urethra of prostatic disease is almost invariably elongated; that the shaft of the instrument will therefore enter it to an unusual depth, and that the handle must be more than ordinarily depressed before the beak reaches the bladder; that the patient is apt to be over fifty years of age, with the characteristic history of partial retention and nocturnal incontinence of urine; and that the obstruction will be found at a distance of more than six and a half inches from the meatus.

The points of normal narrowing of the urethra at the meatus, the middle of the spongy portion, and the membranous region will, of course, be borne in mind, and will not be mistaken for coarctations.

The presence, location, and caliber of a stricture having been determined, its dilatability is to be learned by the use of the conical steel sound, but it is usually well to make this investigation at a second visit.

Results of Stricture.-Unrelieved obstruction of the urethral canal continued for any length of time inevitably produces, in addition to the local conditions already described, a series of changes in the urinary tract posterior to the lesion.

Under long-continued and increasing pressure the urethra gradually enlarges, the mucous membrane becomes thinned and pouched, projecting in places between the bands of muscular fibers, forming diverticula analogous to those seen in the bladder. Sometimes, instead of the gradual escape of urine through minute openings with the formation of smaller abscesses and fistulæ, the urethra gives way more largely at a point behind the stricture, and extravasation of urine follows. The local symptoms of this condition are those produced by the retention of an irritant, and often a poisonous fluid within the tissues. The parts swell and become œdematous, the color of the skin changes to a dusky red or purple, emphysema from the gases of decomposition occurs, spots of gangrene develop, and extensive sloughing takes place. The general symptoms are those of profound sapræmia. There are great prostration, a dry, glazed tongue, a running pulse, frequent shallow respiration, wandering delirium, and finally, if the condition is unrelieved, death in coma. All these occur with greater intensity and rapidity if the bladder was already infected with the pyogenic and putrefactive microbes, and the urine was therefore fetid and purulent before extravasation occurred. This has been shown 
experimentally by Menzel and others (quoted by Keyes, op. cit., page 140), and is a common clinical observation.

The localizing symptoms-those which indicate the point at which the urethra has given way-depend upon the course taken by the urine. In all that part from the meatus to the scrotal curve, extravasation is accompanied by a swelling of the penis, greatest in the immediate neighborhood of the point of escape. In the region included between the attachment of the scrotum and the anterior part of the bulb, the course of extravasated urine is governed by the attachments of the deep layer of the superficial fascia, or the fascia of Colles. Extravasation of urine occurring through a solution of continuity in the bulbous region of the urethra will first follow the space inclosed by this fascia in front and below, and by the anterior layer of the triangular ligament posteriorly, and as it can not reach the ischio-rectal space on account of the attachment of the fascia to the base of the ligament, and can not reach the thighs on account of the insertion of the fascia into the ischio-pubic line, it is directed into the scrotal tissnes, and thence up between the pubic spine and symphysis until it reaches the abdomen.

When it escapes from the membranous urethra, extravasated urine is confined to the region included between the layers of the triangular ligament, and only gains access to the other parts after suppuration and sloughing have given it an outlet, the consecutive symptoms then depending upon the portion of the aponeurotic wall which first gave way. If the opening is situated behind the posterior layer of the triangular ligament-i. e., in the prostatic urethra-the urine may either follow the course of the rectum, making its appearance in the anal perinsum, or, as it is separated from the pelvis only by the thin pelvic fascia, it may make its way through the latter near the pubo-prostatic ligament, where it is especially weak, and may spread rapidly through the subperitoneal connective tissne.

The bladder becomes affected as the evils wrought by stricture progress backward. Occasionally, when the obstruction occurs suddenly, the walls at once become thinned and atrophied by over-distention. As a rule, however, a compensatory hypertrophy takes place first; the muscles become thick and rigid; the capacity of the viscus diminishes; the muscular fibers stand out in bars or ridges, having between them lozengeshaped spaces, over which the walls are greatly thinned. During the frequent and violent contractions of the organ the mucous membrane is driven outward between these muscular partitions, and the bladder finally becomes pouched at a number of places, the projecting sacculi (diverticula, tunicary herniæ) communicating with the interior by narrow mouths, and often containing gravel or calculi. Usually there is also a severe cystitis developed by infection through the urethra and adding greatly to 
the severity of the symptoms. The sacculi occasionally, but rarely, rupture, in which case fatal collapse is apt to follow.

The ureters become dilated at about the same time, partly from the actual backward pressure of the column of urine in cases where there is a distended bladder, partly from the frequent compression of their vesical ends during the-oft repeated acts of micturition. Their oblique course through the walls of the bladder render this compression very effective, and it has been shown (Morris) to be a competent cause for the production not only of ureteral dilatation, but also of hydronephrosis. Sooner or later, however, in addition to the distention of the ureters and kidneys and the mechanical interference thus occasioned with the secretion of urine, microbic infection occurs, and we have the renal alterations due to suppurative inflammation, a pyelonephritis first developing and then foci of suppuration at different points through the cortex and beneath the capsule, until finally the kidney may be converted into a large abscess cavity or into a series of pus-containing sacs, held together by the capsule and by inflammatory lymph, no trace of the secreting structure remaining. This condition has often been called "surgical kidney," but "unsurgical " kidney would be a more accurate term, as it never occurs in cases of stricture when competent advice is early sought and faithfully followed.

Among the possible results of stricture should be mentioned rectovesical fistulæ, vesical calculus, impotence, sterility, etc. Hunter McGuire has recorded (Medical Record, October, 1890) a number of cases in which paralysis, apoplexy, or some form of cerebral disease and spinal sclerosis followed long-standing stricture. He thinks that old strictures, by persistent irritation of one or more nerve-centers, may set up pathological change.

Prognosis.-The prognosis as to life depends, of course, on the stage which has been reached and upon the estimate which may be formed of the secondary organic changes that have taken place. Relief of the obstruction, drainage and antisepsis of the bladder, milk diet, renal antisepsis, etc., work astonishing improvement often in apparently desperate cases. Fenwick has forcibly called attention (Journ. of Cut. and Gen.Urin. Dis., November, 1888) to the fact that text-books of surgery and monographs on stricture are usually silent upon the question of prognosis in those cases of moderate severity in which most doubt would necessarily exist. As he remarks, the pathological effects of backward pressure upon the kidneys, and the results of the extension of inflammation from the bladder to that organ, are faithfully described as the usual outcome of unrelieved or neglected obstruction of the urethra. But no definite rules for ascertaining the progress of these insidious and lidden pressure changes are laid down, and the practitioner is left to surmise or to assume 
the stage of their development by the general condition of the patient, by an examination of the urine, or by the duration of the symptoms and narrowness of the caliber of the canal. The consequence is, that in the practical treatment of stricture we concern ourselves merely with the mechanical removal of the obstruction, and do not pause to ascertain if, or how far, the secreting structure of the kidney has been weakened, or rendered susceptible to the invasion of inflammation from contiguous surfaces.

He believes that just as the necessity for ascertaining the presence of residual urine in the vesical atony due to enlarged prostate or nerve lesions is duly impressed upon the student and practitioner, so likewise ought it to be inculcated that residual urine exists in most cases of stricture of the urethra, and ought to be measured; that its removal is of value in enabling the vesico-ureteric muscles to regain power, and in preventing the establishment of chronic cystitis and calculous formation.

He emphasizes the fact that, in the obstruction offered to the overflow of urine by the unrelieved stricture, three muscular systems-the vesical, ureteric, and cardiac-become successively affected with hypertrophy. This increase of expulsive power is rarely of long duration, for that stage in which the compensatory hypertrophy is insufficient to cope with the resistance is reached, and relaxation and atony supervene. The cardiac condition is contingent upon the renal changes, which in their turn depend upon the failure of the barriers to backward pressure which healthy or hypertrophied vesico-ureteric muscles present. INence the importance of estimating the condition of these dikelike muscles.

Their energy or incapacity may be appreciated by ascertaining the absence or presence of residual urine, and a systematic measurement of the same at each step of the dilatation, besides affording an index to the recovery or atonicity of these muscle planes, will reckon roughly the amount of backward pressure which has already fallen upon the kidney.

With these ideas in mind, Fenwick made careful examinations of the amount and character of residual urine in seventy-five cases of organic stricture of the urethra, with the following interesting results: Residual urine was found in all the cases examined except five. From this we may argue that residual urine exists in varying proportions in ninety-three per cent of stricture. But if the patient is young (under thirty), if his stricture is of short duration, and the caliber only about half diminished (arbitrarily taking the normal size as 24 French), then all the urine can be expelled.

Twenty-eight per cent of the patients were found to retain only a very small amount, about one to two drachms.

It is to be remarked, however, that these patients were mostly young, 
and that the caliber of the canal was of fair size, for in four only was the stricture so small as to require a guide.

In a certain number (i. e., sixty per cent) the amount of residual urine was large. These patients had strictures of small caliber admitting only a fine bougie at the first sitting. It is to be noted that all (except one) were over the age of thirty. Thirty-two ounces was the largest amount withdrawn. The average amount was 6.4 ounces.

Although the duration of the symptoms and the narrowness of the stricture were powerful factors in the production of the atony on which the residual urine depended, yet the age of the patient seemed the most important predisposing cause. The ultimate recovery of tone by the bladder wall seems likewise to be more influenced by the age of the patient than by any other factor. It might have been supposed that in cases which did not recover themselves after release from backward pressure there would be found a history of repeated retention, or at least of a single great over-distention which had materially weakened the bladder wall, but this was not the case. Many of the patients whose atony was the worst had never had retention at all. The bladders of the older patients suffered most without regard to the past histories. This fact is expressed as follows: Given three patients at the ages of thirtyfive, forty-five, fifty-five respectively; let each become the subject of stricture and be examined at the end of six months. It will be found that there will be a diminution of expulsive power for each decennary, and a correspondingly increased accumulation of residual urine. There are, of course, other factors which have to be remembered, and for which allowance must be made - e. g., the lowered vitality of the lumbar center from masturbation, excessive venery, abuse of alcohol, and the loss of control consequent upon cerebral or spinal lesions.

The precautions necessary in studying these cases are as follows: A blood-clot or plug of mucus, or a previously blocked catheter, will cause the practitioner to register no residual urine, while a clear instrument may withdraw many ounces.

It is of the utmost importance, in order to secure accuracy, that the patient should hold his water for some time previous to the examination.

If a sligintly atonic bladder be partially empty when the patient starts to urinate, a great deal of the mechanical force obtained from the counterpressure of moderate distention is lost. The ejected urine will therefore be small in quantity and the residual large.

The conclusions at which Fenwick arrived in his excellent paper, which on account of its practical value I have used freely, are as follows:

In estimating the health of the kidneys from the indications afforded us by the examination of the residual urine, two items have to be clearly borne in mind. There is, first, the amount of pressure which the kidneys 
have been working against. This is to be measured by the quantity of residual urine. Without a careful comparison of the weight, size, and microseopy of the post-mortem kidney with the amount of residual urine noticed before death, and the duration of the stricture, no formula ean be constructed to indicate the amount of damage which a definite quantity of residual urine will effect upon the secreting structure of the kidney. It may be safely assumed, however, that five ounces of residual urine, which is probably near the average of unreleased narrow strictures, would indicate sufficient damage to eause anxiety as to the effects of any intercurrent inflammation or disease; while an amount over ten ounces would make us cautious in operating for stricture by internal urethrotomy, and in giving anything but a grave prognosis of the ultimate effects of the constriction. Secondly, the behavior of the muscles in their progress toward recovery will teach us much as regards the future course of the case. A disposition to relapse, or sluggishness in recuperation, would cause us to look forward with apprehension to that period of life when fatty and senile changes step in to aggravate greatly the weakness of an organ upon the health of which old age is mainly dependent. Lastly, we are amply justified when the initial amount of residual urine is under five ounces, when the initial recovery is smart, and the duration of the ultimate recovery short, in giving a good prognosis, provided the full caliber of the urethra be maintained.

Treatment of Stricture.-The various plans which may be employed in the treatment of stricture may be divided into-1. Gradual dilatation. 2. Internal urethrotomy. 3. External urethrotomy. 4. Combined internal and external urethrotomy. 5. Perineal seetion. 6. Miscellaneous methods, including divulsion, rapid dilatation, electrolysis, excision, urethrectomy, etc.

Gradual Dilatation.-The instruments required for the gradual dilatation of stricture are few and simple. They consist of a set of whalebone filiform bougies with straight, angular, and spiral ends (Fig. 5), a set of

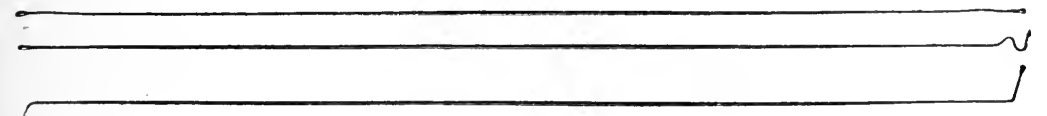

FIG. 5 .

tunneled catheters (Fig. 6); ranging from No. 8 or 10 to No. 18, French; a set of Thompson's conical steel sounds, rumning from about No. 12 to No. 36 or 38 French * (Fig. 7) ; and a number of flexible bougies of different sizes, the best being the black ones of French manufacture, and

\footnotetext{
* For economy only alternate numbers need be procured at first by young practitioners, although it is always convenieric and occasionally necessary to have the entire set.
} 
with slightly bulbons tips (Fig. 8). The principles of urethral antisepsis should be rigorously applied in the use of these instruments. They should always be vigorously scrubbed with soap and water both before and after

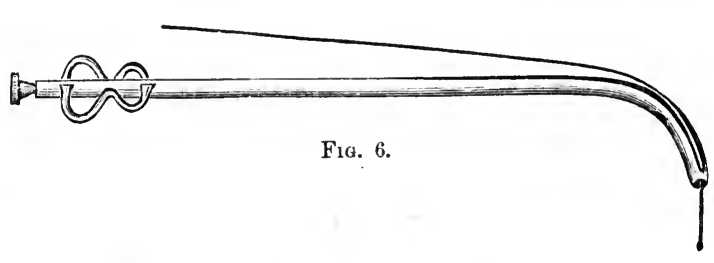
use. The steel instruments should be boiled from fifteen to twenty minutes in water containing one per cent of washing soda, to prevent rusting. The rubber and whalebone instruments should be soaked before using in a 1-to-1,000 sublimate solution. The lubricant employed should be either aseptic or antiseptic. An ointment containing one drachm of boric acid to an ounce of carbolated vaseline or cosmoline is extremely useful for this purpose. Freshly prepared carbolized olive-oil (one part of the acid to thirty or forty of oil) is also useful, but loses its antiseptic properties in a short time. Whenever the bladder has been already infected, if the urethra is permeable it is an advantage to precede the

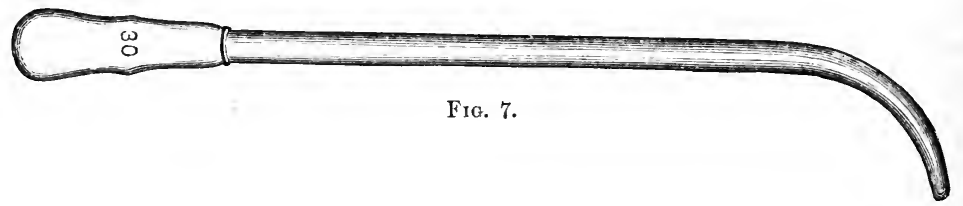

systematic treatment of the stricture by irrigation with a dilute sublimate solution (from 1 in 50,000 to 1 in 20,000); potassium permanganate $\left(\frac{1}{10}\right.$ to $\frac{3}{10}$-per-cent solution), boric acid (10 to 20 grains to the ounce of boiled water), hydrogen peroxide (from 1 in 10 to 1 in 2), or silver nitrate (from 1 in 15,000 to 1 in 500). These are among the best antiseptics to be used for this purpose.

At the same time the internal administration of antiseptics is of great value.

I have learned within a few years to place much dependence upon salol and boric acid, in ten-grain doses fonr to six times daily.

Sahli (La Semaine med., April 7, 1886) showed some years ago that the resultants of the intestinal decomposition of salol are salicylin and

Fig. 8.

carbolic acid, which are eliminated by the kidneys. He had exposed to the air for some weeks the urine of a patient under the influence of salol without the least decomposition occurring. Nencki had made the same observations. Bouchard (Therapent. des mal. infectieuses, antisepsie, 
1889, page 247) called attention to the value of the simultaneous employment of several antiseptics in internal administration. Dreyfous (Gazette hebd. de med. et de chir., January 4 and 11, 1890) further emphasized the value of antisepsis of the urinary organs by the internal administration of remedies; and Ernest Lane (The Lancet, March 22, 1890) followed him with corroborative statements.

Palmer had already shown (Amer. Med. Pract., August, 1887) the value of boric acid in sterilizing the urine, having had but one case of urethral fever in forty urethrotomies, and that occurring in a patient in whom by accident the boric acid had not been administered. The wellknown effect of quinine in preventing urethral fever is probably due largely to its antiseptic action and to its elimination through the urinary apparatus (Reginald Harrison, Lettsomian Lectures, 1888).

These facts led me long ago to the systematic employment of these drugs in all my urethral and vesical cases, and I am entirely convinced that I have noticed in all of them a more or less marked influence for good. On the other hand, Albarran (Ann. des mal. des org. genito-urinaires, January, 1890) claims to have demonstrated that urine passed by patients who have absorbed large quantities of salol has no bactericidal power and contains pathogenic microbes. Petit and Wasserman (ibid., July, 1891), at Guyon's instigation, conducted a series of experiments in urethral antisepsis by the direct method, and inoculated bouillon tubes with mucus taken from the urethra after thorough irrigation with sterilized water-four-per-cent boric acid solution and 1 to 1,000 nitrate-ofsilver solution. In every instance the tubes showed numerous microorganisms at the end of a week. Keyes, who quotes these statements (Med. News, Philadelphia, 1891, ii, page 505), hopes, however, that surgeons will not be deterred by these facts from employing all antiseptic precautions. He has been using, in addition, diuretin, and has employed it in thirteen cases. His plan is to give sixty grains of salol per day for forty-eight hours before operating, and commence diuretin on the day of the operation, giving it in ten-grain doses every four hours for two days. Its value requires to be tested by fuller experience, but he thought the evidence pointed to a certain degree of usefulness to be expected from its employment.*

This preliminary preparation having been attended to wherever possible, and the stricture, if its caliber will permit, having been located and measured by the bulbous bougie in the manner above described, a sterilized conical steel sound, a few sizes larger than the bulb which has passed the stricture, should be warmed to the temperature of the body and oiled

\footnotetext{
* He still more recently (July, 1892) notes a failure to prevent urethral fever by means of diuretin.
} 
with an antiseptic ointmert or with 1 to 40 carbolized oil, and should be carefully introduced through the stricture.

The fixed curve of the urethra-i. e., the curve assumed by the majority of adult male urethræ-in a condition of rest is measured from just in front of the triangular ligament to the neck of the bladder. It is that of a eircle three and a quarter inches in diameter, and is represented by an are of such a circle subtended by a chord two and three-quarter inches in length. This curve may be lessened somewhat by depressing the urethra by means of a finger at either side of the root of the penis; it is always lengthened in cases of hypertrophy of the prostate, or when the bladder is greatly distended. It may be temporarily obliterated, as when a perfectly straight instrument is introdnced into the bladder.

Catheterism.-The passage of a properly made steel sound or silver catheter, the curve of which corresponds, as it should, with that given above, is one of the minor operations of surgery most frequently required, and therefore one of the most important. For its performance the patient should, whenever possible, be placed in a recumbent position, the head and shoulders slightly elevated, the knees a little separated, the muscles relaxed.

The surgeon, if right-handed, stands at the left side of the patient. The sound or catheter, thoroughly sterilized, well warmed, and lubricated with some aseptic oil or ointment, is taken in the right hand, the penis between the thumb and fingers of the left hand. The organ is gently put on the stretch, care being taken to keep the dorsum facing the abdominal wall and to avoid any twists in the urethra which would constitute obstacles to the instrument, the point of which is then engaged within the meatus. At this time the shaft of the sound or catheter should be parallel to the line of the groin. This direction is important chiefly in cases of catheterism of persons with large, protuberant bellies, in whom, if it is not followed, the point of the instrument will be made to catch in the upper wall of the triangular ligament, owing to the elevation of the handle necessitated by the protrusion of the abdomen. The handle should in any event be kept low until the tip of the instrument is about to enter the membranous urethra. The penis is drawn up with the left hand while the instrument is gradually pushed onward, the handle being finally swept around to the median line, the shaft being kept parallel to the anterior plane of the body and nearly touching the integument. The instrument is now pressed downward toward the feet, while the left hand still steadies the penis and makes slight upward traction. After four or five inches of the shaft have disappeared within the urethra, it will be found that the downward motion of the instrument is arrested. The fingers of the left hand can then be shifted to the perinæum, where the curve of the instrument can be felt behind the scrotum. 
The handle may then, and not until then, be lifted from its close relation with the anterior abdominal wall and swept gently over in the median line, describing the arc of a circle, while the left hand acts as a fulcrum over which the curve of the instrument glides. After the shaft has reached and passed the perpendicular, the handle should be taken in the left hand, and the index and middle fingers of the right hand should be placed one on either side of the root of the penis, making downward pressure, while the left hand, depressing the handle, carries the point of the instrument through the membranous and prostatic urethra into the bladder. Its entrance into that organ will be recognized by the free motion that can be given the tip of the instrument when the handle is rotated, and by the latter remaining exactly in the median line and pointing away from the pubes when the hold upon it is relaxed.

The whole manœuvre should be done with gentleness; no force whatever is necessary.

If there is spasm of the circular fibers of the urethra at any point, or, as is often the case, of the compressor urethræ at the bulbo-membranous junction, a little delay will be followed by relaxation. At this same region, where the large and movable bulbous urethra contracts as it passes through the ligament, the onward pressure of a bongie or catheter is more often arrested on account of unskillfulness on the part of the operator than at any one other point. If the handle be lifted too soon from its proximity to the abdominal wall, the tip of the instrument catches in the subpubic ligament above the urethral orifice; if the handle is not raised at the proper time, or if the fingers in the perinæum do not give the curve of the instrument the gentle upward pressure that it needs, the tip buries itself in the loose and movable floor of the urethra below the orifice. In either case the curve protrudes unnaturally in the perinæum. The gentle withdrawal of the instrument for an inch or two and its reintroduction, raising or lowering its tip as may be required, will usually suffice to overcome this obstacle.

In cases of enlarged prostate a catheter with a much longer shaft and larger curve will be required, or a Mercier catheter (Fig. 9) will be found useful. In stricture a large variety of sizes and shapes may be employed. If the contraction is small and tor-

FIG. 9.

tuous, the most useful instruments are the whalebone filiform bougies, which may be used straight; or, better, with a slight angle given them near the tip (Fig. 5), as the orifice of a stricture is usually eccentric-i. e., not in the middle or center of the obstruction. It is very rare that one of these will not pass. The tunneled catheter (Fig. 6) and the ordinary steel sound are indispensable instruments in the treatment of these cases. 
If the instrument is used with ordinary care and gentleness and has been properly sterilized, and if in passing it through the deep urethra the fingers of the left hand of the surgeon are used as a fulcrum in the perinæum and the long end of the lever is depressed with slowness, while the conical point representing the short end is made to follow accurately the subpubic curve of the urethra, the production of prostatitis, epididymitis, or urethral fever, the three most common complications of rough or clumsy instrumentation, will follow with the extremest rarity. In the majority of cases these complications are due to the use of force in the introduction of the bougie (when it practically becomes a divulsor and is very objectionable), or to a slovenly disregard of antiseptic details in the use of urethral instruments, which is not uncommon.

Sometimes a few drops of blood will follow the withdrawal of the instrument. Usually the next act of urination will be slightly painful, and often the gleety discharge, which has caused the patient to seek treatment, will increase for a day or two. The use of an instrument in this manner is always followed by a slight and transitory hyperæmia of the region about the stricture, during which period in many cases, particularly recent ones, appreciable softening and absorption of stricture tissue occur. This period lasts for from three to four days, and only when it begins to subside should the instrument be reintroduced. Ordinarily, an advance of one or two numbers of the French scale may be made each time, but occasionally the same instrument must be introduced at several sittings before it can be exchanged for a larger one. This should be determined by the degree of resistance experienced during its introduction, the pain which it excites at the time and subsequently, and the presence or absence of bleeding. Personal experience soon becomes the best and safest guide as to the degree to which dilatation may be carried at any particular sitting. The feelings of the patient should always be consulted. I am distinctly of the opinion that the statement often made (Keyes, op. cit.) that there is no advantage in the retention of the instrument after its passage through the stricture is a mistaken one. It is not reasonable to suppose that the full effect of dilatation is obtained by the presence of a sound for a few seconds. It is well known that in continuous dilatation the continued presence of even a filiform bougie greatly softens and enlarges the stricture, and to a certain extent the same effect may be obtained by leaving a steel sound in situ for five or ten minutes. The point may be withdrawn a little during the time, so as to avoid irritation of the prostato-vesical region. When the full size has been reached (following the table given on page 283 as an approximate guide) the symptoms will usually disappear, and after this it is only necessary to carry on the dilatation at longer and longer intervals to maintain the cure. Most surgeons whose patients are of average intelligence have no difficulty in 
teaching them to use such an instrument for themselves, and the majority of my patients do so without the least discomfort or inconvenience. A certain proportion of cases under this plan of treatment will get entirely well, so that years afterward no trace of stricture can be discovered. Others, if the intervals between the introduction of the sound are too long, will have slight recontraction, evidenced possibly by a recurrent gleet, but the rule is that with ordinary care a practical cure is attained by this method in the great majority of cases. The process which is set up by this treatment is that long ago described by Voillemier as "inflammatory atrophic dilatation." Desnos and Kirmisson (op. cit.) do not think that in the present state of our knowledge it can be definitely stated whether or not the inflammatory reaction softens the fibrillar cells and brings about their absorption, thus robbing the stricture tissue of its contractile power, but consider it certain that some physio-pathological process occurs, which gives a certain degree of permanency to the results of slow dilatation, either continuous or gradual, which is not seen to follow the various methods of rapid dilatation.*

The practical advantages of the method are obvious, and have for many years held for it the first place in the estimation of those surgeons whose aim is to effect a cure or at least cause the disappearance of all symptoms, while at the same time they minimize the danger and inconvenience to the patient, who even in those cases in which an entire cure is not brought about remains master of the situation.

The introduction of a bougie into any stricture which it fills without causing laceration is accompanied by certain phenomena. There is felt at the end of a few minutes a difficulty in withdrawing the instrument. Soon the spasm disappears, and movement of the bougie becomes easy again. Afterward a muco-purulent discharge is established in the canal, and usually the stricture permits the passage of a larger bougie. The enlargement obtained is evidently not the effect of the pressure exercised by the bougie, since this may have been in no wise tightly grasped; it is the result of the inflammation excited in the stricture by the presence of a foreign body. By the action of bougies of gradually increasing size

* Taylor thinks that the great majority of soft strictures can be cured by a slow intermittent course of gradual dilatation. This treatment, however, takes much time, and many patients will not faithfully follow it. When, however, it can be carried out, a permanent cure will result in many cases. Even in cases of penile stricture there are many that can be cured with a straight sound aided by pressure and manipulation of the stricture over it. Keyes agreed as to the value of dilatation, and considers the operation of cutting as one of necessity to be used only in cases of exigency. He is sure that moderate strictures are cured by dilatation. Bryson heartily indorses the method of dilatation in treating strictures, characterizing it as the ideal way, and the one procedure that could not be eliminated from any method we may adopt for the treatment of these lesions, which it sometimes cures (Jour. Cut. and Gen.-urin. Dis., July and August, 1889). 
the work of resorption continues, the contractile elements atrophy, and the urethra sometimes resumes its normal caliber (Duplay, International Encyclopædia of Surgery, •vol. vi, pages 465,466 ).

These remarks apply to any stricture in which the conditions of marked resiliency, or of peculiar susceptibility to instrumentation giving rise to rigors and urethral fever, do not exist, and in which the stricture is not complicated by the existence of abscess, fistula, urinary extravasation, etc. As to the treatment of such a stricture, if it be of large caliber and if it occupy the region at or behind the bulbo-membranous junction, there is scarcely any difference of opinion, the above views being those of the vast majority of surgeons. If, however, it is in the pendulous urethra, gradual dilatation is rejected by a number of very competent men of large experience in favor of internal urethrotomy. The details of that operation will be described later (see page 304), but it will be necessary briefly to discuss here its alleged merits, which in my opinion are somewhat extravagantly vaunted by its advocates, while its undoubted dangers are sometimes ignored. I do not refer so much to the danger to life, though, as has been conclusively shown, that does exist in a definite percentage of cases, but rather to the curvation of the penis, the defective expulsive power causing dribbling after urination, etc., which not infrequently follow extensive urethrotomy.

It must be remembered that no special advantage is claimed for this operation unless it is extensive, the figures of Otis being usually adopted by the few practitioners who habitually employ urethrotomy in stricture of large caliber in which no contra-indication exists to the method of dilatation above described. As to the mortality, Watson's figures (collected by an advocate of the operation) (Boston Med. and Surg. Jour., December $29,1887)$, show fifty-one deaths in twenty-five hundred and forty eases, or two per cent; but they include the statistics of all the extremists, whose operations were often on physiological narrowings, and therefore in patients with sound urinary tracts. There is not a more able or skillful genito-urinary surgeon in Europe than Guyon, and his experience is enormous; but we find that he had twenty deaths in four hundred and fifty-nine operations, or about $4 \cdot 1$ per cent. Stein places the mortality in internal urethrotomy of the penile urethra at from two to five per cent (Trans. of the Am. Assoc. of Genito-urinary Surgeons, 1889). Thompson had six deaths in four hundred and thirty operations.

A review of a large number of reported cases, and familiarity with a considerable number even less favorable and not reported, lead me to believe that these figures rather underestimate the mortality, and that the practitioner who decides to cut a stricture anterior to the bulbo-membranous junction must do so with the full knowledge that there are at the very least two chances in the hundred of losing his patient. There 
should certainly be definite and well-grounded reasons for accepting this risk, and the operation which involves it should show results unmistakably superior to those of gradual dilatation-a procedure with practically no mortality at all.

It may be admitted that as regards stricture of the penile urethra scrupulous attention to antiseptic precautions may possibly lessen somewhat the rate of mortality given above; and the evidence, though not conclusive, is also in favor of the assertion that the probability of effecting a permanent cure by internal urethrotomy is much greater here than elsewhere; but such a cure can only be expected in a limited proportion of cases. It is not in accord with other pathological observations to suppose that the mere division of a dense and old contractile band of fibrous tissue will result in its absorption, and my belief is that the majority of the true strictures of the spongy urethra which are cured by internal urethrotomy are those in which the division of the stricture is supplemented by the use for some time of full-sized bougies. The relief of tension afforded by the section of the stricture gives full play to the so-called "inflammatory atrophic dilatation," and in a certain proportion of cases either retrograde metamorphosis and absorption take place, or at least a thinning and weakening of the fibrous band, which results in its practical disappearance as a cause of obstruction. I am compelled to believe, however, on both clinical and pathological grounds, that the great majority of socalled strictures of the pendulous urethra which are cut by the extremists in urethrotomy are points of physiological narrowing, and that the so-called "cures" are merely illustrations of the fact that by a linear incision into its long axis we can put in the normal urethra a longitudinal splice of fairly healthy tissue which has but little tendency to contract afterward, and can thus more or less permanently enlarge the urethral caliber. This fact also explains the freedom from fatal results claimed by some surgeons who find strictures and do urethrotomies in the great majority of their cases of chronic urethral discharge. As there has been no real interference with the genito-urinary functions, there has been no development of renal or vesical disease, and the urethra can be operated upon and the "splice" introduced with comparative impunity.*

* "Slight encroachments upon the urethral caliber when submitted to internal urethrotomy are not, as a rule, attended with the same inconreniences that follow the operation upon narrow strictures, but strictures of large caliber rarely require cutting unless indeed a superficial contracting band occasion sufficient reflex disturbances to require prompt relief. As to the ultimate results, thorough dilatation compares most favorably with urethrotomy" (Stein, op. cit.).

Agnew says (Principles of Surgery, p. 492): "However valuable may be incision, it must not be deemed a radical cure for stricture, notwithstanding what is said to the contrary by sanguine urethrotomists. The 'splice,' of which so much is said, and which occupies the gap between the sides of the divided parts, is granulation tissue; and the re- 
It is difficult to see, however, why such a splice should prevent the steady contraction of a mass of old cicatricial tissue, such as occupies the wall of the canal and the periurethral space in strictures of some standing.

Mr. Harrison has called attention (op. cit.) to the damage that the urine is capable of exerting while the process of repair in a wound is going on. He adds :

"And what applies to the healing of wounds inflicted accidentally upon the urethra internally, applies equally to others similarly inflicted on the canal for surgical purposes. I have elsewhere stated that for many years past $I$ have taken pains in collecting and noting cases of urethral stricture with the view of estimating the permanency or otherwise of the treatment to which the patient may have been previously submitted by various surgeons. The testimony that I have thus gathered from the examination of many hundred cases is certainly not favorable either to the permanency or the character of the relief that internal urethrotomy usually affords. Among the worst cases of stricture that I have thus met with have been those which have been treated by an internal section."

The proportion of cases in which true strictures of large caliber in the pendulous urethra require internal urethrotomy varies with the character of the patients among whom the surgeon practices. In hospital and dispensary service and among the poorer classes generally, strictures will quite frequently be found to have been neglected, and will almost always be of long standing before the patient presents himself for treatment, even if they are not of small caliber. They will therefore require urethrotomy in a much larger percentage of cases than will be found among private patients of good social position. Among the latter perhaps not more than one in eight or ten in my own practice need such operative measures, gradual dilatation, as above described, amply sufficing in the remainder to cause the disappearance of all symptoms, and often of the stricture itself when it is of recent formation.* Indeed, even in older cases, as Keyes and others have observed, a notable amount of absorption of the indurated band constituting the stricture can often be seen, and its diminution from day to day can be verified by the touch when the urethra is stretched over a full-sized sound.

We may now consider the application of gradual dilatation to the

sulting eicatrix will always retain that invincible power of contraction. The tendency to coarctation in the canal, it is true, is lessened, but it is not destroyed; and hence the patient must be instructed to pass a metal sound once or twice every month if he expects to keep out of the hands of the surgeon at some future time."

* Watson (op. cit.) finds that the proportion in his practice would be one case of urethrotomy to ten treated by gradual dilatation. 
stricture of small caliber (less than 15 French), situated at or deeper than the bulbo-membranous junction.

The diagnosis of these strictures, which are surgically most important, can be made either by means of a bulbous bongie, if it is possible to pass one through the contraction, or by introducing a sterilized sound, well warmed and oiled, down to its anterior face. They will usually be accompanied by gleet and marked vesical symptoms, increasing in severity with the tightness of the stricture. The choice of treatment lies between dilatation and some form of urethrotomy. Divulsion is so clumsy, so uncertain, and so dangerous as to have to-day almost no advocates, and to enter into an argument against it is therefore, in my opinion, a waste of time. I have never believed in it even when, many years ago, it was the favorite method among my seniors at the Philadelphia Hospital, and I have still less confidence in it now.

In beginning the treatment of such a stricture as we are considering, I prefer to attempt first, with great gentleness, to pass through it a steel sound, provided its introduction requires no force whatever. Below No. 8 or 10 of the French scale I rarely go in the use of metal instruments, believing that in the most skillful and experienced hands there is a distinct and unavoidable danger of laceration of the inflamed and degenerated mucous membrane around the strictured region. It is under these circumstancesi. e., in the presence of deep stricture of small caliber-that "false passages" are usually made, and almost always with

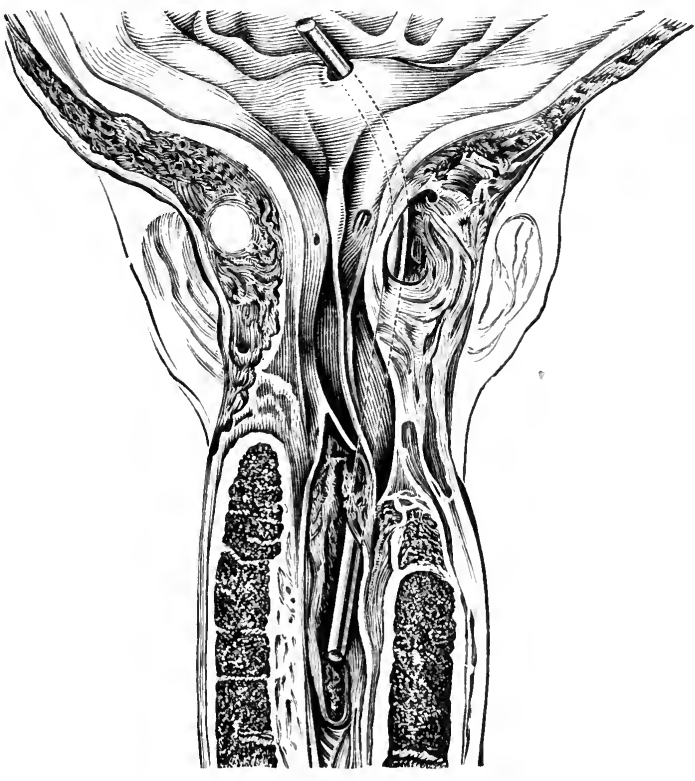

FIg. 10.-(Dittel.)

small metallic instruments, either sounds or catheters. The mucous membrane in front of a tight stricture is often inflamed and softened, and if fistulæ have formed behind the stricture diverting the course of the urine, the anterior portion of the strictured region itself, no longer subjected to constant irritation, undergoes atrophy, and a thin-walled dilatation is 
frequently found there, which offers but little resistance to the point of an instrument. If, then, the handle of the instrument is depressed too soon, the tip may perforate the upper wall; if too late, the lower wallthe former accident occurring more frequently just anterior to the subpubic ligament, the latter in the region of the bulb (Fig. 10).

The sensation conveyed to the hand of the surgeon who has been unfortunate enough to make a false passage differs markedly from that attending a successful catheterism.

The point of the instrument is not in the median line and is held with unusual firmness; there is usually free bleeding almost immediately; the finger in the rectum will usually detect easily the deflection of the instrument, and often the absence of the normal thickness of urethral and prostatic tissues beneath its curve.

The immediate treatment, if there is no retention of urine, should consist in rest, urethral and urinary antisepsis, and avoidance of instrumentation for some weeks.

If I succeed in passing a sound of whatever size through the stricture, I allow it to remain for from five to ten minutes, and then withdraw it. If it is the first experience with the patient, it is best to avoid further instrumentation for from twenty-four to seventy-two hours, in the meantime administering five-grain doses of salol or boric acid four times daily, with a full dose of quinine night and morning. At the next sitting it is often well to recommence with the same instrument, after which one, two, or three larger sizes may be used in succession, if their introduction is easy and is not accompanied by pain or followed by bleeding. Pain and hæmorrhage are unmistakable indications for lengthening the intervals and for proceeding with greater slowness in the use of larger instruments. Savory calls attention to the fact that many cases of stricture of the urethra are overtreated, and that some cases of supposed stricture require no treatment at all.* I have seen many such cases of overinstrumentation, always attended by aggravation of the symptoms.

Once fairly established, howerer, the treatment by dilatation is carried on as described above, the full normal caliber being usually reached in two or three weeks. If the stricture is not resilient or irritable, or tranmatic in its origin, it will be found that all symptoms have disappeared, unless perhaps the gleet persists for a time; but this, too, will finally subside. If the stricture were a recent one, it also may undergo absorption, but in any event the occasional introduction by the patient of a steel sound will always keep the case under control.

* He illustrates his view by the apt story of the physician who, having in consultation discerned a pericarditis, consoled his colleagne who had overlooked it with the remark, "My dear fellow, if you had found it out you would have treated it" (The Lancet, March 3,1883 , p. 357). 
In the case of resilient, irritable, or traumatic stricture in this region, or of stricture which for any reason, as the occurrence of rigors, is nondilatable, external perineal urethotomy is the operation of choice.

In certain cases no steel sound and no ordinary soft instrument can be made to pass the stricture, but a persevering trial with filiform bougies made of whalebone will result in the passage of one into the bladder. This trial should be made persistently and patiently, and in the absence of retention of urine may be repeated on successive occasions. At the first sitting, a filiform having been passed down to the stricture but refusing to enter it, it should be withdrawn and an angle of $45^{\circ}$ put into it by bending it across the thumb-nail from one quarter to one third of an inch from the end (see Fig. 5). As the orifice of a tight stricture is frequently not in the middle of the obstructed urethra, but is to be found at some point around its circumference, this little manœuvre will often enable the surgeon to enter it when with a perfectly straight filiform he can not do so. If this does not succeed, several filiforms should be passed by the side of the first one so as to impinge upon the irregular anterior face of the stricture at a number of points. By attempting to pass first one and then another of these, the instrument bearing the right relation to the orifice will usually be found and passed into the bladder. If this fails, or if one filiform can merely be engaged in the stricture, but can not be made to pass through it, it is often best, in the absence of retention, to tie it in place and allow it to remain in situ for twenty-four hours. In the great majority of cases at the end of that time it can be passed with comparative ease into the bladder. There is no objection to still longer delay, or, as I have said, to repeated trials if no urgent symptoms be present. This is certainly better than the repeated attempts at catheterism with small metallic instruments which so often, even in not unskillful hands, result in false passages.

After the first instrument is introduced in such a case four courses are open to the surgeon: 1 . He may allow it to remain in place, with the certainty that in one or two days others may be slipped alongside of it and may be used as guides for the introduction, first, of a tunneled eatheter, and later of the ordinary soft or steel bougies. 2. He may attempt to conduct a tunneled catheter over it into the bladder at once, to be followed by gradual dilatation. 3. He may conduct over it a tunneled and grooved staff, and then proceed to the performance of external perineal urethrotomy. 4. He may use it as a guide for a Maisonneuve urethrotome, and may immediately perform internal urethrotomy.

I have mentioned these procedures in the order of preference and of safety. If the stricture which is being dealt with is not of traumatic origin, and is not known to be specially resilient or irritable, the first method will lead up to the adoption of gradual dilatation with the great- 
est degree of comfort and absence of anxiety to both patient and surgeon. Even if there had been moderate retention it is absolutely certain that the urine will pass with increasing freedom by the side of the filiform, and that the danger of the case so far as retention is concerned is at an end.

Gueterbock (Deutsch. Zeitschr. f. Chirurgie, 1891, page 237) recommends this method of continuous dilatation not only in the cases we are now considering, but also in those of deep stricture with great irritability and sensitiveness to the introduction of an instrument, which is accompanied or followed by hæmorrhage. He thinks it likewise indicated whenever it is advisable rapidly to restore the caliber of the urethra, but contra-indications to urethrotomy exist.

If retention has been complete for a long time and is threatening, and the need for immediate relief is marked, it is well to adopt the second method and endeavor to catheterize at once. Failing in this, the third procedure should be employed in cases of urgency, and in all cases with a history of frequent rigors after instrumentation.

Internal urethrotomy should, in my judgment, be employed only in those cases in which the patient refuses to have the external operation performed. It is attended in the best hands with a distinctly larger mortality than any of the other methods mentioned, and there is to my mind no reliable evidence that it is followed by any larger percentage of real and permanent cures.

This completes the consideration of those strictures to which some form of gradual dilatation is applicable. It leaves, however, a considerable number which may best be treated by other methods.

2. Internal Urethrotomy.-The chief variations in the methods employed in the internal division of stricture have reference to the direction and the location of the incision. This may be made $(a)$ from before backward, or (b) from behind forward; and it may be made $(c)$ upon the roof or $(d)$ upon the floor of the urethra.

For operations upon the meatus and within the "navicular" portion of the urethra an ordinary blunt tenotome with convex cutting edge

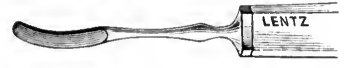

Fig. 11.

(Fig. 11) is all that is required. For operations at a greater depth a number of instruments have been devised, for each of which some special merit is claimed.

Internal Urethrotomy from before Backward.-Of those intended to cut from before backward, Maisonneuve's urethrotome (Fig. 12), or the modification of it introduced by Teevan (Fig. 13), are the best. They are used with flexible screw-tipped filiform bougies, which are first passed through the stricture into the bladder and then attached to the end of the urethrotome, after which they are pushed in advance of it until the stricture is reached and entered by the tip of the instrument. The knife 
is then protruded and made to divide the stricture, cutting on the roof of the urethra.

Teevan's urethrotome and its method of use are described as follows (British Med. Journ., September 20, 1884) :

The instrument consists of a grooved staff, which is introdnced into the bladder. A triangular blade, with a stylet attached, is made to slide along the groove, and the blade is contained in a double sheath, from which it is protruded when the stricture is reached. In Maisonneuve's original instrument the blade was furnished with a knob, and the groove extended to the end of the staff. The groove in this instrument terminates two inches from the end; it is likewise fitted with a wire stylet, on

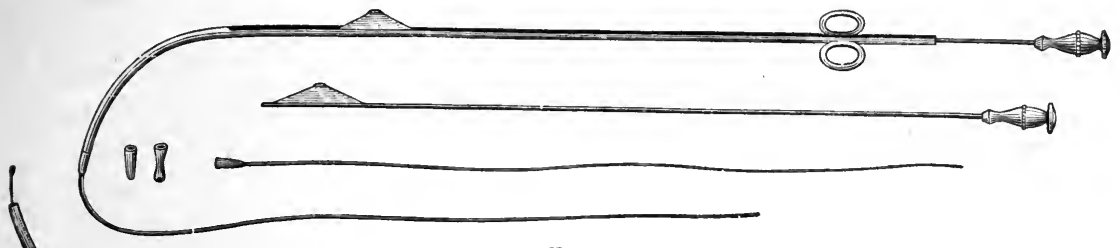

FIG. 12.

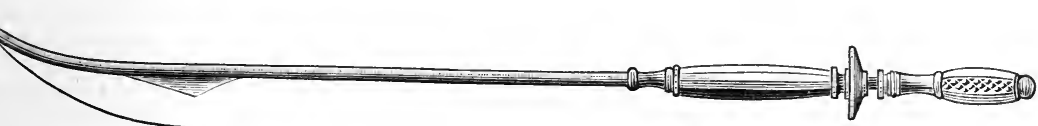

Fig. 13.

the withdrawal of which urine will flow if the instrument be really in the bladder. The knob on the blade is replaced by the double sheath, which incases the knife. The instrument terminates in a small screw, by means of which it can be attached to a conducting bougie which guides it into the bladder. Before making the incision it is of great importance to be quite certain that the instrument is really in the bladder ; for, in the absence of such a proof, a false passage might be divided instead of the stricture. After this instrument has been passed, the stylet is withdrawn, and urine will then escape if the bladder have been reached.

The operation is performed in the following manner: A fine flexible guiding bougie, equal in size to No. 4 or 5 French, is passed into the bladder. The staff or sheath of the urethrotome is then screwed on to the guide, passed throngh the stricture into the bladder, and held in position by an assistant. The operator with his left hand seizes the penis just behind the glans and draws it forward; with his right hand he pushes the stem of the sheathed knife down the urethra until the obstruction is reached; the knife is then protruded, and all resisting 
tissue in front of it is divided. The incision is thus made in the roof of the urethra. The knife is then withdrawn into its sheath, which is pushed along the urethra in order to ascertain that the division has been complete. If it be so, the instrument is immediately withdrawn. A full-sized silver catheter is then passed, the bladder is emptied, and the catheter is also withdrawn.

It occasionally happens that some difficulty is experienced in the introduction of the catheter after the operation. In some cases this is due to the staff having been held too far out by the assistant, so that the stricture is not completely divided; but this difficulty will not be experienced if the sheath be found to pass freely through the stricture after division. If it meet with an obstruction, the incision may have to be repeated, but this is undesirable.

With regard to the after-treatment, the ordinary routine is as follows: Immediately after the operation the patient of course remains in bed; hot bottles are applied to the feet, and three grains of quinine are given with half an ounce of brandy. A little beef tea is also given from time to time. If no rigors occur, the patient has a hot bath at night, and forty-eight hours afterward a French bougie corresponding in size to the catheter used after the operation is passed along the urethra. The patient is then allowed to get up. The bongie is passed every three or four days, and at the end of ten or fourteen days the patient is taught how to use the instrument himself. He is kept under careful observation for as long a period as possible.

This represents the best of the methods by which the urethra is divided from before backward.

b. Internal Urethrotomy from behind Forward.-Sir Henry Thompson (The Lancet, June 14, 1884, page 1061) describes as follows his method of performing internal urethrotomy, using his own modification of Civiale's urethrotome; the bulbous end has a mean diameter of No. 5, the stem is about two and a half or three inches; the patient being under the influence of an anæsthetic, a small catheter which has been tied in is withdrawn, and the urethrotome is first introduced as far as to the deep-seated stricture, through which the terminal bulb is then insinuated. It must now be passed fully half or three quarters of an inch in-that is, beyond the stricture-and the blade, being exposed in the direction toward the floor, is pressed firmly thereon, and drawn forward until all resistance, sometimes considerable, is completely overcome. A touch on the button near the handle completely sheathes the blade, and the outward movement proceeds until the site of the second stricture is reached, when another incision is made in the same manner as before. The urethrotome is then withdrawn, and the meatus freely divided by a scalpel or a short urethrotome. A blunt metal bougie or dilator, No. 15 or No. 16 
English size, is next used and if possible passed into the bladder. It generally passes at once without obstruction; if, however, its progress is arrested at any point, the situation of this is carefully noted, when the dilator is withdrawn, the original urethrotome reintroduced, and the opposing tissue divided. But this is seldom necessary. The stricture should permit the free and easy passage after the operation of a metallic sound, about No. 27 French in size; and in some cases No. 28 or even No. 30 may remain for forty-eight hours, with an extra twenty-four hours or forty-eight hours if the incisions have been deeper than usual, or if hæmorrhage is free or continuous, the latter being a very exceptional occurrence.

A further modification of Civiale's urethrotome is one known by the name of the late Prof. Gross, of Philadelphia, which I have used occasionally and with satisfaction for many years (Fig. 14). The acornshaped head permits of the accurate location of the stricture, and its

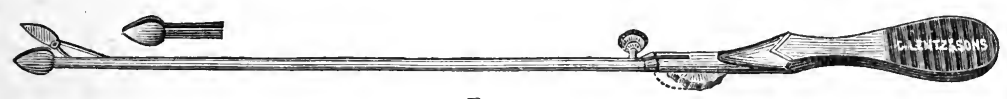

FIG. 14.

thorough division may be ascertained at the same time without withdrawal of the instrument. It is, of course, like all the other urethrotomes that cut from before backward, only applicable to strictures that permit of a certain amount of dilatation, the large majority of which, if my views are correct, should be treated by further dilatation and without urethrotomy.

The only other urethrotome which requires mention is also intended to cut from behind forward and at the same time so to dilate the strictured portion of the urethra as to insure the complete separation of every portion of the constricting bands. The method of its employment is described by its inventor, Dr. Otis, as follows: The dilating urethrotome is an instrument (Fig. 15) (constructed on the principle of the ordinary

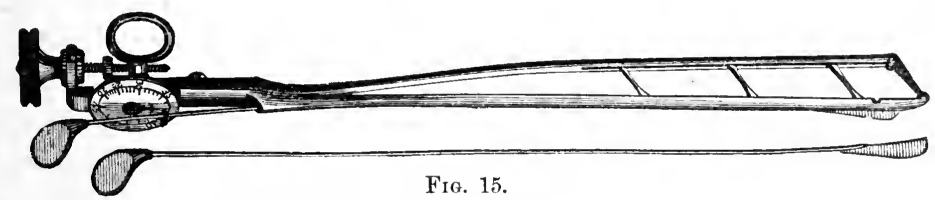

parallel rule) which, when closed, measures 18 (French); the bars are separated by means of a screw apparatus at the handle, the amount of separation being registered on a dial at the handle of the instrument. A knife two millimetres in breadth runs in a groove down the upper bar of the instrument, guarded after the manner of the urethrotome of Maisonneuve. The size of the urethra having been ascertained by 
means of the urethrometer (or by assuming it from the proportions of the flaccid penis), the urethrotome is introduced beyond the stricture; the screw at the handle is turned, dilating the instrument up to and a millimetre or two beyond the normal caliber, in order to make the strictures completely salient; then the blade is drawn through them, cutting through the entire mass of cicatricial tissue, severing the strictures completely. The diagnosis of stricture is made in every case by the bulbons sound; and freedom from stricture after operation is demonstrated by the free passage of a bulbous sound of a size corresponding with the previonsly ascertained caliber of the urethra under operation.

$c$ and $d$. As to the choice between the roof and the floor of the urethra as the preferable site of urethrotomy, it may be said with considerable dogmatism, and in spite of the opposing views of such eminent authorities as Sir Henry Thompson, Mr. Buxton Browne, and others, that for the anatomical reasons already given (see page 282) the roof is to be preferred in the portion of the urethra anterior to the bulb. It is claimed in addition that, as the floor of the urethra is the region chiefly affected by the "stricture disease" (Bryson), the incision on the roof is preferable, as permitting of the better and more thorough division of the urethral wall and thus favoring the insertion of the "splice," of which so much has been written.

The discussion as to the best site for the incision in stricture posterior to the bulb is not of so much importance, as the operation is performed in that region with much greater rarity.

Fenwick says (Annals of Surgery, August, 1889) that, owing to the close proximity of the dorsal vein and the plexus of Santorini, hæmorrhage is apt to be extremely profuse and difficult to control when a urethrotomy has involved the upper wall of the deep or membranous urethra. He gives a fatal case of this character. The bulb and its arteries are liable to be wounded by incision in the reverse direction.

The antiseptic details of the operation have been formulated by Bruce Clark (The Lancet, October 13, 1888) as follows: 1. The urethra is rendered as pure as possible by previous irrigation, and for several days beforehand, both with hot water and a 1 to 2,000 solution of corrosive sublimate. 2. The instrument which is to be employed should be taken to pieces and carefully scrubbed in soda and water, and soaked in carbolic acid (1 to 20 ) for at least ten minutes before the operation, and only put together at the last moment, just before it is to be used. 3 . When the urethra has been more freely divided a full-sized catheter should be passed into the bladder and retained there for twenty-four hours. The eatheter should be a new one, and should be allowed to soak in carbolic acid ( 1 to 100$)$ for at least twelve hours previous to the operation. In 
his series of fifteen cases there was but one rigor, and that was not attended by any severe constitutional disturbance.

Southam (The Lancet, June 14, 1890) has adopted the following plan of preliminary treatment: Internal urethrotomy having been decided upon, the patient is confined to bed and kept upon a milk diet for a few days previous to the operation, the urethra being meanwhile left at rest, and free from the irritation caused by the passage of instruments. To relieve any tendency to congestion of the urethral mucous membrane and parts about the neck of the bladder, the bowels are kept freely open by small doses of magnesium sulphate. A mixture containing boric acid in tengrain doses is also given three times daily; for in the majority of cases of stricture where operative interference is required chronic cystitis is also present to a greater or less extent, the urine containing more or less pus, and, if not alkaline, being either neutral or only faintly acid in reaction. Under a course of boric acid given internally it will generally be found that the urine, at any rate in the less severe cases, soon regains its normal acidity; at the same time a portion of the boric acid being eliminated by the kidneys, and passing away in the urine, the antiseptic action of the drug is exerted directly on the mucous lining of the bladder. Thus the cystitis is relieved, the pus is diminished in amount or entirely disappears, and the decomposition of the urine in the interior of the bladder is also prevented. The result is that the urine, if toxic, is sterilized or rendered aseptic, and its contact with the surface of the urethral wound after operation is consequently innocuous.

This confidence in boric acid is confirmed by the experience of Palmer (already quoted), and by that of many other genito-urinary surgeons, including myself.

We may now consider the indications for the performance of one or the other of these forms of internal urethrotomy. I have already sufficiently stated the reasons which seem to me to justify the rejection of internal urethrotomy as a routine procedure in cases of stricture of large caliber of the pendulous urethra, and a fortiori in cases of similar or of narrower stricture in the deep wrethra. Of those which remain, we may first consider the strictures of the meatus and of the neighborhood of the fossa navicularis. In this region dilatation is peculiarly unsatisfactory. Owing to the intimate relation between the spongy tissue of the glans and the urethra, to the exceptionally rich nerve-supply to the part, and to the extreme sensibility characteristic of muco-cutaneous outlets, the stretching of the stricture by means of sounds gives rise to pain, irritation, and inflammation. For these reasons, and on account of the absolute safety of the procedure, it is better to divide all such strictures rather than to attempt to dilate them. I have done so for many years, and have never had an alarming symptom. I desire, however, to record my protest 
against the most unnecessary and ill-judged zeal which, as Keyes says, makes it of late years the rule, rather than the exception, for cases of obscure genito-urinary disease to present themselves with traumatic hypospadias, and with the history that their whole anterior urethra has been cut to a size among the thirties, under the assurance that this would cure the malady. He adds, "This is the natural result of the modern fashion of meatotomy and urethrotomy, which has run riot in the profession and led to much unnecessary surgery and to some positive injury." I agree with him and with other competent observers who believe the cutting of true strictures in the anterior urethra to be good surgery, but who think that the division of every point at the meatus and in the first few inches, simply because those points are (as they should be normally) narrower than other points in the canal, is routine surgery of a very unprofitable sort.

The operation of meatotomy is best done with a probe-pointed tenotome with the usual convex-cutting edge. The incision should be made upon the floor of the urethra, and should be sufficient to entirely remove all sense of resistance upon the withdrawal of a full-sized bulbous bougie. It should be a little larger than the caliber which it is desired to establish permanently, so as to allow for subsequent contraction. Like all other operations upon the urethra, it should be done with scrupulous attention to antiseptic details. A short, straight, conical bougie, the so-called meatus sound, should be gently inserted once in twenty-four hours during the healing process. Deeper troubles, unless urgent in their character, should be ignored until healing is complete. Sometimes, but much more rarely than is generally supposed, such troubles, although previously thought to be organic, will be found to have disappeared. In those cases they have probably been due to reflex irritation, but this condition never occurs except in conjunction with a pin-hole meatus or with a distinctly strictured condition of the urethra in its vicinity. I doubt greatly the frequent production of deep urethral spasm as a result of the so-called anterior stricture of large caliber, either at the meatus or elsewhere. I am the more desirous of emphasizing this point as I at one time strongly advocated the opposite view ; but, in the light of wider experience and longer observation of cases, I believe I did so upon insufficient grounds.

If strictures of large caliber in the pendulous urethra are of long standing, distinctly fibrous in character or non-dilatable, internal urethrotomy is indicated. Resiliency or resistance to dilatation is indeed the chief indication for a cutting operation on strictures at any portion of the urethral tract, and is far more important in determining the choice of treatment than their caliber; but internal urethrotomy in the pendulous urethra is particularly satisfactory on account of the comparative freedom from danger which has already been alluded to. As to the probability 
of effecting thereby a permanent cire, while, as I have already said, it is greater here than elsewhere, such a cure can only be expected in a limited proportion of cases.

Strictures of small caliber (less than 15 French), situated in advance of the bulbo-membranous junction, unless seen very early and found to be unusually soft and dilatable, furnish the typical condition for internal urethrotomy, that in which it is attended with the minimum of danger and with the greatest prospect of effecting a permanent cure. The exceptions to this rule will be noted when the operation of combined internal and external urethrotomy is described. The operation may be performed with the instrument which the surgeon happens to prefer, the essentials to success being a linear division in the roof of the urethra of every portion of strictured tissue, the incision extending from the normal parts behind to the normal parts in front of the stricture. If the contraction is of very small caliber, a Maisonneuve urethrotome may be passed over a filiform guide and a preliminary urethrotomy done, so that one of the various forms of dilating urethrotomes may be passed through it and the stricture freely divided from behind forward. A bougie à boule should then be used to demonstrate the complete division of the stricture, a large-size gum catheter passed and tied in the bladder, and bleeding controlled, if necessary, by the application of a firm bandage. The moderate risk attending this operation is reduced to its smallest proportion by the employment of sterilized instruments, the use of urethral irrigation before, and if necessary after, the operation, the administration of internal remedies which tend to sterilize the urine, and by attention to the various details of antisepsis, as applied to genito-urinary surgery.

A few days after the operation a full-sized bougie should be gently passed and should be used afterward, as in cases of dilatation, for some weeks; often it will be necessary to use it at intervals for a much longer period, and in a fair proportion of cases an apparent cure will follow.

These indications for internal urethrotomy will surely be regarded by some surgeons as entirely too restricted, and it seems right to give some illustration of the range which the operation takes in the hands of those who favor its performance. Buxton Browne, who represents Sir Henry Thompson's teaching and practice as well as his own, gives (Brit. Med. Jour., April 16, 1887, page 823) the following indications for its employment:

1. When time is an object. The patient is perhaps ordered on foreign service, or perhaps, on the eve of marriage, finds that he is subject to stricture.

2. When the stricture is at the urethral orifice, or in the penile urethra, 
and will not yield permanently to either continuous or interrupted dilatation, but must be divided.

3. In cases of stricture where the gentlest instrumental interference is followed by rigors and great prostration. If the fibers of the stricture are freely divided, the use of a bougie will cease to be followed by rigor. If, after internal urethrotomy, the use of a bougie is still followed by rigor, it will be because the operation has been incomplete, and it must be repeated more thoroughly.

4. Internal urethrotomy is required when a stricture rapidly recontracts after dilatation. If such stricture is obviously large and dense, dilatation is useless, and the stricture must be cut, and sometimes requires more than one cutting operation before a satisfactory result is obtained.

5. When renal or other calculus is impacted behind a stricture, the stricture had better be divided internally, and, if possible, the calculus extracted per vias naturales; should this prove impossible, the calculus may be cut down upon, and the division of the stricture and subsequent treatment will prevent the opening thus made from becoming fistulous.

6. No urethral fistula will ever heal as long as the urethra is contracted in front of the fistulous orifice. Divide the stricture and keep it open by periodical instrumentation, and usually the fistula will close.

7. For stricture complicated by prostatic enlargement.

8. Occasionally, as a prelude to lithotomy, to permit the introduction of the grooved staff.

9. In a patient with a tight stricture who is dereloping a perineal abscess. The urethrotomy is followed by the introduction of a largesized catheter, and the perinæum is then opened until pus issues, the wound is enlarged and dilated a little with the fingers.

Bangs, in this country, has recently (Medical News, December 12, 1891) formulated the views of the followers of Otis by reserving gradual dilatation for soft, non-fibrous strictures of the posterior urethra and for those of similar pathological character in the bulbous urethra. He states that it is also sufficient in some of the soft, not well-organized strictures in the penile urethra that are practically adhesions of mucous surfaces. In all strictures of large caliber, requiring interference, ${ }^{*}$ he advocates internal urethrotomy, making for simple, uncomplicated strictures of small caliber a reservation in favor of the combined operation in certain cases to be described later (see page 319).

Internal Urethrotomy in Children.-De Pezzer (L'Union méd., 1887, page 193) says that in literature he has been able to find only two allusions to internal urethrotomy in children-those of Holmes, who advocates it, but reports no case of it, and Eugene Boeckel, who reports a case of this

* The italics are his. 
operation in a boy of twelve, which was successful (Gaz. méd. de Strasbourg, 1876, page 122).

De Pezzer has had two cases-one in a boy fifteen years old, for stricture appearing soon after contracting gonorrhœa, the other in a boy of ten, with traumatic stricture in the perineo-bulbar region. Dilatation and electrolysis failed, but internal urethrotomy was followed by a good result.

He concludes that internal urethrotomy is not contra-indicated, and has the same applications in the child as in the adult, but that the curve of the urethrotome must be modified in degree and length to suit the age of the individual patient.

3. External Urethrotomy.-There has always been more or less confusion in the nomenclature of the perineal operations for the relief of stricture. The old boutonnière operation (of which the so-called "Cock's operation" is a modification) had for its object the opening of the urethra behind the obstruction, was purely palliative, and if it ever effected a cure did so by accident, as the procedure itself did not include of necessity any division or enlargement of the strictured portion of the canal. It was an "external urethrotomy," to be sure, but it is not related either in its purpose or its method to the other operations known by that name.

Since Syme revived and popularized the formal operation of external urethrotomy-the division of a stricture upon a grooved staff passed through it into the bladder-more system has been introduced into the terms employed, but there is still much objectionable looseness. Hunter, Grainger, C. Bell, and others had, before Syme's time, formulated the operation known as "perineal section," which was also an external urethrotomy, but which was then restricted to those cases in which no instrument whatever could be made to pass the stricture. The term should still be reserved for such cases, and on account of its brevity is perhaps preferable to its synonym, "external perineal urethrotomy without a guide."

The opening of the urethra in its penile portion is, as a formal operative procedure, practically unknown. I shall therefore employ the term external urethrotomy to indicate those operations in which the urethra is opened through the perinrum upon some sort of guide. It will only be necessary to describe the two chief varieties of this procedure.

a. Syme's Operation.-The instrument needed will be a grooved staff (Fig. 16) with a narrow terminal part which is passed through the stricture. When this narrow portion joins the shaft there is a "shoulder" which, when the instrument is in position, rests against the anterior face of the stricture. The groove of the staff runs to its extreme tip. A scalpel, a probe, a broad-grooved director, or, better, a Teale's probe 20 
gorget (Fig. 17), and a catheter, are all that will be required. The operation is described as follows (Treves, Operative Surgery, vol. ii, page 626):

The patient is placed in lithotomy position, and the staff is introduced with the care already advised in performing that operation. An incision

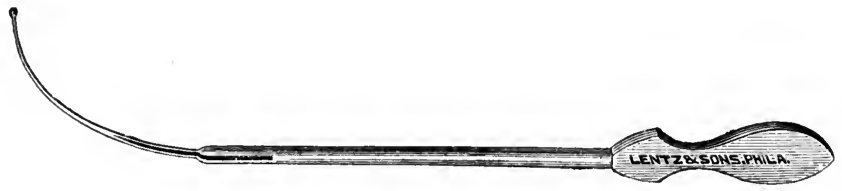

FIG. 16.

is made precisely in the median line of the perinæum, and the knife is so directed that its point shall hit the shoulder of the instrument.

The surgeon must convince himself that this portion of the staff is laid bare. He then engages the point of the knife in the groove of the staff, and, keeping most carefully to the groove, thrusts the knife toward the neck of the bladder until he has divided the whole of the stricture. A director or probe, or Teale's probe gorget, is now introduced along the convexity of the staff into the bladder, and the staff is removed. A gumelastic catheter may then be passed into the bladder through the penis, and be guided by the director or probe gorget aided by the tinger inserted into the wound.

Should the irritability of the bladder prevent the retention of a catheter, a tube should be passed into the bladder from the perinæum, and should be retained in position by tapes.

Syme's curved catheter may be employed for this purpose, or a por-

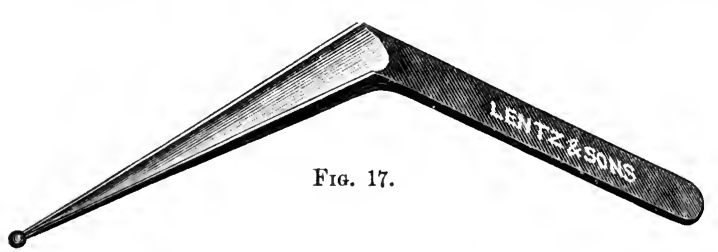
tion of a gum-elastic catheter may be made use of. Whitehead's perineal tube, with sliding adjustable shield, is a useful instrument. As soon as possible, however, a catheter should be passed by the meatus, and the perineal wound allowed to close.

b. Not infrequently eases are met with in which it is possible to pass a filiform bougie into the bladder, but no larger instrument can be made to follow it. In that case a grooved staff similar to Syme's, but with a quarter of an inch of its extremity bridged over so as to convert the groove into a canal—or, better, a " tunneled catheter" staff (Fig. 6)-may be used in the same way.

The operation is performed as follows :

The exact seat of the stricture having been ascertained, the urethra is 
filled with olive oil, and a capillary probe-pointed whalebone bougie is introduced into the canal, an attempt being made to pass it through the stricture. If it enters a false passage, it is retained in situ by the left hand while another is passed by its side. If this second guide finds its way into a false passage, it is treated precisely as was the first, and the operation is repeated until one bougie has been passed through the obstruction into the bladder. The other guides-often five or six in number-are then withdrawn.

The tunneled catheter staff is then threaded over the free end of the filiform bougie and is gently pushed onward toward the bladder, the guide being held in the meantime between the thumb and finger of the left hand. I have found it a useful manœuvre to release the guide when the strain and friction upon it became greatest, and to permit it to be pushed onward with the staff or catheter. Its end will of course disappear within the urethra, but can almost always be found within an inch of the meatus by pressing the penis backward after the metallic instrument is in the bladder. If it should not be in sight then, it will certainly reappear when the staff is withdrawn.

If the latter can be made to pass through the stricture, the operation is completed precisely as in the Syme operation just described. If not, the tip of the staff is held in contact with the anterior face of the stricture by an assistant, who supports the scrotum, and the patient is brought into the lithotomy position. The surgeon makes a free incision in the median line of the perinæum, and brings into view the urethra, which is opened upon the bridged portion of the staff, the knife following the groove in the instrument. A loop of silk is then passed throngh each edge of the incised urethra, close to the face of the stricture, and serves to enable the canal to be held open by an assistant. The catheter staff is now withdrawn a little, so as to bring into view the black guide; then the stricture, with about half an inch of the uncontracted eanal behind it, is divided by means of the beaked bistoury. The last step consists in passing the staff, guided by the whalebone bougie, into the bladder. The subsequent treatment of the case is conducted upon ordinary principles.

The general indication for external urethrotomy is the existence in the deep urethra of a stricture of small caliber, not amenable to dilatation. The following varieties should be specially mentioned:

1. Tough, fibrous stricture, which will not permit of dilatation to a proper extent. 2. Resilient stricture, which recontracts rapidly after dilatation. 3. Hard, narrow stricture, associated with deep perineal indurations and intractable to dilatation both continuous and intermittent. 4. Stricture complicated with fistulæ, in which dilatation has failed to bring about closure of the latter. 5. Typical traumatic stricture, which is 
almost always dense and resilient. 6. Stricture behind which extravasation of urine has occurred. 7. Stricture complicated with perineal abscess, the latter being laid open and the stricture divided by the same incision. 8. Stricture complicated by intense cystitis, so that the advantages of continuous drainage of the bladder may be secured at the same time. 9. Stricture associated with enlargement of the prostate, and refusing to yield to either continuous or intermittent dilatation. 10. Stricture complicated with retention of urine or with the "incontinence of retention." The high degree of atony of the bladder which ordinarily exists in these cases renders perineal drainage exceptionally desirable. 11. Stricture in which urethral fever follows instrumentation, or in which renal congestion or nephritis is known to exist.

It is to be understood, I repeat, that in all these cases the stricture is situated posterior to the bulbo-membranous junction.

As to the results obtained by this method the evidence is conflicting, but is sufficient to show that in by far the larger number of cases the canal must be kept open by the more or less frequent passage of bougies, or recontraction will follow. That cure oceasionally though rarely follows seems to be fairly well established.

Antal (Vierteljahrsschrift für Derm. und Syph., iv, 1887, page 863) states, however, that as his experience increases he is more and more impressed with the view that the permanent cure of stricture is well-nigh impossible. Dittel is of opinion that, if a stricture be cut, it heals lineally and so does not add to the lumen of the urethra. Guyon believes that the elastic fibers run circularly, and when cut, retract, forming a rhomboidalshaped lumen, and, a wide interposed scar results, and teaches that the stricture does not recontract. He does not follow by dilatation. Antal says, however, that this theory holds good only in the early stages, as later the elastic fibers have lost their elasticity. IIis endoscopic studies have shown that, after cutting, a stricture always heals as a linear scar, and that therefore, although the treatment may have been shortened, the lumen is not permanently increased.

Keyes (Med. Record, May 25, 1889) says that he has had some cases in which permanent cure seemed to have followed the thorough division of stricture by perineal section, while at the same time he acknowledges the failure of accomplishment of radical cure in a vast majority of the cases operated on. In seeking for an explanation of the difference, he found that there are different forms of organic stricture-the soft, the inodular, and the purely cicatricial fibrous stricture with no inodular masses. His conclusion is that the soft stricture, in which there is no fibrous organic change below the surface of the mucous membrane, but probably considerable spasmodic element, may be radically cured by dilatation or section; pure fibrous stricture only by thorough section, and 
subsequent use of large instruments for a considerable time, after which he believes instruments may be given up; inodular stricture, not at all radically, except perhaps after treatment by excision.

Similar expressions of opinion could be quoted from many writers, but the general belief is as above stated-viz., in the possibility but extreme rarity of radical cure.

Combined Internal and External Urethrotomy.-This operation, especially advocated by Mr. Reginald Harrison (Brit. Med. Jourual, July 18, 1886, page 91; The Med. Press and Circular, February, 1889, page 131), is performed as follows: The stricture is divided by means of one of the ordinary forms of urethrotome. The patient is then placed in the lithotomy position, a grooved staff introduced, and the membranous urethra punctured with a long straight knife one inch in front of the anus, the back of the knife being toward the rectum. The incision is slightly enlarged forward so as to permit the introdnction of the index-finger; if the staff be found exposed at the bottom of the wound, as it generally is, all well and good; if not, reintroduce along the finger to the bottom of the wound a somewhat blunt though pointed knife (made for the purpose), with which clear away the few fibers that remain between the tip of the finger and the groove; if a sharp knife be thus used, either the wound is made unnecessarily large or the finger may very easily be cut. The plan of the incision is first to make it fit the finger and subsequently the drainagetube; if this be done accurately, there is practically no bleeding. When the groove of the staff is felt, Wheelhouse's small probe-pointed gorget is slid along it, the staff is withdrawn, and the drainage-tube passed along the concavity of the gorget into its position. The proceeding thus described is merely for the purpose of drainage and for placing the urethra at rest.

The tube is retained seven to ten days; after the second day it should be taken out and cleansed daily; the bladder is irrigated twice daily with a $1-10,000$ to $1-5,000$ sublimate solution.

This operation, which Harrison very properly says should be described as a perineal puncture rather than a perineal section, has the great advantage of meeting the indication for the removal of the stricture tissue from the contact with the urine, which, as we have seen, is the essential factor in the production of organic stricture. It shonld therefore be carefully considered in all those cases of anterior stricture requiring division in which there is marked resiliency or a distinct inodular mass, in those which were traumatic in their origin, and in those which are complicated by fistulæ. The following line of argument (The Lancet, London, 1892, vol. i, page 132) in favor of the employment of the combined method in such strictures seems to me incontrovertible: The inflammatory deposit on the surface or in the substance of the mucous membrane, or oftener beneath it, which constitutes the beginning of a stricture, stead- 
ily increases little by little as fresh lymph is poured out, around, and beneath it, involving first the urethral and then the periurethral tissues, until at length it forms a definite obstruction. The problem in the treatment is, how to insure the absorption of this deposit without causing the addition of more. Temporary relief may be obtained by division or dilatation, if the lymph is still soft and cellular so that its absorption can be effected without loss of time; this sometimes leads to permanent cure. Nearly always, however, a hardened patch or an open ring is left in the substance of the wall, less elastic than the tissues near, and unable to yield smoothly and evenly when the folds of mucous membrane are straightened out by the passage of urine. This obstruction, combined with the leakage of urine into the periurethral space, is sufficient to keep up irritation and cause the stricture to relapse. Each act of micturition strains and pulls upon the tender tissues around, and furnishes a fresh source of irritation. More lymph is thrown out, and organization begins and contraction is well established before the original induration has had time to disappear Strictures relapse and recontract not only because they are formed of scar tissue (with a few exceptions), but also because the remains of the old hardened inflammatory deposit act like the little thickened patches or the papillary granulations that are left after gonorrhœa, and begin again the old vicious circle. What is wanted is something that will hasten the fatty degeneration of the old exudation and help the tissues to remove it before the irritation of its presence or the continued contact of urine with tissues, the epithelium of which has been crippled or destroyed, can lead to the formation of any more exudation or cause any fresh trouble. Inflammatory exudation in this region ean not be treated as in other parts of the body. Experience has proved that it is necessary to free the tissues from every source of irritation, and to secure for them a long period of complete physiological rest. If this can be done, the hardened lymph will slowly disappear, and the walls of the urethra become soft and yielding again.

Moullin claims to have shown (The Lancet, London, September 19, 1891) that cure is by no means uncommon after external urethrotomy, and even goes further, and asserts that not only is this possible, but that under certain eircumstances some strictures of the penile urethra are capable of undergoing a process of spontaneous absorption. The occasional success of extreme dilatation and free internal urethrotomy after Otis's plan is probably to be explained in this way: thorough stretching or division of the stricture places the part at rest for a sufficient length of time, and the inflammatory products in the wall undergo fatty degeneration and absorption before they can give rise to sufficient obstruction or irritation to lead to the production of any more; but the result is very uncertain, even in comparatively recent instances. 
Now, in old cases, especially if there is more than one stricture, the only plan that will give sufficient rest is by opening the urethra in the perinæum and allowing the whole of the urine to escape without coming in contact with the affected part. Hence the value of the combined method.

In deep strictures it seems to me less likely to be useful, as external urethrotomy, as ordinarily performed, meets the same indications, the incision being carried behind the strictured region into healthy tissue and the retained catheter thus diverting the urine. If for any reason, however, it is desired to shorten the healing of the external wound, or if an extensive external cutting seems inadvisable, the combined operation will admirably meet the requirements of the case and will certainly greatly diminish the risks of internal urethrotomy in the deep urethra. Harrison especially recommends it in "cicatricial, contractile, and relapsing strictures situated in the deeper portions of the urethra," and claims for it these advantages: (1) It is applicable to the worst forms of urethral stricture when the narrowing is so complete as to reduce micturition to the finest possible dimension, so far as the escape of urine is concerned, and where necessarily only the smallest and most delicate instruments can be used; (2) it provides against rigors and fever and the complications that arise from these; and (3) it tends to improve permanently the conditions of the stricture.

Bangs believes (Medical News, December 12, 1891) that if a stricture of small caliber be complicated by fistulæ, either in the penile urethra or in the perinæum, or if there be indurated cicatricial deposits posterior to four and a half inches, we should employ the combined operation. The external urethrotomy, in his opinion, serves more than one purpose. It enables us to divide all stricture tissue thoroughly and fearlessly, with a definite object in view, and permits us at the same time to abrogate the functions of the diseased urethra by draining away the urine, drop by drop, withont any effort on the part of the urethra or perineal muscles. Thus physiological rest to the whole region is obtained as certainly as we obtain physiological rest for a broken limb when we put it on a splint. The process of healing is rendered a continuous and uninterrupted physiological one, without at any time becoming an inflammatory or disease process. Even in cases that have enormous, hypertrophic, indurated masses in the perinæum and neighboring tissues-masses that are the result of tight or filiform strictures in the bulbous or membranous urethra -the effect of this drainage will be seen in the softening and gradual disappearance of these hypertrophies, sometimes before the perineal wound has begun to heal.

Perineal Section. - This term has been reserved for the operations performed upon stricture which will not adnit of even the smallest in- 
strument. Such strictures, whether gonorrhneal or traumatic in their origin, are usually deeply seated and to be approached through the perinæum.

Two methods of operating may be described (British Medical Journal, June 24, 1876):

a. Wheelhouse's Operation.-A special hooked staff (Fig. 18) is required, with the probe gorget (Fig. 17), needles, forceps, scalpel, etc. The patient is placed in the lithotomy position. The staff is to be introduced with the groove looking toward the surface, and brought gently into contact with the stricture. It should not be pressed much against the stric-

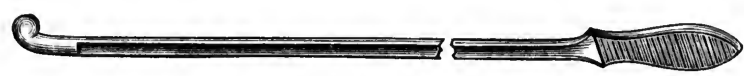

Fig. 18.

ture, for fear of tearing the tissues of the urethra and causing it to leave the canal; this would mar the whole after-proceedings, which depend upon the urethra being opened a quarter of an inch in front of the stricture. While an assistant holds the staff in this position, an incision is made into the perinæum, extending from opposite the point of reflection of the superficial perineal fascia to the outer edge of the sphincter ani. The tissues of the perinæum are to be steadily divided until the urethra is reached. This is now to be opened in the groove of the staff-not upon its point-so as certainly to secure a quarter of an inch of healthy tube immediately in front of the stricture. As soon as the urethra is opened and the groove in the staff fully exposed, the edges of the healthy urethra are to be seized on each side with straight-bladed nibbed forceps and held apart. The staff is then to be gently withdrawn until the button-point appears in the wound. It is then to be turned round, so that the groove may look to the pubes, and the button may be hooked on to the upper angle of the opened urethra, which is then held stretched open at three points, and the operator looks into it immediately in front of the stricture. While thus held open a probe-pointed director is inserted into the urethra, and the operator, if he can not see the opening of the stricture-which is often possible-generally succeeds in very quickly finding it, and passes the point onward through the stricture toward the bladder. The stricture is sometimes hidden among a crop of granulations or warty growths, in the midst of which the probe-point easily finds the true passage. The director having been passed into the bladder (its entrance into which is clearly demonstrated by the freedom of its movements), its groove is turned downward, the whole length of the stricture is carefully and deliberately divided on its under surface, and the passage is thus cleared. The director is still held in the same position, and a straight probe-pointed bistoury is run along the groove to insure complete division 
of all bands or other obstructions. These being thoroughly cleared, the old difficulty of directing the point of the catheter through the divided stricture and onward into the bladder is to be overcome. To effect this, the point of a Teale's probe gorget is introduced into the groove in the director, 'and, guided by it, passed onward into the bladder, dilating the divided stricture and forming a metallic floor, along which the point of the catheter can not fail to pass securely into the bladder. The entry of the gorget into the latter viscus is signalized by an immediate gush of urine along it. A silver catheter (No. 18 or 20 French) is now passed from the meatus down into the wound, and is made to pass once or twice through the divided urethra, where it can be seen in the wound, to render certain the fact that no obstructing bands have been left undivided, and is then, guided by the probe-dilator, passed easily and certainly along the posterior part of the urethra into the bladder. The gorget is now withdrawn, and the eatheter fastened in the urethra and allowed to remain for three or four days, an elastic tube conveying the urine away. After the catheter is removed, it is passed daily, or every second or third day, according to circumstances, until the wound in the perinæum is healed; and after the parts have become consolidated, it requires, of course, to be passed still from time to time to prevent recontraction.

Treves (op. cit., vol. ii, page 630) remarks that the operation requires a good light and infinite patience. There is often some difficulty in detecting the orifice of the stricture, and matters may be complicated by a false passage. The hooking of the button of the staff on to the upper angle of the opened urethra is not always of service. The instrument has to be held by an assistant, and is apt to be in the way. The margins of the urethral wound may be conveniently held aside by long threads which have been passed by means of curved needles in holders.

This operation properly belongs under the head of "perineal section," as it is not only always begun without a guide having been passed throngh the stricture, but is often of necessity continued by dissection until the urethra is fairly opened, even the probe-pointed director not passing through the coarctation.

b. Cock's Operation.-This consists in opening the urethra at the apex of the prostate, behind the obstruction, no guide having been passed. The patient is to be placed in the usual position for lithotomy ; and it is of the utmost importance that the body and pelvis should be straight, so that the median line may be accurately preserved. The left forefinger of the operator is then introduced into the rectum. The bearings of the prostate are examined and ascertained, and the tip of the finger is lodged at the apex of the gland. A sharp-pointed double-edged knife is then plunged steadily and boldly into the median line of the perinæum and carried on in a direction toward the tip of the left forefinger, which lies 
in the rectum. At the same time by an upward and downward movement the vertical incision may be carried in the median line to any extent that is considered desirable. The lower extremity of the wound should come to within half an inch of the anus. The knife should never be withdrawn in its progress toward the apex of the prostate, but its onward course must be steadily maintained until its point can be felt in close proximity to the tip of the left forefinger. When the operator has fully assured himself as to the relative position of his finger, the apex of the prostate, and the point of his knife, the latter is to be advanced with a motion somewhat obliquely, either to the right or to the left, and it can hardly fail to pierce the urethra. If, in this step of the operation, the anterior extremity of the prostate should be somewhat incised, it is a matter of no consequence.

It is of the utmost importance that the knife be not removed from the wound and that no deviation be made from its original direction until the object is accomplished. If the knife be prematurely removed, it will probably when reinserted make a fresh incision and complicate the desired result. It will be seen that the wound when completed represents a triangle, the base being the external vertical incision through the perinæum, while the apex, and consequently the point of the knife, impinge on the prostate. The knife is now withdrawn, but the left forefinger is still retained in the rectum. The probe-pointed director is carried through the wound and passed into the bladder.

It only remains to secure this drainage-tube in place by means of two tapes, which are attached to the sides of the tube on the one hand, and to the perineal strips of a T-bandage on the other.

Through the tube the bladder may be washed ont.

Indications.-The operation of perineal section in some form is indicated in all cases of impassable stricture. If there are no other complications, the method of Mr. Wheelhouse seems to me to meet every indication. The passage of the staff to the stricture shows the exact site of the latter. The insertion of the threads into the divided urethra serves not only to hold it open and give an opportunity for the discovery of the proximal portion, but also fixes the anterior end and renders it easily recognizable during the operation. The least useful direction which Mr. Wheelhouse gives is that of turning the staff with the concavity of the curve upward, so as to hook it into the upper portion of the urethral wound. The instrument is sometimes in the way, has to be held by an assistant, and it seems to me does not afford much help during the operation.

In cases in which a portion of the urethra has been practically destroyed, in which urinary extravasation has occurred and in which the perinæum is riddled with sinuses, and in those of great urgency from ab- 
solute retention and in which no aspirating apparatus is procurable, Cock's operation may be tried. The operation has, however, never been a favorite with me, is sometimes disappointing in the best hands, and, as Treves advises, had always better be abandoned if the urethra is not opened at the first or second trial.

Retrograde Catheterization.-If, during a perineal section. persevering search fails to reveal the proximal end of the urethra in cases of traumatic stricture with practical obliteration of the canal, are suprapubic cystotomy and retrograde catheterization justifiable? I am inclined to answer this affirmatively, though such failures shonld be most exceptional. Retrograde catheterization was practiced for the first time in 1757 by Verguin, a surgeon of Toulouse, who passed a catheter into the urethra through a pre-existent fistula of the bladder, consecutive to suprapubic puncture, since which time a number of surgeons have had recourse to this procedure; and Sedillot formally expressed his opinion that, "in the absence of a pre-existent fistula, if in the course of an external urethrotomy (perineal section) undertaken for an impassable stricture it were found impossible to discover the posterior end, the surgeon would be justified in doing a suprapubic cystotomy at once in order to practice retrograde catheterization."

If the bladder contains urine, and particularly if it is distended, pressure on the hypogastrium or bimanual pressure with the fingers of one hand on the abdomen and of the other in the rectum will often cause a jet of urine to issue from the proximal end of the urethra and will at once disclose its situation. Hæmostasis in the whole wound, by the use of very hot water, will sometimes reveal the urethra by emphasizing the difference between its color and the surrounding parts, the urethra generally showing much paler. The relation to the pubes and to the lower edge of the triangular ligament should be most carefully borne in mind, the search for the torn end in ruptures or for the portion behind the stricture being frequently carried too near the pubes. The membranous urethra in the adult usually runs through the ligament about one inch below the symphysis and about three quarters of an inch above the perineal center. All guides, however, fail occasionally in certain of these cases, and it is then that suprapubic cystotomy is warranted, as an operation with so small a mortality that the slight additional risk is far outweighed by the advantages to the patient of having even an imperfect restoration of the urethral canal.

Drainage after External Urethrotomy and Perineal Section.-Much difference of opinion exists as to the value of a retained catheter after perineal operations opening into the urinary tract. Many authorities advise that in cases of section for stricture no instrument should be employed, or that, at the most, a short perineal drainage-tube be used. Others 
direct that it be kept in for forty-eight hours and then withdrawn. I believe, basing my opinion on a fairly extensive trial of both methods, that the retained catheter is of great value in all operations connecting the bladder with the surface of the body, not excepting certain cases of lithotomy with purulent cystitis. If Spence's caution is observed, and it is not allowed to project far into the bladder, and if it is kept clean and sweet by regular antiseptic injections, it is of the utmost advantage in aiding in the prevention of urethral fever, as has been shown by Keyes, Harrison, Diday, Shield, Davis, Hill, Gueterbock, and many others. The last-named writer suggests (Zeitschrift für Chirurgie, Bd. xxv, Heft 415, 1888) the employment of prolonged and frequent antiseptic irrigation or the use of an antiseptic sitz-bath. Of equal importance, in my opinion, is regular catheterization at short intervals, begun after the removal of the catheter first introduced. I have repeatedly seen a decided febrile movement after urethral operations disappear or reappear in accord with the employment or the neglect of the catheter. The traumatic urethritis, which the retained catheter or the frequent use of the instrument is said to give rise to, has almost disappeared from my practice since I have begun the use of antiseptic irrigation in such cases, and particularly since I have paid especial attention to the sterilization of the urine by the internal administration of antiseptics. The sterilization of urine by boric acid, or by salol, is of great importance in the after-treatment of all these cases, and should never be omitted. These remedies are most effective, in my judgment, if combined with full doses of quinine, but either of them is far more useful than quinine alone. They should be given with more freedom and more regularity in urethral and vesical operations.

6. Miscellaneous Methods.-a. Excision.-A number of interesting cases of excision, usually for traumatic stricture, have been reported. They have met with a varying degree of success. Hensner has reported two cases-one with slight recontraction, one with apparent cure three years after operation. König, Poncet (who reports nine cases), and others, have recorded similar experiences. The latter writer believes that the indications for urethrectomy are to be found in the existence of marked fibrous periurethral induration encircling the urethra in the perinæum, and in a history of previous operations.

b. Excision with Transplantation of Mucous Membrane.-Wölfler has reported three cases in which he employed Thiersch's method of transplanting epidermis for the radical cure of stricture. The cases were of impermeable stricture, which did not seem to him suitable for internal urethrotomy or for perineal section. The cicatricial area of the strictured urethra was first carefully excised, and afterward the granulating surface was entirely covered with mucous membrane derived from a prolapsed uterus (of which there were two cases on hand). The membrane 
was kept in place by a packing of iodoform gauze, lubricated on its inner side with vaseline. (The method of bladder drainage is not mentioned.) Three or four days later the bandage was removed, showing a gray, sticky mass where the mucous membrane had been placed. Three or four days later the granulating area seemed covered as with a veil, and again after four or five days the surface was smooth and shining, like true mucous membrane. One of the patients was seen after a year, having had no intermediate treatment. He urinated in a thick stream. The second did as well, but the observation had not lasted as long. The third died six months after his operation, from double nephritis. Autopsy demonstrated a continuous mucous membrane. The boundary between the old and the new mucous membrane could not be clearly determined. Mensel, of Gotha, Guyon, and others, have reported favorable results.

Mayo Robson (Brit. Med. Jour., May 7, 1885) describes as follows his procedure in a case which six months afterward took a No. 13 (English) sound without a hitch. The man was etherized and placed in the lithotomy position, when Mr. Wheelhouse's staff was passed down to the stricture, the scrotum held up, and the urethra laid open for half an inch in front of the stricture, which was carefully divided and the urethra laid open for half an inch behind this. The stricture, being thus fully exposed, was found to consist of a fibrous cicatricial band about one fourth of an inch wide, involving mucous membrane, submucous tissue, and bulb tissue. The whole of the cicatrix was excised, and the cut ends of the mucous membrane were drawn together over the gap thus formed and secured by a continnous catgut suture. A catheter being then passed into the bladder, the vertical incision into the urethra was united by catgut, thus closing the canal and leaving a continuous and closed urethra. The last sutures were an afterthought, and perhaps unnecessary, for they gave, way on the second day, and the urine partly escaped for some little time by the perineal wound, which healed by granulation, as in the ordinary boutonnière operation. For the first two days after operation the temperature reached $101^{\circ}$, lont was afterward normal.

Keyes (Jour. of Cut. and Gen.-Urin. Dis., July and August, 1888) has recorded a case in which he excised a traumatic stricture and inplanted a portion of the inner layer of the prepuce. A part of the graft took, and the patient made a good recovery. Fourteen months later there were evidences of stricture, but they were slight, and Keyes thought that the result was in favor of the method of treating long, tight, fibrous, and inodular stricture by excision and grafting.

c. Electrolysis.-I have carefully reviewed the entire literature of this subject, including the records of the work of Mallez and Trippier, Newman, and Le Fort, and have no hesitation in saying that the evidence adduced in favor of the method is altogether insufficient to warrant its 
general adoption, and does not justify even a belief in its usefulness in the average case. As to the claim that urethral stricture is radically cured by this method, it seems to me, in the light of the investigation of Keyes (N. Y. Med. Jour., October 6, 1888, page 372), Tilden Brown (Jour. of Cut. and Gen.-Urin. Dis., July and August, 1888), and others, simply preposterous. F. Swinford Edwards (Medical Press and Circular, 1888) and Bruce Clark (Brit. Med. Jour., December 7, 1889) have been the most prominent British advocates of the method, but have failed to convince their colleagues on either side of the water of its efficacy.

d. Divulsion (Holt and Voillemier, Dict. des sci. méd., art. Urethra). - This method, once so popular in the treatment of stricture, is now almost entirely abandoned. It is so clumsy, so uncertain, and so dangerous, that it has to-day almost no advocates, and to enter into an extended augument against it would therefore, in my opinion, be a waste of time and space. Under this heading may be mentioned, only to condemn, the procedures known as forced catheterization (Desault, Chopart, ibid.), immediate progressive dilatation (Maisonneuve, Le Fort, ibid.), tunnelization (Hirschberg, of Frankfort, ibid.), "rapid dilatation by methodical, rational, progressive, and maintained compression "!! (Thiry, of Brussels), progressive divulsion (Lavaux, Congrès Français de Chir., Paris, 1889, page 521), etc. They all involve rupture of mucous or submucous tissues to an indeterminate extent and at an uncertain point, and have all the disadvantages of internal urethrotomy, with the superadded risks of a lacerated and contused wound as compared with an incised wound.

e. Over-distention of the urethra, as advocated by Dr. Tuttle (New York Medical Journal, October 3,1891), is performed by a special instrument. While it avoids many of the dangers of divulsion, it would seem that it must produce at least minute tears of the mucous surfaces. The reported results in thirty-five cases were very favorable, and the method seems worthy of further and careful testing.

$f$. Water (St. Lonis Courier of Medicine, March, 1886) and Air (Félix Gauron, Thèse, 1859) have been employed as dilating agents, being conducted to the anterior face of the stricture by a catheter with a terminal aperture. The method and results of using the former are described as follows: The catheter is attached to a tube four feet long, connecting with a reservoir about four feet above the bed, containing water at a temperature of from $100^{\circ}$ to $106^{\circ}$ Fahr.

The patient holds the penis well compressed on the catheter to prevent reflux of the water, and at the same time keeps the catheter end well down on the face of the stricture for the whole time of the séance, which lasts from three quarters of an hour to one hour.

On the removal of the water-pressure a filiform is introduced, if possible, using the ordinary modifications of direction and motion to enter 
the stricture. The author believes the good result is reached in three ways :

1. Pressure of the water against the opening. 2. Gentle pressure of the instrument, acting as in Guyon's method of "supported catheterization." 3. Effect of the warm water, probably modifying the physiological condition of the stricture tissue.

Thus, in one of his cases he failed three times with cold water, and succeeded the fourth time with warm water. He gives seven cases of gonorrhoal and traumatic stricture, impermeable previously, of which four admitted filiforms after one séance of simple hydraulic pressure of fortyfive minutes; one admitted it after two séances of five minutes each-this was a tranmatic case, in which the liquid went over to the bladder, probably the impossibility to pass before was due to deviation rather than stricture of the canal; one case yielded after four séances of one hour each ; finally, one case of traumatic stricture did not yield to six séances, and was treated by perineal section.

Some of the cases were treated subsequently by various methods to cure the stricture.

They occurred in the Hôpital Necker-six in the service of Guyon and one in that of Trélat-while the author was interne there.

g. Massage has been employed by Antal (Centralblatt für die gesammte Ther., July, 1889), who reports distinct beneficial results in six cases.

$h$. Cauterization, while it still crops out in the literature of stricture now and then, is antiquated and barbarous, and does not require discussion.

My conclusions as to the treatment of organic strictures of the urethra may be summed up as follows:

1. Strictures of large caliber-that is, of more than 15 French, situated at or behind the bulbo-membranous urethra, are to be treated, almost without exception, by gradual dilatation.

2. Strictures of large caliber occupying the pendulous urethra are to be treated by gradual dilatation when very recent and soft, and by internal urethrotomy when of longer standing, distinctly fibrous in character, or non-dilatable. It is to be remembered that the great majority of socalled strictures of large caliber of the pendulous urethra are merely points of physiological narrowing.

3. Strictures of the meatus and of the neighborhood of the fossa navicularis should be divided upon the floor of the urethra whenever it is evident that they are real pathological conditions producing definite symptoms and not normal points of narrowing.

4. Strictures of small caliber (less than 15 French), situated in advance of the bulbo-membranous junction, unless seen very early and found to be unusually soft and dilatable, furnish the typical condition for internal 
urethrotomy, which should be done on the roof of the urethra, preferably with a dilating urethrotome, and invariably with all antiseptic precautions. The operation is especially indicated if the stricture is irritable, resilient, or inodular. Combined internal and external urethrotomy is often of advantage under these circumstances.

5. Strictures of small caliber (less than 15 French), situated at or deeper than the bulbo-membranous junction, should be treated, whenever possible, by gradual dilatation. In a case of resilient, irritable, or traumatic stricture in this region, or of stricture which for any reason (as the occurrence of rigors) is non-dilatable, external perineal urethrotomy is the operation of choice.

6. Strictures of the deep urethra, permeable only to filiform bougies, should be treated by gradual dilatation when possible, the filiform being left in situ for some time and followed by the introduction of others, or used as a guide for a tunneled catheter. If the stricture be not suitable for dilatation, external perineal urethrotomy should be performed.

7. Impassable strictures of the deep urethra always require the performance of perineal section. They sometimes can best be treated by exsection, followed by the implantation of grafts of mucous membrane.

Stricture of the Female Urethra.-This comparatively rare condition deserves at least a mention, although it has attracted but little attention from surgical writers.

Herman, of London, in 1887 reported to the Obstetrical Society his views, after an examination of the female urethra in fifty-five cases, in which no urinary trouble was complained of. He found that in the majority a No. 17 catheter wonld pass, and in all but two a No. 14. $\mathrm{He}$ related six cases of stricture of the female urethra under his own care. He had collected and arranged in tabular form twenty-three others, which were all that he had been able to find reported. He drew a parallel between the two sexes as to the etiology of urethral stricture, and attempted to show that, while it was commoner in males its causes were much the same in the two sexes. In both it might be the result of injury (these cases being proportionately commoner in women on account of child-bearing), or of the cicatrization of chancres. In the woman it was sometimes due to the so-called lupus of the vulva. In both sexes the chief cause in young and middle-aged subjects was gonorrhœa. In old women there was found stricture due to general fibrous thickening and induration of the urethra, occurring without any history of gonorrhœa or other discoverable local cause. The author suggested that, as in women the homologue of the prostate gland was the urethro-vaginal cellular tissue, these cases were possibly analogous to enlarged prostate in the male. As to treatment, he found that rapid dilatation was so simple and successful that it was preferable to any other method. 
Otis, of New York, is convinced that the foundation of at least the largest proportion of the cases consists in cicatricial deposits, due to lithiasis, at periods often long antecedent to the gonorrhœea to which they are attributed. These strictures are productive of severe reflex irritations and neuroses, which may be attributed to causes quite independent of their relations to the urethra. In such cases Otis suggests the desirability of early exploration of the urethra, by means of the urethrometer or the bulbous sound, to demonstrate the condition present.

Van de Warker (Medical News, July 16, 1887) has discussed the subject at length, and gives a brief review of its history, or rather of its absence of history, and urges the importance of examination with bulbous sounds in various urinary difficulties in the female. He offers no opinion as to the etiology of such strictures, but believes that they are both as frequent and as important as in the male. He adds: "Sometimes the constrictions are arranged in series, and Nos. 24 to 27 bulb will slip along in a series of jerks that offer but the slightest resistance to the hands; but more usually one or two of these bands are found located at the middle or upper portion of the urethra. The lumen of the normal female urethra is not the same throughout its length. It is contracted toward the meatus, expanded in the middle portion, and narrows again as it approaches the bladder. The use of the urethrometer proves this. One must not, therefore, allow error to occur from the introduction of too large a bulb, which would move with more freedom at one portion of the urethra than at another. The sensation of meeting and passing one of these bands is so characteristic and unmistakable that there is little danger of error."

In strictures of small dimensions, if the obstruction is sufficient to diminish the stream, this symptom of course persists; but even here it is subject to varying degrees of intensity, amounting occasionally to retention, while it is possible to introduce a fair-sized catheter. In women it is not safe to regard every case of urine retention as hysterical until, after a urethral examination by the bulbs, the passage is shown to be free from strictures, large or small. For the purpose of dilatation he makes use of graduated steel sounds, the same as are used to treat male stricture. The action is twofold - to dilate and to cause gradual absorption of the constricting exudate. In strictures of large size, forcible dilatation or divulsion may be used. The treatment, made two or three times a week, extends over a considerable length of time-from two to three monthsbut the relief given to the patient by the use of sounds is very satisfactory. As the dilatation of the urethra which they produce is no more important than the absorption caused by their use, the latter implies a more or less prolonged treatment. He believes any one can verify this position upon the subject by the habitual examination of the urethra 
with the exploratory bulbs in every case of pelvic disease that presents itself for treatment.

I have no positive opinion based on experience to express upon this subject; but the anatomical conditions seem to me to make it probable that stricture of the female urethra will never occur with great frequency. The subject is, however, worthy of more attention than it has yet received, even if the results of further study should be negative. 


\section{DISEASES OF THE PROSTATE.}

By W. T. BELFIELD, M. D.

wounDs.

THE prostate is so thoroughly protected by surrounding parts that, aside from wounds made by the surgeon, it is rarely the object of mechanical violence, except when contiguous organs, of more vital importance, are simultaneously injured. Its tissue exhibits decided reparative power ; even the lacerations produced by forcible and faulty handling of catheter and sound-bathed as they often are with fetid urine-commonly heal rapidly.

Wounds of the prostate contain three sources of peril : infiltration of surrounding tissues with urine; hæmorrhage ; septic phlebitis, followed by prostatic abscess or even pyæmia.

Urinary infiltration extends backward into the ischio-rectal tissue, forward into the prevesical space, and into the perinæum; the direction depending upon the involvement of the different fasciæ in the wound. I have seen the entire pelvic contents below the peritonæum, and the perinæum, penis, and scrotum, infiltrated with urine after forcible attempts at catheterism through an enlarged prostate.

Hæmorrhage from the large and numerous prostatic veins may be considerable and its extent unsuspected, because the blood flows into the bladder.

Treatment. - In lacerations of the prostate catheterism is suggested by the lesions : urinary infiltration demands free incision and drainage; hæmorrhage requires hot-water injections and pressure with a large sound. In either case a catheter should be tied in for a day or two, to prevent repetition of the injury. In rare cases hæmorrhage may persist in spite of these measures; in such case perineal urethrotomy affords digital control of the bleeding surface as well as drainage of the bladder.

Several cases of enlarged prostate have come under the writer's care, in which prostate and perinæum were so extensively lacerated by forcible catheterism that the urethra could not be identified even after perineal incision. Suprapubic cystotomy and retrograde catheterism were performed in the earlier cases; in later instances I have passed a trocar and cannula from the perinæum through the prostate into the bladder, and left a drainage-tube in the new passage for several days; a catheter intro- 
duced from the meatus has then found the natural channel through the prostate.

Abscess in or around the prostate can generally be evacuated by aspiration from the perinæum; yet the free drainage usually required can be secured only by incision. The body of the prostate, the seminal vesicles, and base of the bladder, can be freely exposed by an incision beginning at the tip of the coccyx and extending around the sphincter ani to the perineal raphé the rectum is separated by blunt dissection and turned to one side. Should the septic infection have involved the prevesical tissue, a suprapubic incision is needed; this space is most thoroughly drained by carrying a tube from the ventral incision behind the symphysis (on either side of the median line so as to avoid the prostate), and through the perinæum, emerging in the median line an inch or more in front of the anus.

Continuous Immersion in hot water is a most important measure for removing toxic products from a septic wound; it is, in fact, the only rapid way of clearing tissues infiltrated with carriers of pathogenic bacteria, such as foul urine and pus. This method, while not novel, has not secured general adoption, probably because of the usual difficulty of execution; this should not excuse the neglect of so essential a measure. Tissues infiltrated with pus from urinary extravasation (or from other cause) should, after necessary incisions, be immersed in hot water continuously for several hours, if facilities permit-certainly for twenty to thirty minutes several times in the first forty-eight hours. A full-length bath may be required; where such is not available, a portable tub of canvas can be constructed at small expense, placed beside the patient's bed, and occupied by him so much of the time as the circumstances of the case may require. The water should cover the entire area infected, and the usual irrigations be made in the bath. The writer has long employed continuous immersion for cleansing septic wounds in general, and in particular for the after-treatment of cystotomies, urinary infiltrations, pelvic abscess, etc., and considers it the one reliable antiseptic method for septic wounds.

\section{ACUTE PROSTATITIS.}

Acute septic invasion of the prostate is commonly an extension of a suppurative process of the anterior urethra; the latter may be acute (gonorrhœal) or chronic. Provocation to the involvement of the prostate may be chilling, sexual indulgence, drink, violent exertion, or the passage of instruments into the deep urethra. Possible contributive factors are direct violence to the perinæum, severe urethral injections, injury by instruments, or calculi.

The symptoms, course, and treatment are elsewhere described (see "Complications of Gonorrhœa.") 
CHRONIC INFLAMMATION OF THE PROSTATE AND APPENDAGES, INCLUDING CHRONIC PROSTATITIS, PROSTATORRHEA, PROSTATIC ABSCESS, AND PELVIC ABSCESS.

All modern works on genito-urinary surgery devote a chapter to chronic prostatitis (often miscalled, by one of its symptoms, prostatorrhœa), and all contain descriptions of chronic abscess in and around the prostate and epididymis. Yet no author that I have consulted notes the fact that chronic inflammation affecting the prostate is commonly not limited to that organ, but invades a part or the whole of the genital tube proper-seminal vesicle, vas deferens, epididymis-and naturally involves also the connective tissue inclosing this tube. The condition is therefore not merely a prostatitis, but a chronic inflammation of the genital canal, or, to paraphrase the term made familiar by gynacologists, of the prostate and its appendages.

There is, indeed, a complete analogy with the chronic inflammatory conditions of the uterus and tubes so far as morbid anatomy is concerned; pelvic cellulitis and abscess (the so-called periprostatic abscess) are probably less frequent than in the female, though by no means rare; possibly they are still sometimes overlooked in the male, as they were formerly in the female.

Etiology.-Among the causes, gonorrhœal infection seems the most frequent; excessive sexual excitement, chilling, injury to the perinæum (horseback and bicycle riding) seem possible causes. Chronic suppuration in the urethra from any cause is very often propagated to the genital canal; hence stricture occupies a causal relation, and the regular catheterism necessitated by prostatic hypertrophy induces a chronic catarrh of the deep urethra, which is often propagated along the genital tube even to the epididymis.

High living certainly favors this morbid condition, either through constant congestion of the pelvic veins or by inducing the gouty diathesis.

Morbid Anatomy.-As the disease is never fatal except.through abscess formation, post-mortem observation of the earlier lesions is rare. In the prostate there have been seen the usual results of catarrhal and suppurative inflammation; the racemose glands and prostatic utricle are distended with secretion mixed with pus, sometimes forming large excavations in the substance of the organ; the parenchyma undergoes either atrophy or a thickening whereby the size and consistence of the prostate are somewhat increased, while its rectal surface may become uneven. Seminal vesicles, efferent tubes, and epididymis may exhibit similar distention, with catarrhal and suppurative products, and are also found to be thickened. The surrounding connective tissue between bladder and rectum, and in the prevesical space, is hard and brawny. 
Kirmesson and Desnos have recently called attention to the fibroid thickening of the anterior rectal wall which may accompany chronic inflammation of the prostate.

Symptoms.-These vary extremely in severity with the cause and extent of the inflammation. Two types may be described: in the first, the patient is a man under thirty-five when the disease begins, and the cause is gonorrhœal infection; in the second, the subject is often elderly, and the excitant nongonorrhœal.

In the former case the symptoms are those commonly described under chronic prostatitis; they are mental and physical, the latter due largely to derangement of the urinary, sexual, and rectal functions. There is a painful sense of fullness in the perinæum; dull pain in the rectum and glans penis ("neuralgia of the urethra"), suprapubic region, and down the thighs, aggravated by jolting in cars and wagons, by sitting on upholstered chairs, crossing the legs, etc.

Urination is unduly frequent, and the call to micturate imperative; at the end of the act pain is felt in the bladder-neck, and a trace of blood mingles with the urine, which is followed by a thick, viscid fluid-not semen, but the contents of the distended prostatic glands. This intermittent discharge of a glairy, viscid liquid is the true "prostatorrhœa," though the name is often given to a continuous watery discharge, which seems to proceed less from the prostate than from the membranous and anterior urethra, which may participate in the chronic catarrhal condition.

Sexual desire is commonly decreased, and pleasure much diminished or even absent; ejaculation may be painful, and the semen tinged with blood; involuntary emissions are frequent. Sterility is a possible effect; for, as Fürbringer has shown, the spermatozoa exhibit their vibratile movement only after contact with normal prostatic fluid. It is to be expected, and I have verified the expectation by actual observation, that the secretion of the chronically inflamed prostate fails to arouse their activity.

Defecation is accompanied with such unpleasant, even painful, sensation that some of these patients seek treatment for piles. One of my cases, with typical chronic suppurative prostatitis, had received local treatment for piles for a year without appreciable effect.

Extension of the inflammation to the epididymis induces thickening, sensitiveness, and fleeting pains ("neuralgia of the testicle").

Physical examination shows undue sensitiveness of the entire genital tube and vicinity, the prostate, the suprapubic region, the space between the bladder and rectum (where a brawny hardness is also perceptible), the spermatic cord, and epididymis.

The mental symptoms are striking and characteristic-despondency, even melancholy, quite out of proportion to the physical ailment. While 
this is sometimes partly due to the erroneous belief that the prostatic discharges are seminal losses, yet it is pronounced in other cases.

The second type of ehronic inflammation of the genital canal is one that I have never seen described. From the fact that all standard authors consider "chronic prostatitis" a disease of men under forty, I infer that this type has not been generally recognized. It occurs at any age from twenty to sixty, though my cases have usually been between forty and fifty years-men who have always been accustomed to high living and sensual indulgence. Gonorrhœa is sometimes denied absolutely, more often admitted in youth, ten or more years before the beginning of present ailment. The symptoms are those of the first type in minor degree, diminution of sexual desire and pleasure and neuralgia of the testicle being among the most constant. Thickening of the prostate and of the epididymis is frequent.

Diagnosis.-Chronic inflammation of the prostate and appendages of gonorrhœal origin is distinguished from other eomplaints by the grouping of the symptoms just enumerated. The usual demonstration of catarrh of the prostatic urethra, by irrigating the anterior channel, and then having the urine passed in two bottles, should ve made. Further proof as to the source of the pus ean be obtained by "milking" the prostate from the rectum. Tuberculosis of these organs is the only morbid condition requiring careful differentiation; it is distinguished by the nodular and irregular hardness and extensive thickening in the prostate, seminal vesicle, and epididymis.

The nongonorrhœal type exhibits usually a less pronounced and complete picture; it differs from tuberculosis in the features already mentioned. It may be, however, confounded with the early stage of prostatic hypertrophy, since both are common after the fortieth year; indeed, they may coexist in the same patient.

Prognosis. - This condition involves no peril to life except through abscess formation, and even then is not commonly fatal. Yet complete local recovery can not always be assured; the more recent and acute cases are the most favorable. Those in which the prostate alone is involved more frequently recover than do those in which the appendages also are chronically inflamed.

Treatment.-Chronic inflammation of the prostate alone can usually be relieved by applications to the urethral and rectal surfaces of the organ and to the perinæum. A large (16 to 20 English) sound is introduced every two or three days, and permitted to remain three to five minutes; it should be used cold, or first quite hot and then withdrawn, quickly cooled and reintroduced.

Irrigation of the prostatic urethra with a quart or more of hot water $\left(110^{\circ}\right.$ to $120^{\circ} \mathrm{Fahr}$.), the bladder being emptied as required, is an effective 
measure. Instillation of a few drops of silver nitrate, two to five per cent solution, is another.

The rectal surface of the prostate is medicated by suppositories, each containing one half grain of iodoform with cocaine or belladonna, and by hot rectal injections.

The perinæum is irritated by mustard or a blister, repeated at intervals of a few days for several weeks. Galvanism of the prostate has been advised, a current of ten to twenty milliampères being passed for ten minutes from a negative pole in the prostatic urethra to a positive sponge on the perinæum. The general health should be most carefully maintained; the extreme despondency is better relieved by evident improvement in the local condition than by good advice, as usually recommended.

Some cases, particularly those in which chronic suppuration of the prostate exists, do not materially improve under these measures. In these I have had the best results by two additional procedures: careful stretching of the prostate by a special dilator, and parenchymatous injections from the perinæum. The stretching is used (in the absence of suppuration) where the characteristic perineal pain and sense of weight are prominent; it is followed by hot irrigation and instillation of silvernitrate solution.

Parenchymatous injections are made from the perinæum with the ordinary hypodermic syringe provided with a strong needle three inches long; iodine-trichloride solution ( 1 part to 1,000 to 2,000 of distilled water) is the agent employed, ten to thirty minims being daily distributed in the gland during the gradual withdrawal of the needle.

When the chronic inflammation has involved the vas deferens and epididymis, a suspensory bandage is a valuable adjuvant.

Chronic Suppuration is a frequent feature of chronic inflammation of the genital canal, though the additional symptoms may be slight and pass unrecognized unless the pus reaches the surface. It occurs in the prostate, seminal vesicle, pelvic connective tissue, and epididymis. In the prostate the pus commonly empties spontaneously into the bladder or urethra; or it may, by causing retention of urine, lead to the introduction of a catheter, the pressure of this instrument bursting the abscess wall. Such abscess formation is not rare in cases of prostatic hypertrophy and of chronic prostatitis from gonorrhœea or stricture.

Chronic suppuration in the seminal vesicles (often associated with the same process in the prostate) may discharge into the urethra; or it may extend to the connective tissue between the bladder and rectum, producing a so-called "periprostatic"-properly speaking, pelvic-abscess. The pus may slowly burrow through the entire pelvic connective tissue even to the iliac crest, or it may remain long localized between the bladder 
and rectum. The abscess ultimately points toward the rectum, bladder, perinæum, or suprapubic region. The suppurative process may proceed along the vas deferens and involve the epididymis, tunica vaginalis, and even the testicle.

These chronic suppurations due to subacute inflammation of the genital canal, and usually extending from the prostate, often remain undetected until they reach the surface or are revealed by autopsy. They are directly responsible for some cases of retention of urine, chronic cystitis, chronic urethral discharge, nocturnal emissions, vesical and rectal irritation and rectal fistula, suppurative epididymitis and orchitis. They can often be detected by careful digital examination, by rectal touch, and bimanual method.

A pelvic abscess, when discovered, should be opened not through the nearest surface, if that be a mucous membrane, but always through the skin. I have seen several chronic rectal and one urethro-rectal fistula result from the incision of an abscess in the recto-vesical septum from the rectum. Abscess in this locality can always be reached by incision from the perinæum, as already described; this route should be followed, even if the pus is found bulging the rectal wall into the lumen of the gut.

Tumors of the Prostate.-Tumors originating in the prostate are : 1, Fibro-adenoma, fibro-myoma, both included under the time-honored name "hypertrophy of the prostate" ; 2, Papilloma; 3, Cancer; and, 4, Cysts.

\section{HYPERTROPHY OF THE PROSTATE.}

Prostatic hypertrophy is a disease of the second half of life; the period at which it commonly begins is undetermined, since it causes pronounced symptoms only indirectly, namely, by interference with the function of the bladder; hence it remains unnoticed until the occurrence of vesical disorder leads to its detection. This does not, as a rule, occur before the forty-fifth year of age, though individual cases have even required operative relief at an earlier age, and unique instances of prostatic hypertrophy have been observed at thirty-six, twenty-seven, and even five years of age.

The teaching of the older surgeons, that this morbid condition of the prostate is limited to advanced life, must therefore be interpreted as meaning that extreme interference with the exit of urine by the enlarged prostate does not commonly occur before the fiftieth year.

Some degree of enlargement is found in about thirty per cent of men who have passed this period; in many of these the vesical symptoms are slight, and are overlooked or misinterpreted. The cases of pronounced symptoms on the part of the bladder are probably not more than one in three or four of those affected with prostatic enlargement.

The etiology has not yet been determined. 
Morbid Anatomy.-Prostatic hypertrophy consists in an overgrowth of all the normal elements-fibrous, muscular, and glandular-the first two predominating. Aside from the uniform enlargement thus produced, there is a characteristic feature in the tendency to arrangement of the fibrous and muscular elements into distinct nodules of spherical form, which can be shelled ont from the cut surface even during life. The gradual growth of these tumors causes them to elevate, and finally protrude from those surfaces of the prostate which offer the least resistance-i. e., the vesical and urethral faces-for these are not hemmed in by firm fasciæ, as are the rectal and perineal boundaries. Hence a hypertrophied prostate rarely retains the original form of the gland; it presents a deformity as well as an enlargement, due to distinct tumors projecting toward or into the bladder and urethra. The greatest diversity in size, shape, and location of these outgrowths is observed; yet so commonly are they found at the floor and sides of the urethro-vesical orifice, that in this locality they were by the older surgeons considered integral parts of the enlarged glands, and were described under the names of middle and lateral "lobes," respectively-terms still retained for convenience. They are found also in the body of the organ; projecting into the urethra; arching the urethral orifice like a collar or horseshoe; and even at times imbedded in the vesical wall, isolated from the prostate proper. In size they are found from the smallest noticeable dimensions to the bulk of an orange, the prostate and its outgrowth sometimes filling the true pelvis.

Pathology.-Whatever the form and location of these overgrowths, three morbid conditions are almost invariably induced:

1. Distortion of the prostatic urethra, which suffers a deviation in direction, decrease in caliber, often, also, elongation.

2. Elevation of the level at the vesico-urethral orifice; and-

3. Obstruction to the return of blood from the hladder, since the vesical veins empty into the compressed prostatic veins.

As a result of these anatomical changes varions morbid symptoms ensue; decrease in the force of the urinary stream ; imperfect evacuation of the bladder, a little "residual" urine remaining after each nrination; slight eatarrhal inflammation of the bladder; and a desire to urinate at intervals shorter than the normal period.

The last is the symptom which often first attracts the patient's attention to his urinary organs. It is due in the early stage to irritation of the bladder-neck from venous congestion, for it occurs during the night (i. e., period of bodily inactivity), before it is manifest during the day (when museular activity improves the circulation). It is also marked during overloading of the pelvic veins from constipation or overeating.

In the course of years further morbid conditions naturally ensue:

1. Dilatation of the bladder with increase of residual urine. 
2. Hypertrophy of its muscular coat and fibrous tissue.

3. Depressions of mucous membrane ("diverticula") between the muscular trabeculæ.

4. Dilatation of ureters and renal pelves, with stagnation of urine in them.

5. Congestion and catarrhal inflammation of entire urinary tract; accumulation of products in the bladder, tending to calculus formation.

6. Ammoniacal fermentation of urine, favored by but not dependent on the use of unclean catheters.

7. Septic inflammation extending from the bladder to the kidneys"cysto-pyelonephritis" and chronic uræmia.

8. Death from uræmia, often provoked by various agencies, particularly the first use of the catheter.

Symptoms. - These vary, of course, with the extent of the disease. Among the earliest are difficulty in starting the flow of urine, feebleness of the stream, and unduly frequent calls to urinate, especially at night"irritable bladder." In the earlier stages other symptoms may obscure the slight urinary disorder; the patient frequently complains that he is "bilious"-has indigestion, dryness of the mouth, loss of appetite, nausea, headache, and pain in the back, or slight fever-all of which may be due to mild uræmia. Another symptom is polyuria, even to six or eight pints daily, the urine showing reduced specific gravity, and sometimes a trace of albumin; such cases have been miscalled "diabetes insipidus."

With the advent of catarrh of the vesical neck the bladder symptoms become more prominent; frequent calls to urinate are felt by day as well as by night; the normal sense of satisfaction after micturition is lacking; the urine becomes cloudy and deposits a white sediment; dull pain may be felt along the urethra, in the perinæum and rectum.

The quantity of residual urine slowly increases, amounting sometimes to a quart or more; the overtaxed sphincter often yields to the pressure, permitting an involuntary escape of urine. This dribbling of urine indicates not "paralysis of the bladder," but an organ habitually distended in consequence of an impediment to the exit of urine. Thus far the patient may have been fairly comfortable, and the nature of his disorder unsuspected; but he may now be awakened to the gravity of his condition by the supervention of either of two events-a sudden and severe aggravation of the heretofore mild cystitis, or a complete retention of urine. Each is frequently provoked by chilling and wetting, particularly of the feet; excessive eating or drinking, constipation, undue physical effort. In either event the patient becomes usually the subject of chronic cystitis, which presents varying degrees of severity, now remaining mild for weeks or months, again increasing to an acuteness which confines him to bed. 
Diagnosis.-A patient over forty years of age, whose history includes any or all of the symptoms narrated, may justly be suspected of prostatic hypertrophy; yet direct evidence should be elicited, since prostatic hypertrophy, like other frequent and familiar morbid states, is made to cover a multitude of sins in diagnosis-of omission as well as of commission; for the symptoms are primarily those of cystitis, which may be due to any one of many causes other than prostatic enlargement.

The examiner's oiled forefinger is gently insinuated into the patient's rectum, and notes carefully the size, smoothness, symmetry, and consistence of the prostate. Undue size or firmness of the organ (or both, for they usually coexist), furnishes proof that the prostatic disease may be responsible for the vesical trouble. Yet it is the urinary, not the rectal surface, which offers an impediment to the exit of urine, and there is no constant relation between the diffuse enlargement discoverable per rectum and those deformities of the organ which prevent the evacuation of the bladder; these are accessible only from the vesical side. A general idea of the conformation of the urinary side of the prostate may be sometimes obtained by the gentle manipulation of a sound, particularly when supplemented by a finger in the rectum. Yet this measure, to which much importance was formerly attached, is often disappointing.

The cystoscope sometimes enables the examiner to see distinctly the contour of the vesical surface of the prostate; but in many cases this instrument can not be introduced through the distorted and elongated prostatic urethra.

Yet the essential information sought is not the contour of the enlarged prostate, but whether or not the evacuation of the bladder is complete. This is ascertained by directing the patient to pass (in the upright posture) all his urine; a clean, soft catheter is then inserted; a flow of urine (which should be stopped at two ounces) proves that voluntary evacuation of the bladder is incomplete-that there is residual urine.

Having demonstrated prostatic enlargement and residual urine, the physician should not jump to the conclusion that he has made a complete diagnosis; for other serious morbid conditions may coexist, contribute largely to the patient's symptoms, and yet be obscured by the prostatic enlargement. The examiner must proceed to detect or exclude degeneration of the bladder walls, prostatic cancer and tuberculosis, vesical calculus, tumor and tuberculosis, renal calculus and tuberculosis, and urethral stricture.

Degeneration of the Bladder Wall-sclerosis-is a not infrequent coincidence with prostatic hypertrophy, because both are frequent features of the second half of life. The French school, indeed, following Civiale and Guyon, regards the prostatic disease as merely one manifestation of general senile sclerosis involving arteries, kidneys, and bladder. While 
this conception seems entirely refuted by clinical and anatomical observations, yet the frequent coincidence of prostatic enlargement and general sclerosis in elderly subjects should be remembered; since degeneration of the vesical wall may be a potent factor in the retention cystitis of prostatics.

Cancer of the prostate, when advanced, is recognized by the uneven hardness and nodular projections perceptible per rectum, the infection of inguinal glands, emaciation of the patient, hæmaturia and prostatic pain, microscopic and cystoscopic appearances. In the earlier stages the disease is doubtless often undetected because passed as simple prostatic hypertrophy. The writer made this mistake a few years ago in a case which a year later showed the typical features of cancer-a diagnosis subsequently confirmed by autopsy.

Tuberculosis of the prostate is recognized by the nodular rectal surface, thickening of the seminal vesicles, epididymis, etc. It may coexist with prostatic hypertrophy and be overlooked in consequence. The writer once made a perineal prostatotomy, and discovered only when the finger entered the prostatic urethra that there was a tuberculosis of the prostate; subsequent observations proved that the pronounced general hypertrophy was the minor factor in the production of vesical irritability in this case.

Vesical Calculus is the natural and frequent sequence of retention cystitis from prostatic hypertrophy. Its detection by the sound or cystoscope should follow, though notoriously difficult because of the frequent concealment of the stone in the various pockets which abound in such bladders.

Urethral Stricture is a possibility that should not be ignored.

Renal Calculus and Tuberculosis may induce the symptoms of cystitis, and be overlooked in the presence of the more evident prostatic hypertrophy.

Having determined by exclusion of these possibilities that prostatic enlargement is alone responsible for the urinary symptoms in a given case, the physician should then thoroughly and invariably examine the patient; therapy and prognosis are based upon the general condition rather than that of the prostate. The points to be especially investigated are the upper urinary tract, the arteries, and the general nutrition.

The condition of the kidneys can not always be ascertained with certainty; renal sclerosis may exist though no casts be found, the latter being dissolved by alkaline urine. The quantity of urine in twenty-four hours, its specific gravity, and the relation of albumin present to the quantity of pus, should be ascertained. Polyuria or low specific gravitywith or without renal albuminuria-suggests involvement of the kidney 
either as sclerosis or as pyelonephritis, and therefore demands caution in both prognosis and treatment.

Arterio-sclerosis, indicated by hard and tortuous arteries, polyuria, possibly cardiac hypertrophy, is a most serious coincidence; for the subjects of this disease are poor material for therapentics, whether medical or surgical.

The patient's general condition, weight, flesh, color, vigor, dryness of skin, digestion, excretion, his occupation and habits, are factors about which full information should be secured.

Prognosis.-Prostatic hypertrophy involves peril to life only indirectly, through disorganization of the urinary apparatus. While restoration of the bladder function can not be expected, except through surgical interference, yet the eatheter function, so to speak, may be successfully and comfortably substituted for an indefinite time-ten, fifteen, or more years. The course of events varies extremely, and is influenced by many conditions other than the prostatic hypertrophy.

Treatment. - The first object of rational treatment would naturally be the removal of the prostatic obstacle to urination and restoration of the normal relations at the bladder-neck. All efforts to this end (other than surgical, to which a special chapter will be devoted)-internal medication, parenchymatous injections, galvanism-have failed. Treatment must therefore be palliative, aiming alike to retard the destructive changes in the urinary tract, and to secure the patient from the discomforts incident to the urinary impediment.

He should be instructed in personal hygiene; assimilation and excretion should be promoted. Good digestion, daily and easy defecation, warm baths, and friction for the skin, warm elothing, plenty of food, sunlight, air, and exercise, are cardinal principles for the prostatic. $\mathrm{He}$ should drink at least three pints of good water daily (hot, if preferred), and plenty of milk, unless specially contraindicated. He should be warned that the process commonly termed "taking cold," exposure to inclement weather, excess in eating and alcohol drinking, extreme bodily effort, and habitual constipation, have a special and immediate danger for him, in that acute cystitis and complete retention of urine are commonly provoked by one of these.

Local Treatment varies in its details with the degree of urinary derangement. The cardinal principles are always-

1. To retard the progressive distortion of the prostatic urethra.

2. To promote complete evacuation of the bladder-i. e., prevent the accumulation of residual urine.

3. To prevent or arrest septic processes in the urinary organs.

1. Efforts to retard the progress of the prostatic distortion are perhaps never entirely successful; yet, in the earlier stages especially, they should not be neglected. 
The plan followed in gradual dilatation of urethral stricture shonld be applied to the prostatic urethra; the passage of steel sounds every four to six days, beginning with the largest that enters easily, and increasing the caliber until the full size (18 to 22 of the English seale) has been introduced. More complete stretching of the prostate can be secured by the judicious use of special dilators. The writer has been convineed by experience of the value of this neglected measure in facilitating the exit of urine.

2. To prevent the accumulation of residual urine the catheter is the chief reliance. The patient should be at once tanght to pass the instrument, and the necessity for its daily use explained. Because some of the older surgeons advised that catheterism be deferred as long as possible, it must be expressly stated now that the detection of residual urine renders the daily use of the catheter imperative, even though the patient urinates voluntarily and with apparent freedom. In cases where the bladder is habitually distended with residual urine, the evacuation must be gradual and cautious, as will be presently explained; but in any case, after the bladder has been completely emptied by the physician, the patient must keep it empty, supplementing Nature's effort by the use of the catheter one or more times daily as may be required.

Catheters, it is understood, shonld be flexible and clean. The soft Nélaton catheter (velvet eye preferred) will usually enter the bladder; if it fail, the stiffer coudé instrument of Mercier, commonly called prostatic catheter, generally succeeds. Metallic instruments, and those armed with a stylet, can easily be made to lacerate the urethra, and are rarely needed except in cases of extreme hypertrophy.

All instruments used should be surgically clean-aseptic. The soft as well as the metal catheters can be sterilized in the usual way, by boiling. Elastic instruments are destroyed by hot water, and must be rendered aseptic by thorough mechanical cleansing and prolonged immersion in fiveper-cent carbolic or similar solution. For lubrication, a five-per-cent borax solution in glycerin has evident advantages over the usual dirty oils; in it, moreover, catheters can be kept clean when not in use.

It is never amiss to warn the patient that flexible catheters are prone, when given the opportunity, to disappear into the urethra. When one is inserted, the outer end should be constantly held. He should also be advised to discard a catheter whenever its surface shows cracks or its eye becomes rough.

3. To combat septic inflammation in the bladder many means have been recommended; only the following need consideration: Hot water, boroglyceride, nitrate of silver, bichloride of mercury, and trichloride of iodine.

From the earliest stage the patient is instructed to bathe the bladder 
daily with water as hot as can be comfortably borne, say from $98^{\circ}$ to $108^{\circ}$ Fahr. A few ounces-not enough to provoke a feeling of distentionshould be gently injected, the eye of the catheter being first withdrawn into the prostatic urethra, so that the latter as well as the bladder is washed. (Many prostatics can inject the bladder direct from the meatus without a catheter.)

So long as the urine remains acid, an excellent local application is boroglyceride, a teaspoonful to a quart of warm water. After two or three washhings with plain water the boroglyceride solution is injected, allowed to remain a few minutes, and withdrawn.

Should decided irritation of the bladder-neck occur, as indicated by frequent desire to urinate with pain along the urethra, the bladder washing may be followed by a deep urethral injection of silver nitrate, one quarter to one half grain to the onnce of water; or three grains of cocaine muriate in a drachm of water may be instilled into the deep urethra, or both may be profitably used.

When the urine has become ammoniacal, the washing of the bladder with hot water must be very thorough, and followed by a solution either of mercury bichloride ( 1 to $10,000,15,000$, or 20,000 ) or of iodine trichloride (1 to 2,000). The latter has in my hands superseded the former. It is important that the prostatic urethra as well as the bladder receive these applications-that is, the catheter should be withdrawn until the eye has receded one to two inches from the bladder. The fluid then injected enters the prostatic urethra, and passes through this canal into the bladder; the eatheter is then pushed back until the outward flow is reestablished. Should the eatheter have been withdrawn too far, notice is given, so soon as the injection commences, by the appearance of the injected fluid around the catheter at the meatus. Washing of the prostatic urethra in this way should always be included in the washing of the bladder; in these pages the latter phrase is meant to designate both.

The method of evacuating the bladder during irrigation is also a matter for consideration. As ordinarily done it is quite inefficient, because left to the unaided expulsive effort of the bladder. Now it is admitted that this organ in the prostatic, when it has reached the stage under discussion, does not and can not expel all its contents; the heavier pus and mucus particularly gravitate to the base below the urethral orifice and behind the protruding prostate, whence expulsion by the natural effort is most difficult and least probable. Moreover, such a bladder usually presents diverticula of the mucous membrane between the muscular fibers, which pockets are distended rather than collapsed by vesical contraction, and are consequently reservoirs for stagnant urine, pus, mucus, and blood. The emptying of the bladder should therefore be assisted by two simple measures: (1) the position of the patient, who turns as much as possible on his 
breast, the hips somewhat elevated, or take the "knee-elbow" position; and (2) the suction of a siphon, easily secured by disconnecting the tube from the fountain syringe (the best instrument for irrigation) and conducting it to a basin of water on the floor.

When the cystitis becomes very acute and painful the bladder may be rested by continuous drainage for a few days through a soft catheter, the instrument being retained in position by a rubber "catheter-holder"; in its absence, a good substitute can be made with a strip of dentist's rubberdam five or six inches long, perforated in the center to slip over the end of the catheter against a safety-pin near the meatus; the ends of the strip are applied to the sides of the penis and retained by adhesive plaster strips, which should not entirely encircle the penis. Twice daily the catheter should be removed and cleansed, and the anterior urethra irrigated, to diminish the urethritis and irritation which usually ensue. This drainage of the organ through a catheter (with or without the siphon attachment) is an efficient and simple means of relief during aggravations of the cystitis. A patient whom I saw with Dr. Seelye, of Chicago, became so enamored with the method that he wore the catheter continuously for two years, meanwhile performing his duties as a mechanic ; his act of urination consisted simply in withdrawing a plug from the end of the instrument.

In some cases catheter drainage fails to relieve the cystitis, and in others the constant contact of the catheter can not be endured; these should be promptly relieved by puncture of the bladder through the prostate from the perinæum, the cannula, guided by a finger in the rectum, being made to enter the post-prostatic pouch. A soft catheter is introduced through the cannula, which is then withdrawn; the catheter can be retained indefinitely by adhesive plaster. This simple operation can be practiced without anæsthesia.

Attempts to improve the condition of the urine by internal medication have not, in the writer's observation, been attended by pronounced success; yet there can be no objection to the proper use of the various demulcents and diluents, from slippery-elm and buchu to corn-silk and pichi. An emulsion containing small doses of powdered cubebs, turpentine, and belladonna is worthy of trial. For arresting fermentation in the urinary cavities, salol, boric acid, saccharine, and microcidine have each been highly lauded. Perhaps the most efficient means is a combination of borax, boric and benzoic acids, three grains each, taken four to eight times daily. As the digestive organs of the prostatic are, as a rule, easily deranged, over-medication must be avoided.

Such is, in brief, the palliative treatment by which most subjects of prostatic hypertrophy are enabled to pass many years in comparative comfort and safety. They are, of course, liable to sudden exacerbations 
of cystitis and sudden œdemas of the prostate, troubles which generally subside under rest in bed combined with the treatment already outlined.

Complications. - Yet deviations from this general course are by no means infrequent-conditions fraught with suffering and danger to the patient, and commonly requiring interference by the surgeon; they may be thus enumerated :

1. Complete retention.

2. Interference with defecation by the prostatic tumor.

3. Persistent cystitis of great severity.

4. Such distortion or stenosis of the prostatic urethra as renders catheterism extremely difficult or painful.

Complete Retention.-Many prostatics seek no medical advice until, after chilling, excesses in eating and drinking, or other cause, they suddenly find themselves unable to void any urine. Rest and a hot bath often re-establish the flow, but frequently all domestic remedies fail.

This is an epoch fraught with danger to the patient and responsibility to the physician. Many a prostatic experiences serious, even fatal illness after such a crisis, because the physician sees in acute retention no indication except to empty the bladder as soon as possible.

When called to such a case, the physician should observe three rules of vital importance :

1. Never use force, nor lacerate the urethra with the catheter; in other words, use flexible instruments.

2. Observe strict aseptic precautions as to instruments, etc.

3. Never empty the bladder completely at one sitting.

The patient's entire urinary tract above the prostate is probably in a state of chronic inflammation and overdistention with urine; there are therefore three calamities that may be directly and immediately provoked by the catheter: 1 . An unclean instrument may start a septic inflammation of the entire urinary tract-cystitis, pyelitis, nephritis, one or all. 2. Lacerations of the urethra-" false passages" - not only increase greatly the difficulties in reaching the bladder, but also offer an opportunity for acute urinary intoxication and infiltration. 3. The immediate evacuation of the bladder, even with antiseptic precautions, causes a collapse of the distended urinary tract and consequent engorgement of its surface. Within a few days the urine contains a little blood (independent of mechanical injury), becomes turbid and scanty; the temperature rises, the tongue becomes dry and brown, the mind a little unsettled-in short, the patient sinks into the condition known as the typhoid state. The term "catheter or urinary fever" has been promiscuously applied to these morbid states, which are, however, essentially distinct; the cause of the fever being in the first case a septic infection of the urinary tract, in the second an intoxication from urinary absorption, in the last uræmia from 
congestion of the kidneys caused by sudden removal of the habitual pressure of the retained urine-a " hyperæmia ex vacuo," as it has been termed. Doubtless two or all of these may be induced in the same patient by attempts to relieve complete retention.

The patient suffering from complete retention should have the rectum emptied by a hot-water injection, to be followed by a suppository containing one-eighth grain of morphine, or an enema of thirty grains of chloral in a half pint of warm water. A hot sitz-bath, or, in lieu of this, hot-water fomentations should be employed for twenty or thirty minutes. The effect of these measures is often to cause the escape of some urine; even if this occur, catheterism is still necessary. The patient is warmly covered in bed, the hips elevated above the shoulders. The anterior urethra is irrigated with hot water and injected with warm, clean oil ; a clean, soft catheter (No. 10 or 12 English) is filled with the oil and introduced, the penis being drawn firmly forward. Steady, persistent pressure on the instrument-slight as the transmitted force necessarily is-is often rewarded by a gush of urine. Should this effort fail, an elastic coudé catheter of smaller size is next tried; flexible instruments of various sizes and shapes may be afterward employed. In most cases one of them enters the bladder.

In exceptional instances, all flexible instruments having been arrested before reaching the bladder, rigid catheters may be used. The metal or stylet-armed instrument selected should be of good size (14 English, or larger), of very long curve and clean. In the attempt to introduce it force must be scrupulously avoided, for no one can know the direction of the distorted and swollen prostatic urethra. The catheter is guided less by anatomical knowledge than by the resistance it encounters, and the attempt to force the rigid instrument results usnally in sinking its point into or beyond the urethral wall. False passages are made, blood flows freely, and the difficulties of the situation are multipiied. The physician, naturally disliking to acknowledge himself baffled, yields to the temptation to employ more force, until finally the prostate is perforated with holes.

While prostatic retention, until maltreated with instruments, is usually relieved with ease, yet after false passages have been made it is sometimes impossible for even a skillful hand to find the natural way to the bladder with any catheter. In this case suprapubic aspiration should be made, the needle entering at right angles with the spine, about an inch above the upper border of the symphysis. A half or a third of the estimated contents of the bladder is withdrawn, hot fomentations again applied, and the patient left in bed for some hours. The relief of bladder tension and reduction of prostatic cedema by this time often results in eaşy catheterism or even voluntary urination. Should retention persist 
for a day or two, aspiration must be superseded by some one of the methods of operative relief to be presently described.

Should the physician succeed in introducing the catheter, he must not evacuate the bladder completely at the first sitting; about a third of the estimated quantity, say from six to ten ounces; may be withdrawn and two or three ounces of some antiseptic solution injected. Five or six hours later a larger quantity may be withdrawn, and thus in the course of two to four days the bladder may be gradually emptied. If the first introduction is extremely difficult, the catheter may be left in place for a day or two, tightly corked, until complete evacuation is secured.

Even with the greatest care some fever frequently follows complete retention, requiring the first use of the catheter; yet the illness is kept at a minimum by the measure described.

Persistent cystitis of high grade, difficult catheterism from prostatic distortion, and rectal impediment from prostatic tumor, should be relieved by operative treatment.

\section{OPERATIVE TREATMENT OF PROSTATIC ENLARGEMENT.}

In a considerable number of prostatics the palliative measures already enumerated fail to avert constant suffering and a fatal result. These cases were formerly regarded as beyond surgical aid; aside from the relief obtained by suprapubic puncture, they were abandoned as hopeless.

During the last decade temporary drainage of the bladder by different routes has become a standard operation for the relief of these patients. Yet this measure, though gratifying in its immediate results, is in theory unsatisfactory, and in practice but too often of only temporary benefit. The relief so obtained is of uncertain tenure; the factors which originally induced the malady remain undisturbed; they may, and as experience shows they often do, cause a recurrence of the vesical inflammation and difficulties of catheterism.

A rational procedure should aim to give permanent relief by removing the cause of the urinary difficulty. This has been attempted upon two plans:

1. The institution of a permanent artificial exit for the urine-a ven. tral or perineal fistula-whereby the obstructing prostate is aroided. 2. The restoration of the natural urinary channel through the prostate-a socalled radical operation, or "prostatectomy."

The former is evidently the simpler method. The suprapubic fistula has been often made in the past half century, usually by puncture with a trocar, sometimes by cystotomy, a tube being more or less constantly worn subsequently. A perineal fistula, kept open by a soft catheter passing through the incision and the prostatic urethra, has also been found useful. 
The permanent fistula is a mutilation obviously to be employed only when the restoration of the urethral channel seems impracticable. Aside from the objections on the score of sentiment and comfort, the fistula can not be relied upon to secure immunity from cystitis; thus, Bennett May mentions three instances of such failure in his own practice. The explanation of the failure is, perhaps, that the projecting portions of the prostate prevent complete evacuation of the vesical contents; and probably that these tumors, like other neoplasms and foreign bodies, induce mechanical irritation of the bladder. The latter condition seems to have been exemplified in one of May's cases, which, after the fistula had failed to give relief, was entirely cured by the removal of a large middle lobe.

The theory of the radical operation assumes that the cause of chronic retention is mechanical obstruction by the enlarged prostate; that such prostatic obstacles are capable of removal; and that after such removal the bladder will resume its function of voluntary evacuation.

These propositions have been separately and collectively denied by three of the foremost among living genito-urinary surgeons-Thompson, Guyon, and Socin. Guyon maintains that prostatic enlargement is but a local manifestation of a general senile "sclerosis" which pervades the entire urinary tract; that the chronic retention is chiefly due not to prostatic obstruction but to the coincident impairment of vesical contractility caused by sclerosis of the bladder muscles; hence, that the removal of prostatic obstacles is irrational, and futile to restore voluntary urination. He asserts further, as the result of post-mortem examination, that the prostatic obstacle is rarely of such contour as to permit its removal by the surgeon. Socin advances essentially the same opinions. Thompson, while repudiating sclerosis as the cause, and assuming that enlargement of the prostate is an attribute of senility, indorses Guyon's conclusions.

These are, however, conjectures unsupported by clinical experience. Thompson and Socin have not yet performed-or at least have not published-a single radical operation. Guyon made his first attempt in July, 1889 , and found a prostatic growth readily removed.

The accumulated clinical observations of the past five years prove that these traditions of the earlier surgeons, upheld by Guyon and Thompson-which have dominated surgical opinion and practice-must be restricted to a minority only of prostatics; for it is clearly demonstrated :

1. That there is a larger class, entirely distinct from these, in whom the failure to evacuate the bladder is due in no wise to degeneration of the vesical muscles, but solely to the mechanical obstruction offered by prostatic growths; since the removal of such obstacles has been followed in over three fourths of the cases previously dependent upon the catheter by restoration of the vesical functions. 
2. It is shown that the enlargement of the prostate, commonly called "senile" hypertrophy, is not limited to advanced life. Thompson has strenuously taught that "it never appears but in advanced years," the minimum age being fifty-six. "I have never been able to meet with an instance of its occurrence at so early a period as fifty-three years of age." Many others have seen it in younger men. McGill removed a prostatic collar, "the size of a large walnut," from a man aged fifty-three; $M$. Schmidt, a large middle lobe from a man of fifty-two; the writer has removed a prostatic growth from a patient aged forty-nine; Packard operated a case forty-three years old ; Moullin one at forty-one; Dunn excised a tumor of the lateral lobe from a man of forty-five; Iversen described a case at thirty-six year's; H. G. Mudd exhibited to the Association of Genito-Urinary Surgeons, 1890, an extreme example of prostatic hypertrophy from a negro aged twenty-seven; the prostate and its outgrowths literally filled the true pelvis, and on microscopical examination were found to be fibro-myomatous, without a suspicion of malignancy. On the same occasion Dr. Mudd mentioned a case of true prostatic hypertrophy, under the observation of Dr. H. II. Mudd and himself, in a child of five years.

3. That the prostatic obstruction is usually-contrary to the opinions of Guyon and Thompson-of such form as to permit excision, is shown by the fact that in four fifths of the cases of suprapubic incision such obstacles were found and removed.

The assumptions upon which the radical operation is based are therefore justified by experience. It is proved that in many prostatics the source of the evil is to be sought not in sclerosis or senile degeneration of the bladder, but in mechanical obstruction by prostatic tumors; that these tumors are not a product of selerosis, nor of senility, nor even limited to advanced life; their greater frequency after forty years of age is paralleled by that of other tumors, particularly the fibro-myomata of the homologous organ, the uterus, which are not considered an attribute of senility; that these tumors are in most instances susceptible to removal; and that in such cases a well-executed radical operation restores voluntary urination.

It is indisputable, however, that in many prostatics of advanced age arterio-sclerosis and degeneration of the vesical walls coexist; the contractility of the bladder is seriously impaired, and removal of the prostatic obstruction fails to restore voluntary evacuation of the organ. In nearly one fourth of the cases on record the radical operation failed to restore voluntary urination; while in several it is evident that the removal of the obstacle was incomplete, yet in others the explanation of failure seems to have been impaired vesical contractility.

Differentiation between the obstructive and degenerative factors in a 
given case should naturally precede operative measures; at present such differential diagnosis can not always be accurate. The subject of pronounced sclerosis exhibits rigid arteries-polyuria; his prostate is very hard, his bladder not dilatable. In the presence of these symptoms restoration of voluntary micturition ean not be expected? the radical operation is useless except to facilitate difficult catheterism.

Modes of Operation.-Prostatic obstacles to urination have been attacked by four routes :

1. The urethra (Mercier, Bottini).

2. Perineal incisions (Harrison, Keyes, Dittel).

3. Suprapubic cystotomy (Belfield, McGill).

4. Combined perineal and suprapubic incisions (Belfield).

The operation should secure three results : Restoration of a low-level urethra by removal of prostatic obstacles; temporary drainage of the bladder; and stretching of the prostatic urethra. The choice of operation is therefore a mechanical problem, determined largely by the accessibility of the prostatic obstruction. Now, these present the greatest diversity in size, shape, and location. Whether the obstruction is a distinct tumor, median or lateral, sessile or pedicled, intravesical or intraurethral; or a so-called bar; or a combination of these-can be in only exceptional cases more than conjectured by any means other than digital contact, which is, therefore, the first essential of every accurate radieal procedure.

This condition obviously excludes from serious consideration all operations from the meatus, and shows the limitations of operations through any perineal incision, since the average finger is too short to explore or even to reach the vesical orifice from the perinæum when the prostate is much enlarged. Operations from the meatus are evidently, in the light of present experience, tentative, haphazard procedures; they were based upon the faulty conception that the prostatic obstacle is always a bar or other median obstruction; and that the division of this supposed bar would effect relief. These operations of Mereier secure no one of the three essentials sought; they contain, moreover, elements of danger foreign to the later procedures, where the cutting edge in the bladder is directed and controlled by digital contact. His followers have been few, and his operations seem now to possess only historic interest. Bottini's modification of Mercier's operation-the substitution of a cauterizing for a cutting edge-is equally unsatisfactory, and seems to have found no adoption.

Perineal urethrotomy has been employed in some forty cases as the avenue of access to the prostatic obstacle; small median tumors (middle lobes), transverse overgrowths (bars at the bladder-neck), have been incised and excised; even lateral masses of considerable size have been 
enucleated (Wishard). Yet many cases are not capable of satisfactory treatment by this incision; the elongation and rigidity of the prostatic urethra prevent complete operation or even exploration at the bladderneck. The surgeon may therefore fail to detect the obstructing portion, or find its removal impracticable. Since it is impossible to distinguish in advance the cases operable by this incision, it is apparent that a better method, one that shall be applicable to every case, is required.

Suprapubic cystotomy seemed at first to fulfill every requirement; it secures minute exploration of the entire vesical surface of the prostate, the easy recognition and complete removal of the intravesical projections, and thorough drainage of the bladder. Prostatectomy by this incision has been performed over eighty times with satisfaction to the operator, and often with results most gratifying to the patient. Yet in several instances this operation has failed to detect or to remove the prostatic obstruction; failures which direct attention to the fact-rather obscured by the many brilliant successes in removing intravesical growths - that the object is to restore a low-level channel through the prostate, and not simply the excision of salient tumors. To this end exploration of the urethral is quite as important as that of the vesical surface of the prostate; for the canal may be distorted by growths imperceptible or inaccessible from the bladder; especially frequent is a pronounced thickening of the suburethral prostate, whereby the urethra is elongated and the vesical orifice displaced upward. In five recorded cases such a mass has baffled the surgeon who approached it by suprapubic incision; in two (McGill) it could not be satisfactorily removed; in a third (Guyon) the partial removal was insufficient to restore the channel; in the remaining two this obstruction was overcome by perineal incision and stretching (M. Schmidt, Belfield).

By suprapubic incision alone the surgeon may find himself unable to restore the patency of the urethral channel; the intravesical projections may in a given case constitute but a part of the prostatic obstruction, and the removal of such outgrowth is not necessarily a successful operation. The prostatic urethra should always be explored by the finger; and the usual discovery of a hard, suburethral mass or rigid ring should be the signal for perineal urethrotomy, incision or excision of the resisting tissue, and thorough stretching.

It seems, therefore, that the combined operation by both suprapubic and perineal incisions should be the rule rather than the exception; the addition of the boutonnière increases but slightly the injury to tissue and the time of anæsthesia, while it affords an access to the entire prostate, which may convert an utter failure into a complete success.

Results. - The results of the various radical operations have been carefully collected and discussed by Watson in an excellent monograph 
published in 1888 ; by the present writer in the American Journal of the Medical Sciences, 1890 ; and by Mansell Moullin in his admirable Hunterian Lectures for 1892, In all, about one hundred and fifty cases are now on record ; while pioneer results, performed by many surgeons acquiring experience with any operation, naturally failed to exhibit the full value of the measure, yet even these early experiments show the value and success of radical operations in relieving a hitherto incurable and most distressing condition. In about three fourths of the cases voluntary urination has been restored in patients previously dependent upon the catheter; in most of the remainder catheterism has been made easy.

The mortality of prostatectomy (thirteen per cent) seems somewhat greater than that of cystotomy in'elderly men for other purposes, particularly the removal of calculi, though due to the same causes-uræmia, shock, and irritation of the respiratory tract by the anæsthetic. In other words, the hazard of the operation is entirely due to the pre-existing disease of the urinary tract as well as to the enfeeblement of vitality from age and protracted suffering; and its excess of mortality over lithotomy must be ascribed to the greater delay in operating. In only one case was serious local disturbance caused.

The obvious deduction is, that the operation should be performed earlier in the course of the disease, and not, as heretofore, deferred until the last stage. Corroboration of this inference is found in the paradox that operations upon the prostate complicated with calculus extraction furnished a much smaller mortality than the uncomplicated prostatectomies. A calculus was therefore an advantage rather than an injury, since its presence led to early operation.

Opportunity is still lacking-because the operation is so recent-to determine decisively whether the restoration of voluntary urination is permanently assured ; further experience must show whether new tumors may not frequently develop from the unexcised prostate, causing a recurrence of the original morbid condition. Actual observation on this point is limited as follows: (1) In twenty-four cases, unobstructed urination and catheterism have persisted for periods ranging from six to thirty-two months after removal of prostatic obstacles; (2) in two cases, actual inspection of the bladder three months after operation showed no further tumor formation.

On the other hand, Schmidt observed at an autopsy, nine months after the removal of a median enlargement, that a new growth had appeared near the site of the one removed. The description suggests a malignant tumor rather than a prostatic myoma, but no microscopical examination is recorded. An important deduction from these considerations is the indication for thorough enucleation of all circumscribed masses within as well as abore the general prostatic surface. Such tumors can 
be enucleated after incision of the mucous membrane with surprising facility. McGill especially has demonstrated the feasibility of this measure in many cases of his extensive experience. Vignard, experimenting upon the cadaver, corroborates this observation; he asserts that in six out of ten cases he has enucleated the entire prostate-hypertrophied or otherwise-with reasonable ease.

This process of enncleation obviates the necessity for the operation suggested by Dittel, under the title of "lateral prostatectomy." After emphasizing the importance of the neglected lateral lobes in the production of retention, and criticising Harrison's plan of treating them by prolonged pressure as inefficient, Dittel shows that they can be reached by separation of the rectum from the prostate; and by operation upon a cadaver with lateral enlargement of the prostate, he proved that the excision of portions of the lateral lobes from the rear-withont opening the bladder-relieved the prostatic obstruction by permitting the vesical surfaces of these lobes to fall apart. He admits that suprapubic cystotomy would also often be necessary for the removal of intravesical obstacles; and that the two operations, even if separated by an interval of weeks, would rarely be sustained by the same subjeet until patients are educated to submitting to them at an early stage of prostatic disease.

Dittel makes no allusion to the fact that the lateral lobes can be and have repeatedly been removed by enucleation after suprapubic cystotomy. The ingenious operation suggested by Dittel seems therefore to have been anticipated by a much simpler, safer, and more practicable means of attaining the same end.

Küster has, however, performed the operation on three patients, with a fair degree of relief to the bladder symptoms, though in two of them a permanent urinary fistula resulted.

Operation.-The salient points in prostatectomy may be thus enumerated :

When time is allowed, the patient may be for two weeks previously prepared by restriction to a diet of milk and vegetables, with copious ingestion of water. Antisepsis of the urinary passages may be attempted by the internal and local remedies already described. Chloroform is preferable to ether for anæsthesia, because less irritating to both kidneys and lungs.

During the operation the body is inclined so that the hips rest six to eight inches higher than the shoulders; the bladder is moderately distended (eight to ten ounces); the rectal bag is both unnecessary and dangerous; aside from mechanical injury (bruising, even rupture of the rectum), the distention may cause alarming decrease of the blood-pressure. This depression of the circulation by rectal irritation, first observed by the writer in experiments on animals (Du Bois-Reymond's Archiv für 
Physiologie, 1882), was subsequently witnessed in two of his patients, in marked degree, while a rectal bag was being filled in preparation for suprapubic cystotomy. Wishard has since observed three similar cases, one of which did not rally.

After suprapubic incision, further procedures are determined by the size and location of the growths. Pedicled tumors, such as middle lobes, are removed by cautery wire, snare, tonsillotome, or scissors (bleeding may be profuse when edged tools are used); sessile tumors, such as lateral lobes, can usually be enucleated with the finger or spoon, after the mucous membrane is incised. Protruding parts of the prostate that can be neither excised nor enucleated safely should be treated by sinking a cautery point into them-a measure which induces subsequent absorption.

After treatment of intravesical projections, the vesical orifice and prostatic urethra are examined with the finger. Usually a suburethral growth is found; a perineal urethrotomy is then made, one forefinger inserted through the incision and the other through the vesical orifice until they meet; obstructions detected are removed according to indications, since the object of the operation is to restore a low-level, unobstructed channel from the bladder to the membranous urethra. The suprapubic wound may often be closed, except for drainage of the prevesical space, and the bladder drained by a large perineal tube extending at least an inch into the vesical cavity.

The dangers of prostatectomy are :

1. Hæmorrhage.

2. Intoxication and sepsis from contact of fetid urine with prevesical tissue.

3. Uræmia.

Hæmorrhage is minimized by the substitution of the cautery for the cutting edge, and may be checked by packing with iodoform gauze.

Intoxication and infection from urine are combated by prior cleansing of the urinary passages by iodoform and drainage. This great risk is, however, best reduced by making the operation in two stages: at the first, merely exposing the bladder and packing the wound with iodoform gauze until granulation is established (five to seven days); then opening the bladder and completing the operation.

Uræmia, due to existing pyelonephritis or renal sclerosis, is a frequent cause of death; nervous shock and the anæsthetic seem to be exciting causes. Hence chloroform should be used as sparingly as possible.

Palliative Operations-While the radical operation for prostatic hypertrophy gives great satisfaction to both patient and surgeon, its utility is as yet restricted by the fact that many patients requiring it are, when they reach the surgeon, too feeble to endure it without great risk.

Education of both physicians and patients to the necessity for earlier 
interference, will in time extend the usefulness of this life-saving operation; meanwhile help in some form must be extended to the large class who can no longer obtain relief through the catheter, and yet must not be submitted to prostatectomy.

For such, great relief at far less risk is afforded by drainage of the bladder through a suprapubic or perineal opening, which can be maintained as a permanent fistula if desired. (The ventral opening is preferred as a permanent exit, because more easily endured and managed by the patient.) If anæsthesia be permissible, a suprapubic cystotomy or perineal urethrotomy is made for the insertion of a soft-rubber tube; if anæsthesia be deemed unsafe, the opening can be made with a trocar, plunged above the symphysis, or from the perinæum through the prostate.

As a rule, great comfort is immediately derived from any of these methods of draining the bladder; the cystitis and prostatic œdema subside; sometimes spontaneous urination or easy catheterism follows in a few weeks; the tube is withdrawn, and the patient enjoys comparative comfort for many months.

But when the bladder is small and undilatable or catheterism very difficult, such lasting benefit can not be expected from temporary drainage; the fistula must usually be made permanent. While the patient greatly prefers this mutilation and comfort to his former suffering, yet the evident annoyances are many and constant. In the hope of diminishing these, I have devised a plan which, in the only case as yet so treated, succeeded perfectly, and which seems worthy of repetition, especially in cases of difficult eatheterism too feeble for a radical operation. A staff with broad groove is introduced until its point rests in the prostatic urethra; a trocar slightly curved, the convexity of its cannula being open, is plunged into the perinæum (mesial line) about an inch and a half above the anus, its point, guided by the finger in the rectum, is made to enter the groove of the staff at the apex of the prostate, it is pushed through the substance of this gland nearly horizontally (the patient lying on his back) into the bladder. The trocar is withdrawn from the cannula, the staff from the urethra. An elastic catheter armed with a straight stylet is passed through the urethra into the cannula and thence to the bladder. The cannula is then withdrawn, its track serving the important purpose of drainage, to prevent urinary infiltration.

This low-level channel through the prostate seems to have the advantages of the usual external fistula, without the annoyance common to the latter from constant dribbling of urine (prevented by the sphincter action of the membranons urethra). When necessary, the bladder can be kept empty by tying in a catheter. That such a channel, made by bungling and forcible catheterism, can even form a good substitute for the radical operation, has been occasionally revealed on the post-mortem table. 
Summary.-I. The prostatic suffering from persistent severe cystitis, or difficulty or pain in catheterism, should early receive operative relief; delay is destructive to his urinary apparatus and hence to his chance for life and comfort.

II. The choice of operation must be determined partly by the patient's vigor and partly by the condition of the bladder: fair health, renal integrity, a distensible and contractile bladder, warrant the radical operation; debility, renal disease, a small, undilatable bladder, restrict the operative interference to drainage, temporary or permanent.

III. The safest and simplest method of drainage is puncture from the perinæum through the prostate, with little or no anæsthesia. This may be profitably performed in any of these cases, even if more radical measures are subsequently undertaken.

IV. Between these extremes - the radical operation and perineal puncture-are several measures of intermediate gravity, one of which may be selected according to the vitality and needs of the patient:

1. Perineal prostatotomy after Harrison-i. e., urethrotomy, incision of the urethral floor at the vesical orifice, drainage for six to twelve weeks through a large tube. (A distinct middle lobe would naturally be removed when easily accessible.)

2. Perineal urethrotomy or suprapubic cystotomy for drainage, temporary or permanent.

\section{HYPERTROPHY OF THE PROSTATIC SPHINCTER.}

By this tentative term I have designated a morbid condition hitherto unrecognized in standard works. Post, of Boston, has described it in one case as a "fibrous ring" in the prostatic urethra. Neither name is founded upon post-mortem examination, and both may hereafter be shown to be inaccurate.

I have demonstrated this condition as the cause of obstinate cystitis in three subjects, each under thirty years of age; the last two were essentially identical with the first, which is here outlined: A boy of nineteen was referred to me, complaining that so long as he could remember he had suffered from frequent and painful urination; intervals of micturition less than an hour, day and night, except that occasionally they would lengthen for a day or two to three or four hours; pain was felt, during urination only, at bladder-neck and along the urethra; the urine was acid and contained considerable pus. Tuberculosis was suspected, but no abnormality of epididymis, prostate or seminal vesicle detected; urethral infection and injury were denied. A sound was arrested in the prostatic urethra by an obstacle that permitted only a fine bougie to pass; this condition remained during anæsthesia.

The finger introduced through a perineal incision found the distal 
end of the prostatic urethra closed and resisting all the force that could be safely applied; by means of conical bougies and forceps the channel was gradually dilated until the finger passed the obstruction, which was found to be a band of firm tissue surrounding the outer third of the prostatic urethra. Nothing abnormal was detected in the bladder.

Drainage for a few days was followed by complete recovery of the normal vesical condition and disappearance of pus from the urine. Eight months later there was no return of the former symptoms.

While I have by digital contact proved this condition to exist in only three cases, there is reason to suspect that it is not rare ; for stretching of the prostatic urethra from the meatus has in some additional instances relieved a mild cystitis for which no other cause was found.

\section{PAPILLOMA OF THE PROSTATE.}

This relatively rare tumor is for clinical purposes identical with the more frequent papilloma of the bladder, since it commonly arises from the vesical surface of the prostate (see Tumors of the Bladder). Small tumors of this variety have been discovered post mortem on the urethral surface of the prostate. Grünfeld recognized and removed such a growth through the endoscope. The writer excised one at the urethro-vesical orifice through a perineal urethrotomy.

\section{CANCER OF THE PROSTATE.}

Under this term are included two histological varieties of malignant growth-carcinoma and sarcoma. Clinical distinction between the two can rarely be made, unless particles of size sufficient for microscopical examination are found in the urine or removed in the eye of a catheter.

Cancer of the prostate arises (1) by extension from adjacent organs (rectum, bladder), (2) by infection (metastasis) from cancer of a distant organ, and (3) as a primary tumor. Only the last requires consideration here.

Primary cancer of the prostate occurs chiefly in patients under ten and over fifty years of age, though the intermediate periods of life furnish not infrequent instances. Jolly found, out of 35 cases, 7 in boys under ten and 21 in men over fifty. Engelbach found, out of 89 carefully observed cases, 9 in boys under ten ( 3 less than one year of age) and 49 in men over fifty. About seven eighths of the tumors are found to be carcinoma, the remainder sarcoma.

Morbid Anatomy.-When sufficiently developed to attract attention, the disease is usually found diffused throngh a large portion of the gland; in some cases a well-defined neoplasm is observed. For a time the disease remains limited by the capsule of the prostate, though sometimes distending this to the size of an orange; then it invades adjacent 
lymph-glands and adjacent organs, often filling the entire true pelvis, compressing the rectum and raising the bladder; perhaps extending through the various pelvic outlets.

Destruction of the walls of the cavities adjoining the prostate-urethra, rectum, bladder, and seminal vesicles-follows, the escape of their contents into surrounding tissues inducing suppuration. Invasion of the vascular channels may occur, followed by metastasis of the growth in distant organs.

Symptoms.-Intermittent hæmaturia at intervals of weeks may be the first and for a time the only symptom. Some difficulty and pain in urination; pain in the perinæum, down the thighs, in the rectum or sacral region; cystitis, complete retention ; profuse hæmorrhage with the urine, are often observed later. In other cases attention is directed chiefly to the rectum by pain and difficulty in defecation, tenesmus, and dysenteric discharges. Sometimes both bladder and rectum participate in the disturbance of function.

No one of these symptoms is invariably present.

Diagnosis.-Cancer of the prostate frequently fails of recognition, partly from neglect to make digital examination per rectum, partly because cancerous enlargement is not distinguished from the more frequent and familiar hypertrophy of the prostate. Three of the most important and constant diagnostic points are (1) progressive emaciation and pallor; (2) hard enlargement of lymph-glands in the groins, within the pelvis (detected by bimanual examination), and in Scarpa's triangle; (3) irregular, nodular enlargement of the prostate. The discovery of recognizable cancer tissue in the urine, or inspection of a malignant ulcer through the cystoscope, is conclusive evidence.

Differential diagnosis need take cognizance only of simple hypertrophy, from which cancer in the early stage may be difficult to distinguish; indeed, malignant growth may be grafted upon hypertrophy; its advent may be detected by the symptoms enumerated.

Failure to distinguish between simple and cancerous enlargement of the prostate led the older surgeons to the belief that prostatic cancer is very rare; more accurate observation in recent years reveals it relatively often.

Duration. - In children the fatal termination is reached in a short time, even three months to four weeks after the first pronounced symptoms. In elderly men the progress of the disease is less rapid-from one to three, four, even five years.

Treatment.-Palliation of distress should be attempted. Pain is mitigated by morphine; cystitis and urinary retention by the treatment outlined for prostatic hypertrophy ; rectal disturbance by avoiding constipation and by hot-water injections. The iodine trichloride is especially 
valuable for bladder irrigation because of its hæmostatic properties. The catheter must be used with extreme care, since the friable tissue of the prostatic tumor is easily wounded. Czerny even passed a catheter through a prostatic cancer into the rectum. When other measures fail to relieve the vesical disturbance, a permanent suprapubic exit for the urine should be made.

The tumor sometimes completely occludes the rectum, compelling the institution of an artificial anus in the groin or loin.

Extirpation of the cancerous prostate has been performed ten times; the patients who survived the operation have all died of recurrence within a few months. While improved diagnosis and consequent earlier operation may in the future furnish better results, the present record offers but little encouragement for the operation.

\section{CYSTS OF THE PROSTATE.}

These are of three kinds: 1 . Hydatids, which must be rare, as only three authentic instances are furnished by post-mortem records. 2. Cystio dilatation of the prostatic utricle from occlusion of its orifice. English found five such cysts during the examination of the bodies of seventy newborn infants, and suggests that this retention may be the occasional cause of urinary retention in the newborn. 3. Cystic distention of occluded prostatic glands. These retention cysts, containing prostatic secretion, are not infrequently found post mortem, especially in elderly subjects. Clinical recognition of them is very rare. The writer reported a case to the American Medical Association in 1886. The patient, a man about thirty years old, had for months experienced extreme difficulty and pain in expelling the urine; examination showed no obstruction, a catheter entering the bladder readily. Upon perineal section, the finger detected a cyst suspended from the prostate above the vesical orifice, which it almost completely closed. Removal by the finger was followed by restoration of the normal resical condition. 


\title{
THE FUNCTIONAL DISORDERS OF MICTURITION.
}

\author{
By JOSEPH D. BRYANT, M. D.
}

URINATion, or the normal act of passing urine or an analogous fluid, is a function that characterizes the more important forms of animal life. However, in the accepted sense of the term, the act is especially identified with the higher orders of this form of life. In the human family the proper performance of the act appeals not only to the bodily comfort of the individual, but to the sense of propriety as well, in the vast majority of responsible beings. Therefore, the irresistible demands of urination not infrequently impose great physical and sentimental burdens, both in health and disease. The urgent and varied importance of this demand instantly justifies the attempt to treat of the disturbances of this function somewhat independently of the morbid states that cause them. In the adoption of this course, the established morbid modifications of urination are employed in an interrogative sense, with the view of eliciting the abnormal conditions that are peculiar to each of them.

Physiology of Urination.-In order to interpret properly the abnormities of an act, the physiology of the act should be well understood in each of its recognized parts. The component parts that influence the act of urination may be classified practically as follows: 1 , The canal for the escape of the fluid-the urethra ; 2 , the receptacle for the fluidthe bladder; 3 , the fluid itself-the urine. The urethra and the bladder are of muscular structure, lined with sensitive mucous membrane, and vivified with nervous motor influence; and, too, each of these parts is animated and modified in its function by supplemental influences of voluntary and involuntary nature. The normal urine varies in amount, chemical constituency, and rapidity of production, and therefore often modifies the act of expulsion, within the limits of health. If, now, these three elements of the act of urination be normal, and their supplementary influences be rightly attuned, physiological urination ensues. If, on the other hand, discordant action, or disturbed sensibility of one or more of the controlling influences of the act happen, then disordered micturition is the result. Normal urination takes place about once in five hours during the twenty-four hours of a day. It is commonly passed on retiring at night and rising in the morning, the remaining acts being distributed 
through the day. The amount voided daily varies from thirty to sixty ounces (about 900 to 1,775 cubic centimetres). The average quantity voided per hour is two and one tenth ounces (62 cubic centimetres). Physiological urination is induced by the presence in the bladder of a certain amount of urine, which causes the sensation that leads to its expulsion. The contraction of the muscular walls of the bladder, supplemented by the contraction of the diaphragm and abdominal muscles, cause the evacuation of its contents. The relaxation of the sphincter vesicæ and the actions of certain ones of the urethral muscles (accelerator urinæ and compressor urethræ) co-operate harmoniously in aiding and completing the execution of the act. At first the act of urination is voluntary, but when begun it is continued by the involuntary contraction of the bladder alone. Voluntary effort increases the flow at all times, and especially is this influence noticeable at the completion of the act; then the abdominal effort and the contraction of certain perineal muscles complete the expulsion of all the urine except the few drops that remain behind in the bladder. The flow of normal urination can be arrested by voluntary effort at any stage of the act, if the capacity of the bladder has not been taxed by the unwonted amount of urine it contained. The center of the reflex influence that incites the bladder is thought to be located in the lumbar region of the spinal cord, and its action is modified by the inhibiting influence of the sympathetic filaments which arise from a ganglion located near the same place. It is difficult, indeed, to determine definitely the frequency of normal urination, as it varies in different individuals, and is influenced by trivial causes. The amount of fluid taken, the action of the cutaneous surface, the chemical constituency of the urine, and the influences of mental emotions, exercise an action in modifying the frequency of urination within the limits of health. But, as already indicated in the preceding text, the normal act of urination commonly takes place once in five or six hours during the twenty-four, being less frequent at night. When, however, the healthy habit-limit of the individual is frequently or continuously encroached upon, either by the effect of avoidable or unavoidable causes, then abnormal urination ensues.

\section{ABNORMAL URINATION.}

Abnormal urination, or micturition, is modified as follows: 1 , as to the length of time between the acts; 2 , as to the length of time associated with the act; 3 , as to the effort necessary to the performance of the act; 4 , as to the unnatural sensations connected with the act; 5 , as to the physical appearance of the stream.

1. The modifieation of the length of time $\int$ Inereased frequency of micturition. between the acts gives rise toDiminished frequen
Retention of urine. Overflow of urine. 
Frequency.-The frequency of the act may be morbidly increased or diminished. The former state is dependent on any condition that incites the bladder to a more frequent expulsion of urine than normal, and it is commonly related to those influences that exaggerate the normal sensation which stimulates the organ to contraction. These influences may act directly or through reflex channels. Stone, gravel, foreign bodies, and morbid growths in the bladder, stimulate it to contraction directly, or by reason of the secondary inflammatory changes which their presence causes in the mucous and muscular structures of the organ. Inflammation of the lining membrane and muscular walls of the bladder, from whatever reason, also inflammation of the prostate, are common causes of this manifestation, and the more acute the cause the more urgent is the demand and the briefer the intervals between the acts. Irritability of the neck of the bladder following gonorrhœea, or dependent on the venous congestion of the neck and floor of the bladder in prostatic hypertrophy, or disease of the rectum, and vesical irritation from irrecognizable causes, lead to increased frequency of micturition. The presence in the bladder of blood, pus, and other fluids foreign to it, alone or mixed with urine, excite abnormal frequency of its contraction. In fact, it ean be safely asserted that "the bladder will retain unheeded a greater amount of normal urine than of any other fluid, both in health and disease" (Gouley). Incomplete emptying of the organ, caused by prostatic, urethral and other obstructions to the urinary flow, and by diminished power of the bladder walls, hastens the frequency of micturition by causing a rapid over-repletion of the viscus. Concentric hypertrophy of the bladder, with or withont cystitis, sexual excesses, and neuralgia of the neck and body of the bladder, unduly hasten the frequency of micturition. Diseases of the spinal cord and tumors of the medulla cause frequent micturition by a modification of the reflex center of the former, and the center of urinary excretion of the latter structure. The expulsive sensation of the bladder is incited also by an increase in the amount of urine from any cause, by changes in its specific gravity and chemical constituency, and by the presence of foreign matters and decomposing changes in it. An increased amount attends hysteria and such emotional causes as fear, apprehension, etc.; saccharine and insipid diabetes; it follows the administration of diuretics, also chronic interstitial nephritis and amyloid degeneration of the kidneys. All varieties of cystitis and of marked prostatic enlargement stimulate a more rapid excretion of urine than normal, and therefore increase its amount, and thus add correspondingly to the amount of suffering attending its expulsion. Changes in the specific gravity and chemical constituency of the urine go practically hand in hand together. Acute general inflammatory diseases, dyspepsia, saccharine diabetes, lithæmia, oxaluria, and certain 
medicines, as cantharides, add elements to the urine in a greater amount, or of an unnatural character, which so modify its nature as to increase the frequency of micturition by the influence of augmented vesical stimulation. Suppurative, tuberculous, and malignant diseases of the kidney often add pus, blood, and other products to the urine, which, like decomposing urine, cause frequent micturition by direct action of the lining membrane of the bladder itself. Certain other disorders increase the frequency of micturition through the reflex influence they exercise on the bladder, as renal calculi in the kidney pelvis, and especially so during their passage through the ureters into the bladder; also, diseases of the rectum, anus, perinæum, and spinal cord, possess a potent influence in this respect.

Diminished Frequency of Micturition.-This disorder is of less frequent occurrence than the former one. Other things being equal, any influence that lessens or destroys the normal sensation that stimulates urination, or impairs or delays the motor influences that accomplish the act, decreases its frequency; as, the blunted sensation attending the stupor of various diseases, especially typhoid and typhus fevers, and cerebral diseases; also, following the use of narcotics, alcoholics, etc. Persons of indolent habits urinate less frequently than do those of an active life. The recumbent posture, in health and disease, lessens the frequency of micturition, not only by lessening the amount of urine produced, but also by delaying and diminishing its pressure influence upon the neck of the bladder. The entrance to the bladder of a small amount of urine is attended by diminished frequency of micturition, for, if other things be equal, the slow collection of fluid in the bladder delays the sensation that stimulates expulsion. Still, in this instance, if the specific gravity be greatly increased, or if the urine contain blood, pus, or other foreign matter of undue stimulating nature, then the acts of micturition are not diminished in frequency, and may be much increased in this regard. Diminished excretion of urine attends kidney inflammations, and suggests the danger of impending suppression. Free perspiration, the ingestion of a small amount of fluid, the administration of medicines that delay metabolism, and profound lesion of the spinal cord, diminish the production of urine, and may therefore lessen the frequency of micturition.

Retention of Urine.-This disorder is distinguished by the presence of a morbid accumulation of urine in the bladder, which should remain only for a time, but which continues there until removed by the aid of mechanical agents, or such therapeutical measures as heat, narcotics, and anæsthetics. This condition is divisible into two classes-viz., complete and incomplete retention. Retention is dependent either on obstruction of the urinary channel or diminished expulsive force, or on both combined. The obstructing causes are located at the urethro-vesical orifice 
and in the urethra itself. The prostatic enlargements of old age, tuberculosis of the prostate, prostatic concretions, and tumors, are the common impediments at the urethro-vesical orifice. Acute congestion of a chronically enlarged prostate is a most fertile source of retention. Acute prostatitis of the parenchymatous or follicular forms, incited by gonorrhœa and other causes, together with abscess and cancer of the prostate, frequently close this opening. Pedunculated tumors of the bladder, stone, and foreign bodies in it; bony displacement from fracture and dislocation of the pubes; inflammation of the seminal vesicles; bloodclots, and the products of cystitis, not infrequently exercise a similar influence. Stricture is the most frequent form of urethral obstruction, and when acute congestion is added thereto, the canal is often promptly closed. Foreign bodies and polypi in the urethra may suddenly close the canal. Atresia, false passages, rupture, and other formed wounds of the urethra often effect its closure. Large and open valves within the canal are assigned as a cause of retention, especially if looking backward and modified by inflammatory changes. Diminished expulsive force is the result of lessened muscular and nervous action. Acute over-distention of the bladder so paralyzes its muscular coats that for some time thereafter retention ensues. Repeated over-distention, voluntary or otherwise, is frequently followed by atony of these coats; and, too, atony arises from unknown causes, and in both instances retention is common. Spasmodic contraction of the urethro-resical orifice and of the deep perineal muscles not infrequently cause temporary retention of urine. Prostatic enlargements and prostatic tumors may so interfere with the action of the bladder walls as to canse incomplete retention. Paralysis of the body of the bladder alone so cripples the act of expulsion that the normal neck of the organ offers an obstacle to the escape of urine, and retention thus ensues.

Modified nervous action leading to retention follows inhibition of the lumbar centel by a strong stimulus, eaused by surgical operations on the urinary organs, rectum, and contiguous structures. Severe injuries here, and the pain attending micturition, cause retention for the same reason. Obviously it should be remembered that retention commonly follows operations for hæmorrhoids, prolapse of the rectum, fistula-in-ano, etc. Retention of the urine is frequently a complication of severe injuries and surgical operations on distant parts of the body, and of acute over-distention of the bladder due to exhaustion of the lumbar center. Hysteria, mental emotions, and some forms of syphilitic and inflammatory disease of the brain; diseases that blunt the sensibilities, as typhus and typhoid ferers, paresis, etc., are quite frequently, indeed, complicated with urinary retention dependent on general disordered nervous influence. In the preceding enumeration of the causes of retention no differential dis- 
tinction is made between those causing complete and incomplete retention.

Complete retention is rare indeed, for it is unusual to have an obstruction or loss of power so profound as not to permit the escape of a few drops of urine from time to time. Complete retention must be discriminated from rupture of the bladder and from suppression and extravasation of urine.

While neither time nor propriety will permit me to enter into the details of a differential diagnosis, still I can not forego the opportunity of strongly impressing one fact in this connection-viz., the great importance of a rectal digital examination as the first means of determining the presence of an over-distended bladder.

Incomplete or partial retention is often mistaken for involuntary and frequent micturition, as it is characterized by frequent urinary acts, feeble streams, and perhaps dribbling of urine. However, the presence of residual urine in the bladder, as demonstrated by the introduction of a catheter, establishes conclusively the presence of retention.

Overflow of Urine.-This expression contemplates very properly the pre-existence of retention. Overflow of urine results from its damming in the bladder, the same as the damming of water elsewhere than in the body causes overflow; for, when the receptacle is filled and the supply continues, overflow necessarily follows in all instances. It is now apparent that the causes of overflow are the same as those of retention, except perhaps in cases of complete retention, and even then the difference is one of degree rather than of variety. In overflow the discharge is commonly continuous and dribbling, hence it is often mistaken for true incontinence. Sometimes brief interruptions of the flow happen, due to temporary impediments at the urethro-vesical opening or within the urethra itself. While these cases are rare, yet they may be mistaken for increased frequency of micturition. However, a thoughtful examination of the case will lead to prompt recognition of the true state of affairs.
2. Modification of the length of time as- (Irrepressible micturition (Gouley). sociated with the act gives rise to $\{$ Retent micturition.
Retarded micturition.
Interrupted micturition.

The time required for the act of physiological urination is brief and usually uneventful, being modified only by the amount of urine passed, and the length of time given to the purpose. Abnormally, the length of time between the demand for and the execution of the act may be so brief as to admit of no delay, the urine being voided in defiance of the will. This is known as-

Irrepressible Micturition.-Chiefly the bladder and its nervous supply, and incidentally the urine, are coneerned in the causation of this 
form of morbid micturition. In this disorder the normal urination sensation is so exalted as to cause the motor impulses of the act to be irrepressible. While cystitis itself is the chief cause referable to the bladder, still, the exciting causes of the cystitis frequently add the direct and baneful influences peculiar to their nature to the former infliction. Stone, prostatic enlargement, and morbid growths are striking and common examples of the exciting causes. Acute cystitis, with or without polyuria, often causes this disorder. Catheterism of an acutely inflamed bladder produces irrepressible micturition, and therefore should be avoided when possible. Over-distention, due to improvidence or bad judgment on the part of the patient, or to carelessness of the physician, may provoke its occurrence.

The nervous element of this disorder is frequently illustrated by the catheterism of neurotic patients; also by the so-called incontinence of children. In the latter it is thought to depend on inordinate and uncontrollable contraction of the bladder, hence denominated chorea of the bladder by some authors.

Urgent Micturition. - In urgent micturition there exists a strong yet controllable desire to urinate. The term is a relative one, and represents a lesser degree of disorder than the irrepressible variety, as well as an exaggeration of the normal desire to urinate. It is a much more frequent manifestation in morbid conditions of the bladder and nervous system than is imperative micturition. It happens, too, from causes that are foreign to any established disease whatever, but are incident to mental emotions, preoccupation, suggestive sounds, ideas, etc. Inasmuch as urgent micturition is akin to the irrepressible form, it is not likely to be due to dissimilar canses, but rather to similar ones acting in a less emphatic manner. It is characteristic, therefore, of all forms of cystitis, prostatic enlargement, and inflammation, especially with instrumental interference. Inflammation and irritability of the urethra and hypera sthesia of the external genitals cause it. In a word, the step from increased frequency of micturition to urgency of the act is often a brief one, for the causes of the former are provocative of the latter, especially when the sensation exciting the latter is exaggerated by a greater diseased action, or by inattention, heedlessness, or inability to meet the requirement of increased frequency on the part of the patient. Urgent micturition is common in many cases in which disease of the bladder and urethra and their appendages acts no special part, as in polyuria, irritating urine, moderate over-distention of the bladder, due to neglect and preoccupation; mental suggestions, as the sound of falling water; mental emotions, as apprehension, fright, etc. It is proper to say, however, that the diuretic effects of mental emotions add much, indeed, to the urgency of the act. Urgent calls to micturate occur frequently in rupture of the bladder. 
Retarded Micturition.-This signifies an abnormal increase in the length of time essential to the performance of the act of micturition. The retardation may depend (1) on delay in "starting the stream"; (2) on slowness of emptying the bladder; (3) on a combination of these influences. This variety of micturition does not contemplate the presence of a degree of interference of sufficient moment to call for a distinctly marked voluntary effort to overcome the obstruction influence, as a decided effort is a part of difficuit and obstructed micturition rather than of the retarded variety. The approach of the retarded form of micturition is so stealthy, especially when it is dependent on a chronic cause, as to escape for a time the notice of the patient himself. Actually its presence may be first appreciated only when the time permitted for a special performance of the act fails to completely accomplish the purpose. Not infrequently a patient is reminded of the previous existence of retarded or delayed micturition in his own case when he is brought suddenly face to face with severe obstruction to the act, caused by the influence of some indiscretion he has recently committed. It follows, therefore, that many of the causes of retarded micturition will, in becoming more pronounced, or when supplemented by other agencies, lead more or less promptly to difficult and obstructed micturition. Retardation from delay in "starting the stream " arises from slight obstruction in the urinary canal, and also from diminution or delay in the action of the expulsion-forces of the bladder. Enlargements, tumors, concretions, and abscess of the prostate cause this disorder in a more or less appreciable degree during the course of their development. Urethral stenosis from stricture, and from the external pressure of periurethral abscess and tumors, exhibit it; also, obstructions from foreign bodies, concretions, and blood-clots in the urethra and urethro-vesical orifice, exercise a similar influence. The presence in the bladder of a small stone often retards the escape of urine at the outset; usually, however, its influence is noticed more frequently near the termination of the act, as then the stone is pressed more directly against the urethro-vesical orifice. Delay in "starting the stream " and emptying the bladder are jointly associated with atony of the bladder, after over-distention from voluntary and involuntary causes. It happens with diminished vesical power arising from special nerve-lesions, independent of genito-urinary disease ; with mental emotions, as shame, etc., seen during the efforts of micturition before a class of students; with blunted sensibilities from the effects of shock, narcotics, and asthenic diseases. The congestive swelling at the urethro-vesical orifice which is incident to the hours of sleep and the horizontal posture, causes retarded micturition on arising in the morning. However, while retardation disappears during the day, still it may be repeated again from time to time on arising. This form of retardation is not dependent on established struct- 
ural changes of the bladder, prostate, or urethra. Retardation of the emptying of the bladder, due alone to diminished capacity in the urethral canal, follows urethral stenosis from all causes, also urethritis and urethral inelasticity caused by gonorrhœa, and neuralgia, and congestive swelling of the urethro-vesical opening.

Interrupted Micturition.-The stream of normal micturition is a free and continuous one until near the completion of the act; then the spasmodic efforts attending its conclusion cause the urine to be discharged in jets, either with or without a complete interruption of the flow. The interruption of the stream in this disorder is usually transitory, yet, it may soon become permanent, and quickly lead to retention of urine. The causes of interrupted micturition relate to the bladder and the urethra. The mechanism of these causes, in a large majority of instances, is like that of a valve which, when thrown by the force of a stream into or against a canal or opening, causes its complete closure, until a change in its position, or a modification of the forces directed against it, removes the obstruction for the time being, and thus permits the resumption of the flow. Stone, blood-clots, pus, stringy mucus, pedunculated growths, foreign bodies, etc., act in this manner when forced against the urethro-vesical orifice. Over-distention of the bladder may be attended with interrupted micturition, which in this instance is probably due to the improvement of the bladder tone, which results from the interruption of the efforts to expel its contents. A movable growth at the neck of the prostate, and the presence of obstructing valves in the urethra, cause this modification of urination. Superadded congestions at the seat of disease, especially of inflamed prostate, and urethral stenosis, cause matutinal interference with the stream, in the following manner, it is thought: In the morning, when the bladder is over-repleted, the patient strains with beginning micturition, and thus engorges the vessels still further, which engorgement is often sufficient to close the already narrowed canal, and the stream is arrested. A few minutes after this, however, if the patient makes a further attempt, a small amount of urine is passed. Perhaps this is repeated several times, until finally the bladder is emptied by installments. This cause of interrupted micturition often leads promptly to difficult micturition.

3. Modifieation of the effort necessary for $r$ the performance of the act leads to- $\left\{\begin{array}{l}\text { Difficult micturition. } \\ \text { Obstructed micturition. } \\ \text { Incontinence of urine. } \\ \text { False incontinence. } \\ \text { Involuntary micturition. } \\ \text { Unconscious micturition. }\end{array}\right.$

Normally, no effort is necessary for the performance of urination, except the physiological effort attending the completion of the act, which has been described already. 
Difficult Micturition.-This form is commonly associated with the retarded variety of micturition, and often, though less frequently, with the painful form. In brief, anything that weakens the forces causing evacuation of the bladder, and anything that obstructs the flow of urine, is apt to cause difficult micturition. When the difficulty is constant at the beginning of the act, it is an indication of urethro-vesical contrac tion and of prostatic obstruction. When it occurs constantly at the close of the act, it is, in a general way, indicative of stone in the bladder, and also of the stone's close proximity to the urethral orifice. Difficult micturition may be defined, therefore, as a form of micturition that calls for greater efforts of expulsion than does the retarded variety, or the act is made difficult by the pain and apprehension associated with it. Prostatic enlargements and prostatic tumors, urethral obstruction of all kinds-stone, blood-clots, and foreign bodies in the bladder-are established causes of difficult micturition. Atony and paralysis of the bladder from special nervous lesions and general neurosis are causal connections of this variety of micturition. Acute inflammation of the urethra and of the prepuce, when complicated with phimosis; chancroid of the glans or foreskin with a contracted preputial orifice, cause marked retardation, and frequently difficult micturition. Since the nature of the causes of retarded and difficult micturition are substantially similar, the more infrequent causes of the latter will not be repeated here, as they are placed already under the former title.

Obstructed Micturition. - In this variety of micturition the greater effort necessary to expel the urine depends on the presence of obstacles in the urethral canal and at the urethral orifice. These obstacles are similar in nature and situation to those that cause retarded and difficult micturition, and therefore the reader is referred to those titles for further information in this regard. As heretofore stated, the degree of hindrance is greater in the obstructed than in the retarded form of micturition. It is not practical, however, to make a distinction, as to degree of obstruction between the difficult and obstructed varieties of micturition, and therefore these terms are often used interchangeably in connection with impedimentary interference with urinary discharge. After this, these expressions diverge somewhat in their application, for, while obstructed micturition signifies always a greater or less difficulty in emptying the bladder on account of obstruction to the flow, yet difficult micturition happens without the presence of any such appreciable lesion. As an apt illustration of the latter assertion, the difficulty of voiding urine in the presence of real or prospective pain, caused by the act, is cited.

Incontinence of Urine.-This expression signifies the inability of the bladder to restrain the escape of its normal contents. As commonly understood in this disorder, the urine escapes as soon as it reaches the 
bladder, and the small quantity that may be retained in the bladder by the force of gravity, or by the opposition offered to its outflow by the curves of the urethra, does not conflict with the above definition.

Incontinence is commonly though unwisely divided into two varieties, viz., true and false incontinence. The condition of true incontinence is accepted as similar to that of incontinence, and is therefore properly defined above under the title of the latter expression. False incontinence as employed indicates the same modifications of urination as those happening in involuntary and unconscious micturition and overflow, with which expressions it is often interchangeably employed. The term "false incontinence" is objectionable, not only on account of its nosological inaccuracy, but also by reason of the confusion it causes in many minds as to its exact application. If incontinence be present at all in a case, then, indeed, the conditions causing it must conform to the definition applied to that term, otherwise incontinence can not exist. Moreover, the conditions assigned as causes of false incontinence and the phenomenon attending this act are such as permit of their proper and ready classification under other common and simple titles. While the term false incontinence will not be excluded from consideration, still, the inconsistent and unnecessary use of the term will appear as the assigned causes and the manifestations of this disorder are placed before the reader. Incontinence depends on any modification of the urinary bladder which permits the early, continuous, and uninterrupted flow of urine from it, irrespective of the patient's will. In fact, the urinary bladder becomes a passive part of the urinary canal, owing to the loss of power to retain its contents, which, when favored by gravity, escape as fast as received within the organ. This variety of incontinence is caused by unsymmetrical development or hypertrophy of the prostate, which prevents closure of the sphincter vesicæ, and permits the urine to dribble away at once on entering the bladder. A degree of concentric hypertrophy of the bladder that will not permit its distention, and for this reason all the urine above a few drachms must flow away at once, is incorrectly regarded by some as incontinence. And, too, this illustration seems similar to frequent and involuntary micturition, but there is this difference between them : in concentric hypertrophy of the bladder, the patient not only urinates without willing it (involuntary micturition), but he must urinate in spite of the volition not to do so (irrepressible micturition); therefore, this is not a proper example of incontinence. Paralysis of the sphincter vesicæ and the sphincter muscles of the urethra, with or without loss of power of the detrusor urinæ muscle, dependent on a lesion in the course of the nerves themselves, or of the nervous center, malformation of the bladder, exstrophy, etc., and of the urethra, as the opening of the ureters into the urethral canal, especially near the external meatus, will cause incontinence. 
The last abncrmity excludes the bladder from the urinary tract, and, although the symptoms are typical of incontinence, still the conditions do not conform to the definition given of incontinence. Fistulous communications between the bladder and rectum; relaxation of the sphincter vesicæ in hysteria, and perhaps in grave disease, as typhus and typhoid fevers, cause incontinence. It is well to be reminded of the fact at this time that paralysis of the body of the bladder alone causes retention of urine; and that paralysis of the neck of the organ only causes incontinence and not retention.

So-called False Incontinence.-In this form of disorder the contents of the bladder are wholly or partially discharged, owing to the loss of control of the expulsion forces, during a conscious (involuntary micturition-overflow) or unconscious state (unconscious micturition).

Nocturnal incontinence of children is a good illustration of unconscious micturition, and the causes provoking it may be of either a direct or reflex nature, such as: fissure of the neck of the bladder and vesical calculus; increased irritability of the bladder from unknown causes; increased quantity of urine, as in diabetes, nephritis, and with increased ingestion of water; irritant nature of the urine, from undue acidity, hyperalkalinity, and medical agents ; anal irritation, due to pin-worms, fissure, eczema, etc.; hyperæsthetic state of the external genitals dependent on stricture, phimosis, balanitis, etc.; and the psychical influence of dream impression -e. g., a child dreams he has found a suitable place in which to urinate.

General debility, spinal disease, injuries and diseases of the spinal cord, diseases of the vesical nervous supply, and acute febrile diseases are also among the many common atonic causes of this variety of unconscious micturition.

Involuntary micturition in children, distinctive of chorea of the bladder, is a source of great annoyance, not only by reason of the uninviting catastrophe it inflicts, but also on account of the uncertainty as to the time of infliction. In these cases, though the urine is properly retained for a while, still, with scant warning often, it may be expelled at any moment with a gush. Varions theories are advanced to account for this phenomenon in children, among which may be mentioned that of Oberländer, who believes it is only due to reflex irritation in the urethral and anal openings. Fauboren says the cause is insufficiency of the sphincter vesicæ, which permits a little urine to enter the upper portion of the urethra, and its presence there causes a further performance of the act by the excitation of reflex contraction. Enuresis in these cases is attributed by some to a lack of power of retention and enfeeblement of the voluntary power of the sphincters at the neck of the bladder and commencement of the urethra. In addition to this particular variety of involuntary micturition, many of those conditions recognized as potent 
in the production of unconscious micturition are not infrequently associated with the involuntary act in a causative sense.

Involuntary Micturition.-In adults this arises from both hyperæsthesia and anæsthesia of the mucous membrane of the bladder and urethra. The former condition occurs not infrequently in those who suffer great hardships and deprivation, as noted during the French campaign of Moscow. The presence of an irritable bladder, due to the common causes of this condition already mentioned, gives rise to involuntary micturition also. It is not difficult to understand the influence of a hyperæsthetic state of the bladder mucous membrane on the act of urination, since the irritability incident to the hyperæsthesia excites expulsive efforts that overcome that sphincteric control, and involuntary or irrepressible micturition ensues. Skene, in speaking of micturition of the anæsthetic state, explains it thus: "The stimulation is sufficient to excite contraction, but the sensation is not acute enough to weaken it." The involuntary micturition of those afflicted with typhus and typhoid fevers, and of other grave asthenic states, properly exemplify this phase of the disorder.

Over-distention of the bladder is a most fertile cause of so-called false incontinence (overflow), especially in old men who are affected with prostatic obstruction; and it may follow over-distention from any cause, irrespective of the age of the patient. It is instructive to note the fact at this time that overflow and so-called false incontinence can be employed synonymously with perfect propriety in connection with over-distention of the bladder from obstructive causes. To avoid repetition it may be stated that the causes of unconscious or involuntary micturition in children act as causative agents of these disorders in adults as well. However, acute febrile diseases, general debility, fright, vesical calculus, cystitis, and the effects of spinal traumatism, are more pronounced causes in this regard in adults than in children. Senility often leads to incontinence, regardless of the prostatic obstruction so frequently connected with it at this period of life.

Retention is classified also under this division of the general arrangement, for the obvious reason that the majority of its associated disorders here are of such a nature as to suggest at once the probability of the occurrence of retention, when the causes of the associated disorders are more fully advanced in an unhindered course.

$$
\text { 4. The unusual sensations attending } \begin{gathered}
\text { Time of sensation, }\left\{\begin{array}{l}
\text { Before micturition. } \\
\text { as, } \\
\text { During micturition. } \\
\text { After micturition. }
\end{array}\right. \\
\begin{array}{c}
\text { Seat of sensation } \\
\text { (direct and re- } \\
\text { flex), as, }
\end{array}
\end{gathered}
$$


Painful Micturition. - In normal urination no annoying sensations are in any way connected with the act. In fact, a pleasurable feeling only attends the evacuation of the bladder and the flow of urine through the urethra. However, in disease of the bladder, urethra, and of their appendages, and also attending abnormities of urinary composition, painful sensations, varying in nature, degree, situation, and relationship with the act, often take place. As to its nature, the sensation may be a cutting, burning, or itching one, etc., as characterized by the appreciation of the patient, and depending on the variety, situation, and acuteness of the disease. In degree it varies from that of an annoying sensation only to one of pain and intense suffering even, being controlled in this respect largely by the acuteness of the diseased process; for, as a rule, the acuter the' process, the severer is the pain arising from it. The situations of these abnormal sensations are commonly located at the seat of the disease, but this fact must be accepted in a general sense only, as reflex sensations frequently happen at a considerable distance from the lesion that causes them, and where no other evidence of disease exists. The relationship of abnormal sensations to the acts of micturition should be carefully noted, since the occurrence before, during, or following the act is very important for the establishment of a correct diagnosis.

Discomfort and pain of the urinary track before micturition frequently arise from the local action of ultra-irritating urine; from increased sensibility of the prostate and mucous membrane of the bladder from various causes, plus the direct pain, peculiar to the cause itself. The mechanism of the production of painful micturition is a simple one, being due to the contact of slowly accumulating fluid with the abnormally sensitive structure within the bladder, and to the contact of irritating agents with these structures when normal. The urine is made irritating by those diseases and conditions which destroy the proper equilibrium between its fluid and solid constituents. An abnormal increase in the solids or diminution of the fluids leads to similar results in this respect. Acute inflammatory diseases; profuse perspiration; small ingestion of fluid ; frequent copious liquid alvine discharges, oxaluria, pyuria, lithuria, alcoholics, certain medicines, as cantharides and turpentine; vesicles of kidney hydatids, and inordinate alkalinity and acidity, cause irritating urine. Both acute and chronic cystitis and prostatitis; prostatic hypertrophy and prostatic tumors, cancer, etc.; sympexious prostate; inflam. mation of the seminal vesicles; gravel; circumscribed and general vesical irritation; over-distention; contraction with hypertrophy and ulceration of the bladder walls, etc., cause more or less pain and discomfort before micturition. Pyonephrosis, calculous, and tuberculous kidney are sometimes indicated in their earlier stages by pain before and during micturition. The acute diseased processes of the bladder, prostate, and urethra 
especially, add their own peculiar pain to that caused by the contact of urine with their diseased surfaces during the act of micturition.

Pain during micturition depends on diseased action within the bladder and within the urinary canal. Those diseased conditions of the bladder just enumerated as causing pain before the act frequently cause more or less pain and discomfort during the expulsion of the fluid, and the acute varieties of these diseases are more pronounced in this respect than are the chronic. The contraction of the bladder in the presence of acute inflammation of its lining membrane and of the prostate causes pain of the bladder, due to the contraction itself, and of the prostate from the increased pressure attending the contraction. The pain caused by these acute conditions, by the presence of stone and foreign bodies in the bladder, and of ulcer and fissure of the neck of the bladder, are increased during the completion of the act, as at this time they are brought more closely within the grasp of the bladder, and are therefore subjected to a more direct pressure than when urine intervenes between them and the bladder wall. Neuralgia of the neck of the bladder is of fickle presence and severity, and while it may happen at any stage of the act, yet it most frequently attends that of expulsion. Pain in the urinary canal during the discharge of urine attends all varieties of urethritis, circumscribed and general; also urethral chancre and chancroid and simple ulceration, stricture of the urethra, balanitis, and occasionally phimosis cause it. Changes in the chemical constituency of the urine, and the presence of irritating substances in it, as already enumerated, frequently cause more or less pain during the act, and may be followed by pain and disagreeable sensations after its completion. This is especially true in the presence of increased sensibility and inflammatory and ulcerative processes of the canal.

Pain diminished after micturition indicates that contact of urine with the bladder and urethra, or distention of the bladder, was the probable cause of the pain. Pain at the time of micturition may be at the seat of the disease causing it, or be referred to a distant parte. g., pain at the end of the penis may be referred there from the neck of the bladder; pains radiating down the thigh or occurring in the testicles or groin are often referred from the pelvis of the kidney or the ureter. Pains in the sole of the foot, calf of the leg, and the thigh are a part of the history of urethral stricture. Usually prostatic pains are felt in the perinæum and lower portion of the rectum; bladder pains over the pubis; kidney pains in the loins, etc. While some of the illustrations here given are not exactly in line with the subject under consideration, still their mention now will serve to emphasize the importance of those manifestations in the actual as well as in a differential diagnostic sense. Many of the examples just cited are common ones, and there- 
fore serve the more emphatically to impress the diagnostic importance of a detailed consideration of both the local and reflected pains of disordered micturition in connection with the diseases themselves. The diagnostic significance of painful micturition in various abnormal conditions can be best considered for practical purposes in the following manner:

(1) The disease causing it; (2) the seat and kind of pain commonly produced by the disease; (3) the relation of the pain to the act of micturition.

Acute Cystitis. - In this disease pain is located most frequently in the bladder itself, being often more severe at the neck than elsewhere. The severity of the pain is in direct proportion to the process that causes it, being much more pronounced in acute than chronic diseases of the membrane. Abnormally irritating urine, from chemical decomposition and other causes, adds to the intensity of the suffering in all phases of the disease. The relationship of the occurrence of pain with the entrance of urine to the bladder is governed by the situation of the disease and the site of greatest sensibility, as modified by the position of the patient, and the degree of distention of the organ. If the inflammation be limited to the neck and base of the bladder and the patient be erect, pain follows quickly the entrance of urine to the organ, since it is then brought in contact at once with a diseased surface. However, if the position of the patient be so changed as to delay or limit the degree of urinary contact with the diseased surfaces, then pain is lessened, or it disappears until sufficient accumulation ensues to encroach on the seat of disease, and then the pain returns or increases, and is relieved only by prompt micturition. Distention of the bladder increases the pain if the disease be general, and produces it when sufficient urine collects to cause impingement on circumscribed disease of the membrane. If the inflammation be general and of equal degree and uncomplicated, pain comes promptly, increases with uniform intensity, and, although augmented during micturition, is relieved or greatly lessened by the act. Also in this disease pains are often felt, conjointly or separately, along the urethra, in the perinæum and rectum, above the pubis, at the end of the penis, and radiating down the thigh, and, as a rule, the acuter the diseased process and the severer the bladder pain, the more constant and pronounced are these reflex sensations. As to the kind of pain, it is variously characterized in this disease, and is apparently modified in this respect by its situation. The local pain in the bladder is sharp, pricking, smarting, and burning in character, and it is entirely relieved or greatly lessened by the emptying of the organ. Not infrequently a straining pain (tenesmus) follows micturition, especially if the neck of the bladder be much involved by disease. The referred pains in the perinæum, above the pubis, in the 
rectum, at the end of the penis, and radiating down the thigh, are classed as heavy burning pains. As may be readily inferred, the painful manifestations of chronic cystitis are less vigorous - the pain being aching and dull in character-and they do not lead so soon to uncontrollable results. Still, a general dependent connection of the phenomenon of painful micturition in acute cystitis can be easily distinguished in the chronic form.

Neuralgia of the Neck of the Bladder.-In this disease, while the pain is always located at the neck of the organ, still, it not infrequently shoots from there into the urethra, rectum, and perinæum. It exhibits the same uncertainty, as to the time of occurrence, that distinguishes similar pains elsewhere in the body. It is sharp and darting in character, and although it happens during the intervals of micturition, still it presents itself most frequently during the act.

Fissure and Ulcer of the Neck of the Bladder.-These conditions, especially the former, are more frequently present than they are commonly considered to be. The fissure not infrequently extends into the upper end of the urethra. The pain attending them is located at the neck of the bladder, is of a burning character, more or less continual, and attended by the feeling of an unvaried desire to micturate. Much straining or tenesmus is present, and after micturition the pain and burning are most intense.

Stone and Gravel in the Bladder.-Each of these agents causes painful micturition by contact with the bladder wall; the former, as a rule, causing the greater disturbance.

In stone the pain is dull and commonly located in the bladder during the intervals of micturition, if it be present at all. However, even then dull reflex pains may be present along the urethra and behind the glans, or may radiate to the hips and thighs, the perinæum, rectum, sacrum, and above the pubes. Still, these painful manifestations during this time are modified by the position and activity of the patient. If pain be caused while standing, the horizontal posture often lessens or relieves it. If the patient be quiet, no pain may be present, irrespective of his position; but on walking, running, or riding, pain quickly occurs, and the intensity is proportioned directly to the degree of jarring and jolting he experiences. The pain with stone is caused not only by the presence of the stone itself, but likewise, and in great part often, by the cystitis which its presence excites. Pain with stone is usually present before, during, and after micturition, and especially are these facts true if cystitis have ensued. While in rare cases of stone no pain at all is present, yet practically it varies in degree from that of an exaggerated urinary sense to one of agonizing intensity. The dull pain before micturition is due to the contact of the accumulating urine with the inflamed surfaces 
caused by the stone, and also in a degree to the presence of the stone itself. The pain during the act is the severest of all, especially at its termination, for then the stone is spasmodically grasped by the sensitive bladder walls. During this time sharp and severe reflex pains invade the urethra and glans, and violent tenesmus often ensues, together with dull, severe, deep-seated pubic and perineal and rectal pains. If any be present, the pain following the act is the least of all, and is largely the result of the physical violence inflicted on the neck and walls of the bladder by the stone itself during the act of micturition.

Gravel in the bladder causes no distinctive pain in that organ before micturition, and is usually washed out by the escaping urine during the act. Therefore, the smarting, burning pain suggestive of the presence of gravel in the urine takes place in the urethra during the emptying of the bladder, and rarely remains but a short time thereafter.

Hydatid Cysts of the Kidney.-In this instance the pain and disturbance of micurition depend on the presence in the urine then in the bladder of the vesicles and fragments that have passed down from the growth above. The discharge of these agents does not take place rapidly in the majority of instances, therefore periodical attacks ensue at intervals of days and even of months. Their presence in the urine may cause retention with the dull and bursting pain of this disorder, followed by severe strangury with radiating burning pains of the urethra and glans penis. Evacuation of the bladder is followed by instant relief.

Acute Prostatitis.-The pains experienced in this disease differ somewhat according to its variety, and the differences are more a matter of degree than otherwise, their comparative relationship to the act of micturition being still quite pronounced. In the parenchymatous form the suffering arises from the disease itself, from the irritating effects of the urine on the diseased organ, and from the act of micturition. Heat and throbbing pain are present in the prostate, attended with dragging pain in the scrotum, and an aching sense of fullness in the perinæum and rectum. These pains are present at all times during the acute stage of the disease, and are increased often by the irritating effects of accumulated urine, and are always exaggerated by the act of urinary expulsion. Pains independent of these precede, attend, and follow the act of micturition. The burning, smarting pains before the act are due to urinary contact, and are located at the neck of the bladder and upper extremity of the urethra. As the urine increases in amount, the consequent pressure from it increases their severity, and often gives rise to similar shooting pains in the urethra, the glans, the perinæum, and scrotum. During micturition these painful expressions are increased in severity, and at its finish often become excruciating, being attended with violent tenesmús, severe reflex pains in the glans, and deep-seated subpubic pains, caused by the vigorous 
contraction of the bladder on the inflamed prostate itself. The phenomena of acute prostatitis and of stone in the bladder are similar during micturition, especially if cystitis be present with the latter. Following the act the pains incident to it quickly subside, leaving the patient to suffer only from those sensations peculiar to the disease alone, and with apprehension born of the memory of the past and sustained by the fear of prospective suffering. In other varieties of prostatitis the painful phenomena of micturition bear a similar relationship to the act as in the acute form, but are less severe in character and often incomplete in sequence.

Acute Inflammation of the Seminal Vesicles.-Burning pain and discomfort in this disease are present at the base of the bladder, and may be referred to the spermatic cord, glans penis, perinæum, and rectum. The accumulation of urine in the bladder increases the vigor of these sensations, and the act of its expulsion adds greatly to the suffering, being often attended by severe vesical and rectal tenesmus. However, marked relief follows at once the evacuation of the bladder.

Prostatic Hypertrophy of Old Age.-The degree of painful suffering in this disease depends on whether or not cystitis or other complications have taken place. If uncomplicated, then a dull pain or a sense of weight and fullness is present in the hypogastrium, rectum, and perinæum, and perhaps in the bladder as well. These sensations are constant exhibitions, and, as a rule, are not modified in any essential particular by the act of micturition. However, in some cases, as urine collects in the bladder, the sense of weight and pain increases, and the desire to micturate is soon incited, and during the performance of the act a burning pain occurs at the neck of the bladder and in the course of the urethra, followed by a dull pain behind the pubes, in the perinæum and rectum, and perhaps the glans itself, near the completion of the act. These disappear promptly after the cessation of vesical contraction and the emptying of the urethra. If the urine have undergone decomposition changes, then these latter exhibitions are more often present and better pronounced. If cystitis or stone be present with prostatic hypertrophy, then pain attends the accumulation of urine, and it is increased in proportion to the amount contained in the bladder, the grade of cystitis, and the size and roughness of the stone. The discharge of urine under these circumstances causes increased pain, referable to the neck of the bladder, urethra, and perhaps more distant parts, and, at the completion of the act, severe subpubic, perineal, urethral pains, and pain at the glans penis take place, attended not infrequently by tenesmus of greater or less degree. After micturition, in this as in preceding instances of prostatic involvement, the more distressing symptoms rapidly subside, leaving belind only such as belong to the diseased bladder and prostate during the intervals of micturition. 
Prostatic Cancer and Tumors of the Prostate and Bladder.-In prostatic cancer pain depends primarily on the cancer itself, and secondarily on obstruction to the flow of urine and the consequent cystitis induced by the presence of the cancer. The same may be said of other tumors of the prostate, and bladder tumors as well. But, inasmuch as these growths differ in no important particular from other forms of prostatic disease and cystitis, as regards the pain with the act of micturition, it seems useless to give to them a more detailed consideration in this connection.

Retention and Contracted Bladder.-In either of these conditions the pain is described as a splitting one, and it is referred properly to the bladder alone. The mechanism of its causation is similar in both instances, being dependent on painful distention of the bladder from overrepletion by urine. The painful micturition incident to a contracted bladder happens more frequently than that due to retention, for the obvious reason that, when a diminished capacity receives a normal supply, overdistention occurs much more quickly than when the capacity and supply are proportionate to each other. The pain in both of these conditions precedes micturition and is promptly relieved by it.

Urethral, Inflammatory, and Structural Changes.-In all varieties of urethral inflammations, whether general or eircumscribed, painful micturition ensues. Urethral ulcerations cause a similar exhibition, irrespective of their nature, extent, and location. Urethral strictures, likewise, often cause painful micturition. The degree of pain experienced during micturition in all varieties of urethral disease is proportionate, of course, to the sensibility of the diseased surfaces, and also, in a measure, to the amount of urethral distention attending the act. These pains are of a smarting, burning, or cutting nature in the great majority of instances. They are located in the urethra, and correspond to the extent and situation of the diseased surface of that canal. These pains are direct and are cansed by the contact of the passing urine as it escapes from the bladder. The pain is manifest, therefore, during the act of micturi. tion, and may be present for a short time thereafter. Balanitis, posthitis, and ulcerative changes within the foreskin cause pain during micturition, and this pain is increased in severity if these diseases be complicated by phimosis.

Irritating urine, dependent on decomposition, hyperalkalinity, and hyperacidity, and other chemical changes of it, causes painful micturition. The presence in the urine of pus, cancerous and hydatid products, and the effect on it of cantharides, turpentine, cubebs, etc., often produce a like result. The presence in the bladder of irritating urine begets discomfort and painful sensations there before micturition, and similar exhibitions often attend at the neek of the organ and in the urethra during its discharge, but thereafter they quickly subside. Tenesmus may attend and 
follow the act of micturition in many instances, especially after the use of the remedial agents just mentioned.

5. The character of the stream may be modified as to its-

$$
\begin{aligned}
\text { Force. } & \left\{\begin{array} { l } 
{ \text { Increased force. } } \\
{ \text { Diminished force. } }
\end{array} \left\{\begin{array}{l}
\text { Feeble stream. } \\
\text { Perpendicular stream. } \\
\text { Dribbling stream. } \\
\text { Slobbering stream. }
\end{array}\right.\right. \\
\text { Size. } & \left\{\begin{array}{l}
\text { Increased size of stream. } \\
\text { Diminished size of stream. }
\end{array}\right. \\
\text { Form. } & \left\{\begin{array}{l}
\text { Oval stream. } \\
\text { Flat stream. } \\
\text { Spiral stream. } \\
\text { Bifurcated stream. }
\end{array}\right. \\
\text { Direction. } & \left\{\begin{array}{l}
\text { Radiating stream. } \\
\text { Deflected stream. }
\end{array}\right.
\end{aligned}
$$

The characteristics of the normal stream during urination are not easily to be described. The periodical appearance of the stream forms such a very important though unappreciated part of one's every-day existence, that its normal aspects are recalled only when something unusual happens to it. It belongs really to the order of natural, every-day objects which, though noticed by us, their presence makes no distinct impression on the perceptive sense except something quite unusual attends them.

Force.-This element of urination depends, on the one hand, on the degree of energy exercised by the bladder and the abdominal muscles; and, on the other, it is controlled by the restraining conditions obtaining in the urethra. The degree of force is estimated by the distance to which the stream can be propelled.

The force is much increased by the powerful and uncontrollable contractions of the bladder in those conditions recognized as causing irrepressible micturition. The causes of urgent micturition and not infrequently those of the involuntary act increase the force of the stream, especially when no obstruction to the escape of urine obtains.

Diminished force is the result of indolent or weakened vesical contraction, and also of the presence of obstructing influences in the urinary canal. Diseases of the spinal cord, brain, and of the nerves supplying the bladder directly; also such asthenic diseases as typhus and typhoid fevers, and atonic states for other reasons, are suitable illustrations of the conditions producing diminished force of the urinary flow due to an enfeeblement of the propelling power. The obstructive influences of the flow that serve to weaken the force of the stream are present at the neck of the bladder in the urinary canal, and are often connected with the prepuce alone, especially in children. Stone in the bladder, hypertrophy, tumors, and enlargement of the prostate from all causes ; narrowing of the urethra from constrictions, growths, and obstructions, and phimosis, congenital and acquired, weaken the urinary flow. In a few 
words, the diseases that give rise to overflow and retention of urine, to retarded, difficult, interrupted, and obstructed micturition, are causative as well as of diminished force of the urinary discharge.

Size, Form, and Direction.-These characters of the stream can be advantageously considered together. If the conditions in the urethra be such that during micturition the meatus is fully distended by the stream, then to the distended meatus the stream will owe both its size and shape (oval). Urethral stenosis, urethral narrowing from any cause and at any point, will surely modify all these qualities, causing the stream to be flat, small, or twisted (spiral); if near the external meatus, curved (deflected) or divided into two streams (bifurcated), each propelled in different directions; or one driven forward and the other tumbling perpendicularly down (perpendicular); or into several streams, each with a different direction (radiating).

Continuity of the stream is an important quality for consideration. Normally the stream is full, oval, and vigorous, the size being increased when additional expulsive efforts are made. Toward the completion of the normal act the stream becomes flattened, owing to diminished force, and, too, it is flattened during disordered micturition, if diminished force be a characteristic feature of the disorder. With obstructive disease of the prostate the stream may be spasmodic, or the fluid be passed in a feeble, perpendicular, dribbling, slobbering manner. The abnormal conditions of certain portions of the urinary tract and their modifications of the stream are submitted to the attention of the reader as illustration of the mechanism of stream variation in connection with obstruction and paretic changes associated with urinary flow.

Prepuce.-In phimosis, if the preputial opening be smaller than the meatus, the stream is modified in size, shape, and sometimes in direction. The reasons for these changes are too obvious to require any further mention.

Meatus Externus.-Such changes here as epispadius, hypospadius, multiple urethral openings, or a contracted or deflected single opening, will exercise an evident influence on the size, shape, and direction of the stream.

Urethral Inflammation causes congestion and swelling of the mucous membrane of the urethra, and therefore occasions a change in the size of the stream, it being smaller; in the shape, it being flat, forked, or twisted, etc.; and often in direction and continuity being devious and attended by some dribbling at the end of the act.

Organic Stricture.-This formation influences the size of the stream, and for this reason the force, form, and direction of it also. If the diminution in size have been coming on gradually, the presence of increasing stricture is quite surely demonstrated. An inordinately small stream in a 
case usually points to one of three conditions, viz., contraction at the urethro-vesical orifice, urethral stenosis, or impaction of calculous matter in the urethra. In stricture the form of the stream may be twisted, forked, or radiating, as when divided into several streams. The direction of the stream may be upward, downward, etc., or two streams may be exhibited, one perpendicular, the other propelled. Impairment of the force of the stream happens, too, and this effects its continuity, causing the last fow drops to dribble away, because the expulsion-wave does not pass the stricture.

Prostate.-Hypertrophy modifies the force of the stream, causing it to be weak and slow. The lessened force diminishes the size of the stream, which, failing to distend the meatus, prevents the proper form at once. A bifurcated stream is not infrequently present in this connection. In hypertrophy the continuity of the stream is often changed, due at first to delay from congestive swelling, but later it may be interrupted or entirely stopped by the valve-like action or increased growth of the hypertrophied organ. This valve-like action may affect both the size and form of the stream, especially with straining, causing dribbling and slobbering of urine. In parenchymatous prostatitis and in prostatic and periprostatic abscess and prostatic cancer the stream becomes gradually small and forceless; if combined with vesical irritability, the continuity of the stream is modified by sudden and premature vesical contraction.

Neuralgia of the Vesical Neck.-If the bladder act without power, the force and size of the stream are diminished; but if it act spasmodically, with power, then these peculiarities are increased.

Spasmodic stricture of the urethra interrupts the continuity of the stream, it being normal between the spasms.

Incontinence, from whatever cause, modifies the continuity of the stream, and dribbling of urine ensues; but in the great majority of cases dribbling indicates overflow.

Bladder-In concentric hypertrophy impeded bladder contractions diminish the force of the stream, causing it to be feeble and slow.

Atonic Conditions. - In these conditions the force of the stream is lessened, and if they be complicated by causal obstruction, then the stream is weak and dribbling.

General Neuroses.-If these exist alone, then the stream is feebie and slow.

Vesical Paralysis.-When vesical paralysis is complete and limited to the body alone, dribbling of urine ensues as the result of retention with overflow. If incomplete, then the stream is feeble and slow and followed by dribbling. Limited retention may be present in these cases and cause increased frequency of micturition. If only the neck of the organ be paralyzed, then uninterrupted incontinence ensues. 
Cystitis and Trachelo-cystitis. - In these diseases the accompanying spasmodic and vigorous contractions of the bladder increase the force and size of the stream, change its form and direction, and may also affect its continuity.

Stone in the Bladder.-If the urethra be normal and the stone movable, the continuity of the stream is interrupted or enfeebled, but a change in the patient's position may restore the stream to its normal characteristics, if complications be not present. Foreign bodies in the bladder cause similar phenomenon as stone, when movable.

\section{DISORDERS OF MICTURITION TABULATED.}

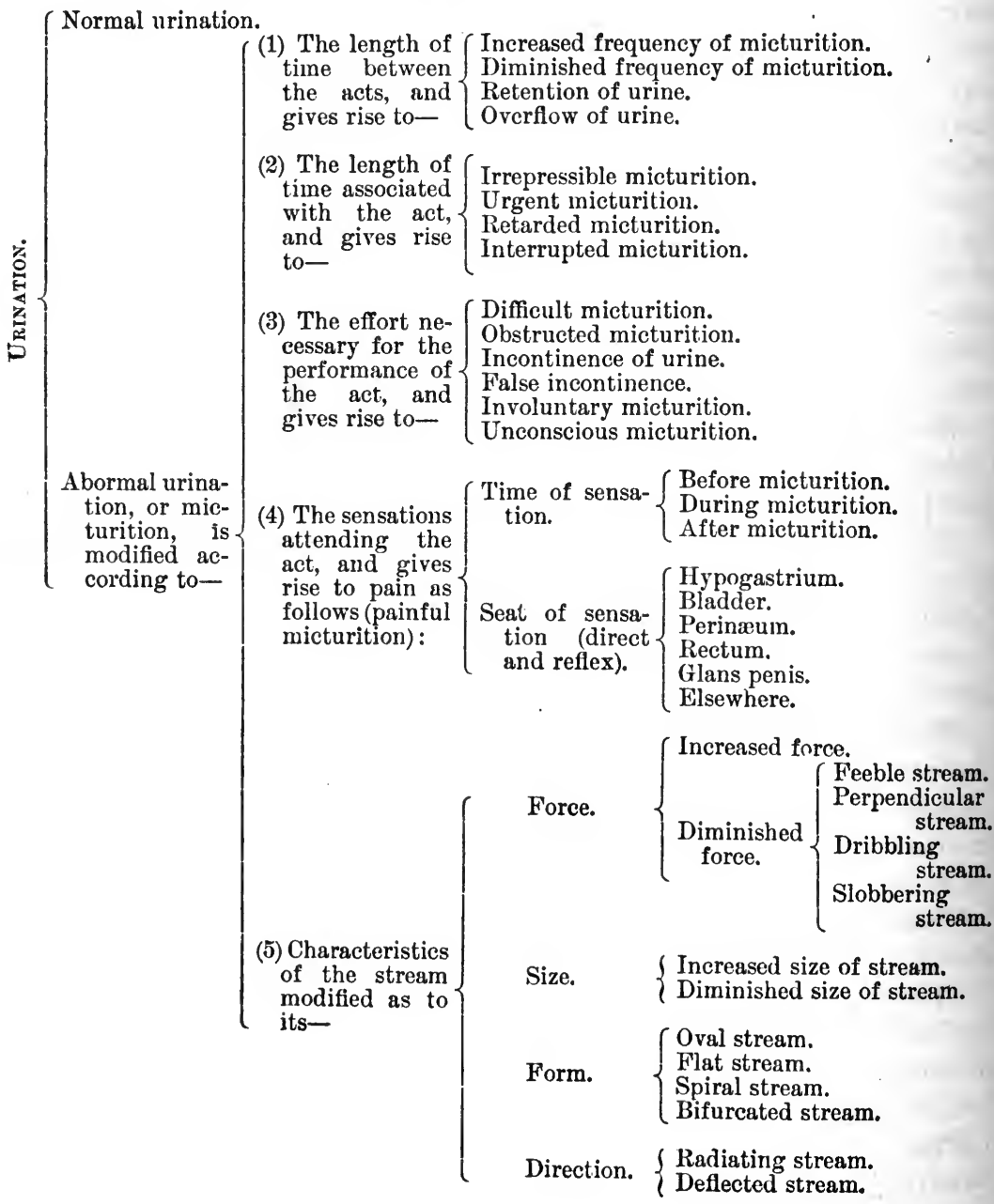




\title{
DIAGNOSTIC SIGNIFICANCE OF PATHOLOGICAL MODIFICATIONS IN THE URINE.
}

\author{
(INCLUDING THE MOST PRACTICAL METHODS OF URINE ANALYSIS.)
}

\section{Bx EUGENE FULLER, M. D.}

The above-entitled article, owing to the space allotted, can not be exhaustive in character ; consequently, the attempt will be made to bring out as clearly as possible only the points which are apt to confront the busy practitioner, and the methods by which he can deal with them. It has seemed well to classify the subject as follows :

1. General characteristics of the urine.

2. Chemical constituents of the urine, subdivided as follows :

$a$. The normal chemical constituents.

b. Those that are normal only when present in very small amounts, abnormal when abundant.

c. The abnormal chemical constituents.

3. Organized sediments.

4. Miscellany.

\section{GENERAL CHARACTERISTICS.}

These include color, odor, transparency, chemical reaction, volume, and specific gravity.

Color.-It is customary to classify urine with reference to color as pale (i. e., colorless to straw color), normal (i. e., amber yellow), or high color (i. e., reddish yellow to mahogany). Other colors are abnormal. The coloring matter of the urine and bile taken together are supposed to represent approximately the amount of decomposition of the red bloodcorpuscles. Just what the coloring matters are is still a subject of some discussion among chemists. An occasional specimen of pale urine in a given case is of no moment. The continuous voiding of such, however, would lead one to suspect either one of the conditions where a great volume of urine is habitually secreted, thus reducing the normal amount of color by dilution, as in diabetes mellitus or insipidus, contracted kidney, etc., or some condition of anæmia where there are few red bloodcorpuscles to undergo the change. 
High-colored urine is, vice versa, indicative of concentration. Such a condition, which is of moment only when continuous, exists with individuals who drink little, and in eases where the seeretion of the kidney is interfered with, as in fever, parenchymatous nephritis, surgical suppression, and in some neuroses.

Dark-colored urines generally indicate some abnormal pigment, such as blood or bile; more rarely the action of some drug, usually carbolic acid or one of its derivatives; sometimes rhubarb or senna. Blood in urine soon loses its red color and becomes dark. These dark shades vary all the way from a smoky tinge, where there is little present, to an inky black, where there is mueh.

In the great majority of instances where the urine is so colored, the red blood-corpuscles are present and contain all the pigment. There is, however, a rare condition described as hæmoglobinuria, where the urine is discolored by the blood-pigment only, there being present no eorpuscles. Such a condition sometimes exists in diseases where there is a great dissolution of the red corpuscles, as in scurvy, typhus, and malignant malarial fevers, and after the inhalation of arseniureted hydrogen gas. The microscope confirms the diagnosis only in the first class of cases. In the cases where the pigment exists without the eorpuscles, on adding a very little acetic acid and boiling, a brownish coagulum forms. The spectroscopic test is, however, for such conditions more exact. Bile in the urine gives a dark-yellow or brownish color, usually with a greenish tinge. It is always found where there is jaundice, and occasionally in cases of phosphorus-poisoning. A rough and valuable test to prove the presence of bile is to shake a specimen of the urine in a partially filled bottle, the greenish tinge to the bubbles being very characteristic. Another good demonstration is to spread out a drop of the suspected urine on a white porcelain surface, and into the middle of this, by means of a glass rod, to place a little concentrated nitric acid. There will be promptly, in ease bile is present, a play of colors, the yellow changing first to green, then to blue, violet, ruby red, and back to yellow again.

Some observers have called attention to the fact that in cases of melanotic cancer, frequently the urine on standing will turn rapidly brown, or even black (Neubauer and Vogel, Analysis of the Urine, translated by Cutler, page 368).

Odor.-Little information is to be gained from the odor of freshly secreted urine aside from suspecting or detecting fermentative changes. Many drugs, such as sandal-wood, asparagus, turpentine, etc., lend their characteristic odors to the urine.

Transparency.-Urine, when voided, is normally transparent, with, on standing a few minutes, a little cloud of mucus near the middle of the vessel. If the urine freshly passed is turbid and cloudy to any marked 
degree, then we commonly suspect one of two causes, either a deposit of earthy phosphates due to an allaline condition of the urine, or free pus. (Chiluria, a rare condition, will be considered later under another heading.) A few drops of acetic acid will dissolve the phosphates and render the urine clear in the first instance, thus deciding the question. If, however, the urine has had a chance to cool slightly after being passed, amorphous urates may be thrown down, causing at times great opacity. If this urine is now heated again in a water-bath, it speedily becomes clear, such not being the case in the other two conditions. A water-bath is recommended rather than the flame, for by heating with a flame albumen, if present, may be thrown down, and thus obscure the test. There is another cause for turbidity in freshly voided urine which occasionally exists largely by itself, but which is usually associated with pus, namely, bacteria. Here the turbidity is apt to be rather slight. There is no sparkle to the urine, but a slightly ground-glass appearance, and on standing there is no tendency for the opacity to settle, leaving a transparent zone near the top. The microscope demonstrates this condition. It is seen in cases where bacteria have been introduced through instrumentation. Some urines on voiding show a great general turbidity due to pus. It will be found after they liave remained standing a few hours in a glass that all the pus has settled at the bottom in a sharply defined, not adhesive, solid mass, leaving the supernatant fluid perfectly clear. This condition is, in a general way, indicative of pus coming from the kidney region.

Chemical Reaction. - Normal urine is moderately acid. A urine may be temporarily neutral or even slightly alkaline, and not be pathological. Just what causes the acid reaction of normal urine is still rather a mooted question among chemists. It is probably only due in small measure to the presence of free acids, but chiefly to the acid salts. To test the acid reaction of urine a piece of blue litmus-paper is dipped partially in, the part immersed rapidly turning red in case the fluid is acid. If it is alkaline or neutral, there is no change. Red litmus-paper to test the question between a neutral and an alkaline reaction is rarely necessary, for one can readily detect alkalinity from decomposition by the odor, and normal alkalinity is sufficiently demonstrated for practical purposes by the failure of the fluid to change the color of the blue paper. One of the functions of the kidneys is to separate the chemically acid salts which occur in the urine from the alkaline blood. When, however, the blood is rendered excessively alkaline by medicines or herbivorous diet, enough alkaline material passes through the kidneys to neutralize or more than neutralize the natural acidity of the urine. This explains how it is possible for a normal urine to be alkaline.

Any concentrated urine is likely to be very acid, and, conversely, any 
diluted urine is apt to be neutral, simply from the abundance or absence of the solid elements which go to make up the reaction.

Decomposed urine is alkaline. Certain fermentation germs acting on urea cause its decomposition into carbonate of ammonium. When this decomposition takes place within the body, the urine becomes putrid and alkaline before being voided. In such an instance the germs of fermentation have been introduced mechanically from without.

All urines for examination should be voided in clear vessels. If a number of such urines are watched and examined from time to time, it will be found that alkaline decomposition occurs much earlier in some than in others. As a rule, the urine of females decomposes earlier than that of males, probably due to the admixture of vaginal material. One class of urine, it is often found, will keep for many days, frequently for a week, and still remain strongly acid. Such a condition of affairs, there being pus present, is a diagnostic point in favor of disease of the pelvis of the kidney.

Volume.-The quantity of urine secreted in a given space of time, usually twenty-four hours, is a matter of importance in many conditions. Care must be taken in making measurements that there be no loss at stool. In a rough way the amount secreted in twenty-four hours in health varies between forty and fifty ounces. Large individuals secrete more than small ones, those who drink freely more than those who do not. As a general rule, it may be said that the quantity of urine secreted depends largely on the watery condition of the blood, and the activity of the renal secreting epithelia. It has been calculated that ordinarily about one half the fluid drunk passes out of the system through the kidneys, the other half being eliminated by the skin, lungs, and bowels. During active exercise, hot weather, or in case of diarrhœea, very little of the fluid drunk may be eliminated by the kidneys. More urine is secreted during mental and bodily activity than during sleep, dne in the former case to the activity of the renal cells. Many spring-waters, teas, drugs, etc., by increasing the renal activity, act as diuretics. To test the efficacy of these, comparative measurements before and while taking are most important.

The volume of urine is decreased in the early stages of acute febrile affections Then later, as the fever subsides, it is apt to be increased beyond the normal. In most diseases, as death draws near, the quantity of urine is much diminished. This is often an important point. In the active stages of acute nephritis little urine is passed. This is also the case in all kidney affections accompanied with dropsy, while the dropsy is on the increase, the opposite condition existing while the dropsy is on the decrease.

After surgical operations on the urinary tract, it is most important to 
watch carefully the amount secreted. Complete surgical suppression at times ensues, and not a drop of urine is secreted for a considerable period. These cases are usually fatal. After these operations, if the volume of urine is good, the greatest danger is averted. It is very important to measure carefully the amount of urine passed in these cases, and, in fact, in all cases, in a measured glass graduate, and not depend in the least on the testimony of the patient or attendants ; for frequently, in the cases where the amount passed is very small, the desire to urinate is most frequent, and consequently the common impression is that the quantity secreted is great. In some forms of kidney disease where there is no dropsy the volume of urine passed is apt to be very great, as in the ease of interstitially contracted kidneys. In diabetes mellitus the volume is generally much inereased-so much so that often one's attention is directed to the disease by the amount of urine passed. Many text-books would lead the student to believe that a considerable increase in the volume of urine was one of the eardinal acempaniments in this disease. This, however, is not so. There may exist in this eondition even a diminution in the volume. I have had under observation for several years a case of this trouble in an elderly lady, where the amount of urine passed in twenty-four hours is invariably in the neighborhood of thirty-two ounces. In diabetes insipidus the amount of urine is increased, generally much so, together with the amount of solid ingredients which the urine contains. Such a condition of affairs is almost always associated with some form of nervous debility. The nervous system plays a great part in regulating the quantity seereted, and if an obseure case arises in practice bearing on this point it is always well to examine carefully the nervous system. Hysterical individuals at times pass a great abundance of urine.

Specific Gravity.-The specific gravity of the urine which is so easily read off from the urinometer is closely associated in clinical investigations with the volume. It is so much easier, however, to find the specific gravity than the volume, that it is very much more eommonly employed in diagnosis. From the specific gravity can be inferred the amount of solid material in the urine. In a general way urea represents half the solid constituents. Normal specific gravity varies from 1,015 to 1,025 or thereabout. Any one can bring the specific gravity of his urine temporarily down to the neighborhood of 1,005 by imbibing freely, especially of diuretic fluids. So a temporary increase of volume and decrease of specifie gravity suggest nothing pathological. A permanent condition of this sort, however, points strongly to an interstitially contracted kidney or to a decrease of a dropsical condition, and sometimes to anæmia. The opposite state of affairs is found in both diabetes mellitus and insipidus. In these eases the specific gravity is liable to be quite high, often considerably over 1,040 . In office practice where the volume is not known 
a high specific gravity together with a high color (no other evidence of anything wrong being found) suggests a small volume and general concentration.

\section{CHEMICAL CONSTITUENTS OF THE URINE.}

Under this heading only the substances will be considered which with our present knowledge by reason of their variations or presence are of diagnostic value to the practitioner. For a thorough treatise which would be of special interest only to the physiologist or chemist, reference is made to Nenbauer and Vogel's Analysis of the Urine, or a similar work.

a. Normal Chemical Constituents (Urea).-This is the most important as well as the chief organic constituent of normal urine. It represents the final product obtainable by oxidization of a nitrogenous substance in an alkaline fluid similar to the blood. It thus represents the amount of bodily waste or combustion; much in the same way as ashes represent the amount of fuel consumed. The more work done, the more food digested, the more combustion; hence the more urea. This combustion in the body, owing to adverse conditions, is not always complete, and, as a result, substances representing incomplete combustion or oxidization are found in more or less amounts in the urine. Chief among these are kreatinine, kreatine, xanthine, uric acid, oxaluric acid, etc. Many of these are rare and hard to demonstrate, and of no practical importance. Uric acid and calcic oxalate are of importance, however, and will be mentioned further on. Urea itself is very soluble, and therefore one never sees its crystals among the urinary sediments. It is a white crystalline substance tasting much like saltpeter, neutral in reaction. On heating these crystals further combustion takes place, and finally nothing but carbon remains.

A healthy man on a mixed diet taking a fair amount of exercise ought to secrete from twenty-five to forty grammes of urea in the twentyfour hours. There is, however, considerable variation to this. Individuals on animal diet, for instance, secrete much more urea than those on regetable. In any condition where there is great bodily wear, as after violent exercise, or during an acute febrile disease, the urea is increased. In making an examination into the quantity of urea only a mixed specimen of urine passed during the twenty-four hours should be taken, as the amount of urea varies greatly at different times, from being abundant after eating and exercise to being scanty after rest and sleep. Urea when not eliminated from the body, as in diseased conditions of the kidneys, acts as a poison, and sets up one of the many toxic trains of symptoms classed under the rather general heading of uræmia. It is with reference to uræmid almost wholly that the question of the quantity of 
urea secreted becomes of importance to the general practitioner. By quantitative tests from time to time in these conditions the working power of the kidneys can be carefully noted, and thus the relative values of different eliminative drugs can be accurately judged, and the course of the disease often prognosticated. There are several methods of estimating the quantity of urea. Most of them are inaccurate or so difficult outside of a well-equipped laboratory as not to be practical. Dr. Charles Doremus, of this city, has rendered quite practical the bromine method by means of his ureometer, which is manufactured, together with directions for use, by Eimer and Amend. By carefully following these directions (which have been embodied here with a few alterations and explanatory clauses), fairly accurate results can be obtained.

Reagents necessary.-The sodium hydrate solution (100 grammes to 250 cubic centimetres of water, or six ounces to one pint of water) will keep indefinitely when tightly stoppered. Bromine, twenty-five cubic centimetres added to the above amount of sodium hydrate solution, will make the concentrated hypobromite of sodium solution. This solution does not keep very well; so, if but few tests are to be made, it is not well to make up but a small amount, observing these proportions. The bromine may be removed from the bottle in which it is kept by means of the nipple pipette. One cubic centimetre of bromine suffices for a test. More can be removed if a quantity of hypobromite is to be made up. Some care must be exercised in handling the bromine, since it gives off irritating fumes; but by the above method of procedure no inconvenience ought to be experienced.

This concentrated hypobromite must be diluted with its own volume of water. This can be done approximately.

The long arm and the bend of the ureometer must be filled with the hypobromite.

Having washed the pipette, draw up exactly one cubic centimetre of urine, pass the pipette through the bulb of the ureometer as far as it will go in the bend; compress the nipple gently and steadily. The urine will rise through the hypobromite, and the urea will instantly decompose giving out nitrogen gas.

Withdraw the pipette after the urine has been expelled, taking care not to press the nipple hard enough to drive the air out after the urine, and read the volume of gas after allowing the froth to subside. The ureometer indicates according to its graduation either in milligrammes of urea in one cubic centimetre of urine or grains of urea per fluidounce of urine.

It also indicates, by the signs + and - on either side of the central division, whether the urea is present in a normal quantity or is increased or diminished. 
When the total quantity voided in twenty-four hours is known, the calculation of the amount of urea is very simple. Multiply the result found in milligrammes by the number of cubic centimetres voided, or the grains per fluidounce by the number of ounces voided.

The percentage by volume, or the milligrammes of urea per one hundred cubic centimetres of urine, is ascertained by multiplying the milligrammes of urea found by the test by one hundred.

Lowering the ureometer in a vessel of water until the water and hypobromite inside and out of the graduated limb are on a level, will give a more correct reading. The ureometer is graduated for $65^{\circ}$ Fahr., and upon an experimental basis.

It is well to impress the fact that only urines which have not undergone decomposition are available for accurate estimations of the quantity of urea, for, as has been previously noted in such conditions, urea is rapidly converted into carbonate of ammonium.

Chloride of sodium is quite abundant in the urine. Its presence can be demonstrated by dropping into the urine a solution of nitrate of silver, a thick cloud of chloride of silver being precipitated. It diminishes much in quantity in some acute diseases, especially, it is stated, in pneumonia. It is not, however, of much diagnostic importance.

Phosphates of calcium and magnesium, otherwise called earthy or amorphous phosphates, are abundant and of some importance. These are the salts which render undecomposed alkaline'or neutral urines turbid. Urines which when passed usually or frequently contain these salts in a state of precipitation suggest in the individual concerned some neurotic disturbance or the ingestion of much alkaline material. A few drops of acetic acid added to the urine will quickly clarify it with a slight effervescence. Under the microscope this precipitate appears as an amorphous sediment.

b. Uric Acid.-Uric acid, as has been stated, represents one of the products of imperfect combustion of nitrogenous substances, urea representing perfect combustion. It is pathological and of considerable diagnostic importance when it is abundant. At such times it can generally be easily seen by the naked eye, as the ordinary so-called brick-dust deposit at the bottom of the vessel in which the urine has stood for some little time. Uric-acid crystals, as found in urinary sediments and seen by the microscope, are always colored, the color varying from very light yellow to deep yellow, orange red, or dark red. The color is very diagnostic. The shape and size of the crystals vary greatly. They usually occur in pointed oval, lozenge, barrel, or dumb-bell shapes. They also have a tendency to agglutination at all angles, forming grotesque masses. They are very brittle, and when large are often fractured between the microscopic slides. The chemical test for uric acid is important. It is 
frequently of use in analyzing urinary calculi, which contain uric acid or urates. It is well known as the murexid test. Evaporate the urinous residue containing the suspected uric acid in a white porcelain or glass dish placed in a water-bath. Then add a few drops of a fairly concentrated nitric-acid solution, and evaporate this residue as before. Then moisten the last residue with an ammonia solution, and the purple-red murexid appears. If now a drop of potassic hydrate is added, the color changes to a purple blue. Uric-acid crystals, owing to their strong tendency to conglomeration, often form calculi. These crystals may occur in abundance at the onset of a sharp cold, also in acute febrile conditions, in gouty and rheumatic subjects, in subjects devoted to high living, especially where little exercise is taken, in dyspeptics, in many neurotic individuals where a debilitated condition of the nervous system has been brought about as the result of overwork, worry, some acute disease, anæmia, etc. In many of these neurotic individuals the urine is subject to change suddenly from concentration with high color and uric acid to dilution with pale color and an abundant precipitate of earthy phosphates. To remedy these conditions one of the numerous causes must be sought out and corrected.

The Urates of Potassium, Sodium and Ammonium.-These exist in abundance under much the same conditions as uric acid. They are rather easily disintegrated, setting free uric acid. Urines which contain an abundance of urates rapidly become turbid on cooling, the urates becoming insoluble in the cold fluid. The color of the precipitate is frequently light, but sometimes quite reddish. Most of these urates under the microscope are amorphous in character but sometimes crystalline, which condition is seen in the clumps of spicules which form the so-called hedgehog crystals of urate of ammonium. For the diagnostic significance of the urates the remarks on uric acid apply. They at times help to make calculi.

Calcic Oxalate.-A small amount of this substance exists in normal urines in solution, the acid phosphates of sodium and a few other substances which are present in small amounts holding it in solution. Calcic oxalate, however, is of interest to the practitioner only when it exists in abundance, in which case it does not remain in solution, but appears in the urinary sediment as white, transparent, sharply defined erystals. These crystals are usually in the form of an envelope and their shape is very characteristic. No chemical test is needed to confirm the diagnosis as revealed by the microscope. Rarely a few appear rounded like blood-disks. Calcic oxalate forms calculi at times, though not nearly so often as does urie acid. Such calculi are commonly extremely hard-not very large but very rough, hence often called " mulberry calculi." Only in the case of calculi is the chemical test for this substance necessary. This test is to 
fuse some of it, quicklime being left as the residue if the heat is powerful. Red litmus-paper being moistened and applied will, such being the case, turn very blue. No individual can be in a good state of health in whose urine there exists more than a stray calcic-oxalate crystal. The existence of these crystals is very demonstrative of an unhinged condition of the nervous system; and then again their presence in the urinary channels, by reason of their sharp cutting edges, sets up local symptoms due to direct irritation. They are rather frequently found associated with uric acid in neurotic individuals. Sometimes one of these sets of crystals will disappear for a time, the other variety taking the field. One very frequently finds these crystals in cases of spermatorrhœa-so frequently, in fact, that in those cases where the nervous symptoms associated with spermatorrhœea exist, calcic-oxalate crystals being present, the finding of free spermatozoa would seem to follow almost as a sequence.

In these calcic-oxalate cases there is often found an early history of mental worry or strain, youthful excesses or some debilitating disease where proper attention was not paid to convalescence. Then, again, this tendency is sometimes transmitted by inheritance, an instance being recalled where it has appeared in a marked degree through three generations. The local symptoms set up by the presence of the crystals in the urinary tract are usually in the form of paroxysmal attacks of sharp pain across the lumbar regions, often radiating down the thighs or toward the testicles or end of the penis. At such times frequent urination is of common occurrence. A stray blood-corpuscle and a considerable increase in the cloud of mucus are generally associated in the urinary sediment.

Cystine.-This substance exists in small quantities in most urines in solution. When it is in larger quantity than usual, it is precipitated in the form of whitish or light yellow, transparent, six-sided plates. These crystals can be confounded with nothing unless it be a rare form of uricacid crystal. If there is any doubt, the murexid reaction can be tried to determine the question. Cystine, like mic acid, is one of the intermediate products in the combustion of nitrogenous substances. It differs from uric acid chiefly in the fact that it contains sulphur. Sulphur goes to make up about a fourth of its composition. Under just what pathological conditions cystine occurs in quantity is not known. It is very rare. The author during the last year has had occasion to observe one case. Here the cystine crystals existed in connection with uric acid. The patient when first seen was in a miserably weak, nervous condition, having been so since an attack of scarlet fever some few months previously. Under tonic treatment and rest he soon recovered. The chief interest that the practitioner has in cystine is in the fact that it forms, 
though rarely, a calculus. Such a calculus is yellowish in color, rather soft, and usually small. The test for this substance is to dissolve some of it in potassic hydrate, then add a solution of acetate of lead, and boil; a black precipitate of sulphate of lead being quickly formed.

Indican, which seems to be a chemical combination of a saccharine substance with indigo, is normally present in small quantities. Its demonstration by setting free the indigo is as follows: Into a wine-glass pour half an ounce of concentrated sulphuric acid; to this add three drops of concentrated nitric acid; then from a dropper add thirty drops of the urine, and stir with a glass rod till everything is well mixed; then set on a shelf, with a piece of white paper for a background. In about fifteen minutes the solution, if the normal amount of indican is present, shows an amethyst color. When there is an increase in the indican, the color is more blue. Sometimes where the increase is great the shade of blue is very strong. Rarely indigo red takes the place of indigo blue. The blue (or red) tint gradually fades in the course of some minutes, leaving the solution about the same color as when it was first mixed. Jaffé (Pflüger's Archiv, vol. iii, page 448) has found that any causes whieh interfere with the action of the small intestine occasion an increase in the amount of indican. Mention is made of cholera, peritonitis, strangulated and incarcerated bowel, etc. Some diagnosticians, especially the Freneh, lay considerable stress on an increase in indiean, considering that, other causes for its increase being absent, some internal cancer or growth, especially of the brain, should be suspected. I have in mind the case of a gentleman who had consulted several leading men in this country for some indefinite nervous symptoms, without any positive diagnosis being made. He went to France, and a large amount of indican being found, a diagnosis, chiefly from this point, of tumor of the brain was made. This diagnosis was later sustained by the symptoms which developed, and was demonstrated as correct by autopsy. One should, however, be very careful in drawing any such radical conclusions as the preceding, since I have found a large amount of indican repeatedly in simply highly nervons and hysterical individuals.

c. Products of Chemical Decomposition of Normal Ingredients. - Triple phosphates, otherwise called ammonio-magnesian phosphates, are the chief products of decomposition, and appear as crystals in the form of rhombic prisms when alkaline fermentation sets in. When this process takes place in the bladder these crystals are deposited in great abundance, and if careful washing and disinfecting are not resorted to, phosphatic calculi quickly form. This state of affairs is very frequent in connection with catheter life where rules of cleanliness are not rigidly adhered to. This substance forms the outer coatings of many calculi. Such calculi are apt to be soft and mushy, and therefore after 
the operation of litholopaxy it is difficult to be positive that all the fragments have been washed out. Carbonate of ammonium and other produets of decomposition are secondary in amount and importance to triple phosphates, and have no individual importance in diagnosis. The chemical test for these phosphatic deposits is the addition of an acid, the precipitate being quickly redissolved.

Albumin.-The presence of any appreciable amount of this substance in the urine is pathological. One of the chief functions of the kidneys in their action as filters is to prevent the passage of albumin through them into the urine. Albumin in the urine does not necessarily indicate that it got there by reason of defective kidney secretion. It may have been introduced by reason of an injury to the mucous membrane in some portion of the urinary tract, with which condition blood or pus is usually associated. In small quantities also it may be present in cases where the urine contains considerable prostatic or seminal fluid.

The detection of albumin in the urine is, as a rule, a simple matter. The test with concentrated pure nitric acid is the standard one. Two to three drachms of the urine are poured into a sherry-glass. Then the glass being tipped, the nitric acid to the extent of half a drachm to a drachm is allowed to trickle slowly down the tipped side of the glass to the bottom, its specific gravity being much greater than that of urine. The line of division between the two fluids is sharply defined. If albumin is present, a distinct white layer will appear at this line of division. The more the albumin the greater is the density and thickness of this layer. This test is important, for by it not only is the presence of albumin detected, but the practiced eye can, by observing the layer quite accurately and at a glance, reckon the percentage by weight of albumin in the specimen. There are a few conditions which interfere with or obscure this test. In urines rich in uric acid or urates a zone of opacity is formed, consisting of nitrate of urea, which can easily be confounded with the albumin zone. This zone of nitrate of urea rarely forms immediately except when the specimen tested is quite cold. If this layer of nitrate of urea is carefully examined, it will be found that it is not at the line of division between the nitric acid and the urine where the albumin is always situated, but that it is above in the urine, and that a little clear area of urine is present between the bottom of this nitrate of urea deposit and the line of division between the two fluids. Sometimes both albumin and nitrate of urea exist together, causing some confusion. In such a case it is well to try one of the confirmatory tests. Once in a while some other substance which chemists have not at present determined, but which is thought to be myocin or mucin, is deposited by the nitric-acid test much as albumin, except that in these rare instances the upper layer of this pseudo-deposit is not at all defined in the way 
albumin ought to be when the test is carefully and properly made, and generally the turbidity of the false deposit quickly extends, involving the whole specimen of urine. If the urine is decomposed the nitric-acid test is much interfered with, owing to the turbidity of such urines and also to the effervescence caused by adding the acid to the alkaline fluid. Then, again, pus or blood interferes. In the case of pus it is well to filter and then test. Where, however, there is enough blood present to interfere with the test, it is of no value to make any test for albumin, for the selfevident fact that if red blood-corpuscles can get into the urine, necessarily albumin can and must. If urine is turbid, owing to earthy phosphates, clarify it with a drop of acetic acid before testing. If turbid, owing to urates, heat the specimen in a water-bath till it is clear and then test. Owing to these few conditions which interfere with the nitric-acid test, the careful observer always has resource to another test for the sake of verification.

The heat-test is valuable and is somewhat more delicate than the nitric acid. Fill a test-tube about half full of urine (previously filtered only in case pus is present). To this urine add a drop of acetic acid to make sure of the presence of a little free acid. Then expose the upper area of the urine to a Bunsen or alcohol flame and let it come to a boil, turning the tube from time to time so that it will not crack. If the urine was turbid at the start the acetic acid will clear up phosphates and the heat urates, these last disappearing long before the boiling-point is reached. When the boiling-point is reached, albumin, if present, shows itself as a white turbidity. In some cases where the amount of albumin present is very small, the tube has to be held up against a dark object, as the coatsleeve, to detect its presence, the difference then between the natural cool fluid in the bottom part of the tube and the slight turbidity of the boiled portion being better determined. This slight whitish turbidity in cases where the amount of albumin is very small increases to a condition where the boiled fluid resembles buttermilk, or even sometimes to a white, curdy mass which will not pour out of the tube. In such conditions the amount of albumin present is very great.

Millard's test is highly recommended for its delicacy in detecting and clearly demonstrating albumin when it exists in small amounts. Nothing under one twelfth per cent by weight of albumin can be seen by the nitric-acid test, while with the Millard test amounts very much smaller can be plainly detected. The following are the reagents used :

Acid. carbol. concent. ............. 20 c. c.

Acid. acetic glacial............... 70 c. c.

Liq. potass................... 220 e. c.

Here a drachm of the test reaction fluid is poured into a sherry-glass. About a drachm of the urine to be tested is sucked into a pipette. Then, 
the finger being placed quickly over the end of the pipette as soon as suction is suspended, the urine is allowed to flow slowly down the inclined edge of the glass on to the fluid in the bottom. If any albumin is present, a very fine distinct white layer is deposited just at the line of division of the fluids.

Quantitative Estimation of Albumin.-The old-fashioned and rather common method of estimating albumin by employing the heat-test, and then, after the precipitate has settled, calculating the amount of albumin by the relation which the volume of the fluid containing the deposit bears to the whole volume, is very inexact and unsatisfactory. Esbach's method, by means of a test solution of picric acid, is well spoken of. I have never thoroughly investigated its accuracy. The method used by the writer is that taught by Prof. Wood, of the Harvard Medical School. Here the percentage of albumin by weight in a specimen is calculated by the appearance of the zone in the nitric-acid test. In applying this method it is necessary to add the nitric acid with as little agitation as possible, and to inspect the zone as soon as it forms. By neglecting to take these precantions the zone will appear heavier and denser than it otherwise would, consequently leading the observer to overestimate the amount of albumin present. The general rules are that the faintest zone that can be seen indicates one twelfth per eent by weight of albumin. On looking vertically downward one can see the bottom of the glass through any zone where there is less than one per cent of albumin. If the zone is too dense to be seen through, there is present one per cent or over. In cases where there exists a great amount of albumin-i. e., two to five per cent (the latter amount being the greatest per cent possible)--the zone is not only very dense and thick, but it very quickly tends to lose its defined borders, and to present a curdy appearance. The amount of albumin between two and five per cent can not be calculated by the eye; but as cases very rarely get beyond two per cent, this is not important. When this method is mastered by practice, small variations in percentage can be accurately reckoned, and such conclusions verified by filtering out, drying, and weighing the albumin in a given amount of the urine.

The objections to be urged against this method are that it requires considerable practice before one can be accurate; and to facilitate matters the beginner ought to have the help of an expert, as it is impossible to give an exact written statement by following which accuracy can be assured. A good way, however, to perfect one's self (and this can be done without a teacher) is to observe carefully a zone, and then by precipitation, drying, etc., to verify matters by actually weighing the albumin. If one will take this trouble the method can soon be mastered, and then its value to a busy man will readily be seen, for the presence and amount 
of albumin can be determined at a glance. Hereafter in this article, where percentage of albumin is mentioned, that by weight is intended. Much has been written lately in support of the theory that a small amount of albumin (that is, in the neighborhood of one twelfth per cent) can appear from time to time in the urine from mere physiological and not pathological causes. Such statements should, however, be taken with suspicion. It is safe to say that when albumin to the amount of one fifth to one fourth per cent exists in urine, blood and pus being absent, its source is from the kidney. This fact ought to be verified by microscopical examination of the sediment, and it can be in almost all cases if time and patience are employed.

Small traces of albumin, blood and pus being absent, usually have their source from the kidney, but may arise from certain conditions of the prostatic or seminal organs. In these latter conditions the microscope reveals considerable quantities of prostatic, vesicular, or seminal fluid. In some cases a small amount of albumin is found in the urine secreted during exercise, while in that secreted during sleep, or perfect quiet, none appears. Such individuals are usually in perfect apparent health, and probably have had their attention first drawn to the presence of albumin on applying for life insurance. This condition may be accounted for by the fact that a little gritty or calculous material, or perhaps a cyst, exists in the kidney, which exercise stirs up, slightly congesting the immediately surrounding renal tissue.

Some drugs which act as irritants to the kidneys frequently cause a little albumin to appear when given in physiological doses. When taken in poisonous doses, much albumin with suppression results. This applies to such drugs as phosphorus, cantharides, turpentine, and the like. In sharp fevers, especially in scarlet, typhoid, and the septic fevers, it is customary to find some albumin; also in passive congestive conditions of the circulation, such as exists in pregnancy, some tumors, portal, cardiac, or pulmonary obstruction, etc. In most of these preceding conditions the presence of albumin is temporary, dependent on something that has its location outside of the kidneys, the albumin disappearing together with the outside cause.

Then, again, albumin exists in the urine as a result of disease located in the kidneys themselves. In such condition the percentage of albumin is to some extent a differential diagnostic feature. A large amount, generally one per cent or over, is usual in the early stages of acute nephritis, and throughout the course of chronic parenchymatous nephritis, where degenerative changes take place in the kidney-secreting cells, usually of a fatty character. In amyloid degeneration and early tubercular invasion, in gouty kidney, and in the early stages of chronic interstitial nephritis (contracted kidney), one expects a consider- 
able amount of albumin, usually from one fourth to one per cent, according to the extent and involvement of the disease.

In the convalescence of acute nephritis the albumin gradually decreases and disappears with complete resolution. A very small amount of albumin-so little, in fact, that it often escapes the observation of a careless examiner-frequently oceurs in the later stages of contracted kidney. I have in mind two such instances where practitioners of good repute, judging from examinations of the urine only, the patients never having been seen by them, sent written opinions to sufferers bedridden by this slow and fatal disease, stating that no disease of the kidneys existed. In both these cases death from uræmia followed in a few months. If this form of disease be watched, however, exacerbations will be found rather frequently where as much as one fourth or one fifth per cent of albumin is present. At those times also the microscope finds many confirmatory evidences of disease which are very few and far between in the intervals where the albumin largely disappears. This is the disease in which mention has been made of the low specific gravity and great increase in volume of the urine. In this form of trouble general arterio-capillary fibrosis co-exists with the consequent hypertrophy of the heart. If in such cases one uses a delicate test, such as Millard's, the albumin is readily detected. In conditions of hydronephrosis a small amount of albumin, often only a trace, exists, probably due to a low-grade inflammatory condition set'up in the secreting tubules owing to the pressure of the confined fluid. If this pressure is suddenly taken off, much albumin and active inflammatory changes usually ensue. Such conditions exist in connection with the sudden relief of retention of urine, more especially with reference to old men suffering with prostatic obstruction, where the increase in the amount of residual urine, with the accompanying dilatation of the ureters and pelves of the kidneys, has been a matter of considerable time.

In elderly individuals where there are extensive atheromatous changes a trace of albumin usually occurs. Such cases as a rule do not do well after operation or instrumental interference in connection with the urinary organs, probably owing to the fact that the kidneys are prevented by these changes from adapting themselves to altered conditions.

Albumin is also to be considered when associated with pus, no blood being present. When pus exists in the urine, albumin is necessarily there also in some amount. In vesical catarrhs where the urine is acid, as is the case with a smooth, hard calculus, or with prostatic obstruction without much tenesmus, also in cases where gonorrhœa has extended to the neck of the bladder, considerable free pus may be present, but the amount of albumin in a filtered specimen is not great, usually about one fifth to one sixth per cent, or less. This rule also holds for tubercular 
affections confined to this region before ulcerative changes with great tenesmus have taken place. In this last condition, however, nuch albumin may be expected, probably due to blood serum. The same is the case sometimes in old men with severely inflamed prostates where there is great tenesmus. In such conditions over one per cent of albumin may exist with the pus, making the surgeon fear considerable kidney involvement. Still, speedily after the tenesmus has been relieved by a suprapubic incision, the albumin often almost wholly disappears, and no involvement of the kidneys can be traced. Aside from these instances, however, where from one half to one per cent of albumin exists in a filtered acid specimen of urine, involvement of the pelvis of the kidney in the inflammatory process may be suspected. In decomposed urine containing pus, little can be inferred from the albumin tests, as under such conditions they are, as has been explained, unsatisfactory. Where blood to any extent is present in the urine, either by itself or associated with pus, the tests for albumin are of little or no significance, as it is always present in abundance, owing to the blood serum.

Sugar.-Sugar in the urine is always pathological and indicative of a diabetic condition. This important pathological state can not be positively diagnosed except by the chemical demonstration of the presence of grapesugar in the urine. Not only is the chemical demonstration of the presence of sugar important, but also the quantitative estimation, for by this latter procedure both the severity of the disease can be noted, and also the modifications and results which dietetic, therapeutic, and hygienic measures are able to effect. The test recommended for the detection of sugar is a modification of Fehling's test with cupric sulphate. The trouble with Fehling's cupric sulphate solution is that it is unstable. The modification test to be described was recommended to the writer several years ago by Dr. Keyes, who states that he first adopted it on the strength of an indorsement which appeared in one of the New York medical journals. Search has been made for this article in order to introdnce its author, but without success. This test is very reliable in all cases where sugar is present to any considerable extent. For small traces it is well to confirm the results of this test by the fermentation test or by the polariscope. The three reagents necessary for the copper test to be described are (1) a solution of cupric-sulphate, grains $x l$, in pure glycerin, $z j$ (this is perfectly stable, care being taken to have the glycerin pure), (2) liquor potassæ, (3) tartaric acid. Into a test-tube pour a drachm to a drachm and a half of the liquor potasse. Into this liquid in the test-tube drop three or four grains of the tartaric acid, reckoned in a rough way on a knifeblade. Heat the fluid in the tube to boiling over a Bunsen or alcohol flame. To this add six to ten drops of the glycerin and cupric-sulphate solution. Shake and heat a little more. The fluid in the tube is now a 
deep robin's-egg blue. To this fluid add the suspected urine, $\dot{a}$ few drops at a time, shaking and heating between each addition of drops until about half as much urine in volume as there was blue fluid has been added. If sugar is present in a larger quantity than a trace, an ochre-yellow, with sometimes reddish and sometimes grayish tinges, appears in the form of a precipitate. If much sugar is present, the addition of the first two or three drops of urine to the blue solution will demonstrate the reaction very clearly; if but little sugar is present, then the larger amount of urine must be added. The colors in the end reactions by this test are exactly similar to those obtained by the tests with Fehling's solution. Sometimes uric acid and allied substances which have not been thoroughly oxidized into urea act as reducing agents on the copper solution, changing the color from blue to reddish, no precipitate usually being found. In such in-

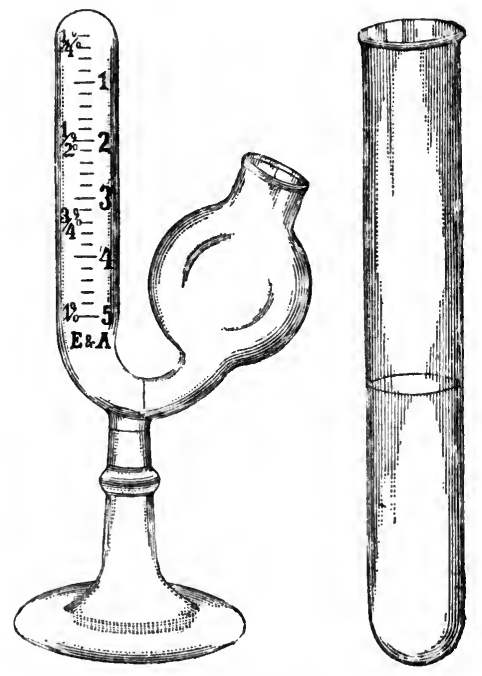

Fermentation Saccharometer. stances by this test alone the presence of a trace of sugar can not be determined, and another test is resorted to for confirmation.

Fermentation Test.-This test is based on the fact that the yeast-fungus acting on solutions of grape-sugar causes their decomposition into alcohol and carbonic-acid gas, one equivalent of grape-sugar being decomposed into two equivalents of alcohol and four of carbonic-acid gas. Dr. Max Einhom, of New York, has rendered this test very practicable by means of his tubes, which are manufactured by Eimer and Amend, with directions for use. The cut representing the tubes and the directions sold with it, which seem explicit, are embodied here. There is one important statement to make in regard to this test, and that is, never to use dry yeast-cake. Of the wet compressed cakes, Fleischmann's have been used, and have given accurate results.

Directions.-Take one sixteenth of a cake of Fleischmann's yeast, shake thoroughly in the graduated test-tube with ten cubic centimetres of the urine to be examined. Then pour the mixture into the bulb of the saccharometer. By inclining the apparatns the mixture will easily flow into the cylinder, thereby forcing out the air. Owing to the atmospheric pressure, the fluid does not flow back, but remains there.

The apparatus is to be left undisturbed for twenty to twenty-four hours in a room of ordinary temperature. 
If the urine contains sugar, the alcoholic fermentation begins in about twenty to thirty minutes. The evolved carbonic-acid gas gathers at the top of the cylinder, forcing the fluid back into the bulb.

On the following day the upper part of the cylinder is filled with carbonic-acid gas. The changed level of the fluid in the cylinder shows that the reaction has taken place, and indicates by the numbers-to which it corresponds-the approximate quantity of sugar present.

If the urine contains more than one per cent of sugar, it must be diluted with water before being tested.

Diabetic urines of straw-color, and a specific gravity of 1,018-1,022, may be diluted twice ; of 1,022-1,028, five times ; 1,028-1,038, ten times. The original (not diluted) urine contains in proportion to the dilution two, five, or ten times more sugar than the diluted urine.

In carrying out the fermentation test, it is always advisable to take, besides the urine to be tested, a normal one, and to make the same fermentation with it. The mixture of the normal urine with yeast will have on the following day only a small bubble on the top of the cylinder. That proves at once the efficiency and purity of the yeast. If there is likewise in the suspected urine a small bubble on the top of the cylinder, then no sugar is present; but if there is a much larger gas volume, then we are sure that the urine contains sugar.

If the percentage of sugar in a mixed specimen of the urine passed in twenty-four hours is known, together with the number of ounces secreted during that time, the exact number of grains of sugar in the urine can be most easily calculated.

The quantitative estimation of sugar, together with the demonstration of its presence can be quite accurately obtained by means of the polariscope. Théodore and A. Duboseq, of Paris, manufacture a beautiful instrument for this purpose. The expense of this instrument, however, stands in the way of making this method popular, and as the simple fermentation method just described seems to answer the purpose very well, it has not seemed judicious to take up space here to explain the steps necessary in connection with the use of the polariscope.

The appearance of sugar in the urine is associated with the nervous centers, and has nothing to do with the actual condition of the kidneys, though frequently in cases where much sugar is habitually secreted there is found a little albumin and a stray hyaline cast in the urine, probably indicative of a slight inflammatory or congested condition set up in the kidneys by the passage through them of sugar. A small amount of sugar often appears temporarily during dietetic and venous excesses in highly nervous individuals. A little less commonly a mental or nervous strain not assoeiated with any excess may cause it to appear. In this latter elass of individuals often uric-acid and calcic-oxalate erystals and sometimes 
spermatozoa, appear at such times associated with the sugar. In some individuals simply an excess of saccharine food, with no accompanying nervous tension, will cause sugar to appear. In such cases the prognosis is not so good as in the former class. Then, again, there are the confirmed diabetics, in whom no dietetic régime, together with nervines and climatic changes, can cause an entire disappearance of the sugar except possibly for a short and very occasional interval. It is in these cases that the quantitative estimations are important in calculating the effect of the different methods eniployed to insure relief, as it is impossible frequently to judge accurately of the condition of these individuals by their symptoms or by their statements.

\section{ORGANIZED SEDIMENTS.}

The study of organized sediments is accomplished by the eye, aided in large measure by the microscope, together with certain surgical procedures, chemistry playing a very minor part. This subject will be subdivided as follows: epithelia, prostatic fluid, seminal and vesicular fluid, mucus, casts, pus, and blood. Any of the rare conditions which are not included in these subdivisions will be treated in the final chapter, under Miscellany.

Epithelia.-Epithelial cells from different parts of the urinary tract differ in one or more of the three following particulars-namely, size, shape, and structure. The same statement can be made to apply to a considerable extent to the different layers of epithelia from the same region according as the cell is new, signifying from a deep layer; or old, signifying from an outside layer. One has rarely to consider the layer of the epithelial cell except in inflammatory conditions at the pelvis of the kidney or the bladder. In some cases the location of inflammatory disturbances can be definitely determined by the nature of the epithelia found in the urinary sediment along with the inflammatory exudations, and in all cases valuable hints as to the source of the inflammation may be obtained from the study of the epithelia even though the cells may not be characteristic enough to admit of definitely locating the trouble. Anterior urethral epithelia are not very important in diagnosticating inflammations in that region, since it is rarely necessary to have recourse to the microscope as an aid, the discharge from the meatus of itself furnishing all the evidence that is usually necessary, the exact location being determined by the endoscope. Under the microscope these cells usually appear spindle-shaped or oval, sometimes irregularly rectangular, and occasionally caudate. The nucleus is large and very distinct. The cells themselves are rather large, and their structure is firm and compact. They are well seen in beginning convalescence from an acute urethritis entangled together with pus-corpuscles in the stringy mucus when one of the fine mrethral shreds is 
taken from the urine and placed under the microscope. These cells can be confounded with those from the deep urethra. The only difference is, that those from the deep urethra are proportionately more inclined to the oval shape, quite a number of them being almost round. It is common for them to be associated in inflammatory conditions with prostatic fluid, and thus their source can frequently be verified.

The typical bladder-cell is quite large. It has a generally round or rectangular shape, with irregular, roughened edges. Frequently several of these pavement-cells are seen attached, their irregular lines of union being plainly visible. These cells are inuch lighter in structure than those from the urethra. They are from the outer epithelial layer of the bladder, and are consequently the most matured. In the region of the neck of the bladder there is a gradual blending of cell characteristics ; in that part of the neck nearest the deep urethra the cells resemble to a considerable degree their neighbors in the deep urethra, this resemblance growing less and less, and the bladder characteristics becoming more and more marked on the passage backward to the bladder proper. Caudate and spindleshaped epithelial cells from the bladder wall come from the median epithelial layers. These cells are lighter in structure than the urethral cells, and their nuclei are not so distinct. They resemble much, and can not be well distinguished from, the cells from the ureters and the cells from the external epithelial layer of the pelvis of the kidney. These caudate and spindle-shaped cells from the median epithelial layers of the bladder are not frequently seen in large numbers, except occasionally in cases of some hard, smooth vesical calculi, which cause a constant moderate friction on the bladder walls, consequently scraping off the epithelium before it reaches the last stage in its development. These cells are not found in cases where calculi set up severe grades of inflammation.

In severe vesical inflammations cells from the deep vesical layers of epithelium appear in the urine, associated with much pus. These cells are oval or round, resembling in a marked degree cells from the neck of the bladder, the distinction being that the average size of the deep-layer vesical cells is smaller and the structure lighter than that of the cells from the neck.

The deep-layer vesical cells may also be confounded with the cells from the deeper layers of the pelvis of the kidney. Here, again, the distinction is that the pelvic cells average somewhat smaller in size and are lighter in structure than the deep vesical. Cells from the ureter are rarely seen in the urine except immediately following a sharp passage of gravel or a small calculus down the nreter, in which case a little blood and pus are associated in the sediment with the cells. These cells, as has been stated, are customarily caudate or spindle-shaped. They resemble very closely the onter-layer pelvic cells and the middle-layer 
bladder-cells. The cells from the outer layer of the pelvis of the kidney appear in the urine only after some acute inflammatory disturbance in that region, such as may be set up by calculous irritation or acute congestion from cold, etc. They bear so close a resemblance to ureteral cells that a differential diagnosis can not be made. The cells from the deeper layer of the pelvis of the kidney and from the large, straight tubules of the kidney are identical, and are important in diagnosticating chronic inflammatory conditions of that part. The practiced eye can in most cases positively identify them under the microscope. Dr. Heitzman, of this city, has, more than any one else, called special attention to these cells. They are round in outline. Many of them, however, have the least bit of an attempt at a caudate extremity, which can be seen only by careful observation, and which bears about the same relation to the cell proper that the stem bears to the apple. These cells are about one third larger than a natural pus-cell. They are most delicate and light in construction, and under the slide of the microscope they seem to float about more readily than the associated pus-cells. They are so delicate that they soon disintegrate, in which condition they can not be distinguished from puscells that have absorbed much fluid. It is therefore necessary, in looking for them, to examine a rather freshly passed urine in order not to risk disappointment. These cells frequently seem to have several nuclei, as do pus-cells. Cells from the vesical neck, and from its deep epithelial layers, may be confounded with the cells under consideration. These vesical cells, however, average larger and are much denser in structure. Cells from the small tubes of the kidney are almost exactly like pus-cells, except that they have no visible nuclei. They can therefore but rarely be differentiated from pus-cells, except when they appear adherent to casts. They are consequently by themselves of little diagnostic importance.

Vaginal epithelium resembles in a marked degree that from the outer vesical layers. The vaginal cells are almost always irregularly rectangular in shape, showing a great tendency to appear segmented together in groups. When one sees in a sediment a great number of such cells and very little pus, indicative of existing inflammation, the presumption is very strong that the cells are vaginal and not vesical. These cells appear in great numbers in the normal vaginal secretions, consequently they easily and naturally become mixed with the urine as it is voided. They often serve to confuse the microscopist. It is always well, therefore, to direct women patients to carefully donche themselves before voiding the specimen to be examined. Occasionally heterologons cells are found, indicative of some foreign growth.

Prostatic Fluid.-This fluid, when present to any extent in the urine, is visible to the naked eye, floating about as a viscid, quite transparent, 
stringy mass. When clear it is usually of a light specific gravity, and consequently remains generally near the surface of the urine. It appears as a white, clumpy, opaque mass when pus-corpuscles are intermixed, as occurs in inflamed conditions of that part. Under the microscope the fluid itself presents a finely striated appearance, and in this mesh-work are seen the prostatic epithelial cells, which are rounded or oblong, closely resembling those already described from the deep urethra. Associated with these cells in the fluid one often sees irregularly shaped, smooth, rather highly refractive amylaceous bodies called sympexions. With our present knowledge no special diagnostic significance is attached to these bodies. Prostatic fluid in the urine not associated with pus points to over-activity of the gland. This condition is often observed in individuals addicted to sexual irregularities and excesses, a morbid sensitiveness of the gland being produced. It also occurs in highly nervous individuals as a result of mental and nervous strain due to overwork. Urethral stricture sometimes causes it, owing to the congestion of the parts behind the stricture; also rectal and vesical tronbles which occasion much spasm of those parts. During or following an active inflammation of the deep urethra, such as occurs often in gonorrhœa, the prostatic follicles are involved, and then an abundant flow of prostatic fluid, associated with pus, results.

Vesicular and Seminal Fluid.-Seminal fluid is gradually collected in the vesicles, thence to be ejaculated as occasion requires. Consequently, seminal fluid when found in the urine is associated to a greater or less extent with vesicular secretions. In normal conditions of the vesicles, however, the amount of fluid generated in them is very slight, and consists of a little mucus and a few desquamative cells not to be distinguished from prostatic cells. In normal urine neither this nor seminal fluid is found, except in the urine first voided after sexual intercourse. When the seminal vesicles are inflamed, they secrete a mucus which is thick, glairy, and abundant, looking much like prostatic fluid. In fact, it could not be distinguished from prostatic fluid were it not for the presence in it of spermatozoa, due to the admixture of seminal fluid. In this vesicnlar fluid, secreted in inflamed conditions of the glands, a considerable number of epithelial and pus cells are intermingled, the greater the inflammation the greater the number of the cells. It is important from a diagnostic point of view, in case spermatozoa are found in the urine, to determine whether they are associated with an abnormal amount of vesicular fluid or not. If they are associated with this pathological vesicular fluid, the diagnosis points to vesiculitis; if they are not so associated, the diagnosis points to spermatorrhœa-two distinct diseases, which differ much in prognosis and treatment. Pure seminal fluid is hardly a urinary sediment, and so will not be further considered here. If one 
suspects vesiculitis, and has trouble in finding evidence of it in the urine examined, it will be of advantage sometimes to examine a specimen voided after or during the passage of a large stool, or after digital pressure on those parts per rectum. Occasionally the spermatic cord connecting with the corresponding vesicle becomes impervious, as after gonorrhœal or tubercular inflammations of that region, in which case, should vesiculitis occur, no spermatozoa would appear in the sediment in connection with the vesicular fluid, and the fluid would probably be mistaken for prostatic fluid. In such a case the diagnosis would have to be made by means of surgical explorations

Mucus from the urinary tract is rarely important from a diagnostic point of view. It is always increased during inflammations, and often it is seen as quite a cloud in urines where there have been active inflammations, the pus largely disappearing before the mucus. In the urine from a bladder where there is much inflammation, together with putrefactive changes, the mucus, on standing, becomes as it were coagulated, gluing the sediment together into an elastic, gummy mass.

Casts are molds formed of exudative inflammatory material in the secreting tubes, generally of the kidney, rarely of the prostate. This exudative material, pure and simple, is a hyaline substance, translucent and sometimes semi-transparent, easily molded, but at the same time of enough consistency to preserve its molded form in the urine. Hyaline material, which makes up the body proper of the cast, frequently is so stained by or mixed with blood, blood-pigment, and degenerative material, such as fat, epithelium, etc., that its hyaline characteristics are to a great degree or wholly masked According to these different appearances, casts are classed as hyaline, waxy, blood, brown granular, coarsely granular, finely granular, fatty, and epithelial.

A waxy cast is a pure hyaline cast of dense structure and with little transparency. A blood-cast is one with adherent blood-corpuscles. A brown granular is one stained with blood-pigment. Coarsely granular and finely granular casts are those mixed with amyloid degenerative material of varying degrees of fineness. Fatty and epithelial casts, as their names imply, are those covered respectively with fat-globules and epithelial cells. A fat-globule on a cast is distinguished from a speck of granular material by its marked refractive properties.

Casts vary in caliber according to the size of the tubes in which they are molded; consequently casts from the straight tubes of the kidneys are larger than those from the convoluted tubes. In children they are naturally all small; and in an adult, where the casts are all of small caliber, a contracted kidney is suspected. In diseases of the kidney, pure hyaline casts are found in convalescence from acute nephritis, in passive congestion, and in many chronic diseased conditions of these organs in 
the absence of acute exacerbations. Hyaline casts are liable to present a waxy appearance in amyloid disease of the kidney, and in other diseases of the kidney when the vitality of the individual is low. Blood-casts appear in acute nephritis, in calculous nephritis, due to the wounding of a blood-vessel, and after injuries from the same cause. They also occur occasionally in acute exacerbations of a chronic disease. Brown granular casts often appear with, and usually persist after, the disappearance of the blood-casts. They would also appear in any individual with kidney disease whose red blood-corpuscles are undergoing rapid degeneration, as occurs at times in scurvy, typhoid, etc. Coarsely and finely granular casts are found in the commencement of convalescence from acute nephritis, and in chronic conditions generally, where the disease shows some activity. Fatty casts are found in chronic parenchymatous nephritis, usually associated with much albumin, and, when abundant, are indicative of a serious state of disease. An occasional epithelial cell often adheres to a cast as it slips out of its tube. It is only when very many of these are found adhering to a cast that it is called epithelial. In acute nephritis following scarlet fever such casts are numerous, as the name "desquamative nephritis," often applied, would signify. In order to find casts, urine should be allowed to stand for several hours, and then the fluid for examination should be taken by the pipette from the bottom or very near the bottom of the glass.

If, from the presence of albumin, casts are suspected, many slides should be examined before a negative opinion is given. Sometimes, in cases of chronic interstitial nephritis, for instance, the finding of a cast is extremely difficult, because not only few casts are present, but also, owing to the great transparency of those present, detection is difficult even when in the field of the microscope. In such cases the finding may be facilitated by staining the fluid slightly with a dye like methyl violet, which color is quite readily taken up by the hyaline material. The demonstration of disease involving kiduey-tissue to any extent is never perfect until casts have been found.

Casts are cylindrical in shape. The parallel sides and the well-defined ends should be carefully focused under the microscope, and determined before a positive opinion is advanced; for many times, if this is not done, a roll of stringy mucus may deceive one who is not very expert. In the case of the mucous roll, however, the sides will rarely be found to be parallel except perhaps for a short distance, and one or both ends will be found, under careful observation, to be spun out into a fine, wavy tendril. Prostatic casts are occasionally seen. These casts are generally finely granular, some nearly hyaline, and taken by themselves they can not often be distinguished from kidney-casts. They, however, rarely exist alone, floating freely about, as is usually the case with kidney-casts, but 
are found imbedded in a mass of prostatic fluid. The urine also has no albumin, unless it be a trace revealed by a delicate test, due to the prostatic material present. These prostatic casts may apparently occur in the case of any prostate where a moderately inflamed or congested condition has persisted for some time.

Pus.-Pus is found in the urine as a result of inflammation in connection with the urinary tract. It is easily detected by the eye when it is present in any quantity. When it is in great abundance, the urine resembles gruel. When there is but little, the urine simply loses its clear, transparent appearance, and, instead of looking like sherry, as it should, it resembles cider. The eye frequently can not differentiate between pus and earthy phosphates or urates, and chemical measures liave to be resorted to in order to determine this point. These measures, however, have been already described in connection with the consideration of earthy phosphates and urates, and so need not be referred to again.

Bacteria also may be confounded with pus, and this question must be settled by the microscope. Of course, when bacteria are present in freshly voided urine, inflammation to a greater or less extent must of necessity exist in the urinary tract, and as a result pus must also be present in quantity proportionate to the existing inflammation. In some chronic conditions, where the bacteria have been present for a long time, the results of the acute inflammation set up when the bacteria were first introduced may have largely passed away, leaving a low-grade chronic inflammation behind, in which condition the urine may be loaded with bacteria and yet contain but little pus. Such a state of affairs is rather frequent in the case of old men long habituated to catheter life, where great nicety with reference to cleanliness has not been observed. In the majority of these bacteria cases, however, the pus is present in amount sufficient to mask the bacteria except where the microscope is employed.

After the presence of pus in the urine is established, the main point to be determined is the source of the pus. To accomplish this, both inspection, aided by the microscope, and surgical methods, are employed. Only a few observers claim by the microscopical study of the pus-cells themselves to be able to determine their source; for, of course, all pus-cells are much alike, and whatever differences they may have seem to depend on their stage of development and on processes of disintegration which set in after death rather than on their source. It is, however, on the sediment which is found in connection with the pus that opinions as to its source are usually based. Thus, if one finds epithelium from the pelvis of the kidney in considerable amount along with the pus, it is proper to infer that the pelvis of the kidney is the seat of disease. So, likewise, if casts are found, the kidneys are considered; if vesical epithelium, the bladder, etc. 
There are a few points which it is well to mention here with reference to the general appearance of pus. Some of these points have been already referred to in the consideration of acidity and transparency. Very often pus from the pelvis of the kidney settles in a compact, firm mass, leaving a quite clear if not perfectly clear fluid above. If, now, the vessel is agitated, the pus is easily stirred up again, rendering the fluid turbid and homogeneous in appearance, showing that the settled mass had little or no adhesive qualities. The pus in such urines is apt to preserve these qualities for a considerable time, owing, probably, as has been observed, to the persistence of the acidity in these cases. Pus from the bladder region generally does not settle well. In most cases of moderate vesical inflammation it appears at the bottom of the glass, after standing. as a very loose, flocculent mass, taking up considerable space, and having an irregular, wavy outline to its upper border. In some cases the upper border of this sediment can not be well defined, it being impossible to detect where clear urine commences. When, however, no clear upper space appears on standing, bacteria are associated with the pus. Then, again, if these urines after standing a time are agitated, the sediment is not likely to be readily stirred up, owing to adhesive qualities which have been developed. In such instances, if the fluid is vigorously agitated, the sediment leaves the bottom bodily in a twisted, ropy mass. This adhesive quality is probably due to fermentative changes, which set in promptly, as a rule, in cases of vesical inflammation.

The surgical methods employed in detecting the source of pus are as follows: If the source is from the anterior urethra, pus appears at the meatus to a greater or less extent as a continuous flow. In such conditions, if the flow is slight, stroking the urethra from behind forward will bring some pus to the meatus. If the source of pus is from the deep urethra, there may be no flow of pus forward at all, the meatus being perfectly dry. In such cases, however, there is liable to be at irregular intervals, probably due to spasmodic contraction of an expulsive nature, some pus at the meatus. In cases of urethral inflammation, in fact, as a preliminary step in the investigation of all inflammations of the urinary tract, the patient, the bladder being fairly distended, should be directed to pass his urine in two parts as equally divided as possible. By this means the washings of the anterior urethra are all in the first specimen. If there is active inflammation of the deep urethra the products of that inflammation by natural muscular action flows backward into the bladder. In this condition of affairs the second specimen of urine is just as turbid as the first.

In some chronic conditions of the deep urethra where there is little free pus, clumps of prostatic material and shreds containing pus are all that is present to mark the inflammation. Frequently these clumps and 
shreds adhere to a certain extent to the deep urethra, and are not pressed back into the bladder by the natural muscular contraction, and consequently appear in the first flow of urine, the second remaining clear. Sometimes-and this occurs occasionally in prostatic inflammations-the greatest turbidity of the urine will be in the last half-ounce passed, the muscular spasm becoming more violent as the vesical walls contract on an empty bladder, giving the inflamed and congested prostate a squeeze, thus setting free an extra amount of the products of inflammation. This condition of affairs must be distinguished from the following one, and it can easily be done by the absence of vesical irritability and spasm in the condition to be considered. In some cases of pyelitis, or vesical atony, urine, loaded with pus, collects in a non-irritable bladder, and if the individual is not active the pus settles largely at the bottom. Consequently, the first urine passed may be quite clear, the latter portion being very muddy. This condition of affairs is seen in elderly men who have prostatic enlargement, which causes the urethral outlet to be somewhat above the normal level. It can never exist in the young subject, where the urethral outlet is at the most dependent portion of the bladder. When the second specimen contains pus the deep urethra, together with the vesical neck, the bladder proper, and the kidney region have to be taken into consideration. Usually the deep urethral region can be ruled out as the seat of trouble if there is an absence of tenderness in that region, and no blood follows on the passage through it of a good-sized blunt steel sound, or on pressure exerted there by means of the finger in the rectum.

In order to settle the question as to the source of the pus between the kidney region and the bladder, a good procedure is to introduce a soft catheter into the bladder, and through this instrument to wash the cavity perfectly clean, the last washing being poured into a glass in order that its cleanliness can be the better demonstrated. The bladder after the washing should be dry. Then the patient is left for an hour or so, at the end of which time he is directed to pass his urine into a glass, and this is compared with the specimen voided before the washing. If the specimen passed after the washing is much clearer than that previously passed, the evidence is good that the pus comes wholly or in great part from the bladder. If the specimen after the washing is just as turbid as the one before it, then the strong presumption is that the pus comes from the kidney region. This is a very practical and useful test. It is often very necessary to determine whether the pus comes from one or both kidneys, and if from one only, to locate that one positively. This can frequently be done with great nicety by washing the bladder and filling it with clear water and then introducing a cystoscope. By means of this useful instrument the mouths of the ureters can be watched, and the pus can be seen as it is ejected from them. In case the cystoscope is not to be had, the 
history of pain and tenderness on one side, together with palpation (better under an anæsthetic), which frequently shows an enlargement of the affected organ, have to be relied on in making the differential diagnosis. Sometimes the pus in the urine does not come from the urinary tract at all, but flows into it through some sinus connecting an abscess cavity. In such a state of affairs, if the sinus makes its entry into the vesical cavity, it can frequently be discovered by the cystoscope, otherwise the diagnosis must be made largely by the exclusion of other conditions.

After pus has been located, its diagnostic significance is to be considered. When from the urethra, gonorrhœa or some of its sequelæ, tranmatism, and tubercle are the causes usually to be considered; rarely urethral chancre, malignant disease, etc. In diagnosticating the first of these troubles and its complications the presence of the gonococcus should be demonstrated. Pus in abundance from the prostate would indicate gonorrhœal infection, tubercle, traumatism (usually due to a calculus lodged in that region, or to instrumentation), seldom malignant disease. A small amount is found at times in prostatic hypertrophy and in some of the neuroses, and after sexual excesses. Pus from the seminal vesicle is usually indicative of a vesiculitis, resulting from gonorrhœea, sexual excesses, the use of tight condoms, or tubercle, rarely malignant disease. Pus from the bladder would lead one ordinarily to consider gonorrhea (or one of its sequelæ, chiefly stricture), traumatism (occasioned by the presence of a foreign body, or the passage of an instrument, or external violence), tubercle, defective drainage (such as exists in prostatic hypertrophy, atony, and stricture), benign and malignant growths, and bacterial infection. Pus from the kidney region is generally indicative of gonorrhcal infection, calculus, tubercle, defective drainage, inducing hydropyonephrosis, traumatism, ascending bacterial infection, abscess (as may occur in pyæmia and low fevers), and occasionally a benign or malignant growth.

Blood can readily be detected by the eye when present in any appreciable amount, owing to the color it imparts to the urine, as has been already considered. Under the microscope the red corpuscles are easily distinguished, and can hardly be confounded with anything else, unless it be very rarely with an extremely unusual and very stray form of calcic oxalate crystal. This condition, on account of its rarity, is hardly worth consideration.

Red corpuscles, which are alive and have amœboid movements, are rarely seen in the urine, as the acidity of the fluid quickly kills them. These corpuscles usually present a round nummular appearance, the disk being evidently rather thicker than the center. In fresh, very acid, and concentrated urines they are apt to present a shriveled, crenated appearance, probably due to the loss of some of their fluid under such conditions 
from exosmosis. In very faintly acid and diluted urines the opposite condition is liable to exist, the corpuscles being quite full from endosmosis. When a moderate amount of blood is present in the urine, the microscopical examination of the associated sediment is of value in determining its source, as in the case of pus. When, however, blood is present in abundance, very little idea regarding its source can be derived from the microscope, since the blood is liable to mask the associated deposits in the sediment. Resort, therefore, must often be had to surgical procedures similar in some respects to those adopted with reference to pus. The same procedures, however, which are successes in investigating the source of pus, may be failures in the case of blood, because blood may collect-there being a fresh hæmorrhage-very quickly, while pus collects slowly. Thus, a bladder may be washed free of pus, no matter whence its source, while this can not be done as regards blood during a vesical hæmorrhage. It is very rare for hæmorrhage from the urinary tract to be continuous. It is therefore of importance to watch these cases, and examine under the microscope specimens of urine secreted during the cessation from bleeding, all elements in the sediment being then visible.

Blood appearing at the meatus in the absence of vesical spasms has its source in the anterior urethra. If the bleeding is from the deep urethra, and is free in amount, it will flow backward and distend the bladder, not appearing at the meatus at all, provided there is an absence of vesical spasm. Such a condition of affairs, however, is very unusual, unless it be directly after the administration of a strong anodyne, for, as a rule, much inflammation attends a free bleeding from the deep urethra, and consequently there is a rapid succession of spasmodic contractions of that part, forcing the blood forward at frequent intervals. Generally in the absence of resical spasm, probably due to the puckered condition of the deep urethra, the hæmorrhage from that part is very slight. It is usual in hæmorrhages from the deep urethra to find all or the great bulk of the blood in the last half-ounce of urine passed, a few drops of quite clear blood in such instances dropping from the meatus at the very end of the act This is because of the tight spasm of these parts toward the end of micturition, which does violence to the inflamed surface, thus setting up the bleeding, which in turn is soon checked by the increasing tonic contraction after the act is finished. Then at the next urination, in the first gush of urine, some blood-clots are liable to appear from the source of the preceding hæmorrhage, which again repeats itself at the end of the urinary act. In such a condition, if one attempts to pass a sound through this region great muscular resistance with much pain is encountered. The preceding remarks apply also to prostatic hæmorrhage, except in cases of senile hypertrophy of that organ, in which conditions the 
hæmorrhages usually take place directly into the vesical cavity, and will consequently be considered as vesical.

Hæmorrhage from the seminal vesicles is rare, and slight in amount. Its occurrence is demonstrated by bloody seminal emissions. It now remains to distinguish between vesical and kidney hæmorrhages.

Hæmorrhage from the bladder proper, in the absence of existing irritable conditions, frequently does not disturb the functions of that organ or cause pain unless its source involves the vesical neck. Free hæmorrhage from the kidney region is almost always followed by nephritic colic, due to the distention of the pelvis and ureter by bloodclot. Palpation also in such conditions usually shows tenderness and enlargement of the affected organ. In case the hæmorrhage is from the bladder, vesical lavage and instrumentation would in all probability affect it-that is, by increasing the bleeding-except in cases where hæmostatic agents are employed in the lavage, under which circumstances there would be a diminution in the hremorrhage. Such is not the case if the kidney region is involved. If the hæmorrhage is from the kidney, the bladder can, in a great majority of cases, be washed clean enough so that with the cystoscope the blood flowing from the ureter can be observed. In most bladder cases, by taking advantage of an interval during which there is little or no hæmorrhage, the cystoscope can be used to advantage in the inspection of the cavity, and in the observation of any existing pathological condition. Occasionally, in hrmorrhage from the pelvis of the kidney, the blood-clot as it passes down the ureter will be molded into a long, stringy cylinder, looking like an earth-worm. The moldings are called "urcteral casts," and are of diagnostic value when observed.

Dr. W.K. Otis, of New York,* speaks highly of a method depending on the resorption of a solution of iodide of potash in determining vesical hæmorrhage. In a case of suspected vesical hæmorrhage the bladder is filled with a solution of iodide of potash; then, after an interval, the saliva is tested for iodide of potash. If this substance is detected by the test, the hæmorrhage is supposed to be vesical in origin; if it is not detected, the source of the hæmorrhage is not vesical, it being considered that no resorption of iodide of potash takes place if the continuity of the vesical mucous membrane is intact.

Prof. Guyon, of Paris, has called attention to the fact that in cases of renal cancer free bleeding often takes place at night during repose, while by day the urine may be comparatively clear. The same author also

* Dr. Otis writes me that the late Dr. T. A. McBride, of New York, in 1885 suggested the idea of this test to him, and that he experimented with it successfully, supposing himself to be the pioneer worker. In 1887 , while in Vienna, Otis found Ultzmann using the same test; so the question of authorship is not determined. 
states that the blood in these cases usually gives the urine a black rather than a red color.

It is not within the scope of this artiele to eonsider in detail the subjeetive symptoms of patients afflieted with hæmorrhage; a eareful study of whieh, together with the histories, is of great help to the practitioner, aside from the,investigation of the urine in making a final diagnosis.

Hæmorrhage from the front urethra would suggest traumatism due to instrumentation, a foreign body, the forcible straightening of the organ in the state of ereetion, chordee being present, injury during coitus, the injection of irritants, ete. ; also gonorrhoea in its aeute stage, or a strietured or granular eondition resulting from that disease, benign or malignant growths, and tubercular or syphilitic lesions. Hæmorrhage from the deep urethra suggests gonorrhœa or one of its sequelæ (stricture or uleeration), traumatism (generally due to instrumentation, the impaction of a foreign body, or external violenee, as from a kiek or a fall), tubercle, benign or malignant growths, prostatic hypertrophy in old men, and rarely a reflex spasmodic eondition of that region.

Hremorrhage from the seminal vesicle might indieate gonorrhœal, tubercular, or malignant involvement.

Hæmorrhage from the bladder suggests benign or malignant growths, foreign bodies, tuberele, prostatie hypertropliy assoeiated with atony, the vesical drainage being very defective (in such instances the hæmorrhages usually oeeurring after the sudden (lrawing off of much fluid), traumatism from instrumental or outside violence, rarely gonorrhœal ulceration, febrile conditions, syphilis, and parasitie disease.

Hæmorrhage from the ureter may follow violence, as caused by the passage of a ealculus.

Hæmorrhage from the kidney region leads one to consider acute general nephritis, calculous disease, tranmatism, benign and malignant growths, and tuberele, rarely syphilis, febrile conditions, parasitie disease, ete. Numerous surgeons have reported instances where nephrotomies have been performed for persistent hæmorrhage from a kidney (I myself liave seen two such cases in Dr. Keyes's practice), for which no cause could be discovered at the time of the operation. Nevertheless, the operative proeedures in such cases generally serve, for some unknown reason, to cheek all further annoyanee from this souree.

\section{MISCELLANY.}

Bacilli.-A great variety of bacilli are found in urine, but with our present knowledge special diagnostic importance is attached to but two of them, namely, the bacillus of gonorrhea, commonly known as the gonoeoccus, and the tubercle bacillus. The gonocoecus was discovered by Neisser, of Breslau. Any urethral discharge eontaining these germs 
associated with its pus is considered contagions, and such a discharge has its source from outside contamination. No conditions within the urethra can give rise to a discharge containing them. If, however, these bacilli have been once introduced into the canal, they may remain there a long time in a state of quiescence, as has been shown by the investigations of Prof. Goll and others (Correspondenzblatt für Schweizer Aerzte, Basel, April 15, 1891); and while this is the case, they will appear in any discharge which may intervene, no matter how innocent its apparent cause may have been. Nany social questions affecting marriage, chastity, etc., depend on the presence or absence of the gonococcus. In fresh cases of gonorrhœa, gonococci are very abundant, and consequently their presence is easily demonstrated. In old chronic conditions, however, oftentimes a long and careful search among the pus-cells of the stray urethral shreds has to be made before the germs are found, or should be made before they are pronounced absent. In order to demonstrate the presence of gonococei, the staining methods recommended in the article on the "Etiology of Urethritis" may be employed in preparing the specimen.

If gonococci are present they will be seen in the affected pus-cell, stained by the methyl violet as little black, cylindrical bodies, always lying together in pairs, parallel and with even ends. Generally quite a colony of these bacilli are gromped together. They are never found outside of pus-cells. They usually incolve the lumen of the cell, rarely its nucleus. They colonize certain individual cells, the others being left perfectly free. The percentage of pus-cells affected is very small, even in the most acute stage of gonorrhcea.

Tubercle Bacillus.-This bacillus, when found in urine, is of great importance, as it definitely determines the character of the existing inflammation. In some tubercular conditions, howerer, it is very difficult to demonstrate the presence of the tubercle bacillus by means of the methods of investigation in use at the present time. If, therefore, tubercular germs are not found in a case of suspected tubercular inflammation, this evidence should be treated simply as negative, and should not be used to exclude a diagnosis of tuberculosis based on physical signs. The tubercle bacillus in urine can not, as in sputa, usually be demonstrated directly in the specimen to be examined, but cultures in gelatin have to be resorted to. This is owing to the fact that but few bacilli are apt to exist in a specimen of urine. It is very difficult to find these bacilli when they are few and scattered. By cultivating them their number is so increased that they are easily recognizable. This process of cultivation requires so much care and skill that it falls to the lot of the bacteriologist rather than the practitioner. For full particulars and directions regarding the tubercle bacillus, reference is made to Friedländer's Microscopical Technique (Putnam). 
Parasites. - Filaria Sanguinis Hominis. - These are the parasites which cause chyluria; they are consequently of importance in connection with urinary analyses. The urine secreted during the period of activity of these parasites in individuals so affected presents the appearance of milk, or rather cream, with here and there a speck of blood. These parasites are active only when their victim is at rest. Therefore it is in the night urine that chyluria manifests itself; that passed during the day being quite clear. The microscopical appearance of chyle is the same as that of milk. Under the microscope the parasites themselves are seen thrashing about, if the specimen is fresh; otherwise motionless and dead. They are sharp-pointed at both ends, and resemble earth-worms in shape. They are about five times as long as spermatozoa. This disease was thought formerly to be confined to the hot countries of the East. Numerous instances of it, however, have been reported in our Southern States, in the West Indies, and in Brazil. Its diagnostic appearance is so marked that it can not be confounded with any other disorder. Dr. W. M. Mastin, of Mobile, Ala., has considered this subject in a very complete and classical article (Annals of Surgery, November, 1888).

Echinococcus cysts are rarely seen in the urine; sometimes, thongh more rarely, the echinococcus heads and hooks. These cysts vary from the size of a pea to that of a hen's egg. Their source is usnally from the kidney, though rarely they find their way into the bladder from a perivesical tumor by means of nlceration.

Bilharzia homatobia is a parasite indigenous to sections of Africa, chiefly Egypt, and was first described by Dr. Bilharz, of Cairo. Hæmaturia is associated with the presence of this parasite in the urinary tract.

Bits of tissue from benign and malignant growths are sometimes found in the urine. These bits should be picked out and placed in a drop of glycerin on a glass slide, and gently teased apart and unfolded, so that their structure may be distinguishable under the microscope. Occasionally a positive diagnosis from this procedure alone can be made. This applies especially to villous vesical growths. Very rarely pseudomembranous vesical molds and diphtheritic gangrenous detachments from the bladder appear in the urine. In such conditions inspection aided by the microscope should be relied on to determine the diagnosis. For full consideration of these conditions, reference is made to articles by F. Gnyon (Annales des maladies des organs genito-urinaires, Juillet et Août, 1887). Occasionally fecal material and gas may be introduced into the urine through inflammatory action attended by ulceration; so, likewise, necrosed bits of bone, etc. It is well to guard the practitioner against the occasional introduction of foreign material into the urine sent for examination. 


\section{URINARY FEVER.}

\section{By J. A. FORDYCE, M. D.}

THE various terms used by writers to designate the phenomena recognized by surgeons for a long time as sometimes following operations or instrumentation upon the urethra or bladder, indicate in a certain measure the obscure and conflicting views that have been and are yet held regarding their nature. While all authors agree that more than one class of cases, or pathological condition, may be present, a confusion of terms has obscured to some extent a general conception of what is meant by urinary fever. Shock of a mild and severe form, urethral fever, urinary fever, catheter fever, urinary poisoning, uræmic poisoning, urinary infection, are some of the many names given to these disturbances.

The association of micro-organisms with cystitis, often with pyelitis and pyelonephritis, has been recognized for a number of years, and their etiological relationship with these affections pretty definitely established.

In the light of the investigations of the Necker school and others, it is probable that many cases of urinary fever ascribed by the older writers to reflex nervous influence are dependent upon bacterial or ptomaine poisoning, although it is impossible in our present knowledge to ascribe all disturbances which follow operations on the urethra or bladder to an identical cause, to local infection, or to deny absolutely the possibility of a nervous febrile reaction. Septic infection, which differs in no respect from the septic accidents after other surgical operations, follow operations on the urinary organs, or may complicate one of the typical forms of urinary fever, so that it is sometimes difficult to sharply differentiate one form of infection from the other. Still the intimate relationship of the urethra with the bladder and the kidneys, both by continuity of tissue as well as nervous connection, introduces a factor which plays no small part in the development and progress of the malady, furnishing certain conditions which favor, complicate, and follow local infections from the urethra and bladder, and permitting by reflex nervous influence (?) a disturbance in the vascular supply of the kidneys and a resulting disturbance in their functional activity.

Shock.-Immediately following the contact of an instrument with the sensitive urethra, the patient may become faint, lose consciousness for a 
few minutes, or have a slight chill or convulsion. As the patient grows accustomed to the introduction of an instrument, the attacks may not be repeated.

It is impossible to explain such attacks, except to call them cases of shock, resulting from an unusual impression made upon the sensitive nerves of the urethra, transmitted to the brain and reflected to the inhibitory nerves of the heart or along the motor nerves. The attacks are quite analogous to shock which results from ordinary surgical injuries, or to the fainting spells which follow an unusual cerebral impression in a sensitive subject. When one keeps in mind the peculiar sexual sensibility of the urethra and its nervous connection with the brain, it is not surprising that such reflex phenomena manifest themselves. Aside from these transient fainting spells, or slight chills or convulsions which are recovered from within a few minutes, a severer form of shock has been recognized in which death has rapidly followed the introduction of a sound or a slight operation on the urethra.

Sir Henry Thompson has met with two cases in which deatl followed in twenty-four and forty-eight hours after the introduction of a small catheter through a narrow stricture where no visible lesion was produced by the instrument. In one of the cases, in which suppression of urine and death in forty-eight hours took place, the autopsy revealed only highly congested kidneys. The author regarded the fatal issue as the re'sult of suppression of the kidney functions, through some nervous influence transmitted from the urethra.

Mr. Mitchell Banks relates the history of a case in which the passage of a No. 4 metallic bougie through a tight penile stricture was followed by vomiting and rigor within half an hour; a second rigor occurred in two and a half hours, and death in six and a half hours. At the postmortem no injury was seen in the urethra, and only a slight congestion of the kidneys. A tablespoonful of thick, muddy urine was in the bladder.

Similar cases have been reported by Lister, Velpean, Fayrer, Harrison, and others. A number of years ago I had the opportunity of closely observing the case of a man aged about forty years in whom a convulsion, coma, and total suppression of urine followed an hour after the passage of a small flexible bougie through a tight stricture of the membranous urethra. Death without a re-establishment of the urinary secretion took place twenty-four hours afterward. There was no elevation of temperature, but for several hours before death a subnormal temperature was found. Although no autopsy was made, it is probable that a previous damaged condition of the kidneys, with insufficiency, was present, otherwise one would not expect death to follow so rapidly from suppression of urine. 
Unfortunately, these cases occurred before the importance of a bacteriological examination of the previons condition of the urine or genitourinary tract was recognized, so that the autopsies made are in a great measure valueless. It has been held that death has taken place too rapidly to be accounted for by an infection, as a longer period of incubation has been looked upon as necessary to such an infection. It is recognized now, however, that the healthy urethra contains micro-organisms which are pathogenic, and that the urethra, which is the seat of an old stricture or other pathological condition, is pretty constantly inhabited by pathogenic germs with their chemical poisons, capable of rapid absorption into the general circulation after an abrasion or other traumatism of the mucous membrane.

Albarran reports in detail the very instructive case of a man fortythree years old, the subject of a traumatic stricture in the bulbous urethra. Following an internal urethrotomy by Guyon without a retention catheter, he was seized, an hour and a half after the operation, with a violent chill. The temperature rose to over $104^{\circ}$ Fahr., and the chills were repeated during the day. In the evening, twelve hours after the operation, the patient died with marked asphyxia. At the antopsy ecchymoses were found beneath the capsules of the kidneys; they presented a marked congestion, as did the other viscera.

Behind the urethral stricture pus was found which contained the $3 a c$ terium pyogenes, now thonght to be identical with the bacterium coli commune, unmixed with other organisms. The same microbe was found some hours after death in the blood, the kidneys, the spleen, and in the urine of the pelvis of the kidneys. There can be little doubt, in view of the dissemination of this organism, that its entrance into the genera] circulation through the wrethral wound was the cause of the patient's death.

Clinically this case is identical with the ones of Thompson, Banks, and others, and without the bacteriological examination could be classed with these as an example of reflex kidney congestion. In this ease, however, we must regard the kidney lesions as the result of their attempts to eliminate from the blood these pathogenic microbes or their chemical poisons. Cases are reported in which, almost immediately after the entrance of a sound into the bladder, the patient has been seized with a rigor, followed by collapse and death in a few moments. It would be difficult to account for such a rapid death on any other supposition than as the result of some severe shock. Where to draw the line between these cases of severe shock and the others of rapid septic poisoning is, in our present knowledge, a matter of extreme difficulty.

Although we must recognize the influence of shock, both in its light and grave forms, it is necessary to bear in mind the probability that all 
the cases of malignant urinary fever, in which a chill followed by fever and rapid death have occurred, were cases of rapid septic or ptomaine poisoning, and not of reflex suppression of the functions of the kidney from an impression made on the sensitive nerves of the urethra. Clinical evidence, however, strongly favors the view that in a previously diseased kidney, with deficient elimination of excrementitious matter, urethral instrumentation may, by disturbing the normal kidney innervation, precipitate a grave and fatal form of uræmic poisoning without a chill or elevation of temperature.

Symptoms of Urinary Infection.-Clinical observers have recognized several types of urinary infection, which may, according to Guyon, be classified as follows:

1. The acute form $(a)$ with a single chill, fever, and sweat; $(b)$ one or more repetitions of the acute paroxysm, with remission or intermission in the fever. 2. Chronic urinary fever.

Acute Urethral Fever.-After operative interference with the male or female urethra, or the simple introduction of a catheter into the bladder, the patient may experience within an hour or two symptoms of indefinite character, followed, generally after the first urination, by a chill of varying intensity, and lasting from a few moments to several hours. During or after the chill a rapid rise of temperature

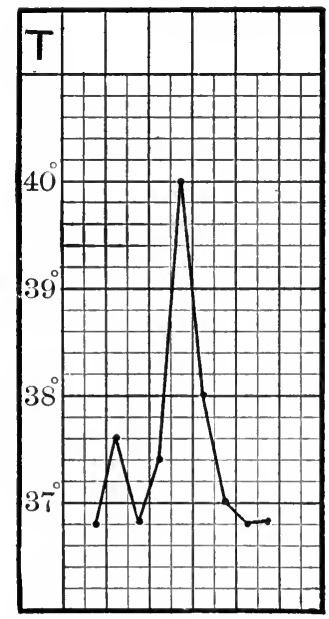

Fra. 1.-Acute urinary fever after internal urethrotomy;
recovery. (After Guyon.) takes place $\left(103^{\circ}\right.$ to $106^{\circ} \mathrm{Fahr}$.), of somewhat indefinite duration, with severe headache, backache, and rapid action of the heart, attended or followed by profuse perspiration, and ending in an equally rapid decline in the fever. (See Fig. 1.)

Within twenty-four hours, as a rule, the entire paroxysm is over, leaving the patient with only a slight lassitude, which rapidly passes away, with a complete restoration to health. The chill may be so intense and prolonged as to be alarming, the surface cold and cyanotic, and the respirations labored, or of so mild a character as to pass almost unnoticed. The regularity and mutual relationship of the three stages are, according to Guyon, of importance in prognosis.

If the chill is prolonged and out of proportion to the fever and sweat, the prognosis should be reserved, and one should be on the alert for a repetition of the attack.

Quiet or active delirium may be present during the chill or fever. It is not of grave import, and does not materially affect the prognosis. 
Nausea, vomiting, or diarrhœea may likewise be encountered. Dyspnœea and cardiac irregularity may, in exceptional instances, be met with.

Instead of a rapid return to the normal condition, as is the rule, the patient may have a recurrence of the paroxysm on the following day, or after three or four days; these paroxysms may be repeated without obvious cause, such as the dilatation of a stricture or the introduction of an

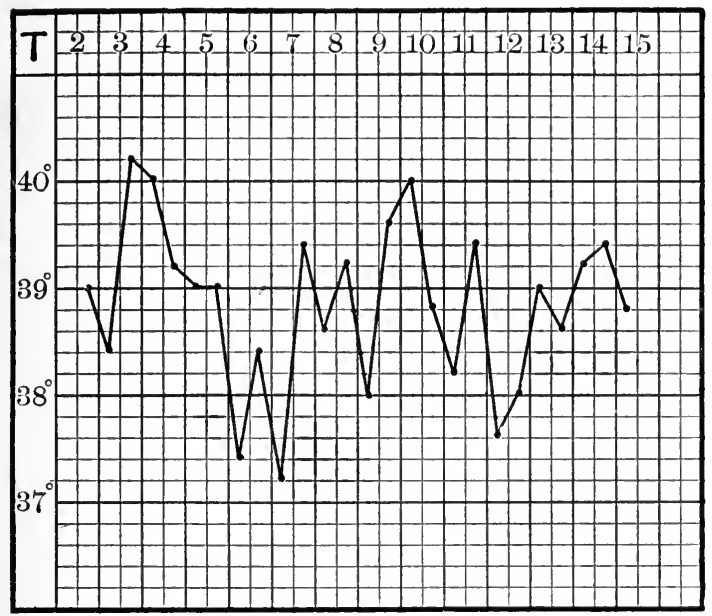

Fig. 2.-Acute recurring urinary fever. Pyelonephritis revealed at autopsy. (After Guyon.)

instrument into the urethra. In case no local lesion can be found to account for the repeated attacks, they may soon disappear, and the patient be left in a normal state of health. (See Fig. 2.)

Thompson has called this variety "acute recurring urinary fever."

Cases are encountered where every attempt made to dilate an irritable or resilient stricture is followed by a chill and febrile paroxysm. On complete division of the stricture these manifestations disappear. It is possible that the free drainage afforded by the complete division of the stricture may account for the disappearance of these attacks. The febrile state may persist with exacerbations and remissions, or may remain more or less constant. When a repetition of the acute attack takes place there is not the same relationship between the three stageschill, fever, and sweating-as in the frank variety. One of the stages is apt to be exaggerated, while the others may be incompletely developed. With a persistence of the fever the tongue becomes red and dry, and in aggravated cases brown or almost black; and covered with a thick coating, which may involve the pharynx and soft palate. Vomiting and diarrhœa are in certain cases such marked symptoms that some writers have made a distinct type called "choleriform." 
Oppression in breathing and cardiac irregularity are more frequently noted than in the first variety of acute urinary fever. Congestion of the base of the lungs, with numerous râles, is often met with.

Contrary to the observations of Morris, Thompson, and others, Guyon has detected neither albumen, blood, nor tube casts in the urine during the attacks; the quantity of urine is diminished only as the result of the fever and perspiration.

This writer has carefully examined his patients with the view of determining the presence of pain in the kidneys. He has found spontaneous pain rare, and pain provoked by pressure over the organs in from one third to one half the cases. The nervous disturbances are similar to those met with in the frank variety of urinary fever. In grave cases the delirium becomes more continuous, and before death the patient passes into a state of coma. While it is the rule for complete recovery to take place in the first variety, death is not so exceptional in this second class of cases.

In certain cases more frequently met with after operative interference on the urethra, certain complications arise which are more closely allied to pyæmia than to true urinary fever. Among these may be mentioned circumscribed induration of the subcutaneous tissue and muscles, with or without abscess formation, pustular skin eruptions, suppuration of the articulations, parotid gland, etc.

The duration of this variety is somewhat indefinite; when death takes place it is seldom delayed longer than twenty days.

Chronic Urinary Fever.-The chronic form of fever may follow the variety just described, from the development of a cystitis, a pyelonephritis, or a general infection. It occurs more frequently as the result of catheterizing an over-distended or atonied bladder in a subject affected with prostatic hypertrophy or an old stricture. In many such cases the bladder contains varying quantities of residual urine, and the kidneys are already the seat of a pyelonephritis or other chronic changes producing renal insufficiency.

This condition of the bladder and kidneys offers a favorable soil for the development of germs introduced by unclean instruments or carried in from the urethra. Independent of interference with the urethra or bladder, these old prostatics, patients with long-standing stricture, calculi, or bladder tumors, may develop irregular chills and fever, or a continuous fever of a mild type (see Fig. 3), in connection with progressive impairment of their strength, loss of appetite, with dry and coated tongue, vomiting, diarrhoea alternating with constipation, headache, a cachectic look, and other symptoms of chronic uræmia.

No fever may be present, or of so mild a type as to be overlooked. When noticed in connection with acute exacerbations or chilly sensa- 
tions, it is often attributed to malaria or other influence than its true character.

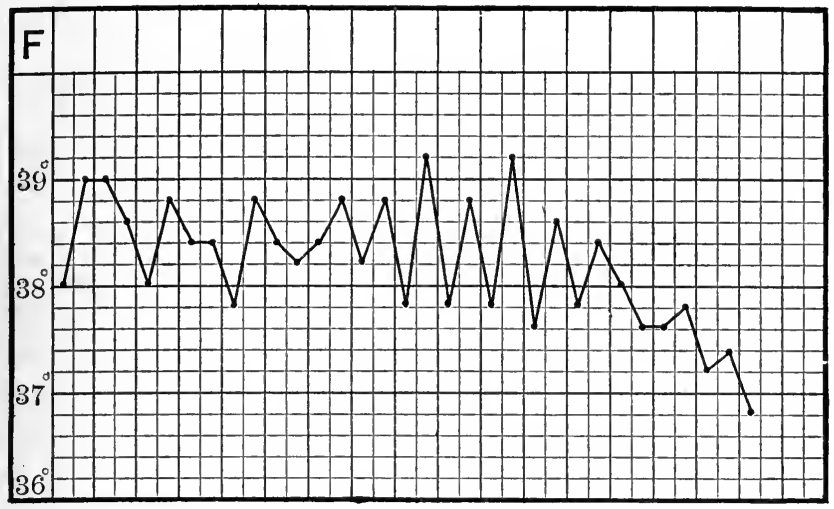

Fig. 3.-Chronic urinary fever. Incomplete retention of urine from prostatic hypertrophy. (After Guyon.)

Guyon has devoted considerable space to the discussion of the dyspeptic symptoms presented by such patients. They readily succumb to operations on the urinary organs, or even to catheterism performed in the most careful manner.

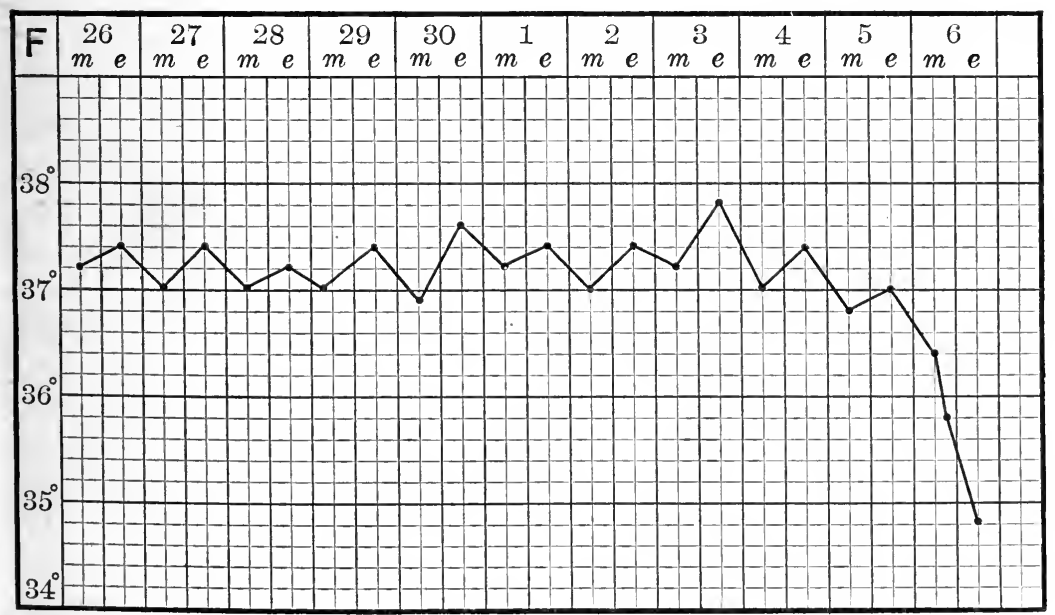

Fro. 4.-Non-febrile urinary eachexia, terminating fatally, with subnormal temperature. (After Guyon.)

If the use of the catheter is begun before the amount of residnal urine becomes large-not exceeding twelve to fifteen ounces-it is unusual to find symptoms indicating a local infection. When the amount 
of residual urine is larger, with the resulting bladder and kidney changes correspondingly advanced, a cystitis may readily be excited by the use of the catheter, which can produce a general infection with secondary deposits in the kidney, an ascending inflammation of the kidney, or remain confined to the bladder. Such patients may die from pyæmia, septicæmia, or, as is generally the case, from uræmic coma.

In chronic urinary poisoning from obstructive lesions in the urethra or bladder, a cachectic condition may develop in connection with vesical distention and pyelonephritis, in which no fever is present, but where death with a subnormal temperature may follow instrumental interference (see Fig. 4).

Etiology.-The more frequent occurrence of urinary infection in males probably depends upon the fact that they are more often sub. jected to instrumentation than females. The anatomical structure of the female urethra, while more readily admitting of infection from the vulva or vagina, is less liable to obstructive lesions and urinary stagnation.

A case came under the observation of the writer in which the acute recurring variety of fever was excited by removing a caruncle from the female urethra. A nephritis occurred which, after persisting for several months, disappeared, leaving the patient in good health.

The fever which is seen with acute inflammatory affections of the prostate, epididymis, testicle, etc., presents no special characteristics; septic complications may occur which differ in no way from ordinary surgical sepsis. Injuries of the external genitals other than the urethra, although producing shock of a severe kind, are not followed by the chill, fever, and profuse sweating seen in acute urethral fever.

In rupture of the urethra with urinary infiltration, localized destrnction of tissue with abscess formation and pyæmia is met with, but no true urethral fever. The same holds true in intraperitoneal and extraperitoneal rupture of the bladder, and in urinary infiltration after suprapubic cystotomy.

Operations on the kidney, followed by the passage of urine mixed with pus and blood over a freshly made wound, do not produce these complications. The observation of these facts has led Bryson to make a distinction between urethral and urinary fever, and to limit the former to the manifestations following urethral instrumentation alone. So many exceptions surround the occurrence of urethral fever that it is difficult to formulate any laws regarding it. It is met with after the most careful operative manœuvre, and absent following prolonged and painful attempts to pass a tight and long-standing stricture.

Some writers have indeed claimed that slight operative interference is more apt to induce urethral fever than more severe injuries. When 
all the conditions of the urinary apparatus are taken into consideration, this statement will scarcely hold good.

It may be stated, as a rule, that in cases of long-standing stricture or prostatic hypertrophy, in chronic brain and spinal cord affections, with stagnation of urine, the conditions are more favorable to infection than in more recent urethral troubles with an intact bladder. A bladder whose contractile power is unimpaired can resist the invasion of micro-organisms, and free the urethra of toxic agents, which by remaining might readily excite febrile reaction.

Strictures with tortuous passages and diverticuli afford opportunity for the retention of urine and excellent soil for the growth of microorganisms. Internal urethrotomy on strictures posterior to the bulb are more likely to be followed by fever than operations on strictures in the pendulous urethra, although exceptions to this rule are met with. After external perineal urethrotomy, the combined internal urethrotomy with perineal drainage (Harrison), and lithotomy with proper bladder drainage, such attacks are rare. Wounds on the floor of the urethra are more frequently followed by these accidents than on the roof. Urethral fever is seldom encountered when proper precautions are taken to prevent contact of the urine with the urethral wound; this especially is true if the urine contains bacteria.

It has been noted by a number of writers, and quite recently by $\mathrm{Mr}$. Harrison, that on removing the drainage-tube after perineal operations or the retention catheter after an internal urethrotomy, the first normal micturition along the canal has been immediately followed by a chill, fever, and sweat. Mr. Harrison, while admitting the influence of microorganisms in producing the more common forms of urethral fever, is disposed to regard this variety as due to a reaction on the part of the nervous system, and not to a bacterial or toxic infection.

Aside from the presence of bacteria and pus in the urine, certain chemical changes in this fluid are favorable to urinary infection. It is well known that diabetes mellitus predisposes its possessor to local and general septic arcidents.

One of the severest eases of urethral fever terminating in recovery which ever came under the observation of the writer occurred in a patient with a long-standing diabetes insipidus. A bulbous bongie, 32 French, was introduced for exploratory purposes as far as the bulb, and was followed by a trifling hæmorrhage only. Shortly after the first urination in the evening a prolonged chill took place, which was repeated during the night, the temperature going up to $105^{\circ} \mathrm{Fahr}$. On the following morning a convulsion with rapid and feeble pulse and general cyanosis was followed by a comatose condition, persisting for three days. Irrigation of the urethra with a boric-acid solution during the second day after the 
introduction of the bougie was immediately succeeded by a tonic convulsion and a further elevation of the temperature.

It is difficult to resist the belief that some relationship existed between the diabetes insipidus and the phenomena which followed the urethral instrumentation, although the observation of an isolated case will not permit of any generalizations.

The urine which in health is an aseptic fluid in certain infectious diseases contains extractives allied to the toxalbumens and ptomaines capable of producing in animals fever, coma, and apoplexy, followed by death.

Binet has discovered a thermogenic substance in the urine in certain pathological conditions, and in normal urine to a less extent, which causes in guinea-pigs a rise of temperature from $1.8^{\circ}$ to $3.6^{\circ} \mathrm{Fahr}$.

While many conditions not understood surround the occurrence of urethral fever, it may be stated in a general way that the greater the force used to pass the urethral obstruction and the deeper in the canal it is situated, the more liable are we to produce it.

The presence of cystitis with or without ammoniacal urine, although sometimes benefited by removing the urethral obstruction to free drainage, usually renders its possessor more liable to fever after operations.

All the conditions which produce chronic retention of urine, such as old strictures, prostatic hypertrophy, vesical calculi, and tumors compressing the ureters, are predisposing causes to chronic urinary poisoning. The stagnation of urine by producing changes in the structure and functional activity of the kidneys exposes the patient to the dangers of chronic uræmia if no infection of the urine has occurred, otherwise, to one or more of the septic conditions classed under chronic urinary fever.

Pathogenesis. - The discussion of the theories which have been advanced to account for the occurrence of urinary infection has been entered into at length in the very elaborate and excellent article by Guyon, a brief reference to which can only be made in this article. Velpeau, in 1840 , was among the first to attempt to explain its nature by attributing it to the absorption of some of the constituents of urine into the general circulation. Chassaignac believed it to depend upon a phlebitis of the periurethral spongy tissue and of the plexus of the urethro-prostatic veins. Icard, in 1858, upheld this theory of Chassaignac as the general cause of the affection.

Reybard and Bonnet (of Lyons) regarded these accidents as allied to nervous shock. This theory, with some modifications, has been adopted to account for a certain class of urinary fevers by a number of German, English, and French writers, among whom may be mentioned Roser, Morris, Dittel, etc.

While explaining certain exceptional occurrences after urethral and bladder operations, these theories are not sufficiently comprehensive to 
account for the general febrile accidents met with. It is admitted that shock of a mild and severe type may occur, and that phlebitis of the urethral veins is an exceptional pathological condition, causing a pyæmia rather than a true urethral fever. A previously diseased state of the kidney, or a passing congestion of these organs interfering with their proper functional activity, caused by a reflex nervous influence having its point of departure in the urethra or bladder, were regarded by Verneuil, Philips, Marx, and others as sufficient to explain the phenomena of urinary fever. On the other hand, Perdrigeon, Civiale, Sédillot de SaintGermain, Reliquet, and Gosselin, in 1879, believed it to be owing to the absorption of urine from some lesion in the mucous membrane of the bladder or urethra. While clinical evidence sustained the view that urinary absorption was responsible for many of the accidents denominated urethral or urinary fever, the conditions which rendered the urine a bland aseptic fluid in one case and a virulent poison in another were not understood until revealed by modern bacteriological methods.

The toxicity of the urine as the result of imperfect tissue metabolism, from gastro-intestinal absorption of alkaloids resulting from the activity of putrefactive organisins (Bouchard), or from the presence of bacteria or their chemical poisons excreted by the kidneys in certain infectious diseases, may, in exceptional instances, be responsible for certain phenomena connected with urinary fever, or favor its evolution.

The rapidity of the development and intensity of the manifestations of urethral fever in the majority of cases can not be accounted for, however, on the supposition of a previous toxic urine resulting from an elaboration of a ptomaine, except as the result of a local infection of the urethra or bladder.

It is to the Necker school especially that we are indebted for the careful observations and experiments which have established the infectious theory of urethral fever on a scientific basis.

Starting with the demonstration of Pasteur in 1859, that the ammoniacal decomposition of the urine was due to the influence of organisms introduced into the bladder from without, a great number of researches have been made to determine the influence exercised on the bladder and kidneys by the action of micro-organisms.

It is recognized now that the decomposition of urea and the formation of carbonate of ammonia are brought about by micro-organisms of various kinds, and also that micro-organisms which have no power to effect this transformation of urea are the most potent agents in producing a local and general infection.

Klebs, Virchow and Lancereaux demonstrated the important rôle played by bacteria in the production of nephritis, ascending from the bladder along the ureters to the kidneys. 
Omitting reference to many important communications bearing directly on this subject, we come to the modern observations and experiments which have led to the infectious theory of urinary fever.

Clado in 1887 made a bacteriological study of a bacillus which he obtained from pathological urine. He was able with cultures of this organism to produce in animals a general infection and death by injection of them into the peritoneal cavity, and a cystitis following their introduction into the bladder after ligature of the penis. The same author obtained a similar organism from puncture of the spleen in two cases of urinary fever with which he was not able to obtain any positive results on animals.

Berlioz succeeded in producing a nephritis by injecting this organism into the blood of rabbits.

Halle found a similar bacillus in the urine, the kidneys, spleen, and blood of a patient who died as the result of attempts to pass a tight stricture. The same bacillus produced rapid death in guinea-pigs from general infection.

Albarran and Hallé, as the result of bacteriological studies of cases of urinary fever at the Necker Hospital, found this organism in forty-seven out of fifty cases of pathological urine in a state of purity or mixed with other germs.

In nineteen autopsies they found the same organism in the urine eighteen times; in peri-urethral abscesses, perinephritic abscesses, in infectious nephritides, in the blood, liver, and spleen it was found alone or associated with other micro-organisms.

These investigators succeeded in producing with this bacillus, which they named bacterium pyogenes, a cystitis, general infection, localized suppuration in the cellular tissue, and pyelonephritis with renal abscesses. They observed, during the course of a general infection, miliary cortical abscesses in the kidneys after the injection of the bacillus in the blood, and after the production of a pyelonephritis following its injection into the ureters afterward ligated.

The observations have in part been confirmed and supplemented by the investigations of others who have found other micro-organisms in the urine and kidney lesions produced by urinary fever, alone or associated with the bacterium pyogenes.

Achard and Renaut found a bacillus in the kidneys of a woman who died of nephritis, which presented all the characteristics of the bacillus coli communis (Escherich), and which they regarded as identical with this organism and with the bacterium pyogenes. This observation was confirmed by Reblaud, who recognized certain differences in the growth of the two germs on gelatin; he was inclined to think that a residence in the urinary organs had produced certain modifications in the germs. 
Krogius, after a bacteriological examination of the urine from seventeen cases, arrived at the same conclusion as Achard and Renaut regarding the identity of the bacterium pyogenes and the bacterium coli commune.

In addition to this bacillus, which seems to be a pretty constant inhabitant of the urinary organs, Rovsing has described three other microbes which are capable of infecting the kidney by way of the ureters or the blood.

While much progress has been made in the clinical, pathological, and experimental proof regarding the important part played by bacteria in the production of urinary infection, it is probable that many other microorganisms not yet described will be found, which may assist or play an important rôle in its production, and that we will soon possess more definite knowledge regarding the respective influence of the germs and their soluble products.

The rapidity of development of the chill and fever, after an injury to the urethra or bladder in certain cases of acute urinary fever, would seem to speak decidedly in favor of the absorption of a toxine already formed in these organs. It is in such cases, however, which have developed with extreme rapidity and proved rapidly fatal that the presence of microbes has been demonstrated in the blood. The communication of the urethra with the air, its frequent seat of chronic inflammation, and the excellent culture medium afforded by the urine, especially in cases of incomplete retention, offer the most favorable condition for the propagation of germs either introduced spontaneously or by instruments. The changes which have taken place in the bladder wall in long-standing obstructive lesions with residual urine and the congestion of the blood-vessels, both in the bladder and kidneys, following the too rapid removal of the support to the vessel which such retained urine afforded, is soon followed by local and general infection unless care is used in antisepsis and in the gradual emptying of the bladder. It is in such cases also that an ascending bacterial pyelonephritis most frequently occurs.

It is difficult to explain why, under the same conditions, one patient will rapidly develop febrile manifestations following the slightest interference, while another will escape such reactions after prolonged and careless instrumentation. Under these circunstances it is necessary to consider certain variations in the virulency of the micro-organisms as well as in the resisting power of the patient. Albarran and Halle have noted marked differences in the infective power of the bacterium pyogenes, where the cultures from different sources were placed under the same conditions. In a case of gangrene of the penis this bacillus was found in a state of purity, and gave rise to localized gangrene when injected into the cellular tissue.

Guyon, having observed that many patients with long-standing sup- 
puration of the bladder were exempt from general febrile accidents following operative interference, has proposed the theory that they have acquired immunity by a kind of auto-vaccination from absorption of toxines or bacteria in a state of modified virulency.*

Diagnosis.-As a rule, it is a matter of no difficulty to establish a diagnosis of the various forms of urinary infection.

A patient who is the subject of an obstructive disease of the urinary apparatus, and at the same time exposed to malarial poisoning, may develop a train of symptoms which are often referred to the wrong cause. The fever and chills which depend upon a spontaneous urinary infection are irregular in their manifestation and are uninfluenced by quinine. The loss of appetite, vomiting, diarrhœa, and emaciation which occur in the chronic form of urinary fever may be and often are attributed to chronic gastro-intestinal infections rather than to their true cause. An examina-

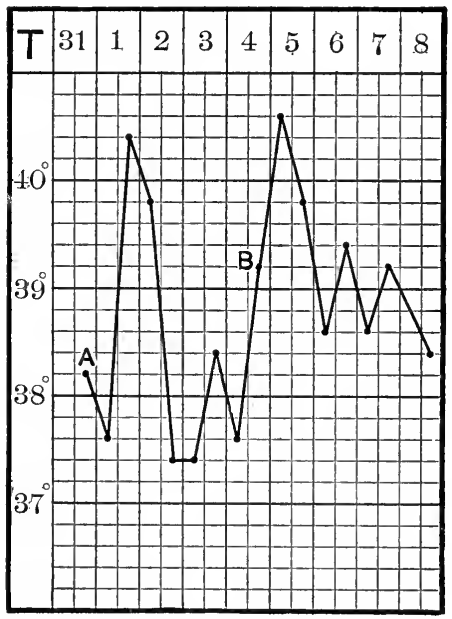

FIG. 5.-Combined renal infection by microcoeei and the bacterium pyrogenes in a patient with stone in the bladder. Attempted lithrotrity (A), followed by rapid elevation of temperature; suprapubie eystotomy (B). Death after four days. (After Albarran.) tion of the bladder and of the urine should be sufficient to guard against an error in diagnosis.

Prognosis.-While the prognosis in the acute urinary fever with a single paroxysm is generally favorable, the outlook in the recurring variety is not so good, as the repetition of the attacks indicates, as a rule, the secondary involvement of the kidneys. In a certain percentage of cases, even when the kidneys are affected, a favorable termination may be looked for. In case the kidneys, the seat of one of the forms of nephritis due to obstructive disease, are in addition infected by the microbes in the blood (descending nephritis), the prognosis is extremely grave. (See Fig. 5.)

A young man who develops a febrile paroxysin after dilatation of a stricture or internal urethrotomy has infinitely more chance of recovery than an old prostatic with long-standing residual urine. In the latter the prognosis depends on the duration of the obstruction, the changes which have taken place in the urinary organs, and the amount of residual urine.

* For a more extended account of the pathogenesis of urinary infection, the reader is referred to an article by Dr. Noël Hallé, in the February (1892) issue of the Annales des Maladies des Organes Génito-Urinaires, of which liberal use has been made in the foregoing article, and to a report by Prof. Guyon in the May number of the same journal. 
The temperature is not always to be considered as of decisive prognostic value, as death may be preceded in subjects of long-standing obstructive trouble by a condition of apyrexia or by a subnormal temperature. Malignant forms of urinary fever begin with a prolonged and intense chill, sometimes repeated, followed by a high fever, convulsions, asphyxia, coma, and death, with suppression of urine. The intensity and rapid sequence of the symptoms speak in favor of a quickly fatal result.

Treatment.-A recognition of the infectious origin of the various forms of urinary fever, and of the conditions of the urinary organs underlying their development, has proved of signal prophylactic service.

A correct asepsis of all instruments introduced into the urethra or bladder, the use of Thiersch's solution or other antiseptic in irrigating the anterior and posterior urethra and the bladder, the internal use of boric acid (suggested by Dr. E. R. Palmer), salicylic acid, salol or benzoic acid for a period of twenty-four to forty-eight hours before operating, have, in the hands of many operators, reduced the frequency of these accidents to a minimum.

Bryson states (loc. cit.) that since adopting these measures he has had a comparative immunity from the urethral fever. In addition to the precautions mentioned, he carefully washes the glands and prepuce with Thiersch's solution before operating, uses borated glycerin only as a lubricant, and boils his instruments in a soda solution. The rules which govern the performance of operations on the urethra or bladder should be followed, care being taken not to use too large instruments in beginning the progressive dilatation of a urethral stricture or in relieving a distended bladder, so that an abrasion or tear of the mucous membrane may, when possible, be avoided. The urethra may be irrigated with Thiersch's solution after an internal urethrotomy when indications do not point to the performance of an external perineal urethrotomy or the use of a retention catheter.

In beginning the systematic use of the eatheter in prostatics, all the precautions should be taken which have been mentioned; in addition, one should avoid completely emptying the bladder, or replace a part of the evacuated urine with some mild antiseptic, in order to avoid the congestion of the bladder and kidneys which is apt to follow the withdrawal of the accustomed support to their blood-vessels. Should urinary fever develop in spite of the precautions used, the patient should be kept warm in bed with blankets and hot-water bags, and an effort made to encourage perspiration, which is the natural termination of a paroxysm, by the use of hot drinks and jaborandi. After the attack is over, a saline purgative may aid in eliminating the toxic agent. Little faith is now placed in quinine, aconite, or other remedies, which formerly were recommended as prophylactic or curative in this affection. 
In the subject of renal insufficiency from obstructive disease, the use of milk with Vichy water, with careful attention to the condition of the alimentary canal and the functional activity of the skin, constitute acces sory measures of great value.

\section{BIBLIOGRAPHY.}

Sir Henry Thompson. Clinical Lectures on Diseases of the Urinary Organs, London, 1888.

Mitchell Banks. Edinbnrgh Medical Journal, June, 1871.

Lustgarten und Manneberg. Viertelesschrift f. Dermat. u. Syph., October, 1887.

Rovsing. Die Blasenentzündungen. Translated from the Danish. Berlin, 1890.

Legrain. Les microbes des écoul. urèth. Thèse de Nancy, 1888.

Giovannini. Die micropar. des männl. Harnnöhrentrip. Centralbl. f. med. Wiss., No. 48, 1886.

Wasserman et Petit. Annales génito-urin., 1891.

Albarran. Étude sur le rein des urinaires, p. 122. Paris, 1889.

Guyon. Leçons clinique sur les maladies des voies urinaires, second edition. Paris, 1885.

Morris. Surgical Diseases of the Kidneys. London, 1885.

Bryson. Influence of Asepsis and Antisepsis on the Prophylaxis of Urethral as distinguished from Urinary Fever. Journal of Cutaneous and GenitoUrinary Diseases, September, 1892.

Reginald Harrison. Remarks on some Forms of Acute Urine Fever in Surgical Practice. London Lancet, May 21, 1892.

Binet. La Semaine Médicale, August 5, 1891.

Guyon. Loc. cit.

Velpeau. Leçons orales de clinique chirurgicale, 1840, vol. iii, p. 324.

Icard. Des rétrécissements de l'urèthre. Thèse de Paris, 1858.

Reybard. Traité pratique des rétrécissements du canal de l'urèthre, 1853.

Bonnet (of Lyons). Leçons orales citées par Bron. Gaz. Méd. de Lyon, 1868.

Roser. Archiv der Heilkunde, 1867.

Morris. Loc. cit.

Dittel. Die Stricturen der Harnröhre, 1880.

Pasteur. Des éléments figurés comme cause de l'urine ammoniacale. Ann. de phys. et de chimie, 1859 .

Klebs. Handbuch der pathol. anatomie, vol. i, Berlin, 1868.

Clado. Étude sur une bactérie septique de la vessie. Thèse de Paris, 1887.

Berlioz. Recherches clinique et expérimentales sur le passage des bactéries dans l'urine. Paris, $188 \%$.

Hallé. Bull. soc. anat., October, 1887.

Albarran et Hallé. Note sur une bactérie pyogène et sur son rôle dans l'infection urinaire. Acad. Méd., August 21, 1888.

Achard et Renaut. Société de biologie, December 22, 1891.

Reblaud. Société de biologie, December 29, 1891.

Krogius. Note sur le rôle du bacterium coli commune dans l'infection urinaire. Archives de Méd. expérimen., January, 1892.

Rovsing. Loc. cit 


\section{ON CYSTOSCOPY.}

By WILLY MEYER, M. D.

History. - The problem of illuminating and inspecting the human bladder has been practically discussed by many medical men within the present century. A number of interesting instruments have been constructed by them for this purpose, and are to be found in the armamentarium of genito-urinary surgery. The invention of later years is always more or less based upon the principle and merits of the one of earlier date, and shows the author's desire to simplify the difficult task before him. The attempt has been made to explore visually various cavities or canals of the human body with the same new instrument. Thus, bladder and urethra were considered together, especially in the early beginning of this new endoscopic research.

We will in our brief perusal, as far as feasible, confine ourselves to the more important instruments which have been devised to facilitate the examination of the bladder-cystoscopy. But we must not fail also to make due mention of the work of the pioneers in this formerly dark field of surgical diagnosis, no matter of what little "practical" value it may be to-day.

In 1807, an article by Bozzini, a German physician of Frankfort-on-the-Main, was published at Weimar, with the title "The Light-Conductor, or Description of a Simple Contrivance for Illuminating the Internal Cavities of the Human Body."

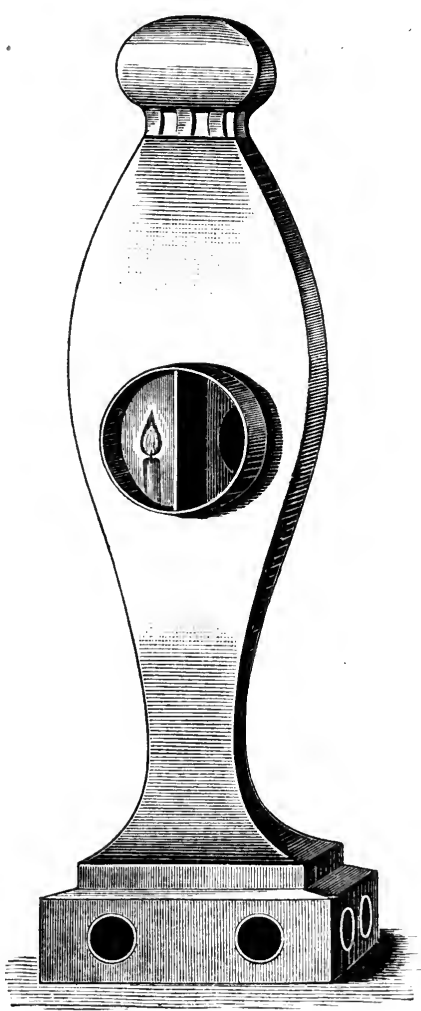

FIG. 1. The instrument devised by him is described and illustrated in this paper. It consists, briefly, of the chamber which contains the light and of the light-conductors, differently shaped 
for the various organs. The former (the light-conductor, Fig. 1), of the shape of a vase, presents a round opening at its anterior wall, which is divided into two equal parts by a vertical septum running through to the posterior wall. From the one emerge the rays of the candle which distributes the light; through the other these return to the eye of the observer, which is to be posted at an opening in the posterior wall of the instrument. There is a projecting ring at the anterior opening on which fit the various light-conductors. These are introduced into the cavities of the body. The light was to be thrown through the conductor into the respective cavities and reflected from its walls into the observing eye.

Although these instruments did not and could not well find general recognition (they were even condemned by the Vienna faculty), they - certainly mark the beginning of the many to-day so well-developed endoscopic methods.

Bozzini's instruments and article had long since fallen into oblivion, when Ségalas (Rev. méd. France et Etrang., Paris, 1827, i, 157) presented his "speculum urethro-cysticum" to the French Academy of Medicine in 1826.

Two years previous John Fisher had completed his instruments for inspecting the urethra. On the principle on which these were based, Désormaux, "the father of endoscopy," constructed his endoscope, which was laid by him before the Academy of Paris in 1853. He succeeded by his enthusiasm to also interest others in the endoscopic examination of bladder and urethra. His well-known work, De l'endoscope et de ses applications au diagnostic et au traitément des affections de l'urèthre et de la vessie, Paris, 1865, was the result of many years' hard work and studies. As this endoscope has been the model for a long series of later instruments, it deserves a short description.

As seen in Fig. 2, a lamp, which furnishes the light, occupies the greatest part of the apparatus. At a level with the light runs a transverse tube, which again carries another one attached to it in a right angle. This latter one can be turned around the transverse part in such a way that it runs in a horizontal line when the observer looks through it (Fig. 3). Its anterior (in Fig. 2 superior) opening carries the ocular lens, and a little below its middle the perforated reflector just opposite the lamp. With the help of an optic apparatus the light is concentrated before it reaches the mirror. It is then reflected from it toward the posterior (in Fig. 2 inferior) end of the tube, and further on, by means of the endoscopic tube which is fastened to the tube by a screw, into the urethra (Fenwick, The Electric Illumination of the Bladder and Urethra, second edition, London, 1889 , pp. 3-10). The manifold modifications, or rather improvements, of this endoscopic apparatus may be divided into those which maintain the original as a whole and only change the light (Cruisc, 
of Dublin, and Fürstenheim, of Berlin, substituting petroleum; Stein, magnesium), and into those which divide the complicated apparatus into its various components: the lamp or light and reflector and endoscopic

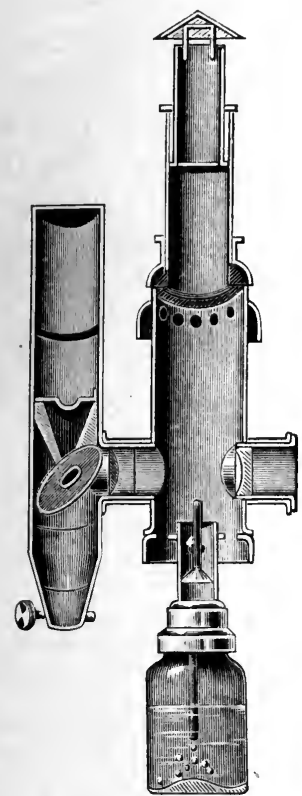

FIG. 2.

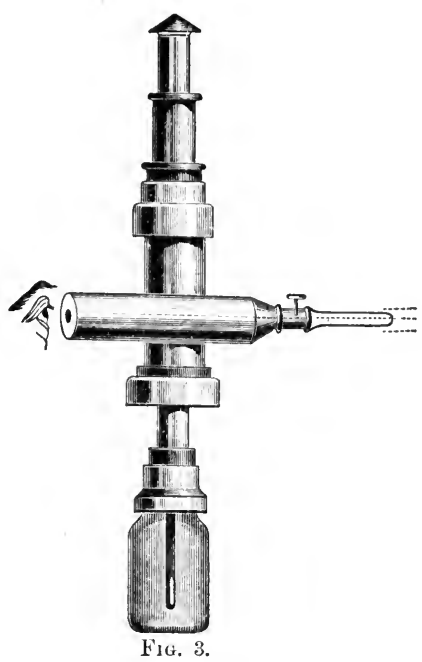

tube. This division is by far handier. Among the host of others who tried the latter in every imaginable variety, Gruenfeld, of Vienna, deserves to be especially mentioned. He has greatly improved the endoscopic tube.

Quite original and different from the former was the method of Bruck, a German dentist of Breslau, Silesia, who proposed to effect the illumination of various parts and cavities of the body by making use of the diaphanic qualities of the tissues (Das Urethroskop und Stomatoskop durch galvanisches Licht, Breslan, 1867). To examine the bladder, he advised to introduce a strong light-the white-hot platinum wire-into the rectum and a tube through the urethra into the bladder. Through the latter he then hoped to inspect the diffusely illuminated interior surface of the bladder. The heat produced by the hot wire was to be counteracted by a continuous circulation of cold water around the latter. It is not known whether Bruck or any other man ever carried out this plan.

Nine years later, in 1876, Dr. Max Nitze, of Dresden, now of Berlin, began his studies. He proposed and at last introduced two new principles into the method under consideration-the introduction of the light 
itself into the cavity to be examined, and an optic apparatus which magnifies the spot coming into view.

It is the combination of these two devices which has mainly effected the immense progress of recent times. Nitze accepted the incandescent platinum wire for illumination which had been proposed by Bruck, but his first instruments remained quite defective. They were improved, however, and made available for use when Nitze moved to Vienna and interested the well-known instrument-maker of that city, the late Joseph Leiter, in the matter. By the combined work of these two ingenious men, of which the patience, perseverance, and technical skill of Leiter was by no means the least important factor, the "cysto-urethroscope of NitzeLeiter" was at last completed, after many months of expensive and troublesome experiments. On the 9th of May, 1879, Nitze presented it to the Society of Physicians at Vienna (Wiener med. Wochens., 1879, No. 24, and Wiener med. Presse, 1879, No. 26).

Later a controversy arose between Nitze and Leiter in regard to the invention of the instruments. There can, however, be no doubt that Dr. Max Nitze is the real inventor. The medical profession is greatly indebted to him for his continuous efforts, which now cover a period of sixteen years, to improve the instrument, and for his never-abating zeal to construct, in addition to the original cystoscope, others which might prove useful in diagnosis of urinary troubles and in the treatment of bladder-diseases. Leiter's work will also be forever appreciated; but we must never forget that the modern cystoscope is "Nitze's cystoscope." All the others are variations of his original ideas, whether manufactured at Vienna or at Paris.

Instruments.-As the fundamental construction of the original cystoscope of 1879 is the same as that of newest shape, it may be described here.* As seen in the accompanying sketch (Fig. 4), it consists of a silver tube of the shape of a catheter, in the short beak of which a platinum wire is fastened. The latter is made incandescent by means of an electric current, which passes through it and darts its rays upon the wall of the bladder throngh an open window in the concavity of the beak covered with a pane of rock-crystal. To convey the current of electricity to the platinum, an insulated wire runs through the whole length of the shank; the metal of the tube forms the connection with

* In the following, and throughout this treatise, I have freely used the material contained in my former articles on cystoscopy: On Cystoscopy, and the New Cystoscope of Nitze and Leiter, New York Medical Journal, A pril 20, 1888; A Contribution to the Surgery of the Bladder, New York Medical Journal, February 23, 1889; Review of Nitze's Text-Book on Cystoscopy, Annals of Surgery, June, 1890; and The Progress of Cystoscopy in the last Three Years, New York Medical Journal, January 30 and February 13, 1892. 
the opposite pole. The platinum wire is surrounded by a combination of pipes, through which a current of cold water runs to keep the heated instrument perfectly cool. They measure only one millimetre and a half in diameter, and pass through the whole length of the sound, entering at its collar. An optic apparatus is introduced into the straight silver tube.

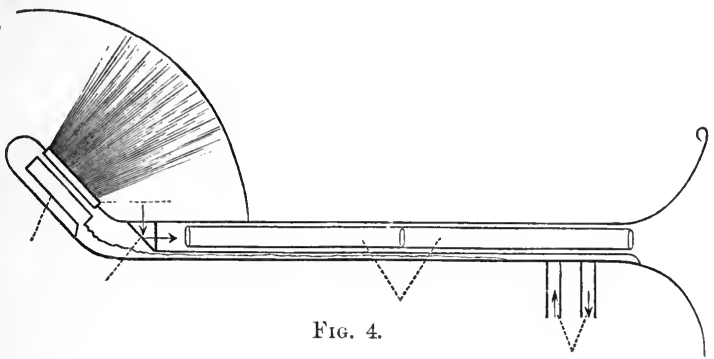

The combination of its lenses enlarges and magnifies the spot which comes into sight.* Without it we should not see much more at the time than a spot of about the size of a pea; with it we are enabled to inspect a portion as large as a silver dollar, and even more. At the junction of beak and shaft, corresponding to the concave side, a rectangular prism is cemented in, the hypothenuse-plane of which acts as a mirror on account of the total reflection of the rays. Thus is developed in the shaft a diminished inverted real picture of that wall of the bladder which is situated at a right angle to the longitudinal axis of the instrument and opposite the prism. It is again inverted by means of the lenses of the telescope and thrown to its outer end, where the examining person looks at the now upright picture through the magnifying ocular of the optic ap-

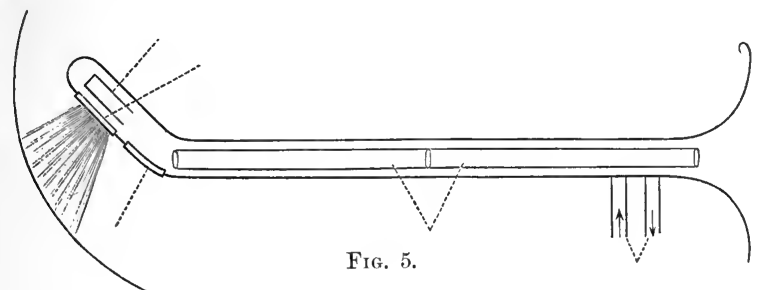

paratus. At the middle of the upper circumference of the funnel-shaped handle, called "funnel," a small knob is soldered. By this the operator can always tell to which side the beak points.

If the fundus of the bladder is to be inspected with this instrument, it must be turned $180^{\circ}$, and its handle in some cases slightly, in others

\footnotetext{
* It is by others called "telescope," to which term, however, Nitze emphatically objects. It is no telescope in the ordinary sense.
} 
deeply, depressed between the thighs of the patient, who is put in the recumbent (lithotomy) position, the best for examination with the cystoseope. This manipulation is sometimes painful, especially in patients with a sensitive or enlarged prostate. To avoid this, a second instrument (Fig. 5) is made with the window for the incandescent platinum on the convex side of the beak. There is another window at the end of the straight tube, through which the observer looks with the optical apparatus. Of course, there is no prism.

In using the original cystoscope there were needed, besides a Bunsenbattery with a special fluid, a rheostat, to give the current with the necessary strength in each case, and a reservoir containing about two or three gallons of cold water; the latter continuously circulated around the heated platinum wire in order to keep the instrument cool. These accessory apparatuses complicated the instrument very much, and made it cumbersome and very expensive. In consequence of this, cystoscopy was formerly rarely employed. The new instrument was only bought by some larger clinical institutions and hospitals, and used by a few specialists and surgeons who interested themselves in the investigation of genito-urinary diseases, such as Nitze, von Dittel, Nicoladoni, Ultzmann, Sir Henry Thompson, and Maas. They all, however, repeatedly praised the method as a splendid means of diagnosing diseases of the bladder by direct inspection.

The enormous advance in electro-technique made in the last few years also improved the cystoseope. Edison's lamps of a very small caliber, the so-called mignon lamps, took the place of the platinum wire, and did away with the many unhandy accessory apparatuses, especially with the water-pipes. The new cystoscope is very handy, and not expensive. It is not larger than an ordinary short-beaked calculus sound (Mercier's), No. 22, French gauge.

In adopting the Edison lamp for illumination, Nitze and Leiter entered into a very bitter polemic, which later also extended over the claim to priority in regard to the invention of the original instrument. The consequence was, that Nitze had his modern cystoscope manufactured by Mr. Hartwig, of Berlin (Markgrafenstrasse 79), whereas Leiter produced another one at his factory, of course following Nitze's original ideas. Although it varies from Nitze's only in some trifles, it has entered the market and come into the hands of many medical men as "Leiter's cystoscope." This is a wrong term and an injustice to Nitze, as far as priority is concerned, which is now by all unhesitatingly conceded to Nitze. The latter therefore demands that the Leiter-instrument be called the Nitze-cystoscope, manufactured by Leiter. I agree with him.

The Modern Nitze-Cystoscope and its Varieties, manufactured by Hartwig, Berlin. - Nitze put the mignon lamp at the end of the beak unprotected. He found that the lamps are very strong and do not break by shock 
or heat-a fact which has been, moreover, corroborated by a five years' experience of many cystoscopists. This arrangement permitted the use of larger lamps, and gives a free opening for the light.* It became possible also to make the beak shorter than before, as it is found in Mercier's catheter-a very important point. The lamp is fitted in a silver tip, which can be easily screwed on or off the end of the beak. Thus a lamp whose carbon-filament has given out or has been destroyed can be replaced in a moment (Fig. 6). The ocular end of the so-called funnel presents at its

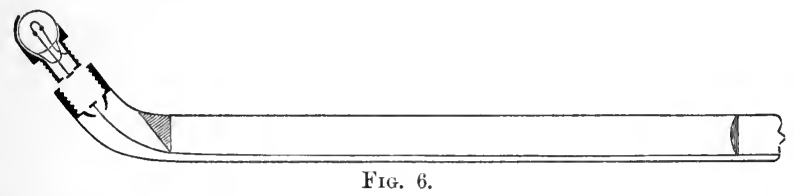

base two isolated metal grooves (see Figs. 9, 10, and 11.) To these are attached the so-called tongs, a metal handle which is connected with the battery wires and carries a slot-key on its surface. This key slides under the pressure of the thumb, and makes or breaks the current. The lenses of the optic apparatus of the cystoscope give a very nice perspective picture, and cover a larger field than those of the other instruments to be mentioned later.

Nitze recommended three different cystoscopes for a thorough inspection of the entire inner surface of the bladder :

Cystoscope No. 1, which carries the lamp and prism at its concave side (the latter at the junction of beak and shaft). It represents the main instrument, "the" cystoscope, and is the one which is generally sold (Fig. 7). With it we are in most cases able to inspect the entire

FIG. 7.

bladder. The shaft of this instrument is also made one inch longer. This will be found of advantage in many instances, especially in cases of hypertrophy of the prostate, when the urethra is materially lengthened.

Cystoscope No. 2, for the inspection of the fundus, constructed according to the principle of the second one of 1879. The beginner

* To heat the beak as little as possible the carbon filament of the lamps is longer and thinner than in the ordinary mignon lamps. On this account it offers more resistance to the current, and requires more electromotive force (9 to 10 volts) than that sold with the Vienna instrument ( 6 volts). To preserve his lamps, the cystoscopist should make it a rule always to determine the electromotive force required by each lamp previous to examination. 
should not buy this; the experienced cystoscopist may need it in a few instances.*

Cystoscope No. 3, for illumination of the internal orifice of the urethra and its immediate neighborhood: lamp and prism on the concave side of the beak, which latter is about half an inch longer than in the others, and bent in nearly a right angle to the shaft. A small mirror, situated at the convexity of the curve inside the tube, reflects the picture which is thrown into the telescope from the reflecting plane of the prism. The picture seen with this instrument is indistinct. It is to be hoped that it will soon be improved. Although we are able to diagnosticate the hypertrophy of the prostate "in the picture" with No. 1, yet a thorough inspection of the internal urethral orifice and its surroundings under electric illumination would be very valuable in a number of cases.

The Irrigating Cystoscope.-This excellent instrument permits of changing the fluid in the bladder without itself being removed + (Centralblatt für Chirurgie, 1889, p. 949). It contains (Fig. 8) two small tubes in its shaft, which is thus increased in size to 25 gauge, French. (The size of the beak is 22 , its length also the same, as in the others.) The one tube ends just in front of the prism, with three small holes side by side $(a)$. It carries fresh water into the bladder. The other tube ends, or rather begins, with a single oval-shaped opening at one side of the lower end of the shaft $(b)$; through it the water passes out of the bladder. Both tubes are carried to either side of the upper end of the instrument $(c, d)$, and their current can there be shut off by a small stop-cock. Both these stopcocks are attached below the funnel to a metal ring (e), which also carries the handle with the slot-key. To put the whole into working order, rubber tubes are attached and tied on the end of the two tubes of the instru-

* Nitze has lately constructed No. 2 according to the plan brought out in his No. 3 and thus removed the annoying sharp angle which is found in No. 2 of the old pattern (Fig. 8).

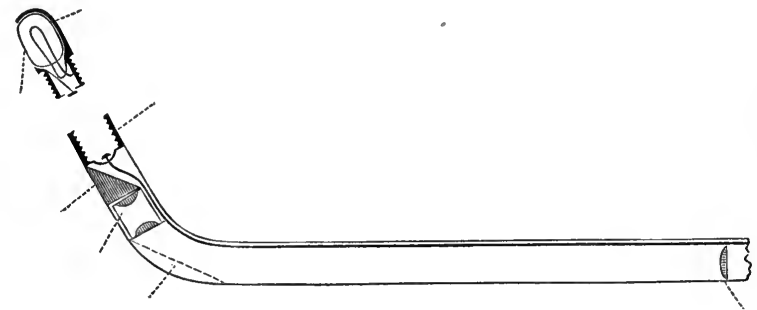

FIG. 8.

† Nitze had two irrigating cystoscopes made, a "simple" and a "more complicated" one. The former, oval in shape, only permits of throwing more water into the bladder; the latter, which is round, enables us to really change the water. Only the latter is to be recommended. 
ment. It will be found of advantage to have the one which carries the water out of the bladder cut so long as to hang into a basin under the table. If the water is changed and the examination continued-which is frequently of great importance-an assistant or the patient must pump the water in. This latter manipulation is best done intermittently by sudden brief pressures on the handle of a syringe.

Advantages.-1. The instrument is round. 2. Its caliber is only three numbers larger than No. 1 . 3. It is easily handled. 4. Irrigation can be effected without regard to the turning of the shaft. 5. The water, thrown in with the help of a hand-syringe, passes with considerable force over the surface of the prism, thus washing it and removing bloodcoagula or pus shreds, which so frequently settle there and render a successful cystoscopy impossible. This is a very clever arrangement. 6 . It often enables the observer to diagnose the solidity, and especially the insertion of a growth. The jet of water propelled across the prism and beak will make a pedunculated growth swing, while it leaves the sessile growth undisturbed. (The same result can sometimes be obtained in using cystoscope No. 1, by pressing with one hand in sudden short shocks on the epicystic region.) 7. In cases where papillomatous growths, inserted around the internal urethral orifice, cover the lamp and prism of the instrument as soon as it has entered the bladder, and darken the field of vision, thus rendering an examination impossible, the forcibly injected fluid pushes them aside. 'They will then be suddenly seen in bright illumination, swaying in the fluid. 8. Concrements and foreign bodies lying in the pouch behind a hypertrophied prostate gland, and not to be detected there by the examining eye, can sometimes be thrown by the water ont of the recessus and thus diagnosticated. 9. The instrument enables us to view the bladder in different degrees of distention. In a certain number of cases the ureteral

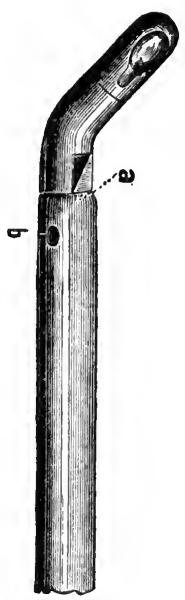
openings can be seen only by this means. It also enables us, by letting off some of the fluid in the bladder during the examination, to finish the exploration of a very obstinate one in cases where No. 1 would decidedly fail. 
Drawback.-The three holes in front of the prism, through which the fresh injected water is intended to enter the bladder, are too small. It takes quite a while before one single ounce has passed them. I therefore inject only a very small quantity by this way first, just enough to wash off prism and lamp, and make the rest pass the larger tube, which generally gives exit to the fluid contained in the bladder. This is, of course, not quite as convenient. The arrangement for irrigation does not enable us, however, to flood the viscus or wash it out in the ordinary sense. Still, I have found it sufficient and of the greatest value in enabling me to detect an obscure bladder trouble, as well as in the diagnosis of renal disease, pyuria and hæmaturia. In examining the bladder of patients who suffer from such diseases, the originally transparent medium becomes rapidly and suddenly turbid, and the outlook is at once clouded by a dense fog; nothing can be distinguished. I succeeded, in one of these cases, where I had to perform nephrectomy for pyonephrosis and cystic degeneration, and therefore wanted to determine the condition and excretory power of the remaining kidney, in establishing the fact, just after fresh water had been thrown into the bladder, that the urine descending from the other kidney was clear. In other troublesome cases I could make out only with the help of the irrigating cystoscope that the large amount of pus which always turned the injected water murky in a few seconds was ejected from one ureteral orifice only. In abundant vesical hæmaturia, where the blood more easily coagulates, the irrigating cystoscope of the present size will be of little or no use at all, as the blood-clots generally block at once the canal which carries the water out of the bladder. Fresh water is then pushed into the vesical cavity, but the turbid fluid can not get out. If the hæmaturia be slight, the irrigating cystoscope is the only one which will enable the observer to finish the examination to his satisfaction.

The instrument just described is indispensable with proper cystoscopic work. I use it wherever I can, and would advise to only buy this one, were it not that on account of the slightly increased size of the shaft its use is only practical when the urethra is of a certain dimension (25 French).

Advantages of the Berlin Pattern in general.-Short beak, long shaft, nice perspective picture, variety of useful instruments.

Drawbacks.-1. A somewhat less brilliant light.* 2. The silver tip, the carbon filament of which has given out or has been destroyed, has to be sent to Berlin for putting in a new incandescent lamp. If in every large city an instrument-maker would take a number of new silver tips

* Still the light is white and entirely satisfactory in every case. Where we can not see with it for special reasons, we shall most probably also not succeed with one manufactured elsewhere. 
in stock so that useless tips could there be exchanged for new ones, this disadvantage would at once be remedied.* 3 . The screw of the different tips in stock will sometimes be found not to fit exactly on the thread at the lower end of the shaft; now and then it overruns the limit of screwing, or does not reach it at all. The surface of the lamp then points to another direction than the prism. The tip becomes simply useless. This is no real drawback to the instrument proper, but a mistake which can and has to be remedied by a greater accuracy of the instrumentmaker. The latter must have a standard instrument on which the tips, before being sent away, should be carefully fitted. $\dagger$

The Operating Cystoscope (Centralb. für Chir., No. 51, 1891, page 993). A cutting forceps is attached, by a peculiar mechanism, to the

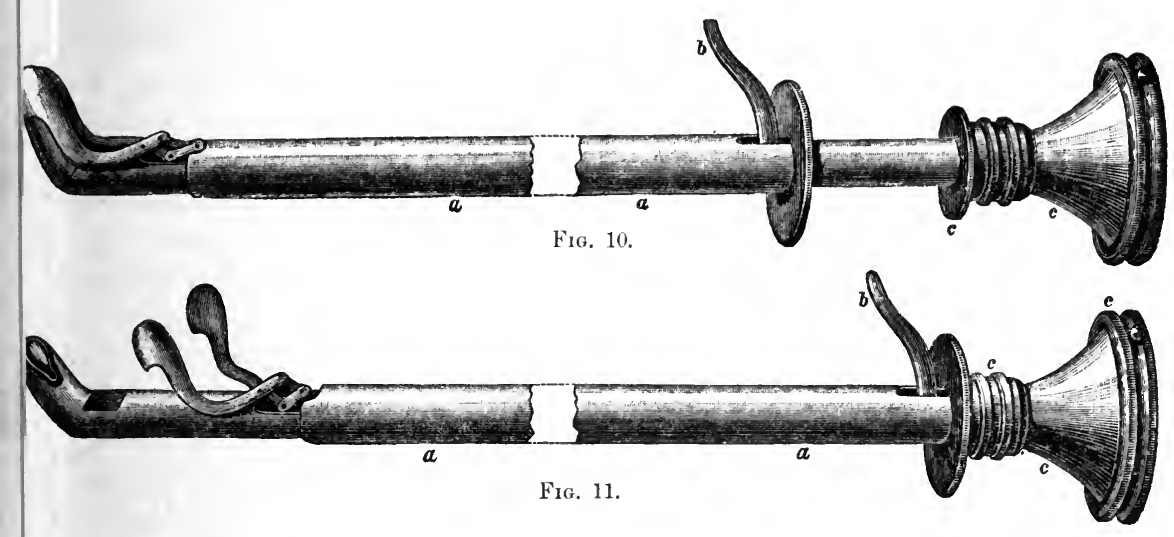

lower circumference of a cylindrical tube. Its two blades carry at their end a small, sharp scoop. They are opened and closed by a lever, which moves in a longitudinal slit at the upper end of the same tube. The whole is slipped on the shaft of the ordinary cystoscope No. 1, and can be moved on it, downward and upward (see Figs. 10 and 11). A few more instruments of a similar pattern have been designed for intravesical topical treatment.

To enable one to disinfect these different instruments, and also to

* I have tried to induce the Edison Manufacturing Company, of New York, to make these lamps. They have answered that they "can not at present undertake their manufacture." Mr. Hartwig has lately (August 10, 1892) informed me that the tips are now made of Nickelin, which is so much cheaper than silver that burned-out lamps can be thrown \&way with the tips. The expense is not greater, considering the necessary outlay involved by sending the silver-tips to Berlin and back, duty, etc. The main reproach to the Berlin pattern, made by cystoscopists not residing in Germany, would thus be eliminated.

$\dagger$ After some experience, with proper care and a good battery the same tip can be used for many months. 
make the armamentarium which is needed for intravesical surgery as simple and comparatively cheap as possible, the funnel of the cystoscope has been made movable. It can be screwed on or off the shaft. Thus, we can use the same cystoscope for all the manipulations, and only need a number of the cylindrical tubes, which carry the proper mechanism. The cystoscope for this kind of work has, besides the movable funnel, a smaller caliber and a longer shaft than the ordinary No. 1. The instrument armed with the forceps has the size of an evacuator as used in litholapaxy; if armed with the other accessories, it corresponds to about Nos. 21 to 23 of the French gange.

Before introducing the operating cystoscope, the cylindrical tube $a$ is shifted down toward the prism, and the forceps (for instance) closed by pressing the lever $b$ upward. The two blades then surround the beak in such a manner as to form one solid body with it (Fig. 10). The instrument will now easily pass a urethra which is not too narrow. As soon as it has entered the bladder the tube is slipped back toward the funnel of the cystoscope (Fig. 11), the light is turned on, the forceps, etc., opened, and everything is ready for the work. In looking through the telescope, the motion of the two blades of the forceps or of the cold or red-hot wire of the snare-écraseur can be thoroughly observed and controlled by our eyes.

This new instrument is not yet completed. It will doubtless greatly advance cystoscopy. The main improvement in the special cystoscope is, that the instrument, which hitherto could only be used for diagnostic purposes, can also be used for local intravesical treatment. With the same precision as in laryngoscopy, we can apply medicinal solutions to definite areas of the bladder mucosa without touching any other spot; we can cauterize (with the galvano-cantery) ulcers and flat tumors ; * can tear off pedunculated growths with the forceps; can seize and extract foreign bodies, small stones, or the fragments of larger ones, which have been previously crushed. And all this under the direct guidance of our eyes.

Cystoscopes according to Nitze's Principles, manufactured by Leiter, of Vienna.-The So-called "Leiter Cystoscope."-In modifying the original cystoscope, Leiter adhered to the old pattern of 1879 (Fig. 12). He re. tained the hood with the oval window for the passage of the rays of light, closed by a piece of rock-crystal (Fig. 13). It can be screwed off the

* Of course, this can not be done if the bladder is filled with water. For such purposes it has to be expanded by air. As Nitze's lamp is situated in the cxtreme end of the beak, and heats comparatively little, it can burn in the open air for about two or three minutes without spoiling the prism. I presume Nitze intends to proceed in this manner. He promises, in his preliminary article, soon to give full particulars in a more elaborate essay. 
shaft of the instrument in order to allow of access to the incandescent lamp, which has been substituted for the platinum wire. The terminals of this lamp fit into two sockets, which are in direct communication (by means of insulated surfaces) with the battery (Fig. 14). The carbon-filament of the mignon-lamp in the Vienna cystoscope is less delicate. Fol-

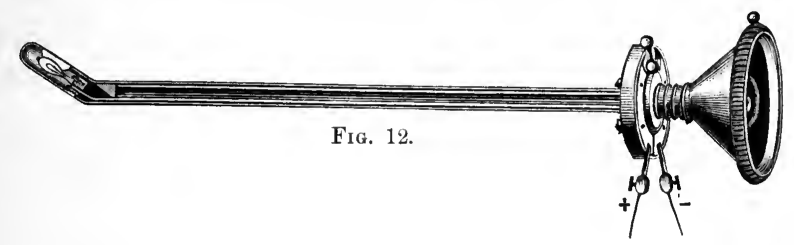

lowing suggestions of Hurry Fenwick, of London, many essential advantages of the Berlin instrument have later been secured, while many defects were eliminated. The length of the beak is now reduced to less than one inch. The elbow is rounded. The length of the shaft is increased to seven and a half inches. Fenwick also proposed to change the connec-

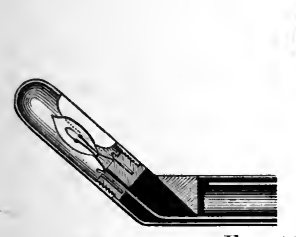

FIG. 13.

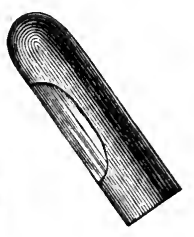

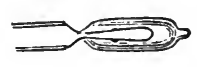

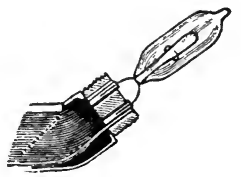

FIg. 14.

tion with the battery. The ocular end is now fitted with a rotary plate, carrying the binding screws for the battery wires; a small screw upon the face of this plate forms the switch (Fig. 15). In the instrument of 1887 the beak was nearly half an inch longer, the elbow presented an

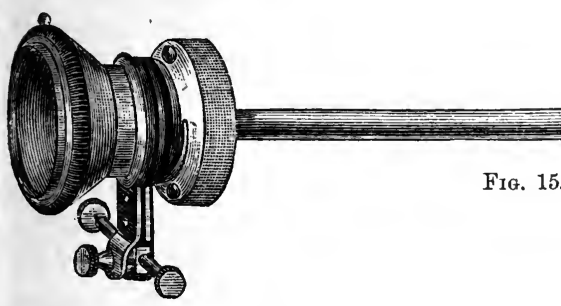

angle, the shaft was too short, and the battery wires had to be fastened in the binding screws, which were immovable upon the instrument, and thus would twist if the latter was turned around its longitudinal axis in the bladder (Fig. 12). The instrument is elegant and highly finished.

Advantages.-1. Carbon-filament of mignon-lamp less delicate ; gives a bright light; requires less electromotive force. 2 . If one lamp is de- 
stroyed, only the lamp itself is changed; this is less expensive. 3. Every lamp fits (in the sockets).

Drawbacks in comparison with the Berlin instrument: 1 . The lamp heats the beak more. 2. The beak is one quarter of an inch longer. 3. Length of that part of the shaft which alone must be considered with reference to the length of urethra (from inner brim of funnel to lower border of prism) is one inch shorter. 4. Optic apparatus slightly diminishes. Its lenses give a less perspective picture and cover a smaller field.* 5. Only one useful instrument is offered (corresponding to the Berlin pattern No. 1 short).

Fenwick's Modification.-Instrument as before. Silver cap perfo-

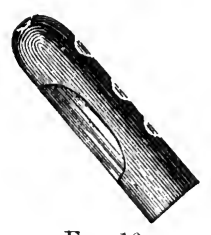

Fig. 16 rated by three small holes on the side opposite the pane of rock-crystal, "to allow of a free current of water to surround the lamp, whereby the hood is kept perfectly cold" (Fig. 16).

Advantage.-Impossibility of burning the mucous membrane of the bladder, if examination of the anæsthetized patient is performed.

Drawbacks.-1. Can not be used in cases of hæmaturia (a non-perforated hood must therefore also be on hand. 2. More lamps are broken in its use. In buying a Vienna cystoscope it will be advisable to also order the perforated cap, which is, no doubt, a useful addition.

Variation introduced by Whitehead, of Manchester.-Instrument No. 1 of the Vienna pattern of 40 French gauge. Window of observation and incandescent lamp present double the size. light.

Advantages.-1. A wider field of vision. 2. Increased brilliancy of

Drawback.-Can be used only in the female; in the male it must be introduced through a median incision in the membranous portion of the urethra. Such an application directly annuls the special advantage of cystoscopy, namely, that "it affords a visual diagnosis without a cutting operation."

Brenner's Modification for Catheterizing the Ureters.-Cystoscope No. 2, of 28 French, which carries a separate small channel on the convex side of the shaft. This channel terminates just below the window, and can also be used for changing the water in the bladder (Fig. 17). It is occluded by a mandrel when the instrument is introduced. The mandrel later is extracted, and replaced by a minute English catheter or an

* Take Nitze's instrument in one hand, turn its prism to the window, and hold the palmar side of the slightly flexed fingers of the other hand at a distance of about two inches from it; then look through the telescope: you will see at once the fifth to second fingers and a part of the ulnar side of the thumb. Do the same with Leiter's cystoscope: you will see only two fingers and a half. 
elastic metal sound. When the catheter or sound is in the ureter, the instrument itself may be slipped back over it.

Brenner thus succeeded in pushing the catheter into each ureter of a female patient exposed by the light, but failed to do the same in the male.

Advantage.-Allows of irrigating and sounding the bladder. The former must be done, however, through the same channel. In exceptionally favorable cases the observer may be enabled by it to catheterize the ureters.

Drawbacks. - 1. It presents all those of the original Vienna pattern; at least, the instrument does which is in my possession. Shaft too short, six and a half inches; beak too long, one inch and a half; binding screws for the battery wires fixed upon the instrument; the old key for opening and shutting circuit. 2. Angle between shaft and beak too obtuse to permit of an easy instrumental access to the mouth of, and especially instrumental progress in the direction of, the ureters. 3. The field of vision covered by the system of lenses is small. 4. Continuous heavy leakage alongside the instrument which is introduced through the channel. It does not last very long and the bladder is found to be empty. The instrument in its present shape is useless, especially in the male.

Cystoscope according to Nitze's Principles, modified by Boisseau $\mathrm{Du}$ Rocher, and manufactured by Collin, of Paris (so-called mégaloscope)* Vienna pattern No. 2, as used for an easy inspection of the fundus, elongated (Fig. 18). Compared with the latter its special striking features are: A longer beak; a longer telescope (Fig. 19), which causes the length of the instrument; an additional combination of pipes for irrigating the bladder, and also for passing the telescope, or passing instruments for catheterism of the ureters.

Probable advantages in comparison with the Berlin or Vienna instruments: 1 . On account of the length of its telescope it is six to seven inches longer, and

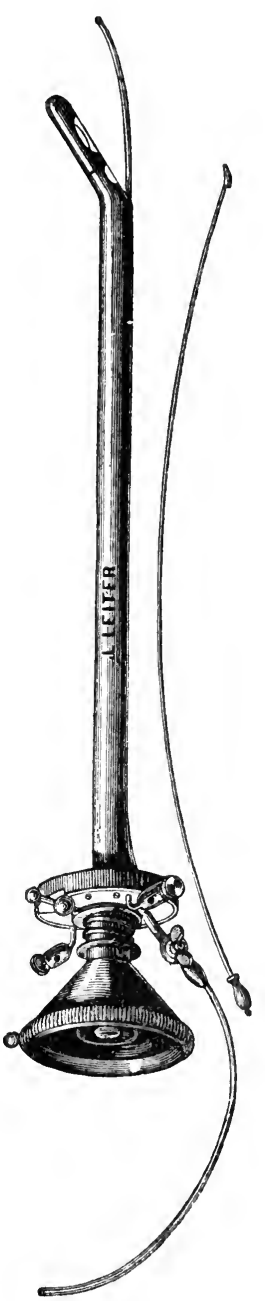

FIG. 17. the face of the observer is therefore farther removed from the genitals than is possible in using the other cystoscopes. (But, on account

* The medical profession should not accept this name, which was introduced by Rocher. We should only have modifications of a "cystoscope." 
of the great length, a slight motion of the handle will result in a by far greater one of the beak, which thus will often touch the wall of

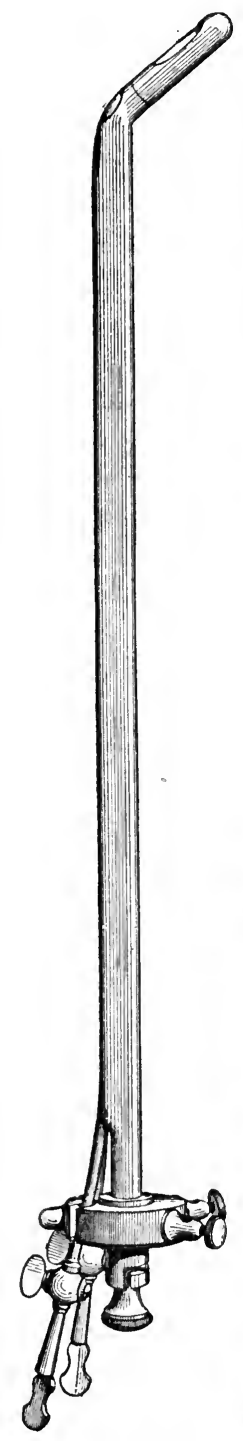

Fig. 18.

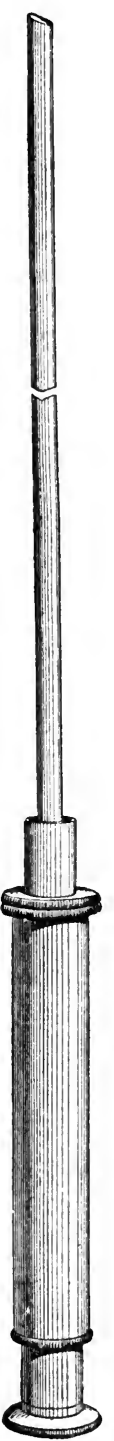

Fig. 19. the bladder.) 2. We see everything in the same upright position as our eyes would see it without the telescope. (The latter advantage is also offered by the Nitze pattern No. 2.) 3. The pipes which run alongside and inside of the lower aspect of the shaft enable us to wash out the bladder before, and apply permanent irrigation during, the cystoscopic examination. There is a wider canal in the center of the instrument for passing the telescope. It is filled out by a steel mandrel while the instrument is introduced into the bladder. If we make use of this canal for irrigation, the viscus can be very thoroughly flushed. Of course, examination is during that time impossible 4. The larger one of the small pipes can be utilized for passing instruments of minute caliber for catheterism of the ureters. 5 . The telescope is introduced, after the whole instrument has passed the urethral canali.e., is in the bladder. The objective lens can thus never be dimmed by an adherent mucus or pus-shred, or a small blood-coagulum.* 6. The instrument can be sterilized by boiling water, the cement, which is used for fastening the rock-crystal pane in the window of the beak, etc., being such as to stand a great heat. The other cystoscopes can not be boiled.

Drawbacks-1. We can not inspect the anterior surface of the bladder with this cystoscope, which, besides, is quite clumsy, and not at all as easy to handle as the others. Two instruments become necessary for viewing the entire bladder. Both can be bought at Collin's. 2. The caliber of the shaft is

* Compare Nitze's irrigating cystoscope. If we make it a rule always to inject some glycerin into the posterior portion of the urethra with the help of a Nélaton catheter after a careful irrigation of the anterior portion of the urethra, of the neck of the bladder, and of the latter organ itself, and before introducing the cystoscope, we ce:- 
No. 27 of the French scale, that of the beak No. 23. The increase of the size of the shaft is caused by the pipes for irrigation. It is to be mentioned, though, that they are situated at the lower aspect of the shaft, and thus give the tube a conical shape (cf. Brenner's modification). The top of this cone corresponds with the lower circumference of the urethra, which can be stretched. (The size of the other cystoscopes is No. 22; that of Nitze's irrigating cystoscope, the shape of which is round, No. 25.) 3. The beak is very long-half an inch longer than that of the Vienna and twice as long as that of the Berlin cystoscope. 4. The angle at the junction of beak and shaft is $130^{\circ}$, and abrupt; in the other two instruments only $145^{\circ}$, and well rounded. (The smaller angle seems to be of advantage, for the sake of catheterizing the ureters; see further down.) 5. The caliber of the two pipes used for irrigation and passing catheters for catheterizing the ureters is extremely small (No. 6, French). 6. There is no key or screw for opening and shutting the electric circuit. We always have to put in or unscrew one of the conducting wires for this purpose. 7. It is difficult to thoroughly cleanse the inner surface of the objective lens of the long telescope. This lens can not be detached from the tube, but has to be reached by a long conductor which holds at its end a piece of maple-marrow, etc.* 8 . The spherical aberration of the lenses of the telescope. 9. A constant dripping of water out of the upper end of the instrument during examination. The intraresical pressure constantly forces the water alongside the telescope, which does not snugly occlude the lumen of the central canal. On account of the more perpendicular position of the instrument during examination this is not as marked and annoying as in the Brenner modification. The leakage is increased, if we use the smaller tube for catheterization of the ureters. $\dagger$

The Paris instrument decidedly has a few important new features, which will make it desirable for the cystoscopist to be in possession of it. But, until the defects mentioned above shall have been remedied, we certainly shall always need the additional use of one of the other cystoscopes in the market, if we want to be ready to thoroughly perform a cystoscopic examination in cases where this method can be applied.

In closing this section, a tabulated comparison of the size of the different parts of the cystoscopes which are manufactured at Berlin, Vienna, and Paris, and attract special interest, may perhaps be welcome.

tainly shall rarely meet with this annoying occurrence when using the ordinary cystoscope.

* I have not, so far, succeeded in removing some particles of dust from the inner surface of this lens. A compliance with this need by the manufacturer would mean an improvement of the telescope.

† This latter leakage will be present in every cystoscope with a special channel for passing instruments for catheterizing the ureters. 


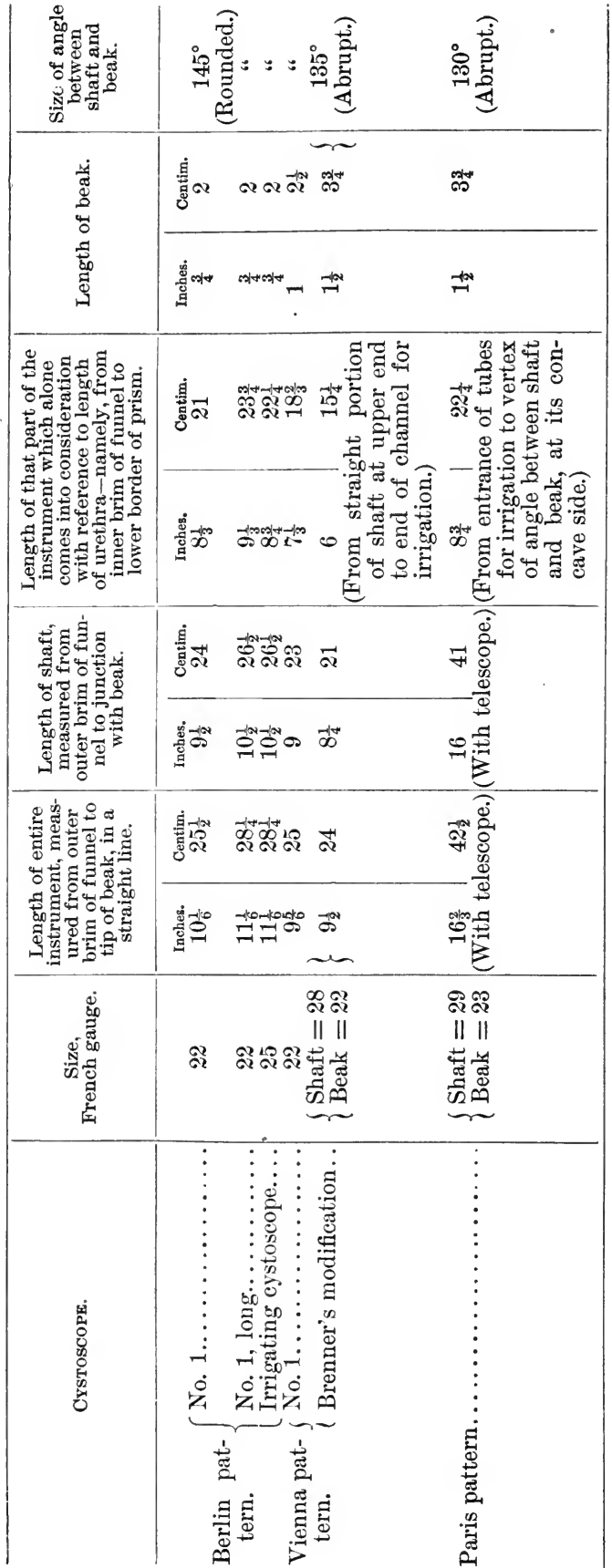

As I have been frequently asked, by many colleagues throughout the United States, which instrument or instruments had best be bought by the beginner, I should like to state here my opinion. I decidedly advise to buy the Nitze cystoscope, as manufactured by Hartwig, of Berlin. This for the reason, that Nitze has given us three useful cystoscopes, according to pattern $\mathrm{No}$. 1 , which must be in the hands of every one who wants to practice cystoscopy thoroughly and to be satisfied with it; and that I deem it of preeminent importance, in order to avoid mistakes, to keep to one pattern from the beginning. Personally, I have found the annoyance slight of sending my few burned-out reserve tips to Berlin for repair once in a year; although it is sometimes disappointing to find that the screws of only, say, six out of ten new tips fit on the thread at the lower end of the shaft or shafts. The pleasure of being enabled to work with the three cystoscopes has amply re-

* See above. 
warded me for these little inconveniences. Still, the more experienced may often find advantage in using the elegant instrument manufactured by Leiter in its new shape, as also that made by Collin, in spite of its manifold yet uncorrected drawbacks.

The Batteries.-In regard to the batteries, a great variety is now at our disposal. Hartwig, as well as Leiter, sells a battery with the cystoscope which fully answers the purpose (Nitze's Text-Book, p. 62 ; Leiter, Catalogue, 1889, pp. 13-17). The fluid is a mixture of pure chromic acid (trioxide of chromium, manufactured at wholesale by Sullivan \& Co., Limited, British Alkali Works, Widnes, Lancashire; to be ordered of Messrs. Churchman \& Co., Philadelphia), sulphuric acid, and water. (Formula for Hartwig's battery: Chromic acid, 375 ; sulphuric acid, 300 ; water, 3,000. For Leiter's: Chromic acid, 500 ; sulphuric acid, 375 ; water, 3,000.) Hartwig's battery, large size, is an eight-celled battery with Grenet elements. It is the one which I mostly use. The two original Leiter batteries, with hard-rubber cells, are not to be recommended, as they will surely crack and leak after a short while. The repair of such a crack is troublesome and always unreliable. Lately the cells have been made of glass, by which a very annoying disadvantage is at last eliminated. Fenwick uses a battery with a rheostat supplied by Schall, of Wigmore Street, London. Schall sells two batteries adapted for the purpose, one of very small size and light weight, which can be easily carried; and another large one, which only needs refilling every nine to twentyfour months. It is not intended for frequent transportation in private practice. Both are well spoken of. For cystoscopists on this side of the ocean who do not want to import the original Nitze battery, I would recommend the cheap and easily manageable, portable, small six-celled battery of the Galvano-Faradic Manufacturing Company, New York city. Not to destroy with it the incandescent lamp at once, the elements must be screwed very slowly and carefully into the fluid until the light is bright. In all these batteries there is no rheostat attached. I so far have never seemed to actually need the latter. In a more than four years' practice of cystoscopy the number of lamps destroyed by me is a very small one (except in the last four months). However, a rheostat is a useful addition to a battery. A very fine storage-battery, Gibson's (three different sizes), for office-work, is sold by the W. F. Ford Surgical Instrument Company, 315 Fifth Avenue, New York city. It contains four large cells, and has a rheostat. Its lighting power is ample. It can be arranged to permit of endoscopic and galvano-canstic work at the same time, which will be found especially convenient in urethroscopy. Recharging from a dynamo once in two to six months.* I have now used

* This is, of course, a disadvantage, as it makes the cystoscopist dependent upon the electrician. In houses that are connected with the street electric-light system, charging 
this battery in my cystoscopic work for the last four months, and have destroyed more lamps with it than in the last four years combined. I have therefore returned to Nitze's fluid-celled battery, and use the Gibson's only for urethroscopy with Leiter's pan-electroscope.

Fixing the Picture as seen with the Cystoscope by Clay or Wax-modeling, Colored Drawings, and Photography.-Before entering the practical part of our subject, I have to call attention to the various attempts which have been made in this direction.

Fenwick, the originator of the first-mentioned method (Brit. Med. Jour., January. 5, 1889 ; and the Electric Illumination, etc., loc. cit., p. 88), has proved, by the very pretty pictures in his work, how nicely and thoroughly the various pathological conditions of the interior of the living bladder, especially of tumors, if modeled in some plastic materialwax or clay-(and then photographed), may be recorded and demonstrated to others who could not attend the examination.

E. Burckhardt very lately gave us a fine collection of colored drawings of bladder images in health and disease (Atlas der Cystoskopie, mit 24 Tafeln in Farbendruck, Basel, 1891; see also the few excellent colored drawings at the end of Nitze's Handbook, and the three nice pictures in Albarran's work, Les tumeurs de la vessie, Paris, 1892, pp. 252-254); every one of them was observed by himself, and drawn at once with the cystoscope in position. The Atlas will especially be useful to the beginner, and explain to him many a picture which was seen but could not be at once identified.

Instantaneous photography, the ne plus ultra of cystoscopic delineation (Fenwick), is still in its infancy. Nitze has theoretically laid out an interesting method of best getting a small negative and then magnifying it. Want of time has not permitted him to make practical experiments (Text-Book, p. 325). Géza von Antal (Internat. Ctrlbl. für Phys. u. Path. der Harn u. Sexualorgane, Bd. 1, Heft 1, p. 18), by his assistant, B. Hermann, published the photographic picture of the cystoscopic appearance of a black hair-pin in a female bladder. It is, however, entirely indistinct.

Fenwick (in connection with Mr. Pearson-Cooper, of the London Camera Club) has succeeded in obtaining good negatives of artificial growths both in the dummy and the dead bladder. But the negatives of the living bladder were too indistinct. A number of mechanical obstacles have hitherto offered an almost insuperable barrier to the practical success of such methods.

R. Kutner, a former assistant of Nitze, lately succeeded in overcom-

can be done at home. It also must be said that one set of lead-plates will generally not last longer than five years. 
ing the principal obstacles to photography (Deutsche med. Wochens., 1891, p. 1311). It seems that we shall now be enabled to "graphically record the many new and interesting clinical facts which the electric cystoscope is constantly revealing" (Fenwick). Two pictures in Kutner's article demonstrate what we may expect from cystophotography in the future.

Literature.-The literature on cystoscopy, which has appeared mostly within the last four years, is already very large. The fundamental and first work, upon which all the later various writings on the same subject, books, and articles, necessarily are based, is that of Max Nitze, which appeared in 1887: Contribution to the Endoscopy of the Male Bladder (Archiv f. klin. Chir., vol. xxxvi, p. 661). Nitze left there hardly anything unsaid that could even be thought of in regard to the future progress in diagnosis and treatment of urinary diseases with the help of his instrument. In his Text-Book on Cystoscopy (Wiesbaden, J. F. Bergmann, 1889) he continued his original work. A similar thorough book is only found in the English language. It is the admirable and extremely interesting work of E. Hurry Fenwick (Electric Illumination of the Bladder and Urethra, London, 1889), who added many new and interesting facts from his large cystoscopic material to those first published by Nitze. A great many other articles bearing on cystoscopy, written by different men all over the globe, have besides been published, mainly within the last four years. All show original work. It is to be hoped that every cystoscopist will continue to publish his experience. If the facts laid down in these publications coincide with those observed by others under similar circumstances, certain fundamental pathognomonic "cystoscopic" symptoms of different diseases will have been established, and forever remain of value and importance.

The Employment of the Cystoscope.-There are three cardinal conditions :

1. The caliber of the urethra must be sufficiently large to allow of passing No. 22 French gauge.

2. The bladder must have a capacity of at least two, better four to five, fluidounces $(60-150$ c. cm.).

3. The fluid in the bladder must be transparent.

To comply with the first condition, the male urethra should, in suspicious cases, be first tested with the sound No. 22. (Nitze reports cases where the stricture was first diagnosed by passing the cystoscope, and following dilatation with the sound cured the entire trouble. Of course, cystoscopy was here simply not indicated.)

A narrow meatus is to be cut inferiorly, under cocaine. This procedure will be very rarely necessary.

With reference to the second point, it is important to remember that 
the beginner should always endeavor to have five ounces of fluid thrown into the bladder. We know, from Nitze's investigations, that this amount just expands all the folds and grooves of the intravesical surface.* Only in this way, by training one's eyes to inspect the surface of the properly and more or less always equally expanded viscus, can one hope to learn gradually how to avoid mistakes in cystoscopic diagnosis.

(I know of two cases of chronic ulcerative catarrh of the bladder where, owing to an insufficiently distended bladder (two or three ounces only were borne), the observer was misled to diagnose a tumor. In both cases a portion of the bladder wall, not yet expanded, had been mistaken as such. In one of the cases, that of a young man of thirty-one years, the bladder surface showed an irregular cicatrization, especially around the spot in question. The scar-tissue seemed to prevent a more regular distention. In the other case, that of a young lady of seventeen years, there were numerous florid ulcerations.)

The more experienced practitioner, however, will not find cystoscopy contra-indicated, even if the bladder be irritable and expel the larger amount of fluid. With the irrigating cystoscope in hand success is still possible. The minimum amount of fluid, however, is two ounces. A bladder which holds less can not be illuminated (for reasons, see under "Posssible dangers," etc.). On the other hand, the cystoscopist should be cautioned against injecting much more than five ounces. The anterior wall of the bladder will then be so far away that it can not be properly inspected.

But the filling of a diseased bladder to the proper extent is sometimes rendered impossible on account of its irritability. What means are then at our disposal to facilitate the examination?

Cocaine.-Nitze is in the habit of using local cocaine anæsthesia in nearly every case, in order to render the manupulations nearly painless. $\mathrm{He}$ injects in all fifty cubic centimetres (one and two-thirds ounces) of a two-per-cent solution (=fifteen grains of the drug), and takes pains that this fluid reaches every part of the urethra and bladder.

For this purpose a few cubic centimetres of the solution are first injected into the anterior urethra with an ordinary urethral-syringe. Then the bladder is emptied with a soft-rubber catheter, and washed, if the condition of the urine render it necessary. About half of the anæsthetizing fluid is then thrown directly into the bladder with a hand-syringe, and the rest made to pass the posterior urethra by slowly withdrawing the catheter until the fluid again appears at the external meatus. In short, we perform an irrigation of anterior and posterior urethra with a cocaine solution, as we do in a case of chronic specific urethritis with one of the ordi-

* This is especially important for examining the mouth of the ureters. 
nary antiseptic lotions. At last the catheter is pushed back into the bladder, and closed by a stop-cock. After five minutes the cocaine solution is replaced by one-half-per-cent solution of carbolic acid (sterilized physiological salt solution answers the same purpose; boric acid lotion, which is slightly muddy, not as well). A sufficient degree of anæsthesia will now have been effected.

Fenwick injects a drachm or more of a twenty-per-cent solution of cocaine (about the same amount of the drug as Nitze does) into the water which had been poured into the bladder. "It there rapidly diffuses itself throughout the water, and serves not only to deaden the sensibility of the vesical mucous membrane in spasmodic cases, but also to allow of a still larger introduction of the boracic solution, if a difficulty in tolerating the necessary quantity is experienced by the patient." Besides, he also " sometimes squirts a little of a twenty-per-cent solution of cocaine into the urethral canal, anæsthetizing especially the prostatic section." He adds that "no apprehension need be felt about this application," as he had never seen any case presenting symptoms of so-called cocaine-poisoning.

Up to six months ago, in a few years' large cystoscopic practice, I also had never seen anything which might have induced me to look at cocainization of irritable bladders as a possibly dangerous affair. I had always made it a rule to use cocaine, if indicated (otherwise not), according to Nitze's plan-that is, I always made the solution run off before properly expanding the bladder for examination. For special reasons, I left in one (the first) case the fifty cubic centimetres of the two-per-cent solution in place, adding three more ounces of sterilized $0 \cdot 6$-per-cent salt solution, and barely escaped having a death from cocaine poisoning in my office.

Mr. W. P., sixty-eight years old, came to my office through the courtesy of Dr. W. B. Graves, of East Orange, New Jersey. He suffered from intermittent retention of urine with occasional slight hæmaturia, prostate enlarged. February 23, 1892 : Cystoscopy, with local cocaine-anæsthesia, as mentioned above. Fifteen minutes later the following sudden serious attack set in : Convulsive twisting of right lower extremity, less of left, and of both arms (flexors exclusively involved); also twisting of muscles of face, strabismus, turning of head to the right, perspiration of head and scalp, hallucinations-saw birds and bugs). Pulse 156, comparatively full and regular; respiration rapid. Symptoms lasted unabated for forty-five minutes, then began to pass off. One hour later, still twitching of muscles around mouth and orbits. Pulse 158, respiration 32. Aphasia. Continuous inhalations of nitrate of amyl proved of the greatest benefit. Besides, digitaline ${ }_{1 \frac{1}{0} \overline{0}}$ of a grain was given subcutaneously. About one hour later patient was still weak, but able to leave the office.

Albarran (loc. cit., page 235) reports a death under these cireumstances:

In a case of irritable stone-bladder only two ounces of a one-per-cent solution of cocaine (equal to nine grains of the drug) had been thrown into the bladder, 
and the greater part of it a few minutes later withdrawn as soon as the first symptoms of poisoning had been noticed, and the bladder thoroughly washed. Two minutes later convulsions set in, the patient became cyanotic, and died barely fifteen minutes later, in spite of the most vigorous attempts at saving his life.

These two cases show beyond a doubt that even a small dose of cocaine, if injected into the diseased prostatic portion of the urethra and the vesical viscus, may prove dangerous or even fatal, and can be reabsorbed by its mucous membrane with the same rapidity as if it had been given by hypodermatic injection. It will therefore be our duty to be careful, in that procedure, certainly not to leave the solution in the bladder longer than five minutes. But even then we can not always rest assured to be on the safe side.

Morphine.-The application of morphine by subcutaneous injection or suppository generally quiets an irritable bladder markedly. For obvious reasons, it is only in place if the examination is made at the patient's house, where I have often made use of it with advantage-more so, if it was combined with a careful application of cocaine.

Narcosis. - The cases are comparatively rare in which we have to resort to its help. In a large number of patients who were submitted to cystoscopy by Nitze during a period of nearly three years, only three had to be narcotized (Text-Book, page 118). Still, in view of the possible dangers of cocainization, I must confess that I shall henceforth oftener use general anæsthesia in patients with a very irritable bladder, where local anæsthesia might at last also succeed, except in office practice. I can not see that this possible necessity for narcosis offers any objection to cystoscopy, especially not in the so-called obscure urinary troubles of old standing with a very obstinate bladder, which compose a large part of the cystoscopist's material. Does not the gynæcologist make it a rule to examine his patients under an anæsthetic, in order to clear up by thorough "painless" bimanual palpation the pelvic trouble, to get at the correct diagnosis in its different parts as closely as possible? Is the result of the cystoscopic examination less important? A suprapubic cystotomy, nephrotomy, or nephrectomy, generally confines a patient longer to the bed than the extirpation of a pyosalpinx or a cystic ovary.

Certainly the different forms of anæsthesia should only be employed according to precise indication. The majority of cystoscopic examinations can and should therefore be performed without it.*

* Nitze divides the cases with irritable bladder into three classes:

1. If the bladder be greatly contracted, and its walls so infiltrated that the slighest artificial distention is not borne, even chloroform will be of no avail. Cystoscopy can not be performed.

2. Capacity also small; frequent desire to urinate. Causes: Superficial catarrh, reflex irritation, real smallness of bladder. 
Fenwick employs anæsthesia : $a$, in young females for reasons of delicacy; $b$, in tuberculosis or similar cases where the prostatic urethra is extremely sensitive; $c$, when it is necessary to demonstrate some particular disease to a number of visitors ; $d$, in order to make a leisurely prognosis of a discovered growth, so as to determine the expediency of operating for its removal (loc. cit., page 73). Albarran proposes to try the rapid bromethyl narcosis (loc. cit., page 236).

As it has been seen, grave obstacles to cystoscopic examination will be sometimes encountered. Frequently these will be overcome by experience, patience, and careful handling; now and then they will prevent the examination. In nearly every case these obstacles depend on the catarrh of the bladder. In cases of tumor this accompanying catarrh has "always" been carried into the bladder by the doctor, with the sound or catheter. Nitze therefore absolutely forbids the introduction of such an instrument into the bladder of patients where a vesical growth is suspected. The cystoscope must in future be the first instrument which is resorted to in these cases. The general practitioner therefore must realize the eminent pathognomonic importance of a spontaneous symptomless hæmaturia.

The third condition, that the fluid in the bladder be transparent, and that the beak may enter the bladder without dimming the prism and lamp, was formerly in many eases not possible of fulfillment. If, for instance, we examined a case of suppurative pyelitis or of hæmaturia with cystoscope No. 1, the originally transparent medium rapidly, often suddenly, turned turbid, and the light seemed to be placed in a yellow or red fog. Everything was obscure. It was necessary to withdraw the cystoscope, and reintroduce it after replacing the murky medium with clear water. One glance in bright illumination, and again a dense fog suddenly came up and threw a heavy veil over the whole landscape. This would be a case where cystoscopy "failed"! Or, we had an older patient with an enlarged, easily bleeding prostate gland and vesical catarrh. Sometimes the hæmaturia was quite abundant. Was the latter perhaps caused by an accessory trouble-tumor, stone, etc.? Urethra and bladder were very

$a$. Mild cases: With cocaine little improvement. Morphine; wash with small quantities; at last inject the five ounces intermittently; compress penis until cystoscope is introduced.

b. Severe cases: Preparatory treatment advisable-rest, suppositories, oleum santali; instillation of nitrate of silver. Must individualize. Systemic stretching of bladder with "hand-syringe." For examination proper: Combine cocaine with morphine; if necessary, chloroform.

3. Capacity ample; painless irritability after washing had been started.

Give first morphine; do not force injection of the five ounces at once. If, during injection, contraction sets in, wait until it has passed. Use hand-syringe (this is necessary in every case). Eye of catheter must be in the cavity, not at the neck. Compress penis until cystoscope is in urethra. 
carefully washed, glycerin injected into the posterior urethra, the cystoscope put in. The entire field of vision appeared red; everything was indistinct. Sometimes the mucus or pus shred or the small blood-coagulum, which had settled on the prism or lamp on their way into the bladder, could be successfully removed by mechanically wiping these parts of the instrument on the fold of mucous membrane which surrounds the internal urethral orifice (Nitze, Text-Book, page 110). If they stuck in place, however, the disgusted cystoscopist had to stop the examination. The instrument, when extracted, showed the cause of the failure as just stated. The patient refused a second trial. Conclusion: "The instrument fails under certain conditions, unhappily usually there where its use seems to be mostly needed." Nitze's irrigating cystoscope has obviated such a disappointment. Its prism and lamp are thoroughly washed, and a murky medium is easily changed. (Of course, for lubricating the cystoscope, glycerin must be used; vaseline would dim lamp and prism.) We therefore have to change the chapter on "Limitations of the Cystoscope," established by those authors who so far only worked with the Vienna instrument. The only condition in regard to the medium which is really liable to limit the use of the cystoscope, even in the hands of the experienced, is an obstinate and abundant vesical hæmaturia. In such cases the cystoscope is best introduced after the bleeding has ceased, and when a sufficient amount of urine is probably in the bladder. Washing will frequently start the hæmorrhage again. If, for any reason, waiting is impossible, Nitze's advice should be borne in mind, not to empty the bladder completely while washing it. The definite contraction of the empty organ will always produce new hæmorrhage. Blood-clots will be best removed through the lithotrite catheter. To get a satisfactory picture, the examination is then made under constant irrigation (Albarran). If the instrument has to pass a prostate which bleeds at the slightest touch of a solid instrument, irrigation should be started when the beak passes the posterior urethra and neck of the bladder.

Possible Dangers in using the Cystoscope.-Burning the Mucous Membrane.- "Although the heat emitted by the cap or hood with its contained incandescent lamp," says Fenwick (loc. cit., page 50), "when in action, is not so great as that given off by the platinum-wire lamp, yet it does become very quickly hot if it is allowed to burn in air instead of under water. Thus, it becomes warm after four seconds, unbearable after ten, and scorches the skin severely after fifteen seconds. If, however, the tip of the instrument is placed in water, the heat is rapidly absorbed and the cap or hood remains cool, and may be touched with impunity even after an hour's use. This is exactly what happens in the bladder, for the urine carries off the heat of the lamp as fast as it is formed." Its temperature is not perceptibly raised, even if the lamp is burned for an hour in the 
bladder; but as soon as it comes in contact with the sensitive mucous membrane of the bladder wall, the patient invariably has a burning sensation. It will be readily understood that a longer unintentional contact, for instance, during narcosis, may really burn the mucous membrane. This will easier occur with the Vienna instrument. Its carbon-filament is shorter and thicker than in the Berlin, and the lamp, situated in the middle of the beak and covered by the rock-crystal pane, heats the entire cap. On this account Fenwick devised his perforated cap, as above described. The short-beaked Nitze cystoscope, manufactured at Berlin, carries the incandescent lamp in the tip uncovered in direct contact with the surrounding medium. If its beak tonch the bladder wall it also creates a slight burning sensation. For this reason it becomes necessary to constantly have surrounded the beak by a sufficient quantity of water, in which it may freely move without touching the vesical wall. This is the reason that cystoscopy is impossible if the bladder retain less than two ounces (cf. above, page 164). If the cystoscope is carefully handled, the danger of burning the mucous membrane is certainly not great. In a large number of cystoscopies, with or without anæsthesia, I have so far never had a mishap.

Breakage of the Lamp.-(Fenwick, loc. cit., page 51). This is a groundless fear, and decidedly no objection whatsoever to the Berlin pattern. The latter is at present used in every country of the globe. But a case of breakage of the lamp "splintering the glass" has never been reported. It certainly would, had it ever been observed.* Such an occurrence necessarily would condemn the entire arrangement. Nitze carefully compared the resistance to mechanical injuries of the mignon-lamp, as used in his Berlin pattern, with that of the rock-crystal pane. The result was in favor of the lamp. The fear is therefore "happily quite groundless," not only for the Vienna lamp, but also for that of Berlin.

Infection of the Bladder.-This accident is easily possible if the cystoscope is carelessly handled. As mentioned above, the Berlin and Vienna patterns can not be sterilized by heat. $\dagger$ Prism and lamp make

* In my four years' practice it has happened to me twice that Nitze's lamp, which had given a bright light just before examination was started, burned darker and darker when turned on inside of the bladder. The cireuit was broken and the instrument pulled out. It was seen that the lamp itself had filled with water. (Probably the lamp had been injured on its trip across the ocean.) The glass had cracked in all directions, but not one single splinter was loose, or could be artificially loosened in spite of manifold attempts.

† Nélaton's soft-rubber eatheter can not be boiled either. Still, it is daily in the surgeon's hands, and, if properly attended to, is considered a safe instrument as far as asepsis is concerned. In adopting the cement as used by Collin, of Paris, the cystoscopes manufactured in Berlin and Vienna might in future perhaps also be rendered aseptic by boiling them. 
the surface of the instrument irregular: there are grooves and depressions at their borders. Nitze's irrigating cystoscope has a system of minutest pipes. Septic material can easily settle there. Being aware of this, the conscientious cystoscopist who is used to aseptic work will take double pains to have his instrument thoroughly disinfected as soon as the examination has been finished.

Rules for disinfecting the Cystoscope after each Examination.- $a$. No. 1 (or 2). Rub the instrument thoroughly with a piece of aseptic gauze or a small soft brush soaked in a five-per-cent solution of carbolic acid; pay special attention to the various grooves. (If an infected bladder had been explored, the lower half of the instrument should be besides immersed in a five-per-cent carbolic solution for about one hour.) Dry well with another piece of gauze, and polish with a piece of chamois; then put it away.

b. Irrigating Cystoscope.-Throw a good deal of five-per-cent carbolic solution forcibly with the hand-syringe through the pipes; then put the instrument, nearly up to the funnel, in the same solution for about one hour. (A long, narrow glass jar, used for measuring fluids, answers this purpose very nicely.) Afterward irrigate with sterilized water; dry as before.

By carefully following these precautions I so far have never had the mishap of infecting a bladder or aggravating an already existing catarrh by the cystoscopic examination.

Hints on the Preservation of the Lamps, and on keeping the Berlin Instruments in Good Working Order.-Always determine the proper strength of current which is necessary for amply lighting the lamp, before the instrument is introduced into the bladder. Do this by holding the beak under water in a bowl.

Screw the elements slowly down into the fluid until the contour of the carbon-filament is unrecognizable, and the entire surface in the cutout of the tip emits a bright, white light. The resistance offered by the carbon-filament to the current is different in each lamp. Use for the purpose as few elements as possible; in Nitze's battery generally from four to seven will be required. No new element should be added until those already in circuit have been entirely immersed in the fluid.

If the fluid has been recently renewed, the strength of the current is greatly increased for a short time. One to two elements less should then be used first. Do not shake the battery.

If the cystoscope is new, smear some varnish mixed with red lead (vermilion) around the borders of the prism. This mixture should have the consistency of honey. It should be done a few times only within the first months.

Keep clean and dry the two circular grooves at the upper end of the 
instrument, as well as the concave surface of the rotatory handle, which, when attached to these grooves, conveys the current from the battery to the instrument. This refers especially to the irrigating cystoscope.

Turn now and then the screw home which holds the slot-key in place. If it works too easily under management of the thumb, the light will be less brilliant. Sometimes the cystoscope turns too easily in the handle. Then leave the left hand at the upper end of the cystoscope during examination, while pressing the handle with the other hand tightly against it.

If the lamp of a new tip does not burn at once when the current passes its filament, straighten the little cork-screw-like silver wire at the base of the tip before the latter is screwed on. This will often be sufficient to secure a bright light.

Small bubbles of air will often arise with a peculiar noise from the junction of the shaft and tip. They are produced by the decomposition of the water caused by the electric current. Smear a bit of wax upon the lower groove of the screw at the tip, with a hot wire dipped in the wax, previous to its being adjusted. This is best done whenever a new tip has been put on.

Do not clean the instrument with silver-powder. It might scratch the prism. Do not extract the optic apparatus.

If the instrument has to be repaired for one or the other reason, send it back to the maker.

Rules for performing Cystoscopy.-The cystoscopist should accustom himself, especially in cases which he sees in consultation, first to obtain a thorough history; then to make a general examination of the patient, especially with reference to tuberculosis; to carefully analyze the urine; to palpate the lumbar region of each side, the testicles and the prostate, the bladder bimanually if necessary, and test the caliber and length* of

* It is of importance to measure the length of the urethra, especially in cases of hypertrophy of the prostate, to be sure that the prism will not only enter the bladder, but can be pushed back to its posterior wall. This manipulation is a sine qua non for a thorough examination (see Important Points in examining the Bladder). It is true we can gain a few centimetres by compressing the pendulous portion of the patient's urethra: but if the prostatic portion be materially lengthened, this compression is not only painful for the patient, but is a mechanical impossibility. We therefore have to measure the length of the urethra, and in accordance with it select the proper instrument. If the length is eight and one third inches (twenty-one centimetres), we can use cystoscope No. 1; if longer, we will take the longer No. 1. Nitze very cleverly made his irrigating cystoscope according to this last size. 'The latter will therefore, also in this reference, always be the preferable instrument in such cases, if the urethra is of 25 French. An easy way to measure the length of the urethra is the following: Measure length of Nélaton catheter from outer end to eye; introduce same until urine just begins to run off; measure length of portion of catheter in front of external meatus; subtract from original length (Nitze). 
the urethra. The operator should always start with the case as if he did not yet possess the eystoscope to enable him to view the interior of the bladder. He should push his means of diagnosis as far as possible by rational signs and examination of the urine. But the first instrument he then takes in hand, provided its use is indicated, should be, not the sound, but the cystoscope. Only when a stone in the bladder is strongly suspected should the sound be used first.

Before introducing the cystoscope, we put its beak into a glass bowl under water, push the slot-key on the handle forward and turn the winch of the battery, slowly immersing the elements into the fluid. The full strength of the current allowable for getting a bright light is determined and the circuit broken (see above, Hints, etc.). Previous to this the patient's bladder had been prepared according to indication (see above, Conditions indispensable for the Employment, etc.). The patient is in the recumbent (lithotomy) position at the edge of a high upholstered table or examining-chair, with flexed thighs and knees, the feet resting upon adjustable holders. I always have my male patients take off trousers and drawers, but keep on shoes and stockings. (Fenwick has his patients sometimes lie on the back, "with the trousers unbuttoned and thrown a little way down. A clean towel is placed across the trousers, upon which the right side of the observer's face can rest while he is looking down the cystoscope.") Once more we close the circuit for a moment, to be sure that the connection with the battery is in order and the light sufficiently bright. Now the meatus is wiped off with a ball of cotton moistened in a two or three per cent solution of boracic acid, the beak lubricated with glycerin and introduced.* This manœuvre is just as easily performed as the passing of a sound. As in that case, it must be gently done, without any undue violence. The position of the instrument, the length of the portion already introduced, and a certain slight jump in passing the neck of the bladder, will tell the experienced when the beak of the cystoscope is in situ. Involnntarily he will push the key forward and look into the bladder, which now presents itself in brightest illumination. The novice had better first turn the beak laterally, to be sure that it moves freely in the fluid, before he lights the lamp. $\dagger$

* The funnel is for this purpose firmly grasped between the thumb and second finger of the right hand, while the same fingers of the left hold the anterior portion of the glans in such a way that the meatus is gaping. Attention is paid to the point that the small knob which is soldered to the outer brim always points directly upward. If the irrigating cystoscope is used, the passing of the instrument through the urethra may be facilitated if vaseline is smeared on the shaft as soon as the beak and a little of the shaft have entered. With cystoscope No. 1 this is not advisable, as changing of the fluid and reintroduction of the instrument may be required.

† Of course, the circuit is broken before the cystoscope is extracted from the bladder when the examination is finished. 
Important points in examining the Bladder with the Cystoscope.-It is the observer's task to inspect the entire inner vesical surface as quickly as possible and with the least annoyance to the patient; also to bring into sight every spot of the interior of the bladder with mathematical exactness. This can only be done if the instrument is scientifically handled after it has entered the bladder. According to Nitze (page 91 and following pages), the beak in the bladder must be put for this purpose into five different positions, and then moved in each of them in a determined direction.*

Fig. 20 (Nitze, page 95) presents a schematic frontal cut through the middle of the body of the bladder ; $x$ is the frontal cut through the shaft of the cystoscope.

(A) Beak points upward.

1. As soon as the beak has passed the internal sphincter muscle it is turned to the right side of the patient for an angle of $22 \frac{1}{2}^{\circ}$ from the vertical line (Fig. $20, a$ ) and pushed forward until it touches the posterior wall, the funnel of the instrument being slightly raised during this time. To bring most of the posterior wall in view, the funnel is still more raised as soon as it is close at it. The segment observed in this manipulation is marked $\mathrm{A}$ in

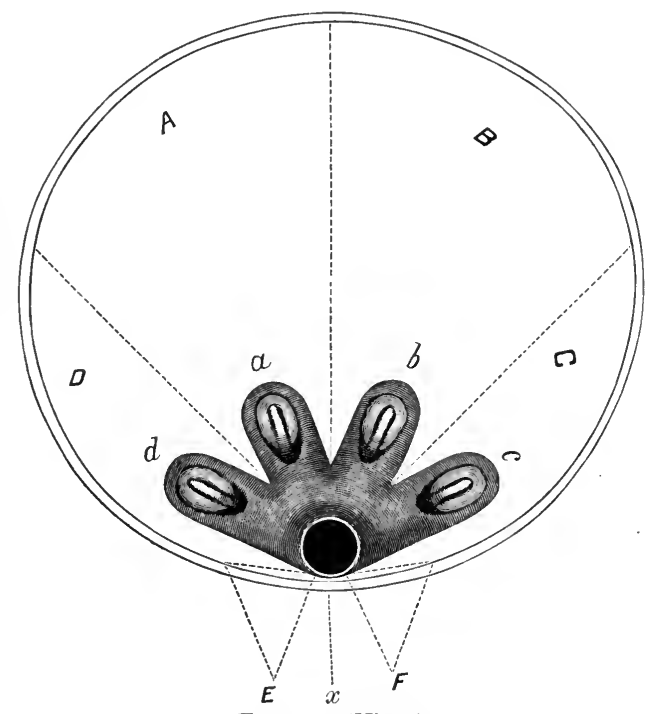

FIG. 20.-(Nitze.) the figure.

2. Beak turned to the left for $45^{\circ}$ (position $b$ ) and pulled back to the internal urethral orifice, gliding over the fundus (this latter can only be accomplished by raising the funnel). Segment viewed $=\mathrm{B}$.

3. Beak turned for $45^{\circ}$ more to the left $(c)$ and pushed again slowly forward to the posterior wall. The funnel is gently pressed to the left. Segment examined $=\mathrm{C}$.

* The motions which can be made with the cystoscope in the bladder are threefold: $a$. In the antero-posterior direction; that means in the direction of the longitudinal axis of the instrument. $b$. By turning the shaft around its longitudinal axis. (In these two motions the axis itself remains steady.) $\quad c$. Changing the position of the axis itself by moving the funnel upward and downward or from one side to the other. By combining these latter motions we will get a kind of funnel-shaped ones.

+ This latter manipulation will make visible the small strip F, on the opposite side also $\mathrm{E}$, marked in the figure on each side of the instrument. 
4. Beak turned for $135^{\circ}$ to the right $(d)$. Funnel also conducted toward this side. Cystoscope withdrawn to the internal urethral orifice. Portion viewed $=\mathrm{D}$.

So far that portion of the bladder has been inspected which is situated above a plane put through the longitudinal axis of the instrument

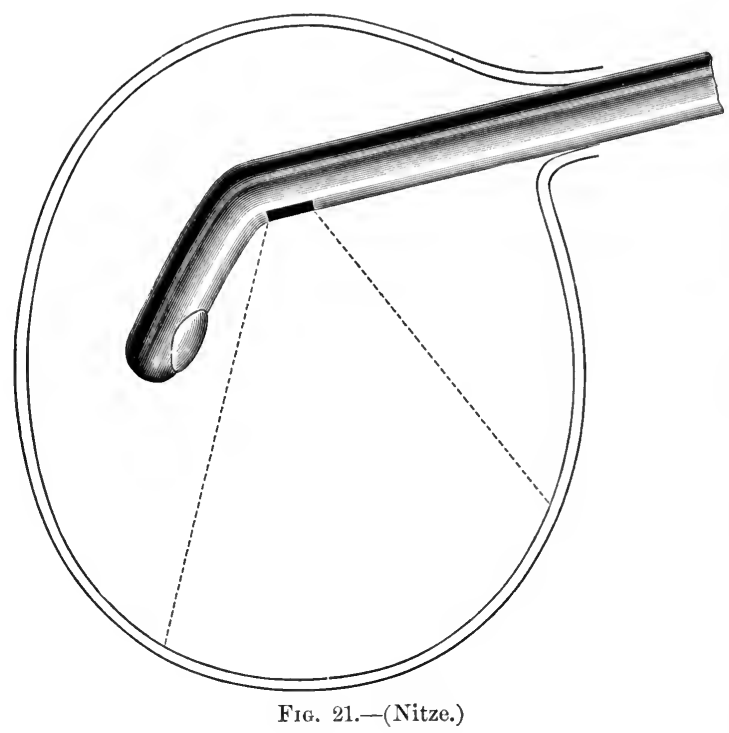
transversely. It is the largest, and embraces the entire anterior wall, the vertex, and the upper larger portion of the posterior and the two lateral walls.

To view the portion below this plane, the beak is-

(B) Directed downward by turning it (from position $a$ or $b$ ) $180^{\circ}$; the funnel is pressed down, and the instrument pushed deep into the cavity of the bladder close to its anterior wall (Fig. 21).

I deem it correct to give this rather elaborate and theoretical descrip. tion here to prove that in the majority of cases we can really examine the entire inner surface of the bladder with cystoscope No. 1. (Only in exceptional cases we have to use, besides, Nos. 2 and 3, the former for the fundus in cases of marked hypertrophy of the prostate, the latter if the immediate neighborhood of the internal urethral orifice presents pathological conditions). It may easily impress the reader as if the examination "in praxi" is a difficult manœuvre; but that is not at all so. It is by far simpler and easier. The above theoretical points are more for the beginner than for the experienced cystoscopist, to be sure that really the entire surface of the bladder had been inspected. The turning around the longitudinal axis for $221^{\circ}, 45^{\circ}, 135^{\circ}$, etc., is also not to be taken literally. The size of the angle in which the field of vision appears is not exactly $45^{\circ}$, as represented in Fig. 20. In most cystoscopes it is $45^{\circ}$ to $65^{\circ}$. Thus the border-strip of mucous membrane, examined in position $a$, will come into view for a second time in position $b$. The border-line of $d$ will cover that of $a$, and so forth. In praxi, moreover, the positions described have to follow each other differently. The last (Fig. 21) has to 
be the first. In other words, we at once direct the beak to the fundus of the bladder, "for it is this section of the bladder which contains or conceals for the most part those diseases which rank as 'obscure.' "The inferior zone of the bladder is to vesical endoscopy what the optic disk is to ophthalmoscopy, the most important area in the examination" (Fenwick). To complete the latter, we will then afterward direct our attention to the less interesting middle and superior zones, by quickly turning and moving the cystoscope in the other positions described above.

A few more general points have yet to be emphasized:

Make first examination always with the prisin far away from the object. If, then, something has attracted special attention, approach it with the prism, but bear in mind the inversion and twisted appearance of certain pictures, and the nearer the prism the larger the object, and vice versa. The knob soldered to the upper brim of the funnel should be frequently touched during the examination, to be always informed where the object just observed is situated.

The distance between the prism and object is estimated by the space in which the beak has been moved forward from the internal urethral orifice into the bladder, by the position of the shaft, and by the amount

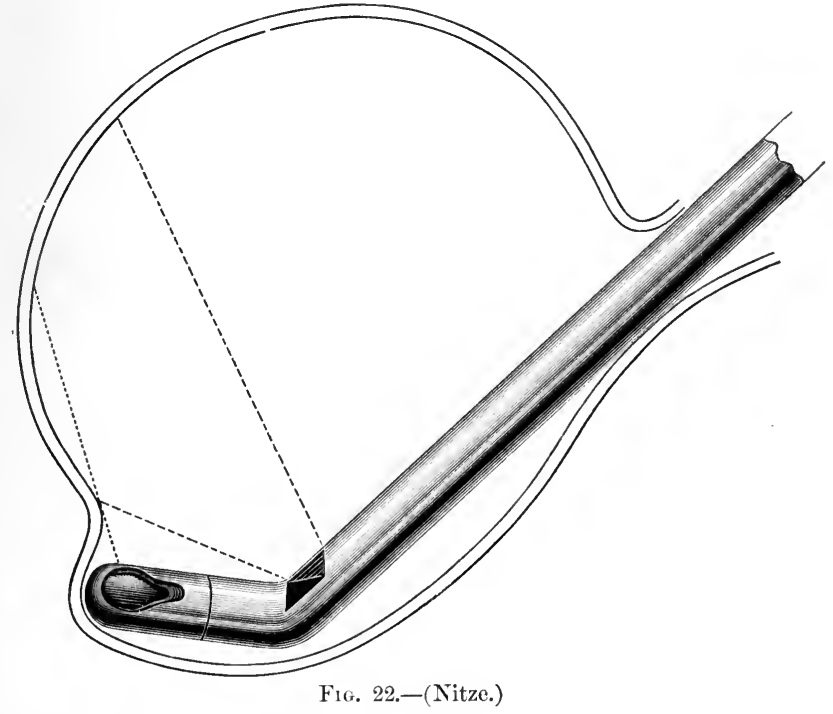

of light that is thrown on the field of vision. Difference of level is signified by a great difference in the degree of illumination of neighboring portions.

Size and shape of a prominent object (tumor, etc.) are diagnosed by the 
size and shape of the shadows thrown upon the vesical wall in various positions of the lamp.

The moment when the surface of the lamp touches the mucous membrane is marked by sudden darkening and deep reddening of the field of vision (Fig. 22), also by the burning sensation soon experienced by the patient; then move beak in opposite direction, as done just before, and the former bright illumination will return.

Summary of Former Scattered Remarks with Reference to the Use of the Cystoscope in Patients with Hypertrophy of the Prostate.-1. If possible, use the irrigating cystoscope. Of course, the general surgical rule for this trouble must be strictly obeyed-namely, to press the handle gently down as far as possible before the beak is made to enter the bladder. The short (Mercier's) beak of the cystoscope is excellently fitted for this purpose.

2. Start a gentle irrigation while passing the prostatic portion of the urethra. If the caliber of the urethra is less than 25 French, introduce

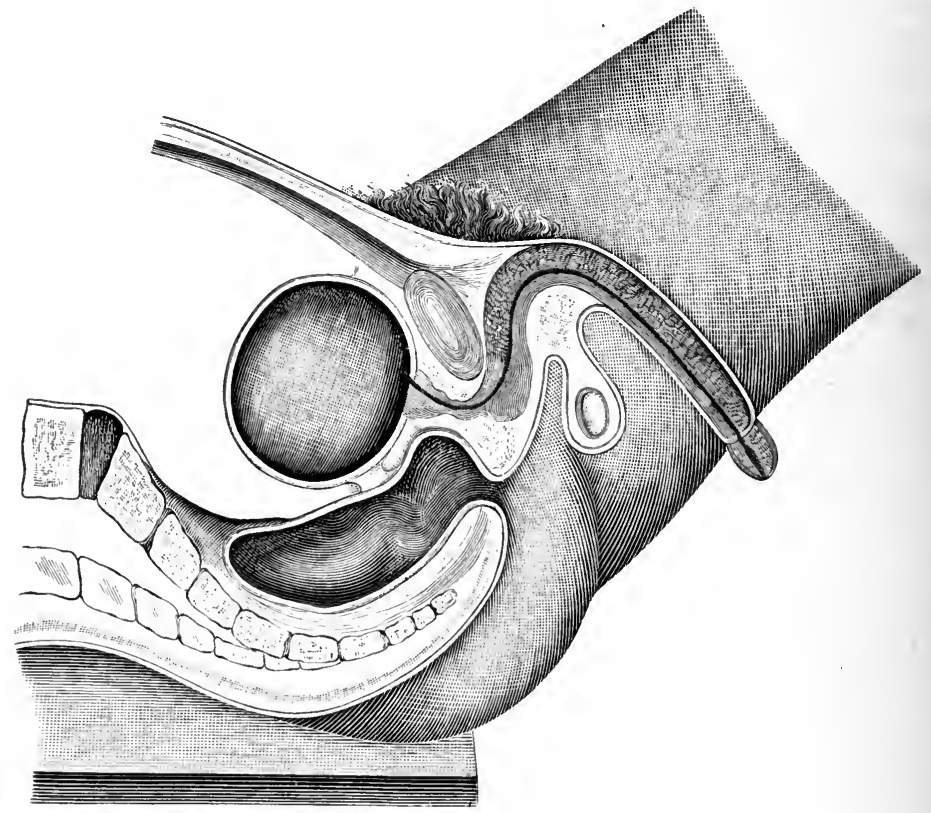

Fig. 23.-(Nitze.)

cystoseope No. 1 long, and just previously inject some glycerin into the posterior urethra. If this latter instrument must be used, a failure may be expected.* Bleeding is easily started when a solid instrument passes

* For quite exceptional cases we need a still longer instrument. As Nitze asserts, a cystoscope can be manufactured as long as 35 centimetres (equal to $13 \frac{3}{4}$ inches). Its cali- 
the prostatic portion. The blood settles on prism and lamp. The field of vision appears diffusely red.

3. The larger the prostate the more firmly is the instrument held in its changed equilibrium. The necessary funnel-shaped motions (see above)

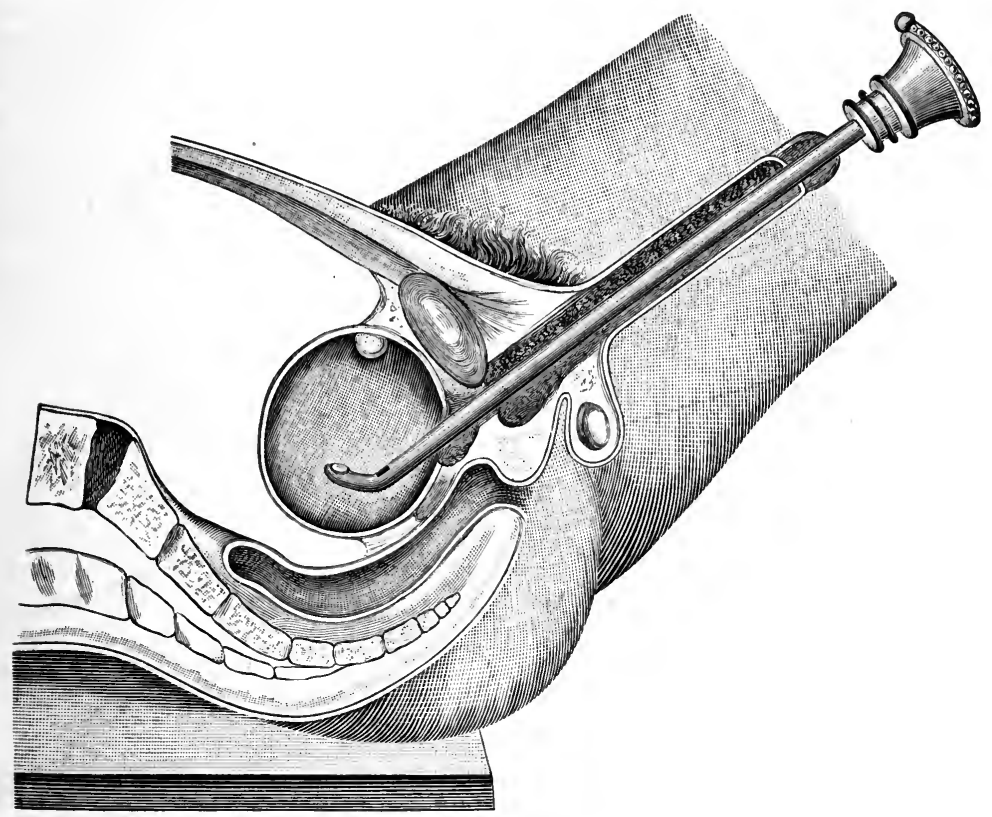

FIG. 24.-(Nitze.)

are in these cases less extensive, besides being more painful to the patient and quite tiresome for the cystoscopist. It is difficult to explore the bladder thoroughly. In obstinate cases narcosis should therefore be used. It will be a great help. In very pronounced cases we may need cystoscope Nos. 2 and 3.

(Every solid instrument (sound or catheter), if introduced through a normal male urethra, shortens the latter for about three quarters to one inch. This is caused by flattening the curve which the normal urethra makes in its way around the lower border of the symplysis (Fig. 23). This flattening depresses the prostate and stretches the ligamentum suspensorium penis (Fig. 24). Thus a double-armed lever is formed. The

ber has then, however, also to be increased, otherwise the illumination would be insufficient. (The note may find a place here that, on the other hand, a good cystoscope can be constructed for use in ehildren as small as No. 16 French.) In such exceptional cases we should make it a rule to explore the urethra with a prostatic sound a few days previous to the examination, if this is feasible. It will tend to spare trouble to the patient and disappointment to the cystoscopist. 
relative strength of its two arms determines the middle (equilibrium) position of the sound which was introduced and left to itself. In a normal bladder this position is such that the shaft does not touch the fundus, but forms with it an angle of about $20^{\circ}$, open posteriorly (Fig. 24). In cases of hypertrophy of the prostate the prostatic portion of the urethra is rigid, the ligamentum suspensorium penis, as in all older patients, less

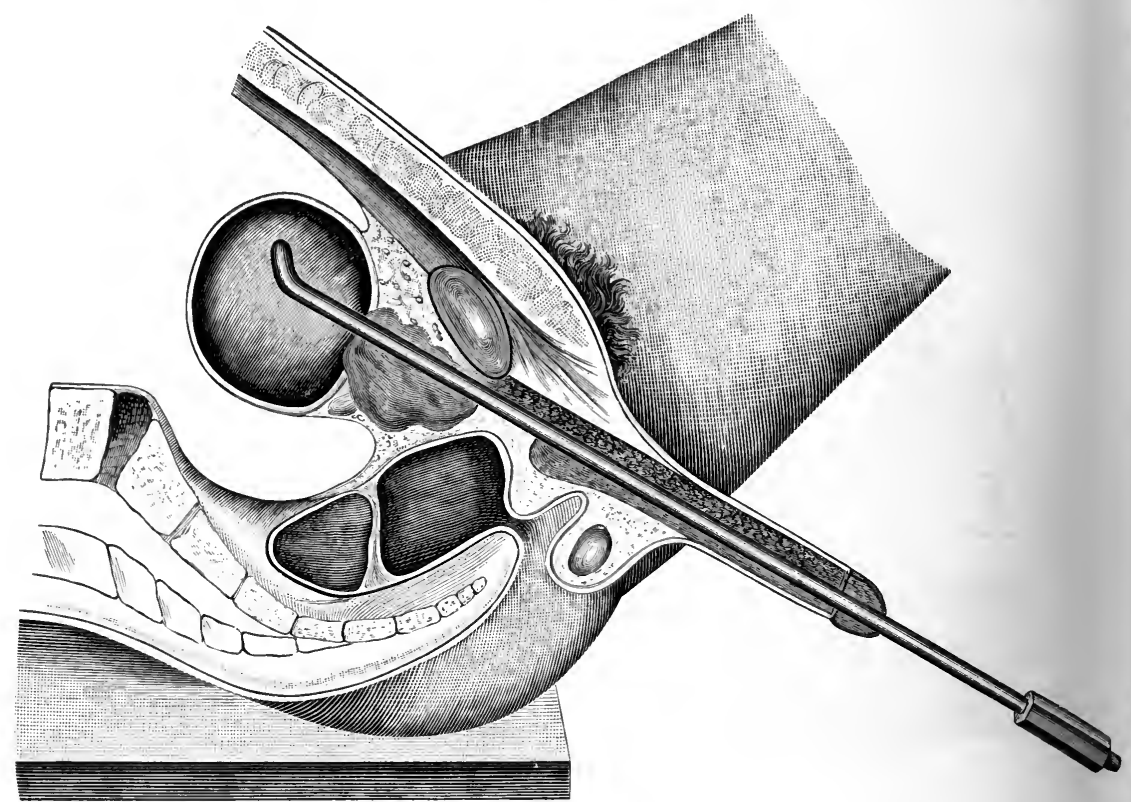

FIG. 25.-(Nitze.)

firm. Thus the equilibrinm of the sound is changed, as demonstrated in Fig. 25. The axis of the latter runs parallel with that of the body, or is even deviating posteriorly.)

4. Be eareful in estimating the size of the different lobes of the prostate, especially of the median. They can be observed with No. 1, lying in the shade. No. 2 illuminates them. The prism is near, the object therefore greatly magnified.

Cystoscopic Appearance of the Healthy Bladder.-To avoid grave mistakes in interpreting the pietures observed in the diseased bladder, the cystoscopist should first earefully study the normal viscus. Only if he gets no chance to inspect the interior of the healthy living bladder ought he to make use of the eadaver. The former is by far preferable. A number of important phenomena are to be seen only there.

The medical man interested in genito-mrinary surgery, who once has the chance to leisurely explore the bladder surface with his eyes, no doubt 
becomes an ardent admirer of this newest branch of surgical diagnosis. Not that the coloring (pale, yellowish-red, rarely pink) and surface of its mucous membrane (uneven, hillocky, on account of the slightly projecting bundles of the detrusor muscle), or the fine ramifications of minutest blood-vessels, similar to those seen on the retina with the ophthalmoscope (Figs. 26, 27, and 29), or the peculiar picture of the fold of mucons membrane around the internal orifice of the urethra (Figs. 26 and $27^{*}$ ), will especially strike him; not that the rhythmic pulsation of the same spot of the wall, especially on the lateral sides of the bladder (transmission of the pulse of the external iliac artery), or the glistening bubble of filtered air at the vertex (Fig. 26), intentionally injected for easier localization of the latter, and slightly rolling up and down according to the displacement of the intra-abdominal organs during respiration, will astonish him so much.

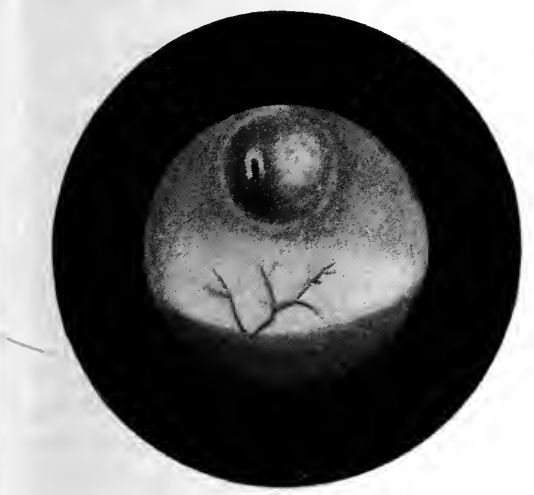

Fig. 26.-(Nitze.)

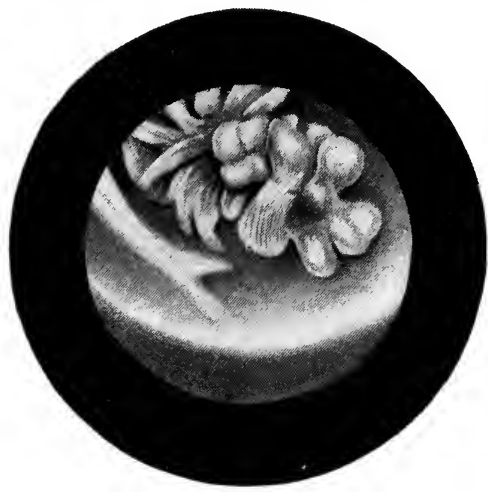

Fig. 27.-(Nitze.)

Nay, it is the cystoscopic picture of the fundus of the bladder, the trigonum with the mouth of the ureters and their folds of mucous membrane, which will arouse his most intense interest. Nearly every surgeon will once have had the opportunity of observing the small fountains of urine emitted from the everted mouths of the ureters in congenital exstrophy of the bladder, or perhaps in a specially favorable case of vesico-vaginal fistula. Often he may have watched the same picture, after the bladder had been opened by the suprapubic incision and the patient put in Trendelenburg's posture, and have then eren catheterized the small channels with ease. Yet it is like a revelation to watch this phenomenon under ordinary circumstances in the healthy living bladder which is filled with transparent fluid; to see the glycerin-like jets of urine, appearing intermittently about every thirty to sixty seconds, sometimes not oftener than every five to six minutes (and that in non-pathological cases), not simply

* Fig. 27 shows at the same time the cystoscopic picture of a benignant growth. 
run down as in the congenitally or artificially opened viscus, but create, physiologically, sudden whirls, which last a few seconds and then slowly disperse in the clear medium.

When I observed this for the first time with the cystoscope (January, 1888), no mention had yet been made, in the very few articles bearing on cystoscopy, which had been published up to that time, with reference to the peculiar, physiological, intermittent motions of the month of the ureters, and their adjacent fold of mucous membrane. On April 9, 1888, I expressed myself with reference to this observation in my paper "On Cystoscopy and the New Cystoscope of Nitze and Leiter" (New York Medical Journal, April 21, 1888), as follows: "In watching more closely the orifice of either of the ureters for some time, I have easily been able to see how the urine entered the bladder at intervals of from thirty to sixty seconds. I have been enabled to do so by the whirls which the urine-viz., a solution of salts-produced in the water that had been injected into the bladder (we can see the same arise at any time when we pour urine into a test-tube filled with water). On the same occasion I observed quite an interesting physiological phenomenon, which is not mentioned, as far as I have seen, in any one of our handbooks on physiology. It is well known that the urine enters the bladder at intervals with a rush, and so much so that it forms a real little fountain when the bladder is opened by incision. The urine is pressed forward, firstly, by the vis a tergo; secondly, by the difference in pressure between the pelvis of the kidney and the cavity of the bladder; and, thirdly, by the peristaltic contractions of the ureteral muscles. Now, as there are in the walls of the ureter more muscular fibers running longitudinally than transversely, the orifice of the ureter, and with it the elevation of the mucous membrane in which it ends, is drawn inward at the end of each conveyance of urine into the bladder, the orifice and its nearest adjacent parts then taking the shape of a funnel. After a few seconds it returns to its former position." Two years later I said (Annals of Surgery, June, 1890): "When the urine begins to enter the bladder, the mouths of the ureters with their elevation of mucous membrane keep their position, or are sometimes pressed a little forward. Only, at the end of each conveyance of urine into the bladder the orifice is drawn inward, and assumes with its nearest adjacent parts the shape of a funnel. As soon as the last drop of urine has been passed into the bladder the mouths of the ureters return to their former place." I mention this, because Nitze has seen the different phases of this phenomenon in rather the opposite succession: (loc. cit. page 147) "The mouth of the ureter first slowly contracts to the size of a very small groove, and then again gradwally enlarges to its former size, at this moment suddenly giving exit to the urine; it then remains in this middle position for a short while, until 
the recurrence of the same movement determines a repetition of the phenomena." Fenwick states (loc. cit. pages 82, 83): "If one of these orifices be watched carefully, the curious phenomenon of efflux will be seen. The little slit will suddenly gape and a tiny swirl of fluid will be emitted. . . . Not infrequently the observer will notice a rhythmic contraction in the ureteral orifice and the surrounding bladder wall. Should the orifice be projecting inward as a cone, the apex of the body will alternately recede and protrude." I think that I am not mistaken in assuming that the funnel-like retraction and contraction of the ureteral "orifice" generally immediately follows, not precedes, the jet of urine. It announces its end, not the beginning.*

Cystoscopic Appearance of the Diseased Bladder.-Nitze gives a very good résumé of the capabilities of the cystoscope in pathological conditions of the bladder. The present electric illumination of the bladder, he says, gives us the means of establishing a strict differential diagnosis between the various forms of catarrh of the bladder; it is easy to see with it ulcerations, to demonstrate diverticula, to find and localize foreign bodies; it seems almost unnecessary to mention how plainly we can now see stones, make out their number, size, shape, and mobility, and percuss them with the beak of the instrument; how encysted stones, too, will not escape the examining eye ; especially the diagnosis of tumors of the bladder is now easy, and can be made early.

The rapidly following development of this new method, which is illustrated by manifold brilliant results in clearing up previously obscure urinary diseases with electric illumination of the bladder, is laid down in the already comparatively large literature on cystoscopy. I would by far trespass the limits of this article-which is mainly written to illustrate and describe the best and most improved instruments, the practical application of the cystoscope for diagnostic and other purposes, as well as its limitations-should I try to enumerate everything of interest that has so far been seen in the bladder, and described in published accounts. It would, besides, be of no intrinsic value. Certainly it is not exaggerated to state that the results obtained by cystoscopists of nearly every nationality within the last four years sustain Nitze's original statement and predictions in every particular.

The brief report of a few cases of vesical disease may illustrate the different chapters in their cystoscopic appearance :

1. Catarrh.-As in other organs, catarrh of the bladder manifests

* The ureteral "cone," this elevation of mucous membrane produced by the obiique direction of the lower end of the ureter in penetrating the vesical wall, often shows one or more rather slow peristaltic contractions which precede the efflux of urine. If these contractions are confined to the immediate neighborhood of the mouth of the ureter, the latter is pushed forward (erecting) before the whirl of urine appears. 
itself by redness and œdematous swelling of the mucous membrane, and in the production of a catarrhal secretion. The pictures show a great variety, and yet do not distinctly differ from each other, in accordance with the various degrees of cystitis, so much the more as the latter is so often combined with and especially dependent upon other vesical troubles.

a. Acute: Symptoms well known. Cystoscopy not indicated. Pictures only of pathologico-anatomical interest; especially studied in gonorrhœal cystitis by Finger (Wiener medizinische Presse, 1880, page 997). Mucous membrane, in proportion to the extent and intensity of the process, more or less swollen and turgescent, especially at the neck of the bladder, is marked with anastomosing, greatly dilated vessels (injection thus irregular, circumscribed, with interposed large healthy portions). In very acute cases the mucous membrane is studded with hæmorrhagic spots, streaks, or blotches (ecchymoses), or it appears of a uniform dark-red color. Epithelium scaled off, either collected in clumps or in long, thready streamers, which float in the contents of the viscus. The diseased surface bleeds if touched with the beak of an instrument. Other cases of acute cystitis have very nearly the same appearance, only the extent is sometimes more marked.*

b. Hæmorrhagic : A subacute, formerly not well-known, but important form of cystitis. Symptoms often precisely similar to those evoked by growth in the bladder; obstinate to cure. Character usually benign. According to Fenwick, "a certain form of hæmorrhagic cystitis precedes the formation of that stunted nodular carcinoma of the mucous membrane which accompanies interstitial malignant growth of the vesical wall" (compare the precancerous stage of the mucous membrane).

c. Chronic: Great variety of characteristics, according to the degree of the attending inflammation. If that be slight, the mucous membrane is strikingly pale, nowhere reddened, but thickened. After short irrigation only a few clumps of muco-pus are seen to adhere to its surface. In other cases the color of the mucons membrane is dark gray, probably resulting from often-recurring inflammatory attacks which had been marked by sub- and intra-mucous sugillations. Here rugæ are often swollen, of velvety appearance, especially on their surface, running parallel in the shape of longitudinal thick folds. They often appear circumscribed in irregular groups; strips of sticky mucus adhere to their surface and float in the fluid. They are easily mistaken for a tumor when near the internal urethral orifice (magnified) and inspected from the side. If an acute attack is grafted on such a chronic form, mainly the top of these folds and the portions around the neck of the bladder appear deep

* If pathognomonic symptoms are well defined, the use of the cystoscope is here as well prohibited as that of a catheter or sound. 
red. As just stated, the cystoscopic pictures of chronic cystitis show the greatest variety. Their study is very necessary to develop a skilled cystoscopist.

Note.-In many patients, male and female, who mainly complained of frequent micturition (every ten to thirty to sixty minutes) and tormenting tenesmus, having besides clear urine of low specific gravity with a large amount of bladder epithelium, sometimes of all the three layers, the cystoscope always revealed the same condition, namely, perfectly normal bladder; from the injected trigonum to the neck a continuous dense network of dilated capillaries. Whether this be primary or secondary, I should at present not venture to decide.

d. Ulcerative: (Non-tubercular.) Very rare, only occurring in very grave forms of vesical disease. Bladder always very irritable, barely holding two ounces. Cystoscopy therefore often impossible.

CASE.-Girl, seventeen. Enuresis nocturna, since childhood; great vesical, painless irritability (capacity, two to three ounces); hæmaturia, formerly intermittent, lately continuous. Urine ammoniacal. No tuberculous history. Cystoscopy: Many irregular deep-red patches of a granulating surface, partially covered with deposits of snow-white mucus spread over the whole interior of the bladder, especially numerous on the right side and vertex, still more in the fundus around the right ureteral orifice; intermediate mucous membrane slightly hyperæmic, near the neck of the bladder velvety; otherwise not materially changed. No bleeding during examination. Examination rather hurriedly performed, as the four ounces of fluid originally injected under pressure continuously flow away again alongside the cystoscope. Microscopical examination of deposit in urine seemed to reveal sarcomatous elements. Probable diagnosis : multiple sarcoma of the bladder. Soon afterward the surgeon who had brought the patient to my office performed suprapubic cystotomy, and carefully scraped the bladder as thoroughly as possible. Report of pathologist with reference to the removed scraps of tissue also in favor of sarcoma. Subsequent observation; however, excluded a new growth. Diagnosis (by exclusion): chronic non-tuberculous, ulcerative cystitis.

2. Tuberculosis.- $a$. Tubercular cystitis without ulceration. Pathognomonic cystoscopic picture of this trouble has not yet been definitely established. If tubercle bacilli have been found in the urine before cystoscopy is tried,* a localized hyperæmic spot of the vesical mucons membrane seen through the cystoscope has to be diagnosticated as being most probably one of tuberculous character (tuberculous infiltration).

An interesting case of tubercular cystitis, diagnosed in its beginning cystoscopically, is reported by Burckhardt.

Man, thirty-seven. Chronic gonorrhœeal urethritis, with following cystitis (for nine months). No gonococci, no tubercle bacilli. Local treatment with-

* They will be by far easier found, even in a relatively clear urine, and, if present in a very minute quantity, by the centrifugal method of Stenbeck, modified by von Frisch (Zur Diagnose der tuberkulösen Erkrankungen der Urogenitalsystems. Internat. klin. Rundschau, 1891, Nos. 28-30; also, Wiener klin. Wochenschr., 1891, No. 23, and Berl. klin. Wochenschr., 1892, No. 22, p. 531). 
out improvement. Cystoscopy : Mucous membrane hyperæmic, dotted with numerous minute ecchymoses ; their circumference dark red (Fig. 28). Many anastomosing vessels. Tubercular cystitis suspected. Diagnosis later corroborated, as tubercular epididymitis develops bilaterally, and necessitates castration.

It is to be hoped that such cases, if made out in this manner, will henceforth be published in their cystoscopic detail. Only then we may hope to diagnose the tubercular cystitis with the cystoscope, "even if no tubercle bacilli had been found in the urine."

b. Ulceration.-If no catarrh of the bladder is present, one or more characteristic ulcerations will be seen upon an otherwise perfectly healthy mucous membrane.

CASE.-Man, forty-five ; sick twelve years. Intermittent hæmaturia and increasing trouble since two years and a half. Suppurating right kidney. Tubercle bacilli abundant. Is the bladder also involved? Cystoscopy, April, 1890: Immediately above the swollen mouth of the right ureter and its elevation of mucous membrane an ulcerated spot of about the size of two silver dollars. Purplish-red, broad elevations (evidently the inflamed fibers of the detrusor muscle) cross each other in different directions. Small particles of snowy, shining mucus adherent to their surface float in the fluid. Depressions between these elevations of a lighter hue, also covered with mucous flakes. In between them numerous very small and larger sessile growths of grayish coloring, a few about as large as a pin's head, undoubtedly miliary tubercles. On one spot a small, rhomboid-shaped, dark-red (hæmorrhagic) spot. The entire other inner surface of the bladder perfectly normal, of grayish-white color, rather anæmic, corresponding with the condition of the patient.-(AUTHOR.)

In all these cases the cystoscope has to be used with great gentleness and care. The trouble of patients who suffer from tuberculous vesical disease is sometimes greatly aggravated by introducing a solid instrument into the bladder. If the diagnosis is clear, cystoscopy is contra-indicated.

Hypertrophy of the Prostate, especially its Median Lobe-Trabesular bladder (vessie à colonnes).-Although cystoscope No. 1 does not "illuminate" the internal urethral orifice, still we can see with it a swollen prostate gland, and after a sufficient experience estimate its size. On the fundus, or rather between it and the internal urethral orifice, a small portion of the gland-in the picture a massy projection situated between prism and lamp-is of high red coloring, on account of the oblique direction of the rays. The other part, especially the prominent median lobe, is lying in the shade.* Frequently we see the internal urethral orifice surrounded by a collarette of the hypertrophied gland. If it be sore, I have often seen blood oozing from its surface in a continuous small, shadowed stream, or a greatly magnified coagulum, adherent to its surface, streaming in the fluid.

* With cystoscope No. 2 all three lobes can be illuminated. (Fig. 29.) 
Very important for diagnosing hypertrophy of the prostate is the (diaphanoscopic) picture of the fold of mucous membrane around the upper circumference of the internal orifice of the urethra, to be briefly called "inner fold." Under ordinary circumstances this fold presents itself at the moment the prism enters the bladder, in the shape of a sharply cut half-moon. On account of the peculiarities of the optic apparatus its picture is seen inverted, and therefore observed in the lower half of the cystoscopic picture, its concavity pointing upward (Fig. 26). If the prostate is in the state of hypertrophy, this neatly and everywhere equally outlined thin fold is thickened, irregular, and nodulous. The gentle concave curve is represented by a deep angular notch with two upwardly divergent thick folds (Fig. 30).

Especially prominent in the picture, but greatly enlarged, appears the median lobe of the prostate gland.

CASE.-Man, seventy. Relies entirely upon catheter, which has frequently to be passed. Passage attended by difficulty and pain. Urine clear; no albumin, no casts; great local distress. Cystoscopy : Slightly hypertrophied

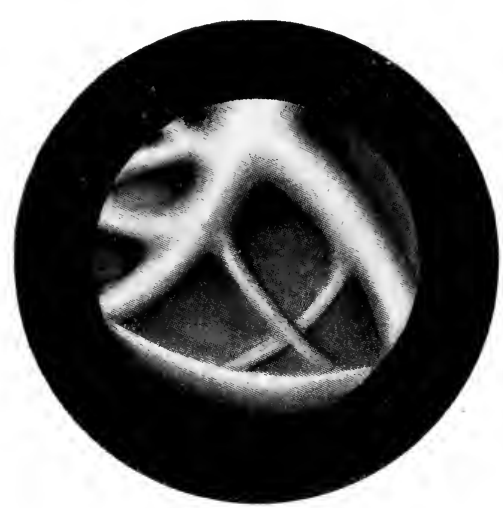

FIG. 31. (Nitze.) median lobe of about one half to three quarters the size of the third phalanx of the middle finger, easily diagnosticated; the bladder presents a vessie à colonnes in a most beautiful manner (Figs. 30, 31 , and 32). On suprapubic cystotomy, performed by another surgeon, the third lobe was found as diagnosticated.-(AUTHOR.)

Diverticulum.-Its formation is closely connected with trabecular hypertrophy; therefore mostly found in such cases, solitary or multiple, sometimes in all stages of development (Fig. 32).

CASE.-Man, sixty-four; diabetic, hypertrophy of the prostate, chronic catarrh. Cystoscopy: All symptoms of chronic catarrh; trabecular bladder. A little above the mouth of the left ureter a large dark spot of about the size of a five-cent piece; its lining mucous membrane wrinkled; no more dark spots visible. Light of cystoscope turned off, and tip posted right in front of this spot, gently pushed forward. It evidently enters a cavity, but touches no concrement. Diagnosis: Diverticulum (Fig. 33).* Two years later epicystotomy becomes imperative, and shows a large diverticulum at the diagnosticated spot, (then) filled with phosphatic débris.-(AUTHOR.) Stones.")

* The stones, as seen in the picture, were not present in this case. (Cf. "Encysted 
Foreign Bodies.-It is self-understood that foreign bodies, not to be detected by the sound even in the hands of a well-experienced surgeon, can not only be seen but localized.

Case I.-Man, fifty-four; tabetic, who catheterized himself. Having done so one night, patient fell asleep. On awakening, catheter nowhere to be found. Entered von Dittel's clinic. Gruenfeld's endoscope, tried first, gave negative result. On using the old Nitze-Leiter endoscope, December 16,1883, there came

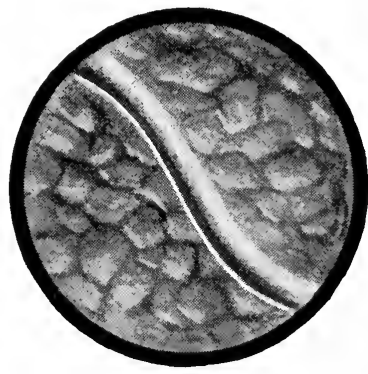

FIG. 34.

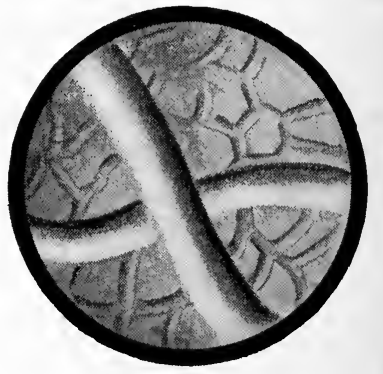

FIG. 35.

into view " a symmetric, elongated, yellowish body, somewhat like an 'ascaris lumbricoides,' which was evidently the catheter covered with a yellow urinary deposit" (Fig. 34). In one spot it was seen as reflected over itself (Fig. 35). It was extracted with a lithotrite.

CASE II.-Lad, eighteen: had introduced a medium-sized pin, head forward, into his urethra. Nine days later, on account of great pain, admitted to Nico ladoni's clinic. Different instruments failed to detect the body. By means of the old-shaped cystoscope, pin plainly seen fixed in the right part of the anterior wall, close to the vertex. It cast a shadow on the opposite wall of the bladder, and could be well demonstrated to a large assembly (Fig. 36).

CASE III.-Lady, thirty-five; ovariotomy, by Martin, of Berlin, April, 1885; pedicle and vessels tied with silk. Recovery. Two years later, serious sickness. At last an intraperitoneal abscess perforated into the bladder. Shortly afterward stone discovered. A portion removed with the lithotrite. Patient then evacuated a silk thread. One month later, sound demonstrated presence of stone, left at the first operation: crushed by Nitze, August 18th. A few silk ligatures passed and extracted. Two days later, neither sound nor lithotrite could detect anything further in the bladder. Cystoscopy (Nitze): On the left side another ligature discovered. "One of the ends projected out from the walls, glistening in the brilliant illumination of the light; a smaller and shorter end was found below, and the middle, or loop and knot part, was buried in a rosecolored granulation-papilla, which sprang from a well-marked, crater-like depression in the mucous membrane (Fig. 37). The longer thread threw a distinct shadow on the subjacent surface, which traveled to and fro according to the movements of the lamp. The ligature had exactly the same appearance as the one removed the previous day in the blades of the lithotrite. The condition thus being diagnosticated and localized, another thread two and a half inches long was cleverly caught with the blade of the lithotrite and removed by Nitze. The knot with the shorter end remained in the wall, as seen again through the cysto- 
scope. Patient had then to leave for America. Condition explained to her. November 11th, the same year, a small stone was extracted by Prof. Gross, of Philadelphia, hanging on that silk thread which had to be left in situ by Nitze three months previously. Entire recovery.

Stones.-It seems almost unnecessary to mention that we can plainly see stones in the bladder, make out their size, shape, and mobility, and percuss them with the beak of the cystoscope, by the guidance of our eyes.

CASE.-Man, fifty-three; short-lasting lumbar pain on left side, in fall of 1886. One year later developed symptoms of stone, but no hæmaturia, no stoppage of flow of urine. Repeated examination by a very able surgeon failed to detect stone. Condition getting steadily worse, patient was sent to me for cystoscopy. On patient's urgent request, cystoscope at once introduced. In the fundus an oval-shaped, brownish body covered with white spots (evidently phosphatic deposits) of the size of an almond at once detected. It lay in the pouch behind the enlarged prostate, and threw a distinct shadow upon the opposite wall of the bladder. Light turned off, and body touched with tip of instrument. Unmistakable click. Suprapubic lithotomy. Stone easily removed. It presented the characteristics as seen with the cystoscope.-(AUTHOR). A very good cystoscopic picture of stone is given by Nitze (Fig. 38).

Encysted Stones.--Fenwick reports the following case (Intern. Med. Annual, 1892, page 119):

Man, sixty; symptoms of an enlarged prostate, complicated with stone, present for many months. Sounding, on four separate occasions, with and without ether, resultless. Cystoscopy: A "pile of small concretions lodged deep in a sac" (Fig. 33). A small-jawed lithotrite was guided to the mouth of the pouch and the beak edged in ; calculi were seized, pulled out and crushed. Complete recovery. Cystoscopy one year after: Sac greatly contracted; free from stone.

Tumors.-In establishing their " early diagnosis" cystoscopy celebrates its greatest triumph. Many cases have been reported where the cystoscope easily revealed this formerly obscure trouble. (Cf. Albarran, loc. cit., page 422 and.following pages.)

CASE.--Man, fifty-three: for six months pain in glans, frequent forcible micturition, continuous hæmaturia. Cystoscopy: Round sessile growth with an uneven surface, not unlike a large, round strawberry, on the left wall of the bladder about an inch above the mouth of the left ureter (Fig. 39).* Blood oozed out of its surface. Bladder otherwise healthy; symptoms of slight catarrh. Diagnosis: Cancerous sessile tumor of the bladder. Suprapubic eystotomy. March 25, 1890: Growth on the spot localized before, with all the characteristics as diagnosticated with the cystoscope; cut out with knife, base burned with Paquelin. Recovery. No recurrence to date (AUTHOR).

* Picture corresponded exactly to the accompanying cut, which is taken from Von Antal, loc. cit., page 406 . 
NотE.-Fenwick believes he has seen and found a peculiar condition of the vesical mucous membrane which he calls "precancerous." Four interesting cases are reported for illustration. He rightly advises the cystoscopist to be guarded with his report in eases where a projecting tumor or a very definite change of the mucous membrane is absent, but the obstinate character of the symptoms arouses suspicion. The cystoscopist "will be acting wisely by simply declaring the bladder to be free from any projecting growth or tumor, and by stating that operative interference is not at once called for. At the same time, he will do well to hint that such conditions of mucous membrane are likely to degenerate into carcinoma, and that a re-examination at the end of three months is advisable" (loc. cit., p. 160).

How greatly such exact diagnosis * at once facilitates both prognosis and treatment of bladder diseases, is obvious. The more thorough knowledge of the various forms of catarrh of the bladder will demand a modified treatment. The diagnosis of ulcerations of the wall of the bladder, especially those of a tuberculous nature, will force the knife into the surgeon's hands to perform suprapubic cystotomy, to scrape the ulcers under the guidance of the eye, and burn their base, or to make a radical extirpation which includes the wall of the bladder. Foreign bodies, plainly localized, can be removed with absolute certainty by a proper method. If, after litholapaxy, any detritus remains in the bladder, the cystoscope will find and localize it, and therewith greatly facilitate its removal. The only plausible reproach which has been addressed to litholapaxy has thus lost its foundation. Litholapaxy, with the help of cystoscopy, will forever rank highly among operations for stone. If a catheter with a curvature adapted to the locality of a diverticulum is introduced into the bladder, it may be washed out, and the catarrh caused by its presence may be thus improved. If a diverticulum has been seen, the bladder must be very carefully distended for suprapubic incision.

The treatment of tumors of the bladder has been especially benefited by cystoscopy. "Early diagnosis and removal" characterize the progress in this class of cases. To remove benignant pedunculated growths, Nitze proposed not to incise the bladder, but to crush the growth when localized with a long forceps introdnced through the urethra. The curvature of the forceps must correspond with the insertion of the growth. $\dagger$ The operation may be performed in one sitting or more. The result will be easily controlled by repeating cystoscopy after some time. Von Antal and Fenwick have successfully operated according to this method; the tumors should be torn off, however, not crushed (Von Antal). In view of the continuonsly vanishing dangers of epicystotomy, and the sometimes can-

* We may rightly presume that the near future will define the eystoscopic picture of beginning tubercular cystitis, and probably that of syphilitic condition of the mucous membrane.

$\dagger$ G. G. IIopkins, of Brooklyn, has constructed a new curette for the male bladder, which, as it seems, can be well used for this purpose (Brooklyn Med. Jour., January, 1890). 
cerous base of an even small, seemingly benign, villous growth, this latter operation will, in most cases of this kind, be the preferable interference. The removal of growths through the urethra should be confined to polypi. Still, it seems that the strict visual diagnosis may in the future allow operative interference by way of the urethra in many instances. Nitze's operating cystoscope (not yet finished) is the latest and boldest ontcome in this direction. With its aid we may look forward to be able to carry on intravesical treatment without any cutting operation, under the direct guidance of our eyes.

Certainly it has been sufficiently established to-day, that if any doubt exists of the diagnosis in a case of vesical disease, and the three cardinal conditions which enable a cystoscopic examination to be made are fulfilled, it is the duty of the attending physician to submit his patient as early as possible to this ocular inspection. This for the same reasons as would lead him to use a mirror to look at the interior of the larynx, eye, nose, or ear, in their respective disorders.

Visual Fallacies of the Cystoscope, and how they may be avoided.-The fact must be emphasized, that the cystoscopic diagnosis in bladder diseases is not at all always easily made. Cystoscopy requires in praxi a great deal of patience, and much experience, in order to avoid mistakes. "It is perfectly true," says Fenwick (loc. cit., page 93), "that a novice may recognize a typical growth or a glistening stone without difficulty, but it is also equally true that the inexperienced cystoscopist will be readily and more often deceived by the perplexing appearance which the mucous membrane is wont to assume under the varying conditions of relaxation, congestion, extravasation, and infiltration, and may be tempted to interfere, operatively, to his own and the patient's detriment, under the belief that he has to deal with a growth, when no such morbid condition is present." The above-given rule should also be borne in mind, always to make first a careful general examination, and let cystoscopy be the last leelp in the attempt at getting down to a correct and well-sustained diagnosis.*

Here are a few selected examples for the beginner, as well as for him who uses the cystoscope without a previous general examination:

The beginner will easily mistake the taggy shreds of necrotic tissue,

* To avoid mistakes in performing cystoscopy, the important peculiarities of the optic apparatus should be closely studied: 1 . In moving, for instance, the cystoscope from the internal urethral orifice toward the posterior wall of the bladder, the different areas appear in the opposite direction. They enter the field of vision at the beak and move toward the observer. 2. If the beak is held parallel to the fundus of the bladder, objects, which really run transversely from the right to the left, appear in the cystoscopic picture to run from upward down, and those the longest dimension of which passes from the front backward, appear to run in the transverse diameter (for particulars, see Nitze, loc. cit., pages 52-56). 3. If the prism is moved near to the object, the latter is magnified; if it is withdrawn, the opposite effect is produced. 
hanging at the edge of a tuberculous ulcer and floating in the fluid, for a polypus, without at all noting the ulcer, especially if the prism is brought close to it. But the experienced and careful cystoscopist has probably found, in a previous examination, tuberculosis of the testicles, or an enlarged kidney, or tubercle bacilli in the urine, or such detritus under the microscope, as will lead him to suspect the existence of an ulcer, and thus make him look out for it.

Again, the beginner may take a very projecting ureteral cone, or a prolapse of the ureter, for a sessile growth. But if the prism be carried near to the latter, this growth is found to be entirely transparent, and on careful inspection it will be seen that out of one spot of its perfectly round and smooth surface a whirl of urine will suddenly be expelled. The pathognomonic symptoms of the ureteral cone are, "that its position corresponds to the postero-external angle of the trigone; that the summit is slightly flattened, depressed, and occupied by a small orifice, whence tiny jets of glycerin-like fluid issue at various intervals; and, moreover, that the apex not infrequently protrudes and recedes rhythmically" (Fenwick, loc. cit., page 97 ). . . . The beginner will take an incrusted growth for a stone-a touch with the beak will generally dispel all doubt. . . . He will be apt, in seeing the picture of the enlarged median lobe of the prostate, to diagnose a benign or malignant growth in this position; . . . or, in observing the elongated, oval, or roundish elevation, of a deep-red, gelatinous aspect, caused by a submucous hæmorrhage, to diagnose epithelioma, being unaware of the importance of the differently shaped hæmorrhagic spots so often seen in the neighborhood of the former. ... He will be puzzled by a deposit of coagulated blood around one of the ureteral cones, while the experienced will take it as a hint at hæmaturia of renal origin, most probably on the same side.*

Success will also not be with him if he only works with cystoscope No. 1, and the just injected clear fluid turns murky at once after a longcontinued irrigation, or is found to be so as soon as the cystoscope has entered the bladder. In such a case the experienced man at once suspects kidney trouble, will exchange the ordinary for the irrigating cystoscope, and watch, after a quick glance at the interior of the bladder, the ureteral orifices. Most probably pain in one lumbar region or a swelling there, already perceptible to palpation, will guide him on which side to look first.

Of course, an infiltrated spot in the wall of a bladder which can not be properly distended, or greatly hypertrophied and easily bleeding

* The above-quoted pitfalls have all once puzzled the author. A few more are enumerated by Fenwick in his book, selected from the immense cystoscopic material which came under his observation. 
rugæ in a case of localized chronic catarrh, will also sometimes mislead the well-trained eye, and induce one to diagnosticate a tumor where the suprapubic incision will merely show infiltration. Also many other mistakes may occur. But they will become rarer with increasing experience; and if, nevertheless, they still occur, the physician may console himself with the reflection that mistakes occur just as often and as easily in the other and older branches of surgical diagnosis.

Cystoscopy with Reference to Kidney Diseases. - That the diagnostic capabilities of the cystoscope are not limited to diseases of the bladder, but extend in many cases with admirable exactness also to those of the kidney, is an established fact. Not only can we with its help often localize the seat of the trouble-negative vesical evidence giving a positive diagnosis of renal disease-or distinguish whether there are two working kidneys, whether both are affected or only one, and then which of the two. Nay, the cystoscope gives us the means in certain cases to even go one step further-to determine whether the other kidney already is doing the work for the one which is diseased.

All this is accomplished by observing the character of the jets of urine at the ureteral orifices (transparency, color) and timing their frequency and duration, and also by noting the position, shape, and injection of the ureteral orifice itself (now and then with its fold of mucous membrane) in comparison with that of its fellow.

Under ordinary circumstances it is not difficult to determine whether the urine propelled from the ureteral cones is clear, murky (purulent), or bloody. We simply have to place our prism just opposite and comparatively close to the mouth of the ureter and then carefully watch.* Some experience and patience, a quiet hand, and close attention are all that is needed. But, as mentioned above, this task at once grows more difficult if the injected clear water rapidly becomes turbid. The inspection can then only be effected by means of the irrigating cystoscope.

A few cases may illustrate this :

1. Renal Hcematuria.

CASE I.-Female, forty-six; painless hæmaturia of twelve months' duration. Cystoscopy, made at the time of bleeding: Bladder perfectly healthy. In watching the left ureteral orifice, a red, rapidly propelled whirl was seen to cross the prism and slowly mix with the transparent water, slightly coloring the same. After a short while the phenomenon reappeared. Diagnosis: Unilateral renal hæmaturia; according to the symptoms, evidently caused by a new growth. Nephrectomy proposed and declined. The patient died not long afterward after an abundant hæmorrhage.-(AUTHOR.)

CASE II.-Man, thirty; intermittent hæmaturia since two. years. Urine

\footnotetext{
* Even with the strongest eyes a close watch of a ureteral orifice lasting longer than a
} few minutes (five to six) is impossible. The eyes get tired, and vision is blurred. 
often more bloody toward the finish. Cystoscopy: "The entire bladder was healthy. I was just giving up the examination in despair, when I saw a stream of brightish blood shoot right across the prism. Keeping the instrument fixed, I waited until the medium became clear again, and then I found that I was
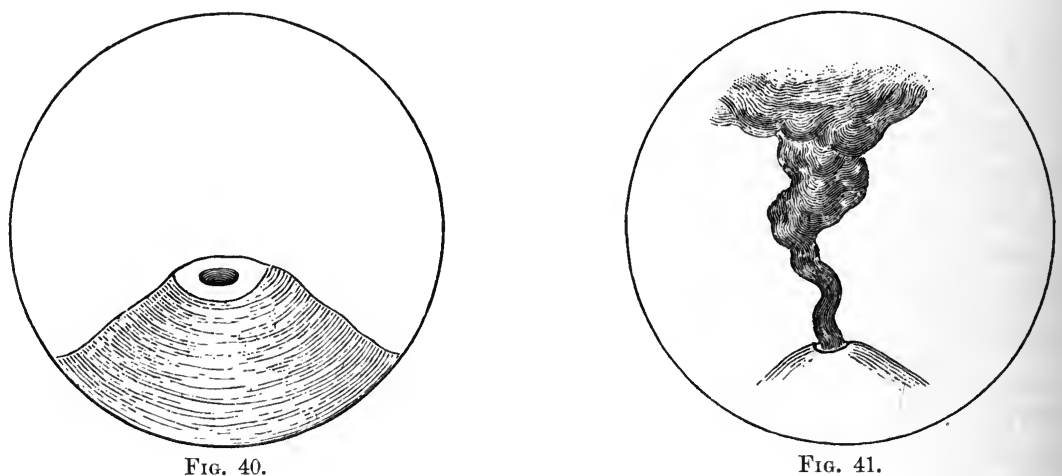

watching the orifice of the right ureter. In another second a jet of bloody urine burst from the tiny opening, and, after forming many rings, it paled by diffusion and disappeared, only to be replaced by a successor (Figs. 40,41). The right renal source of the hæmorrhage was at once indicated" (Fenwick,*loc. cit., page 93).

\section{Renal Pyuria.}

CASE I.-Lady, forty-five; cloudy urine for years, lately thick. Since a few days high fever. Tumor palpable in left groin. Cystoscopy under irrigation: Prism turned to the left, and murky fluid allowed to run off while handsyringe threw in short jets of clear water. The fog lifted; left ureteral opening clearly perceived. Out of it, nearly at the same moment, a forcible eruption of a snowy-white, milky fluid. Spurting forward into the medium, contained in the bladder, in the shape of a fire-sheaf, the first rather thick mass at once dispersed and was dissolved into myriads of minutest snowflakes, which slowly came down and at once put a stop to all further examination. Manœuvre repeated with same result. Jets descend frequently. Urine of right kidney clear. Diagnosis: Left suppurating kidney, probably stone in pelvis. Nephrotomy, later nephrectomy. Perfect recovery. Specimen: Pyonephrotic kidney with a large stone.-(AUTHOR.)

CASE II.-Lady, forty-five. May, 1887, nephrotomy for left (floating) cystic, partially suppurating kidney. Condition satisfactory for two years; then

* In another case of profuse hæmaturia, also diagnosed and localized with the cystoscope as being of renal origin. Fenwick extirpated the kidney two months and a half after the onset of the symptoms. On making a section of the gland, he discovered "a small monkey-nut-sized growth fungating into the pelvis. This was proved to be the apex of a carcinomatous degeneration which occupied the middle of the anterior half of the kidney. It did not approach the surface, and was quite unfeelable and unrecognizable by means of the finger while the organ was in situ" (International Med. Annual, 1892, pages 118 and 119). 
chills, fever, unbearable vesical irritability. Marked ureteritis. Cystoscopy: Catarrh. At seat of orifice of left ureter a succulent and curiously folded growth-viz., the swollen and inflamed fold of mucous membrane belonging to and surrounding the ureteral opening; whole mass resembling a large lampshade. Short jets of fluid, mixed with smaller and larger yellow flakes and shreds, come from a somewhat retracted spot, a little to the left from the top of the mass. A few minutes later a long thread of thick, yellow pus was slowly making its way out of the same opening into the bladder. Orifice of right ureter rather rounded; pumps frequently, but comparatively regularly. Urine clear. Efflux timed: Right, every twelve to twenty-five seconds; left, every six to eight minutes; the period of the emission of the jet on the right somewhat longer than on the left side. Conclusion: Left kidney nearly useless; right, seemingly healthy, doing from twelve to sixteen times as nuch work as the left. Specimen obtained by successful nephrectomy finally corroborated the cystoscopic diagnosis. - (AUTHOR.)

CASE III.-Lady, forty-eight; urinary trouble for fourteen years. Lately urine very offensive, murky, two thirds of volume sediment. Cystoscopy, December 18, 1890: Mucous membrane hyperæmic, and around left ureteral orifice papillomatous. Out of the latter a very long, worm-like, under the electric illumination snow-white, shining thread of thick pus of the size of a thin pencil very languidly makes its ways at short intervals. No jet whatever. 'The long strings settle and curl in the bladder fundus-a very characteristic picture. Right ureteral orifice occupied by a round red growth of the size of a cherry. On putting prism quite close to it, it appeared transparent. Out of its left (median) side jets of seemingly clear urine thrown at short intervals. Prolapse of ureter. Cystoscopic diagnosis: "Left suppurating kidney. Its secreting tissue entirely gone. Right kidney already does double duty for its destroyed fellow. Some irritation in the pelvis of the right kidney." Nephrectomy. Kidney greatly enlarged, sclerosed, with many cavities; in them a large ramified stone. Uninterrupted recovery. On thirty-ninth day intense right renal colic and abundant hæmaturia. After five days of serious-nearly hopeless-illness, a stone was passed, an excellent "demonstratio ad oculos" of the pathognomonic correctness of prolapse of ureter. Rapid, perfect recovery.-(AUTHOR.)

CASE IV.-Gentleman, fifty-three; suprapubic lithotomy in 1888. Three years later symptoms of left renal calculus with irritable bladder. Cystoscopy, April, 1891: Bladder comparatively healthy. Prostate large, easily bleeding. Right ureter pumping at regular though rather short intervals; ejects clear fluid. Whirlings propelled from left ureteral opening by far less frequent; last nearly as long as three of the opposite side combined. Phenomenon can be noticed with so much greater precision, as urine of corresponding kidney is slightly turbid. No renal hæmaturia present. Diagnosis (also with reference to history, general condition, etc.): "Stone in pelvis of left kidney large enough to partially block the upper ureteral opening." Second cystoscopic examination fully corroborated result of first. Explanation: Urine has to gather in greater quantity, and has to distend pelvis of left kidney more than ordinarily before finding, or rather making, its outlet on one or more spots alongside the necessarily irregular surface of a probable stone. Nephrolithotomy, May 5, 1891. Large stone two inches long, presenting perfect cast of the enlarged pelvis of kidney and upper part of ureter. Lower pole flattened in a shape not unlike the mouth-piece of a flute. Suture of pelvis. Primary union. Rapid 
recovery. Sixteen days later, at home, very intense renal colic on opposite (right) side, lasting six hours. To-day free from pain. Still under observation. -(AUTHOR.)

CASE V.-Man, sixty-eight; bladder trouble for ten years. Intermittent retention and slight hæmaturia; depends upon catheter; marked vesical catarrh; irritability; pain in left lumbar region.

Irrigation of bladder for one week. Then cystoscopy: Trabecular bladder. Mucous membrane pale. Prostate enlarged in entire mass. Right ureter pumps at regular intervals. Jets at mouth of left not marked. There is a continuous exit of whirling, slightly turbid water, which did not stop during an at least five minutes' examination. Mouth of ureter always in same position; does not protrude nor recede; widely gaping. Picture resembles exactly that of a slightly smoking chimney. Eyes being tired, light was turned off and examination stopped. Phenomenon then watched again; same result in most elegant precision. Diagnosis: Hypertrophy of prostate, chronic catarrh, dilatation of left ureter with loss of muscular power. Urine simply runs down into the bladder as soon as formed, mainly on account of its gravity. Pyelitis sinistra.-(AUTHOR.)

CaSe VI.-Male, thirty-nine. Sent for diagnosis by an eminent specialist. Fifteen years ago, gonorrhœal discharge for four months; bilateral epididymitis. Seven years later, chancre. Five years ago, again urethral discharge. Two weeks later suddenly acute right pyelitis with grave bladder symptoms. In eight ounces of urine at least six ounces of thick, gelatinous pus. For three months seriously sick. Since then condition bearable, sometimes satisfactory; intervening times of general malaise. Irritable bladder. Slight hæmaturia. January, 1891, chill; recurrence of serious character. External and internal urethrotomy. Lately, sound 32 French passes. Questions to be answered: Is neck of bladder affected? Are both kidneys diseased, or only one; if so, which? General examination: No lumbar tumor; prostate slightly enlarged. Urine: Chemical analysis negative: microscopical: a few pus-cells; micro-organisms of various kinds; no tubercle bacilli. Scolices and hooks of the parasite "tænia echinococcus" (hydatid).* Cystoscopy: Bladder healthy. Fundus hyperæmic. Prostate irregular. Right ureteral orifice oval, gaping, about three times as large as on the opposite side, not on top of cone, but drawn backward from the fold of mucous membrane. Jets clear, now and then with a few long white shreds. $\dagger$ Left ureteral opening and kidney urine normal. Diagnosis: Echinococcus (hydatid) of right kidney, probably multilocular cyst or cysts, communicating with pelvis of right kidney. Right chronic ureteritis. Indication for radical cure: Extirpation of right kidney. Whether this operation has been performed meanwhile I am unable to tell.

* Patient, when asked later, stated that as long as he can remember he had dogs around him. He often petted and "kissed" them.

† I want to state here that, even with the able help of the irrigating cystoscope, it is extremely difficult, if not in many cases impossible, to make out a urine which is only very slightly turbid, or which is clear and contains long, tiny shreds of tissue. Generally a certain amount of turbidity is required before it becomes visually apparent and perceptible. Bimanual, sharp palpation of the suspected diseased organ during examination will frequently help in making the cystoscopic diagnosis of renal pyuria. 
Note.-For making a urethro-cystoscopic report, I should venture to propose the following scheme for the cystoscopist:

I. Urethra: 1, Meatus: 2, caliber ; 3, discharge from anterior urethra; 4, discharge from posterior urethra: 5, urethroscopic result.

II. Prostate gland: 1, Amount of residual urine; 2 , rectal palpation: 3 , cystoscopic appearance (also of fold of mucous membrane around upper circumference of internal urethral orifice, so-called "inner fold").

III. Bladder: 1 , Urinary analysis; $a$, chemical ; $b$, microscopical ; 2 , eystoscopic picture; $a$, vertex, posterior and lateral walls; $b$, fundus; $c$, right ureteral opening and cone; $d$, left ureteral opening and cone.

IV. Ureter: Rectal or vaginal palpation; $a$, right; $b$, left.

V. Kidney: 1, Bimanual palpation, etc.; 2 , jets of urine; $a$, as seen with the cystoscope; color; transparency; frequency; other characteristics: $b$, as gathered separately through catheterization of the ureters (dream of the future).

VI. Remarks.

VII. Cystoscopic diagnosis.

As is seen, the cystoscope has literally carried a bright light into this hitherto dark field of urinary surgery. It has elevated the diagnosis of an obscure urinary disease from mere guesswork on a scientific base. It has thus greatly widened and strengthened our means for making the indication and prognosis of nephrectomy. The time has more or less passed where the surgeon, after a nephrectomy, must anxiously wait to know whether the other kidney would now kindly do the work for its removed fellow, or whether this organ of last resort were also diseased. To-day these vital questions can nearly always be settled before the operation, by cystoscopy. Its results are reliable; they may, will, and must guide the surgeon. If the latter has previously ascertained that the opposite kidney is working well and that its urine is apparently healthy, and if, then, immediately after nephrectomy, the urine voided through the urethra suddenly appears very turbid, this will be proof to him that the second kidney is also diseased.* He may conclude that the sudden hyperæmia of the remaining kidney, setting in after the removal of the other (Author: Nephrotomy for the relief of sudden total suppression of urine occurring some time after nephrectomy. Annals of Surgery, April, 1892), has aggravated the slumbering trouble and made it thus apparent. Prognosis then doubtful. Still, it is to be expected that the sudden increase of the disease in the remaining kidney, especially if it be of a surgical character, will gradually wear off with the re-establishment of a more normal circulation. If, however, the urine, which in the cystoscopic examination before the operation had clearly been seen descending from the opposite kidney, is suddenly suppressed after nephrectomy for longer than twenty-four to forty-eight hours, the surgeon has still resources by which he may try to save the patient's greatly endangered life. If the anuria is not due to nervous reflex, it may be caused

* Tubercular urinary disease is excluded from these considerations. 
by a mechanical occlusion of the pelvis or ureter of the remaining kidney. And this mechanical occlusion may still be removable by a promptly performed nephrotomy on the second kidney.

These cystoscopic facts bearing on the condition of the kidneys will also tend to make superfluous, in the majority of cases at least, a suprapubic or perineal incision for diagnostic purposes, as well as a nephrotomy performed for determining the action of the other (not diseased) kidney, as also determined opening of the peritoneal cavity during nephrectomy, to prove, by palpation, the presence of the opposite kidney.

Of course, not all exploratory operations will thus become obsolete. It is obvious that there will still be a number of cases where obstacles will render the ocular inspection of the bladder and of the descending jets of urine impracticable; where an insufficient capacity of the bladder, or an abundant vesical hæmaturia, will make the electric illumination of the bladder useless. In such cases suprapubic cystotomy for catheterizing the ureters in renal disease (and even Sir Henry Thompson's digital exploration of the bladder for diagnosticating vesical troubles) may still be indicated. But these cases will henceforth be exceptions, and their number will continue to lessen with every advance in endoscopic diagnosis, with every increase in the dexterity and experience of the cystoscopist, and with the additional construction of a really useful cystoscope for catheterism of the ureters.

Catheterization of the Ureters.-The careful observation and analyzation of the "character" of the jets of urine with the cystoscope at the month of the ureters in the bladder is already a great means to diagnose disease of the kidney. In many cases this "visual analysis" of the fresh "kidney-urine" has proved to be sufficient for the establishment of a strict diagnosis and prognosis. Still, it would be very desirable in every case, and really necessary in many, to be in the possession of comparatively easy means by which the urine of each kidney may be gathered separately for careful microscopical and chemical analysis.

Attempts at solving this problem are manifold.* Yet I am fully

* Tuchmann, Ueber ein neues Mittel zur Diagnose der Blasen u. Nierenkrankheiten, Wiener med. Wochenschr., 1874, Nos. 31 and 32. Ueber den kuenstlichen Verschluss u. ueber die Sondirung des Harnleiters, Deutsche Zeitschr. f. Chirurgie, Bd. vi, p. 560. 0. Silbermann, Ueber eine neue Nethode, der temporaeren Harnleiterverschliessung u. ihre diagnostische Verwertung für die Krankheiten des uropoëtischen Systems, Berl. klin. Wochenschr., 1883, No. 34. In the female, our non-operative diagnostic means in this respect have been more ample, however difficult to practice, for a number of years. Cf. G. Simon, Ueber die Methoden, die weibliche Urinblase zugaengig zu machen u. ueber die Sondirung der Harnleiter beim Weibe. Volkmann's klin. Vortraege, No. 88. Lewers, Lancet, 1886, November 13th. K. Pawlik, Ueber Harnleitersondirung beim Weibe und ihre praktische Verwendung, Wiener med. Presse, 1886, Nos. 44-51. 
convinced that the cystoscope is the instrument which is going to solve this practical problem. We are still in need of a really useful cystoscope for catheterization of the ureters. The method of introducing a thin bougie, catheter, or sound alongside the cystoscope into the bladder, and then trying to make its tip enter the mouth of the ureter, is a bad one. The secondary instrument is greatly hampered in its motions by the presence of the cystoscope in the same channel. Moreover, it can only be practiced in the female, and even there the manipulation is very difficult. The catheter for the kidneys must be passed through a separate channel of the cystoscope proper. But it can not fit in the channel as snugly as a mandrel does. It has to remain freely movable. Consequently there must be leakage, as the intravesical pressure is greater than the atmospheric. The fluid in the bladder is therefore slowly ebbing away, the clear appearance of the cystoscopic picture changed, the position of the ureters annoyingly influenced. The instrument must therefore enable us to easily replace the fluid which is running away (as this is the case in the Paris instrument), and the thin catheter must be engaged in the mouth of and pushed forward into the ureter as quickly as possible. For this latter reason it may be advisable to first find and localize the mouth of the ureters with cystoscope No. 1 before attempting catheterization. The pictures as seen through the latter are more familiar to the eye of the cystoscopist, as the prism and lamp are on the concave side.

The only partially useful instrument for the purpose under consideration has so far been that manufactured at Paris. Its advantages and drawbacks have been described elsewhere.

It is to be regretted that Nitze's inventive faculty already began to apply his cystoscope for the treatment of bladder diseases before he had entirely exhausted the diagnostic side of its usefulness. All cystoscopists are anxiously waiting for a cystoscope for catheterizing the ureters "according to the Berlin pattern." Nitze should give it to us. Its "practical" construction will really complete the diagnostic capabilities of his instrument from a mechanical standpoint.*

If Nitze's irrigating cystoscope were built according to the pattern No. 2, and if the tube which at present gives exit to the water could be

* One might say, Why is the construction of such an instrument not tried in this country Why is it necessary for us to wait until Nitze bestows upon us this new, greatly needed gift The answer is simple. The manufacture of the cystoscope would require a factory which is specially fitted up for this purpose. A trial to make the cystoscopes here would consume an immense amount of money, and would probably at the end be a failure (as partially demonstrated by the trial of Messrs. F. A. Reichart \& Co., of New York City, Author, Progress of Cystoscopy, loc. cit.). And the finished instrument, even if useful, would be by far more expensive than that made abroad. 
arranged so as to run at the lower aspect of the shaft and to end at the convexity of the junction of beak and shaft (as in Brenner's and Boisseau's modification), the problem would, I believe, be best solved. The amount of water lost by the continuous leakage could then be easily replaced through the afferent tube. Perhaps such an arrangement would make the now rounded shape slightly oval, but that would be immaterial.*

The catheterism of the ureters with the help of special cystoscopic instruments will, I trust, soon be an extremely important and neverto-be-omitted factor in cystoscopy for renal disease. Its results will greatly reflect upon and vastly increase the correctness and exhaustiveness of such a cystoscopic diagnosis. The catheterism of the ureters in this way will easily, simply, and happily solve the great problemthe bloodless, separate collection and analyzation of the secretion of each kidney.

* Brenner, Poirier, and others have catheterized the ureters, in a few instances, with the help of a channeled cystoscope. My own trials in this respect have so far only been made in the female with Brenner's and Boisseau's modifications of the Berlin pattern' The former instrument is badly fitted for the purpose under consideration. Its curve at junction and shaft is too slight. I succeeded in three patients in engaging the thin catheter in the mouth of the ureters, and in passing it slightly forward.

\section{EXPIAANATION OF FIGURES ON PLATE IV.}

Fig. 28, tubercular cystitis; primary stage. (Cystoscope I.) Numerous minute ecchymoses, surrounded by a hyperæmic spot; many ramified vessels.

Fig. 29, partial hypertrophy of the prostate. (Cystoscope II.) Enlarged median lobe, projecting into the bladder.

Fig. 30, marked bilateral hypertrophy of the prostate; trabecular bladder. (Cystoscope II.)

Fig. 32, trabecular bladder; diverticulum of the lateral wall. (Cystoscope I.)

Fig. 33, encysted multiple stones-four only are shown. (Cystoscope I.)

Fig. 36, pin fixed in the anterior wall of the bladder, near its vertex; shadow on opposite wall. (Cystoscope I.)

Fig. 37, silk ligature adherent to the wall of the bladder; it projects from a red papilla (granulation tissue). (Cystoscope I.)

Fig. 38, two fragments of stone which remained in the bladder after lithotrity; in the larger one the nucleus of uric acid is seen. (Cystoscope I.) 
PLATE IV.
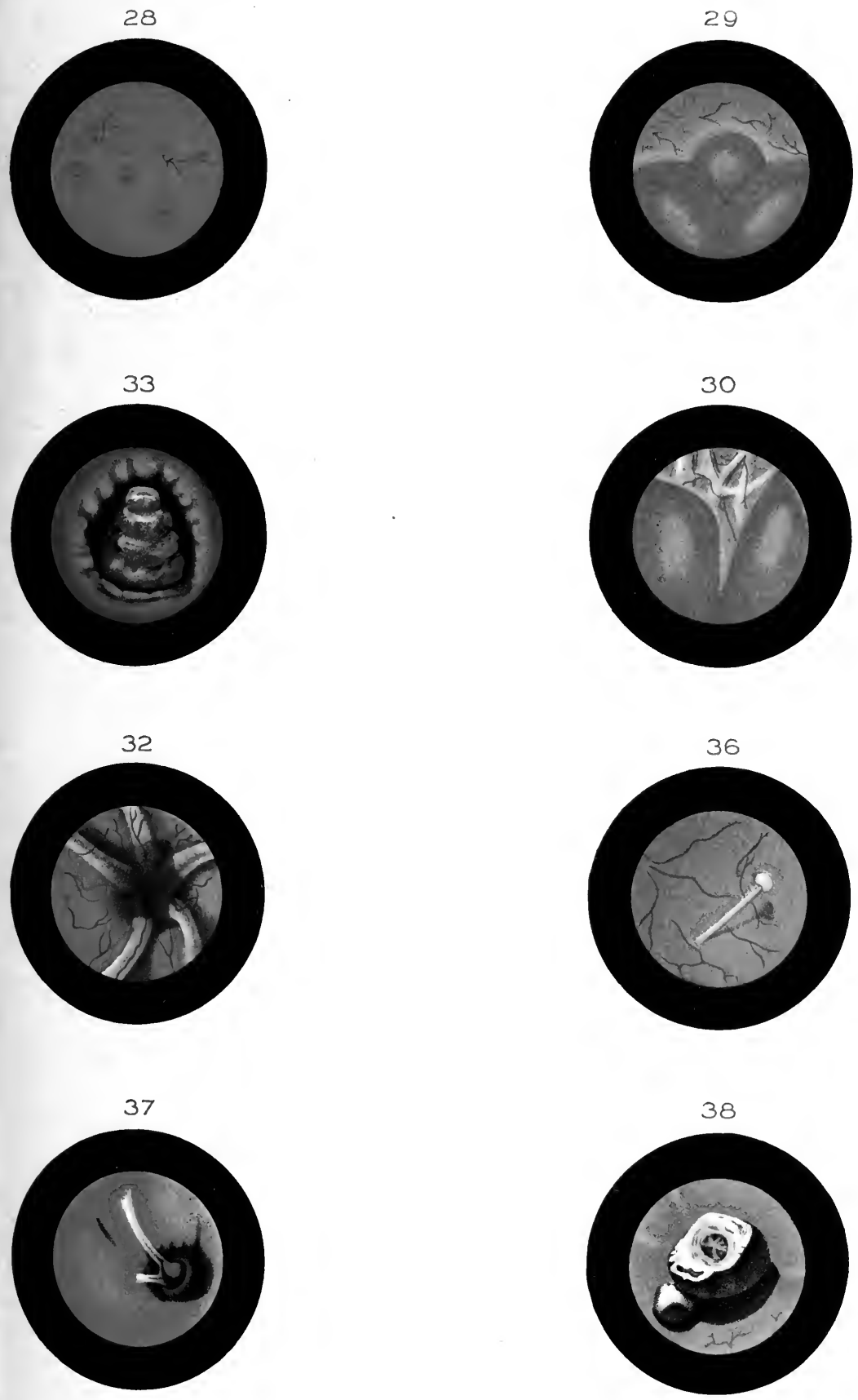

CYSTOSCOPIC PICTURES. 


\section{THE CYSTITES.}

\section{By SAMUEL ALEXANDER, M. D.}

DURING the first half of this century the nature of inflammation of the bladder was imperfectly understood. Civiale was the first to call attention to the constant discharge of pus in the urine of cystitics as the principal clinical distinction between inflammation and certain non-inflammatory conditions of the urinary tract that formerly had been regarded as forms of cystitis. Until quite recently, however, the study of inflammation of the bladder was confined almost exclusively to its clinical aspect. The study of the pathology and pathological anatomy of its lesions was neglected. Much of the vagueness and mystery that formerly existed in regard to the nature of inflammation has been cleared away by the advent of modern pathological doctrines. Recent investigation in regard to the nature and causes of urinary infection justify us in regarding all suppurative lesions of the bladder-i. e., the cystites-as forms of local microbic infection.

Definition.-The term cystitis has a precise significance in modern pathology. It indicates local infection or inflammation of the urinary bladder. The suppurative lesions of the bladder will, in this article, be described collectively as the cystites.

Classification.-On account of the number and variety of the lesions of cystitis, the clinical aspect of the disease is subject to great variations. A satisfactory classification is impossible at the present time, owing to an imperfect knowledge of the pathological anatomy of the lesions, and of the relation of the different lesions to the clinical aspect of the disease.

As a result of the numerous classifications that have been made, a great number of terms have been introduced into modern surgical literature, for the purpose of indicating different varieties and sub-varieties of inflammation of the bladder.

Definition of Terms. - The terms acute and chronic are used by many writers as if they described types of cystitis. These terms have a relative not a positive meaning, and should be used to indicate either the duration or the intensity of an inflammation. They refer more to symptoms than to the lesions. There are no special lesions characteristic of 
an acute or of a chronic inflammation, and the line of demarcation between them is not sharply defined, as is shown by the use of the term subacute.

An acute cystitis is one in which pain, frequent micturition, etc., are prominent symptoms, and which is of comparatively recent occurrence. A chronic cystitis may develop acute symptoms, owing to increased congestion.

The terms purulent cystitis and hcemorrhagic cystitis should be given up. All the cystites are characterized by pus in the urine, the character or the amount of the pus taken alone is not characteristic of any type of the disease. A similar objection may be made to the term haemorrhayic cystitis. The term painful cystitis has been used by Guyon to describe a clinical type of inflammation in which the symptom of pain is a marked and persistent feature; there are no special lesions, however, which characterize this form of the disease.

The distinction between cystitis of the neck of the bladder and cystitis of the body of the bladder has no pathological or clinical signifieance, and should be given up.

The suggestion has been made recently by Denys to classify the different varieties of cystitis upon the basis of the bacteriological character of the urine. Such a classification has very little practical value, since the morphological character of many of the microbes is not distinctive. A careful study of the different lesions of inflammation of the bladder, and of the clinical aspect of each, is the only scientifically accurate and satisfactory basis upon which to classify the different varieties of cystitis; but as a classification upon this basis is at the present time impossible, some other, for the time being, must be adopted.

There are certain diseases in which cystitis, although not an essential feature in their clinical history, plays frequently an important part. These diseases determine and modify to a great degree the course and the character of the vesical inflammation, and may be regarded as predisposing causes. We may recognize with propriety and practical advantage the following clinical types of cystitis: 1, The cystites of gonorrhœa ; 2, The cystites of tubercle; 3 , The cystites of stricture; 4 , The cystites of calculus ; 5 , The cystites of tumor; and, 6 , The cystites of prostatics.

It must be understood that these terms do not indicate that special inflammatory lesions are peculiar to any of these varieties of cystitis. In addition to the varieties which I have mentioned, there are two subvarieties of cystitis which merit special mention, since they are characterized by well-marked lesions and a more or less well-defined clinical course. I refer to the pseudo-membranous and the membranous cystites, and to "nodular eystitis." 
Sensibility of the Bladder.-The normal bladder has very little sensibility to contact. It is wholly insensitive to the contact of unirritating fluids at a temperature of about $100^{\circ} \mathrm{Fahr}$. The urine as it enters the bladder produces no sensation, and the same is true of fluids injected into the bladder through a catheter. The healthy bladder likewise is almost entirely insensitive to the contact of solids or semi-solids. A soft catheter or bougie produces a distinct sensation while passing through the urethra, but its contact with the bladder causes no pain and very little if any sensation, unless the contact be repeated and violent. There is a slight difference between the contact sensibility of the mucous membrane about the internal urethral orifice and that of the body of the bladder, the former being a little more sensitive. The sensibility of the normal bladder is a sensibility to tension. The normal capacity of a healthy bladder is the amount of urine which it can contain without producing the desire to micturate. When the normal capacity of the bladder is exceeded, the bladder contracts, and this causes the desire to pass water, the intensity of the desire being in proportion to the force of the contraction. Repeated and prolonged or violent contact of solids also may cause a desire to pass water, but contraction is necessary to produce the sensation. The normal bladder is very sensitive to the contact of irritating fluids, and to extreme heat or cold. The intense pain which an irritating injection causes is in fact a vesical colic, and is due to the violent contraction of the bladder. If analyzed, the sensation thus produced will be found to be an intense and prolonged and painful desire to pass water.

The sensibility of the bladder to tension and to contact depends upon the degree of hyperæmia or congestion. A metal or a soft eatheter or bougie causes pain when brought into contact with the inflamed mucons membrane. Distention of the organ when inflamed causes a violent and painful desire to pass water.

The Physiology of Congestion.-The normal urinary bladder is exceedingly vascular; the blood-vessels which traverse the mucous membrane and submucous connective tissue anastomose with each other, and form a very rich capillary plexus; the superficial vessels of the latter lie directly beneath the epithelium; these vessels have an arboreseent arrangement, as seen through the cystoscope. The blood-vessels are larger and more numerous in the neighborhood of the trigone, about the ureteral orifices, and the internal orifice of the urethra, than elsewhere. In the neighborhood of the trigone there is also a very rich plexus of veins, which converge toward the neck of the bladder, and empty into the plexus of Santorini. In the vessels of the normal bladder the amount of blood varies at different times, according to the tension of its walls. When empty, the bladder is comparatively anæmic; the mucous membrane is 
of a pale, pinkish color, notwithstanding its great vascularity. As the walls of the bladder distend, the amount of blood in the vessels, especially in the veins, gradually increases; when the capacity of the bladder is exceeded the detrusor muscle contracts, and the blood in the vessels is increased still further. If the bladder is not emptied the contractions increase in violence, and the congestion becomes more and more marked. Under these circumstances the mucous membrane, especially over the trigone and about the urethral orifice, becomes reddened, the superficial vessels are engorged, and small extravasations of blood may occur as the result of this excessive hyperæmia. The superficial capillaries may rupture, and a more or less severe hæmorrhage result.

The principal causes of congestion of the bladder are, distention, in. creased muscular effort, frequent, repeated, and long-continued or violent contacts of solids, and chemical irritation.

Pathological Anatomy. - The frequency of cystitis, the number and variety of its lesions, and the clinical importance of the disease, are sufficient reasons to demand a most careful and thorongh study of the pathological changes which occur in the bladder as the result of local microbic infection. Guyon was not understating our present knowledge when he said that the entire pathological histology of the chronic cystites had yet to be written.

The following is a description of the location and general character of the principal lesions :

Situation of the Lesions. - The prostatic urethra, which is to be regarded pathologically and physiologically as part of the urinary bladder, is involved in all cases of cystitis. The inflammation is more intense in this situation when the disease is due to the extension of a urethritis backward along the mucous membrane. The entire bladder may become inflamed during the course of a cystitis, but the degree of inflammation and the distribution and arrangement of the lesions is not uniform. There are certain situations which are always inflamed in cystitis, and whatever may be the cause or duration of the disease the lesions in these situations are always more prominent than elsewhere. These are, first, the trigone and the mucous membrane about the internal ureteral orifices; second, the region about the internal orifice of the urethra; and, third, the prostatic urethra. The inflammation nearly always begins in the mucous membrane, and thence may extend through the entire thickness of the bladder wall; the mucous membrane, however, is the site of the principal lesions in most cases. The muscular coat is involved to an appreciable extent only after the disease has continued for some time; the serous coat is involved as the result of extension from the muscular coat or from somewhere without the bladder. The intensity of the inflammation varies; it may be acute, subacute, or chronic, depending 
upon the degree of congestion. An acute or subacute inflammation may become chronic, and a chronic inflammation may develop acute symptoms. The lesions of cystitis may be transient, or they may result in more or less serious structural changes.

Description of the Lesions. - In describing the pathological anatomy of the lesions the following classification is convenient: 1 . The superficial cystites. 2. The interstitial cystites. 3. The productive cystites.

The superficial cystites include those lesions which are situated almost exclusively in the mucous membrane. They are transient, and do not usually cause permanent alteration in structure. The inflammation may be acute or chronic, depending upon the cause and degree of congestion. Almost every cystitis begins as a superficial inflammation.

The lesions of the interstitial cystites involve the mucous membrane, and to a greater or lesser extent the connective tissue and muscle. Inflammation of the serous coat, or pericystitis, may be included under this head. The lesions of interstitial cystitis may or may not result in permanent injury to the bladder.

The productive cystites include the lesions of tubercle, enlargement of solitary lymphatic follicles, diphtheritic membranes, ulcerations, villosities, and fungo-vascular excrescences. These lesions usually are chronic as to the time of their appearance, but acute symptoms often develop. They are associated either with the superficial or interstitial lesions.

This classification is purely artificial. There are no sharply defined lines of demarcation between the superficial and interstitial lesions. The inflammation may continue to be superficial throughout its entire course, or it may become interstitial or productive.

The Superficial Cystites. - These lesions vary according to the intensity and duration of the disease. In recent cases of acute cystitis all the vessels of the bladder, but especially those which are superficial, are intensely congested. . Seen through the cystoscope, they appear as interlacing lines of a bright-red color, the arborescent arrangement being marked. Small extravasations of blood appear upon the surface of the mucous membrane as dark-red points. The mucous membrane itself is reddened, especially along the course of the capillaries. The vascular injection and reddening, which are not uniform, but occur in patches, are most marked in the region of the trigone. The mucous membrane is swollen and œdematous. Early in the disease the urine is acid in reaction, but decomposes rapidly outside the bladder. During this stage the urine does not contain albumin unless blood is present, but pus and epithelial cells from the surface of the membrane are present in a greater or a lesser number, according to the duration of the disease. The epithelial cells are more or less altered, being granular, with two or more nuclei. Bloud is present when the cystitis is very acute, there being more blood in the urine passed at the 
end of micturition. Small granulations or exulcerations may appear on the surface of the mucous membrane. Patches of membrane resembling that of diphtheria may be present when the inflammation is very intense. Small vesicles, resembling those of sudamnia, may appear in certain cases. In chronic cases the mucous membrane is softened and thickened. Its surface is covered when there is ammonuria, with a thick, grayish-white or yellowish-white gelatinous material, consisting of altered pus and urine. The color of the mucous membrane may be nearly normal, or it may be yellowish or grayish. The superficial vessels are more or less prominent, depending upon the intensity of the inflammation. The surface of the mucous membrane is lusterless, and here and there irregular reddish areas may be seen from which the epithelium has been stripped.

When the inflammation has existed for some time, the folds of the membrane are elevated and reddened, especially along their tops. When there are congenital diverticula or pouches, these contain a mixture of pus and urine which may be squeezed out. The epithelium is softened and is thrown off in shreds, leaving bare patches of the mucous membrane proper. From the edges and surfaces of these patches shreds may float out into the urine, resembling small, dirty villi. The urine is neutral or alkaline, usually of a yellowish or grayish color, and deposits a heavy sediment of pus, or a viscid, mucoid substance consisting of altered pus and epithelium, triple phosphate crystals and bacteria. The epithelium is from the lower layers of the mucous membrane. Sometimes upon the denuded areas of the membrane the urinary salts are deposited. These patches are then of a dirty-white or brownish color, and may be mistaken for calculi. The urine may contain albumin, but it is usually absent.

The Interstitial Cystites. - The appearances of the mucous membrane do not differ from those described above. When the inflammation extends into the muscular coat, it usually attacks the interstitial connective tissue rather than the muscular trabeculæ; on this account the latter become loosened and undermined. When the mucous membrane is destroyed at any place, small shreds of muscular tissue are sometimes cast off into the urine, or they may appear as floating shreds attached to the bladder wall. The muscular trabeculæ are swollen, and a roughened appearance is given to the surface of the nucous membrane. The trabeculæ themselves may be involved and become destroyed by the suppuration. The whole bladder wall may be converted into a mass of suppurating tissue. Small abscesses may form in the substance of the mucons membrane, or in the submucous tissue, or in the muscle itself. The openings to these abscesses are usually small. Abscesses may open into the normal diverticula, when these exist, or upon the surface of the mucous membrane. Inflammation may involve the entire thickness of the bladder wall and result in a pericystitis. This, however, is rare, and when it 
does occur the latter is usually a localized inflammation. The urine is of a dirty yellowish color, usually ammoniacal and very fetid, and contains an enormous number of micro-organisms; it deposits a thick material, consisting of altered pus and epithelium. This is mucilaginous in character, very thick, and adheres to the bottom of the vessel and to the walls of the bladder. As the process of repair begins, new connective tissue forms between the muscular trabeculæ; and if the suppuration has extended into the substance of the muscular trabeculæ themselves, the latter are more or less destroyed. The newly formed connective tissue contracts; the anatomical capacity of the bladder is diminished; the elasticity of its walls is destroyed. This is the condition known as contracture of the bladder, and is the only one in which the anatomical capacity of the bladder is lessened.

The Productive Cystites.-For a description of these lesions the reader is referred to special works upon pathological anatomy, since a full description can not be given satisfactorily in a clinical article.

Pathology and Etiology.-The study of experimental pathology and bacteriology has cleared away much of the vagueness and obscurity that formerly existed in regard to the nature of inflammation. Recent investigation by various observers has established the principal facts upon which the modern doctrines of local infection are based, and the microbic origin of suppurative lesions can not any longer be regarded as a mere theory, but must be accepted as an established fact.

General Doctrines as to the Nature of Suppurative Lesions:

1. All suppurative lesions are due to local microbic infection.

2. The microbes having pyogenic powers are numerous and varied.

They may be divided into two classes: (a) Those microbes which always produce pus when they exert any pathogenic action. This class includes the staphylococcus pyogenes aureus, albus, and citreus, and the streptococcus pyogene; and $(b)$ those microbes which exert a pyogenic action only under special conditions. These may produce other pathogenic effects, under different conditions. It is certain that many of the microbes having pathogenic power may become pyogenic under favoring conditions.

3. The normal tissues in health are able to resist and to dispose of most pyogenic microbes.

4. The power and action of pyogenic microbes are modified-that is to say, increased or lessened by a great number and variety of conditions. Local infection can occur only when the soil is favorable to the growth and development of the microbe; the simple introduction of a pyogenic germ, the tissues being normal, does not cause suppuration.

The following are the principal factors which modify the action of pyogenic microbes : 
A. Local: The anatomical and physiological condition of the tissues at the time of infection, especially as to (1) the vigor of the epithelium and its power to resist the poison; (2) as to the normal and regular performance of function; (3) as to the condition of the local circulation; and ( 4$)$ as to the character of the secretions and their antiseptic properties; and-

B. General: The condition of the general constitution, especially as this affects the antiseptic power of the blood plasma.

C. In addition to the above factors which favor infection, there are the following which relate especially to the agent of infection: 1 . The species of microbe, and the number introduced at the time of infection. 2. The vigor of the microbe, and its power to produce poison. This latter is modified, to a great extent, by the source and previous environment of the germ.

Conditions necessary to cause Local Infection.-In every local infection three factors are necessary: 1 . The presence of microbes having active pyogenic powers. 2. A medium favorable for their development. 3. The power of the tissues must be weakened.

Pathology of Local Infection of the Bladder.-It has been known for a long time that the normal urine contained in the bladder of a person in perfect health is an aseptic fluid. Cultures of such urine remain sterile. This fact, discovered in $1858-59$ by Pasteur, has been repeatedly confirmed since then by numerous observers. On the other hand, we know that the purulent urine in an inflamed bladder always contains numerous micro-organisms. A simple microscopical examination of the urine in a case of cystitis shows, in addition to the pus-corpuscles and other inflammatory products, a variety of microbes, usually in great numbers.

The entire absence of germs from the normal urine, and their constant presence in the purulent urine of cystitics, naturally raises the question as to the relation between these microbes and the occurrence of inflammation of the bladder. According to the general doctrines respecting the nature of suppurative lesions, as stated above, the cystites must be regarded as forms of local microbic infection.

In considering the pathology and etiology of cystitis upon the basis of these doctrines, the following questions are presented: 1 . What pathogenic microbes are found habitually in the purulent urine of cystitis, and which of these microbes have pyogenic power in the bladder? 2 . What is the action of these microbes in causing cystitis? 3 . What is the origin of these microbes, and how do they invade the bladder? 4 . What are the conditions which favor and what those which retard infection?

The study of microbes found in the urinary tract was begun by Pasteur. His discovery in $\mathbf{1 8 5 9}$ of the micrococcus urece, and the investigations subsequently made by Van Tieghem as to the power of this microbe 
to cause decomposition of urea, attracted widespread attention to the study of the pathogenic microbes in the urinary apparatus.

For many years after the publication of Pasteur's discoveries, however, the investigations of nearly all observers were confined almost exclusively to the ammoniogenous action of the microbes found in the urine. But it has been shown recently, both by experiment and clinically, that nearly all the microbes found in the urine have this power to a greater or less degree, but that their pyogenic action in the urinary tract does not depend upon this.

From the discovery of the micrococcus urece in 1859 to the present time, no less than thirty species of micro-organisms have been found in pathological urine, and have been described by various observers. Many of these have no known pathogenic power; some have been found only in exceptional cases. It is not necessary, for the purposes of a clinical article, to give a description of all these microbes. I shall mention only those which have been found by different observers in the urine of eystitics, and which are known to have distinct and definite pyogenic power in the urinary tract.

\section{Microbes that have a Pyogenic Action in the Urinary Tract:}

1. Micrococci.-The micrococci habitually present in suppuration, viz., the staphylococcus pyogenes albus, aureus, citreus, and the streptococcus pyogene. The action of these germs is general, and it is not surprising, therefore, that they should be found in purulent urine.

2. Bacilli.-Two species of bacilli, viz., urobacillus liquefacius septicus and a bacillus, which since its discovery by Bouchard in 1879 has been described by various names, and recently has been identified with the bacillus coli communis.

Of these microbes, the bacillus coli communis is by far the most common, and next in frequency is the staphylococcus pyogenes anreus. The presence of the former in the urine of cystitis is almost constant. To give an idea of its frequency, I append the following table taken from Guyon's report, showing the result of various observations made in respect to this point :

Albarran and Halle found it in 47 cases of cystitis, out of 50 examined. In 30 cases, studied by cultures, it was found 15 times alone, and 15 times it was associated with other microbes. Albarran found it in 23 cases out of 25 ; in 16 of the cases it was alone. Morelle found it in 13 cases out of 15 ; in 6 it was alone. Verogius found it in 12 eases out of 17 ; in 11 it was alone. Denys found it in 17 cases ont of 23 ; in 15 it was alone.

The Gonococcus and the Tubercle Bacillus.-There are two species of microbes found in pathological urine of cystitis in addition to those mentioned above, which I have omitted purposely from the list. These are 
the gonococcus of Neisser and the tubercle bacillus of Koch. Although these microbes have undoubted pathogenic powers in the urinary tract, they can not be regarded as direct causes of cystitis. The gonococcus has unquestionably very active pyogenic powers in the urethra; alone, or associated with other microbes, it causes suppuration in this situation, but it is very doubtful whether the gonococcus has the power to cause any of the complications of gonorrhœa, such as cystitis, epididymitis, or pyelitis. Cystitis has never been produced experimentally by injecting cultures of the gonococcus into the bladder. In the so-called gonorrhœal cystitis, the urine taken directly from the bladder, without any chance of contamination from the urethra, contains very few gonococci. Other microbes which are known to be the cause of other varieties of cystitis, are habitually found in the urine of so-called gonorrhœal cystitis. The rapid manner in which gonorrhœal cystitis yields to treatment is in marked contrast to that of gonorrhœal urethritis. Gonorrhœal cystitis; so called, should be regarded, therefore, as either a mixed infection, or as a simple infection in which the gonococcus takes no part.

The local action of the tubercle bacillus upon the bladder is unique, and it requires a separate consideration. It is claimed that the lesions in the bladder due to the tubercle bacillus when acting alone are not themselves suppurative; these lesions precede the cystitis, and a second infection by other microbes than that of Koch must occur before pus appears in the urine. 'The cystites of tubercle are the result, first, of infection by the tubercle bacillus, and subsequently by other of the microbes that have pyogenic power in the bladder. It is said that pure cultures of the tubercle bacillus do not cause cystitis experimentally.

Simple and Mixed Infection.-The microbes found in the urine of cystitics are extremely varied. In some cases a single species is found, in others several species may be associated together. In the former case, a cystitis is spoken of as a simple infection; in the latter, as a mixed infection.

That the micro-organisms which I have mentioned as being habitually found in the urine of cystitis are the essential cause of inflammation of the bladder there is abundant proof. It has been shown by repeated experiments that pure cultures of these microbes injected into the bladders of animals after temporary ligation of the urethra, is always followed by cystitis, and that injection of these cultures into the cellular tissues always produces suppuration.

Action of Pyogenic Microbes in the Bladder.-There are two theories which have been advanced to explain the action of micro-organisms in producing cystitis, viz. : 1 . That the inflammation is due primarily to the action of the microbes in producing ammoniacal decomposition of urine, the carbonate of ammonia resulting from this fermentation acting directly 
as an irritant upon the tissue. 2. That the microbes or their ptomaines act upon the tissues.

The latter theory is true. For practical purposes of treatment it makes little difference whether the germs act directly upon the tissues, or whether their ptomaines are the essential poison. That the germ must have the power to decompose urea in order to cause cystitis, is certainly not true.

While ammoniacal decomposition of urea increases the intensity of an existing inflammation, it is never the canse of cystitis. It has been conclusively shown, by Guiard and Guyon, that ammoniacal fermentation in the bladder occurs as the result of the cystitis, and that unless there is cystitis, ammoniacal decomposition in the bladder does not occur. This question will be discussed more at length in considering ammoniacal fermentation as a symptom of cystitis.

Modes of Infection. - A priori, it is evident that the bladder may become infected from two sources-first, through the urethra, and, secondly, through the general circulation, the germs gaining entrance by way of the kidneys and ureters. The former is direct infection, the latter is indirect infection. Direct infection through the urethra is the rule; indirect infection through the circulation is rarely observed clinically in man.

Indirect Infection.-That indirect infection may occur, has been experimentally demonstrated, but it has not yet been shown that the bladder in man can be infected indirectly without disease of the kidneys. It is by indirect infection that certain cases of cystitis which occur during the progress, or as sequelæ, of general infectious diseases, may be explained. These cases, however, are rare, and before regarding a cystitis as the result of indirect infection we should exclude all forms of direct infection through the urethra.

Direct Infection.-Infection through the urethra may be of two kinds -first, spontaneous infection, and, secondly, provoked infection. Spontaneous infection occurs when the microbes invade the bladder without mechanical assistance. Provoked infection occurs when microbes are introduced into the bladder mechanically, as upon catheters, or by injection.

Spontaneous Infection.-Spontaneous infection of a healthy and normal bladder occurs clinically only when the urethra is diseased, as in gonorrhœa. On the contrary, spontaneous infection of the bladder is impossible in man when the urethra is healthy and normal. The length of the urethra, and the barrier which the sphincter-urethræ et vesicæ muscles offer, protect the bladder from microbic invasion so long as the urethra is healthy. Spontaneous infection of the female bladder, however, may occur even when the urethra is healthy. Spontaneous infection may occur by the 
direct extension of an inflammation along the mucous membrane of the urethra, or microbes may invade the bladder as the result of an ascending infection. When the urethra does not empty itself entirely, the urine retained furnishes the necessary medium for the transmission of microbes into the bladder; and the presence of blood in the urethra after operations, or of pus, may serve the same purpose. In like manner, in cases of retention of urine after the power of the sphincter vesicæ has become weakened the urine escapes, the constant flow of urine (overflow) from the distended bladder furnishes a column of fluid in the urethra, a condition particularly favorable to infection.

Provoked Infection.-Provoked infection through the urethra is much more common clinically than is spontaneous infection. It is most often the result of surgical interference, and usually follows the improper use of instruments, or the use of instruments without aseptic precautions.

Microbes may be carried into the bladder from without by the instruments; but as infection occurs sometimes after the introduction of instruments which have been thoroughly sterilized, it can not be doubted that in such cases the microbes causing the infection existed previously in the urethra, and were conveyed into the bladder by the introduction of the instrument.

All observers agree that the normal urethra contains microbes of various kinds. Petit and Wassermann failed to find, however, any of the pyogenic microbes in the healthy urethræ which they examined. Rovsing, Lustgarten, and Manneberg, hold the opposite opinion as the result of their respective observations. It is certain that the microbes having pyogenic powers in the bladder may be found in diseased urethræ, or in urethræ which have been diseased, but which apparently are healthy at the time the examination is made. This is in accord with our clinical observations.

Conditions which favor Local Infection of the Bladder.The fact that the injection of pyogenic microbes into a healthy bladder does not cause cystitis has been demonstrated repeatedly by experiments upon the lower animals, and it is confirmed by the results of daily clinical observations. To produce local infection of the bladder by the injection of pure cultures of the pyogenic microbes found in the urine, it is necessary to ligate temporarily the urethra, so as to produce retention of the urine and distention of the bladder; or to produce a mechanical or chemical traumatism. The proof that local infection of the normal bladder is impossible by the introduction simply of pathogenic microbes is shown clinically by the repeated introduction of instruments without any aseptic precautions, which so constantly occurs. Thus, the passage of catheters which have not been sterilized, the needless or rough use of urethral instruments, injections, etc., fail to produce cystitis in some cases, not be- 
cause pathogenic microbes are not introduced thereby into the bladder, but because the bladder is healthy, and its normal mucous membrane is able to resist the poison of these microbes. The study of microbes by culture is most instructive in this connection, and shows to how great an extent the growth and development of pathogenic germs depend upon the character of the medium in which the culture is made. The conditions which favor local infection of the urinary bladder are (1) congestion, (2) retention of urine, and (3) trauma.

Congestion.-It may be stated, as a general axiom, that congestion always precedes inflammation; local infection without congestion never occurs. I have called attention already to the principal causes of congestion of the bladder, and, therefore, need only mention them here. They are (1) distention, (2) increased or repeated muscular contraction, (3) frequent or violent contact of solids, and (4) chemical irritation. Speaking generally, congestion predisposes to cystitis by lessening the resistance of the tissues, and supplies in them the media for the development of the microbes. A congested bladder is, therefore, always in danger of infection.

Retention of Urine.-Retention of urine may be caused by obstruction in the urethra, which may be due to stricture, or to nervous causes, or to an enlarged prostate; or the retention may be due to a lack of muscular power from overdistention, or from paralysis of the bladder. The retention may be acute and complete, or partial and chronic. Retention of urine predisposes to cystitis by causing distention, and thereby increased muscular effort, resulting in congestion. The urine, although itself an aseptic fluid, soon becomes a favorable medium for the development of microbes, if retained in the bladder when the latter is congested. Acute retention of the urine is much more favorable to local infection of the bladder than chronic or partial retention, because the congestion which it causes is greater.

Trauman-This includes the repeated or violent contact of solids with the bladder, such as occurs in vesical calculi or are cansed by the rough use of instruments. Trauma predisposes to cystitis by causing congestion, and by affording an opportunity for microbes more easily to enter the tissues owing to the destruction of the epithelium, by favoring the growth of microbes in the urine, and by weakening the power of the tissues to resist the poison. Inflammation of the bladder, however, occurring as the result of trauma is not necessarily more intense than when due to other causes. In addition to these local causes there are certain general constitutional conditions which predispose to local vesical infection. These will be mentioned more fully in the next section.

Clinical Causes of Cystitis.-Although the gonococcus acting alone can not be regarded as the essential cause of cystitis, yet gonor- 
rhœa is one of the most common of the conditions which predispose to local microbic infection of the bladder. Cystitis may occur as a result of an acute or a chronic gonorrhœa.

Acute Gonorrhœe.-Cystitis rarely occurs as the result of a first gonorrhœa until the disease has reached its height-i. e., about the end of the third or the fourth week. Spontaneous infection of the bladder may then occur where there is a constitutional predisposition to suppuration, by direct continuation of the inflammation along the mucous membrane of the urethra. In an acute gonorrhœea, the infection of the bladder is much more commonly provoked by the improper use of urethral injections, or the improper introduction of surgical instruments withont aseptic precautions. When the inflammation has extended into the posterior urethra, sexual excitement or sexnal intercourse, or the use of alcoholic stimulants, may provoke a cystitis during the acute stage of a gonorrhœa. The swelling of the mucous membrane of the urethra in acute cystitis obstructs to a certain extent the ontflow of urine, causing an increased muscular effort of the bladder to overcome this obstruction and to empty the bladder, and this increased muscular effort, if long continued, results in congestion and favors infection. Or, retention of urine may occur during the course of an acute gonorrhœa, as the result of muscular spasm of the compressor urethræ. The passage of a catheter at such a time for the purpose of relieving the retention, without strict antiseptic precautions, both as regards the instrument and the anterior urethra, is almost certain to be followed by cystitis.

Chronic Gonorrhœa.-Cystitis as the result of a chronic gonorrhœa rarely occurs when the inflammation is in the pendulous urethra only. When the bulbous portion and the posterior urethra are the seat of chronic inflammation, spontaneous infection of the bladder may occur as the result of apparently slight causes. Thus, sexual intercourse or prolonged sexual excitement, or the use of alcoholic stimulants, or even a sudden check of perspiration, or fatigue, may cause infection of the bladder by direct extension of the inflammation from the deep urethra.

The infection is usually provoked by the introduction of instruments, or by the improper use of injections. The microbes may be carried from without into the bladder, or the instrument may carry germs from the urethra. The rough use of an instrument in a chronically inflamed urethra may increase the intensity of the inflammation, which then may extend backward to the bladder.

Stricture.-Stricture is usually put by most writers as among the commoner causes of cystitis, It is of rare occurrence, however, unless there is great obstruction to the outflow of urine, which has existed for a long time. The cystites of stricture, therefore, occur late in the course of the disease, but rarely as the result of stricture of the pendulous portion of 
the urethra. They are most commonly caused by a stricture situated at or near the bulbo-membranous junction. Stricture favors local inflammation of the bladder (1) by causing obstruction to the outflow of urine, and increasing the force of the contractions of the detrusor muscle, thus producing congestion; (2) by causing retention of urine and distention of the bladder; (3) by favoring the growth of microbes in the urethra, just behind the stricture, and by favoring their introduction into the bladder, as the result of surgical treatment. Cystitis is more likely to result from a stricture, when the latter causes retention of urine, especially when the retention is acute, as in cases of traumatic stricture. The sudden obstruction to the outflow of urine produces intense eongestion of the bladder, and under these circumstances, if a catheter is introduced without the strictest aseptic precautions, it is almost certain to be followed by cystitis. The dilatation of the urethra which occurs immediately behind a narrow stricture of long standing favors the growth and development of pathogenic microbes. In such a dilatation a little urine always remains behind after each act of micturition ; this decomposing, increases the urethral inflammation at that point. In such a dilatation, containing a fluid favorable to the development of microbes, the latter develop slowly or rapidly, and may at any time be carried into the bladder by iustruments introduced through the urethra.

Chronic Prostatitis. - Inflammation of the bladder is very common as a result of prostatic hypertrophy. The cystites of prostatics may be divided into two classes, viz.: (1) those in which there is retention of urine, complete or partial, without distention of the bladder ; (2) those in which there is partial or complete retention of urine with distention of the bladder. The conditions which favor local infection of the bladder in prostatics are: (1) the congestion due to frequent micturition, and to the increased muscular effort necessary to overcome the obstruction caused by the enlarged prostate; $(2)$ the anatomical state of the bladder dne to this increased muscular effort; (3) the age of the patient, the latter condition weakening the power of resistance; $(4)$ retention of urine.

In the second class of cases, namely, those in which the bladder is distended, in addition to the causes just mentioned, the congestion is increased by the distention of the bladder. In both classes of cases the retention of urine supplies a favorable medium for the growth of pathogenic microbes. In cases of partial retention of urine due to prostatic hypertrophy, the microbes are usually introduced into the bladder upon the instruments used for purposes of diagnosis or of treatment without thorough aseptic precautions. Cystitis is much more likely to occur from this cause, however, in cases in which there is acute retention of urine, such as occurs from chilling of the surface of the body, or from sexual excitement, or from alcoholic stimulation, or from errors in diet. 
Calculus.--It is an error to suppose that inflammation of the bladder is a common result of vesical calculus because cystitis is found in the majority of cases of stone. Phosphatic or secondary calculi often occur as the result of cystitis associated with partial retention of urine. When the calculus is primary - that is, of renal origin-cystitis is the exception, and not the rule. The contact sensibility of the bladder being slight, a smooth calculus may remain a long time in the bladder without producing any serious results. The constant violence, however, done to the bladder by the repeated contact of a calculus after a time causes congestion, and thus predisposes to cystitis. In cases of vesical calculus the congestion of the bladder is increased by exercise and by jolting, as in riding or driving. In the majority of cases of calculus cystitis the infection of the bladder is provoked by the improper use of unsterilized instruments, employed either for the purpose of exploring the bladder or removing the calculus. It is likely to follow operations where the entire calculus is not removed. The repeated contact with the bladder of the sharp fragments remaining behind increases congestion and favors infection.

Vesical Tumors.-Morbid growths of the bladder favor local infection by causing congestion and by weakening the power of the tissues to resist the action of the microbes. The hæmorrhage, which is so constant a symptom of tumors of the bladder, furnishes a favorable medium for the development and growth of micro-organisms. Cystitis rarely occurs spontaneously in cases of tumor, the infection being usually provoked by the introduction of surgical instruments. Cystitis occurs more often in cases in which hæmaturia is a prominent symptom.

The Action of Cold. - Cold is mentioned as one of the common causes of cystitis. It can not be doubted that the sudden chilling of the surface of the body often determines cystitis when the conditions favorable for infection are present, but the influence of cold alone can not produce a cystitis. When the surface of the body becomes chilled, congestion of the internal organs occurs, and the general vitality and power of resistance of the tissues are diminished. The action of cold in causing cystitis is frequently seen clinically in cases of stricture or prostatic hypertrophy where the conditions favorable for infection have existed for a long time, and where the action of the cold, by increasing the congestion of the bladder and by diminishing the resistance of the tissues, determines the infection.

Sexual Excitement.-Prolonged sexual excitement or sexual intercourse may become a factor in causing cystitis by increasing the local congestion of the bladder, especially in cases in which the posterior urethra is inflamed. In such cases the bladder is involved by the direct extension backward of the inflammation. 
Gout and Rheumatism.-Grout and rheumatism are often mentioned by authors as direct causes of cystitis. If they exert any influence, it is simply that of weakening the general resistance of the tissues to infection. There is certainly no separate form of cystitis characteristic of these diseases, and many cystites occurring during an attack of gout or rheumatism are not affected by anti-rheumatic treatment. The irritating effect of very acid urine, and of urine containing crystals, may predispose to cystitis.

Infections Diseases. - Cystitis occurring during the course or convalescence of certain acute infectious diseases is frequently dne to retention of urine, which may occur as the result of all febrile diseases. This retention is of nervous origin, and is due to spasmodic contraction of the compressor urethræ muscle. If not immediately relieved, intense congestion of the bladder is likely to occur, and thus the conditions favorable for local infection are developed.

In addition to these local causes the power of the tissues to resist infection are weakened by the disease, and the action of microbes, introduced as the result of careless catheterism, is favored. The increased quantity of urea is another predisposing factor in these cases.

Paralysis.-In cases of paraplegia, where there is retention of urine, the sensibility of the bladder is lost; and there being no contraction of the detrusor muscle, there is little active congestion. Passive congestion due to tension occurs. The power of the tissues to resist infection is weakened as the result of trophic disturbances resulting from the lack of nervous force. These conditions favor infection, which is provoked usualiy by catheterism.

Symptomatology.-The clinical aspect of inflammation of the bladder is subject to great variations. The evolntion, comrse, duration, and severity of the symptoms differ in the different types of cystitis, and in different cases of the same type, according to the cause and intensity of the inflammation. There are certain symptoms which, when present, are characteristic of cystitis. There are other symptoms which are not characteristic, individually, of inflammation of the bladder, but which taken together and in their relation to each other have a diagnostic value.

The symptoms of inflammation of the bladder are local phenomena. There are no general constitutional symptoms which per se are characteristic of the disease. It is impossible in a brief clinical article to describe separately the symptoms in each of the clinical types. I shall therefore describe separately the principal symptoms of inflammation of the bladder, calling special attention to their physiology and to the variations to which each is subject in the different forms of the disease, and to their diagnostic value.

There are three principal symptoms which, when they are all present, 
are characteristic of inflammation of the bladder. These are, frequent micturition, pain, and pus in the urine. These symptoms vary in their evolution, in their intensity, in their duration, and in their relation to one another. The presence of any one of them is not distinctive of inflammation of the bladder. Their clinical importance depends upon the presence of two or more of them in the same case, and upon the relations which they sustain to each other. Pus in the urine is the only symptom which is never absent in the cystites.

Another important symptom is homaturia. This symptom, although very common, especially when the cystitis is acute, may not occur during the entire course of the disease.

In addition to these four symptoms there are others which occur more or less frequently. These are: First, modifications in the character of the urine, such as albuminuria and ammoniacal decomposition; second, changes in the character of micturition; third, certain general constitntional symptoms. The latter, however, are rather the result of the complications of cystitis than symptoms of inflammation of the bladder.

Frequent Micturition.-This is a constant symptom in cystitis, unless there is atony or paralysis of the bladder. Its intensity varies according to the sensibility of the bladder-according to the degree of local congestion. The desire to pass water, therefore, is much more frequent when the inflammation is acute than when it is chronic. In certain cases of acute cystitis the desire to pass water may occur so frequently as to be mistaken for incontinence of urine. In the cystites of prostatics the desire to pass water is usually more frequent during the night than during the day. In nearly all cases of inflammation of the bladder, whatever the cause, the desire occurs with greater frequency when the patient stands. Exercise or jolting, as by driving or riding, usually increases the desire to pass water, especially in the cystites of calculus. The desire to pass water is made more frequent by anything which increases the congestion of the bladder. Frequent micturition is not due to diminution in the anatomical capacity of the bladder, nor is it induced by the contact of the urine with the influenced mucous membrane. In certain cases, however, which I have mentioned in the section on pathological anatomy, under the title of Contracture of the Bladder, the anatomical capacity of the bladder is diminished as the result of prolonged interstitial suppuration. In cases of acute cystitis the desire to pass water increases in frequency as the disease progresses, because each act of micturition increases the local congestion of the organ.

Pain.-Pain, as a symptom of cystitis, is of two kinds, spontaneous and provoked.

Spontaneous Pain.-The symptoms of pain and frequent micturition are closely related to each other, the intensity of both depending upon 
the sensibility of the bladder. As a rule, the more frequent the desire to pass water the more intense is the pain. In many cases the pain is most severe at the moment immediately preceding the act of micturition, and is due to the tension produced by the urine in the bladder, and is usually relieved to some extent by the discharge of urine. In many cases, especially in acute gonorrhœal and calculus cystites, the pain is most intense at the end of micturition, and continues after the act; the desire to pass water is increased greatly by violent and repeated contractions of the detrusor muscle, and is accompanied by ano-vesical tenesmus. Pain at the end of micturition also is a marked symptom in the acute cystites of prostatics. In certain cases in which there is ammoniacal decomposition of urine, pain during the act of micturition is especially severe, on account of the passage of the altered urine through the urethra. As has been shown already, the sensibility of the bladder to the contact of irritating fluids is much less marked than that of the urethra, but the contact of ammoniacal urine with the urethral mucous membrane usually causes pain, although the same urine produces no sensation in the bladder. In addition to the pain associated with the act of micturition, there is in many cases a feeling of weight and heaviness above the pubis and in the perinæum which is referred to the bladder.

Provoked Pain.-The normal bladder, as has been said, is almost wholly insensitive to the contact of unirritating fluids and to the contact of solids. The sensibility of the inflamed bladder, on the contrary, is very intense; if the contact be violent or repeated, it produces, in addition to pain, a desire to pass water, the latter being due to the contraction of the detrusor muscle. The sensibility to tension of the inflamed bladder is always increased. The injection of even a small quantity of an unirritating fluid into a bladder that is acutely inflamed causes an intense and painful desire to pass water. This increased sensibility of the inflamed bladder both to contact and to tension is characteristic of cystitis, and may be taken advantage of in doubtful cases for purposes of diagnosis.

Pus in the Urine.-The presence of pus in the urine is a constant symptom of cystitis; its absence is positive evidence that the bladder is not inflamed. The amount of pus is very variable; at the very beginning of a cystitis it may be so small and so intimately mixed with the urine as to be imperceptible to the naked eye, and can only be discovered with the aid of the microscope. When pus is present in sufficient amount to be perceived by the eye, it is mixed with the entire quantity of urine, giving to the latter a cloudy appearance. If all the urine passed at a single act of micturition be received into three separate glasses, the larger part of the fluid being passed into the first glass, the pus in the first glass may be relatively greater than that in the second or third, or there may be a larger 
amount of pus in the first and third glasses than in the second. Pus is always found in the third glass when there is cystitis. When the urine passed at the beginning of micturition contains a relatively larger amount of pus than that passed at the middle or at the end of the act, it is an evidence that the prostatic urethra and that portion of the bladder nearest the internal urethral orifice is the principal seat of the inflammation. On the other hand, a relatively large amount of pus at the end of micturition is not characteristic of inflammation of the base of the bladder, for it may be due to inflammation of the body of the bladder. The pus from this latter portion naturally flows toward the base, and is expelled therefore at the end of micturition. A large amount of pus in the urine passed at the end of micturition is therefore not a symptom of much value in determining the seat of the vesical lesion.

The appearance of the pus in cystitis varies. When the urine is acid, as it is at the outset of an acute superficial cystitis, the part passed in the first glass may contain, in addition to the free pus which appears as a cloud in the urine, shreds from the urethra. In the second glass the pus is intimately mixed with the urine and gives it a uniformly cloudy appearance. The sediment in both of these specimens consists of a more or less dense white precipitate, which settles to the bottom of the glass, and above there is a superficial floating cloud composed of muco-pus. In the urine received in the third glass the pus is more plentiful than in the second, and in certain cases, at the end of the act of micturition, there may be passed a number of drops of pure yellow pus, or of pus mixed with blood.

In cases of cystitis associated with ammonuria, the action of the ammonia upon the pus converts it into a glairy, mucilaginous substance, which adheres to the mucous membrane of the bladder or to the bottom of the vessel into which the urine is passed. The appearance of this glairy material is extremely characteristic of that form of chronic inflammation of the bladder to which the term "vesical catarrh" has been given.

Blood in the Urine.-Blood in the urine is not a constant symptom of cystitis. It is a symptom of intense congestion, and therefore usually occurs when the inflammation is acnte. The cause of the blood is due to the rupture of the smaller capillaries in the upper layers of the mucous membrane. The diagnostic importance of this symptom depends upon the circumstances under which the hæmorrhage occurs, and whether the blood was passed at the beginning or at the end of micturition, or is equally distributed throughout. Blood may discolor the entire quantity of urine passed at a single act of micturition, it may appear only in the urine passed at the end of the act, or it may follow the discharge of the last few drops of urine. It may be passed as clots or in the fluid state. In color it may be a bright or dark red, or, in cases where there is 
ammoniacal decomposition, the blood may appear as a dark brownish or blackish material, which rapidly sinks to the bottom of the vessel into which the urine is received. The amount of blood passed at any time depends very much upon the cause of the hæmorrhage ; it is usually increased by exercise and decreased by rest. The hæmorrhage may be provoked by the contact of instruments or by distention with fluids. When blood precedes the flow of urine it is not a sign of vesical disease, but is due to disease in the urethra or in the prostate.

The source of blood in general may be determined by employing the test with the three glasses described above. In acute cystitis blood usu. ally follows the act of micturition, and is the result of the forcible contraction of the bladder walls upon each other. In the cystites of calculus hæmorrhage likewise is most marked at the end of micturition, as the result of the contraction of the bladder upon the stone; it is increased, as a rule, by exercise and by jolting, and in some cases by distention. In the cystites of tumor, blood is mixed with the whole quantity of urine passed, the hæmorrhage being increased by distention of the bladder, and by its contraction at the end of micturition. In the cystites of tubereu. losis and of tumor there is often a history of hæmorrhage preceding the occurrence of the cystitis.

Albuminuria.-Albuminuria is not a constant symptom of cystitis ; when it occurs early in the comrse of the disease, it is usually due to the presence of blood in the urine or to renal disease. The pus-corpuscles usually do not yield albumin in a sufficiently large quantity to be appreciated. Albumin, as the result of inflammation of the bladder, when there is no blood in the urine, does not usually occur until the disease has been present for some time. When albumin is found in urine in which there is not sufficient blood to be appreciated by the naked eye, a further examination with the microscope should be made. When there is no blood in the urine, and where the kidney is free from disease, the cystitis is usually of long standing, and the upper layers of the epithelium have been destroyed over a greater or lesser area, thus allowing the serum of the blood to escape into the bladder.

The amount of albumin found in the urine of cystitics when there is no blood or renal disease is very small. When ammoniacal decomposition is present, it sometimes is difficult to detect the presence of albu$\min$ in the urine.

Ammoniacal Fermentation.-Ammoniacal decomposition of urine is a fermentative process due to the growth and development of certain microbes in the urine. Nearly all the microbes found in normal and pathological urine are to a greater or lesser degree ammoniogenous. By the decomposition of urea, carbonate of ammonia is set free, and the latter, acting upon the pus, converts it into a thick, glairy, gelatinous mate- 
rial, which sinks rapidly to the bottom of the vessel into which the urine is passed. When this change occurs, the urine is strongly alkaline and has a very fetid, ammoniacal odor, and is of a sirupy consistence, due to the changed condition of the pus.

Ammoniacal decomposition of urine formerly was regarded as the cause of cystitis, and this theory is still entertained by some authors. It has been proved conclusively, however, by the investigations of Guiard and Guyon, that ammoniacal decomposition is a symptom and not a cause of cystitis. It occurs in cystitis only when, in addition to the presence of ammoniogenous microbes in the bladder, there is either complete or partial retention of urine.

The condition of the urine and the amount of pus are important factors in the production of ammoniacal decomposition. When urine is of low specific gravity - that is, contains little urea-and of acid reaction when it enters the bladder, the fermentative process is slight. A relatively large amount of urea and of pus in the urine favors ammoniacal decomposition. The effect of this fermentative change is to increase the intensity of the cystitis, owing to the irritating qualities of the carbonate of ammonia set free. Ammoniacal decomposition in the bladder is pathognomonic of cystitis.

Diagnosis. - A thorough diagnosis in every case of cystitis is of the utmost importance. Cases are often treated for cystitis when there is no inflammation of the bladder, the diagnosis having been made upon a single symptom. In order to treat a cystitis intelligently it is necessary to know, in addition to the fact that the bladder is inflamed, its cause, its intensity, the anatomical and physiological state of the bladder, and the character and extent of its lesions.

To determine whether the bladder is inflamed is usually an easy matter. Frequency of passing water, and pain associated with the discharge of pus in the urine, make the diagnosis of cystitis probable. None of these symptoms alone, however, are distinctive of cystitis. Frequent micturition is a common symptom in almost every disease of the urinary tract. Painful micturition, resembling that which occurs in cystitis, may result from a calculus in the pelvis of the kidney, or from certain nervous affections of the bladder, or from inflammation of the prostatic urethra, the bladder itself being entirely free from inflammation. Pus in the urine is a symptom of any local infection of the urinary tract. The diagnostic importance of pus in the urine depends upon its source. In every case where cystitis is suspected the urine should be examined in three glasses, as described above. When pus is found in relatively larger quantities in the first and third glasses than in the second, the presence of cystitis may be assumed, especially when there is associated with this symptom pain and a frequent desire to pass water. A large quantity of 
pus at the end of micturition may be misleading, since this is found in certain cases of pyelitis. In the latter case, however, the urine usually retains its acid reaction; whereas in cystitis, the urine is usually neutral or alkaline.

The microscopic examination of the urine is of great value in determining the presence of cystitis, and it should be made in every case. The presence of the cystitis may be determined in this way by the character of the epithelium, and possibly by the character of the micro-organisms found in the urine. Ammoniacal decomposition of urine, when present, is a sure symptom of cystitis. In order to establish the diagnosis of inflammation of the bladder, it is necessary often to make a physical and instrumental examination of the patient. If pressure by the finger be made upon the neck of the bladder through the rectum in cases of cystitis, it will cause pain in proportion to the intensity of the inflammation. The sensibility of the inflamed bladder to the contact of solids may be taken advantage of in doubtful cases. A sterilized bougie à boule or a searcher brought into contact with the mucous membrane of the inflamed bladder causes pain. So also the increased sensibility of the bladder to tension is diagnostic of cystitis. This may be determined by the injection of fluids into the bladder.

The cause of a cystitis can be determined usually from the history of the case. In young or middle-aged persons cystitis is usually the result of urethral inflammation. A history of recent gonorrhcea or chronic urethritis is suggestive of this form of cystitis. In cases where there is a history of previous disease of the urethra, examination of the urine or an instrumental examination of the urethra may discover a lesion in the deep urethra, from which the bladder has been infected. In the cystites of stricture there usually is a history of progressively increasing difficulty in micturition or of sudden retention of urine. The occurrence of ammoniacal decomposition of urine in the bladder of a patient under fifty years of age can be taken as evidence of probable stricture cystitis. In a patient over fifty years of age, where preceding the cystitis there is a history of frequent micturition, the desire to pass water being more frequent at night than during the day, prostatic cystitis may be inferred. Rectal or instrumental examination of the urethra will determine this point. In the cystites of tuberculosis and of tumor there is often a history of hæmorrhage preceding the occurrence of the inflammation. A cystitis in which the cause can not be determined by the most careful examination should suggest its tuberculous origin, and repeated examination should be made for the tubercle bacilli.

The intensity of a cystitis may be determined by the symptoms of pain and frequency. The more intense the cystitis, the greater the pain, and the more frequent the desire to pass water. Hæmorrhage is also a 
symptom of the intensity of a cystitis, especially when the blood appears at the end of the stream.

It is necessary, in addition to the points mentioned above, to determine also the anatomical and physiological condition of the bladder, especially as to its power to discharge completely its contents, and as to its eapacity to retain fluids. In cases of cystitis due to stricture or to enlarged prostate, it is important to ascertain the amount of residual urine. This can be done by the introduction of a catheter into the bladder, after the patient has passed all the urine that he can. In cases where the muscular power of the bladder is diminished as the result of overdistention (atony) the flow of the urine through the catheter will be slow. The degree of atony may be ascertained by placing the patient upon his back, and by instructing him to take a deep breath while the urine is flowing through the catheter. This will canse an acceleration in the flow of urine when atony is complete. In cases of acute cystitis the contractility of the bladder is greatly increased, and urine is discharged through the catheter very forcibly. In cases of chronic cystitis in which the sensibility of the bladder is not greatly increased, and where there is hæmorrhage the cause of which can not be determined by the ordinary means of examination, a cystoscopic examination should be made in order to ascertain its source.

Prophylaxis.-In view of the facts already presented, it is evident that inflammation of the bladder is never a primary affection, but is always secondary to some pathological state of the urinary tract, and that the determining cause of a cystitis not infrequently is the careless or improper surgical treatment of this condition.

According to the doctrines of surgical pathology relating to suppurative lesions, the following general principles may be formulated: First, all operations or surgical procedures, however slight, should be performed with thorough aseptic precautions: and, secondly, all pathological states of the urinary tract which favor the local infection of the bladder should be removed as soon as possible and with the least mechanical violence.

Under the first of these heads I may call attention briefly to the method of performing operations aseptically upon the urinary tract. In the first place, the external genitals, especially the glans penis and the cavity of the prepuce, should be thoroughly cleansed and washed with an antiseptic solution, such as Thiersch's fluid. The pendulons and bulbous portions of the urethra should be thoroughly irrigated with Thiersch's solution.

In cases where the anterior urethra is inflamed, although it can not be rendered thoroughly aseptic by irrigation, the chance of infecting the bladder from the urethra by the introduction of instruments is greatly lessened when the attempt is made. 
Preparation of Instruments. - All instruments should be sterilized by boiling, or by passing them through the flame of an alcohol-lamp. The soft instruments, catheters, etc., should never be immersed, even for a short time, in any of the stronger antiseptic solutions, such as solutions of bichloride of mercury or carbolic acid.

The following simple rules for sterilizing catheters will be found effcient: The instruments, having been washed in hot water, should be boiled for about twenty minutes in a solution of chloride of sodium, one drachm to the ounce. Upon their removal from this solution they should be dried with sterilized gauze, and should then be placed in a case made of sterilized Canton flannel or gauze. Before using, the catheter should be placed for a moment in boiling water.

Local infection of the urinary tract occurs frequently, I believe, from the use of unsterilized oil employed for the purpose of lubricating instruments. In order to avoid this, the oil used should be sterilized by boiling.

Treatment. - In the treatment of the cystites the principal general indications are: 1. To remove the cause. 2. To relieve the pain and frequent micturition by subduing and preventing local congestion. 3. To modify the character of the urine, so as to prevent as much as possible the development in it of pathogenic microbes. 4. To control suppuration.

Treatment is both medical and surgical. The latter is of the most importance, but in nearly all cases medicinal remedies are indispensable.

In most cases it is necessary to use some form of intravesical medication, and, although in certain cases the disease will subside under medical treatment alone-providing the cause is removed-still the proper use of local treatment makes the cure more certain and more rapid.

In every case of cystitis we should seek to discover whether the inflammation is the result of a urethritis, acute or chronic, or of stricture, or of an enlarged prostate, or of a vesical calculus, or of tumor of the bladder, or of tuberculosis, etc.; and when we have decided that any one of these conditions is present, it should be removed if possible. In nearly all cases of inflammation of the bladder surgical interference is necessary to remove the cause-the use of a sound, or the introduction of a catheter, internal or external urethrotomy, litholapaxy, lithotomy, cystotomy, and deep instillations, are among the various operations which are thus indicated. There are two questions of importance to which I desire to call attention in this connection. The first of these is in regard to the risk of operating upon the urinary tract of cystitics, and the second relates to the choice of operation.

To regard cystitis as a contraindication to operative procedure is an error. So long as the cause of a cystitis remains, it is impossible to 
cure the inflammation, and clinical experience shows that the removal of the cause by operation, when properly and aseptically performed, is much less dangerous than to allow the cystitis to continue. It is true that the intensity of a cystitis may be increased by surgical operations improperly and unskillfully performed to remove the conditions which favor local vesical inflammation, and that disregard of aseptic precautions in these operations may expose the patients to serious risk. On the other hand, if these causes are not removed, disease of the kidneys and general urinary infection may occur. But, although a prompt removal of the cause is imperatively demanded to cure a cystitis, there are certain conditions in which it is advisable to postpone the operation, but not to abandon it.

In calculus cystitis, when the inflammation has been intensified from any cause, the operation may be postponed until the acuter symptoms have been subdued by appropriate treatment.

The conditions which determine the choice of operation are fully considered in the article on Stone in the Bladder.

In the cystites of stricture the question of operation and the choice of the method depend upon the character of the stricture, the intensity of the cystitis, and the anatomical and physiological condition of the bladder. In cases of acute retention of urine due to stricture the surgeon has no choice: the bladder should be relieved at once, and its thorough and regular evacuation insured for the future. The operative treatment of stricture is fully treated in the article on Stricture of the Urethra.

Speaking generally, dilatation should not be performed when, in spite of thorough asepsis, the cystitis is increased by the introduction of an instrument, or where dilatation is followed by urethral or urinary fever. Nor should gradual dilatation be resorted to when there is retention of urine, unless it is possible at the time the urine is drawn to overcome the obstruction sufficiently to insure at each subsequent act of micturition a complete evacuation of the bladder. The choice between external and internal urethrotomy, I believe, should be determined by the intensity of the cystitis as much as by the character of the stricture.

Rest and General Hygienic Treatment.-In the treatment of any locainflammation, rest and position are important factors. The special indication for rest is the character and severity of the pain, the object of rest and position being to relieve this symptom by decreasing the congestion. When the pain is intense, or there is much hæmorrhage, the patient should not get up, even to pass water or go to stool. The desire to pass water, and the pain, may be relieved in many cases by placing the patient upon his back, with the thighs and knees flexed, this position being maintained more easily by supporting the knees with a firm cushion or pillow. In this way pressure upon the bladder is removed. Pain is often lessened if the patient lie upon his side when making water. 
The diet of cystities is an important factor in the treatment. The food should be bland in acute cases, and to a large extent should consist of fluids. When milk agrees, it is best to confine the patient to this diet exclusively until the acuter symptoms subside; the milk may be mixed with Vichy or any of the carbonated waters, and taken ad libitum. In chronic cases the diet may be more varied, but the food should be easy to digest, and all stimulating condiments, such as eurry, mustard, and vinegar, should be avoided; green vegetables and ripe fruits can be taken with advantage. Asparagus and rhubarb are harmful in some cases. The ordinary nonalcoholic beverages may be taken, but those which contain much sugar should be avoided. Most careful attention should be given to the condition of the lower bowel, which should be emptied at least once a day without straining. An enema of hot water, or a dose of Carlsbad salts or of Hunyadi water before breakfast, or a mild laxative should be given at bedtime. The constipation certain to result from the use of opium should be counteracted by a laxative, and in addition to the latter an enema should be given before the stool. Cystities should avoid violent exereise, both because the latter causes an increased excretion of urea, and therefore favors ammoniacal decomposition, and also because when overheated the surface of the body is likely to become chilled by any sudden ehange of temperature, and the congestion of the bladder thereby increased. The use of alcoholie stimulants is contraindicated in the cystites, except in chronic cases where the patients are old and feeble, and where the habit of using stimulants has been long indulged. Sexual intercourse and sexual excitement are to be avoided, and to this rule there are no exceptions.

Although the cause of a cystitis is the principal factor in determining its treatment, the intensity of the inflammation, and in a less degree the character of the lesions, indicate the special mode of treatment. The intensity of a cystitis depends upon the degree of local congestion, and is indicated clinically by the symptoms of pain and frequent mieturition.

The Use of Anodynes.-In the treatment of pain and frequency, the remedy par excellence is opium and its alkaloids. The use of opium should be regarded as a temporary form of treatment, which in most cases must be accompanied or followed by local treatment of the bladder. The choice of the method of administration depends upon the effect which is to be produced, the length of time during which the drug is to be employed, and the effect which it produces upon the digestive tract. It should be given by mouth or rectum when it is necessary to employ the drug for several days. The use of rectal suppositories of opium, with or without belladonna, is a valuable method when the drug is not well borne by the stomach. Opium is indicated in all cases of cystitis in which the symptoms of pain and frequency are severe, and in 
which the former is not relieved by rest and hygienic treatment. The more intense the pain, the greater the indication for the opium, and to liesitate to give it is a serious mistake. In acute gonorrhœal cystitis, and in the cystitis of calculus, where the pain is severe, the use of opium is indispensable. In most cystitics the pain and frequency create a tolerance to the drug which is in proportion to the symptoms. In administering the drug, provided the patient has no known idiosyncrasy which contraindicates its use, no limit should be placed upon the dose, the object being to give rest to the bladder, the dose should be repeated sufficiently often to accomplish this. Its use, however, as I have said, should be temporary. In most cases of acute cystitis it is not necessary to continue the drug more than three or four days, when it is combined with intelligent local and hygienic treatment.

Instillations.-There are two modes of intravesical medication in the treatment of cystitis, exclusive of those which necessitate the performance of cystotomy. These are, first, injections; and, second, instillations. Injections are contraindicated in all cases where the bladder can not retain at least an ounce of fluid withont creating a desire to pass water, and in all cases associated with great pain and frequency of micturition. The use of injections in acute cystitis is certain to intensify the inflammation, owing to the pain and increased muscular effort which the introduction, of the fluid provokes. Instillations, on the contrary, are useful in all inflammations of the bladder, but especially in those conditions in which injections are contraindicated. The lesions of cystitis are usually most intense in the prostatic urethra and the neighborhood of the trigone. Fluids injected into the deep urethra flow backward into the bladder. These facts are taken advantage of in the treatment by instillation. A few drops of fluid are introduced into the prostatic urethra when the bladder is empty, and thus are brought into contact with its inner surface.

Instillations of the nitrate of silver made with Ultzmann's or Keyes's deep urethral syringe, give the best result. Instillations of solutions of bichloride of mercury in the tubercular cystites have been recommended by Guyon. I have not found these of any greater value than those of the nitrate of silver. The bladder should always be emptied immediately before the instillations are made. Nitrate of silver is valueless in certain cases of tubercle cystitis.

The immediate effect of the nitrate-of-silver solution upon the bladder is to canse contraction of the detrusor and increase the desire to pass water. When the inflammation is intense, it is sometimes necessary to control the pain cansed by the first instillation by a hypodermic injection of morphia. The pain, however, if the solution is not too strong, is temporary, and soon passes away. The congestion that immediately foilows the application of the nitrate of silver and causes the symptoms of pain and 
frequency is followed by a reaction, and within a few hours the congestion is reduced and the pain and frequency appreciably relieved. Exaggerated tension sensibility of the bladder is likewise blunted. The best effect is obtained by the frequent use of weak solutions, rather than by stronger solutions used at longer intervals. The action of the instillations in cystitis is analogous to the effect produced by the drug in conjunctivitis. In beginning treatment, a solution of one grain, or even one half grain, to the ounce should be used and the effect produced by this noted. If the solution is too strong, pain and a desire to pass water will continue for several hours. The pain does not continue usually more than twenty minutes or half an hour; but in peculiarly sensitive patients this period may be longer. The strength of the solution may be in. creased gradually at each operation, but only in exceptional cases is it necessary to use solutions of greater strength than eight or ten grains to the ounce. In acute gonorrhœal cystitis, where this mode of treatment is peculiarly valuable, from one to four grains to the ounce is usually strong enough. When a solution of a given strength is found to relieve pain, the strength should not be increased until the weaker solution fails to produce the effect desired. The amount of fluid injected should not exceed twenty-five or thirty minims. Instillations of ten minims are usually sufficient, especially at the beginning of a course of treatment. In the treatment of acute cases the instillations can be repeated every day or every other day, according to the effect produced.

Instillations of the silver nitrate in the treatment of cystitis are of more value than other forms of local treatment in the vast majority of cases.

Instillations are contraindicated in the cystites of stricture, when the caliber of the urethra is much diminished. In the cystites of prostatics they often may be combined with injections.

Baths and External Applications.-The use of baths and hot-water applications over the hypogastrium and perinæum are of unquestionable service for the relief of pain in the treatment of acute cystitis. To be of any service, the bath should be hot, at a temperature of $100^{\circ}$ to $110^{\circ} \mathrm{Fahr}$. The patient should not remain in the bath over ten minutes. Poultices, or the application of the hot-water bag over the bladder or perinæum, are often very comforting.

Local Anæsthetics.-The application of local anæsthetics to the mucous membrane of the bladder is, I believe, of little value in the treatment of cystitis. Intravesical injections of cocaine have only a temporary effect, and the ultimate result of these injections is to increase the congestion. The same is true of intravesical injections of opium or morphia.

Vesical Drainage.-There are two forms of vesical drainage: first, temporary; and, second, permanent. 
There are three modes of employing the temporary form of vesical drainage: 1. By way of the urethra. 2. Through the perinæum. 3. By suprapubic cystotomy.

Drainage through the Urethra.-This mode of vesieal drainage is made by introdueing a eatheter into the bladder through the urethra. The best eatheter to employ for this purpose is the Guyon's drainage catheter, which is open at both ends. It should be of small size, not larger than No. 16 French. Before introducing it, the catheter should be thoroughly sterilized and the urethra washed out. This mode of drainage is of little value in the eystites, and should be employed only as a temporary expedient. The indieations for its use in the treatment of cystitis are after operations upon the urethra, to prevent the contact of purulent urine with the wound, and in eases of difficult eatheterism.

The Perineal Method.-This eonsists in opening the membranous portion of the urethra in the median line of the perinæum, and through this opening introdueing a large rubber eatheter (No. 27 to 32 French) into the bladder. The latter is kept in place by means of tapes, which are tied about it as near as possible to the edges of the perineal wound, and the ends of these are then secured to a broad waistband.

It is unnecessary for me to deseribe here the operation in detail, but there are certain points to which I desire to call attention. The tube should have only one eye, which should be as near to the extremity as possible. The lumen of the tube should be large without weakening its walls. When there is hæmorrhage from the bladder, or when the pus is very gelatinous, the tube should be of larger size (30 to 32 French), as otherwise it is likely to be stopped up. After opening the urethra, and before introducing the tube, the prostatic urethra and neck of the bladder should be dilated. This, in many eases, may be done with the finger or a blunt gorget, or a Sims uterine dilator may be used. This dilatation of the resical neck is of the utmost importance, especially in cases of intense vesieal tenesmus. When this is negleeted, the tube causes painful contractions of the bladder, and in some eases pain and a desire to urinate so severe as to necessitate the removal of the tube. The latter should be placed so that its eye is just within the internal urethral orifice. The length of time which a tube introduced through the perinæum may remain in the bladder ean not be positively stated. In a number of cases of old prostaties who were bedridden, and whose bladders were contractured, I have maintained this mode of drainage for many weeks at a time. In most cases the sensibility of the bladder to tension and the amount of pus are the best guides to determine this point. When necessary, the tube may be removed temporarily at any time after the first twenty-four hours, and replaced without diffieulty. It should be kept scrupulously clean. Intravesieal injections may be made through the tube. After 
finally removing the tube, the fistula remaining should be refreshed by scraping its.sides if the drainage has been continued for a long time. These fistulæ usually heal kindly within two weeks, providing the caliber of the urethra be maintained by passing, on every fourth or fifth day, a full-sized conical steel sound during cicatrization.

The Suprapubic Method.-Drainage of the bladder by suprapubic cystotomy may be performed either for the purpose of temporarily draining the bladder, or to establish and maintain afterward a permanent fistula through which the urine may flow. The operation of suprapubic cystotomy has been elsewhere described, and the details of its technique need not be given here. The way in which drainage should be made when the bladder is opened above the pubes is still a subject of discussion. The principal points at issue are: First, as to the number of tubes; and, second, as to their disposition and the manner of securing them in place. The method most commonly employed is that with two tubes, the bladder being emptied by syphonage. This mode of drainage is undoubtedly the best. One of the tubes can be removed at any time, while the other is retained. When both tubes are removed finally, it is best for the first few days to empty the bladder by the use of a catheter; the fistula remaining closes gradually.

Permanent Drainage.-Various methods of establishing permanent drainage of the bladder above the pubes have been suggested. At present, the rules which should govern the choice of a method can not be stated definitely. The best method at present is that which consists in introducing a soft-rubber catheter, bent at an angle, through a fistula previously established above the pubes, and securing it in place by passing it throngh a hard-rubber pad which is molded to the surface, the pad being secured by tapes passing around the waist. Permanent drainage is indicated in cases of cancer or in cases of very difficult catheterism in prostatics. The choice between permanent drainage and prostatectomy presents itself in these cases.

Indications and Choice of Operation.-Drainage by suprapubic cystotomy is to be preferred to the perineal method in most cases where an operation is undertaken for the removal of morbid growths or foreign bodies; but unless during the first few days the attendance of a competent nurse can be secured, the perineal method is to be preferred even in these cases. It has been claimed that the division of the detrusor muscle in the suprapnbic operation is an advantage in cases of cystites with intense pain. The same relief, however, may be obtained, I believe, in most cases from the perineal operation, providing the vesical neck be thoroughly dilated.

Vesical drainage through the perinæum is to be preferred in most cases of cystitis. Its advantages over the suprapubic method are: First, 
that the operation is safer; second, that, if necessary, it may be performed under local anæsthesia (cocaine); third, that it is more rapidly performed; fourth, that the tube requires less care subsequently than when suprapubic cystotomy is performed.

Vesical Dilatation. - The theory that the capacity of an inflamed bladder can be increased by dilatation is contrary to physiology and anatomy. To attempt by forced injections to relieve frequent micturition can not be too strongly condemned, and it is a surprising fact that this method of treatment should be suggested at the present day by any author.

Treatment of the Urine.-As before stated, the normal urine in health, as it flows from the kidney, is an aseptic fluid. In cystitis, the urine, upon being discharged into the bladder from the kidneys, becomes modified by the pus, and serves as a medium for the development of pathogenic microbes. When there is obstruction to the outflow of urine, causing partial or complete retention, the fluid undergoes ammoniacal fermentation. Three factors are necessary to cause this change: 1 . The presence of ammoniogenous microbes in the urine. 2. The presence of pus. 3. Retention or stagnation of urine.

This is the result, as I have shown, of decomposition of urea, caused by the development in the fluid of certain micro-organisms. The carbonate of ammonia set free by this fermentation acts as an irritant upon the inflamed mucous membrane. This decomposition of urine within the bladder is pathognomonic of cystitis. It occurs, however, only when in addition to the pus and microbes there is also stagnation of urine. This condition is favored by the amount of urea which the urine contains, as well as by the amount of pus.

Since the growth and development of micro-organisms in the urine are favored by the quantity of urea which the latter contains, the object in treatment should be to diminish the quantity of urea, and to increase proportionately the amount of water. This is best accomplished by attention to the rules of diet which have already been given, and by the use of certain mineral waters and diuretics. The Poland, Bethesda, Contrexéville, and Vichy waters are the best for this purpose. These should be taken in large quantities between meals, and in some cases may be advantageously combined with the use of saline diuretics, the citrate of potassium being the best. It is a mistake, however, to give doses of the latter sufficiently large to make the urine alkaline, as this favors infection.

The second indication is to render the urine antiseptic by the use of certain remedies which are eliminated through the kidneys. Salol and salicin, either alone or in combination, in from five to fifteen grain doses, three times a day, may be given with advantage. Napthalin, in doses of from two to five grains, three times a day, in the form of capsules, acts 
well in some cases; and the same in true of the oil of gaultheria, in doses of from five to fifteen minims. The latter, combined with salicin, is specially indicated in cystites occurring in patients who are gouty or rheumatic. The oil of sandal-wood and the balsam of copaiba in the treatment of the cystites are not of much value. In cases of ammonuria, in addition to the treatment just indicated, it is necessary to prevent stagnation of urine by the regular and thorough evacuation of the bladder.

Intravesical Injections. - These are of two kinds: First, those used for the purpose of cleansing the bladder; and, second, those used to produce some local effect upon its mucous membrane.

The value of intravesical injections in the treatment of cystitis is very great. They are contraindicated, however, in many cases, and should not be used indiscriminately in all forms of the disease.

Fluids used for Injections.-In washing the bladder the fluid used should be aseptic, should be hot (temperature $100^{\circ}$ Fahr.), and should contain no solid particles. The best fluids for the purpose are Thiersch's solution; a solution of borax, one drachm to the pint; a saturated solution of boracic acid; a solution of chloride of sodium, two drachms to the pint; and a solution of peroxide of hydrogen, of three or four volumes, or stronger. The stronger antiseptic solutions, such as those of corrosive sublimate and carbolic acid, should never be used within the bladder. All the fluids mentioned above are useful, and each has advantages in certain cases.

Washing the bladder is indicated where there is ammoniacal fermentation of the urine, if the sensibility of the bladder to tension is not very much increased. In such cases, especially where the ammoniacal fermentation has been of long standing, the best results are obtained from injections of Thiersch's solution. For ordinary cases I prefer this or the borax or boracic-acid solutions. The use of all these, however, may be followed in some cases by a feeling of irritability or a burning sensation in the bladder. In such cases the simple salt solution, made with boiled water, may be employed. The peroxide of hydrogen is of value in cases of hæmorrhage, where there are organized clots in the bladder. These are disintegrated by the action of the peroxide, and the bladder then may be washed clean with less chance of congestion. It may be stated as a general rule that the solution used for washing the bladder should never cause any pain, nor excite increased effort of the detrusor muscle.

In washing the bladder, the latter should never be distended to its physiological capacity; in other words, the fluid injected should not cause pain or a desire to pass water. An injection of two ounces of fluid or less is usually sufficient to inject at one time, and the fluid should be immediately withdrawn. The operation may be repeated as 
often as necessary. A number of small injections in succession clean the bladder far more rapidly than the injection of a large quantity of fluid. When withdrawing the fluid, care should be taken to have as little of the catheter within the bladder as possible, so that the softened mucous membrane may not be forced against the instrument when the bladder becomes empty. The frequency with which the bladder should be washed depends upon the state of the urine and the degree of the cystitis; the object of this mode of treatment is to prevent or to stop ammoniacal decomposition by the removal of the pus, and to arrest the development of pathogenic germs in the urine. Once or twice daily is sufficiently often for washing the bladder in most cases, and after ammoniacal fermentation has been arrested the operation may be performed less often.

The danger of infection from disregard of asepsis in this operation, the danger of increasing the congestion by overdistention of the bladder, and the danger of injury to the bladder from the rough use of instruments or the use of improper instruments, should be kept in view constantly. The urethra should be irrigated before each washing.

Contraindications.-An injection should not be repeated when the moderate distention of the bladder necessary to the operation causes pain or an increased desire to pass water. In cases of acute cystitis, without retention of urine, injections are contraindicated.

Nitrate-of-Silver Injections. - The second class of injections are those used for the purpose of producing some local effect upon the mucous membrane of the bladder. A great number of remedies have been employed for this purpose. I have already said that the use of the more powerful antiseptic solutions, and of solutions for producing local anæsthesia, are contraindicated in the treatment of cystitis. By far the best injection of the second class is that of a solution of silver nitrate. This remedy, as are all forms of intravesical injections, is contraindicated when there are pain and frequent micturition, or when the inflammation is acute. The strength of the solution to employ in the bladder is much weaker than that used by instillation. From one quarter to one half a grain to the ounce is strong enough to begin with. The strength of the injection may be gradually increased, if necessary, up to two grains to the ounce. In using nitrate-of-silver injections the bladder should be washed out first, and then completely emptied. The amount of the nitrate-of-silver solution should not exceed two ounces, and should be permitted to escape immediately. These injections may be given as frequently as every other day, if required. This mode of treatment is specially indicated in cases of chronic inflammation associated with ammonuria, where there is a large discharge of pus in the urine. 


\title{
INJURIES AND DISEASES OF THE BLADDER.
}

\author{
By GEORGE RYERSON FOWLER, M. D.
}

\section{WOUNDS OF THE BLADDER.}

\section{CONTUSION OF THE BLADDER.}

THE elasticity of the anterior abdominal walls weakens to a very considerable extent the force of blows directed over the region of the bladder. The fact that the bladder wall itself, unless the cavity be filled with urine, offers but slight resistance to force applied from in front alone, serves as a still further protection against serious contusions or rupture. In a bladder overfilled and with tense walls, both vesical and abdominal, as frequently occurs in drunken men, a kick or a blow may result in any form of injury, from a slight contusion to complete rupture.

In a contusion of the vesical wall a greater or less number of bloodvessels in the mucous membrane may be torn, and blood enter the cavity of the bladder as a result. This constitutes the condition known as vesicular hæmaturia.

Symptoms and Diagnosis. - The diagnosis of contusion is easily made. The urine clearly shows the presence of blood. Injuries and diseases of the kidneys with hæmorrhage into the capillary canals, and of the renal pelvis, may produce similar discoloration of the urine. In these cases, however, the blood-pigment, before leaving the bladder, is of a hrownish color, while in cases of vesicular hæmaturia due to contusion the urine is blood-red. In cases of renal hæmaturia, microscopical examination reveals the presence of red blood-corpuscles in a very much shrunken condition; while in case of hæmaturia following contusion the corpuscles are colorless, swollen, and globular. In vesicular hæmaturia, also, small fibrinous coagulations of a blood-red color are observed f fibrinous coagulations of kidney origin are deprived of their color before leaving the body. In case of contusion of the bladder, the entire organ may be filled with large blood coagula.

Immediately following the occurrence of a contusion of the bladder of any considerable extent, symptoms of vesical irritability and tenesmus arise. The impulse to empty the bladder is strongly felt, and repeated attempts, with bearing-down efforts, are made. The bladder is but in- 
completely emptied in some instances, owing to the presence of clots checking the outflow of urine. Attempts to empty the bladder by means of the catheter may be frustrated, owing to the fact that the eye of the instrument becomes occluded by the presence of coagula. Water forced throngh the eatheter may serve to clean it for the time being.

The principal dangers of contusion of the bladder are two in number. These are : first, decomposition of blood in the bladder; and, second, persistence of the hæmorrhage to a dangerous extent. The first named may arise from the accidental introdnction of germs of putrefaction from the urine itself, or from the use of a non-aseptic eatheter. From the very incipiency of a septic condition cystitis of a greater or less severity occurs.

Treatment.-In the treatment of contusion of the bladder the indications point to, first, providing against septic infection of the blood already escaped into the cavity of the bladder, and, second, the arrest of the hæmorrhage. The first is to be accomplished by the use of antiseptic irrigations, and, of these, that known as the boro-salicylic of Thiersch will probably be found to best answer the purpose. By this means the coagula which are retained are kept disinfected and thus rendered powerless for harm, while the débris from them, as they break down, are washed away. With a soft catheter and a common glass funnel connected therewith by means of a length of rubber tubing a simple irrigating apparatus may be made, and the process repeated, if gentle manipulation be employed, a number of times daily, until the dangers of septic cystitis are passed.

In cases of hæmorrhage from external injuries hæmostatic measures are, as a rule, unnecessary. As soon as the distention is relieved by emptying the bladder, and the walls of the vessels resume their normal condition, spontaneous arrest of the bleeding takes place. But it sometimes happens that the accident occurs in old persons with chronically diseased bladder walls; hæmostatic measures must be resorted to in persistent hæmaturia following the injury in such.

\section{WOUNDS OF THE BLADDER.}

Gunshot injury may be taken as the type of perforating wound of the bladder. Punctured and lacerated wounds originating from other sources do not essentially differ in their course from that pursued by gunshot-wounds. Although subentaneous wounds or rupture of the bladder will be treated of in another section, it will not be out of place to call attention here to the important fact that, as shown by a study of 285 cases of gunshot-wounds of the bladder by Bartels, there were but 65 fatal results, while of 185 cases of rupture only 17 ended in recovery. It is not difficult to account for this difference. Gunshot-wounds of the bladder almost necessarily provide for escape of urine along their track; 
on the contrary, the occurrence of subcutaneous wound or rupture of this viscus is followed at once by extension and most dangerous infiltration. Although the statistics of Bartels are somewhat contradictory to those compiled from the records of the late civil war, these showing 185 cases with 96 deaths, yet the fact remains that the mortality following open wounds is not nearly so great as that which follows rupture.

A wide distinction is to be made, in the study of wounds of the bladder, between those which occur extra- and those occurring intraperitoneally. Those involving the vesical wall at the apex of the bladder, as well as those occurring posteriorly, as a rule invade the peritoneal cavity, while in those at the lower and anterior vesical wall the last escapes. In the first group a most serious condition is present, and the patient's life is threatened from the invasion of a rapidly fatal septic peritonitis. In those cases of an extraperitoneal character, although even here the condition may become a grave one from infectious cellulitis, the prognosis is more favorable, and, inasmuch as the fatal issue is somewhat postponed, time is allowed for interference. This is particularly true in cases where the wound itself is such as to permit more or less free escape of the bladder contents. Failing in this, however, and in the absence of operative interference, a rapidly spreading infectious cellulitis, almost as fatal as septic peritonitis itself, carries the patient off within a few days.

Treatment of Intraperitoneal Wounds.-In the first class of cases-i. e., those in which the vesical wall and peritonæum are simultalleously wounded-an immediate laparotomy is imperatively demanded. Absolutely no consideration should deter the surgeon from giving the patient, and at once, the benefit of the only resource at all calculated to give him a chance for life. The same principles should govern here as in cases of perforating wound of the bowel. The abdomen is to be opened in the median line, the wound in the vesical wall songht for and sutured by means of the Lembert or Czerny suture, and irrigation of the peritoneal cavity accomplished by means of a sterilized solution of the normal blood-salts, or a chloride-of-sodium solution, one drachm to the pint. Walter, an American surgeon, was the first to put this prompt procedure into practice, and with the result of saving the patient. Following this measure a suprapubic or perineal cystotomy, or permanent catheterization may be added for the purpose of insuring drainage and removing pressure from the sutured portion of the bladder. Of these measures, the most certain to accomplish the object, when the difficulties of keeping a perineal drainage-tube or permanent catheter from becoming occluded are considered, will be found to be that of suprapubic cystotomy, combined with capillary drainage.

Treatment of Extraperitoneal Wounds.-In cases of extraperitoneal wound of the bladder the indications are to insure, at the ear- 
liest possible moment, complete and free drainage of urine from the bladder, and thus prevent infiltration and infection of the para-vesicular connective tissue in the lesser pelvis, and consequent phlegmonous inflammatory deposits in remote and inaccessible regions. At the very outset, therefore, measures should be taken to explore the original wound, and, by means of a proper drainage-tube, insure free escape from the direction which it takes. This should be supplemented, without delay, by either suprapubic cystotomy and capillary drainage, permanent catheterization and siphon, or perineal cystotomy. The latter procedure will be preferable, particularly in instances in which a free flow of urine has taken place, through the rubber tube placed in the bladder through the wound itself. Under these circumstances there is an advantage in opening the bladder at its lowest point and directing the flow of urine away from the wounded portion. In cases in which urinary infiltration has already developed, attempts should be made to follow up, from the perineal or suprapubic wound, the infected planes of connective tissue and to irrigate and drain these as thoroughly as possible. If much time has elapsed since the infliction of the injury, these attempts will be found of doubtful utility.

\section{FOREIGN BODIES IN THE BLADDER.}

Foreign bodies may find their way into the bladder either by accident or design. In the first-mentioned class of cases are to be included instances in which efforts to titillate the sensitive urethra by those in whom the sexual apparatus is no longer responsive to natural influences. These include almost any imaginable article to be found at hand in domestic life, such as pins, needles, shoe-strings, hair-pins, pieces of wire, feathers, pipe-stems, pencils, broom-straws, etc.; in addition to these, bits of broken catheter which become detached, either through the use of defective instruments in the hands of those who have become launched upon what is known as the "catheter-life," or in the hands of those who seek to resurrect the deadened pleasurable sensations of youth by artificial stimuli.*

Symptoms. - Foreign bodies in the bladder give rise, in due course of time, by their presence, to almost precisely the same class of symp-

\footnotetext{
* Defective instruments in the hands of the surgeon may likewise be responsible for the presence of a foreign body in the bladder. The writer has knowledge of a case in which, in the eourse of an exploration of the urethra by means of the Otis urethrometer, the rubber eap covering the expanding portion of this instrument became detached in the deep urethra, and found its way into the bladder. Fortunately, a few days subsequently, the rubber cap became engaged, with its open extremity toward the cavity of the bladder, and its closed and pointed end at the internal urethral orifice at the neek of the bladder: it was thus expelled. distended with urine, during an act of mieturition. The expulsion was accomplished with considerable difliculty.
} 
joms as stone, such as vesical irritability, more or less pronounced ; dysuria, nainly at the end of the act of micturition, and most likely to be referred to the extremity of the penis; alkalinity and putrefactive shanges in the urine when passed. It but rarely happens that the case zomes under the surgeon's notice until most pronounced evidences of their presence manifest themselves, especially in the class of cases in which the foreign body has slipped from the fingers of a masturbator. In females, particularly, foreign bodies have been removed from the bladder of individuals who have affected the most profound astonishment at their presence. In the latter, particularly in the absence of any well-defined cause affording explanation of persistent bladder symptoms, an exploration of the viscus should be made with the sound. Where this fails to reveal the presence of a foreign body, either from the fact that lithic deposits have not yet taken place, or from any other reason, cystoscopy will be found to be a valuable resource. It occasionally occurs that the patient acquaints his medical attendant at once with the nature of the accident which has befallen him, and before symptoms of a very pronounced nature have developed, particularly if the foreign body consists, as it usually does in this class, of a portion of a gum-elastic, waxed linen, or soft rubber catheter.

When a foreign body becomes lodged within the bladder, providing that it is too small, or is so located that its spontaneous expulsion is impossible, one of two things will occur : it will either form the nucleus of a calculous deposit, which will as a rule be phosphatic in character, or, by the pressure which it exercises upon the bladder wall, ulceration will be produced. In the latter case the subsequent course will be determined by the site of the ulceration. If it involve the peritonæum, the escape of urine within this serous cavity will give rise to rapidly fatal peritonitis. If the point of perforation be not such as to involve the peritoneal cavity, extravasation of urine in other directions will give rise to extensive septic cellulitis, sloughing of planes of connective tissue, putrid collections, and death by general infection and exhaustion.

Treatment. - When the patient is wise enough to acquaint the surgeon promptly with an explanation of the precise nature of the accident, suitable means may at once be employed for the removal of the foreign body. With the exception, however, of those cases in which the accident befalls those who are conscious of having employed the article lost within the bladder for a legitimate purpose (broken catheter, etc.), comparatively few will come under the surgeon's notice until acute evidences of ulceration and perforation manifest themselves, or symptoms simulating stone are present.

Probably the best instrument for the removal of a flexible catheter or a portion thereof from the bladder is a small, smooth-bladed lithotrite. 
It is not necessary that the catheter be grasped by the end, for the reason that these instruments are so yielding, they will generally double up after once being grasped, and may be brought away with the exercise of moderately forcible traction. Where bougies of larger size, which have been used for purposes of dilatation, have slipped into the bladder, inasmuch as these do not possess the flexibility of hollow catheters, special instru-

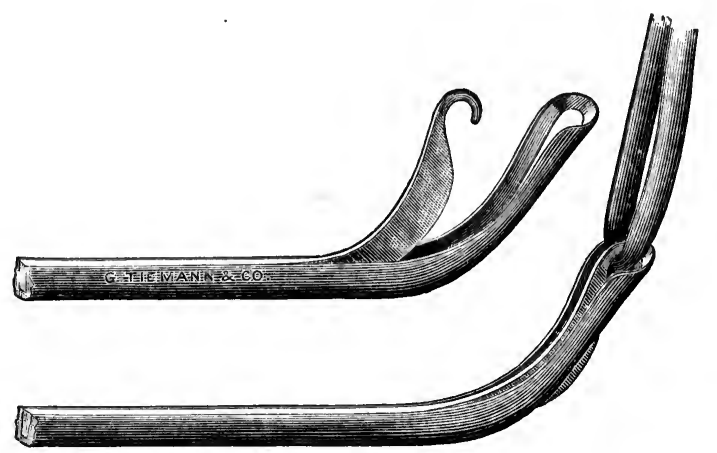

Fig. 1.-Mercier's duplicator.

ments may be employed for their extraction. Mercier has devised an instrument which may be employed for this purpose when the lithotrite fails. This instrument consists of a male and female blade, similar to those of a lithotrite. The female blade is half-tubular-shaped, and fenestrated near its beak; the male blade terminates in a hook-shaped extremity, directed downward, and fitting, when closed, into the fenestrum of the female blade.

In the rare cases in which it will come within the surgeon's opportunity to immediately remove a foreign body other than those just mentioned, the greatest circumspection is to be enjoined both in the selection of the means and the method of employing the same. While special instruments have been devised for the purpose of insuring a grasp upon inelastic cylindrical foreign bodies in the direction of their long axis, the surgeon must be prepared to meet these cases mainly by the exercise of his own ingenuity and the instruments to be found in an ordinarily wellequipped surgical armamentarium. The common lithotrite may be employed, with the patient in deep anæsthesia (and an anæsthetic is to be em. ployed whenever practicable in these manipulations), in such a manner as to favor the extraction by the exercise of care and patience in grasping one of the extremities of the foreign body lightly, and "coaxing" it, so to speak, to the urethral opening. In this manner Reginald Harrison succeeded in extracting a lead-pencil three and a half inches long from the bladder of a male patient the day following its introduction. 
Where the foreign body has produced ulceration and perforation of the bladder, the treatment must be based upon general principles and the conditions present in the case under notice. Should the case come under the surgeon's observation sufficiently early, even here prompt operative measures may meet with success. An immediate laparotomy, followed by cleansing the parts, their isolation by means of antiseptic gauze packing, thus providing means for drainage as well as favoring the formation of protecting adhesions, may, combined with perineal section, serve as a forlorn hope in cases of peritoneal perforation and extravasation, and is amply justified by the desperate straits in which a patient is placed by the occurrence of such an accident. In cases in which the foreign body lodges in such a fashion as to make pressure against the anterior vesical wall, the parietal and visceral layers of the peritonæum may become agglutinated sufficiently to protect the peritonæum, in which case the abdominal walls will become the seat of the urinary extravasation. In any case, extensive incisions promptly made along the lines in which the urine dissects up the connective-tissue planes are imperatively demanded, combined with efficiently maintained perineal drainage, antiseptic irrigation, either constantly maintained or frequently repeated, and absorbent dressings.

In cases in which a foreign body has remained in the bladder sufficiently long to become incrusted, the decision as to the best means to be employed for its removal must rest, in some measure, upon the character of the foreign body and the length of time since its introduction. While the use of the lithotrite, employed to crush and remove the calculous incrustation at a single sitting, may be the proper means in case the foreign body be small, or itself of a friable nature, in other cases it may be found that, while the perfect accomplishment of the crushing process as applied to the incrusting deposit will be all that could be desired on the one hand, on the other a lithotomy, either suprapubic or perineal, will be finally necessary for the purpose of removing the original cause. Therefore it would be but a wise precantion on the part of the surgeon, in a given case of vesical calculus with a history of a foreign body as the possible cause, to carefully consider this point, and, if the latter be a hair-pin, piece of wire, or other article in which the blades of the lithotrite may become entangled, to carefully weigh the propriety of performing one of the cutting operations as preliminary to its removal without delay.

The above remarks upon the treatment of foreign bodies in the bladder relate to cases occurring in the male. The female bladder is occasionally found to contain foreign bodies, of which perhaps hair-pins are the most common. Titillation of the region about the clitoris with these lead to their becoming engaged in and slipping from the grasp of the fingers along the urethra and into the bladder. They usually enter the 
bladder with the bent portion first. This causes the free extremities to project forward within the bladder as two prongs, and enhances greatly the difficulties of their removal. This position of the pin may likewise produce ulceration and abscess.

In the removal of foreign bodies from the female bladder dilatation of the urethra will ordinarily suffice. Should this fail, the operation of cystotomy through the vesico-vaginal septum is to be preferred certainly to incision of the urethra, and probably to the suprapubic route. The resulting vesico-vaginal fistula, if primary union fail from any cause, can be subsequently dealt with in the usual manner.

\section{MALFORMATIONS AND MALPOSITIONS OF THE BLADDER.}

\section{ABSENCE OF THE BLADDER.}

Complete absence of the urinary bladder is of very rare occurrence. Instances have been observed of this anomaly, and in these the ureters, one or both, open either into the urethra, vagina, or rectum. Although permanent incontinence is the rule in the case of urethral implantation, yet it has occurred that the patients have lived to a ripe age with an entire absence of a proper bladder, one or both ureters, constricted at their lower portion and dilated, opening into the urethra and forming a reservoir for the accumulated urine. Post-mortem examination alone served to reveal the true state of affairs. It is probable that a certain amount of natural or spasmodic contraction at the point of constriction of the ureters in these cases prevents the occurrence of constant dribbling of urine, which otherwise must have occurred.

In complete absence of the bladder, efforts of an operative nature have not been thus far successful in ameliorating the sufferer's condition. Attempts have been made to divert the course of the urine by implanting the terminations of the ureters into adjacent portions of the intestinal canal in cases of exstrophy of the bladder, and no doubt these have been suggested by Nature's efforts in these cases of congenital absence of the bladder.

Tizzoni and Poggi have reported (Congress of Italian Surgeons, Bologna, April 16-18, 1889) a series of experiments upon dogs, made with the view of determining whether or not it would be possible to construct a urinary bladder from an isolated coil of small intestine. The design, in these experiments, was to replace the organ when removed in its entirety for disease. I am not aware that any attempts have been made to imitate these experiments, which met with a fair measure of success, upon the human subject, either in cases of extirpation of the bladder for malignant disease or in congenital absence of the organ. 


\section{MULTIPLE BLADDER.}

Multiple, or, more properly speaking, divided bladder, has been described. These cases consist of the existence of a septum, dividing the bladder into two cavities. The division of the bladder into two or more cavities, as a result of disease, will be referred to in discussing the condition known as sacculated bladder. An instance of double bladder is referred to by Smith, of Baltimore, in which there likewise existed a double penis, with a separate urethra for each bladder. Blasius mentions a case in which five complete and distinct cavities or sacs existed.

These cases are of interest to the surgeon solely from the fact that calculous or other condition requiring interference may exist in one cavity, while the other may be healthy. The possibility of the existence of the malformation should be borne in mind in obscure cases. In Smith's case, one of the bladders contained a stone, for which a lithotomy was successfully performed.

\section{CONGENITAL EXSTROPHY OF THE BLADDER.}

In total fissure of the bladder the umbilicus is found either at the highest point thereof, or perhaps even upon a still higher plane in the cicatricial tissue; the edges of the vesical and abdominal fissures correspond exactly, and the symphysis pubis is also fissured. Assuming these facts, it is evident that this anomaly occurs somewhere about the second month of fœtal life. It is at this period of intra-uterine life that the centers of ossification of the pubic symphysis are not yet established; the bladder reaches almost to the umbilicus, the abdominal wall is as yet membranous, and its muscular tissue has extended but a portion of the distance toward the median line. The fœtus at this period is suspended by the umbilical cord, the latter lying upon the anterior abdominal wall, and, in cases in which, either from the greater development of the cephalic extremity of the body, or a mere caudal insertion of the cord, the head hangs downward, and pressure is exerted, as the cord passes between the legs of the fotus, exactly at the point where both epispadias and ectopia vesicæ develop. The abundance of amniotic fluid will permit of the fœtus assuming any position under the control of the force of gravity. By pressure the anterior abdominal wall is arrested in its development upon this line, and the intra-abdominal pressure eventually forces the posterior vesical wall into the fissure, where it presents its characteristic convex appearance (Rotgans). Whether the result of pressure of the cord, or from some other cause, the anterior wall of the fœtal bladder is either destroyed, or fails of development entirely, and the mucous membrane of the posterior wall of the bladder unites with the abdominal plates in the line of the defect in the anterior wall, the condition becom- 
ing that of a large fistulous communication between the bladder and external abdominal surface. The posterior wall and base of the bladder are normally developed.

The peculiar and characteristic appearances presented by a case of congenital exstrophy of the bladder are so striking as to scarcely be mistaken for any other condition. The posterior wall, thrown into rugæ by a reversal of its surface from a concave to a convex shape, presents itself at the line of fissure as a rounded prominence, dark red in color from being in a state of chronic hyperæmia. In some instances, particularly where care has not been taken to prevent undue pressure, the point of junction of the abdominal wall with the mucous membrane breaks down, granulations spring up, cicatrization takes place, and a ring of scar tissue, which gradually widens, operates to lessen the area of mucous surface. As time advances, this becomes more and more marked, until finally but a comparatively small area is left.

Upon the lower portion of the red, projecting surface the orifices of the ureters are found, through which urine flows intermittently, and a slender probe can, as a rule, be easily passed up to the pelvis of the kidney upon either side. Exceptionally the posterior bladder wall may present a concave instead of a convex surface anteriorly, in which case the orifices of the ureters are not so readily found (vesico-abdominal fissure without exstrophy).

As a rule, the fissure continues downward, involving the pubic symphysis and extending into the anterior urethral wall, thus dividing the two halves of the bilaterally developed genital organs of the male, in whom this condition is observed much more frequently than in the female. In the rarer instances in which it has been known to occur in the female, the posterior bladder wall ends in a short, open furrow, which corresponds in length to that of the female urethra. In the male, on the other hand, a long furrow, with its open part uppermost, passes along the dorsum of the stunted penis and through its glans. The prepuce itself is likewise divided, the two halves hanging downward upon either side of the furrow like a veil, and converting the normally short preputial frænum into a pendulous mass. The scrotum and testicles may be normal, or but illy developed. Large external inguinal herniæ are commonly observed in conjunction with this abnormity, which are probably due to the inherent weakness of the abdominal wall itself from loss of support at its median attachment. Cryptorchism, resulting from interference with the normal intra-abdominal pressure in its influence upon the descent of the testes, is almost a necessary accompaniment of the condition. Division of the scrotum into halves, whereby the parts somewhat resemble the labia majora, is sometimes observed. The pubic symphysis is never united; as a rule, the fingers can be placed between the undeveloped rami of the 
ubic bones. The rectus abdominis muscle of either side may be parially developed; their separation, however, may continue for some disance above the upper limit of the abdominal fissure, the gap between the sges being bridged over merely by the integument. In cases in which his occurs ventral hernia is very apt to take place.

The miserable condition of patients the subject of this malformation is almost beyond conception. Excoriations of the surrounding parts, and chronic dermatitis from the presence of decomposing urine, giving rise to itching and burning almost beyond toleration, together with the constant presence of a urinous odor in the clothing, combine to exclude the individual from society, and to render life a burden to him. This is particularly true of those in the lower walks of life, in whom the wearing of expensive apparatus, consisting of an accurately fitting rubber funnel and receiver, and frequent changes of clothing, are well-nigh impossibilities.

Treatment. - The resources of operative surgery have been taxed to their utmost in behalf of this class of sufferers, and many operative procedures have been devised for the relief of the disgusting and wretched state in which they are plunged. These all have for their object the closing in of the abdominal wall in such a manner as to replace the absent anterior vesical wall. To these efforts have been added those designed to form a urethra from the furrow upon the dorsum of the penis. To accomplish the first named, the operation designed and carried out by the late Dr. Daniel Ayres, of Brooklyn, N. Y., has proved thus far, in the hands of the writer, the most successful. It consists essentially of taking a flap of skin from the abdominal wall above the fissure sufficiently long and wide, when allowance is made for its contraction, as to cover completely the defect. The hinge upon which the flap turns as it is reversed and laid with its integumentary surface facing the posterior bladder wall, and its raw surface presenting anteriorly, corresponds to the limit of the upper rounded edge of the fissure. Two lateral flaps are now dissected up, their free margins corresponding to the edges of the defect upon side. By a sliding movement these free edges are made to approximate each other in the median line of the body, and over the raw surface of the flap which has been turned from above downward. In this manner each lateral flap covers the corresponding half of the first flap, and an anterior bladder wall, representing in thickness double that of the integument itself, is formed. These flaps are sutured in position by means of silk-worm gut (crin de Florence) rather than silk, for the reason that this material is less likely, during the time when its presence is necessary, to absorb urine and become the site of deposits of the urinary salts, thus increasing the irritation and lessening the chances of union. An opening is left at the root of the penis for the escape of urine in instances in which no effort is made to restore the urethra. 
Where it is decided to attempt the relief of the epispadias as well, the operation of Thiersch, of Leipsic, answers the purpose better than any other. A new track for the passage of urine, extending from the base of the bladder and passing in front of or through the soft structures, marking the site of the pubic symphysis, and thence to the perinæum, may or may not be required preliminarily. If this additional precaution is made use of, there should be passed through this channel a soft-rubber tube, having a cup-shaped flange resting against the bladder wall, to collect and guide the flow of urine away from the base of the penis. This being accomplished, the furrow upon the dorsum of the penis is deepened by a longitudinal cut extending its entire length. A strip of surface is now denuded upon either side of the penis; by approximating these latter and securing them by sutures in position, a soft catheter of proper size occupying the furrow deepened by the above-mentioned incision in its bottom, a covered channel for the transmission of the urine from the bladder is formed.

The flanged catheter should be retained in the artificial urethra opening into the perinæum until the new channel upon the dorsum of the penis is formed. When this is completed the former is to be allowed to heal. In the operation for formation of an anterior bladder wall a permanent catheter should be kept in place in the completed urethral channel upon the dorsum of the penis until the completion of the healing process.

In thus furnishing a flap from the abdominal wall for the purpose of supplying a defect in the vesical wall, it is expected that the integumentary surface thus reversed and made to take the place of mucous membrane would take on more or less of the characteristics of the latter. To what extent this occurs is thus far problematical. In one case upon which the writer operated, in the Methodist Episcopal Hospital in Brooklyn, the child was brought back to the hospital in about eight months after the operation, suffering from a violent rectal tenesmus, the cause of which was found to reside in a formation of calculous material upon the artificially formed anterior wall. This was removed by means of a small curette introduced through an opening made for the purpose at the base of the penis and alongside of the vesical opening of the artificial penile urethra. This opening healed in a few weeks.

After all, the hope of benefit to be derived from these operations is solely that an apparatus may be worn to catch the urine with greater facility. There must of necessity be incontinence, for nothing has as yet been devised to take the place of the sphincter vesicx. This, and the advantage to be derived from relieving from irritation and pressure the theretofore unprotected posterior vesical wall, constitute the sole justification for their performance. 


\section{CONGENITAL DEFECTS OF THE POSTERIOR BLADDER WALL.}

Congenital defects of the vesico-vaginal and vesico-rectal septum have been observed, the condition varying from a complete absence of the septum to the existence of a mere fistulous communication. In the case in the first named the condition is almost necessarily a hopeless one. Where a fistulous communication exists, operative measures of a plastic nature may with propriety be instituted.

\section{PATENT URACHUS.}

A further abnormity of the bladder, congenital in character, consists of a persistency of the cavity of the urachus, this apparently resulting from some obstruction to the free passage of the urine by the normal channel. Early in intra-uterine life the intra- and extra-abdominal portions of the sac of the allantois communicate with each other through the umbilicus. Coincidently with the disappearance of the extra-abdominal portion of the sac the lower half of the intra-abdominal portion becomes the urinary bladder, while the upper half later on (at or about the seventh month) becomes impervious, and connected with a fibrous cord extending from the summit of the bladder to the umbilicus. This tibrous cord, known as the urachus, may fail of complete obliteration, and form a fistulons communication between the navel and the cavity of the bladder. This constitutes the more common form of the affection, and its existence is announced by the occurrence of a discharge of urine from the umbilicus. Calculous concretions may form along this abnormal urinary tract, and inflammatory conditions as well as suppuration occur.

The most aggravated form of this condition consists of a complete prolapse of the vesical wall through the urachus, as observed by Von Gusseron and Froriep. Generally speaking, this form presents itself as a portion of mucous membrane, of the size of a cherry, from some point of which urine is seen to flow from an opening. This is found to be irreducible.

Treatment.- In the treatment of this class of cases the first care must be to insure complete and uninterrupted patency of the normal urinary passages. To accomplish this in the female, it has been recommended to produce artificial incontinence by forcible dilatation of the entire urethral tract. In the male the bladder may be placed entirely in a state of rest by tube-drainage through the perinæum. This being xccomplished, the means employed to close fistulæ in general may be resorted to, such as the application of the actual cautery, the performance of plastic operations, etc.

\section{HERNIA OF THE BLADDER.}

Cystocele in the male occurs most frequently in the inguinal canal. It may be found as a complication of intestinal hernia in this region, or 
the entire protrusion may consist of the bladder alone. In either case it may be complete or incomplete, and occupy simply the inguinal canal or extend into the scrotum. In those instances in which cystocele is found as a complication of intestinal hernia the bladder, as a rule, will be found lying behind the intestine. In uncomplicated cases the protrusion is destitute of a sac for the greater part of its extent, owing to the fact that the bladder is normally only partially covered by peritonæum.

The importance to be attached to the condition under consideration is due mainly to the fact of its comparative rarity, and the dangers to which a patient suffering from hernia of the bladder may be exposed, from an error of diagnosis. It usually presents itself as a rather soft and fluctuating tumor with some elasticity. The especial point to be borne in mind is the fact that it varies in size at different times, that it is capable of being emptied by pressure, and that this emptying of the sac is at once

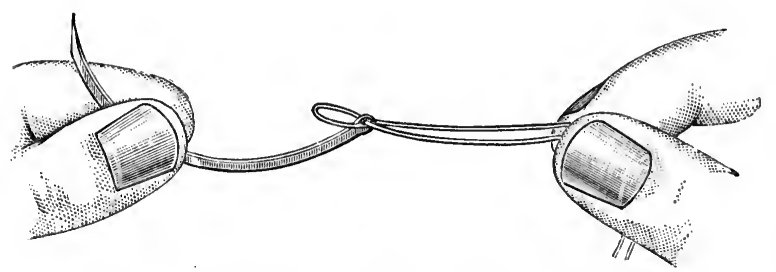

Fig. 2.-The crossed suture. Manner of threading the needle.

followed by a desire to urinate. Indeed, it may happen, as is sometimes found in females suffering from vaginal cystocele, that the act of micturition can not be accomplished without first raising the tumor and other-

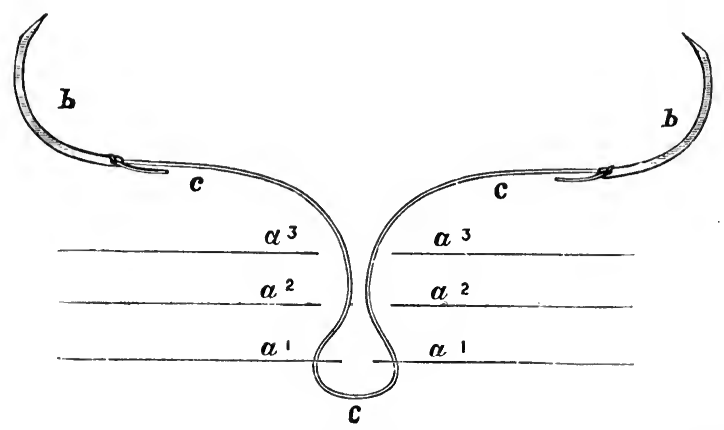

Fig. 3.-The crossed suture. Showing suture passed through lowermost layer. $a^{1}$, first layer; $a^{2}$, second layer; $a^{3}$, third layer.

wise manipulating it. In some respects it may resemble hydrocele of the cord; and no less an authority than Pott relates an instance in which, under the supposition that he was dealing with a diseased testicle, he 
incised a cystocele which had found its way into the scrotum. It is more likely to be mistaken for hydrocele of the tunica vaginalis testis, in

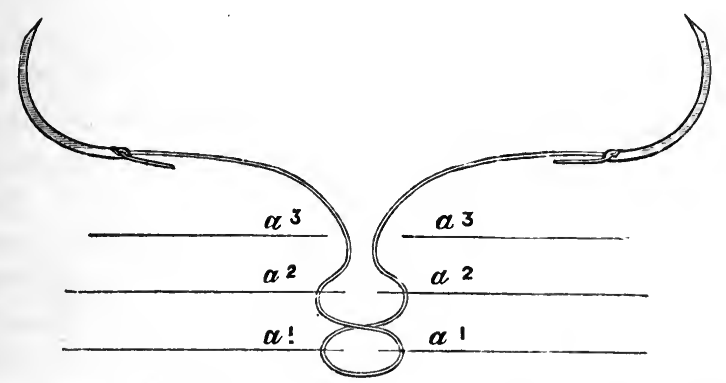

Fig. 4.-The crossed suture. Showing suture crossed and passed through seeond layer from below upward.

which case the operation of tapping would expose the patient to grave dangers.

The complications to which hernia of the bladder may give rise relate mainly to-first, retention of urine ; second, cystitis ; third, calculous dis-

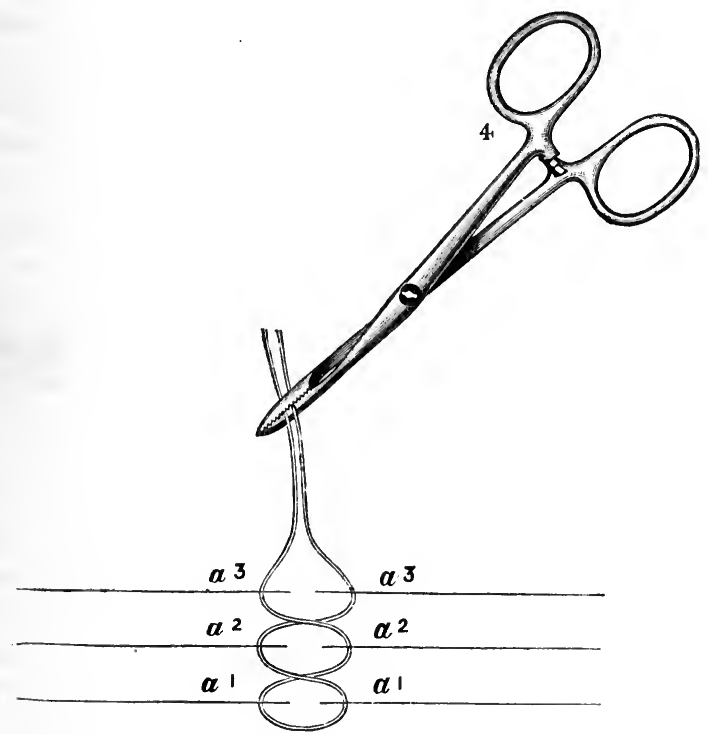

F16. 5.-The erossed suture. Showing all three layers included in the suture ready for tying. 4, ordinary hæmostatic foreeps, temporarily seeuring suture for ready identifieation until all are ready for tying.

ease. The herniated bladder must very rarely become strangulated; at least I am not aware of a recorded instance of the occurrence of this accident. Angulation of the sac may give rise to partial or complete 
retention of the contents, but patients, as a rule, instinctively take care of this by manipulation; hence the surgeon's services are rarely called into requisition because of this complication.

Treatment. - In the treatment of the displacement under discussion, the same principles should govern the surgeon as are applicable to other forms of hernia. Both palliative and radical measures may be called for in different cases. In the former are to be included the use of trusses or other supports. These should be carefully adjusted to the position, nature, and extent of the protrusion. It may occur that, in spite of the most carefully fitted apparatus, the hernial protrusion exhibits a marked tendency to pass beyond the limits of the inguinal canal, and to extend into the scrotum. This circumstance, combined or not with the occurrence of angulation and consequent retention, will be sufficient to justify the surgeon in recommending the operation for radical cure. In fact, so favorable are the statistics of the present day in radical herniotomy that it is a question if all instances may not with propriety be at least offered the opportunity for radical cure.

In selecting the means of bringing about a radical cure, the surgeon will be guided more or less by his preferences for operative procedures intended to cure intestinal hernia ; they are to be dealt with identically. In the present state of our knowledge, it is probable that the procedure known as Potempski's modification of Bassini's operation offers the best chance of permanently relieving the patient. This consists essentially in reducing the herniated portion, after exposing the same by the usual incision parallel to Poupart's ligament, lifting the spermatic cord from the inguinal canal and carrying the same toward the median line, and attaching it to the external oblique muscle, this having been previously bared for that purpose. The cord is here held in position by a loose suture of catgut. The margins of the inguinal groove, as it now becomes, are drawn together, including the arched fibers of the transversalis muscle at the internal ring, leaving but just barely room in the latter for the passage of the spermatic cord. It may be mentioned here that the latter, as it leaves the internal ring, should take a direction first upward and inward for an inch or more, prior to being attached to the external oblique. For the purpose of suturing the canal and ring, chromic gut as a buried suture may be employed; preference may be given, however, to the method known as "cross-suturing." . This consists in including the different layers, from below upward, in loops of silk-worm gut, arranged in figure-of-eight fashion (Figs. 2, 3, 4, 5). The advantage of this method consists in the fact that one by this means may employ a suture which will serve to accurately coaptate the individual layers and yet be capable of removal at will (Annals of Surgery, May, 1892, page 351). These should be allowed to remain for at least fourteen days; it is 
my habit to remove them between the fourteenth and twenty-first days. The entire procedure should be conducted with due regard to aseptic conditions, in which case no drainage should be employed.

Hernia of the bladder occurring in the female, or vaginal cystocele, is usually treated by the gynæcologist; the reader is therefore referred to works upon the diseases of women for the discussion of this condition.

INVERSION OF THE BLADDER.

The condition known by this term is of exceeding rarity, and is only found in females. As its name implies, the viscus is literally turned inside out, and projects as a red, polypoid mass from the urethra. The inversion is, as a rule, but partial, and may be complicated, as in the case of a sixteen-months-old child observed by Ashhurst, with prolapse of the rectum.

The diagnosis of this affection is important. The error may be committed of attempting its removal, under the belief that a polypoid tumor is present. The points to be observed are as follows: Presence of a pyriform, red, vascular elastic tumor between the labia, below the clitoris, and above the vaginal orifice; the urethra is not discoverable, and careful inspection will reveal in the mass the orifice of the ureters with urine issuing therefrom, if the inversion be sufficiently cumplete. As considerable relaxation of the urethral orifices must have been previously present, a history of incontinence preceding the inversion will usually be obtained, and the first appearance of the tumor following a violent expressive effort at micturition or defecation.

As a complication of this condition, rupture of the inverted bladder has been recorded. The ease was under the care of Mr. Croft, and it appears from the description that the inverted and ruptured viscus was returned under chloroform. The patient made a complete and permanent recovery.

The treatment of inverted bladder consists in a reduction of the viscus, and care on the part of the patient to guard against a relapse. Should this occur, scoring the parts about the vesical neck with the actual cautery before reduction, in order to favor increased solidity and hence support of the relaxed parts, has been recommended. In a persistent case, laparotomy and intraperitoneal fixation would be justifiable as a last resort.

\section{OTHER ABNORMITIES OF THE BLADDER.}

\section{HYPERTROPHY OF TIIE BLADDER.}

Hypertrophy of this organ is a symptomatic and never an idiopathic condition, and is to be regarded as the natural result of obstruction to 
micturition, or any condition which persistently stimulates the muscular wall into contraction. Thus, inflammatory changes in the bladder of both acute and chronic character, especially the latter, by giving rise to greater frequency of micturition, and in obstructive conditions of any portion of its outlet, by giving rise to proportionate development in order to make provision for the additional resistance which must be overcome, increase the nutrition of the muscular structure until the latter attains many times its natural bulk.

Muscular hypertrophy may be eccentric or concentric, the first named being attended by general dilatation of the organ, and results from obstructive conditions of the outlet; while the latter is rather marked by a contracted condition, and is the natural consequence of causes which tease the organ into frequent use of its muscular apparatus, such as the presence of foreign bodies, calculus, etc. Chronic cystitis is the most frequent cause of the last-naned condition, and it is commonly designated contracted bladder.

In the eccentric form of hypertrophy there sometimes occur herniæ of the mucous membrane and subcutaneous cellular tissue through adjoining portions of the muscular fasciculi. Thus pouches are formed, and in these sacs or diverticula urine is apt to collect and be retained; this, by undergoing decomposition, produces by its presence irritation and inflammation.

The prognosis depends, as a rule, upon the curability of the condition which gives rise to the hypertrophy. In the form resulting from obstruction, when the latter is removed the hypertrophy generally disappears. Oceasionally, however, it happens that a condition of irritability remains, due probably to the presence of diverticula long after the structure has been sufficiently relieved to no longer act as an obstruction.

Treatment. - Being simply an effect of disease, the treatment of this condition relates almost entirely upon the removal of the primary or determining cause upon which it depends. The exception to this is to be noted in the cases in which, after removal of the obstructing cause, irritability of the bladder and more or less persistence of the hypertrophy remain. Here cystotomy and irrigation are indicated, and have been resorted to with beneficial results. This procedure exerts a favorable influence in two ways: In the first place, perfect rest is given to the organ for several weeks, under the influence of which the increased muscularity diminishes or comparative atrophy ensues. In addition, residual urine undergoing decomposition is prevented, and lessened irritation and consequently lessened frequency of expulsive effort follow.

Limited hypertrophy will be described under the head of Bar at Neck of Bladder. 


\section{ATROPHY OF THE BLADDER.}

This condition of the bladder is but rarely met with save in aged persons who are thin and emaciated, and in certain cases of paralysis from defective nutrition, although both Hunter and Bonnet have recorded cases of rupture of the bladder, even in young subjects, by simple pressnre over the organ during catheterization. In cases in which the organ has been greatly over-distended, fatty degeneration may take place and atrophy ensue. So, also, atrophy may occur as a result of malformation or extensive vesical fistula, the bladder ceasing to perform its function, and being reduced to a rudimentary state.

\section{SACCULATED BLADDER.}

This condition has already been referred to as one of the results of eccentric hypertrophy of the bladder. It is most commonly observed in connection with the hypertrophy due to prostatic enlargement, and occurs as pouches or diverticula, which protrude between separated portions of the muscular coat where the mucous membrane is unsupported. In these, urine lodges and may undergo decomposition. Under the influence of expulsive efforts on the part of the bladder the pouches tend to become constantly deeper, until they at last attain a capacity of several ounces.

Among the inconveniences incident to sacculated bladder, aside from those already mentioned, is to be noted the fact that these pouches may become the receptacles of calculous material. Instances have been recorded in which the lethal result following lithotrity has been attributed to the fact that sharp fragments, following the operation, have lodged in these diverticula and given rise to severe and fatal cystitis.

Diagnosis. - The diagnosis of this condition is not easily made. It may be suspected to exist if, upon introducing a catheter for the purpose of emptying the bladder, it is found that a still further flow can be obtained by changing the position of the patient, or raising him from the recumbent to the upright position. It sometimes happens that the bladder is injected with a measured quantity for purposes of examination, and, upon permitting the organ to empty itself, but a relatively small quantity is expelled. This will lead at once to a suspicion that sacculation exists. The employment of a silver catheter, the beak of which can be rotated into different positions, may lead to the withdrawal of urine or injected fluid containing puriform material from the pouches. This varying of the appearances of the urine during different stages of the flow is held by Cadge to be a diagnostic, although not pathognomic, sign.

The results of sacculation, in addition to the aggravation of cystitis, which already exists, and the impaction of calculi, relate first to the possi- 
bility of an enormous tumor rising into the abdominal cavity and giving rise to erroneous operative interference and, in case of the involvement of the posterior wall, suppuration of the sacculus. In the last-named condition an abscess, which finally communicates with the rectum, occurs as a consequence, with recto-vesical fistula and the passage of fecal matter into the bladder.

Treatment. - In the treatment of sacculated bladder attention should be paid to the subject of drainage and cleansing of the viscus by irrigation following the removal of the cause. If this condition is suspected, complicating a calculus, the latter had better be removed by one of the cutting operations, unless there be some positive contra-indication, and drainage and irrigation practiced until the muscular apparatus is restored to its normal state.

\section{BAR AT NECK OF BLADDER.}

This term has been applied by English and French authorities to a condition believed to occur independently of prostatic enlargement, but usually associated with the latter, and which by its presence gives rise to more or less obstruction to the free performance of the act of micturition. Three varieties of the affection are recognized and described by Mr. Reginald Harrison, namely, (1) spasmodic, (2) mucous, and (3) muscular.

The first or spasmodic variety is found chiefly to exist in persons of a gouty or rheumatic disposition, who possess in a high degree the uric-acid diathesis. It appears to consist of an irregular or spasmodic action of the muscles at the vesical neck during the action of micturition, and is not in any sense due to prostatic involvement, inasmuch as it is frequently found where no hypertrophy is present. Delafosse describes it as a contracture of the vesical neck. It is questionable if the condition so described should be considered as a "bar" at all in connection with permanent structural changes at the neck producing obstruction to urination.

The treatment of contracture of the vesical neck or "stammering of the bladder" (Paget) is in the main purely medicinal. The diathesis upon which its production depends requires especial attention, and the remedies to be employed will include those usually found useful in gouty or rheumatic conditions. It has been thought that the symptoms depend in some measure upon extreme sensitiveness of the prostatic urethra; hence the recommendation by some authorities that a solution of nitrate of silver, two grains to the ounce, be employed, applied occasionally to this part by means of a deep urethral syringe, after a thorough anti-rhenmatic course.

The second or mucous variety is formed, as its name implies, from a more or less distinctly formed duplicature of the mucous membrane, and has always been found to be associated with and dependent upon prostatic 
hypertrophy. This variety appears to result from lateral enlargement of the prostate, and the "buckling" up, so to speak, of the floor of the urethra. It is crescentic in shape generally, and may even form a distinct valve-shaped flap at the vesical urethral orifice. The extent of the mucous bar does not necessarily depend upon the amount of lateral hypertrophy present; a certain extent of elevation of the mucous membrane, called the uvula vesicce, is found at this point in health. The introduction of a sound will reveal a distinct "catch" as its point slips over the bar, while conjoined manipulation with the finger in the rectum simultaneously will show that the distance between the sound and the finger is but slightly increased beyond the normal. Careful examination, however, will reveal more or less increase in the normal lateral boundaries of the prostate.

The third or muscular variety, sometimes known as the limited hypertrophy of Guthrie, always exists in connection with prostatic enlargement, although structurally it is not connected with the prostate. Here a bar is formed at the neck of the bladder by the excessive development of some of the muscular fibers which run transversely across the trigone behind the prostate, thereby forming a more or less prominently marked elevation, and dividing the bladder at this point into two separate and distinct compartments, an upper and a lower one.

The explanation given by Reginald Harrison of the occurrence of limited hypertrophy of the bladder at the trigone, or muscular bar, is prubably the correct one. This eminent authority says that the formation or aggregation of muscular tissue at this point is due to a conservative effort on the part of Nature to empty the pouch which forms at the trigone and the posterior limit of the prostate in cases of enlargement of the latter, and in which urine tends to collect.

As in the case of sacculated bladder, the diagnosis of the condition is attended with marked difficulty. Nor is its differentiation of great importance, so far as the usually employed treatment of impeded micturition in elderly persons is concerned. The treatment of muscular bar is the treatment of the enlarged prostate upon which it depends, for the details of which the reader is referred to the chapter upon injuries and diseases of that organ.

SUPRAPUBIC VESICAL PUNCTURE IN OBSTRUCTIVE DISEASE AT THE VESICAL NECK.

This operation is indicated as soon as catheterization can not be accomplished, and simultaneonsly decided retention of urine is at hand. These circumstances will usually co-exist, for the reason that the abnormal condition which interferes with the passage of the catheter likewise operates more or less completely in preventing micturition. The course of re- 
tention, due to prostatic obstruction, may vary considerably, in some instances the retained urine accumulating rapidly, the vertex of the bladder reaching above the umbilicus within twenty-four hours. On the other hand, it may require weeks to reach this condition in cases in which the patient occasionally passes small quantities of urine. The urgency, therefore, of the demand for the performance of the operation will depend upon these varying circumstances. In any event, the patient should not be permitted to suffer where retention, whether complete, as in the first named, or incomplete, exists. The mere fact that the bladder is overdistended, and no catheter can be passed, is sufficient justification for the performance of an operation so devoid of risk in proper hands.

\section{FISSURE OF THE NECK OF THE BLADDER.}

This condition, analogous in some respects to anal fissure, although much smaller, is of comparative rarity in the male. It is most frequently met with in females, in whom it is properly termed vesico-urethral fissure. It is only within the last few years that this condition has been brought to the notice of the profession.

In the male the symptoms are such as to suggest the variety of spasmodic bar at the neck of bladder, or contracture du col vésical of the French. There is the same frequency of micturition, with a hesitancy, so to speak, during the act. There are these differences to be noted, however: whereas, in spasmodic bar, the "stammering" is more particularly noted in the commencement or middle of the act, in fissure the sensation is often described as an alternate opening and closing at the end of the act, accompanied by the presence of a few drops of blood following the last of the urine, and a sharp, stinging pain, referred to the neck of the bladder. In varying degrees of acidity of the urine the pain in the same individual may vary, becoming more intense as the acidity increases, and lessening in severity when the urine becomes neutral or alkaline in reaction. The passage of an instrument into the bladder occasions great distress, and pressure against the vesical neck from the rectum similarly produces pain at this point.

In females the symptoms are such as to suggest cystitis. There is a feeling of burning at the neck of the bladder, and acute pain during and following the act of micturition and vesical tenesmus. These symptoms continue for some time, finally subsiding, only to recur as soon as urine collects in the bladder. In differentiating, however, it is to be noted that in cystitis the pain is never localized, and the act of urination is followed by a sense of relief, while in tissure the reverse is true. In females particularly the endoscope will be found useful in making the diagnosis.

The treatment consists in producing and maintaining a neutral or alkaline condition of the urine, combined with the local application of a 
solution of nitrate of silver, one grain to the ounce, to the prostatic urethra. In the female, by means of Skene's fenestrated endoscopic tube with closed end, it is possible to touch the fissure directly with the modified nitrate-of-silver stick. Should this means fail after a few applications, the urethra should be dilated after Simon's method, and a condition

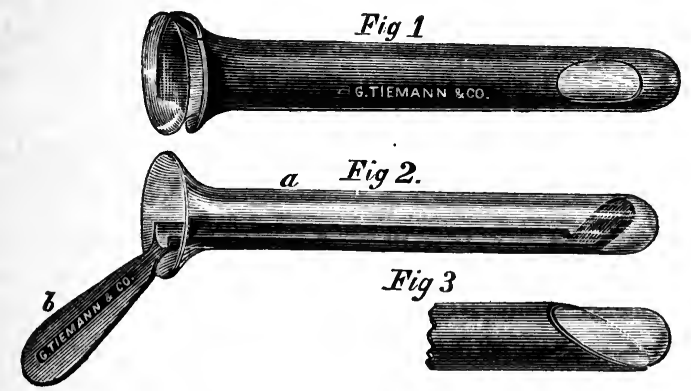

Fig. 6.-Skene's endoseope, for the female urethra.

of temporary incontinence produced. Cases persistent in character and otherwise intractable in the male should be subjected to cystotomy, preferably by perineal section. The object of these operative procedures is to obtain complete rest for the region about the neck of the bladder. The suffering produced by vesical fissure may be so great as to demand inmediate relief by cystotomy.

\section{PUNCTURE OF THE BLADDER.}

In opening the bladder for any purpose, advantage is taken of its but partial serous investment in order to avoid risks incident to section of the peritonæum. With this in view, the following rontes have been devised: (1) above the pubis, (2) by Voillemier's sub-pubic operation, (3) through the symphysis, (4) from the perinæum, and (5) from the rectum.

Suprapubic Puncture.-By all odds the best method of performing vesical puncture is by the suprapubic ronte. The interior of the bladder may be reached in this way through the space immediately above the pubic bone without wounding the peritonæum. As shown by the researches of Ponliot, Bernays, and others, this space varies greatly in extent in different individuals, althongh it is always increased in distention of the viscus. It is well, however, to keep as close to the parts as possible, with the patient in the horizontal position. A general anæsthetic is not required, as the introduction of the trocar occupies but a few seconds, and the rest of the operation is painless. The suprapubic region should be shaved and thoroughly cleansed beforehand. A slight skin incision may be made prior to the introduction of the trocar, but, as a rule, 
when the aspirating needle is employed this may be omitted. When it is tolerably certain that the operation is to be required but temporarily, and the hope is entertained that the natural channel will shortly be re established, the aspirator will be found the most useful instrument. The point of the index-finger should be placed so as to mark the site of the upper limit of the pelvic arch, and by a quick, thrusting movement the aspirator needle, attached preferably to a previously exhausted bottle, made to penetrate to the interior of the bladder. If it is designed to accomplish a more permanent drainage of the bladder, a curved trocar and cannula are selected, and under cocaine anæsthesia a small incision made. This is rendered necessary particularly in fat individuals, and should extend to the muscular layer, to avoid the recession of the abdominal walls before the larger-sized and curved trocar, the passage of which is resisted by the former. The index-finger of the right hand should be placed upon the instrument at the point to which it is intended to introduce it, thus varying in different cases according to the thickness of the abdominal wall. The convexity of the curve should be placed in an upward direction, the concavity of the instrument hugging the posterior surface of the symphysis pubis, thus the more effectually guarding against injury to the peritonæum.

It sometimes happens, whether the aspirating needle or cannula is employed, that blood-clots, resulting from previous attempts at catheterization, obstruct the free flow of the urine. In this event the introduction of a clearing wire into the former, or the trocar into the latter, is necessary. If the outflow is frequently embarrassed in this way, a solution of boric acid, or borate of soda, should be forced repeatedly through the instrument, and the coagulation broken up gradually in this way.

When the incision is employed, care is to be taken that the urine, which under these circumstances is usually decomposed, does not produce infection of the abdominal walls by coming in contact with the wound surfaces. In order to avoid this, pledgets of cotton or gauze, saturated with a 1 to 1,000 mercuric-chloride solution, should be wrapped about the cannula after its introduction and packed into the wound, or a stream of the same solution is to be allowed to run over the parts during the evacuation of the bladder contents. When the aspirating needle is employed, its withdrawal should not be attempted until the bladder is empty, and then only with the inlet stop-cock open, and as complete a vacuum as possible present in the aspirating bottle. By this means infection of the abdominal walls is avoided. A simple strip of adhesive plaster sterilized by being heated, covered with a pledget of cotton saturated with mercuricchloride solution, suffices as a dressing.

If aspiration has been employed, the bladder need not be washed out at this time, but this may be postponed until the viscus can be reached by 
the natural route. If the larger trocar and cannula have been employed, a soft-rubber catheter may be passed through the latter, and irrigation performed by means of Thiersch's boro-salicylic solution. The cannula may be fastened in place by means of tapes passed through openings in its flange, somewhat in the manner employed in securing a tracheotomytube. Either an antiseptic dressing may be placed over the tube, or Dittel's suggestion followed. The latter consists in attaching a rubber siphon-tube to the cannula, and leading it to a receptacle in which an antiseptic solution is placed. Either of these procedures is to be preferred to the old plan of placing a small cork or ball of wax in the cannula outlet, and removing this occasionally to empty the bladder. Urinary infiltration is almost certain to follow the adoption of this method.

In the after-treatment of cases of suprapubic puncture, where a cannula is to remain for a time, the points to be borne in mind relate particularly to, first, the maintenance of a strictly antiseptic regimen; and, second, to the prevention of irritation from the presence of the cannula and the deposit thereon of the urinary salts. The first named is accomplished by several times daily irrigation of the bladder with the boro-salicyilic solution, and an occasional mercuric-chloride solution when the urine is very fetid, in addition. The latter need not be employed stronger than a 1 to 4,000 solution. In order to remove and replace the cannula easily, the following expedient may be resorted to: An elastic catheter is to be passed through the cannula and into the bladder, where it is held while the latter is withdrawn. After cleansing, the cannula is to be replaced by slipping it over the catheter, using the latter as a guide. After the lapse of a fortnight the wound is converted into a granulating track, and the use of the catheter may be dispensed with.

The permanent withdrawal of the cannula depends upon the course of the individual case. It is a frequently observed fact that a very decided and sometimes rapid subsidence of the swollen prostate follows puncture of the bladder. In instances, therefore, in which the operation is performed for retention due to this cause, the introduction of the catheter by the normal route is possible sooner or later. The cannula should not be permanently withdrawn until this is fully assured, and in cases in which false passages have resulted from previous attempts at catheterization, this should be postponed until their closure is believed to have taken place. Upon the fifth or sixth day the catheter may be passed, if this can be accomplished, and repeated upon several subsequent days; drainage by the cannula may then be abandoned. The granulating canal closes very quickly, cicatrization occurring in twelve hours in some cases. The further management of the case will then depend upon the measures selected for the amelioration or cure of the condition producing the obstruction. 
Suprapubic puncture has been employed for the purpose of accomplishing posterior catheterization (Brainerd), and for the locating of an impermeable stricture in instances in which, owing to the presence of false passages, this can not be done with certainty from in front. A metallic catheter, passed through the suprapubic puncture and into the internal urethral orifice, will frequently accomplish the above-named purpose in a very satisfactory manner, and permit a safe and easy external urethrotomy, the catheter serving as a guide. The same manœuvre may be executed in cases of rupture of the urethra, the presence of the catheter passed from behind enabling the operator to identify the central end of the torn urethra after perineal section, therefore avoiding a long and tedious and sometimes fruitless search. When suprupubic puncture is performed for this purpose care should be taken to select a cannula whose curve corresponds to that of the catheter or guide intended to be employed, otherwise some difficulty may be encountered in manipulating the latter.

When aspiration is employed (and this method is to be given a fair trial before the employment of the permanent cannula), the operation may be repeated, if necessary, two or more times daily for several days; the bladder should not be allowed to become over-distended after the first aspiration, for the reason that urinary infiltration may take place along the needle-track, under these circumstances.

Subpubic Operation.-Voillemier's proposal to puncture the bladder below the symphysis has attracted some attention. The advantages claimed for this method are, first, that the adhesions between the anterior vesical wall and the abdominal wall which may follow the suprapubic method are avoided; and, second, that the bladder is opened at a lower point, and is thus emptied more readily. In carrying ont this procedure the penis is drawn strongly downward, and the trocar and cannula passed through the skin and middle pubo-prostatic ligament or deep femoral fascia, which fills the angle formed by the descending rami of the os pubis. The method has never become popular, for the reason that its alleged advantages are more than counterbalanced by the uncertainty of reaching the bladder, owing to the fact that its base is distended the least relatively in retention; and further, by the danger of injuring the dorsal artery and vein. The operation is not easy of performance, and the position of the opening is neither a convenient nor a comfortable one.

Puncture through the Symphysis Pubis.-Puncture of the bladder through the symphysis pubis has been recommended (Brandner). This, like the preceding, has no advantage over the suprapubic method, and is open to the objection that it may, in aged persons, be exceedingly difficult of performance, because of ossification of the intra- 
pubic cartilage. Under such circumstances, as suggested by Coulsen, it would be necessary, in order to carry out the procedure, to perforate the ossified cartilage by means of a bone-drill.

Perineal Puncture.-Tapping the bladder from the perinæum (Cock's operation) is particularly adapted to cases of irremediable stricture of the urethra, complicated with urinary fistulæ, and a perinæum more or less disorganized by inflammatory exudation, and in which but a remote prospect exists of establishing the continuity of the natural passage following the relief of the retention. The cases are but comparatively few at the present day in which this procedure will become necessary, perineal section, the structures being divided layer by layer, with attempts at the restoration of the urethra to a useful condition, having taken its place (see external perineal urethrotomy). This is to be preferred to the method originally suggested by Cock, which consisted in plunging a knife through the perinæum, at the apex of the prostate, along the prostatic urethra and into the bladder.

Direct perineal paracentesis-or Reginald Harrison's operation-is adapted to cases in which the obstruction to micturition has its origin in the prostate itself, and is behind the point at which the urethra is tapped in Cock's operation, as well as in the ordinary perineal section. It consists of puncturing the bladder directly through the prostate from the perinæum, without reference to the line of the urethra. The operation is performed as follows: With the left index-finger in the rectum as a guide, a trocar (see Fig. 7), specially made for the purpose, is introduced into the median line of the peri-
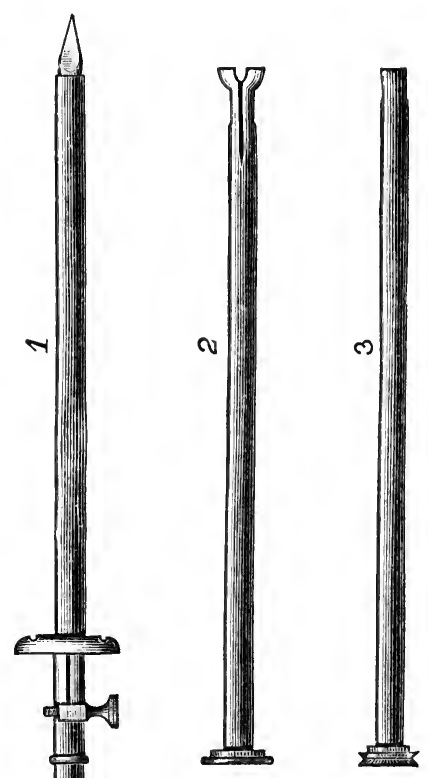

Frg. 7.-Reginald Harrison's troear. A piece of rubber tubing is to be attached below the collar of the eannula on withdrawal of the trocar, to convey the urine into a receptacle. 2 , silver eannula, which is introduced into the tube (1), to form a probe end, and for retaining it. 3 , plain silver tube with which to elean 2 in case it should become blocked. næum about three fourths of an inch in front of the anal margin, and pushed steadily through the prostate into the bladder. The trocar is now withdrawn, and the cannular portion of the instrument retained in position by suitable tapes attached to its 
shield or flange. In one remarkable case narrated by Harrison, the first in which the method was employed, the patient, a man of eighty-four, not only was made comfortable by the operation, but in the course of the following two months the prostatic enlargement almost entirely disappeared, and the tube was withdrawn.

The advantages claimed for this method by Harrison are, first, a convenient position for the external opening in all positions of the body ; and, second, the establishing of a short, low-level urethra, adapted to the altered relations of the urethra and prostate when the latter becomes enlarged, for the purpose of securing the most perfect drainage. The operation need not be limited in its application to cases of retention. It may be employed in cases in which a condition of constant vesical irritability and inflammation render it necessary to establish a permanent opening. Under these circumstances Harrison suggests that a sound should first be passed into the bladder and its beak reversed in such a manner as to lie in the depression behind the prostate. The point of the trocar should be directed against this.

Puncture from the Rectum.-Tapping the bladder from the rectum, first introduced by Fleurant, is but rarely practiced at the present day. The left forefinger is introduced into the rectum and the bulging posterior wall of the bladder identified beyond the prostate. Into this, precisely in the median line, the trocar is to be thrust. The objections to this operation are as follows: There is great difficulty in securing the cannula in position; the latter interferes with defecation, and it is almost impossible to replace it after it has been accidentally displaced. As a temporary expedient it is unnecessary, and as a permanent means for supplanting the normal channel it is comparatively useless, or at the best exceedingly difficult of management. In addition, it is not devoid of danger. Hill makes the observation that injury to the recto-vesical fold of the peritonæum in the cul-de-sac of Douglas has followed rectal puncture of the bladder. 


\title{
RUPTURE OF THE BLADDER.
}

\author{
By ALEXANDER W. STEIN, M. D.
}

Definition.-Rupture of the bladder may be divided into three classes: intraperitoneal, extraperitoneal, and subperitoneal-that is, the lesion may be situated within the limits of the peritoneal covering, and involve that structure; it may be located at some point of the viscus not invested by the peritonæum; or it may be that the lesion occurs within the limits of the serous tunic, but the rupture is incomplete, the mucons and muscular coats alone giving way, allowing an extravasation of urine beneath the peritoneal covering, which latter remains intact. A few cases are on record in which the lesion was both intra- and extraperitoneal. Intraperitoneal ruptures are more frequent than extraperitoneal, nearly as three to one. This finds an explanation in that when the bladder is fully distended, and is subjected to violent or sudden compression, the peritonæum, which is then the most tense and least yielding of the several tunics, will split in advance of the subjacent coats, and carry the latter with it in the laceration; whereas, in situations where the peritonæum is absent, the coats of the bladder are more elastic and yielding. Subperitoneal ruptures are certainly not common, there being but thirteen on record, yet it is probable that they have occurred somewhat oftener than we find recorded. In several cases of recovery there is every reason to believe that the extravasation was partial or subperitoneal ; while the long duration and slow progress of some cases of intraperitoneal ruptures can be accounted for only in the supposition that at first the extravasation was subperitoneal, and that the peritonæum became subsequently involved by pressure or a necrotic process. Such a conclusion would seem inevitable when, following a fall or an injury, symptoms of vesical rupture appear, and continue for several days without being very grave, until suddenly after a straining effort, something is felt to snap or crack in the abdomen, after which rapid and fatal peritonitis ensues. This is the history given in several instances.

Location.-As regards the exact location of the lesion, our histories are definite in only a limited number of cases. In the intraperitoneal variety, we find the superior region of the bladder mentioned in 79 cases. In 59, the laceration is said to have been at the posterior wall; 36 times 
at the upper part, and 19 times at the lower part. The extraperitoneal lacerations occurred anteriorly in 25 , in 7 at the side, and in 6 at the lower posterior portion of the viscus. We thus find that the largest number of intraperitoneal ruptures occur at the upper and posterior part of the bladder, where the viscus is thinnest, and where, in the course of distention, it comes in relationship with the sacral promontory. In these cases there need be no bony lesion; the relationship of the bladder to the bony prominence behind is such that any external violence or pressure may cause the injury, and the reason for the more frequent location of intraperitoneal ruptures in the upper and posterior portions of the biadder is found in the force being directed upon the upper part of the viscus, which is thus compressed against the vertebro-sacral eminence. Extraperitoneal lacerations are ordinarily found in front. The cause of this is manifest in the fact that the anterior surface of the bladder, being in immediate contact with the pelvic zone, is most exposed to injury from lesions of bone. The majority of ruptures situated anteriorly are complicated with fracture of the pelvic bones.

Character of Lesion.-The rent may be perpendicular, transverse, or oblique. It is usually linear. It seldom presents as a gaping wound or hole. When such exists, it varies in size from a minute opening to one admitting the passage of several fingers. The rent is generally ragged and its edges everted. Its length varies from 1 to $12 \mathrm{~cm}$. Extraperitoneal lacerations have, so far as we know, not exceeded $5 \mathrm{~cm}$. in length ; nor is the rent larger in the greatest number of intraperitoneal ruptures, but in several of these the tear was said to be from 5 to $12 \mathrm{~cm}$. in length. The coats may not be torn equally. In intraperitoneal rupture especially, the serous investment is apt to tear more than the other coats. In one such case the rupture in the bladder is said to have been $3 \mathrm{~cm}$., while that in the peritoneal covering was $15 \mathrm{~cm}$. in length. This is no doubt owing to the fact, already mentioned, that the peritonæum is the least yielding of the vesical coats, and, being upon the stretch, splits first and most. As a rule there is but one rent; exceptionally, two or three points have given way simultaneously. In two or three cases the edges of the rent were found to be so overlapped as to form a kind of valvular closure, or a loop of intestine was adherent by recent adhesions, which prevented the further escape of urine from the bladder. Morris (Med.-Chir. Trans., vol. $1 \mathrm{xx}$ ) mentions a unique case of this kind, in which the bladder was twice ruptured, the first time by violence, the second time, seven years after, by giving way of the adhesions between the bladder and rectum.

Etiology.- The causes may be divided into predisposing and determining, or traumatic and non-traumatic. The first and most prominent predisposing cause is distention of the bladder, the danger of rupture being proportionate to the degree of fixation and elevation of the viscus 
above the true pelvis, and in front of the projecting lumbo-sacral angle. While it would appear that distention is not a conditio sine qua non to rupture, a more or less replete state of the bladder no doubt exists in nearly all cases previous to the accident. In the majority of cases the injury occurs while under the influence of alcohol. The etiological influence of alcoholism is manifest-in the diuretic action of the drug rapidly increasing the accumulation of urine in the bladder, and in the obtunding of sensibility, vesical as well as general, whereby this undue accumulation is disregarded. Other predisposing causes of rupture are atony, fatty degeneration, sacculation, and ulceration of the vesical walls. In the majority of cases the autopsy showed that the coats of the bladder were healthy at the time of the accident, but the conditions of the bladder just mentioned render it very liable to rupture, even under circumstances of moderate distention, and from comparatively trivial causes.

It is probable that in rupture primarily depending upon some longexisting mechanical obstacle to the escape of urine, such as urethral stricture or prostatic hypertrophy, some physical or pathological changes have existed in some instances prior to the rupture of the viscus, but in many cases no such changes were found. One would suppose that a degree of thickness or hypertrophy of the vesical walls would be a safeguard against rupture, but this condition of the bladder is specially noted in several cases. A specimen in my possession shows an extensive laceraation through a contracted bladder, whose walls in some places are three fourths of an inch thick. This man had long suffered with stricture of the urethra, and had received a kick in the abdomen causing the laceration. The most common determining cause is some external violence applied over the region of the viscus, a sudden kick or blow over the hypogastrium, a simple tumble, or a fall from some point of elevation, the falling or kneeling of one individual upon the abdomen of another; these are most frequent causes, and are generally incident to drunken brawls. In another class of cases the injury is occasioned by the falling of a beam, the caving in of a wall or of an embankment, the passage of a wagon over the abdomen, etc.; these are frequently complicated with fracture of the pelvic bones, and it is difficult often to determine during life whether the injury to the bladder is the direct consequence of the compression, or due to the laceration of its coats by spicula of bone penetrating its cavity.

In the empty state the bladder is so deeply situated in the pelvic cavity that it is in a great measure protected from external violence, and, as a rule, can only be injured by such force as will separate the pubic symphysis or fracture the pelvic bones. But rupture by contre-coup, as in falling from an elevation upon the feet or buttocks, has occurred even when the bladder was comparatively empty. 
The cause of rupture may emanate from the individual himself, as occasioned by violent straining in micturition, defecation, and in lifting heavy weights. Rupture of the bladder depending upon stricture of the urethra as a primary cause is not common, for the reason that in stricture of the urethra the canal behind the obstruction, having become dilated and attenuated by the constant pressure made upon it during urination, will yield in preference to the bladder, which organ, under these circumstances, is often rather hypertrophied than atonied. Nevertheless, not a few instances of vesical rupture from straining to overcome an obstructed urethra have occurred.

Those instances in which the accident was primarily dependent upon prostatic enlargement were all intraperitoneal. The bladder has been ruptured during parturition - a fact to be remembered in always keeping the bladder empty during the progress of labor. Some cases are recorded as having occurred from retention of urine caused by retroverted gravid nterus. A man with retention, laceration of the urethra, and extravasation of urine, sustained in addition a rupture of the bladder in his struggles while undergoing etherization-a warning not to administer anæsthetics while the bladder is in a condition of distention. A number of times the viscus has been ruptured from the overdistending force of the injected fluid preparatory to a suprapubic cystotomy. Assmuth mentions two cases of the intraperitoneal variety which seem to have been caused solely from pressure of the abdominal muscles upon the distended bladder. There was no impediment to the escape of urine, and apparently no diseased condition of the bladder walls. We are told the bladder was ruptured spontaneously, in one instance, "while the man was sitting at the table," in another "while asleep." It occurs more frequently among men than among women, in the proportion of ten to one. This comparative exemption of the female from this injury is due to the fact that women, from the nature of their occupations, associations, and habits of life, are less exposed to the dangers of such an accident; while the greater width of the pelvis, and the interposition of the uterus and its appendages between the bladder and the sacral promontory tends to mitigate and break the force of the concussion or compression exerted upon the viscus.

In children this is a rare accident, notwithstanding their numerous knocks and falls. This may be ascribed to the fact that, owing to the greater sensibility of the bladder in the young, micturition is more frequent, and the viscus is rarely suffered to become greatly distended. The tissues are more yielding and elastic, and the sacro-vertebral angle is less prominent than in later life. In the adult the injury occurs most frequently between the ages of thirty and forty and between twenty and thirty-that is, at that period of life when man, by reason of his occupa- 
ion, is most exposed to injuries, and when, too, he is inclined to indulge nost freely in intoxicating drink.

Symptoms.-In consequence of the fracture of pelvic bones, or peraaps simply from shock, the patient is unable to walk, or even to rise irom the place where he has fallen; pain severe over hypogastrium; desire to micturate incessant, with an inability to void the smallest quantity of urine, or possibly but a few drops mixed with blood. Constitutional symptoms indicative of great prostration rapidly ensue. Countenance pallid and anxious, pulse feeble and fluttering, perspiration clammy, voice weak and husky, abdomen becomes distended, temperature rises, delirium, coma, or convulsions supervene. If the patient rallies from this shock or collapse, his life may be next imperiled by the development of peritonitis or septicæmia. Infiltration of urine is often remarkably extensive, detaching the pelvic organs from their annexes, descending into the perinæum through the inguinal canal and obturator foramen into the scrotum and thighs, and ascending the abdomen and back, reaching at times as high as the shoulder.

In the majority of cases the symptoms are sufficiently expressive to enable the surgeon to arrive at a prompt diagnosis. Of great diagnostic value is the statement so often made by the patient that, after a blow over the hypogastrium, or while in the act of straining, he "felt something give way within the abdomen," and sometimes adds, "experienced a sudden relief from the discomfort occasioned by the previously distended bladder." The evidence furnished by catheterization is of special value when it is positively known that the individual had not micturated for several hours previous to the accident. Under these circumstances an empty bladder, or one containing but a small quantity of sanguinolent urine, is strongly confirmatory of laceration of the bladder. But the evidence pointing to rupture is by no means always unequirocal. Often the signs and symptoms upon which we would most depend are absent ; those that are present are dangerously misleading, and the case is shrouded in uncertainty and doubt. Especially is this the case in the beginning, when a long interval of time may elapse between the receipt of injury and the development of characteristic symptoms. It is important to watch closely a patient on whom the suspicion of rupture of the bladder rests, for, though the symptoms may for a time be latent, once manifest they are rapidly progressive, and everything depends upon a timely and perhaps bold surgical interference. Not only may the first symptoms be delayed or be insufficiently marked to attract attention, but the vesical rupture may be complicated with other injuries whose symptoms may for the time obscure the more dangerous lesion within. As indicating the difficulties sometimes encountered in arriving at a diagnosis, we find that there is rarely any external sign or mark of injury to be found over the 
abdomen, even where a wagon has passed over the body. We depend much upon the history given as to the nature of the accident, the condition of the bladder at the time of injury, etc.; but if the patient is unconscious or is drunk, as is so often the case, no information can be obtained, or what may be given can not be relied upon. If we will take a lesson from some of the reported cases relative to the constancy and reliability of the most characteristic symptoms, we shall find that standing, or even walking, is not inconsistent with the existence of laceration of the bladder. Neither is the difficulty or inability to micturate a uniform symptom. The patient may have no difficulty in voiding urine, and, indeed, may pass a considerable quantity of water. In some cases it occurred that after the first catheterization the patient regained the power of voluntary micturition.

The absence of difficulty in voiding urine is met with in both extraperitoneal and intraperitoneal ruptures. One patient, with an extraperitoneal rupture an inch long and half an inch wide, communicating with an abscess half full of urine and pus, "passed his urine for three days after the injury as naturally as ever." This man lived thirty-two days ; while another patient, with an intraperitoneal rupture one and a half inches long at the summit of the bladder, "was able to pass water without any difficulty." He died eighty hours after the injury.

The marvelous retentive power of the bladder noted in some other cases would seem explicable only on the supposition that at first the rupture was incomplete, while in others this was accounted for in the closure of the vesical wound by "valvular protrusions" and adhesions, which prevented the escape of urine. An instance is cited of a man who was injured in a fall, and who manifested symptoms which led to the diagnosis of laceration of intestine. The catheter evacuated a large quantity of perfectly clear uine. In twenty-four hours the man died, and the autopsy revealed a large tear in the bladder, into which a coil of intestine had slipped and become engaged. The intestinal tract was found uninjured. There were signs of general peritonitis, and a large quantity of bloody urinous fluid was found in the cavity of the peritonæum. Many cases might be cited to show that the presence of elear urine in the bladder ean not be accepted as an evidence of the absence of injury to the viscus. On the other hand, the sudden advent of blood in the urine is nothing more than a presumptive evidence, strong though it may be, of this lesion. The urine may be tinged with blood from simple contusion of the bladder without laceration.

Diagnosis. - Important as it is to establish the diagnosis early in all eases, it is even more important to ascertain whether the rupture is intraor extraperitoneal. In the early stage of the case, when exact knowledge would be most serviceable, such a differential diagnosis is most difficult, 
often impossible. In a few instances the diagnosis of rupture was established by the accidental passage of the catheter through the vesical rent into the abdominal cavity. If, after entering the bladder and removing perhaps a few drops of urine, the catheter is suddenly felt to slip into a secondary cavity, in which its beak is freely movable, and from which a considerable quantity of sanguinolent urine escapes, it is certain that the laceration extends into the peritoneal cavity, or that the instrument has entered either a subperitoneal pouch, or, if it is situated inferiorly, has passed into an extraperitoneal circumscribed collection of urine. In some cases it was noted that warm water injected through the catheter was felt by the patient in the groin and abdomen, and the escape of the fluid ebbed and flowed with the respiratory movements. As to the significance of peritonitis as an element in diagnosis between intra- and extraperitoneal ruptures, it is nothing more than strongly suggestive of the former. The development of peritonitis in a given case does not necessarily denote a laceration of the peritonæum, as inflammation of this structure may occur in extraperitoneal ruptures either as the direct result of traumatism, independent of the injury to the bladder, or as the consequence of the propagation of inflammation from subjacent parts. When the extravasation is subperitoneal this complication is rather to be expected than otherwise. A further important sign is in the appearance of the urinary infiltration, which in extraperitoneal ruptures is apt to present as a localized or circumscribed swelling, limited perhaps to one side of the body, while in intraperitoneal ruptures the tumefaction is more general, and uniformly globular over the abdomen. The injection of an antiseptic fluid into the empty bladder and the escape of more or less of the fluid injected, is strong presumptive evidence of existence of rupture, provided no time has elapsed for adhesions to form between the seat of the lesion and its annexes. Keen suggests the employment of $I I$ gas, and noting whether it distends the cavity of the peritonæum in general, or only the bladder. In extraperitoneal ruptures the injection of fluid would increase the limit of dullness, while II gas would increase the limit of resonance on percussion.

Digital exploration of the interior of the bladder is in some cases an efficient means of diagnosis, especially when the lower part of the viscus is involved. In the male this is effected through a small medium perineal incision made in front of the prostate. In the female it is accomplished readily enough through the short urethra.

Prognosis.-This, always grave, will depend upon the situation of the rent, whether it is extra- or intraperitoneal, whether at the upper or lower portion of the viscus, upon the size and character of the laceration, and upon the quantity and extent of the urinary infiltration.

Of 315 cases collected there are reported 52 recoveries. Of these, 42 
were doubtless extraperitoneal, and were mostly treated tentatively-i. e., by catheterization, paracentesis, opening of urinary abscesses, some by perineal cystotomy, etc. ; 10 were equivocally intraperitoneal, and were treated by laparotomy. Therefore it may be said that but for this operation intraperitoneal ruptures are uniformly fatal. If left to themselves, they terminate fatally in from a few hours to within three days. Few survive the first week. In extraperitoneal lacerations the fatal result is rarely deferred beyond the second week.

Treatment.-The foregoing facts in symptomatology will tend to disturb our faith in symptoms; and well they may, if, in appreciation of their unreliability, we shall be led without delay to seek the more positive information which an abdominal incision will furnish; for we must bear in mind that upon prompt surgical intervention the success of the case will depend. If the question still exists between intra- or extraperitoneal laceration, all doubt will be dissipated by an incision through the linea alba, at first carefully avoiding the peritonæum. Thus, if the injury prove to be without the peritonæum, we have afforded a direct means for the escape of the extravasated urine. If the lesion be intraperitoneal, we have taken the initial step to a laparotomy. Two or three cases are reported of catheterization of a subperitoneal pouch through the rent in the vesical wall. This, to be successful, must be done soon after the injury-accomplished readily, without prolonged search for the opening, the extravesical cavity emptied, cleaned, and disinfected, and subsequently treated by the catheter à demeure, as presently to be set forth. In certain selected cases, notably when an extraperitoneal rupture is situated near the base of the bladder, perineal cystotomy, or kolpocystotomy, has proved to be an effectual means of affording drainage.

Abdominal section has been performed twenty-eight times-seven extra- and twenty-one intraperitoneal. Of the seven extraperitoneal, three died and four recovered. Of the fatal cases, the bladder was sutured in one. Of those that recovered, Lloyd and Rose treated their cases by the open method, with suprapubic drainage. The other two cases, of Socin-Keser and Garr, the bladder was not completely sutured, but stitched to the abdominal wall. Laparotomy has been performed twentyone times for intraperitoneal rupture. Of these, ten were successful, nine of which occurred since 18s6. Walters and Rose treated their cases by the open method; in all the other successful cases sutures were applied to the vesical rent. The operators were as follows: Walters, Phila. Med. and Surg. Journ., 1S59; MacCormac, London Lancet, December 11, 18s6, two cases; Walsham, London Med.-Chir. Trans., vol. lxxi, 18s8; Hofmokl, Wiener med. Presse, 1S86; Holmes, London Lancet, July 23, 1887 ; Blum, Arch. Gén. de Méd., 1Ss8; Schramm, Wiener med. woch., No. 33, 1890 ; Rose, Deutsche Zeitsch. f. Chir., Bd. 31, 1890 ; Mikuliez, 
given by Rose, Deutsche Zeitsch. Chir., Bd. 31, page 366, 1890. Eleven were unsuccessful. The cases of Willett, St. Barthol. Hosp. Rep., 1876 ; Heath, Med.-Chir. Trans., London, 1876; Sonnenburg, Centralbl. f. Chir., 1885; Bull. Annals of Surgery, January, New York, 1885 ; Duncan, London Lancet, August 28, 1886 ; McGill, London Lancet, November 20, 1886 ; Teale, London Lancet, June 6, 1887 ; Keyes, New York, Med. Rec., December 24, 1887; Symonds, London Lancet, May 10, 1888 ; Lloyd, London Lancet, February 6, 1892 ; Lane, London Lancet, April $16,1892$.

From a careful study of these cases we may formulate our deductions as follows :

In most of the unsuccessful cases an avoidable cause of failure is apparent. The cases of Teale and Lloyd must be altogether excluded in determining the merits of the operation. Teale did perineal urethrotomy, for the purpose of drainage, from which it is said there occurred "oozing of blood, from which the patient died." The perineal incision was apparently alone responsible for the fatal result. Lloyd's patient died before the operation was completed. Duncan and Sonnenburg did not suture the bladder, and, in the cases of Willett, Heath, Symonds, and Bull, the sutures gave way, or were, owing to some difficulty, not completely adjusted in the first place. In the patients of Willett and Heath, sutures were passed through the entire thickness of the bladder wall-an expedient now known to be a mistake. Lane attributes the death of his patient (also complicated with fracture and dislocation of the pelvis) to pneumonia, for, at the autopsy, both lungs were found to be in a condition of gray hepatization; the wound in the bladder had perfectly healed, and there was no evidence of peritonitis. "Except for the onset of pneumonia, he might have recovered." McGill sutured the intraperitoneal wound, but made an incision three quarters of an inch long on the anterior surface of the bladder, for the purposes of drainage (?). Died from shock seventeen and a half hours after the operation.

It is yet an open question whether an extraperitoneal rent should be or should not be sutured. Certain it is that the open method gives good results in suprapubic cystotomy, and equally certain it is that extraperitoneal wounds do not respond to cystorraphy as readily or as promptly as do the intraperitoneal wounds. In either case, therefore, a tube should be placed in the external wound, so that, if treated by the open method, or the sutures give way, free and uninterrupted drainage may be effected. On the other hand, an intraperitoneal wound once securely sutured, there is no danger of its giving way. Nature begins her work at once, and the quantity of plastic material thrown out in the vicinity of the injury in a few hours is remarkable.

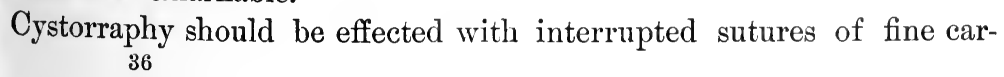


bolized silk, with the ends cut short. The stitches should be sero-muscular - that is, each suture should only involve the peritoneal and muscular coats of the bladder, care being taken to exclude the nucous membrane, the edges of which must be inverted. The sutures should be introduced at intervals of not more than a quarter of an inch apart, beginning and ending well beyond the limits of the wound, for it is here where there is most danger of leakage.

When, from the situation of the rent, the application of the sutures is attended with difficulty, the distention of the rectum by means of the air tampon will materially raise the bladder, and bring the lesion within reach. If it is still inaccessible, transverse incisions of the parietal peritonæum on each side, as recommended by MacCormac, will enable the operator to draw the bladder farther out. Before the abdominal wound is closed, the accurate coaptation of the vesical rent should always be tested by moder-

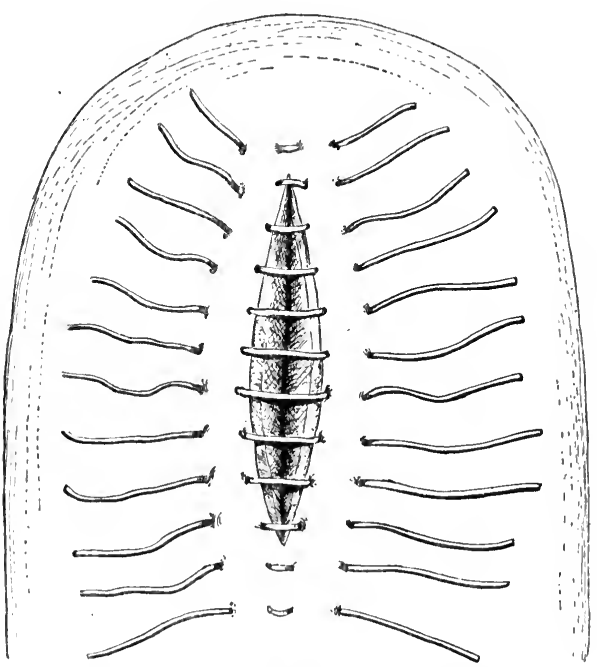

Fig. 1.-The manner of placing the sutures. (After MacCormac.) ately distending the bladder with water, and, if there is any escape between the sutures, an additional stitch applied. So firm are the adhesions between the coaptated surfaces that the catheter à demeure has been dispensed with in some cases in a few hours after the operation, and the patient directed to micturate by his unaided efforts every two or three hours. Of course, if any impediment exists to the outflow of urine, and it is not feasible to remove the catheter at once, it then becomes an important factor in the treatment, and I believe in such cases the catheter $\grave{a}$ demeure is preferable to the periodical introduction of an instrument; care must, of course, be exercised not to disturb the reparative process already begun. A soft (Nélaton), velvet-eyed catheter open at the extreme end, and secured so that the eye reaches just within the vesical orifice and no farther; to the catheter is attached a rubber tube of sufficient length to reach into a bottle on the floor; the bottle and the tube are filled with carbolized water before the apparatus is attached; thus a vacuum is created in the tube and catheter, and a gentle but continuous aspiration is established, with a suction force depending upon the height of the column of water. The height 
of the ordinary hospital bed is sufficient. The bottle is allowed to overflow into a basin, which may be emptied as it is filled without displacing the apparatus.

The clinical and experimental evidence is that peritonitis does not always and at once ensue upon the escape of healthy urine in the peritoneal cavity. This was apparent in the patient of Schramm, with a laceration of $2 \frac{1}{2} \mathrm{~cm}$. Fifty hours elapsed from the time of the injury to the time of the operation; and, what is even more significant, the peritoneal cavity was not washed out, but the urine simply soaked up with sponges - the patient's low condition necessitating a rapid completion of the operation. This patient was discharged cured in ten days. In the patient of Lane, too, the blood and urine in the pelvis was sponged out, but no attempt was made to wash out the peritoneal cavity; yet there was no evidence of peritonitis. That MacCormac, Thomas, Vincent, Esmarch, Billroth, Juillard, Atlee, Homans, Possi, Sonnenburg, and others mention cases of recovery after intraperitoneal injury of the bladder occurring chiefly during ovariotomy, and Schramm in intraperitoneal eystotomy for stone, is suggestive of the fact that the danger lies not in the vesical lesion, but in the presence within the peritoneal cavity of a decomposable and septic fluid; and when this is absent (for they injured the bladder when empty), or when removed shortly after such extravasation has occurred, we may expect a successful termination of an otherwise uniformly fatal lesion. The disposition of intraperitoneal wounds of the bladder to union is so great, that it may be regarded as certain if the edges of the same are accurately adjusted. Therefore, as a rule, the toilet and disinfection of the peritonæum are of primary importance; this should be made with great care, but as expeditiously as possible; the cavity of the peritonæum washed out with a one-per-cent solution of boracic acid, warmed to blood-heat, the irrigation being kept up until the fluid returning is perfectly clear. All blood-clots and foreign material must, of course, be removed, but this should be done with as little sponging as possible. In the absence of signs of peritonitis; the drainage-tube may be dispensed with. When peritonitis is undoubtedly present, the insertion of a drainage-tube may be advisable; but we must hear in mind that if any fluid exists, it is diffused over the entire abdominal cavity by the movements of the intestines and the suction action of the diaphragm, and never accumulates at one point, unless encapsuled by peritoneal agglutinations.

When, in consequence of the uature, position, and extent of the wound, its successful closure by the ordinary method is doubtful, cystoabdominorraphy may be practiced - that is, the vesical and the abdominal wounds stitched together. This may interfere with the functional activity of the viscus, endangering the occurrence of cystitis, etc. Indeed, I ob- 
served some years ago, in experimenting on a number of dogs, that in each case the bladder had become considerably hypertrophied in a few days, no doubt in consequence of its abnormal attachments, and therefore cysto-abdominorraphy must be a dernier ressort. But, as such, it must be kept in mind, for, in the few cases that it has recently been practiced on man, it commends itself for repetition. 


\section{TUMORS OF THE BLADDER.}

By FRANCIS SEDGEWICK WATSON, M.D.

Pathology and Etiology of Bladder Tumors.-Beyond the influence of the conditions which are stated below, we have practically no knowledge of the causation of bladder tumors. The influence of age, sex, and the liability of certain portions of the bladder to be affected by preference, will be seen in the following :

Age, with Reference to the Occurrence of Bladder Tumors.-With the exception of myxoma, which is more often encountered in children, all forms of bladder tumors are more frequent after middle life. Of eightynine cases of papilloma collected by the author, the age of occurrence is as follows :

Papilloma (eighty-nine cases) :

Males, between 10 and 20 years of age, 1 case.

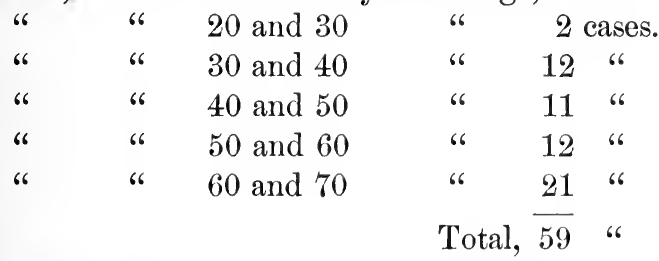

Females, between 1 and 2 years of age, 1 case.

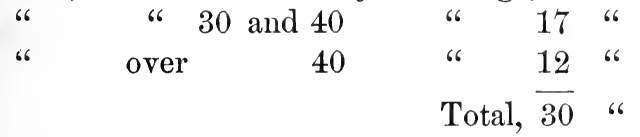

Cancer.-In over 100 cases of cancer collected by the author, the youngest patient affected was 30 years of age.

There were 12 cases between 40 and 50 years of age, and the rest were over 50 years old.

Sarcoma.-Of 20 cases reported by Hinterstoiser in which the age is mentioned, the youngest patient was under two years.

Under $20 \quad$ years of age, 5 cases.

$\begin{array}{crrrr}\text { Between } & 20 \text { and } 30 & \text { " } & 3 & \text { “ } \\ \text { “ } & 40 \text { and } 50 & \text { " } & 2 & \text { “ } \\ \text { " } & 50 \text { and } 60 & \text { " } & 6 & \text { “ } \\ \text { Over } & 60 & \text { " } & 4 & \text { “ } \\ & & & \text { Total, } 20 & \text { “ }\end{array}$


Myoma.-In four cases of myoma, of which two are reported by Guyon, two were in young men, one in a patient aged 30 , and another in an old man.

Sex.-All computations agree in declaring the greater liability of the male than of the female to the formation of bladder tumors. Pousson, in the April (1889) number of the Jour. Des. Mal. Des. Org. Gen. Ur., reports 141 cases. Of these, 83 were in men, 58 in women. In the Lancet, March 10, 1888, Fenwick states that, in a series of 634 cases of all sorts of bladder tumors, men were found to be affected thrice as often as women. Of 201 cases tabulated by Conner, 133 were in men.

The following figures show the relative frequency of occurrence in the two sexes, with reference to special classes of tumors:

Papilloma.-Of 99 cases of papilloma, 66 occurred in men.

Cancer:-Guyon quotes Feré as stating that, of 138 cases of this disease, 110 occurred in men. Of 35 cases in Conner's tables, 28 were in men.

Sarcoma.-This growth is generally asserted to be more frequent in the female, but the following does not bear out this statement fully: Thus, of 17 of Hinterstoiser's cases, 13 occurred in men. Of 11 cases noted by Conner, 6 were in men. Of 5 eases reported by Küster, all were women; and of 11 cases reported by Sperling, 8 were in women.

Situation of Bladder Tumors.-Fenwick (op. cit.) states that, of 60 cases of papilloma, 60 per cent were single, and that of these 43 per cent were growing about the right ureteral orifice, and 86 per cent of the single growths occupied the lower zone of the bladder. These observations agree with those of other authors in the main, and it is generally conceded that the lower third of the bladder is much more frequently occupied by bladder growths of all sorts than the upper two thirds. Beyond these facts the etiology of bladder tumors is exceedingly obscure.

General Pathological Considerations.-A division of bladder tumors according to their structure, which is convenient and simple, is as follows :

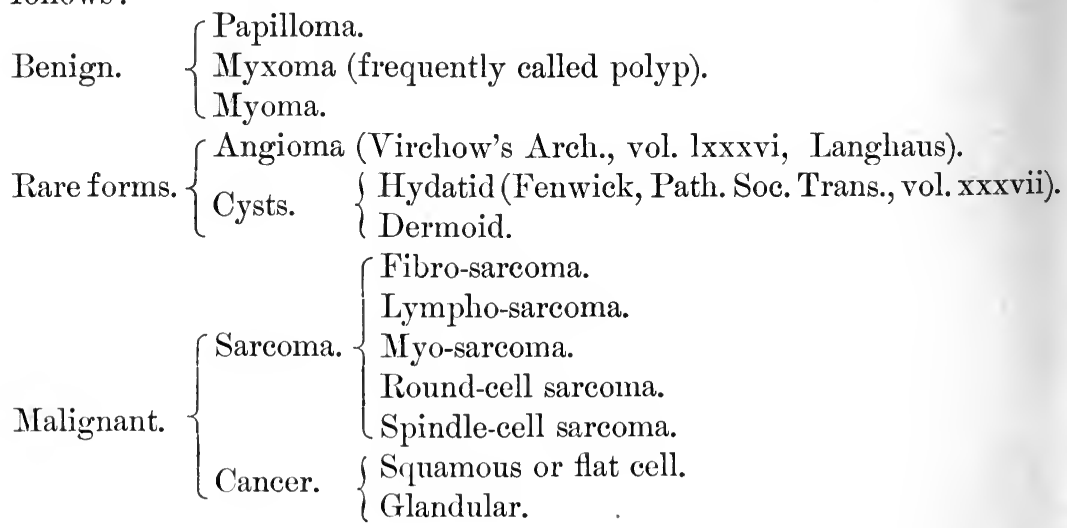


The various terms, encephaloid and schirrous, etc., as descriptive of gross appearances, may be retained if so understood. The terms epithelioma and alveolar cancer may be better superseded by squamous cell and glandular, though not absolutely corresponding to them.

The relative frequency of benign and malignant growths:

Of 100 cases, 60 were benign (Sir Henry Thompson).

$\begin{array}{llrll}\text { Of } 208 & \text { " } & 106 & \text { “ } & \text { (Clado). } \\ \text { Of } 205 & \text { “ } & 138 & \text { “ } & \text { (Pousson). } \\ \text { Of } 107 & \text { “ } & 81 & \text { “ } & \text { (Conner). }\end{array}$

It is generally agreed that of benign growths papilloma occurs most frequently. Next in frequency is myxoma. Of malignant growths, sarcoma was formerly considered to be a very rare form, but of recent years the number of well-authenticated cases has increased. (See Küster, Volkmann) (Hinterstoiser, Wiener Klinische Wochenschrift, 1890, iii, 304).

\section{PAPILLOMA.}

Any tumor of the bladder may have a papillomatous (villous) surface, but this must not be confounded with the true papilloma. Papilloma may be single or multiple, pedunculated or sessile. Fenwick (op. cit.) states that " of 60 cases of papilloma, 60 per cent were single and 40 per cent multiple, and of the 60 cases two out of three were more or less

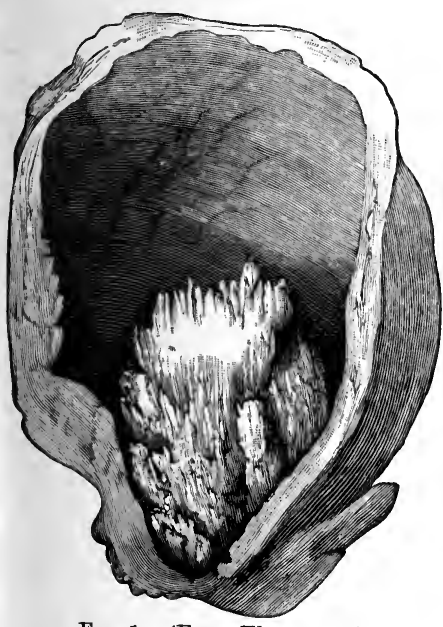

FIG. 1.-(From Thompson.)

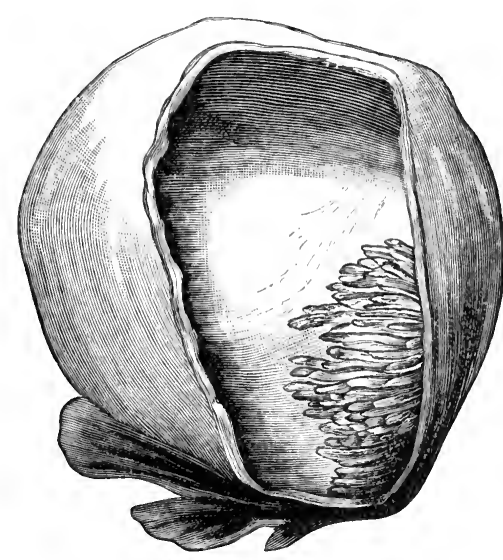

FiG. 2.-(From Thompson.)

pedunculated; also notes that cancer has been found to coexist in the bladder with benign papilloma."

A frequent form of papilloma is that in which there is a well-defined pedicle, from the summit of which branch out a number of papillæ bearing heads-a typical cauliflower-growth (see Fig. 1). In contrast to this 
are the rarer cases in which the papillæ spring separately or in small groups without pedicles directly from the mucous membrane (see Fig. 2). In the latter the papillæ are sometimes very delicate, and float out in fluids like fine threads. In other cases (sessile growths) the papillæ are short and thick, lying close to the surface in clumps. (Plate V.)

The pedicle, when present, varies in length and thickness and structure. It may be as long as an inch or more. It varies in size from that

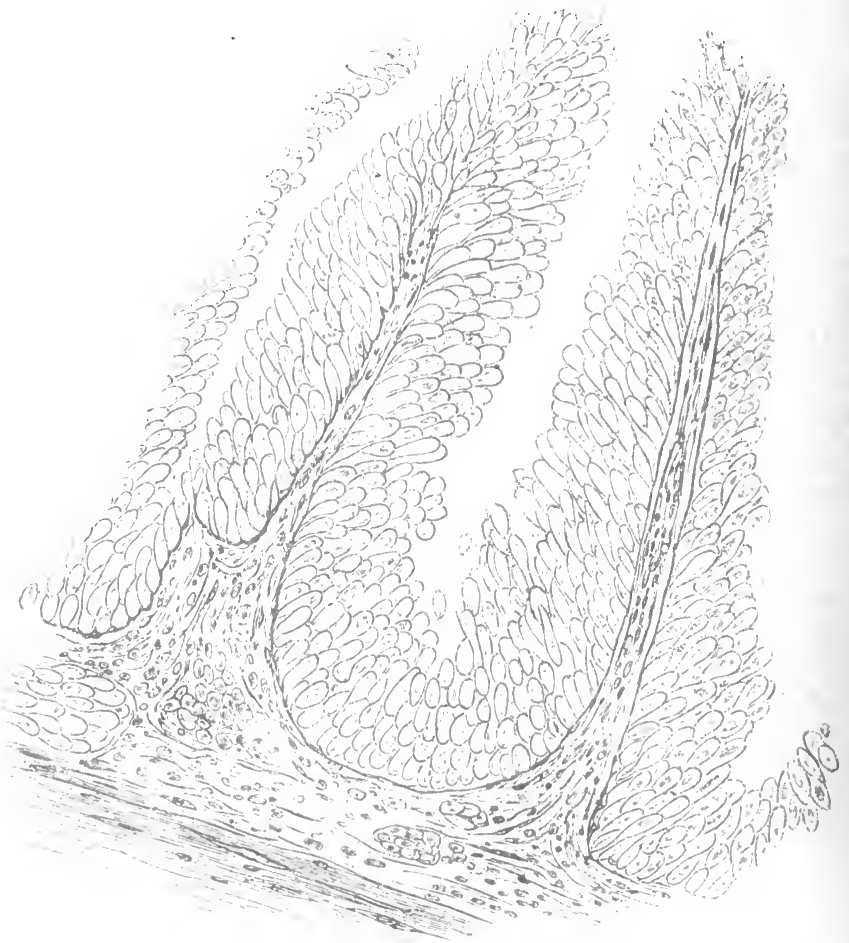

Fig. 3.-Fimbriated papilloma, showing two of the slender papillomatous processes, or so-called "villi." (From Thompson.)

of a knitting-needle to that of the thumb or larger. It is more or less dense, according to its proportion of connective tissue. In color, a fresh growth generally resembles that of the inner surface of the lip. The structure of the papillæ is as follows: Their base is made up of unstriped muscular fiber and connective tissue. From this spring the individual papillæ. Each of the latter consists of a delicate framework of connective tissue, through the center of which run loops of capillary bloodvessels with very delicate walls, lined with endothelium bearing endo- 


\section{PLATE V.}

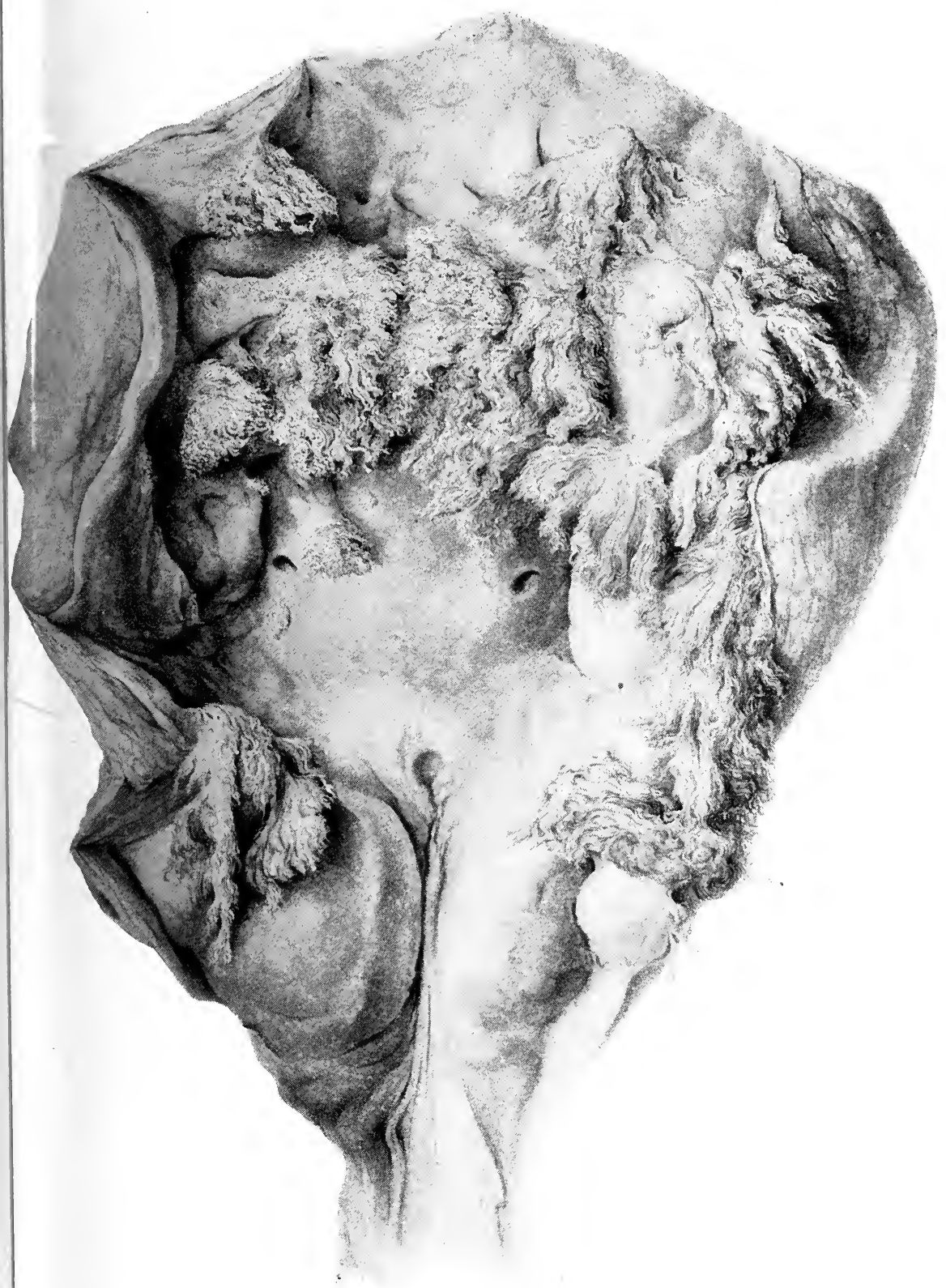

Multiple Villous Papilloma.

Histologically : True benign papilloma (Albarran). 

thelia with large nuclei. Externally each papilla is covered with one or more layers of epithelium of columnar form with large nuclei; these cells are of the same type as the cells of the deeper layers of the normal mucous membrane of the bladder.

The papillæ may be long and delicate, and constitute the chief part of the growth, or the connective tissue may preponderate. To those specimens in which the proportion of connective tissue is very large, Sir Henry Thompson has given the name of fibro-papilloma.

The same author also speaks of a special form of growth which he considers as a papilloma undergoing change into a malignant growth

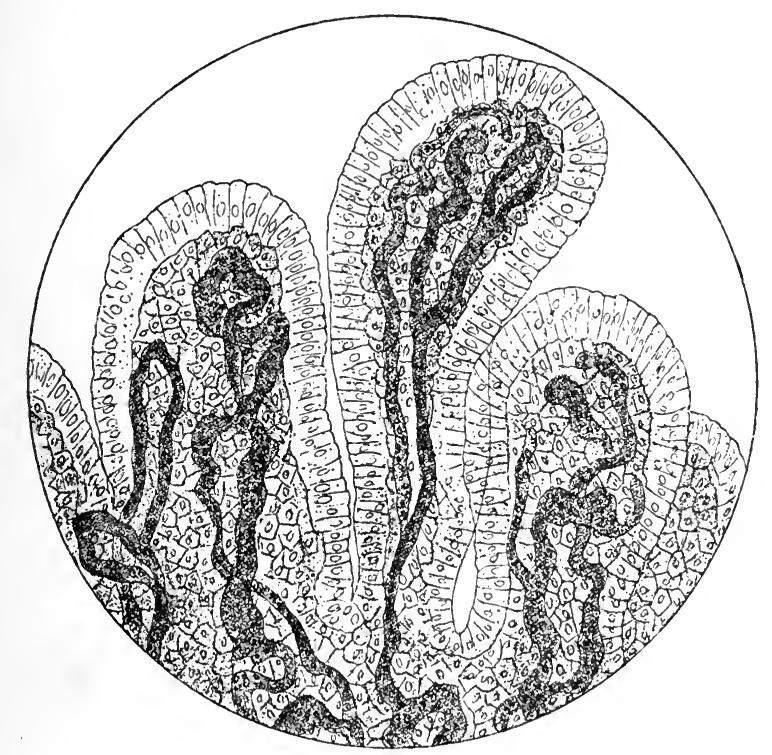

Fig. 4.-(From Ultzmann.)

(sarcoma). Of this, he quotes the description of Dr. Heneage Gibbs, who says: "These tumors are characterized by a dense, fibrous groundwork of very irregular growth, and by the presence in this groundwork of variously shaped cells, generally arranged in different groups. In some, there are small, round cells; in others, large, irregular cells. The surface of these growths is covered with columnar epithelium, resembling that of the normal bladder. The one feature which differentiates them from the ordinary papilloma or the fibro-papilloma is the arrangement of the ground substance, and the presence in it of the irregularly shaped cells, which do not belong to the normal tissue on the one hand, or to that of distinct new growth on the other (see Fig. 5). 
"To such growths" Sir Henry Thompson gives the name of "intermediate transition varieties."

The possibility of such a change from benign to malignant growth is also asserted by Reginald Harrison, who, in support of this theory, cites a case in which a growth, originally benign, became, after repeated removals, malignant at the end of ten years.

Guyon, on the other hand, combats this idea.

The special characteristic of the true papilloma is that it never invades the submucous tissues. In the form, however, called fibro-papilloma by

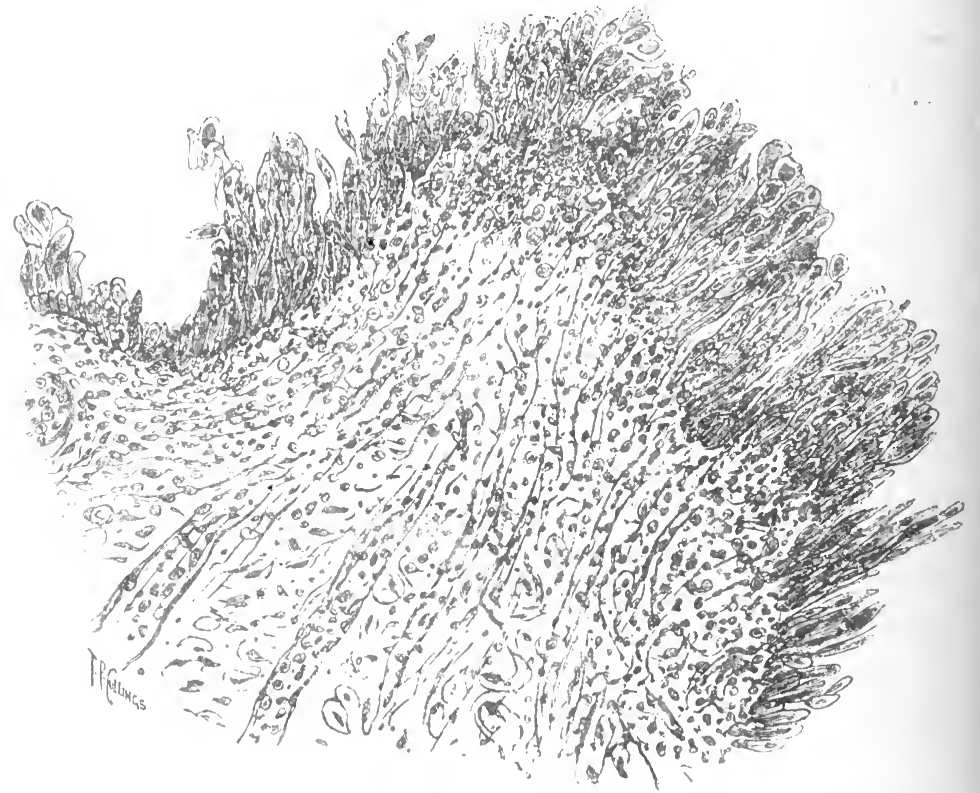

Fig. 5.-Example of the "transitional" type of tumor: showing the ground-substance infiltrated with variously-shaped eells; and numerous blood-vessels running to the surface: one has given way, and effused blood is well shown at the highest point. (From Thompson.)

Sir Henry Thompson, he says the base is made up of unstriped muscular fiber and connective tissue from the submucous tissue of the vesical coat.

\section{MYXOMA (Polyp).}

The structure of this growth is reported in three instances, by Butlin, Beck, and Shattock, as follows: In one "the growth is composed almost entirely of small, round cells of a lymphoid type, imbedded in a base, homogenous or nearly so on the surface, but becoming more and more fibrous toward the pedicle, until, at the lowest part, fibrous tissue forms the bulk of the growth." Shattock's specimen is described as displaying 


$$
\text { - }
$$


PLATE VI.

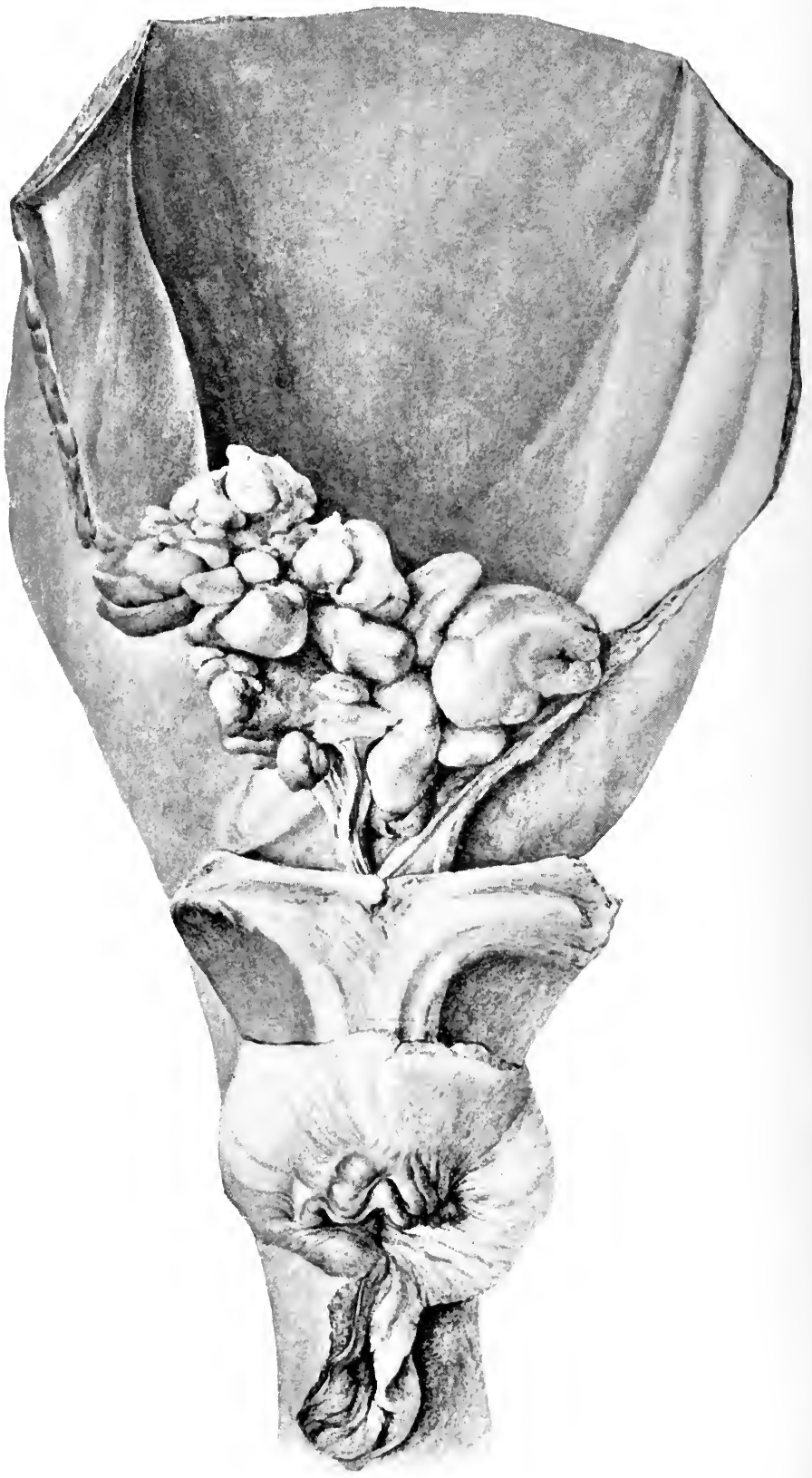

Mucous Polypi from a Young Gird.

Histologically: Myxo-sareoma (Albarran). 
oval and more elongated cells lying in an abundant substance, either albuminous or mucous. The myxoma is a single growth, pedunculated, and resembles in its gross appearance nasal polypus. It sometimes has a gelatinous texture, sometimes is dense, owing to a greater proportion of

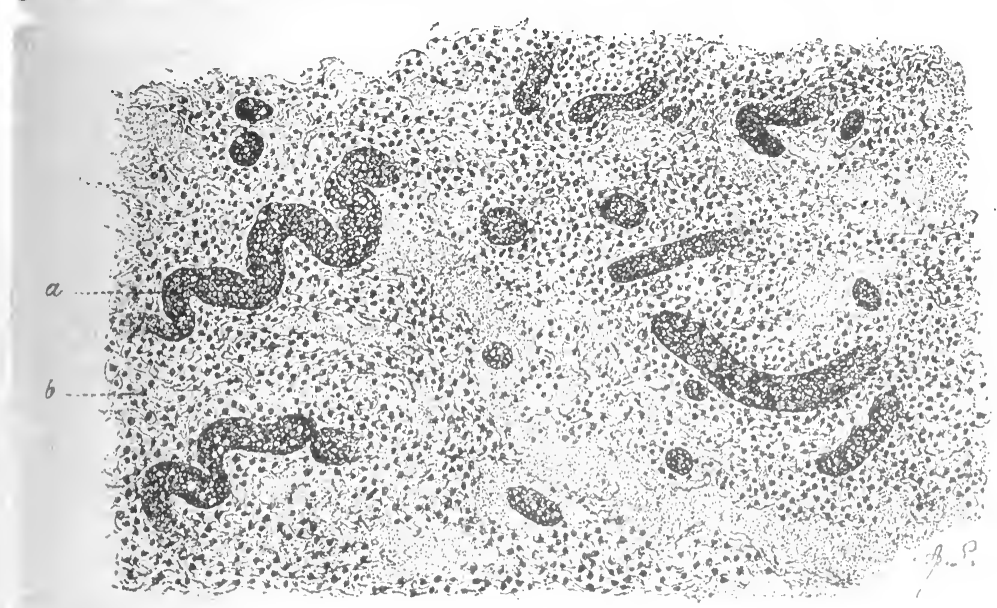

Fia. 6.-(After Albarran.)

connective tissue, in which case it approaches more nearly a true fibroma. Myxoma is essentially a fibroma, which is undergoing or has undergone a mucoid transformation. (Plate VI.)

These growths occur most frequently in childhood, and it is reasonable to believe that in some instances they are congenital. In one or two cases they have been recognized by the fact of their being spontaneously protruded from the female urethra.

\section{MYOMA.}

This growth is single generally, and shows but a moderate tendency to pedicle formation. Its chief constituent is organic, muscular fiber, intermixed with connective tissue, and in size it has been noted as large as a Tangerine orange.

\section{CYSTS.}

Serous, hydatid, and dermoid cysts of the bladder have in a few instances been recorded.

\section{CARCINOMA.}

The simple and excellent account of these growths given by Reginald Harrison, in his Surgical Disorders, third edition, 1857, page 555, will suffice for their description : 
"The epithelial lining of the bladder is composed of a single layer of large-sized, squamous cells, with interlying pear-shaped and round-cell layers. It is also provided with racemose mucous glands, most numerous near the neck of the bladder. It will be seen that we may thus expect to meet two varieties of cancer of the bladder: $a$, the squamous, or flatcell carcinoma ; $b$, the glandular-cell carcinoma. Speaking clinically, the former would be termed encephaloid cancer; while the latter might be encephaloid or schirrous; in other words, hard or soft. These clinical terms give no information relative to the intimate structure of the tumor, but indicate only its rapidity of growth and probable degree of malignancy.

"The vesicle glands might be liable to benign adenoma as well as to malignant carcinoma. So far as I know, only one case of the former is recorded, viz., a case of a papillary growth, removed by Kaltenbach (Langenbeck's Arch., xxx, 1884) from the bladder of a woman aged 44.

"The stroma of a carcinomatous growth, composed of the tissue of the submucous and muscular layers, with more or less round-cell infiltra-

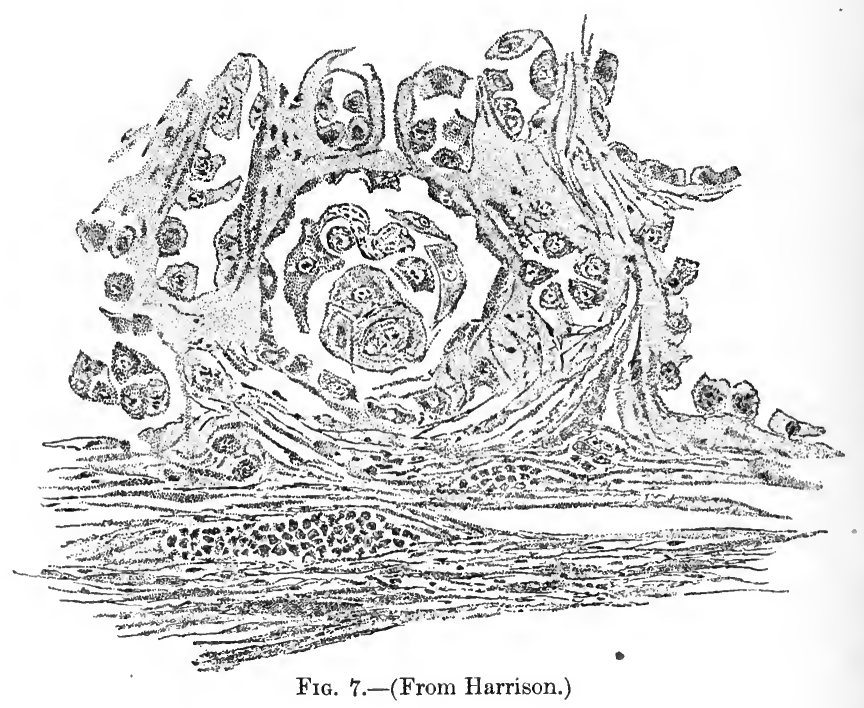

tion, is full of alveoli, well packed with epithelial cells, following with more or less accuracy the types of the bladder epithelium. The formation of ' cell-nests' varies much in different specimens, and often in different parts of the same specimen (Fig. 7). Colloid degeneration may occur in primary cancer of the bladder, but is by no means common."

The special characteristic of carcinoma, here as elsewhere, is its tendency to invade all contiguous tissues of whatever nature; but the tend- 


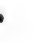


PLATE VII.

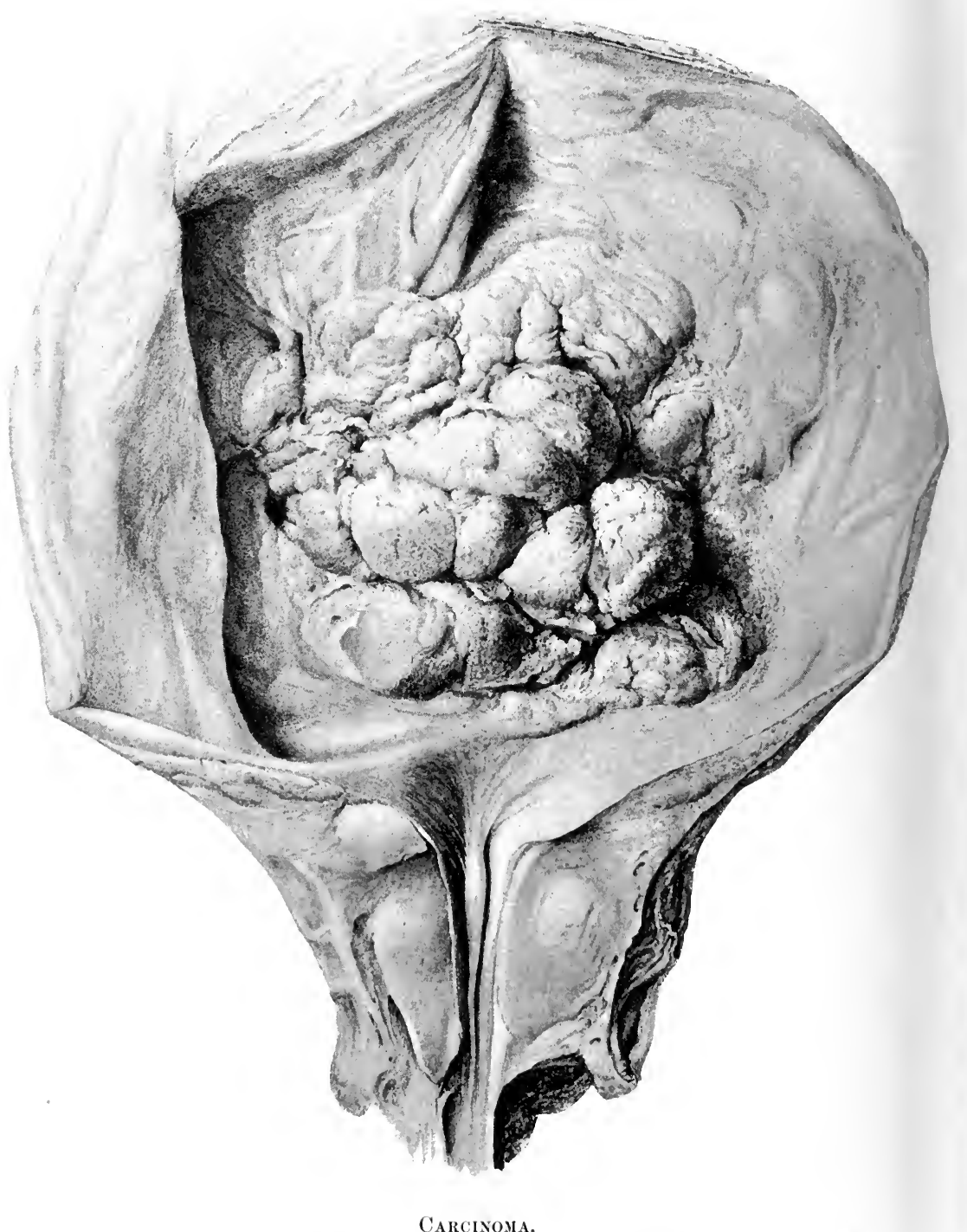

Histologically : Epithelial cancer (Albarran). 
ncy to remain local in the bladder for a long period of time is a notevorthy feature. Where direct extension does occur, perforation of the ntestine, the involvement of the peritonæum, etc., may follow. The first ase in the author's experience was an example of the immense destrucion by gradual extension that may take place. In this patient, a man of 36 years of age, the first symptoms of the disease dated back six years. Tn his death, almost the entire bladder was found to be involved by can(3erous growth. The inner surface was covered with papillæ. From the right side of the bladder the disease had extended outward, attacking all the tissues between this point and the right acetabulum, which it had destroyed, allowing the head and neck of the femur to enter the pelvis as far as the great trochanter. The head of the femur about the round ligament was also eroded by the disease.

The surface of the cancerous growth within the bladder may be smooth, the mucous covering seemingly intact, or more or less ulcerated. In form these growths are either flat, with ragged, raised, indurated edges, or sprouting more or less into the bladder cavity, which they may entirely fill. In the latter form they are often pedunculated to a greater or less degree. The surface is not infrequently covered by a papillary growth which resembles in structure exactly that of the benign papilloma. Cancerous growths are sometimes single, and limited to one portion of the bladder; sometimes they exist in disseminated patches. They originate in a majority of instances, as with other growths, in the lower half or third of the organ. (Plate VII.)

Barling (Birmingham Med. Rev., March, 1890) states that in only 3 out of 15 cases of carcinoma of the bladder did extension occur to neighboring organs; and in the same series of cases secondary deposits were found in other organs in 9 . Of 49 cases 33 had, as secondary changes, hydro- or pyo-nephrosis, or both.

Clado states that there exists in some cases of carcinoma vesicæ, at a certain period of their development, an adipose layer between the cancerous base and the sound tissue beneath. This Guyon observed while operating in one instance. In the specimens examined by Barling, with reference to this point, he failed to confirm this observation.

The remarkable tendency of carcinoma of the bladder to remain localized for very long periods has been commented on by various writers, and is generally explained by the absence, according to some investigators, or by the meagerness, according to others, of the supply of lymphatic channels in the bladder. Barling (op. cit.) quotes Hoggan with reference to this matter as follows: "There is but one set of lymphatic vessels over the bladder except at the trigone. This single layer commences on the deep or mucous surface of the muscular coat, beginning as loops or chains around the smaller arteries or veins within that coat, as though 
destined to absorb their exudations rather than those of the mucous membrane. These lymphatics concentrate into two main sets, one going back to the neck of the bladder, the other toward the urachus." 'These are said by the text-books to empty into the internal iliac glands, but Hoggan says this is incorrect. At the trigone there is a second set of purely collecting lymphatics arising in the mucous membrane and connecting with the deeper set. This lack of direct connection of the larger portion of the lymphatic channels with the mucous membrane explains the failure of the neighboring glands to become involved in some cases, or of their tardy infection in others. This also explains the extraordinarily long course of the disease that has been noted.

Symptoms and Diagnosis.-The most characteristic and constant symptom of bladder tumors is hæmaturia in a large majority of cases. Exceptionally it is slight, and may be absent during the entire course of the disease, as has been noted by Stein, Guyon, and others. Its absence has been more frequently noticed in cases of cancer, less frequently in cases of papilloma. Hæmorrhage and its consequences may be the only symptoms of importance throughout the disease. As a rule, in benign growths hæmorrhage precedes the occurrence of pain. In malignant disease pain or bladder irritability is apt to precede or accompany hæmorrhage. There have been numerous cases reported, however, in which this was not true, and occasionally in malignant disease pain is absent throughout. Reginald Harrison attributes much diagnostic importance to the relief of pain, when it has been present, which follows an attack of bleeding, especially in malignant disease. This symptom he considers as almost pathognomonic of the affection.

Special Characteristics of Hæmorrhage from Bladder Growths.-In general the most distinctive characteristics of hæmaturia from bladder tumors are :

1. Its occurrence wholly independent of any exciting cause, such as exercise. It is not infrequent to see some of the most severe attacks take place during sleep.

2. Its abundance, with the few rare exceptions of cases of hæmorrhage from an ulcerated surface of the hypertrophied prostate, of stone in the bladder, of renal calculus, and of malignant disease of the kidney-the bleeding from bladder tumors is far more abundant than that arising from any other disease in the genito-urinary tract. (One case of gonorrhœal cystitis associated with abundant hæmorrhage has been described by Guyon.) Sometimes the bladder becomes filled with clots, urinary retention and vesical tenesmus resulting.

3. The progressively increasing frequency and abundance of the hæmorrhage as the disease advances.

Occasionally the hæmaturia is constant after the first attack during 
the entire progress of the malady, the blood flowing in small quantity, perhaps, yet never wholly absent, but, as a rule, intermittence of the hæmorrhage is observed. The length of the periods of remission varies greatly. An interval of seven years has been noted between the first and second attack of bleeding, and in the early stages of the disease many months often intervene. There have been a few cases reported in which the interesting observation of the occurrence of but one or two hæmorrhages has been made, with no recurrence during lifetime. These have been taken as indicating the possibility in rare instances of spontaneous cure either by atrophy or exfoliation, but the evidence is of doubtful value, owing to the lack of positive proof of the existence of the bladder growth.

When blood is present in microscopic quantities, the microscopic appearance of the red globules was formerly thought to furnish evidence as to the origin of the bleeding. It was asserted that, when such bleeding took place from the kidneys, the corpuscles, owing to their longer retention in the urinary tract, took on a spherical form by imbibition, whereas when from the bladder they preserved the biscuit-shaped contour of fresh blood-corpuscles. The fallacy of this latter statement was demonstrated to the author some years ago by a case in which, during repeated examinations of the urine, red blood-corpuscles were invariably found, and always presenting spheroidal form. The origin of the bleeding in that case was thought to be renal, but the post-mortem revealed two tumors of the bladder situated around the orifices of the ureters.

The color of the blood when the hæmorrhage is abundant is generally bright, but may be dark, or, if in clots, dull red. Should any obstructive disease of the prostate or urethra have caused retention in the bladder, sometimes the blood may not be bright. It is, however, decidedly rare to see the light, smoky, or greenish-brown color evenly disseminated through the whole urine that is noticed in connection with some cases of renal hæmorrhage. Evidence of the renal origin of the blood is sometimes furnished (when the bleeding has been sufficient to form clots in the pelvis of the kidney), by the occurrence of attacks of renal colic accompanying the hæmaturia. Also, of course, renal calculus as a cause of the hæmorrhage is to be suspected when there has been a well-marked history of attacks of renal colic. The presence, in addition, of renal casts and crystals of urid acid, the urates or oxalate of lime, makes the probability very great that one has to do with a case of renal calculus.

The presenee of renal casts alone in connection with hæmaturia need not mean that the bleeding originates in the kidney; for, as has already been pointed out, many bladder tumors grow about the ureteral orifices, and may cause hydro- and pyo-nephrosis. 
Guyon describes a manœuvre for the purpose of ascertaining the presence of a renal tumor in cases in which there is doubt as to the origin of the hæmaturia, which he calls renal ballottement. The palm of one hand is laid upon the abdomen above the kidney, while that of the other is placed over the kidney in the back. A quick pressure is then made by the hand on the back, when, he says, the tumor has in several cases been driven against the abdominal wall, giving to the hand resting there a sensation similar to that felt in ballottement of the foetus.

Instrumental manipulation within the bladder is liable to produce hæmorrhage, or increase it if present, in cases of bladder growth, and considerable diagnostic importance is attached to this sign by Reginald Harrison, Coulson, and various writers. It is well to remember that there have been one or two instances of fatal hæmorrhage caused by such instrumental manipulation.

Pain and Disturbances of Micturition.-These symptoms, as a rule, in cases of benign growths do not precede the occurrence of hæmorrhage, unless in a very slight degree. In malignant growths, on the contrary, most writers agree that pain precedes or accompanies the first appearance of blood. If a growth be situated near the vesical orifice, it sometimes announces its presence first by interfering with the act of micturition. This was noticed by the author in a case in which a papilloma with a moderate pedicle was situated about the upper margin of the vesical orifice, and first attracted the patient's notice by occasionally interrupting the urinary stream, and in giving rise to a slight twinge of pain at the same time. With malignant growths pain, as a rule, sooner or later, becomes a prominent symptom, and is frequently of a radiating character, felt in the sciatic region, along the front of the thighs, over the symphysis pubis, or about the sacrum and perinæum. Exceptionally pain may be entirely absent throughout the progress of a malignant growth. The pain referred to above is intended to indicate that which is due to the presence of the growth per se. Any growth, benign or malignant, may of course have pain associated with it when a cystitis, which is so apt to occur at some period of its course, supervenes, or when hæmorrhage has been so excessive as to cause retention of urine and vesical tenesmus.

Unlike the pain caused by stone in the bladder, or the bladder irritability sometimes noticed in connection with renal calculus, that arising from bladder tumors is generally not increased by exercise. In malignant growths pain is not necessarily associated with ulceration. Guyon mentions a case in which pain was absent while this condition existed. Frequency of micturition, unlike that arising from prostatic hypertrophy, is not characterized by being more noticeable at night. 
Evidence furnished by the Urine.-The most characteristic and conclusive evidence of the existence of a bladder growth furnished by urinary examination is the presence in the urine of bits of the surface of the growth which have become detached and are passed out in the urinary stream. These, when large, are often noticed by the patient. If not decomposed, they resemble fleshy lumps, varying in size from a pin's head to that of a pea, and look as a bit of the gum would, if detached from the mouth. Sometimes to the naked eye they present the characteristic appearance of finger-like projections floating in the urine. In many instances, however, these fragments are not to be found.

Inasmuch, as has been mentioned already, any tumor, benign or malignant, may have a papillary surface of essentially the same structure in either case, no inference as to the nature of the growth can be drawn from the microscopic examination of such fragments. They are only proof positive of the presence of some form of bladder growth. Such fragments are more commonly thrown off from papillomata and cancerous growths, so that when found they show a probability of the presence of one or the other of these varieties. Hæmorrhage as a rule is associated with the casting off of these fragments. Occasionally the reverse is true, as in the case of cancer reported by Landers, and referred to by Guyon, in which fragments were frequently seen, although hæmorrhage was entirely absent throughout the course of the disease. Besides actual fragments of the growth, the urine very often contains a more or less abundant sediment, in which are found large numbers of epithelial cells of great variety of shapes with large nuclei. Although no forms have been observed which are absolutely different from those of the epithelial cells of the normal bladder in its various layers, and while, therefore, theoretically there is no reason why they should not be also found in any disease in which desquamative or deep-seated ulcerative processes are present, nevertheless, the author has failed to find them in such quantities and so repeatedly in the urinary examinations of any other conditions as in those of bladder tumors, and therefore considers that they are of distinct diagnostic value. In this opinion he is confirmed by Prof. E. S. Wood, of Harvard Medical School.

As has already been pointed out, secondary changes in the kidney or its pelvis are liable to follow upon the presence of a bladder tumor, especially if it be seated at the ureteral orifice. In this case casts and renal epithelium are likely also to be found in the urine.

Physical Examination. Rectal Touch.-It is rare to be able to detect a benign growth, unless it be very large and dense, by rectal touch. Cancerous growths, on the contrary, can usually, except in their early stages, if situated within reach of the finger in the rectum be felt as areas of induration, and by giving a sense of increased resistance. 
Any considerable hypertrophy of the prostate will make a rectal examination unavailing. The rectal examination should be made with the bladder nearly or quite empty; for if full, a growth not situated near the vesical neck and upon the floor of the bladder will be carried beyond the reach of the finger.

In individuals with thin abdomens who have a large and dense tumor of the bladder, bimanual examination with one finger in the rectum and those of the other hand pressing deeply in above the symphysis pubis, may assist in the detection of the growth. Otherwise, it is not of much assistance.

Instrumental Exploration of the Bladder.-The failure to detect a bladder tumor by exploration with the searcher or sound is no evidence of its absence, for, unless the tumor be prominent and dense and resistant, no characteristic sensation is given to the fingers holding the instrument; rugæe or projecting portions of a hypertrophied prostate may lead to a false inference that a tumor is present. In cases, which are not very infrequent, in which the surface of a tumor is covered with a deposit of phosphatic salts, the error is likely to be made of mistaking the condition for a stone. Such instrumental examination, therefore, should be discarded because of the far more accurate means at our disposal.

Some writers have advised that an attempt should be made, with an instrument of catheter form, furnished with a large eye, having a moderately sharp edge, to detach fragments of the growth in suspected cases by active movements of the instrument within the bladder. This practice the author considers most injudicious, for the reason of its liability to produce hæmorrhage, or to give rise to a cystitis, to which condition such cases are peculiarly liable upon interference, and also because it is rendered unnecessary by the cystoscope.

The Cystoscope.-Among the various writings upon the cystoscope and its uses, a most satisfactory exposition of the subject is in the admirable work of Fenwick (on the Electric Illumination of the Bladder and Urethra, as a means of diagnosis of obscure vesical urethral diseases. A. Churchill, 11 New Burlington Street, London, second edition, 1889). What follows is taken almost wholly from this author. It is out of place in this article to enter into the history of the gradual development of the cystoscope, from its original conception by Bozzini, of Frankfort, in 1805 , to a practical instrument constructed by Desormeanx, in 1853 , and down to the instruments in their more approved forms now in use. Without entering into a discussion of the claims of authorship of the modern instruments over which some dispute has arisen, it is fair to say that Dr. Max Nitze should be credited with being the practical inventor and introducer of the modern instrument; that to the instrument-maker, Leiter, of Vienna, also belongs credit for having produced a technically admirable instrument following Nitze's ideas. 
The instruments best suited to their purpose that are in use to-day are the Nitze "Incandescent-lamp Cystoscope," the Leiter "Incandescent-lamp Cystoscope," Fenwick's Cystoscope with a perforated hood to keep the lamp cool, and Whitehead's Cystoscope for use through a perineal boutonnière. (A detailed description of the instruments is given elsewhere.)

Schall, of Wigmore Street, London, has a battery for cystoscopic illumination, which has given entire satisfaction to the author ; it has the practical advantages of a dry-cell battery, and in the use of it during one year it has proved wholly reliable. It is a six-cell battery, the cells intercommunicating by silver wires, and also connecting with the cystoscopic wires. There is no lever, the plates being sunk constantly in the fluid of the cells. The intensity of the current is regulated by a rheostat, which is cleverly arranged so as to form a part of the handle by which the battery is lifted. The cells are sealed with a metal cover, which is pierced by a pin-hole opening only, and so the one great inconvenience of the fluid-cell batteries, such as that of Leiter, for example, is done away with. The battery measures only about eight inches by five, and is light and easily carried.

Objections to the Use of the Cystoscope.-Two objections might reasonably be made to the use of the incandescent-lamp cystoscope :

1. Burning the Mucous Membrane.-Although the heat emitted by the cap or hood, with its contained incandescent lamp, when in action, is not so great as that given off by the platinum-wire lamp, yet it does become very quickly hot if it is allowed to burn in air instead of under water. Thus, it becomes warm after four seconds, unbearable after ten, and scorches the skin severely after fifteen seconds. If, however, the tip of the instrument is placed in water, the heat is rapidly absorbed, and the cap or hood remains cool, and may be touched with impunity, even after an hour's use. This is exactly what happens in the bladder, for the urine carries off the heat of the lamp as fast as it is formed. "They may be burned for an hour in a male bladder, holding seven ounces of fluid, without perceptibly raising the general temperature" (Brenner). It is therefore a sine qua non in electric cystoscopy that the bladder shall contain five or six ounces at least of urine or water. Now, although the urine or water absorbs the heat of the cap, if it be freely surrounded by the medium, yet this does not happen if the cap be held in contact with the vesical wall.

The perforated hood, which is adapted to my own (Fenwick's) cystoscope, does away with much of the danger of burning the mucous membrane, for the water circulates through the small holes, and is in direct contact with the lamp.

2. Another objection is the breakage of the lamp. It might be feared 
that the contact of the water with the heated hood would crack the small lamp, or might even split the rock-crystal plate which covers the aperture for light. If there was any chance of such an accident happening, it would be fatal to the use of the cystoscope altogether. The fear is happily quite groundless in the Leiter pattern. If an ill-made lamp should shiver-and I have never heard or known of such an event-it would still be inclosed in a metal hood, the aperture in which is occupied by an unbreakable plate of rock-crystal two millimetres thick.

Mr. Whitehead, of Manchester, has introduced an improvement in the size of the instrument. The cystoscope which Mr. Leiter has made at his request is identical in principle with the new pattern, but of 40 $\mathrm{F}$. gauge instead of $22 \mathrm{~F}$. The advantages of this increased size lie in the wider field of vision obtained, and the employment of a larger incandescent lamp, with correspondingly increased brilliancy of light. The window of observation in the new, instrument is six sixteenths of an inch square, at least twice the area of that in the original one, and the lamp is double the size. Mr. Whitehead introduces this instrument through a median incision in the membranous urethra. Mr. Whitehead speaks well of the instrument, saying that "the illumination and the field of vision left nothing to be desired." The innovation is a valuable one, although it directly traverses the raison d'être of cystoscopy, which is that it affords a visual diagnosis without a cutting operation.

It at once suggested the employment of a larger instrument upon females. This has proved invaluable, for, with slight dilatation of the female urethra, a No. 40 French cystoscope is easily passed. Leiter has made, at my suggestion, such an instrument, to be used exclusively for the female bladder. Its shaft is four and a half inches in length.*

Even with a slight experience of this larger size of incandescent-lamp cystoscope, one can conceive that it will be of the greatest value in that class of cases in which the exploring finger can not reach the bladder through the membranous urethra by reason of certain mechanical obstacles, such as (1) the stoutness of the patient, by which the depth of the perinæum is increased ; (2) an enlargement of the prostate, by which the bladder is pushed farther from the surface: (3) a considerable narrowing of the pelvic outlet, which prevents the hand being well pushed into the perinæum.

* The teaching of Dr. F. N. Otis, of New York, viz., that the average male urethra has a caliber of 32 (French scale) after the meatus has been freely divided, and that there are but few cases in which it is smaller than this, is so generally accepted in America, that there is no reason why the cystoscope-tube should not be made of that size, or at least of a 30 caliber, and used through the male urethra without opening the perinæum, in the absence of stricture or prostatic hypertrophy.-(Author.) 
The management of the cystoscope requires, as we have said, but little technical knowledge, but the power of gentle and purposive manipulation of the instrument. when in the bladder can only be acquired by extensive practice. Those who are constantly in the habit of using the calculus sound, and of passing solid steel instruments or metallic catheters, will have nothing to learn in the actual introduction of the cystoscope into the bladder, for the latter is as easy as the former; but the pointing of the beak, and due rotation of the instrument, so that a certain patch of the bladder wall may become illuminated and examined, requires a great deal of practice and much patience. It is wise to use the instrument freely on the dead subject first, for we gain thereby a more rapid knowledge of the capabilities of the instrument than we can expect to acquire on the living.

Again, although we may become rapidly proficient in placing the light, it is far otherwise in the just appreciation of what is seen. A stone or needle incrusted with phosphates, or a typical growth, is as readily recognizable by a novice as are some well-marked conditions of the retina, tympanum, and larynx, when examined by means of the ophthalmoscope, otoscope, and laryngoscope.

There are, however, certain conditions of the mucous membrane which are at first exceedingly puzzling, and which require some experience to determine their nature. One of these pitfalls consists in swollen mucous membrane; another in rugæ of the mucous membrane of the insufficiently dilated bladder simulating outgrowth from the wall. Lastly, it is very difficult to appreciate the exact size of the object we examine. The size of the object as seen through the instrument varies according to the distance of the prism from it. If the prism be near, the object is magnified, and we are deceived as to its proportions; if the prism be withdrawn, the opposite effect is produced.

The cystoscope fails under certain conditions: 1 . It can rarely be passed without undue violence in patients the subjects of irregularly enlarged prostates-i. e., in which the prostatic canal is very devious, and blood becomes smeared on the window and mixed with the urine in the bladder. An ordinary enlarged prostate offers but little impediment to the introduction of the instrument, but it is impossible to examine the pouch behind the prostate if that depression is deep. Nor can the base be thoroughly searched if the intravesical prostatic outgrowth be large. 2. Stricture of the urethra, of course, offers an impediment to the passage of the cystoscope. This obstruction can easily be removed by dilatation. It may happen, moreover, that in dilating the stricture to facilitate the introduction of the instrument. we may remove the cause of the obscure symptoms which have suggested the electric-light exploration of the bladder. If the meatus be small, it can easily be cut inferiorly, 
under cocaine, to the proper size by a touch of a blunt-pointed bistoury. 3. If the urine contain blood or pus, the wall can not be distinctly made out, for the light seems to be placed in a red or yellow fog, and everything is obscure; this is, however, generally obviated by washing out the bladder and replacing the murky medium with clear water. 4. It will be found that the cystoscope is difficult to work in contracted bladders, or in those in which the capacity has been greatly diminished by pressure from without. 5. Occasionally spasmodic contraction of the bladder (especially in cases of tuberculous or other ulceration) will not allow a sufficient amount of water to be tolerated. Cocaine or anæsthesia usually overcomes this difficulty. 6. Certain deformities, such as ankylosed hip, a rickety pelvis, or a kyphotic spine, will give the observer some difficulty in approaching the ocular end with his eye; a little management of the head is needed in these cases to obtain a good view.

Summing these points up briefly, we may say that there are three conditions indispensable for the employment of the cystoscope: 1. The urethral canal must have a caliber of 22 French catheter gauge. 2. The bladder must have a capacity of at least four ounces. 3. The water in the bladder must be translucent, and ought to be perfectly transparent.

Summary of Rules for using the Instrument. - 1 . See that the bladder contains at least six ounces of clear fluid. 2. Regulate the lamp beforehand, and do not start the lamp until the elbow is well within the bladder. 3. Do not keep the cap in contact with the wall. 4. Let the manipulation be gentle and purposive. 5. Let the instrument remain half a minute in the bladder after the current has been shut off, in order to completely cool the hood before you withdraw. 6. Let the base of the bladder be examined first; for the inferior zone of the bladder is to vesical endoscopy what the optic disk is to ophthalmoscopy-the most important area in the examination. It is to the inferior zone that the cystoscope is first directed, for it is this section of the bladder which contains or conceals for the most part those diseases which rank as "obscure."

(The special appearances of disease are described in the article on "Cystoscopy."')

The Value of the Electric Cystoscope.-The value of the electric cystoscope may be estimated in various ways. The instrument can either afford us a clearer insight into pathological conditions of the vesical mucous membrane, and enable us to watch the progress of the disease and the behavior of the same under varying forms of treatment; or it may allow us to control our elinical observations and speculations by direct visual research, and rightly to assign the more prominent symptoms to definite causes; or, finally, it may at once elucidate for us the cause of obscure symptoms of urinary disease, of which we otherwise could obtain no certain clew without a cutting operation. 
The following three illustrations, taken from Albarran, give an impression of the cystoscopic picture of three forms of bladder tumor.

Digital Exploration.-The last measure at our command for arriving at a diagnosis, and as opening the way to operative treatment (in which latter connection it will be considered later), is digital exploration of the bladder through a perineal boutonnière.

Although this manœuvre had been previously performed by many surgeons, Sir Henry Thompson should nevertheless be credited with

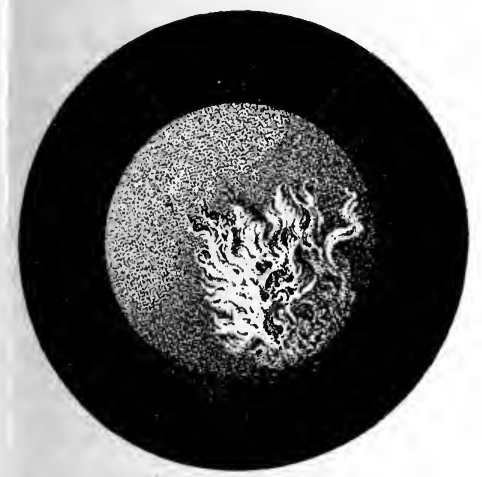

Fig. 8.-Villous epithelioma.
(From Albarran.)

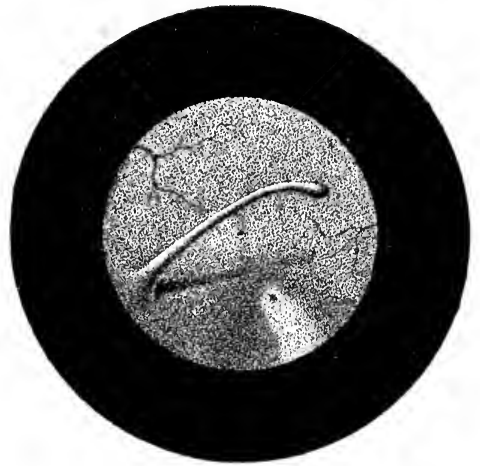

Fig. 9.-Small, smooth tumor, with long pedicle. (From Albarran.)

having proposed and established it as a diagnostic step in cases of bladder tumors, and so supported it with ample experience as to give it a definite and valuable place among the surgical procedures in this class of cases. This he did in 1882 and 1883. His proposition was to employ this manœuvre only in cases of obscure bladder disease in which other means for diagnosis had proved unavailing. The urethra should be opened in the usual way at its membranous portion through the perinæum, sufficiently to admit the index-finger, which thereafter is passed through the vesical sphincter until its tip lies within the bladder, which for the purpose of exploration should be empty. By then pressing the fingers of the other hand deeply in

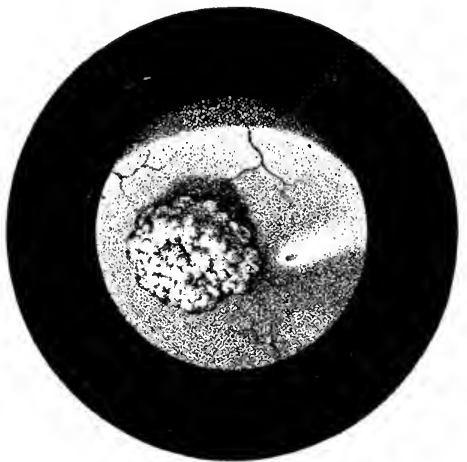

FIg. 10.-Lobulated epithelioma (From Albarran.) above the symphysis pubis upon the summit of the bladder, the interior of the latter can, by rotary movements of the hand which presses upon it from above, be made to pass 
over the tip of the finger lying in its interior. The exploration may be supplemented by a finger in the rectum. In this way the entire cavity may be brought within the reach of the examining finger. By this means Sir Henry Thompson was led in a considerable number of obscure cases to a certain diagnosis of bladder tumors, and in some to their subsequent successful removal, inasmuch as he advocated at that time for the majority of such cases their removal through this wound already made.

The procedure was thus of value, not only for the diagnostic but in a certain proportion of the cases for operative purposes. This is one of its chief advantages, provided one wishes to attempt removal of the growth through this road; and Sir Henry further stated that, if the growth was of such a nature as to preclude the possibility of its removal in this way, the boutonnière in nowise deterred the surgeon from proceeding immediately to the ligh cut in such a case.

The sense of tonch he also considered as practically unerring in the detection of bladder growths. The objections to be urged against this manceuvre are, that it is incapable of being employed in any case in which there is a considerable bilateral hypertrophy of the prostate or a very narrow ontlet to the pelvis. A number of examples of the failure of touch to detect growths are on record; also, among others, the follow. ing case of the author's :

The growth was in this instance a papilloma made up of fine, delicate papillæ which sprang from a very ill-defined base about the size of a half-dollar between and above the ureteral orifices. The finger wholly failed to appreciate its presence, which, however, was established by scraping at hazard with the curette, which brought away an unmistakable bit of new growth, and enabled the operator thus guided to accomplish its entire removal successfully.

Where the cystoscope has satisfactorily demonstrated a tumor, it is unnecessary to resort to digital exploration. The author is in accord with Sir Henry Thompson's expressed opinion as to the harmlessness, however, of the boutonnière operation in such cases, and it may serve a useful purpose as an extra means of drainage subsequent to the high operation.

In the author's belief, however, the special value of digital exploration as described is in those cases in which there has been a failure to make a diagnosis by the usual means, or by the cystoscope, owing to the conditions which render impracticable the successful use of the latter instrument, or through failure to see the growth even in cases in which the conditions are favorable, should such an occurrence happen. Under these conditions, and in the belief of the existence of bladder disease of unascertainable nature, the digital exploration should be resorted to, and it takes a place of much value, involving almost no risk, as the last of the 
steps in making a diagnosis. Sir Henry Thompson's recent views in regard to the value of cystoscopy and digital exploration are expressed thus in the Medical Press and Circular, August 13, 1890: "But it may be said that the diagnosis may be readily established by means of the Leiter cystoscope. I long ago tested its value. Let me say, first, that the employment of it in the cavity of the bladder involves much irritation in some cases, far more than that which follows the use of the sound.

"Secondly, that in the presence of a tumor which the slightest mechanical contact causes to bleed the observation is mostly an imperfect one.

"Thirdly, that the instrument is rarely necessary to determine the fact of the tumor's presence, that being ascertained in nine cases out of ten by the ordinary methods.

"Lastly, it is assumed to furnish the means of insuring a view of the growth which shall enable the operator to determine whether it is pedunculated and easily removable, or the reverse.

"In regard to this important matter I admit that the object may be obtained, but in the majority of cases I am certain it can not be thus decided, as when the tumor is not seen in profile, and when the bleeding occasioned permits only an imperfect view, a far more accurate idea can be obtained by 'digital exploration,' which never produces a cystitis, becoming chronic and confirmed, which unhappily too often follows prolonged manipulation with a rigid cystoscope in the diseased bladder of an elderly patient."

The following comparison, as opposed to the above view of the value of cystoscopy and digital exploration, is made by Fenwick:

"1. Digital exploration is a cutting operation, needing confinement to bed. Electric cystoscopy does not need such confinement, although it is wiser and safer to have the patient in bed for the rest of the day. I have used the cystoscope in ont-patient and private practice, favorable cases being examined without any preparation. Certain cases, however, as will be seen in the third statement, are not examined except when in bed.

" 2 . The former operation needs an anzesthetic. In the latter it is not absolutely necessary. In the greater number of cases I have neither used gaseous narcosis nor cocaine. I employ anæsthesia $(a)$ in females for delicacy, $(b)$ in tuberculosis or similar cases where the prostatic urethra is extremely sensitive, $(c)$ in order to make a leisurely diagnosis of a discovered growth, so as to determine the expediency of operating.

"3. Digital exploration is not absolutely free from risk or hamorrhage, and frequently either a troublesome fistula or a hypersensitive scar is left in the urethra. Cystoscopy is in most cases, if it be gentle and purposive, as free from risks as routine catheterism or sounding. There is, as every surgeon is aware, some risk in the first introduction of any instru- 
ment through the deep urethra-a risk which must not be made light of. There is also some danger to be incurred in washing out the bladder for the first few times if residual urine be present; and, lastly, there are certain diseases, such as tuberculosis of the urinary tract, which resent at once any instrumental interference.

"The surgeon must therefore select cases for routine observation with care and judgment-those which his knowledge of the idiosyncrasies of urinary disease can assure him will be suitable. He must regard the cystoscope in the same light and with the same deference that he should regard the bougie, the somnd, or the catheter, for, as Sir Henry Thompson has so tersely and so wisely expressed it, "The introduction of an instrument is more or less an evil, never to be resorted to unless a greater evil be present, which its employment may probably remedy.'

"I myself avoid using the cystoscope if the following conditions are present: (1) Obvious tuberculosis of the urinary tract; (2) traumatic renal hæmaturia of recent origin ; (3) irregularly enlarged prostates ; (4) residual urine due to ataxia; (5) phosphaturia.

"I include traumatic renal hæmaturia on the strength of a fatal case which occurred quite recently in my own practice.

"4. In most cases the educated eye is to be preferred to and relied upon rather than the finger.

" 5 . It must be readily admitted that digital exploration allows of the bladder being subsequently drained, but the rest thus afforded is not always necessary, nor is it always productive of benefit.

"A belief has been expressed that the early use of the cystoscope in hæmaturia will tend to limit the size and number of vesical papillomata by enabling us to detect and remove these and other growths in their very infancy.

"I do not quite agrce with this belief, for a careful examination of the histories and pathological specimens of vesical growths has convinced me that vesical tumors do not obey a fixed law of development, for they do not increase in size pari passu with the duration of symptoms. The increase depends upon the character of the growth, its position in the bladder, and its method of attachment to the wall. It is therefore possible for a growth to attain a considerable size before any evidence is afforded of its presence. It thus lies 'latent' until a chance congestion or aceident develops the characteristic hæmaturia."

Duration of the Disease, and Prognosis.-In the majority of cases of both benign and cancerous growths the progress of the disease is usually slow. Guyon refers to an instance of benign growth in which characteristic symptoms had been present for twenty-seven years before death occurred, and other instances, in which the nature of the growth was presumably cancerous, in which the disease had lasted fifteen years 
before death. These are, however, exceptional. It is probably fair to assume that the average duration of the disease after the appearance of the first symptoms is from three to five years.

On the other hand, exceptionally, a patient may die within a few months, from the presence of the bladder growth. In such cases death is usually due to frequent and repeated hæmorrhages alone, or from the effects of cystitis and nephritis superadded.

Cancerous growths kill the patient by exhaustion, hæmorrhages, or by extension or metastasis to other organs. Ordinarily the patient fails slowly, often recuperating, however, wholly in the intervals between the attacks of bleeding in cases of benign growth, if the intervals are sufficiently long.

No definite prognosis as to the duration of the disease can be given. It can only be said that, practically speaking, death is the inevitable result in all cases of bladder tumors if left to themselves. The prognosis after surgical operation will be dealt with in considering the operative treatment.

Treatment. Palliative Treatment.-Palliative treatment, it may be said at once, is wholly unavailing so far as cure is concerned. Some measures, however, may be employed with temporary benefit to check hæmorrhage, and perhaps by so doing enable the patient to recover sufficient strength to undergo surgical operation, if appropriate. For this purpose in the author's experience long-continued irrigations of the bladder through a soft-rubber catheter with very hot water, $110^{\circ}$ to $120^{\circ} \mathrm{Fahr}$, best accomplishes the purpose. The use of strong astringents and of styptics within the bladder can not be advised, as they are liable to produce tough coagula, and to render the evacuation of the bladder more difficult than it already was.

In cases in which there has been already a severe hæmorrhage and a coagulation of blood within the bladder, the author believes that it is wiser to allay vesical tenesmus and pain by the administration of opium rather than to attempt to remove the clots by breaking them up and washing them out with large catheters or evacuators. The latter practice is likely to result in renewal of the bleeding as fast as the clots are removed, whereas the pressure caused by the filling up of the bladder with clots tends to check the hrmorrhage after a certain time of its own accord, and in the course of a few hours disintegration of the coagula and their evacuation will occur spontaneously.

If this expectant treatment, however, be followed in such a case, a careful watch should be kept to see that the flow of blood and retained urine in the bladder does not increase to a dangerous point, which can be determined by frequent examinations of the organ above the symphysis pubis. The patient's general condition also furnishes a satisfactory 
guide. The occurrence of such an attack of bleeding should lead to as speedy an operative treatment as the patient's condition will allow.

Any palliative measures, such as the administration of drugs by the mouth or by the rectum, are of too little value to notice.

Operative Treatment of Benign Growths. - It may be stated as an axiom that in all cases of benign tumors removal of the growth should be undertaken at the earliest available moment after the establishment of the diagnosis, except in the presence of definite contra-indications. (The question of operative measures in cases of malignant growth will be considered under a separate heading.) The only contra-indications in cases of benign growth that seem worthy of much consideration are such exhaustion of the patient as to render the issue of the operation hopeless, or the presence of grave renal disease, or some other complicating malady which on general principles would contra-indicate any surgical operation.

Although isolated instances of the removal of bladder tumors occurred in the hands of various surgeons from the time of Corvilard, who in 1639 removed a bladder tumor by the lateral cut for stone, down to 1880 , the operative treatment of this condition practically dates from about the latter year. Since that time this condition, which formerly was considered as among the rarest of diseases, has been shown to be much more common than it was supposed to have been, and within the last eleven years the number of cases observed has been very large, and the number of those operated upon has been sufficient to furnish a series of data upon which to base satisfactory conclusions as to the results of treatment by operation in the various classes of tumors, and the relative advantages of the different methods.

For the removal of growths from the bladder one of two routes is most commonly employed: that through the perinæum, as proposed in 1883 by Sir Henry Thompson, and the suprapubic. In women we have in addition colpocystotomy. And there have also been proposed by Langenbuch and Helferich special operations. That by the perinæum is described by Sir Henry Thompson as follows: (The urethra is opened in the median line, as described under "digital exploration," and the finger is pushed into the bladder and its interior explored.)

"When the survey of the vesical cavity has been completed, the operator has to determine the following question: Am I capable of completing my design of removing the tumor before me wholly or partially, as the case may be, through the incision made, or should I do so more efficiently through a suprapubic opening? For it may be taken for granted that a case may occasionally present itself in which the latter course may appear to be preferable, and if so there is no reason whatever for not selecting it. The perineal incision made will not add to the risks of the 
case, and it has afforded that most important element, the means of obtaining an exact diagnosis.

We shall now consider the means which it is desirable to employ in order to remove the tumor, when, having examined it fully, we have decided to make an attempt through the perineal opening. First, if we have the good fortune to encounter a single growth, polypoid in form, and therefore with a pedicle of no great width, there need be no hesitation whatever in introducing a pair of forceps into the cavity of the bladder, and employing them there without any aid from the operator's finger. The forceps to be used for this purpose are to have rather wide and serrated margins where the blades meet, so as to crush but without any power to cut the tissues seized. These forceps should be provided in different forms. The first or simplest pattern should be straight, resembling an ordinary lithotomy forceps; others should be curved for seizing
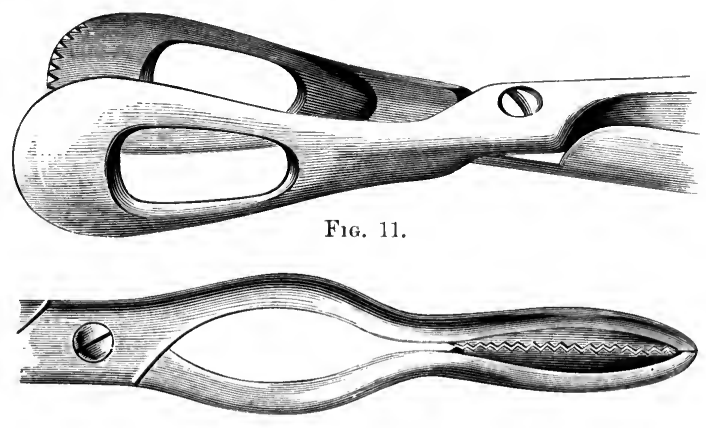

Fig. 12.
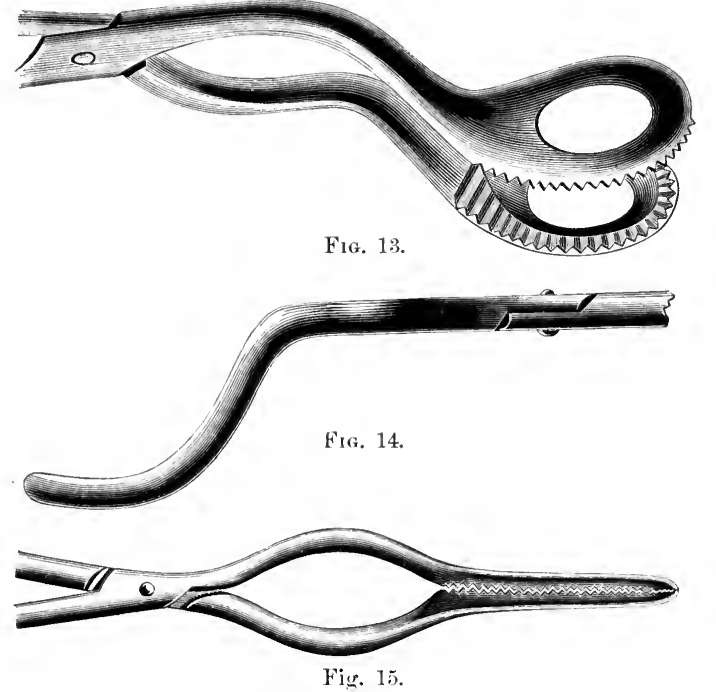

Figs. 11 to 15 , from Thompson.

tumors which are situated laterally and near to the neck of the bladder, in which last-named position the straight forceps are powerless to grasp the tumor.

A pair of each pattern should have cutting edges for exceptional cases when, for example, the growth is more firm and solid than usual. When the blades are free in the cavity, they have merely to be opened easily and widely, and on closing them it is almost certain that they will 
grasp the polypus more or less completely. The proceeding thus advised is more likely to prove successful in accomplishing its object than is the attempt to seize a stone in the bladder. But here let it be observed that no suprapubic pressure should be made during this act; nothing should be done to interfere with the natural contour of the bladder. The operator, by light and easy movements of the forceps in varied directions, ascertains that he has the growth within their grasp. My advice is that, above all things, he is not now to pull forcibly, but that he is to press firmly the blades together, biting or chewing a little, if I may use the terms, with the extremities of the blades, without changing the original situation of the bite or grasp. Then a little twisting movement may help to disengage the mass, which, if accomplished, the forceps will be felt to be free, and may be withdrawn with their contents; after which the finger enters to feel what remains, and what more must be done in order to complete the removal. Let me remark, whenever the forceps has removed a portion, however small, the instrument should never be reintroduced until the finger has again examined the interior, and enabled the operator to form a fresh estimate of the portion remaining, if any, to be removed. But, supposing that the tumor lias not been separated by the moderate amount of forceps action described, the operator may disengage and remove them; when on introducing the finger he will probably find the part so nearly severed that the actual division may be completed with the finger-nail. There is really no difficulty, nor is there any risk, with ordinary care, in removing a growth or two of pedunculated form, sufficiently near to the walls of the bladder to render the operation a permanently successful one. I suppose that the ultimate result of dividing the pedicle of a tumor in the manner described is such a cicatrization of the wound as to prevent the recurrence of outgrowth from that spot hereafter. (The different forms of forceps are shown, Figs. 11-15.)

"Other means may be used according to the judgment of the surgeon. A very small écraseur, with violin-string ligature, may be manipulated by the side of the index-finger, and used in polypoid forms of tumors. In the cases of women, such a tumor may sometimes be slowly and carefully brought into view by traction on the forceps, and then the pedicle may be ligatured. I have succeeded in doing this in one case; so has Mr. Bryant.

"In another I drew a polypoid mass within view, and found it was a completely encysted calculus; I then opened the mass, when the calculus escaped.

"In the male it has occasionally happened that the tumor can be drawn throngh the wound, as happened with Mr. Davies Colley, and also with Mr. Morris, in whose case the growth seemed to have been extruded 
by natural vesical efforts on the day after operation (having been left untouched on the first), and was then removed, the patient making a good recovery.

"When the growth takes a more complex form-as when several lobes spring from a wider base, the forceps must be depended upon for removing them by repetitions of the same process which is employed for the single growth. But when the base is wider still and the growth is less prominent, it is doubtful whether complete extirpation is possible by any proceeding, either through a perineal or through a suprapubic opening. The wide base involves such intimate continuity of tissue between the coats of the bladder and the structures of the growth, that I believe separation to be mostly impossible; and that ablation of the prominent portions of the growth, when feasible, should be undertaken only with the view of retarding its progress, but with no prospect of affecting complete separation of the disease from the body.

"These portions are to be seized and destroyed, partly by removal, on the plan just described, and partly by crushing, and thus indncing sphacelus and sloughing of the growth. The question of applying some chemical astringent arises in such eases, and its utility may be regarded perhaps as twofold. A solution of the perchloride of iron may tend to check the hæmorrhage, which is almost invariably very free for a few hours after the removal of wide-based growths, and it may partially destroy the portion which remains after the forceps have done their work. For this purpose I have contrived a straight and also a curved glass syringe containing a small sponge, saturated with the solution; the sponge being pressed by the piston permits the escape through small perforations at the extremity of from 30 to 60 minims of the solution, at the precise spot where it is desired to make the application.

"There is one circumstance important to be noted, especially in dealing with the less prominent growths; and that is, the effect of strong suprapubic pressure made by an assistant in relation to their apparent situation, and their mode of presentation to the finger of the operator engaged in exploring the bladder, and in estimating their size and form. If that pressure is considerable, it forces the upper wall of the bladder into its own cavity, and thns gives to the growths a larger contour than they possess, and makes them apparently salient to a much greater extent than they really are. Thus an eager or inexperienced operator, unaware of the effect of strong suprapubic pressure, might be led to seize the mass offered to the forceps, through the inflnence of this pressure, and, under the belief that it was a large growth, he might inflict a fatal wound by crushing a double fold of the coats of the bladder, and so making an opening into the peritonæum. To avoid such a catastrophe, it is only necessary, first, to decline the attempt to destroy any growth 
which is clearly not sufficiently salient to admit of complete or nearly complete removal; and, secondly, never to employ the forceps while forcible suprapubic pressure is made-at least, no more pressure than is desirable just to steady and support the bladder and the parts adjacent."

The Suprapubic Operation.-The revival of suprapubic cystotomy since 1880, owing to the introduction of the Garson-Petersen technique, and the application to the operation of the antiseptic regimen, and the successful modifications and improvements that have followed from time to time, have made it one of the most useful of the surgical operations of to-day, and its greatest benefits are to be found in connection with the removal of bladder tumors.

The suprapubic operation properly had its origin in the hands of Pierre Franco, who performed it with success in Lausanne about 1560. Franco in this case rendered the extraction of the stone more easy by pushing it upward by two fingers in the rectum. The case of Jean Doot, of Amsterdam, may be quoted among the early operations as a matter of curiosity, he having removed a stone from his own bladder through an incision above the pubes, made with a shoemaker's knife.

In 1581 Rousset recommended the injection of the bladder with water, with a view to elevating it above the symphysis, previous to incising it. Piètre, in 1635, tried to accomplish the same result by the introduction of a sound into the bladder.

The operation was not much heard of until Douglas and Cheselden, in England, almost a century later, revived it, both practicing it successfully several times. It now was frequently performed, meeting with varying success and failure, until it came into the hands of Frère Côme, who in 1799 had operated one hundred times, with only nineteen deaths. Perineal section was practiced in combination with the high cut by Côme and by Sermes before him.

In our century many surgeons have done the operation, among them Dupuytren, Souberbielle, Sir Everard Home, Leroy d'Etiolles, Nelaton, Civiale, etc., but it may be said that throughout this time, until of recent years, the operation steadily lost ground.

Up to 1880 but little change was made in the technique. Since then three notable advances have again placed it in the category of favorite surgical operations; these steps are: (1) Antiseptics; (2) vesical suture or drainage; (3) vesical injection combined with the use of the rectal balloon.

Rectal Balloon.-In 1880, at Copenhagen, Petersen described his method of raising the bladder and with it the peritoneal fold by means of an oval-shaped rectal balloon. (Garson had previously demonstrated the same possibilities on the cadaver.)

The process consists, after the patient is prepared in the usual way 
for operation, in passing a catheter into the bladder through which about ten ounces of a four-per-cent boracic-acid solution is injected, the rubber balloon greased with vaseline folded upon itself longitudinally, having been previously introduced by an assistant into the reetum, so that its outer end is securely placed above the sphineter. Ten ounees of warm water are then injected into the balloon (some operators prefer to make the preliminary incisions through the abdominal muscles previous to inflating the balloon, and this is the author's own preference, the object being to reduce the period of rectal distention to as short a time as possible). By this manœuvre the peritoneal fold at its attachment to the anterior surface of the bladder is raised from two to four fingers' breadth ahove the symphysis pubis, and thereby is avoided one of the principal dangers, as already pointed out, of the operation-namely, the wounding of the peritonæum. In a few cases the peritonæum will have an abnormally lower attachment, and will not be raised sufficiently above the symphysis pubis under these eireumstances.

In a few others, owing to previous inflammatory processes, the peritonæum may be found to be attached to or about the symphysis pubis. In such cases it must necessarily be wounded before entering the bladder.

In still another class of eases, ehiefly those in which a long-standing cystitis has been associated with prostatic hypertrophy, resulting in great thickening and rigidity of the bladder, the bladder will be found to have lost its elasticity, and be incapable of sufficient distention to allow of its being elevated above the symphysis pubis. With these exceptions the bladder will rise, as already described, when Petersen's method is used.

Various experiments, notably those of Garson, of Fehleisen, and recently of Dr. A. B. Strong, of Chieago, with reference to the degree of elevation of the bladder by the use of the rectal balloon in eonjunction with the injection of varying quantities of fluids of different kinds and of air into the bladder, have established the fact of its more or less successful accomplishment in the large majority of instances, and given it a valuable position in the eyes of many surgeons.

Dr. Strong's conclusions, derived from his anatomical study of the subject, are as follows:

" 1 . In the normal condition, the bladder and rectum being empty, the apex of the bladder and peritoneal reflection are a little above the arch of the pubes.

" 2 . In moderate distention of the bladder, the anterior peritoneal reflection is below the arch; with the same degree of distention and the bladder pressed against the abdominal wall, the peritonæum ascends higher.

"3. Suprapubic eystotomy ean be most easily and safely performed 
when the bladder is lifted from the pelvic floor and moderately distended against the abdominal wall.

"4. Distention of the rectum alone elevates the base of the empty bladder, but does not raise materially the vesico-abdominal fold of peritonæum.

" 5 . Distention of the bladder alone, in thin subjects particularly, requires relatively a greater amount of fluid to elevate the peritoneal reflection. The biadder is not erowded against the abdominal wall, but rather falls away from it.

" 6 . Moderate distention of both rectum and bladder meets the indication the best; from ten to twelve ounces in the rectal bag, and eight to ten in the bladder, is generally enough, and seems to be a safe quantity to use.

"7. Dilatation of the rectum first and the bladder afterward lifts the peritoneal reflection the highest.

" 8 . The object of the rectal bag is to elevate the distended bladder and press it against the abdominal wall and so crowd up the peritonæum.

" 9 . To meet the indication, the gut should be dilated from the anus to near the promontory of the sacrum.

"10. The rectal bag should be sausage-shaped, of rather thin rubber, rather than pyriform and thick, for the thinness enables it to follow up the gut, and the shape makes uniform pressure throughout the entire length.

" 11 . In a very fleshy subject, with a flabby or pendulous belly, the bladder is relatively freely movable. In such a case it will easily rise out of the pelvis against the abdominal wall, when alone distended with a moderate quantity of water; the reetal bag may then be safely dispensed with.

"12. Air injected into the bladder of a subject lifts the bladder and its peritoneal reflection out of the pelvis better than water.

"13. In one case the gut was ruptured opposite the promontory of the sacrum with twenty-three ounces in the rectal bag; in no case was the bladder ruptured.

" 14 . In the cases reported an average of fourteen ounces in the rectal bag and twelve in the bladder elevated the anterior peritoneal reflection an average of one inch and a half above the erest of the pubes; the apex of the bladder was one inch higher."

In twenty-five experiments reported by Dr. Helmuth, when the bladder alone was injected with twenty-seven ounces of water, the peritoneum was lifted two inches and the apex of the bladder four inches above the crest of the pubes. In five cases rupture of the bladder occurred with an injeetion of fifty-nine fluid ounees.

Of recent years there has been a tendency on the part of various 
surgeons to omit the use of the rectal balloon, and to operate without it, in the ordinary position of the patient, or to adopt the postural method of raising the bladder introduced and practiced by Trendelenburg, who omits the rectal balloon entirely, and seeks to secure the elevation of the bladder by raising the legs and pelvis of the patient. When this is done, Trendelenburg says that, the intestines and diaphragm falling toward the thorax, draw the bladder after them out of the pelvis, and that the latter organ and its anterior peritoneal fold are in this way as satisfactorily raised as by means of the rectal balloon; and that the additional advantage is offered in that the bladder maintains its elevated position after it is opened, and is thus rendered easier of access for instrumental manipulations in its interior.

Trendelenburg has devised a special form of operating chair, by which the inverted posture of the patient is secured. In its absence the same end may be attained by placing cushions beneath the pelvis, and having the legs raised by assistants. The objections to Trendelenburg's method are the necessity of possessing the special operating chair, or, in its absence, of being obliged to maintain the awkward position of the patient at the cost of considerable labor, but more especially, so far as the

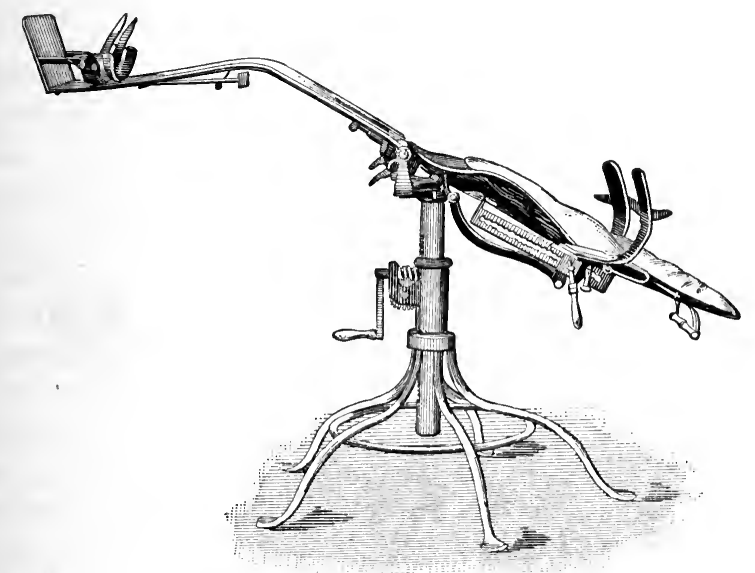

Fig. 16.-Trendelenburg's operating-chair.

author's experience goes, the elevation of the bladder is far less satisfactory than that secured by Petersen's method. (Trendelenburg's chair is shown in Fig. 16).

In the author's experience, the rectal balloon is of the greatest assistance and value in elevating the bladder and its peritoneal fold, and pushing the whole organ forward against the abdominal wall, and making it much more accessible. 
The dangers attending the use of Petersen's method, that have been dwelt upon, are rupture of the bladder and rupture of the rectum. Examples of such occurrences are noted in the Annals of Surgery, vol. xii, 1590 , page 129, by Dr. Fowler, of Brooklyn, who publishes an instance of this accident occurring in his own practice. This happened in a man sixty-three years of age, who was the subject of an hypertrophied prostate. The rectal colpeurynter and the bladder were each injected with eight ounces of fluid. The rectum was ruptured in this case, a rent of four inches having occurred upon its upper wall. The autopsy showed that the rectal wall was unusually thin and weak. The author then quotes the following cases:

That of Cadge, reported in 1886 (Proc. Roy. Med. and Chir. Soc'y, London, page 97,1886$)$, in which the rectum was ruptured by a colpeurynter containing eighteen ounces of fluid.

Nicaise, in 1888. A rupture of the rectum, the rectal bag being filled with ten and a half ounces, and the bladder with eight and a half ounces of fluid. This accident happened in a man sixty-five years of age.'

In old people, with trabeculated bladders, there are occasionally found diverticula with very thin outer walls, which moderate distention of the bladder would be quite sufficient to rupture. It is impossible to have a knowledge of the existence of this condition beforehand, and, as it is rare, the surgeon may justifiably take the risk of its being present in any given case. In adults the danger of rupture of the rectum will be practically never incurred, if the quantity of fluid injected into the balloon and bladder is limited to ten counces in each, and this is almost always sufficient to raise the bladder satisfactorily.

If, during the injection of either the bladder or rectal balloon, great resistance is experienced, it should be taken as an indication to eease at once from the attempt to force in any more fluid, and the operation should be proceeded with as well as may be under the conditions. Such resistance will be encountered in cases in which there is a small bladder with thickened, rigid walls, such as are seen in certain patients, as the result of chronic cystitis, or hypertrophy of the prostate, and will he also noticed in the rarer condition of extreme bladder irritability, which sometimes persists even during the anæsthesia of ether, the organ contracting violently after a few ounces have been injected into it.

In a few cases, failure to elevate the peritoneal fold occurs, owing to its adherence, from previous inflammatory action, to or about the symphysis pubis. But the above conditions are relatively rare, and Petersen's method is applicable and innocuous in the vast majority of cases.

In children, three ounces of fluid in the bladder and three in the rectal balloon will be ordinarily free from risk, and sufficient. 
The abdominal incisions are next made in the following way: The incision is begun in the median line about three or four inches above the symphysis pubis (in fat subjects the incision should be longer, in lean ones shorter), and is carried down to and a little over the upper margin of the symphysis pubis. The fascia covering the recti muscles is next divided, and the incision carried, if possible, through the linea alba down to the prevesical space. This is at once recognized by the presence within it of more or less yellow fat. Immediately beneath this lies the bladder. At this point of the operation there are two methods of procedure advised. Most operators counsel the lifting up, toward the upper angle of the wound, of the presenting fat, with the index-finger, until the bladder is exposed to view. The object of this manceuvre is to carry upward with the finger, together with the fat, the peritoneal fold which may lie within it.

The objection to doing this lies in the disturbance of the contents of the prevesical space, for, in some instances, especially where there is a good deal of fat, holes and pockets may be made by pulling up the fat as described, which will give lodgment subsequently to the fluid draining from the bladder, and incur the risk, which is one of the most serious ones, as already mentioned, of originating in these spaces, septic phlegmon and subsequent pus-burrowing. It is a rule that can not be too strongly adhered to that there should be as little disturbance as possible of the anatomical relations of the tissues lying in and about the prevesical space, during the operation.

This can be better secured by dividing cautiously the fat-layer with blunt-pointed scissors, until the bladder is laid bare. The peritoneal fold, should it be placed low, and encountered in the course of this division, can be recognized and avoided by lifting it upward out of the way. In either case the bladder when reached is easily recognized as a dense, rounded body bulging upward, of a dark-red or purple color, bearing on its surface a number of veins; its appearance is oftened likened to that of the head of the fœtus presenting at the vulva. The author has never encountered bleeding of any importance, in exposing or opening the bladder.

The usual step now is to insert a tenaculum as high up upon the presenting surface of the bladder as the position of the peritoneal fold will allow without injuring it. This instrument in the hands of the assistant serves to maintain the bladder in its high position after it is opened. As soon as the tenaculum is placed, the bladder is opened by one clean incision, extending from immediately below the tenaculum downward for an inch or an inch and a half in length. The fluid contents of the bladder now immediately rushes out through the wound. As soon as possible sutures should be placed about the middle of either edge of the bladder incision, 
in order to separate its edges and to sustain it and prevent its falling downward behind the symphysis pubis. The rectal balloon is now emptied, and spatulæ or wire écarteurs are inserted within the bladder to open its eavity to view and to expose the growth. For this purpose two or more single-bladed Sims specula may be used, which are employed by Bazy, in France, but strong wire instruments which can be bent to any desired form are preferable. There is almost always a tendency of the summit of the bladder to bulge downward and obscure the cavity of the organ. The most important speculum, therefore, is that which is placed in the upper angle of the wound, to obviate this difficulty. There should be as little manipulation as possible within the bladder at this time; otherwise, bleeding may be excited from the growth, and so obscure the view. When the bladder is emptied of its contents, the cystoscope-tube of Leiter or any other convenient electric lamp may be introduced within the bladder from above through the wounds. Eigenbrodt has proposed (Archiv für klinische Chirurgie, 1889) a special form of speculum and electric lamp combined to answer this purpose.

The author has not personally used this instrument, but from its description it seems too complicated and too little adapted to its purpose to be of especial value. It has the great disadvantage of an exposed lamp, which is readily broken by contact with fluid. The author's own method of exposing the bladder and the advantages which he has found it to afford over and above those offered by the other methods in use were described at the Berlin Congress in Angust, 1890, and published as follows, in the London Lancet, October 18, 1890, under the title of-

A New Means to assist in the Removal of Intra-vesical Growths through a Suprapubic Cystotomy. In spite of the various improvements that have been applied to suprapubic cystotomy since its revival in 1880 , which have brought its technique so nearly to perfection, I have thought that a still further advance might be made in regard to some of its steps, when the operation is undertaken for the removal of intra-vesical tumors or prostatic hypertrophies. The steps of the operation which I have sought to improve are those which relate to the exposure of the interior of the bladder after it has been opened, and to the removal of the growths. I was led to make these modifications by the objections experienced in the use of the methods at present employed. With reference to the exposure of the interior of the bladder, the method of Trendelenberg seems to me cumbersome and awkward. The nse of tenacula, or threads, or spatulæ, to sustain and open the bladder to view, calls for a number of assistants' hands, which are in the operator's way. The assistants frequently displace the spatulæ or écarteurs, and let them assume faulty positions. Furthermore, solid-bladed écarteurs like those used by Bazy, cover up a portion of the bladder wall and interfere with 
the view of the growth and its removal by instruments. To obviate these difficulties I have devised a bladder speculum (Figs. 17 and 18) which seeks to accomplish, by one instrument, all the objects, both for sustaining the bladder and exposing its interior to view, now gained by tenacula, threads, sutures, écarteurs, or posture of the patient, while doing away with the objections to the use of the latter methods, enumerated above.

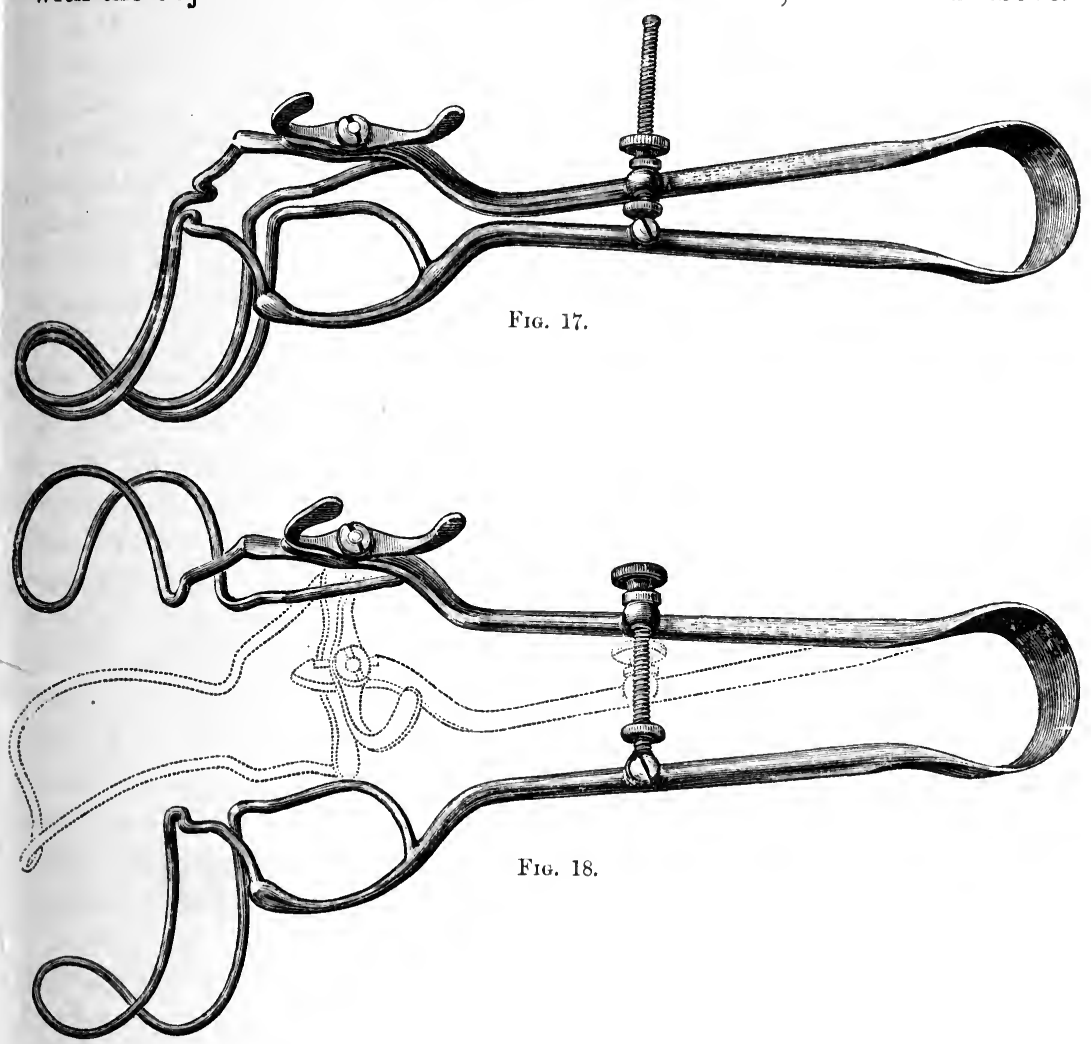

Fias. 17 and 18.-Author's bladder speculum, for exposing the interior of the organ after suprapubic cystotomy.

The speculum consists of two strong wire blades, which, when closed, as in Fig. 17, can be readily introduced into the bladder throngh an incision of one inch or a little more in length. Above, the wire blades are attached to either side of a stout steel shaft or handle which terminates at its farther end in a strong spring, the action of which is to separate the two sides of the shaft, and consequently, also, the wire blades of the speculum attached to either of them. About the middle of the shaft, and connecting its two sides, is a screw by which their separation can be controlled by governing the action of the spring. The blades of the instrument 
are introduced closed (as in Fig. 17) into the bladder. The speculum is then opened (as in Fig. 18) by rotating the inner blade upon a pivot by the thumb and one finger; this blade then assumes a position opposite to its fellow, at the same time moderately distending the cavity of the bladder. The sides of the abdominal and bladder incisions can now be still further separated, and the bladder at the same time further distended by turning the screw outward along its thread, thus allowing the spring to push the handles apart. The instrument then takes the position shown in Fig. 18, and between its blades a free view of the field of operation is obtained. To withdraw the speculum, its blade is rotated back again and the sides of the shaft approximated by the screw; the position is then again as in Fig. 17, and it can be easily withdrawn. With the blades opened, the inner surface of the bladder incision rests upon the shoulder formed by the angle at the junction of the upper and lower portions of each wire blade. The bladder consequently can not collapse cr fall downward behind the pubes, but is held close up to the abdominal wound, and firmly fixed in that position, even when no hand holds the handle of the instrument at all. This does away with the necessity of having two or more hands to sustain the bladder with tenacula, or threads, and dispenses with sutures also, which require time to place, and may tear out under strain. By grasping the shaft of the instrument with one hand the operator can raise or lower the bladder at will, and by turning the handle from side to side can expose to view and operative manipulation all parts of the interior of the organ. In using the speculum the bladder should be sustained by a tenaculum below the peritoneal fold while it is incised, and until the speculum is introduced and its blades opened. The tenaculum is then withdrawn. The open, widely fenestrated wire blades allow the best view; do not cover up portions of the surface against which they rest, and give room between their sides for the passage of instruments.

To recapitulate. The advantages gained by this speculum are: The placing of the bladder for all purposes of sight and instrumental manipulation under the control of the single hand of the operator, and the doing away with the objections to the other methods already mentioned. The contrivance is simple, inexpensive, easily cleaned, and efficient; it saves time, and facilitates decidedly the removal of bladder growths. In order to allow for the differences in the capacities of different bladders, and also for the varying thickness of the abdominal wall in different individuals, the speculum is made in three sizes; the medium and smaller sizes will be found, I think, generally sufficient. There are, on the other hand, certain limitations to the use of this speculum, to which special attention should be given, else one may be disappointed in the instrument. It will be found inapplicable should the growth be an unusually 
large one, with a salient, firm base, beeause in that case the rotation of the inner blade may become impossible, and the instrument, therefore, can not be opened. The same is true if there be a very small, contracted, inelastic bladder, for the same reason. If the patient be not completely anasthetized, the blades ean not be opened, owing to their being held together by the recti muscles in contraction. Onee opened, however, the blades ean be locked in that position by the serew-head on the inner side of the handle. The second step relates to a device for the removal of the growths, and is based on the assumption that, when praeticable, it is desirable to effeet their removal by galvano-cantery action. The instrument shown in Fig. 19 is designed as a more convenient and more easily applied form of eautery than we have at present.

It has the form of a short, right-angled-bladed pair of seissors, the curves of which are such that the burning surfaee, which is a platinum

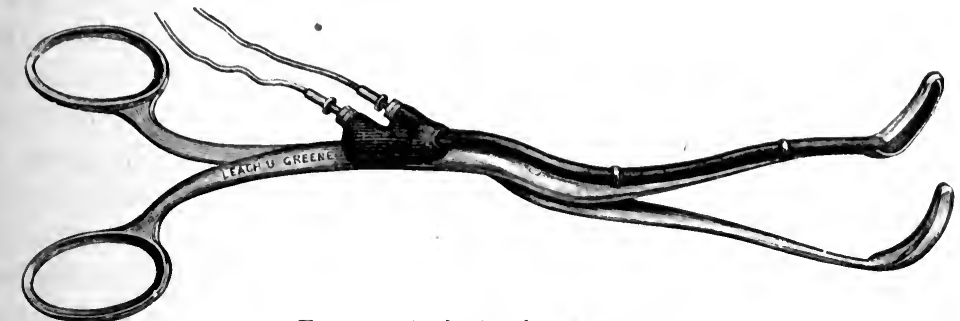

FIG. 19.-Author's seissors cautery.

wire lying in the center of one of the blades, can be applied easily to a growth in whatever part of the bladder it may be situated. This is its prineipal advantage. The wire is insulated by a bit of slate of triangular form, upon the apex or inner surface of which it lies; the opposite blade is similarly furnished with another piece of slate. The triangular form of these bits of slate serves to protect the bladder wall from injury by the heated wire by holding it away from the mueous membrane by about one eighth of an ineh. The cautery can be passed between the blades of the speculum or through their sides, and the growth seized and burned off; as an extra precaution against burning the bladder wall, it is well to interrupt the current frequently, especially if dealing with a tough-based growth, which requires some time to burn through. If the growth be too large to be burned off by one application of the cautery scissors, it ean be removed piecemeal by successive applications. This instrument also has its limitation. It can not be successfully applied to very broadbased, sessile growths, but with this exception it will, I think, be found most useful. The bladder may, of course, be illuminated in using these instruments, as is now usually done, by the electrie eystoseope lamp, introduced through the suprapubic incisions or through the urethra. I have 
used these instruments in four cases-two of bladder tumors and in two prostatectomies. These patients all made a good recovery from the operation, the performance of which was greatly facilitated by these new devices. (The instruments can be obtained of Leach \& Greene, Tremont Street, Boston, Mass., U. S. A. ; or of Krohne \& Sesemann, 7 Duke Street, Manchester Square, London.)

The choice of instruments for the removal of the growth must depend on the nature of the latter, its position, etc. If markedly pedunculated, the pedicle may be caught with forceps, such as those used by Sir Henry Thompson in the perineal operation, or encircled by the wire or galvano-cautery wire snare, or by the author's cautery scissors just described, or divided by scissors. If sessile, it is best removed by the curette, or if of delicate structure, by the finger-nail. The base, unless the removal has been made by a cantery, should be touched lightly with the paquelin cautery, which serves the double purpose of checking hæmorrhage and of destroying the growth more thoroughly. If scissors, forceps, or snare be used, care should be taken not to include the bladder wall beyond the base of the growth in their application; otherwise there is danger of perforating it. The growth, however, should be removed down to the level of the surrounding mucous membrane at least. Another advantage in using the paquelin cautery for treatment of the base of the growth is that it gives a very good illumination of the interior of the bladder. In cases of large, dense growths it will be sometimes necessary to remove them piecemeal. Thorough exploration should always be made by sight and by touch of the whole interior surface of the bladder, in order that no small beginning growths may escape detection which may readily occur when they are multiple. Hæmorrhage, as a rule, is not a serious complication in these operations. Occasionally in a growth with a large, dense base, one or more arterial vsssels of considerable size may be severed and may sometimes be successfully ligated. The best means, however, as a rule, for checking bleeding is by application of the cautery as already mentioned. In case of a free and persistent oozing of blood from the seat of removal of an extensive sessile growth, it is best to pack the interior of the bladder, for a few hours at any rate, with iodoform or sterilized gauze. As a rule, more or less oozing of blood will take place from a site of operation for from one to four or five days, and in exceptional cases for a longer period.

A modification of the bladder incision has been proposed by Antal, of Buda-Pesth, by making a cross cut instead of the perpendicular one, and by dividing the bladder, layer by layer, by successive incisions. One of the advantages claimed for this incision is that in cases in which the peritoneal fold is low it may be avoided, while still obtaining ample room through the wound in which to work. This is of distinct value 
in cases in which the peritoneal fold is found to be low. The wound, however, ordinarily does not give any more room in which to work than the perpendicular cut, especially if the latter be increased, as it may be without harm, by the slight lateral incision at its middle point on either side of the edge.

The after-treatment of the wound is a matter of importance. There are three methods in vogue: immediate suture of the entire wound, with the intention of securing prompt union; partial suture of the bladder wound, with drainage; and the absolutely open method, with drainage. Among those who have used the suture with success are Ultzmann, Parker, Zezas, Antal, Kummel, Orlowsky, Makawejar, Zancarol, Julliard, Reverdin, Kispert, Von Bergmann, Bassini, Koenig, Corradi, Petersen, Mickulicz, Pilchar, Dulles, Lister, Keyes, Lindrier, Lucas Championnière, Pean, Schwartz, Pozzi, Tuffier, Marcsee, Guiard.

As a rule, French surgeons are in favor of the open treatment of the wound, or of partial suture with drainage, as are also Sir Henry Thompson, Dittel, Meyer, and Trendelenberg. In the open method the upper and lower angles of the outer wound only are sutured, the bladder wound left entirely open, and the double-siphon drainage-tubes as used by Perier (Perier employs two large rubber drainage-tubes which he inserts down to the lowest part of the bladder; they lie side by side, and should have, beside the open ends, another hole cut in the bladder extremity of each just above its end) are inserted into the bladder, attached to the margin of the outer wound and led through the antiseptic dressing to a receptacle placed within the bed or on the floor beneath it. In using these tubes the author prefers to cut them off immediately above the antiseptic dressing, and to insert into each a short bit of glass tubing, attaching to the outer ends of the glass tubes long rubber tubes which go to the receptacle. This has the advantage of enabling one to judge more readily at what point in the course of the tubes any stoppage has taken place, should such occur by coagula, and, also (by detaching the glass tubes at the points at which they join the rubber drains leading into the bladder), of securing a shorter circuit for fluids used in subsequent washings of the bladder through them. These washings should be employed daily, as a rule, during at any rate the first few days after the operation; and if there is evidence of cystitis or much débris within the bladder, they should be continued. The better washes to employ under these circumstances are the milder antisepties, such as a four-per-cent solution of boracic acid and a solution of permanganate of potash of the strength of 1 to 3,000 or 4,000 .

If the tubes perform their work properly, as in the anthor's experience they have invariably done, they act as complete siphons, and the fluids of the bladder flow through them completely, keeping the patient 
entirely dry. After the fifth or sixth day the author has found it advantageous to replace the larger drains by two of smaller ealiber, and, unless bleeding still persists, or cystitis is present, at the end of the eighth or

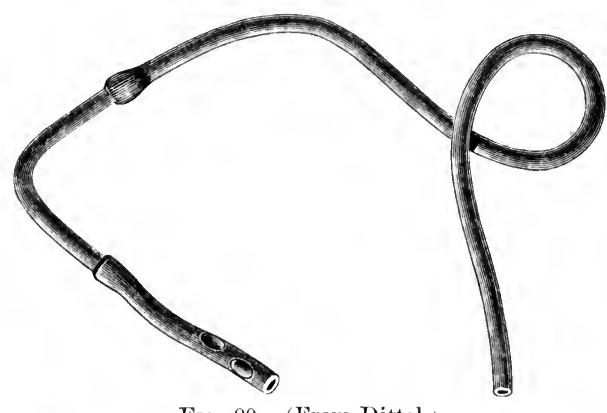

Fig. 20.-(From Dittel.)

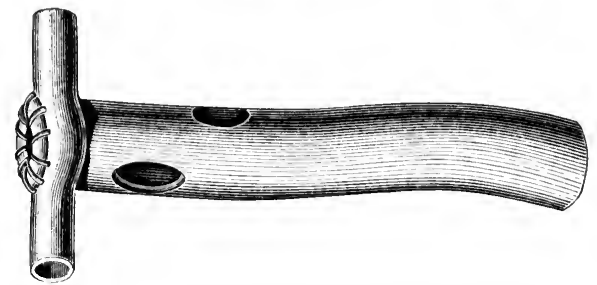

Fig. 21.-Trendelenburg's suprapubic drain. ninth day to remove one of the tubes, taking ont the remaining one from the tenth to the fourteenth day, according to circumstances.

Dittel has made a modification of the drainage-tube, and employs one of hard rubber, which is bent upon itself above the symplissis pubis in such a way that its outer portion points downward between the thighs.

Trendelenberg employs a T-shaped tube. Both of the above are illustrated in the accompanying cuts.

At a meeting of the New York Academy of Medicine, January 12, 1891 (Medical

Record, March 7, 1891), Dr. Willey Meyer describes a form of drainagetube used by him after a suprapubic prostatectomy. This consists of a soft-rubber tube led through a hard-rubber shield, by which it is secured in place. Dr. L. B. Bangs, in the same meeting, also described a drainage-tube of similar pattern.

The advantages of the open method and drainage are: (1) Avoidance of the danger of urinary infiltration into and about the prevesical space; (2) the shorter time required for the completion of the operation as compared with that when vesical suture is applied; (3) the beneficial effect of drainage and rest to the bladder where cystitis is present; (4) the hæmostatic effect of leaving the wounds open.

The one disadvantage of the open treatment which is urged against it by advocates of the closure of the wounds by suture is that of prolongation of convalescence. It is generally agreed that this is true, and that the time before complete healing occurs, when the wound is left open, is increased on an average abont ten days as compared with that of the closure of the wound when successful. The average length of time required for healing of the wounds when they have been left open is from twenty-one to thirty days.

A compromise between total suture and the open method has been 
often practiced in partial suture of the bladder wound, closing it so far as to leave room only for the passage of the drainage-tubes. Examination of the results in point of time and in point of success of this method as compared with the entirely open method, leaves but little to choose between them.

In an original memoir in the Annales des Malad. des Org. Gen. Urin., October, 1890, M. de Dellefosse presents in a very admirable manner the subject of bladder suture as compared with the open methods of treatment. The following is a summary of his remarks :

"In 1883 Dr. Bouley published a statistical report of twenty-three cases of vesical suture after suprapubic cystotomy. In this series of cases failure to unite occurred in ten; silk phéniqué was used eight times, with three failures; catgut was used eleven times, with seven failures; silver wire twice, with no failures; and ordinary silk twice, with no failures. In the cases of failure it was generally upon the sixth day that leakage began."

In 1884 Terrillon published twenty-five cases of suture of bladder wounds which had been made accidentally for ovariotomy. There were fourteen deaths in this series, but in the large majority they were wholly independent of the bladder injury.

Bladder wounds in this class of cases offer the most favorable conditions for the success of the suture, for the bladders are generally healthy, and the wounds are within their peritoneal surfaces, the healing of which by rapid agglutination is far more likely to occur than when the wound lies without the limits of the peritoneal covering.

In 1884 Truffier published a collection of one hundred and twenty cases of suprapubic cystotomy undertaken for various purposes between the years 1879 and 1883. There were of these twenty-two cases of stone in which the suture was applied to close the bladder wound. Among these were only two successes. In the failures, leakage occurred from the first to the ninth day. In one of the two successful cases, interrupted catgut sutures were used; in the other, silk pléniqué. In these two cases the wounds healed finally on the thirtieth day. In sixteen of the failures, three serious accidents can be attributed to the suture.

In 1884 Garcin reported seven cases of suture of the bladder of Boeckel, of which five died of urinary infiltration. In the same year Malharb Webber reported forty-one cases of suprapubic lithotomy and suture, with sixteen successes. There were eight deaths; seven were septic, and one was from erysipelas.

In 1886 Dr. Gross, of Nancy, reported forty cases of vesical suture after suprapubic cystotomy ; of these only two died, 5 per cent as compared with the mortality of the operation, which he states to be 21 per cent, from which he concludes that the suture is less dangerous than 
it has been supposed. In fifteen cases of complete suture there were five unions by first intention, and ten failures, but no accidents. Complete suture of both bladder and abdominal wounds gave the best results, namely, nine cases with four complete successes. Since 1887 the showing has been more decidedly in favor of the suture. In children the results of the suture are especially good.

Dietz, 1890, reports forty-seven cases of suture collected since 1880, with the following results: Two deaths, fourteen failures of union by first intention, thirty-one cases of union by first intention; average duration of treatment seventeen days in the successful cases, twenty-six days in the failures. It is to be remarked that from eleven cases in this series in children all were successful. Dellefosse goes on to say that there are certain conditions in which the majority of surgeons agree that the suture should not be applied. These are, first, in cases of tumors; second, in cases of chronic cystitis with vesical changes; third, in tuberculous cystitis; fourth, in cases where there is a tendency to phosphatic deposit. The advantages of the suture would be certain if, first, it can be shown that with it cicatrization is much more rapid; second, that the cicatrix is more firm; third, that it renders the wound more septic ; and, fourth, that is is without danger. In regard to the first, the advocates of the complete suture claim that, when successful union occurs about the fifth day, yet, nevertheless, the duration of the after-treatment of the wound is seen to continue from eighteen to twenty-six days, and these statements are based upon the results of the most favorable cases. At best there are only about 60 per cent of recoveries after the operation in all classes of cases when the suture has been applied. This is certainly no better than when the wounds have been left open. With the latter method there is the great safeguard that the urine escaping either through or around the tubes does not infiltrate, while with the suture, especially if the outer wound be closed, the condition is one that favors infiltration, by which a distinct danger is thus encountered.

Dellefosse's conclusions are, first, that the vesical suture ought to be rejected in more than two-thirds of all cases of suprapubic cystotomy; secondly, it is only actually indicated in the cases of intra-perineal wounds, and then only when the bladder and kidneys are thought to be normal; thirdly, the partial suture with siphon drainage gives quite as good results.

In the Dictionnaire Encyclopédique, Hache makes the following comments: "What does one gain by suture? If successful, the duration of convalescence is shortened by perhaps ten days; but this advantage is secured at the price of a decided prolongation of the anæsthesia, and especially at the risk of urinary infiltration subsequently."

The author's belief in regard to the application of the vesical suture 
is that until it has been so far perfected as to secure union by first intention, with very few exceptions, it should not be employed for the purpose of total closure of bladder wounds except, of course, in cases of traumatism, or accidental injury during operations upon abdominal tumors, and perhaps in children in whom it is attended with much more success. In cases where cystitis is present, it is of distinct advantage to prolong the drainage, and, moreover, success with the suture in such cases is less likely to occur. Cases of bladder tumors are least of all suited for the employment of total suture, although several entirely successful results are recorded.

In one of the author's cases, in which the bladder wound was closed after the removal of a papilloma, the vesical tenesmus which was produced by the retention of clots within the bladder decidedly increased the shock of the operation, endangered the patient's life, and produced great pain. Relief followed upon the removal of one or two of the stitches, but convalescence was prolonged by the foregoing accidents. If the vesical suture is to be used, that form should be selected which, while it effectually closes the wound, is at the same time capable of being rapidly applied. For this purpose the suture of Brenner, which is in the form of a double purse-string, as indicated in the accompanying figure, is well suited. The application of this suture is described by Brenner thus: "The usual incision is made in the bladder; the mucous membrane is then separated from the muscular coat above it for a slight distance around the margin of the wound; a continuous silk suture $(a)$, as in Fig. 22, is then passed around the wound in the submucosa. A similar suture $(b)$ is previously passed around the wound in the muscular laycr, the suture $(a)$ is then drawn tight and tied, and the same is done with the suture $(b)$."

(The author has twice recently employed this suture to close wounds made in healthy bladders during the course of an abdominal hysterectomy ; the patients died on the sixth and on the fourth

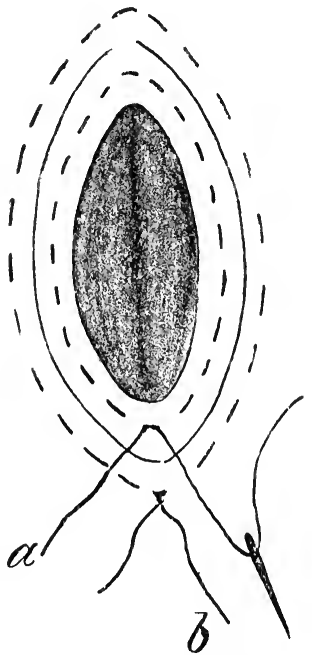

Fig. 22.-Brenner's suture. days respectively, there having been no symptom of bladder disturbance or of leakage in that interval. On examining the organ after death, the closure by the suture was found to be perfect, and the wound was in the process of healthy healing.)

The only objection to its use lies in the fact that it compromises a larger portion of the bladder than others. Unless, however, the wound is of large extent, this is an insufficient objection. It is very ready of 
application, and one of the simplest in use. The form of suture which the author prefers is the right-angled suture suggested by Dr. H. W. Cushing, of the Boston City Hospital, for the closure of intestinal wounds, which is illustrated in the following figure (23).

If the complete vesical suture be applied, the abdominal wound may be left open through the greater part of its extent or wholly closed. In this

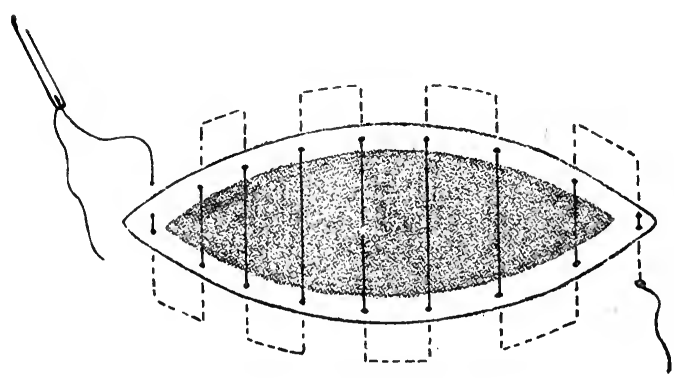

FIg. 23.-Cushing's suture. connection the report of Alexandron (Arch. für klinische Chir., 41. Band, 4. Heft, 1891, page 877) should be noted. In eighteen out of twentysix cases in which he sutured the bladder after a sectio alta, he left the lower angle of the abdominal wound open, and drained it; in none of these cases did he secure a complete union by first intention. The.remaining eight he sewed all the wounds tight. In seven of these he got a complete first intention without any leakage of urine whatever. In one case the sutures were removed owing to an intravesical hæmorrhage. These cases were all in children; but so were the first series of eighteen, and it must be admitted that the uniformly better result in the second series of eight is suggestive of the desirability of further trials in the direction of total suture of all wounds; but a further experience is needed to support the same view in regard to the advisability of this plan in the varying and more difficult conditions encountered in adults; and in bladder tumors, in which connection the subject is here considered, the desirability of closure of the wound is most open to doubt.

Up to the present time it can not be said that the total suture of the abdominal wound is free from danger to life, and certainly the percentage of successes does not at the present rating exceed 70 per cent in adults in the most favorable computation. The author's experience has been so distinctly favorable to the employment of the open or partially open method of treating the bladder wound, that he is strongly prejudiced in its favor. Of five operations for the removal of benign bladder tumors treated by siphon drainage and open method, the wounds, abdominal and bladder, closed in the following periods of time:

The first, on the fifteenth day; the second, on the eighteenth day; the third, on the eighteenth day; the fourth, on the sixteenth day; the fifth, on the twenty-third day. The patients were out of bed at the end of these times. 
Helferich's Partial Resection of the Symphysis Pubis.-In the Arch. für klinische Chirurgie, Band 73, part third, page 625, 1888, Helferich proposes in certain exceptional cases a partial resection of the symphysis pubis. He advises the performance of the operation in the following way:

A horizontal incision is made just above the upper margin of the symphysis pubis, extending laterally on either side to a point slightly beyond the tubercle of the os pubis, taking care not to wound, of course, the vasa deferentia. The soft parts are now divided down to the bone, and separated thoroughly from it. The periosteum need not be preserved except at the extremities of the exposed bone on either side. The bone is now divided to the desired depth by a chisel applied just beyond the tubercle of the os pubis on either side, taking care not to open the obturator foramen; the portion of the bone included between the two chisel-cuts is now removed by the chisel applied in a horizontal direction, or sidewise along its anterior surface. The finger is placed on the under surface of the symphysis during the chiseling, so as to guard against any injury to the bladder by the instrument. As soon as the bone is divided, the soft parts attached to the inner surface of the symphysis are drawn to either side by hooks, and the anterior surface of the bladder becomes widely exposed.

Helferich originally added a perpendicular eut to the horizontal one, making a T-shaped incision, but has subsequently decided that the cross cut alone is sufficient.

(Helferich favors the cross incision, in the ordinary suprapubic operation, in preference to the usual perpendicular cut.)

The partial resection gives a much better opportunity than the ordinary method to strip back the peritoneal covering, where this is desired (as in the extra-peritoneal method of resecting the portion of the bladder in the case of malignant growth of the apex). Helferich advocates this partial resection as described, in any case in which either the size of the stone or tumor, or low attachment of the peritoneal fold, or its adhesion from inflammatory action or a non-distensible bladder, makes a large wound desirable, or the bladder inaccessible by the usual suprapubic cut.

Helferich adds the report of two cases, in one of which he performed the above operation for disease of the bone of the symphysis, the patient making a good recovery. In the second he removed a carcinoma of the bladder, and states that the growth was much more readily dealt with than would have been the case by the ordinary incisions.

He claims that no interference of locomotion from weakening of the pelvic arch ensues from his operation.

The author has no experience of this operation, but has performed it on the cadaver, and it certainly gives freer access to the bladder, and commends itself for further trial under the special circumstances for which it was designed. 
In 1888 Langenbuch proposed another route to the bladder, which he called "sectio alta subpubica." By this he proposed to expose the anterior surface of the organ by an incision between the pubic arch and the root of the penis. Langenbuch claims for this method the following advantages: (1) The opening of the bladder is at the lowest point; (2) avoidance of the peritonæum ; (3) avoidance of urinary infiltration; (4) avoidance of the necessity of a catheter in the urethra for subsequent drainage, and of the need of bladder suture; (5) the possibility of union by first intention.

The incision is made in the form of an inverted $Y$, the vertical incision extending from the root of the penis over the symphysis, the two sidecuts right and left on either side of the penis. The penis is loosened from its attachments to the symphysis. The triangular ligament is then divided beneath the arch of the symphysis, and the urethra with its attachments is pushed backward; by this means a free space is given in which to reach and open the bladder, which is done by an incision above the plexus of Santorini.

Langenbuch tried this method on a patient with impassable stricture; hæmorrhage was free, the space opened was not large enough until a portion of the symphysis was resected; urinary infiltration and suppuration followed. There seems little to recommend this method.

\section{OPERATIVE TREATMENT OF BLADDER TUMORS IN THE FEMALE.}

In women the removal of bladder tumors is rendered easier by the greater accessibility of the organ. In the case of malignant growths where drainage only, is sought for relief, the most natural means to employ is an opening through the vesico-vaginal septum. To secure continued patency of the wound, if desired, the edges of the wound may be sutured throughout their extent, or a large button, of the form of a shirtstud, with a hollow center, may be inserted through the wound, one plate resting upon the inner surface of the bladder, the other upon the vagina.

The only advantage of the suprapubic route in malignant disease, as compared with the vaginal, is that, if extension of the growth occurs through the wound, it is more easily dealt with in the former.

The dilated urethra affords, for small and easily removable tumors, the safest and most satisfactory way, and in any case, except those in which it has been already determined that there is a large growth present, the urethra affords an easy and satisfactory road by which to determine the situation and characteristics of the tumor by digital exploration-the objection raised against the latter procedure in the male, that it involves an additional cutting operation, being absent in the female. If removal be impracticable by this route, the suprapubic or 
colpocystotomy remain. Of the last two, the advantage certainly lies with the former, in that it gives a satisfactory view of the interior of the bladder, and more room in which to work. The additional risk in a colpocystotomy, also of a vesico-vaginal fistula remaining, though small, is still something.

\section{ULTIMATE RESULTS OF OPERATION FOR THE REMOVAL OF BENIGN GROWTHS.}

It is difficult to obtain a satisfactory judgment of the final results of these operations, owing to the facts that but few years have elapsed since these operations have been systematically practiced in considerable numbers of cases, and also that in but few of the cases reported has the subsequent course been noted.

The following series of cases, in which the author has found the history subsequent to operation indicated is small, and can give, therefore, but a very partial idea of what may be expected from the treatment, and it is presented in this sense only. Sir Henry Thompson gives the following summary of the results, immediate and final, of the operations which he had performed in forty-one cases, of which thirty-four occurred in male and seven in female patients:

" 1 . In seven cases there has been no reappearance of symptoms of any kind, three years having elapsed since the most recent, and nearly ten years since the first case of the series. All are living and well except one, who met his death by accident some two years after; but the bladder was examined by his medical attendant, after death, and sent up to the museum of University College Hospital, London, where it now is, together with the tumor originally removed; no trace of reappearance can be found in it. In every case but one the nature of the tumor was papilloma, the exceptional case being probably a myoma. In three cases the growth was removed by forceps through a perineal incision; in one female case dilatation of the urethra sufficed, and in the three remaining cases by suprapubic operation.

" 2 . In fifteen other cases death occurred at different periods, varying between three days and four months after the operation. Ten of these were the subjects of malignant growth of some kind, epithelioma and sareoma in two or three varieties. Two were papilloma, apparently simple. The rest were myomatous, but contained nuclei which aroused suspicions of a grave character. It is worthy of remark that in five of this series papilloma was also present on the surface, and therefore probably as a secondary product to a more important growth, as myoma or sarcoma.

" 3 . Nineteen patients remain who lived after the operation for periods varying from about a year or a little less up to four years, one of 
these being now in good health (two years after the operation), much stronger and stouter than before, but is just beginning to note the reappearance of a little blood, and is, therefore, not entitled to be regarded like the first elass, or those in whom the recovery was permanent."

In addition to Sir IIenry Thompson's report, the eleven following cases of benign growths have been found, in which the result as to recurrence or death at a period exceeding four months has been noted:

1. Winckel. Female, aged thirty-nine. Papilloma. Removed per urethra by finger. Recurrence in five years.

2. Schutz. Female, aged eighteen. Telangiectasic fibro-myoma, three times operated. First, broken down and removed. Second time, same. Third, extirpated and base destroyed. First, great relief for one year. Second, operation for two years and six months. Third, recovery.

3. Marsh. Female, aged two. Papilloma. Removed by ligature. Death from exhaustion sixteen months later.

4. Alexander. Female, aged thirty-six. Papilloma. Removed by finger-nail per urethra. Recurred twice. Final result not stated.

5. Balleray. Female, aged two. Papilloma. Removed by ligature and scissors. Death six months later from exhaustion.

6. Davies Colley. Male, aged thirty-two. Papilloma. Long pedicle. Removed with scissors through a lateral lithot. Excision. No recurrence in four years.

7. Tiffany. Male. Papilloma. Removed through median perineal incision. Recurrence four years later.

8. Bernard Pitts. Male, aged forty-seven. Papilloma. Removed by a suprapubic cystotomy. Recurrence at end of two years after the first operation.

9. Watson. Male, aged thirty-one. Single papilloma, size of a Spanish chestnut. Removed by snare through a suprapubic cystotomy. No evidence of recurrence five years later. (Patient reported at that time.)

10. Watson. Male, aged thirty-six. Papilloma. Removed by curette through a median perineal incision. Recurrence one year later. Same operation repeated. Five growths removed by same means. Recurrence one year later, six growths were then removed through a suprapubic cystotomy by cautery scissors, curette, and ligature. Two years since last operation. Hæmaturia has just reappeared.

11. Watson. Male, aged twenty-four. Papilloma. Removed by curette through a median perineal incision. Died two years later of pyonephrosis. Autopsy showed the bladder to be absolutely free from any recurrence of the growth. Patient, who had had profuse hæmaturia, had no return of it after the operation. 


\section{TREATMENT OF MALIGNANT GROWTHS.}

The question of treatment in a case of malignant growth is less easily decided than in that of a benign one.

The means of determining the nature of the growth which were set forth under the heading of diagnosis may be briefly recapitulated as follows: If an area of induration can be felt by the finger in the rectum, if the patient be over fifty years of age, and if the cystoscope shows a growth with an ulcerated surface, which has raised irregular, ragged, everted, and œdematous edges. If pain be a marked symptom, the chances are strongly in favor of the disease being malignant.

The decision as to operation will depend, in such a case, upon the amount of pain or hæmorrhage present. It is difficult to see what objection can be raised to performing a palliative operation with a view to relieve pain or check hæmorrhage in this class of cases; and it is the anthor's decided belief that such should be done whenever either of these indications are present.

\section{DRAINAGE FOR RELIEF IN MALIGNANT DISEASE.}

As has been mentioned, pain sooner or later becomes a prominent feature in most cases of cancer of the bladder, either from the presence of the growth itself, or from the cystitis which so frequently arises during its progress; to relieve this symptom or to check hæmorrhage, and to ease the vesical tenesmus which accompanies the latter when excessive, we can drain the bladder either from the perinæum or through a supra pubic cystotomy. Of the two, the author has found the latter the more satisfactory. The former may be entirely efficient if it is simply a question of relieving pain, but fails to give exit to the clots in cases of severe bleeding, and so fails to relieve. If the perineal operation is decided upon, the urethra is opened in the perinæum by the ordinary median incision upon a grooved staff; the neck of the bladder is then stretched by large sounds, and finally by passing the index-finger through it, and a large drainage-tube is inserted into the bladder through the wound and secured in place. For this purpose a large-eyed, soft rubber, or webbing catheter may be used, or, better, the large straight double tube devised by Reginald Harrison for perineal drainage, or the author's perineal drainage-tube, which was suggested for bladder drainage three years ago, and described as follows :

The perineal drainage-tube, which the accompanying cuts illustrate, was designed to secure thorough drainage of the bladder after external perineal urethrotomy, whether this operation be performed for stricture of the urethra, or as a preliminary step in the removal of bladder tumors, or for prostatotomies or prostatectomies, or for perineal cystotomy for any cause. 
The essentials in such a drainage-tube are, firstly, that it shall have a thin wall and a large caliber. These are obtained by making the tube large and of thin hard rubber. Secondly, cleanliness and ease in keeping

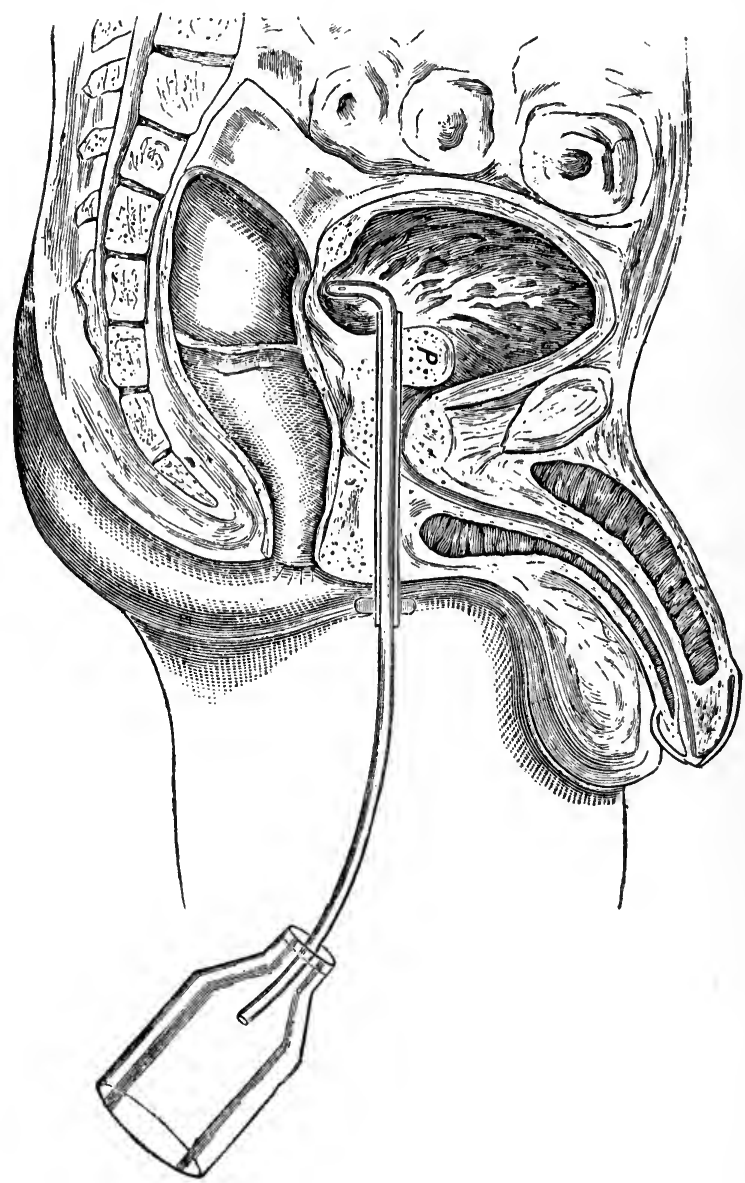

Fig. 24.-(After Ilarrison.)

clean. Placing a large eye elose to the end of the tube, so as to have no cul-de-sac beyond it, and the polished surface of the hard rubber accomplish these objects. Thirdly, the tube must have such a curve that its bladder end shall occupy the lowest part of the bladder, while the outer end shall lie in a parallel plane to the bed when the patient is on his back; the intermediate shaft must have the form and direetion of the posterior urethra between the vesical orifice and the perineal wound.

To establish this eurve, measurements were taken upon twenty cada- 
vers (soft metal being used for the purpose). The shape and direction of the tube, as seen in the figures above, represent the average result of these measurements in an adult between the ages of thirty and fifty.

Fourthly. Inasmuch as the distance from the outer surface of the perineal wound to the bladder orifice varies more or less, owing to individual peculiarity, or to prostatic hypertrophy, and consequent lengthening of the prostatic urethra; and further, as the depth of the bas fond below the vesical orifice also varies in different instances, it is necessary, to meet the first indication, that it should be possible to shorten or lengthen this distance at will, to suit the individual case. This is secured in the con-

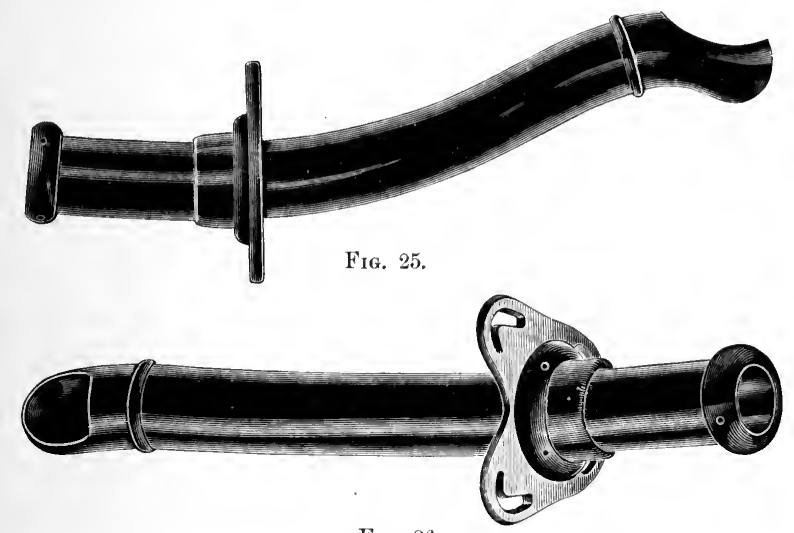

FIG. 26.

Figs. 25 and 26.-A A thor's perineal drainage-tubes.

struction of the plate and collar which surrounds the tube, which can be pushed forward or backward on its shaft by exerting some force, but will stay at the point at which it is placed.

To drain a deep bas fond, such as often occurs behind a median prostatic enlargement, the curved end of the tube which lies in the bladder is made longer in some of the instruments.

Fifthly. The collar is made to fit the perinæum, and has slots through which tapes are passed, by which it is secured to a waist-belt.

Sixthly. To guard against hæmorrhage-as, for instance, may occur in the course of the deep urethra after prostatotomy or prostectomy-some of the tubes have been made with a ridge just above the eye, so that they can be used as a cansla à chemise. A raised edge at the outer end of the tube allows a bit of tubing to be attached, through which the urine can be led into a urinal.

The practical application of these tubes has been made in many cases by the author and by other surgeons, among them Reginald Harrison, who speaks favorably of them. 
Where hæmorrhage from the bladder is likely to be abundant, as after removal of a bladder tumor, the large, smooth-sided tube gives the best ehance for the exit of large clots.

These drainage-tubes are made of different ealibers and lengths, and with or without the ridge which permits of their being eonverted into a canula à chemise, by Messrs. Codman \& Shurtleff's, No. 13 Tremont Street, Boston.

These tubes require somewhat more care in their adjustment and more attention than the ordinary soft-rubber tubes or catheters do. Only ordinarily intelligent eare, however, is needed to keep the tube in its proper place, and to have it perform its work satisfactorily.

In the presenee of severe intravesical bleeding, however, the perineal operation is unsatisfactory, for the reason that it is difficult and often impossible to reach by this route the souree of the hæmorrhage, and to check it; and if this is not done, the bladder will continue to be filled with clots, sometimes so dense and large that they can not make their exit through the perineal tube, no matter how large it may be, or what form it may have. In such cases, therefore, the suprapubic route should be selected by preference, and when this is done the operation should be a free cystotomy and not a puncture with the retention of a canula, or the insertion through a small opening of a catheter or single drainagetube.

The danger of urinary infiltration into the prevesical space after the latter operation has been done was insisted upon by the author in his monograph upon The Operative Treatment of the Hypertrophied Prostate in 18ss; and, moreover, if hæmorrhage is present, the bladder will not be efficiently drained by this means. The operation should be performed, if the purpose is for drainage for relief alone, just as though the intention was to remove the growth. By this means the dangers of the puncture are avoided, and the additional advantages are gained: (1) Of having room to seek out the source of the bleeding, and to check it by the application of cautery, curette, or ligature. (2) Of being able to empty thoroughly, and thereafter drain the bladder by the double siphontubes of Perier (already described). (3) Should an error of diagnosis have been made, and it be discovered on actual inspection that a benign and not a malignant growth be present, its removal ean be undertaken. (4) Even if malignant growth be present, its partial removal (if the patient's condition permits) can be performed, and in some cases, especially those in which there is a large neerotic salient mass present, this should be done. For this substitutes a cleaner surface for the foul one, for a time at any rate, although it is true that these sprouting growths recur oftentimes very rapidly, and may invade the edges of the wound, and extend ontward throngh it. 


\section{RESECTION OF THE BLADDER.}

In certain cases of cancer an attempt at removal of the growth, together with that portion of the bladder which it involves, is warranted by past experience, slight as it is. The cases suitable for such resections are necessarily rare, for they are only those in which the growth is situated at or near the summit of the bladder, and of limited extent. This implies an early diagnosis, and the site mentioned is, as already demonstrated, a rare one for the primary occupation of the growth. When the growth has deeply infiltrated all the layers of the bladder, and has involved the peritonæum as well, or, not having actually included the latter, has gone so far as to cause its intimate adhesion to the outer layers of the bladder, the peritoneal covering must of necessity be included in the resection. In this case the peritoneal wound should of course be at once carefully sutured.

If the fissure has not penetrated so far, the extra-peritoneal operation, as advised by Von Antal, may be performed, and offers a chance of success. This operation consists in the stripping off the peritonæum, and then resecting the affected portion of the bladder, uniting the wound so made by suture.

Another plan which may occasionally prove successful is that practiced by Guyon, of dissecting the growth from the deeper layers of the bladder without penetrating its outer coat. In one case Guyon states that he recognized the adipose layer described by Clado as sometimes to be found interposed between the base of a cancerous growth and the sound tissues beyond.

Barling, in an interesting article in the Birmingham Medical Review, cites the four following cases of bladder resection for cancer:

1. Bruce Clarke. Elderly man. Suprapubic cystotomy. Growth, a flat-celled cancer of the vertex of the bladder. Touched with cautery. A few days later the growth and a piece of the bladder wall three inches by two inches, including their whole thickness, were resected. The operation involved a cancerous piece of the peritonæum, wound of which was closed by suture. Death in a few days. No peritonitis.

2. Sonnenberg. Female, aged sixty. Suprapubic cystotomy. Resection two thirds of the bladder. Peritonæum extensively injured. Peritoneal part of the wound sutured. Death at the end of one month by exhaustion.

3. Von Antal. Male, aged fifty-one. Suprapubic cystotomy. Peritonæum stripped off. Growth size of child's fist on vertex of the bladder. Peritonæum stripped far back. One third of bladder resected with growth. Peritoneal cavity not opened. Recovery from the operation. Recurrence in one year. 
4. Guyon. Male, aged fifty-five. Suprapubic cystotomy. Resection of growth about the size of last joint of thumb from right side of the bladder. Recovery. Death four years later from local recurrence. In this case the adipose layer, described by Clado as lying between the peritonæum and the deeper part of the bladder, was seen by Guyon.

Barling's conclusions as to operation in cases of cancerous growths are these :

1. The hope of cure by excision lies in the early diagnosis and operation before the patient's powers have been sapped by the disease, and before secondary changes due to obstruction or secondary growths have occurred.

2. For the rare cases in which the tumor is seated at the vertex. Antal's extra-peritoneal method (stripping back the peritonæum) should, if possible, be followed. By it about one half the bladder may be resected without opening the peritonæum (in favorable cases). Some of the muscular fibre is likely to be stripped off with the peritonæum. The bladder should be full during the attempt to strip off the peritonæum, as it is much more easily done when this is the case.

\section{SARCOMA.}

Of twenty-one cases of sarcoma in which operation was undertaken, reported by Hinterstoisser, the results were as follows : Six extirpations through the female urethra; two deaths due to the operation (one peritonitis from bladder perforation); two deaths after a few months by recurrence : four deaths; two recoveries. Both were cases of small pedunculated tumors. One colpocystotomy, recovery. Seven median or lateral cystotomies. Three deaths; two recoveries; two not known; deaths due to 1. Peritonitis. 2. Rapid recurrence. Five sectio alta : two deaths due to operation; two from rapid recurrence ; one not recorded. Two lateral cystotomies immediately following sectio alta, which failed to allow of removal of growth: one death from recurrence in eight months; one recovery. Total deaths following operation in twenty-one cases, twelve. Six recoveries. Three unrecorded.

The longest period withont recurrence which the author has found recorded is that of five years in one case.

In connection with the subject of the removal of malignant growths, the following remarkable case of Paulik's is of especial interest, as indicating still further possibilities of a successful issue by the most radical of procedures.

\section{EXTIRPATION OF THE BLADDER IN THE FEMALE.}

"The patient applied to his clinic on account of persistent hæmaturia. By catheterizing both ureters, hæmorrhage from the kidney was ex- 
cluded. Digital exploration of the bladder revealed the presence of a polypus the size of an almond, which was removed per vaginam through an incision, its base being thoronghly cauterized. The patient was discharged cured. A year later she returned, extremely anæmic, stating that after enjoying good health for eight months the hæmaturia returned and had never ceased. Through the endoscope a diffuse papillomatous growth was seen, portions of which were removed for microscopical examination and proved to be malignant. The writer determined to extirpate the entire bladder, after turning the ureters into the vagina, in order to form a new bladder from the latter and the remains of the urethra. The preliminary operation was performed by inserting metallic catheters into both ureters (the patient being in the knee-elbow posture), incising the vagina opposite to the terminal extremity of each ureter, dissecting out the duct, and then ligating and dividing it two fifths of an inch from its opening into the bladder. The eut end of each ureter was then stitched into the corresponding vaginal wound after removing the catheter; after healing had occurred there were thus formed two utero-vaginal fistulæ. Three weeks later the radical operation was performed. An incision was made as for a suprapubic lithotomy, the peritonæum was dissected from the bladder, and the latter was separated from its attachments as low down as the urethral opening, the organ having been previously distended with an iodoform emulsion in order to render it more prominent. At this stage the bladder was emptied, and the cavity already made was packed with iodoform ganze on account of the oozing. The anterior vaginal wall was now incised just above the most prominent part of the urethro-vaginal septum, the bladder was drawn down through the wound and was cut away at the vesical orifice of the urethra.

"Here the patient collapsed, but was revived by the subcutaneous injection of ten ounces of warm salt solution, and the operation proceeded. The anterior vaginal wall was sutured to the anterior border of the urethral wound, the posterior border being united to a freshened surface at the entrance of the vagina in such a way as to produce a kolpokleisis. Before the sutures were tied, elastic catheters were introduced into the urethra and carried into the ureters. The abdominal wound was closed, except at its lower end, through which were carried the ends of the gauze for drainage. The patient made a good recovery, but a fistula remained which communicated with the cavity that had formerly been oceupied by the bladder, which did not heal for eight months. A second unsuccessful attempt at kolpokleisis was made ten months after the primary operation. This was repeated a month later, the anterior and posterior walls being united transversely; a minute fistula remained behind the urethra, through which urine escaped only when the patient was in an 
erect posture. She was able to retain control of her artificial bladder until twelve ounces of urine had accumulated, when she could empty it by contracting the perineal muscles. The woman made a perfect recovery and was able to undertake a journey from Prague to Berlin, and to spend several days in sight-seeing."-American Journal of Medical Sciences, January, 1891, p. 101, No. 225.

In this connection also are to be mentioned the following cases which antedate the case of Paulik's reported above, and are, so far as the author knows, the first examples of the total extirpation of the bladder or its mucous membrane in the living subject. They are reported by Brohl (Wiener Med. Presse, 1889, Nos. 27 and 28), the operations having been performed by Von Badenhener :

$\mathrm{C}_{\text {ASE }}$ I. Fifty-seven-year-old patient; a tumor occupied the larger part of the base of the bladder - the bladder was extirpated (the symphysis pubis being partially divided to expose the organ); for ten days the progress was favorable. Death on the fourteenth day from uræmia.

$\mathrm{C}_{\Delta \mathrm{SE}}$ II. Girl, seven years old ; primary tuberculosis of the bladder, mucous membrane of the bladder extirpated. Recovery. Death eighteen months later from tubercular peritonitis.

CASE III. Man sixty-four years old. Entire interior of the bladder occupied by disseminated cancerous (?) growths. Excision of the whole of the mucous membrane of the bladder, and resection of a large area of the posterior wall, which was occupied by a large single tumor. Recovery.

CASE IV. Man, aged thirty, multiple small tumors of the bladder; total excision of the mucous membrane. Recovery. 


\title{
STONE IN THE BLADDER, PROSTATE, URETHRA AND URETERS.
}

\author{
BY ARTHUR T. CABOT, M. D.
}

\section{STONE IN THE BLADDER.}

Definition.-It is not easy to state concisely what constitutes a stone in the bladder; and Surgeon-Major Freyer was criticised by Sir Henry Thompson for including in his statistics stones weighing less than twenty grains. In reply, he pointed out the fact that under some circumstances the removal of small stones was a matter of considerable difficulty and danger; and it is to be borne in mind that before the introduction of lithotrity even small stones required serious cutting operations for their removal. A stone in the bladder may be defined as a concretion which the bladder is unable to expel by its natural efforts, and which therefore requires surgical interference for its removal.

Chemical and Physical Characteristics. - These concretions are composed mainly of salts, or solid constituents of the urine, which are thrown down in a more or less crystalline form, as will presently be shown. Urinary calculi may be classified according to the principal ingredient which goes into their composition, and may accordingly be divided into the following groups :

1. Uric stones, composed of uric acid and the acid urates.

2. Oxalic stones, composed of oxalate of lime.

3. Phosphatic stones, which contain phosphates and carbonates, not infrequently combined with urate of ammonia.

We have also in the urine certain organic substances which sometimes exist in such amounts as to be deposited in the shape of calculi. Of these, cystin is the most common, but concretions of it are of very unusual occurrence. Xanthin and indican calculi have been reported, but are exceedingly rare. Occasionally hæmatoidin and coloring matters from the urine are found in stones, but rarely in any quantity. Cholesterine and fatty materials have been observed, and a few instances of fibrinous masses in the urine are reported. These rarer constituents of urinary concretions have a scientific interest in connection with the chemistry of the body, but are so infrequent as to be of little practical importance. For ordinary purposes the division into uric, oxalic, and phosphatic stones sufficiently distinguishes the different kinds we meet. 
While a calculus is to be referred to one or other of these classes, according to the character of its main constituent, it is not unusual to find one belonging partly in one group and partly in another. Thus, for

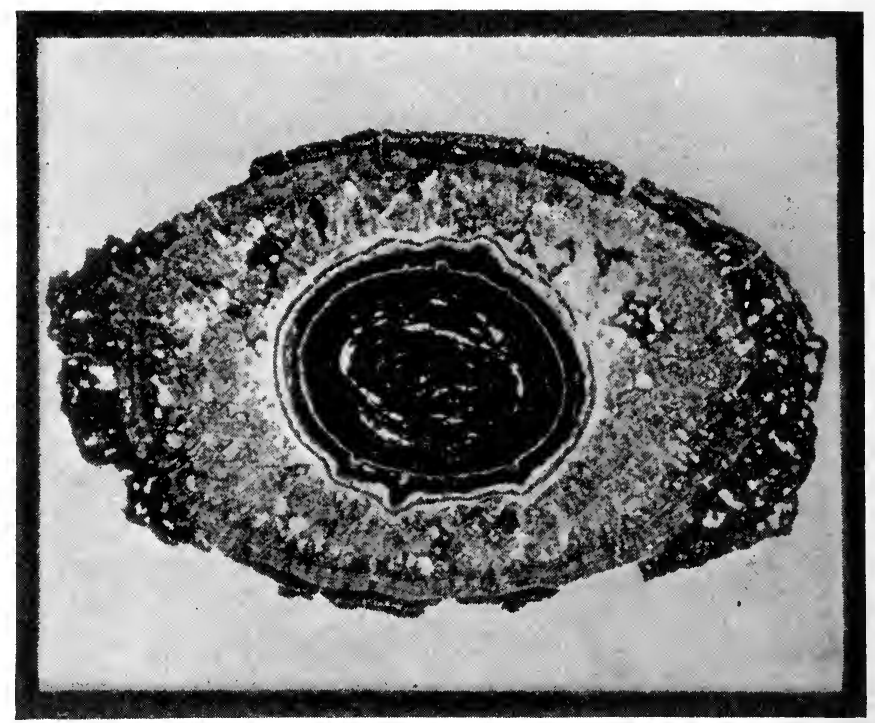

FIG. 1.-A stone having a nucleus of urate of soda, a middle layer of oxalate of lime, and an outside layer of earthy phosphates.

instance, a stone with a uric-acid nucleus will often have an oxalate of lime sheet or layer around it, and outside of that again a phosphatic layer. Sometimes several constituents are mixed more or less evenly throughout the stone. Thus a uric-acid calculus may contain oxalate of lime distributed through it. The phosphates and carbonates are usually thus combined.

If the crystalline matters are dissolved ont of a stone, an albuminoid mass is left, which has served as the skeleton in which the salts were deposited. This framework or basis substance exists even in the minutest bits of sand, and in oxalate stones is partly made up of cells, which Ebstein believes to be epithelial cells, and in which he has shown the oxalate of lime to be actually deposited. Cystin stones have less of this albuminoid skeleton than the other urinary calculi.

The researches of Rainey, Harting, Ord, Ebstein, and others indicate that the formation of stone is not a simple process of deposition of salts existing in excess in the urine. Indeed, instances are of every-day occurrence in which uric acid, the urates, oxalate of lime, or the phosphates are present in solid form for long periods of time in the urine without 
leading to stone formation. The investigations of these observers have shown the crystalline substances of the urine to follow the law of "molecular coalescence" laid down by Rainey and elaborated by later observers; which is," briefly, that "in the presence of colloid or albuminoid snbstances crystalline materials become spheroidal in shape and coalesce in rounded form." Following this law, in the urinary passages crystals of uric acid in their usual rhomboidal shape may for long periods be thrown down, washed along, and passed out with the urine without showing any tendency to form concretions. Finally, the irritation of the urinary tract leads to the exudation of albuminoid material, which, acting on the crystals, changes their molecular form and creates in them the tendency to coalesce. The necessary albuminoid material may be supplied in other ways. Necrotic bits of tissue are, as is well known, likely to be incrusted with lime salts. Masses of bacteria and bits of blood-clot are also found

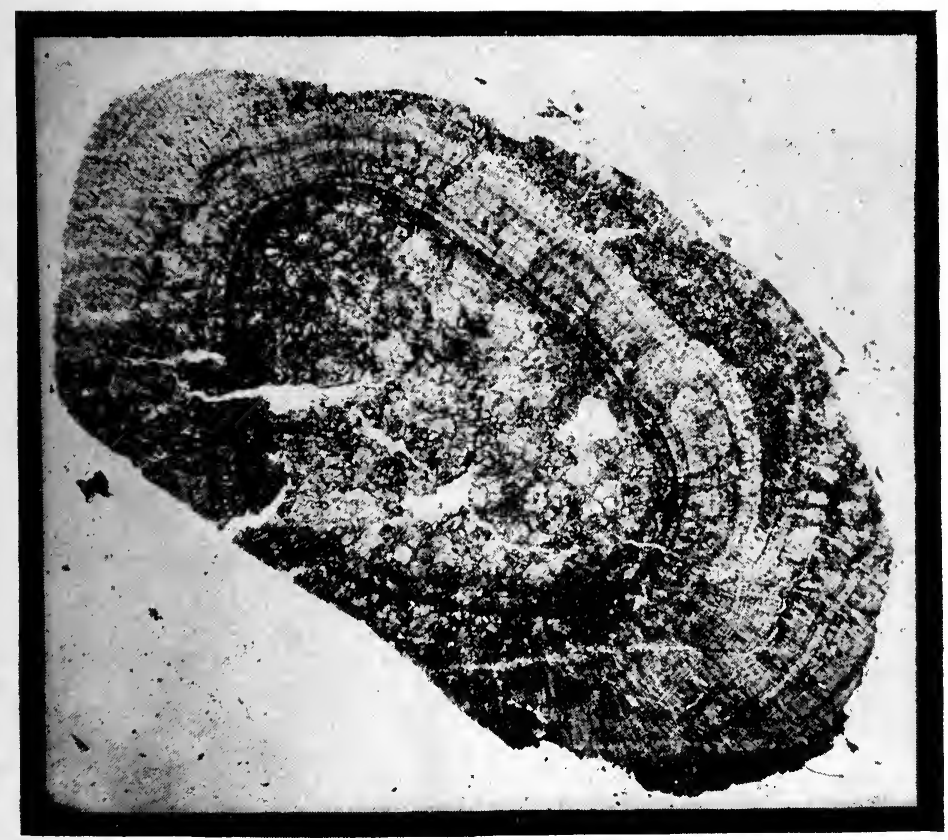

Frg. 2.-A uric-acid stone, showing a nucleus, partly granular partly crystalline, surrounded by well-marked concentric layers.

in stones, but what part they play in the formation of the stone is not clear. The concretion, once started, continues to act as an irritant to the wall, and so continues to be supplied with an albuminoid envelope, in which successive layers of spheroidal crystals are deposited. 
Ultzmann (Die Harnconcretionen des Menschen und die Ursachen ihrer Entstehung), by a study of thin sections of stone, arrived at conclusions somewhat at variance with these. He obtained sections thin enough to make good microscopical preparations, and his study of these led him to think that "stone formation in man does not consist in a simple agglomeration of sedimentary substances held together by an organic basis material, but that it is a real cyrstallization (Massen Krystallisation), which follows common crystographic laws." Unfortunately, Ultzmann seems entirely ignorant of the researches of Rainey, Ord, and others, on changes in crystalline form brought about by the presence of an albuminoid in the mother-liquid; and, having shown in his thin sections that there is often a more or less regular crystalline form, he jumps to the conclusion that he has hereby disproved all of the hypotheses that require an organic basis substance in the building of a stone. He even denies the existence of such an organic skeleton, on the ground that he sees no traces of it in his thin sections. Apparently, he never tried the experiment of dissolving out the solid constituents, and so demonstrating the existence of the albuminous framework.

Although he draws such hasty and unproved conclusions from his study of his sections, they will be found interesting and worthy of close inspection, and some of them are accordingly copied here. Being re-

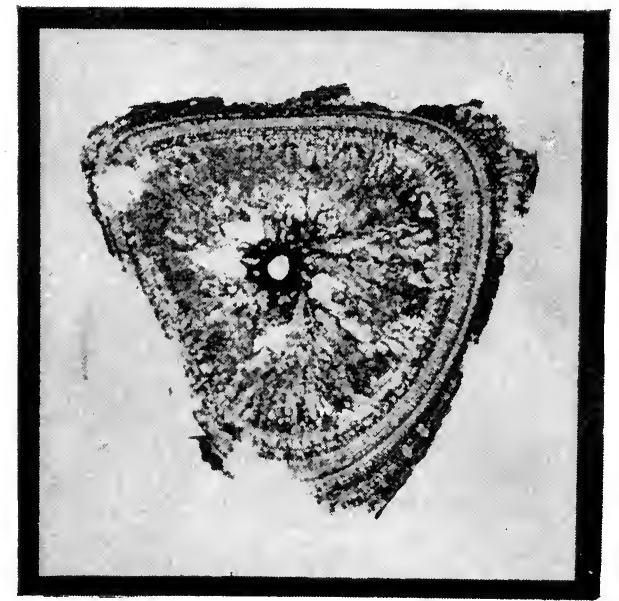

Fla. 3.-A stone composed of earthy phosphates. In the central portion it shows the radiating strice, surrounded by outer coneentric layers. ners of the crystals are usually rounded, as if the molding tendency of the albumen had been partly felt. produced from micro-photographs, they tell the truth as far as the imperfections of the photographic process allow. In many of them (Figs. 2,3 , and 6 ) the globular character of the crystals may be seen, and they confirm the accepted opinion as to the effect of the surrounding albuminoid on their manner of crystallization. In some sections, which show these rounded forms quite well, are to be found places in which the crystallization has more of an angular character, and it is on these appearances that Ultzmann founds 
A superficial examination of the plates shows the concentric formation of these stones, with more or less plainly marked rings or zones (Figs. 2, 3, and 4). This appearance reminds one of the rings seen on

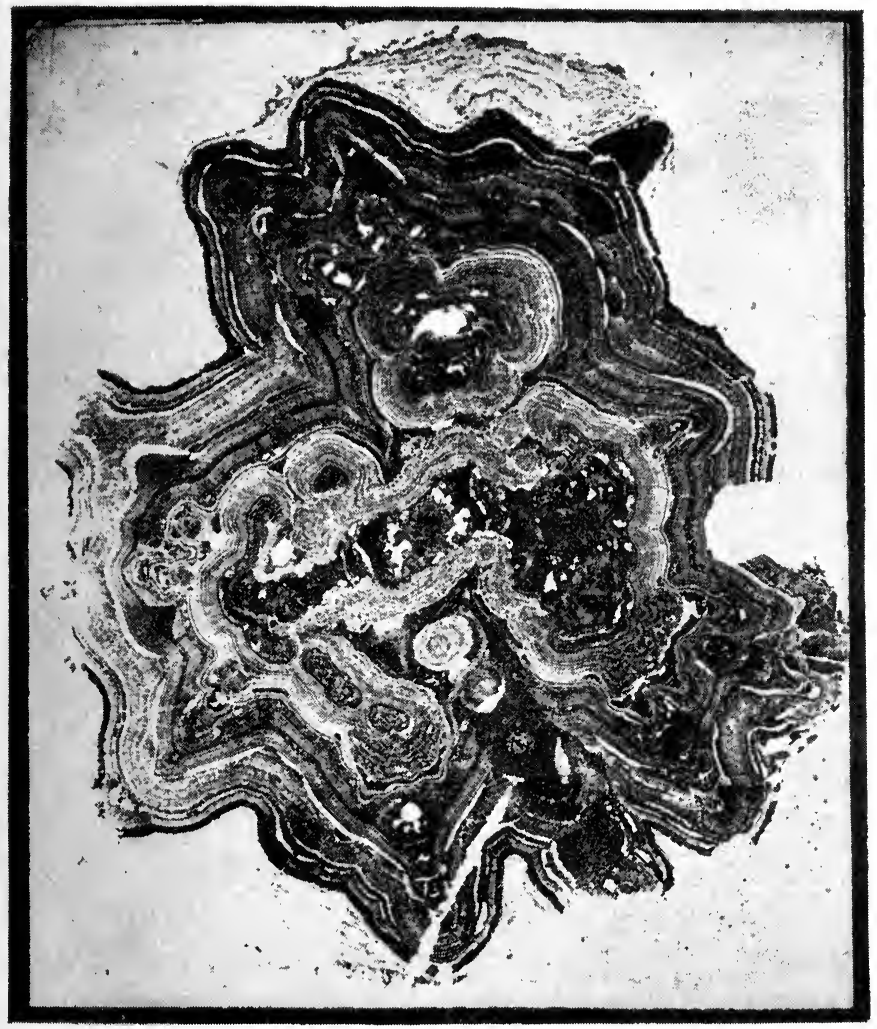

Frg, 4.-A stone composed of oxalate of lime and uric acid, showing the manner in which several nuclei may coalese to form one stone.

the bole of a tree when cut across; and, as the rings on the tree mark the cycles of its growth, so the layers of the stone give evidence of varying conditions which surrounded it during its formation-variations in the rapidity of the deposition of salts at different times causing changes in the compactness of the layers.

Further inspection will show in most of them an indistinct striation radiating from the center, and extending out through the concentric rings. Figs. 3 and 6 give an indication of this formation, and in the section of an oxalate of lime stone (Fig. 5) these striæ are seen as distinct lines of cleavage. Considerable portions of these stones show an irregular, 
loosely crystalline character, which may be seen in the central portion of the sections in Figs. 2 and 3, and which prevail throughout Fig. 6, being

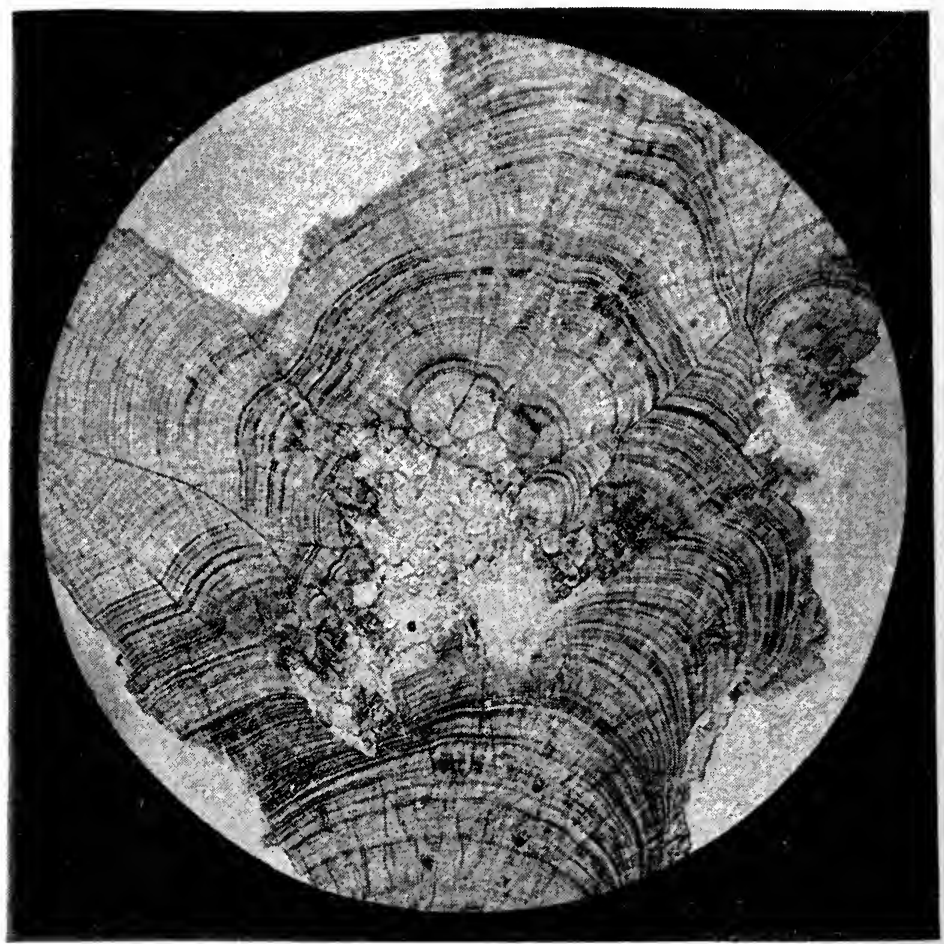

FIG. 5.-A highly magnified section of an oxalate-of-lime stone, showing the delieate concentric layers and the radiating strix.

only partly interrupted here and there by more compact rings. Ebstein calls attention to the fact that in some stones the nucleus is made up of an irregularly grouped mass of little rounded bodies, while the outer portions are arranged concentrically, and show radiating striæ. A condition somewhat approaching this may be seen in Fig. 2, although here the nucleus has a more crystalline appearance than he describes.

From these variations in structure it may be inferred that the conditions under which the crystallization takes place are not constant, and it is reasonably certain that the amount of albuminoid about the stone has a decidedly molding effect on the crystals, which, when this is in relatively small amount, may keep more nearly their ordinary crystalline form.

Bearing upon this point I have made the following observation: In a patient with some residual urine, who was constantly passing uric-acid crystals, a stone presently formed. The urine remained clear to the eye, 
although under the microscope it was seen to contain a few blood and pus cells. The stone was crushed and pumped out. It was very friable, and consisted of almost pure uric acid. There was so little cohesion in this stone that the fragments, after being dried and bottled, fell apart into fine dust, simply from the shaking they underwent during a few hours in my coat-pocket. An examination of this dust under the microscope showed that the uric acid had a distinctly crystalline form, not globular. The crystals were mostly of rod or needle shape, with here and there rhombic plates. This stone, which formed in a urine almost free from pus and albumen, thus retained a more decidedly crystalline character than usual. Its extreme friability showed the absence of cohesion be-

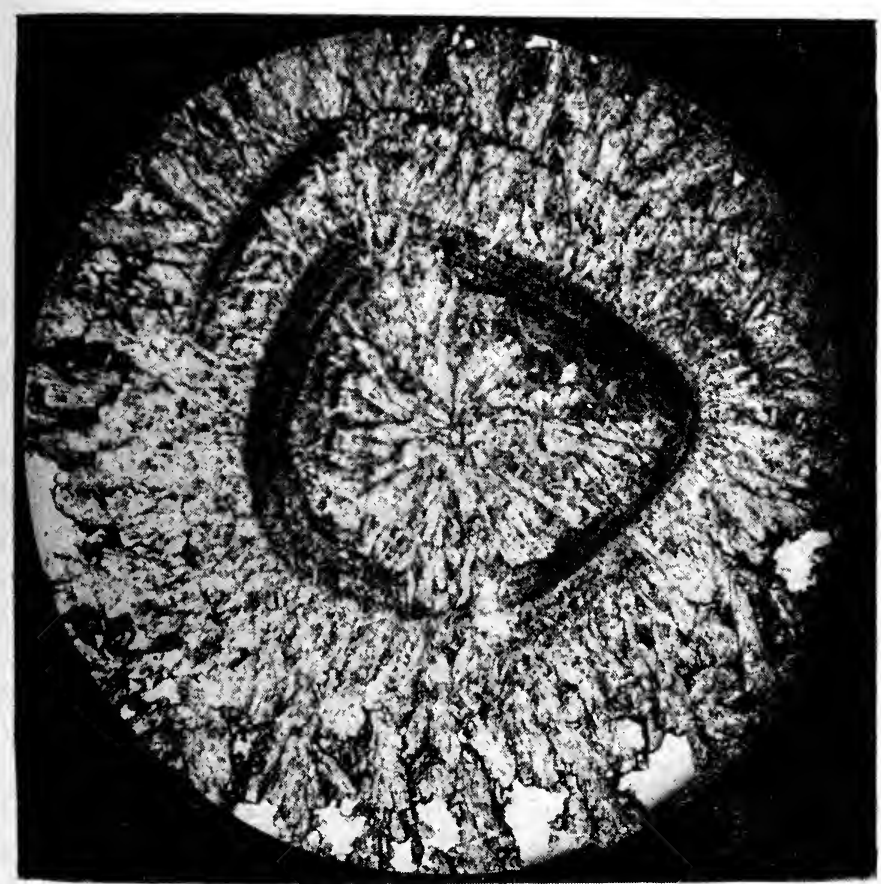

Fro. 6.-A eystin stone, showing the radiating striæ, partially interrupted by imperfeet eoncentrie layers.

tween crystals of this form, when not held together by an albuminous binding material.

Color.-The color of a stone varies according to its constitution. Uric acid and the urates make a red or yellowish-red stone. Oxalate of lime is brown or gray in color, often so dark as to be practically black. Phosphates and carbonates are almost white, shading off into gray. 
Consistence.-They differ greatly also in their degree of compactness and hardness. Oxalate-of-lime stones are the hardest, often being so firm as to require great force to erush them with the lithotrite, and resisting any but the strongest instruments. Stones made up of urates are somewhat less hard, while phosphatic stones, and some of those consisting of pure urie acid, are the softest, offering usually but little resistance to crushing instruments.

Shape.-The shape of a stone is governed, in the first place, by the more or less even deposition of the crystalline substances about it; secondly, by the frietion of the containing walls ; thirdly, by friction of other stones; lastly, by the molding of surrounding walls, as when it forms in a eanal such as the ureter or urethra, or in a pocket in the bladder. This molding is particularly well seen in the stones which form in the kidney, which often take the exact shape of the ealyces of that organ. Occasionally a stone is made up of an agglomeration of several stones, which, after existing independently for a time, cohere. This is seen in Fig. 4, which further shows the irregularity of shape which may arise from this arrangement.

Surface.- The surface of a stone varies aecording to the constitution of its outermost layer. If this is eomposed of uric acid, or the urates, it gives a tolerably smooth surface. A phosphatic stone is somewhat rougher, and an oxalate-of-lime stone has an extremely rough, often prickly or knobby, surface (mulberry caleulns).

Number.-Stones usually exist singly in the bladder, but it is not an infrequent oeeurrenee to find more than one; and sometimes several hun-

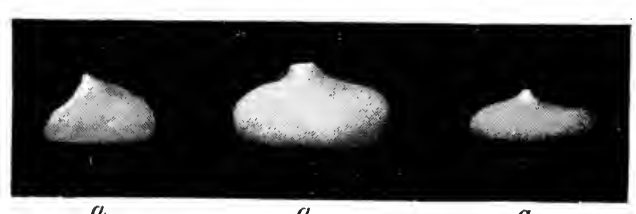

$a$

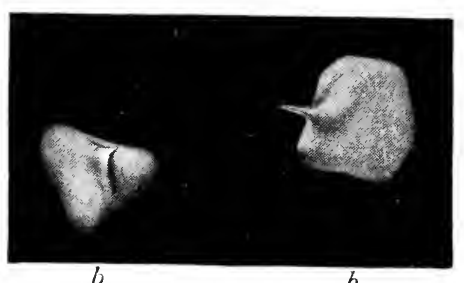

FIG. 7.-Multiple calculi with curious projections. dreds are found in one bladder. When many exist, they are ordinarily small, with rounded outlines. As they become larger and rub against each other more evenly and firmly, they wear off surfaces and become faceted. This action is particularly noticed when the stones are confined in a pocket where they are held in close apposition. Sometimes this mutual molding leads to the formation of regular prominences on some of the calculi which are not of satisfactory explanation. In Fig. 7 we have a drawing of such calculi, in which curious projections have been developed quite symmetrieally on many stones: $a, a$ 
are drawn from stones removed by me in a case of multiple calculi; and $b, b$ represent similar stones obtained by $\mathrm{Mr}$. Buckston Browne in a similar case.

The Spontaneous Fracture of Stones. - This phenomenon had already been recognized and somewhat investigated by earlier writers (Von Schaden, Schmidt), but the researches of Ord were the first to bring forth an adequate explanation of it. Rainey, when investigating the laws of "molecular coalescence," found that spheres of carbonate of lime which had been formed in a solution of gum would split up radially and again disintegrate if they were placed in solutions of a different specific gravity. This effect was even more noticed when the spheres were composed of mixed carbonate of soda and triple phosphates. Following this suggestion, Ord believes that the splitting up of a urinary calculus is, in a similar way, due to the action of urine of a different specific gravity and reaction from that in which the calculus formed. He thinks that the colloid material in

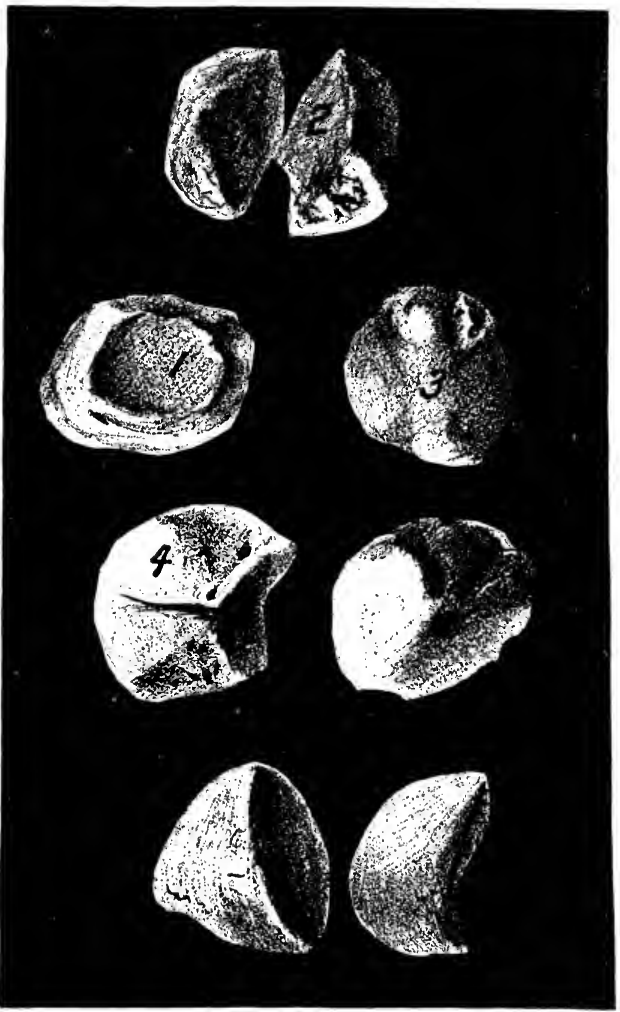

FIG. 8.-Fragments of a stone spontaneously fractured in the bladder. the stone swells by imbibi-

tion of urine of a different density, and that the uncleus, becoming thus enlarged, breaks the outer enveloping layers by an "explosive disruption such as would occur in a thick-walled shell." In three of Ord's cases the fragments, which were of uric acid, were covered with a little urate of ammonia, by which fact he was led to surmise that the urine had become alkaline, and that to that change was partly due its action on the colloids of the stone.

The splitting of calculi under these conditions follows the radiating and concentric lines which we have seen to exist, and the fragments show a tendency to assume wedge-like forms. The accompanying figure (8), taken 
from Watson, shows very beautifully the shapes thus formed. In his case, from which these fragments came, the disintegrated uric-acid stone was found in the bladder after death. The débris weighed in all about 160 grains. In No. 1 it may be seen that the outer phosphatic layer has shrunk somewhat in drying, and has formed little radiating cracks through it, which suggest very well the way in which it would break up if the stone within swelled from any cause.

Ord has recorded a case in which an actual molecular disintegration of a calculus seemed to have occurred, similar to that observed experimentally by Rainey in his carbonate-of-lime spheres. It seems probable that some of the reported cases of the solution of stone in the bladder by the drinking of certain lithia or other spring waters, in which the patients have passed large quantities of débris, are instances of spontaneous fracture, brought about by the great alteration of the specific gravity of the urine, due to the continuous drinking of an excessive amount of water.

Some observers have thought that several calculi might be so pressed together by the contracting bladder, or might strike against each other in such a way as to lead to their mutual fracture. In a specimen of spontaneous fracture met with by Croft, there was a soft, friable layer between two harder layers ; and Ord points out that " this is a condition favorable to fracture, when two or three calculi of about the same size and the same constitution are present in the bladder together. They crack each other's shells, like eggs carried loose in a bag."

Etiology.-In considering the circumstances that lead to stone formation we have two sets of causes to investigate: First, the general conditions and tendencies, diathetic and otherwise, that induce the overproduction in the system of the materials which are deposited from the urine in the shape of calculi ; and, secondly, certain local conditions which favor the deposition of these solids out of the urine. We will first consider the general conditions concerned in their formation.

Locality.-The irregularity of the distribution of stone through different countries and localities has given rise to much speculation, and various theories have been advanced to account for it. The frequent occurrence of these cases in the central states of North America, and in other localities having a similar geological formation, was thought by some writers to be due to the limestone underlying those places, and causing a strong impregnation of the drinking-water with lime-salts. This theory falls to the ground, however, when it is seen that the tendency to stone is not evenly distributed throngh the countries where lime abounds, and that it shows itself at times in but one part of such a district.

Heredity.-That heredity may have an influence in determining a predisposition to stone is undoubtedly true, and many instances are reported 
in which the uric-acid diathesis has existed through several generations in a family, and has made its members liable to the occurrence of calculous disease. Whether a tendency to oxaluria or phosphaturia is similarly transmissible is not so clear. The influence of heredity has been evoked as one factor leading to the frequency of stone in certain neighborhoods, where generations of intermarriage might fix calculous tendencies on the people. The amount of effect that this may have is not easy to trace.

Diet and Habit.-The influence of diet and of habits of life upon the excretion of uric acid and oxalate of lime is unquestioned. Thus, the customs of a people, their ways of living, and their choice of food, may have a decided influence on the frequency of stone among them. Uric acid and oxalate of lime are often found abundantly in the urine of poorly nourished children, in whom the processes of digestion and of oxidation of tissues are imperfectly accomplished; also in older individuals who take large quantities of nitrogenous food, and whose functions are sluggishly performed. It is therefore in these two classes that acid stones most commonly form. Thompson has pointed out the fact that stone is common among the children of the poor, while among the rich it spares the children and appears among the old men. This observation refers only to primary calculi ; for secondary calculf, due to fermentation of the urine, are more common among the aged poor, who pay but little regard to a moderate cystitis. In the case of oxalate of lime, its appearance in the urine may come about in a way even more direct; for the ingestion of certain articles of food that contain oxalic acid abundantly often leads at once to the deposition of large amounts of oxalate of lime in the urine. Chismore has found oxalate-of-lime stones disproportionately abundant on the Pacific coast of North America, and is inclined to ascribe this to the fruit-eating habits of the people.

Recent investigations of Ebstein and Nicolaier throw some light on the manner in which a chemical substance may be carried through the body without change, and be deposited out of the urine in the form of a stone. They fed animals (dogs and rabbits) on oxamid, an ammonia derivative of oxalic acid, and succeeded in causing the formation of numerous oxamid stones in the urinary passages. As oxamid never appears in the urine under normal conditions, these experiments are convincing proofs of the possibility of this method of stone formation directly from substances contained in the ingesta.

Diathesis.-Besides the general conditions of life which have an influence on the frequency of primary stone formation, we have also to consider certain tendencies peculiar to the individual which favor the occurrence of calculous disease. The existence of a uric-acid diathesis has been alluded to as having been invoked to explain the frequency of stone in certain localities, and the hereditary transmission of this diathesis is 
generally recognized. Its association with various gouty lesions is so constant that it is properly looked upon as one manifestation of gout; and it is not uncommon to find, in a family with a strong inheritance of gout, one or more individuals suffering from uric-acid gravel, or from stone in the kidneys or bladder. It has been observed that patients suffering from hepatic disease are liable to an excess of uric acid, and the same is true of diseases of the spleen. In leucocythæmia the presence of large quantities of uric-acid crystals in the urine is of frequent occurrence, and the formation of stone in the bladder under these circumstances has been recorded.

When we come to the consideration of phosphatic stones, we find quite a different condition of things; for, although it may occasionally happen that the phosphatic diathesis gives rise to the formation of a calculus, it is far more common to see a stone of this kind the result of a local process.

Alkaline Fermentation.-In chronic inflammation of the bladder, sooner or later the urine is likely to become alkaline, and to throw down crystals of triple phosphate as well as of other alkaline salts. These are thickly deposited in the gelatinized pus, erroneousiy called mucus, and in this they undergo the molecular changes that lead to their coalescence, and agglomerate themselves into little masses, around which the subsequent formation of the stone goes on quite rapidly. Any condition that predisposes to chronic cystitis and alkaline fermentation of the urine may be regarded, therefore, as an etiological factor in stone formation. The condition most favorable to alkaline fermentation is that of partial retention (residual urine). This is usually the result of some obstruction, rarely of atony. The common cause of obstruction is an enlarged prostate, but a stricture often acts in the same way. I have met with a number of cases of stone which have thus formed behind a stricture. The production of alkaline fermentation is not the only way in which an obstruction to the flow of the urine favors the tendency to the formation of stone. In the healthy bladder, which completely empties itself at each act of micturition, any erystals which are deposited in the urine are washed out with it, and any little stones that come down from the kidneys are usually expelled in a similar way. When, however, owing to an obstruction, the bladder ceases to thoroughly expel its contents, the chance of a commencing concretion being thrown ont is diminished, and thus the formation of a stone in the bladder is mueh more likely to occur.

Sometimes a patient is for years subject to attacks of renal colic, and during adult life the small stones which pass down from the kidney are sucessfully voided, until finally as he advances in age the prostate becomes enlarged, the bladder ceases to entirely empty itself, and a renal stone, being retained, forms the nucleus of a stone in the bladder. In the 
same way a sacculated condition of the bladder favor's stone formation, for in the pouches, fermenting urine and mucus are retained, thus producing conditions suitable for the origination and rapid progress of calculous deposition.

Symptomatology.-A stone may exist for a considerable time in the bladder without causing the least discomfort to make its presence known. I on one occasion touched a stone in the bladder, and, because the patient could not then attend to it, left it undisturbed; but the man went for several months in perfect comfort before symptoms appeared which finally led to an operation. In this case the stone lay in a postprostatic pouch, and did not come in contact with the sensitive vesical neck either during motion or after urination. Such cases are not very uncommon. Occasionally we see a case almost the exact opposite of this, in which most of the uncomfortable symptoms that are ever associated with a stone in the bladder are heaped upon the unfortunate victim. Most of our patients, however, occupy the middle ground between these extremes, and, while presenting some of the symptoms in a more or less characteristic form, may be wholly without other sensations and phenomena that we usually expect.

The following are the symptoms that we have to look for in these cases, and I have taken them up in the order of their importance :

Pain is a very constant aecompaniment of stone, and, although other evidences of irritation may precede it, it is perhaps the most characteristic symptom that we have. The pain of a stone varies greatly in intensity, and is referred to somewhat different localities in different cases. There may be merely a sense of discomfort and weight in the perinæum and rectum, or a burning may be felt in these same regions. When more severe, a lancinating or pricking pain may be felt; and I have had a patient insist that he had exactly the feeling as if a paper of pins were in the lower bowel, just within the anus. These pains show a tendency to shoot out along the urethra and into the testes, or down the thighs. Not infrequently the pain is all referred to the end of the penis, and the dragging upon this organ in the effort to relieve discomfort sometimes causes a considerable elongation of it. In children a greatly lengthened prepuce results from the same habit.

Wherever the pain is felt it is almost invariably aggravated during and after urination. As the bladder closes down on the surface of the stone, the irritation is increased. At this time the pain is almost certainly felt, in part at least, in the glans penis. This is dne to the fact that the stone is pressed down against the prostate as the bladder empties itself, and irritations at the bladder face of the prostate are referred to the glans penis. Occasionally sharp spasms are set up in the bladder as its walls come in contact with the stone. As might be supposed, the increase of 
pain at the end of urination is often absent in a patient who does not completely empty the bladder; and, in that case, it may make its appearance if the residual urine is drawn with a catheter.

Another characteristic of this pain is that it is aggravated by motion. If the stone is movable in the bladder, it is natural that it should be more irritating when rolling about than when lying still. Riding in a jolting vehicle is the most trying kind of motion; but often the sensitiveness is so great that quiet walking can not be endured, and some patients even suffer greatly from turning over in bed.

Other things being equal, a stone causes more pain when resting near the neck of the bladder than when lying back toward the fundus, so that these patients are often more comfortable when lying in bed with the hips raised than when in a more upright position.

Increased Frequency of Urination is so constant a symptom in all inflammatory troubles of the bladder that its appearance would not arouse a suspicion of stone. The fact, however, that in stone cases the frequency is much increased by motion may be of assistance in deciding on the probable diagnosis. Especially may a study of this symptom assist in differentiating between stone and enlarged prostate; for, while the stone patient will probably be less called upon to urinate at night than in the day, the reverse is apt to be true in the prostatic case, for the passive congestion of a hypertrophied prostate is aggravated by recumbency, and is usually somewhat relieved by exercise. The frequency, dependent on the presence of a stone, may be slight in degree, or may be so extreme that the patient urinates every few minutes, and sometimes suffers from a true incontinence. The efforts at urination often induce painful spasms of the bladder and rectum, and prolapse of the bowel sometimes follows se vere straining.

Sudden Stoppage of the Urine in Full Stream.-It sometimes happens that the stone rolls over the internal orifice of the urethra during urination and thus causes a sudden stoppage of the urine, accompanied by a sharp twinge of pain, with often considerable straining following it. Under these circumstances the patient usually finds that, after a moment's pause, especially if he changes his position, the urine will again flow and the bladder will empty itself. In the recumbent position this stoppage is less likely to occur, for the obvious reason that the stone then lies at a distance from the vesical neck. This symptom is more common in young and middle-aged patients, and is rarely observed in the old. The reason of this is easily seen; for in the undistorted bladder of youth the vesical orifice is dependent when the body is erect, and the stone readily falls upon it. In old age, on the other hand, after the development of the post-prostatic ponch, the stone gravitates into this, and can not easily roll forward on to the urethral opening. Furthermore, the irregularities of 
the vesical orifice in old men, caused by projections from the prostate, especially of the third lobe, make it difficult for a stone to fit into and close it tightly. And, lastly, when the bladder has lost its force, the slowly flowing stream is less likely to carry the stone along with it.

Hæmaturia.-The presence of blood in the urine is a usual accompaniment of calculus, and is the direct result of the injury done to the bladder walls by the stone. It is usually slight in amount, often requiring the microscope for its recognition. Occasionally in patients having the hæmorrhagic diathesis, or in old persons with congested prostates, it may assume serious proportions.

Violent motions have a marked effect in increasing the hæmorrhage. The blood may be mixed evenly in the urine, giving it a smoky or red color. It may, however, come at the end of urination, the first portion of urine passed being clear and the last few drops containing much blood, which is pressed out as the bladder closes on the stone. This expulsion of a few drops of blood at the end of urination is not, however, at all characteristic of stone, for the same thing is observed in inflammatory troubles about the prostate, and also sometimes in cases of tumor of the bladder.

Cystitis.-It is common, sooner or later, to see a cystitis develop itself in connection with a stone, and the symptoms are then complicated by those of inflammation of the bladder. Under these circunstanees the pain is usually more intense, and the frequency of micturition is a more prominent symptom.

The Condition of the Urine often gives important indications in eases of stone.

Before a cystitis develops the urine may be quite clear to the eye, but even then a microscopical examination will usually show the presence of crystals of uric acid or oxalate of lime, with a few blood and pus cells. Patients sometimes state that for a long time the urine contained red sand, but that finally this disappeared, and soon after the symptoms of stone began. This would seem to show that, after the stone formation has started, crystallization around it removes in some cases the over-abundance of uric acid. This observation depends, as far as the author is concerned, on the statements of patients, and it is to be remembered that, in persons who have no stone, crystals of urie acid will appear intermittently in the urine. Still, in view of what is known about the change which takes place in the crystallization of uric acid when albuminoid materials have become mixed with the urine, it seems possible that this observation is founded on more than a mere coincidence, and that the change marks the commencement of "moleeular coalescence" of the urie acid.

When cystitis develops, the urine becomes more or less cloudy, owing to the admixture of pus and bladder epithelial cells. Usually crystals are 
found in connection with the evidences of bladder inflammation. If the urine continues acid, as it not infrequently does, the crystals may be those of uric acid or calcic oxalate. If the urine becomes alkaline, the uric acid usually disappears, and we have crystals of the triple phosphates, of the carbonates and urates. The alkaline condition of the urine also induces a change in the condition of the pus, which becomes slimy, and clings together in stringy, coherent masses, which often adhere to the bottom of the vessel in which the urine has stood. The pus, thus altered, usually passes by the name of mucus, but it is not the excretion of the mucous glands.

When there is ulceration of the bladder walls the amount of pus in the urine is often very large, and it is frequently so mixed with blood as to be distinctly stained by it. There is always some albumin in the urine when pus or blood is present. The albumin is sometimes out of proportion to the amount of blood or pus, even when no serious condition of the kidneys exists.

Priapism.-Together with other symptoms of bladder irritation that have been enumerated, we sometimes see erections as another evidence of the irritation caused by the stone, and the consequent congestion of the vesical neck. These are more common in the young, and, in connection with the pulling on the penis to relieve the pain felt in it, may lead to the practice of masturbation.

Reflex Pains.-Besides the pain directly caused by the irritation of the stone, there are occasionally distant reflex pains in parts having no close nerve association with the bladder. These pains are distinctly neuralgic in character. They are felt in the hips, thighs, and even run down to the lower leg or foot. Podalgia, pain in the sole of the foot, may be due to vesical irritation from any cause, and may therefore exist as a consequence of stone. These pains, well described by Dr. T. B. Curtis, usually affect the great toe or the ball of the foot. The writer has seen it in the hollow of the instep in a case of prostatic disease. It is described as a burning pain confined to one small spot. Sometimes the feeling is one of intense coldness, or the pain is lancinating in character. These reflex pains, due to bladder irritation, are not confined to the lower extremities, but may be felt through the shoulders and arms.

Diagnosis. - There are varions conditions of the urinary organs that may produce symptoms closely resembling those of stone in the bladder. Of these may be mentioned inflammatory conditions, tuberculosis, and tumors of the bladder and prostate. Other conditions, such as stricture, chronic inflammation of the urethra, stone or auberculosis of the kidney, and neuralgia of the neck of the bladder, may produce one or two of the symptoms associated with stone, but do not often give rise to groups of symptoms that are seriously misleading. 
The only certain means of settling the diagnosis is by physical examination. This examination may be conducted in a variety of ways :

1. Digital exploration through the rectum or vagina.

2. Exploration with searcher or sound, including examination with a lithotrite.

3. Exploration with a litholapaxy-pump.

4. Examination with a cystoscope.

5. Digital exploration through the dilated urethra in woman, or through a median perineal opening (boutonnière) in man.

6. Digital and ocular examination through suprapubic opening.

This list of manœuvres is arranged in the order of their severity, and usually the information sought will be satisfactorily got by one of the less serious procedures.

1. The examination by the vagina or rectum is that made familiar by gynæcological and other pelvic explorations. With the usual bimanual manipulation a stone may not infrequently be felt and its size correctly judged. This examination is especially applicable to children. Also, during the search with instruments in the bladder, the finger in the rectum or vagina may be of great assistance in locating a stone--as, for instance, one protruding from the opening of a ureter, or a sacculated stone.

2. Exploration with a searcher is the operation familiarly known as "sounding for stone," so called because the instrument used is named a

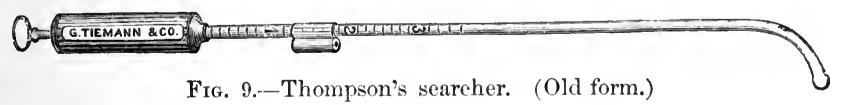

sound, rather than because the ear usually plays any important part in the detection of the calculus; for, although the ear can often catch the click of a sound upon a hard calculus when they are brought sharply together, yet the trained hand will perceive the contact even more sensibly and surely. If the calculus is a small one, or has a soft surface, the touch will often readily recognize the impact of the searcher upon it when no sound can be elicited. The sensations that are perceived through the sound are of two kinds, and are produced by the shock of impact, or by the scratching of the metallic instrument over the rough surface of the stone.

The instrument should have a shape that permits of its easy manipulation in the bladder, and it will be found that a hard metal gives a more distinct sense of contact than a soft one.

The shape of the instrument generally preferred is well shown in the accompanying figures. The shaft should be thin (15 to 18, French scale) 
for adults, smaller for children. This is in order that the urethra may not hold it firmly, and thus prevent easy rotation and sliding to and fro. The beak may be somewhat larger, and if the end is rounded and blunt it will slide more easily along the urethra. The beak, moreover, should be short, and should form a very moderate obtuse angle (about $120^{\circ}$ )

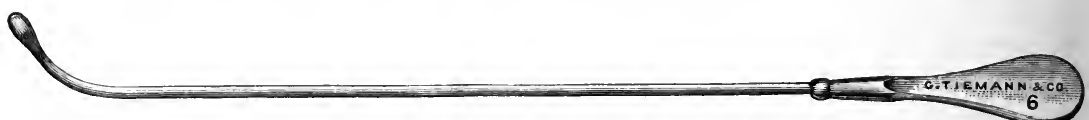

Fia. 10.--Steel seareher.

with the shaft. An instrument of this shape will rotate readily in the bladder, and will sweep around so close to the vesical face of the prostate that it is not easy for a stone close behind the prostate to evade it.

The handle of the searcher varies in shape according to the fancy of the surgeon; but as much of the manipulation of the instrument is by rotation, a round, barrel-shaped handle, with corrugated surfaces, is perhaps the most convenient; and it should always be marked on the side that corresponds to the point, so that a glance will tell where the beak is directed. A flattened handle, such as is familiar in the urethral sound, is also a good form, and has the advantage that the operator is never for a moment unaware of the direction of the beak. Recognizing the advantage of each of these handles, Thompson has combined them in his latest searcher (Fig. 11).

As it is often desired to change the amount of water in the bladder during a search, the shaft of the instrument may be hollow, like a catheter. This has an additional advantage, that it makes the instrument light, and therefore more susceptible to slight vibrations. The searcher, if hollow, may be made of silver or German silver; or, if solid, of zinc, aluminum, or steel. It has been suggested that a soft metal of high polish

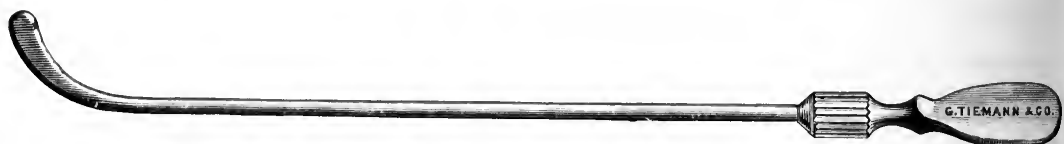

Fig. 11.-Thompson's searcher. (Latest form.)

would show the scratches of the stone, but it would be indeed a clumsy hand that did not feel a contact slighter than that necessary to mark metal. The softer metals (zinc and aluminum) have the advantage that they can be bent to a special shape for a particular case; they transmit vibrations, however, somewhat less rapidly. $A$ steel searcher in the shape of Fig. 10 is a very delicate instrument, and especially useful in searching children where the urethra is small. 
The Operation of Sounding should be conducted with the patient in a horizontal position. In old men, where there is presumably a pouch behind the prostate, the hips may with advantage be raised higher than the shoulders, to roll the stone back toward the fundus.

The question of anæsthesia for sounding must be decided in each case for itself. In children who are restless, hard to control, and whose bladders are so sensitive that they ordinarily empty themselves on the entrance of the instrument, anæsthesia is always to be used when possible. In adults, unless there is unusual sensitiveness, sounding may be accomplished without anæsthesia, or, where thought desirable, the use of a five-percent solution of cocaine in the prostate and bladder may be employed. If, however, there is much rigidity in the deep urethra, or spasm of the bladder, interfering with the manipulation of the instrument, the search will be very imperfect and unsatisfactory without general anæsthesia. In case this is used, it is well to make preparation for the immediate removal of the stone, if found. In this way the shock of two sittings of anæsthesia and instrumentation is avoided. Indeed, the chance of serious reaction following an ordinary sounding is so great, that it has been my habit, in cases of old men with large prostates, and with symptoms strongly suggesting stone, to make the first examination with anæsthesia, and with all preparations for operation. I have never regretted this course, for in such cases I do not regard the search without ether as satisfactory, and I have on several occasions found stones under anæsthesia which had eluded me without. This being the case, I feel that it is better to spare the patient the pain and shock of a preliminary examination, and to resort to ether at once.

To proceed now with the method of conducting the search. It is well that the bladder should be moderately distended with fluid, to permit the ready manipulation of instruments within it, and to preclude the possibility of the stone being concealed within its folds when collapsed. The patient being in position, the searcher is carefully introduced through the urethra. The similarity of its shape to that of the lithotrite makes the introduction of the searcher very like that of the larger instrument, and the manipulation will be fully described in the consideration of lithotrity.

The instrument, being in the bladder, is carried down to the most dependent part toward the fundus, and here the stone is usually found. If it is not at once touched, the searcher is to be rotated first to one side and then to the other, so far that its beak comes down against the floor of the bladder. It is then to be drawn forward a little and again rotated from side to side, and so on ; little by little the floor of the bladder is investigated, till the beak comes up against the face of the prostate. In cases of enlarged prostate it is here to be completely rotated, so that the point sweeps through the post-prostatic ponch. If the point catches during this 
rotation, the handle of the searcher should be depressed between the thighs, until the beak is lifted clear of the floor of the bladder. If the stone is still not touched, the lateral portions of the bladder are to be searched by carrying the instrument along first one side and then the other, tapping along the bladder wall on each side. In examining the sides of the bladder, the handle of the searcher should be carried close to the opposite leg.

The upper walls of the bladder may be searched by carrying the instrument well into the viscus and then depressing the handle between the thighs, and, with the point on the roof, rotating it gently from side to side.

Finally, if the search is still unsuccessful, the bladder may be emptied, and, as it collapses, the stone may be brought down against the instrument. Keyes recommends that this final manœuvre be performed with the patient in the upright position, leaning slightly forward. As the water runs out, the beak of the searcher should be kept near the neck of the bladder and gently rotated from side to side. He says that in this way a stone is rarely missed.

Sources of Error in Sounding.-It so often happens that a stone is missed by what seems a thorough search, that a moment's consideration of the sources of error is worth while.

a. The searcher may not enter the bladder. It sometimes happens, in cases of enlarged prostate, that the sound, after getting into the prostatic urethra, has a considerable degree of mobility, and when the third lobe of the prostate is somewhat enlarged, so as to considerably resist the further passage of the instrument, it is not infrequently thought that the sound is in a contracted bladder. This mistake is much less likely to occur in an examination under ether.

b. A light stone, of a specific gravity very close to that of the urine, may give too little shock of contact to be perceived by the sound.

c. A stone may be so enveloped in mucus or blood that the sound can not be brought in contact with it.

d. An encapsulated stone may be out of reach of the searcher. A stone in a pocket on the floor of the bladder may sometimes be lifted, by the finger in the rectum, to a point where the searcher can touch it.

$e$. Sometimes the opposite mistake is made, and it is thought that a stone is felt when none exists. A tumor incrusted with lime-salts may be mistaken for a stone. This may be suspected, if the stone is always felt in a part of the bladder where it would not be kept by gravity. A stone partly projecting from a pocket, and a stone formed upon an ulcerated surface and so adherent to the bladder wall, will give the same sensation; so that a diagnosis between these conditions may be impossible.

$f$. When a hollow searcher is used, the rush of water into it as it reaches the bladder may give a shock so exactly like the contact with a 
stone as to deceive the most practiced touch. It once happened to the writer to meet this sensation when introducing a glass catheter into a female bladder, and each time the tube was slipped in and out the sensation was reproduced again and again, exactly as if the end were tapping on a stone. The introduction of a solid sound showed the bladder to be empty.

Some of the above sources of error may be avoided by using a lithotrite for sounding. A stone too light and small to be felt with a searcher, or one enveloped in mucus or blood, may be grasped in the jaws of the lithotrite. When the sounding is done with this instrument, it is possible to tell whether more than one stone exists; for, with one stone held firmly in its jaws, the lithotrite may be used for further search.

When a stone is touched it is generally possible to tell whether its surface is rough or smooth, and something may be learned of its consistence by the sharpness of the ring which the instrument makes against it.

To judge of the size of a stone by the searcher, the beak should be carried beyond it, and then, while in contact with the farther end of it,

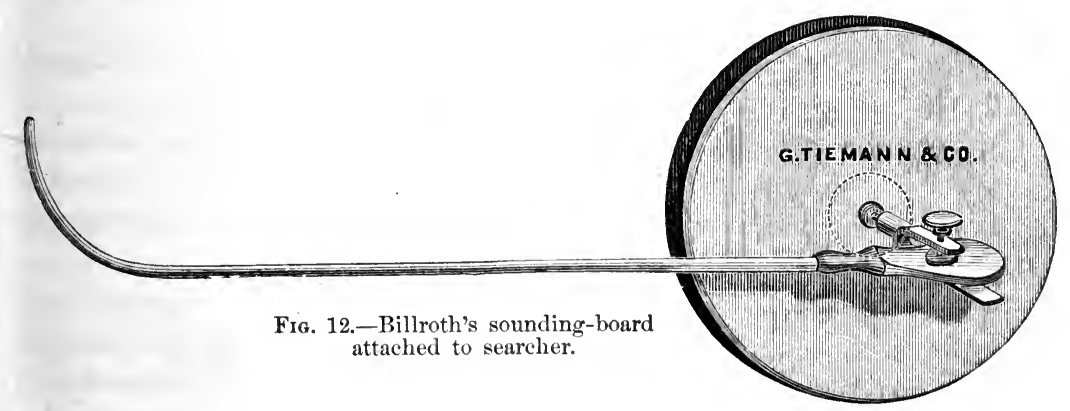

the position of the meatus on the shaft should be marked. Many searchers have a sliding collar to slip down against the meatus for this purpose. Now the instrument should be withdrawn along the side of the stone, tapping gently against it until the nearer end is reached, and, noting then the distance that the meatus has moved along the shaft, we have a rough measure of one diameter of the calculus. With a lithotrite, or similar instrument, an exact measure may be taken of the stone in several diameters.

Efforts have been made to add to the delicacy of perception by the searcher by the attachment to it of a sounding-board. This is of no assistance to the operator, but may enable him to demonstrate the existence of the stone to the by-standers. Stethoscope-like tubes, connecting the instrument with the ear of the surgeon, are also of no practical assistance. 
Exploration with Litholapaxy-Pump.-For a small stone, that eludes searcher and lithotrite, Dr. Bigelow showed the evacuating pump to be the best possible sound. The current brings small calculi violently against the orifice of the tube, and the elick is both perceived by the hand and heard by the ear. One souree of error is to be noted, namely : that sometimes, when the bladder wall is suddenly sucked against the tube, the shock stirs some of the joints of the apparatus, and gives a click very like that produced by the contact of a stone. A mistake from this

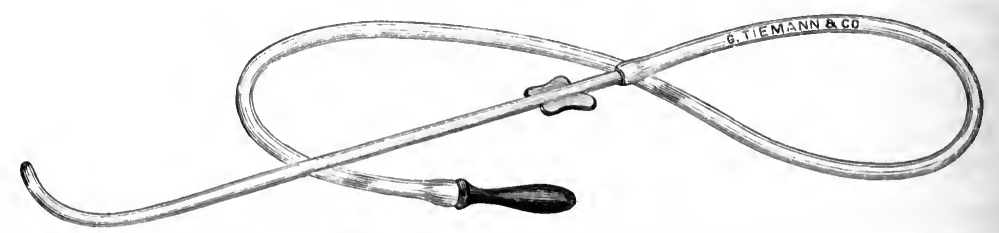

Fig. 13.-Andrew's seareher, with a tube to convey the sound to the ear.

cause can be avoided by tightening the joints and then prolonging the seareh without being able to reproduce the sound. If air is contained in the evacuator during the search, its bubbling to and fro may make the perception of a slight elick almost impossible.

4. Examination with the Cystoscope is rarely necessary, as the information sought may be usually obtained by simpler methods.

To decide the diagnosis between an incrusted tumor and an adherent or encysted stone, ocular inspection may be of use. The eharacter of a foreign body serving as the nucleus of a stone might be thus ascertained, though the matter wonld rarely be in doubt.

5. Digital Exploration of the Bladder.-Other kinds of diagnosis failing in eases of suspected stone, a digital exploration of the bladder may be readily made through the dilated urethra in the woman, or through a median, perineal incision with dilatation of the prostatic urethra in the male. In most cases the interior of the bladder can be thoroughly searehed in this way. In order that the more distant parts of the viscus may be reached, the bladder should contain no water, but should be collapsed on the end of the finger. Pressure with the other hand into the pelvis is often necessary, in order to bring the upper part of the bladder down on to the examining finger. It occasionally happens in men that the prostate is so much enlarged that the perineal route into the bladder is too long for the ordinary forefinger to satisfactorily reach through it.

6. Exploration through Suprapubic 0penings.-In cases of doubt, when the bladder ean not be reached throngh the perinæum, a suprapubic opening may be required in order to establish a diagnosis. It will often happen, too, that a high operation may well be chosen in exploration, when there is a strong probability of a condition of things requiring 
ready access to the parts for their proper treatment, as in some cases of incrustation of the bladder wall, or of sacculated stone. This matter will be considered at further length under the head of Suprapubic: Cystotomy.

Treatment.-In approaching the treatment of stone in the bladder, we may consider it under the following heads :

1. Prophylaxis-preventive treatment.

2. Solvent treatment.

3. Operative treatment.

In the care of most cases all these methods will be more or less combined, and the intelligent application of them will do mueh to relieve the discomforts of the calculous diathesis.

1. Preventive Treatment. - The presence of crystals (sand or gravel) in the urine, or the occurrence of renal eolic, may give the warning which leads to the adoption of preventive measures. Often, however, this is disregarded, and it is only after the removal of a stone that treatment is instituted to prevent its recurrence.

This treatment will be varied, according to the kind of stone formation which is imminent. If the tendeney is to the formation of urie aeid or oxalate of lime, the fault is a constitutional one, and must be met by appropriate general measures of treatment. Phosphaturia, too, may be constitutional, and should then be treated generally. Usually, however, alkaline stone formation is secondary, and depends, as has been shown, on some local conditions, which should be met by appropriate local treatment. When a tendency to uric-acid formation is shown, by the appearance of erystals in the urine, or by symptoms indicating a gouty diathesis, efforts should be made to counteract this by a striet attention to the patient's habits in regard to food, drink, and exercise. It was formerly thought that, to prevent the formation of an excess of uric acid, all nitrogenous articles of diet should be avoided as far as possible. Later experience has not fully borne this out, and Lehmann and others have found that a meat diet, unless immoderate, does not increase the excretion of uric acid.

The following rules may well be borne in mind while preseribing for these patients:

It is of the first importance to avoid overeating. In judging of this, the normal requirements of the individual must be sought. These are so various in different subjects that they can only be learned in each person by study and experiment. In each case, too, these requirements vary greatly at different times, and an amount of food which a man may easily bear during a life of aetive exercise in the summer, will be too much for him in the winter, when shut up indoors and engaged in sedentary oceupations. 
A relatively light diet should then be adopted, and, while meat is taken, it should not be in excess. This remark applies well to other forms of animal food, such as eggs, fish, etc.

Fatty foods should be used very moderately. It is true that experiments conducted upon a normal individual have shown that butter, up to three ounces a day, does not increase the amount of uric acid excreted. But it must be remembered that people differ greatly in their power of disposing of fat; and while these experiments show that fat in moderation, or even in considerable quantity, does not directly increase the amount of uric acid, they do not show that fat, taken for a considerable time, may not do so by an indirect method. When fat is taken for a long period, especially by a person whose digestion is not vigorous, the presence of the fat among the other food interferes with the digestion of it, and in this way leads to an acid dyspepsia which has a considerable effect in producing uric acid. For this reason many articles of food, which would not be avoided on account of any direct effect in producing uric acid, are to be avoided on account of their influence in producing dyspepsia. Ainong these may be mentioned pastry, fried articles of food, new bread, etc. Highly seasoned dishes are harmful in two ways: First, they are ordinarily indigestible, and give rise to acid dyspepsia; and, secondly, they stimulate the palate and lead to overindulgence. Sugar and saccharine articles of food are decidedly injurious, and seem to directly increase the excretion of uric acid. They should be wholly given up when there is any appearance of uric acid in the water, and at other. times should be used very sparingly by a person of uric-acid tendencies.

Alcoholic drinks should be avoided, as they decidedly increase the excretion of uric aid. Especially is this true in regard to sweet wines and malt liquors, which contain large amounts of saccharine material, in addition to the alcohol. If stimulants are called for in any case, welldiluted spirits, either brandy, whisky, or gin, are to be preferred. Of the wines, the least injurious are very dry champagne, light claret, or thin Rhenish wine.

Systematic exercise is of the greatest importance. This is useful, both by stimulating the digestion and assimilation of food and by furthering the tissue-changes of the body. When possible, the exercise should be violent enough to give rise to a good perspiration, and the skin should be afterward cleared by a vigorous rubbing down. This greatly favors the elimination through the skin, and thus somewhat relieves the kidneys.

Much may be done in reducing the uric acid passed through the kidneys by proper management of the bowels. The influence of a course of treatment at Carlsbad upon a patient of gouty tendencies is well known; and this treatment consists largely in the administration of laxative waters. Much of the benefit ascribed to the waters is, no doubt, more 
properly to be laid to the credit of the regulation of diet and exercise which is insisted upon, and to which the patient cheerfully conforms, at one of these great sanitaria. Still, the promotion of elimination through the bowels plays undoubtedly an important part.

The sulphates of sodium and magnesium, with which the saline waters are charged, are believed to act upon the liver, as well as upon the smaller intestinal glands, and to be efficacious in removing portal congestion. How much they owe their beneficial effects to their action upon the liver is somewhat problematical, but it is undoubtedly true that they are of great benefit to plethoric patients of constipated habit, with tendency to gout or uric-acid formation.

To be of use, these laxatives should be administered every day in sufficient quantities to insure at least one good, relaxed movement of the bowels. It is not desirable to excite sufficient cathartic effect to cause decided weakness. When this plan is carried out at the patient's home, the Carlsbad salts, Hunyadi Janos, Friedrichshalle, or other similar water, may be administered each morning in the dose that is found to produce the desired effect. It should be taken early on an empty stomach, and usually acts more satisfactorily when warmed or mixed with hot water. If there is evidence of sluggishness of the liver, a three- or five-grain bluepill may be given occasionally at night, being followed in the morning by the usual saline dranght.

The habits of the patient in regard to taking water should also be inquired into. Much water taken at the time of a meal tends to hinder digestion and to favor fermentation and acidity. On the other hand, it is important that sufficient fluid should be taken in the twenty-four hours to keep the solid excreta of the kidneys completely in solution.

When the presence of crystals in the urine shows that the solids are not held in solution, and that the danger of stone formation is imminent, it becomes important to administer some solvent agent which shall as quickly as possible remove the danger. For this purpose the salts of lithia, of potash, or of lithia combined with potash, may usually be depended upon to prevent the further formation of crystals. Piperazine has lately been bronght into notice as a powerful solvent. The further consideration of this question will be found under the head of "Solvent Treatment." Probably, however, one of the best ways of getting rid of crystals is by administering large quantities of water, producing a flushing of the urinary tubes and reservoirs, and thus washing them out.

The above remarks concerning the control of uric-acid tendencies may be applied, with but slight variation, to the cases showing a tendency to oxaluria. It is to be remembered, however, that in prescribing the diet of these patients fruits containing oxalic acid should be specially forbidden. I have seen sharp attacks of oxaluria follow indulgence in rhubarb 
(Rheum ponticum, familiarly known as pie-plant throughout New England). Oranges, strawberries, and tomatoes should be omitted from the dietary while oxalate crystals are present in the urine, and should be used sparingly at all times by patients of oxalic proclivities. Phosphaturia may usually be relieved by the exhibition of nitro-muriatic acid, and its recurrence is to be opposed by hygienic measures, including adequate exercise and a relief from care, if that is possible. General nerve tonics, such as iron, quinine, and strychnine, will sometimes be of distinct benefit. When cystine has been detected in the urine, Beale recommends the use of ammonic carbonate.

As has been said, in considering the etiology of stone, not only the deposition of crystals, but also the presence of albuminoid materials, is necessary for the formation of a calculus. These are ustally supplied in the form of mucus, or muco-pus, which is exuded from the walls of the urinary passages in consequence of the irritation of the crystals in the urine. Anything which tends to lessen this irritation will have some effect in hindering the formation of stone, and soothing diuretics may therefore play some part in the direction of prophylaxis.

Local Treatment directcd to the Prevention of Stone.-Besides these general measures directed to the constitutional conditions that lead to stone formation, much may be done by direct treatment to correct the conditions which locally favor the accumulation of crystals and their concretion into stones.

When the bladder does not completely empty itself, the residual urine should be drawn off from time to time. Even when the tendency is to the deposit of uric-acid crystals, this may prevent their collecting and having time to agglomerate themselves into a nucleus. It is, however, in the prevention of phosphatic stones that the removal of residual urine plays the most important part. It is in a fermenting residuum of urine that the deposition and coalescence of phosphates goes on most rapidly, for the accompanying cystitis liberally supplies the albuminoid skeleton in which the crystals build themselves up into a stone. At such times thorough irrigation, removing crystals and mucus and bringing the fermentation to a stop, may act as a most effective preventive measure.

It may be appropriate to mention here certain drugs which, when administered internally, impart to the urine some antiseptic qualities, and which therefore check fermentation, and also have indirectly a topical effect upon the bladder.

Benzoic acid and the benzoates of soda and ammonia may be given in doses of from 10 to 15 grains, or even, if needed, in much larger quantities. Over 300 grains of the benzoate of soda have been given in a day without unpleasant effects. Under their use the ammoniacal tendencies of the urine are largely corrected. Benzoic acid is eliminated by the kid- 
neys, either unchanged or as hippuric acid. It is, however, not well borne by the stomach, and it is usually preferable to administer one of its salts. These are broken up in the body, and furnish benzoic acid to the urine.

Boracic acid, administered internally, produces a decided antiseptic effect on the urine. It may be given in doses of 10 or 15 grains three or four times a day, either in capsules or in a very dilute solution. It is not well borne by a delicate stomach.

Eucalyptol may be given in an emulsion in doses of from 3 to 5 minims. It is excreted by the kidneys, and imparts its odor, together with its antiseptic properties, to the urine.

Salol, salicylic acid, and the salicylates, have also some antiseptic effect upon the urine. When used for this purpose, the dose shonld not be pushed to the extent that is usual when they are given as anti-rheumatics. It is a good rule to administer all these antiseptic remedies midway between meals, as, if taken when there is food in the stomach, they are likely to interfere with the digestion of it.

Even after a small concretion has formed in the bladder, or has found its way down from the kidney to the bladder, there are certain manœuvres that assist its passage through the urethra by the natural efforts. Before the operation of litholapaxy was devised, attempts in this direction were of some importance, and patients were advised to drink liberally of water, to hold their urine until the bladder was distended to its full capacity, and then in passing water to assume a position slightly bent forward, so as to bring the urethral opening into the most dependent part of the bladder, so that the stone should rest over or close to it, and be carried into the canal by the first rush of the stream.

This plan, when successful, subjects the patient to the risk of impaction of the stone in the urethra, and a small concretion can be pumped out so readily with a litholapaxy-bulb, that it is unwise to waste time with less efficient methods.

Solvent Treatment of Stone.-Although experience has given but little encouragement to those who have attempted to bring about the solution of stones in the bladder, still their efforts have brought out interesting facts, and are worthy of a moment's attention. In these experiments the solvent fluid has been brought in contact with the stone in one of two ways: either (1) the urine has been so changed by the administration of drugs as to itself become a solvent fluid, or (2) the dissolving agent has been injected directly into the bladder.

Before considering what has been accomplished in these attempts, let us look a moment at the conditions under which the work must be done. In the preceding pages we have spoken of the different methods of bringing about the solution of free crystals in the urine; but when we approach 
the question of the possible solution of stones already formed, we find the situation a more complicated one.

In the first place, a stone presents a comparatively small surface upon which the solvent can act. This surface is to a considerable extent protected by the mucus which adheres to it. Even if the outer layer of calcareous matter could be dissolved, this would leave the stone covered by a tenacious albuminoid envelope, which would serve as a protection against the further action of the solvent. Moreover, stones are not usually made up of one kind of earthy salt, so that the agent designed to dissolve one constituent of the calculus would have no action upon the others in it.

Besides these obstacles offered by the stone itself, the sensitiveness and delicacy of the bladder introduce a difficulty hitherto insuperable. For no fluid has been discovered which can be used in a sufficient concentration to have any powerful solvent effect on the stone without being dangerously irritating to the urinary organs.

(1) Efforts to dissolve stone by the administration of drugs which shall act through the urine date back at least to the time of Pliny. He speaks of burned snail-shell as a remedy for stone, and from that time to this the mixtures that have obtained any popular repute have usually contained alkalies, either potash, lime, or soda in some form. Probably these, as well as the vegetable diuretics and mineral waters, obtained their reputation largely from their soothing properties rather than from any solvent action; and it seems fair to suppose that the cases which made them celebrated for a time were often instances of bladder inflammation without stone.

The early essays at stone solution have not left any accurate data from which to draw conclusions as to their efficiency. Recently this subject has received some attention, and has been investigated in a more thoroughly scientific manner. Roberts (Treatise on Urinary and Renal Diseases, fourth American edition, 1885, pages 315-334), who has made a careful study of the subject, believed that his results, while not indicating the general possibility of substituting a solvent for a mechanical treatment of vesical stone, suggested essential improvements in the treatment of renal calculi.

Each form of calculus has to be considered separately. Taking first the more common uric-acid calculi, Roberts started his investigations by accepting two propositions as already proved: First, that solutions of the alkaline carbonates exercise a solvent action on uric acid; second, that urine can be rendered alkaline from alkaline carbonates by the administration of certain salts by the mouth. Examining now the comparative solvent powers of the various salts, he found that outside of the body carbonate of potash was decidedly more solvent than carbonate of soda; also, that a solution of from 40 to 50 grains of the carbonate to the pirit 
gave better results than one either weaker or stronger than this; also, that the quantity of the solution allowed to flow over the stone in twentyfour hours made but little difference in its daily loss in weight. The rate of solution obtained by fluids of the maximum solvent power he found to be from ten to twenty per cent of the calculi in the course of twentyfour hours.

Encouraged by these results, he next turned his attention to finding the best way of alkalizing the urine to the strength which he had found best in his experiments outside the body. He found that by administering from 40 to 50 grains of the acetate or citrate of potash every three hours he obtained an average alkalization equal to about 50 grains of the carbonate to the pint. With urine thus rendered alkaline he experimented upon uric-acid calculi outside of the body, and found that they underwent solution at the mean rate of $12 \frac{1}{2}$ grains in twenty-four hours. If, however, the urine became ammoniacal, it was found that the calculus became covered with a crust of mixed phosphates, which protected it from any further solvent action. This points to the practical importance of avoiding any alkaline fermentation of the urine while applying alkaline treatment.

These experiments are interesting as showing that the alkalies thus administered have decided solvent powers. Later observations by Garrod and others have seemed to show that the lithia salts have even more power in rendering uric acid soluble, and they have also found that lithia is well borne by the stomach. Garrod speaks of slight muscular tremors as occasionally produced by large doses of lithia salts. These have been met with by the writer, but have never caused serious inconvenience, and are only to be taken as a warning of the need of a cessation of the medicine for a time.

Another substance that has claimed some attention as a solvent of uric acid is piperazine. It is said to dissolve, in cold solution, twelve times as much uric acid as carbonate of lithia. Vogt thought that the amount of urea increased and uric acid diminished under its use, but these observations have not been verified by other investigators (Modern Materia Medica; Helbing, London, 1891). Biesenthal and Schmidt (Berliner klin. Wochenschrift, No. 2, 1892), from clinical and experimental researches, attest its value as a solvent of uric acid in the system, and have found that it increases the elimination of it.

It should be given dissolved in water, in a daily dose of 15 grains. They also recommend its use in a one to two per cent solution, as an irrigating fluid in the bladder, for the solution of calculi. It is asserted also that it dissolves the albuminous substances that hold concretions together, and so, it is thought, may be useful as a solvent of mixed stones, containing other salts besides uric acid or the urates. 
Oxalate of lime and phosphatic stones have proved quite intractable to the action of solvent remedies administered by the mouth.

Cystine is, according to Roberts, quite soluble outside the body in a solution of carbonate of potash, 40 grains to the pint. He found, however, clinically, that urine rendered alkaline by potash salts had little or no solvent power, and even contained crystals of cystine floating in it. Beale (London Lancet, August 30, 1884) has found that large doses of carbonate of ammonia have the power of causing cystine to disappear from the urine. One hundred and tifty grains a day was the quantity taken by one of his patients.

(2) The solution of stone by agents injected into the bladder has not succeeded to an encouraging degree. As the retention of injections in the bladder can not be prolonged over any considerable length of time, the solvent strength of the injected fluid must be very great in order that anything may be accomplished by this method. Practically, however, strong solutions are not borne by the bladder. The most successful application of this method is seen in the solution of phosphates by the injection of diluted nitric acid. This proceeding, suggested and carried out by Sir Benjamin Brodie, is no doubt available against small bits of phosphatic material, and very probably the astringent and stimulating action of the nitric acid improves the condition of the bladder, and thus helps to prevent the recurrence of phosphatic deposition. There are, however, no observations to show that stones of any considerable size can be thus disposed of.

Finally, attempts have been made to aid in the solution of stones by the electrolytic action of the galvanic current. Some slight effect was produced in this manner on stones outside of the body, but all attempts to make the process of use have been found unsuccessful, both on account of the time required in the manipulation, and because the stones proved unexpectedly resistant, owing to unlooked-for qualities in their composition; for stones composed of but one constituent were found to be not suited to electrolytic action.

The recent improvements in operative procedures for the removal of stones make the mechanical treatment so sure and easy that there is little inducement to try any of these tedious methods of solvent treatment in ordinary cases. Under exceptional circumstances, when operative treatment seems contra-indicated, they may occasionally be of service. Their legitimate field of action is in the treatment of stones in the kidneys, which can only be reached surgically by more dangerous operative measures. 
THE OPERATIVE TREATMENT OF STONE.

History.-Operations for the removal of stone in the bladder have always occupied a large share of surgical attention. The history of this subject has been so often and thoroughly written that it is not intended here to treat it exhaustively, but only to trace broadly the evolution which has led up to the present methods, and to dwell for a moment on the changes which have marked eras in the progress of this branch of operative surgery.

Before the present century the only operations practiced for the removal of stones were by cutting. It is true that in the early Arab writings the idea is advanced of wearing down a calculus by the friction of a diamond fixed on a wire, and thus introduced into the bladder along the urethra, and that other equally inefficient plans for rubbing down a stone were thought of in somewhat later times. The imperfection of instruments and methods in those early days prevented, however, these operations from obtaining a foothold among the accepted procedures of surgery.

The cutting operations for the removal of stone may be divided into two classes: first, those performed through the perinæum; and, second, those done above the pubes-high or suprapubic operation.

The perineal operations are by far the most ancient, dating back at least to the beginning of the Christian era ; while the suprapubic operation originated with Pierre Franco, in 1560. The earliest operations through the perinæum were done withont a guide. The first authentic and detailed report of these is given us by Celsus, of the school of Alexandria. It appears that the operation, as practiced, had come down from antiquity without special rules, the technique being passed on from one operator to another. In the operation described by Celsus, the stone was pulled downward by the fingers in the rectum, and was then cut down upon through the perinæum. The incision employed appears to have been either transverse, bilateral, or lateral, and the bladder was entered either through its body or its neck, according to accident. This is the method known as " cutting on the gripe," the apparatus minor-so called because but few instruments were required.

Eginus, describing what is practically the same operation, says that the external incision should be made on the side of the perinæum toward the left buttock. The intention was that the incision into the bladder should be made through the neck and not through the body of the organ; for it was thought that a wound of the wall was not likely to heal saticfactorily.

According to Celsus, only children from nine to fourteen were suitable subjects for the operation. Eginus speaks of it as applied to all ages. 
A variation of the perineal operation without a guide, consisted in plunging a grooved director into the bladder through the ischio-rectal fossa, and then passing the blade of a bistoury along the groove and with it making an incision of the required length. This operation was so dangerous as never to come into good repute.

After these early descriptions, surgical literature is for a long time silent in regard to lithotomy - a fact which suggests that it was not accepted by the authorities, but was relegated to specialists and empirics. It was not until 1533 that any movement in the direction of better methods was made. In this year Marianus published a description of the operation known under the name of the apparatus major, on account of the large number of instruments which it required. This operation was not original with him, but his description is the first reliable one that we have of it. It was a distinct advance over the operation of " cutting on the gripe," in that a grooved staff passed through the urethra was used as a guide, and gave the operation much greater precision. The operation of Marianus consisted in a median incision, opening the deep portion of the urethra, through which the neck of the bladder was stretched either by a two-branched dilator, or by the introduction of a gorget. It was practically the operation now known as the "boutonnière," and differed from the present median operation in the fact that the prostate was not incised. While a far safer operation than those which went before it, it was very unsatisfactory for the removal of large stones. This was the operation practiced by Franco, who modified it somewhat, and added to it the use of a concealed bistoury for incision of the prostate.

While the perineal operation was in this stage of its development, Franco was led by an accident to adopt the incision above the pubes, and thus became the inventor of the suprapubic operation, of which, however, he did not recognize the value.

At the beginning of the eighteenth century, Jacques Banlieu, better known as Frère Jacques, operated in Paris in a manner which he had learned from an itinerant lithotomist. In this operation the incision through the perinæum was made on the left side, and the operation was done upon a staff in the urethra. Curiously enough, Frère Jacques at first employed a round staff for his guide, and it was only after this had been adversely criticised that he adopted the grooved staff which had so long been in use for the median operation. With his method thus improved, he obtained many satisfactory results, and, with further modifications by Cheselden, the modern lateral operation was brought to perfection.

During the eighteenth century and the early part of the nineteenth, under the stimulus of success which now attended the operative treat- 
ment of stone, many surgeons sought to further improve existing methods and to discover still safer routes into the bladder. Frère Côme introduced the use of a concealed bistoury in the lateral operation, with the object of gaining mathematical exactitude for the deep incision. The cutting gorget was also nsed for the purpose of exactly limiting the incision in the prostate, and the, blunt gorget was introdnced to dilate the deep incision. These instruments were made in various shapes, and were much recommended by their inventors; but to-day most surgeons use the scalpel for the whole incision in the lateral operation, and it is rare that a lithotome or gorget finds its place in a modern armamentarium.

In the search for better incisions, the notable modifications devised were the bilateral operation, published by Dupuytren, in which a semilunar incision with its concavity backward was made transversely across the perinæum, and the prostate was incised with a double lithotome. The mediobilateral, Civiale's modifieation of Dupuytren's operation, differed from it in that the incision through the skin was on the median line, while the incision in the neck of the bladder was bilateral. The recto-vesical operation consisted in a median incision through the anterior inargin of the anus and anterior rectal wall, and so into the bladder, either through the prostate or through the trigone just behind it. The mortality of this operation, and the frequency of troublesome fistulæ which followed it, prevented its general adoption. None of these variations have proved to be of any especial value, and the median and lateral operations still hold their places as the best perineal methods.

While perineal lithotomy was passing through this gradual evolution, the suprapubic or high operation had various periods of favor and discredit. It proved its value for the removal of large stones, but showed itself to be much more dangerous than the perineal operations, and so did not succeed in displacing them for the majority of cases. Later, however, in 1878, as will be described in the chapter on Lithotomy, snch improvements were made in the technique of the high operation, that it has now to a considerable extent displaced the operation through the perinæum.

In 1824 the first serions effort was made to remove stones through the natural passages by pulverizing them in the bladder with instruments introduced through the urethra. In that year Civiale operated publicly with a three-branched instrument which he had derised some years before, and successfully crushed a small stone, fragments being passed by the natural efforts. Even with his imperfeet instruments Civiale produced results which showed the possibility of treating stone in this way.

This opened a new era in the treatment of stone, and immediately the mechanical ingenuity of surgeons and instrument-makers was taxed to 
the utmost to devise new and more efficient lithotrites. The gradual evolution of these instruments will be traced in the discussion of lithotrity.

Finally, in 1878, Bigelow showed that it was possible to combine thorough crushing and complete evacuation of the fragments in one operation. With this well-proved method it is now possible to quickly remove large stones from the bladder through the natural passages, without any serious injury of the soft parts-an almost ideal result.

After this brief glance at the history of their development, we will proceed to an examination of those methods of operation which have survived. In determining the comparative merits and applicability of the various operations for stone, it is evident that only statistics of recent date can be relied upon for forming an idea as to the comparative mortality of different methods. The general adoption of antiseptic precautions has so greatly diminished the dangers of all cutting operations, that the old rates of mortality can no longer be depended upon in estimating the dangers of modern lithotomy. It would be impossible to reflect the opinions of all the recent writers upon the subject as to the present standing of the various operations for stone removal, but we will attempt, in the description of the various operations, to give to all a fair consideration of their merits, and to consider the special conditions under which each one is applicable.

\section{CHOICE OF OPERATION.}

Before proceeding to the separate examination of the various operations, let us look for a moment at the considerations that are to be taken into account in deciding upon the operation proper to a given case; for the surgeon who best appreciates the influence which varying conditions should have upon his choice of procedure, and who decides each case for itself, selecting the operation which most surely avoids the dangers surrounding that particular patient, will arrive at better results than the advocate of a special operation, however expert.

The question of comparative danger is not the only one that the surgeon has to take into account in choosing an operation. He must also have regard to the interference with bodily functions which the various operations entail ; and, lastly, he must decide as to the probable completeness of cure which he can obtain by one or another method.

First, in regard to the comparative danger of the different operations. A glance at the rates of mortality shows us at once that we can not properly compare the results in patients of very different ages. The mortality of children and young adults after any operation upon the bladder is distinctly less than it is in advanced life, and there are at different ages changes in the organs concerned which make marked differences in the 
manner in which the various measures are borne. For the sake of convenience in this study, cases may be grouped in three categories:

Children, from birth to fourteen years of age.

Adults, from fourteen to fifty.

Old men, from fifty upward.

This division of cases is somewhat arbitrary, but the ages of fourteen and fifty are selected as marking, more or less accurately, certain epochs in the development and decay of the genito-urinary organs. At about fourteen we look for changes in the size and sensibility of these organs, which accompany the arrival of puberty; and at fifty senile changes in the bladder and prostate begin to make their appearance, which often interfere with the healthy performance of the functions of those parts. In the collection of statistics, those tables only should be used in which the operators have published all their results, and reports of single cases should not be included. This is to avoid the danger of forming tables of exceptional results, for single cases are more likely to be reported when successful than when they resulted unfavorably.

As I have been unable to find any ready-made statistics showing the comparative results of the different modern methods of operation, I have collected a series of cases from accessible published reports, and from the private reports of unpublished cases which I have been able to obtain in the time at my comniand. I lave succeeded in obtaining 2,562 cases in which the ages of the patients were given, so that they could be classified in the manner above indicated.

All these cases have been operated upon since 1878, and have thus occurred during the time when they would have all the advantages of modern surgical methods.

Perineal Lithotomy.-This operation has not shown any modern improvement in technique beyond the introduction of aseptic methods. A comparison of results under old and new methods is interesting.

Keyes, in an article on Urinary Calculus, contributed by him to the International Encyclopædia of Surgery, gives the following table, which contains the death-rate calculated by Coulson from an analysis of 2,972 cases, and by Thompson from an analysis of 1,827 cases :

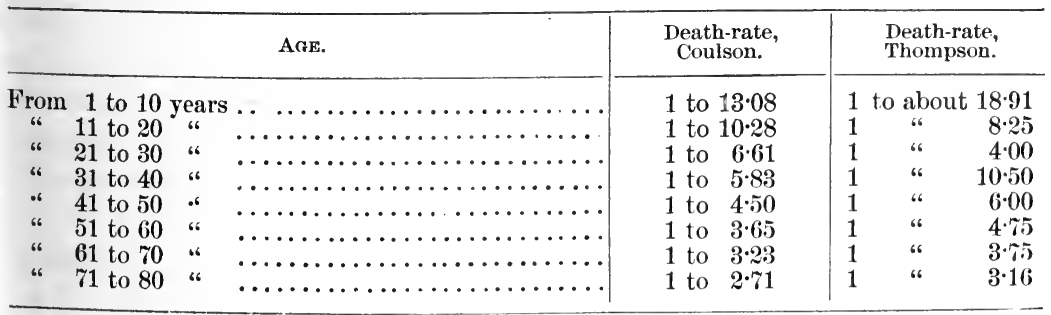


Thompson's cases may owe part of their improvement in results to the commencement of antiseptic habits in surgery. Better methods of handling the stone, of controlling hæmorrhage, and of conducting the aftertreatment, are, however, mainly responsible for the lower death-rate.

I have collected 764 cases of perineal lithotomy. They resulted as follows :

Children under 14, 539, with 16 deaths; $2 \cdot 96$ per cent of mortality.

Adults, 14 to $50,17 \%$ " 19 " $19 \quad 11 \cdot 30$ " “

old men,

48 " 8 " $16 \cdot 66$ "

The number of operations upon old men is too few to give a reliable percentage of mortality. The operation is probably more dangerous in old age than is here indicated.

Freyer gives the following rates of mortality after lateral lithotomy, arranged according to age. They are calculated from 987 cases occurring during the year 1883 in the Northwest Provinces of India:

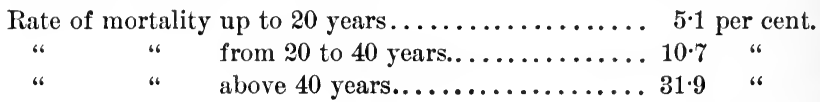

Rosenthal, from a collection of 400 cases, deduces the following rates:

\begin{tabular}{|c|c|c|c|c|c|c|}
\hline \multicolumn{2}{|c|}{ Mortality from } & \multicolumn{3}{|c|}{ 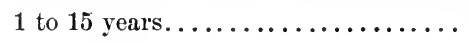 } & \multicolumn{2}{|c|}{3.5 per cent. } \\
\hline “ & “ & 6 to 11 & “ & & $2 \cdot 1$ & “ \\
\hline “ & “ & 12 to 16 & “ & & $8 \cdot 4$ & “ \\
\hline " & “ & 17 to 29 & “ & $\ldots \ldots \ldots \ldots \ldots \ldots \ldots \ldots$ & $15 \cdot 7$ & “ \\
\hline "6 & "6 & 30 to 66 & “ & $\ldots \ldots \ldots \ldots \ldots \ldots \ldots$ & $38 \cdot 8$ & “6 \\
\hline
\end{tabular}

Harrison, from more than 100 cases, taken irrespective of age, gets a mortality rate of 1 in 12 .

Keyes says that his "perineal lithotomies before puberty have been few in number, and have all recovered." In those cases after puberty he has found "the mortality much higher than for the crushing operation."

Suprapubic Lithotomy.-I have obtained data of 744 cases of this operation, from which the following table has been made:

Children under 14,591 , with 74 deaths; 12.52 per cent of mortality.

Adults, 14 to $50, \quad 100$ " 12 " 1200 "

Old men, " $53 \quad 17$ " $\quad 32.07$ "

Comparing these with other statistics, the results in children and adults are more than usually favorable.

Garcin, from a collection of 106 cases of all ages, calculates a deathrate of 24.4 per cent. Tuftier, from 120 cases without regard to age, has a death-rate of 27 per cent. Dulles, among 231 adults, finds a mortality of $32 \cdot 4$ per cent; while among 132 children there was a death-rate of 21 per cent.

Besides the cases tabnlated above, I have obtained reports of 165 cases, unclassified as to age, in which the death-rate was $15 \cdot 15$ per cent. 
Litholapaxy.-I have classified reports of 1,074 cases of litholapaxy, as follows:

Children under 14, 241, with 4 deaths; 1.66 per cent of mortality.

$\begin{array}{llllllll}\text { Adults, } 14 \text { to } 50, & 400 & \text { " } & 13 & \text { " } & 3.25 & \text { " } & \text { " } \\ \text { Old men, } & 433 & \text { " } & 26 & \text {. } & 6.00 & \text { " } & \text { " }\end{array}$

Guyon had a mortality of $5 \cdot 2$ per eent in 647 cases of all ages. Usigli calculates a mortality of 4 per cent, while Tuffier places it at only 3 per cent.

Keyes, in 100 cases, lost 7 , but almost all his patients were old men, many of them with desperate disease of the urinary tract, and even under these unfavorable conditions the later eases are showing better results.

From these statistics we see that in childhood, judging from the results as to mortality, there is little to choose between lateral lithotomy and litholapaxy, the advantage, however, being slightly with the latter. Suprapubic lithotomy is more dangerous, with a death-rate of about 12 per cent.

In adult life the death-rates alter very decidedly in favor of litholapaxy. As the prostate and urethra enlarge, and the parts about the neck of the bladder become more vascular, the dangers incident to cutting through them increase. On the other hand, the increase in the size of the parts makes the performance of litholapaxy comparatively easy and safe. Suprapubic lithotomy keeps its place as a more dangerous operation than either. In old age, the rates of mortality are overwhelmingly in favor of litholapaxy. While the dangers attending all the cutting operations have increased very greatly, the mortality after crushing is very little higher than it was earlier in life.

\section{INTERFERENCE WITII THE FUNCTIONS OF THE PARTS.}

It is somewhat exceptional to see a serious loss of function follow any of the operations for the removal of stone. The parts are more injured by some of the operations than by others, but usually the restoration of function is satisfactory, if not complete.

A litholapaxy, carefully performed, should never cause any lasting injury of the genito-urinary organs. It may sometimes happen, in an old man with an enlarged prostate, that the instrumentation so irritates the neck of the bladder as to lead to a still further swelling of the prostate and thus causes retention, which may subsequently require systematic catheterization. This course of events is only likely to occur in patients who had already a partial retention, with loss of expulsive power in the bladder, who were therefore close to the time when they would require the constant use of the catheter in any event.

The suprapubic incision does not traverse important parts, and therefore rarely causes after-trouble. Occasionally a fistulous opening remains 
which ean not be closed, and therefore is a source of discomfort to the patient; but this accident can usually be avoided by the timely removal of the drainage tube.

The perineal operations, entering, as they do, through the neck of the bladder, are more likely to cause serious trouble. The seminal ducts, situated in the lower part of the prostate, are exposed to injury by incisions in the floor of the prostatic urethra, and impotence from this cause is to be feared. The median operation avoids this when the stone is small enough to be removed by stretching the neck of the bladder, but even then lacerations are likely to occur. The lateral incision has the advantage that, while giving more room, it endangers only one of the ducts. The erectile tissue, known as the caput gallinaginis, is also liable to injury by perineal incisions, and this, too, may lead to sterility. I have seen a man who was cut by the lateral method when a child, whose semen escaped into the bladder during coition, and was afterward passed with the urine. This seemed to be due to the failure of the erectile tissue to close the neck of the bladder behind the ejaculatory ducts.

Incontinence of urine is another occasional result of perineal operations. Both sphincters of the bladder are often completely divided, and, although the restoration of function is usually complete, it sometimes happens that the healing is in some way interfered with, and a permanent loss of retentive power is the result. Persistent fistula following perineal lithotomy is not very uncommon. Werewkin records this as occurring seven times in 147 cases of lateral lithotomy in children.

It is claimed that stricture does not result from this operation, but the writer has met with a decided narrowing in the membranous urethra in two cases. One of these was a man who had been cut in childhood, and had been subsequently subject to attacks of cystitis. The membranous urethra was narrowed to No. 25 French, while the rest of the canal would admit a 31 French sound. The other case was a child in whom the persistence of a perineal fistula led to an examination of the urethra, and a decided narrowing was found at the junction of the bulbous and membranous portions of the canal.

Injuries of the rectum during lateral lithotomy are accidental and unnecessary; still, they happen often enough in the hands of expert operators to make it worth while to take this possibility into account in deciding upon an operation.

It would not be proper to close this consideration of the chances of a loss of function after these operations without calling to mind the fact that some of these methods open the way for a restoration of function by allowing opportunity for the removal of obstructing portions of the prostate, and thus tending to the recovery of the power of urination. This is especially true of the suprapubic operation. 

OPERA'TING.

It is a not uncommon experience to see a second or third attack of stone in the same patient. In order to understand how far this reappearance of a calculus is dependent upon the operation by which its predecessor was removed, let us consider the ways in which a recurrence of stone may come about:

1. A uric-acid stone may be followed by another on account of the persistence or reappearance of the uric-acid diathesis. This may be true, though less commonly, in the case of an oxalic stone, and may even occur with a phosphatic stone due to phosphaturia of constitutional origin. When cystine is present in the urine, the frequent appearance of small calculi is a characteristic feature of the disease.

2. The successive escape of several stones from the kidneys may give rise to consecutive attacks of stone in the bladder. These stones may be uric, oxalic, phosphatic, or composed of cystine.

3. A soft, phosphatic stone may be reproduced after removal, if the chronic cystitis and alkaline condition of the urine, which led to the original formation, persist. This is not uncommonly seen in those cases where an obstruction to the complete emptying of the bladder perpetuates the fermentation of the urine.

4. Lastly, if a fragment is left after operation it may serve as the nucleus for another stone. The danger of this mischance is greatly increased by any obstruction to the flow of the urine, such as is caused by an enlarged prostate. The bladder in such cases is often sacculated, so that fragments are more likely to escape removal by the evacuator after litholapaxy, or by the lithotomy scoop and forceps after lithotomy; and if such a fragment be left, it is very unlikely to be voided by the natural efforts of the bladder, but remains in the residual urine. A healthy bladder that completely expels the urine at each act of micturition usually frees itself from such small fragments.

It is plain that recurrences due to the patient's diathesis, in which a new stone forms years after the removal of a former one, can not be laid at the door of the operation, being as likely to follow one method of removal as another. And the same is true when successive escapes of renal calculi from the kidneys give rise to recurrent attacks of stone. In cases where a recurrence is due to a persistent cystitis, with consequent deposition of phosphatic material, the later attacks of stone can not be ascribed to incompleteness in the operation, but rather to neglect in the aftertreatment. It is obvious that, to prevent this sort of recurrence, it is important to entirely relieve the cystitis before allowing the patient to pass from under observation, and then to send him away with a clear 
understanding of the importance of immediately correcting any tendency to alkalinity of the urine or to pus-formation. (See Preventive TreatMeNT OF Stone.)

Finally; a stone which has for its nucleus a fragment of an earlier stone, is obviously the result of an incomplete operation, and no method can be regarded as satisfactory which is often followed by such recurrences.

It has been charged against litholapaxy that this accident of the retention of a fragment is very likely to follow it. The means of preventing this will be considered at length in the description of that operation; and suffice it here to say, that since the possibility of this occurrence has been generally recognized, the measures taken against it have been almost uniformly successful in preventing it. Moreover, it is an accident by no means confined to this operation. It is not uncommon to have stones broken, or fragments chipped off of them by the lithotomy-forceps, and, as may be supposed, this is more apt to occur in perineal lithotomy, where the stone has to be dragged through a narrow wound, than in the suprapubic operation, where more space is offered. Fragments so left are often very difficult of removal with the scoop, or by irrigation, and instances of their retention after lithotomy, and of subsequent stone formation about them, are not infrequently reported.

\section{SELECTION OF OPERATION.}

From what has been said above, it will be seen that many matters have to be taken into consideration in making the proper choice of operation in each case. Indeed, much of our knowledge in regard to the treatment of stone is so new that we are only now accumulating the data on which our judgment must be based.

In childhood, as has been shown, the mortality after any operation for stone is small. Lateral lithotomy and litholapaxy are very nearly equal in this respect, and both are decidedly safer than suprapubic lithotomy. The crushing operation has the great advantage that it avoids injury to the seminal ducts and rectum; also, that it does not give rise to fistula or to the incontinence of urine-all of which are occasional results of perineal lithotomy. An ample experience has shown that the urethra and bladder of a child will tolerate a considerable amount of instrumentation. It would therefore seem wise to use litholapaxy for all small stones, or stones of moderate size (from one and a half to two and a half centimetres in diameter), and for stones larger than this to do lateral lithotomy, except when they are very large (three and a half centimetres and upward in diameter), and then suprapubic cystotomy is to be resorted to.

The ease with which bimanual palpation can be practiced in children, with the finger in the rectum and a hand on the abdomen, makes it pos- 
sible to judge pretty closely the size of the stone, and so select intelligently the best method for its removal. The consistency of a stone is also to be taken into account when litholapaxy is to be thought of, and stones of considerably larger size than is above indicated may be crushed, if they are soft and friable. The quality of a stone in these regards may usually be determined with some degree of accuracy by the sensation imparted to the sound and by a knowledge of its probable constituents, which can often be gained by an examination of the urine. Certain other conditions which should lead us to employ some other method than litholapaxy will be spoken of in considering the operation in adults.

Whether we consider the danger of the various operations for stone in the adult, or the likelihood of the disturbance of functions following them, we are led to regard litholapaxy as the operation of choice for stone removal. With the efficient lithotrites and evacuator which make "lithotrity at one sitting" possible, it is now usual to remove stones of considerable size and hardness, and practically it has been found that, under ordinary conditions in adults, any stone which is suitable for lateral or perineal lithotomy is suitable for litholapaxy, and that even stones so large that they would require a suprapubic incision if they were removed with the knife, may, when reasonably friable, be pumped out. A number of instances are on record in which stones between 2,000 and 3,000 grains in weight have been successfully removed in this manner.

The exceptional cases in which litholapaxy can not be used are as follows:

1. A very large and hard stone may resist every attempt at crushing, especially if it is tightly grasped by the spasmodically contracted bladder.

2. A stone may have as a nucleus a foreign body, such as a bit of necrosed bone, or a bullet, too hard to crush and too large to pass out through a tube.

3. An encysted stone may be out of reach of the lithotrite.

4. Some writers hold that stricture of the urethra may prohibit litholapaxy. This can not often happen, for strictures, however close, yield readily to divulsion, which may be immediately followed by the crushing and evacuation of the stone. I have so often seen these operations successfully done on an etherized patient, that I can not but think this the best practice. While it economizes time, it saves the patient much needless manipulation.

5. False passages may exist, which interfere with the introduction of instruments, so that the dangers of the operation are greatly enhanced, and the question of lithotomy is to be entertained.

6. The hip may be anchylosed in a position which interferes with the use of urethral instruments.

7. A stone may be so lodged in the entrance to the urethra that it 
can not be pushed back into the bladder, where it can be seized by the lithotrite.

In any of these exceptional cases in which litholapaxy can not be applied we have to make our choice between a perineal and a suprapubic incision. The danger attaching to the perineal incision is, according to present indications, decidedly less than that after the high operation, so long as it is applied to small or medium-sized stones; but when large stones are dealt with the facts are reversed, and the perineal operation becomes the more dangerous of the two. Under ordinary circumstances, as has been said, litholapaxy disposes of stones of a size suited for perineal removal, and these operations through the perinæum have therefore fallen largely into disuse in adult cases. They find occasional application in case of stones of moderate size, where false passages, anchylosis of the hip, or the presence of a foreign body, make litholapaxy impossible. A stone impacted in the neck of the bladder, if it can not be dislodged, may be properly removed through the perinæum.

Suprapubic lithotomy is to be employed in cases where the stone is too hard and large to be crushed, or where an impervious urethra makes the introduction of a lithotrite or staff impossible. In cases of encysted stone also the high operation is the best, as the thorough inspection of the bladder which it makes possible enables us to treat the conditions intelligently.

In old age the same indications are to be followed as in the adult, except that it is to be remembered that perineal incisions are especially dangerous for old men, and are not to be undertaken for the removal of a stone without urgent reasons. The suprapubic operation will therefore be called upon to deal with most of the stones that are unsuitable for litholapaxy. In cases of enlarged prostate, when it is desired to combine prostatotomy or prostatectomy with the removal of the stone, the high operation affords ample room for thorough inspection of the condition and the correction of it. It is in old men that the crushing operation has its most signal advantages; for, while the mortality of the cutting operations is very great, the ample size and the tolerance of the urethra and bladder make litholapaxy easy and comparatively safe.

\section{COMPLICATIONS WHICH MAY MODIFY OUR CHOICE OF OPERATION.}

In what has been said, we have examined the general considerations which must influence our selection of an operative procedure. It remains to consider certain complications which sometimes exist in these cases, and which may modify our choice.

Occasionally the existence of some general disease not connected with the stone may so add to the danger of the operation as to make it wiser 
to palliate. Thus, affections of the heart and lungs may add great risk of death from anæsthesia. In these cases careful preliminary treatment may considerably modify the danger. Certain other general diseases make the system intolerant of cutting operations. Diabetes mellitus, Bright's disease, alcoholism, anæmia, and gout are prominent instances of general disease which interfere with the healthy healing of wounds, and in the presence of which one would give an added preference to litholapaxy.

In those cases of interstitial nephritis in which large quantities of urine of low specific gravity are excreted, the crushing operation is well borne; and I have known three litholapaxies to be done at intervals on such a patient, who died uræmic two or three years after the last one. To cutting operations, on the other hand, these patients frequently succumb. When to this interstitial nephritis is allied a chronic pyelitis, with dilated pelves and ureters, we have a far more grave condition. Unfortunately, this state of the urinary organs is not infrequent, brought about by those obstructive diseases of the prostate and urethra which also give rise to stone. Even under these unfavorable conditions, with careful preparation, an operation may be undertaken, and litholapaxy is much safer than any form of lithotomy. In fact, the increased relative safety of litholapaxy late in life is probably due to the renal complications so common in these old patients. which makes them very intolerant of cutting operations, while they bear lithotrity at one sitting remarkably well.

As has been said, a stricture in the urethra may be corrected as one step in the removal of a stone without seriously complicating the performance of a litholapaxy. In case of a serious stricture, however, which can not be properly treated by rapid dilatation or divulsion, and which therefore requires an incision for its correction, it may be wise at the same time to cut for the stone.

With an incision throngh a stricture in the perinæum, the posterior urethra may be dilated sufficiently to allow of the removal of a small stone with the forceps. If the calculus is too large for this, crushing instruments may be introduced through the perineal incision after the manner to be described later under Perineal Lithotrity. If the stricture is very close, so that the operation upon it would be difficult, and would add considerably to the shock of the lithotomy, it may be wise to remove the stone at once by suprapubic lithotomy, and, after the patient's condition has been improved by relief from the pain of the stone and by drainage of the bladder, to then undertake the correction of the stricture.

The enlargement of the prostate usually offers but slight obstruction to the introduction of instruments. It does, however, in some cases, considerably interfere with their use after they are in, and the changes in the 
shape of the bladder that it brings about may somewhat hamper the lithotritist. The way in which these difficulties are to be met will be considered in the description of that operation.

In the very rare cases in which the obstacles to lithotrity from this cause are too great to be overcome, a lithotomy must be done, and as a large, hard prostate greatly complicates and lengthens the route into the bladder, it will usually be found best to use the suprapubic incision for these cases.

In connection with a stone, conditions may exist in the bladder which themselves require operative treatment, and the proper operation being chosen for their correction, the removal of the stone is merely a secondary matter. Tumors of the bladder and tumors or outgrowths of the prostate may be mentioned here, and, as their treatment is fully considered elsewhere, I will not go into that question, but will only remind the reader that the symptoms of an enlarged prostate are greatly aggravated by the presence of a stone in the bladder, and that their discomforts often wholly subside upon the removal of the source of irritation. It is well, therefore, always to consider this, and in cases of doubt to do the comparatively safe litholapaxy first. By this course the patient will often be saved from the added danger of a suprapubic incision.

Severe chronic cystitis is also mentioned as a reason for opening the bladder. Keyes says: "Cystotomy, under such circumstances, deals with two maladies at once; and the greater risk of lithotomy is more than compensated for by the prospect of curing the cystitis; while the danger of acutely aggravating the chronic cystitis by the necessarily harsh manœuvres of litholapaxy makes the latter operation a second choice." I quote this statement at length, because, coming from so high an authority, it carries great weight; and it seems to me that such a rule, applied by men of less sound judgment than Prof. Keyes, might often lead to the choice of severer measures than the case demanded. In most of these cases the stone is the cause of the cystitis, and usually the removal of this cause allows the rapid subsidence of the bladder inflammation. It has not been my experience that the manœuvres of litholapaxy greatly aggravate even severe chronic cystitis; and it is well to remember that in old men, in whom these cases of cystitis usually occur, lateral lithotomy is about three times as dangerous as litholapaxy. A better way of meeting the indication for drainage of the bladder, when that is evidently important, is, I think, to remove the stone by litholapaxy, and then to introduce drainage through an opening in the membranous urethra (boutonnière), which operation leaves a much less extensive wound than a lateral lithotomy. 


\section{PREPARATION FOR OPERATION.}

An operation having been decided upon, it is important to put the patient and his urinary organs in the best possible condition before subjecting him to instrumentation. If the patient is strong, and the bladder reasonably healthy, but little preparation is required. When, however, the patient is old and broken in health, with urinary organs more or less disabled, a wise preliminary course of treatment will often considerably increase his chances of recovery.

First, the general health should be looked to. It is often possible sensibly to add to the strength of a feeble patient by putting him in bed and supplying the fullest quantity of very digestible food that the stomach can properly dispose of. Extra meals, the moderate exhibition of stimulants, with a liberal supply of milk, will accomplish much in this effort to artificially manufacture strength. In old men with diseased kidneys, the action of the heart is often seriously enfeebled, and cardiac stimulants are then of prime importance. Aside from this, the ordinary tonic treatment suitable for each case is to be applied, and the regular action of the bowels is to be provided for.

It is next important to investigate the action of the kidneys and the condition of the bladder. The daily amount of urine, taken together with the specific gravity, or a quantitative test of the urea, enable one to determine whether the kidneys are accomplishing a sufficient amount of work. If the urine is small in quantity, and of high specific gravity, or if it is about normal in amount (between forty and fifty ounces), but of low specific gravity, an effort should be made to increase the amount by the free administration of water and milk. Digitalis may act as an efficient diuretic, if the heart's action is feeble.

Next, an examination of the urine should be made, and if evidence of serious disease of the kidneys exists, or if sugar is present in any considerable amount, the proper treatment should be instituted to correct these conditions as far as may be done. If the urine is ammoniacal or putrid in odor, efforts should be made to stop the fermentation and to restore its normal acidity. This is as important a compliance with the rules of antiseptic surgery as the effort to clear any field of operation of infective germs. The measures to be adopted to this end are both general and local. The general measures consist in the administration of large quantities of water to flush the urinary passages, with the addition of certain drugs which are excreted through the kidneys and have some effect in supplying an antiseptic quality to the urine. The benzoates, salicylates, boracic acid, eucalyptol, and sandal-wood oil, may be mentioned as the more efficient of this class of remedies.

The local treatment of these conditions is even more important than 
the general, and consists in the systematic removal of the residual urine with a catheter and irrigation of the bladder. The advantages of irrigation are largely due to the mechanical effect of removing the last remains of the urine and mucus. Certain drugs, however, in the irrigating fluid may, by being mildly antiseptic or astringent, add to the efficacy of these injections. The more useful irrigating fluids are borax, or boracic acid, one drachm to the pint of water, permanganate of potash, or carbolic acid in very dilute solution, and acetate or sulphate of zine well diluted. Oftentimes very weak solutions of nitrate of silver, one-half grain to one grain in an ounce of water, exercise a very beneficial effect in these cases. This is better used as an occasional wash, some milder solution being employed in the intervals. When the urine is strongly ammoniacal and loaded with triple phosphates, dilute solutions of nitric acid will often be of great service.

These injections must be properly managed with eare and judgment, in order that they may not be a source of irritation rather than of soothing to the bladder. If there is a strong tendency to spasm, and the bladder shuts down painfully on to the stone at each emptying, the use of irrigation is of doubtful advantage. In such a case the quantity injected each time should be small, so that it may not distend the bladder and put it in a condition of spasm. When such a patient can not bear the injections, the correction of the urine may be accomplished, although more slowly, by putting him in bed with the hips raised, so as to roll the stone away from the sensitive neck of the bladder, and then instituting the general treatment outlined above, with the addition of opium or belladonna to keep the bladder at rest.

If these measures fail of success, and it is evident that the irritation of the stone is more powerful to keep up the inflammation of the bladder than any measures at our command are to diminish it, the operation must be proceeded with at once, and the stone, the exciting cause of the cystitis, must be removed, even under these unfavorable conditions

Some operators prepare the urethra before lithotrity by the passage of large sounds, and by the operative treatment of stricture, if such exists. The previous use of sounds to prepare the urethra for instrumentation seems to me unnecessary, and the operation upon any existing stricture can be readily and properly combined with the operation for the removal of the stone. A sufficient preliminary examination of the urethra to ascertain the character of any such complications is wise, as a knowledge of them may modify the plan of operation.

As a precaution against the occurrence of chill after the operation, the previous administration of quinine and morphine has been recommended. Keyes prefers a combination of pilocarpine and morphine. I have for a long time operated without any such preparation, and have no 
reason to regret the omission. On the morning of the operation the stomach should be kept empty, but a little spirit may be administered, if the patient is feeble.

We have shown the crushing of the stone to be the operation of choice whenever it is possible, and we will consequently consider this method first, even though it is the most modern.

\section{Lithotrity.}

History and Development.-The idea of mechanically pulverizing a stone by instruments introduced through the urethra is by no means modern. In early Arab writings the suggestion is made of wearing down the calculus by attrition with a diamond fixed on a wire and thus introduced into the bladder. Mention of these procedures is made in the works of Albucasis in the twelfth century. In 1580 Sanctorius suggests the possibility of extracting little calculi with three-branched forceps. According to Haller, Sanctorius even proposed perforating them with a stylet, when they were too large to be removed whole. The imperfection of methods, however, in those early days prevented the operation from obtaining a foothold among the accepted procedures of surgery, and practical lithotrity, therefore, waited to be an invention of the nineteenth century.

In 1813 Gruithuisen demonstrated the possibility of eatheterization with a straight instrument, and showed that the urethra was much more dilatable than had been previously supposed. He introduced straight sounds over three and a half lines in diameter, and proposed to operate on stones by snaring them with a wire projected through the end of a straight tube and then perforating them with a drill. He never carried this plan into practice.

A few years later Civiale began to interest himself in this operation, and after several attempts produced an instrument in which a threebranched forceps held the stone, while a drill perforated it. As has been

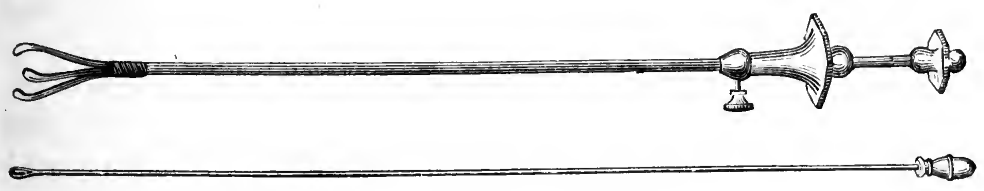

FIG. 14.-Civiale's trilabe or litholabe.

said, Sanctorins proposed such a plan centuries before, but to Civiale is due the credit of applying it practically and making it succeed. Leroy was working on parallel lines with Civiale, and produced at about the same time a forceps with three branches which held the stone, while it 
the general, and consists in the systematic removal of the residual urine with a catheter and irrigation of the bladder. The advantages of irrigation are largely due to the mechanical effect of removing the last remains of the urine and mucus. Certain drugs, however, in the irrigating fluid may, by being mildly antiseptic or astringent, add to the efficacy of these injections. The more useful irrigating fluids are borax, or boracic acid, one drachm to the pint of water, permanganate of potash, or carbolic acid in very dilute solution, and acetate or sulphate of zine well diluted. Oftentimes very weak solutions of nitrate of silver, one-half grain to one grain in an ounce of water, exercise a very beneficial effect in these cases. This is better used as an occasional wash, some milder solution being employed in the intervals. When the urine is strongly ammoniacal and loaded with triple phosphates, dilute solutions of nitric acid will often be of great service.

These injections must be properly managed with care and judgment, in order that they may not be a source of irritation rather than of soothing to the bladder. If there is a strong tendency to spasm, and the bladder shuts down painfully on to the stone at each emptying, the use of irrigation is of doubtful advantage. In such a case the quantity injected each time should be small, so that it may not distend the bladder and put it in a condition of spasm. When such a patient can not bear the injections, the correction of the urine may be accomplished, although more slowly, by putting him in bed with the hips raised, so as to roll the stone away from the sensitive neck of the bladder, and then instituting the general treatment outlined above, with the addition of opium or belladonna to keep the bladder at rest.

If these measures fail of success, and it is evident that the irritation of the stone is more powerful to keep up the inflammation of the bladder than any measures at our command are to diminish it, the operation must be proceeded with at once, and the stone, the exciting cause of the cystitis, must be removed, even under these unfavorable conditions

Some operators prepare the urethra before lithotrity by the passage of large sounds, and by the operative treatment of stricture, if such exists. The previous use of sounds to prepare the urethra for instrumentation seems to me unnecessary, and the operation upon any existing stricture can be readily and properly combined with the operation for the removal of the stone. A sufficient preliminary examination of the urethra to ascertain the character of any such complications is wise, as a knowledge of them may modify the plan of operation.

As a precaution against the occurrence of chill after the operation, the previous administration of quinine and morphine has been recommended. Keyes prefers a combination of pilocarpine and morphine. I have for a long time operated without any such preparation, and have no 
reason to regret the omission. On the morning of the operation the stomach should be kept empty, but a little spirit may be administered, if the patient is feeble.

We have shown the crushing of the stone to be the operation of choice whenever it is possible, and we will consequently consider this method first, even though it is the most modern.

\section{Lithotrity.}

History and Development.-The idea of mechanically pulverizing a stone by instruments introduced through the urethra is by no means modern. In early Arab writings the suggestion is made of wearing down the calculus by attrition with a diamond fixed on a wire and thus introduced into the bladder. Mention of these procedures is made in the works of Albucasis in the twelfth century. In 1580 Sanctorius suggests the possibility of extracting little calculi with three-branched forceps. According to Haller, Sanctorius even proposed perforating them with a stylet, when they were too large to be removed whole. The imperfection of methods, however, in those early days prevented the operation from obtaining a foothold among the accepted procedures of surgery, and practical lithotrity, therefore, waited to be an invention of the nineteenth century.

In 1813 Gruithuisen demonstrated the possibility of catheterization with a straight instrument, and showed that the urethra was much more dilatable than had been previously supposed. He introduced straight sounds over three and a half lines in diameter, and proposed to operate on stones by snaring them with a wire projected through the end of a straight tube and then perforating them with a drill. He never carried this plan into practice.

A few years later Civiale began to interest himself in this operation, and after several attempts produced an instrument in which a threebranched forceps held the stone, while a drill perforated it. As has been

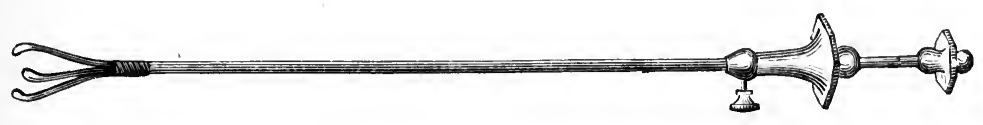

Frg. 14.-Civiale's trilabe or litholabe.

said, Sanctorins proposed such a plan centuries before, but to Civiale is due the credit of applying it practically and making it succeed. Leroy was working on parallel lines with Civiale, and produced at about the same time a forceps with three branches which held the stone, while it 
was operated upon with a saw. It fell to Civiale, however, to be the first to put his instrument to practical use, and in 1824, before the Academy of Medicine in Paris, he operated upon two patients successfully. These fortunate results of Civiale aroused the mechanical inge-

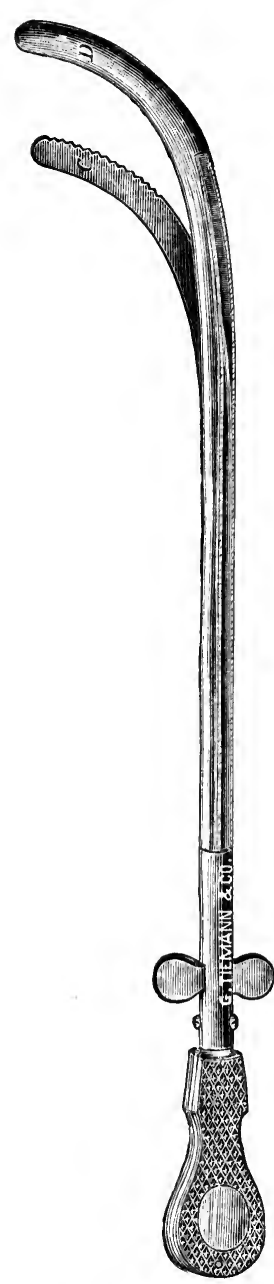

F19. 15.-Weiss's first lithotrite. nuity of other surgeons, and at once the greatest activity was shown in devising improvements in the form of crushing instruments.

While Civiale and Leroy were interesting themselves in perfecting instruments which had for their object the perforation and gradual wearing away of the stone, other inventors were addressing themselves to the discovery of means for the direct crushing of stones, either by percussion or pressure. In 1822 Amussat had brought out his "brise-pierre," which was a straight tube through which a two-branched forceps was worked, the branches being separately movable. This instrument was designed to catch the stone and then rub it down between its jaws. The brise-coque of Heurteloup was a somewhat nicer adaptation of the same principle. But neither of them worked in practice.

The difficulty of introducing a straight instrument led to many efforts to adapt a curved lithotrite to the requirements of the crushing operation. In 1824 Weiss constructed such an instrument, with two blades, with shafts working parallel, and with blades which were in principle the same as those of the present instruments. The force in this instrument was applied by a screw. Retoré and Haygarth devised similar instruments in 1825. In all these early lithotrites, however, the shafts were insecurely fastened together, and force which could be safely applied was not sufficient for the purpose of breaking the stone.

In 1830, Jacobson, of Copenhagen, introduced a lithotrite which had one solid blade and one blade articulated like a chain-saw. The stone was to be caught in the loop made between these two, and crushed by screw force drawing the articulated blade down on to the solid one. This instrument, while useful for small stones, proved insufficient for large ones, and with it it was difficult to find and seize fragments after the first crushing.

In 1832 Heurteloup brought out his lithotrite, called a percuteur, in 
which the force was applied by the blows of a hammer. The blades in this instrument were practically like those of Weiss's and Retoré's lithotrites, but the shaft was somewhat differently arranged for the purpose of

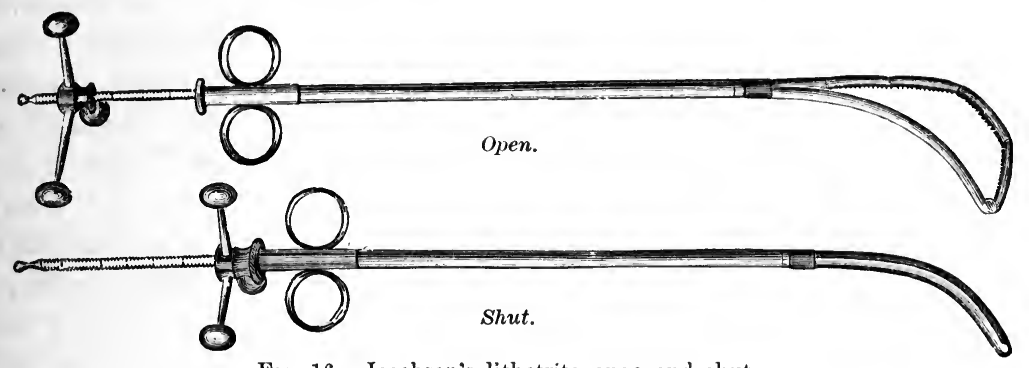

Fig. 16.-Jaeobson's lithotrite, open and shut.

giving more strength. The shaft was made of three pieces, the outer ones of which were attached to the female blade, and the middle one, which slipped to and fro between them, carried the male blade. The two outside pieces of the shaft were joined in the lower part by a cross-rivet, and in the shaft of the male blade was a long slit through which this rivet passed, which was designed to prevent the riding up of the male

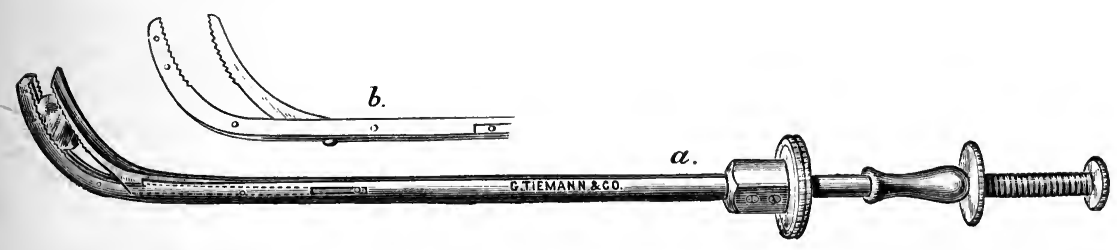

Fig. 17.-Heurteloup's pereuteur. $a$ shows the riding up of the male blade when pressure is applied; $b$ shows eheek-button added by Costello.

blade when pressure was brought upon the stone between its jaws. In practice this arrangement was found not strong enough to deal with stones of any hardness, and in one disastrous case Sir Benjamin Brodie

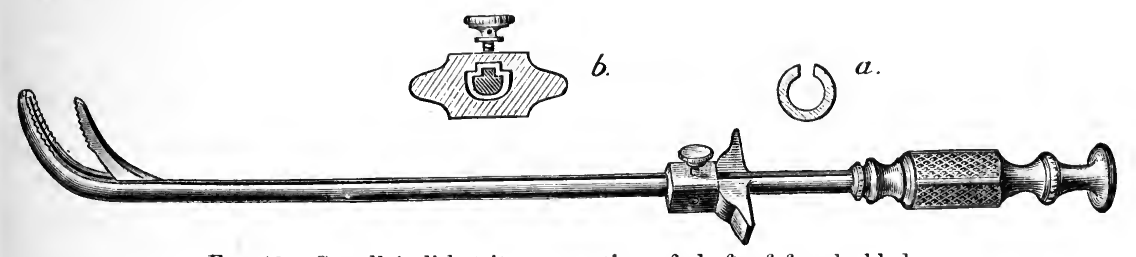

Fig. 18.-Costello's lithotrite. a, seetion of shaft of female blade.

was obliged to do lithotomy to release the jaws of the instrument, which had sprung apart in this manner.

To remedy this defect, Costello fastened a button on the lower side 
of the male blade, close down to the heel of the instrument, which, overlapping the lateral pieces of the female shaft, prevented this riding up to a considerable extent (Fig. 1 $6, b$ ). A little later (1832), Costello, finding that even with this modification

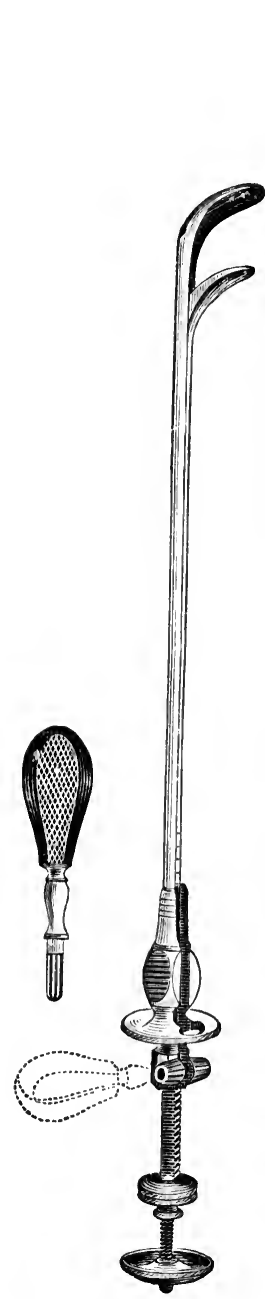

Fig. 19.-Ferguson's lithotrite.

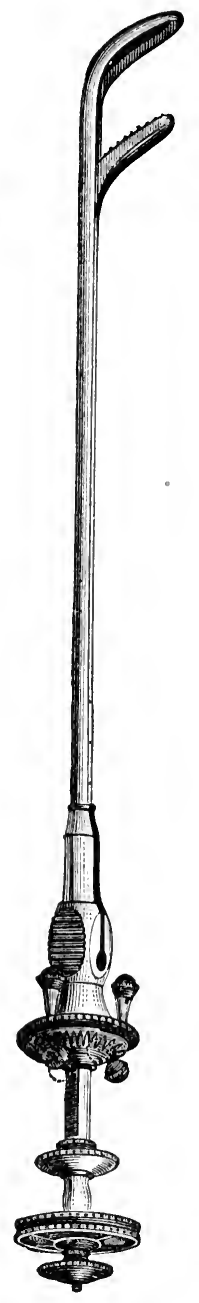

Frg. 20.-Civiale's lithotriptor. the instrument did not work satisfactorily, devised, and himself made, an instrument in which the shaft of the male blade ran within a corresponding groove in the female blade, the shoulders of which groove held it down (Fig. 18), and thus entirely prevented the

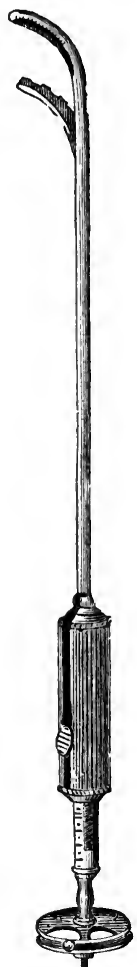

Fig. 21.-Thompson's lithotrite. riding up which had been so troublesome in Heurteloup's percuteur. This final modification has been retained up to the present day, and no essential change has been made in the shaft of the instrument since that time. This lithotrite of Costello's was operated by percussion.

The instrument now possessing blades suitable for eatching the stone, and a shaft strong enough to exert crushing power safely, the attention of inventors was turned to the management of the force, and screw power came very quickly into general use. At first fear was felt that the bladder would be injured by fragments flying off, but this was found, in a bladder filled with water, not to be the case. It was evidently important that the blades should slide easily upon each other while searching for and seizing the stone, and that then the force should be in some way applied to them. Ferguson accomplished this 
by a rack and pinion upon the handle of the instrument (Fig. 19), but very soon Charrière devised an arrangement in which a twist given to the lock threw the screw in or out of action (Fig. 20). Later, Thompson added a cylindrical handle, which surrounded the screw and gave a firm grasp to the left hand. Weiss added to this the contrivance by which the screw was thrown in and out of action by sliding a button, and this arrangement is still preserved in Thompson's lithotrite (Fig. 21).

Now attention was again turned to the blades of the instrument, which were made stronger; and, with the object of preventing clogging by dé-

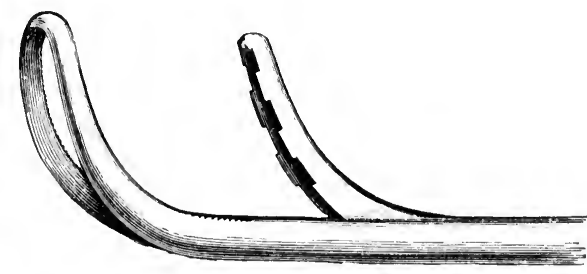

Fig. 22.-Fenestrated blade. bris, the fenestrated female blade was devised, the male blade being ronghened in various ways to enable it to hold fragments from slipping during the crushing. The accompanying cuts present most of the impor-
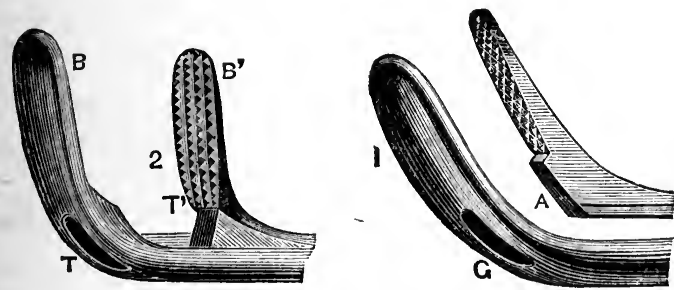

Frg. 23.-Solid blades of lithotrite. tant modifications which had appeared up to the year 1878 , when the introduction of litholapaxy increased the need of powerful, rapidly working and non-clogging instruments, and these will be considered when describing the operation.

From the time of the introduction of lithotrity, the practice was to crush the stone by numerous short sittings, and the natural expulsive power of the bladder was relied upon to throw ont the débris thus produced. The operator usually contented himself with seizing the stone but two or three times in one sitting. After one of these crushings it was the practice to have the

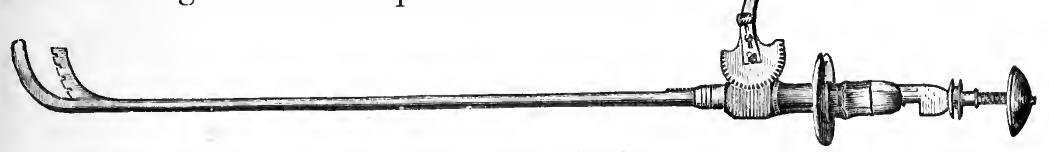

FIG. 24.-Lever lithotrite.

patient remain in bed on his back for a few days, in order to let the sharp edges of the fragments become rounded off before they were expelled through the urethra. 
The frequency with which stone is complicated by an obstruction to urination soon set the early lithotritists to devising means for the removal

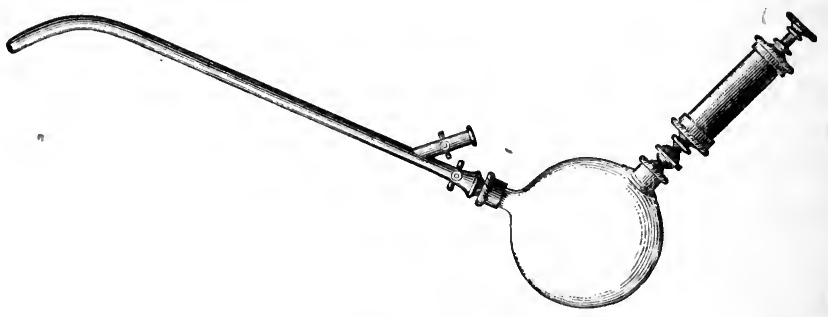

FIG. 25.-Cornay's washing-bottle.

of crushed fragments by means of scoops and by the use of large catheters; and presently by the adaptation of suction to the catheters. Figs. 25, 26,

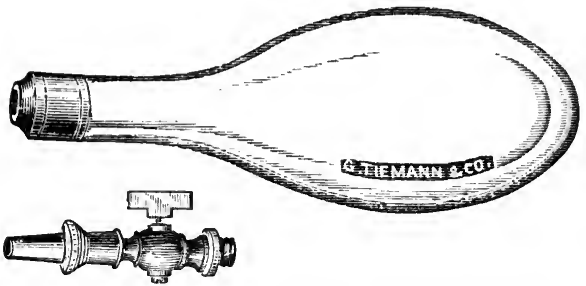

FIg. 26.-Crampton's bottle, used with an exhaustpump.

27,28 , and 29 give the more important of these early aspirating instruments; and, as will be seen, they owed their suction either to a piston (Cornay, Crampton, Nélaton), or to the expansion of a rubber bulb (Mercier, Clover, Van Buren); and these instruments were designed especially for those cases in which, either through atony or obstruction to the urethra, the bladder had lost the power of satisfactorily voiding its contents.

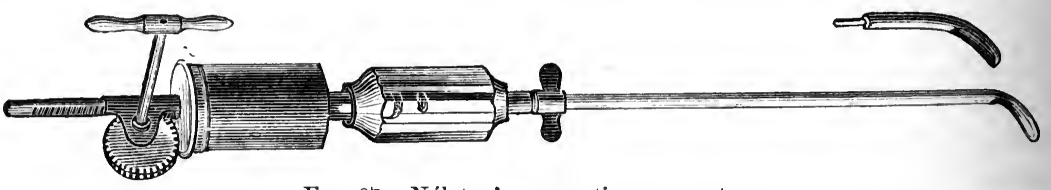

F1g. 27.-Nélaton's evaeuating apparatus.

The employment of evacuating apparatus was, however, up to the year 1878 regarded as of doubtful advantage, or even detrimental. Gross, in 1876 , says: "The practice of injecting the bladder to wash out the detritus is obsolete. . . . This apparatus of Mr. Clover should not be employed, if it is possible to dispense with it, as its use is quite as irritating as lithotrity itself."

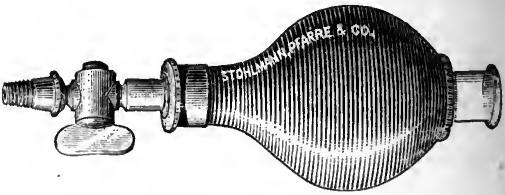

FIG. 28.-Mercier's washing-bottle.

Contemporaneous French writers are almost equally strong in condemning the practice; and Thompson, holding a somewhat more favorable 
opinion, said in 1871: " Having used it (Clover's bottle) very frequently, I would add that it is necessary to use all such apparatus with extreme gentleness ; and I prefer to do without it, if possible."

These quotations reflect as well as may be the commonly received opinion at that time upon this procedure; and the caution with which surgeons then approached the bladder is further shown by the shortness of the sittings that were thought proper in lithotrity. Two to three, or at most five minutes, was the

Fig. 29.-Clover's evacuating apparatus.

usually prescribed range of time. After the year 1871 it became more usual to employ an anæsthetic and to spend more time, and so do more crushing at one sitting; but even these sittings were comparatively short, usually not more than ten or fifteen minutes.

During this time, while lithotrity was slowly establishing itself as the proper operation for small and soft stones in comparatively healthy bladders, the operation of lithotomy was not standing still; for, although no new operative procedure was added to its technique, the introduction of antiseptic measures did so much to rob it of its dangers, that it began to be a not uncommonly expressed opinion that the cutting operation, which at once thoroughly removed the stone, was likely to displace the crushing operation, which was more tedious in its performance and less certain in its results.

Such was the position of things when, in 1878, Bigelow published his operation of litholapaxy, in which thorough crushing was combined with complete evacuation; and this method of stone removal has proved itself of such great practical value that cutting operations are now the exception, and not, as formerly, the rule.

Such is the brief résumé of the history of the crushing of stone in the bladder, and it offers as interesting a chapter of surgical evolution as can be found in any branch of our art. Even this short statement will give some idea of the amount of thought devoted to the technique of the operation, and of the mechanical skill and ingenuity expended in devising instruments for furthering the crushing of the stone and the removal of the fragments.

The modern operation of litholapaxy, or lithotrity in one sitting, has absorbed the older methods, and stands as the representative of them all. It is rare indeed now for a surgeon to plan the crushing of a stone, with- 
out including in his scheme of operation the immediate evacuation of the fragments.

The description of litholapaxy which follows is intended, therefore, to include what seems valuable of the experiences gathered in the field of lithotrity, together with all that has been added to it since the evacuator came to the assistance of the lithotrite.

\section{Litholapaxy.}

The name of this operation was derived by Bigelow from $\lambda i \theta o s, a$ stone, and $\lambda a^{\prime} \pi a \xi \iota s$, evacuation. Thus it covers not only the operations in which a stone is crushed and pumped out, but also those not infrequent cases in which small stones are drawn ont through the tube without crushing.

Bigelow, having convinced himself that the inflammations which followed erushing operations were due rather to the irritation of the fragments which were left than to the contact of the instruments, and believing that the bladder was much more tolerant of instrumentation than had been supposed, devoted his attention to the devising of more perfect ap-
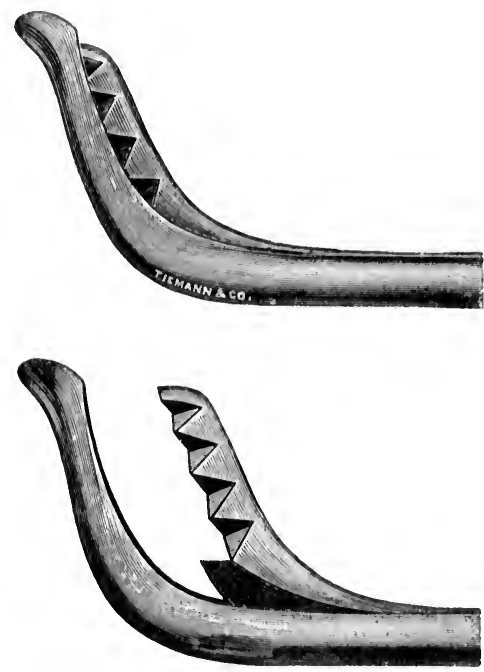

Fia. 30.-Blades of Bigelow's lithotrite, open and shut. paratus for the evacuation of the fragments after crushing. His experiments showing him that the débris could be rapidly removed, provided that the crushing had been thorough, he then set about improving the lithotrites with the object of making them more efficient.

For convenience of arrangement we will describe first the alterations that he made in the lithotrite; we then will examine the mechanism of the evacuator; and, finally, will consider the methods of using these instruments in the operation.

Lithotrite. - The alterations in this instrument were, first, in the blades; and, second, in the lock and handle:

1. Prior to this time considerable difficulty was experienced from the impaction of the mortar-like stone between the blades or jaws. This, as has been said, was obviated by making the female blade fenestrated, so that the male blade pushed the délris directly through it. This worked well, so far as the erushing of 
the stone went, but, for certain reasons which will be dwelt upon in the description of the operation, the fenestrated instrument required more care in its manipulation, and was more likely to damage the bladder walls than an instrument with a solid blade.

To provide, then, a solid-bladed lithotrite that should be self-clearing and could be used for an indefinite time without becoming clogged, Bigelow had the male blade made with a series of alternate, triangular notches, by whose inclined planes the detritus should escape laterally, after being crushed against the floor and rim of the shoe, or female blade.

The rim of the female blade he made low, so that this detritus should easily escape over it, and also that the fragments of stone should readily fall or slide upon it. At the heel of the shoe, where most of the stone is usually comminuted, and where the impact is therefore greatest, the floor was made high, so that it should more easily discharge itself laterally, while the customary slot (Fig. 31) was made to work effectively.

To facilitate the introduction of this instrument, the point of the female blade was turned over and blunted. The advantage of this form in enabling the instrument to slip easily along the urethra is illustrated in Fig. 32.

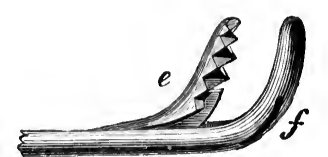

Fig. 31.- Ileel of Bigelow's lithotrite, slowing the slot for the escape of detritus.

Keyes objected to this form because, with the instrument reversed for the purpose of picking up fragments with the tip of the blades, the snout prevents the seizing part of the blade from coming down closely upon the bladder floor. This manouvre, however, which is thus interfered with, is one of the most dangerous in the manipulation of the in-

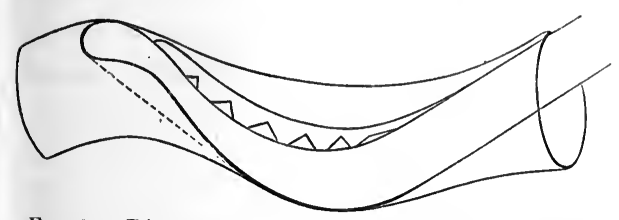

Fic. 32.-Diagram of urethra with blade of lithotrite within it. strument, and, though proper in skillful hands, should not be used if it can be dispensed with.

2. The handle of the instrument was also modified in important particulars. In place of the wheel, Bigelow substituted an egg-shaped, fluted handle of hard rubber that comfortably fits the palm of the hand. Immediately below this handle he placed a revolving cylinder cap which is attached to the screw-guard, and which, being held between the forefinger and thumb of the right hand, enables the operator to turn the lock by a half-turn of the wrist without changing his hold on the handle. This arrangement gives the surgeon the great advantage of being able to lock and unlock the instrument at will, and even to complete a protracted crushing without disengaging the hold of either hand. It thus saves time and lends accuracy to the manipulations. 
In selecting the proper lithotrite for a given case, the size of the urethra should be taken into account, and the largest instrument that will pass without undue stretching of the canal should be used. A powerful

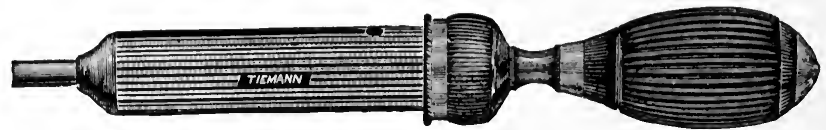

Fig. 33.-Handle of Bigelow's lithotrite, elosed.

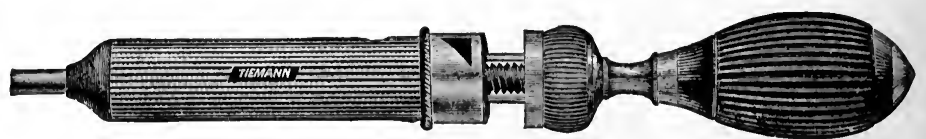

FIG. 34.-Handle of Bigelow's lithotrite, open, showing the cylinder-cap and the bars connecting it with the screw-guard.

lithotrite works more rapidly and easily than a weaker one, and there is less danger of its breaking. On the other hand, it should not fit the urethra so tightly as to interfere with its free manipulation.

Bigelow's lithotrites for the adult are made in three sizes, namely, Nos. 25, 27, and 31 of the French catheter scale. This measurement is taken round the heel of the blades, which is the largest part; the shaft is several sizes smaller. With well-made instruments of these sizes moderate stones of any degree of hardness may be disposed of. For a large and hard stone that offers more than usual resistance a fenestrated instrument may be employed for the first crushing, as it cuts through the stone more readily. After the calculus is divided into fragments the instru-

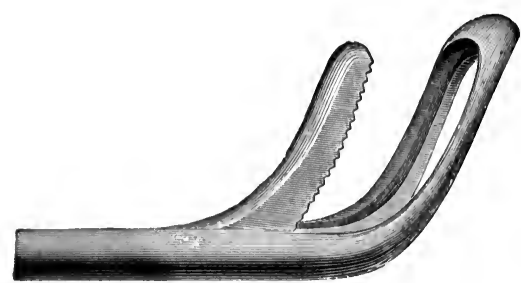

Fio. 35.-Bigelow's fenestrated blades. ment with the closed female blade may be taken for the further comminution of them, as it can be operated with more freedom and speed.

In operating upon children, instruments much smaller than these (Nos. 16 and 20 French) are employed. Here, except with very small stones, a fenestrated lithotrite is an advantage, often a necessity, as it cuts the stone more easily and is therefore less likely to break. To finally reduce the fragments to a size that will pass the small evacuating tubes, however, an instrument with a solid blade is a great convenience.

The Evacuator.-Much ingennity has been expended in devising modifications of the evacuating apparatus. The essential points of this are a tube, or catheter, an elastic ball, and a receptacle for fragments. This last part may even be omitted, the fragments colleeting in the lower part of the bulb. For convenience, however, to enable the operator to 
see what he is accomplishing and to guide his manipulations accordingly, a transparent receiver is an important part of the apparatus.

In an early article Bigelow published a series of figures showing the important combinations which could be made of these three parts; and, after trying several arrangements, he decided upon one which will be described later. Some operators have preferred other solutions of this problem, and have changed the form of evacuator to suit their individual tastes. The accompanying figures present the more important of these alterations of form, in all of which the essential prineiples remain the same.

For the sake of brevity we will first give a description of Bigelow's "simplified evacuator," and will then consider those changes of form which have attained any degree of prominence.

The evacuator consists, as has been said, of a tube, an elastic bulb, and a receptacle for fragments. For convenience of description the tube may be described as consisting of two parts : the movable part, or catheter, and the fixed part, which enters the bulb and is attached to it by a bayonet-joint.

The catheter may be either curved or straight. A straight tube offers less resistance to the passage of fragments, and is therefore better for the evacuation of them. The curved tube, however, is much easier of introduction in difficult cases. $\Lambda \mathrm{t}$ the upper end of the catheter tube is a projecting wing, which facilitates the handling of the instrument, and at the same time serves as a guide to the position of the mouth of the tube.

The fixed portion of the tube, connecting the catheter with the pump, extends obliquely upward to the center of the bulb. This tube is, for convenience, provided with a stop-cock just outside of the bulb, and, if a second stop-cock is attached to the upper end of the eatheter, the patient and bedding can be kept dry wlıen coupling and uncoupling these tubes.

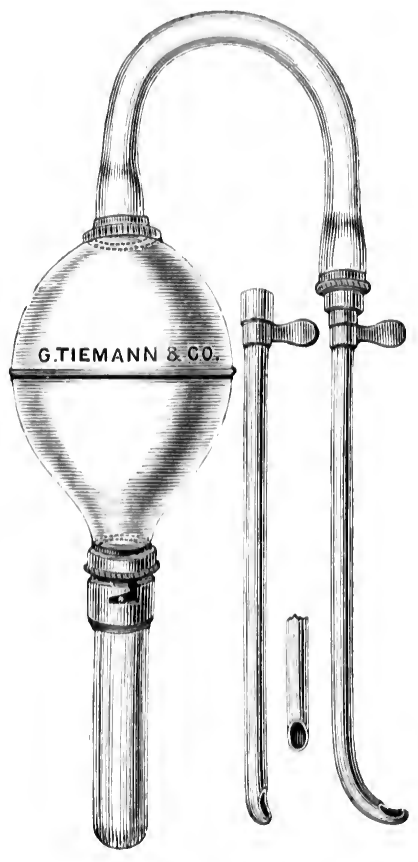

F16. 36.-Bigelow's evacuntor, first form.

The portion of the tube which extends into the bulb is perforated all around with small holes, the aggregate area of which is larger than that of the opening at the end of the tube. The fragments enter the bulb easily through this tube, while, as the water is forced back into the bladder, it naturally seeks the shortest road and freest 
outlet through the perforations in the sides of the tube, which do not admit of the return of the fragments. The solid particles which are thus caught and retained in the bulb naturally gravitate to the bottom of it,

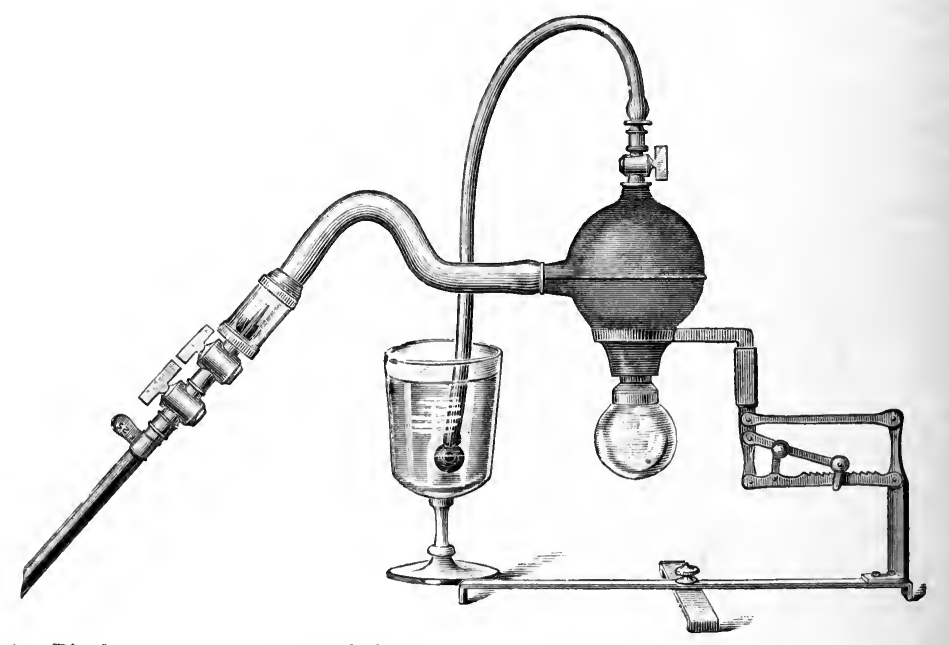

FIG. 37.-Bigelow's evaeuator, seeond form. This form was used but for a short time, as the simplified evacuator followed closely after it.

where they are received in the glass ball or receiver, which can be readily removed and emptied. At the top of the bulb is an opening with a stop-

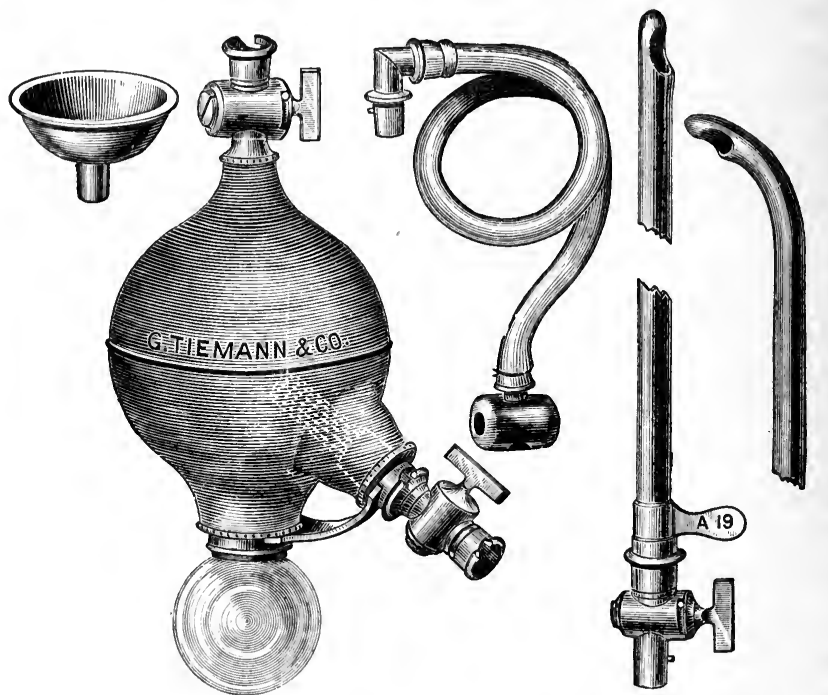

Fig. 38.-Bigelow's simplified evacuator. This form he did not further alter, but with it all his later cases were operated. 
cock to which a hose can be attached. This provides for the expulsion of air from the apparatus, and also for the easy regulation of the amount of water in the bulb and bladder during evacuation-a matter of great importance, as an over-tense bladder is a source of danger and must be at once relieved; while a too empty bladder interferes greatly with the operation, as the lax walls are drawn into and obstruct the mouth of the tube.

In Bigelow's evacuator, as will be seen, the receiver in which the fragments are caught is at the extreme farthermost point of the apparatus from the bladder, and fragments once received in it can not be again put in circulation and returned to the bladder.

In Thompson's, Guyon's, and Otis's instruments, on the other hand, the trap or receiver is between the bulb, or pump, and the bladder. The

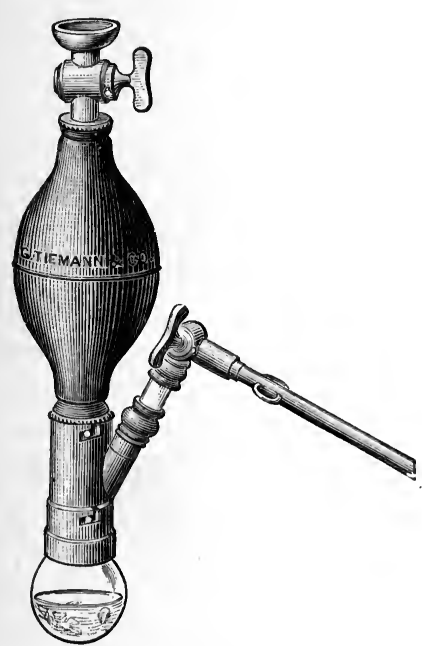

Fit. 39.-Guyon's evacuator.

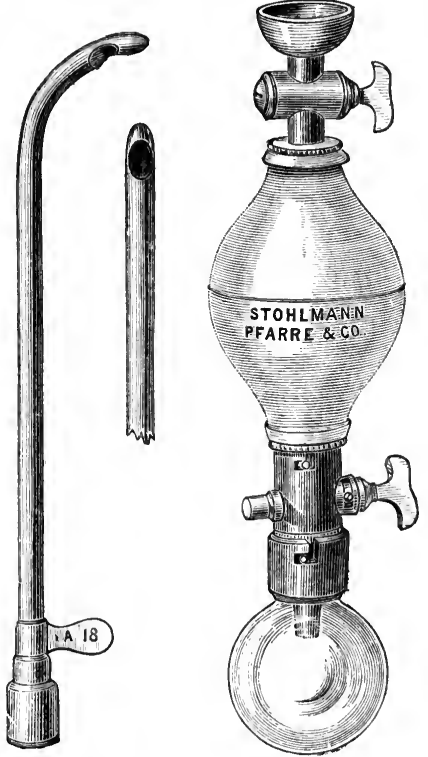

FIG. 40.-Thompson's evacuator, early form.

return of the fragments is guarded against in Thompson's instrument (Fig. 42) by a perforated valve at the end of the tube. The presence of much mucus in the bladder interferes somewhat with the efficiency of this arrangement. In both Guyon's and Otis's instruments there are no valves, the force of gravity being relied upon to separate the fragments and sand from the water; and to assist this, the direction of the current is so arranged as to guide the fragments toward the receptacle provided for them. Walker's and Chismore's evacuators are modifications of Otis's ; at least they follow it in principle. The Otis evacuator serves well for the 
pumping out of fragments, but, as it can not be wholly freed from air, it makes so much bubbling and noise that it prevents the ear from accurately distinguishing the sound of fragments against the tube. This is a considerable disadvantage, for by the ear much may be learned as to the

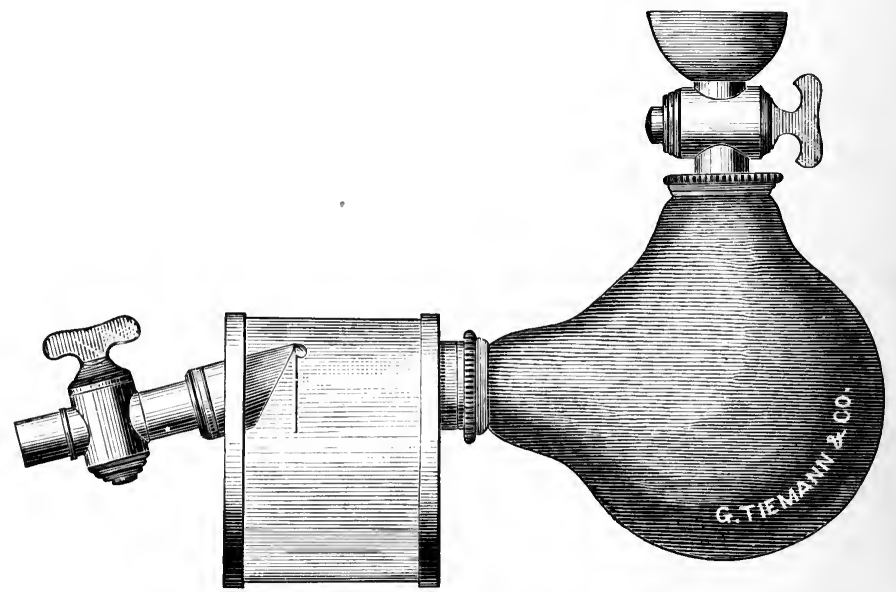

Fia. 41.-Thompson's evacuator, latest form.

working of the instrument and the size and abundance of the fragments. In the search for the last fragment or for a small stone the ready perception of a click is of the greatest importance.

The opening of the tube was a subject of much thought and experiment, and Bigelow endeavored to place it as close as possible to the ex-

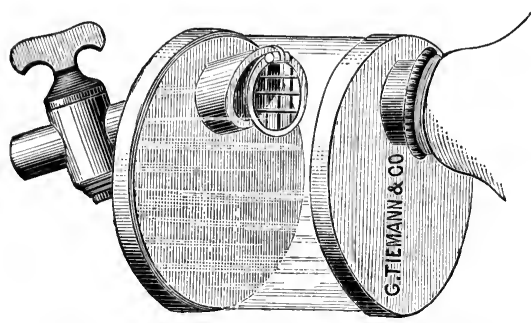

FIG. 42.-Thompson's evacuator, detail of receiver. tremity, so that it could be carried down to the floor of the bladder to collect the last fragments. At the same time he shaped the end so that the mucous membrane should be held away from the opening, and not be sucked into it. He made the opening slightly smaller than the caliber of the tube, so that a fragment which has once passed it shall not be arrested in the tube

higher up. This arrangement prevents the stoppage of the tube higher up by a fragment, but it does not prevent bits of stone from lodging in the eye and obstructing it. The projecting lip below the opening will sometimes hold a fragment quite firmly when it is impacted in the eye, so that the force of the current can not throw it out, and a stylet is required for its dislodgment. 
Keyes, endeavoring to avoid this impaction, has made an evacuating tube entirely open at the end. It requires an obturator for its introduc-

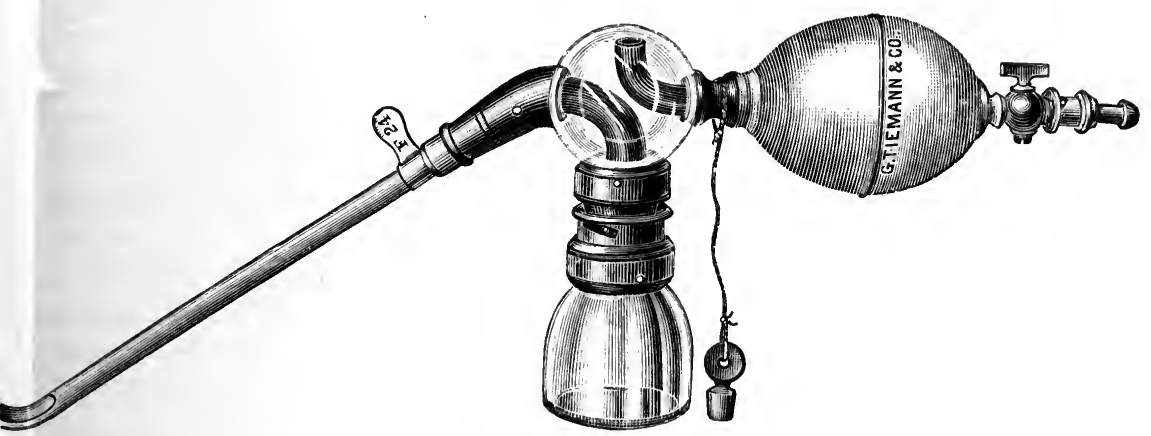

Fig. 43.-Otis's evacuator.

tion, and special skill in its management. The working of these tubes will be considered in the description of the operation.

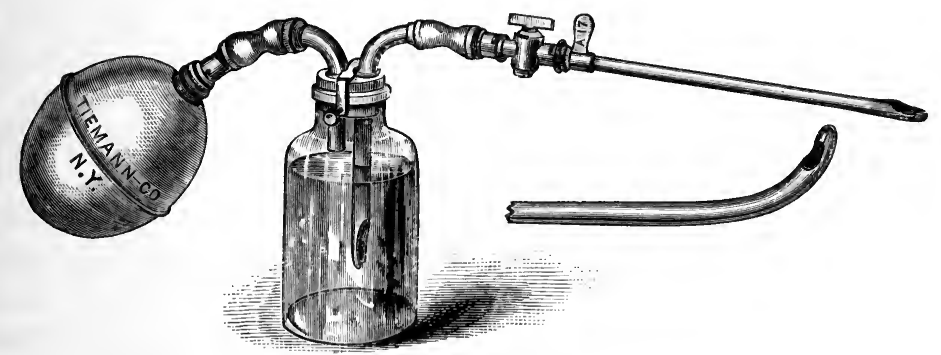

Fig. 44.-Walker's evacuator.

In regard to the size of the tubes, obviously the larger tubes will work more rapidly, and therefore should be used when the urethra will readily admit them.

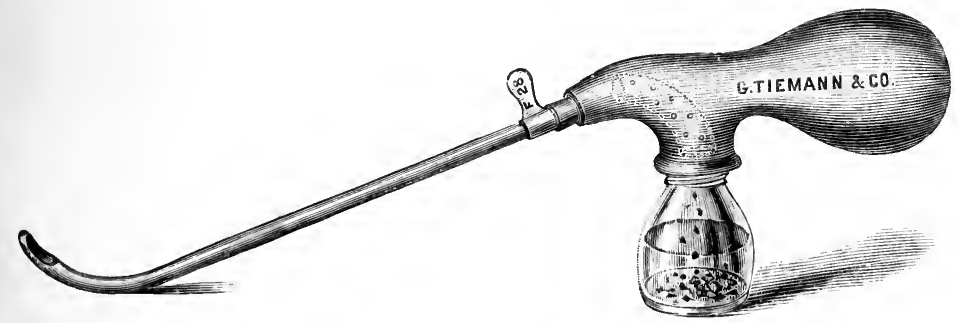

FIG. 45.-Chismore's evacuator.

There has been a mistaken idea that Bigelow advocated the use of very large tubes and lithotrites. He belonged to the older school of surgeons, 
who had been in the habit of using much smaller instruments than those now employed, and when he urged the use of large instruments, he did not propose to go into the largest sizes which Otis has shown that many urethræ will carry. To him, accustomed to look upon No. 21 French as a large sound, the numbers above 25 French seemed very large, and he so spoke of them. As a matter of fact, the largest instrument ever used by him in the operation of litholapaxy was a No. 31 French, and this he only employed on rare occasions, ope-

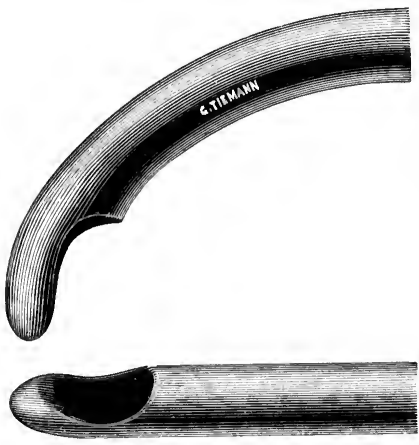

Frg. 46.-Ends of evacuating tubes. rating usually with instruments between 25 and 29 , French scale.

The proper length of tube is another point which has been somewhat discussed. It is obvious that a short tube will work somewhat more rapidly than a longer one. This is not so important as it might at first appear, as the difference between the amount of fluid drawn in through a long and a short tube, at a given expansion of the bulb, is only a matter of one to one and a half drachm, and a slightly deeper compression of the bulb each time will make up this difference. In a fat patient with a long prostate, the tube as designed by Bigelow, measuring nine inches from the wing to the eye, is almost completely buried in the urethra; and it is obviously an advantage to have the receiver sufficiently above the patient to be easily visible, and not to have it buried between the thighs, as it wonld often be if a short tube were employed. If a surgeon has a large number of tubes, it is well to have them of various lengths.

The second stop-cock on the end of the catheter adds to the length of the evacuating tube. This slight disadvantage is, however, more than

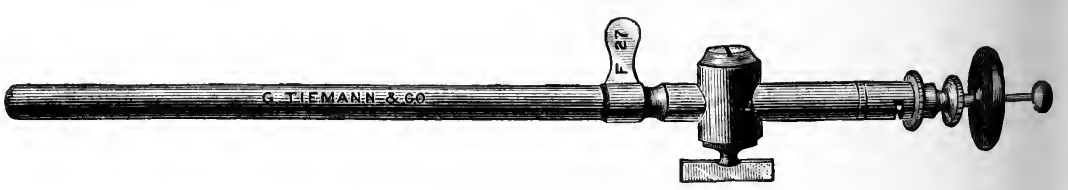

Fig. 47.-Keyes's evacuating tube.

compensated for by the added comfort and convenience which it gives. It saves the patient and his bedding from a wetting, and prevents the loss of unmeasured quantities of fluid from the bladder. This last is sometimes of great importance, for in a small, contracted bladder it is necessary to be always cognizant of the amount of distention. 
THE OPERATION.

Small stones can ordinarily be crushed and pumped out without causing much pain; so that, in a patient who is not especially sensitive, or whose urethra is accustomed to the passage of instruments, no anæsthetic may be required. The pain of the operation may be decidedly diminished by the application of cocaine to the bladder and urethra, and in this way many stones have been crushed without resorting to general anæsthesia. If cocaine is employed, it should be thoroughly applied in a two to four per cent solution, the bladder having been previously emptied and washed out. In order to reach all parts of the vesical cavity, it must be injected in considerable quantity, and there is therefore some danger of toxic effect, especially if the bladder is ulcerated and presents an absorbing surface.

Usually, except in the most trivial cases, it is best to do the operation under general anæsthesia; for the relaxation of complete insensibility makes the manipulations much easier, and adds greatly to the quickness and thoroughness of the operation. Especially is it important to allay the tendency of the bladder to spasmodic contractions, for not only does this force the water out alongside of the instruments, and so reduce the size of the cavity when room within it is necessary, but it subjects the bladder walls to a dangerous strain, which, if the urethra is blocked, may lead to their rupture.

The patient, then, being thoroughly anæsthetized, and the presence of a stone having been established, a large sound may be passed, to make sure that there is no stricture or other obstruction in the urethra. If a stricture is found, it may be rapidly dilated with large sounds, or divulsed. A narrow meatus is to be cut. The urine should then be drawn with a catheter, the bladder should be washed out, and borax-water or a solution of boracic acid should be introduced in sufficient quantity to distend it, and so keep its walls out of harm's way during the crushing of the stone. From four to six ounces is usually a proper quantity. An elastic rubber tube may then be tied lightly around the penis, close to the corona glandis, to prevent the escape of water along side of the instrument. This fills the double purpose of keeping a known quantity of water in the bladder, and of preventing the wetting of the patient and the bed.

Before each introduction of the instrument the urethra should be filled with liquid vaseline from a syringe, in order to lessen the injurious friction as far as possible.

To introduce the lithotrite properly, the point should be carried with considerable gentleness through the constriction made by the rubber tube, and it then slips without difficulty through the movable urethra. After the beak passes below the pubes, the handle should be brought to a verti- 
cal position, and the instrument will then drop almost without assistance, by its own weight, until the point rests just in front of the triangular ligament. Traction upon the penis now effaces the depression made by the extremity of the instrument in the bulbous urethra, and if the handle is then brought down gently between the thighs and at the same time the point is advanced in the axis of the body, the lithotrite usually slips easily into the bladder.

The places where difficulty may be met in a normal urethra are at the triangular ligament and at the prostate.

The point of the instrument may catch on the upper or lower edge of the comparatively rigid opening of the triangular ligament. If the handle is depressed before the beak of the instrument is carried down as far as it will go toward the rectum, the point is likely to catch against the upper edge of this opening; while, on the other hand, if the instrument is pushed too forcibly toward the sacrum, the lax, bulbous urethra is depressed below the aperture, and the point catches on the lower margin. Practically, if the instrument catches at the triangular ligament, it should be passed down with the beak hugging first the roof and then the floor of the urethra, and in one or the other of these ways it will usually find its way through. The finger pressing against the convexity of the curve of the instrument in the perinæum will often lift the point over the lower margin of the opening when it is catching there.

A similar difficulty may be met with at the opening into the prostate. This happens but rarely, and is to be overcome by the same tactics. In cases of much difficulty the finger introduced into the rectum serves as a good guide, and with it the point of the instrument may be lifted into the prostate when it is catching on the lower edge. An enlarged prostate is, as a rule, easily passed by the lithotrite, whose short, curved beak carries the point along the roof of the canal, where it rarely meets an obstacle.

False passages may make the introduction of instruments extremely difficult and dangerous. Even if by long and patient trial they are finally passed, and the stone is comminuted and removed, the danger is not then over, for a serious swelling of the urethra is likely to follow, and under these circumstances the passage of a catheter is almost an impossibility.

A single false passage, if its position is accurately made out, may usually be avoided by carrying the instruments along the opposite wall of the urethra at this point. If, however, several of these pockets exist, in which the instruments are caught, it will perhaps be a wiser plan to resort to lithotomy, which, though a more severe operation, has the great advantage of providing certain drainage for the bladder.

If the stone is free, it rests upon the floor of the bladder, and, with the patient in a horizontal position, it is usually found at a point a short distance behind the prostate. 
The operation of crushing not only demands considerable skill of manipulation, but it is also greatly assisted by an accurate knowledge of the contour of the bladder, and of the changes in shape which may be impressed upon it by varying conditions of the surrounding organs.

Dr. Bigelow, in preparing his work on litholapaxy, made a careful study of these changes. He says: "We get a useful view of the interior of the bladder by examining in position through an opening in its summit. This part of the organ, with the free and thin posterior wall, is mainly concerned in distention. The floor of the bladder is comparatively firm and flat, and, if the subject is in good condition, adheres to a thick mass of cellular tissue in and near the ischio-rectal fossæ, upon which it rests. This mass is traversed by the rectum variously distended; and this canal, in a thin subject, may be advantageously filled with air during an operation, to facilitate its indentation by an instrument-reversing, for the operation of lithotrity, one of the precepts of lithotomy.

"The sigmoid flexure is largely concerned in compressing the bladder behind. The posterior wall of this viscus may be so crowded by the intestines as to become flat, or even concave. A horizontal section of the bladder is then transversely oval, flattened between the intestines behind and the pubes in front, each of these indenting it. A well-filled or tense abdomen tends so to shorten the antero-posterior diameter of the bladder that, while a large stone may gravitate backward into that part of the bladder that is compressed by the intestines, carrying the thin wall with it, it is not so with a fragment, which, unless the floor be artificially depressed, may lie on one side or the other of the vesical orifice more readily than at some distance behind it."

A rectum filled with fæces raises and distorts the floor of the bladder, and so not only makes the search for a stone more difficult, but by resisting the indenting of the floor with instruments adds greatly to the difficulty of crushing. It is for this reason that the bowels should be thoroughly emptied before the operation.

If now the lithotrite be

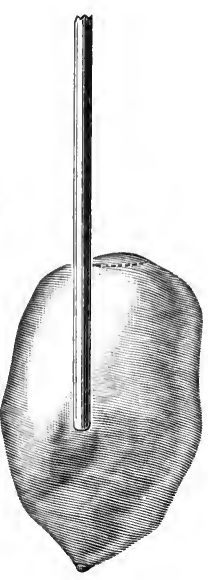

FIG. 48.-Cast of the bladder with an instrument depressing the floor. introduced and carried to the bottom of the bladder, its beak will make an indentation in which it will rest. Fig. 48 represents a plaster cast of the bladder with an instrument in this position.

As the stone rolls to the bottom of this funnel-shaped depression it 
rests on or alongside of the beak, and when the blades are opened it falls over the shoe, or female blade, and is therefore seized as the male blade is again closed down.

If this manœurre does not succeed in catching the stone, the blades should be opened in the upright position, and then turned over on one side and shut along the floor. Care should be taken to always open the

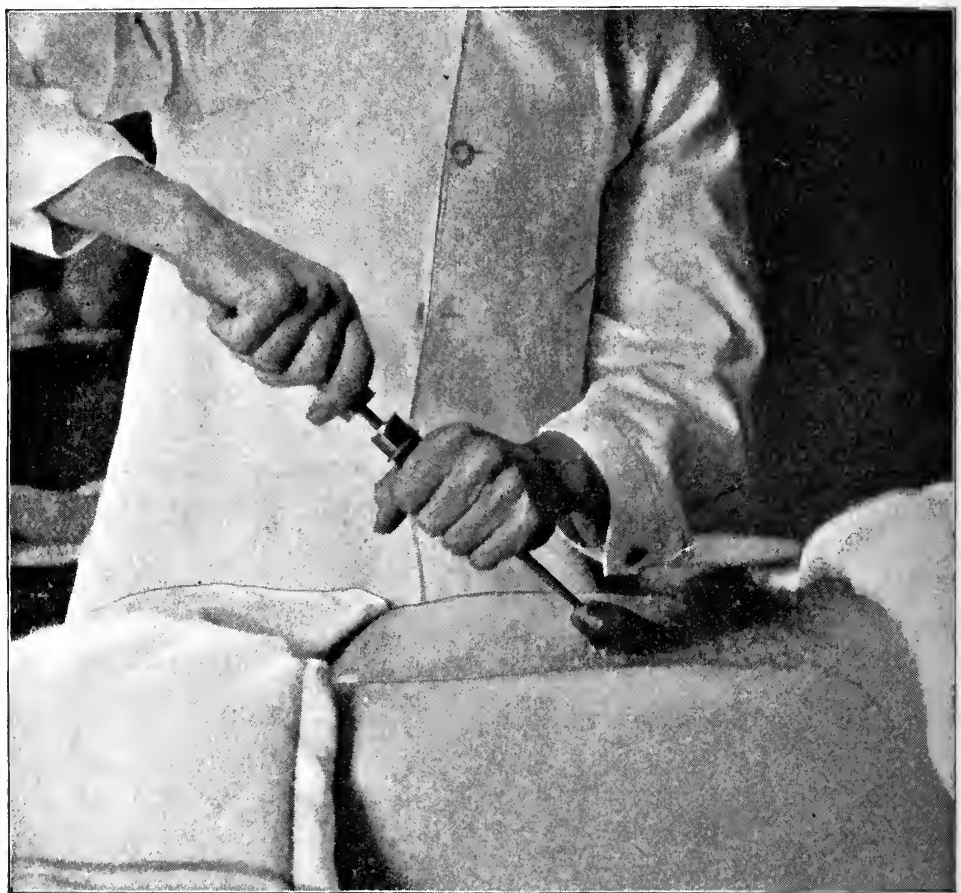

FIG. 49.-Showing the manner of holding the lithotrite when opening and shutting in the search for fragments.

instrument in the upright position, for if it is turned on the side and then opened, the male blade pushes the stone or fragments away, and they are not caught as it is again closed.

If the stone can not be seized, it may be because it lies in the pouch behind an enlarged prostate, so that the instrument enters the bladder over it. In this case it may be caught by opening the instrument and then turning the blades over so that they point down into the base of the bladder, and shutting them in that position. There is some danger of nipping the bladder wall by this manœuvre, and an easier way of obviating the difficulty is by raising the hips of the patient. This change of position usually rolls the stone out of the post-prostatic pocket into a part of the bladder where it can be easily reached and crushed in the ordinary manner. 
When, as rarely happens, the stone is so held in the pouch behind the prostate as not to be easily rolled back, it may usually be displaced by using the closed blades of the lithotrite to pry it out. If this is not successful, it can be pushed up by a finger introduced into the rectum, and if it tends constantly to fall back to a point where it is not accessible, the post-prostatic depression may be effaced by a distended rubber colpeurynter in the rectum, as suggested by Buckston Browne. Mr. Browne met with several cases in which the post-prostatic pocket was a veritable diverticulum beneath an enlarged third lobe, and so concealed the stone that it required a suprapubic operation for its removal.

A stone may be too large to fall into the depression of the instrument, and it is then necessary to depress the handle and so raise the blades, before it can be seized. This sensation of having the stone above the sound or lithotrite sometimes leads to the belief that it is attached to the upper bladder wall, when this is not the fact. In such a case, after the first crushing, the fragments, as they are reduced in size, will be found on the Hoor of the bladder.

The crushing of the stone should be done as thoroughly as possible at the first introduction of the lithotrite. This saves time and irritation to the urethra, and, with the self-clearing instruments described above, may be accomplished by a little skill and patience in searching for fragments. If the stone is a large one, however, sand and small fragments will presently accumulate and fill up the bottom of the bladder, and then the large fragments lying on top of this débris can no longer fall by gravity into the jaws, and they are then not easy to find. When this is found to be the case, it is time to use the pump and clear the bladder of the finer portions. The presence of clotted blood or of thick mucus in the bladder has a similar effect in filling up the depression upon the floor and preventing the fragments from falling into the jaws of the lithotrite. Here, again, the pump is called for.

The form of the female blade, with projecting end and width enough to carry its sharp rim away from close contact with the male blade, makes the nipping of the bladder wall unlikely to occur. This accident is so serious that it should be further guarded against by always, in case of doubt, carrying the blades toward the center of the bladder and slightly rotating them from side to side, to see if they are free, before they are screwed down upon anything that has been seized.

Practically, with the Bigelow instrument, having a floor to the female blade, operating in a moderately distended bladder, the jaws may be safely pressed down toward the trigone and then opened and shut without any fear of catching the bladder wall, so long as they are kept nearly upright. This manœuvre is a valuable one in rapidly reducing the fragments of a soft stone; for no screw-force is required, and the fragments constantly 
falling on to the female blade are comminuted as rapidly as the instrument can be opened and shut.

It must be remembered, however, that if the bladder is so empty as to fall into folds, greater care must be exercised ; and also that, if the instrument is turned toward the side wall, it may perhaps catch a fold, and should be closed with caution. A nip from the blade of a non-fenestrated lithotrite does not necessarily produce a serious lesion of the bladder walls. This accident occasionally happens to every operator without causing troublesome after effects. But the fenestrated instrument with its scissorlike blades, if shut down closely, cuts out the piece of the bladder wall included within its jaws. Especial care should therefore be used in the manipulation of this instrument.

There is also another peculiarity in the working of a fenestrated lithotrite, which should be borne in mind when one is used. It is this: During the early part of the crushing, when the stone breaks and falls apart in the jaws, it is natural to open the lock and again search for a large fragment without fully screwing down. This is a safe manœuvre with a solid instrument, but with a fenestrated one it is important to remember that portions of stone pushed through the female blade will cling to it with some tenacity unless the male blade is thoroughly pushed down through it, and that a rough fragment thus projecting below the blades may do serious injury. Therefore the fenestrated instrument should always be closely shut down on whatever it seizes.

When, finally, no more fragments of any size can be caught, the lithotrite should be firmly screwed together and withdrawn. If there is any difficulty in fully closing it, the jaws may be carried to the center of the bladder, and there with the screw may be opened and shut a few times to work out the impacted stone from between them. This manœurre is of importance, not only on account of the difficulty of drawing out a partially open instrument, but far more because an instrument not wholly freed of débris may have sharp, angular fragments projecting from it which will lacerate the urethra in withdrawal.

We now come to the second part of the operation, the evacuating of the fragments. In selecting the catheter for this, a straight tube should be used, if possible, as it not only affords a straighter and easier road for the fragments, but also is more easily turned in the bladder, when the operator wishes to direct the orifice toward different portions of the viscus.

While the curved tubes are introdnced like an ordinary catheter, the straight tube requires a little manipulation. It should be carried down in a vertical direction until it will go no farther toward the rectum. Then, being brought to a horizontal position, it should be gently pushed upward in the axis of the body. At the moment of bringing the tube down be- 
tween the thighs, pressure should be made over the pubes, pushing the penis downward in order to relax the suspensory ligament. Before advancing the instrument horizontally, it is well to withdraw it very slightly in order to disengage its point from the pocket which it is likely to make in the bulbous urethra. When there is a hitch at the triangular ligament or at the prostate, the tube may usually be carried through by rotation, in the manner of a corkscrew. As all the obstacles likely to be met in the urethra are on the floor, it is usually sufficient to carry the outer end of the straight tube low down between the thighs, in order to enable its point to ride over any obstruction that it meets in the prostate. With care in these regards, it passes readily into the bladder. When, owing to any peculiarity of the urethra or prostate, the straight tube does not pass readily, a tube with the point slightly turned up, after the manner of a coudé catheter, may be tried, and if this fails, the curved tube may be used as a last choice.

When the tube has entered the bladder, its point should be carried gently down toward the base, and it should then be connected with the bulb. On opening communication between them, the air contained in the tube will escape into the bulb, and should be driven out through the hose at the top, and its place supplied by water before the operation begins.

When there is much débris, it is

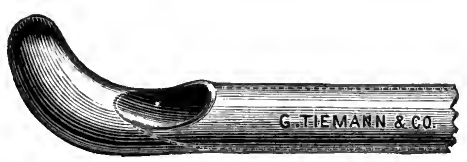

Fra. 50.- Straight tube, with the end turned up to facilitate introduction. well to commence pumping with the point of the tube held a little above the floor of the bladder. During this earlier part of the operation there should be no interval between the compression and expansion of the bulb. The object at this time is to set the fragments whirling, and to catch them while they are suspended. If the end of the tube is buried too deeply in the detritus, it is apt to be clogged at the outset, and the evacuation is thereby considerably hindered. Later, when the fragments are few, the tube is carried to the floor of the bladder, and a few moments should elapse, after pressing the bulb, to give the fragments time to settle into the depression about the end of the tube before the expansion which is to suck them into it. When any particular aspiration brings fragments, the position of the tube should be kept unchanged until they cease to come.

The wedging of a fragment in the tube causes an obstruction which is very noticeable. The compression of the bulb is rendered difficult, and its expansion slow. An angular fragment may lodge so that, while it allows the passage of water, it prevents the entrance of other fragments. This condition may be suspected if there is a constant clicking against the tube, and still nothing appears in the reservoir. The usual point 
where fragments wedge is at the mouth of the tube. When one thus becomes fixed, it may be dislodged with a stylet, and this is the safest method of disposing of it, although with care the tube may generally be drawn out with the fragment in it. This procedure, however, is not de. void of danger, for sharp, projecting angles may lacerate the urethra, or a bit of stone may remain sticking in the passage.

The tube may also be obstructed by the bladder wall, which is some. times sucked into the orifice. The stoppage from this cause is usually

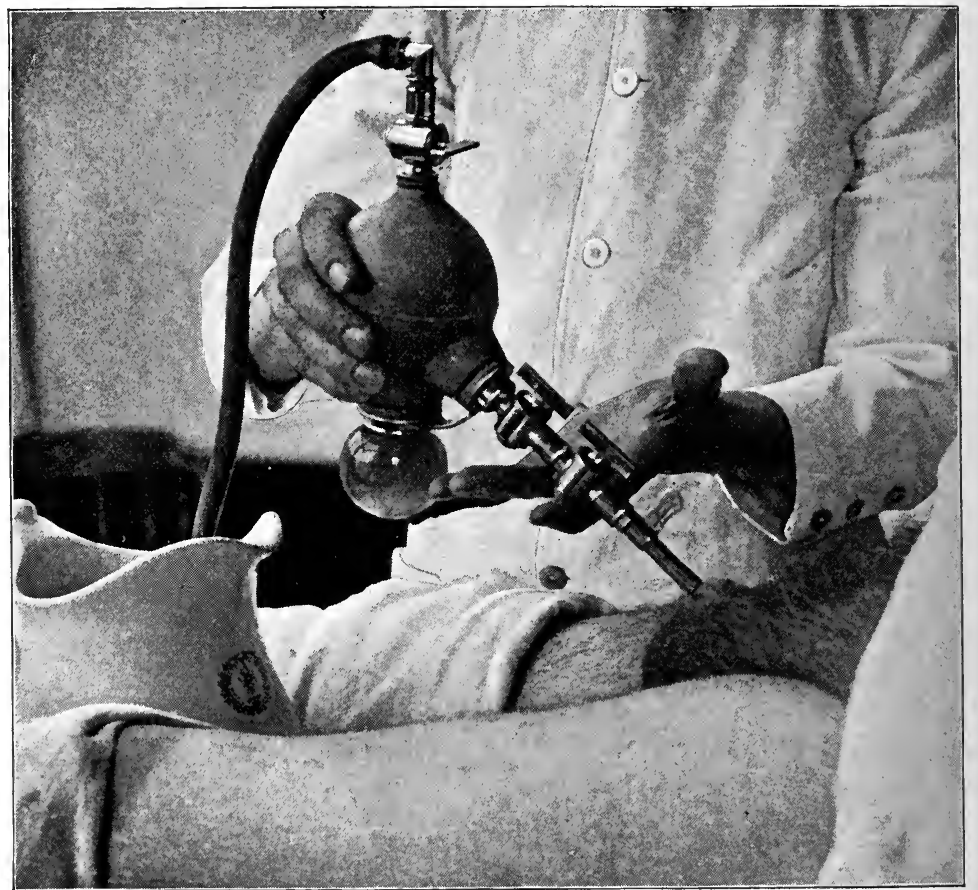

FIG. 51.- Showing the manner of holding the bulb. The left hand holds the weight while the right manipulates it.

not continuous, but the walls flapping against the opening give the instrument a series of jerks which remind one of a fish-bite. When this is felt the instrument should be moved to another part of the bladder; and, if it then occurs, it shows that the bladder is not sufficiently distended, and water should be added through the hose at the top of the bulb. Pumping should be prolonged until the sand and gravel cease to come. Then the lithotrite should be again introduced and the crushing continued, followed again, as before, by the washing out. Finally, after the stone has been thoroughly crushed, and when for several minutes no fragments appear in the reservoir, it is evident that the evacuation is 
almost completed; and now begins a very important part of the operation-namely, the search for the last fragments. The tube should be moved to different parts of the bladder, and the orifice should be systematically turned in all directions in order to wash out any side pockets or corners that have been passed over. The pouch behind the prostate should be especially attended to in this way.

Keyes recommends his tube with open end for the washing out of the last fragments. It requires especial care in its manipulation, and its vesical end must be kept close to the neck of the bladder. If introduced too far, it sucks up the posterior bladder wall uncomfortably; and if drawn out too far, it may bruise the prostate.

It sometimes happens that the last fragment is difficult to find with the lithotrite, and is still a little too large to pass the tube. Great patience is then required in the search for it. I have once or twice succeeded in such a case by drawing the fragment to the tube, and have then dropped it on the floor of the bladder, where the lithotrite readily found it and crushed it.

Chismore has devised a lithotrite which seems admirable for the removal of this last fragment. In it the shaft of the male blade is hollow, the opening being in the heel of the blade. It has an aspirating bottle attached to its upper end, so that when the blades are slightly separated and suction is applied, the fragment is drawn between the jaws and easily crushed on closure of the instrument.

Finally, if after a careful search no more fragments can be obtained and no click of stone against the tube can be elicited, the operation is completed. Before closing, however, it is well to wash out the urethra thoroughly with a catheter of small size, which allows the water to rush out alongside of it. This removes much find sand,

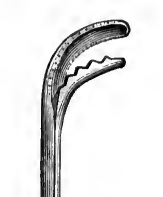
which oozes along the lithotrite and lodges in the canal during the operation. The writer adopted this plan several years ago, and he has found, since putting it in practice, that his patients have suffered less from painful, scalding micturition than formerly.

After-treatment.-If the patient has been able to urinate normally before the operation, little or no after-treatment is required. It is usually well to allow the patient to drink liberally of water, and to keep him quiet for a few days, although this is often not necessary where the stone is 
small and has not caused much eystitis. When, however, owing to en. largement of the prostate or stricture of the urethra, the bladder has not been able to empty itself, but requires the aid of a catheter, it is often well to fasten the catheter in for the first few days and thus provide for the drainage of the bladder, and at the same time spare the urethra the irritation of the frequent passing of an instrument. This also may be required when there has been any laceration of the urethra which may lead to swelling and partial closure of the eanal.

The eystitis which persists after the removal of the stone should be treated by medication, and, if necessary, by irrigation, as described elsewhere.

It sometimes happens that the bladder becomes partly filled with tenacious muco-pus which does not readily pass with the urine, and which leads to an almost constant desire to micturate, with a sometimes painful tenesmus. This can usually be drawn off through a large, soft eatheter, but if this does not succeed, it may require the use of a litholapaxy-pump for its removal.

At the end of ten days, or a fortnight, the bladder should be washed out with an evacuator to remove any sand or gravel that was left at the time of the operation, and which would serve as a nucleus of a new stone. In a sacculated bladder, or where there was much mucus present at the time of the operation, fragments will often be found by the secondary pumping, and in some cases the tendency to the formation of phosphatic material is so strong that repeated pumpings at intervals of ten days will continue to remove little calculi, until the alkaline tendency of the urine has been overcome.

The operator should not be content until he has had a satisfactory washing with the pump without obtaining calcareous material. As a small eatheter can be used for the removal of this fine débris, the operation is comparatively free from pain, and can be done without anæsthesia.

The operation as just deseribed is the litholapaxy of Bigelow, and it remains to speak of one or two variations of it practiced by good operators and liked by them. The changes introduced are mainly in the methods of accomplishing evacuation.

Chismore's evacuating lithotrite has been deseribed, and its usefulness in the search for the last fragment alluded to. It was originally devised for this purpose, but its inventor has gradually pushed his use of it, until now he employs it in all his operations from beginning to end. The light rubber aspirator that he has attached to it does not interfere with its manipulation as a lithotrite, and does not have to be removed during the erushing of a soft stone. When more power is needed in the crushing, the aspirator is removed and a handle is substituted, which gives a firmer hold and permits of the exercise of more force. When the 
stone is very hard, he uses an extra strong male blade with a lock and screw.

Some operators use the elasticity of the bladder walls to accomplish the evacuation of the fragments. Guyon introduces the evacuating catheter and lets the fluid in the bladder rush out, bringing what fragments it can with it. He then with a syringe fills the bladder and again empties it with a rush. After repeating this a number of times and removing as much as possible, he then uses the aspirator to pump out the last fragments.

Keith has recently reported satisfactory results obtained without the use of any aspirator whatever, and seems to have removed many large stones simply by filling and emptying the bladder. He speaks of no cases of rupture of the bladder due to the forcible injection. His practice has been in India, and possibly his patients have been somewhat younger than is the rule among stone patients in other countries. This would account for his finding sufficient resiliency in their bladders to accomplish evacuation in this fashion. Among old patients with atonied bladders it would not seem possible to expect such results.

I have tried this method in a somewhat modified form, after a manner proposed by Dr. F. H. Williams a number of years ago. By means of a two-way cock attached to the end of the catheter, it was arranged so that water could be at will run into the bladder and then run out through a tube over the side of the table. In this way we had a siphon action drawing the fragments from the bladder, in addition to the elasticity of the bladder driving them. The injection of water into the bladder was provided for by a tube connected with the upper opening in the two-way cock and joining it with a raised reservoir. This reservoir was a transparent graduated glass, and thus at each filling of the biadder the operator could see exactly how much water had flowed in, and could judge of the tension by noticing the rapidity of the fall of water in the reservoir. The apparatus was easily and quickly worked by alternately turning the current to and from the bladder.

In the case upon which this was tried the amount of débris was great, and the bladder was dilated and atonied. The clogging of the tube was troublesome, and the outrush was not forcible enough to carry the detritus along in any large amount. After trying this method for a time, I substituted the Bigelow evacuator, with the result of a greatly increased rapidity in the removal of the fragments.

\section{COMPLICATIONS WHICH MAY ARISE DURING LITHOLAPAXY.}

Among the accidental conditions which may arise during a litholapaxy may be numbered the following: Hæmorrhage, laceration of the urethra, injury of the bladder walls, impaction of a fragment in the urethra, and clogging, bending, or breaking of the lithotrite. 
Hæmorrhage. - A slight or moderate amount of bleeding not uncommonly accompanies the operation. It may come from the urethra or bladder. More commonly it is due to the injury done to the congested prostatic urethra, and it is more likely, therefore, to be seen in old men. When the blood comes from the urethra anterior to the prostate, it rarely causes any trouble, and soon ceases. Blood from the prostate runs back into the bladder, and, clotting there, may interfere with the crushing of the stone. It is readily sucked out through the evacuating tubes of ordinary size, and the bleeding usually ceases, or diminishes after the first few minutes. If it continues to be troublesome, the injection of hot water $\left(115^{\circ}\right.$ Fahr. $)$ may be tried.

The bladder walls rarely bleed much, unless seriously diseased or injured. A hæmorrhage of any amount in the vesical cavity leads, therefore, to a strong suspicion of the existence of a tumor, and in such a case the washings from the bladder should be carefully saved and examined for bits of new growth. If these were detached and recognized during the operation, the plan of procedure would naturally be changed, and a suprapubic incision would be resorted to. Keyes mentions one case in which, in a bladder not containing a tumor, the loss of blood was so great as to lead to the temporary abandonment of the operation. Subsequently, when the fragments remaining were washed out, there was very little hæmorrhage.

In patients of a hæmorrhagic diathesis the operation would naturally be approached with a good deal of hesitancy; but I have seen no account of serious trouble from this canse.

Laceration of the Urethra may be caused either by the introduction of the instruments or by the withdrawal of them when clogged with projecting sharp fragments. In skillful hands an uninjured urethra ought not to be torn during the introduction of instruments of a proper size. An ulcerated or partly strictured canal may be torn even when due care is exercised. It is a good safeguard against this accident to pass a conical steel sound one or two sizes larger than the lithotrite and tubes. This gently stretches the canal, so that the rougher, pointed instruments are afterward less likely to eatch. In the event of having troublesome false passages, either pre-existing or made at the same time of the operation, the surgeon may wisely decide to change the plan and do a lithotomy.

The withdrawal of a clogged lithotrite or of a tube holding an impacted fragment may considerably lacerate the urethra. When this occurs, the ragged tear offers a favorable surface for septic or urinary infiltration, and this accident adds, therefore, considerably to the danger of the operation. This danger is lessened if the urethra is thoroughly washed out with an aseptic solution at the end of the operation.

Whenever so much injury of the urethra results as to lead to the fear 
of urinary absorption, or of retention from swelling, it is well to fasten in a catheter for the first two or three days. This is especially desirable in a case requiring catheterization, or when a long and tortuous prostatic urethra has been thus injured. Usually, with this precaution, lacerations of the urethra do not lead to serious consequences, especially if antiseptic precautions have been carefully observed in the instrumentation, and if the urine has been rendered aseptic before the operation.

Injuries of the Bladder may be caused by catching the walls in the lithotrite. This may usually be avoided by keeping the bladder moderately distended with water during the crushing, so as to prevent its wall from being thrown into folds which might be caught in the jaws of the instrument. The care in the use of the lithotrite which is necessary to avoid this accident has been described in considering the operation. It is conceivable that in a diverticulated or chambered bladder the female blade of the open instrument might find its way into a pocket, or chamber, while the male blade remained in the general cavity, and the intermediate septum would then be caught as the instrument was closed. I have seen bladders in which this might have easily occurred, and have twice during litholapaxy in old men had my instrument so caught and held that I thought it must be thus held in a pocket; and finding, on trying to close it, that a soft resistance was met, I kept the blades partly open, and then, keeping the shaft nearly upright, carried the heel of the instrument close down behind the neck of the bladder and thus was able to close it. In both cases, by operating near the neck of the bladder, I was able to finish the crushing and evacuation of the stone. Fortunately, injury of the bladder walls by nipping is usually followed by no untoward results.

Another accident which may happen to the bladder walls is the rupture of them during litholapaxy.

Rupture of the Bladder Walls.-This may result either from using the pump when the bladder is too full, or from spasmodic contraction of the bladder when the urethra is blocked with instruments. Great care should be taken, when injecting the bladder before the operation, to notice the degree of resistance which it offers to the entrance of the water. This is especially important if the escape of the water alongside of the instrument is prevented by an elastic band. The force required to rupture an undiseased bladder wall is so great that this is very unlikely to occur ; but as many of these bladders are diverticulated in old people, there is considerable danger to the thin-walled diverticulum.

I have had one such case, in which a bladder ruptured itself by its own spasmodic contraction when containing but an ounce or two of urine. In this case a laparotomy was done at once. It was found that the rupture had taken place extraperitoneally on the left side, and the urine had es- 
caped into the cellular tissue up toward the kidney on that side. Drainage was established in the left groin, and the case eventually got well.

Whenever, during the litholapaxy, the bladder is affected by violent spasms, the operation should be delayed until the anæsthesia is so profound as to put a stop to these. If this is found impossible, it may even be necessary to do a lithotomy rather than run the risk of rupture. When a spasm occurs during the pumping, it can be at once relieved by opening the stop-cock at the top of the bulb, and letting out the excess of water.

Impaction of Fragments in the Urethra.-Occasionally, as has been said, fragments lodge firmly in the eye of the evacuating tube, and the temptation is strong to try to draw them ont with the tube. This attempt usually succeeds, but, besides its scratching the urethra, there is danger that the fragment may be dislodged from the tube and remain sticking in some part of the canal. Also, if the bladder is contracting strongly, a fragment may be forced into the urethra at the time of withdrawal of some of the instruments.

The points of usual arrest of fragments are in the prostate, at the triangular ligament and just behind the meatus. From this last situation fragments are usually removed by incising the meatus and using forceps. A fragment lodged in the prostate may often be pushed back into the bladder with a large, blunt-pointed sound. When the bit of stone lodges in the urethra just anterior to the prostate, it may be very difficult of removal. In a recent case, where a fragment was dropped by the tube in the membranous urethra, I succeeded in pushing it back into the bladder by the following manœuvre: Passing a straight tube with a long under lip (Fig. 50) down to the fragment, I then introduced the finger into the rectum, and, while lifting the bit of stone, worked the lip of the tube under it, so catching it in the mouth of the tube and readily pushing it back into the bladder. (For further consideration, see Urethral Calculi.)

Clogging and Breaking of the Lithotrite.-The strong modern lithotrites of the Bigelow pattern do not easily become clogged. A tough foreign body, as the nucleus of a stone, may, however, resist complete crushing and become entangled in their jaws. I have met one case in which a leather shoe-string formed the nucleus of a stone, and became so fixed in the jaws of the lithotrite as to make a very considerable mass to be drawn out with it. Though the urethra was much rasped by its passage, no ill effects followed. It is conceivable that in some such way the jaws of an instrument might be so held apart that they could not be withdrawn without great violence. Under such circumstances the blades should be carried to the center of the bladder, where there is no danger of eatching the walls, and should there be many times opened and shut 
with the screw in order to gradually grind up or cut through the offending body.

If a lithotrite is tested outside of the bladder on a hard stone, it will be found that its blades are often twisted considerably during the crushing, but being of well-tempered steel, they spring back into correct shape when the pressure is taken off. With a small instrument suitable for a child, this twisting is often very great, and it may happen that the resistance of the metal is overcome, and the jaws are so distorted as to be afterward difficult of withdrawal. Usually, however, they break rather than bend. A number of cases of this accident have been reported. The fracture may occur at the heel of one of the blades, or on the shaft of the female blade, a short distance above the bend.

This last point is the position of the greatest strain in crushing a large stone, for the obliquity of the blades causes the force against the female blade to act rather downward, so that there is greater leverage exerted upon the shaft above than at the heel of the blade. When a hard stone is encountered, which resists all the force that it seems safe to apply, it is best, after cutting into it somewhat in one diameter, to seize it in a new place. It thus presently becomes weakened and gives way.

The breaking or serious clogging of a lithotrite may oblige a resort to lithotomy. A perineal incision will usually give room enough for the extraction of a broken blade, or for the clearing of a clogged instrument. If the perinæum is very deep, or the prostate large, the suprapubic route will probably be preferable, and, in case of a twisted or distorted instrument, the high operation gives more room for the exercise of force sufficient to bend the blades back into place.

\section{COMPLICATIONS AFTER OPERATION.}

Hæmorrhage rarely causes trouble after litholapaxy, except that, in cases requiring the use of a catheter, this may be plugged by the clots. It may be necessary to free the bladder by the evacuator, if ordinary injections and irrigation fail to bring them away.

Epididymitis occasionally follows an operation in which the prostate has been much irritated. The only case in which I have seen epididymitis follow the operation was one in which the urethra was considerably scratched by a rough fragment which was drawn out in the eye of the evacuating tube.

Urethritis, Prostatitis, and Cystitis.-Inflammation of the urethra, of the prostate, or of the bladder, may follow a litholapaxy. In fact, such conditions existing before the operation may be temporarily aggravated by it. The treatment of them is considered in another part of this work, and does not require special attention here.

Urinary Fever is a complication to be occasionally expected, especially 
in those cases in which the kidneys are gravely affected, and something has been already said in regard to the best prophylactic measures to be taken against it.

Phlebitis.-Rarely, an inflammation may extend from the small venous sinuses about the neck of the bladder to neighboring large trunks. I have had one case in which the left leg was swollen and tense from this cause. The patient was a middle-aged man, who made a good recovery from an operation for the removal of a hard uric-acid calculus of considerable size. Fourteen days afterward he was on the lounge, and the bladder was washed out with the pump without ether. This caused him rather more pain than usual, though no difficulty was experienced in the manipulation. A few hours later he was seized with severe pain in the left leg, which at once began to swell, and ran the ordinary course of a rather extended phlebitis of the femoral vein.

Treatment after Litholapaxy.-After an uncomplicated litholapaxy, in a case with reasonably sound urinary organs, the relief is often immediate and the recovery uneventful. It is well, however, even in such a case, to keep the patient quiet in bed for a few days, as the urinary organs are often slow to show inflammation, and a febrile condition may set in on the second or third day after a litholapaxy, when up to that time everything has seemed to go smoothly.

Usually some pain and febrile reaction follows the operation. Hot applications to the hypogastrium and perinæum will do something to relieve the pain. The treatment commenced before the operation to render the urine aseptic may be continued, and for a diuretic the free administration of spring-water is usually sufficient.

If there is a tendency to retention of urine, and the bladder empties itself incompletely, the catheter must be used. Mucus and irritating ammoniacal urine must be removed by irrigation, with appropriate solutions of borax, permanganate of potash, or nitrate of silver. If there is a tendency to phosphatic deposit, it must be met by injections of dilute nitric acid, and by the occasional use of the evacuator to remove granules too large to be destroyed by solvents.

Other complications must receive appropriate treatment.

At the end of ten days or a fortnight, when the irritation caused by the operation has subsided, the evacuator should be used, to make sure that there are no retained fragments or sand. At this time, after the mucus has largely disappeared from the urine, it is surprising how easily fragments are removed which escaped the most diligent search at the original operation. 


\section{Lithotomy.}

The cutting operations by which stones are removed from the bladder naturally divide themselves into two classes:

1. The perineal operations, in which the incision approaches the bladder along the urethra and enters it through its neck.

2. The high, or suprapubic operation, in which the incision is made in front above the pubes, and the bladder is opened through its anterior wall just above the prostate.

\section{PERINEAL LITHOTOMY.}

The perineal route is a comparatively long one and beset with anatomical difficulties, consequently the ingenuity of surgeons has been much exercised in devising methods by which the dangers may be avoided and at the same time the most room may be obtained.

Of these methods and modifications we will consider those which have had sufficient merit to retain a place in modern practice.

The space in the perinæum within which the lithotomist must work is bounded by the rami and tuberosities of the ischia, and posteriorly by the sacro-ischiatic ligaments.

The structures within this space which the knife must avoid are the internal pudic arteries, which lie close to the tuberosities and rami of the ischia ; and the rectum, which lies in the middle line just below or behind the centre of the pelvic outlet. It is also well to avoid an extensive incision into the bulb of the urethra, as its spongy tissues sometimes bleed in a troublesome way.

The field of possible operation is therefore a crescentic one, encircling the rectum anteriorly, coming well down on each side, and extending back even to the sacro-sciatic ligaments. Within this space all the external incisions may be made safely. As we approach the bladder we reach more important structures, which may not be incised so freely.

The early Marian operation contemplated an incision into the body of the bladder, and the effort at that time was to avoid the neck of the bladder, if possible. It was found, however, that this was a needless fear, and the introduction of the grooved shaft as a guide for the knife led the incision directly through the neck of the bladder. This practice soon demonstrated that the prostate could be freely incised, and the belief gained ground that the danger of the operation was considerably increased when the deep incision reached beyond the prostate and opened the loose cellular spaces behind it, thus preparing the way for urine infiltration.

Thompson, while accepting the general truth that the incision should remain within the prostate when possible, points out the fact that in 
children, before the prostate is developed, these spaces are opened, and yet that urine infiltration is rare; and he urges that the deep incision should be sufficiently free to permit the removal of the stone without bruising the tissue, even if the limits of the prostate are overstepped. Another disadvantage in carrying the incision into the bladder wall behind the prostate is that the seminal vesicles may be injured. It may be regarded then as a proper matter for attention during the operation to keep the deep incision within the prostate, if possible.

There have been in recent times various attempts to get more room for removing large calculi through the pelvic outlet by carrying incisions through the rectal wall into the bladder. Some of these contemplated openings through the prostate, while in others it was intended that the trigone should be incised. These recto-vesical operations, although they gave much room, had, however, a high rate of mortality; and since modern improvements have made the supra-pubic operation a safer method for the removal of large stones, they have no longer any title to surgical consideration.

The perineal operations which are still more or less in vogue are the lateral, the median, the medio-lateral, and the medio-bilateral.

\section{ANATOMICAL CONSIDERATION OF THE VARIOUS INCISIONS.}

Lateral Lithotomy.--In this operation the space in the left half of the perinæum is utilized for the incision. The knife enters the perinæum just to the left of the raphé, at a point an inch to an inch and a quarter in front of the rectum, and is carried backward over the ischio-rectal fossa. The urethra is opened in the membranous portion, the knife then enters the bladder along the urethra, and the incision in the prostate is made out through the greatest diameter of the lateral lobe.

Thompson has pointed ont the fact that the incision in the prostate does not follow the oblique downward direction of the external incision, but runs more horizontally.

The parts divided in a typical lateral operation are the skin and superficial fascia, the transversus-perinei muscle, the posterior portions of the accelerator-urinæ muscle, the membranous-urethra and the compressorurethræ muscle, the anterior and posterior layers of the deep perineal fascia, and the left lateral lobe of the prostate. In the child, who has practically no prostate, the body of the bladder is incised.

The vessels and nerves that are cut are of little importance, the transverse perineal artery being the only blood-vessel of appreciable size that is necessarily divided.

If the incision is carried too far forward, the bulb of the urethra or the artery of the bulb will be cut, and may give rise to troublesome hæmorrhage deep in the wound. The pudic artery is usually so far out under 
the ischia that it can only be injured as the result of carelessness; but cases are reported in which it runs across the field of operation, and is necessarily wounded. Occasionally, too, the artery of the bulb starts farther back than usual, and so runs directly across the line of incision. The hæmorrhoidal veins, or the venous plexus about the prostate, may be much dilated in old men and bleed profusely.

The rectum, if distended with freces or flatus, may encroach on the line of incision, but, if empty, is easily avoided. In case of a large stone that will not pass through the opening provided by this incision, it may be somewhat enlarged. The narrow part is not in the lax onter tissue, but in the neck of the bladder, and with the object of gaining more room here, the incision may be carried beyond the prostate, or a second incision may be made into the other lobe.

Bilateral Lithotomy.-The bilateral operation consists in an incision curved crescentically in front of the anus, with the concavity looking backward. It crosses the raphé at the point where the lateral incision begins, and the tissues divided are the same as in the lateral operation, with the difference that the similar structures are divided on both sides of the middle line, and the ends of the incision are not carried so far back over the ischiatic fossæ. The opening into the prostate is made by simultaneous incisions into both lateral lobes, for which part of the operation Dupuytren devised his double lithotome caché.

Median Lithotomy. - In this operation the incision is made on the raphe immediately in front of the rectum, and the parts divided are the skin, the superficial fascia, fibers of the accelerator urinæ and of the sphincter-ani muscles, the membranous urethra, and the two layers of the deep perineal fascia. The prostate is incised in its posterior commissure. In the case of a small stone or a foreign body, it is sometimes possible to get sufficient room for its removal by simply dilating the prostatic urethra. Dolbeau, when devising his apparatus for perineal lithotrity, tested the dilatability of the prostate, and found that it could be stretched to a diameter of from twenty to twenty-four millimetres withont tearing. Keyes has verified these figures. It is therefore possible to extract through the uncut prostate a foreign body which, with the forceps holding it, does not measure quite an inch. Anything larger than this will cause tearing; and it is better to make an incision and thus divide the tissues without violence, rather than to make bruised lacerations, with the chance that they may occur in unfortunate directions. The median operation affords but little room at best, and Reynaud combined the median incision through the skin and superficial tissues with a lateral incision in the prostate (nediolateral operation).

Medio-bilateral Lithotomy.-Civiale, with the object of still further enlarging the opening, devised the medio-bilateral operation, 
in which, through a median opening, the prostate is enlarged bilaterally.

Selection of Incision according to the Size of the Stone, - In making choice of a perineal incision for the removal of a stone, we have only the median and lateral operation to consider, including under the median those modifications of the incision through the prostate, either unilateral or bilateral, which are designed to give more room. The other methods of incision, such as the bilateral, the pre-rectal, or the recto-vesical, have all fallen into disuse, and may be left out of the present consideration.

The median operation, while it best avoids injury of important structures and is therefore the safest operation, gives the least room. Dolbeau, as has been said, found that dilatation could be carried to a diameter of one inch. A median incision in the prostate increases this possible diameter somewhat, and with a lateral or bilateral incision considerable room is gained. The stones, however, which can be removed through this opening are all of them of a size to be easily managed by litholapaxy. Under exceptional circumstances, when a digital examination of the bladder is desired, or when drainage for the relief of a long-standing cystitis is planned as one result of the operation, the median incision may be used, and through it a stone or foreign body, having a diameter in two directions of not more than one and a half inch, may be removed.

The lateral operation gives a much larger and more distensible opening, and the incision into the bladder may, when necessary, be carried outside of the prostate with more safety than can be done by the median incision. If through a median incision the limits of the prostate are overstepped and the spaces behind it are opened up, the drainage of these cellular interspaces is not readily accomplished through the opening in the skin; whereas, if the same spaces are opened up in a lateral operation, the dependent character of the external wound does provide good and sufficient drainage to these same cellular spaces.

Very large stones have been removed through the perineal incision. Keyes ealculates that a stone two inches in diameter may be drawn through a prostate which has been incised bilaterally; but that, when a stone larger than this is removed, the incision must extend into the body of the bladder. These figures may be somewhat increased in cases of enlarged prostate, where more room is afforded within the body of the gland.

If the operator, having entered upon the perineal operation, finds a stone more than two inches in diameter, it is better to crush the stone and remove it piecemeal, rather than to make extensive incisions into the bladder wall. It is always to be remembered, however, that a clean incision which allows of the easy removal of the stone is less dangerous than a smaller opening through which the stone or its fragments have to be 
dragged with considerable force. As in the case of the median operation, the stones which are suitable for lateral lithotomy are usually suitable for litholapaxy, unless some special condition makes a cutting operation advisable.

If a stone is too large for perineal removal-and any stone over two inches in diameter may be considered so-the suprapubic incision may be selected. Through an opening above the pubes, stones of any size which may form within the bladder may be extracted. Although, when all cases are taken into consideration, the statistics of the high operation show it to be more dangerous than the perineal incision, if the statistics of the removal of large stones through the perinæum and above the pubes are contrasted, the advantage is found to be vastly in favor of the high operation. So true is this that if an operator, beginning with the perineal operation, finds a stone so large that great force is evidently needed for its extraction by that route, he will give his patient a better chance by changing his plan of operation and making the suprapubic incision, using afterward the perineal wound for drainage, if it seems desirable.

\section{DESCRIPTION OF THE DIFFERENT OPERATIONS.}

Perineal Lithotomy.-Lateral Operation.--Instruments required.-The difficulties which the surgeon may encounter in this operation are so manifold, that it is important that he should be thoroughly familiar with the use of instruments and appliances suitable for all cases. The following instruments are those with which the surgeon must be provided, and with the use of which he must be familiar when undertaking a lateral lithotomy.

Fig. 53.-Grooved lithotomy-staff.

(1) The Staff.-The staff should be grooved on its lower side, and may either be curved like an ordinary catheter, or may be made more in the form of a hook, so as to bulge somewhat in the perinæum. It

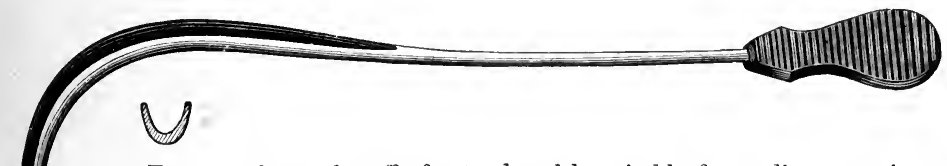

Fig. 54.-Grooved staff of extra breadth, suitable for median operation.

should be broad enough to be readily found, and the groove should be so deep that the knife can not easily slip out of it. 


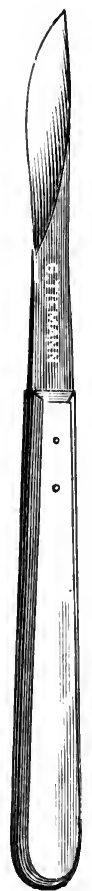

FIG. 55.

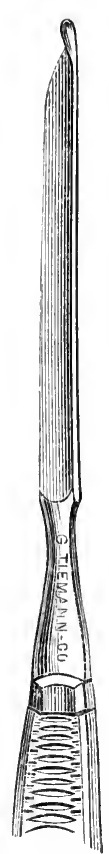

FIG. 56.

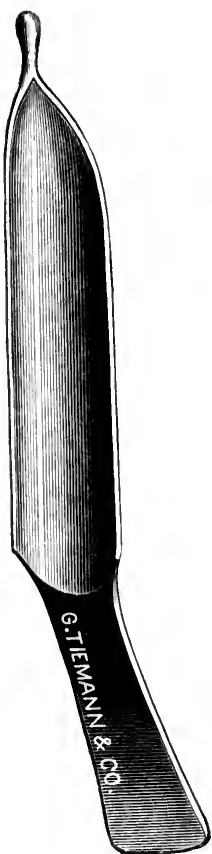

FIG. 57.

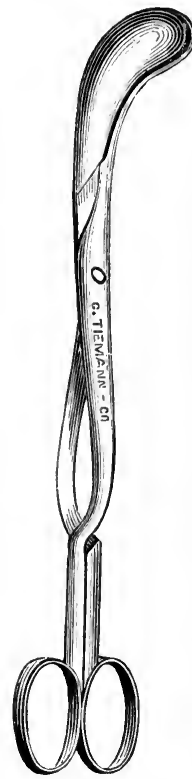

FIG. 59.

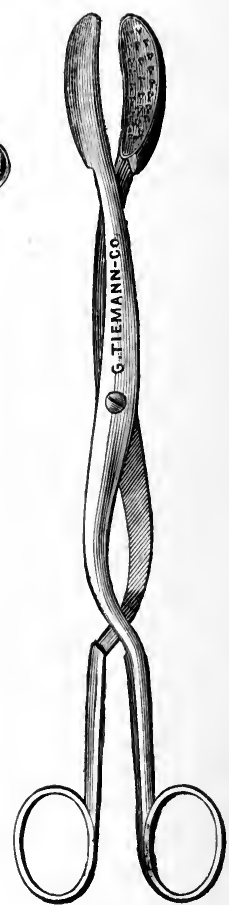

FIG. 60.

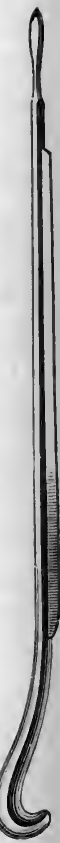

FIG. 61.

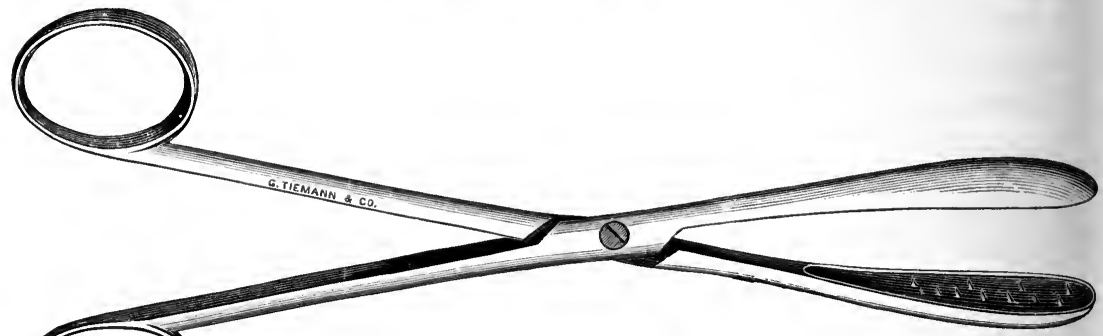

Fig. 58.

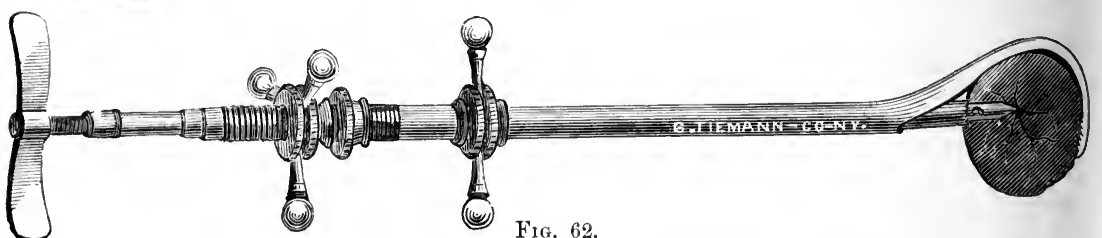

Figs. 55-62.-Fig. 55. Lithotomy-knife. Fig. 56. Probe-pointed lithotomy-knife. Fig. 57. Blunt gorget. Fig. 58. Lithotomy-forceps, straight pattern. Fig. 59. Lithotomy-forceps, curved. Fig. 60. Lithotomy-foreeps for deep perinæum. Fig. 61. Crested lithotomy-scoop. Fig. 62. Maisonneuve's lithoclast. 
Some operators prefer a lateral groove in the shaft, but this is not at all essential.

(2) The Lithotomy-Knife.-This should have a more solid handle than the ordinary scalpel, with a blade from three to three and a half

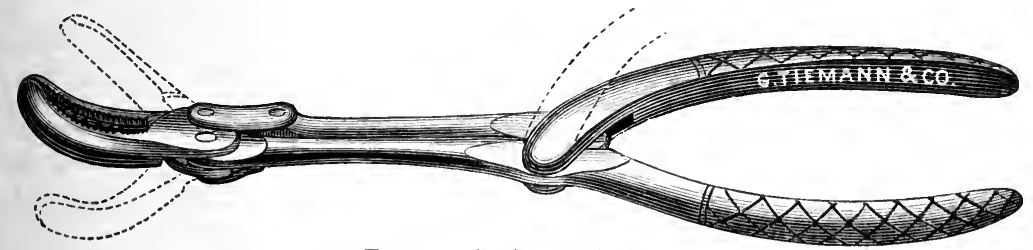

FIG. 63.-Gouley's lithoclast.

inches in length. Besides this, it is well to have a second knife of similar shape, but with a probe point, for use in case it is necessary to enlarge the incision after the bladder has collapsed. Thompson also advises a blunt gorget for dilating the incision in those cases in which the peri-

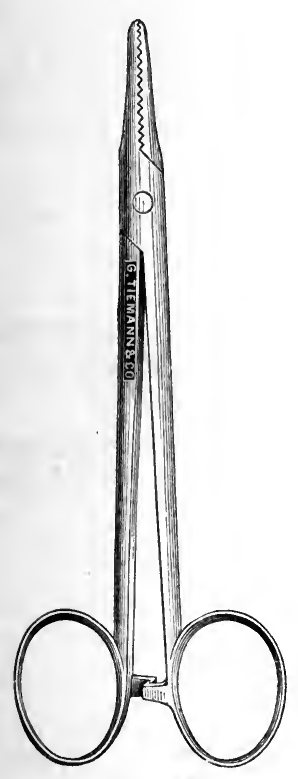

Fig. 64.-Artery pressureforceps.

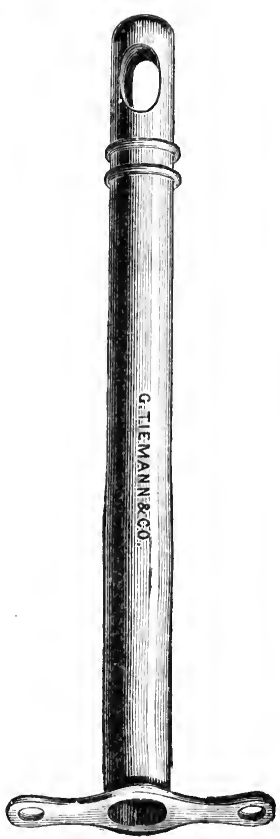

FIG. 65.-Chemise cannula.

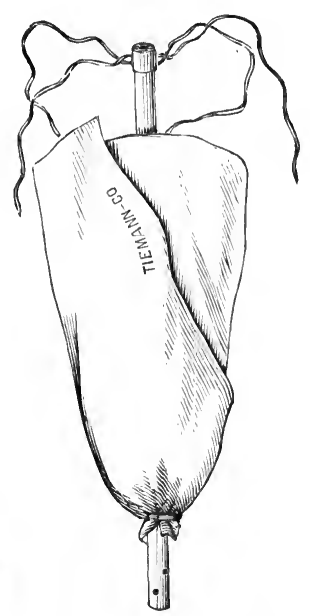

Fig. 66.-Cannula with chemise attached.

næum is so deep or the prostate so large that the finger can not reach the bladder. In these cases the groove of the gorget serves as a guide for the forceps. 
(3) Lithotomy-Forceps should be of various sizes and different curves. The straight forceps are usually sufficient for cases in which the bladder is of normal shape and size; but in old men, where there is likely to be a pouch behind the prostate, or when the stone lies to one side of the vesical neck, a somewhat sharply curved

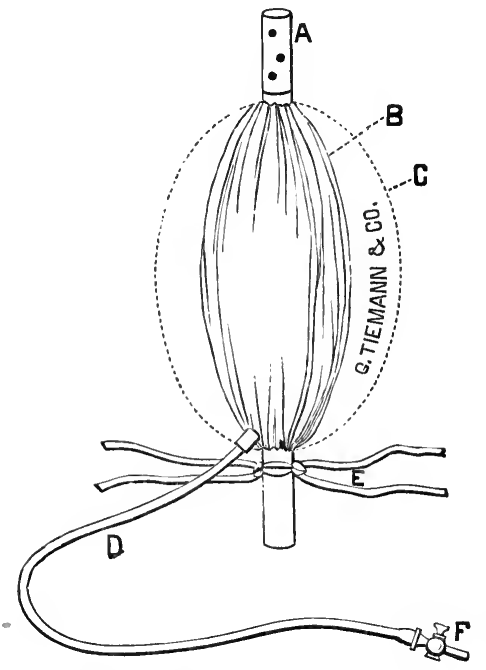

Fig. 67.-Browne's air-tampon and cannula. pair of forceps may be useful. When the perinæum is deep, it is well to have a pair of forceps the handles of which shut by each other, so that the blades can be well opened without widely separating the handles.

(4) The Scoop should also be at hand for prying the stone out of a pocket, or for removing fragments too small to be easily seized by the forceps.

It is well also to have crushing instruments of sufficient strength. Many ingenious special forms of instruments have been devised for this purpose, but a strong lithotrite would usually be sufficient. The use of these instruments will be considered under perineal lithotrity.

For washing out débris, a large tube should be provided. To check hæmorrhage, pressure-forceps are useful; and Thompson advises the occasional use of a tenaculum which can be detached from its handle and left in the wound, if necessary. This is to be used by hooking up deep vessels upon it, and then tying a ligature firmly around it and leaving the tenaculum in place.

For stopping oozing which does not come from vessels of a size to be easily tied, a chemise cannula is useful, or Buckston Browne's air-tampon.

Preparation for the Operation. - The general preparation of the patient has been considered, and the importance of examining into the activity of the kidneys has been pointed out. It is especially important to render the urine as aseptic as possible, as foul, putrid urine may do harm when coming in contact with freshly wounded tissues. It is to be supposed that the surgeon has informed himself as to the special circumstances surrounding his case; that he has determined the size of the stone as far as possible; that he knows the size of the prostate and the depth of the perinæum. The condition of the urethra is also of importance; and it is well to know whether the bladder is closely contracted about the stone, or whether it is readily distensible. That the rectum should be emptied and collapsed so as to be readily avoided, has been said. 
The perinæum should be carefully shaved and thoroughly disinfected on the day before the operation, the corrosive poultice being afterward applied and continuously worn up to the time that the patient is on the table.

A suitable strong table should be provided, and, if possible, this should be from two to two and a half feet wide, so as to allow the assistants to stand closely upon each side of the patient. The chair of the surgeon should be of a height to bring the upper part of his chest opposite the patient's perinæum. A tub should be placed at the end of the table, and a rubber cloth should be so arranged that, when the patient is brought down to the end of the table for operation, it shall conduct the blood and fluids into the tub. A convenient rubber apron has been devised for this purpose.

Enough assistants should be provided to give the surgeon all the aid that he requires. One assistant administers the ether, and one

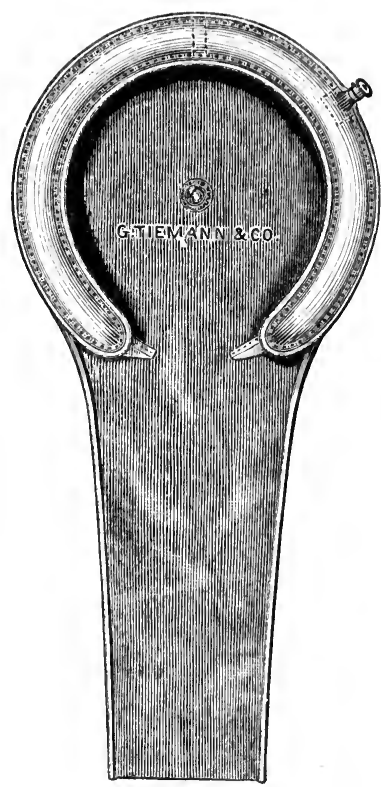

Fig. 68.-Rubber apron to be put under the hips. is essential to the care of each leg. To the one holding the patient's left leg can be intrusted the care of the staff. It is well to have a

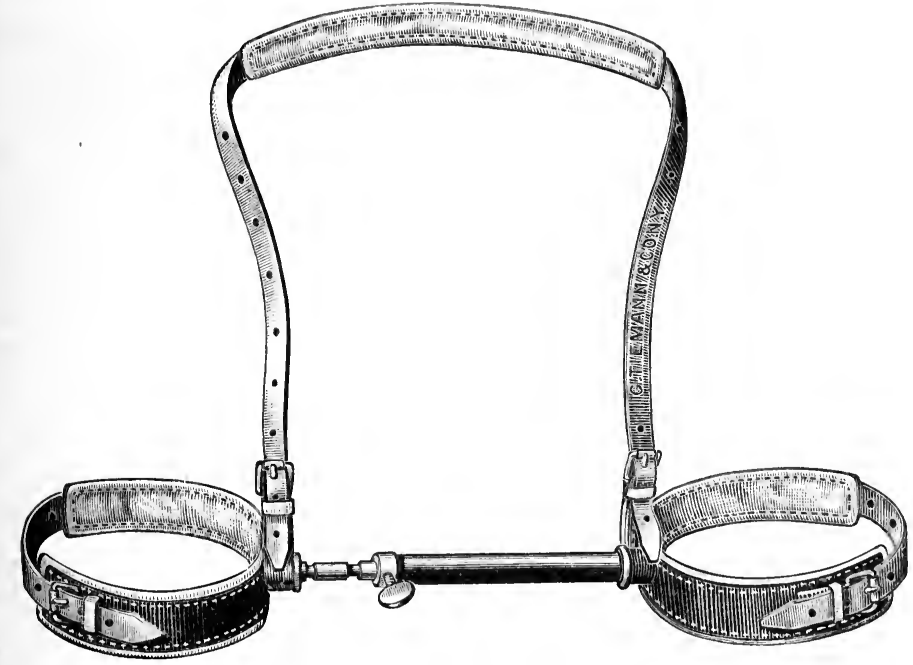

Fic. 69.-Bar to hold the legs, with strap to go over the shoulders. 
fourth assistant, to give the surgeon such aid as is required; but if one is not fortheoming, the one holding the patient's right leg can sponge and give all the help that is absolutely necessary.

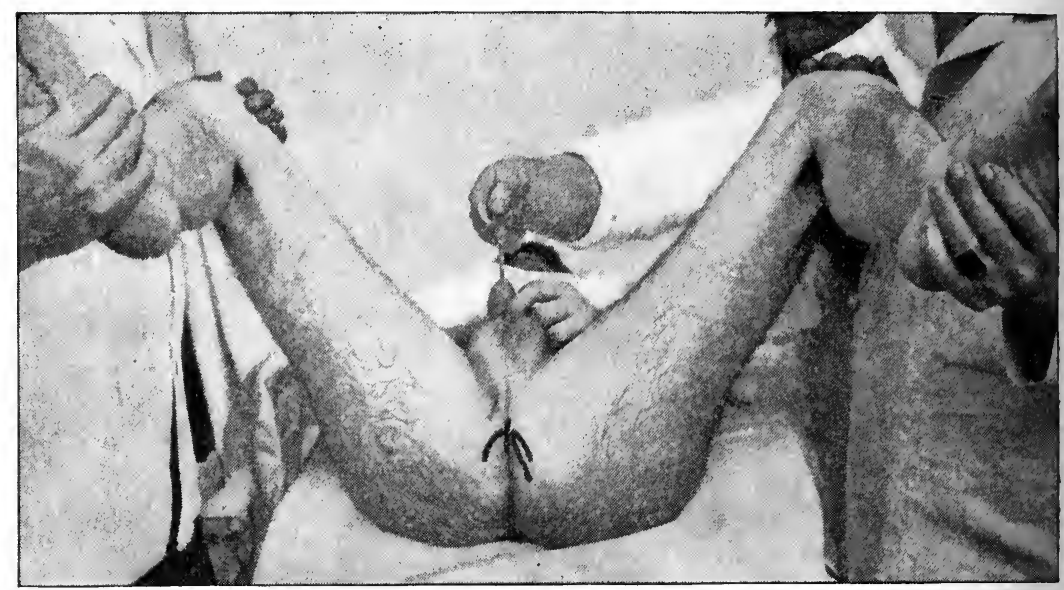

FIG. 70.-Patient in lithotomy position, with the staff eorreetly held. The lines of incision for the median, the lateral and the bilateral operations are marked.

The instruments should be on a table at the right hand of the surgeon, within easy reach.

It being important to hold the patient steadily and firmly after he is once in position, it is well to provide a bar to go between the legs,

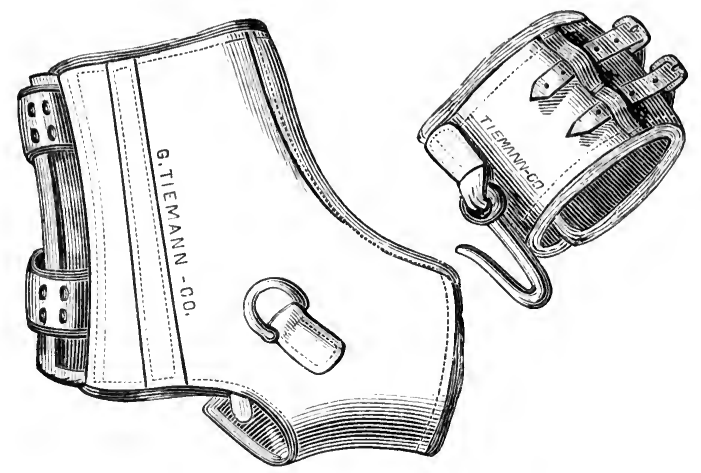

Fig. 71.-Anklets and wristlets.

with a crutch at each end for the leg to rest in. This, if provided with a band to go around the calves, holds the legs satisfactorily, and it only remains for the assistants to preserve them in their proper relative position. Another way of securing the patient is by fastening the ankles 
and wrists together, which may be done by a soft piece of webbing, or by the anklets and wristlets manufactured for this purpose.

The Operation.-Ether having now been administered, the patient is placed upon the table, the bladder is emptied and washed out, and moderately distended with a boracic or other aseptic solution. The grooved staff is then entered and the stone is touched. This is an important precaution in this stage of the operation, for, besides the chance that the staff may be in a false passage and not in the bladder, there is also a possibility that no stone exists; and a review of the cases in which it has happened to thoroughly competent and skillful operators to find no stone after the bladder was opened, attests the importance of this precaution.

The patient is now brought into position at the foot of the table, and there secured. The staff, being properly in place, is intrusted to the assistant, and the same assistant may with his left hand hold up the scrotum during the operation.

Everything being now in readiness, the surgeon should examine the rectum to make sure that it is properly collapsed. The importance of keeping his hands absolutely clean may lead him to intrust this examination to an intelligent bystander, or to an assistant who can afterward give time to the disinfecting of his hands.

Being now seated in front of his patient, he informs himself of the position of the various landmarks mapping out the perinæum, reassures himself as to the position of the staff by making it prominent where it is easily felt in the perinæum, and then, carrying in his mind the plan of operation and the point at which he intends to strike his staff, he makes the incision.

This should commence a little to the left of the raphé, and from one and three quarters to one inch in front of the anus. The incision which begins farthest forward falls over the bulb of the urethra, and, in order to avoid wounding this, great care must be taken that the upper portion of the first incision be not carried too deep. The lower incision admits the operator more directly to the urethra at the point where he intends to open it, and less care is necessary to avoid wounding the bulb. As Thompson says, it is therefore a safer practice to err, if it be an error, by placing the incision too low rather than too high. Commencing at the point selected, it should run back over the ischio-rectal space for three inches. The superficial layers and the loose cellular tissues in the ischiorectal fossa should be rapidly divided. The bulb of the urethra, if it comes into view, can be drawn somewhat upward, and the staff should be sought just below it. When the urethra is approached, the forefinger of the left hand should be introduced into the wound, and with it the groove in the staff be sought. When this is felt, the finger-nail should be pressed 
into it, and along this as a guide the knife should be entered until it can be felt scratching the metal of the staff.

Being sure, now, that the knife is firmly placed in the bottom of the groove, it may be carried into the bladder by one of two manœurres. Either it may be slid along the groove and so through the prostate until it reaches the vesical eavity, or the operator may take the staff from his assistant, and, holding that firmly in his left hand, with the knife held fixedly in the groove so that there is no danger of slipping, he may then by a combined movement of both hands advance the staff and the knife into the bladder at the same time.

Having arrived at the vesical cavity, the prostate may be incised by simply depressing the handle of the knife, the point of the blade being still fixed in the groove of the staff. In making this cut the blade of the knife is not to be carried directly down in the line of the external incision, but should be directed more horizontally, so as to make the incision through the thickest part of the lateral lobe. In this way an incision of any desired extent may be made in the prostate, and the knife may then be withdrawn at once, care being taken not to cut with it during the withdrawal. In this way a more exact control can be had over the extent of the incision made in the prostate than when the cut is made during the withdrawal of the knife by sweeping the point outward. It is difficult in a deep wound to exactly calculate where the point of the knife is, and there is danger of making a more extended incision than is intended by the latter manceuvre.

The knife, being withdrawn, is laid down, and the right forefinger is introduced through the wound. If the incision in the prostate is found to be too small, the probe-pointed knife may be entered along the groove of the staff, and the incision enlarged by depressing the handle of the knife in the same manner as before. The finger is then introduced into the bladder, the stone felt, its size estimated, and the staff may then be withdrawn, the right forefinger being kept in the bladder. The staff then being laid down, the left forefinger may be introduced along the right, and with both fingers in the bladder, placed back to back, a separation of the finger-tips will produce a gradual and easily regulated stretching of the vesical neck to the extent desired. This having been accomplished, the right forefinger is withdrawn, the forceps are seized and passed into the bladder along the left forefinger, which is retained as a guide. The forceps, being carried well into the vesical cavity, are widely opened in a transverse direction, rotated in a quarter of a circle, and then closed. In this rotation one blade sweeps the floor of the bladder beneath the calculus, and when they are closed the stone will usually be found in their grasp. If difficulty is found in seizing the stone, owing to any peculiar location, forceps especially adapted to reach it may be 
selected, and under the guidance of the finger it may be seized and withdrawn. An irregularly shaped stone should always be so caught by the forceps that the smaller diameters shall pass through the opening. A stone having a length of three inches, if the other diameters do not measure more than one or one and a half inch, may be readily removed through the perinæum.

Traction in withdrawing the stone should be downward, the stone being worked through the soft tissues by a side-to-side motion of the forceps, while the soft parts are pushed back with the fingers of the other hand. Any real contraction of the soft parts is overcome by further incision with the knife, rather than by tearing with the forcible traction of the stone.

If the stone breaks in the forceps, it is to be removed piecemeal. This is sometimes rather difficult. The smaller fragments, which are not readily seized with the forceps, are best removed with the scoop, the finest sand and bits of stone being finally washed out by means of a syringe and tube passed to the bottom of the bladder, so that the current shall flow from within outward, carrying the débris before it.

An examination of the interior of the bladder should follow the removal of the stone, in order to make sure that there are no other calculi present, either loose in the bladder cavity or contained in pockets. The interior of the bladder may be brought down upon the examining finger by pressure above the pubes, in the method adopted in other bimanual pelvic examinations.

The stone being out, the stopping of any hæmorrhage which is going on is the next duty of the surgeon. All vessels in sight should be tied, and the deep portions of the wound should be explored if there is a persistent flow of blood from them. With well-arranged retractors this can usually be done quite satisfactorily. Occasionally a deep vessel may be found which it is difficult to put a ligature about. The tenaculum of Thompson may serve a good purpose here. It is hooked into the vessel and a ligature being tied beyond it, the tenaculum is left in place ; or the pressureforceps may be clamped on to the vessel, and left for a day or two projecting from the wound.

In case of a considerable oozing surface, as is sometimes left when the bulb of the urethra has been injured, it may be necessary to take a stitch in the bleeding portion and tie the tissue en masse. If this is done, great care should be taken not to encroach upon the caliber of the urethra. Usually the oozing from the bulb may be checked by packing the wound, and in this case a tube stiff enough to resist the pressure of the packing must be introduced into the cavity of the bladder for drainage. The best method of applying this packing is with the dilatable tampon of Buckston Browne, or with a chemise cannula. 
The first of these is readily understood from the figure. The chemise cannula is prepared by binding a piece of cotton or linen cloth on to the tube between the encircling ridges near the eye. The point of the tube then being carried well within the bladder, the cuff of cloth that lies in the wound is lightly packed with strips of gauze, so as to make an even pressure in all directions. This packing may usually be removed in from twenty-four to forty-eight hours, and the cannula can then be easily withdrawn.

If the water used for washing out the bladder is hot, a good deal can be accomplished in the way of allaying the oozing by irrigation. When there is not sufficient bleeding to necessitate the use of a cannula, the wound is left to fall together, and may be protected by a loose pad of iodoform gauze laid over it, care being taken that this shall not be pressed in firmly enough to hinder the outflow of urine.

The patient may then be put to bed, and should be laid upon pads of oakum, wood-wool, or pine-fiber, sufficiently large to catch the urine as it flows. The greatest care should be exercised to protect the skin as far as possible from the effect of contact with the urine, by frequent washing with alcohol, and by smearing it with vaseline or some other oily substance.

At first the urine flows freely through the wound, but after a day or two the inflammatory swelling somewhat hinders its escape, and it may partially pass through the urethra. Later, as this swelling subsides and suppuration is established, the urine again may, for the most part, escape through the perinæum.

The patient should be watched somewhat closely for the first few days, as occasionally secondary hæmorrhage appears during that time.

Attempts at primary union of the wound have occasionally been successful, the approximation of the wound in these cases being accomplished by bringing the thighs snugly together and securing the legs, so that the wound shall not be stretched apart. These efforts have not succeeded often enough, nor is the time which is occasionally gained in this way of sufficient importance to warrant the additional risk of urinary infiltration. It sometimes happens that the outer part of the wound quickly heals or agglutinates, while the neck of the bladder, which has suffered the most injury during the extraction of the stone, does not so quickly unite. This condition is, of course, very favorable to urine infiltration.

The patient should be kept in bed usually from two to four weeks, although sometimes, in the case of children, recovery is much more rapid than this. The further treatment follows the same lines as that after lithotrity, with the addition of the care of the wound.

Accidents and Complications during Operation.-Hæmorrhage. -An unusual position of the perineal arteries may give rise to a profuse 
hæmorrhage at the first incision. This should be at once stopped by ligature, as much blood may be lost if it is postponed until the end of the operation.

Hæmorrhage from the deep incision may usually be left until after the removal of the stone, as the pressure of the fingers and the instruments with which extraction is being made prevents any very great loss of blood in the narrower or deeper parts of the wound. The methods of controlling it after the operation have been considered.

Failure to enter the Bladder.-If false passages exist in the urethra, the sound may, during the operation, slip back out of the bladder and enter one of these. This, of course, is prevented by a skillful assistant, if he directs his attention to preserving the sound in the proper place, but in careless hands it is not impossible. When it occurs, it may be the source of serious trouble. If the sound is advanced into the bladder at the same time that the knife enters, the surgeon will usually recognize that it is caught in a false passage, and can then rectify its position. If, however, the knife is slipped along the groove of the staff while that is held stationary, its faulty position may be discovered only after an extensive wound has been made. To correct this unfortunate state of affairs, the staff should be partly withdrawn and carefully guided into the bladder, where it will again touch the stone, and the proper incision can then be made upon it.

It occasionally happens that, even after the urethra has been incised, the opening into it is so small that the finger can not readily enter it. In a child the too violent effort to push the finger on into the bladder has occasionally forced the bladder so far backward as to separate it from the membranous urethra. This is so serious an accident, that the greatest care should be taken to avoid it.

To the skilled operator the sensation imparted to the finger as it enters the urethra and passes through the neck of the bladder is so characteristic that it can not be mistaken. If difficulty is encountered, it is better to introduce a director along the line of the staff, and, upon this as a guide, to enlarge the incision. If the attempt is made to enlarge the incision simply by again using the sharp-pointed knife in the groove of the staff, there is danger of entering the staff through another opening, and thus making two incisions into the urethra.

Injury to the Bladder.-This may be caused by a sharply pointed staff, and Thompson has recommended that the staff be curved on a rather short axis, to avoid this accident. It is unlikely to occur if the bladder is moderately distended with water, but is to be looked for in case of a contracted bladder. Also, in a small bladder care should be taken that the point of the knife does not come in contact with the posterior wall; and the same precaution should be observed after the urine has 
escaped and the bladder has shut down. When the prostate is of sufficient size to admit of the removal of the stone through an incision made into it alone, care should be taken not to carry the cut into the body of the bladder; but practically the cases in which this occurs usually do well, if there is not much laceration of the parts. In cases of encysted stone, the bladder wall may be torn in efforts to dislodge the stone. If it is discovered that the bladder wall has been injured, especial care should be taken to provide for drainage, so as to prevent any infiltration of urine.

Wounding the Rectum.-The importance of great care to avoid this mishap is shown by the fact that it has so often happened to the most skillful operators. It is especially liable to happen to old men with lax, dilated rectum, especially if dilated with flatus. The precautions which should be taken to avoid this are : First, to see that the staff is not pushed downward toward the rectum while the incision is being made; second, to make the deep incision laterally, not downward; lastly, in a difficult case, the rectum should be held down with the fingers of the other hand while the lateral incision is being made. If the point of the knife is swept along the lower angle of the incision as it is withdrawn, this accident is more likely to happen than when incision is made in the way recommended in the description of the operation, by depressing the handle of the knife and not entting during withdrawal.

It is usually recommended that the wounded rectum be left to itself, and in many of the cases thus treated healing finally occurs. In case, however, the opening can be distinctly brought into view, there seems to be no objection to the application of stitches bringing the rectal wall together, but not inclnding the mucous membrane. In any event, care should be taken to keep the wound clean by thorough irrigation, and to regulate the bowels by laxatives and enemata.

When healing does not occur, it is likely to leave a urethro-rectal fistula. Systematic catheterization, by absolutely preventing the contact of the urine with the fistulous tract, will sometimes allow this opening to close. When it does not, if the fistula is within reach from the outside, attempts may be made to close it by plastic operation, and the mucons membrane of the rectum may be slipped down over it and stitched. In this case, too, the urine should be diverted from contact with the wound during the healing process.

Excessive Depth of Perinæum.-This may be a serious complication, and make the execution of the operation very difficult. If the finger can not reach the bladder, the forceps may be guided into it along the groove of a blunt gorget, as advised by Thompson, or along the ridge of a crested scoop, which is the method advised by Keyes. The forceps for such a case should have exceptionally long handles, and the shafts of the handles should shut by each other, so as to allow them to be opened widely in the 
bladder, even while the handle is in a confined space. The difficulty of the operation through such a perinæum is so great, that, if the condition is previously recognized, it would usually be proper to choose some other method of operating on that account.

An Enlarged Prostate may similarly so lengthen the route to the bladder that the finger can not reach it. Similar devices should be used in this case; and as, in cases of enlarged prostate, the bas fond is likely to be deep, a curved pair of forceps are usually necessary in order to reach the stone. Sometimes, in case of an enlarged prostate where the third lobe is prominent, it will be caught in the forceps when closed, and may make the use of the forceps difficult. If it is easily accessible, it may often be proper to remove it; and cases are reported in which this procedure has been followed by the return of normal urination, after such had been absent for some time.

Occasionally tumors will be felt in the prostate, which may be shelled out. In each case the operator must judge from the circumstances whether this is advisable. It has been done many times with good results. Gross reports one case in which a suppurating pocket was left by such a procedure. The present position of surgeons in the treatment of enlarged prostate favors this operation.

A Tumor of the Bladder, if met, may be operated upon with forceps and curette, provided it can be made accessible. If not, it may be wise to change the plan of operation, and add the suprapubic incision to that through the perinæum.

Unnatural Rigidity of the Neck of the Bladder is mentioned by Thompson as occurring in old men. Allied to enlargement of the prostate, it adds to the difficulty of getting sufficient room for the extraction of the stone. The tissues yield but little to efforts at dilatation, and free incisions must be made on both sides of the prostate before the requisite space can be obtained. Fortunately, the tissues, when in this condition, will bear more violence than when in a normal state.

Encysted Stone.-A stone may be encysted by irregular contractions of the bladder, in which a part of it shuts down about the calculus and holds it entrapped in an offshoot from the main cavity, or the stone may form in a diverticulum which existed previously. If the stone is not readily felt when the finger enters the bladder, careful search should be made over the whole cavity of the viscus.

As the finger can not readily reach all parts of the wall, this examination should be made with the vigorous help of the other hand pressing in over the pubes and forcing the various parts of the bladder wall down on to the examining finger.

If the encysting of the stone is only caused by muscular contraction, the neck of the sac which is formed can usually be dilated, and the stone 
then falls into the general cavity, from which it can be readily removed. If it is not easily dislodged, it may be seized with a pair of suitable curved forceps and withdrawn.

When the stone forms in a diverticulum, it may project into the cavity of the bladder, or it may be wholly contained in the side pocket. If such a condition is suspected before operation, the suprapubic incision would usually be selected rather than the perineal. Occasionally, when the perineal incision has been made for exploration, an encysted stone may be found, and the possibility of removing it by that route will then depend on the position of the stone and the ease with which it can be reached, and also on the relative size of the neck of the sac and that part

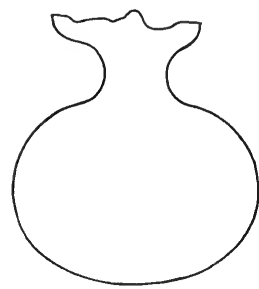

FIG. 72.-Outline sketch of encysted stome, in which only the small rough surface above projected into the bladder. of the stone contained within it. Unless the neck of the sac is large in relation to the stone, it is best to make at once the suprapubic opening; and the same is the best procedure when the pocket is so far from the perinæum that it can not be easily reached.

When the conditions are favorable for perineal removal, it will usually be found that a small spoon, under the guidance of the finger, is the best means of dislodging it. If the forceps are used, care should be taken not to seize a portion of the bladder wall with the stone, and also not to lacerate the neck of the sac by too forcibly dragging the stone through it. If the bladder wall is injured, an unfortunate result may usually be averted by providing thorough drainage until the rent is healed. If the stone is adherent to the bladder wall, it may be scraped out with a scoop and removed. In that case, it is well to thoroughly eurette the surface to which it is attached, in order to remove any bits of calcareous matter with the granulations upon which they were deposited. In such a case it is important to afterward guard against the alkaline fermentation of the urine. Keyes advises the after-use of irrigation of the bladder with dilute nitric-acid solution.

Stone too large for Perineal Removal.-If the stone is so large that it can not be safely removed throngh the perinæum, it may either be broken by instruments introduced through the wound, and so removed piecemeal, or it may be removed by suprapubic incision. The method of fragmentation will be considered under perineal lithotrity.

\section{COMPLICATIONS WHICH MAY ARISE SUBSEQUENTLY.}

Hæmorrhage.-As has been said, the patient should be watched for secondary hæmorrhage. If this appears, it may usually be stopped by the use of the cannula with an air-tampon, or by the chemise cannula, with the packing of the wound. I have seen one case in which a young 
man had several attacks of hæmorrhage which packing failed to stop, and which compelled a reopening of the wound and ligature of an artery believed to be that of the bulb.

If the bladder fills up with bloed-clot, it is an uncomfortable complication, and may prove serious. Irrigation should be tried, and hot solutions of pepsin, or other digestant, have been used to dissolve the clot. Opiates should be given to allay the spasm, and usually the escape of urine is sufficiently free to allow of waiting till the clot breaks up. If, however, the urine does not find its way out, and there seems to be danger of suppression, the clot may be broken up and evacuated. This may be accomplished with the litholapaxy-pump and a large tube introduced through the perineal wound. The bleeding usually comes from the deep wound, and this, therefore, should be again carefully packed after the blood is removed.

Usually hot water is a sufficiently good astringent, but, if more constricting action is required, alum may be added to it. If there is a tendency to a continuation of the hæmorrhage from the mucous membrane, ergot may be administered internally, to assist the contraction of the bladder wall and prostate, as well as of the small vessels.

Retention of Urine, if it occurs, should be relieved by the temporary introduction of a tube, and it should be remembered that provision for the free escape of urine is one safeguard against the occurrence of suppression. The treatment of this, if it occurs, should follow the lines laid down in the consideration of its possible appearance after lithotrity.

Calculi or Fragments left in the Bladder.-This accident is to be feared in cases of multiple calculi, especially in a sacculated bladder. If the first stone or stones remored are faceted, others are to be looked for. When a stone breaks in the grasp of the forceps, the fragments are often very hard to find and thoroughly remove, even with the intelligent use of the scoop and irrigation.

If a fragment or other calculus is left, its presence may be suspected if the irritation in the bladder does not subside. It may best be sought by the introduction of a litholapaxy-tube, and, if it is small, it may be quickly removed by the evacuator. If somewhat larger, it may be crushed and pumped out, or it may be seized and drawn out with forceps.

Phosphatic Incrustation of the Wound.-This occurs occasionally, appearing from the seventh to the fourteenth day. Thompson, who has met with this condition a number of times, advises hip-baths and irrigation with acidulated water. He says that sometimes the accumulation of phosphatic material is considerable in the deeper parts of the wound, and may require the use of the forceps for its removal. On one occasion he was obliged to crush a little calculus that formed at the bladder end of 
the wound. The curette may do good service in removing the calcareous matter and the granulations on which it is deposited.

\section{WOUND COMPLICATIONS.}

The lithotomy wound is naturally liable to various wound diseases, the more so as absolute asepsis is practically impossible. Erysipelas, diphtheria, pyæmia, and septicæmia are to be occasionally expected, and when occurring should be treated on general surgical principles. Inflammation arising in the wound may also extend to the parts about, and give rise to cellulitis, phlebitis, prostatitis, or cystitis. Occasionally even peritonitis may arise after perineal lithotomy, being caused either by perforation or injury of the bladder wall, or by wounding the posterior fold of the peritonæum, which sometimes comes low down over the prostate, and may be reached if the incision is extensive. More remotely, the inflammation may reach the kidneys, or the epididymis and testis. Extension in this last direction may be especially looked for if the seminal ducts or vesicles are injured.

Besides these more serious complications, the wound may heal slowly, the granulations may be sluggish or exuberant, and there may be a tendency to the formation of a fistula. When this is the case, the track may be cauterized with a hot iron, or with nitrate of silver. The importance of diverting the urine from such a sinus has already been alluded to in reference to the treatment of recto-urethral fistulæ.

It should also be borne in mind that the tendency to the formation of a perineal fistula is greatly increased by any narrowing of the anterior urethra which obstructs the flow of urine through it. Such a stricture, if it exists, should be dilated by the passage of sounds, or in such other way as seems best.

Injury to the Seminal Ducts or Vesicles.-Cases of impotence from this cause have been alluded to in the consideration of the choice of operation. This possibility should be borne in mind in guiding the incision through the prostate, which should be directed outward rather than downward. The loss of sexual power after the operation may appear either as impotence, or, what is more common, as sterility. Numerous instances of the latter are on record. In many of these the evidence is not good that the trouble is due to injury of the ducts; for when, as has happened in many of them, the fault is in the power of ejaculation at the time of intercourse, the seminal fluid dribbling away after the act is over, it seems probable that this failure in the act is partly, at least, due to injury of the ejaculatory apparatus, or of the erectile tissue in the prostate that directs the flow of semen.

There is little or nothing to be done in the way of treatment, although it would be fair to try the use of sounds and of mild currents of elec- 
tricity, in order to restore the tone of the muscular apparatus of the deep urethra.

Incontinence of Urine is fortunately of rare occurrence. Although both the sphincters of the bladder are divided in the operation, the healing is generally sufficiently complete to restore their function. When this is not the case the incontinence may depend partly upon general debility, and should be treated with tonics, among which strychnia should hold a place. The use of ergot, too, may strengthen the contraction of the voluntary fibers in the prostate. In addition to these, electricity may be of use. These measures failing, the patient should wear a urinal.

Stricture of the Urethra.-It has been claimed that this never results from the incision for stone, and it is probably true that a snug stricture is never so caused.

Since, however, Otis has called attention to strictures of larger caliber, and has shown that they may occasionally give rise to discomfort and inconvenience, there is need that this subject be again reviewed. It seems not improbable that slight narrowing of the membranous urethra would occasionally be found were old stone patients carefully examined with reference to this point. I have met with one case in which a man who had been cut many years before suffered from an intractable cystitis, and a slight narrowing of the membranous urethra was found.

The use of large sounds in this case showed that it was not a spasmodic stricture, but a dense, organic narrowing which could only be dilated by the use of considerable force, and which could never be brought quite up to the caliber of the rest of the canal.

\section{BILATERAL OPERATION.}

After the same preparation of the patient as for the median operation, the incision is made in a crescentic way across the perinæum, crossing the raphe at a point about an inch in front of the anus. It is carried

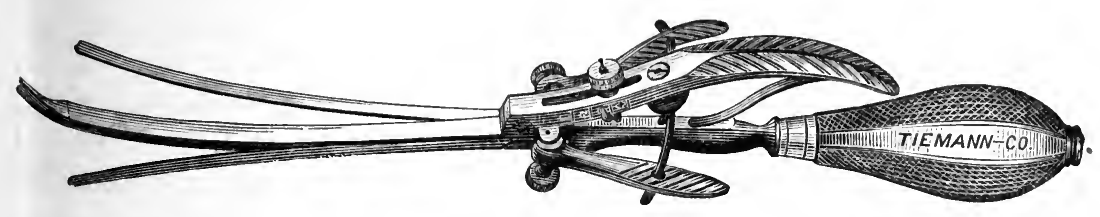

Fig. 73.-Dupuytren's double lithotome caché.

down until the bulb is exposed; this is drawn upward, and the urethra is opened in the membranous portion. The staff, which has a central groove, is here exposed, and along it Dupuytren's double concealed bistoury is slipped into the bladder. The staff is now withdrawn, and the blades of the bistoury being pressed ont sideways to a distance previously 
arranged for with a set screw, this instrument is withdrawn, cutting into the lateral lobes of the prostate. This withdrawal should be in a line somewhat directed downward, in order to have the blades traverse the thickest part of the gland. The finger is then introduced, and the stone is extracted just as in the lateral operation.

\section{MEDIAN OPERATION.}

In this operation a staff in the urethra with a wide groove on its under surface is used (Fig. 54). This may be reached either by an incision from the outside on the median line, rapidly dividing the tissues down to the urethra, or by a direct thrust the knife may be carried into the groove of the staff and the incision then made from the urethra outward, while withdrawing the blade.

If the latter method is intended, the left forefinger is introduced into the rectum with its palmar surface upward. With it the apex of the prostate is sought and the staff is felt entering it. A narrow, straight bistoury, with the cutting edge directed upward, is now entered in the middle line about half an inch in front of the anus, and carried by direct thrust into the groove of the staff. The bistoury employed has a short cutting edge on the back, and as it is pushed along the staff this cuts into the apex of the prostate. Now, while withdrawing the knife, the cut is made upward along the raphé. A straight grooved director is now introduced into the bladder, the staff is withdrawn, and along the director the finger is introduced.

If the fingers are not sufficient to produce enough dilatation to allow of the removal of the stone, more room may be gained by making incisions either directly downward into the prostate, which is the direction in which the median incision is made, or by lateral incision added to this, if more room is desired. Various methods have been employed to distend and enlarge the space obtained by the median incision. Forcible dilatation with special instruments has been practiced to some extent, but has little or no advantage over the use of the fingers, with the addition, if necessary, of a probe-pointed bistoury.

In the medio-bilateral operation the incision is to be made from with-

- out inward, until the membranous urethra is opened, and then through the groove of the staff the double lithotome caché of Dupuytren is to be introduced and the incision made through the lateral lobes of the prostate, exactly as in the bilateral operation.

Other methods of performing perineal lithotomy might be mentioned, such as the prerectal, the operation upon an angular staff of Buchanan, the operation upon the straight staff of Aston Key. These operations are, however, at the present time, practically out of date, and descriptions of them would be out of place in this account of modern procedures. 
They can be found fully described in Keyes's exhaustive article on Stone in the Bladder, published in Ashhurst's Encyclopædia of Surgery, and also in Thompson's Practical Lithotomy and Lithotrity.

\section{Perineal Lithotrity.}

In the performance of lithotomy it has always been a frequent occurrence to have the stone break or crumble in the forceps. The idea has therefore forced itself upon many operators, that by fragmentation much larger stones could be removed through the perinæum than would otherwise pass this route.

Many instruments have been devised to accomplish this end, some of which are here represented. While this method

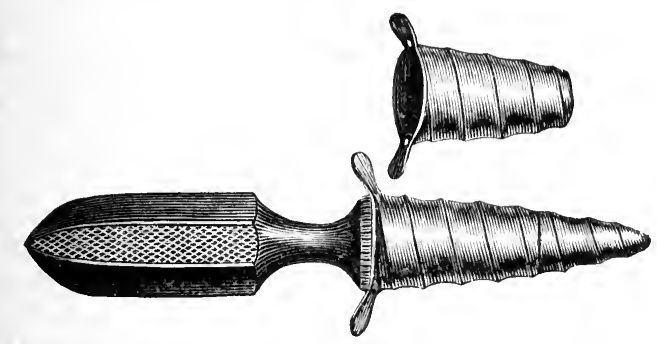

Fig. 75.-Dolbeau's shield to protect the walls of the wound during erushing.

has been used by many surgeons to supplement the ordinary operations for stone removal, and to help them out of a hard place when they were brought face to face with a large stone and insufficient room for its removal, the suggestion that perineal lithotrity should be made a regular procedure to be often resorted to in preference to other methods, has been made by a few operators.

Dolbean devoted much thought and ingenuity to the elaboration of this operation, and, with his ingenious dilator introduced through the median incision, he proposed to dilate

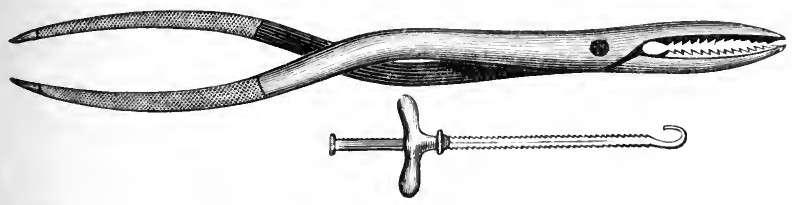

Fig. 76.-Dolbeau's lithoclast.

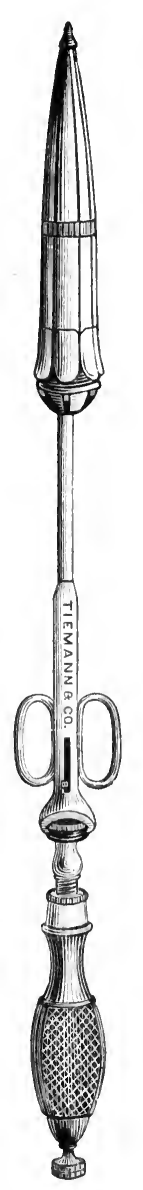

Fig. 74.-Dolbeau's dilator.

the neck of the bladder to a size sufficient for the introduction and operation of his powerful lithoclast. His endeavors, however, were never successful in establishing this operation on a firm basis. 
At the time when the treatment of stone was in its transitional state. in the seventh decade of this century, Harrison again brought forward the question of crushing stones through the perinæum, and urged its wide

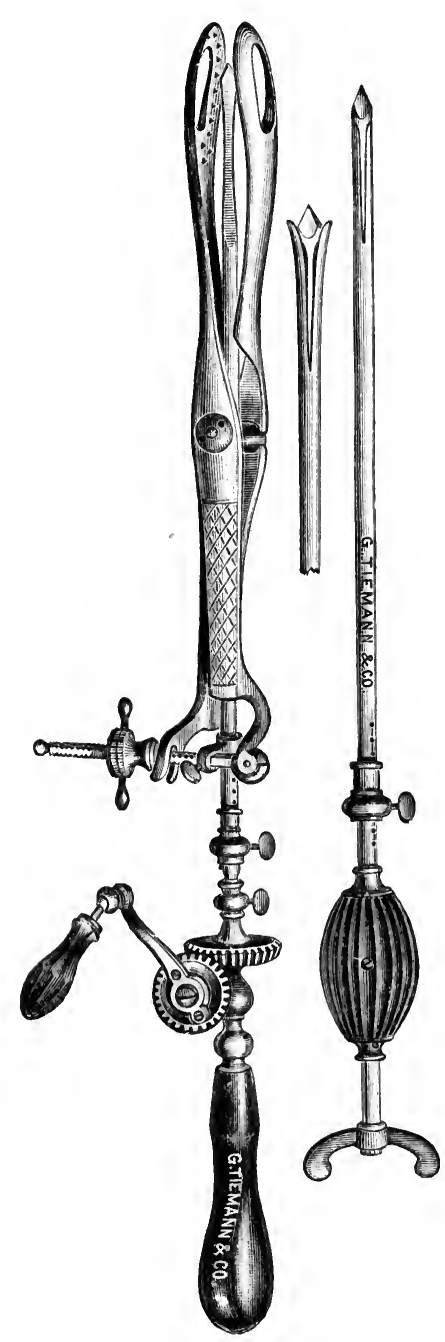

Fig. 77.-Rigal's stone perforator and crusher. adoption. Soon after, however, the appearance of litholapaxy, and the papers of Garson and Peterson on suprapubic technique, turned the attention of surgeons in other directions.

Now, again, a strong advocate for this operation has appeared in the person of Surgeon-Major J. R. Keith. His experience in stone operations has been gathered in India, where he has had a considerable experience. Allusion has already been made to his method of evacuation after crushing. Having met with several cases in which the stones were too large to be grasped by the ordinary lithotrites, owing to the separation being so great that the screw would no longer lock, he had a larger lithotrite constructed with a longer screw, with the purpose of using it through a perineal opening.

He has operated upon several cases in this way, simply by incising the membranous urethra, and conducting the whole operation through the thus shortened route. Occasionally he has met a stone so large that it can not be grasped even by this large lithotrite. In such cases he somewhat enlarges the incision in the perinæum, and dilates the membranous and prostatic urethra to a size through which he can easily introduce a pair of strong lithotomy-forceps. With these he seizes the stone, and while holding it firmly he introduces a chisel, fixes its edge against the stone, and then, with the blows of a hammer, breaks it. At the same time he forcibly closes the forceps, and thus brings out any fragments that remain between the jaws. This proceeding he repeats until he has brought the whole stone away. He says that in this way he on one occasion removed a stone weighing seventeen ounces. His reported 
results have certainly been very good, but his methods have not yet been tested by other operators. Whether they constitute an advance in the treatment of stone, time will show.

\section{Suprapubic Lithotomy.}

Turning now from perineal operations, we find that the only other portion of the bladder wall uncovered by the peritonæum through which an incision can be made lies in front of the prostate, and rises a short distance above the pubes in front. Many anatomical operations have been devised for entering this portion of the bladder. Attempts have been made to push the penis downward, and enter beneath the pubic arch. Other plans have contemplated a division of the pubes. These theoretical routes have had so little to recommend them that they have never been put to practical use.

The suprapubic incision, however, which enters the bladder just above the pubic arch, has lately become an operation of the greatest importance in the consideration of surgeons.

The recent advances in technique and in wound-treatment have, within the past decade, lifted this operation out of comparative obscurity into the position of being one of the surest and most satisfactory of surgical methods. This subject has been also treated in the article upon the removal of bladder tumors. To avoid repetition, therefore, as far as possible, the account which follows will leave out much of the history and many considerations which arise in connection with this operation, and will only emphasize those points which are particularly to be remembered in connection with the treatment of stone.

History and Development.-The first recorded instance of suprapubic lithotomy is that of Pierre Franco. In 1561 he cut a boy two years old for stone, and finding the calculus too large to remove through the perinæum, and urged by the importunities of the parents and the necessities of the case, he pressed the stone upward with the fingers in the rectum, and cut down upon it over the pubes. The child, though very sick, ultimately recovered. Franco was so impressed with the serious nature of this operation, and with the then common belief that wounds of the body of the bladder were almost necessarily fatal, that he strongly advised against a repetition of the procedure which had proved so successful in his hands.

In the year 1580 François Rousset perceived the importance of distention of the bladder to increase the space between the peritoneal fold and the pubes, and advised that the bladder be filled with barley-water, milk, or some mucilaginous fluid, the escape of which should be prevented by compression of the penis. When the bladder was small and contracted, he counseled efforts at gradual distention by diuretic drinks, coupled with 
efforts at forced retention of urine. He recommended that the operation should be done with the patient on his back and the pelvis raised, so that the force of gravity should draw away the intestines, and that the liquid in the bladder should gravitate to the fundus and draw it out of the pelvis. He advised that the incision should be in the median line between the recti and pyramidales muscles, and that while the peritoneal fold was retracted and protected by the forefinger a small opening should be made in the bladder, and afterward enlarged at need with a probe-pointed bistoury. We have in these recommendations of Rousset an outline of the best modern treatment. He saw and urged the advantage of the Trendelenburg position, with distention of the bladder, and his method of reaching the bladder is the one now employed. The completeness of his understanding of the situation is the more remarkable when it is known that he was not a surgeon, and never performed the operation that he so ably planned.

Suprapubic lithotomy did not obtain a firm foothold in surgical practice until the eighteenth century, when it was revived by Douglas (1719), Cheselden (1723), and Morand, but then only as an exceptional method.

Toward the end of the century Frère Come (Baseilhac) introduced the sonde-à-dard, with the aid of which he did many successful operations. His method of using this instrument was as follows:

When the median incision close above the pubes had been carried down to the bladder wall, the sound, with its concealed dart, was introduced into the bladder. The operator then put the forefinger and thumb of his left hand to the bottom of the wound, and with his right hand carried the beak of the sound with a seesaw movement along the anterior bladder wall, until its point came under the

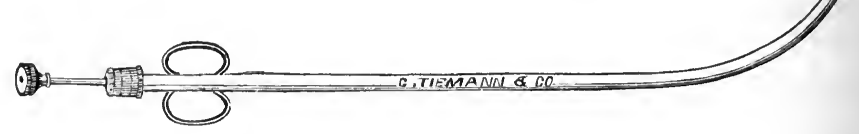

FIG. 78.-Sonde-à-dard.

middle of the wound. The handle of the sound was now depressed, and the point raised a nipple-like projection of the bladder wall, which was seized with the left finger and thumb. During this part of the operation the peritonæum was carefully pushed toward the umbilicus.

The sharp-pointed director was now pushed out of the sound, appeared through the bladder wall, and served as a guide to a bistoury, which made a small opening, through which the index-finger was at once slipped to hook and hold up the bladder, and to serve as a guide for further incision and for the manipulations of removing the stone.

Frère Come also practiced and recommended a perineal opening for purposes of drainage in patients cut over the pubes. 
For more than a century after this the operation remained practically at a standstill in the matter of technique; and in the estimation of sur-

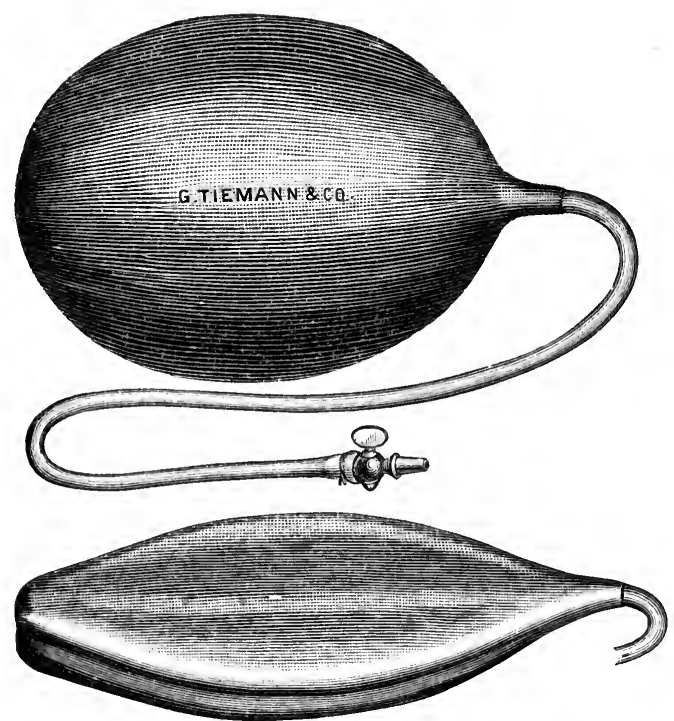

FIG. 83.-Rubber bag for distention of the rectum. geons it steadily declined in importance on account of its high mortality, and of the improved methods of treating large stones through the perineal opening with powerful crush. ing instruments.

In the Dictionnaire de Médecine, published in Paris in 1844 , is a fair summary of the feeling of the time. The advantages of the operation in the avoidance of hæmorrhage and of interference with the urethra and prostate are dwelt upon, as well as the fact that it is the only possible operation in cases of very large stones. On the other hand, the author draws a gloomy picture of the course and fatal effects of urinary infiltration, and closes by saying, "It is, then, owing to the danger of urinary infiltration, after this operation, that the great majority of surgeons have preferred, and still prefer, the narrow and embarrassed route that the perinæum presents to ordinary calculi."

This opinion of the operation, expressed in 1844, was not materially altered during the subsequent thirty years. The technique usually followed during this period was essentially that of Frère Come with the sonde-à-dard, although occasionally the operation was done after the manner recommended by Rousset over a full bladder.

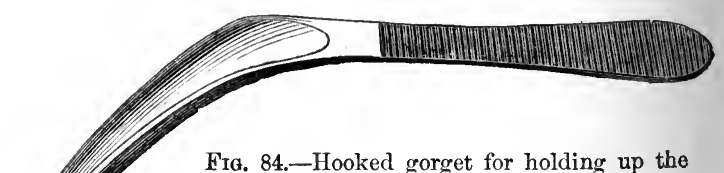
bladder while searching its interior.

In October, 1878, Garson, of Edinburgh, published some investigations made in the laboratory of Braune, of Leipsic, upon the changes of position of the pelvic organs when the rectum was distended by an in- 
flated rubber bag. The surgical importance of his observations was at once manifest, for he found that the bladder was lifted out of the pelvis to a considerable degree, and that the prevesical peritoneal fold was widely separated from the symphysis pubis, thus giving room for the performance of the suprapubic incision without fear of injury to the peritonæum. At about the same time Braune brought this matter to the attention of German surgeons, and in 1880 Petersen appeared with an article in Langenbeck's Archiv für klinische Chirurgie, recommending the procedure as a good one surgically, reporting two cases operated on in this way, and recounting a number of personal observations made on the cadaver.

This paper of Petersen's gave a great impetus to the operation, which since that time has continued to make its value more and more felt.

Anatomy of the Prevesical Space.-The peritonæum, as it is reflected from the anterior abdominal wall on to the top of the bladder, does not descend as low as the upper edge of the pubes. The loose, cellular space between this fold of the peritonæum, extending down between the abdominal wall and the posterior surface of the pubes in front, and the bladder wall behind, is known as the prevesical space, or the space of Retzius (cavum Retzii). The distance between the peritoneal fold and the upper edge of the pubes varies considerably in different individuals and at different ages. It is relatively greater in children, for in them the pelvis is small, and the bladder lies for the most part in the abdomen. This interval, too, varies greatly with different degrees of distention of the bladder; and in the Garson-Petersen method, the bladder being forced up and forward by the dilatation of a balloon in the rectum, the peritoneopubic interval is still further increased.

In some cases there is a distinct layer of fascia, connecting the peritonæum with the pubes (transversalis fascia, fascia propria of Velpeau), and if this.is well developed it may do much to frustrate attempts to artificially increase the distance between the two.

Preparatory Treatment.-If the bladder is much contracted, efforts may be made with warm injections to stretch it to a reasonable capacity; and if there is cystitis present with fermenting urine and mucus, an emollient and mildly antiseptic solution of borax or boracic acid may be used for this irrigation. On the day of the operation the bowels are to be thoroughly emptied by laxatives and enemata.

Description of the Operation.-The patient being fully etherized on a table of convenient height, an oval or pear-shaped rubber bag is introduced into the rectum. This is easily done before the bladder is filled. Before distending the rectal bag the bladder should be thoroughly irrigated and then filled with a borax solution.

The filling of the bladder should be done with extreme care, as rupt- 
ure of the viscus has occurred in several instances during this procedure. If a bulb syringe is used, the hand recognizes the amount of resistance, and can judge with considerable accuracy of the tension of the bladder. It is to be borne in mind that these bladders have usually been long diseased, and are not infrequently weakened by ulceration or sacculation. In most cases from six to ten ounces may be safely thrown in. To prevent subsequent leakage of water from the bladder an elastic band may be tied round the penis, but this had better be dispensed with if the bladder shows any tendency to spasmodic action. The rectal bag is now to be slowly filled with water, and here, too, great care should be taken against over-distention, for there is danger not only of rupture of the rectum, but also of so increasing the tension of the bladder as to cause a rupture there. During the filling of the rectal balloon the rise of the bladder into the abdomen may be watched by palpation and percussion.

Some operators prefer to dispense with this rectal distention altogether, and operate either withont special effort to increase the distance between the pubes and the peritonæum, or endeavor to accomplish this object by putting the patient in the Trendelenburg position, with the hips raised, so as to cause the abdominal contents to fall away from the pelvis, and to allow the bladder, drawing in the same direction by its weight, to separate the peritoneal fold from the pubes.

The incision is now made in the median line, extending two and a half to four inches upward from over the symphysis pubis. The length of the opening will be varied according to the thickness of the abdominal wall. The incision is carried down between the recti and pyramidales muscles into the prevesical space (cavum Retzii). In many cases the bladder wall is now at once in plain view, but in others a layer of cellular tissue, containing more or less fat, has to be separated. This should be done by dividing it directly down toward the bladder, and any dissection or separation of these loose tissues laterally should be carefully avoided, as stich handling of them opens up the way for infiltration of urine and septic absorption.

Sometimes veins of considerable size lie at the bottom of the prevesical space, and these, when seen, may be clamped and cut, or, if possible, pushed to one side. If they are injured and give rise to troublesome hæmorrhage, it should be borne in mind that this bleeding usually stops when the bladder wall is incised, much as the venous hæmorrhage in the neck, in the operation of tracheotomy, ceases when the trachea is opened; so that, if the bleeding points are not easily seen and caught up, the search should not be prolonged, but the bladder should be at once opened.

If the peritoneal fold is held down in close proximity to the pubes by the prolongation of delicate fascia which attaches it to the bone, it can be easily separated with the scalpel and drawn upward. 
The bladder, when uncovered, should be opened by a clean thrust of the knife. The left forefinger, which is during this part of the operation protecting, and, when necessary, holding up the prevesical fold of the peritonæum, should be at once slipped into the opening while it is distended with the escaping urine. The incision can then be enlarged according to need, either by stretching, or with a probe-pointed bistoury. The opening should be sufficiently large to allow the passage of the calculus without undue bruising of the edges of the incision. The finger that is in the bladder serves as a guide for the forceps, and also appreciates any tightness of the wound which needs to be relieved by incision.

The stone being removed, careful search should be made to see that there are no bits of calculous matter left, and to detect, if possible, the existence of sacculi, in which stone formation may have taken place. Any further operative measures, such as the removal of a tumor or a prominent prostatic lobe, may now be undertaken.

If the stone is contained in a diverticulum, care should be exercised not to unnecessarily lacerate the bladder wall in its removal. The opening should be dilated as far as possible by gentle force, and if the stone can not be removed through it, it is perhaps better to crush it in situ and remove it piecemeal, than to run the risk of urinary infiltration, which would attend any incision into the bladder wall. Such incisions have, however, been used with success, and Bryson speaks of laying open these pockets in the hope of obliterating them. This was not accomplished in his case, though the patient did well. When diverticuli are present, it is to be borne in mind that these are sometimes symmetrically arranged, a knowledge of which fact may lead to the detection of stones in ponches so small that they would otherwise be overlooked.

The After-Treatment of the Suprapubic Wound.-The first important question we have to decide in the matter of the after-treatment is, whether to apply sutures to the bladder wound or not. There is considerable difference of feeling among operators as to the wisdom of doing so. Statistics are too meager to prove either plan decidedly superior, but, as far as they go, they show that patients have done better when the bladder wound was not closed, but was used for drainage. The added danger in the cases where the wound has been closed seems to come from the increased chance of infiltration of urine and septic infection, when the bladder leaks after the outer part of the wound has partly united.

The failure to unite has no doubt been due, in many instances, to the fact that improper cases were chosen for closure. It is evident that when the bladder wall is much inflamed, especially in old and broken-down subjects, the chances of healing by first intention are very small, and the dangers of urinary infiltration are proportionately large. Only those cases, 
then, should be selected for suture in which the bladder is tolerably free from inflammation.

Further, if there is much hæmorrhage into the bladder which can not be checked at the end of the operation, this is to be looked upon as a contra-indication to the closure of the bladder wound by sutures. For it is difficult in such cases to conduct off the urine, owing to the stoppage of the tubes with clots; and when these are clogged, the efforts of the bladder to free itself soon reopen the wound.

In spite of these drawbacks in the use of sutures of the bladder, the quick recoveries that are obtained in those cases in which the suture does hold are so striking that operators are constantly endeavoring, by improvement in technique, to make first intention of the suprapubic wound a comparatively sure thing, and many are so sanguine as to believe that this effort will finally be successful, and will place the suprapubic operation easily at the head of the procedures for the treatment of the interior of the bladder. Many forms of the continued and interrupted suture have been used in these wounds, but perhaps the most useful has been the interrupted Lembert suture, which is easily applied, and has the advantage of bringing broad surfaces together.

In applying a suture, it is a good plan first to fix a thread at each end of the incision, by which the wound may be steadily held in view during the application of the intermediate stitches. Some operators have advised that the outer surface of the bladder wall be dissected off for a short distance around the incision, in order to prepare a fresh, raw surface which shall readily unite when drawn together by sutures. Antal suggests that an oval surface be thus denuded before the cavity of the bladder is opened, and that the incision be then made in the middle of this refreshed area. Whatever plan is used, great care should be taken that none of the stitches penetrate the cavity of the organ, for not only would they favor escape of urine and thus make first intention almost impossible, but they would also be likely to serve as a nucleus around which calcareous deposits would form.

When the bladder wound is sutured, provision has to be made for the escape of urine, either by passing a catheter through the urethra and securing it there, or by putting a tube out through the perinæum. The latter plan affords the best drainage, but involves an additional operation. Keyes has sought to reduce this operation to a minimum by the following procedure: He passes a sound from the bladder into the membranous urethra, and, making its point prominent in the perinæum, cuts down upon it by a very small incision. Through the opening thus made a probe is passed up into the bladder, which draws after itself a thread, which in turn pulls in the catheter.

The other plan for the treatment of these wounds, and the one which 
thus far has had the most success, is that of keeping the wound open and draining the bladder through it. It is well to use two tubes for this, and their ends should be carried down to the bottom of the bladder when it is collapsed in the pelvis, after the rectal bag has been removed. The use of the two tubes is a safeguard against the escape of urine being stopped by clots, and they are useful for irrigation. They should be secured to the skin, and their ends should be left long enough to dip down into a bottle between the thighs; or, if preferred, they may have attached to them a longer piece of tubing, which hangs down over the side of the bed and acts as a siphon to draw the urine out of the bladder. Some operators prefer to keep their patients on the side or belly, to favor drainage, but this is usually not necessary

After thorough irrigation, the wound should be dressed antiseptically, with iodoform gauze covering it closely around the tubes, and an absorbent dressing outside of this. For the first few days the bladder may be irrigated with a solution of boracic acid several times in the twenty-four hours. After a few days one tube may be left out and a catheter tied in. When this is working satisfactorily, and after the wound has contracted pretty well, the remaining tube may be removed, and the suprapubic wound soon closes.

Accidents and Complications in Suprapubic Lithotomy.Rupture of the Bladder.-The over-zealous distention of the bladder has led to rupture in a number of instances. Although this accident is most apt to occur in the weakened, sacculated bladders of old men, still it has happened in some instances in children. It is to be borne in mind that the pressure in the bladder is very considerably increased as the rectal bag is filled, and as much care should be exercised in the distention of this as in that of the bladder.

Especially is this care of importance, in view of the fact that the rectum itself has been ruptured not a few times by over-distention, and fatal results have followed. The knowledge that these accidents may happen should be a sufficient safeguard against their occurrence in skillful hands, except in conditions of weakness which could not be suspected.

Septic Infiltration.-This is a complication that may arise in the wound. The precautions that should be taken against it should comprise, first, the preliminary purification of the urine by internal medication and by irrigation; secondly, the thorough observance of antiseptic methods during operation; and, lastly, the adequate provision for drainage subsequently. The loose, cellular spaces about the neck of the bladder should be disturbed as little as possible during the operation, for pockets are easily created in it by too much handling, and the way is thus prepared for penetration of urine into the deep parts. 


\section{STONE IN THE FEMALE BLADDER.}

A stone forms in the female bladder far more rarely than in the male. This is due in part to the anatomical arrangement of the parts. Through the soft and distensible female urethra small renal calculi which reach the bladder, and sand and gravel which form there, are quickly and easily passed. Moreover, the rarity of stricture in the female urethra, and the absence of any prostate to cause obstruction by enlargement, make the occurrence of retained (residual) urine very infrequent; and this fruitful canse of fermentation and consequent formation of secondary stones in the male is therefore not so active in the female. On the other hand, women are subject to hysteria, and the short urethra is favorable to the introduction of foreign bodies about which calculi may form.

After operation upon a vesico-vaginal fistula a stone may form either as an incrustation upon granulations projecting into the bladder at points where the mucous membrane has healed imperfectly, or a wire stitch may serve as a nucleus. If a stitch cuts through one margin of the wound and remains attached to the other, its end may fall within the bladder and the opening may close outside of it, leaving it projecting into the resical eavity. I have met with one such case in which two stitches thus escaped skillful operators, and in which a tendency to stone formation persisted until they were discovered and removed.

Instances are reported in which large stones have by ulceration gradually worked their way through the vaginal septum and have thus escaped; in other cases they have gradually worked their way out along the urethra by a process of combined dilatation and ulceration, and have thus finally been expelled. Stones thus voided have reached a weight of from two to twelve ounces. Keyes gives an account of many such. In most of the cases incontinence has followed, but Agnew reports a case of spontaneous expulsion of a stone through the vagina, in which, though the calculus was as large as a goose-egg, no incontinence remained; and in another case, mentioned by Keyes, a stone having a circumference of five inches was passed by a woman of eighty years, and only temporary incontinence followed.

Instances are recorded in which a stone has proved a serious obstacle to labor, and in which both mother and child have been lost through this complication.

The symptoms of stone in the female do not differ essentially from those observed in the male, although an appreciation of their significance may be interfered with by the coincidence of uterine or other pelvic disorders. In the case above alluded to, in which stones formed on wires projecting into the bladder, it was noticeable that as long as the stone remained attached to the wire in the vesical wall it did not give rise to 
sharacteristic symptoms; but, the moment it became detached, all the symptoms were at once present in their intensity.

The physical examination for a stone in the female bladder is an easy one. If large, it may be made out by vaginal touch, and with a finger in the vagina and a sound in the bladder, all parts of the vesical cavity may be thoroughly searched.

Operative Treatment. - The only methods of removal of stone from the female bladder that meet with modern approval are: 1. Dilatation of the urethra. 2. Litholapaxy. 3. Vaginal lithotomy. 4. Suprapubic lithotomy.

Dilatation of the Urethra.-This method is applicable to the treatment of small stones. Bryant states that stones up to two inches in circumference may be thus removed without causing incontinence; and Keyes places practically the same limit when he says that he has dilated up to a diameter of three quarters of an inch.

If the urethra is dilated further than this, the fibers of the sphincter are apt to be ruptured and incontinence to result, so that for stones which, when in the grasp of the forceps, have a diameter greater than three fourths of an inch, it is best to choose some other method.

The dilatation may be carried out first with large sounds or nterine dilating stems, and then with the fingers, if they are sufficiently slender. Keyes has found the dilator of Dolbeau a convenient instrument. Having attained a sufficient degree of dilatation, a slender pair of forceps may be introduced and the stone seized and removed.

Litholapaxy.-This operation is easily performed in woman, and is to be chosen for the great majority of stones. Even those small enough for removal through the dilated urethra may best be treated by crushing, which causes even less injury of the parts.

In using the lithotrite it is usually convenient to have the patient in the lithotomy position. The contour of the bladder is now to be remembered, for in woman the presence of the uterus behind the bladder makes the posterior wall of the latter irregular and flatter than in man, so that the fragments lying upon it do not naturally gravitate to the center, as in the male bladder.

Difficulty in seizing the fragments will then be met, if the handle of the lithotrite is held at an angle of forty-five degrees with the horizon. To obviate this, the instrument should be held almost vertical. The blades then rest on the yielding vaginal wall, close to the urethral opening, and depressing this they make it the most dependent part of the bladder, into which fragments gravitate and where they may be readily seized. Occasionally, in cases of difficulty, the fingers may be used to raise the vaginal septum, and thus to bring the stone into reach.

For the evacuation of the débris, a short, straight tube should be 
used, and if the urethra is distensible, one of good caliber may be chosen. If there is reason to believe that a stitch remaining from a vesico-vaginal operation is the cause of the stone, a careful search should be made along the line of the cicatrix. This may well be done with the forceps or sound in the bladder and the finger in the vagina. In the case alluded to, one stitch was felt and removed through the vagina, and the other, which could not be felt, was finally, after a long search, caught with the forceps in the bladder and twisted away from its attachment. The granulations which may exist along the vesico-vaginal scar should be thoroughly curetted away.

Litholapaxy may seem unsuitable if the stone is too large or hard to crush, or if it is too tightly grasped by the bladder to allow of the manipulation of instruments. A foreign body too large to come through the urethra, and resistant to crushing, may require lithotomy ; and an encysted stone which can not be dislodged with the lithotrite must be reached by cutting. Occasionally other conditions may coexist, such as a tumor or an inveterate cystitis, which are treated by an opening into the bladder, and the removal of the stone is incidental to this operation.

Vaginal Lithotomy. - This is a comparatively safe operation, and is suitable for any of the conditions enumerated as not proper for litholapaxy. It is so simple an operation, and so much in the line of gynæcological work, that it is frequently chosen by gynæcologists into whose hands cases of stone have fallen, even when litholapaxy might be applied. The possibility of a persistent fistula, or of the subsequent formation of stone on stitches or granulations, as well as the greater severity of the operation and the length of time required for convalescence, should all be considered before choosing this operation needlessly.

The operation may be done with the patient on the back, or in Sims's position. The vagina is held suitably open with specula; a sound is introduced into the bladder, and the tip is made prominent against the vesico-vaginal septum just within the bladder. The wall of the vagina is then steadied with tenacula or forceps, and an incision is made directly down on the tip of the sound. This opening may be enlarged by scissors and by stretching till it is large enough for the removal of the stone. If the stone is very large, it may be crushed in situ and removed piecemeal.

The wound in the bladder may then be at once closed by stitches, or it may be left to heal spontaneously, any fistulæ which remain being afterward closed by operation. If this plan is followed, the vagina and bladder must be constantly washed out, to prevent phosphatic incrustation of the wound.

The tendency to spontaneous healing is so great in some cases that, where it is desired to keep up bladder drainage for a time, Emmet advises attaching the mucous membrane of the bladder and vagina together 
around the incision, thus making the opening permanent until it is thought wise to close it by operation.

Suprapubic Lithotomy.-Rarely it may happen that a stone is too large for vaginal lithotomy and too hard for crushing. In such a case a suprapubic incision may be chosen.

A colpeurynter in the vagina might be of assistance in this operation, but I am not aware of any instance of its employment. The technique is the same as in the operation on the male bladder.

\section{PROSTATIC CALCULI.}

The prostatic urethra at times contains concretions which, originating in the kidneys or bladder, have lodged in the prostatic sinus. These will be considered under the head of "Stone in the Urethra." Besides these, there are to be found in the prostate stones which have their origin in the gland, and which are wholly distinct from the other urinary calculi.

The so-called corpora amylacea are almost constantly present in the glands and ducts of the prostate. In a series of one hundred prostates examined by Thompson, these bodies were found in every instance. In the younger prostates they were of microscopic size, while in older organs they were almost always visible to the naked eye. These are quite different from the bodies of the same name that are found in the brain. They are yellow, brown, or black in color, and are apparently formed by the inspissation of the prostatic secretion. They are usually so small as to give rise to no symptoms, but occasionally they have lime salts deposited in them, and may then grow very considerably in size and give rise to much trouble. Thompson regards the deposition of lime salts in them as evidence that they are felt by the surrounding tissues as foreign bodies, and, by the irritation that they cause, lead to the precipitation of lime about them.

They exist either in an isolated form, each calculus occupying a little pocket of its own in the gland, or, not uncommonly, several concretions may lie together in a single pouch. In this case they are closely applied to each other, and fit together with corresponding facets. Sometimes they coalesce to form composite stones. In this way large calculi, result, and they may even project back into the bladder.

The salts of which these prostatic calculi are made up are the phosphate and carbonate of lime. They are very hard, and have a smooth, polished surface like porcelain, which distinguishes them from the rougher urinary calculi. When they lie in pockets connecting with the urethra, so that the urine has ready access to them, they may become coated with 
other urinary salts. In this way mixed calculi result, which may attain great size. Thompson cites four instances in which a large number of such calculi were removed from pouches which they had formed for

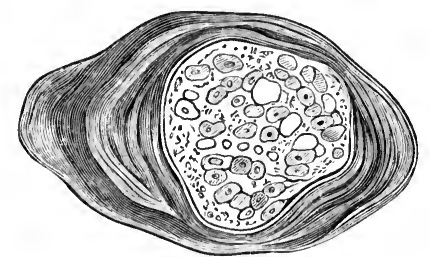

FIG. 85.-Prostatic calculus. Showing the granular, nucleated centre surrounded by laminated layers. themselves in the prostate and surrounding tissues. They sometimes by irritation give rise to suppuration, leading to an abscess in the prostate which may open into the urethra or may perforate the gland in some other direction; the pus may appear in the perinæum, or may break into the rectum or bladder.

The symptoms arising from them are those of prostatic irritation, and if they press or encroach upon the prostatic urethra they may lead to obstructive symptoms such as are present in enlarged prostate.

Diagnosis. - It is extremely difficult to determine the existence of one of these calculi in the prostate, unless some portion of it so projects into the prostatic urethra that it can be detected with the sound. Occasionally their presence may be suspected when, by the rectal touch, very hard bodies are felt in the substance of the gland, especially if these are observed early in life, before hard, fibrous growths are likely to appear in the prostate. If abscesses have already formed about the stone, and have broken or been opened externally, a search with a probe through the fistulous openings that are left may lead to the discovery of calcareous matter.

Treatment. - The only treatment of these cases is either by palliation or operation. The stone should always be removed if the patient is in fit condition for surgical interference.

It may rarely happen that the stone projects so far into the prostatic urethra that it can be pushed back into the bladder and there crushed. Usually, however, the stone is so inclosed in the prostate that it can not be dislodged or reached through the urethra, and can therefore only be removed by incision.

The median operation is the suitable one for these cases, for it affords a direct route to the stone, and usually gives sufficient room for its removal. If the stone is too large for removal through the median opening, a lateral or bilateral incision may be used. If the stone lies wholly in a pocket in the prostate, the bladder will not be entered by the operation. It should always, however, be explored, to see whether it contains a urinary calculus as well. 


\section{STONE IN THE URETHRA.}

It is a rare occurrence to have a stone originate in the urethra. Occasionally calcareous salts (phosphates and carbonates) are deposited on ulcerated patches in the urethra posterior to a stricture, and these may accumulate till they assume the proportions of a stone. In the same way stones may form in pockets, glandular or otherwise, communicating with the urethra, in which the urine stagnates.

Usually, however, stones which are found in the urethra have been formed either in the kidneys or bladder, and have been arrested in the urethra during their passage outward. This arrest takes place either at a point of physiological narrowing, or behind a stricture which has resulted from previous urethral disease or injury.

The prostatic sinus, the membranous urethra just behind the opening through the anterior layer of the deep perineal fascia (triangular ligament), and the fossa navicularis are the more common points where stones lodge. At the peno-scrotal angle is also a slight narrowing that may catch a stone, which has successfully passed to this point.

If the stone completely fills the urethra, it may cause a stoppage of water; and this forced retention may lead to great distention of the bladder. This course of things is favored by the tendency of the urethral walls to close spasmodically upon any foreign body that acts as an irritation to them. After the overfilling of the bladder and the urethra behind the stone becomes extreme, the urine usually finds its way past the stone, and a constant dribbling is the result of this overflow. Keyes has recorded such a case.

When the stone does not completely fill the urethra, or if it lies in a pocket to one side of the canal, it may be carried for a term of years without leading to any serious condition of things. Under less favorable circumstances it causes ulceration, and may lead to an abseess, or even work its way out and be discharged externally.

Sometimes several stones are found in the mrethra at one time. In the Warren Museum of the Harvard Medical School is a set of calculi, four in number, removed from the urethra by Dr. J. T. Boutelle, of Hampton, Va. The patient was a negro, and had had discharging fistula in the perinæum for ten years. They are of a glossy surface, of porcelain-like hardness, and articulate one with another by ball-and-socket joints in a very beautiful manner. Several stones may exist together in pockets alongside of the urethra. They are then faceted, much like those which form in a similar way in prostatic pouches.

Symptoms. - The symptoms caused by urethral calculi vary greatly. If a renal or vesical stone enters and is stopped in the urethra, it produces a sudden painful stoppage of the urine that is very characteristic. On the 
other hand, slowly forming stones make their presence known by the irritation and partial obstruction that they cause.

If they are situated in the anterior part of the canal, they do not usually affect the frequency of urination, and may be accompanied by but little pain. When, however, the stone lies in the prostatic urethra, particularly if portions extend back into the bladder, the tenesmus and pain may exactly resemble what is seen in stone in the bladder.

Diagnosis. - This can usually be made sure by a physical examination. All parts of the urethra are accessible to the finger, either from the outside or through the rectum. The stone is felt as a hard body somewhere in the course of the canal, and a sound passed down to it shows it to be calcareous. When it has ulcerated its way into a side pocket, there may be difficulty in bringing the sound into contact with it, even by combined manipulation with a hand on the outside. If it lies in an abscess which has opened externally, a probe will usually touch the stone through one of the fistulous openings.

Treatment. - An attempt may be made to wash the calculus along by an over-forcible expulsion of urine. Keyes suggests that under the relaxation of an opiate and a warm bath the stream be stopped suddenly during urination by compression of the meatus, and says that in this way the anterior urethra, being dilated, may provide room for the passage of the stone. When this manœuvre does not succeed, resort should be had to surgical interference.

A small stone or fragment, recently arrested in the anterior portion of the urethra, may perhaps be worked forward and moved along the

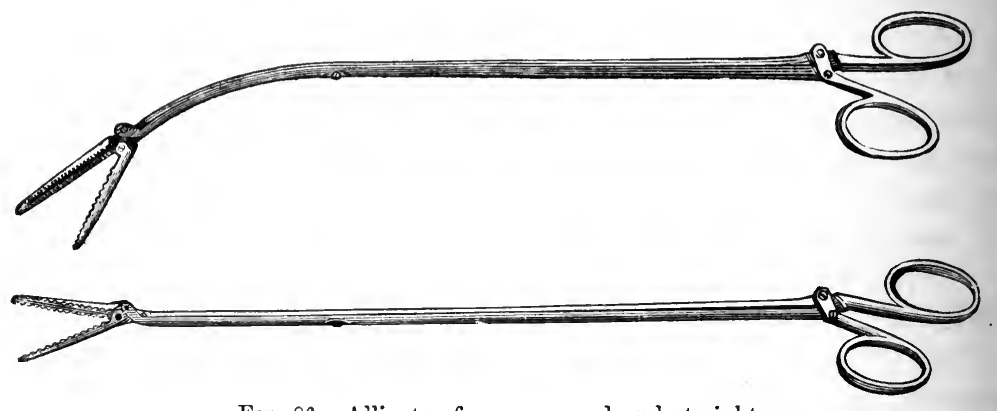

FIt. 86.-Alligator foreeps, curved and straight.

canal by manipulation. It sometimes crumbles under the fingers, and is then washed out with the next passage of urine. If it is not thus readily dislodged, it must be removed either by instruments passed along the canal or by incision.

A sound passed down to the calculus, and then hastily removed while the patient passes urine, may so distend the passage in front of the cal- 
culus as to allow of its being driven out. If this fails, attempts may be made to seize and draw it out.

The instruments to be used along the urethra are the alligator forceps or the urethral scoop. The stone should be held steady with the fingers outside, while trying to grasp it with the forceps, or when passing an instrument, designed to act as a scoop, beyond it.

Keyes describes an ingenious use of Thompson's urethral dilator in accomplishing the removal of a calculus from the urethra. The slender

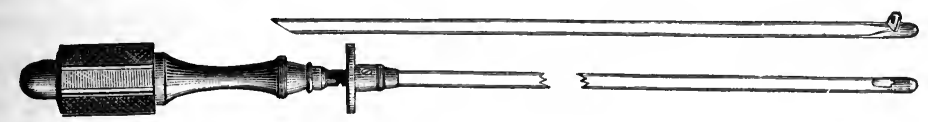

Fia. 87.--Leroy's urethral scoop.

shaft of the instrument is split and may be separated by screw force. When this is carried down past the calculus and then forced open, the stone falls between the two parts of the shaft, and in closing them again
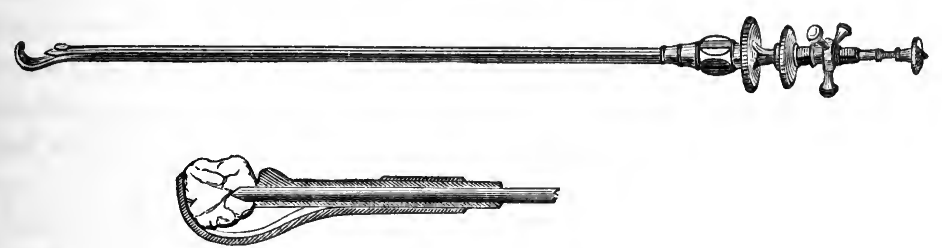

Fra. 88.--Reliquet's urethral stone-crusher.

it is firmly held, and may be withdrawn. He gives the credit of the first use of this expedient to Dr. Westmoreland, of Georgia, although he was himself not aware of its previous use when he first employed it.

When a stone is lodged in the urethra posterior to the constrictor muscle, it may be possible to push it back into the bladder, where it can be crushed. A large sound may be used for this purpose, or an evacuating tnbe. The use of this has been described. (See Litholapaxy.)

When a stone is so fixed in the urethra that it can neither be drawn out nor pushed back, or when it is located in a pocket to one side of the urethra, it must be cut down npon and removed. Incisions made for this purpose usually heal well. It is better practice, I think, to remove them thus than to try to crush them in situ. Urethral lithotrites work in too confined a space, and are apt to lacerate the surrounding parts. 


\section{STONE IN THE URETER.}

The stones which lodge in the ureter and lead to trouble there have their origin in the calyces and pelvis of the kidney, and are carried down with the stream of urine. A stone never forms in the ureter unless it has a foreign body for a nucleus, but concretions may increase in size while arrested there. Usually renal calculi of small size and smooth surface, when they once get started along the ureter, find their way down into the bladder. Occasionally, however, when of large size or of a prickly, irregular surface, they are caught in the canal and remain there for a longer or shorter time. Even comparatively small stones, if of sharply angular shape, may become thus fixed. For not only do the sharp projections catch in the mucous membrane and thus hinder their progression, but by their irritation they may, as M. Le Dentu suspects, excite the ureter to a contraction that holds them fixed. It is also probably true that their irregular shape prevents their so closely stopping the ureter that the urine collects behind them. This would render inoperative one of the most efficient agencies by which their progress downward is effected. For it is the gradually accumulating pressure of the retained urine that forces the concretion along the canal, and, if the urine constantly leaks past the stone, the accumulation behind it is impossible.

A calculus may be arrested at any part of the ureter, but there are certain points where this arrest is likely to take place. First, at the entrance of the pelvis into the ureter, there is a narrow place, and a calculus too large to enter is apt to become impacted here. Also, about two inches below this there is a point at which calculi are prone to lodge. The writer has met with one instance of this sort.

Farther down, just below the brim of the pelvis, there is often a slight narrowing; and, lastly, as the ureter perforates the bladder wall it penetrates first the muscular coat, then running for from one half to three quarters of an inch between that and the mucosa, it finally opens through the mucous membrane on a little nipple-like projection into the cavity of the bladder. Just in this portion of the canal calculi frequently become fixed. Numerous instances are reported in which they have been removed from this point.

Occasionally the ureter contains more than one stone; for, as it is not unusual to see several calculi in the kidney, it will sometimes happen that one stone working its way down and dilating the ureter prepares a way which others easily follow. It also sometimes occurs in calculous patients that both ureters will contain stones at the same time.

When a calculus becomes fixed in the ureter it produces effects both general and local. Its general effects are brought about by its me- 
chanical obstruction to the flow of urine, and also apparently in some cases through the reflex effects of the irritation. Thus it has happened that a calculus lodged in one ureter has led to complete suppression of urine, owing, first, to the stoppage of one kidney; and, secondly, to the inhibitory effect produced upon the other. If the kidney whose ureter is stopped has a partial outlet for the urine, either intermittently or constantly, by the leakage past the stone, its function is usually not destroyed, althongh much interfered with. In such a case we may have a simple hydronephrosis, or an inflammatory process may establish itself in the kidney, and this may either take the form of a pyelitis, and lead to a pyonephrosis with perhaps a pyelonephritis, or the kidney substance may be mainly affected, and we then have a parenchymatous or interstitial nephritis.

When, however, the stone produces a complete stoppage, we have an effect much like that which follows the ligature of the ureter, and with the greater or less formation of a hydronephrosis the secreting portion of the organ is gradually destroyed. The writer has met. with such a case, in which a kidney that had been subject to attacks of renal colic from the passage of stones for many years, had its ureter finally completely blocked by a large calculus, and in consequence lost its secreting power, so that when the stone was removed urine no longer came from that side. Usually, under these circumstances, the remaining kidney becomes hypertrophied and performs the functions of both. It is a not uncommon circumstance, however, in the existence of a calculons diathesis to finally have the ureter of the remaining kidney suddenly blocked, and complete suppression result.

The pain which a stone causes is also to be taken into account among its serious general effects. This is sometimes so severe and prolonged as to gradually wear a patient out with nervous exhaustion and loss of sleep. Unider these circumstances, a long-delayed operation will have less chance of success than one undertaken while the patient's forces are well preserved.

Besides these general changes which lead to a partial disabling of the urinary excretory organs, a stone lodged in the ureter produces also serious local lesions which may gravely affect life. When a stone thus becomes fixed the first effect is a stretching of the ureteral walls, making them thinner; and if at the same time the irritation of the stone sets up an inflammation, they may be perforated, and the escape of urine and even of the stone into the parts about may lead to the formation of an abscess.

If, however, the pressure and irritation of the stone do not lead to perforation, the walls of the ureter gradually become thickened by the formation of fibrous tissue in and around them. At the same time the 
ureter behind them is subjected to an increase of pressure proportionate to the degree of completeness with which the calculus stops the flow of urine. The canal is dilated, and if this condition of things persists, the walls gradually become thickened. Especially is this the case when a suppurative inflammation of them is set up.

Not uncommonly a stone, after lodging for a time, moves on before the accumulating pressure behind it, and, again lodging, sets up the inflammatory changes of the wall at this new place. Thus a ureter through which many calculi have passed may be much dilated and thickened through its whole extent, with here and there points of comparative narrowing where more active irritation has set up a peri-ureteritis and consequent stricture.

The abscesses which occur in connection with impacted stones may, as has been said, appear soon after the impaction of the stone, but usually do not form till late, after the wall of the ureter has been weakened by a slow process of ulceration. The pus may find its way in several directions. Spreading in the loose connective tissue behind the peritonæum, it may run down into the pelvis about the bladder, or it may find its way upward and even penetrate into the pleural cavity, or open into a bronchus. Posteriorly it may perforate the muscles and point in the back, or anteriorly it may open into the colon-a coil of small intestine-or even into the general peritoneal cavity.

Symptomatology.-The pain caused by the impaction of a stone in the ureter is the most characteristic symptom of the condition. Coming as a sudden attack of nephritic colic, it is felt in the side and back, or may be referred to the abdomen. Of a severe, lancinating character, it shoots downward toward the bladder, or into the scrotum and thigh. If the calculus enters the ureter and works down, the pain may sometimes be felt to shift downward, but this is not always the case, as the pain in the kidney often remains severe as long as the ureter is blocked. The pain is usually associated with a frequent desire to urinate.

When the stone becomes fixed at some point, the pain may gradually abate in severity, and may take on an intermittent character, periods of comparative comfort being interrupted by attacks of pain, recalling that of the first onset in their severity.

Nausea and vomiting are the usual accompaniments of the severe attacks. An examination of the urine will commonly reveal the presence of blood and albumen, and later pus-cells are also present. If the stone wholly occludes the ureter, so that no urine comes from the affected side, these evidences may be absent, and the urine will then appear quite normal in character but scanty in amount.

As has been said, the stoppage of the ureter is at times partial or intermittent. It is well, therefore, to save the urine from each micturition in 
a separate glass. A comparison of all the specimens thus collected through the day will often show great variations in quality, some of them being quite clear, while others are clouded by the presence of blood and pus. Even when all of them are clondy there may be considerable differences in the amount of sediment in the different glasses.

The quantity of the urine in twenty-four hours should always be carefully watched, and the search for casts and albumen should be frequently made. There is usually but slight fever at first in these cases of impacted stone; but if inflammation is set up either in the kidney or in the parts about the stone, the temperature becomes elevated. The formation of an abscess gives the daily fluctuation of temperature characteristic of suppuration.

Diagnosis. - The diagnosis of a stone in the ureter is not always an easy one to make; for, while the pain of renal colic is usually very characteristic, it sometimes lacks salient features and is not easily recognized. Moreover, when the pain is obviously caused by an obstruction or irritation in the ureter, it is not even then always easy to determine that the cause is a calculus, for the passage of sand or of clots of blood or mucus through the canal, the pressure of growths from without, or the existence of a stricture in its walls, may give rise to an almost similar train of symptoms, and the differential diagnosis can only be made by a painstaking study of each case, and is then not always feasible. The possibility of confusing these cases with some obstructive lesion of the bowels should not be lost sight of. If the pain is most felt in the abdomen, the accompanying vomiting, and the constipation which almost certainly follows the exhibition of opiates, combine to form a very misleading picture, and the careful examination of the urine must be relied on to prevent a mistake. For purposes of treatment it is also important, after the presence of a calculus is assured, to make out, if possible, the exact seat of the concretion, and for this we must rely on a very careful exploration.

Physical Examination.--Unfortunately, the greater part of the ureter lies so deeply that it can not easily be explored by the touch. The lower part of the canal can be reached through the vagina in woman, and by the rectum in the male, although even this is impossible in the case of a fat man with a thick perinæum. Palpation through the vagina is not difficult, and the stone can usually be recognized in the last two or three inches of the ureter. The portion of the male ureter that can be reached through the rectum is much less considerable than this, probably not often more than the last three quarters of an inch, that lies in the wall of the bladder, being accessible.

Palpation through the abdomen may be snccessful in a thin subject, especially if the stone is a large one. In a fat patient, or with a small 
stone, this search would probably be fruitless. In case the stone has so blocked the ureter that a hydro- or pyonephrosis has formed behind it, the upper dilated portion of the ureter may sometimes be felt below the enlarged kidney, and the site of the stone would naturally be at the lowermost point of this swelling.

Even when nothing can be felt by the examiner the probable seat of the stone may sometimes be determined by the patient's sensations to palpation. A constant tender spot over one part of the canal is always suggestive of the seat of the stone: The pain at such a point is usually of a pricking character, and is especially to be elicited by a sharp push of the fingers. When a stone is lodged in the upper part of the ureter the tender point may also be sought in the lumbar region, and the writer has succeeded in removing a stone from this portion of the canal which was located by such a point of tenderness in the back. When the indications do not point with sufficient distinctness to any one spot, and the condition of things is so serious that some interference is demanded, the position of the stone may be sought by an abdominal incision. With the hand in the abdomen, the whole course of the ureter can be easily palpated, and the stone thus discovered. Recently it has happened to two operators * to successfully locate a stone in this manner, and in each case to remove it with good results.

Treatment. - The treatment of these cases may be divided into preventive, non-operative, and operative measures.

The preventive treatment, directed in general against the calculous diathesis, is considered at length in the article on Stone in the Bladder.

The non-operative treatment has for its object, first, the relief of the pain, and, secondly, the furtherance of the passage of the calculus through the canal.

The pain is often so severe that full opiates fail to give relief. As vomiting is apt to occur, and as the absorption through the stomach is thus rendered uncertain, it is well to give morphia subcutaneously, so that the exact quantity administered may be known and the absorption may be rapid. It is also to be borne in mind that the pain may at any time cease if the calculus escapes into the bladder. This cessation of pain at once removes a powerful antidote to the morphia, and an amount of the drug which was producing no toxic symptoms may, after this relief, throw the patient into a profound and dangerous coma. The severity of the pain and the danger of over-administration of morphia make it wiser ofteri to use anæsthetics by inhalation; and sulphuric ether may be used continuonsly or intermittently for many hours when necessary. Locally the application of heat, dry or moist, is of use in mitigating the sufferings,

* Hall, N. Y. Med. Record, October 18, 1890 ; Lane, London Lancet, November 8, 1890. 
and may assist in relaxing spasm. The passage of the calculus along the canal may be helped by the liberal ingestion of water, and this is also useful in keeping up the action of the kidneys and preventing the suppression of the urine.

When the amount of urine is decidedly diminished, diuretics may be required. Those which are not irritating to the kidneys should be selected. Sugar of milk, in doses of one drachm, of a saturated solution every two hours, or diuretin, gr. $\mathrm{x}$, frequently administered, will sometimes be of service. The citrate and acetate of potash may be tried also, but the liberal administration of water is perhaps the most efficient means of keeping up the action of the kidneys. Local counter-irritation by mustard poultices and hot fomentations over the kidneys will greatly assist in promoting their action.

Whether solvents, such as the salts of lithia or piperazine, can have any effect in reducing the size of an impacted stone, is doubtful, but they may be properly given in the hope of breaking up a loosely made concretion, and of bringing into solution crystals that would otherwise be deposited upon it. Supporting measures, to assist the strength of the patient to hold ont under the strain of extreme pain, should not be neglected.

Operative Treatment.-Before proceeding to the consideration of serious measures for the relief of a stone in the ureter, it will be well to consider the history of these cases somewhat carefully, and to endeavor to lay down some rules by which we shall be decided when to operate and when to let the case alone, and leave it for the efforts of Nature to cure.

When we have a condition of high fever, with other indications of the formation of an abscess around the calculus, our duty is clear to open the abscess and remove the irritating cause. Also, when the obstruction has led to. a pyonephrosis with a gradual loss of the patient's strength and flesh, the indications for the surgeon are again plain, and an operation should not be delayed.

On the other hand, it is not easy in acute cases to determine at first which ones are likely to go well to a successful issue, and to result in the final expulsion of the stone, and which must be assisted by operative measures. If in such a case the amount of urine becomes dangerously small, and especially if the presence of albumen and casts in that which is passed indicates a serious implication of the kidneys, an operation should be undertaken at once.

It is to be remembered, that if one kidney contains stones it is not improbable that the other does also, and that the added work is being borne by an organ already partly disabled. The serious impairment of a kidney whose ureter is blocked, and the possible permanent deterioration 
of so important an organ, should not be lost sight of, and full weight should be given to these considerations in deciding for or against operation. If an enlargement of the kidney and of the ureter above the seat of the calculus can be made out, it is to be inferred that the stoppage is quite complete, and that the kidney is sure to suffer.

If, on the other hand, the urine finds its way past the calculus, an examination of that passed will tell us whether the kidney is undergoing injury. When the urine is at times clear and then again clonded by the presence of pus and blood, showing that the stoppage on the affected side is complete at times, an examination of the clear specimens will tell the condition of the other kidney, while in the cloudy specimens will be found evidence as to the state of the kidney on the affected side.

While it is undoubtedly true that a calculus will occasionally remain fixed for a long time in the ureter without giving rise to serious symptoms, the fact that it almost inevitably leads to a gradual deterioration of the kidney makes its removal when firmly impacted always desirable. It may sometimes be questioned whether the operation is too dangerous to be undertaken, or is justifiable under the circumstances in an individual case.

These operations have not been done in a sufficient number of cases to establish any ratio of mortality, and the decision must be based upon general surgical principles. Certain parts of the ureter are much more difficult to reach than others. That portion lying in the true pelvis has been hitherto thought inaccessible, but in a recent paper (in the American Journal of the Medical Sciences) the writer has endeavored to show how all parts may be reached; and as some of the procedures in the plan proposed have not yet been tried in actual practice, they are presented here merely as anatomical suggestions, which, when tested by dissection, seem to have some value.

In deciding upon the best method of operation, we have before us an anatomical problem, which is, How can the ureter be reached and opened in the different parts of its course without injury to the peritonæum?

This is one of the eases in which it seems unwise to conduct the operation through the abdominal cavity; for, although the canal can be easily reached and examined through the abdomen, and although the removal of the stone in this manner wonld be a comparatively simple matter, yet the condition which would be left of an intraperitoneal wound of the ureter introduces so much danger of urine leakage into the abdomen that surgeons have for the most part avoided it, and have songht to reach the canal extraperitoneally. The opening of the abdomen for the purpose of locating the stone and thus properly guiding the extraperitoneal incision is quite a different matter, and of undoubted value. 
By what incision, then, can we best reach the ureter extraperitoneally? Let us glance a moment at its anatomy.

The ureter, leaving the pelvis of the kidney by a funnel-shaped opening, runs downward on the anterior surface of the psoas muscle, crosses the common iliae artery and vein at the entrance of the pelvis, and then, running in Douglas's fold of the peritonæum, converges toward the opposite ureter and enters the posterior walls of the bladder. Here, in the male, it crosses the vas deferens. In the female the ureters pass around the neck of the uterus to reach the bladder. As the ureters approach the pelvis in the lower part of their course through the abdomen, the left ureter lies close to the spine and in the angle between the bodies of the vertebræ and the psoas muscle. On the right the ureter is somewhat separated from the spinal column by the interposition of the vena cava inferior. The vein and ureter lie in close apposition.

While this description serves as a fairly reliable gnide to the ureter in most parts of its course, still, in a surgical search for the canal deep in the tissues, particularly if the subject be a fat one, it is extremely hard to find the lax tube and to recognize it in its collapsed condition. Anything, therefore, which enables the surgeon to locate the ureter more exactly may be of great aid in his search.

There is a relation of the ureter to the peritonæum a knowledge of which will greatly simplify this search. This is, the fact that the ureter is adherent to and always separates with the peritonæum, as it is stripped up from the parts behind. The reason of this adhesion of the ureter to the peritonæum may be seen upon microscopical sections across a ureter separated with its peritonæum. It will be found that the ureter is more or less closely bound to the under surface of the peritonæum by fibrous bands, and thus this intimate connection of the ureter to the membrane over it is explained. Moreover, the relation of the ureter to that part of the peritonæum which becomes adherent to the spine is, within a slight range of variation, pretty constant, the ureter lying just outside the line of adhesion; so that, if the surgeon has stripped up the peritonæum and come down to the point where it refuses to strip readily from the spinal column, he will find the ureter upon the stripped-up peritonæum at a short distance outside this point. On the left side the distance from the adherent point to the ureter is from half an inch to an inch, while on the right side it is somewhat greater, owing to the ureter being displaced to the outside by the interposition of the vena cava between it and the spine.

If now a stone is lodged in the abdominal portion of the ureter, it may be reached by an incision on the line mapped out by Israel, which starts at a point on the anterior edge of the sacro-lumbar mass of muscles, a finger's breadth below the twelfth rib, is carried parallel to the rib as far as its tip, then turns down toward the middle of Poupart's ligament 
till the line of usual incision for tying the iliac artery is reached, then again turns toward the middle line, and ends on the external border of the rectus muscle. According to the seat of the calculus, the incision will be made on the posterior, middle, or anterior third of this line.

In the first three inches of its course it may also be reached by an incision along the outer edge of the quadratus lumborum muscle, and this opening gives sufficient room for examination of this part of the canal, except in cases where the ribs and iliac crests are unusually near together. The dissection should be quickly carried down to the peritonæum, and this membrane should be separated from the parts beneath, the finger pushing readily in behind it. The ureter, as has been said, adheres to the peritonæum and is lifted up with it.

The relation of the ureter upon the right side to the vena cava inferior should be remembered, and care should be taken in searching for it. If a stone is impacted in the ureter, it can usually be easily found by palpation through the wound, and this greatly simplifies the search for the canal. In the upper part of the pelvis the ureter may be reached by an incision along the lower end of Israel's line, and in a child or thin person it might be accessible in this manner, even to a point behind the bladder. Practically, however, in adults of ordinary development, the lower three or four inches of the eanal can not be reached through an extraperitoneal incision in front.

In one case (Lane, loc. cit.) in which an incision had been made into the peritoneal cavity for the purpose of diagnosis, where a stone had been found in the pelvic part of the ureter, it was found possible with the hand in the abdomen to lift the stone toward the crest of the ilium, where it could be reached by the extraperitoneal incision. This case suggests, and it seems probable, that the combined incision might safely be used in some of these cases where the calculus is otherwise out of reach; and it would even seem possible, in a favorable case, to work the calculus along the ureter by manipulation to a point where it could be reached by an extraperitoneal opening.

When we come to the last two or three inches of the ureter, however, we reach a region which can not be operated upon through the anterior incision. This lower end of the canal, too, is a point where calculi are very apt to become impacted. Occasionally they even project into the bladder, and in such a case they may sometimes be reached and removed either by dilatation of the urethra in the female, or by suprapubic incision in the male, through the bladder. There is always danger, however, if the bladder wall is considerably incised, to release one of these calculi, that urinary infiltration will take place.

In the female, this last two or three inches of the ureter runs for the most part within the broad ligament, and calculi in this region can be 
removed by an incision through the vault of the vagina. If the calculus is readily felt near the cervix uteri, and the incision is made outward from the cervix toward the side of the pelvis, there is little danger of opening into the peritoneal cavity, and the incision can be readily earried down to the stone. If it lies deeply, the parts may be separated with the finger, until the stone lying in the ureter is reached.

In one such case the writer removed a stone from this region weighing one hundred and ninety grains. The difficult step in the operation was the dislodging of the stone after it was fairly uncovered. This was finally accomplished by passing a blunt hook up over the calculus within the ureter, and by traction upon this, at the same time pushing the tissues aside with the finger of the other hand, the calculus was finally drawn down and delivered.

In the male, the removal of calculi from this region is much more difficult. It would seem possible, however, that it might be accomplished by an incision over the sacrum, with the removal of a part of that bone after the manner practiced by Kraske in cases of cancer of the rectum. Dissections made by the writer show that the ureter is readily accessible in this way.

The incision for thus reaching the ureter may be made over the margin of the sacrum on the side of the ureter which is sought, commencing on a level with the sacro-lumbar articulation, and ending just below the tip of the coccyx. After dividing the sacro-iliac ligaments, the cocey $x$ and the lower portion of the side of the sacrum are removed. This may be readily accomplished with large gnawing-forceps, and, if the side of the bone is cut away as high as the third foramen, a good opening and easy access to the interior of the pelvis are obtained.

In order that the view of the pelvic contents shall be satisfactory, the patient should lie partly on the side, with the abdomen dependent, as in Sims's position. The cavity of the wound is then ballooned out by the pressure of the air, just as happens to the vagina in the examination for which this position is commonly used.

A sound introduced into the rectum now maps out its outline, and serves to hold it to one side during the subsequent search.

The converging lines that the ureters pursue through the pelvis lie pretty closely over the lateral edges of the sacrum, and this relation will help somewhat in fixing their location when approaching them from behind. The normal, collapsed ureter is, under the most favorable conditions, hard to find, and care is required to avoid wounding the peritonæum during the search for it.

In the cases we are considering, however, we have the stone to guide us to it, and, when the calculus is found, an incision may be safely made over its lower surface, which is not covered by the peritonæum. 
In some one of these ways we may hope to reach and remove a stone from any part of this deep-lying canal, by incision directly upon it. It may often, however, be easier to make our incision into the dilated pelvis of the kidney, or the upper enlarged portion of the ureter, and then with long forceps to reach and remove the stone. This plan has frequently been resorted to when the surgeon, having opened the pelvis of the kidney, has found that the calculus that he expected to find there is already in the ureter. It might also be a possible means of approach to a stone in the pelvic portion of the ureter, provided the canal above was sufficiently dilated to allow of the manipulation of instruments through it.

Lastly, how shall we treat the wound in the ureter, after the removal of the calculus?

In one case (Lane, loc. cit.) it was closed with a continuous silk suture with success. In other eases this has not succeeded; and considering the difficulty of applying a suture, and the possibility that it may serve as a nucleus for future calcareous deposit, this plan would seem to be not without inconvenience and danger.

On the other hand, in several cases the wound in the ureter has healed quickly, without trouble, when left to itself. It would seem, therefore, best not to suture the canal, but to provide adequate drainage for the urine escaping from it till the wound in its wall closes. The only exception to this is when the ureter is opened through the vagina in its lowermost part, where it is in intimate relation with the vaginal wall, and where it is therefore possible to get sufficient thickness of tissue for the application of stitches without encroaching on the cavity of the tube. 


\title{
THE SURGICAL DISEASES OF THE KIDNEY.
}

\author{
By LEWIS A. STIMSON, M. D.
}

\section{WOUNDS AND INJURIES OF THE KIDNEY.}

Simos's Chirurgie der Nieren, the two parts of which were published in 1871 and 1876 respectively, was the first attempt to group the various surgical diseases of the kidney, and formally to recognize this branch of the general subject of surgery. Heretofore the only monographs on the diseases of the kidney had been the work of physicians, and with reason, for but little had been done in the way of surgical treatment of such affections as were amenable thereto. The two decades of years that have followed this publication have brought an amount of surgical experience in this department that is simply enormous; our journals and the records of our societies are filled with the reports of cases, and a number of attempts have been made to collect this scattered experience and to treat the subject with more or less completeness and system. The papers and articles that have concerned themselves with certain portions of the subject will be referred to in connection with these portions; those that have songht to cover the entire subject, exclusive of the corresponding chapters in treatises of general and special surgery, are, more particularly, Morris, 1885; Newman, 1888; and Le Dentu, 1889. Of these three, the latter is distinctly superior in orderly arrangement, proportionate distribution of space, and utilization of material coming from all sources. His classification and arrangement have been here adopted, and his work has been made the basis of this article.

Adopting Le Dentu's definition, under surgical diseases of the kidneys we here include all those treatment of which may at any time require surgical or, better, operative interference.

\section{TRAUMATISMS OF THE KIDNEY.}

The principal papers of the last twenty years upon this subject are those of Maas, "Klinische und experimentelle Untersuchungen über die subcutanen Quetschungen und Zerreissungen der Nieren," in Deutsche Zeitschrift für Chirurgie, Bd. x, 1878 ; Clement Lueas, "On Injuries of the Kidneys," in the Lancet, April 19 and 26, 18s4; Edler, "Die 
traumatischen Verletzungen der parenchymatösen Unterleibsorgane," in Archiv für klinische Chirurgie, Bd. xxxiv, p. 738, 1886-'87; and Edward O. Otis, "Injuries of and Operations upon the Kidney," Boston Medical and Surgical Journal, October, 1887.

Edler collected 152 cases, divided as follows :

Subcutaneous injuries of the kidney.......... 90

Gunshot-w.ounds of the kidney............ 50

Incised wounds of the kidney . ........... 12

Of the 90 subcutaneous injuries, 71 were collected by Maas in 1878; the remaining 19 were those reported between 1878 and 1887 . The relative frequency, in comparison with injuries of other viscera, is shown by Edler's tables relating to spleen and liver: thus, he collected-

Subcutaneous injuries of the liver, 189 ; of spleen, 83

Gunshot-wounds of the liver .... 289; " " 42

Incised wounds of the liver...... $65 ; \quad$ " 35

Totals............ $\overline{543 ;}$ " $\overline{160}$

Leaving the details of the different classes for later mention, the following may be here quoted from Edler's summary: Shock is present more often and is more severe than after injurics of other viscera; local pain is constant and radiating, especially to the testes; colicky pain is due to the plugging of the ureter by blood; hæmaturia is a common symptom (70 per cent), and often recurrent. The mortality of the 152 cases was 47.3 per cent; of the extraperitoneal cases, 30.4 per cent; of the intraperitoneal, 80 per cent ; the most frequent cause of death after subcutaneous injury was primary hæmorrhage, after open wounds, peritonitis or suppurative processes.

\section{OPEN WOUNDS.}

The wound of the kidney may be caused by a bullet or by a sharp instrument, or by a blunt one, as a fragment of shell, the horn of an ox, the prong of a fork, etc. The injury may be limited to the kidney and the overlying soft parts of the back and loin, or it may be complicated by associated injury of the peritonæum and of other viscera, or by the prolapse of the wounded kidney through the wound. Incised and punctured wounds are rare, actually and relatively, and are usually received from behind or from the side, the knife, sword, or bayonet penetrating to the kidney with more or less extensive division of the wall on the way; in some cases it has passed through the lower part of the chest from behind, in others through the anterior abdominal wall. The same is to be said of bullet-wounds, which are much more frequent, and more commonly associated with the injury of other viscera. In eases in which 
the kidney alone lias been wounded the course of the bullet has been oblique from the neighborhood of the median line of the back to that of the anterior end of the eleventh or twelfth rib. In a case that came nnder my own observation; the bullet entered between the eighth and ninth ribs on the left side in the mid-axillary line, passed through the pleural cavity without wounding the lung, through the upper end of the left kidney, and through the peritoneal cavity to the body of the twelfth dorsal vertebra.

The injury to the kidney varies from a slight incision to an extensive laceration, with the immediate loss of a large part of the organ; the escaping blood spreads through the adjoining connective tissue and into the peritoneal cavity, if that has been opened, in quantities that vary with the extent and character of the injury; the parenchyma of the organ apparently does

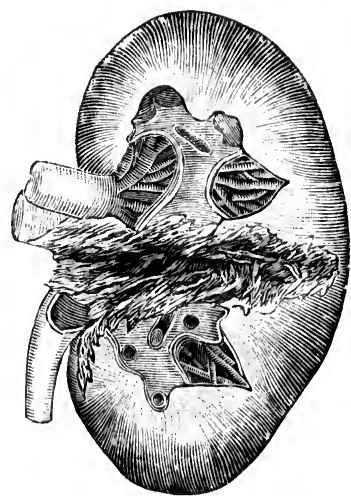

FIG. 1.- Section of a kidney with a shot perforation from before backward. not often bleed freely enough to endanger life, but if the larger arterial or venous branches at the hilum have been divided the hæmorrhage may be promptly fatal. If the pelvis of the kidney is opened the blood makes its way into the ureter; if in small amount, it gives rise only to the symptoms of hæmaturia; but if the quantity is large, the blood clots within the ureter, giving rise to colicky pains or to complete obstruction of that organ, or rarely, it may flow freely to the bladder and there form a large clot.

A number of specimens obtained after sufficient intervals show that repair takes place with the formation of a retracted, puckered cicatrix. Tillmanns has sought to show, by ingenious but not very satisfactory experiments, that the kidney itself takes no part in the process of repair, but that the cicatrix is formed by the evolution of leucocytes migrating from adjoining vessels. Clinical evidence, which is now rapidly accumulating through operations on the kidney, indicates that wounds of the parenchyma will usually heal promptly and without the formation of a urinary fistula, but that the definitive healing of a wound of the pelvis is more likely to be delayed than to fail.

Symptoms. - The immediate local symptoms are the presence of a wound of the abdominal or thoracic wall, perhaps large enough and so situated as to permit the recognition of the wound of the kidney by the eye and finger, or having a depth and direction which suggest an injury on anatomical grounds. In exceptional cases the injured kidney protrudes through the wound.

Hæmorrhage, pain, and shock are present in varying degree. 
Hæmorrhage may be free through the external wound, or the blood may be extravasated amid the loose circumrenal connective tissue, or flow into the abdominal cavity, or down the ureter to the bladder. It is ordinarily slight when only the cortex of the kidney has been wounded. Secondary and late hæmorrhages have been observed.

The escape of the blood through the ureter to the bladder is shown by homaturia, which may appear promptly or tardily, continue for a longer or shorter time, or be intermittent. Intermittence may coincide with paroxysms of pain, kidney-colic - that is, blood disappears from the urine, and then, after a longer or shorter interval, paroxysmal pains occur, and their cessation coincides with a reappearance of blood in the urine. This sequence of events is explained by the supposition of transient obstruction of the ureter by clots, the paroxysmal pain being excited by the forcing of the clot through to the bladder.

Pain may be slight or severe, and so far as it is local it is apparently referable to the general wound rather than to the kidney alone. It is often noted that the pain radiates to the epigastrium, the groin, the testicle, or the thigh. Paroxysmal pain radiating to the testicle and accompanied by the retraction of that organ is distinctly significant of injury to the kidney or ureter, and, as has been stated, is supposed to be provoked by the passage of clots. Vesical tenesmus may be of reflex origin, or may be due to the presence of clots in the bladder.

Urine may escape through the external wound in large or small quantities, but, as a rule, this occurs only when the pelvis of the kidney or the ureter has been opened. If with this opening of the urinary tract coincides plugging of the ureter by a clot, or its retraction after division, all the urine thereafter secreted by the kidney must find its way to the exterior through the wound, or must accumulate in the peritoneal cavity, or in some space made for itself by pushing back the connective tissue surrounding the kidney, and will manifest its presence by corresponding symptoms.

The general symptoms are those of ordinary traumatic shock and hæmorrhage, and, as is the case with other injuries also, those belonging to shock do not correspond with the extent of the injury. Some authors attribute the nausea and vomiting, which have been so frequently noted, directly to the kidney and not to the shock, basing their opinion upon the frequency with which the same symptonıs accompany other painful affections of that organ.

In two cases of gunshot-wound (Hennen, Demme) a piece of cloth was voided through the urethra, after a lapse of seven months in one of them.

If the patient does not promptly succumb to hæmorrhage or the effects of other injuries, and if uneventful repair does not immediately 
follow, the later consequences appear: suppuration of the wound, perinephritis, pyelitis, general or local nephritis, cystitis, and possibly peritonitis. The extravasation of blood into the surrounding connective tissue favors the extension of such suppurative processes as may be set up in the track of the wound or in the kidney, with the production of more or less extensive perinephritic abscesses, and the septic condition thus established is increased by the decomposition of such urine as escapes into the wound. The resulting abscesses may burrow to a great distance in various directions if a free outlet for the pus is not provided. If the patient survives the immediate dangers' of this condition, the cavities contract, and a fistula, which may or may not discharge urine, results. The co-existence of complications is so common that it is not easy to say how much of the admitted gravity of these injuries arises from the wound of the kidney itself. The records of the war of the rebellion contain 26 alleged recoveries from about 70 gunshot cases, but the diagnosis is by no means certain in all of them. Of 50 gunshot cases collected by Edler, 28 recovered; of 20 uncomplicated cases only 5 died; 8 uncomplicated incised wounds gave only 1 death, while of 4 complicated cases all died. In 2 cases the kidney was prolapsed in the wound; both patients recovered.

Post-mortem examinations, made where death had followed after a long interval, or as the result of associated injuries, have shown widely different conditions. In some cases the kidney-wound has been entirely healed; in one with the bullet incapsulated; in others the bleeding had stopped, the wound was filled with a clot, and repair was apparently progressing in the usual way; in others, abscesses have been found in and about the kidney. Sympathetic inflammation of the other kidney has been occasionally noted.

Treatment.- In cases that are probably uncomplicated and in which the hæmorrhage is not serious, cases in which the diagnosis of a wound of the kidney rests on the position and direction of the peritoneal wound and on the presence of hæmaturia, the treatment shonld be that of an incised or gunshot wound of any indifferent region or tissue-namely, cleansing of the surface and of the superficial part of the wound, and an occlusive dressing. But if the flow of blood from the wound is free, or if there is good reason to suspect an important internal hæmorrhage in the perinephritic tissues or the peritoneal cavity, the interference should be prompt and active; the wound should be enlarged sufficiently to give free access to the kidney, so that the extent of its injury may be determined and suitable measures adopted. If the bleeding comes mainly from the parenchyma of the kidney, it can be readily checked by sutures that hold the sides of the wound together; if, on the other hand, it comes from the large vessels near the hilum, they must be secured by ligature, 
or the kidney must be removed. The narrowness of the space between the twelfth rib and the crest of the ilium, and the depth at which the kidney lies, may make the exploration and search very difficult, and the surgeon should therefore give himself the benefit of free incisions in the most favorable positions and directions (see Nephrotomy and Nephrectomy); and it may be necessary temporarily to arrest the hæmorrhage by large clamps thrust to the bottom of the wound in order that a suitable exposure and examination of the organ may be made.

If the wound of the kidney should be so extensive that the removal of that organ is necessary, it must be thoronghly exposed and freed from its fatty capsule and the peritonæum, and then the artery isolated and tied, if that is practicable, or the pedicle tied en masse; the latter is uncertain and correspondingly dangerous because of the difficulty of drawing the ligature tightly enough to control the vessels; it is safer to use an elastic ligature, or to tie the pedicle in sections.

Plugging of the wound with iodoform gauze, so valuable a means of opposing hæmorrhage in many places, is to be distrusted here, for the bleeding may go on beyond it into the peritoneal cavity, and may also be accompanied thither by urine.

If the pelvis of the kidney is opened into through the cortex, the escape of the urine may usually be prevented by suturing the latter; but if it is directly opened into, provision must be made to carry the escaping urine freely to the surface. The opportunity should be utilized to remove blood-clots from the pelvis and the ureter; the latter can be done by passing a soft catheter or bougie throngh to the bladder if it will pass, or by directing a stream of water into the ureter to wash the clot out backward.

Prolapse of the injured kidney in a large wound has been observed in a very few cases, and has been given more prominence in some systematic treatises than it appears to deserve. In four cases quoted by Le Dentu, the kidney was removed in two and replaced in two; all the patients recovered. It is taught by some that if the injury is recent and the main vessels not divided the kidney may be replaced, but if several hours have clapsed and infection has probably taken placé, it should be removed. The recommendation of removal is based upon the assumed probability of setting up a dangerous septic process in the perinephritic tissues by the return of an organ presumably covered with infectious microbes; but the argument is purely theoretical, and there seems to be sufficient ground to maintain that the risks conld be reduced by antiseptics, iodoform packing, and drainage to such a degree that they would not outweigh the advantages of retaining the organ. Of course, if the vitality of the kidney has been serionsly compromised by division of its main vessels, or if it has been so extensively lacerated that there is but little probability that it 
would be able to perform its functions if it were saved, the prudent treatment would be immediately to remove it.

The treatment of uncomplicated gunshot-wounds is guided by the same principles as that of incised wounds ; in complicated cases the character of the complication will probably dominate the treatment.

The inflammatory and suppurative conditions which arise in consequence of a wound of the kidney will be considered in another section; it is sufficient here to say that the treatment is to be conducted according to the principles governing the treatment of similar conditions in other regions: free evacuating incisions and drainage, the removal of foreign bodies and necrotic tissue, and possibly an ultimate nephrotomy, as the most expeditious and effectual means of bringing to an end processes that are endangering the life of the patient.

\section{SUBCUTANEOUS LACERATIONS AND RUPTURES.}

The statistics above quoted show that these are much more frequent than open wounds of the kidney, and doubtless this numerical superiority would be considerably increased if the statistics shonld include the due proportion of the slighter cases.

The injury appears to be caused much more frequently by direct than by indirect violence, fifty-six and thirty cases respectively, according to Edler. The common direct canse is a fall from a height. Some authors describe as laceration of the kidney by indirect violence those not uncommon cases in which transient hrematuria follows violent contraction of the abdominal muscles or prolonged exercise on horseback; but othersmore plausibly, I think-class them as cases of lithiasis, and attribute the bleeding to injury of the somewhat congested mucosa of the pelvis of the kidney by small uric-acid crystals or actual gravel. It is, however, to be remembered that such muscular efforts appear to be a cause of perinephritic abscess. Among the direct causes are blows, kicks, crushings betwean moving bodies, the passage of the wheel of a wagon, a fall against some prominent object, like the corner or the edge of a table. Simon noted in his first nephrectomy that the handling of the kidney in the course of the operation caused several subcapsular hæmorrhages; and Maas has shown experimentally that notable laceration can be effected by the pressure of the fingers.

The injury may vary in extent from slight subcapsular hæmorrhage to complete rupture of the organ. Apparently the cortex is involved in all cases, but its laceration may or may not extend to the medullary portion. Extensive lacerations are commonly transverse. A case of complete longitudinal rupture cansed by the passage of a wagon-wheel occurred during the war of the rebellion, and is reported in Circular No. 3, 1871, S. G. O., page 106, and Edler notes two others. The superficial and slighter 
lacerations vary indefinitely in direction and extent. The surface of the rupture may be almost as regular as that of an incised wound, or it may be irregular and pulpy. The anterior and posterior surfaces are torn with equal frequency (Edler). Of Morris's twelve cases, seven were partial ruptures, extending through half the thickness or less of the organ; two were complete divisions; in one the entire kidney was crushed to pulp; in one there was only subcapsular hæmorrhage; and in one there was blood in the pelvis, but no injury of the kidney substance could be detected.

The mechanism of the laceration is not always clear. Transverse rupture in a fall from a height has been attributed to the momentum of the less firmly attached lower portion (Le Dentu), or to the forcible bending of the trunk when the body strikes the ground (Morris). Rupture of the anterior portion has been explained on the theory of forcible dorsal flexion of the trunk in which the kidney is stretehed across the twelfth rib, and rupture of the posterior portion on the theory of direct pressure of the kidney against and past the ribs.

The peritonæum covering the kidney appears to be but rarely torn; more frequently, it is claimed, in the young than in the old.

Hæmorrhage may be entirely within the substance of the kidney, or it may be confined within the fatty envelope, sometimes pressing it back and forming a large hæmatoma; or this envelope may itself be torn, and the blood pass far and wide along the intermuscular planes, in the retroperitoneal tissue, or into the peritoneal cavity. Blood that gets into the pelvis, either directly from the torn parenchyma or through a rent in its own wall, may pass to the bladder or may clot and obstruct the ureter. Morris alleges that such obstruction can produce permanent structural occlusion of the ureter.

Associated injuries are frequent, and, indeed, are almost constant in the severer cases. Among them may be mentioned rupture of other viscera-liver, spleen, ureter-contusion or rupture of the intestines, fracture of the ribs.

A very striking and important complication or consequence has been noted in two cases by Poland and Moxon (Guy's Hospital Reports, Third Series, vol. xiv), namely, complete thrombosis of the renal vessels.

If the individual survives the immediate consequences of the injury, the wound of the kidney may undergo complete repair, or suppurative and degenerative processes may ensue and again place life in danger. It has been satisfactorily demonstrated, both clinically and experimentally, that a laceration of the kidney may heal, leaving a more or less. wellmarked scar. Cases have been reported in which the examination has been made at dates varying from a week to five years after the accident, and has shown either complete cicatrization, or the process so far ad- 
vanced that complete repair would probably have taken place if the patient had survived the associated injuries.

Maas has traced the process in detail by the aid of experiments upon animals. He found the cicatrix retracted and the surface correspondingly depressed, and fine prolongations running from it into the adjoining healthy tissue of the kidney, scattered through which were many small eysts, some of which apparently were the remains of collections of blood, while others were dilated canaliculi. In another series of experiments he found as occasional results atrophy of the kidney, its complete disappearance by the formation of a large, irregular hæmorrhagic cyst, and hydronephrosis. Mounier, quoted by Maas, reported a case in which the antopsy, forty-one days after the accident, showed a blood-cyst weighing seven and a half pounds, extending from the sixth rib to the crest of the ilium, covered by peritonæum, and with a very irregular deeply grooved wall which contained kidney tissue at only one point. Maas attributes the disappearance of the substance of the kidney to thrombus of the renal vessels.

Other late consequences are suppuration in or about the kidney, interference with the secretion or the voiding of urine, nephritis, and the formation of stone. They will be considered under another head.

Symptoms.-Except for these immediate local symptoms which pertain to the external wound, the symptoms of subcutaneous injury are the same as those of open wounds of the kidney. There are the same general symptoms, varying in severity in the different cases-nausea, vomiting, shock, and perhaps the constitutional signs of severe internal hæmorrhage.

The following history is an illustration of the symptoms and course in the milder cases (Notta, Schmidt's Jahrbuch, 1859, No. 10, p. 90, quoted by Edler):

"A powerful peasant, forty-eight years old, was kicked by a horse in the right side. He fell to the ground and was unable to rise; great pain at the seat of injury. Three hours later Notta found him in bed, on his back, pale face, cool surface, pulse 70; constant complaint of frightful pain in right side. No trace of the blow on the surface. It had been received upon the lower ribs, which, however, were not broken, although they and the entire side were extraordinarily tender on the slightest pressure. The abdomen was soft, and not distended; percussion-note clear everywhere. No nausea, vomiting, or chills. The patient frequently voided, with great pain, pure blood through the urethra. The next day the pain continued; the urine was dark, and contained a considerable amount of blood, but no clots. Urination was more frequent than normal; the bladder was emptied. The pains increased in severity; the patient was hardly able to breathe, and felt very weak. On the third day the pains diminished. The urine, which was passed without pain, was turbid, orange-colored, and contained blood. On the fifth day kidney- 
pains were renewed. The urine grew clearer. An ecchymosis four to five centimetres in diameter appeared two centimetres above the anterior superior spine. On the eleventh day the urine was clear. A fortnight later the man returned to work, but he long remained sensitive to deep pressure in the region of the kidney."

Pain, usually severe at first, may be modified by the injuries of adjoining parts. The characteristic radiation to the groin and testicle, with retraction of the latter, is, as we have seen, commonly to be referred to the passage of clots of blood through the ureter. These paroxysms, though common, are not constant; they may be intermittent, and sometimes do not appear until after several days.

The appearance of an ecchymosis in the loin, or in front below the ribs, can have no special signiricance in cases of injury by direct violence; but an ecchymosis in the region of the external inguinal ring has been deemed pathognomonic of injury of the kidney. It has been observed in several cases, in one of which (Letulle, Bull. de la Soc. Anat, 1876, p. 236) the antopsy verified the diagnosis, and proved the connection; the blood had made its way alongside the spermatic vessels to the scrotum.

Hæmaturia is a common and valuable sign. It has been absent in some cases of extensive laceration because of the plugging of the ureter by a clot; and in other cases of assumed injury its absence has been explained on the supposition that the lesion was only a slight laceration of the cortex. Ordinarily it appears promptly, but in four of the cases collected by Edler it appeared first on the third, seventh, ninth, and fourteenth days respectively. It may continue for a few hours only, or for days, weeks, and even months, and may vary in degree from moderate staining of the urine to almost pure blood. Le Dentu says that even when the blood flows into the bladder in large quantities-several quarts -it rarely clots there, so thai retention of urine due to this cause is very exceptional. The voided clots are sometimes long and cylindrical, apparently having been formed in the ureter. In some cases the urine remains albuminous for several days after the blood has ceased to be present in it. This may be due to the admixture with the urine of the serum of a clot that has formed in or in communication with the pelvis of the kidney; in other cases it has been the first symptom of a commencing chronic nephritis.

Urination may be frequent and urgent because of unexplained rcflexes, as observed in connection with injuries of other parts, or because of reflexes originating in the kidney, or because of clots in the bladder. Occasionally there is complete suppression of urine, as when both kidneys have been torn, or when there has been only one kidney; more frequently the amount of urine has been only diminished, the diminu- 
tion lasting for several days; exceptionally the amount has been increased.

The later symptoms have their rise in the later processes and changes that have been already mentioned. If suppuration takes place in and about the kidney, not only do the usual constitutional symptoms of that process appear, but the urine also may contain pus, and the abscess may become large enongh to be recognized by palpation. Pus in the urine may also have its origin in a cystitis set up by the presence of blood in the bladder or by catheterization.

An extensive and persistent hæmorrhage may show itself in ecchymosis in the loin or in the anterior abdominal wall and scrotum, as already mentioned, or as a collection of blood within the fatty capsule or within the kidney itself (hæmatonephrosis), or, rarely, deep in the pelvis behind the peritonæum, within the peritoneal cavity. Such a hæmatoma forms slowly, and is recognized as an elastic globular mass in the loin or hypochondrium ; it is much more frequent as a perinephritic hæmatoma than as a hæmatonephrosis.

A similar elastic globular swelling is exceptionally formed by urine, mingled perhaps with blood, that continues to be secreted by the kidney and is prevented from reaching the bladder by obstruction or rupture of the ureter. There is good reason to think that such urinary collections are extrarenal, the urine escaping into the circumrenal tissue through a rent in the pelvis; and that when snch escape is impossible, the kidney ceases to secrete before any considerable amount of urine has collected within the pelvis, so that a notable distention of the pelvis by simple pressure of urine retained within it is very rare, or perhaps has never occurred.

Diagnosis.-For the diagnosis of rupture or laceration of the kidney the most trustworthy symptoms are hæmaturia and pain radiating to the testicle; but neither is pathognomonic, nor is its absence proof that the injury has not been received. As has been stated, blood may appear in the urine after a traumatism, or after a violent muscular effort which, there is good reason to suppose, has not injured the kidney; but such cases appear to be very rare, and to be associated with a history of chronic irritation or congestion of the upper urinary passages. On the other hand, the absence of hæmaturia does not exclude certain mild lesions limited to the cortex of the kidney, or severe ones associated with rupture or plugging of the ureter. Local tenderness" on pressure is significant only when it is clearly not due to contusion of the abdominal wall, and ecchymosis is significant only when it is tardy and appears at a distance, at points that are in ready communication along connective-tissue planes with the site of the kidney.

Prognosis. - The prognosis, so far as it is shown by the percentages of published cases, is grave. Of Edler's 90 cases, 45 died; of these, 33 
were classed as "complicated" cases, with 25 deaths (75 per cent); 17 patients died within the first two days ; 19 died of hæmorrhage, 14 primary, 5 secondary ; 5 of peritonitis, 5 by the immediate effects and extent of the crushing, 3 by the wound of both (1), or the absence of a second kidney (2), 3 by suppurative processes, and, by various complications. Excluding the cases of early death by bleeding, severity of the injury, and complications, there remain 61 cases, with 13 deaths (21 per cent), from which Edler draws the conclusion that, if a subcutaneous injury is not so severe as to cause death within two or three days, the prognosis is more favorable than after injury of the liver or spleen. Maas's statistics indicate an especial gravity in children under ten years of age, 6 deaths in 7 cases. As has been already intimated, these statistics doubtless do not contain a due proportion of the milder cases.

Treatment.-The first indications, in any case of recognized or suspected injury of the kidney, are to relieve the shock and pain and to arrest such hæmorrhage as may be going on. These are met by absolute rest in bed, the administration of sedatives and anodynes, the avoidance of stimulants except so far as they are clearly indicated on special grounds, and methodical compression of the abdomen, and the internal administration of hæmostatics if it appears that the bleeding is free and persistent. Compression can be made by the usual abdominal binder over a thick layer of cotton or by a sheet of adhesive plaster extending from the middle of the back around the injured side and well across the front of the abdomen. Morris strongly recommends half-drachm doses of the liquid extract of ergot every three hours as " the most certain and rapidly efficacious remedy of this sort." The food should be limited in quantity, and concentrated, in order that movements of the bowels may not be frequent. Vomiting must be immediately checked, lest it provoke a recurrence of the bleeding. Morris attributes a recurrence in one case to the "passage of hardened fecal matter along the colon and thus across the surface of the kidney," and in consequence advises that the bowels should be relieved only by enemata, or left undisturbed for several days. It seems not unlikely that this recurrence, and a few others attributed to changes in diet, may have been simple coincidences. The external application of ice or the cold-water coil is routine treatment to check hæmorrhage, but it can hardly be supposed that the temperature of the deeper parts is thereby lowered sufficiently to have any effect.

A very serious question-Is nephrectomy indicated ?-occasionally arises when the signs of internal hæmorrhage steadily increase and indicate imminent danger to life, notwithstanding the use of such measures as have been mentioned. The only practicable operative method of arresting the bleeding is by nephrectomy, for there can be no thought of seeking and securing a single vessel under such circumstances. What- 
ever is to be done for the exsanguinated patient must be done rapidly and with the least possible draft upon his strength. The operation has been done in a few eases (Newman has collected five, with three deaths), and seems to be fully justified when the condition is clear; but it must be remembered that it is a vastly more dangerous operation than the ligature of even a large artery in continuity, and that it is not to be resorted to so promptly as the latter in cases of persistent or secondary hæmorrhage from some arterial branch in a limb.

The early symptoms which may suggest the propriety of a resort to nephrotomy are those of serions internal hæmorrhage, pallor, sighing respiration, restlessness, and a small, rapid pulse, possibly associated with the frequent voiding of blood from the bladder, or with the formation of a more or less tense, fluctuating swelling in the loin. The best judgment of the surgeon will be required to determine the propriety of operative interference and the time at which it shall take place; for, on the one hand, it may be urgently necessary that bleeding should be promptly arrested, and, on the other, each day's increment of incapsulation of the escaped blood and consolidation of the surrounding tissues adds to the chance of successful interference.

Occasionally the flow of blood into the bladder is so profuse as to create special indications for treatment, for the clots interfere with the voiding of the urine, and at the same time add to the vesical irritability and tenesmus. The catheter does not relieve, for the clots obstruct it, and the distention that results from the accumulation of blood and urine causes severe and constant pain, and introduces new elements of danger-possible rupture of the bladder, or the setting up of a cystitis which may spread to and incapacitate the remaining uninjured kidney. It has been suggested to relieve by breaking up the clot with a lithotrite, or by washing it out with a Bigelow evacuator, or by disintegrating it with injections of a two-percent solution of gallic acid; but it seems probable, in the absence of demonstrative clinical experience, that if the well-proved method of reliance upon the solvent action of the urine should seem too hazardous, it would be better to resort to cystotomy, either perineal or suprapubic. Rawdon (Lancet, May 26, 1882, p. 907) reported a case in which eystitis supervened upon recurrent hæmaturia, and he performed lumbar nephrectomy on the seventeenth day; the cystitis persisting, he did lateral perineal eystotomy four days later and evacuated fetid clots; but the patient died on the fourteenth day, of pyelo-nephritis of the other kidney, which had apparently been excited by extension upward of inflammation set up in the bladder by the retention and the decomposition of the clots.

It is easy to draw the inference that in this case the interference was unduly delayed, and that if it had been undertaken earlier the patient might have been saved; and it is also easy to lay down the principle that 
nephrectomy is indicated when without it the patient will die of hæmorrhage, and that cystotomy is indicated when without it a dangerous cystitis and pyelonephritis will ensue; but it is far from easy in any given case to say that the indications are sufficient to call for the operation. Fortunately, the cases in which the questions will arise are very infrequent.

Later in the course of a case suppuration of a hæmatoma within or about a kidney, or the formation of a urinary cyst, may call for operation. Suppuration requires a free incision in the lumbar region, with the precautions and after-treatment appropriate to the conditions; in some cases nephrectomy, either as a primary or secondary operation, has been done. Hydronephrosis may be temporarily relieved by tapping, with the chance that recurrence may not take place or may be so slow that more radical and dangerous measures will be unnecessary. These various conditions will be considered in detail in another chapter.

\section{NEPHROLITHIASIS-GRAVEL-KIDNEY STONE.}

The scope and character of this work will not permit detailed study of the cause and of the constitutional treatment of the conditions indicated by the titles of this section; for them the reader is referred to works on internal medicine, to which branch of medical science these conditions long belonged wholly because of the powerlessness of the surgical art to aid in them. To that powerlessness has succeded within the last twenty years a notable and increasing efficiency, and the surgeon is called upon to relieve not only those conditions which constitute the later and extreme stages for which the need of an efficient surgery was always recognized, but often also certain slight and earlier ones which were looked upon as purely medical affections.

Under nephrolithiasis-a term which etymologically signifies the presence of calculi in the kidney - we here include the various stages of the local manifestations of the general constitutional condition that underlies most of them, and those other affections in which similar manifestations are of purely local origin. We have to deal not only with those classes of cases in which the stone remains lodged in the kidney and is of considerable size, but also with those others in which the stones are numerous and small, perhaps barely recognizable by the unaided eye, and are voided with the urine, and with those in which the patient presents many of the symptoms associated with the other forms, but whose kidney is free from stone, and whose urine is free from crystals-cases of simple nephralgia, but still cases in which the same constitutional condition is involved, and which ean be surgically relieved.

Nephrolithiasis is observed at all ages, even during intra-uterine life. 
According to Morris, it is most common before the age of fifteen years and after that of fifty, the youthful patients belonging usually to the poorer classes, the older to the richer. It is more prevalent in some countries-England, for example - than in others, and the difference is apparently due to climatic and dietary conditions. It is often inherited, and in its uric-acid form is clearly associated with the gouty diathesis.

The concretions, the different sizes of which are rather arbitrarily classified as calculi, or stones, gravel, sand, and crystals, are found in the pelvis, infundibula, or calices, or in dilated tubules or abnormal cavities in the substance proper of the kidney. In the bodies of stillborn children and of those that have lived for only a few hours, and in the contracted kidney of gouty adults, it is not uncommon to find collections and even small concretions of crystals of uric acid or urates in the collecting tubules of the pyramids and the straight tubules of the cortex. Occasionally, but very rarely, kidneys are found that contain, within completely closed cavities in the parenchyma, gravel and even calculi of considerable size; these are interpreted as later results of the lodgment of crystals or minute concretions within the tubules, the crystals having increased in size by the continuous precipitation upon them of the urinary salts, until finally, by pressure and the resultant irritative changes a closed cavity with fibrous walls had formed about them.

By far the most frequent seat is, however, in the pelvis or one of its prolongations, an infundibulum or calix, and the concretions found in this situation are ordinarily of considerable size, for the smaller ones are likely to escape through the ureter. If the calculi are small they may be very numerous, and even if quite large there may be two or more of them, the pelvis being correspondingly dilated and the parenchyma thinned. Ordinarily there is but one stone of any considerable size, and in only one kidney, but exceptional cases showing many variations have been reported: for example, one in which each kidney was like a bag full of small stones; another in which there were 100 stones in one kidney and 70 in the other; another in which there were 36 stones in one kidney and 6 large ones in the bladder; and others in which each kidney has contained one or more very large stones. The largest that have been reported are (Revue des Sc. Méd., 1875, vol. v, p. 499) one weighing 1,015 gramınes, and accompanied by 60 grammes of small ones; and one, reported by Pohl (De prost. calcul. Quoted by Le Dentu), weighing five pounds.

The shape may be regularly rounded and the surface smooth, or the calculus may closely resemble in shape and surface a branch of coral with a central body and several prolongations corresponding to the calices within which the increase in size has taken place by continuous 
parenchyma, and it is thought that the extreme conditions of atrophy sometimes observed, those in which the kidney is reduced to a small nodule with barely a trace of glandular tissue, are to be explained on the theory of primary dilatation followed by the escape of the liquid contents and retraction of the wall.

Modifications in the shape of the kidney correspond, in general terms, to the size of the calculus, if the latter is large, and to the presence or absence of dilatation. On section the cut surface shows a more uniform appearance than is normal, and is sometimes studded with minute eysts. The large cysts seen on section are usually portions of the car lices that have been crossed by the knife, but some of them are actual closed eysts formed within the parenchyma. They sometimes contain eoneretions.

The question of the effect of the destruction, removal, or incapacitation of one kidney upon its fellow, which was suggested by Rayer and first studied by Simon, has not yet been completely answered. It is known that in many cases the remaining kidney has satisfactorily performed the double duty that has been thrown upon it, and that it has apparently undergone a notable increase in size. In other cases, perhaps equally numerous, the remaining kidney has shown more or less marked evidences of disease, and after abstraction of those cases in which the changes were probably independent of the disability of the other kidney, and were the result of a general eause or of an inflammation ascending from the bladder, there still remain a few cases which have seemed to some observers to justify the theory of reflex nephritis.

Etiology.-The underlying, primary cause of the formation of gravel or of a calculus in the kidney is, in the great majority of cases, a general, perhaps even a constitutional one, and only in a relatively small minority is the cause local. A local cause, which has been clearly established by the study of the formation of vesical calculus, is the existence of a catarrhal or ulcerative condition of the mucosa of the pelvis and its prolongations, the products of which combining with some of the constituents of the urine provoke the precipitation of the phosphatic salts, and thus either originate a phosphatic ealeulus, or lead to the rapid deposition of a phosphatic layer upon a pre-existing ealculus of urie acid or oxalate of lime.

The causes and processes which lead to the much more frequent crystallization of uric acid, urates, oxalate of lime, and the hard phosphates are by no means so clear. In respect to uric acid, it is known to coincide with a diathetic condition (gout) and with certain dietary and hygienic peculiarities which coexist with and apparently have a causal relation to an excess of uric acid in the urine; and to this excess crystal- 
lization within the urinary reservoirs and passages is presumably due, although the immediate cause or provocation thereof is not known.

In like manner crystallization of oxalate of lime is thought to follow an excess of that constituent in the urine; that excess also is thought to be a manifestation of the uric-acid diathesis, and oxalic acid, like uric acid, is thought to be an irregular product of the normal metabolism. It is of course well known that oxaluria is not dependent upon the ingestion of food containing large amounts of oxalates, but that it habitually coincides with a nervous and depressed condition in sedentary, overfed. gouty individuals.

If cystine is a derivative of uric acid, as is now believed, the formation of calculi composed of it should be referred to the same causes.

An excess of phosphates, sufficient to make the urine alkaline, habitually exists during digestion, and may be constant for hours or days in those who are anxious and overworked, or whose food farors the presence of these salts in the urine.

One immediate cause of crystallization may be accepted as proved, namely, the presence in the liquid of some solid body, no matter how minute, such as a clot of blood, a shred of mueus or of fibrin, a fragment of tissue. It provokes the precipitation upon it of the salts held in solution, and if it so situated as not to be carried away by the flow of urine it becomes the nucleus of a calculus. Many such cases have been reported; many calculi when sawn in two have shown a small central cavity containing the remains of a blood-clot or of mueus; others have formed about a fragment of a tumor of the kidney or of a hydatid cyst; and in one singular case, quoted by Le Dentu, a fragment of the carious second lumbar vertebra had been detached, and had made its way by ulceration into the kidney, and had there beeome covered by a complete shell of urinary salts.

To say that a tendency to gravel or kidney-stone can be inherited, is only to say that a ehild can inherit the constitution of which these are a manifestation.

Statistics that have been eollected to show the relative frequency at different ages, can at the best give only approximate results, for many kidney-stones grow so slowly and give rise to so little disturbanee that they may be borne for years without their presence being suspeeted. The opinion of Morris upon this point has already been quoted; to it may bo added the statisties concerning gravel collected by DurandFardel, 280 patients from well-to-do elasses :

Under 20 years of age, three cases; from 20 to 29 , nine cases; from 30 to 39 , forty cases; from 40 to 49 , thirty-eight enses ; from 50 to 59 , seventy-one cases; from 60 to 69 , seventeen cases; from 70 to 79 , twelve cases. 
Finally, it is much more frequent in men than in women, and in those who lead a sedentary life and are overfed. The influence of certain foods and drinks is occasionally very marked, and there are individuals who can not drink a single glass of Burgundy without having a sharp attack of nephralgia, or perhaps even a paroxysm of kidneycolic.

Symptoms. - To describe in detail the widely different forms under which nephrolithiasis may present itself would require much space and much repetition, and it seems best, therefore, separately to describe the signs and symptoms, and then briefly to indicate the ways in which they may be grouped.

Pain.-A dull, aching pain deeply situated in the small of the back and flank, often unilateral, sometimes radiating along the ureter and into the testicle or the thigh, usually increased by exertion or local pressure, is habitual in irritative or eongestive states of the kidney, and is what is commonly indicated by the term nephralgia. It may coincide with any grade of nephrolithiasis, from that of over-acid urine with a few crystals to that of large calculi with at least partial preservation of the structure and function of the kidney.

Not only may pain radiate to the kidney and thigh, but it may persist in these regions after it has disappeared from the loin, and, exceptionally, it may appear there while the loin remains free; at least, in a number of reported cases patients whose kidneys have subsequently been found to contain ealenli, or who have shortly thereafter passed gravel, have complained of sciatica or of a sharp aching or burning pain in the thigh, calf of the leg, and foot, and the pain has been attributed by those who have reported or quoted the cases to the changes in or the irritation of the kidney. The pain in the testicle has occasionally been accompanied by all the signs of epididymitis.

Instead of a dull ache, the pain in the region of the kidney may be sharp and severe, with remissions or intermittences, or with very sharp exacerbations; and the condition of the patients who are thus afflicted presents all grades of severity between a few rare paroxysms at long intervals and a pain that is almost continuous and so violent that men have committed suicide to end it, and women have aborted because of it. In severer cases opium is not sufficient to relieve, and we must resort to chloroform.

To this paroxysmal form, especially when the intervals are completely free from pain, the term kidney-colic or renal colic is especially applied, and some seck even to restrict the term to those eases in which the maximum of pain shifts its seat along the ureter during the paroxysm, and the other signs point clearly to the passage of a stone or other solid substance through this tube. A paroxysm may begin abruptly without the slight- 
est warning, or it may be preceded by dull lumbar pain, or sometimes by symptoms which apparently have no connection with the urinary passages, but which the patients soon learn to recognize as a warning of an impending attack. 'Thus, in one of Le Dentu's patients, a persistent inclination to go to stool habitually preceded a paroxysm for several hours. The paroxysm lasts for a few hours, rarely a day, and usually ends abruptly, and this abrupt termination is thought in most eases to mark the final escape of the calculus from the ureter into the bladder or back into the pelvis.

There is every reason to acept the explanation as correct in those cases in which the paroxysm occurs only once or at long intervals, and is promptly followed by the eseape of the enlenlus through the urethra; but it is not so easy to explain the paroxysmal pain in those eases in which the autopsy or an operation shows a large stone firmly adherent to the wall, or immovably fixed by its many irregularities in an infundibulum or calix, or a large stone lodged in a dilated ureter. The character of the pain suggests that it is excited by the contractions of the museular wall of the cavity within which it lies, and its cessation would then coincide, in the caso of a fixed stone, with the arrest of these contractions. (On the other hand, quite a number of cases have been reported of late years which indieate that typical paroxysms may oceur withont the presence of a stono or any other solid or semi-solid object in the kidney or ureter, and that consequently it is possible that spasmodic contraction of the ureter or pelvis may be sufficient to eause the pain. Such eases are those in which, after several paroxysms not followed by the voiding of a stone, the kidney has been exposed by incision and has been explored by palpation and by puncture with the needle at many points without disclosing a stone, and this explorntion has been followed by the permanent cessation of the paroxysms. In one of my own eases in which this sequence of events took place, the operation was preceded by an experience which at the time I deemed demonstrative of the presence of a stone in the pelvis. I had caused the patient to be inverted for a moment during a paroxysm, and the pain had instantly ceased. On its recurrence a few days later I operated; the exposure of the kidney to sight and touch was excoptionally good, and I felt that there was no calenlus in the pelvis; the latter was small, and showed rhythmical contractions, about thirty per minute, that continued down the ureter; the latter had a sharp turn in its upper portion. The young man remained under observation for a year without recurrence. $\Lambda$ plansible explanation of the curative eflect of these exploratory incisions down to the kidney has been recently offered in the supposition that the pain is due to temporary distention of the pelvis by urine, the ureter having been temporarily bent upon itself by a slight displacement of the kidney. The cicatrix left by the operation 
fixes the kidney and prevents recurrence. (See Intermitting Hydronephrosis.)

Pressure upon the kidney through the abdominal wall from in front or behind may reveal tenderness; it can be made with the patient in dorsal decubitus, or lying on the opposite side on a round cushion so placed under the loin as to keep the spine straight, or even give it an upward lateral convexity.

Special excitants of the pain are occasionally found in the more violent forms of exercise and errors in diet.

Nausea and vomiting habitually accompany the paroxysms and are probably the result of the pain. Whether or not there is any more direct connection by nerve-reflex between the pelvis or ureter and the gastro-intestinal nerve-center which would enable irritation or congestion of the former to excite anorexia, nausea, or vomiting, has not been established. Some authors-Torres, Morris-believe there is, and claim that these symptoms are very common at times when the pain is not severe, and under circumstances that make such a causal relation the most plausible explanation.

The urine, in cases of simple nephralgia and of gravel without severe pain and other obstructive symptoms, is rather scanty, high-colored, and over-acid. The "alkaline tide," which normally should appear two or three hours after a meal, is absent, and the urine if left to stand will promptly show a bluish line at the edge, and deposit the dark-reddish crystals of uric acid along the sides and at the bottom of the vase. The microscope may reveal, in addition, crystals of oxalate of lime, a few red blood-corpuscles, and possibly epithelial cells that can be recognized as belonging to the pelvis or ureter. (Under the much rarer circumstances of primary phosphatic gravel the urine is alkaline and shows an excess of phosphates. The secondary phosphatic gravel is merely an incident of pyelitis.)

Small stones may be voided with the urine without the knowledge of the patient; the larger ones are usually heard to strike against the side of the chamber, or are passed through the urethra slowly and with pain. Occasionally they lodge in the urethra and have to be removed by the surgeon, or they remain in the bladder and become the nucleus of a vesical calculus. Incredible stories are told of the great size of calculi that have been spontaneously passed per urethram; the largest of which I have seen mention was one said to have been in the museum at Strasburg, which measured ten centimetres in circumference, or about one and a quarter inch in diameter. Le Dentu reports a case in which he removed on one occasion seven calculi from the urethra of a man who had just passed seven others spontaneously. Le Dentu found the eighth lodged in the fossa navicularis, and the remaining six in the urethra behind. After 
enlarging the meatus the stones were easily pushed forward and out. The largest measured fifteen millimetres in diameter, corresponding to No. 46 of the French urethral scale.

The intermittent appearance of a moderate amount of blood in the urine is a constant symptom of a stone that is lodged in the kidney. In fact, the appearance of a small amount of blood, after such exercise as a ride on horseback or over a rough road in a wagon, in urine that is at other times free from it, is deemed almost pathognomonic of a stone in the pelvis of the kidney. Again, it may be present in quantities sufficient distinctly. to color the urine, or even to give it the appearance of being almost pure blood. Long, cylindrical clots of blood are generally thought to have been formed in and to take their shape from the ureter. The source of the bleeding in the slighter cases is probably some slight lesion of the congested mucosa by a passing crystal or small stone; in the more severe cases it appears to come from the vessels of an ulcerated surface.

Mucus and pus, when present in notable quantities, and of kidney origin, are incidents of pyelitis, and are symptoms of nephrolithiasis only so far as the pyelitis is a result of it.

Frequency and urgency of micturition, well-known consequences of renal irritation, are naturally found among the transitory and permanent symptoms of nephrolithiasis. This symptom so commonly has its origin in the bladder that it is very easy to overlook the rarer renal cause unless the physician keeps the possibility in mind. Finally, palpation of the kidney may show its enlargement, or may canse recognizable grating of one stone upon another.

Diminution or total suppression of the urine, lasting for a few hours, has been observed, and is attributed to reflex action upon the vaso-motor nerves of both kidneys even when the calculus is unilateral. In the cases in which it lasts a much longer time it must be attributed to the simultaneous obstruction of both ureters, or to obstruction of one ureter when the other kidney is congenitally absent or has been incapacitated or destroyed by disease. The occurrence is extremely rare. It may follow a well-marked attack of nephralgia or kidney-colic, or may begin insidiously and attract no attention until the symptoms of uræmia set in. The amount of urine passed from day to day may show notable variations, and at times may even be in excess of the normal in consequence of the temporary removal of the obstruction. It differs from the highcolored, scanty urine of partial suppression, and is pale, clear, and of low specific gravity. As time passes the local signs of hydronephrosis may appear, to be followed by œdema of the limbs and uræmia. The average duration of life is said to be from ten to fifteen days. In about one fifth of the cases recovery takes place by spontaneous removal of the obstruction, occasionally by its passage through the ureter. 
The grouping of these symptoms in typical cases of the different kinds will not now require much space. Nephralyia, with or without one or more small gravel-stones, is marked by high-colored, over-acid urine, dull lumbar pain, perhaps radiating to the testicle, thigh, or leg, and a few blood-corpuscles in the urine.

A kidney-stone, small enough to pass through the ureter, but large enough to have some difficulty in so doing, will cause a well-marked paroxysm of kidney-colic, which ends abruptly in a few hours after the seat of maximum intensity of the pain has shifted downward along the course of the ureter. The stone will probably be spontaneously voided within two or three days thereafter.

A kidney-stone retained within the kidney may remain there for years without giving rise to any symptoms that arouse the attention of patient or physician,* or it may cause pain more or less severe and persistent, hæmaturia and the other abnormal conditions of the urine that have been mentioned, and may lead to pyelitis, pyelonephrosis, hydronephrosis, perinephritic abscess, and uræmia.

Course and Prognosis. - The course and prognosis vary in accordance with the conditions already described. An individual who heeds the warning given by over-acid urine, nephralgia, or the passage of gravel or of a kidney-stone, and modifies his mode of life and diet accordingly, may pass the remainder of his life undisturbed by a recurrence; and, indeed, many a man has clung to his habits of self-indulgence for years, and yet shown no other sign and paid no other penalty of his nephrolithiasis than more or less frequent recurrences of the "gravel." So long as the crystals and small stones pass out through the ureter without much delay, the affection usually remains in that stage except for the slow contracting changes in the kidney, which are as much the result of the diathesis as of the local irritation. The one early danger that lies in his path is the arrest of some of the gravel or of a small stone in the bladder and the formation of a vesical calculus. It may happen that the persistent irritation of the pelvis sets up a catarrhal and then a suppurative pyelitis, and the course and prognosis are then those of that grave affection.

If the stone remains in the kidney, various widely different results may follow : it may be perfectly tolerated from the beginning and give rise to recognizable symptoms at no time; or, after weeks or months of pain, it may become encysted or immovably fixed, and thereafter give rise to no symptoms and have apparently no influence upon the patient's health.

* At the autopsy of a middle-aged patient, who died in my service at Bellevue Hospital about three weeks after admission, with "spontaneous" fracture of both thighs, and who presented no symptorns of kidney trouble, a large stone was found in the pelvis of each kidney, one weighing (dry) 152 grammes, the other 59. 
Or it may occasion frequent and violent paroxysms of kidney-colic, which, if allowed to continue, threaten life by exhaustion, and by inflammatory changes in and about the kidney. Or, finally, it may excite a suppurative pyelitis. The prognosis in the last two conditions has been greatly improved of late by nephrolithotomy and nephrectomy.

Diagnosis.-The diagnosis of certain forms of nephrolithiasis is easily made, that of others may be very difficult. It is usually easy to recognize the diathesis that is associated with the common forms, the over-acid urine, and, of course, the passage of gravel or small stones. It may also be easy to recognize a pyelitis, but very difficult to determine whether or not a stone is present, or, if present, whether it is the cause or the effect of the pyelitis. In the earlier stages of the affection the diagnostic problem is to determine the presence or absence of over-acid urine, of gravel, or of small stones; in the later stages it is to determine the presence and extent of important changes in the kidney and the cause thereof. Other and very difficult diagnostic questions may arise in connection with complications or with the predominance of certain symptoms.

The symptoms by which the earlier stages may be recognized are generally definite enough when they are all present, but some of them are closely simulated by those of other affections, and if all are not present the diagnosis may be difficult. Thus, high-colored urine that deposits numerous uric-acid crystals on standing or becomes very turbid on cooling, unilateral lumbar pain, and urgency and frequency of micturition, clearly indicate irritation of the kidney and a condition of the urine that makes the formation of gravel or of a calculus possible, perhaps even probable. If to these are added radiation of the pain to the groin and testicle, distinct tenderness of one kidney on pressure, and some blood in the urine after exercise, or, if blood is habitually present, its diminution or disappearance after rest in bed for a day or two, the diagnosis of a kidney-stone may be made with considerable assurance; and if the history of the patient reveals that he has previously (perhaps long before) passed gravel or small stones, all doubt may be dismissed.

Actual paroxysms of renal colic seldom offer any difficulty, for proper attention to the symptoms will ordinarily distinguish them at once from other conditions with which they might be confounded, such as hepatic or uterine colic, ovarian neuralgia, or a local peritonitis in the iliac fossa. A stone lodged in the ureter near the brim of the pelvis might be confounded with an appendicitis. The diagnosis from intermitting hydronephrosis, due to slight displacement of the kidney and kinking of the ureter, may be made by the absence of hrematuria and by the sudden relief by manipulation or change of posture.

These symptoms, occurring separately, have much less significance. Lumbar pain may be neuralgic, showing maxima at certain points distant 
from the kidney ; or it may be that of a lumbago, and will then be aroused or increased by pressure upon the lower part of the erector spinæ or by movements of the trunk; or it may have its origin in the kidney, but in some condition other than that now under consideration.

Tenderness of the kidney on pressure indicates only sensitiveness or inflammation independent of the cause; for example, it may be tubercular. Le Dentu says that methodical percussion along the twelfth rib, and laterally in the flank, causes pain in lithiasis but not in neuralgia, and is very valuable for differential diagnosis.

Blood in the urine may have many other sources than the kidney, and even after its origin at that point has been established the nature of its cause has still to be determined, for renal hæmaturia is not only a symptom of several other renal affections than calculus, such as malignant or tubercular disease, but it may be occasioned by certain drugs and by certain general or constitutional conditions.

Frequency and urgency of micturition suggest renal disease only after origin in the bladder has been excluded, and do not indicate the kind.

If the symptoms are severe and persistent, and are not relieved by measures which diminish the acidity of the urine or favor the escape or possibly the dissolving of small stones, an exploratory incision in the loin, to determine the presence of a calculus and to remove it if found, is justifiable. An additional warrant for making this exploration is supplied by the relief it has given in a number of cases in which no stone was found. After the kidney has been exposed, the search for the stone is made by palpation and by puncture with a needle. Possibly further experience will confirm the growing confidence in the rapid and completehealing of wounds of the substance of the kidney, and it will be deemed proper to extend the search directly into the pelvis and calices by incision through the parenchyma. (For details of the operation, see Nephrotomy.)

The conditions found in the later stages-pyelitis, pyo- and hydronephrosis, perinephritic abscess, etc.-will be recognized by the corresponding symptoms, to be subsequently considered. Their relation to calculous disease can be established by the history of the case and by examination of the urine, for urine containing pus from a pyelitis is acid, and will remain for days, and sometimes even for weeks, without undergoing ammoniacal change and developing bacteria; the pus settles and the supernatant liquid is perfectly clear.

Simulated Lithiasis.-It is to be remembered that occasionally patients seek to deceive by presenting stones which they claim to have passed with their urine. Broquiart (De la gravelle urinaire simulée, etc., Ann. des mal. des org. genito-urin., 1883) collected twenty-five cases, three of which had come under his own observation. The great ma- 
jority (four fifths) were in girls or women, the remainder in boys. It is frequently associated with hysteria, and is probably connected with perversion of the sexual appetite. Ordinarily the deception can be easily detected.

Treatment.-Here again we must distinguish between the different forms and stages of the disease. In the early stage-that of over-acid urine, more or less nephralgia, and the threatening of stone-a moderate regulation of life and diet, with the occasional use of alkalies, will usually be sufficient. The patient must be abstemious in respect both of the quantity and the quality of the food; he must refrain absolutely from the use of malt liquors and certain wines (champagne, burgundy), should eut down on sugars, should exercise regularly but not immoderately, and should promote the action of the skin by baths and dry friction. He should drink water freely, either the common table water or the lighter mineral waters that are alkaline or diuretic. (For a detailed treatment addressed to the predisposing diathesis, the reader is referred to the more strictly medical treatises.)

The principles of treatment are essentially the same when the affection is more marked and the patient is passing sand or gravel. The formation of crystals and concretions is to be opposed, and the escape of such as form is to be facilitated. The first of these indications is to be met in the manner above stated, but the treatment must be carried out more vigorously and with more attention to detail. Game, shell-fish, spiced foods, regetables that contain an exceptional amount of oxalic acid, such as rhubarb, asparagus, tomatoes, must not be eaten ; the patient must live on milk, eggs, beef, mutton, chicken, fish, rice, potatoes, bread. The use of tea, coffee, and alcohol must be guided by consideration of previous habits and the general condition.

In the choice of a mineral water there are two main points to be considered: First, whether an alkali is desired for its effect upon the system, upon the nutritive and metabolic processes, as well as directly upon the acidity of the urine; and, second, whether a simple diuretic effect is desired. In the first case, the physician will resort to the stronger alkaline waters-Carlsbad, Vichy, Vals-or to some of the lithia waters; in the latter, to Contrexéville, Bethesda, Poland, or even to rain-water in large quantities.

When for any reason these waters are not available, the constituents upon which their efficiency is thought to depend, or their equivalents, may be directly administered; thus, the citrate of potash, in doses of ten to thirty grains three or four times a day, or the citrate or carbonate of lithia in doses of two to five grains. It is not proper to substitute for the diuretic waters such mineral or vegetable diuretics as niter or digitalis. It is alleged that ozonic ether (the ethereal solution of hydrogen 
peroxide) inereases the solubility of uric acid in water, and thus opposes the formation of stone. Edes explains the action on the theory of the breaking up of the uric acid into allantoic and carbonic acid.

Whether or not the effect of the administration of certain alkalies or alkaline waters is wholly or partly to redissolve crystals and stones that have already formed in the kidney, is still an open question. It has often been observed that such administration has been followed by the voiding of much sand, gravel, or irregular small stones, and it is argued that the remedy has brought about the disintegration of a large stone, or has rednced the smaller stones to a size that could be passed through the ureter. Another hypothetical explanation of the occurrence is that the remedy has reduced the size of the stones by removing a coating of mucus; and a third is that, by reducing the inflammation or the irritability of the passage, it has quieted the spasm or increased the distensibility of the wall.

The above treatment is suitable for either form of acid gravel-that is, either the uric or the oxalic-and it is also in great part applicable to the much rarer hard phosphatic gravel or stone. In the latter the alkalies may yield place to the diuretics, and, in addition, the special cause of the phosphaturia must receive attention. Soft, secondary, phosphatic gravel, that dependent upon ammoniacal fermentation of the urine, is an exceptional and usually a wholly unimportant epiphenomenon of serious suppurative disease of the kidney, and its treatment is subordinate to that of the latter. In eases in which palliation alone can be attempted, the fermentation may be measurably restrained by the internal administration of the benzoate of soda, salol, or similar remedies.

Pain that is too severe and continuous to permit waiting for the somewhat tardy effect of the above regimen may be relieved by anodynes, hot baths, and hot applications to the loins. Antipyrine has been recommended as more efficient than opium under these circumstances.

In paroxysms of kidney-colic the pain is too violent for any dallying with half-measures. Morphine must be at once given subcutaneously, and, if that fails to relieve, ether or chloroform must be resorted to. Under their influence the patient is kept quiet and half unconscious, and the paroxysm is apparently shortened, for the relief of pain (and with it, presumably, of spasm of the muscular coat of the ureter) is the most efficient means we possess to hasten the passage of the calculus to the bladder. It has been proposed that large quantities of water be drunk in order to create a greater pressure of urine behind the stone and thus force it along; but as the patient usually vomits constantly during the paroxysm, the treatment could hardly be carried out, even if its efficiency and safety had been demonstrated.

The grave and fortunately very rare complication of obstruction of the ureter by a calculus, when the other kidney is already incapacitated 
or destroyed, may call for prompt surgieal interference because of the mminent danger to life. Either the obstrueting calculus must be renoved, or a way for the escape of the urine must be opened above it. The first of these alternatives will be discussed in the section on the surgery of the ureter; the seeond can be met by a lumbar nephrotomy, with incision of the pelvis of the kidney. This has the advantage of being a comparatively simple and safe procedure, and it may even be possible for the surgeon to remove the obstructing stone by the aid of forceps passed down the ureter, or by the reflux of a stream of water directed into it. By its aid, also, time may be gained, and perhaps a more favorable opportunity for direct approach to the ureter.

Surgical interference is called for when a stone is lodged (or thought to be) in the kidney, and has given rise to important symptoms which have not been relieved by prolonged use of the measures above described; or when suppurative or obstructive changes have taken place and have produced conditions that seriously interfere with health or comfort, or actually threaten life. The interference may be an exploratory incision, in search of a stone; or a nephrolithotomy, the removal of a stone; or a nephrotomy, incision of a diseased kidney for the drainage of purulent or cystic collections; or a nephrectomy, removal of the kidney. Morris thinks it desirable to distinguish sharply between nephrotomy and nephrolithotomy, to limit the use of the latter term to eases in which the operation is done primarily for the removal of a stone from a (in the main) healthy kidney, and to use the former to designate operations in which the main indication is the incision and drainage of a seriously diseased kidney, and the following removal of a stone, if one is found, is secondary and incidental. It is clearly desirable that the cases should be differentiated; but it seems improbable that it will be done by this use of the terms, for it is already usual to describe as a nephrolithotomy any operation in which stone is removed from the kidney without regard to the extent of disease of the organ or to the primary object of the operation.

The justification of an exploratory operation must be found, if at all, in the value of the remedial measures made possible by the information which it furnishes. The remedial measure in the circumstances we are considering is the removal of the stone discovered by the exploration, consequently the exploratory operation must stand or fall with nephrolithotomy. The latter, using the term in the narrow sense recommended by Morris, is of recent origin, having been first done in 1880 by Morris; but it has already been proved an efficient and safe operation; * and as the exploration itself, in case a stone is not found and the kidney is not

* Newman (Surg. Dis. of the Kidney, 1888) collected thirty-eight cases in which calculi had been remored by operation from otherwise healthy kidneys without a death. 
incised, has even less risk, it may, I think, very properly be resorted to with even less hesitation than is usually shown. I refer, of course, to the lumbar operation, not to the one in which the kidney is approached through the peritoneal cavity. Few, I think, would be as conservative even as Le Dentu, when he says that "after a long continuance of the pain, or the frequent recurrence of paroxysms not followed by the expulsion of stones, has shown that the spontaneous or medical arrest of the affection is not to be hoped for, and after the long series of counter-irritants has been exhausted-iodine, the actual cautery, moxas-then begins the rôle of surgery." Unwelcome as is the suggestion of the knife, the actual suffering and danger involved in the resort to it is infinitely less than that of the ghastly series suggested in the quotation, not to speak of the prospect which it holds ont of a prompt and permanent cure in the place of the simple palliation offered by the other. Morris quotes with some bitterness of tone the remark of a medical author, suggested by the occasional spontaneous escape of a calculus by ulceration through the loin: "The cautious surgery of Nature is surrounded by safeguards which rarely attend the rougher operations of art," and points ont that by "Nature" is meant a prolonged, often painful, and always destructive pathological process; he suggests as a substitute for the aphorism the following: "The cautious and well-timed assistance of the surgeon is surrounded by safeguards which rarely attend the operations of disease."

Moreover, as has been already stated, the exploration has itself proved curative in a number of cases in which no stone was found, and in which nothing was done to the kidney except palpation and perhaps puncture with a needle. In one of Le Dentu's cases the needle touched stone at first, but, as it conld not be found again, the operation was carried no further; the next day the patient passed several gravel-stones through the urethra, having never done so before, and remained thenceforth free frum the continuous pain that had led to the operation. The case is hardly sufficient to justify Le Dentu's proposed establishment of another kind of operation, dislodgment of stone. In like manner, the relief which has followed in a few cases in which the fibrous capsule of the kidney has been intentionally split does not appear to have been greater than that which has followed simple exposure of the kidney; more definite experience is needed before it can be formally recommended.

It seems, then, to be justifiable to recommend an exploratory lumbar incision to a patient who is suffering with a constant or recurring pain, probably of calculous origin. It is objected that this is a confession of inability to make a diagnosis. If the inability exists, it had better be confessed; and, moreover, the measure is the only thing that holds out any promise of supplying the information that is needed for a diagnosis. 
Nephrolithotomy; Nephrectomy.-The presence of stone having been determined, it should be removed, either alone or with the kidney. The choice depends partly upon the condition of the kidney, whether it is capable of sufficient functional activity to make its retention desirable, and if existing abscesses can be satisfactorily drained, and partly upon the possibility of making a complete and safe removal of the stone or stones. As a general rule, the kidney should be left if there is reason to believe that any considerable portion of it can do its work. If the abscesses do not heal, if a urinary fistula forms, a secondary operation can be done and the organ removed more easily, often, than by the primary operation. On the other hand, if any stone is left behind, a second operation will certainly be required for its removal, and there is no reason to suppose that its removal except by nephrectomy is any more probable than at first. The details of the operation will be described in another chapter. I shall here only call attention to the frequent difficulty of recognizing the presence of a stone, and the consequent necessity of making a very careful and systematic search for it by pressing upon all parts of the kidney with the finger, or, if possible, between the finger and thumb, in order to recognize differences of resistance, and by puncturing with a needle through almost every square centimetre of its surface. In cutting for a stone, it is better to make the incision through the parenchyma than through the wall of the pelvis; the bleeding is not severe, and the probability of the establishment of a urinary fistula is much less.

The treatment of cases in which the most prominent condition is a pyelitis, or pyelonephritis, or perinephritis, or in which a renal fistula leading down to a stone exists, will be considered in the corresponding sections.

\section{PYELITIS, PYELONEPHRITIS, PYONEPHROSIS, NEPHRITIS, TUBERCULOSIS.}

\section{A. PYELITIS, PYELONEPHRITIS, TUBERCULOSIS.}

As the result of the most varied general and local causes, an acute, subacute, or chronic catarrhal or suppurative inflammation may attack the pelvis and its prolongations or the parenchyma of the kidney, and may spread from either to the other. Pyelitis is an inflammation of the pelvis; a nephritis is an inflammation of the kidney itself; and a pyelonephritis is a combination of the two. In pyonephrosis the distended pelvis and the thinned and atrophied kidney together form the irregular lobulated wall of a cyst with purulent contents. In certain forms the affection may exist for months, or even years, without giving rise to symptoms that attract much attention, and without seriously affecting the health of the patient; in others, it is one of the last manifestations of a 
general and fatal illness, as in the infectious fevers, pyæmia, etc.; in others it is an extension, perhaps unnoticed or unrecognizable, of neighboring local disease, as ascending pyelitis following cystitis, and is dominated in importance by the latter; while in others it may be a purely local and isolated affection, as in calculous and sometimes even in tubercular pyelitis, and one which in itself deserves all the attention and care that can be given it.

It is mainly an affection of adult life, although occasionally seen in childhood, and is much more frequent in men than in women, because of the great preponderance in the former of conditions obstructive to the escape of urine from the bladder.

Etiology.-The most common causes are the extension of inflammation from the bladder, obstruction to the flow of urine through the ureter or from the bladder, nephrolithiasis, especially the retention of a calculus in the pelvis, and tuberculosis of the kidney. The rarer causes are traumatism, many general and infectious diseases, the ingestion of certain irritating drugs, foreign bodies in the pelvis, and possibly chilling.

The possible occurrence of pyelitis as a consequence and complication of a traumatism has been mentioned in a preceding chapter. Like other suppurative complications of wounds, it is the result of infection by pyogenic germs, nsually through an open wound, which by providing at the same time an outlet for the pus separates this form widely from the others. The traumatism may first set up a suppurative perinephritis which ruptures into the pelvis.

Another rare local cause, somewhat akin to a traumatism, is the presence of a foreign body, possibly introduced from without, once a fragment of bone from the carious second lumbar vertebra, parasites of different kinds, blood-clots, malignant tumors. These appear sometimes to act by the simple irritation of their presence, at other times by interfering with the flow of the urine from the kidney and thus causing distention of the pelvis.

Certain irritating drugs-cantharides, turpentine, cubebs-may, in the course of their elimination by the kidneys after having heen taken into the stomach, set up catarrhal pyelitis, which, however, is usually prompt to disappear on cessation of the cause.

The group of general causes contains all the acute infectious diseases, osteomyelitis, typhoid, diphtheria, puerperal septicæmia, pyæmia, cholera, etc., and also others, such as carbuncle, scurvy, Bright's disease, and diabetes mellitus. In many of these there is but little tendency to suppuration; the pyelitis remains catarrhal, and without notable influence upon the course of the primary disease; in others, the process begins as multiple foci of suppuration in the substance of the kidney, the small collections run together to form large cortical or medullary abscesses, 
and by the rupture of the latter into the pelvis a suppurative pyelitis is set up.

Some authors claim that a spontaneous pyelitis, the result of chill. ing, is not uncommon; thus Rosenstein attributes the frequency of pyelitis in Holland to its damp climate. Most authors quote the opinion with much reserve, although the occasional action of the cause is admitted (A. Robin, De la pyélonéphrite primitive, Gazette médicale, 1885, p. 215).

The most common local cause is nephrolithiasis, which can set up a pyelitis (and pyelonephritis) either by irritation or by obstruction; that is, the over-acid urine, or the recurring crystals and gravel, or the retained calculus, may irritate the mucosa of the pelvis and thus originate a catarrhal pyelitis, which may remain such for an indefinite time or may become suppurative; or the retained stone may obstruct the escape of urine from the pelvis, and give rise to the common pathological changes through the mechanism of over-distention.

The same consequences may follow obstruction of the ureter by a lodged calculus, the pressure of a tumor, or of the gravid or otherwise enlarged and degenerated uterns), or, more remotely, obstruction to the escape of urine from the bladder (enlarged prostate, tight urethral stricture); but in the cases of obstruction to the outflow from the bladder the pathogeny is complicated by the addition of another factor, namely, the extension upward along the ureter to the pelvis of the inflammation that is first set up in the bladder by the obstruction. This class of ascending pyelites is a large one, recruited as it is so freely from the vast number of elderly men who are affected with prostatic disease, and also from those younger men who have long neglected their strictures. To it are to be added also the eases of direct extension of a gonorrhœa from the urethra through the bladder and ureter to the kidney. Whether such gonorrhœal cases are numerous, or so rare as almost to be unknown, is still in dispute. Fournier says he has never seen a case; Keyes speaks of them as not rare. I presume that all those who question the fact admit that a gonorrhœal cystitis is sometimes followed by a pyelitis, and that their denial is only of the gonorrhœal character of that pyelitis, of the presence in the kidney and direct agency of the gonococci.

A remaining and far from infrequent cause is tuberculosis; and, although the process when thus originated is essentially chronic from the beginning, it bears a close resemblance in lesions and symptoms to the other common varieties. The form of tuberculosis which we have in mind, and which alone will be here considered, is that marked by slowly developing cheesy nodules, with changes in the neighboring tissues similar to those in and about tubercular joints; we shall not con- 
sider the acute miliary tuberculosis (the disseminated tuberculosis of Morris) which appears by preference in the young, is characterized by multiple minute miliary nodules scattered through the organ, is usually part of an acute general tuberculosis, gives rise to no symptoms specially referable to the kidney, and calls for no special treatment.

Some idea of the relative frequency of tuberculosis of the kidney may be gained from the following statistics, although they are by no means in accord. Morris (loc. cit., page 474) states that in 2,610 postmortem examinations made in the Middlesex Hospital in the course of ten years, 44 showed tubercular disease of the kidney, of which 29 were of the miliary form and 15 of the cheesy. Steinthal (Virchow's Archiv, 1885, vol. c, p. 81) states that 28 cases of tuberculosis examined in the pathological institute at Geneva showed implication of the kidney in 18, in 16 of which the form was miliary. The latter author made a careful analysis of 24 cases of cheesy tuberculosis of the kidney, and, although the number is perhaps not large enough to justify very positive generalization, his conclusions are interesting and valuable. They are, that with tuberculosis of the kidney is always found tuberculosis of the lungs or bronchial glands, possibly quiescent, and that the latter is primary. The first infection of the kidneys is commonly in the medullary substance, at the base of the pyramids, and it spreads thence to the cortex and into the calices and pelvis. After the process has reached the free surface of the urinary passages it spreads with great facility along them, apparently by transport of the bacilli in the urine, for intervals of uninfected mucosa are found, and when the bladder is reached it develops first and most rapidly in the trigonum immediately about the orifices of the ureters. In the kidney the process may continue until the organ is completely destroyed, transformed into a bag of cheesy pus. In the pelvis and ureter the process may be ulcerative on the surface of the mucosa, or may show itself only in great thickening of the wall. The process rarely extends to the testicle, and tuberculosis of the testicle rarely extends upward to the kidney. In 12 of the 24 cases both kidneys were involved, but not to the same degree. Of Morris's 15 similar cases both kidneys were involved in 8 ; the ages show the affection to be one of young and middle adult life, although it occurs in the old.

Pathology.-From the anatomical stand-point the different forms of pyelitis may be classified, following Cornil, as (1) catarrhal, (2) acute or chronic purulent, and (3) calculous.

The catarrhal form shows a thickened and injected mucosa with rapid formation and desquamation of the epithelium, and the process involves also by direct propagation the tubules of the pyramids. In the more severe forms there is a fibrinous exudation, that adheres in part to the surface and in part is carried away as shreds in the urine. 
In the acute purulent form there is a round-cell infiltration of the wall of the pelvis, the mucosa is greatly thickened and irregular, with numerous small extravasations of blood and scattered points of superficial sloughing and uleeration. The kidney substance on seetion shows small white streakings through cortex and medulla, and later punctate foci of suppuration or larger abseesses formed by confluence of the small ones. The suppuration appears to begin in the pyramids by the agency of bacilli that have made their way directly into the tubules from the pelvis. The pus that forms on the free surface is either carried away in the urine, or, if the ureter is obstructed by the inflammatory swelling of its own wall or by a fibrinous plug, it collects within the pelvis.

The chronic purulent form may be the later stage of the acute, or it may be chronic from the beginning. It gradually destroys the parenchyma

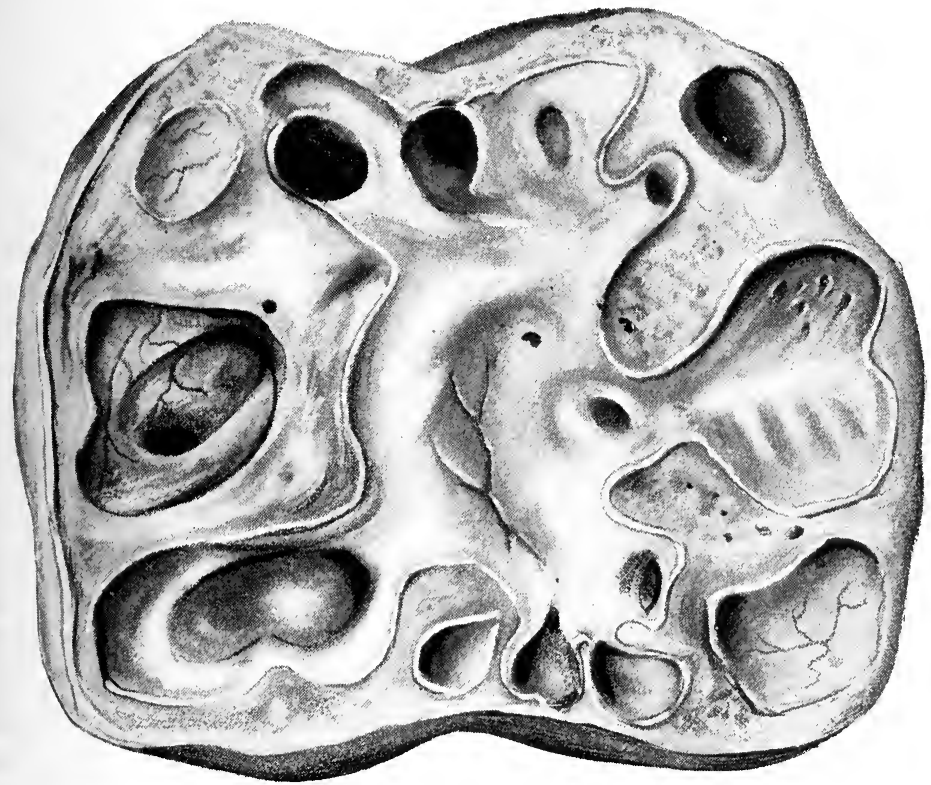

Fig. 5.-Calculous pyelitis. (Rayer.)

of the organ, partly by suppuration and partly by the formation of interstitial connective tissue, and as the escape of the eontents of the pelvis through the ureter is always opposed and at times entirely prevented, more or less distention of the kidney ensues. The resistance of eertain portions of the parenchyma to this distention eauses it to be irregular, with formation of pouches of variable size separated by partitions and spurs often of notable thiekness (Fig. 5). The mueosa may be smooth, 
velvety, or papillomatous, pale, injected, or so vascular that hæmorrhages are frequent and profuse.

If the obstruction to the escape of the contents is permanent, the condition becomes that known as pyonephrosis, and the kidney is transformed into a vast irregular sac filled with pus.

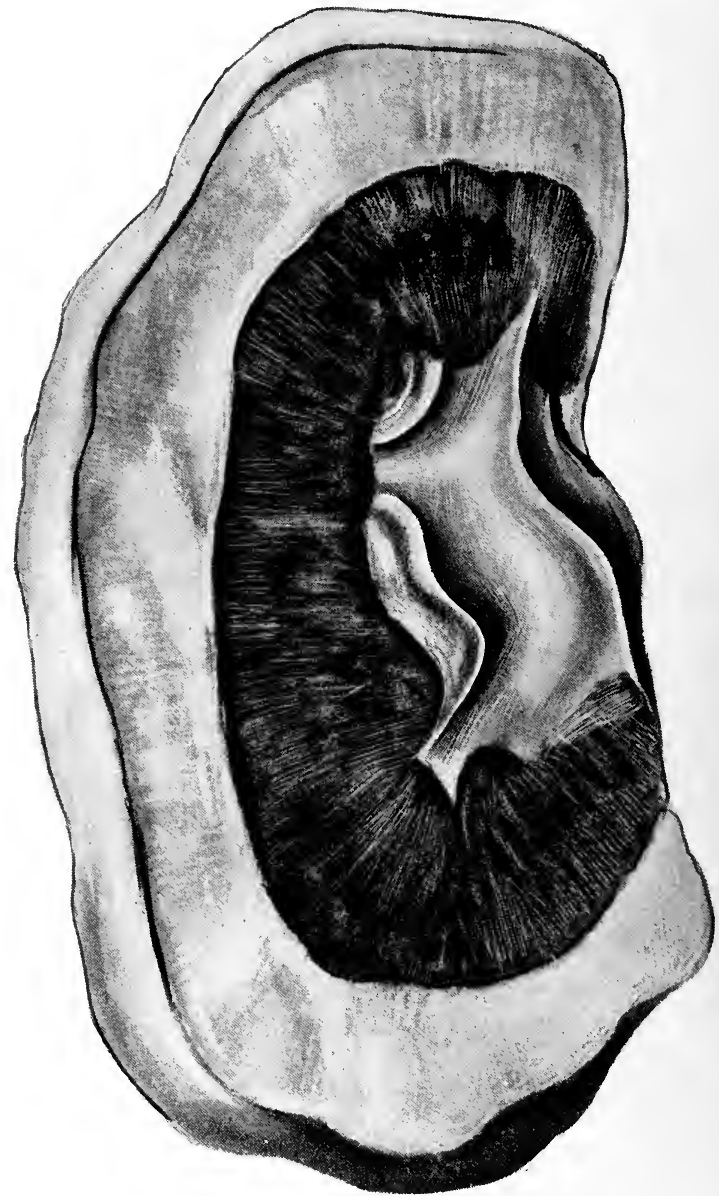

Fig. 6.-Pyelonephritis; the outer layer is the thickened and condensed fatty capsule. (Rayer.)

The process involves the adjoining tissues either by ulceration and perforation of the wall of the pelvis, or perhaps simply by bacillary infection, with production of a perinephritic abscess, or more slowly by direct non-suppurative extension, with constant thickening and condensation of the fatty capsule, that may be so marked that it is transformed 
into a thick, fibrous layer that bears no resemblance to its normal condition (Fig. 6). The same process may result in the formation of abnormal adhesions to neighboring parts, such as the peritonæum, loin, intestines, and vena cava, that create important difficulties and dangers in case nephrectomy should be attempted.

In case the imperfectly evacnated urine undergoes ammoniacal fermentation, the triple phosphates are formed and precipitated, often in large amount; they may form a putty-like mass, or adhere to the wall, or mat together in large, friable stones.

The calculous pyelitis is restricted to those cases in which the formation of the stones precedes and causes the pyelitis, in contradistinction to those just mentioned, in which the formation of a phosphatic stone is a late epiphenomenon in the course of a pyelitis. The pyelitis thus set up is at first catarrhal, and may long remain so, and, as was explained in the preceding chapter, may be so slight as to give rise to few or no recognizable symptoms. It may, however, become suppurative, and is thought to be more especially prone to ulceration and consequent perinephritic abscess. The sudden and permanent blocking of the ureter by the calculus occasions prompt distention of the pelvis by the urine that is subsequently secreted (hydronephrosis); this may result in a simple interstitial, non-suppurative nephritis, with progressive atrophy of the parenchyma, or, in case of bacillary infection, in diffuse or circumscribed suppuration.

In the tubercular form the process begins, as has been said, with the formation of one or several nodules (ultimately cheesy), usually in the medulla, at the base of the pyramids, but occasionally in the cortex ; if suppuration ensues in them, the nodules become abscesses, and, according to the varying activity of the process, either remain as independent, purulent, cheesy foci, or coalesce. At the same time new nodules form and pursue the same course, with increasing destruction of the parenchyma, and finally rupture to the exterior, or more commonly into the pelvis. The destruction of the kidney may be complete, the thickened and tubercular capsule inclosing only pus or a mass of friable or putty-like, cheesy matter. The wall of the pelvis and ureter is greatly thickened by tubercular degeneration, and the mucosa correspondingly degenerated or ulcerated.

Symptoms. - In describing the symptoms of pyelitis and pyelonephritis, it will be advantageous to treat separately of the acute forms; of those chronic ones in which the affection is of local origin and uncomplicated by disease of the bladder ; and, finally, of those, the "ascending" cases, in which it is a complication and result of cystitis.

In the acute form, which is rare, the onset may be sudden, with chills, headache, fever, nausea, the usual signs of an acute visceral infiammation, accompanied or promptly followed by local signs and changes in the 
urine which show the kidney to be the organ involved, namely, pain in the loin, tenderness on pressure upon the kidneys, frequency of micturition, increase of mucus in the urine, possibly hæmaturia, and the presence of the small, tailed, imbricated epithelial cells characteristic of the pelvis. If the case is one of pyelonephritis, the urine is diminished in quantity, and albuminous.

In the chronic form of local origin-for example, the pyelitis of nephrolithiasis - the catarrhal stage is marked mainly by nephralgia and local tenderness and by changes in the urine, which latter become more marked and varied as the affection passes into the purulent stage. The quantity of the urine may be notably increased, at first without change in its appearance, but later it becomes turbid by the admixture of pus. It remains acid, and if allowed to stand may retain its acidity for days, while the pus sinks to the bottom of the vase and there forms a greenish, oily-looking stratum; and the supernatant urine remains perfectly clear, without development of bacteria-a group of characteristics which absolutely distinguish the affection from any of the forms of cystitis. There may be a few exceptions to this rule, as where the kidney is almost destroyed as a secreting organ, and the urine, retained for a time within its dilated pelvis, has had an opportunity to undergo ammoniacal fermentation. The urine is albuminous, sometimes more so than the amount of pus would account for, and contains mucus, pus, possibly blood, and epithelial cells, which are thought to come from the tubules of the kidney and to be pathognomonic of the affection. These cells are round, with a single nucleus, and about one half larger than a leucocyte. Accepting the theory of their origin in the tubule, their presence indicates the extension of the inflammation to the parenchyma of the kidney. Some of the pus-cells also are agglutinated in short, cylindrical masses, as if they too had come from the tubules. The amount of pus in the urine may vary from day to day, and may even be intermittent, the intermittences coinciding with exacerbation of the local and general symptoms, and being due to the temporary obstruction of the ureter.

The amount of blood in the urine varies greatly in the different cases and at different times in the same day. It is habitually absent in some, habitually present in others, and is most frequent and most marked in calculous pyelitis, in which it is easily produced or increased by such causes as sudden movements, muscular efforts, and jolting. The blood, when in small quantity, is uniformly distributed through the urine; when abundant, it may be in clots which are sometimes long and cylindrical, as if formed in the ureter.

Dull pain in the loin is constant, with occasional exacerbations; to it may be added the pain excited by the presence of a calculus, or by the difficult passage through the ureter of a large fibrinous plug. 
More severe pain is provoked by pressure upon the kidney through the anterior abdominal wall, or by percussion in the loin and along the floating ribs.

Bimanual palpation may show the kidney to be enlarged; and if a perinephritic abscess has formed, it may cause a swelling in the loin that is recognizable by the eye as well as by the hand.

The general symptoms vary greatly. So long as suppuration is absent or slight, the general condition remains fairly good ; but as the disease advances the health deteriorates, there is fever every evening, chilly sensations, perhaps a regular chill; the patient loses his appetite, and grows pale, thin, cachectic.

In the ascending chronic form, that due to extension of inflammation of the bladder, the same symptoms are present, but they are in great part masked by those of the cystitis; and, as Morris says, "pyelonephritis, when secondary to disease of the bladder, is too often unrecognized until discovered at the post-mortem examination." The frequency of micturition, the pus, mucus, and blood in the urine, the fever, and the deterioration of the general condition, are all equally symptoms of cystitis, and the ammoniacal change which the urine undergoes in the inflamed bladder destroys the combination of pus and acidity which is so characteristic of pyelitis, even if the ammoniacal ferments have not already made their way up to the kidney. There remain only the local symptoms in the region of the kidney to point to its association in the disease. It may be said, however, that a sudden change for the worse-fever, dry tongue, quick, small pulse, chills, prostration-in a patient who has long been suffering with cystitis, especially of obstructive origin, clearly indicates the appearance or the dangerous increase of a pyelonephritis.

The following description of such patients is quoted from Halle: "Pale, with yellow, earthy, dry skin, our patients rapidly lose strength and are soon confined to the bed. The digestive functions are profoundly affected. Appetite is completely absent; there is often even an actual repugnance for food. Only liquid food can be taken, and the digestion of that is often difficult. Nausea and gastric distress at once follow the least attempt to take solid food. On the other hand, thirst is great. The tongue, usually white and coated, becomes red and dry during the febrile exacerbations, which ordinarily indicate extension of the nephritis. Fever is not constant. There are long periods of apyrexia, especially when the affection is left to take its natural course. Then, there are irregular febrile movements, the cause of which is always easily to be found. A transient retention of pus in the kidney, the slightest surgical traumatism inflicted on the urethra, causes a chill and high fever."

Course and Prognosis. - These are largely determined by the cause of the affection and by the extent to which the kidney parenchyma is 
involved. The acute forms due to the action of irritating drugs cease promptly on the cessation of the cause. Those that occur in the course of infectious diseases are rarely severe or serious in themselves except when the primary disease is almost inevitably fatal. If the patient can recover from the latter, the pyelitis is not likely to last long or to give hims much trouble.

There is a similar relation with the cause in the chronic form. A calculous pyelitis will last so long as the stone remains in the kidney, and one that is the result of an obstruction in the ureter or urethra will last while the obstruction remains. Unfortunately, we can not add that it will always cease on the removal of the cause, but the affection may persist in one form or another even for years without endangering life. This is especially the case when only one kidney is involved, and when the process in it has advanced so far that the function is entirely abolished, and the organ is reduced to a sac of inspissated pus, or to a shrunken abscess wall that discharges a moderate amount of pus through some spontaneous or artificial opening. Such suppurating sinuses are, of course, as potential for evil when they lead to a degenerated kidney as when they have originated under other circumstances. There is the same possibility of retention of the pus, of septicæmia, amyloid degeneration, erysipelas, etc.

Cases are not uncommon in which a pyelitis that had advanced even to the condition of a pyonephrosis has ceased to give any symptoms after a single or perhaps repeated evacuation of its contents through the ureter, or through a spontaneous opening into the intestine or the lungs or through the skin. Strange as it may seem, not a few cases of recovery by spontaneous opening into the lung and evacuation of the pus through the mouth have been reported. Opening into the intestine is thought not to be quite so favorable; that through the skin of the loin more so. Still, the prognosis in all purulent cases without surgical treatment is bad. It is worse in some than it is in others. It is worse when both kidneys are involved, worse in pyelonephritis than in pyelitis, and worse when the pus is fetid and can not be freely evacuated. Under surgical treatment the prognosis is notably improved, and in some cases it may be fairly claimed that the interference restores the patient to health. Even if it does not do so much as that, it often removes an immediate serious danger and enables the patient again to become self-supporting. The benefit is particularly clear in calculous and tubercular pyelitis. In the one, the removal of the stone is the removal of the cause, and may be followed by complete restoration to health; in the other, the removal of the diseased organ-nephrectomy-is the removal of a focus of incurable disease, and, while it may not prevent later manifestations of that disease at other points, there is good reason to believe that it delays them, and that it has 
prevented in a considerable proportion of cases an early outbreak of miliary tuberculosis.

Diagnosis. - The difficulties in diagnosis are mainly found in distinguishing between pyelitis and cystitis, or in recognizing the co-existence of the former with the latter and in distinguishing between the different forms and causes. Not to repeat the account of the symptoms already given, I shall only call attention again to some of the more important diagnostic points.

Pyelitis has, in common with cystitis, frequency and urgency of micturition, pus, mucus, albumen, and blood in the urine, and in the severer forms the general symptoms associated with fever. The distinguishing features (not all present in every case, of course) are, in pyelitis, sndden changes in the amount of pus; the presence in the urine of epithelial cells peculiar to the kidney, and of cylindrical masses of agghtinated pus-cells resembling urinary casts; acidity of the urine, which persists for several days if the urine is allowed to stand; absence or very tardy appearance of bacteria in the standing urine, and consequent clearness of the urine above the thick, greenish, oily deposit; purulence of the urine drawn by the catheter during ten or fifteen minutes immediately after the bladder has been thoroughly washed; pain and tenderness on pressure in the region of the kidney, and perhaps its recognizable enlargement.

The intercurrence of pyelitis in the course of a cystitis is indicated by a sudden change for the worse, especially if the patient has just been subjected to some instrumentation or operation upon the urethra or bladder. The characteristics of the change have been described above.

Ascending pyelitis, calculous pyelitis, and tubercular pyelitis are to be distinguished from one another mainly by the clinical history. In the first, if the patient is an elderly man, there is enlargement of the prostate and a cystitis ; if younger, a long-existing tight strieture of the urethra, or a history of gonorrhoa and cystitis. In the calculous form there may be a history of the passage of gravel or of nephritic colics, perhaps many years before. In the tubercular form there is the family history, possibly evidence and history of tubercular trouble in other organs, and possibly the recognition of tubercle bacilli in the urine by the microscope, or their demonstration by inoculation upon an animal.

If the kidney forms a large tumor, and the question is between a pyonephrosis and a solid tumor, the aspirating-needle should prove the presence of pus, and possibly of stone or tubercle, or an exploratory incision make the condition plain, and at the same time prepare the way for appropriate treatment.

Treatment.-The principle of treatment which indicates the removal of the cause of disease wherever practicable, and when the cause is a continuing one, has an important corollary in the present instance, 
namely, prophylaxis. The prevention of pyelitis is possible to a considerable extent in those classes which furnish the largest number of casesthe calculous and ascending forms. For the former the reader is referred to the chapter on Nephrolithiasis, to the measures recommended to correct over-acidity of the urine and diminish the tendency to the formation of stone. In the latter, in which the pyelitis is secondary, through cystitis, to an obstruction to the flow of urine from the bladder, especially enlargement of the prostate and stricture of the urethra, prophylaxis consists not merely in the removal of the obstacle or the cure of the cystitis, but also in the removal in such a way that it will not itself be the occasion for the outbreak which it is designed to prevent. It will be remembered that a slight urethral traumatism or surgical operation upon the urethra or bladder is often the immediate determining eause of pyelitis in those who have been prepared for it by long-continued obstructive disease of the bladder or urethra. Consequently, such operations must be avoided so far as possible, and, when necessary, must be done in such a way as to reduce the risk to a minimum. The urinary passages should be disinfected, so far as that can be done, by the internal administration of drugs. Among those which are believed to be thus efficient are salol, the benzoates, and especially diurethin, or, what appears to be its equivalent, sodiosalicylate of theobromine, in ten-grain doses every four hours. If the cause of the obstruction is a deep stricture of the urethra, it should unquestionably be divided only by perineal section. Internal urethrotomy or divulsion is full of danger. Chloroform is to be preferred to ether, and local anæsthesia by cocaine is vastly better. If the patient is an old man and the obstruction is at the prostate, if there is considerable residual urine, or if the cystitis is severe and the urine fetid and ammoniacal, suprapubic eystotomy is, in my judgment, much the safest way to establish drainage and disinfect the bladder.

In the acute pyelitis of cantharides, turpentine, etc., and the rare spontaneous form, rest in bed, diluent drinks, hot fomentations, an alkali to diminish acidity, and enough opium to relieve pain, constitute the treatment; and there is not much more to be added for the general and in. ternal treatment of the chronic forms. Difficult as it may be to appreciate it when we think of the marked alkalinity of the urine when it is passed, it is habitually acid at the kidney, and the administration of an alkali will ordinarily relieve pain and diminish the urgency of other symptoms. Benefit sometimes appears to follow the administration of tannic acid in two-grain doses when there is much mucus, and of small amounts of quinine and opium (Morris). When there is polyuria, Morris recommends $3 \mathrm{ss}$. doses of the liquid extract of ergot. The muriated tincture of iron in large doses has also been claimed to have a beneficial effect. 
The surgical treatment includes measures undertaken to remove ob. structions to the free escape of urine from the bladder, to relieve cystitis, to remove renal calculi, evacuate renal abscesses, or to remove the kidney itself.

The methods employed to carry out the first two indications will be described in other sections of this book, and we have here to consider only the indications for nephrotomy, or nephrolithotomy and nephrectomy.

Nephrolithotomy is clearly indicated in calculous pyelitis when the general condition is seriously affected by persistent pain or suppuration, or by the establishment of a pyonephrosis, which is in itself an indication for surgical interference. Probably a near future will establish wider indications for the operation, and show the advisability of the removal even of stones that cause but few symptoms. Occasionally it will happen that the calculi are so numerous, and so encysted or so adherent, that it will seem better to remove the kidney, to do a nephrectomy rather than a nephrotomy. The relative advantages of the two operations will be discussed in detail elsewhere, and it is sufficient here simply to indicate some of the points to be considered. On the one hand, there is always a doubt as to the condition of the other kidney and its ability to do all the work that would be thrown upon it by the removal of the first, and there is the greater danger to life; on the other hand, it can be claimed with some justification that if any stone is left the operation is practically a failure, that the pyelitis will persist, with an added sinus. Increased experience will probably enable us to draw the lines more sharply.

The second clearly established indication for surgical interference is found in the existence of renal abscess, pyonephrosis, with marked general symptoms; and here, too, the choice is between nephrotomy and nephrectomy. Probably here, too, increasing experience will show the advisability of interfering even when general symptoms are not marked, for a change for the worse may rapidly supervene, and a condition arise in which operation is powerless to save. The conditions or symptoms which are at present accepted as positive indications for operation are intermittent pyuria, with fever during the intermissions, persistent fever, and other signs of septicæmia, notwithstanding the constant escape of pus and increase of the tumor coincident with the disappearance of pus from the urine.

The preferable operation, in the light of our present knowledge, is clearly nephrotomy. Aspiration may relieve temporarily, and repeated aspiration may occasionally cure, but nephrotomy is not more dangerous, and is more efficient. As between nephrotomy and nephrectomy, the choice is at present decidedly in favor of nephrotomy as the easier and safer operation, and also because it makes a subsequent resort to nephrec- 
tomy safer than if that operation had been done primarily. This is satisfactorily established by the statistics already at hand. Doubtless there are cases where the kidney is so completely destroyed as a secreting organ, and where it is so loosely held within its fatty capsule, that its removal involves no additional risk, and, as it favors rapid and complete recovery without persistent fistula, it is then to be preferred. There was a tendency at first to make it a rule that if both kidneys were diseased only one of them should be operated upon, and the operation should be a nephrotomy; but experience has not only confirmed the frequent difficulty of determining the condition of the second kidney, but it has also shown that nephrectomy can be successfully done when the second kidney is undoubtedly diseased. The extent of the disease in the kidney to be operated on is of more importance in determining the choice, for if it has wholly lost its function as a secreting organ, its removal is not the removal of a kidney, physiologically speaking, and throws no additional work upon the other.

In tubercular disease fairly limited to the kidney, nephrectomy finds a special indication, the removal of a focus of disease in which a general infection may originate. König thinks the operation is clearly called for, and adds that its execution appears to be somewhat easier because of the loose attachment of the fatty capsule to the kidney in these cases.

\section{B. PRIMARY SUPPURATIVE NEPHRITIS.-GANGRENE OF KIDNEY.}

Primary suppuration of the kidney is very closely allied by its causes and symptoms to pyelitis, and the only forms in which surgical treatment is indicated are those in which the differential diagnosis, even postmortem, is difficult or even impossible.

The affection usually appears as a complication and result of the more serious infectious fevers-typhoid, osteomyelitis, ulcerative endocarditis, diphtheria, pyæmia, and septicæmia, especially when it has originated in disease of the bladder or urethra. It is therefore an infectious disease in a stricter sense than that which would include all suppurations. It may also follow a traumatism of the kidney limited to the parenchyma.

The suppuration may be diffuse or circumscribed, with the formation of a larger or smaller abscess. In the former case the kidney on section is yellow, exudes pus on pressure, and is friable; the microscope shows a general round-cell infiltration and a tendency to the collection of the leucocytes in miliary abscesses; if sufficient time elapses, large abscesses may form, giving the kidney an appearance similar to that observed in some forms of pyelonephritis - that is, it is a mass of cavities separated by complete or incomplete septa, and containing pus. The circumscribed form may be a stage of the preceding, the coalescence of miliary foci to 
form a few abscesses, or it may be the result of an infection limited to a few points, with the same result.

Ordinarily these abscesses increase until they open into the fatty capsule or into the pelvis, or more rarely into the peritoneal cavity or the intestine; but they may cease to grow, and undergo partial absorption and caseation of the residuum. It is claimed also that transformation into a serous cyst is possible.

Symptoms. - The symptoms are in the main those of severe pyelonephritis, except for the absence at the beginning of pus or renal epithelium from the urine; these appear as soon as the pelvis becomes secondarily involved and the abscess opens into the pelvis. The general symptoms are severe-high fever, chills, lumbar pains, and perhaps the rapid development of a typhoid condition. In the chronic form the symptoms may be less severe and too vague to permit diagnosis.

In two cases quoted by Simon (loc. cit., second part, page 173) a piece of the kidney of considerable size, one weighing 20 grammes, the other as large as a pigeon's egg, was passed per urethram. One patient recovered; the other died eleven weeks later, and the autopsy showed that both kidneys were affected, and that in each there were several half-detached fragments similar to that which had been voided; this patient was a boy eleven years old, and the nephritis was secondary to an attack of scarlatina.

Gangrene of the whole or a large part of the kidney has also been noted.

Treatment.- The treatment at the beginning can be only symptomatic, even if a diagnosis that is more than a suspicion can be made. If the patient survives long enough, and the abscess grows to a sufficient size to be recognizable, or if it breaks through and becomes a perinephritic abscess, the evacuation of its contents by the knife is indicated. The condition then is so nearly that of a pyonephrosis that the same principles govern the method of treatment.

\section{PERINEPHRITIS, PERINEPHRITIC ABSCESS.}

By perinephritis is meant an inflammation in the connective and adipose tissue about the kidney. Although the condition was known to the ancients, and is mentioned by several of the older classical writers, the first systematic description was by Rayer (1839), and it is only since 1860 that it has received much attention. It is apparent, too, I think, that much that has been written about it will need revision, especially in respect of the classification and etiology; for some of the recorded cases, described and quoted as perinephritic abscesses of "spontaneous" or unknown origin, appear now to have belonged to that affection, appendicitis, of which our knowledge has so greatly increased during the last few years. 
The pus that has formed about an inflamed and perforated appendix not infrequently spreads upward to and is opened in the region of the kidney; it is to that extent a "perinephritic abscess," "an abscess in the connective tissue about the kidney," but nothing is to be gained, and much may be lost in precision of knowledge and in treatment, by grouping such cases with those which originate in the neighborhood of the kidney, by giving them a name which suggests a kidney origin, and by taking them out of the class of inflammations of the appendix. So, too, in the case of inflammations in the region which originate in some other organ than the kidney, as the liver, the intestine, the body of a vertebra, it seems to me desirable to classify them in accordance with their origin rather than with the accident of their location, although, of course, so long as that origin remains unknown we can have no other name for them than perinephritis. It has been proposed to give them a name which defines the situation equally well, and yet conveys no suggestion of origin-to call the affection abscess of the lumbar fossa. The suggestion has been accepted by some writers of authority, but has not yet established itself. With this reservation we may follow the usual etiological classification, and group the varieties of perinephritis as follows (Le Dentu):

1. Primary perinephritis. $\left\{\begin{array}{l}a . \text { Traumatic. } \\ b \text {. Spontaneous. }\end{array}\right.$

2. Secondary perinephritis. $\left\{\begin{array}{c}a . \text { Due to a general cause (infectious peri- } \\ \text { nephritis). } \\ b . \text { Due to a local cause (perinephritis by ex- } \\ \text { tension). }\end{array}\right.$

Etiology.-Perinephritis may be caused by a local traumatism, or by the extension of an inflammation or the burrowing of pus from the kidney or other adjoining organ, or, apparently, by the local action of some general infection, or of some undetermined influence which excites suppuration in the fatty capsule of the kidney. It is in this last group that the probability of error through failure to detect the actual cause, already referred to, exists.

As predisposing causes may be mentioned those which favor the occurrence of suppurative inflammation of the kidney, as tuberculosis or the gouty diathesis, with its tendency to over-acid urine and the formation of stone or gravel. The alleged predisposing influence of certain modes of life, and occupations which call for violent and repeated muscular efforts and expose to cold and wet, is probably exerted, if at all, through traumatisms or through a depravation of the general condition which favors the action of some specific cause. In some cases the pre-existence of some affection of the kidney, such as a calculus or tuberculosis, has clearly enabled some comparatively slight traumatism to provoke suppuration. 
The determining causes of the primary forms are traumatisms (open wounds, contusions, and larger or smaller extravasations of blood caused by exaggerated displacements of the kidney in jolts and violent efforts) and exposure to cold and wet: Open wounds involving the kidney have been considered in another chapter; those that extend into the fatty capsule but not into the kidney provoke suppuration, as do other primarily or secondarily infected wounds; and the only features of special interest in the process are those that are connected with the depth of the wound, with the laxity of the connective tissue, and with the ease of propagation to other parts-features that are shared with suppurative processes in the same region arising from other causes, and will be subsequently considered.

Contusions are deemed a relatively frequent cause. Le Dentu attributes to them one quarter of the primary cases. In some cases, especially those in which a long period-many months-has elapsed between the contusion and the first symptoms of perinephritis, the causal relation can not be accepted as proved, and in others there is evidently present a predisposing cause which has favored the action of the violence. Thus Richet (quoted by Le Dentu) reports a perinephritis following a blow in the flank; when he opened the abscess he recognized and removed several calculi from the kidney. Morris (loc. cit., page 221) reports a similar case. He quotes another (loc. cit., page 206) from Dr. Dickinson as an instance of the effect of slight repeated contusions, but in which the predisposing, or perhaps directly causative, influence of tuberculosis seems more probable: A man forty-three years old, "a free liver, and of tubercular tendency"; the cause, "the severe bruising of the loin by the rolling and tossing of the vessel during a very rough voyage from Queensland to England; two months after the perinephritic abscess was opened hæmoptysis occurred, and was followed by profuse purulent expectoration, and death in three months."

External violence presumably acts by causing larger or smaller extravasations of blood in the loose connective tissue, by rupture of vessels that are put upon the stretch either by the direct action of the violence or by the displacement of the kidney. A violent muscular effort may perhaps act in the same manner or by rupture of a portion of the muscle. The collection of blood causes irritation by its presence, which is increased by the repeated, unguarded movements of the patient, and thus a condition is created which is favorable for the development of such pyogenic germs as may chance to be lodged in the region.

The agency of chilling has long been admitted ; in many of the reported cases the relation can hardly be considered as proved, but in some it appears to be sufficiently well established.

Perinephritis secondary to a general cause, such as pyæmia, septicæmia, 
The liver, spleen, pancreas, suprarenal capsules, and even the stomach, have either been directly invaded by the suppuration, or have softened under the influence of its proximity; the colon has been occasionally perforated.

The pus may be thin by admixture of urine, but this appears to be quite infrequent; or it may be very fetid, in consequence of proximity to the intestine; this fetidity is not an indication of perforation of the intestine.

In exceptional cases the suppuration may be of the diffuse or infiltrated form, of rapid course and grave prognosis, killing by septicæmia, or leading to the sloughing of large portions of the tissue if the patient survives.

If the inflammation is of a lower grade and chronic in its course, the tissue enveloping the kidney may become closely adherent to it and greatly thickened and hardened (Fig. 7). Morris compares it to an anatomical preparation imbedded in plaster-of-Paris.

Symptoms. - The beginning of the affection varies in accordance with its cause; in the primary forms it may be sudden or gradual, and in the secondary forms it is usually masked by the symptoms of the affection to which it is secondary.

One of the earliest symptoms is pain, referred more or less vaguely to the region of the kidney; it is usually dull and continuous, but may be very severe or remittent; it increases as the affection advances, and it is exacerbated by movements or by jarring and by pressure, and in some cases especially by extension of the hip, because of the implication of the psoas. It may radiate to the lower part of the abdomen, the external genitals, and the thigh, presumably through the irritation of the trunk of the nerves of the lumbar plexus.

The general symptoms are those of fever with its usual accompaniments, the fever of suppuration, with its remissions, exacerbations, and occasional chills, and its more or less marked signs of continuous or recurrent septic absorption.

Palpation carefully made by deep pressure through the anterio: abdominal wall, or bimanual with one hand on the loin, reveals tenderness, and sooner or later a tumor in the region of the kidney-a tumor which is globular, smooth, elastic, and perhaps fluctuating. As the abscess grows it fills out the usual slight hollow in the loin between the ribs and the crest of the ilium, and as it approaches the surface there it makes the skin slightly œdematous, and, later, reddened and adherent.

If left to itself the pus establishes a spontaneous opening at some point, usually in the loin, which becomes the orifice of a persistent sinus, through which the abscess empties itself incompletely, with occasional periods of recurrence or exacerbation of the general symptoms excited by obstruction to the flow. 
If the pus spreads in the other directions mentioned in the preceding section the symptoms will be correspondingly modified; if it descends into the iliac fossa or the pelvis, or, still more rarely, into the thigh, it will be there manifested by the usual signs and recognized by the usual manipulations. Its escape into the peritoneal cavity is followed by an acute suppurative peritonitis; its escape into the ascending or descending colon by pus in the stools and the diminution in size of the lumbar tumor. Its spread through the diaphragm and adherent lung to a bronchus leads to the sudden and violent coughing up of a large quantity of pus, and this is repeated from time to time so long as the affection lasts; sometimes the patients can bring up pus at will by bending the body in different directions. If adhesions between the lung and diaphragm fail to protect the pleural cavity from infection by the pus, an acute suppurative pleurisy follows, and usually runs a rapidly fatal course.

The course of the affection after the opening of the abscess differs according as it is primary or secondary. In the former case it is usually that of an ordinary phlegmon, the evacuation of the pus is immediately followed by relief of the general and local symptoins, the discharge grows steadily less, and the cavity finally closes; in the latter the affection to which the perinephritic abscess is secondary may persist and oppose the healing of the abscess, in which case the relief of the symptoms will be less marked, the improvement will not advance beyond a certain point, and it will give place from time to time to exacerbations.

Exploration of the cavity after spontaneous or surgical opening may show calculi or other foreign bodies within it or within the kidney. In one case that has remained unique the abscess appears to have opened into the stomach, and renal calculi to have been discharged through the mouth by vomiting.

In primary perinephritis, and in secondary perinephritis not of renal origin, there are rarely any symptoms eonnected with the urine, but sometimes the frequency of micturition is increased; in secondary perinephritis of renal origin the urinary symptoms are those of the original cause.

Among the rarer symptoms are albuminuria transient or persistent, pain or anæsthesia in the hypogastrium, scrotum, vulva, or thigh, jaundice when the affection is on the right side, and persistent flexion of the hip, simulating hip-joint disease. Gibney has called attention in several articles to the close resemblance in a number of cases, on superficial examination, to hip-joint disease, because of the immobility of the hip in flexion, the lordosis, and sometimes even pain in the knee.

Diagnosis. - A positive diagnosis of perinephritis can hardly be made before sufficient pus has formed to constitute a distinct tumor recognizable by palpation. The signs which precede that condition may 
the eruptive fevers, has nothing but the accident of its situation to distinguish it from other abscesses complicating the same general diseases. It is claimed to appear with special frequency as a complication of puerperal fever, but probably some of the abscesses that have been opened in that region in puerperal women have not originated there, but have spread upward to it from the neighborhood of the uterus or tubes.

The most frequent cause is undoubtedly that of the last group in the table: an inflammation in a neighboring organ, almost always the kidney, which either spreads to the fatty capsule and there ends in suppuration, or produces an abscess at the original site which ruptures into the loose tissue of the fatty capsule. The modes in which perinephritis can be caused by a calculus in the kidney, or by tuberculosis of the kidney, or in the course of an ascending pyelonephritis, have been already described. The other organs in which the suppurative process appears most frequently to originate are the gall bladder and the ascending or descending colon. Biliary calculi occasionally perforate the bladder, and, working backward and downward under the protection of peritoneal adhesions, reach the neighborhood of the kidney and cause an abscess in the loin. The colon may be perforated by a simple or specific ulceration, perhaps as an incident in the evolution of a carcinoma, the perforation taking place directly into the tissue enveloping the kidney between the layers of the meso-colon or through opposing and adherent peritoneal layers. Pus coming from the diseased body of a vertebra may easily make its way into and remain in the same region, and it seems not improbable that some of the perinephritic abscesses of unknown origin, and some that have been attributed to cold or to slight traumatisms, were simply cold abscesses originating in a tubercular process in a vertebra.

Pathology.-The loose cellular tissue in which the kidney lies is situated almost wholly behind, while in front the kidney is rather closely attached to the peritonæum and the thin intermediate fibro-cellular layer known as the fascia propria. In this posterior layer adipose tissue begins to form at about the age of ten years, but the anterior layer is usnally free from it throughout life. Inferiorly the loose tissue is directly continuous with the retroperitoneal tissue of the iliac fossa and the pelvis, and posteriorly it is in communication with the different layers of the abdominal wall by the connective-tissue envelope of the vessels. Superiorly it appears to have equally free relations with the connective tissue between the peritonæum and the diaphragm. As the result of these anatomical conditions pus forming within this layer can spread with great ease upward, downward, or backward if a barrier is not formed by the condensation, the matting together, of the tissue. It forms behind the kidney, very rarely in front, and may form a single small abscess, but ordinarily, if a little time is given it, it invades the entire area and forms 
a collection that is bounded posteriorly by the abdominal wall, above by the diaphragm, and in front by the thickened peritonæum; inferiorly the limit is variable. A very few cases of small circumscribed abscesses have been reported.

Posteriorly the pus may make its way through the fascia by following the vessels and other lines of least resistance and become subcutaneous. It is not very rare to see quite a large subcutaneous collection that communicates with a deeper one through a small opening in the wall close above the crest of the ilium.

Inferiorly the pus may burrow close beneath the peritonæum and collect above Poupart's ligament, or it may get beneath the iliac fascia and

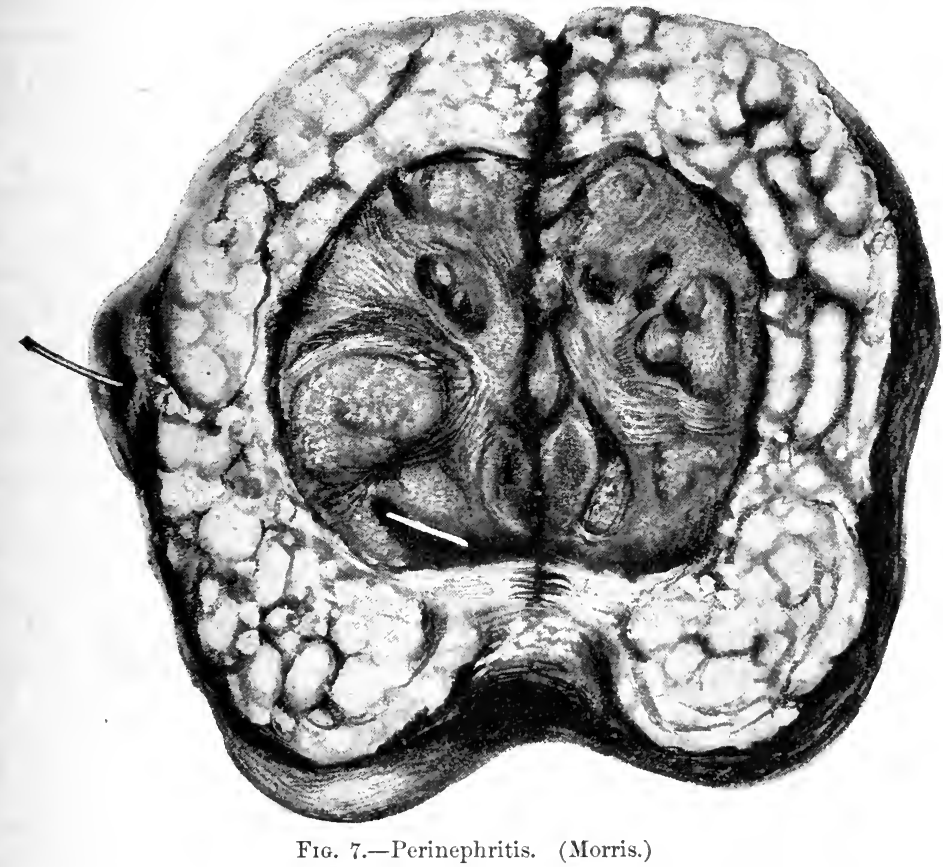

follow the psoas into the thigh; or it may turn aside into the cavity of the true pelvis, still keeping beneath the peritonæum.

Superiorly the pus may perforate the diaphragm, either directly or by following the psoas upward under the ligamentum arcuatum, make its way into the adherent lung, and ultimately escape through the bronchial tubes; or, more rarely, on perforating the diaphragm it may escape into the free pleural cavity and set up a suppurative pleurisy.

Perforation of the peritonæum and escape of the pus into the peritoneal cavity is very rare. 
be sufficiently marked and significant to justify a strong suspicion, or they may be so vague, or so masked by those of the primary cause, as not even to suggest perinephritis. The early symptoms are local pain and fever in combination; either without the other (except perhaps at the very beginning) could hardly be said to justify even a suspicion. The danger of overlooking the affection is apparently greatest when the fever is high, the general symptoms correspondingly marked, and the pain moderate, diffuse, or not clearly described. If a case is not thoroughly examined, perinephritis may easily be confounded with a variety of diseases, as can most others; but when the tumor in the region of the kidney can be felt, the signs that indicate the presence of pus, and of pus behind the peritonæum, can usually either be found, or can be positively declared to be absent. The change in the outline of the loin, subcutaneous œdema, possibly fluctuation, and, above all, the exploring needle, give very positive indications. A previous history of inflammatory or painful affections of the kidney is always suggestive.

Solid tumors of the kidney are differentiated by their consistency, by their more anterior position and the absence of implication of the posterior abdominal wall, and by the absence of fever. Hydronephrosis and kidney cysts have the same situation as solid tumors, and the exploring needle (introduced, of course, from behind) shows the character of the contents. With pyonephrosis an error may easily be made if puncture is employed, but it could hardly have serious consequences; and, moreover, the signs furnished by the urine and history should help to avoid it.

After the presence of an abscess has been recognized, its character and its origin may still remain in doubt. As has been said, the majority originate in and about the kidney, but they may come from the liver, the colon, or the spinal column, from the pelvis or the appendix below, and even, as in three cases quoted by Duplay (Pathol. externe, vol. v, page 779), from the pleural cavity. The differential diagnosis must be made, if at all, by the aid of the history and of associated symptoms. Dr. J. B. Roberts (Am. Jour. Med. Sc., April, 1883) has made an interesting analysis of the signs by which the position of the abscess with reference to the kidney may be indicated.

Prognosis.-The prognosis, which should always be guarded, varies with the cause, the course, and the treatment. Of 28 cases collected by Poland (Brit. and For. Med.-Chir. Rev., 1871, vol. ii), thought to be primary, 20 were treated by free incision (15) or by puncture with trocar (3), or opening with caustic (2), with 2 deaths; of the 8 not operated upon, 6 died. Of 10 cases of secondary abscess, 3 were opened, and the patients recovered; the other 7 died.

The prognosis appears to be graver in secondary than in primary 
eases, evidently because of the persistence of the cause; it is also grave in those which occur in the course of some general febrile disease.

Treatment. - It is claimed that attacks have been cut short, that resolution has been obtained by leeching, cupping, hot baths, and local fomeritations, but the diagnosis in such cases must always remain in some doubt. The indications in the early stage are wholly symptomatic -to relieve pain and diminish the fever.

In the later stage, when the presence of pus is recognized, the indication to evacuate it is peremptory, and this is best done by a free incision in the loin. Indeed, it may be proper to make the incision even if the presence of pus is only suspected, and if an exploratory incision seer,s safer or more likely to yield positive results than exploratory puncture. The graver the general symptoms, the more urgent is the incision.

The incision should be large enough not merely to provide free escape for the pus, but also to permit thorough exploration of the cavity of the abscess for the detection and, if possible, the removal of the primary cause if the abscess is secondary. It may be longitudinal, transverse, or oblique, and should include division of the outer fibers of the quadratus lumborum, if the abscess is deep, both to favor exploration and to insure that the flow of pus shall long be unimpeded. The only objection to a long, transverse incision is the possibility of a subsequent hernia in the cicatrix; this has happened in one or two cases, but the contingency seems remote.

If the pus is fetid the cavity of the abscess may be irrigated with an antiseptic solution; in any case, it should be drained and lightly packed with iodoform gauze, and care should be taken, as always, that deep healing should be complete before the opening in the skin is allowed to close.

\section{RENAL AND PERINEPHRITIC FISTULÆ.}

The various suppurative processes which originate within or about the kidney lead, as has been shown, to the formation of abscesses, which, after having opened spontaneously at one or another point, or having been opened by the surgeon, may leave a permanent fistulous communication with the exterior or with some mucous canal. When this fistulous track communicates with the interior of the kidney, it is known as a renal fistula; or, if urine escapes through it, as a urinary fistula; if it terminates in a blind end near the kidney, it is a perinephritic fistula (or sinus). Of course, the distinction can not always be made during life.

As a fistula is simply the undue persistence of an opening which is necessary to the healing of a pre-existing suppuration, the cause of a fist- 
ula is usually that which maintains the suppuration, and thus causes a constant or intermittent flow of pus through the fistulous track. Among the commonest of these causes are calculi and tubercular foci; among others are foreign bodies introduced from without, and obstruction to the flow of urine through the ureter, in consequence of which the urine is diverted into the fistulous track and keeps it open. Traumatic fistulæ are rare, and, as will appear in the examination of the results of operation upon the kidney, wounds which involve the parenchyma of the kidney have a well-marked tendency to complete healing, while those of the pelvis are much more likely to leave a fistula.

Irregularity and rigidity of the wall of the abscess, as in extreme cases of pyelonephritis, favor the persistence of a fistula by opposing the obliteration of the cavity, and this fact makes the results of late operations less satisfactory than those of earlier ones. The statistics quoted in the preceding chapter show how frequently nephrotomy is followed by fistula.

If the fistula opens upon the surface of the body the opening is in the great majority of cases in the loin; but, as has been stated in the preceding chapters, the opening may be in the groin, the perinæum, or the thigh.

Fistulæ opening into the intestines-intestino renal-are not very uncommon. In almost all of them the communication has been with the colon, but in a few with the duodenum, or possibly some other portion of the small intestine. The diagnosis of communication with the duodenum has been confirmed by autopsy in one or two cases, and in a few others the clinical evidence has been satisfactory, but in other supposed cases the patients have practiced a fraud upon their attendants.

Fistulæ communicating with the stomach-gastro-renal-have been diagnosticated in a few cases, and proved by autopsy in at least one, that of Morris (loc. cit., page 261), in which the communication was between the left kidney and the greater curvature of the stomach. In a case reported by Chadwick to the Obstetrical Society of Boston (Boston Med. and Surg. Jour., Sept. 18, 1884, page 274 ), the patient, a lady sixty-two years of age, had had attacks of vomiting and gastric pain at intervals of from one to six weeks for seven years, and her urine contained particles of undigested food. It was believed that the communication was between the kidney and the stomach. In some alleged cases the romited matter appeared to contain urine, and in one the patient is said to have vomited renal calculi.

The escape of urine or pus from the kidney into the peritoneal cavity is usually fatal within a very short time; but there are a few cases on record in which life has been prolonged sufficiently to make it proper to term the opening a fistula. 
Fistulous communication with the lung and bronchi, or with the pleural cavity, and even through the latter with the exterior, has been noted in a number of cases.

Symptoms. - When the fistulous opening is in the loin the discharge may be slight or profuse, constant or intermittent, and may or may not be mingled with urine. The presence of urine may be indicated by the odor or by the special chemical reactions of some of its constituents, or by the detection in the discharge of some substance administered by the mouth to be eliminated through the kidneys-iodide of potassium, for example, which will give a blue stain to starched cloths wet with the discharge.

Tranmatic fistulæ tend to ultimate, even if long delayed, healing, unless a foreign body has been left in the wound; it is not uncommon for other fistulæ to close and reopen frequently. It must be remembered that not all urinary fistulæ in the loin communicate directly with the kidney; the deep communication may be with the ureter, or the bladder, or even the urethra (Desault).

In the case of fistulæ which open internally, the symptoms are the constant or intermittent discharge of pus, possibly mingled with urine, by the corresponding channel, and the formation or increase of a lumbar tumor when the discharge is arrested, and its diminution when the discharge is resumed. Chadwick's case shows that such a communication with the alimentary canal can exist for years without seriously interfering with the general health ; but ordinarily the general symptoms are important-fever, chills, inappetence, and loss of strength.

The recognition of the renal (or at least of the urinary) origin is very easy when urine is discharged through the fistula, no matter where the external opening may be, but in other cases it may be difficult or impossible, and depends mainly upon the history and upon the presence or absence of associated symptoms connected with the urine passed through the natural channels. For example, a history of nephrolithiasis, of pyelitis, of tuberculosis, and the occasional or constant presence of pus in the urine not otherwise accounted for, indicate a renal origin for the fistula.

Treatment.-When the fistula is maintained by the presence of a foreign body or calculus, the removal of the latter is necessary; but even this is not always sufficient, for, as has been already explained, the rigidity and irregularity of the sides of the abscess may be such as to delay or wholly prevent their approximation and union. Little is to be expected from the use of stimulating injections; occasionally persistence with them is followed by the closing of the fistula, but it is by no means clear whether this is a coincidence or an effect. In tubercular cases the persistent use of iodoform in glycerin is beneficial, and in simple sup- 
puration much may be gained by the use of pure carbolic acid and the aseptic blood-clot.

Operative methods consist of incisions to facilitate drainage and of the removal of the kidney-nephrectomy. Occasionally it may be necessary to remove a calculus or a fibrinous plug from the ureter, and thus turn the urine aside from the fistula. Recent experience, which has shown the readiness with which wounds of the renal parenchyma heal, suggests that possibly the healing of an opening in the pelvis of the kidney might be facilitated by making another through the parenchyma into the pelvis, and that the latter would then heal notwithstanding the flow of urine through it. Thorough scraping of the wall and removal of rigid septa, spurs, and bands, are necessary adjuncts of the operations that seek to heal by favoring free drainage.

Nephrectomy has been done in a number of cases, and is a justifiable operation if there is reason to believe that the kidney is not necessary to the continuance of the patient's life.

In the case of fistula opening into the cavity of some viscus-intestine, stomach, lung - if the symptoms are urgent, it will be proper to seek to turn the flow of pus aside by an incision (nephrotomy) in the loin, and eventually to remove the kidney, if the former plan does not prove sufficient.

\section{HYDRONEPHROSIS.}

The term hydronephrosis was created by Rayer to designate the distention of the pelvis and calices by urine more or less changed. The condition is, therefore, to be differentiated from pyonephrosis, in which there is a similar distention with purulent contents, and from cysts of the kidney, the contents of which are watery but not urinous. Terrier and Baudouin, in an elaborate article (Revue de chirurgie, 1891, page 719) on "intermittent hydronephrosis," give a definition which contains an important pathogenic suggestion, namely, that it is " an aseptic dilatation of the pelvis by urine, the flow of which is obstructed by some mechanical obstacle." If infection takes place, usually from below upward, the liquid becomes purulent, and the hydronephrosis is transformed into a pyonephrosis.

Experimental ligation of the ureter has shown that its complete aseptic occlusion does not produce the dilatation of the pelvis and the sacculation and atrophy of the kidney that are found in well-marked cases of hydronephrosis. The increase of pressure within the kidney by the retained urine promptly arrests the secretion of urine, and the process does not go on to marked dilatation. On the other hand, complete temporary obstruction does produce a certain degree of dilatation; and a 
series of symptoms, among which pain is prominent, which have long been attributed to other causes-abnormal mobility of the kidney, for example-are now coming to be looked upon as dependent upon such temporary obstruction, and the condition is classed as an intermittent hydronephrosis, thus enlarging the series of changes to which the term is applied by inclusion of these cases of simple temporary obstruction.

The statistics of autopsies published by Morris show a frequency much greater than clinical experience would indicate. Thus, in the records of 2,610 autopsies made at the Middlesex Hospital between 1873 and 1883, he found 142 cases of marked hydronephrosis, in all of which "there was very advanced change in the kidney." Terrier and Bandouin collected 83 cases of intermittent hydronephrosis. The affection is nearly twice as frequent in females as in males.

Etiology.-The cause, which is always some obstacle to the flow of urine, may be situated in the ureter, bladder, or even the urethra, and may be congenital or acquired ; but when congenital it does not always produce its effect promptly, so that a distinction must be made between a congenital hydronephrosis, one existing at birth, and a hydronephrosis having a congenital cause - a cause existing at birth, but not producing the hydronephrosis until after the lapse of some time, perhaps many years. Morris thinks that probably one third of the cases in which a palpable tumor is present have a congenital cause. Roberts's (Urinary and Renal Diseases) statistics indicate a still larger proportion -20 out of 52 cases.

The congenital causes are numerous and varied, and almost always involve the ureter. This tube may be imperforate, or obstructed by an anomalous branch of the renal artery, or folded upon itself from above downward, or obstructed by valvular folds of its mucous membrane or by cysts in its wall, or may have a minute vesical orifice or a very oblique communication with the pelvis. Occasionally the ureter is double, each portion draining a separate portion of the kidney, and if one is incomplete or obstructed the corresponding portion of the kidney becomes hydronephrotic. Rayer quotes a case reported by Billard, of a still-born child with imperforate urethra, and dilatation of the bladder, both ureters, and both kidneys; and Porak (France Méd. 1885, page 1441) attributed the enormous dilatation of the bladder (a liter), the ureters, and kidneys which he found in a still-born child to " a valve situated in the membranous portion of the urethra." Phimosis has also been alleged as a cause.

The congenital origin of the valvular folds noted in several cases has been questioned, and the folds or kinks in the upper portion of the urethra have also been interpreted as secondary to a congenital abnormal mobility of the kidney, which permitted it to sink somewhat in the abdomen and thus fold the ureter upon itself. So, too, the oblique angle of 
communication between the pelvis and ureter found in some cases has been claimed to be the result and not the cause of the unsymmetrical distention of the lower part of the pelvis.

The apparent contradiction between the intra-uterine production of a hydronephrosis by total obstruction of the ureter and the impossibility of thus producing it after birth, is explained by the difference in the physiological activity of the kidney at the two periods; during intra-uterine life the secretion of urine is slow and scanty, and the obstruction does not at once produce, as it does after birth, an intrarenal pressure that arrests the secretion, but the pelvis has time to dilate and thus keep the pressure for some time below the point at which the physiological activity is arrested.

The acquired, non-congenital, causes are still more numerous and varied. A rather common one is a calculus lodged in the ureter; others involving the ureter are clots, hydatids, or papillomatous growths within it, traumatic narrowing, twists, pressure upon it by inflammatory exudates, abdominal tumors, or the displaced uterus, and pressure upon the vesical orifice by growths within the bladder; those involving the bladder are mainly prostatic enlargements ; and, finally, there are those which obstruct the urethra.

Morris groups his 142 cases above mentioned as follows: 1 . Cancer of the pelvic organs-uterus, vagina, bladder, or rectum-in 116 cases. 2. Cancer of one or both ovaries in 2 cases ; of these, 94 were double, 17 affected the right kidney only, and 7 the left kidney only. 3 . Of the remaining 24 cases the cause was: unknown, 4 ; cystitis, 3 ; vesical calculus, 3 ; villous growth of bladder, 1 ; enlarged prostate, 3 ; ovarian cysts, 4 ; constriction of ureter, 3 ; pressure on ureter by abdominal cancer, 3 . Of these, 12 were double; 9 affected the right kidney only, and 3 the left kidney only. He adds that, of the 32 cases recorded by Roberts, the cause was impaction of a calculus in the ureter in 11, and was supposed to be that in 3 others; in 5 there was a narrowing or obliteration of the ureter near one or the other end, presumably due to inflammation or ulceration; in 6 the ureter had been compressed by pelvic tumors, and in 3 by peritoneal inflammatory bands. Simon thinks calculus in the ureter the most frequent cause.

Exceptional causes observed or quoted by Morris are abscesses about the lower part of the ureter, enlarged lymphatic glands pressing upon it, abscess of the bladder following lateral lithotomy, obstruction of the ureter by the dragging upon it of a sacculus of the bladder, retroflexion of the uterus, and contraction of the bladder.

Whether hydronephrosis of a portion of a kidney alone is possible, exclusive of the cases of double ureter and corresponding segmentation of the kidney, has not yet been satisfactorily determined. Le Dentu refers 
to a case (Fenger) in which "the upper part of the kidney was transformed into a vast cavity containing urine; the pouch represented a single distended calix, the orifice of which was obstructed by a valve.

Pathology. - The typical changes consist in a dilatation of the pelvis and calices, and a stretching, thinning, and atrophy of the parenchyma, so that together they form an irregular sac, with translucent lobules sepa-

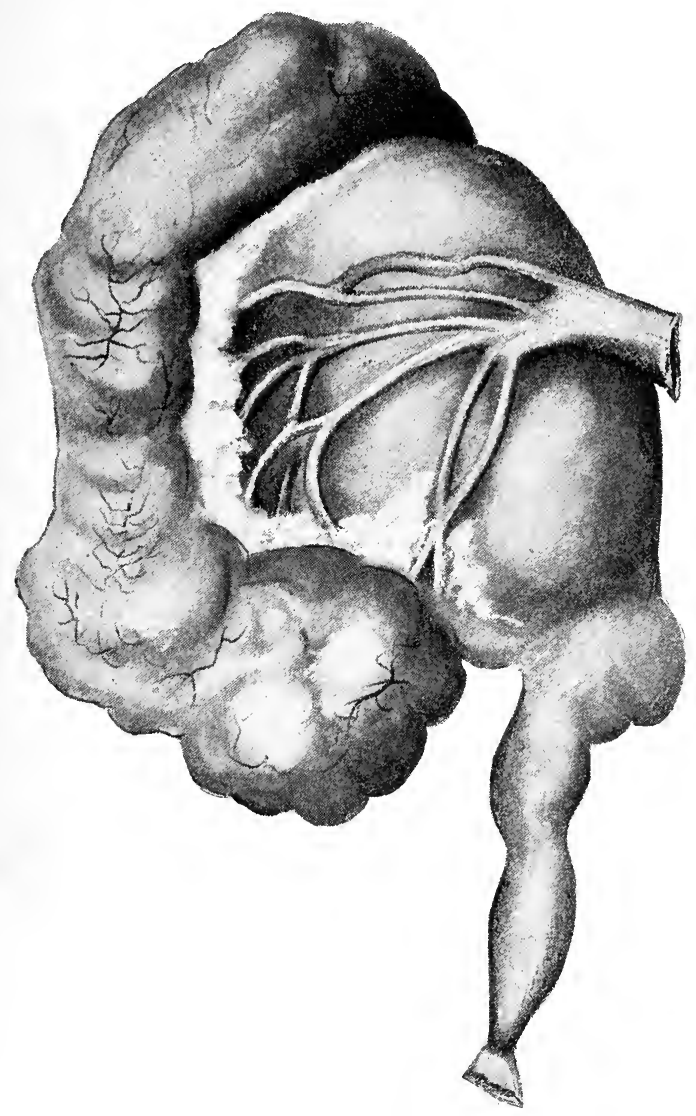

FIG. 8.-Hydronephrosis ; first stage. (Le Dentu.)

rated by fibrous tracts showing on the surface. In the first stage (Fig. 8) the parenchyma is clearly recognizable as a thick layer capping the distended pelvis at its upper and outer part, but in the second (complete) stage (Fig. 9) nothing remains of it but a few scattered patches in the wall. When laid open, the interior shows incomplete septa limiting diverticula or pouches that correspond to the lobules on the surface, and represent, in part at least, the dilated calices; these pouches communi- 
cate with the main eavity of the pelvis, and possibly with the ureter, and are filled with liquid; occasionally one or more of them may be shut off from the rest by obliteration of the orifice of communication. The condition of the ureter varies according to the seat of the obstruction, being distended above it and contracted below it.

Sometimes the dilatation involves the pelvis above, leaving the parenchyma practically intact; and in some cases of double hydronephrosis

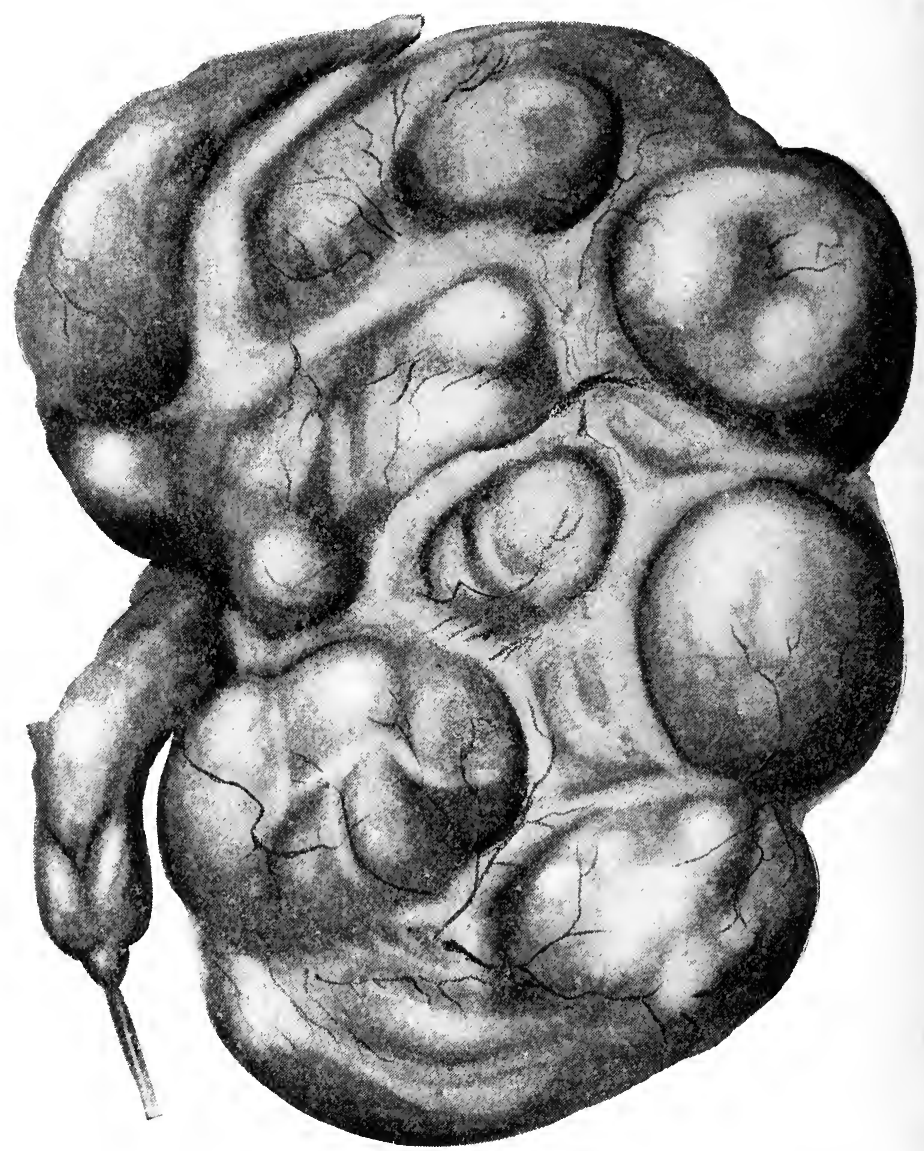

FIG. 9.-Hydronephrosis; second stage, the kidney substance being completely atrophied. (Le Dentu.)

the parenchyma of one kidney has appeared to be hypertrophied; probably it underwent compensatory hypertrophy while its fellow was first to become hydronephrotic, and its own subsequent implication was not sufficient to produce the usual atrophy.

The wall, instead of being thin and translucent as just described, may 
be thick and opaque. Morris says fibro-cartilaginous nodules have been found in some.

The size of the sac may be less than that of a normal kidney, or it may be so large that the tumor occupies a considerable portion of the abdominal cavity, and may even resemble a very large ovarian cyst. In some of the congenital cases the tumor has been so large that it interfered seriously with delivery.

The composition of the contained liquid varies with the degree of atrophy of the parenchyma, the completeness of the obstruction to its escape, and with other chance conditions. So long as the kidney continues to secrete urine, the liquid will contain the urea, uric acid, and the other constituents of normal urine in larger or smaller proportions; and if the interference with its escape is not complete, the liquid is usually nearly normal urine of low specific gravity and neutral or alkaline reaction. When the obstruction is complete and the kidney ceases to excrete urine, the liquid already present in the pouch undergoes change by the gradual absorption of the urinary constituents held in solution, and tends to assume the character of the contents of simple cysts, showing more or less albumen, epithelial cells, and possibly blood. Exceptionally, the contents are colloidal (Dumreicher), and in one case cholesterine was found (Coghill). In another (Terrier), one of the subordinate pouches was shut off from the rest and contained pus. Sometimes the liquid is turbid, and contains mucus, pus, or blood. If infection takes place the contents become purulent (suppurative hydronephrosis).

The amount of liquid varies from a few ounces to several gallonsthirty in Glass's case, quoted by Rayer ; and in several cases in which a fistula has been established by operation it has yielded several ounces of nearly normal urine daily.

Symptoms.-These vary greatly, in accordance with the size of the tumor, the cause and character of the obstruction, and the degree of atrophy of the kidney. The evolution may be so quiet and arrest of growth may come so soon, that the existence of the affection will be betrayed by no symptom during life, and it will be discovered only at the autopsy. On the other hand, the symptoms associated with the causelodgment of a calculus, for example-may be so severe as wholly to mask those which arise from the hydronephrosis. Of the 142 cases collected by Morris from the post-mortem records of the Middlesex Hospital, "an abdominal tumor was clinically observed in but very few"; and of 42 cases of well-marked non-congenital hydronephrosis, which he quotes from Roberts, "there was a palpable tumor in 25, and in 19 of these the tumor was unilateral, in 6 double." Probably, if the condition was suspected and an examination made with the aid of anæsthesia, the presence of the tumor would be recognized in most cases. 
The symptoms of the onset are usually vague, and consist of dull pain in the loin, and possibly frequent micturition and diminution in the amount of urine. If the tumor is sufficiently large, pressure-effects, especially upon the colon, may be noted.

When a recognizable tumor exists it occupies the region of the kid. ney, can be felt at first in front by pressure through the anterior abdominal wall, and afterward may bulge distinctly in the loin. It is flat on percussion, the colon being displaced first forward and then inward, and is usually fluctuating. It may be smooth and globular or irregular. As it grows it spreads to and beyond the median line and downward into the iliac fossa. Morris quotes Dickinson's estimate, that "in about one fourth of the cases of single hydronephrosis in which a swelling is observed during life the tumor extended beyond the median line of the body, and that in one third of these it has at length

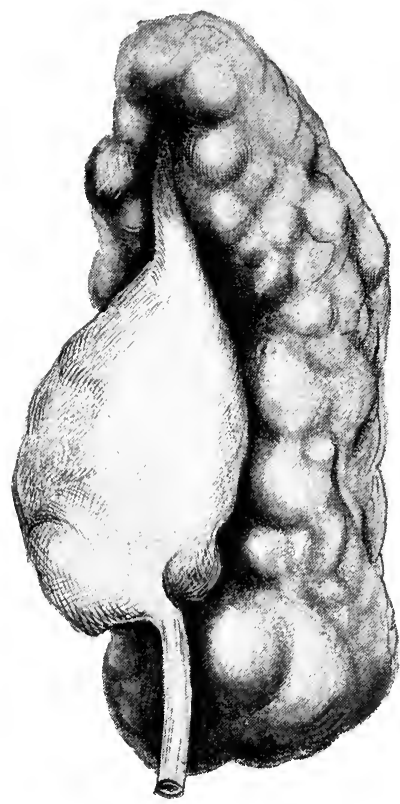

Fig. 10.-IIydronephrotie right kidney; posterior view. The mode of termination of the ureter is seen in the figure. (Revue de Chiurgie.) occupied the greater part of the belly." In Glass's case, above mentioned, much the largest on record, the patient was a tall woman, twenty-two years old. The belly, measured after death, was six feet four inches in circumference, and a little more than four feet from the ensiform cartilage to the symphysis pubis. The cyst contained thirty gallons of liquid; the heart was pushed up to the clavicle, and the lungs were reduced by pressure to the size of those of a new-born child. In Terrier's case the circumference at the umbilicus was one metre, and the distance from the ensiform to the pubes thirty-nine centimetres.

The increase in size, especially if it is rapid, may be accompanied by severe paroxysmal pain.

Sudden diminution in size, coincident with the evacuation of an unusual amount of urine, is not very uncommon, and is characteristic of "intermittent hydronephrosis." The name was given to the affection by Cole in 1874, and was established by an important paper published by Morris in 1876. This form of the affection has received much attention of late, especially from Landau and Israel in Germany, and Terrier and Baudouin in France, whose paper has already been quoted. The latter authors consider the form a frequent one, and believe it often to be the first stage of a permanent (or 
"closed") hydronephrosis. They consider abnormal mobility of the kidney to be a frequent cause of it by producing a sharp bend in the upper portion of the ureter (Figs. 10 and 11). This arrests the passage of the urine and produces a temporary hydronephrosis, which is relieved by the return of the kidney to its place. Repetition of the occurrence leads to structural irritation and to fibrous adhesions which bind the sac to the upper part of the ureter and ultimately make the obstruction permanent.

The occurrences are marked by violent attacks of pain, the appearance of a tense tumor in the flank, and diminution of the amount of urine passed. Each attack lasts several hours, and ends suddenly, rather closely resembling an attack of renal colic. I recall such

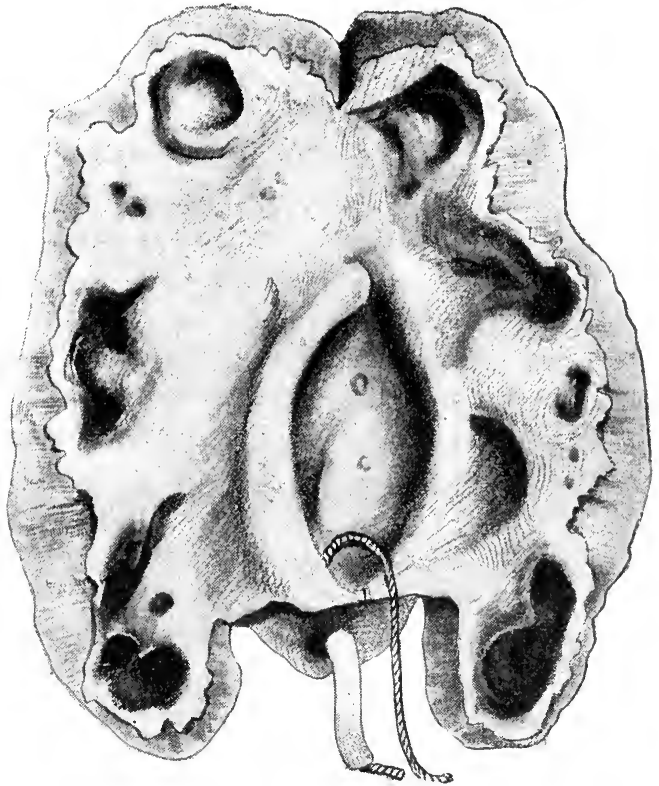

Fig. 11.--Hydronephrotie right kidney opened on its outer edge. Its anterior half is to the right; thread is passed into the ureter. (Revue de Chiurgie.) an attack, supposed to be due to a calculus, that was instantly relieved by inversion of the patient. Subsequent exploration through an incision showed a marked bend in the ureter, and no calculus.

Prognosis. - The cause and prognosis vary in accordance with the changes that have been described. The affection may remain stationary and even unrecognized for years; it may grow rapidly, causing intense pain and various pressure-effects upon other causes, and may even rupture into the peritoneal cavity; or, if double, it may cause death, with the symptoms known as uræmia, by the gradual destruction of the kidneys. In a few cases of intermittent hydronephrosis spontaneous cure has taken place. The prognosis is grave because of the tendency of the affection to produce complete arrest of the physiological action of the kidney, and also in many cases because of the gravity of the primary cause, such as abdominal cancer or obstructive cystitis.

Diagnosis. - The diagnosis, which is very difficult or impossible at the beginning, may become easy when a recognizable fluctuating and not too large tumor has formed. The position of the tumor, its close rela- 
tions with the posterior wall, the presence of the colon or small intestine in front, show its real character, and it then remains only to distinguish it from other liquid collections within or about the kidney. The history will sometimes throw light upon it, and if it shows that the tumor has at some time suddenly diminished in size coincidently with the voiding of a large amount of urine, the diagnosis can be confidently made. Le Dentu gives two signs of diagnostic importance: first, that a hydronephrosis is more distinctly fluctuating than any other variety of renal cyst; and, second, that no solid mass can be felt at any point in it. Exploratory puncture in the loin may make the diagnosis by yielding a liquid that contains urea or uric acid; but, as we have seen, in cases that have existed for some time, the solid constituents are likely to have disappeared by absorption, and then the liquid yielded by the puncture is similar to that found in many other simple cysts.

When the tumor is very large it is likely to be mistaken for an ovarian cyst. Morris states that of twelve cases, in women, which he col. lected in 1876, seven had been diagnosticated as cysts of the ovary, and five of them had been subjected to laparotomy.

Treatment. - The ideal method of treatment-removal of the obstacle and re-establishment of the flow of urine-is one that can not often be carried out. Morris quotes three cases in which massage of the abdomen was followed by the more or less sudden disappearance of the tumor. In one or two acute cases, lodgment of a calculus or a fibrinous plug in the ureter, the occluding body has been removed through an incision in the loin; and it is now believed that a calculus lodged in the lower part of the ureter may possibly be recognized and safely removed through the vagina. Where the hydronephrosis is caused by pressure upon the ureter of a misplaced uterus or an abdominal tumor, the causal indication is clearly to be followed if the primary disease is suitable for operation.

Intermittent hydronephrosis, simulating kidney colic, and due to displacement of the kidney, may be temporarily relieved by inversion of the patient, or by such manipulation of the kidney as will straighten the ureter, and may be permanently cured by an operation for fixation of the kidney-nephropexy, nephrorrhaphy.

If the affection is double and progressive and the tumor fairly large, the plan of treatment is clear: one or both sacs should be opened by incision in the loin (nephrotomy) and a permanent fistula established. Inconvenient as such a condition may be, it is the only one that holds out a hope of arresting the destruction of the kidneys and preserving the patient's life. Possibly aspiration of the liquid would in some cases permit re-establishment of the patency of the ureter, and it may therefore properly be tried; but if reaccumulation follows, nephrotomy should be 
done. Such aspiration should be done in the loin, not through the peritoneal cavity.

If the hydronephrosis is unilateral and progressive and the tumor not very large, and if the symptoms are urgent, or if the case has been under observation long enough to make it certain that it is not of the intermittent variety, the choice of operation is between aspiration, nephrotomy, and nephrectomy. As has just been stated in the preceding paragraph, but little is to be hoped for from aspiration, and it is not wholly free from danger, so that the choice is practically between incision and extirpation Most of the few successes of single or repeated aspiration have been obtained apparently in cases in which the tumor was very large and the kidney-tissue wholly destroyed. The former, practiced in the loin, is much the simpler and less dangerous operation; it restores the kidney to some degree of functional activity, and to that extent is advantageous to the patient; on the other hand, it creates a fistula the discharge from which, and the consequent annoyance, will vary directly with the amount of kidney-tissue restored to activity. For some, the annoyance of such a fistula would outweigh the greater risks of nephrectomy and those inherent to the possession of only a single kidney, but experience has shown that it can sometimes be very satisfactorily controlled by suitable dressings even when the daily discharge amounts to ten or twelve ounces. Moreover, if the annoyance becomes intolerable, nephrectomy can still be done, and with no more risk than if it had been done primarily.

If, on the other hand, the tumor is very large, the risks attendant upon an attempt to remove it are so great that nephrectomy is hardly justifiable. More is now to be hoped for from aspiration; a number of cases thus treated have been cured; and if the operation is done judiciously, and the needle not passed through the peritonæum, the attendant risks are probably. no greater than those of incision and drainage.

Incision and drainage may be made in the loin or through the peritoneal cavity; the former appears to deserve the preference, as involving less danger, although it must be admitted that with proper precautions the dangers of the transperitoneal method are slight. Le Dentu thinks the latter has an important advantage, one that dominates all other considerations, in that it gives greater facilities for satisfactory drainage. It is probably to be preferred in cases of doubtful diagnosis; and when the abdomen has been opened through an error in diagnosis, and the supposed ovarian cyst found to be a hydronephrosis, many surgeons would probably prefer to complete the operation in that way rather than to close the anterior incision and open the cyst through a second incision in the loin.

If the lumbar route is chosen, the incision should be transverse or oblique, and deepened layer by layer until the sac is reached; the latter, 
after evacuation of its contents through a free incision, should be stitched to the skin, and its interior explored as far as possible with the finger, with the object of opening up all pockets, detecting the presence of a calculus, and properly placing the drainage-tubes.

If the anterior-transperitoneal-route is chosen, the incision should be made with reference to easy access to the tumor and to subsequent drainage. It is assumed that the tumor is in contact with the wall at the seat of the incision. After protecting the adjoining peritonæum with sponges, a large trocar is thrust into the cyst, and as its contents escape the sac is seized with volsella forceps and drawn out through the incision ; finally, the sac is incised, and the edges of the incision stitched to the skin. Drainage-tubes are then appropriately placed, and the usual dressings applied. Possibly a counter-opening for drainage in the loin would be advantageous.

After these operations, also, a secondary nephrectomy may be successfully done to close an annoying fistula.

\section{CYSTS OF THE KIDNEY.}

There are three kinds of cysts to be described: (1) simple or isolated cysts, (2) conglomerate cysts, (3) hydatid cysts. This classification is not to be deemed complete from the pathological standpoint, but it is sufficient for clinical purposes. It is not designed to include the small cysts so often found in granular kidneys, or partial hydronephrosis, the dilatation of a calyx shut off from the pelvis, which resembles a cyst much more closely than it does the common hydronephrosis.

\section{ISOLATED CYSTS.}

The term isolated is employed, for lack of a better, to distinguish these from conglomerated cysts; it signifies, not that they are always single, but that they are not grouped; that, when multiple, they are separated from one another by unchanged portions of the kidney-substance. They originate apparently in the cortex, and project beyond its surface; they occasionally attain a great size (Fig. 12), but ordinarily are not large enough to attract attention during life. Morris states that 2,610 autopsies at the Middlesex Hospital furnished 5 cysts of this kind, and 3 conglomerate cysts; the largest of the 5 was the size of a Tangerine orange, the smallest that of an olive. One patient was nineteen years old ; the others forty-nine or more.

The wall is usually thin and translucent, but is sometimes thick and opaque; the contents are usually watery, with a little albumen, some salts, and occasionally traces of the specific elements of urine; sometimes the amount of albumen present is so great that heat will change the 
whole into a jelly-like mass, and sometimes the contents have the consistency of thin glue. Admixture of blood with the contents correspondingly changes the appearance and character. Cornil has pointed out that some specimens thought to be sanguineous cysts are actually neoplasms (epithelioma, adenoma, sarcoma) that have undergone cystic degeneration.

The pathogeny of these cysts has not been made out; they are thought to originate by dilatation of a glomerule, or of a tubule, or within the cellular tissue surrounding the vessels.

Le Dentu quotes from Lannelongue a case in which the cyst contained gas. The patient was eight years old; the tumor large, and occupied the right flank. Exploratory puncture yielded a pint of greenish liquid, that contained nothing that threw light upon the character of the cyst. It was then noticed that gas was present, and it was withdrawn by another puncture, and shown to consist of 8 parts of oxygen, 7 parts of nitro-

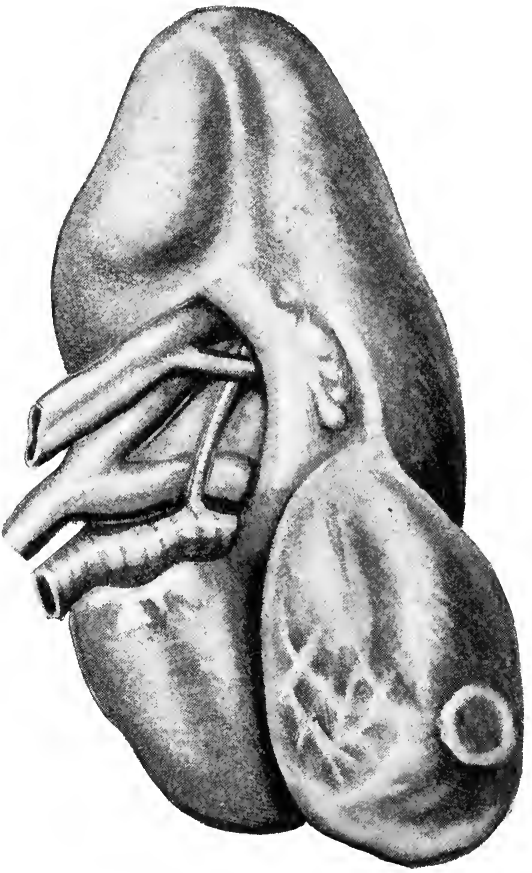

Fig. 12.-Isolated eyst of the cortex of the kidney. gen, and 1 part of carbonic acid.

There was no indication of any communication with the intestine. After the child's death by some intercurrent affection, the autopsy showed that the sac was situated in the kidney, that it had recently opened into the pelvis, and that the gas had not passed down to the bladder. The origin of the gas was not ascertained.

Multiple small vesicles are occasionally found in the mucosa of the pelvis and ureter (Fig. 13); they are supposed to originate in the mucous glands.

One or two cases of atheromatous or calcareous cysts have been reported.

Symptoms.-A cyst gives no symptoms before it is large enough to make pressure upon adjoining viscera. When its position and size are such that it can be palpated, it will be found to be firm, elastic, and globular when of medium size, and to resemble other large cysts when large. It does not modify the urine, or the frequency of urination, except per- 
haps by pressure. The differential diagnosis between a serous cyst, a hydronephrosis, or a hydatid cyst, may sometimes be made by exploratory puncture, the liquid in the first place being albuminous, in the second possibly urinous, and in the third free from albumen and containing hooklets. Some of the larger cysts have been mistaken by experienced surgeons for cysts of the ovary.

Treatment. - As the cysts are not malignant, and as they affect the health of the patient only through their size, the propriety of interfer-

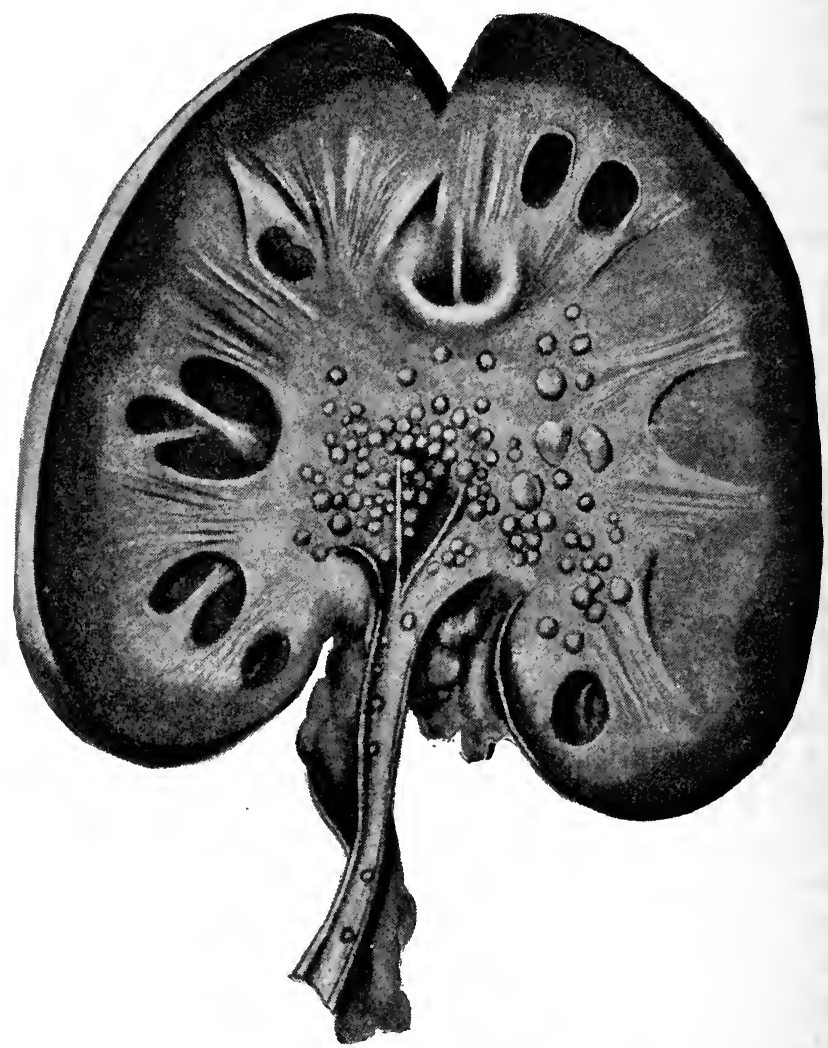

FIG. 13.-Vesicular cysts of the mucosa of the pelvis and ureter. (Le Dentu.)

ence will be determined mainly by the urgency of the pressure symptoms. The choice of operation lies between aspiration of the contents, incision and drainage, and nephrectomy. The latter, according to Le Dentu, has yielded, in 11 cases, 7 deaths and 4 cures.

Extraperitoneal aspiration in the loin is said to have cured once (Duplay). Theoretically, its dangers should not be very great, and it seems proper to employ it, and to repeat it in case the cyst refills. The 
risks of following it with the injection of an irritant, such as tincture of iodine, are probably considerable.

Incision with drainage in the loin is probably but little, if any, more dangerous than aspiration; the edges of the incision in the cyst should be stitched to the skin.

Incision with drainage through the peritoneal cavity, under proper protection, would not involve much more risk; and in cases in which the development of the cyst has been toward the peritoneal cavity, and not along the posterior and lateral abdominal wall, it would be the only practicable method. It is applicable only to large cysts in contact with the anterior abdominal wall. In cases in which the abdomen had been opened under the impression that the cyst was ovarian, it seems probable that most surgeons would complete the operation in this way.

Nephrectomy should be reserved for cases with special indications. Quite recently Tuffier (Arch. gén. de méd., July 1891) has successfully practiced extirpation of the cyst and reunion of the kidney parenchyma by suture.

2. CONGLOMERATE CYSTS, OR CYSTIC DEGENERATION, OR LARGE POLYCYSTIC KIDNEY.

A kidney thus affected is readily distinguishable from one containing multiple isolated cysts, as just described, (r from one studded with the minute cysts of interstitial nephritis. Its surface is lobulated, its size often very great, its shape nearly that of a normal kidney, and on section its parenchyma is seen wholly to have given place to a multitude of closely agglomerated cysts of various sizes (Fig. 14). In Hare's case the kidney was fifteen inches long, and weighed sixteen pounds. The smallest cysts are microscopical, the largest rarely larger than a grape. Some of the larger ones appear to have been formed by the coalescence of several smaller ones, the wall showing on its inner surface the remains of the septa that previously separated them. This inner surface is covered with pavement epithelium. Of the kidney-substance, only traces can be found here and there in the larger tumors; in the smaller ones it is present in varying amount, and shows the changes of interstitial nephritis.

The contents of the cyst vary greatly in color and consistency, and are sometimes purulent, bloody, or urinous.

Usually both kidneys are involved, but unequally; and in a few cases the liver has shown a similar degeneration.

The pathogeny of the affection has not been ascertained; the principal theories suggested are obstruction of the tubules as the result of an interstitial nephritis (Virchow), or of lithiasis (Lecorché), or epithelial degeneration similar to that which leads to ovarian or testicular cystomata (Malassez). The condition is sometimes congenital, sometimes acquired, 
and in the latter case is most common between the ages of forty and fifty years.

Symptoms. - The symptoms, when any are present, are in the main some of those of Bright's disease : pain in the loins, occasional hæmaturia,

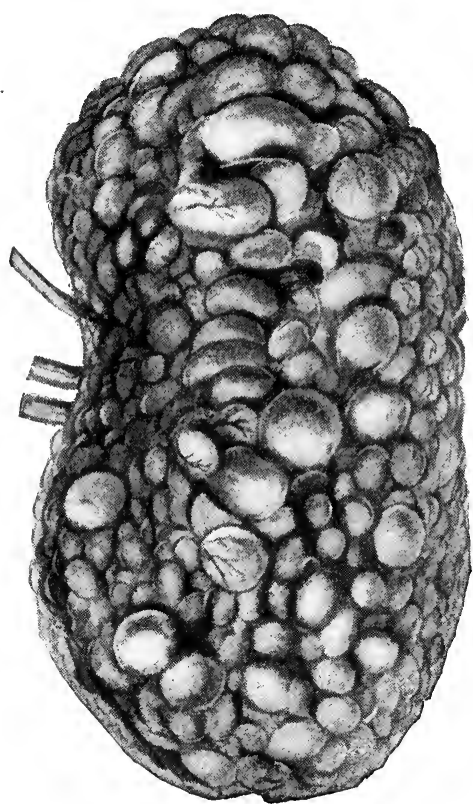

FIG. 14.-Polycystic degeneration of the kidney. (Le Dentu.) the urine sometimes scanty and bloody, sometimes pale, light, albuminous, and abundant; hypertrophy of the heart. When the destruction of the kidneytissue is sufficiently advanced uræmic symptoms appear. Occasionally the tumor is recognized on palpation.

Diagnosis. - The diagnosis might be made by recognition of enlargement of both kidneys with lobulation of the surface, the changes in the urine indicative of nephritis, and œedema limited to the lower limbs and dependent upon pressure made by the kidney upon the large abdominal veins.

Prognosis and Treatment.The prognosis is grave, because of the inevitable ultimate destruction of the kidney, and the possibility that any almost indifferent intercurrent cause may lead to a fatal uræmic outbreak; but it is somewhat mitigated by the slow progress of the disease.

No surgical treatment is possible if both kidneys are involved, or desirable in the exceptional cases in which only one is diseased.

\section{HYDATID CYSTS.}

Although hydatid cysts of the kidney have long been known, the first attempt to collect and analyze the scattered cases was made in 1861, by Bérand, in a graduating thesis. He collected 41 cases, 7 of which had not been published. Roberts, of Manchester, collected 63 ; and quite recently (1887) J. Boeckel (Etude sur les kystes hydatiques du rein) added 20 new cases to the list.

Compared with those of other organs, hydatid cysts of the kidney are rare : 30 cases in 566 collected by Davaine, 80 of 983 collected by Neisser. Béraud found the left kidney more frequently affected than the right, and, although some of the subsequent statistics have reversed this predominance, it has been re-established by the latest and largest. Occasionally, but very rarely, the other kidney or another organ is simultaneously affected. 
The cyst may form immediately beneath the capsule or deep in the kidney-tissue; and it may not exceed an orange in size, or it may become very large. It shows a distinct tendency to grow toward the peritoneal cavity, and thus to drag the kidney from its place and make it abnormally movable. According to Roberts, there is a strong probability of ultimate rupture of the cyst into the pelvis of the kidney and discharge of its contents through the ureter and bladder; this was observed in 52 of the 63 cases collected by him. Rupture into the intestine has been observed in 4 cases, into the stomach in 1, into the lung in 4 , and into the muscular planes of the back in 3 . The cyst may suppurate, or it may shrink after death of its contents, as in hydatids of other organs. By lodgment of a fragment or of a daughter-cyst in the ureter, hydrone. phrosis may be caused.

Symptoms and Diagnosis. - A hydatid cyst gives no symptoms, unless it is large enough to make pressure upon adjoining organs or to be recognized by palpation, or unless it ruptures into some hollow viscus through which the hooklets or daughter-cysts may be evacuated. The tumor is globular, elastic, and flat on percussion. The hydatid thrill appears to be rarely recognizable; the manipulation for its detection is thus described by Newman (Surgical Diseases of the Kidneys, p. 140): "The cyst should be compressed by the second finger of the left hand, and a slight, sharp tap should be given to it with the finger of the right hand. The finger should be allowed to rest for a short time, when a peculiar vibratory thrill will be communicated to the left hand. When felt it is of great value, but its absence is of no significance. Its duration is uncertain-sometimes brief, but frequently prolonged."

Rupture into the pelvis of the kidney is followed by the symptoms of nephritic colic, and the appearance in the urine of the characteristic hooklets or cysts. This appearance in the urine, while positive evidence of the presence in the abdomen of a hydatid cyst, is not proof that the cyst occupies the kidney, for cysts of the liver may rupture into the pelvis or ureter, and a cyst developed in the connective tissue between the bladder and rectum has ruptured into the former. Rupture into the lungs is indicated by the expectoration of the characteristic elements, and rupture into the intestine by their presence in the fæces in case other circumstances lead to the necessary examination. The collapse of the cyst after rupture may be so complete that even the most careful palpation will not determine its position. Exploratory puncture of a recognizable tumor may serve to determine its character. Still, it is to be remembered that the diagnosis, both of the nature and of the character of the tumor, is extremely difficult and uncertain, and the records of published cases are full of errors. Le Dentu noticed in his cases that he could distinguish one portion of the tumor as globular and fluctuating or elastic, and an ad- 
joining portion as solid and resembling a kidney in shape and consistency, and he thinks that a similar finding would justify the diagnosis of a hydatid cyst.

The evolution of the affection is slow, but the tendency is distinctly toward a fatal result. Of 22 cases of spontaneous rupture into the pelvis of the kidney, in which the final result is recorded, 6 appear to have been cured, in 6 the condition remained stationary, and 10 died. Of Roberts's 63 cases the ultimate result is known in 39 ; of these, 19 died, but it is

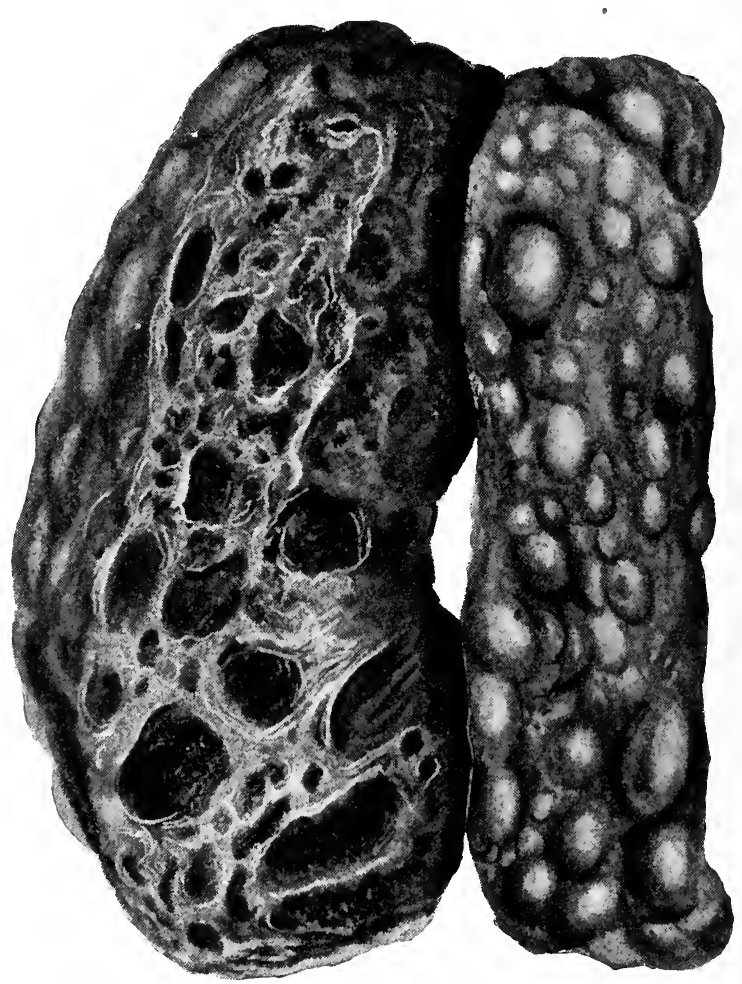

Fig. 15-Cystic degeneration of the kidney. (Morris.)

said that in 9 of them the causes of death were unconnected with the hydatids (Morris). On the other hand, cases are recorded in which patients have continued "to pass vesicles at intervals for twenty and even thirty years." According to Morris, there is no fatal case on record in which vesicles have escaped per urethram from a renal hydatid cyst which had not given rise to an abdominal tumor.

Treatment. - Simple aspiration of the contents of the cyst has been followed in a few cases by an apparently permanent cure, or at least by a long period of quiescence; it is therefore entitled to a trial in any case 
in which the needle can be introduced without passing it through the peritoneal cavity. This failing, incision of the cyst, in the loin or through the peritoneal cavity, followed by excision of as much of the sac as can be conveniently removed, and by suture of the edge of the remainder in the parietal wound, is the method of choice, and has given excellent results.

Nephrectomy may be proper if the kidney is wholly destroyed, and if the tumor is free and movable in the abdomen.

Experience with hydatid cysts of other organs indicates that possibly the injection of a 1-1000 solution of the bichloride of mercury into the i sac after evacuation of its contents, or the injection of a few grammes of the solution by a hypodermic syringe, without previous evacuation, may effect a radical cure.

\section{SOLID TUMORS OF THE KIDNEY.}

\section{A. MALIGNAN'T TUMORS.}

It will be convenient in this chapter to employ the term cancer as a synonym of malignant tumor, including under it the observed varieties of carcinoma, sarcoma, and lymphadenoma.

Cancer of the kidney may appear at any age, but is most frequent in infancy and in advanced life, and is more often seen in males than in females. It may be primary or secondary, the latter, according to Morris, being five times as frequent as the former-twenty-five secondary and five primary having been found in the 2,610 successive autopsies which he analyzed. Of the secondary cases, which, of course, are entirely beyond the resources of our art, I shall only say that they appear to be more common in females than in males, and that in Morris's 25 cases the seat of the primary disease [carcinoma] was the female breast in 5 ; the female genital organs and bladder in 4 ; the rectum in 2 ; the omentum, duodenum, and liver, 1 each; lymphadenoma in stomach 1, and prostate 1 ; sarcoma in arm, axilla, pleura, orbit, spinal dura, and a fibroma of the uterus, each in 1 ; epithelioma in the tongue 1 , and cervical glands 1 .

Primary cancer appears in three forms-carcinoma, sarcoma, and lymphadenoma, sarcoma occurring mainly in the young, sometimes congenital, and carcinoma in the old; lymphadenoma is very rare. Of the carcinomata, the encephaloid is the more common variety, then the melanotic; scirrhus and colloid are so rare that it is questioned if the few reported cases were correctly understood.

The common forms of sarcoma have been observed, including the myosarcoma or rhabdomyoma, which is characterized by the presence of striped and unstriped muscular fibers, and is rarely seen except in the kidney. 
Until a late period in its evolution the neoplasm is confined within the capsule proper of the kidney, appearing either as a single mass, or as a number of scattered nodules varying in size from that of a pea to that

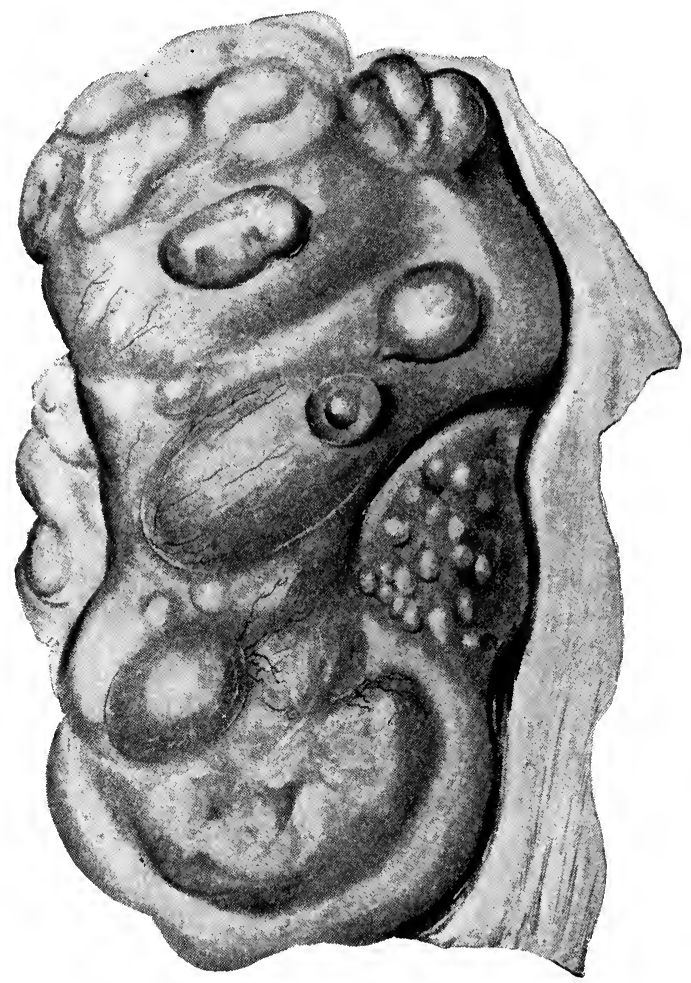

FIG. 16.-Cancer of the kidney. (Rayer.) of an egg, or as a general infiltration of the kidney. The capsule thickens over the growth and its veins enlarge, and ultimately it gives way and allows the tumor to appear on the surface as a cauliflower growth, or it contracts adhesions with adjoining parts, and the cancer spreads directly through it into them. On the other hand, generalization of the disease takes place promptly, affecting first the lymphatics at the hilum and the neighboring lumbar retroperitoneal glands. Roberts found generalization to have taken place in 31 cases out of 51; Dickinson in 14 out of 19 ; Rohrer in 50 out of 115 ; Ebstein in more than half; Laschmann in 26 out of 39 ; and Guillet in 47 out of 70. (Quoted by Chevalier, Tumeurs malignes du rein, Thèse de Paris, 1891.)

In the adult the size of the tumor is not often great, but in the child it may reach ten, twenty, even thirty pounds. The growth is forward toward the peritoneal cavity, and then, as its weight increases, downward; on the right it tends to pass completely to the outside of the ascending colon; on the left it is habitually crossed by the upper part of the descending colon. The tumor may compress the renal vein or the vena eava, or obstruct it more or less completely by direct growth through its wall into its lumen, and in the latter case may give rise to fatal embolism of the pulmonary artery, the embolus being either a blood-clot or a fragment from the projecting portion of the tumor. Sooner or later it spreads to the pelvis of the kidney and the ureter, or it may originate in the 
former in a form apparently identical with the "villous tumor" of the bladder.

Symptoms.-In the typical form, as presented in about half the cases in adults, the patient complains of vague pain in the lumbar region, that appears and disappears without apparent cause, and lasts for longer and shorter periods; occasionally it is sharp and paroxysmal, as in nephritic colic; at the same time he voids blood at intervals with his urine, and the amount of the bleeding, its appearance, and its cessation do not appear to be influenced by exercise or rest. The blood is uniformly mixed with the urine, but sometimes long clots are present. The frequency of micturition is not increased, and the general health may not be greatly deteriorated. A tumor may be recognizable in the flank-a rounded, firm, movable tumor. Varicocele may be present on the same side. In other cases, pain and recurrent hæmaturia exist but the tumor is absent; and in others the tumor is present and the other symptoms absent. Exceptionally, all these symptoms may be absent, and a steadily increasing cachexia may be the only indication of the disease.

The tumor, when small, may be detected by careful palpation, one hand being placed transversely against the liver, the other longitudinally upon the anterior wall, with the tips of the fingers close below the free border of the ribs. If, by steady pressure in this position, the kidney can be felt, it is to be considered enlarged or displaced. Guyon attaches much diagnostic importance to ballottement of the kidney, obtained while the hands are in the position just described, and moderate pressure is made by the anterior one by pressing sharply forward with the other fingers in the loin; the kidney, if enlarged, can be felt to strike against the anterior wall. Another method is to grasp the flank with one hand, the thumb in front, the fingers behind, and to press the tips of the fingers of the other hand deeply in beside the median line at and above the level of the umbilicus; the kidney can then be felt to move downward in deep inspiration.

Varicocele is so common an affection on the left side that it is significant only when on the right.

Hæmaturia occurs at irregular intervals, without recognizable cause, although sometimes it follows vigorous palpation of the kidney, and usually lasts for several days; it may cease for a few hours coincidently with colicky pain, and reappear after cessation of the pain. It is a more common symptom in adults than in children. Occasionally fragments of cancer-tissue have been voided with the urine.

Pain is ordinarily dull, but may at times be severe. Pressure upon the stomach or intestines may produce gastric symptoms, and pressure on the vena cava may cause odema of the lower limbs.

Diagnosis. - The diagnosis, as to both the seat and the nature of the 
tumor, is full of difficulty, and the records show many mistakes made by skillful and experienced surgeons. The source of hæmaturia can usually be satisfactorily determined by the use of methods elsewhere described, and when it coexists with a recognizable tumor in the flank the renal origin of the latter may be safely assumed. The differential diagnosis from renal calculus, pyelonephritis, tuberculosis, and hydronophrosis, must be made by the absence of the groups of symptoms indicative of those affections. As between sarcoma and carcinoma, the differential diagnosis is not possible, and, indeed, is not worth making, since the clinical course is the same. The urgency of an early diagnosis is great, for it is only in the early stage of the disease that surgery can hold out any hope of a cure; for that reason an exploratory laparotomy, or lumbar incision, seems fully justifiable whenever the symptoms indicate or reasonably suggest a beginning cancer.

Treatment. - The only operation that can be considered, according to our present knowledge, is the removal of the tumor and the kidneynephrectomy; and the record, which comprises more than one hundred cases, is not encouraging. Remembering the promptness with which generalization takes place, it may fairly be claimed that when the tumor is large enough to be easily recognizable through the abdominal wall its removal would not be a removal of all the disease, and that, merely as a palliative measure, without hope of a radical cure, nephrectomy is too dangerous to be undertaken. This practically excludes it in all cases occurring in children, for in them, as a rule, attention is first called to the disease by the recognition of an abdominal tumor.

In cases in which pain and hæmaturia have led to a diagnosis early in the course of the disease, nephrectomy is a justifiable although hazardous procedure. The lumbar-extraperitoneal-operation has shown a larger measure of operative successes than the transperitoneal method, and therefore would probably be preferred by most, notwithstanding certain rather important advantages that are offered by the other. With a constantly improving technique, and with earlier accurate diagnosis, it may be hoped that a larger measure of success will attend operative interference in the future.

\section{B. BENIGN TUMORS.}

Benign tumors of the kidney seldom have any other than a pathological interest; clinical importance comes only from the size which they sometimes attain, and in certain diffuse lipomata from their compression of the kidney-tissue. The only indication for operative interference, consequently, comes from the size of the tumor; certain large fibromata and cysto-fibromata have been successfully removed by transperitoneal nephrectomy. 


\section{DISPLACED KIDNEY.-MOVABLE KIDNEY.}

The kidney may congenitally occupy a position other than that in which it normally lies, one even as low as the true pelvis where it has been found behind the rectum and between the rectum and bladder. That the ectopia is congenital, is proved by the origin of its artery, at a correspondingly low point in the aorta or one of the iliac arteries, and the emptying of its vein into the nearest large venous trunk. The condition has no surgical importance except in diagnosis; it is important that it should not be mistaken for an acquired and remediable displacement.

An acquired displacement with abnormal mobility is known as movable kidney or floating kidney. These two terms are generally used interchangeably, although it is sought by some so to use them as to distinguish between two different anatomical conditions-to apply the term movable to those cases in which the kidney is freely movable behind the peritonæum, and the term floating to those in which the kidney has moved forward, away from the posterior abdominal wall, and has become completely invested by the peritonæum and has a distinct mesonephron. Although it is admitted that the two conditions can not be distinguished from each other except by direct examination, it is claimed that the surgical importance of preserving the distinction is great. As the second condition, that of complete peritoneal investment with formation of a mesonephron, is so rare that only two or three authenticated cases are on record; and as the term "floating kidney" is in almost universal use to designate the clinical condition of abnormal mobility which includes both forms, the attempt to discriminate may well be abandoned, and the two terms be used indifferently. Such a result would be in harmony with the conclusions of a committee of the London Pathological Society appointed to report on the subject.

Acquired ectopia (which alone will be considered in the remainder of this chapter) is developed in most cases between the ages of twenty and forty years, is very much more common in women than in men (55 to 9 , Lancereaux ; 82 to 14, Ebstein), and on the right side than on the left (65 to 14, Ebstein). Catamenial congestion, parturition, and tight lacing, are thought to be frequent causes; but when we reflect how common these alleged causative influences are, and how rare the condition under discussion ( 5 cases in 3,658 autopsies, Ebstein ; 22 cases in 5,500, Rollet), we must admit the probability of some predisposing or associated cause. There is good reason to think that when there is but one kidney (congenitally) it is particularly liable to become displaced, presumably because of its greater weight.

The displaced organ appears to suffer but slight changes, if any, as 
the result of its displacement. It has been found congested, sometimes larger, sometimes smaller, than normal, with indications of interstitial nephritis. In the chapter on hydronephrosis mention has been made of the production of a sharp turn or kink in the upper part of the ureter by displacement of the kidney, and the consequent interference with the flow of urine.

Symptoms.-In a well-marked case the kidney, recognizable by its shape, size, and consistency, presents itself as a freely movable tumor at a greater or less distance below the free border of the ribs. Pressure upon it causes pain, or a peculiar sickening feeling. The patient com. plains of dull or sharp dragging pain in the abdomen, provoked by standing, walking, on the approach of the menstrual period, or perhaps of sharp, paroxysmal pain resembling kidney colic. With these are associated other more or less vague sensations referred to the stomach, intestines, or liver. These symptoms are generally relieved by recumbency, and sometimes by wearing a tight girdle. Pressure upward and back. ward upon the kidney when the patient is in dorsal decubitus causes it to disappear, or at least to become much less easily recognizable. Its independence of the liver can usually be determinęd by palpation and percussion, and it is to be distinguished from other renal tumors, solid or cystic, by its size, shape, consistency, and mobility. It may be confounded with stercoral accumulation in the colon, or with tumors of the mesentery or omentum Cases of slight displacement and sharp paroxysmal pain, due to obstruction of the ureter by kinking, are to be distinguished from renal calculus by the absence of blood from the urine, and by the promptness with which the pain will sometimes cease when the displacement is corrected by a change in the patient's attitude, such as raising the hips above the shoulders, or by pressure upon the kidney.

Treatment.-In the slight cases it may be sufficient to avoid the forms of exercise which excite the pain; in others, specially fitted and padded abdominal bandages have effectually opposed recurrence of the displacement, and enabled the patient to go about as usual. One device that has been especially recommended is the addition to such a bandage of a rubber bag, which when inflated would hold the kidney in place. Special trusses, with adjustable pads and arms, are said also to have afforded relief; but the tyranny of such dressings seems intolerable when it can be avoided, and experience has shown that it can be escaped in many cases by a simple operation that involves but little risk.

This operation-nephrorrhaphy or nephropexy-consists in exposing the kidney by an incision in the loin, drawing it back to its proper place, and fixing it there by sutures. It is urged on theoretical grounds that the operation is impracticable when a mesonephron exists; but no operation that has yet been begun has had to be abandoned on that ground, 
and it is not easy to see why, if that very rare condition should be encountered, the mesonephron should not be split, or even the peritonæum beside it opened, and the kidney drawn out through the opening.

An alternative operation is the removal of the kidney. That has been practiced in a considerable number of cases. Newman (loc. cit., p. 62) collected 30 ; in 6 of them the kidney was removed through the loin, all successfully; in the remainder it was removed by laparotomy -24 cases, with 9 deaths. In one of these cases the removed kidney was the only one the patient had; she survived eleven days. In two of the 10 cases of lumbar nephrectomy collected by Brodeur the patients died. In one of them the second kidney was lacking, and in the other it was functionally incapacitated. The possibility of such a state of things, or of the other kidney being seriously diseased, and the risks inherent in the operation itself, should limit resort to nephrectomy to cases in which all other means of relief have failed, and to those in which it is indicated by the co-existence of some other disease, such as pyelonephritis or tuberculosis. That in this small number of nephrectomies the kidney should twice have been found to be the only one, shows that the chance of encountering the condition in this affection is vastly greater than it would ordinarily be. The opportunity which it affords to determine the presence or absence of the other kidney by direct palpation is the only reason for preferring transperitoneal to lumbar nephrectomy.

\section{OPERATIONS UPON THE KIDNEY.}

These comprise nephrotomy, nephrolithotomy, nephrectomy, and nephrorrhaphy or nephropexy.

The kidney may be approached for exploration, incision, or removal through the loin, or through the anterior abdominal wall and across the peritoneal cavity. When the conditions that make the operation necessary are such that either route may be taken, the preference is usually given to the lumbar incision as the least dangerous; and of late it has even been so extended and combined with opening into the peritoneal cavity when necessary, that it has acquired for itself some of the advantages that have heretofore been thought to be obtainable only by the anterior incision. As the details of the incision through the abdominal wall are in the main the same for the different operations, it will be convenient first to describe them, and then to give the subsequent steps of each operation under its own title.

The Lumbar Incision. - This may be longitudinal, along the outer border of the sacro-lumbalis, or transverse, between the twelfth rib and the ilium, or a combination of the two. If the former is used, the patient should be placed in a position that is inclined $30^{\circ}$ or $40^{\circ}$ from the prone 
toward the opposite lateral position, and the trunk should be laterally flexed so as to enlarge the space between the twelfth rib and the crest of the ilium on the side of the operation. If the transverse incision is used, the inclination toward the opposite lateral position may advantageously be greater.

The Longitudinal Incision is made along the outer border of the sacrolumbalis mass, which ean be readily recognized by the finger, about two and a half to three inches from the median line, and should extend through the skin from the level of the eleventh rib to that of the crest of the ilium. It is deepened, layer by layer, until the strong middle layer of the lumbar fascia, or aponeurosis of the transversalis, is reached, after division of which the posterior surface of the quadratus lumborum is exposed. The outer border of this muscle is cleared and drawn toward the spine, and then the fat enveloping the kidney can usually be seen through the thin anterior layer of the lumbar fascia, moving up and down with the acts of respiration. Space can be advantageously gained by dividing the outer portion of the quadratus close to its attachment to the ilium. On division of the thin intervening fascia the fatty capsule of the kidney is reached, and by tearing through it and stripping it toward the sides the posterior surface of the middle and lower portions of the kidney and its pelvis is exposed to sight and touch.

Except in persons who are very fat this incision gives ample room for exploration and nephrotomy, and even for nephrectomy when the kidney is not very much enlarged. It has the advantages of not weakening the abdominal wall and thereby predisposing to hernia, and of not carrying much risk of accidentally opening the peritoneal cavity. On the other hand, the irregular relations of the pleura, and the absence or incomplete development of the twelfth rib in a certain proportion of cases, expose to accidental wounding of the pleura-a complication that has occurred and has proved fatal. Holl found the twelfth rib absent in three of sixty cadavers examined (five per cent)-a proportion so unexpectedly large, that it is now deemed an important precaution to count the ribs before beginning the operation.

A portion of the twelfth rib, and even of the eleventh, has been resected in a few cases in order to gain more room upward-a procedure which of course involves additional risk of wounding the pleura. Some room can be more safely gained by drawing the rib forcibly upward with a blunt hook.

The Transverse Incision is begun just within the outer margin of the sacro-lumbalis, a little below the twelfth rib, and carried outward parallel to the rib for about four inches. The muscular and aponeurotic layers are successively divided, after recognition, until the retroperitoneal layer is reached and the kidney exposed by division of its fatty capsule, 
as in the preceding description. Additional space can be gained by a short longitudinal cut at the inner end of the main incision.

The especial advantages of this incision appear in nephrectomy, when the kidney is much enlarged. When an exceptional indication for resort to it arises from the great size of the kidney, or from its close inflammatory attachment to the peritonæum, this incision permits an easy and free opening into the peritoneal cavity, through which the hand can be introduced for exploration or to draw the kidney outward, and to facilitate the securing of the pedicle, and other later steps of the operation.

The combination incision consists of the longitudinal with a transverse at either or even at each end, or of a long curved incision, the convexity directed downward and backward, beginning at the posterior part of the twelfth rib and running for some distance around the flank.

Some surgeons recommend for nephrectomy a more external longitudinal incision, one running from the outer end of the eleventh rib to the crest of the ilium, because through it the anterior surface of the kidney can be more readily reached and followed inward to the hilum, and the vessels there secured.

The anterior incision does not differ from that of an ordinary laparotomy, except that it is usually made along the linea semilunaris instead of in the median line.

\section{NEPHROTOMY.}

The kidney having been exposed by the lumbar incision, it remains only to cut into it to evacuate the abscess or cyst that has made the operation necessary. This may be done with the knife or the thermo-cautery. Then, if the cavity is very irregular, or if there are separate pouches, the septa should be freely divided whenever it is necessary to secure efficient drainage, and the interior of the cavity thoroughly. scraped with a sharp spoon if its condition requires it. Occasionally it will be possible and desirable to draw the edges of the sac into the parietal wound and stitch them to the skin. Drainage is provided for by large rubber tubes packed around with iodoform gauze.

\section{NEPHROLITHOTOMY.}

The posterior surface of the kidney having been freed as far as possible from its fatty capsule, the surgeon proceeds to seek for signs of the presence and location of a calculus. He may be able to recognize it by touch, grasping the kidney between the thumb and finger; or its position under the thinned cortex may be indicated by a rounded bulging of the surface and a deepening of the color; or the stone may be touched with the point of a needle introduced through the parenchyma 
or the wall of the pelvis. This latter mode of exploration has been proved to be free from serious risk, and when employed should be made systematically, so as to explore all accessible portions of the kidney, the needle being introduced at intervals of about half an inch.

The incision for the removal of a stone has usually been made through the posterior wall of the pelvis, and it has been made with the knife rather than with the cautery; but some recent experience has shown that an incision through the substance of the organ into the pelvis has some advantages, and does not expose to troublesome hæmorrhage. Such pressure as can be readily made by sutures checks the bleeding, and it is believed that a urinary fistula is then less likely to be established than after incision of the pelvis. If the stone lies under a changed and projecting portion of the cortex, it should be reached by an incision through the cortex radiating from the pelvis to the convex border.

If the stone is small, and free in the pelvis, or in a dilated calix, or in a pouch formed abont it in the cortex, its removal is easy-it may be picked out with the fingers or forceps; but if it is large, irregular, or adherent, its removal may be very difficult-it may be necessary to break it into pieces, to divide intervening septa, or to open into separate, adjoining pouches. For the division of septa, strong scissors are said to be better than the knife or the cautery. To break a large, irregular stone, a lithotrite or strong sequestrum forceps may be used. To remove stone from cavities with irregular approaches, scoops of the most varied and bizarre shapes have been designed, few of which have received the approval of others than the inventors, and all of which might still leave the surgeon unprovided with the particular shape that was desirable for the case in hand. In a number of reported cases the surgeon has expressed the opinion, or the autopsy has shown, that stones have been left undiscovered or unremoved.

Occasionally the stone has proved to be so large, or the stones have been so numerous and so difficult of removal, that it has seemed wiser to remove the entire organ-to do a nephrectomy instead of a nephrolithotomy.

The orifice of the ureter should next be sought and that canal explored, to determine whether it is free, or whether plugged by a stone or a mass of fibrin. If such an obstruction is found, it may perhaps be pressed downward into the bladder, or washed out, as was very ingeniously done by Dr. Lange, by a stream of water directed into the distended ureter through the wound.

The stone or stones having been removed, the incision in the pelvis or in the substance of the kidney may be closed with catgut sutures, to hasten its repair and diminish the chance of the establishment of a 
urinary fistula. Provision for the drainage of the parietal wound, and the prompt removal of urine or pus that may escape into it, is made by rubber drains and iodoform packing; the external wound is partly closed by sutures, and a large absorbent dressing is applied.

\section{NEPHRECTOMY.}

After three impromptu removals of the kidney by laparotomy through an error in the diagnosis, in 1861 (Wolcott), 1867 (Spiegelberg), and 1868 (Peaslee), all of which terminated fatally, Gustav Simon, of Heidelberg, first did the operation deliberately and successfully by the lumbar incision on August 2, 1869. His patient was a woman, and the condition for which the operation was undertaken was a urinary fistula following ovariotomy. During the twenty-three years that have since elapsed a large number of operations have been done for suppurative disease-tumors, cysts, calculus, urinary fistula, and abnormal mobility. The removal has always been total, except in some suppurative cases where the ligature could not be got entirely around, or where the anterior portion has been left as a disorganized shred adherent to the peritonæum. Quite recently a formal partial excision was done by Tuffier (Arch. Gén. de Méd., July, 1891); he excised a cyst, and closed the gap in the parenchyma by suture. Recent experience with exploratory incisions through the parenchyma indicates that excision of considerable portions of the organ can safely be undertaken.

The kidney may be removed through either of the two incisions above described. When the lumbar incision is used, the operation is known as lumbar or extraperitoneal nephrectomy; when the other is used, as abdominal or transperitoneal nephrectomy. The mortality of the former has thus far been much smaller than that of the latter, but there are conditions in which the latter alone is practicable, and both operations must be retained.

Lumbar Nephrectomy.-In making a choice between the different incisions used for approaching the kidney in the loin, it will be remembered that the larger the kidney the more advisable will it be to use a transverse incision, either alone, or combined with a short, longitudinal one at its posterior end; it can be more advantageously extended in case of need, and the peritoneal cavity can be more readily entered through it, if that be more desirable.

The details of the enucleation of the kidney vary in accordance with the varying conditions in which it may be found. If inflammation has not caused fusion of the fatty capsule and the cortex into a single lardaceous layer, or united the kidney closely to the peritonæum or colon, it is comparatively easy, after having reached the posterior surface of the kidney, to work the fingers around its convex border and its two extremi- 
ties and separate it from all its attachments except the sort of pedicle constituted by the renal vessels and the ureter. This pedicle may be ligated in sections, the ligatures being passed by means of a large, full-curved aneurism or pedicle needle, or occasionally the artery can be isolated by sight or touch and tied separately, which is much to be preferred when possible, or the entire pedicle can be tied en masse. If the latter course is followed, an elastic ligature should be used. In some cases, instead of a ligature, a long, strong clamp has been placed on the pedicle. In making the enucleation the operator must proceed with great gentleness, especially at the extremities, where abnormal vessels may be encountered; and he must not draw the kidney too forcibly into the wound, lest he tear the vessels.

If the kidney is much altered and adherent, the need of care in detaching it is still greater. The accidents that have happened are varied and serious-laceration of the peritonæum, colon, and even, in several cases, of the vena cava, with immediately fatal result. Where the adhesion of the anterior surface of the kidney to adjoining tissnes has been very close, it has been successfully practiced to remove the posterior half of the organ and then to scrape away from the remaining cut surface as much as it seemed safe to remove. The healing of the wound may require a somewhat longer time, but that is not an excessive price to pay for the greater security.

If the ureter has been separately divided, it is well to close it with a ligature, and, in case of need, to disinfect the stump.

The pouch left by the removal of the kidney should be drained and packed with iodoform gauze, and a large absorbent dressing applied over all.

Abdominal (Transperitoneal) Nephrectomy. - As has been said, the place of election for the parietal incision is along the linear semilunaris, the outer border of the rectus. The abdomen having been opened, and the intestines pressed aside with flat sponges, or brought out of the cavity and wrapped in hot cloths, the surgeon proceeds to strip the peritonæum from the kidney and secure the vessels. It is very desirable that the second incision in the peritonæum should be made beyond, on the outer side of the colon, so as not to interfere with its blood-supply, and this should always be done unless the size of the tumor and the position of the colon make it impracticable. The incision should be longitudinal, and sufficiently long to make the enucleation as easy as possible. It is advisable to proceed at once along the antero-internal face of the kidney to the hilum to secure the vessels, and it may even be advantageous to go directly to the artery through a special incision in the peritonæum, and secure it as the first step in the enucleation. The vein and the ureter should each be tied separately and divided between two ligatures. The 
size, relations, and friability of the mass introduce elements that can not be adequately treated in a general description, and it has more than once happened that very skillful and experienced surgeons have felt compelled to abandon the operation.

Enucleation is effected, as in the lumbar method, mainly by the fingers, and with preliminary ligature of threatened vessels whenever practicable. Every effort must be made to avoid infection of the cavity by the contents of the kidney or ureter.

In case the kidney is fully pedunculated and invested by the peritonæum, "floating kidney," its removal will be conducted as in the case of any other pedunculated abdominal tumor, without stripping off the peritonæum.

The sides of the gap in the posterior parietal peritonæum may be rapidly drawn together with a continuous suture, and lumbar drainage of the retroperitoneal space provided by a small incision in the loin carrying a rubber drain. The primary incision in the anterior abdominal wall is sutured in the usual manner.

\section{NEPHRORRHAPHY OR NEPHROPEXY.}

The operation by which an abnormally movable kidney was fastened by sutures to the abdominal wall at the point where it should lie, was devised and first practiced by Hahn (Berlin) in 1881. He named it nephrorrhaphy, making use of a term which, with the meaning of suture or union, had already secured a place in medical terminology, as, for example, staphylorrhaphy. Trélat suggested as a more appropriate term for a similar operation upon another organ-fixation of the uterus to the abdominal wall-hysteropexy, from the Greek $\pi \dot{\eta} \gamma \nu v \mu$, signifying to attach. The suggestion has been very generally accepted, and has been so far extended that it seems probable that nephropexy will wholly take the place of nephrorrhaphy.

Hahn placed his sutures in the fatty capsnle, but, as experience soon showed that the fixation thus obtained was not permanent, surgeons ventured to pass them through the fibrous capsule, and finally through the substance of the kidney itself ; in addition, some have excised a portion of the twelfth rib in order to place the sutures at a higher level.

The kidney is exposed, by the longitudinal lumbar incision above described, at the outer border of the sacrolumbalis, and the fatty capsule divided longitudinally and stripped back from the surface of the kidney. Three or four stout silk sutures are then passed with a curved needle from the anterior to the posterior surface, just within the convex border, at intervals of about half an inch, and then through the lumbar fascia in the line of the wound, so that when tied they hold the kidney snugly up against the abdominal wall. The wound may then be closed for 
primary union, or packed with iodoform gauze to heal by granulation.

Guyon sought to strengthen the cicatricial connection by removing a long strip of the fibrous capsule; and Sulzer (Deutsche Zeit. für Chir., vol.xxxi) recommends that the capsule be split and reflected, so as to form a flap which can be stitched in the parietal wound. The latter's statistics contain 80 cases, in 37 of which the sutures were passed through the substance of the kidney, and in 23 through the fibrous capsule. 


\title{
TUBERCULOSIS URO-GENITALIS.
}

\author{
By JOHN P. BRYSON, M. D.
}

Definition.-The series of pathological studies which began with the work of Baillie in 1794, and reached definite conclusions at the hands of Robert Koch in 1881, have put us of the present day in a position to define tuberculosis as an infectious disease, caused by the tubercle bacillus, and characterized by the production of tissue and of inflammatory products, which appear both in the form of nodules and as a more diffuse infiltration, and which rapidly undergo caseation (Reference Handbook of Medical Sciences, vol. vii, page 292). The initial etiological factor-the bacillus Kochii-may have been in the body at birth, or it may have gained entrance afterward in a great variety of ways; it may, after having done but slight damage in the way of tissue-change, lie dormant for years or even a lifetime, or, under certain favorable circumstances, be aroused to an activity which results in wide-spread disaster to tissues, organs, and life.

This disease may attack any part of the uro-genital organs. Kraske has observed a tubercular ulcer on the dorsum of the glans penis of a patient aged forty-nine, which was well demonstrated microscopically after amputation. Other equally well proved cases have been recorded. The disease may invade the uro-genital organs by way of the kidney-one or both-the bladder, the testes, the prostate, etc. As a rule, to which I think there are very few exceptions, when one of the uro-genital organs is found, clinically, to be affected, a careful search will reveal evidences of the existence of the disease in other parts of the same system. With the possible exception of a (so far as the whole body is concerned) primary tubercular infection of the external genitalia, the great mass of evidence goes to show that, at the very best, when one part is involved, the disease spreads so rapidly that for all practical purposes one may say several were simultaneously involved. There must go with this, however, the statement that the primarily involved tissues may only give clinical evidence of disease long after the moment of infection; and that this is true we have abundant pathological evidence. The chief point to observe is, that if not simultaneous the order of involvement is quite rapid. When the genito-urinary organs are attacked secondarily there is no ques- 
tion of the very rapid, if not simultaneous, involvement of more than one part of the tract.

In this, clinical observation coincides in all essential particulars not only with the pathology of the disease, but with both anatomy and physiology. One will hardly speak positively about primary infection-atria - when he remembers that the bacilli may enter through the lungs, leaving no trace of their passage; attack the bronchial glands first, in which case the disease is extremely insidious; develop foci there, from which they may be carried by the blood-stream to find lodgment in the epididymis or testes, there to develop, in turn, a nodule which, on account of its situation, offers palpable evidences of its existence long before the disease can be recognized in the lung by the most expert diagnostician (Bollinger, Munchener Med. Wochenschr., 1890, No. 43).

Or, to take another example, the primary lesion may be in one or more of the solitary glands of the ileum; thence the disease may extend to the overlying peritonæum. From a focus here the bacilli may drop into the recto-vesical pouch, there to set going mischief which may quickly reach the prostate by way of the post-prostatic lymphatics. Here palpation would quickly reveal the existence of a disease which had previously escaped a search even of the most careful kind. Every surgeon of experience knows how insidious and difficult of diagnosis is tubereulosis which takes this course; for if exploratory laparotomy has, as claimed by some, done little in the way of life-saving, it has taught surgeons, in the most striking manner, the evolution of tubercular peritonitis.

Varieties.-All the pathological varieties of tuberculosis are to be seen in the genito-urinary organs. They are:

1. Acute, general miliary tuberculosis. Of this form nothing more need be said here than that it is but a part, and a small part, of that general bodily infection which is abont to terminate the life of the individual; and that it is the condition toward which all tubercular disease tends, though fortunately it is not always reached, even when the primary infection-focns may be an ulcer on the dorsum of the glans penis.

2. A distinctly circumscribed miliary tuberculosis. Here a certain area of tissue is involved in the miliary tubercular infection, the amount so affected being variable in extent and irregular in outline. At first this area is not clearly defined in outline, but gradually, as caseation goes on, the healthy tissue about the periphery offers barriers which so delay the advancement as to make this more easy, and in this way a more or less. discrete tubercular nodule may be developed. This form is most frequently met with in the glands, testes, kidneys, and prostate, and generally has an infected lymphatic gland for its center.

3. A diffuse inflammation, with caseation of the inflammatory products, generally involving a considerable amount of tissue, sometimes the 
whole of an organ, as, ex: gr., the testis. This is by far the most frequent form of tuberculosis met with in the genito-urinary organs, especially in the testes and kidneys.

The second and third forms of the disease, as seen clinically, tend to develop nodules which are, as a rule, quite easily differentiated from the surrounding healthy tissues in those parts accessible to palpation. These nodules vary in size from that of a mustard-seed to that of a chestnut; they are wooden in density, generally painless on moderate pressure, and the larger ones are irregular in outline. If the whole of an organ, as the testis, has been involved in the caseation, the irregular and nodulated appearance and feel are quite distinct. In this respect it differs from syphilitic inflammatory change, which produces an enlargement that is smooth, even, and tends to retain the shape of the organ. I have never observed a syphilitic testis which offered that wooden or stony hardness to the touch which seems to me to characterize the tubercular nodule.

The disease is typically chronic, always begins as a localized affection, and in its progress toward general infection shows a predilection for the lymphatic channels and glands. Most important to a correct appreciation of its course is a recognition of the fact that its rate of progress is, in a marked degree, irregular. One may frequently see a certain, easily observed portion of an organ which has been attacked where the tubercular nodules have remained, to all appearances, dormant for months or years, until from some cause or combination of causes a greater rate of advancement is taken on. This may proceed a certain distance, and again either cease or become so slow as to be imperceptible. At what moment some of the poison may be released from this focus to set up a similar action in a neighboring or far-distant part is not known. Already this may have happened, and in some deeper-seated organ a focus may be in existence, and, in the same quiescent state, lying in wait to infect the whole body; or, on the other hand, this deeper-seated mischief may have been the primary one, unrecognized on account of its situation. Thus the difficulty of correctly appreciating the effects of surgical interference may be clearly seen from the standpoint of the general pathology. This difficulty lies in the uncertainty of declaring any recognized tubercular lesion to be the primary one. There is not only the best authority, but the best reason in pathology and in practice, for saying that this, in the present state of our knowledge, is clinically impossible.

Avenues of Infection. - Too much attention can not be paid to the question of the channels or gateways through which the pathogenic micro-organisms may reach the genito-urinary organs. Upon a determination of this, not only the question of the disease being primary or secondary body-infection will depend, but also, and in consequence, the question of treatment. In fact, these two questions-viz., primary or sec- 
ondary infection-infection-atrium and channels of invasion, hold paramount sway throughout the domain of surgical tuberculosis. It is therefore necessary to review at some length the experimental, pathological, and clinical evidence bearing on the point.

It is clear that the pathogenic microbes can only reach the genitourinary organs in one of the following ways :

1. By primary infection occurring as a local, tubercular lesion on the external genitalia or by way of the urethra.

2. By the lymphatics.

3. By extension from a neighboring organ (auto-infection).

4. By way of the blood-stream.

5. By inheritance.

It is impossible to deny that a man with a tight foreskin might, in coitus, get a few bacilli rubbed into the inner fold of the prepuce, whence they could be taken up by the lymphatic vessels and carried to a deeper gland, which would be infected, without leaving any trace of its point of entry; but it is difficult to see how this could take place without leaving some trace of its course in the way of a tuberculous lymphangitis. Still, one so often sees a lymphangitis occurring in the course of an inflammatory balanitis during a clap that this mode of invasion seems quite possible in the face of our knowledge of the influence of ordinary inflammation on tuberculosis. And, again, pathologists have taught us that the point of entry of the virus is not always visible in the shape of an ulcer (Bollinger, loc. cit.). In such a case the deeper-seated, primary, tubercular lesion might appear in the lymphatics about the prostate. This would be a case of hetero-infection by way of the lymphatics, and it seems all the more probable when we call to mind the fact that only a few bacilli might so enter, and the resulting deeper lesion would but slowly advance, accordingly as the rate of tubercular inflammation is so markedly influenced by the number of bacilli which are introduced. It can only be said that this mode of infection seems possible; I have been unable to find a complete and satisfactory demonstration of such a case, so commonly is the lymphatic system overlooked in autopsies. Experimental tuberculosis gives color, also, to this supposition. Baumgarten speaks of the rate of infection of the lymphatics which are in connection with the primary lesion as being "astonishing" (Baumgarten's Lehrbuch der patholog. Mykologie, vol. ii). Is it possible that a few bacilli might enter, from the same point, a vein, and be carried back to set up, in the periprostatic plexus, a tubercular inflammation? Such a thing seems far-fetched, indeed, but less so than some of the methods suggested in explanation of primary genito-urinary infection. It is certain that in practice nothing is more common than to find isolated and distinct cheesy nodules in the meshes of the vesico-prostatic venous plexus. Such a course of invasion 
would, again, be a primary hetero-infection by way of the blood-vascular system. Whether or not these methods of infection exist can not be determined clinically, but must be left to the experimental pathologists. Certainly, if either of these modes of entry is possible, the frequency of chronic and acute balanitis due to neglect, filth, trauma, tight foreskins, urethritis, etc., open wide the doors for entry of the virus.

It seems a priori far more probable that if primary genito-urinary tuberculosis arises in any of these ways, we would find more frequently than we do tubercular ulcers behind the foreskin, on the glans penis, in and about the fossa navicularis urethræ, and on the cutaneous covering of the external genitalia. It has been suggested that the tubercular nature of these ulcers has been overlooked, so commonly are all ulcers about the external genitalia of both sexes regarded as due to some other virus, and this seems quite probable. Since tubercular ulcers, which are external, and liable to mixed infection on that account, often heal, it is not impossible that in this way some of those cases of chancres that are not followed by general syphilitic infection may be explained. Such so-called chancres may not be followed by syphilis, but may, in the course of a greater length of time, be followed by genito-urinary tuberculosis; and in this way another of those now quite commonly mentioned resemblances between the two diseases be recognized.

That primary infection can or does ever take place by way of the liealthy urethra-that is, infection of the prostate, kidney, testes, and their adnexa-seems to me in the highest degree improbable, unless by some mechanical aid, such as the introduction of uncrean instruments, injections, etc. Even in those frequently occurring cases of acute and chronic suppurative inflammation, which might offer a foothold, it seems much more likely that the gateway of the lymphatics and blood-vessels would be opened. The anatomy and physiology of the urethra, the obstacle offered by the compressor urethræ muscle, the fact that the tubercle bacilli do not multiply in the urine, and have no power of motion of themselves, all speak against the view, seemingly held by some, that the bacilli travel backward along a urethra to develop a primary tuberculosis of the prostate, vesiculæ, epididymis, or even the renal pelvis. There is no doubt whatever of the fact that careless catheterization, injection, and surgical manipulation generally will bring about this result; but that hetero-infection, per urethram, may occur from coitus is justly held to be far-fetched. Increasing knowledge of the pathogeny and more accurate observation are likely to show that those cases of apparently primary tuberculosis of the prostato-vesical region occurring in old men, who, being prostatics, use catheters which are not clean, are really such. When we see tuberculosis of the uro-genital organs of an 
old man, we are naturally surprised, since we are aware of the fact that the disease belongs to adolescence and early manhood, and we are entitled to suppose some unusual cause. The only two cases of apparently primary uro-genital tuberculosis which $I$ have seen were in prostatics who had used the catheter, and who were both unclean in their habits. In one, an apparently primary tubercular prostatitis developed rather suddenly. The more acute inflammation subsided, leaving tubercular nodules in the gland. In three months tubercular epididymo-orchitis had progressed so far that I castrated him. Five months later the opposite testis was almost completely disorganized, when it too was removed by curetting. The wounds healed well. Six years afterward the peritonæum and liver became involved, and eighteen months after this (in June, 1891) the patient died of acute general miliary tuberculosis. The kidneys remained free of disease up to the last fourteen days of his life, when albumin and casts appeared in the urine. In the second case the disease also began in the prostate, and there was already a mild, chronic, simple pyelonephritis, from extension. The onset was acute, and an abscess formed in the prostate, discharging through the urethra. There was an extensive destruction of the prostate and membranous urethra, and the tubercular ulceration extended to the meatus urethræ externus, and upon the glans penis. Its tubercular character was well demonstrated. The renal disease did not seem to be aggravated, and the testes remained healthy up to his death, which occurred from exhaustion nearly two years after the onset of the tuberculosis. It will be noted in this case that the cause of death was not generally miliary tubereulosis, but chronic uræmia from the simple pyelonephritis.

These cases bear all the clinical appearances of primary genito-urinary tuberculosis, but that they can not positively be so identified the following, which but for the autopsy would have gone on my case-book as one of primary urethral tuberculosis, will show: A man aged thirty-four applied to my clinical assistant complaining of an intense pain and burning on urination. He could distinctly locate the painful spot about an inch and a half behind the meatus externus. There was no discharge and no thickening of the urethral wall to be felt from the outside. His previous history was not obtained. An attempt to pass a filiform instrument failed, because of its point eatching at the painful spot and exciting the greatest distress. No further attempt at instrumentation was made, but an alkaline diluent was given and the patient advised to go into hospital. The next morning he was found dead, sitting over a vessel into which he had passed some few ounces of urine. At the autopsy, made under the coroner's directions, there was found a tubercular ulcer the size of a fivecent piece on the floor of the urethra at the point where the patient located the painful sensation. The prostate, vesiculæ, kidneys, and the 
other uro-genitalia were sound, at least not diseased, so far as gross inspection could detect. The urethral ulcer was demonstrated microscopically to be tubercular; it was not deep or indurated at the base, and did not appear to be of long standing. There was no stricture in front of it or behind it; the meatus was not narrow, nor the foreskin tight. Though in no way accounting for the death of the patient, it would nevertheless have been looked upon as a primary infection had not distinct and old tubercular foci been found in the lungs (bronchial glands) and in the post-cervical lymphatics. Unfortunately, the brain-case was not opened. While the urethral ulcer in this case might be regarded as a hetero-inoculation, it certainly was not the primary tubercular lesion for the body. A more careful research would doubtless have shown beginning tuberculosis of other organs, as well as the cause of death.

In all the recorded cases where primary tuberculosis of the prostate has been alleged to have followed an acute inflammation (notably a gonorrhœa), and where the inflammatory process, extending backward, has been supposed to have carried with it the infecting agent, the evidence of such a mode of invasion has appeared to me entirely inadequate to support the assertion so commonly made. Certainly this is true of the cases which have been observed by me. So often do we see cases of tuberculosis, the first demonstrable evidence of which is about the prostato-vesical region, in young men or boys who have never had clap, who have never had an instrument passed or an injection, and who present no evidences of any previous disease of the uro-genitalia whatever, that it seems far more in consonance with our present knowledge to say that the acute inflammation, acting after the manner of a traumatism, has roused into activity a slumbering or latent tuberculosis, which was present long before the acute attack, perhaps dated even from birth. That this latter actually occurs I have had occasion to observe in several cases. That a tubercular lesion, clearly demonstrable, may remain quiescent for many years-from birth to the accession of puberty in some cases, past puberty in others, and still in others even into old age-is, I suppose, within the observation of all surgeons of experience.

The genito-urinary apparatus having once been invaded, in whatever part, the question of the spread of the disease to the other parts becomes of the greatest importance. Only one part of this question will be considered here-viz., the passage along the surface of the healthy mucous membranes of the infecting agent, from one point to another, as from the bladder by way of the ureters to the renal pelvis, or from the prostatic urethra to the epididymis along the vas deferens. The other or affirmative parts of the evidence bearing on this point will come more properly under the heading of Spread by the Blood-Vascular Channels.

Thronghout the entire literature of surgical tuberculosis two observa- 
tions are quite commonly made: first, that the testes, the kidneys, and the prostate are most commonly first affected; and, second, that the prostate is rarely-some say never-missed. Long ago Dron urged the value of frequent digital examination of the prostate by way of the rectum in doubtful cases, evidently recognizing the great frequency of the affection of this organ. In order to explain the infection of one organ after another by way of the genital and urinary passages, the most ingenious and far-fetched arguments have been brought forward, and we are asked in each one to accept as proved, statements which have in them elements of the highest improbability; and as our knowledge of the pathology of the disease advances these become all the more glaring. That the kidney being first invaded, the bacilli may be carried by the urine downward to infect the bladder, prostatic urethra, and thence the gland itself, seems in every way easy of belief, but a critical search of the literature does not show, so far as I know, a single recorded case where this has been proved to be the course of the disease. All that have come under my observation fail to show that the disease did not reach the lower passages in some other of the well-known ways. The frequent occurrence of the disease in the periprostatic tissues instead of on the surface of the prostatic mucous membrane, when the course of the infection has been supposed to be along the urinary channels, has not been given sufficient importance in this connection. Nevertheless, that such a course is occasionally taken we are not permitted to doubt; it is in regard to the frequency of such a course of infection that objection is here made. Reports of such cases invariably fail to eliminate one chief source of error-viz., the existence of tubercular disease in other parts of the body and the point of primary body infection.

What has just been said applies equally and in all respects to those cases where the disease is alleged to have leaped from the testis to the prostate by way of the vas deferens, without visibly extending along this duct.

Back of all this lies the question of whether we can ever have a primary body infection occurring in the testis or kidney. On this point pathologists are at variance. For my own part, I fully accept the statement of Baumgarten (loc. cit., vol. ii, page 625), that there are cases of congenital tuberculosis.

Much more difficult of belief is the statement, so frequently made, that the disease may spring from the bladder to the kidney by way of the ureter withont invading that duct in its course from below upward. Only the existence of very exceptional conditions could bring about any such result. The bacilli, having no power of independent motion, must in such a case mount the duct against an almost constantly flowing physiological current, having gained access to the ureter by way of one of the 
most perfectly contrived valves in the body. We know nothing of a toand-fro movement of urine in the ureter, and are equally ignorant of an antiperistalsis, such as we see in the bowel, which might carry the inert bacilli up to the pelvis. The whole duct, being collapsible, acts as a valve in any part of its course. Nor does the fact that other forms of inflammation reach the pelvis and kidney from the bladder by extension avail as an explanation. The tubercle bacilli do not multiply in the urine, as do all those other micro-organisms which, as is well known, bring about this result. On the whole, one must reject this method of extension on the grounds of improbability, and all the more readily since there are no well-ascertained clinical facts which require its aid in explanation. Almost as much can be said of that other alleged method of extension, viz., from the prostate or vesiculæ to the testis by way of the vas deferens. Here, however, there may be an antiperistalsis to overcome some of the mechanical difficulties; but, on the other hand, the tubercle bacilli have not yet been demonstrated in the semen. The ciliary movement would seem to stand opposed rather than in favor of such a course. Again, one may say that there are no clinical facts which, in the presence of our knowledge of other well-ascertained methods of extension, require the dragging in of a hypothesis which involves so many difficulties. More accurate clinical observation, more care in autopsies, and advancing knowledge of pathology will soon-if they have not already-render such explanations unnecessary.

That the disease may and does advance both up the ureter from the bladder and down the vas deferens from the prostate, continuously or by dissemination, according to its well-known course on the surface of mucous membranes, there is, of course, no intention here to question.

Of the methods of invasion of the uro-genital organs by way of the lymphatics, there is no need to speak further here, so well has this field been worked over by the pathologists. The predilection of the pathogenic organisms to the lymphatic tissues, and the manner of the dissemination of the disease along these avenues, does, however, require more attention from the clinical student and more care in autopsies than it has heretofore received (Councilman, loc. cit.). I will only mention the fact that extension of the disease by way of the lymphatics offers an adequate explanation of the fact, often to be seen in practice, of the appearance of disseminated tubercles under, instead of in the form of an ulcer upon, the mucons membrane.

The manner of the invasion of the uro-genital tract by extension from neighboring and surrounding organs and tissues is now, thanks to the work of pathologists, well understood, though it is not so extensively recognized or searched for clinically by surgeons as it might be with 
advantage. The kidneys, the ureters, the bladder, the prostate, the seminal vesicles, the vasa deferentia may all be invaded by extension of the disease from surrounding structures. Often, indeed, the appearance of tubercular inflammation in one of the uro-genital organs offers to the surgeon the first satisfactory indication of the nature of a disease which has long been known to affect some neighboring part.

It is by way of the peritonæum that the disease most frequently reaches the uro-genital tract by extension from neighboring organs, though the kidney is sometimes invaded from a tubercular bone lesion of the spinal column. The use of the cystoscope has greatly enlarged our means of diagnosis in these cases, and has enabled me to recognize in two instances the advancement of the disease from the peritonæum and its gradual spread, by dissemination, under the vesical mucous membrane, in one case on the anterior and in the other on the posterior vesical wall toward the fundus. In both cases the bladder wall toward the cervix was for a long time free of disease. In another case of kyphosis from tubercular disease of the spine a tubercular abscess of the kidney developed. But in all three of these cases disease in and about the prostatovesical region was demonstrable long before the other lesions were made out clearly. And this, in men, has been my constant observation: that so far as the uro-genital organs are concerned, disease apparently taking this course always appears-or is demonstrable-first about the prostatovesical region.

In women the anatomical separation of the genital and urinary organs offers a much better opportunity to observe a distinct primary invasion of the bladder from the peritonæum. Several such cases I have been able to observe and to diagnosticate by means of the cystoscope long before the bacilli could be identified in the urine, though in due time this was done in each instance.

My own observation leads to the opinion that extension to the urogenital tract from the peritonæum is of much more frequent occurrence than is generally believed by surgeons. The fact that the peritoneal fluid is a medium in which the bacilli may multiply; the dependent position of the recto-vesical fold ; the ease with which the bacilli can pass through this thin membrane; its close relationship to the vesiculæ, the vasa deferentia, and the vesico-prostatic, venous and lymphatic plexuses, and the well-known relationship between genito-urinary and peritoneal inflammations-all afford explanation of the clinical observation. The insidious character of tubercular peritonitis, slowly and for a long time advancing, as it often does, without even a fever-rise to give warning of its presence, and with little or no pain, taken in connection with the fact that surgeons do not so frequently as they might take into consideration that there is a peritoneal fold (vesico-rectal) within reach of the finger-tip, leads also to 
the belief that this course of tubercular progress is not so generally recognized as its importance justifies.

Contrary to what appears to be generally believed, the hæmatic channels offer a commion avenue for the invasion by the disease of the genito-urinary system ; and, besides offering a better explanation of the clinical phenomena, this mode of infection, better than any other, accounts for some of those cases of supposed primary infection of the deeperseated organs, such as the testis, the kidneys, etc.

The strong predisposition of certain parts of the genito urinary apparatus, viz., the testes, the prostate, and their adnexa, to tubercular disease has been noticed by clinical observers for the longest time. Naturally the testis, offering as it does the most favorable opportunities for observation, took first rank in the beginning for frequency of attack. It was not long, however, until the prostato-vesical region attracted attention, and at the present day competent observers are entirely agreed that this is, by many times, the most frequently affected of all the genitourinary system. My own observation in a large number of cases is in accord with the opinion expressed by many, that this portion is never skipped in genito-urinary tuberculosis. And not only in clinical observation but in pathological research have we the confirmation of this fact. Jani, whose observations were published (after his death) by $\mathrm{C}$. Weigert (Virchow's Archiv, Bd. ciii, 1886), and confirmed by the latter, earefully studying the genitalia of nine consumptives whose sexual organs presented no evidences of tubercular disease whatever, found tubercle bacilli five out of eight times in the testis and four out of six times in the prostate. The bacilli lay, in the testis, partly within and partly close beside the cellular and granular (crumbling) contents of the seminal tubules; in the prostate, always in the neighborhood of the glandular epithelium. "Great numbers of bacilli" were also observed by him in the prostatic juice (secretion?) in another case (of miliary tuberculosis). In still another case of chronic pulmonary phthisis with severe intestinal tuberculosis, he found several tubercle bacilli in the folds of the mucous membrane of the seminal tubules. He judges that they reached this locality from the peritonæum by means of the ciliary movement. In none of these cases was there the least evidence of pathological alteration of the tissues of the testis. Nasse (Virchow's Archiv, Bd. cr, 1886) found in the kidney local disseminated tuberculosis, whose origin, he thinks, must be sought in the rupture of a tubercle into an artery. DurandFardel (Archiv de Physiol., Norm. et Patlı., 1886, page 393), who claims to have been the first to demonstrate the transportation, in men, of the tubercle bacilli by the blood-current, in examining a tuberculous human kidney found collections of the bacilli, sometimes in very great quantities, in the vessels as well as in the glomeruli, partly such as were involved in 
the tuberculous process, but partly also those in whose neighborhood the tissue, was still perfectly free from the histological appearances of the tubercular process. In the vessels, the bacilli lay imbedded in thrombotic material. In the glomeruli, they occupied partly the walls, partly the lumen of the capillary loops. This position of the bacilli in the lumen of the eapillary loops, or in the walls of the glomeruli, which showed no listological evidences of being diseased, is of the greatest importance, since they could only reach that situation by the blood-vascular channels. According to Weigert, there may be a direct embolism of bacillus-containing thrombi frum the pulmonary veins, and Haller says such a condition may come from the aortic valves. Haller, Weichselbaum, and others had already demonstrated microscopically the presence of the tubercle bacillus in the post-mortem contents (blood) of the heart and great vessels, and in the flowing blood of men. C. Bollinger (loc. cit.) says that, "as regards primary tuberculosis of the testicles, joints, and bones, we must consider that a latent hæmatogenic infection is understood, which leaves as little trace of its point of entry as does a primary septic endocarditis or an osteomyelitis." Birch-Hirschfeld and Baumgarten both accept a hæmatogenic infection of the kidney and testis.

I am fully aware of the fact that good observers (Korzyurcki, Salzman, and others) can be quoted against the infection of the uro-genital organs by way of the hæmatic channels, but a careful examination of the evidence upon which the assertion is based reveals a failure in each case to demonstrate the condition of other organs in such a way as to clearly exclude an auto-infection. In fact, it is only by ignoring the now welldemonstrated power of the blood to carry from point to point, from organ to organ, in the body the infecting agent, that one could feel justified in drawing the conclusions deduced by some. The disposition to generalize on a few imperfectly observed cases is quite as apparent in genito-urinary tuberculosis as in any other. For my own part, I can but say that the conclusion to which the clinical observation of a large number of cases had been steadily driving me was hastened and confirmed by the results obtained by the experimental, pathelogical, and clinical observations of the authors quoted and referred to above. To me it seems that we are now in position to say with much confidence that in the great majority of cases the pathogenic factor reaches the parts of the genitourinary organs which are involved in the tubercular process by way of the hæmatic and lymphatic channels or by extension from neighboring organs or tissues; that the order of frequency of invasion would place the hæmatic channels first; and that a true primary (for the body) genitourinary tuberculosis is one of the rarest of occurrences.

Heredity.-Hereditary infection has not heretofore been considered in this article, but it must be of frequent occurrence. I have myself seen 
six cases of tubercular nodules in the testes of young children, which clearly dated from birth. I have also seen two cases of tuberculous testis in old men, whose histories indicated that the disease dated from early childhood. In one, there never had been any vesical symptoms, though the periprostatic tissues were involved. He consulted me on account of an annoying balanitis which arose during a transcontinental railway journey, but has never had any uneasiness except frequency of urination to attract his attention to the sexual organs. In the other, there has existed for many years a chronic cystitis of the neck withont hæmaturia. In both these cases there is not only testicular but prostatic tuberculosis, and both declare that the hard nodules in the testes have been noticed by them since boyhood. They are brothers, and not only have a family history of tuberculosis, but some of the children of each have succumbed to this disease.

More careful and systematic study of these cases will undoubtedly increase the list of cases of hereditary genito-urinary tuberculosis, and will serve at the same time to clear up some of the mooted points in regard to primary tuberculosis of the testes. This seems all the more certain when we call to mind the fact that it is in hereditary surgical tuberculosis that we see the most marked tendency to remain latent; so that we can readily see how it is that a latent or quiescent tuberculosis of the testes or epididymis may escape observation until the evolution of puberty determines an increased activity of the disease as well as of the affected organ in a way to bring it under observation. I have already urged the necessity of exercising much reserve in pronouncing as to primary genito-urinary tuberculosis, and I would now advise equal caution in declaring as to the duration of any tubercular lesion that may be discovered in the genito-urinary cycle, and more especially when that lesion is situated in the testis or, as it most frequently is, in the periprostatic region. More extended clinical observation of these cases has led me to give much greater weight to the opinion expressed by Baumgarten. He says (loc. cit.): "We can not regard the infection by the digestive tract as the principal source of human tuberculosis, although it is of more importance than the infection by means of the respiratory tract or the skin and outer mucous membrane; and infection by sexual intercourse, which must a priori be considered, must be judged to be of no moment, on account of the extremely rare occurrence of a spontaneous, undoubtedly primary tuberculosis of the outer genitalia-or of the genitalia at all-therefore only one mode of infection remains possible, that by inheritance."

We are now in position to discuss the more positive evidence bearing on the question of the early-perhaps simultaneous-involvement of more than one part of the genito-urinary system, when once it is invaded, and 
the nearly related subject of the greatest tendency to early involvement shown by the prostato-vesical and periprostatic region. The necessity hardly exists of urging the vital importance to diagnosis and the influence on (surgical) treatment which is here apparent.

As is well known, there is great difference of opinion among pathologists as to the portion of the uro-genital organs first affected in tuberculosis. Virchow, Ziegler, Forster, Steinthal, and others hold to the opinion that it always begins in the kidney; while Rokitansky, BirchHirschfeld, Bardenheuer, etc., take the opposite view, thinking the initial point is not only in the testis, but in the epididymis. H. Eilers (Baumgarten's Jahrsbericht, vol. v, 1889), who thinks the disease begins in the tail and not, as is generally supposed, in the head of the epididymis, joins with the last-named authors. He (Eilers) thinks that testicular tuberculosis very seldom exists alone, other parts being ushally found diseased at the same time, and he also speaks of primary testicular tuberculosis as being only "probable." Korzyurcki (Centralblatt für Bakteriologie, vol. iv, 1888), who believes in an infection per urethram, declares that the prostate seems never to be skipped. Steinthal, along with all others who hold to the opinion that the kidney is most frequently the site of the initial lesion, thinks that the prostate is very early involved in the tuberculous process. In fact, all authors of note, no matter how widely they may differ on other points in the pathology, as on the question of primary lesions and the initial points in the genito-urinary organs, are agreed in asserting the great frequency of prostatic involvement and the rarity with which we fail to note the missing of this region in advanced tubercular disease of the uro-genitalia.

If, now, we take into consideration the facts that some tissues reveal a far greater predisposition to tubercular disease than others, as for instance the lungs, which show this even by metastasis (Bollinger); that vigor of onset and the rate of progress of the diseased foci are most markedly influenced by the number of bacilli introduced, and the local power of resistance; that the bacilli have been demonstrated in the blood as well as in the healthy testis and prostate (Jani), and in the healthy portions of the kidney (Durand-Fardel), which they could reach only by way of the hæmatic channels; that the rate of progress in the affected areas is essentially irregular, with a tendency to remain latent or quiescent for an indefinite time; that tubercular inflammation is not suppurative inflammation, and that suppuration takes place only after a mixed infection with pyogenic bacteria (Garri, Baumgarten, etc.); that infection by way of the blood-vascular system does not always mean miliary tuberculosis, but that a lymphatic gland may even be infected from the blood; that the bacilli do not multiply in the urine, and that they have not yet been demonstrated in the semen; that a tubercular nodule may exist for an in- 
definite time in the periprostatic tissues without exciting any subjective symptoms more than it does when situated in the caput epididymis or the rete testis, and hence might easily escape detection; that in extension from the peritonæum tubercular disease is most apt to reach the periprostatic tissues first, on account of the favorable circumstances of the dependent position of the recto-vesical fold; and, finally, that other inflammation, both acute and chronic, slight traumata, and even the evolution occurring at puberty, as well as increased functional activity (sexual excesses), are well known to be capable of exciting a sudden increased activity of the tubercular process - we ought to be in position to see how it is that the disease inost frequently reaches the genito-urinary organs by way of the hæmatic channels, or the lymphatics by extension; how it is that the prostate and its surroundings and adnexa are most frequently first of the genito-urinary organs to show evidence of the disease; and how it is that they seem never to be missed, as well as to comprehend nearly all the other clinical phenomena of tuberculosis uro-genitalis.

General Diagnosis. - The foregoing is intended chiefly to apply to the questions of diagnosis and treatment; indeed, it serves its best purpose in the narrower field of pathological as distinguished from the anatomical diagnosis. The latter, with the means at present at hand, is not difficult in the great majority of cases, and is arrived at by the same means and methods employed in locating any chronic inflammatory process occurring within the genito-urinary cycle. When, as quite commonly occurs, a few disseminated tubercular nodules exist in the rete testis, the epididymis, and in the periprostatic tissues, the subjective symptoms may be almost or quite nil, so that such a disease may remain undetected for the longest time. Generally, however, there exists some frequency of urination, for which one has to account, and such patients are generally told that they have "nervous" bladders, an opinion which can be easily confirmed by the failure to find anything out of the way in the urinalysis, except, perhaps, slight polyuria, with rather low specific gravity. If instrumentation is resorted to, or irrigation, all the vesical symptoms are aggravated, and slight hæmorrhage may be excited. More likely, the deep urethral irritation exciting thereto, the patient indulges in fornication, and this may set the process going more rapidly, and bring it into notice; or, what is still more likely, he contracts a clap which, running backward, quickly arouses the slumbering tuberculosis, with disastrous results to the prostato-vesical region. It is in such cases that, after the acute symptoms have subsided, the error is apt to be committed of supposing that the clap has brought abont a prostatic tuberculosis, with extension to the testes by way of the vasa deferentia, and especially is this likely to occur if the surgeon has never seen the patient previously, or has failed to make a thorough examination. In children we may not 
have, in such a case, even so much as a frequency of urination to attract attention-a fact that may be accounted for on the grounds that, owing to the rudimentary or undeveloped state of the prostate, tuberculosis of that organ does not occur. Neither is enuresis in children ever, so far as I have been able to observe, caused by tuberculosis-a fact which, to my mind, seems easy of explanation. If tuberculosis affects the child's bladder-neck at all, it is the mucous membrane, or the submucosa, which is involved, and this causes not only frequency of urination but a dysuria, which rouses the attention, and there is an absence of that irritability of the vesical neck, so eharacteristic of enuresis. It is in children that we see those cases where the tubercular disease seems to be most clearly confined to the testis. Nevertheless, no case that has been examined by me has ever failed to reveal evidences of the involvement of the vesical neck, either by frequency of urination, without polyuria and dysuria, or a tendency to bleed on the gentlest introduction of an instrument. Another evidence of the frequency of the involvement of the bladder in these cases is the frequency with which they are examined for stonesymptoms of which they present--and the history given that such examinations have been negative, with the result only of aggravating the symptoms and causing bleeding. In these cases, where even the fewest and smallest shot-like nodules can be felt in the testes, suspicion of tubercular disease about the vesical neck ought to be excited, and, besides frequent and careful palpation per rectum, repeated examination of the urine for the bacilli should be made. For it is in these (the most difficult for the purpose) cases that the early (perhaps simultaneous) involvement of the vesico-prostatic region and the testis affords means of differentiating between syphilis and tuberculosis. Syphilis is known not to attack the prostate and bladder, while congenital syphilitic orchitis may so closely resemble tubereulosis that it may easily be mistaken for it. In any doubtful case, therefore, a careful investigation of the vesico-prostatic region is imperative-all the more so when we call to mind the fact that the two diseases may exist at the same time (mixed infection). This holds good equally in adult and acquired as well as in infantile and congenital syphilis and tuberculosis. In the case of adults, however, the differential diagnosis is not difficult.

The early (to say the least) involvement of the prostatic region in all tubercular cases affords valuable assistance in differentiating between cancer or sareoma testis and tuberculosis-a distinction which it is sometimes impossible to arrive at on any other grounds; for, as is well known, both eancer and sarcoma advance toward a general or bodily infection by another route than the prostato-vesical region.

In children we sometimes see tuberculosis of the resical neck without any discoverable testicular involvement. I have observed one such case, 
where the tubercular cystitis was complicated by secondary (phosphatic) calculi, in a child aged four. When the patient, who had been very much neglected, came under my observation a tubercular peritonitis was present, and the history seemed to show that the resical disease was by extension from the peritonæum. The patient succumbed to tubercular meningitis. Neither in the testes, epididymis, nor cords could I detect the least evidence of tuberculosis.

By many times the most difficult problem to solve is when a differential, pathological diagnosis is to be made between primary cancer and socalled primary tuberculosis of the prostate; for, if there is a resemblance between testicular syphilis and tuberculosis, there is a closer one between cancer and tuberculosis of the prostate. I do not find this point discussed in the literature of the subject, and am therefore compelled in my treatment of it to rely chiefly on my own observations, which, in the case of cancer of the prostate, are by no means extensive. Leaving for the present outside of this consideration the detection and identification of the pathogenic bacilli, a comparison of the symptomatology of the two diseases leads to the conclusion that our chief reliance must be on the relative differences in the pain and hæmorrhage; for we must not overlook the fact that neither of these diseases of itself is pyogenic, but that both of them may have the suppurative process ingrafted upon their clinical courses. From the beginning to the end cancer bleeds, as we might expect it to do, seeing the extreme vascularity of the malignant growth, and also that the growth begins from the surface in a way to favorably dispose to hæmorrhage. This disposition to hæmorrhage also increases as the disease progresses. Contrary to what is generally supposed, the disposition to hæmorrhage is not great in tubercular disease. There may be periods in its course when we have sharp bleedings, but these are short, and usually belong to the earlier stages and to those cases where the disease is making progress in or immediately under the mucous membrane; hence, so far from growing worse, the hæmorrhage diminishes as the ulceration proceeds toward the deeper tissues. I have seen cases where the entire prostate had been destroyed, the rectum opened, and a perineal fistula found with only a few insignificant bleedings. Even in these cases of disseminated tuberculosis about the cervix one may irrigate, fill the bladder sufficiently, introduce the cystoscope, and have a look without exciting sufficient bleeding to obscure the field of vision, a thing one could never do in cancer. To say the very least, the bleeding in tuberculosis is intermittent, while in cancer it is continuous. The pain also is quite different. The lightning-like, lancinating pains of cancer, requiring the vigorous. exhibition of opiates for their relief, are, in my experience, never seen in tuberculosis of the prostate. In the latter, burning and scalding on urination are present in the beginning, but grow 
less also as the disease advances to complete destruction of the mucosa, where most of the nerves are distributed; but in cancer there is, if any difference, a decided increase. Again, cancer of the prostate is well known to be one of the most painful of diseases, while tuberculosis, far more frequent, has not that repute. It may be said, then, that, while in their earlier stages a differential diagnosis between cancer and tuberculosis of the prostate may for a time be impossible, a continued and careful study will enable the surgeon to distinguish them, even in those cases where there is failure to identify the bacilli, with sufficient certainty to avoid an exploratory operation, which on the one hand would be useless, and on the other distinctly detrimental.

Those pathological changes wrought by tuberculosis, and which are to be distinguished by palpation in those organs accessible to this mode of examination, are of the highest importance for diagnosis. As the surgeon sees it, tuberculosis appears in the shape of quite hard, usually painless nodules, ranging in size from small shot or mustard-seed to that of a horse-chestnut. Where a larger size is attained, as often happens in a testicle, it will on careful examination be found to be made up of several of the larger-sized nodules, either coalesced or held firmly together by fibrous bands or meshes. This gives the whole mass the feel and even the appearance of being lobulated, or else so nodulated as to be quite irregular in outline, though the general appearance and shape of the organ may be to a certain extent retained. One never sees such large nodules in the prostate as in the testis; long before they are developed to this size ulceration has taken piace, and a more or less rapid destruction by coagnlative necrosis is taking place on the free surface of the ulcer. More frequently one makes out a few (two or three) nodules the size of a pea in the head or body of the epididymis or in the substance of the gland. There is generally a difference in their sizes if more than one are present.

In the testis one frequently encounters fine, shot-like disseminated tubercles, scattered on the capsule and about the rete testis. These might exist, of this size, within the gland, without being detected by palpation. They rarely occur in the cord or on the prostatic capsule. The vas deferens, when affected at all, gives the impression to the touch as if it were a string of beads, or, in other cases, as if bits of tubing were strung together, the indurated parts being longer than they are thick. Most frequently two or more of these conditions exist in the same testicle. In no case which has come under my observation has either syphilis, cancer, or sarcoma of the testicle presented the same evidences as are described above. Neither has acute or chronic non-tubercular inflammation.

Where tuberculosis testis begins and where the disease may be first discovered clinically, may be very different things; H. Eilers (loc. cit.) is responsible for the statement recently made, that the tail and not the 
head of the epididymis is the most frequent starting-point. I know of no clinical confirmation of this statement; and only introduced it here in order to more clearly bring into contrast the differences in diagnostic value of the small, hard mass in the cauda often left after an acute epididymitis following gonorrhœa-which may persist for a lifetime without being followed by any eridence whatever of tuberculosis-and those disseminated nodules, which one always finds either in the body or head of the epididymis, in the testis proper or scattered over its capsule, in tubercular disease of this gland. These small knots following epididymitis from extension are frequently the cause, when bilateral, of an obstructive sterility, while it is well known that bilateral tubercular epididymitis is very rarely the cause of azoöspermism, even when the organs are extensively involved. These nodules at the tail of the epididymis are not to be confounded with the tubercular ones occurring elsewhere in the testis.

If the kidneys were amenable to palpation, we should donbtless have the same evidences of tubercular disease presented by them as in the case of the testes. Warrant for this is found in those cases brought to autopsy, where the nodular condition is to be made out very clearly in the softly elastic organ, before section. The small, shot-like nodules scattered over the capsule are also to be felt here, and in one case seen by me, where an exploratory lumbotomy permitted thorough palpation, both the larger nodules within the substance and the disseminated tubercles on the capsule offered satisfactory evidence of the nature of the disease before section into the gland was made.

Nowhere throughout the whole genito-urinary system does palpation offer the same advantages to diagnosis as those parts haring the prostate for a centre and the range of the finger in the rectum for a circumference; for it is in this region-the one rarely if ever missed by tuberculosis uro-genitalis, no matter where the starting-point may be-that the tactus eruditus can be brought to the investigation of the greatest number of organs in a way to detect those larger and smaller nodules, which are so characteristic of the disease. Moreover, there is here, within reach of the finger, the point of junction of the urinary and genital tracts as well as the point where, to a large extent, the vascular and lymphatic systems come together; so that one might say there are anatomical and physiological grounds, as well as pathological observation, for an explanation of the great frequency of tubercular attack. The well-known influence of blood-stains in localizing and favoring the development of the pathogenic bacteria has free play here in the oft-repeated vascular changes occurring in the different stages of sexual activity. In no inflammation, either acute or chronic, in which the tubercle bacilli play no part, do we find those small, hard, almost painless nodules in the prostatic substance, in 
the walls of the seminal vesicles, on the prostatic capsule, at the prostatovesicular junction, in the intervesicular angle, in the portion of the bladder-wall bounded by the prostate and the vesiculæ, or in the meshes of the surrounding vesico-prostatic plexus. There may be only one or two of such nodules, or, as in the case of the testis, there may be many of them matted together, but their detection here is of the highest diagnostic importance; so much so, indeed, that in doubtful cases of genitourinary disease careful and repeated palpation should always be made; and in cases where they are detected, a guarded prognosis is demanded.

For a final decision, based on the discovery of the tubercle bacilli in the urine, a long time may be required.

In prostatic tuberculosis one may often be able to detect small, hard masses, about the size of a pea, which are clearly developed in the vesicoprostatic plexus of veins, for they are movable over the capsule of the gland and are not attached to the rectal wall. They are of much diagnostic value also, a fact to which I have called attention some years ago (St. Louis Courier of Medicine). A. T. Cabot, of Boston, who has also studied them, thinks they may be infected lymphatic glands, but my belief was that they were perivascular tubercular nodules similar to those found in the lungs. In one case, which I had studied clinically, and which was brought to autopsy, the specimen was so carelessly handled and became so spoiled that the microscopist to whom it was submitted declared his inalility to make a positive statement. The cheesy mass which was squeezed out on section seemed to come from a periprostatic vein.

The nonsuppurative character of tuberculous inflammation is also of diagnostic value in the early stages of the disease, and would be of much more service in this regard if suppurative inflammation were not so frequently ingrafted upon its course by mixed infection through careless and septic instrumentation. When we remember that one of the most common ways of assisting in a diagnosis, as well as treatment, is the introduction of an instrument of some kind, often by the patient himself; that even if the surgeon is scrupulously careful of his instruments, he is not equally so of the anterior urethra through which they must pass to reach the bladder; that a pot of dirty vaseline often supplies the lubricant; and that suppurative anterior urethritis treated by injections is of very common occurrence-there is left little room for surprise that one so rarely sees a purely tubercular formation about the vesical neck and on the surface of the inucous membrane.

That which renders the diagnosis absolute and certain is the identification of the pathogenic tubercle bacilli in the urine; and in all cases-and more especially doubtful ones-careful and persistent efforts should be made with this end in view. But that such efforts fail of success in 
clearly tubercular cases, for a long time at least, is well known to surgeons having experience of these cases-so well known, in fact, that throughout the literature of the subject it is a common observation that absence of the bacilli from the urine is no evidence of the nonexistence of tuberculosis. This absence of the pathogenic micro-organisms, at first sight so striking as to cause surprise, receives, however, adequate explanation at the hands of pathological anatomy. As is well known, the bacilli have never been demonstrated in the seminal fluid. Jani (Baumgarten's Jahresbericht, 1886), quoted above, failed to find them in the contents of the seminal vesicles of nine consumptives, though they were present in the testicles, prostate, and "prostatic juice," these glands being free of evidences of tubercular disease. I caused the seminal fluid of four young men having tubercular disease of the testis and prostate (one had a nodule apparently in the seminal vesicle, and two had nodulated vasa deferentia) to be repeatedly searched for the bacilli by competent microscopists, who failed to find them in any case. In one of these cases the bacilli were tolerably abundant in the urinary sediment and in all, the spermatic filaments showed that the testes were active. In one case I removed the testicle, and in one the organ had been removed by another surgeon before the patient came under my care, the presence of the active spermatozoa showing that the fluid examined came from a tubercular gland. Where the testes, the prostate seminal vesicles, and periprostatic tissues are alone the seats of the disease, we could not expect to find bacilli by an examination either of the urine or semen. Neither may we expect to find them in the urine in those cases where the tract is invaded from the peritonæum, so long as the infected portions remain beneath an intact mucous membrane. In a case, still under my observation, in which the cystoscope revealed a submucous tubercular patch on the anterior of the fundus, over four months elapsed, and there occurred three attacks of hæmaturia-clearly of vesical origin-before bacilli appeared in the urine. There were nodules in and around the prostate, and symptoms of tubercular peritonitis, when he came under observation.*

It is in those cases of extensive tubercular ulceration of the urinary passages, which are freely washed by the urine and which are not sup-

* In this case there was clearly an interference by the disease with the detrusor vesica, several tests showing residual urine to the amount of four to six ounces, without any prostatic or other obstruction to account for it. The urine was rather abundant, of low specific gravity (1008-1012), and contained no pus. The diagnosis, made on the clinical history and the physical examination, was subsequently confirmed by the discovery of tubercle bacilli in the urine. Great care was taken to avoid infection with pus microbes, and the nonsuppurative character of the inflammation with the ability to exclude malignancy were relied upon as strong evidence in the pathological diagnosis. The case is one of $\mathbf{a}$ group constituting the basis of a clinical lecture to be found in the International Clinics, Philadelphia, Lippincott. 
purating, that one may expect to more quickly and frequently find the bacilli in the sediment. One can only say, however, that this is probably true, for these cases are not ordinarily watched clinically in such a way as to determine the point. No systematic attempt to estimate the number of bacilli present in the urine is known to me, but my own observations lead to the belief that the number is never large. Where they have been found at all, from one to four, or at most five, on one cover-glass, is quite the average. One never sees here the same numbers as in the sputum from a tuberculous lung, even in those cases where destruction seems to be going on at a rapid rate. In practice, even the flocculent discharge from a sinus connected with a tubercular testicle has, in my hands, often yielded negative results with the microseope when the pulpy mass subsequently removed with a curette contained them in large numbers.

Here, again, a reference to the pathological anatomy of tubercle seems to offer an adequate explanation. Koch, Baumgarten, and others have demonstrated the fact that the bacilli lie, not on or near the surface of an ulcer, but underneath that portion which is undergoing the coagulation necrosis; hence they are not easily washed up from below by the discharges, or off the surface by the flowing urine. In the caseous center of an imbedded tubercle, the bacilli are very scant, sometimes entirely absent, while the mural arrangement is quite marked. Moreover, unless there is suppuration from mixed infection, the discharge from such a tubercular ulcer is quite small. To trust, then, to the caseous detritus thrown off from the surface of such an ulcer into the urinary stream is, as actually happens in practice, to be often disappointed. When suppuration takes place, we usually have urinary decomposition, with the addition to the urine of such a large number of other micro-organisms as greatly to add to the difficulty of identifying the specific bacilli, and the differentiation by culture-experiments is hardly applicable in clinical work.

In spite of all these difficulties, a persistent search of the urinary sediments will rarely fail to determine the question of their tuberculous character, when the inflammation is situated upon or very near the mucous surface of the urinary passages.* In no instance, where the clinical symptoms, the history and physical examination offer a slight hint, especially in those chronic cases of inflammation along the urinary

* In practice, the finding of the tubercle bacilli, especially in those cases where there is no suppuration, and where the urine is clean and contains only the normal sediment, is greatly facilitated by the addition of some egg-albumen, so as to obtain such a viscid deposit as will enable the microscopist to get a good "spread." It seems hardly necessary here to say that the operator should be sure that the albumen which is expected to entrap and carry to the bottom of the urine-glass the bacilli should itself be free of those organisms. 
passages which do not yield to other treatment, should this persistent and systematic search for the tubercle bacilli be neglected.

In my own observation, embracing six cases (five of which were demonstrated by post-mortem and one by lumbotomy), the bacilli were found in large numbers when the kidney is involved in the tubercular process. Not only were there numerous bacilli in these cases, but they were readily demonstrated. I should therefore be inclined to suspect a renal lesion in any case where considerable numbers were readily demonstrable in the urine. The question of the kidney being attacked is, however, one of anatomical diagnosis, to be determined by the presence or absence of casts in the urinary sediments, and needs but slight mention here. In the case of primary (for the urinary organs) renal tuberculosis the pathological diagnosis is not ordinarily difficult unless there is an accompanying suppurative pyelitis, for the confusion caused by a suppurating cystitis can be readily eliminated by the well-known method of vesical irrigation previous to the withdrawal of the urine for examination.

When the bladder is the first of the uro-genital organs to be attacked, the fact that the disease spreads by dissemination, and not by diffusion, serves as a diagnostic help, now that its walls have been brought into view by means of the cystoscope. The pale, salmon color of the normal vesical wall, and the transparency of the epithelium, enabling one to see the vessels coursing underneath, offer exceptional advantage for the detection of those pathological changes which advance from below. Changes in color and in the vessels are especially easy of detection.

Finally, it can be said that, since one has so frequently in practice to make a diagnosis without that positive evidence furnished by the identification of the tubercle bacilli in the urine, and since we can not expect the seminal fluid to furnish it, it is still possible to arrive at sufficiently accurate conclusions for all practical purposes by a comparison which will bring prominently into view the differences between the grosser manifestations of tubercular and other forms of inflammation. For this purpose there is not a well-demonstrated point in the pathology, the pathogenesis, the rate of progress, or the mode of its invasion which is not both available and important. A diagnosis thus made, by squaring the clinical history and the results of physical exploration with the now wellascertained facts of an advanced pathology, will be found in the end to depend in large measure upon what are even yet too often spoken of as "fine points." And such a method, requiring a more extended knowledge of the pathology and evolution of the disease, is of greater avail in enlightening our prognosis and directing our treatment than the mere fixing of a label after the discovery or the essential etiological factor could possibly be. 
In view of the fact that improved methods and means of diagnosis have lately come into vogue, and of the manifest advantage to be derived from an early recognition of the pathological nature of a complaint which so closely mimics other and different states, as well as for purposes of reference and proper classification, it seems advisable to give some brief account of the symptoms and treatment of tuberculosis of the different organs composing the genito-urinary cycle. And here again it is necessary to eliminate from our consideration the general or miliary form of tuberculosis, for the reason that its rapid progress, and its all but simultaneous invasion of extensive areas, puts it beyond the reach of surgical help. In what follows, especial attention is given to the symptoms that announce the earliest periods of invasion, and the term "primary" has reference only to the invasion of the genito-urinary system.

\section{PRIMARY RENAL TUBERCULOSIS.}

Here, as is well known, groups of miliary tubercles irrupt from beneath the mucous membrane of the pelvis, or in the glomerular zone of the cortex. In the present state of our clinical knowledge it is not possible to distinguish the symptoms evoked by difference in the localities primarily involved. Nor is it greatly important to do so, since one locality is quickly invaded after the other, and the different sets of symptoms blend. Fenwick expresses the belief that, in the pelvic form of the disease, blood and pus appear in the urine in small amounts either coincidently with renal pain or very soon after its appearance; that renal colic appears relatively earlier than in the cortical form; that the stages are passed through more rapidly; and that the bladder is affected earlier. Where, however, the cortex is primarily invaded, polyuria would first appear; owing to the irritation of the excreting tissues, hæmorrhage would be slower to begin; and, later than in the pelvie form, the frequency of urination, caused by resical irritation, would take the place of the increase of quantity. The hæmorrhage from the different localities would also vary. In the pelvic form it should be slight and intermittent, but in the case of a sloughing out of a portion of the cortex, bleeding should be profuse but more transient.

Symptoms. - In any case the symptoms taken in their entirety closely resemble those of renal calculus, and in this resemblance the mimetic character of tuberculosis of these organs is well illustrated. For the purpose of distinguishing between the early stages of these two diseases, Fenwick has constructed the following table: 
PRIMARY RENAL TUBERCLE IN EARLY STAGE.

Family history of phthisis or cancer.

Age between twenty and forty.

Personal history: Perhaps tuberculous bones, joint or gland disease; very rarely any previous urinary symptoms.

Symptoms, onset: Polyuria of a murky type (the frequency of micturition being due to the quantity passed, not to irritation), vague lumbar pain, or a sudden chill, and severe pain in one kidney, but rarely colic. Some frequency of micturition in the early stages at night, from irritation of acrid urine.

Colics appear later, are usually less severe, are more easily under the control of drugs, do not usually retract the testicle, and are preceded by rose-red blood, if they are induced byulceration and not blockage.

Hæmaturia: Slight in amount, more or less persistent, uninfluenced by rest.

Urine: Cloudy at the outset from admixture of mucus and pus. Acid, low specific gravity. Light-colored, depositing a thin layer of pus with streaks of blood, débris of connective tissue. Small clumps of caseous material. Albumin appears early.

Tubercle bacillus found, but with difficulty and after much search, in acid urine.

Inoculation of urine deposit into animals (Rovsing, Jacobson).

General condition: Patient ailing, " never feels well," is anæmic, easily tired; no loss of appetite.

\section{RENAL STONE \\ IN EARLY STAGE.}

Family history negative, or of gout or gravel.

Age about forty.

Personal history: Negative, except perhaps the passage of gravel ; of a "weak" loin; of testicular neuralgia or "sciatica."

Symptoms, onset: Vague lumbar aching or a sudden colic. Frequency of micturition, due to irritation ; small quantities passed often, and in day-not at night-relieved by rest.

Colics: More or less severe, according to composition and size of stone, followed by blood.

Hæmaturia: Intimately mixed; much more marked than in tubercle; dependent on exercise.

Urine: Clear at outset, containing evidences of calculus in the shape of crystals. Acid, normal specific gravity. Pus later in course of case, and then only forming a deposit when pyelitis has been induced.

General condition : Patient may enjoy first-rate health between colics. 
No matter where the process begins in the upper urinary passages, the bladder will quickly show signs of irritation. Both the bacilli and the products of their growth and activity are swept downward with the urinary stream and are held long in contact with the vesical mucous membrane. Here at the anterior portions of the trigone and about the ureteric orifices, the ptomaines, probably, by their irritating action, prepare the way for a true bacillary surface-infection. Be this as it may, we very soon have a highly irritable vesical neck.

Diagnosis. - The cystoscope comes efficiently into play for diagnostic purposes. But it should be employed with the greatest care, and with all the precautions to be presently mentioned in connection with the use of instruments in vesical tuberculosis.

The presence of tubercle bacilli in the urinary sediments should be persistently songht for. They will usually be found.

It is apparent that many of the points for diagnosis can only be developed by judicious cross-questioning, and that much will depend on the intelligence with which the patient has observed the symptoms. For these reasons it is advisable to go over the history several times, allowing a proper interval between the examinations for refreshing the memory. Owing to mixed infection, pus appears in the urine rather early in these cases, generally before the vesical irritation has tempted to a resort to local treatment. Septic catheterization is especially to be avoided, for a cystitis at once clouds the clinical picture and leaves one to grope in the dark during many days of valuable time.

As to whether one or both kidneys are diseased may be determined partly by the localization of the pain and distress in one or the other loin, but our main reliance must be in the catheterization of the ureters, with the aid of the new and improved cystoscope.

Treatment. - It is of primary importance to avoid as far as possible all instrumentation, all washings, and all injections of caustic or antiseptic solutions into the middle and lower urinary passages. Even in the earliest stages of renal tuberculosis frequency of urination and vesical irritability are quite common. This tempts the surgeon to fly to local treatment, especially if, as is not infrequently the case, the patient is a young man and has been the subject of a chronic urethritis, or thinks he has. The judicious surgeon who has his patient's interests at heart will resort to the use of instruments in the urethra and bladder neck with the greatest reluctance in these cases. This caution can not be urged with too great insistence. The surgeon should keep constantly in mind the fact that frequent urination caused by the polyuria does not, and that the frequency caused by vesical irritability, even when accompanied by slight bleeding at the end of the act, may not, mean vesical infection. The former is easily accounted for and does not attract so much attention, but 
the latter is often erroneously taken to indicate the necessity of local treatment. In the earlier stages this frequency of irritability only means congestion and swelling about the interior portion of the bladder, due to contact with urine loaded with ptomaines or other products of renal tuberculosis. This is the moment in the evolution of the disease when the greatest advantage is to be gained by the use of general or constitutional anti-tubercular treatment. Renal and vesical distress are best quieted by codein. This drug acts more like belladonna in its quieting effects on the excitable detrusor, and has at the same time the analgesic properties of morphine. Moreover, there is less danger of the dreadful opium-habit from its use. Cod-liver oil, the hypophosphites, malt, fatty foods, creasote, or, better still, sandal oil, with change to a warm and dry climate, are now of the greatest service, and, I am persuaded by observation, will more frequently result in cure or at least obsolescence of the disease than is generally believed.

Surgical Interference.-This is not only of advantage in certain cases, but is imperatively demanded in others. If the disease remains fairly localized in one kidney, and does not improve under the treatment just named, an exploratory lumbotomy may be done with the view of clearing up the diagnosis, removing any stone that may be causing or adding to the trouble, freeing any sloughing portions of the renal substance, or draining the renal pelvis and diverting the stream of tuberculous urine, in order to avoid infection of the ureters and lower urinary passages. Where the onset is sudden and vigorous, or where an old and "latent" tuberculosis of one kidney is suddenly lighted up into an acute trouble, splitting the renal capsule may be the means of saving a large portion of the kidney which is about to be destroyed by undue pressure. It seems quite possible to remove, as in the case of the testis, only the diseased portions of the gland, leaving the healthy parts to functionate. My own rather limited observations in these cases lead me to think that whenever the tuberculous kidney is incised, it is better to open the pelvis and drain for a long while through a lumbar fistula. In one case under my care, where there was frequent renal colic from the blockage of the left ureter with sloughs, and in spite of the fact that the bladder was extensively infected, the right kidney being apparently healthy, I opened the loin, removed a quarter of the kidney substance which was softened, opened and drained the pelvis for a fortnight, with the result of apparently saving the remaining portion of the gland. Gradual obsolescence of the resical tuberculosis followed, and the patient has now, in a fair state of health, resumed his business in a Southern eity.

In one case, in the practice of another surgeon, violent general tuberculosis followed quickly on the incision of tubercular nodules in the right kidney, causing death within the fortnight. In this ease the pelvis was 
not opened, and there was no drainage. The lower urinary passages were already affected, and it was probable that the patient, a woman, was on the eve of a general tuberculosis at the time of the operation.

Even when the lower urinary passages are infected and both kidneys are involved in the tuberculous disease, surgical interference may yet be of some service. Here it is of the greatest importance to save all the remaining healthy renal tissue. As is well known, the tubereular process, except in the general miliary form, is erosive in its action, often leaving healthy functionating gland tissue in the immediate neighborhood of that which is destroyed or irretrievably diseased. In those cases of nephro-phthisis where the destruction of gland tissue is proceeding in the direction of the cortex from the pelvis, there can be little doubt that drainage would be of advantage, as it is in the case of the bladder. This drainage through the loin would, by diverting the stream of tuberculous urine, enhance the opportunities to benefit the diseased bladder by local and general treatment. By improving the general health, obsolescence at least, and, in a certain number of cases, cure, may come most unexpectedly. The advantage of keeping the tuberculous areas of the lower urinary passages as free from moisture as possible is manifest.

The involvement of other organs which are essential to the maintenance of life in the tuberculous process, has the same bearing on the question of operation as in other forms of surgical tuberculosis. In any event, the strictest attention is to be paid to the state of the digestive organs, to diet, and to climatic influences.

If the use of "tuberculin," or other similar treatment, is to be instituted with the intent to cause a casting off of the tuberculous tissue, it seems indispensable that the pelvis of the kidney should be opened, in order to provide a means of exit other than the ureters.

\section{TUBERCULOSIS OF THE BLADDER.}

Tuberculosis of the bladder is rarely primary in the urinary cycle, this viscus generally suffering in consequence of disease of a neighboring or associated organ. Where the disease does primarily attack the bladder, it seems beyond question that the trigonal submucosa is by far the most likely to suffer.

Symptoms and Diagnosis.-The irruption of the tubercles in this situation develops a series of symptoms closely resembling those of vesical calculus. The patient is generally young - fifteen to twenty-fivegives a history of masturbation, to which he attaches much etiological importance, and has a family history of tuberculosis or cancer and a personal one of enuresis up to four or five years of age. There is a story of gradually increasing frequency of urination for a few months, chiefly during the day. Later on the nights are disturbed, and abont this time 
blood appears at the end of the act. There is pain also in the mid-penis, with a decided vesical tenesmus, and an occasional sudden stoppage of the stream with an increase of distress. As the disease progresses the nights are more and more disturbed by calls to urinate, and an increase is noted in the hypogastric pain when the bladder is disturbed. If the disease is active, there may be some sloughing out of portions of the mucosa, with bright but transient hæmorrhages.

The points to be noted for differential diagnosis from stone are : (1) the absence of history of the passage of renal calculus; (2) the fact that exercise, while showing some effect, does not make so marked an increase of vesical irritability; (3) the situation of the pain in the mid-penis, and the fact that it does not pass forward under the glans; (4) the sudden arrest of the stream by a voluntary contraction of the compressor urethræ, so as to relieve the pain caused by the passage of urine along the urethra, instead of the reverse condition of the pain being caused by the sudden blockage of the meatus internus by the stone; (5) the more rapidly increasing nocturnal frequency of urination, and its clear dependence on distention reflex; and (6) the growing evidences of a contracting bladder.

The cystoscope and bacteriological research of the urinary sediments will, in the great majority of cases, quickly resolve any doubts.

The bladder may be invaded from above in two ways: first, by surface inoculation by the stream of tuberculous urine from the kidney or its pelvis; and, second, by continuity along the line of the ureter. The former mode of extension is most frequent. The urine from a tuberculous kidney is loaded with ptomaines and other irritating products of tubercular inflammation. These first irritate the trigonal mucous surface, causing swelling and congestion, the symptoms of which are frequency of urination, due to irritation of the vesical neck and not to distention reflex. Later on there is excoriation of the surface, and some blood issues with the last drops when tenesmus appears on the scene. Day and night frequency are almost equal. There is scalding urination, the appearance of the fluid voided depending upon the extent and severity of the renal disease. The state is one of more or less intense surface irritation of that portion of the bladder most richly supplied with sensory nerves. The symptoms are almost exactly similar to those caused by an extension backward of an acute urethritis (gonorrhœa). The patient "wants to void the urine all the time," and no relief comes from emptying the bladder.

The soil being thus well prepared by a bath of ptomaines, tubercular infection of the submucosa easily takes place through an excoriated patch or a rent made by an instrument. Such patients should not, except it be unavoidable, be sounded for stone, and judicions cross-examination will 
generally elicit a sufficient discrepancy to place the surgeon on his guard. There are generally both pus and blood as well as tubercle bacilli in the urine. There is also turbidity from abundant detritus.

Where there is extension by continuity from the ureter, swelling, congestion, excoriation, and finally ulceration creep in by the ureteric orifice and extend along the corresponding trigonal limb. It is by the aid of the cystoscope alone that we can observe this process, which, so far as the bladder is concerned, may be almost symptomless, and must be frequently obscured by the clinical manifestations belonging to the state just described above.

True to its mimetic character, this form of the disease often closely simulates renal calculus.

All these forms of tuberculous cystitis are surface infections, and symptoms appear promptly after invasion. Where the attack comes from the other direction the disease may progress considerably before it is recognized, or, indeed, before the patient is aware that there is any bladder ailment at all. In the case of a primary invasion of the middle coat of the bladder from the blood-vascular channels, the symptoms are almost nil, and when they appear at all consist of (1) weakening of the detrusor muscle-plane, showing itself in a slowness to start the stream, weakness of flow, and difficulty in emptying the bladder; (2) accumulation of some residual urine in the later stages; (3) a gnawing pain behind the pubes when the bladder is disturbed, which is not quickly relieved by urination; and (4) by slight hæmorrhages from the over-distention. Here there is no frequency of urination, no pus, no bacilli, and rarely any blood in the urine. When this occurs, as I have seen in a man above fifty-five, the mimicry of obstructive prostatic disease is striking.

When the infectious process takes the course of a primary tuberculous enteritis, localized peritonitis over an infected solitary gland of the ileum, and peritonitis of the anterior or posterior peritoneal folds of the bladder from the bacillary accumulation in the dependent pouches, the disease reaches the muscular coat before the mucous membrane is involved. In such a case we have the same symptom-group, with the enteric and peritoneal set added. The bowel-disease is not easily recognized, and localized tubercular peritonitis is often symptomless.

Moreover, several months may intervene before any visible symptoms appear, the first of which to attract attention may be hæmaturia, without pus or bacilli in the urine. In such a case the cystoscope offers the only means of resolving the doubts. It shows a patchy, disseminated tuberculosis under the transparent mucous lining of the bladder, sometimes on the anterior portion of the fundus, sometimes over the vault itself. I am persuaded from observation that this course is more frequently taken by the disease than is generally suspected, as it was impossible to observe 
it in precystoscopic practice. The post-mortem table would hardly enable pathologists to trace backward this line of attack.

The bladder is sometimes invaded from an infected seminal vesicle, and here again the mimiery of calculous disease is so close as to frequently tempt the most careful to a highly injurious, even dangerous, instrumentation. In several cases I have noted that, owing to the matting together of the tissues of the affected side, the disturbed bladder called urgently for urination, being unable to hold more than five or six ounces; the pain ceased when about half of this was voided; there was at the end of the act much straining, and the extrusion of a few drops of bloody urine at the last, and the distress slowly subsided as the bladder was partly filled again. The distress at the end of urination seemed to me to be due to the bending of the stiffened seminal vesicle and to the pressure about the inflamed meatus internus. As differing from the symptoms of calculous disease, the day and night frequency are about the same. The cystoscope shows a patchy inflammation along the line of the affected vesicle, the other part of the bladder being free of disease. Though more frequent in early life, this form of the disease may be seen in persons of advanced age.

Coming from the prostate, the infection creeps rather uniformly up from the anterior angle of the trigonum, probably climbing by the lymphatics of the submucous coat. The symptoms are those of cystitis of the neck, with frequently bright but transitory hæmorrhages. A finger in the rectum will detect an unusual sensitiveness of the intervesicular space, especially if the bladder be partly filled. Distention - reflex is marked. Nodules may be sometimes felt in the bladder wall below the anterior angle. Detection of the prostatic disease ought to do away with the necessity of cystoscopy or other instrumentation.

Cystoscopy, an invaluable ageney in diagnosis, prognosis, and in directing treatment, should be practiced with the utmost care and gentleness, and only after the most thorough precautions have been taken to avoid aggravating the disease. In no disease is the integrity of the protective epithelial lining of the urinary tract of more service.

A very slight tranmatism of the trigonal mucosa may convert a mere surface irritation into an infection, when tinder becomes fire. In case the attack is coming from the other direction, a tear will admit the urine in such a way as to convert a dry and sluggish infected area into a moist and astonishingly active focus. The following mode of procedure will be found to serve the best purpose. Boil the instruments, and use only a mildly borated glycerin for a lubricant. The anterior urethra should then be washed with a one-to-two-per-cent solution of carbolic acid (the salicylic-acid solutions do not act well here, giving much pain, and softening the epithelium). This is best done by injecting the urethra from the 
meatus several times and allowing it to empty itself. Next an Ultzmann's syringe-point is introduced into the membranous part and a syringeful or two of an aqueous solution of five-per-cent cocaine hydrochlorate and one per cent of carbolic acid thrown into the vesical neck. Then, and not until then, should a catheter be introduced to empty the bladder and distend it for inspection. If these precautions be taken, an anæsthetic will rarely be required; and it is of advantage not to give one, for we want the patient's sensations felt and noted as diagnostic aids. In the vast majority of cases/ the writer has succeeded in getting satisfactory observations without other anæsthetic than cocaine used in this way. In estimating the cystoscopic picture, however, one should keep in mind the fact that a five-per-cent solution of cocaine blanches the mucosa by causing vascular contraction, and that it also causes paresis of the detrusor vesicæ, so that a bladder that would previously project a smart stream from a catheter will, after cocainization, often allow it to flow out only by siphonage. This is a matter of importance in a disease one of the chief symptoms of which is often a weakened bladder force.

The use of the cystoscope has also the advantage of enabling us to do away with the stone-searcher, one of the most dangerous instruments that may be used against a tuberculous bladder.

Treatment.-The treatment of vesical tuberculosis is much the same, no matter whether it be primary or secondary, and without regard to surface or deep infection. One should not lose sight of the fact that the disease often becomes obsolescent without treatment, and true phthisis of this viscus is one of the rarest occurrences.

Caustic application-silver nitrate and zinc solutions-are only mentioned to be condemned. Antiseptic and bactericidal douches do no good, but are positively harmful by spreading the infection. We can never stamp out the disease by such means. Iodoform has been weighed in the balance and found wanting. Corrosive-sublimate solutions give frightful pain, and spread the disease. Counterirritations over the pubes or in the perinæum, still used by some, add to the patient's discomfort, and are powerless to help. The mineral waters, buchu, etc., are of no service, and often disturb the digestive organs.

When the disease becomes obsolete, the bladder wall is thickened, stiffened, and contracted. It is highly irritable and friable, and holds only a few ounces. The reaction of such a bladder to distention is marked. This makes fluid distention, which is of so much service in the concentric hypertrophy of simple cystitis and stricture, inadmissible and dangerous.

In the early stages of surface irritation gentle aseptic washing of the bladder with simple warm water is safe and very soothing. Oil of sandalwood and cubebs, by the stomach, do good service in any stage. Our 
main reliance must be in general treatment. Climate, diet, and the antitubercular remedies often improve the patient in the most astonishing way, hastening a quite satisfactory obsolescence. Cold and damp climates are to be avoided, and a sedentary employment chosen whenever possible. An appeal to surgical means is very doubtful in all, and very disastrous in the majority of cases. Drainage is not beneficial. If the pain and sloughing demand cystotomy for relief, the sectio alta is preferable. The perineal section often becomes infected, and an obstinate fistula with burrowing backward, even into the rectum, will occur with uncomfortable frequency. The suprapubic cut often heals with a soft (infected ?) redbanded scar, which easily spreads and permits a hernia to protrude after the patient has assumed the erect posture. On the whole, surgery offers very little to these patients, and meddlesome surgery does much harm.

\section{TUBERCULOSIS OF THE SEMINAL VESICLES.}

Tuberculosis of the seminal vesicles is treated under the heading of diseases of these organs. It is proper to call attention here to the fact that, owing to their close anatomical relations with the bladder, symptoms appear ratler promptly after infection, and these symptoms have to do chiefly with urination.

\section{TUBERCULOSIS OF THE PROSTATE.}

Symptoms and Diagnosis.--Where there are a few small tubercular nodules on the capsule or near the periphery of the gland, the disease may be symptomless. They are to be felt easily, however, from the rectum. Nodules situated abont the vesiculo-prostatic junction are apt to obstruct the duct, and determine an engorged condition of the corresponding vesicle. Here, too, the proximity to the bladder wall develops symptoms of cystitis of the neck, and the vesicular peristalsis during coitus sets up a painful, throbbing sensation, of which the patient is apt to complain. In some of these cases bloody or "currant-jelly" semen may be emitted.

In other cases a tuberculous nodule of moderate size, and not active, may be imbedded in the substance of a prostatic lobe, and still cause but slight symptoms. Where they are discrete they can usually be felt from the rectum as indurated spots in the soft structure of the gland. There must, however, be many cases where they are indistinguishable by palpation, and where symptoms appear only after the infection has crept upward by the lymphatics to the vesical neck. Some authors allude to an enlarged and indurated condition of the gland produced by early and excessive masturbation, which may be confounded with this form of tuberculosis. It seems much more plausible, in the absence of exact pathological knowledge, to attribute the excessive masturbation as well as 
the enlarged and indurated state to tuberculosis. Aside from atrophy, I am not aware of any pathological state that can be clearly connected with masturbation, however excessive or early practiced, as the sole etiological factor. It seems but right, then, that in all such cases tuberculosis should be strongly suspected, though a positive diagnosis may be properly withheld to await developments. This condition occurs in early life, about the time of the evolution of puberty, and the functional activity, along with the growth and development of the organ, will soon resolve the doubt. The precaution is all the more important when we consider the dangers of instrumentation in these cases.

Some of these cases do not come under the surgeon's care until they have an acute, parenchymatous (gonorrhœal) inflammation ingrafted on the chronic ("latent") tubercular disease. At that moment the diagnosis as to tubercular disease is entirely impossible while one is face to face with the most disastrous consequences; for it is in these very cases that we see total destruction of the prostatic mass by gangrene.

In still another form the tuberculous onset is acute, the gland swelling rapidly from the irritation (ptomaines?) produced by a very few bacilli, resulting in a smooth, symmetrical, and painful enlargement. Rectal and vesical symptoms are prominent from the beginning. Acute retention may necessitate the nse of the catheter, which aggravates the disease. Here the diagnosis is impossible except by the exclusion of other canses, or the implication of associated organs in the tubercular process. The presence of gonorrhœa in any stage, especially if undergoing local treatment, entirely obscures the picture. A tubercular nodule in the testis or cord may give a valuable hint, but these are frequently absent.

In this form two determinations are possible: (1) the acute swelling may subside, leaving one or more hardened nodules in the parenchyma, and the disease become obsolete; or (2) the gland may soften in places, abscesses form, break into the urethra, and a true prostatic phthisis be set up which gradually erodes the entire organ by cheesy necrosis. I have seen such destructive process hollow out the prostate as lupus might do, destroy the membranous urethra, open into the perinæum, while at the same time it burrowed into the post-prostatic tissue without ceasing until the rectum had been invaded. These patients usually go on until they succumb to general miliary tuberculosis. Obscure as it may be in the beginning, the diagnosis is not difficult after the disease has well set in on its way. The prognosis is most unfavorable.

Treatment. - In the acute forms active antiphlogistic measures are required. Where the patient could bear it, I have apparently seen good results from the internal administration of tartar emetic in doses of $\frac{1}{24}$ th grain every three hours until the heart showed signs of being affected, as was first suggested by Milton, of London, for perineal abscess. Hot 
douches to the perinæum have a soothing effect. It is best to move the bowels freely in the beginning with a cathartic, and leave them alone for several days. Pain is to be quieted with opiates, preferably codein. After the acute stage has subsided, or in the chronic forms of the disease, obsolescence is to be courted by a general antitubercular treatment, which should also include such remedies as quiet sexual activity.

All instrumentation, except such as is absolutely required, is to be avoided, and the same is to be said of caustic and antiseptic applications to the prostatic urethra. Surgical means are of no service here; a prostatic abscess may need opening from the perinæum. When an incision is required, the surgeon should, if possible, avoid opening the urethra. Dry applications, which are at the same time astringent, such as tannic and boric acids, are best for local applications to these open ulcerations. The curette and the cautery serve rather to irritate the parts and thereby spread the disease.

\section{TUBERCULOSIS OF THE URETHRA.}

Tuberculosis of the Urethra is doubtless very rare as a primary infection, either of the body or of the uro-genital cycle. The posterior part of the duct may be invaded from the prostate by continuity, or, less frequently, by the tuberculous products of cystic or nephritic disease. Mere surface irritation of the deep urethra by tuberculous urine is one of the common signs of disease of the urinary passages posterior to the prostate. Peripheric prostatic tuberculosis reaches the duct, even in its posterior part, very slowly, and often lets it go free entirely. Ulcerative destruction of the membranous portion is not very uncommon, and tuberculous ulcers of the anterior portion may occur. Miliary urethral tuberculosis is quite common when the disease is about to put an end to the patient's life.

Symptoms and Diagnosis.-The chief symptoms of urethral tuberculosis are excessive pain on urination or instrumentation, strong tendency to bleeding on slight manipulation, and the disposition to urethral fever on very slight provocation. In the few cases that have come under my observation satisfactory cocaine anæsthesia was not possible. This rendered the use of the urethroscope quite difficult and unsatisfactory, but taught me to suspect the disease in those cases where there was a disproportionate amount of pain in a urethra not acntely inflamed.

The urethral discharge is often purulent, and tubercle bacilli are found, if at all, very rarely. A deep and very painful ulcer may exist with very little or eren no discharge from the meatus, but the first ounce or two of urine passed will nearly always contain tubercular detritus. In several cases seen by me the ulcers were remarkable for their clean and smooth appearance and feel. 
Fenwick very properly calls attention to the fact that this disease may easily be mistaken for stricture, and adds that "we recognize the difference by the extraordinary amount of pain which is caused by the most delicate instrumentation, and this even after a strong solution of cocaine has been applied." In a case that remained doubtful, general anæsthesia for urethroscopy would be justifiable.

Treatment.-This should be symptomatic and supportive. Instrumentation is to be avoided. Topical application of astringents dissolved in distilled extract of hamamelis, and washes of mild thallin solutions, have been of most service in my hands. Iodoform, extolled by some, proved useless. Morphine, dissolved in the washes, seemed to have more effect is quieting pain than cocaine.

\section{TUBERCULOSIS OF THE TESTIS AND CORD.}

The disease is encountered here in three forms: (1) where the onset is sudden and acute, the gland and epididymis swelling rapidly, becoming hot, red, and painful, this subsiding and leaving hardish nodules of varying sizes in the gland-substance or epididymis; (2) where the process is more chronic and less active, the hard nodules forming gradually, apparently from the coalescence of several small foci of infection; and (3) the gradual and often quite painless development of very small spotlike nodules upon the tunica albuginea or in the meshes of the epididymis. In the acute form the swelling may be so rapid as to cause gangrene, unless the fibrous capsule be split, and in this case the testis may "fungate."

Symptoms and Diagnosis.-The clinical picture is much like that of an acute gonorrhœal epididymo-orchitis, and is not to be distinguished from it in those frequent instances of the lighting up of an acute upon an old or "latent" tuberculosis by gonorrhoeal extension. When this form occurs in a patient without gonorrhœa and after some slight traumatism, the inference is easily drawn. These cases can also be separated from syphilitic orchitis by the rapidity of the process and the greater amount of pain in the tubercular disease.

There is rarely any difficulty in distinguishing between obsolete or quiescent tuberculosis and syphilis of the testis. The diffuse orchitis of syphilis, swelling the gland and epididymis alike, developing a smooth, painless, and heavy oval tumor, which undergoes involution by atrophy of the organ, presents to palpation a very different object from the nodulated, irregular, often fistulous, tuberculous testis. Gummatous deposits, however, of the testis may closely simulate tubercle. The exclusion of syphilis and the existence of tubercular nodules in other organs-especially the prostate-most frequently settle the diagnosis. In fact, as already mentioned, the all but constant involvement of more than one of the uro-genital organs in the tubercular disease is our safest means for 
differentiating the affections. Where all other means fail, the touchstone of treatment may be applied. Gummatous lesions of the testis which remain any length of time in the same condition are rare. One should not lose sight of the fact that syphilis and tuberculosis are not infrequently concomitant diseases.

The diagnosis of tuberculosis of the epididymis is dependent on the discovery of the hardish nodules, from the size of small shot to that of a pea, in these soft structures. Here, owing to the elasticity of the organ, the nodules can be readily felt, and one may often recognize the irregular shape of even quite small masses. Even when the whole testis and epididymis are indurated by the tubercular infiltration, the nodulated and irregular feel is quite characteristic.

Treatment.- This should be supportive and antitubercular. The organ should be carefully protected against even the slightest traumatism, by suspension and attention to the clothing. Sexual quiet should be courted and enjoined.

Surgical measures should be held as a last resort. In the present state of aseptic surgery, castration, even when unilateral, is rarely necessary. The high importance to man's intellectual and moral state of keeping at least a small share of testicular substance in physiological connection with the circulation and with the cerebro-spinal centers; the suspicion that the opposite organ or the prostate is already implicated; the strong doubt, almost amounting to a convietion, that the disease is never primary, for the body, in the testis, as well as the now common acknowledgment on the part of the most experienced observers, that castration does not have an appreciable influence on the progress of the disease toward a general infection, should make us hesitate long before removing even one of these organs which have so striking an influence on the general nutritive processes. The chances of obsolescence, the wonderful recuperative power of the gland, and the now well-demonstrated efficacy of the removal of the tuberculous foci without serious damage to the remaining healthy portions, leaves only a very small number of eases to demand castration.

When the tubercular nodules remain latent in the testes, especially in those cases where there are evidences of coincident prostatic tuberculosis, they can safely be let alone. If they take on an activity that even threatens trouble, the organ can be incised, the tuberculous areas curetted, cauterized, washed freely, dusted with iodoform (I have lately used aristol with good results), and the wound closed, with or without drainage, and just as good results obtained as where the whole organ is removed. It is quite astonishing how large a part of the testicle can be removed in this way without danraging that which is left behind; and so long as even a small portion of the glandular tissue is left in connection with the nervous system, potentia coeundi remains to the patient even if sterility from 
azoöspermism results. Entire excision of the epididymis may be done in this way with only a minimum of damage to the individual. Moreover, this method has the advantage of being applicable to both testicles at the same time, if the conditions require it, while every one, including the patient, shrinks from double castration.

Where the testis is extensively disorganized, the scrotum riddled with discharging sinuses, and there are evidences that the patient's health is being depressed by the drain, castration with removal of the diseased parts of the scrotum is demanded, but it can not be hoped in this way to do more than arrest the discharge, and to that extent improve the general health of the individual. Radical cure can not be looked for. In such cases, the disease has already attacked parts inaccessible to surgical measures. When the cord is diseased, one must also be prepared to see a fistulous tract remain after the operation, so that in such cases one does not even get palliation of the patient's condition to an extent which will justify the operation.

Yet it is in testicular tuberculosis that surgical interference has heretofore been supposed to offer the best promise of success after removal of diseased foci. The greater the length of time these cases are observed after operation, the more clearly it is seen that but little if any benefit has resulted. 


\section{DISEASES OF THE SCROTUM.}

By CHARLES W. ALLEN, M. D.

Anomalies.-Deviations from the normal scrotum may be either congenital or acquired. Thus, in instances of hermaphrodism, pseudohermaphrodism, or pronounced forms of epispadias, as well as in extroversion of the bladder, we have congenital defects, while in asymmetry due to ectopic testicle the condition is gradually acquired.

Complete absence of the scrotal sac has been observed, and an instance of this abnormity has been put on record by Godard (Recherches Tératologiques). We expect, however, always to find at least a fold of thick and furrowed skin occupying the scrotal region, or an infantile development of the organ with lack of hair-supply, even when ectopy of the testicle exists upon both sides. When anorchidia is single, instead of this rudimentary development of the sac there exists, as a rule, only a slight inequality in the two sides, that corresponding to the lacking testis being the less developed. This asymmetry may be acquired quite late in early life.

Often the bag seems to accommodate itself to the single testicle, and by contraction comes to fit it snugly, while the raphe is either obliterated or is much displaced to one side.

Contraction of the scrotum, as well as of the penis, is also a result of certain neuroses of the prostate.

Another anomalous condition due to lack of development is seen in the congenital separation of the two lateral halves of the scrotal sac. Some of these eases strongly suggest by their peculiar configuration the female labia majora. If at the same time congenital defect of the penis is present, hermaphrodism is more or less closely simulated. Absence of testicles often accompanies cleft scrotum. Adhesions sometimes exist between the penis and the scrotum, binding the organs so firmly together as to interfere with genital functions, and an operation becomes necessary to separate the parts.

Atrophy.-This condition is found in anorchidia and in ectopy of the testicles. Here the scrotal tissues shrink and contract, adapting themselves to the remaining testicle if one only is contained in the sac. In old age, too, the scrotum often becomes thinned as well as elongated, the base becoming stretched out into a cord-like pedicle, while the hairs and folli- 
cles take part in the general atrophy which affects the skin, and pigmentar tion becomes increased.

Hypertrophy.-Simple hypertrophy from mechanical obstruction of the lymphatics is occasionally observed, and may be mistaken for elephantiasis.

Sturgis has reported quite extensive hypertrophy after syphilis. Such enlargement is usually associated with some other manifestation of the disease.

Abscess.-Abscess of the scrotum may follow direct injury of the tissues, especially if an open wound is present, permitting germs to enter, or may be secondary to tuberculosis of the testes, suppurating gumrna, and occasionally other pathological conditions.

When pus has formed in the scrotum, it should be evacuated early. The process of repair to the tissues, as a rule, goes on rapidly when the scrotum has been damaged by abscess formation, provided all the pathological tissue has been removed; but in the case of tuberculosis a fistulons tract usually remains for a long time. Hæmatocele may result in abscess, if an open wound permits the entry of septic germs.

Injuries.-From the exposed position of the scrotum, it not infrequently suffers injury along with its contents, and often, too, is the seat of damage when the testicles escape injury by reason of their mobility.

Incised and lacerated wounds may follow falls upon the perinæun, and occasionally one is called upon to treat those which have been selfinflicted. When deep, wounds often expose the testicle, the elasticity of the scrotal tunics permitting retraction. In contusions resulting from horseback exercise, gymnastics, blows, kicks, etc., there is often an extravasation of blood, causing extensive ecchymosis. This variety of wound is quite common.

Lacerated wounds may further result from bayonet thrusts or from gunshot wounds, though the latter are rarely seen excepting where the testicles have been injured at the same time. The testes may be laid bare by the scrotum being torn away wholly or in part, and a plastic operation be called for.

Punctured wounds from the prongs of pitchforks, falls upon nails or splinters, and surgical punctures not carefully made, offer great chances for infection, and erysipelas, phlegmon, gangrene, and sepsis not uncommonly result. The indication here is for free incision if any signs of sepsis become manifest.

Wounds of the scrotum are frequently produced by the surgeon in operation for varicocele where a portion of the sac is dissected out, or in the procedure for castration; and it is found that here, as in accidental injury, the resulting scars are small as compared with the size of the wound. 
Hæmorrhage into the loose dartos spaces, from retraction of the severed arteries, is to be guarded against. The arteries lie near the raphé, and if cut should be at once ligated or compressed with clamp forceps. In injuries as well as in operations implicating the scrotal sac the possibility of the presence of hernia and wound of the intestine must not be overlooked. As a rule, the scrotal tissues heal promptly, but a good precaution in operation is to pluck out the hairs or cut them short over the operation-field, that they may not interfere with subsequent repair.

In contused wounds the parts should be brought together as well as may be in front of the testicles and maintained in this situation with antiseptic dressings, so as to secure, in healing, the best covering for these organs. If extravasation has taken place, the treatment must be amplified by the application of cooling lotions, rest, and support of the parts, under which absorption usually takes place.

Infiltration of urine may cause injury to the scrotal tissues severe enough to result in sloughing and even loss of life from blood-poisoning. The dartos readily sloughs notwithstanding its great vascularity. The indication is for early and free incision, while the strength is kept up with iron, quinine, and other tonics.

Burns of the scrotum are comparatively rare. If the degree of heat has caused sloughing, the same treatment is applicable as in death of the parts from infiltration of urine. In either case, if extensive loss of tissue has occurred, a plastic operation may be called for.

Tight strapping may result in such injury to the scrotum that sloughing will occur.

Frost-bite, likewise, may be followed by mortification of an area of scrotal tissue.

Fungus of the Scrotum.-Wounds of the scrotum are occasionally, though rarely, followed by a superficial fungous growth. Usually the wound is of such a nature as to permit of partial hernia of the testis, and through neglect in applying suitable sutures after replacing the testicle the surface of the albuginea presenting at the open wound becomes granular. A permanent cicatrix may result, binding the scrotum to the testicle. Benign superficial fungus may be likewise induced by the irritating effect of secretions which are permitted to flow over the skin or remain upon it, causing redness and infiltration, with nodular formation and subsequent vegetating outgrowth or fungus. It is to be treated by thorough cleanliness, cautery, and cicatrizing dry dressings.

Edema_-In general anasarca from heart, kidney, or other disease, the scrotum seldom escapes, and, indeed, it is often here that the fluid in the tissues first manifests itself. CEdema of the scrotum usually attains comparatively large proportions, because of the abundance and looseness of the subcutaneous connective tissue. 
Localized œdema of the scrotum may take place from causes which constrict the apex of the sac, such as occur in hydrocele; and this is often seen in the hydrocele of young children, while œdema may occur in youth, from slight causes, in the absence of hydrocele.

Obstruction of the return circulation from enlarged glands in the groin, syphilitic infiltrations, infiltration of urine, etc., may result in a more or less temporary dropsy of the parts.

This, too, may be caused by the rupture of a hydrocele, or it may be due to acute suppuration of the tunica vaginalis.

Acute anasarca of the scrotum, if due to general debility, has a pinkcolored surface; while cedema, as usually seen, has a transparent, waxy look.

Edema also accompanies elephantiasis, erysipelas, eczema, ivy-poisoning, and other skin diseases.

The diagnosis of this condition from that of extravasation is made by the perinæum being implicated in the latter.

Hard or soft odema, such as is occasionally seen upon the skin in association with urticaria, or as an independent affection, I have not observed upon the scrotum, nor have I found cases recorded. So-called acute œdema of the scrotum is probably, in the majority of cases, a phlegmonous or erysipelatous condition, coming on in the course of certain infectious diseases. Inflammatory œdema, in which the skin becomes stretched, glistening, and red, is seen at times in tuberculosis of the testicle.

Emphysema.-In complete subcutaneous emphysema the scrotum becomes inflated with gas, at times to an enormous extent, just as we have seen that very extensive cedema of these parts is the rule in general anasarca. Emphysema of the scrotum alone may be occasionally observed as the result of local injury from the puncture of a trocar or accidental wound, and the condition may be brought on intentionally for purposes of deception - a fact well to be borne in mind in obscure eases. A peculiar crackling of the tissues is called forth by manipulation of the parts. The percussion note will be resonant.

Emphysema may accompany gangrene of the tissues. Verneuil makes two classes of the affection: 1. Benign aërian, subcutaneous or serous pneumocele, due to injury - $(a)$ local ; (b) remote, as in wounds of the air-passages and neiglıboring cavities of the head and neck, intestinal wounds, etc. 2. Malignant bacterial pneumocele, due to phlegmonous inflammation, implicating the genitals or regions adjacent to them. Benign cases recover spontaneously, or after puncture. The malignant forms are grave and require energetic measures, including incision, drainage, disinfection, and at times castration. 


\section{CUTANEOUS AFFECTIONS.}

Eczema.-This is one of the most frequent, most tenacious, and most discomforting of the cutaneons troubles which affect the scrotum. It occurs often in subjects of gout or rheumatism, and sometimes in diabetics, though it may develop in the absence of any diathesis.

It may present itself in the acute or chronic form, and be of the dry squamous variety, lichenoid, rhagidiform, or exist as an eczema rubrum, crustosum, or seborrhoicum.

The itching is usually most intense, and in the lichen-like form is almost unbearable, especially at night. When deep fissures form, the pain and burning become very distressing. There is considerable thickening of the skin from infiltration, and the volume of the sac is often much increased, while contraction of the dartos may markedly increase the depth of the furrows. The skin may be uniformly reddened or present a raw-looking surfaces from which a sticky discharge takes place. Unless the greatest cleanliness is observed, the odor from such an oozing eczema becomes very offensive.

Treatment.-Acute cases require soothing, slightly astringent and antipruritic dressings, while the chronic demand stimulating applications, especially when the infiltration is marked. For the former, equal parts of aqua calcis and oleum lini, to which may be added five per cent or more of iodoform, dermatol, aristol, europhene, or two per cent of resorcin, meet the requirements for most cases. Zinc oxide, bismuth or lead in ointment to which one per cent of menthol, five of naphthol, or carbolic acid are added, suffice often to allay the itching and hasten the cure. When the skin has become much thickened and thrown up into ridges so that it must be put upon the stretch to bring into view the parts between them, and especially when this condition is a chronic one, such strong applications as the liquor picis alkalinus recommended by Bulkley, or the caustic potash solution in strength of half a drachm or more to the ounce as suggested by Taylor, will often be of benefit; strong nitrate-ofsilver solutions may also be employed in similar chronic cases.

In less severe instances, inunction of fatty substances such as lanoline, vaseline, or simple ointments, perhaps with the addition of a small percentage of tar or oil of cade, may suffice, while in other cases the diachylon ointment of Hebra will give good results.

Internal medication should be directed to any diathesis which may exist.

In the dry and papular varieties arsenic will be found very useful, the dose being gradually increased until physiological effects begin to be noted.

General tonics, including cod-liver oil, sirup of the iodide of iron, 
etc., are to be given according to the requirements, just as in eczemas of other regions, and diet must not be neglected.

Eczema Marginatum.-Though usually beginning upon the inner surface of the thigh, and only attaining the skin of the scrotum by invasion after extending over the fold of the groin, eczema marginatum occasionally appears to develop first upon the lateral surface of the scrotum, and subsequently spreads to the contiguous surface of the thigh.

Certain occupations, such as that of cavalryman and riding-master, favor not only the development of the affection but its rapid spread and tenacity. The natural moisture of the parts thus increased, and especially in warm weather, presents the conditions favorable to the growth of the parasite on which the disease depends. It often proves most rebellions to treatment for the same reason.

It is to be distinguished from ordinary eczemas by the abrupt termination of its margin in the healthy skin, by the somewhat infiltrated border, the comparatively slight itching, and the almost constant presence of a characteristic area upon the adjacent thigh. The chief subjective symptom is pruritus, and this is not always pronounced.

Treatment is best carried out by keeping the parts clean and free from perspiration, while strong parasiticides are employed. In recent cases the compound tincture of iodine is efficacious, while in the more chronic, mereurials in strong percentage, or, probably best of all, ten per cent of chrysarobin in lanoline and lard are recommended.

Pruritus.-Itching of the scrotum is the most frequent of all symptoms referable to this organ, and even after excluding the pruritus consequent upon and a symptom of eczema, there still remains a large number of cases of pruritus, a disease per se. While in pruritus of the general surface from any cause the scrotum rarely if ever escapes, there still are forms of pruritus which affect the scrotum exclusively or especially. Thus pruritus scroti may be spoken of as an exclusive affection with as much propriety as one speaks of pruritus ani.

The etiology of pruritus the disease is in many instances obscure, while in others the rheumatic or gouty habit is plainly at the bottom of the trouble, especially in men past middle life. Here exacerbations of itching are observed to follow those excesses which we might anticipate would preeipitate an attack of the constitutional disease.

Pediculi as the exciting cause of pruritus must be carefully excluded, and the itching in eczema must likewise be differentiated from true pruritus. This is not always as simple a matter as it would seem, for the tissue changes due to the irritation of scratching and mechanical injury might readily be regarded as changes produced by eczema.

Pruritus senilis affecting the whole surface often proves very troublesome in the region of the scrotum, and the skin becomes excoriated, pig- 
mented, and stretched from the constant irritation of scratching, friction, and pulling to which it is subjected. In some cases pruritus of the scrotum leads to masturbation, and the patient is not readily broken of the habit until the pruritus is cured.

Occasionally a variety of pruritic affection is encountered in which the itching may be described as subdermic. There is little or no real itching of the surface, but a feeling of formication beneath the skin, coming on especially when the clothing is removed at night. It is relieved not so much by scratching as by rubbing the walls of the sac together or dragging upon them. This condition may persist for years without causing any appreciable changes in the skin or evidences of its existence upon the surface.

To treat pruritus of the scrotum successfully, any disorder of the system on which it may depend, such as diabetes, rheumatism, etc., must be sought for and treated. Locally, antipruritic remedies, such as tar, aconitia, menthol, as well as mercürial preparations, are of much use, but in all cases the patient should be instructed to avoid scratching as much as possible, for in most instances the condition is only intensified by it, and the relief thus afforded is only temporary.

Turkish baths, sulphur baths, and sometimes sea-salt baths are beneficial, and I have at times seen relief from strong nitrate-of-silver solutions painted over the whole surface. As we know from the treatment of orchitis, the scrotal skin is very tolerant of silver, and one or even two drachms to the ounce can be painted over the surface.

Pediculosis. - The special pediculus which by its presence and bite causes the intense pruritus in this affection is the so-called pediculus pubis or crab-louse.

The diagnosis is readily made (though close inspection is sometimes required) by the presence of brownish spots upon the surface near the base of hair-shafts, and of ovoid spheres upon the hairs themselves. The former are the parasites and the latter their eggs or hatched-out shells.

Treatment is best carried out by means of mercurial washes or ointments, applied not only to the scrotum but to the neighboring region, while the whole surface of the body, including the brows, eyelids, and axillæ, are searched for pediculi. If but few lice are found and none elsewhere, they may be removed mechanically with forceps, and the nits stripped off the hairs in the same way. In order to avoid the disagreeable features of mercurial ointment, an ether or chloroform spray may be directed upon the parts, or one of the non-mercurial parasiticides, such as tincture of delphinium or benzine, may be applied.

Herpes.-Chronic recurrent herpes of the scrotum has been reported by Dr. Flatau (Deutsche med. Wochenschrift, No. 22, 1891) in connection with herpes of the mouth and penis. The affection shows a decided 
tendency to recur, and, while the etiology is not known, it is supposed to be a neurosis. Herpes of the scrotal skin incidental to zoster of neighboring parts is rarely observed.

Lupus is not often seen upon the scrotum. When it does occur, it is the same affection and must be treated in the same way as lupus located elsewhere. The lesions, as a rule, assume a ringed form, and to secure good results the tissues for some distance beyond the ring must be destroyed or removed, just as in treating cancer.

Psoriasis in rare instances may be localized upon the scrotum.

After disappearance of the lesions, pigmented spots may remain for a long time at their site, probably because of the tendency on the part of the scrotum to become pigmented after any irritation.

Dermatitis may arise from any local irritant or may follow the ingestion of certain drugs. It is not infrequently due to the use of mercurial ointment for the cure of pediculosis, and is at times found after the employment of strapping for enlarged testicle, due to the irritation of the plaster. Those who suffer from ivy-poison often have the scrotum implicated in the dermatitis along with the face, hands, and other parts. Some persons who possess an idiosyncrasy as to quinine show a special tendency to dermatitis of the scrotum after the use of the drug, while in other cases the first manifestations of irritation are noticed about the prepuce and glans penis. Arsenical eruptions localized upon the scrotum have been reported as occurring among men whose work brings them much in contact with arsenic. The treatment is that for similar dermatitis elsewhere located, and is usually simple and successful when the exciting cause has been once eliminated.

Intertrigo affecting the scrotum and contiguous region of the thigh is especially prevalent in childhood, from slight canses. In the adult it is for the most part seen in the army and in those who walk much or ride horseback. It is especially prone to attack fleshy men and those of uncleanly habits. In the former it often proves a very obstinate condition.

To secure prompt recovery scrupulous cleanliness is necessary, and the frequent removal of all offending secretions which might prove irritating. Resorcin ointment in two-per-cent strength, with twenty-five-per-cent lanolin, usually gives excellent results. A suspensory bag should be worn, and the scrotum prevented from contact with the skin of the groin and thigh.

Simple dusting-powders are useful, and should be continued after all redness has disappeared.

Molluscum Contagiosum.-This disease quite commonly affects the scrotum, and at times this is the sole region on which the molluscum growths are to be found, although the penis is most apt to show them at 
the same time. When large and grouped, the diagnosis lies between this affection and sebaceous cysts. Treatment by scraping out with the curette and applying nitrate of silver to the base, or simply washing with a 1-2,000 bichloride solution, suffices for a cure.

Pityriasis versicolor occasionally, though rarely, occupies the same location upon the scrotum and thigh as an eczema marginatum. It is much more readily cured than the latter, often requiring no more than a few applications of tincture of iodine.

Scabies finds one of its most frequent locations upon the genitals. It may be readily conveyed to the scrotum and penis by the hands. An eczema of the scrotum may result in neglected cases.

Sebaceous Cysts. - Cysts of the scrotum sometimes attain the size of filberts or even larger dimensions, and when occurring in groups present quite a noticeable deformity. They are for the most part situated in the upper portion of the sac. Treatment consists in incision of the skin, enucleation of the cyst with its walls unruptured, or, if this fail, evacuation of the contents and scraping out the sac with the curette, followed by free antiseptic washing. Small cysts can be squeezed out between the thumbs after incision of the skin.

Pigmentary Affections.--The scrotum is very apt to become deeply pigmented after persistent scratehing, or in fact after any irritation, and at about the time of puberty, notably in dark-skinned persons, a natural increase in the pigmentation takes place, amounting in some instances almost to blackness.

Vitiligo is occasionally seen in association with the same disease, so often found upon the penis.

Treatment has little effect upon this condition. Should advice be sought on account of the pigmentation of chloasma, a strong solution of bichloride (five grains to the ounce), to blister and cause the skin to peel, offers the best chances of success. Pyrozone, the name given to a fiftyper-cent solution of peroxide of hydrogen in ether, will effect the same purpose. A drachm each of ammoniated mercury and bismuth subnitrate to the ounce of vaseline has acted well in some-instances.

Ulcers.-Almost invariably ulceration of the scrotum is chancroidal or syphilitic. Tuberculosis of the testicle as well as hæmatoma, various forms of injury, ete., may implicate the scrotum secondarily.

Gross has described an ulcère soudain which in the short space of twenty-four hours destroyed the scrotum of a fifteen-day-old child down to the vaginalis. The nature of the process is not given, but it would seem probable that the condition was akin to the rapidly ulcerating stomatitis in childhood usually called noma.

Erysipelas.-Erysipelas invades the scrotum by spreading from some other region much more commonly than it starts in the scrotal 
tissues themselves; still, in injuries, and, as I have observed it several times, after ritual circumcision, the first manifestations of the infection are seen here. Erysipelas migrans in childhood is very apt to involve the scrotum, and the swelling of the tissues becomes very pronounced, while much tension and pain are occasioned. Primary erysipelas of the scrotum may start from a fistulous tract, an excoriation, bite of an insect, or wound of the tissues accidentally or surgically inflicted. It is rare. Erysipelas may be followed by gangrene of the scrotum, and this is probably the origin of many obscure cases in which the cause of the gangrene is not found.

Phlegmonous erysipelas occurs, as a rule, only in the aged and infirm. The scrotum here becomes mottled, marbled, or black, from excessive extravasation of blood. The enlargement is often extreme, and the tumor thus produced is tense and has a smooth, shining surface.

Phlegmon is rare. Any prolonged local irritation causing injury to the surface may cause its development. Thus it is seen to follow the presence of a foreign body in the scrotal wall, the escape into the skin of an injection used for hydrocele, a urinary fistulous tract, the injury of a contusion, or the friction incidental to a forced march. Circumscribed phlegmon is commonly surrounded by a zone of œdema which subsides as soon as the pus is evacuated, and this should be accomplished as soon as it is detected. Fever and insomnia are apt to be the most marked symptoms. In diffuse phlegmonous erysipelas there is great tumefaction and the process is very prone to spread to the perinæum and lower abdominal region, and it would appear that the anatomical conditions which determine the course and limits of infiltrated urine operate in the same way as in the case of phlegmon of the scrotum. This condition may prove rapidly fatal, and in the more benign cases a typhoid state with high temperature and grave symptoms may be present. Even in apparently mild cases all infiltrated areas should be freely incised, the lines of incision canterized, and antiseptic dressings applied. In simple erysipelas I have found ichthyol in collodion an excellent application. Erysipelas beginning in the scrotum might be prevented from spreading to the general surface by applying an adhesive band firmly about the apex of the bag at the peno-scrotal junction, in such a way as to constrict the lymphatics without making undue pressure on the cord and vessels.

\section{ELEPHANTIASIS OF THE SCROTUM.}

Elephantiasis scroti, with or without implication of the lower extremities, is quite a rare disease in the United States, especially among the native population, being more common among the blacks in the South than elsewhere. 
It is more or less prevalent in Egypt, India (Lower Bengal), China, Samoa, Barbadoes, and parts of South America, and appears to flourish best near the seaboard. Two forms are usually considered to exist-the firm, hypertrophied variety and the soft lymph scrotum, also ealled at times pseudo-elephantiasis, and by Rindfleisch pachydermia lymphangiectatica.

Dr. Fenger, of Chicago, believes that the so-called lymph serotum is only the early stage of elephantiasis, in which the tissues are still soft and œdematous, permitting the formation of thin-walled transparent bullæ over the surface of the tumor. These bullæ may burst, allowing a milky fluid to escape, and be attended with a temporary decrease in the size of the growth. Enlargement of the lymphatic glands is a common accompaniment, and if an abraded surface permits the entrance of pus microbes suppuration may take place. In the succeeding stages the skin beeomes nodular and leathery, and there is a formation of crusts and seales.

The two varieties resemble each other greatly, if they are not identical, and clinically there is no distinction to be made between them. Manson has indeed shown that elephantiasis, lymph scrotum, and chyluria are all related and due to a eommon cause-the filaria sanguinis hominis. The scrotum in this disease may attain enormons proportions and prevent locomotion by its weight, which may equal that of the rest of the body, or may be of even greater weight. In such a case one might speak of an operation as being undertaken to remove the patient from the tumor!

Dr. Browne, surgeon to the General Hospital in Madras, snecessfully removed a growth which measured over six feet and a half in diameter, the urine being passed from an opening at two feet and a half from the pubis. The weight was found to be one hundred and twenty-four and three quarter pounds.

Elephantiasis of the scrotum usually begins as an induration of limited extent in the more dependent portion of the sac, and gradually extends by infiltration of neighboring tissues, slowly at first and then more quickly; but while its development may extend over a period of years, the increase is always more rapid than in elephantiasis of the extremity. It is not contagious nor hereditary, and, although endemic in the countries mentioned, sporadic eases may oeeur anywhere. It develops rarely in youth or advaneed age, the majority of instances beginning between the ages of twenty and forty. The relative frequeney of scrotal elephantiasis is shown by the statistics of Aranjo (Atlas des maladies de la peau, Rio de Janeiro, 1887) to be about five per eent, or twenty-one instances in four hundred eases of the disease observed. Of these, two were of the serotum alone, fourteen of the scrotum and penis, and five of the scrotum and one extremity. 
Symptomatology.-There are few subjective symptoms attending the onset of elephantiasis. It is usually a painless affection, but at first pains in the lumbar region, in the testes or groins may be complained of, together with swelling of the spermatic cord. The first manifestations of the disease may appear after an attack closely resembling erysipelas, and such attacks may recur during its course, or simply erythematous redness with swelling, and anæsthesia of the skin may occur at intervals. The temperature is usually somewhat elevated, and recurrent exacerbations of fever, attended by enlargement of the scrotum, are the rule. This increase in size at irregular periods decreases again when the temperature falls, but each succeeding attack leaves the parts permanently larger than the last. A lymphorrhcea or serous exudation, attended by the production of bullæ over the surface, may occur, and the skin gradually assumes a thick and rough character. The sebaceous glands and hair-bulbs atrophy, and the hairs fall out. The penis gradually becomes lost to view, and a depression forms in the tissues corresponding to the orifice of the urinary canal.

When the growth has reached a large size it begins to cause annoyance by its weight and bulk, which render locomotion difficult, as well as to produce in almost all cases a certain mental distress. The accompanying plate, taken from Dr. Morrow's collection of original photographs, illustrates the latter condition. The rough and uneven nature of the surface, together with wart-like elevations, are well shown.

Pathology.-All the tissues are hypertrophied as a result of inflammation and occlusion of the lymphatics.

The chief pathological change found is an excessive connective-tissue growth. Microscopical examination of a scrotal tumor removed by Dr. Fenger showed thickening of the epidermal and papillary layers. The epithelial cells were normal in shape and size. Thick bundles of nonfibrillar connective tissue and occasional connective-tissue corpuscles of normal size and shape were found. There was enormous thickening of ali vessels, the small veins being five times the normal size. The thickening of the wall was mainly in the external coat, the endothelium not participating. Leucocytes were present in the perivascular spaces, with islands of young granulation tissue. There was no enlargement of the lymph spaces or of the lymphatics, but, according to the observer, some of the islands occupied by embryonal cells were lymph-vessels or spaces in a state of plastic inflammation-a view which seems very probable. There may be increase in the fatty as well as in the areolar tissue, and endoarteritis as well as periarteritis and phlebitis may be present.

Etiology.-The primary cause of elephantiasis in most cases has been shown by Manson, Lewis, Bancroft, and others to be the presence of the filaria sanguinis hominis, and this view is shared by Maitland in his recent work on Elephantiasis and Allied Disorders. 
PLA'TE VIII.

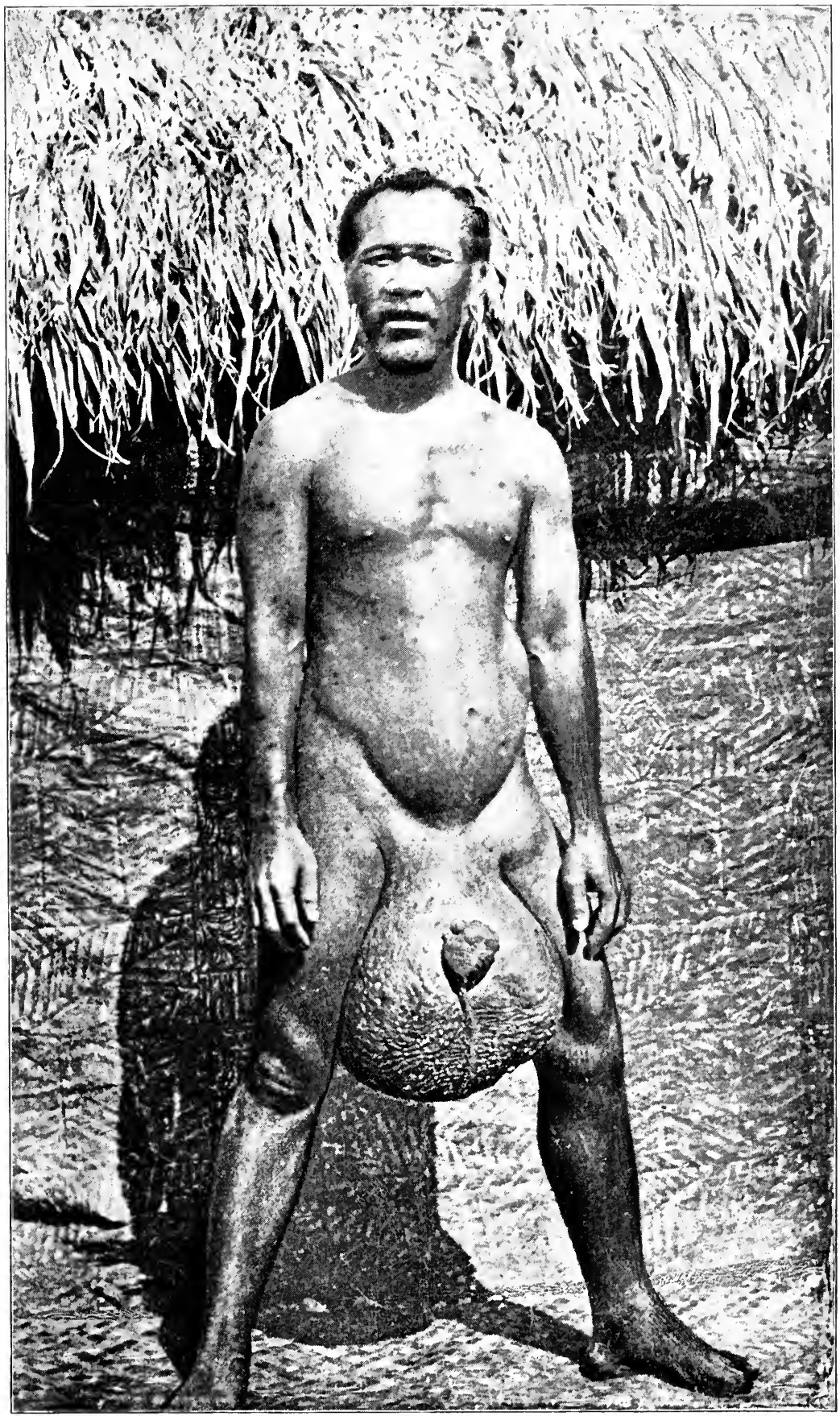

Elephantiasis of the Scrotum (Morrow.) 

Manson believed that all the phenomena of the disease could be explained on this theory. Lewis (Lancet, September 20, 1877) found an adult filaria worm in a lymphatic vessel of a scrotal elephantiasis. The filaria Bancrofti, as it is sometimes called, is a thin, white worm three to four inches in length and of the thickness of a hair. It is capable of laying thousands of eggs. It is thought probable that inflammation may be caused by the chemical products of the excretions of these worms, and in non-epidemic regions that any irritative inflammation, such as that produced by erysipelas or syphilis, may have the same result.

The secondary cause is obstruction of the lymphatic channels either through the production of embolism, by the filaria itself or its ova, or by the occurrence of inflammatory obstruction. Mapother (Philadelphia Medical Times, November 15, 1887) explains the frequency of scrotal implication on the theory that the lymph here has to rise from such a dependent organ against the force of gravity. When obstruction is complete, according to Maitland, the firmer form of elephantiasis results, and, when incomplete, lymphorrhea and lymphatic varix are produced.

Enlargement of the lymphatic glands often precedes the development of the disease, and this is thought by some to have an important etiological bearing on its production.

It is not yet settled that all cases have the same etiology, and many believe that any condition giving rise to lymphatic obstruction and lymphangitis is capable of producing elephantiasis in the absence of filaria.

Diagnosis.-Elephantiasis can only be confounded with other diseases in the early stages. The slow development of the swelling, the exacerbations and absence of marked symptoms pointing to any other condition would suggest this disease, especially in tropical regions. Confirmatory evidence would be furnished by the discovery of the filaria sanguinis hominis in the blood. It has no connection with lepra, though lepers occasionally acquire it. It is to be distinguished from simple hypertrophy, œdema, and fibroma.

Prognosis.-This disease must be looked upon as benign, having little effect upon the general system and little influence upon the expectancy of the individual, not materially shortening life. The prognosis, so far as operation is concerned, is excellent, especially in recent years.

Kocher's statistics (Die Krankheiten der Männlichen Geschlechtsorgane, Deutsche Chirurgie von Billroth und Leucke, 1887) give-

27 per cent mortality for 61 cases (Fayrer).

$\begin{array}{llllrll}9 \frac{1}{2} & \text { " } & \text { “ } & \text { " } & 21 & \text { " } & \text { (Ballingall). } \\ 5 & \text { 6 } & \text { " } & \text { " } & 161 & \text { " } & \text { (Esdaile). } \\ 3 \frac{3}{10} & \text { " } & \text { " } & \text { " } & 61 & \text { “ } & \text { (Manson). } \\ 1 \frac{1}{2} & \text { " } & \text { " } & \text { " } & 138 & \text { " } & \text { (Turner). }\end{array}$

Maitland gives the mortality in the Calcntta Medical College Hospital 
as 18.2 and 17.7 per cent from published statistics, but it is said that more recent statistics bring the rate down to only four per cent. In correction of the above statistics, Dr. Fayrer has recently written to the Indian Medical Gazette, stating that his published reports show one hundred and ninety-three cases operated upon, with thirty-five deaths, or a little over eighteen per cent mortality instead of twenty-seven per cent. These cases were operated in the pre-antiseptic epoch beginning with the year 1859.

Turner's report was made in the Glasgow Medical Journal of June, 1882.

For a good prognosis regarding recurrence after operation all diseased tissue must be carefully removed. In rare instances atrophy of the testicles has been observed, but in most cases the genital functions are not interfered with, and impotence does not follow. In view of this fact an effort should be made in operation to save the penis and testes, as can be done in most instances without much difficulty. Hydrocele and hernia complicate a certain proportion of cases. Suppuration in lymph scrotum is rapidly fatal at times, especially in those of enfeebled constitution.

Treatment.-The early management of a case of developing elephantiasis includes antiphlogistic measures before the occurrence of much hyperplasia, together with pressure made by means of elastic bandages or strapping carefully applied, rest, and antipyretics. If there is anything in the history or appearances that suggests syphilis, mercurials may be advantageously applied locally, while the iodide of potash is given by the mouth, and this form of medication has in fact been found beneficial in the absence of any specific taint.

In the febrile attacks quinine and arsenic have been found useful, and change of climate is often very beneficial. Many cases are never seen by the surgeon until the tumor has attained a considerable size, and then the knife has been considered the only resource. The rule of the older surgeons was to make no attempt to save the genitals in tumors weighing over fifty pounds, but this rule should never be followed. In none of Turner's cases was the penis sacrificed, and in none were both testicles removed.

The testes, if dissected out and left lying upon the perinæum, usually draw up into place and become covered over with granulations. If the tunica vaginalis is hypertrophied, the rule is to remove it along with the growth. Where practicable, flaps should be made to cover in both penis and testes, or, in fact, a new scrotum produced by plastic operation. Fenger advises a median and two smaller lateral flaps, the median for the penis, and the latter to meet a single broad posterior flap and cover in the testes.

The chief source of danger and annoyance in the operation is hæmor- 
rhage. The vessels are numerous, large, and diseased, and success depends very largely upon the thoroughness with which their ligation is attended to. In a twenty-two-pound tumor removed by Fenger sixty ligatures were applied, and more than this are often required for larger growths.

Formerly soft twisted rope was mostly employed to constrict the pedicle and prevent hæmorrhage, and Lister's tourniquet has been used to compress the abdominal aorta. The elastic constrictor has also been in vogue, but it is liable to slip and has therefore fallen into disfavor. The best method of controlling hæmorrhage appears to be by means of the Turner clamp (see Glasgow Medical Journal, June, 18s2, for illustration). It must be applied to the topmost portion of the pediele after the presence of hernia has been carefully excluded. After removal of the tumor one end of the clamp can be loosened at a time, thus giving opportunity to ligate the vessels slowly and make the operation bloodless.

Electricity by means of the constant current is well spoken of by Liebig and Rohé (Practical Electricity in Medicine and Surgery, Philadelphia, 1890). It surely seems a rational means of treatment in eases not too advanced, and one which might render cutting operations much less frequently necessary. Silva Araujo, who has probably had the most extensive experience with electricity as a means of treating elephantiasis, says that while this method requires the greatest perseverance and patience, it is always beneficial if applied regularly in suitable cases. The induction eurrent, by stimulating the vessels, inereases the circulation of both blood and lympl ; and while the continuous current acts as a solvent of the masses of connective tissue, the former determines a rapid absorption of the products of this solution. A combination of these currents has hence been found most useful.

Electrolysis by means of needles introduced into the tissues has also been used with advantage. From three to five needles made antiseptie are connected with the negative pole, while the positive is applied to some portion of the body's surface. Six elements are employed to begin with, and this is gradually inereased to sixty. Electricity can thus be used under either of these forms separately or in combination.

Dr. Araujo reported a case of elephantiasis of the scrotum associated with chyluria, lymphatic scrotum, craw-craw, and erysipelas in the same individual treated successfully by electricity as early as 1877 (Gazeta Medica da Bahia, November, 1877).

\section{TUMORS OF THE SCROTUM.}

Aside from the sebaceous cysts which have been already referred to, cystic tumors of the serotum are not at all common. Dermoid eysts may oceur here as elsewhere, and contain the usual skin elements, hair sebum, 
and teeth, as well as bone and cartilage. Such a tumor may occupy a position between the testes and occasion no annoyance until it becomes accidentally injured. The diagnosis is made from the history, being that of a congenital tumor, and it is to be distinguished from supernumerary testicle by want of subjective sensation on pressure. The diagnosis is rarely made before operation.

Urinary Cyst.-According to Sajous's Annual, vol. ii, 1888, Karewski observed a scrotal tumor the size of the fist, which, on tapping, was found to contain a clear fluid, as in hydrocele. It connected with a urethral fistula, and pressure upon the tumor caused a flow of clear urine from the meatus.

The treatment is excision of the cyst and closure of the urethral fistula.

Cystic tumors are rare and of slow growth. They usually develop in obstructed sebaceous follicles, and, next to the scalp, the scrotum is the most frequent situation for them.

I have seen groups of from ten to twenty distended tumors, from the size of a pea to that of a large grape, occupying the upper half of the scrotum and near the middle line. Under the term scrotal cystoma Curling reports a case in which there was an agglomeration of twenty to thirty cystic spheres, with clear, serous contents.

Hæmatoma or hæmatocele is the name given to the tumor formed by extravasated blood distending the scrotal pouch. It may be located in the dartoid tissue, in the tunica vaginalis, or in the spermatic cord.

True hæmatocele occurs upon one side of the scrotum only. The skin covering the swelling is more apt to be thinned than thickened, and is more or less tensely stretched. There are fluctuation, crepitation, and a surrounding induration, with lack of translucency. Basil has studied hæmatoma (Thèse de Paris, 1890), and finds the seat of predilection to be the cellular tunic of the dartos. The blood constituting the tumor may be fluid or clotted. It is usually caused by trauma. If the blond is diffuse, we have partial hæmatocele by infiltration; if confined within narrow limits, it is said to be by extravasation, or the two conditions may coexist. Extravasation is frequently between the skin and the dartos. Basil has shown that a serous cavity exists in the subdartic cellular tissue, and this probably often presents a favorable location for the extravasated blood. Symptoms complained of are principally heaviness and dragging in the region of the testicles. There may be ecchymosis of the skin, and this may extend from the scrotum over the thighs, abdomen, and possibly the back.

Diagnosis is principally from fibrous tumor. If skin is ecchymosed it is diagnostic.

Prognosis.-Absorption takes place, and there is no suppuration unless there be communication with the outer air through a wound by which 
germs can enter. Gangrene or septicæmia may follow if germs gain entrance. At times the extravasated blood becomes encysted.

Treatment.-Place a cushion or pad between the thighs, or apply a wide strip of adhesive plaster across them for the scrotum to rest upon. Use antiphlogistic measures and cold applications, and, if inflammatory symptoms threaten abscess formation, open freely.

Angeioma or Vascular Tumor is usually congenital. The atten. tion is, however, not directed to it until some injury or other exciting cause stimulates a growth which is peculiar in being intermittent, and attended with some inflammatory symptoms. The tumor may be of firm consistence, or there may be irregular, soft spots over its surface due to the presence of small serous cysts.

Treatment by excision after ligature.

Fibroma is very rare. A case is reported by Ferguson. It occurs as a hard, lobulated, and encapsulated growth, which under the microscope is found to be composed of fibrous or fibro-cellular structure. Such growths may attain a weight of as much as twenty pounds. They are especially seen in old men.

Diagnosis is to be made from hæmatoma, enchondroma, elephantiasis, and cancer.

Treatment.-By removal, as in elephantiasis.

Fnchondroma is also very rare, but instances have been described. The tumor is hard, containing cartilaginous lobes and possibly bone deposits, and may weigh five or more pounds.

Lipoma is of interest and importance from the fact that the testicle at times becomes imbedded in it and may have to be removed. It is rare, and seldom attains a large size. Kimball has reported an instance in which the growth weighed two pounds. When small it may simulate a supernumerary testicle. It may have its point of origin in the spermatic cord.

Epithelioma.-Epithelial cancer of the scrotum is the form of carcinoma usually encountered. It commonly begins as a warty growth upon the surface, which like similar growths elsewhere may remain as an apparently benign affair for a term of years until some irritation or injury excites the malignant tendency. In the succeeding stages the growth softens, becomes more highly vascular, is now for the first painful at times, the surface becomes covered with a crust or scab, which falls off, exposing an ulcerating and possibly bleeding surface. A small indurated nodule may first be noticed in the absence of any previous wartlike growth. The development is slow but surely fatal unless excised freely and sufficiently early. Such a growth beginning on the scrotum, according to Taylor's observations, may invade the penis.

This disease was once quite common in Great Britain, and was called 
chimney-sweep's cancer because of the relative frequency with which it occurred in those following this occupation-the soot being retained in the folds of the scrotal skin until an irritation was produced which led to thickening and subsequent cancerous growth. Now that chimneys are no longer swept in the old way the name has lost its significance here, but according to Volkmann, those who work in the coal-tar products, paraffine, etc., and according to Andrews, those occupied as smelters, are liable to this form of cancer.

Encephaloid carcinoma and scirrhus are rarely observed. The former, if pigmented, is termed melanotic. In any form, if the inguinal glands are implicated they must be dissected out at the time of operation.

A vegetating cancer suggesting in appearance a cauliflower or mushroom, is to be diagnosed from fungus of the testicle. The surface of this form bleeds easily, and the margin of the scrotal orifice through which the cancerous growth escapes is as though pinked.

Sarcoma is extremely rare, both the melano-sarcoma and myxo-sarcoma.

Tuberculosis.-Primary tuberculosis of the scrotum has been studied by Rochette (Thèse de Paris, 1885). It is very rare. In tuberculosis of the testes an inflammatory œdema of the scrotal tissues is observed in which the skin is stretched, glazed, and red. The various layers become infiltrated, thickened, and lardaceous, especially. about the orifices of fistulæ. When the latter have healed this condition disappears. Occasionally secondary tuberculous infection takes place. 


\section{DISEASES OF THE TESTICLE.}

BY JAMES BELI, M. D.

\section{H FMATOCELE.}

Definition.-Hæmatocele, by which we mean a tumor caused by the effusion of blood, may occur into the tunica vaginalis (vaginal), into the substance of the testicle (parenchymatous), or into the spermatic cord.

Etiology. - It is practically always due to injury, and very often occurs in connection with hydrocele. Blood is frequently poured out into the cavity of the tunica vaginalis after the tapping of a hydrocele, generally from the wounding of a vessel by the instrument, but occasionally, perhaps, from the removal of the pressure of the hydrocele fluid upon the vessels of the vaginal walls. Other causes are blows upon the testicle and strains, as in heavy lifting.

Predisposing causes are diseased conditions of the walls of the bloodvessels, hæmophilia, etc. Hæmatocele of the substance of the testicle (parenchymatous) rarely, if ever, occurs alone. It is nsually connected with, and of secondary importance to, hærnatocele of the tunica vaginalis.

Symptoms.-Hæmatocele of the testicle appears as a globular uniform tumor, largest below, painless, except when the tension is great, as in a very large tumor or where the effusion is parenchymatous ; opaque, heavy and resistant to the feel, when recent. Inflammatory thickening of the tissues soon takes place when the tumor becomes dense and hard, and may be mistaken for a neoplasm of the testicle. The inflammatory process which first involves the tunica vaginalis extends to the scrotal tissues, and if acute may end in abscess, especially in aged or debilitated subjects. More frequently, however, the process is of a more chronic nature, and may continue for months or years. In such cases the tunica vaginalis becomes greatly thickened, dense, and hard, and loses to a great extent, or altogether, its character as a serous membrane. Wasting of the testicle may take place, especially if hæmorrhage has occurred in its substance, and diagnosis becomes exceedingly difficult even after an exploratory incision has been made. The irregular sac will be found to contain a brownish, greenish, diffluent substance, often described as resembling coffee-grounds and sometimes contains cholesterine. This is, of course, the remains of the degenerated blood-clots. 
Diagnosis. - In recent cases, and where a reliable history can be obtained of the sudden development of the tumor after injury, diagnosis is sometimes easy. When an acute, intense inflammation has developed and more particularly in the chronic cases, diagnosis is difficult and often im. possible. It is well to remember that the testicle ordinarily preserves the same relation to the tumor as in hydrocele, lying posteriorly and toward the bottom of the scrotum, where it may be distinguished from the bulb of the tumor by yielding its characteristic sensation on pressure.

Treatment. - In recent cases of slight or moderate effusion, rest in bed, elevation of the testicle, restricted diet, and free evacuation of the bowels daily, will usually be found sufficient. If seen early, while the hæmorrhage is still progressing, ice may be applied, and at a later period absorption may be hastened by hot applications containing acetate of lead and opium, to relieve pain if necessary. In very exclusive effusions the sac should be laid open, the clots removed, and bleeding points secured. This operation, performed with modern surgical precautions, is simple and safe, and, in addition to greatly hastening recovery, it is the surest method of preserving the integrity of the testicle. When suppuration has occurred, free incision, washing and draining the cavity are of course indicated.

In the chronic cases free incision and drainage are also indicated, but care must be taken not to injure the testicle, which is often difficult to recognize, unless, indeed, it be already disorganized, when it should be removed, with the thickened and altered tunica vaginalis.

Hæmatocele of the Cord is a very rare affection, but one which may occur in good health and independently of any disease of the genital organs. Its causes are direct violence and sudden and severe strain, as in lifting a heavy weight. Curling mentions that Mr. Pott has related three cases, all of which occurred in this way (from strain); one of these occurred in a young man while straining at stool. In either case the blood is effused into the tissues of the cord, producing a fusiform swelling, which in cases caused by direct violence may be obscured by effusion of blood into the surrounding subcutaneous tissues.

Diagnosis.-Such a tumor suddenly developed, in this situation, after injury or straining efforts, may be mistaken for a hernia. Careful examinations will, however, fail to discover any of the characteristic signs of hernia; and a few hours' delay, during which the hæmatocele will become hard and tense, with probably ecchymoses into the surrounding parts, will greatly aid in clearing up the diagnosis.

Treatment must be conducted on the same principles as in hæmatocele of the testicle; encysted hydrocele of the cord may be converted into a hæmatocele, and should be dealt with in the same way as an ordinary hæmatocele of the tunica vaginalis. 
Free Bodies in the Tunica Vaginalis.-Free bodies have been found in the tunica vaginalis, usually in connection with hydrocele, of which they were probably the cause. These bodies, which are usually small and cartilaginous, and sometimes contain bone elements, correspond to the "floating cartilages" so often found in the synovial sacs, especially of the knee-joint. They have probably a similar origin.

\section{ANOMALIES OF THE TESTICLE.}

Absence of the Testicle.-Cases of congenital absence of one or both testicles (as well as of supernumerary testicles) have been recorded from time to time, but the actual occurrence of such conditions is very difficult of demonstration. Apart, however, from monstrosities, and cases in which the genital system is irregularly or imperfectly developed as a whole, there would seem to be occasional instances of congenital nondevelopment of one or both testicles. One of the best-authenticated examples of this condition is the case reported by Fisher, of Boston (American Journal of the Medical Sciences, vol. xxiii, page 352), which has been quoted by most writers on this subject. In this case the deficiency was noted at birth and confirmed by dissection after death, the man having died of pneumonia at the age of forty-five. It is related that this "man's voice remained unchanged at puberty, he had no beard or. whiskers, the skin of the pubes and scrotum was without hair, the penis was not larger than that of a boy ten or twelve years old, and the scrotum was contracted in size"; also that "lhe never exhibited any amorous propensities or desire for female society" (Curling). Irregular and imperfect descent of the testicle as well as atrophy of the organ must be taken into account in any given case where dissection proves its absence before assuming that such absence is congenital.

Supernumerary Testicles. - A considerable number of cases have been reported of men who are said to have had three or four or more testicles. In very few of these cases, however, was any attempt made to demonstrate this fact by post-mortem dissection, and it is doubtful if it has ever been clearly demonstrated, although Curling quotes Blasius as having examined the body of a man thirty years of age who had two testicles in the right side of the scrotum and one in the left. It is tolerably clear, however, that in most of these cases the supposed supernumerary testicle is a small tumor of some kind, such as an encysted hydrocele, epiplocele, omental hernia, fatty or fibrous tumor (the latter is not rare in the epididymis).

A case came under the observation of the writer a few years ago of a man who appeared to have (and who believed he had) two testicles in one side of the scrotum and one in the other. He was confirmed in this opinion by a great many medical men at home, and by at least one emi- 
nent London surgeon who had examined him. This man has since died, but no dissection was made post mortem.

Size of the Testicle.-The testicles, without doubt, vary considerably in size within natural limits. The mean dimensions of the normal testicle are said by Curling to be one inch and three quarters in length, one inch and a quarter in breadth, and one inch in thickness from side to side. He also states that he has found the ordinary weight of the sound testicle of the healthy adult to be six drachms. The left testicle is generally a little larger and heavier than the right.

Hypertrophy of the Testicle.-Hypertrophy of a compensatory character is believed to occur when one testicle has been removed or remains undescended in early adult life. A certain amount of enlargement of the testicles is also observed when sexual intercourse is regularly indulged in after a long period of chastity. This corresponds with the temporary enlargement of the testicles observed in animals during the rutting season of the female.

Atrophy of the Testicle has been observed to follow upon a great variety of causes. The following are the most important: Orchitis, resulting from injury or disease, as mumps and gonorrhœa, injuries to the brain or spinal cord, pressure as in varicocele, hydrocele, hæmatocele, the pressure of a truss, etc., onanism, and excessive sexual indulgence, syphilis of the testicles, arrest of the blood-supply, enlargement of the prostate gland, and iodism (the latter doubtful).

Atrophy of the testicle has been observed to occur in cretins and idiots, and has been seen in healthy boys, without apparent cause. Not only is there no reason to believe that prolonged chastity canses wasting of the testicles, but the opinions of the best authorities are that it does not do so. It is admitted, however, that when the functions of the testicles have not been called into use they probably cease to be functionally active at an earlier age.

The writer has observed a case in which the excessive use of tobacco at an early age seemed to be the most probable cause of atrophy of both testicles.

A young man, twenty years of age, came under my care in hospital for stricture of the urethra five inches from the meatus. He had a gleety discharge, and attributed the stricture to a gonorrhœe contracted two years before. $\mathrm{He}$ was slightly built and excessively nervous, and the testicles could only be distinguished as the clubbed ends of the vasa deferentia, about the size of an ordinary white bean each. He was, and had been from boyhood, an inveterate tobacco chewer and smoker, and had worked in a tobacco factory from the age of twelve years. He denied having practiced onanism or having indulged too freely in sexual intercourse, and stated that the testicles had always been small, but that they had begun to waste some time before he contracted the gonorrhœa. He had not suffered from orchitis. 
Treatment.-In the majority of cases nothing can be done; but where the cause can be recognized and removed sufficiently early, the further progress of the atrophic change may be arrested.

Seat of the Testicle.-Malposition of the testicle, due to irregular or imperfect descent, is frequently observed. The testicle may remain within the abdomen or be arrested in the inguinal canal or upper part of the scrotum. It is also sometimes found in the groin, the erural region, or the perinæum.

The eauses of these abnormalities in descent are variously attributed to peritoneal adhesions, smallness of the abdominal rings, shortness of the cord, and loss of power of the gubernaculum testis. Testicles retained within the abdomen remain soft and undeveloped, and, where both are retained (cryptorchidism), the individual is generally, if not always, sterile, although sexual power and appetite usually remain unimpaired or even apparently sometimes increased.

[The observations of veterinary surgeons go to show that cryptorchidism in the horse is always associated with sterility, and that such animals show a restlessness not only in the "season," but all the year round.]

When only one testicle is retained within the abdomen (monorchidism), the descended testicle, if normal, is sufficient for procreative purposes.

Misplaced testicles ontside the abdomen (in the inguinal canal, groin, crural region, or perinæum) are especially liable to injury, but seem to be capable, in some cases at least, of a fair if not full functional development, except when they are retained in the inguinal canal, where the limited space, muscular pressure, and constant exposure interfere with development.

The testicle in an abnormal situation is also liable to orchitis from the ordinary causes, and when this occurs the symptoms are much more severe than when the testicle is normally situated within the scrotum. Orchitis occurring within the abdomen may set up peritonitis, and lead to serious consequences.

Treatment.-When the testicle remains within the abdomen, no treatment is indieated. If, however, it comes down at times into the inguinal canal, a truss may be required to prevent its passage through the internal ring. Should the testicle descend with a hernial protrusion, it may be possible to fix it in the upper part of the scrotum while performing an operation for the radical eure of the hernia. This will depend, of course, upon its relation to the hernia and the nature of the adhesions which it has formed with it. When the testicle is aceessible, its reposition within the serotum is a feasible operation, which has been successfully performed in a considerable number of cases. 
Retraction of the Testicle is generally observed as a symptom in connection with lesions of the genito-urinary organs or in pelvic inflammations. It is due to spasmodic contraction of the cremaster muscle, and is most frequently met with in connection with the passage of a urinary calculus along the ureter or during its presence in the bladder.

A form of persistent retraction is also described as occurring in children, and is said to have followed urinary irritation, a kick upon the pubes or groin, a blow upon the abdomen, etc. (Ashhurst's International Encyclopædia of Surgery, vol. vi).

In addition to the foregoing anomalies occurring in persons otherwise normally developed the vas deferens has been found deficient in some cases near the testicle, in others near its distal end.

Curling also mentions a case (recorded by Geoffroy St. Hilaire) of union of the testicles in the abdomen. The subject was an infant, which was registered as a girl, and died at the age of eighteen months. Dissection discovered a complete hypospadias.

Injuries of the Testicle.-When normally situated the testicle is not specially prone to injury. Contusions from kicks and blows, bruises upon the saddle in riding, and from falling straddle legs, are not uncommon, but are very rarely serious. The first effects of such a blow or bruise are a dull, sickening pain and perhaps faintness, which soon pass off. If more severe, hæmatocele or orchitis may ensue, and as a remote result hydrocele or fibroid changes in the organ.

Incised, punctured, or gunshot wounds are occasionally met with, and it is important to bear in mind that under proper surgical treatment such wounds do well; and castration is very seldom called for, even in the worst cases. The following case may be mentioned in illustration :

In 1885 , a man thirty years of age was wounded by a Winchester rifle bullet, which passed through the gluteal fold of the buttock, the perinæum, and scrotum. When seen, the testicle was found lying outside the scrotum, having been pierced through the center from side to side by the bullet. It was cleansed, and the torn and bruised edges of the wound of exit having been removed with scissors it was returned to the scrotum. A complete and uneventful recovery followed, in spite of the fact that the patient went through a severe and prolonged illness from extravasation of urine due to a wound of the urethra, made by the bullet in passing through the perinæum. When last seen, several months after the injury, the testicle, although greatly reduced in size through loss of substance, was apparently normal, and had not undergone any atrophic or other appreciable change. 


\section{DISEASES OF THE TESTICLE.}

\section{BY EDWIN C. BURNETT, M. D.}

INFLAMMATORY diseases of the testicle are separable into three divisions according to their anatomical seat, and are named orchitis, epididymitis, and vaginalitis or periorchitis, respectively. Each of these affections may attack the part after which it takes its name either primarily, or, as is oftenest the case, secondarily to an invasion of parts adjacent or remote, and its course may be either acute, subacute, or chronic.

\section{ACUTE ORCHITIS.}

Etiology.-True orchitis is comparatively rare. The causes are traumatic, idiopathic, and the propagation of disease of the urethra, bladder, and vas deferens. Inflammation of the testicle and epididymis may arise in a case of ectopia testis, when the testicle is placed in a position that exposes it to external violence, or to the pressure of adjacent parts, as when the testicle has been displaced into the perinæum, or is situated in the cruro-scrotal fold, or, partly descended, is arrested in the inguinal canal. English describes as a special form of violence through which inflammation of the testicle and epididymis may arise, that caused by the powerful contraction of the cremaster muscle, which by drawing the testicle upward and against the outer ring of the inguinal canal, brings about such compression of the organ that the injury results in inflammation of it. I have never seen an inflammation that I could trace to this cause, but I have seen a testicle with an inflamed epididymis retracted as far as the outer ring, and held there with such force by the cremaster as to cause excruciating pain out of all proportion to the amount of inflammation, and I shonld not hesitate to ascribe the inflammation of a testicle in such a position to such compression, if there was an absence of all other caises. The clinical picture of a case of this kind which he calls orchitis-epididymitis par effort, is a strong one and better given in his own words: "The scrotum is swelled, in most cases ecchymosed even into the subcutaneous tissue, seldom any fluid in the tunica vaginalis at first, but mostly as a sequel to the supplementary inflammation. In striking contrast to the insignificant initial appearances in the testicle are the subjective symptoms : swooning, nausea, retching, even vomiting, radiating 
pains toward the loin and thighs, convulsions, sudden collapse, even ending in death, as in injuries of the peritonæum."

Injuries are much less frequently the cause of orchitis than of epididymitis, but the result is apt to be more disastrous, as the issue is sometimes a total destruction of the gland through pressure of the inflammatory infiltration upon, and consequent fatty degeneration of, the seminal tubules. Sometimes the destruction is brought about through abscess and gangrene followed by atrophy. In gonorrhœal inflammation of the urethra we have an infrequent cause of orchitis, the disease seldom acting directly, as the epididymis is first affected, the inflammation going over to the testicular tissue in rare cases. Kocher quotes cases in which the testis proper was the seat of the inflammation, the epididymis remaining free. Mr. Hutchinson also mentions such a condition, both testes being affected. I have never as yet seen a gonorrhœal orchitis-that is, one in which the gland was primarily attacked. Després attributed the inflammation in such cases to the accumulation of semen through swelling of the excretory tubes, especially the ductus ejaculatorii. If he meant by this the obstruction to the ejaculation of semen at the time of intercourse, I can agree with him as to the probability of such a condition acting as a cause of orchitis. I believe that obstruction to the emission of semen can play a part in setting up an inflammation of the testicles only when there has been undue and repeated sexual excitement on the part of the patient. Urethritis prostatica, prostatic hypertrophy, cystitis, strictures, operations on the urinary and genital organs (catheterization, lithotomy, evacuation of hydrocele, acute and chronic, especially lithotomy in the aged or enfeebled), form frequent causes. Abdominal strain such as occurs in extreme constipation ( $I$ have seen one case of orchitis from this cause), heavy lifting, prolonged and repeated sexual excitement without gratification-all act as causes of orchitis and epididymitis.

Owing to the very favorable condition in the secreting part of the testicle for the arrest there of morbific elements circulating in the blood, inflammations arise such as are seen sometimes in pyæmia, variola (Chiari), scarlatina, malaria (Simon, Kocher), abdominal typhus, leprosy (Ziegler), often in parotitis, syphilis, and tuberculosis. In orchitis from parotitis we have the so-called metastatic form, wherein, after from four to eight days of the mumps, the testicle is attacked and an acute orchitis set up. This happens most frequently in young individuals, from puberty on to the age of thirty, though the complication may occur in still younger patients; and, on the other hand, cases are reported in much older persons-viz., from the age of forty to forty-five (Kocher, Curling). The testicle is usually attacked after the subsidence of the parotitis, and at times so soon after as to have given origin to the idea of metastasis. In just as many cases, however, the orchitis develops after an interval of from four to 
eight days from the disappearance of the mumps, and there appears to be little ground for the application of the term metastasis in the sense of translation of the disease from the parotid gland to the testicle. Ziegler's term, hæmatogenesis, by which he expresses inflammation of the testis dependent upon the cause in question, as well as that from syphilis, tuberculosis, and allied diseases, is, it seems to me, much more in accord with the present status of our knowledge. Cases of orchitis have been reported which have been followed by mumps, and at the time of epidemics of mumps there has been observed orchitis in patients in whom no trace of parotitis could be found. Kocher reports four such cases, and mentions others observed by different surgeons. Finally, come cases of idiopathic orchitis at a time when there has been no mumps. These have been reported as cases of rhemmatic orchitis, but by Kocher held, as he also holds orchitis from mumps, to be the result of extension of the inflammation with which the urethra and bladder are sometimes affected in mumps, rheumatism, and gout, along the mucous surfaces to the testicle.

The cases of rheumatic orchitis reported by McLeod and Bouison (Curling) are considered by Kocher not to belong to orchitis, as these authors refer the affection to changes in the tunica albuginea and tunica vaginalis. However, Réclus (Curling) describes a case in a patient, aged sixty-one, suffering from acute rheumatism, wherein the two testicles became inflamed and rapidly atrophied, thus showing that here at least the gland proper was the seat of the disease. Mr. Bell quotes a case of gouty orchitis occurring in a friend, a medical man, who relates the case himself in detail, but who gives a perfect picture of an epididymitis occurring after an attack of cystitis and subacute urethritis, which was followed some time afterward by a swelling of the right knee lasting six weeks, after the subsidence of which the testicle again became painful and slightly enlarged.

Keyes also gives gout as an etiological factor in orchitis, and Sir James Paget (Curling) reports several cases of orchitis occurring in gouty patients. Of the above-mentioned general diseases the one most frequently causing orchitis is the mumps. Finally, acute orchitis, and epididymitis also, may occur from causes not demonstrable, especially in little children (Kocher). Fleming saw in a child fourteen days old, and otherwise apparently healthy, an acute, painful swelling of the testis the size of a hen's egg, with protrusion and loss of a mass the size of a walnut, ending in recovery of the normal condition. These cases are reported as being accompanied by sudden abscess formation with and without fungus testis.

Symptoms and Diagnosis.-There are different grades of intensity in orchitis irrespective of cause, so that we may have the mild form, 
even in a severe injury, and, on the other hand, an apparently trivial cause may give rise to quite a severe attack. To the milder forms belong that after mumps, the so-called metastatic variety. Gonorrhœal orchitis attains a more intense grade, as a rule, according to Kocher; but the most acute and most liable to quick suppuration are those cases dependent upon a nonspecific variety of urethritis, such as that which appears after prostatitis, stricture, and operations upon the urethra. He cites Gardner's cases occurring after lithotomy (see Curling) in support of his assertions, that this variety is most liable to quick suppuration and abscess; also a case of Mr. Hutchinson's. There is no change in the shape of the organ in the acute form of the affection, and not so much increase of size, as a rule, as in epididymitis, the enlargement coming about slowly, owing to the unyielding tunica albuginea; twice or thrice the normal is the rule, though there may be enlargement, even in the acute variety, to the size of a goose-egg (Kocher). The tumor is oval, with a smooth and regular surface, which is more or less intensely stretched, according to the extent of the increase in the size of the organ. The swollen gland gives a feel upon palpation as though there were fluid in it, and this pseudo-fluctuation is apt to be misleading. In the beginning the patient is conscious of a feeling of uneasiness and weight, and extraordinary sensitiveness of the part to the touch; this increasing as the inflammation advances, soon becomes acute pain, located in the testicle itself, and which may radiate in the direction of the loin into the groin and down the thigh of the affected side. There is nearly always a slight elevation of the temperature, but in severe cases it rises quite high, and there may be anorexia, nausea, vomiting, hiccough, constipation, which is due usually to the patient's reluctance to make an effort to evacuate the bowels, insomnia, and nervous irritability. These general symptoms may simulate those of strangulated hernia, especially the more faithfully, when through ectopia the part occupies some region common to hernia (Vidal).

The pain may continue severe for three or four days, even longer; after that time gradually becoming less acute, until nothing is left but a soreness upon touch. If suppuration has taken place, the part immediately concerned becomes softened, fluctuation is felt, and if the abscess is not opened by the surgeon the integument and subjacent tissues become thinned by the' pressure and involvement in the inflammatory process, until finally there is a break and the pus discharges itself externally. The abscess cavity usually fills with granulation tissue, and quick healing of the part is the rule.

If the suppurative process has destroyed much of the inter-tubular tissue, prolapses of the seminal tubules may ensue upon the opening of the cavity and discharge of the pus. This condition is caused by the unsupported tubules protruding through the opening of the abscess cavity. 
Rarely the cavity does not heal completely, but fills up with exuberant granulations which grow out and around the opening, forming, together with the prolapsed granular structure of the testis, what is known as fungus testis benignus; or a sinus may be left, through which a discharge of thin pus continues for many weeks, or even months, the testicle remaining chronically inflamed, hard, and tender. After the abscess has been opened and the pus discharged, the severity of symptoms lessens, unless there is another focus of suppuration and another abscess forming. This is sometimes the case.

Occasionally suppuration goes on at the center of the gland and does not make its appearance at the surface. When this occurs, the process is a slow one. The organ becomes less tender, gradually reduces in size; the pus inspissates may even calcify; the function of the gland is destroyed; there are chronic pain and a proneness to repeated attacks of acute inflammation. If the process ends in gangrene through complete strangulation, or septic infection of the parts, the scrotal tissues, already adherent, slough, and the dead portion of the testicle protrudes externally as a dry, yellowish mass in the first instance, or as a putrid semisolid, dirty substance in the last. This is a rare termination.

Pain is comparatively more severe in this variety, and the general symptoms in severe cases arise to a height out of all proportion to the amount of inflammation in the organ itself. This is explained by the unyielding character of the fibrous sheath, the tunica albuginea surrounding the glandular structure, which, resisting the expansion of the inflamed tissue, causes the pain. This condition may result in suppuration, or even in gangrene (dry necrosis) (Ricord, Keyes) of a part of a testicle, and is sometimes announced at the time when the patient's suffering is at its height by a sudden cessation of the excruciating pain (Kocher, Keyes).

To differentiate between an inflamed testicle and periorchitis serosa acuta by simple palpation is difficult. Occasionally the latter affection makes itself known through the transparency that may be present, but it is not a constant feature. Much more determinate is the behavior of the epididymis and the skin. The epididymis is demonstrable to the tonch as normal unless it shares in the inflammation, and then it is slightly harder, but never so hard as in epididymitis from gonorrhœa. The skin of the scrotum in orchitis differs from that covering over an inflamed epididymis. It is stretched, its veins distended, and it may be red, but not œdematous. This condition, which is contrary to that of the skin in epididymitis and periorchitis, is due to the separation of the gland proper from the skin and subcutaneous tissues, by not only a firm fibrous capsule but by a serous cavity. It is true that the tunica enters here and there into the inflammatory process, but at the utmost in the form of a periorchitis adhesiva, in which an effusion is never present (Kocher). The 
spermatic cord is, after the first day of the beginning of the orchitis, usually thickened, irritable to the touch, but without any share being taken by the vas deferens.

Kocher states that in intense cases there appeared upon the corresponding side, near the lumbar vertebral column, a spot very sensitive to pressure, with swelling of the retro-peritoneal lymph-glands. The last is not always to be felt, it is true. In epididymitis, also, that has gone on to suppuration, the same is to be found.

The inguinal pain of the corresponding side, with the same distressing, drawing feeling as in epididymitis, may accompany acute orchitis, though it is not so constant, and the painfulness of the testis itself is often the first and most prominent symptom Pains in the small of the back are frequent, and seem to be increased by the dorsal decubitus (Kocher), while the inguinal pain disappears on assuming this position. The pain in the testicle is not in the least relieved by lying on the back and supporting the scrotum, and the character of the pain serves in a measure as a diagnostic point against epididymitis. This pain is a pulling, stretching one, not as in epididymitis as if caused by pressure; and the explanation of it lies in the stretching of the tunica albuginea by the swelling of the inflamed testis. This is proved by the fact that an incision, or several small punctures, through the tunica albuginea, relieves the pain in a few minutes.

Prognosis.-Acute orchitis terminates in the majority of cases after a course of from fourteen days to four weeks' duration, according to the severity of the case, in a return of the part to the normal state. In some cases, however, there arise conditions which threaten not only to destroy the functioning capabilities of the organ, but also the patient's life. Atrophy is the most frequent cause of destruction of the glandular structure of the organ, and many cases have been reported as the result of acute orchitis, most of these occurring after mumps (Curling, Kocher, Ziegler, Lesser, English). This atrophy follows immediately upon the swelling, and is likened to the somewhat analogons connective-tissue shrinking of the kidney after nephritis (Kocher).

Gangrene as a consequence of orchitis is of rare occurrence. Curling reports a case occurring in his practice, resulting after an attack of acute orchitis, and an abscess which burst spontaneously. He also quotes a case, recorded in Mr. Harvey Ludlow's prize essay, in which gangrene with putrefaction followed acute inflammation of the testicle in a man in feeble health and who had suffered much from stricture. Death, chiefly from peritonitis, occurred. He also mentions another case in an old man from an acute orchitis after lithotrity. Hutchinson (Kocher) reports an analogous case in an old man with prostatic hypertrophy. Kocher claims that after the investigations of Chauveau it may be accepted with certainty 
that the issue in true gangrene may be referred back to infection through the deposit of septic material from the diseased urethra. Gangrene of the testis with putrid decomposition may occur in a similar manner after injuries and inflammation of the epididymis. Quite different is the condition of destruction brought about after injury of the testicle through the bursting of an abscess. Ricord (Keyes) called this necrosis. This condition has already been referred to under symptoms. Patients debilitated by disease or old age are liable to gangrene of the testis after acute orchitis. Spontaneous gangrene in patients hitherto healthy is rare. Bell has reported cases of such a condition. Through septic infection or suppuration extending along the spermatic cord and setting up a pelvic cellulitis or leading to peritonitis the life of the patient is threatened. An acute orchitis may become chronic. This is usually through abscess formation. In absence of the surgeon's aid the abscess breaks its way with difficulty through the tense tunica albuginea. With children, abscess comes more quickly, with formation of fungus, or followed by quick healing. The abscess may become encapsuled, and remain for many years.

Pathological Anatomy.-In four specimens studied by Kocher the following changes were observed macroscopically: The first case was one of acute gonorrhœal orchitis, with unimportant participation of the epididymis, this part exhibiting only a slight thickening. In a second preparation, that of a testis inflamed through violence and undergoing acute suppuration, death resulted. The spermatic cord, which was swollen to the thickness of the thumb, was surrounded by pus. At the height of the under half of the kidney were found pus-infiltrated lymph-glands. In a third preparation of acute orchitis after clap, in which castration was performed three weeks after the beginning of the inflammation, the epididymis showed abscess pockets with raggedly irregular walls throughout its substance. The fourth was taken from a man whose testicle was inflamed by a kick from a horse, and whom it was necessary to castrate. In summing up he says: "The tumor was larger than a goose-egg, and retained exactly the form of a normal testicle. Upon section the tissue sprang into the cut quickly, as if relieved from a strong compression. According to the degree of inflammation in the whole and in places the gland substance appeared hyperæmic, strongly reddened or yellowish in color; especially under the tunica albuginea and along the septa testis were the yellow lines found the soonest." By the formation of circumscribed abscesses they appear lying usually under the tunica albuginea as cavities filled with fluid pus. The albuginea appears somewhat thickened, and the sepa between the lobules of the glandular substance broadened and quite considerably separated. The connective tissue between the tubules is infiltrated with numerous granulated lymphoid cells; its basis substance is granular instead of fibrous as in the normal. In the 
same there appear here and there vessels filled with blood. The wall of the seminal tubules shares in the infiltration with migratory cells; it is less striped, and its outer contour appears less distinctly normal than usual. The contents of the tubules stand out sharply in their dark shading. The seminal cells are no longer distinctly circumscribed; instead, the lumen appears here and there filled only with a strongly granular mass, which, moreover, contains fat-drops, and at a later stage fat-crystals. In the somewhat more advanced parts the cellular infiltration in the interstitial tissue also appears to have undergone in part granular degeneration. There are also seen here large and small fat-drops and separated cholesterine-crystals; through this the color is intensely yellow. Besides the lymphoid cells one also sees those heaps of larger cells with distinct nuclei found in the normal testicle as collections of larger cells arranged in spindle-form. The same appear often as large, homogeneous masses in the form of a cylinder, in a row one upon the other, as when they are stuck in a vessel. In still older places the cells disappear from the interstitial tissue, and this appears a thickened, fibrous-grown connective tissue with numerous strongly prominent nuclei. The wall of the seminal canals shows the same change. The contents of the latter have in part disappeared-in part still exist ; only, however, as a substance consisting of fat-drops and granular matter. In the spermatic cord the vas deferens shows itself unchanged; the infiltration is a phlegmonous one, and belongs chiefly to the connective tissue between the vessels. The same is found in spermatitis after epididymitis. When the epididymis is implicated, there is found the same lymphoid, interstitial cell infiltration as in the testis, with fatty degeneration of the epithelia of the canals; or the migratory cells flow together into abscesses, and press aside the tissue, with partial degeneration of the same. The layers of the tunica are normal, often adherent, in form of an adhesive periorchitis. The foregoing description leaves it to be recapitulated that in acute orchitis it is essentially with an interstitial inflammation we have to deal, just as in epididymitis, with a lymphoid infiltration of the connective-tissue framework, and also of the wall of the testicular canal.

Kocher also calls attention to the rapidity with which the seminal cells undergo destruction throngh granular infiltration and fatty degeneration. The contents of the testicular canal become resorbed, and the walls contract with the interstitial tissue. The return to normal results through the disappearance of the lymphoid cells by way of the lymphatics, perhaps also through the veins, and in part through fatty degeneration. Only when the removal of the infiltration is rapid is a restitutio ad integrum beyond doubt.

Treatment.-Rest in the supine position, with the testicle elevated upon the abdomen and supported there, should be insisted upon. A hot 
poultice of flaxseed-meal applied and changed every two to three hours has, in connection with the hypodermic injection of a half-grain of morphine, followed by a dose internally of an eighth of a grain as often as is necessary to keep the pain down, given me the best results. If the case is seen early, bleeding at some of the scrotal veins, or leeches, ten to fifteen, applied in the neighborhood of the abdominal ring, is still recommended (Bumstead and Taylor, Keyes).

Crushed ice in rubber bags may be applied with a hope of cutting short the process if the case is seen at the very commencement of the inflammation (Curling), but there is nothing to commend this means if the disease has progressed to any extent, and there is always the possibility of its playing a part in the causation of gangrene by its devitalizing power, which will render the employment of it questionable after the inflammatory effusion has taken place. Narcotics in the form of ointments and lotions may be applied, and the efficacy rendered more potent by using them in connection with the hot flaxseed-meal poultice. The bowels should be kept opened, preferably by salines. If, despite this treatment, the pain continues severe, puncture of the tunica albuginea is advised by some. This method of relieving the tension of the inflamed secreting part of the testicle was first employed by Vidal, it being suggested to him by the success of Velpeau, who employed incisions of the tunica vaginalis in cases of epididymitis. Vidal's assertion that he employed it with successful results, and with no case of prolapsus of the testicular substance in four hundred cases, has been severely criticised, and by no one more strongly than by Gosselin, his own countryman, who claims that his cases were not orchitis but epididymitis. However this may be, the principle is a good one, and the method, modified, from one incision one and a half centimetre long, to several punctures through the tunica albuginea, is employed with gratifying results (Keyes, Ashhurst, Wyeth). By relieving the tension the pain ceases in a half-hour or so, and by the continuation of the poultice of hot flaxseed-meal and eleration resolution will usually follow without interruption. Abscess should be opened as soon as fluctuation is detected; antiseptic dressings, and, after the slough has been removed, stimulating applications to hasten granulation and healing in gangrene. If sinuses or fistulæ remain after abscess, and they do not heal through the application of stimulating lotion or injection, they should be slit up, curetted, and packed with iodoform gauze. The general health of the patient should be looked after in these cases of tardy healing. A well-fitting and comfortable suspensory should be fitted on the part by the surgeon as soon as the patient is allowed to be upon his feet. 


\section{EPIDIDYMITIS.}

Etiology.-By far the most frequently diseased of any part of the testicle is the epididymis. We meet with inflammation of this part as the result of injury, the propagation of disease of the urethral mucous membrane onward along the vas deferens to the epididymis, and by infection through the blood. In short, most of those factors given in the etiology of acute orchitis can also give rise to epididymitis. It begins ordinarily as an acute, painful affection running a comparatively rapid course, to end in a few days in recovery, or it may be subacute from the commencement, accompanied by little pain, dragging slowly over a long interval of time, to heal finally, or lapse into the chronic stage. We have in the gonorrhœal inflammation of the urethra the commonest cause of epididymitis. Even after this causative affection has run its acute course, and has become chronic, epididymitis may arise from it, although there may have been at the time no appreciable exacerbation of the urethral trouble; straining at lifting, excess in drinking, or coitus, are very apt to bring it on, especially if there has been at any time prior an attack. It is to be noted that an inflammation of the epididymis from a chronic urethral affection, be it stricture or long-continued clap, is as often subacute as acute in its behavior, and more apt to involve both testicles. Upon the question as to the frequency with which epididymitis complicates gonorrhœa, there is some difference of opinion between observers, and it is a difficult question to settle definitely. In my private practice it is of relative infrequency, but has happened to $12 \cdot 2$ per cent of my out-clinic gonorrhœal patients. The difference is undoubtedly due to the fact that a larger number of the latter class go without treatment for a longer time, and, besides this, continue their irregular habits, such as drinking and having indiscriminate sexual intercourse. Many of these men have the hardihood to continue at their occupation after epididymitis has attacked them, despite the suffering caused by it. I have seen linemen keep at their work, the latter necessitating climbing and clinging with their legs to telegraph poles, throughout the whole course of the disease, the acute stationary stage of which was thus prolonged in most instances into many weeks' duration. A great many of such cases seek the physician only because of the epididymitis, either having paid no attention whatever to the prime cause (the clap), or treated themselves with some uncertain mixture locally, or internally, or both.

Predisposition in some individuals seems to play a part in the frequency, for there are some who seldom escape it whenever they have a fresh gonorrhœa, or an exacerbation of an old one, no matter what precautions they may have taken against it; while, on the other hand, there are those that may commit the grossest indulgences and expose themselves in 
every way, and yet never be attacked. Vidal ascribed to atmospheric change an influence in the production of an epididymitis, and thought that a sudden transition from mild to cold weather causes the testicle to take on inflammation more readily while the patient is suffering from gonorrhœa.

In looking over the record of cases from my clinic I find that there are periods when cases present themselves in groups of greater numbers, but that the same thing happens in respect to chancroids, gonorrhœeas, and syphilitic outbreaks, irrespective of the weather or time of the year. Epididymitis seldom occurs uncomplicated, especially that due to gonorrhœa, in which there is usually an involvement of the tunica vaginalis, with effusion into the cavity, and frequently an inflamed vas deferens.

It occurs at all ages, but oftenest at that period in life when the organ is at its strongest functioning capability. The reason for this increased frequency is obvious, for it is at this time that individuals are almost constantly exposing themselves to conditions most favorable to the origin of the disease.

Rollet (1862) reported 678 cases of epididymitis in 2,425 cases of clap, $27 \frac{9}{10}$ per cent; Jullien (1S86) observed it 381 times in 2,500 cases, $15 \frac{2}{10}$ per cent; Tarnowsky (1872), out of 5,203 cases of gonorrhcea, saw epididymitis 637 times, about 12 per cent; and Finger, during five years' service in a hospital, saw epididymitis 548 times in 1,844 cases of urethritis, 299 per cent. As the latter observer says, all these being taken from hospital material, the percentage is much higher than will be found in all the cases of urethritis, for the reason that the greater number of those suffering from simple urethritis do not go to a hospital. Berg (1882), in his statistics taken from his private practice, gives a percentage of $7 \frac{5}{10}$.

In the greatest number of cases epididymitis attacks but one testicle, and either with equal frequency (Eichorst, Finger, Lesser). It is not uncommon for the sound testis to become affected after the subsidence of the side first attacked, but this is found to occur more often in chronic affections of the urethra, and in syphilis, when the epididymitis is usually subacute from the beginning. Very seldom in acute gonorrhœa are both sides affected simultaneously. Gonorrhœal epididymitis is dependent upon the direct continuation of the urethral inflammation backward to the prostatic urethra, and thence along the vas deferens to the epididymal canals. The usual time for its beginning is in the fourth week of the blenorrhagia, althongh it does commence as early as the end of the second or beginning of the third. Finger has given a collection of statistics, gathered from the reports of numerous observers, which gives the earliest time of attack as the first week and the latest as the seventh year. Cases have been reported as beginning earlier, even before the discharge (Sturgis, Berg); but, as Dr. Finger truly says, there 
is strong probability that there existed a deep-seated urethritis, or a stricture, the result of a former clap. Dr. Keyes believes these cases to have been bastard clap. Out of 1,015 cases occurring at times ranging from the first week to the seventh year, given in this collection, the greatest number, 191, happened in the fourth week. Dr. Keyes generalizes the time by putting it anywhere from the third to the eighth week. The proper treatment of gonorrhœa plays some part in the prophylaxis of epididymitis. Finger gives the following interesting report upon this subject from Lefort:

Epididymitis in cases of gonorrhœa in which there was no treatment, 264 ; treatment with balsam only, 73 ; treatment with injection only, 82 ; treatment with balsam and injection, 60 ; treatment unknown, 97 .

Here of course, as Dr. Finger remarks, the want of treatment is not to be considered as exclusively the cause of the increase in number on the side of epididymitis, for it is this class of careless, neglectful patients that adds to the negative evil of nontreatment, those positive ones of excessive indulgence in drink, in venery, and overstrain at work.

Symptoms. - In the acute form of inflammation of the epididymis there is in the beginning a dull, aching sensation, referable to the testicle itself as if it were too heavy, and which may extend up the cord and into the small of the back. Curling states that the epididymitis is usually preceded by uneasiness in the course of the vas deferens, occasionally accompanied by irritation about the neck of the bladder, causing frequency of urination, which is followed shortly by a dull, aching pain, and slight fullness in the groin. Kocher says that pain in the groin is a constant initial symptom, and that it does not belong to gonorrhœal epididymitis alone, for he has repeatedly observed it in the traumatic form.

Keyes also says that it is rarely absent, especially in inflammation of urethral origin, and that it usually precedes by some hours the pain in the testicle. In my practice, both private and hospital patients refer their first sensation to the testicle itself in the majority of cases, and I have not been able to demonstrate the "doleur susinguinale" of Fournier as a constant initial symptom.

Finger ranks the inguinal pain as among the more infrequent prodromal symptoms. Instead of the uneasy, heavy feeling in the testicle, sudden acute pain may be experienced as the first sign of anything wrong with the part, but this, in my experience, is not so common a beginning as the one above described. Accompanying the uneasy sensation in the testicle there may be a feeling of general malaise, chilliness, loss of appetite, frequent and painful urination, together with pain in the groin and small of the back. The discharge from the urethra usually diminishes very perceptibly several hours before the symptoms of epididymitis appear, 
and may cease altogether, to return upon the subsidence of the acute symptoms of the epididymitis.

Sometimes the urethra gets well during the epididymal trouble, so that by the time the patient is able to be about on his feet he is cured of his gonorrhœa. However, this is a rare and fortunate termination of the urethral disease. Dr. D. D., a dentist, suffering with gonorrhœa, had his epididymis inflame in the third week of the urethral trouble. The discharge ceased upon the sudden appearance of pain in the testis. The patient was confined in bed seven days before I allowed him to get up. The discharge did not reappear at any time during the attack, although the symptoms rapidly subsided from the time of his going to bed; nor was any sign of the urethral trouble discernible thereafter, even the urine being clear of shreds.

If there is much fever, and a tendency to vomit and collapse in the beginning, before the local symptoms become noticeable, it is usually consequent upon considerable inflammation of the vas deferens (Kocher).

In uncomplicated epididymitis the fever comes only with the objective symptoms as a rule, and lasts but a day or two, attaining to three or four degrees above normal, and subsiding, in most eases, almost always before the relief of pain. Soon after the initial symptoms declare themselves are the objective symptoms demonstrable, and there appears the exquisite sensitiveness to the touch of a part of the epididymis. Upon palpation there is found at some part of the epididymis-the globus major the most frequently, according to the observation of Finger, the globus minor according to Curling, Kocher, Bumstead, Taylor, and other's - a small, hard lump, which, increasing as the inflammation advances, soon involves the whole of the epididymis, which may become many times the size of the normal.

The skin of the scrotum upon the side corresponding to the affected epididymis becomes œedematous, hot and red (inflammatory cedema, Eichhorst), and when this œedema is considerable it forms a large part of the tumor. The tunica vaginalis is more or less affected (periorchitis acuta) in acute cases, and there may be considerable effusion into the cavum vaginalis, filling it to its utmost capacity, the increase in bulk smoothing out the folds of the scrotum, thus forming a tense, smooth, oval tumor, in which are masked the constitutent parts of the testicle. The intensely stretched layers of the tunica vaginalis often give rise to excruciating pain, which is not relieved even to a slight degree, by rest on the back, or support of the part, but is subdued immediately upon incising and emptying the sac. Usually there is but a moderate effusion of fluid, and sometimes none at all, the walls of the tunica throwing out a plastic material by which the surfaces are agglutinated (periorchitis 
adhesiva), the scrotal and testicular tunics thus becoming adherent the one to the other.

The inflammation may extend into and affect the spermatic cord more or less acutely. When this part is affected severely the subjective symptoms are intensified, sometimes taking on the character of strangulated hernia through its pressure, in its swollen condition, against the unyielding external abdominal ring. Sometimes the cord is shortened by the inflammation, and the testicle is pulled up to the outer ring with such force as to increase and intensify the suffering of the patient. The usual form of the swelling in epididymitis is when the inflammation is accompanied by little or no effusion into the cavum vaginalis-that of an irregularly long, angular tumor, flattened from side to side, and, as a rule, about the size of a man's fist. The enlarged, hardened, and exquisitely tender epididymis may be felt at the back of the tumor encompassing the posterior half of the testicle, the fore part and sides of which are felt in front, when the effusion is not enough to prevent the contact, as a soft, pseudo-fluctuating part having the sensitiveness peculiar to it. Sometimes, though rarely, the inflammation goes over to the testis proper. When such is the case we have an increase in the severity of symptoms by the addition of such manifestations as go with acute orchitis. Except by this increase in the suffering of the patient, both general and local, we have, in the event of considerable effusion and the participation of the scrotal tissues in the inflammation, no certain means of determining whether or not the testicle proper is involved.

The above-described changes may develop suddenly. In from two to five days the inflammation may have reached its height. After a stationary period, proportionate in length of time to the intensity with which the different tissues have become involved, the pain ceases and a return to normal begins. After the disappearance of the inflammatory symptoms the swelling may remain for some time. The œdema leares the scrotum and subscrotal tissues, any effusion of the eavum vaginalis becomes resorbed, the induration of the epididymis gradually softens and disappears, until there is left only a small, hard lump-usually in the tail, sometimes in the head-which upon pressure is still somewhat sensitive, and which usually occupies weeks in becoming resorbed, or which may remain for years, forming a focus from which relapses occur; or it may undergo suppuration, become encysted, undergo cheesy degeneration, and thus form a so-called gonorrhœal tubercle (Birch-Hirschfeld). Very rarely in acute epididymitis do abscesses form that break spontaneously, or that must be opened by the surgeon. If this should happen, or there occurs suppurative periorchitis, the pain in the parts does not subside so soon as that of a simple inflammation, but tends to increase, and there appears a secondary fever with slight chill-in short, the fever of 
infection. At the site of the most pain, fluctuation after a while is to be felt, and upon incision pus is evacuated, the pain is relieved, the fever subsides, and the part heals. There are such cases as the latter reported (Kocher, Finger, Albert).

The course of epididymitis may, instead of the one above described, be a violent one from the beginning, every symptom simulating those of fulminating peritonitis. The abdomen appears meteoric and distended, extremely sensitive to every movement, to even the pressure of the bedclothes. The temperature becomes high, ranging from $39.5^{\circ} \mathrm{C}$. to $40^{\circ} \mathrm{C}$. The pains increase from hour to hour, radiate toward the lumbar region and into the groin. There are eructations, hiccoughing, nausea; finally, vomiting of greenish, bitter masses of mucus, and collapse (Finger). On the contrary, cases may take on a subacute course from the first, the patient suffering very little pain, the inflammation being limited exclusively to the epididymis, even to a part of it. This is usually the course of epididymitis when it is due to a chronic urethral trouble, either an old, deep-seated gonorrhcea, stricture, tuberculosis, or syphilis. Relapses are, as a rule, subacute. Varieties in the form of the tumor are caused by the malposition of the testis and epididymis. Hernia and varicocele can complicate an epididymitis, as the inflammation of the latter may communicate itself to whichever of the two former is present, thus rendering a reducible hernia irreducible, or, by spreading upward along the peritonæum, cause a peritonitis. A varicocele may be made worse by the epididymis growing to it (Finger).

Diagnosis.--The pseudo-fluctuation of the testicle should not be mistaken for an effusion into the cavity of the tunica vaginalis (Velpeau, Kocher). The latter is more elastic, more sensitive, accompanied by redness and œdema of the serotum over the fluctuating place, and is usually, though not always, translucent. Furthermore, an effusion into the cavity prevents the outlines of the edges of the infiltrated epididymis being felt so distinctly. The characteristic form of an infiltrated epididymis serves to distinguish it from acute orchitis. There is very seldom an exudative periorchitis present in acute orchitis (Kocher).

Prognosis.-Healing of the parts takes place, but in the majority of cases a perfect restitution to the normal does not occur. There have been instances reported of the disease terminating fatally, but in every instance peritonitis or purulent infection has been the direct cause (Velpeau, Kocher, Keyes, Finger). The indurated lump left after subsidence of the general infiltration in the epididymis may be the exciting eause of tuberculous deposit, especially when the epididymitis is of gonorrhœal origin (Bumstead and Taylor). Cases have been reported of acute miliary tuberculosis of the testicle following upon gonorrhœal epididymitis in patients of robust health (Albert). As regards the functioning capability 
of the organ, the prognosis is bad, especially after gonorrhœal epididymitis. Through inflammation of the walls of seminal canals and the interstitial connective tissue, the former are cemented together and their lumen occluded, thus destroying their power of conducting the semen from the testis proper. Liégeois (Kocher) found after unilateral epididymitis important lessening of the spermatozoa; in twenty-eight cases of double-sided epididymitis, he found complete absence of the spermatozoa in twenty-one. Of the seven cases in which he found the spermatozoa, five were not gonorrhœea, and two were quite light cases in which there was no induration left behind. In eighty-three cases of double-sided gonorrhœal epididymitis, in eight only did the power to excrete spermatozoa return (Kocher). Curling reports cases of obstruction to the seminal canals, in one of which, however, the function returned after two years' treatment. It is fortunate for the integrity of a patient's procreative power that the two testicles are not affected oftener, for it is shown on section that, though the hard lump may disappear and leave the epididymis to the palpating finger apparently normal, yet microscopically the lumen of the canal is found to be destroyed. Even though the patient has suffered from double epididymitis, this does not render him impotent. His sexual power and desire are normal ; he still ejaculates a fluid in all respects similar to that secreted before the inflammation, except that it contains no spermatozoa (Gosselin). He is sterile, but not impotent. In many cases this sterility is removed by absorption of the infiltration left after the inflammation, but it is after a long time. When it is due to destruction of the lumen of the canal of the epididymis, of course the defect is permanent.

Pathological Anatomy.-In a similar manner to that of acute orchitis the epididymis becomes infiltrated, only that here there is more room for an interstitial cell infiltration. In gonorrhœal epididymitis the epithelium of the canals may become crowded with round cells, even to the filling of the lumen. At the same time there may be found a mucoid degeneration and a desquamation of the epithelia instead, so that a perfect picture of catarrhal inflammation is present.

Treatment.-For the acute cases, rest on the back, with the testicles drawn up on to the groin of the affected side, application of hot flaxseed-meal poultices, changed every two to three hours according to the amount of suffering, will give relief from the pain in a few hours, in the majority of cases, and hasten the termination of the disease. Narcotics in the form of lotions or ointments, as suggested in the article on acute orchitis, are valuable adjuncts. Keyes recommends a tobacco poultice from the start, and I have found it of considerable value as a narcotic in cases where the patients are not addicted to the free use of tobacco. When this is the case, and the patient is accustomed to the effect of to- 
bacco, it will be of little use to him in this instance. An opiate in the form of an ointment or a lotion should be used instead, in connection with the poultice-a half-grain dose of morphia hypodermically, followed by doses internally of one eighth of a grain every four to six hours, to keep the pain down in extreme cases, especially if orchitis complicates the epididymitis.

When there is much sudden effusion into the cavity of the tunica vaginalis, an incision into and emptying of the same will relieve the pain immediately when due to the stretching of the tunica (Velpeau). Sometimes, though rarely, an abscess forms rapidly (Kocher) and is the cause of the pain; it should be opened as soon as fluctuation is felt. In four to five days most cases will have run their acute course, and the inflammatory symptoms having disappeared, nothing is left but the swelling, due in part to the cedema and effusion, in part to the cell infiltration of the epididymis. Instead of acute pain, there is only soreness upon touch, as long as the patient is in the reclining position; a few minutes in the erect posture, however, serves to bring it back in a measure; and but a few hours upon the feet, without the testicles being supported, will cause a return of all the inflammatory symptoms. If, now, there is applied a proper support, either through strapping, as minutely described by Dr. Keyes, or by means of the Horand-Langlebert suspensory, which has in my practice superseded all other methods of support by reason of the admirable results obtained since I began its use, the patient will be enabled to go about his work without pain, and with but little danger of the return of the inflammation. Those patients that refuse to go to bed at the beginning of the attack can be made comfortable by means of this appliance, and are thus permitted to go on with their business in comparative comfort in all those cases where the cord or the testis proper is not involved. When there is much effusion into the cavity of the tunica vaginalis, tapping should be resorted to before the bandage is applied. Since I began the use of this suspensory in my clinical practice, very few of those patients go to bed throughout any part of the disease. I have had them come to me suffering all the agonies of acute epididymitis, unable to stand erect, and almost creeping into the clinic-rooms, supporting themselves by a cane, going away in ten minutes erect and free from all suffering, relieved by the application of this suspensory bandage. Finger recommends it highly in his book (the edition of 1888), and also in his last book published in 1891. Dr. George Brewer, of New York city, has reported similar results with the original Langlebert, which is not so simple of application. The constituent parts of the bandage are a thick layer of cotton-wool, with which both testicles are enveloped, a piece of guttapercha tissue or oiled silk on the outside of this, and a suspensory bandage of linen placed over the whole. When this is properly applied the testicle 
is lifted up against the front of the pubis, thus relieving the cord of its weight, and it is held there with a firm and soft compressure. Every day this should be repacked, as the cotton soon gets matted and hard, thus lessening the necessary even pressure. It should be worn day and night.

When the inflammation has been allayed, or rather has run its course, and all danger of return is past, we should endeavor to hasten resorption of the indurations left behind. The application of stimulating ointments or lotions, or of three or four folds of linen wet in warm water and placed next to the skin over the callosity, with the bandage adjusted as above described, is highly useful (Finger). I have used of late, with much satisfaction as to results, a thin layer of absorbent cotton saturated with glycerin, to which, if a stronger counterirritant is desired, there may be added, to the strength of one drachm to the ounce, the tincture of iodine, applied to the scrotum over the affected testicle; on the ontside of this, and covering the healthy testicle as well, a piece of oiled silk or gutta-percha tissue is applied, then a thicker layer of cotton, and over this the bandage is applied. This need not be changed oftener than once in twenty-four hours. The internal and external use of iodine locally in the form of a tincture alone, or of Churchill's solution, while the potassium iodide is given internally, is said by some to assist absorption. My experience with it in nonsyphilitic induration has not led me to regard it as much of an aid. When the epididymitis is due to urethral trouble of a chronic nature, either stricture or deep-seated gonorrhœea treatment of these parts with a view to curing them should be begun; and in my experience the instillation into the deep urethra of the nitrate of silver, first recommended in the treatment of this disease by Prof. W. Boeck (Bumstead and Taylor), gives the best results, though I do not limit the medicament to a minute quantity, injecting instead all the way from one drop to a drachm, commencing with one grain to the ounce and going as high as forty. In the event of a small-calibered stricture of the urethra, dilatation must be accomplished up to the size required for the introduction of the injection. This dilatation may be kept up at proper intervals without regard to the presence of the epididymitis, which is in most cases from this cause subacute. Any local treatment of the urethra during an epididymitis arising from an acute gonorrhoea of only two or three weeks' duration is liable to make matters worse, and in my opinion should not be attempted. It has been my custom during the last four or five years to stop the balsams at the appearance of the epididymal inflammation. I can only say in regard to this custom that in a few instancesnot many, it is true-I have seen relapses upon the resumption of balsam copaiba without there being at the time any other cause obvious. In one of my cases it happened three times in succession. 


\section{CHRONIC ORCHITIS.}

Etiology.-The acute form of orchitis may become chronic by the formation of abscesses or through induration. Rupture of abscesses during the acute stage, with a formation of sinuses and fistulæ, often result in a chronically inflamed testicle, with a tendency from time to time to exacerbations and to increased connective-tissue formation; or the acute form may go over to induration through the continual growth of interstitial connective tissue from the albuginea and septa. Kocher describes an orchitis, chronic from the beginning, which is most insidious, and of which there are two forms, the diffuse and the circumscribed. Rindfleisch calls the former orchitis atheromatosa. In chronic diffuse orchitis the principal change is in the collection of round cells in the interstitial tissues, the chronic course of the process showing constantly an increase in the connective tissue surrounding each collection. In this form of the disease the organ may become quite large, even as large as the head of a newborn child. The tumor is evenly oval, with the epididymis in recent cases drawn out along the posterior border, to become later a part of the tumor without definition. By the increased growth of the connective tissue about these cell collections, and the degeneration of the latter, a cyst is formed. Each eyst may become very large. In the circumscribed form there is the presence of abscess. There may be one or more of these abscesses, and the size varies from a pea to a walnut. These abscess points may be situated peripherally, or confined to the center of a testicle. The epididymis is rarely if ever a sharer in this condition, remaining perfectly normal, or at most simply indurated. In some cases of chronic orchitis there may be considerable hydrocele present, which, according to Kocher, is in most cases dependent upon the primary orchitis, although he saw in one case an orchitis developed first, after treatment of a hydrocele by the repeated injection of a small quantity of alcohol. In four cases out of thirteen reported by him, chronic orchitis was consequent upon repeated attacks of gonorrhœea, or in consequence of severe strain or wounds after an early gonorrhœea had existed, or after chronic nonspacific urethritis. It may arise from stricture of the urethra.

Symptoms and Diagnosis. - The insidious manner in which the disease begins in many cases, and the long duration, characterize chronic orchitis. Diffuse orchitis, as well as the circumscribed form, according to Kocher, develops in just this manner. In both the testicle becomes much enlarged-larger in the diffuse than in the circumscribed form. When there is no interruption through exacerbations the course may be a painless one, the patient complaining only of the discomfort caused by the increased weight of the organ. From this perfectly chronic course there are the most different gradations up to an acute orchitis. Not seldom 
to be observed is the interruption of the chronic course through subacute attacks, while, amid passing pains, a spontaneous rupture of an abscess occurs and a fistula forms, which exists, as a rule, for a long time; and rarely there is formed a fungous testis (Curling). The indurated connective tissue surrounding the abscess is not favorable to the production of healthy granulation tissue.

The rupture of abscesses, with subsequent formation of fistulæ, can repeat itself many times within the course of a month or a year. In this case the tumor may become contracted into a hard, fibrous, usually irregular body. A third category of cases consists of those in which there is pain from the beginning, which, through slight strain, blow, or upon pressure of the testicle, is increased. The pain is usually local, though sometimes referred to the lumbar region, and the testicle is strongly sensitive to pressure. The abscess in this variety is long in rupturing, as the tough capsule prevents the breaking through of the pus. Finally, there are cases described that, between the acute and the chronic forms, hold a middle position. Kocher contends that these should be classed as belonging to the tuberculous testis.

Diagnosis. - The differential diagnosis must establish itself between a chronic plastic periorchitis, tuberculosis of the testicle, syphilis, and slow-growing neoplasms. The confounding of the diffuse form with chronic plastic periorchitis is especially easy. There is in both cases a more or less oval tumor, of elastic consistency and uniform surface, often somewhat flattened from side to side. The growth may be in both a perfectly unbroken and very slow one, drawing itself over many years; or there appear from time to time passing pains and slight swelling. Finally, in both a rupture may occur and a fistula form, in which there may be felt in both, upon the passing of a probe, a thick, tough wall, and even here and there places where there is a calcareous deposit. The following are distinguishing points in the differential diagnosis:

In orchitis, in the earlier part of its course, the epididymis is easily palpated in its entire extent. It occupies a posterior region of the tumor. In periorchitis it disappears very soon, hidden by the folds of the tunica vaginalis. In periorchitis one may distinguish in the wall hard and soft spots, corresponding to increased thickening and chalky deposit-ften, also, hard strips running diagonally over the front layer; and frequently there is at the posterior region of the tumor a place of softer consistence, with somewhat greater sensitiveness on strong pressure. In orchitis the consistence is usually uniform, and if certain places appear more solid, and others fluctuating, by the formation of points of suppuration, still the first are not so singularly hard as the circumscribed irregular plates and knots in the wall; an especially sensitive point of softer consistency is not present at the posterior part. Not too much should be expected 
from puncture; only by the emptying of the changed blood elements are there positive signs of periorchitis. Besides periorchitis, the diseases the most in accord with the slow and unbroken course of chronic orchitis are syphilis of the testicle and certain benign tumors of the organ, such as genuine cystoma testis. In the formation of isolated abscesses, together with aggravations from time to time, with or without rupture and fistulæ, lies the danger of confounding chronic orchitis with tuberculosis of the testicle. The chief distinguishing point here is the constancy with which the epididymis is first attacked. The testicle proper is attacked before the epididymis, as a rule only when the epididymis of the opposite side has been affected, or there is tuberculosis of some other organ.

Prognosis. - The prognosis of chronic orchitis is better than that of the acute form, inasmuch as there exists no danger to life. As regards the preservation of the organ it is worse. Recovery from the inflammation, with preservation of the functional capability, must be regarded as an exception. One issue is that in which, without rupture, a degeneration and resorption of the inflammatory product takes place, the testicle again becoming small, soft, insensitive upon pressure, and a fibrous degeneration with destruction of the seminal canaliculæ ensues. Another issne is that of opening with emptying of the inflammatory product, either artificially or spontaneously, and cicatricial contraction of the organ (Curling). Usually the abscess remains open for a long time, the fistula not closing for years. Still, there may be healing, with partial preservation of the organ. Fungous testis is rare in chronic orchitis.

Treatment.-Internal treatment has little or no effect upon chronic orchitis. The patient's general condition should be regarded, and tonics given if there is debility. Treatment of the urethra, if the cause lies therein, should be begun at once. The employment of lotions and salves to the part is a useless waste of time. The testicle should be supported by'a well-padded suspensory bandage. If by the means above mentioned the patient is not benefited, castration is indicated in the diffuse form. Every effort should be made to save the organ in the chronic circumscribed form. Kocher advises incision and removal of the products of inflammation. However, if after incision the fistulæ do not heal-and this, as a rule, is the case-and the organ is thickened and irregular from connective-tissue growth, the question of castration comes up; and as the organ has become a useless one, there is no reason why the patient should not be given the benefit of the operation, as, after the removal, quick healing of the wound follows, and the patient is freed from a disagreeable condition. When the abscess is of limited extent, Kocher recommends a proceeding by which the chronic condition is changed into an acute one, with redness, increased swelling and sensitiveness, and which when incised is usually followed by rapid healing. This proceeding, as carried 
out by him, is the injection of tincture of iodine by means of a Pravatz syringe into the abscess, repeated until the acute symptoms appear. $\mathrm{He}$ employs this method sometimes in acute orchitis with abscess.

\section{CHRONIC EPIDIDYMITIS.}

According to Kocher, the pathological anatomy of chronic epididymitis is still less supported through indubitable investigation than that of acute epididymitis. In a specimen in my possession, taken from a patient who had suffered for two years from the inconvenience of its bulk more than from any pain, the history is that of a chronic inflammation from the very beginning. Six weeks after a gonorrhoeal infection for the first time his right testicle began swelling, became slightly painful and very hard. He was never laid up with it, nor was it painful for any length of time. After a while he noticed that the swelling was increasing, and after a year consulted a surgeon, who tapped him, drawing off considerable fluid, which reduced the swelling about one half, leaving the tumor still very large. About six weeks after the last tapping the patient presented himself at my office with a tumor as large as a baby's head. After removing the fluid, which amounted to eighteen ounces, I found that nearly half the original bulk still remained, and upon palpation thought that I could detect fluid. The tumor was very elastic, and my impression was that it was the testis proper that was involved, with perhaps cystic degeneration. I advised extirpation, to which the patient consented. Upon section, the tumor was found to be comprised of the epididymis, which completely surrounded the glandular structure, the greatest bulk occupying the anterior aspect. The intertubular tissue of the epididymis had undergone fibrous degeneration, and the epididymal canals had expanded into cyst cavities, ranging from the size of a small pea to that of a walnut, containing a perfectly colorless fluid. The testicular structure had undergone apparently no change as to size, but there was a beginning granular degeneration, together with a fatty transformation in streaks.

In chronic epididymitis the increase in size and induration of the organ is quite constant, brought about, according to Kocher, by the growth and increase of the connective tissue between the coils of the epididymal canals. This author describes this newly formed tissue as having the character of a formed connective tissue with distinct striping, a formation of lines in bundles, with cells and nuclei appearing quite in the background. It has the appearance of a callous scar. Great weight should be laid upon this, as especially contrary to syphilitic induration. Still quoting Kocher, there is to be found in certain cases, besides this whitish, callous, true connective tissue, nothing but the remains of the epididymal canals; the walls of the same are melted into the surround- 
ings, the fatty degenerated epithelia presenting as yellowish granular collections of oblong form. In other cases the callous collections appear of yellow color. The connective tissue is infiltrated, with round cells which undergo granular and fatty degeneration. In the last case, finally, there is found in the callous tissues softening deposits with the usual contents of a chronic abscess. The walls are covered by a so-called pyogenic membrane, or there are fungous granulations upon the inner layer. There is a linitation to the inflammatory deposit, which is characteristic of chronic epididymitis, through newly formed connective tissue, of the character of scar tissue, in which there forms around each individual deposit a sort of capsule, that gradually goes over into the surrounding tissue, or in which the lines of firm connective tissue are interspersed among multiple collections.

Herein lies a difference between tuberculosis and syphilis; also where the same leads to points of suppuration.

Kocher has failed to demonstrate satisfactorily, to himself at least, the pseudo-tußerculosis described by Fournier, coming only after chronic gonorrhœa and presenting a picture almost identical with that of tuberculosis. Dr. Albert, in the last edition of his book on Surgery, 1891, makes no mention of pseudo-tuberculosis, but calls attention to the frequency with which gonorrhœal inflammation of the epididymis is followed by miliary tuberculosis of the organ.

Symptoms. - As a rule, chronic epididymitis follows upon an acute inflammation of this part of the testicle, oftenest after gonorrhoeal epididymitis. Instead of the remains of a former acute process-those hard deposits in the tail or head of the epididymis becoming insensitive upon pressure-they remain tender after weeks and months, and upon extra exertion, such as that of lifting, bowling, riding, or long walks, become painful, and inflammation follows. The pain is not always confined to the testicle, but may be experienced in the groin or even in the lumbar region. Objectively, one finds a knotty, hard thickening in the globus minor or globus major, sometimes in the body itself, at some part or other. The whole of the body of the epididymis may be enlarged, but usually, contrary to the condition in the acute form, it is only a part. These knots are roundish, not so angular and irregular as are those in tuberculosis, and they lie in the otherwise healthy epididymis (Kocher). If the whole epididymis is affected, there arises a tumor analogous to that in the acute form. By this it is distinguished from the irregular form of the tuberculous variety. The nodules in the epididymis are painful upon pressure, often extremely so. The tunica vaginalis is usually a sharer in the inflammation, either in the form of an adhesive periorchitis with a gluing together of the two surfaces, or-and this is most commonly the case-there is a serous effusion into the cavity (periorchitis serosa). The eti- 
ology lies mostly in the exciting cause which operates upon an epididymis already changed by former acute inflammation. Still we may have an epididymitis, chronic from the beginning, arising from chronic gonorrhœal urethritis; also, according to Kocher, from urethritis of nonspecific origin and from stricture. Cases arise for which no cause can be recognized. The course of a chronic epididymitis is a long and tedious one, especially when there is abscess. Owing to the encapsuling of the suppurating point, breaking through of the abscess is hindered, but after weeks, or months it may be, there is a rupture, and a fistula forms which will exist for years.

Treatment.-Support of the parts by means of a well-padded suspensory, and the removal of the exciting cause, if possible, and abstention from coitus, tend to alleviate the suffering and to promote a cure, if the process has not gone too far. Local treatment in the form of salves or lotions does little good in this form of the disease, and the internal administration of drugs still less. If by the above means the condition is not remedied, castration is advisable. Kocher recommends incision and removal of the indurated mass, together with all abscess points present. The incision should be made behind. As he says, this proceeding is especially indicated in partial epididymitis-that is, the presence of isolated abscesses. When the whole of the epididymis is involved, either by the induration or through multiple abscesses, castration is the only remedy.

\section{SYPHILITIC TESTICLE.}

Syphilis of the testicle is divided into two forms : syphilitic epididymitis and syphilitic orchitis, diffuse and gummy.

Syphilitic Epididymitis.-When syphilis attacks the testicle, in the early part of its secondary period, it is usually the epididymis that is affected. Although the fact that the epididymis is alone attacked at times by syphilis was recognized by some of the earlier writers, Dron, 1863 (Gosseling), was the first to write an elaborate report of it, in which he attempts to classify it as a variety of syphilitic inflammation distinct from that attacking the testis proper at a later period. This distinction, it seems, was based upon its precocity, syphilis of the testicle being regarded at that time as one of the later manifestations of the secondary period, or of the earlier part of the tertiary period. It has been demonstrated, however, by clinical experience, that the epididymis may be the seat of syphilis in the tertiary stage, and that, on the other hand, the testis itself becomes affected early in the secondary period (Ricord, Reclus, Gosselin). Dron designated as the time of appearance of syphilitic epididymitis that of the third and a half month after the appearance of the chancre. Fournier, who studied this affection afterward, as also did Balme, placed the time at the seventh month from the date of the chan- 
cre; and Balme reported it as occurring even as early as the second month, and as late as the eighth year. I have seen it as late as the twelfth year of the disease. The part of the epididymis most commonly the seat of the attack is the globus major, though the infiltration may spread thence throughont the body into the globus minor, the whole of the epididymis being involved in the process. This does not occur often, the globus major being usually alone affected throughout the course of the attack, especially if treatment is begun soon after the deposit takes place. The globus minor may be simultaneously attacked (Dron, Fournier, Bumstead, and Taylor), as also the cord (Bumstead and Taylor). The deposit is usually in the form of a round or oval tumor, of smooth surface and firm consistency, the size varying in accordance with the amount of epididymal structure involved. The nature of the process is, it seems to me, the same as that characterizing sarcocele of the testis, and consists apparently of an intertubular deposit of plastic material, showing little tendency to change, either generative or degenerative. The epididymal canals are not obstructed by the deposit, as Dron demonstrated in a case in which both testicles were affected simultaneously. He found spermatozoa in the semen of his patient. There is very little pain connected with an attack, the patient oftentimes being made aware that something is wrong with the part through accidental discovery of a lump there. It may affect both epididymes at the same time. Complete recovery ensues under antisyphilitic treatment.

Syphilitic Orchitis or Sarcocele.-There are phases in the behavior of syphilitic orchitis that necessitate a division into two forms-a diffuse and a circumscribed gummy form.

In the diffuse form there is an infiltration of the stroma of the testicle with small cells or nuclei, which after some time develop into connective tissue, the latter at a still later period undergoing a strong fibrous contraction under the pressure of which the testicular parenchyma is destroyed (Ziegler). These cells appear to spring from the inner layer of the tunica albuginea and connective-tissue framework, the intertubular septa of the gland. The process is an indolent one, accompanied by very little pain either spontaneous or upon pressure. The testicle increases in bulk slowly until sometimes the size of a very large fist is attained, retaining throughout the enlargement, however, its normal shape and smooth contour. The whole, or a part only, of the testis may be involved in the process of fibrous contraction. There is no tendency to suppuration exhibited by this form. The epididymis is often involved, though mostly at a later period. This form is also known under the names of syphilitic sarcocele and syphilitic albuginitis. The time when this affection attacks the testicle usually is that in the interval between the second and fourth years, during which time it attains its maximum 
frequency (Gosselin). Cases have been reported as occurring as early as the third and a half month (Nélaton, Ricord, Réclus, Gosselin), while cases occurring in children affected with congenital syphilis have been reported in which the sarcocele existed at birth (Cornil). It may appear very late. Jonathan Hutchinson reports a case as occurring in a patient twenty years after a chancre; and I have at the present writing a patient in whose testicle both forms are present, and it is now seventeen years since a chancre appeared, followed by a secondary eruption.

The second form, the circumscribed gummy orchitis, is characterized by the deposition in places throughout the testicle of nodules, multiple usually, varying in size from that of a point barely discernible by the naked eye to that of a hen's egg, of a yellowish color and firm consistency, and which after a time usually undergo softening. These nodules consist of a collection of nuclei, fat-drops, and granular matter, through which is interspersed a stroma of fibrous tissue. Kocher holds that it is impossible to distinguish more than a quantitative difference between the diffuse and the circumscribed gummy form. In the gumma, he says, there is an extraordinarily stronger increase of nuclei; through this is caused not only the destruction of the seminal tubules at the places attacked, but of the vessels also, and there appears a granular degeneration of the formed elements. To this difference he ascribes the difference in the course of the two forms, the diffuse organizing itself into firm connective tissue, the circumscribed going over into granular degeneration. In the great majority of cases there are found side by side both forms and the view that the gummata are mostly situated in the callous tissue is, according to Kocher, perfectly right, and it affords above all things a most characteristic picture. While the diffuse growth develops itself into fibrous tissue, there goes on, at those places of more intense collection of cells, destruction of the vessels, and thus is brought about granular degeneration. These gummy nodules may be superficial or deep, and they may soften and rupture externally, though, as in the diffuse forms, they may become stationary, finally undergoing contraction (Finger). When these nodules are superficial the smooth contour of the testicle is broken by them, becoming knotty and irregular according to the number and size of the nodules; by the pressure of one or more upon the investments of the testicle, these latter are softened and agglutinated one by one, the skin becomes livid and thin, and rupture with emptying of detritus, flocculent pus, and gummy fluid follows.

The tunica albuginea, and also the tunica vaginalis propria, are involved in the process of infiltration, undergoing thickening, and later on cicatricial contraction. There is nearly always a serous effusion into the cavity of the tunica vaginalis, and quite a large hydrocele may exist. This hydrocele may be masked by a very much thickened and intensely 
stretched tunica vaginalis communis, it being difficult through this to distinguish any fluctuation.

Symptoms and Diagnosis.-Syphilitic testicle can be confounded with gonorrhœal epididymitis only when it is the epididymis that is affected in the former, and when the latter is chronic. The distinguishing points are the slow and almost painless course of syphilitic epididymitis, the involvement of the globus major usually, whereas in gonorrhœa the globus minor is the seat of the affection, and a history of a sore at some time which was followed by an eruption. In chronic gonor. rhœal epididymitis we have more pain, with a history of discharge and an acute beginning. Syphilitic testis has often been mistaken for tuberculous testis, and patients have suffered needless castration in consequence of the mistake. Tuberculosis, when it attacks the testicle primarily, chooses, as a rule, the globus minor of the epididymis as the seat of its action. This fact serves in a measure to distinguish it from the epididymitis of syphilis.

Syphilitis and tuberculous orchitis are distinguished one from the other more particularly by the clinical behavior of the two respectively. The former runs a course comparatively simple in that from the beginning to the end its manifestations are accompanied by very little pain. One peculiarity in this disease is the almost entire absence of pain upon pressure; even in those cases where there is spontaneous pain, and in those where abscess with rupture and fistula formation have occurred, still there is no pain of any consequence upon pressure. Abscess formation with rupture takes place more slowly. The vas deferens and prostate are free from the disease. Tuberculosis testis, on the contrary, has a more rapid course, leads more frequently to suppuration and caseous degeneration, and is accompanied by more pain. While there is very little spontaneous pain compared with the objective symptoms - that is, the sudden swelling and rapid formation of abscess - the organ is usually more than normally sensitive to pressure. The vas deferens, prostate, and seminal vesicles, are usually more or less affected. There remains to be mentioned the greatest of all the distinguishing points, and that is, the result of treatment. Under proper treatment syphilitic testicle gets well, while treatment internal or otherwise has little or no influence on the course of tuberculosis of the testicle. Malignant new formations, such as sarcoma and carcinoma, grow much more rapidly and irregularly, are painful, localize themselves preferably only in the testis proper, carcinoma leading without softening to rupture and rapid decay.

Prognosis.-The prognosis is good. Even after the affection has existed for some time, enough of the parenchyma usually escapes to preserve the function of the testicle. Gosselin found the spermatozoa in the semen of patients after they had been cured of double syphilitic orchitis. 
There is a tendency to relapse if the treatment is not kept up a sufficient length of time.

Treatment.-By the proper administration of potassium iodide and mercury the worst cases as a rule get well. No matter how little of the testicle is apparently left, and even though it is the seat of numerous softening gummata, with or without rupture, or the seat of a fungus, a comparatively good testicle can be saved. There is never a rational excuse for the use of a knife when there is a question of syphilis, at least until proper antisyphilitic treatment has been persisted in for some time and has failed. Locally, a suspensory bandage for support-and, when there are ulcers, some simple antiseptic and protective dressing -is all that is required.

\section{FUNGUS TESTIS BENIGNUS.}

In 1808 Sir William Lawrence (Curling) wrote an article describing for the first time the true nature of this condition, and after him came Malgaigne (Kocher), and later still, in 1840, Jarjavay (Gosselin), the latter separating the disease into two forms-a superficial and a deep. As the superficial form is not in any way connected with the testicular parenchyma -being, in fact, as Kocher has aptly termed it, a prolapsus testis-it is not considered a true fungus testis, therefore will have no place in this article.

The commonest form of fungus testis benignus is that of a reddish or yellowish swelling, which, covered over by a layer of granulation, grows out from the parenchyma of the testis through an opening in the scrotal tissue, to reach a size varying from that of a hazelnut to a walnut, or even to that of a large-sized hen's egg. The skin of the scrotum itself is contracted, thickened, strongly corrugated, and separated usually from the granulating and glistening tumor by a deep furrow, in which runs an undermined edge of atrophied skin about half a centimetre broad (Kocher), or the ring of skin may be hypertrophied and everted (Curling). In the scrotum are to be found the elements of the spermatic cord at the back of the tumor, and a small, firm mass at the lower end, which corresponds to the epididymis.

It will not be often that both testicles will be seen to be the seat of fungus testis simultaneously. Sir William Lawrence reports two such cases (Gosselin and Walther). Instead of protruding in a mass of considerable size, as above described, the tumor may through decay be reduced in size, barely extending beyond the opening in the scrotal covering, its regular smooth outline destroyed by furrows and deep fissures; or the mass, instead of presenting a reddish or yellowish appearance, may, through bleeding into the granulations, become black and marbled; in other cases, through other changes, whitish and lardaceous (Kocher); and Lawrence (Gosselin and Walther) reports a case which was perfectly smooth, and even with no trace of granulation. Syphilis is considered 
by the majority of authors to be the greatest etiological factor in the production of fungus testis.

Kocher gives, as next in order of importance, tuberculosis of the testis, though Ziegler ranks it before syphilis in this respect. Gosselin doubts the formation of fungus from this cause, and Deville (Gosselin), who has shown that the fungus of tuberculosis is superficial-a hernia testis (prolapsus testis of Mocher), in fact-denies absolutely that the deep fungus testis arises from it. Réclus is inclined to rank it as a cause, though he thinks that there has never as yet been a single case described. Kocher refers to a case observed by Herff as one of deep fungus tuberculosis. I have seen two cases of prolapsus of the seminal tubules in tuberculosis testis, but nothing of the nature of a fungus. Simple inflammation of the testis, acute and chronic, acts as an occasional cause through abscess formation with rupture (Curling, Kocher). The formation of the tumor occurs in this wise: Whenever, at the time of inflammation of the parenchyma of the testis, there is formed an opening through the tunica albuginea, tunica vaginalis, and scrotal tissues, either by a considerable incision through abscess and rupture, or through the softening and breaking down of some diseased process, such as in gummata of syphilis, we have an extrusion of the testicular structure, together with such infiltrate as may have taken place, provided the presence of the latter is sufficient to cause an increase of the intercapsular pressure. By this pressure the contents of the capsule are forced through the opening to the outside, where its surface, covered with a layer of exuberant granulations, grows laterally as well as anteriorly through new tissue formation. The scrotal tissues, tunica vaginalis, and albuginea are retracted. The bulk of the tumor is formed of the tubules, intertubular tissue, and new growth; sometimes the whole of the testicle being upon the outside, the stem and base comprised of the epididymis still within the scrotal walls, it increases in size through new tissue formation, and thus is formed a fungus testis benignus. Sometimes, after the rupture of an abscess formed in acute chronic or simple orchitis, the cavity thus left is filled with exuberant granulations that, continuing in growth, protrude from the opening and form a fungous tumor consisting entirely of granulation. This is not a true fungus testis.

Diagnosis.- The diagnosis of fungus testis benignus is not difficult. Especially characteristic is the circumstance, that on the inner part of the scrotum there is to be found, connected with the tumor, the normal or characteristically indurated epididymis as well as the spermatic cord, and the absence of any other swelling. The fungus is covered with healthy, hæmorrhagic, cheesy, or lardaceous granulations, accordingly as it belongs to an orchitis, tuberculosis, or syphilis. If it consists of simple granulations it is soft, solid, and firm, or cleft in syphilis, firm or interspersed with furrows, fistulæ, and cavities in tuberculosis (Kocher). 
Treatment. - When the fungus is due to syphilis it will heal promptly under the proper treatment of the causative disease, it being rarely, if ever, necessary to interfere locally - an antiseptic application when there is sloughing, or a protective dressing, being about all that is indicated. If the tumor is the result of tuberculosis, and there is involvement to considerable extent, no improvement ensuing from building up the patient's general health by tonic, castration is indicated. Removal of the growth by excision, scraping, and cauterizing the cavity which is left, may be employed as a means of healing the trouble, but there is usually a suppurating sinus left which does not heal. Good results may be expected from this procedure only when the fungus is quite small. In fungus which has arisen upon the bursting of an abscess in simple orchitis, application of a cloth wet in silver-nitrate solution and compression, as recommended by Curling, are of undoubted worth. If by these means the fungus is not healed, destruction of the tumor by means of a curette, followed by the cautery, is the plan that Kocher advocates. The operation devised by Syme (Curling), in 1845, which consists in freeing the skin at the base of the fungus, freshening the edges, and, after making an incision at each side of the flaps, thus allowing them to be moved, uniting them over the tumor, may be employed with success in cases due to simple orchitis.

\section{BIBLIOGRAPHY.}

Vidal (de Casis). Traité des maladies venériennes, Paris, 1855.

Curling. Diseases of the Testicles. Edition of 1878.

Cornil. Symes and White, 1882.

Bumstead and Taylor, 1883.

Jonathan Hutchinson. Syphilis.

Eichhorst. Handbuch der special. Patb. u. Ther., 1887.

English. Eulenburg's Real-Encyclopädie, vol. ix, 1887.

Grünfeld. Eulenburg's Real-Encyclopädie, vol. vii.

Gosselin and Walther. Nouveau dictionnaire de méd. et de chir. Paris, 1885.

Birch-Hirschfeld. Lehrbuch der path. Anat. Leipzig, 1887.

Ernst Ziegler. Lehrbuch der special. path. Anat. Jena, 1890.

Lesser. Geschlechtskrankheiten. Leipzig, 1890.

Kocher. Article on Hoden, Pitha und Billroth. Chirurg. Handbuch.

Monod et Terillon. Traité des maladies de testicule et de ses annexes. Paris, 1889.

Eduard, A. Lehrbuch der Chirurgie und Operations-Lehre. Wien, 1891.

Finger. Die Syphilis und die venerischen Krankheiten. Wien, 1888.

Finger. Die Blennorrhöe der sexual Organe. Leipzig, Wien, 1891.

C. Heath. Chronic Enlargement of Testis. London Lancet, 1891, i, 71-73.

Bell. Ashhurst, International Encyclopædia of Surgery, 1886.

Wyeth. Text-Book on Surgery, 1890.

Keyes. Genito-Urinary Diseases with Syphilis, 1890.

Brewer, George E. Jour. of Cutan. and Gen.-Urin. Dis., Jan., 1892.

Martin, E. Art. on Testicle. Hare's System of Therapeutics, vol. iii, 1892. 


\section{DISEASES OF THE TESTICLE.}

BY JOHN P. BRYSON, M. D.

\section{CYSTOMA TESTIS.}

THE testis is sometimes, though rarely, the seat of a growth composed of tough-walled cysts joined together in a compound mass by their walls as they come in apposition, and by an intermediate, apparently fibrous, tissue. The characteristic formations are the cysts, but these may be associated in various degrees, in different cases, with other elements such as sarcomatous, carcinomatous, and cartilaginous tissues. The cysts vary in size from that of a millet seed to that of a pigeon's egg, and are oval in shape. They are sometimes simple, but often contain intracystic cellular growths covered by cylindrical epithelium with or without cilia. The cystic contents vary, being in some cases clear, in others bloody and glairy, while in others still the contents may be cheesy. The whole mass is oval in shape, elastic to palpation, and fluctuates at those points where the larger cysts are close to the surface. The new growth is separated by a capsule from the normal gland-tissue which is spread out on its surface. The epididymis is gradually attenuated by the growth, and eventually becomes indistinguishable from the capsule, though it remains free from disease. Adhesions between the gland and the enveloping scrotum form as progress is made, and between these some hydrocele fluid may appear.

Investigators are not unanimous as to the site of origin of the tumors, but the weight of opinion seems to be with Mr. Curling, whose careful researches led him to the conclusion that they spring from the rete testis. Both clinical observation and microscopic research have enabled pathologists to distinguish two forms of the disease, viz., the malignant and the nonmalignant, the observations of surgeons who noticed that some cases ran an unsatisfactory course ending in general infection, corresponding with the finding of sarcomatous and carcinomatons elements in the new formations.

The disease properly belongs to middle life. The mode of develop ment is much like that of sarcoma of this organ, the patient usually referring the onset to some slight traumatism. Later on he observes that one testis is larger than is natural, that it is flattened laterally, and is causing a dragging sensation in the cord and even in the loin. The 
tumor is not painful, is smooth on the surface, with here and there a fluctuating point to indicate the presence near the surface of one of the larger cysts. The veins in the cord and scrotum enlarge as the growth slowly progresses. The increase in size is at an even rate, different from cancer, which advances by successive leaps.

Diagnosis. - It is impossible to distinguish, in the earlier stages, between the nonmalignant and the malignant cases, or to say at what period in the progress the change from the former to the latter takes place.

Besides the difference in the modes of development, there are other points that serve to assist in distinguishing cystoma. If one of the fluctuating points be punctured, the fluid contents of the cyst wonld issue and diminish the tumor at that point, while in the case of eancer blood would flow freely without lessening the size of the tumor: Cancer, besides increasing irregularly, is painful. Cystic tumor is not translucent, but is said to be heavier than a hydrocele and not so heavy as a malignant growth. Sometimes hard, cartilaginous nodules may be felt. Hæmatocele develops rapidly after a blow, and testicular sensation remains unchanged. It is often impossible to distinguish between this and cystosarcoma. It may be feasible to evacnate one of the cysts and examine the contents under the microscope, in which event the discovery of tessellated epithelium would render the prognosis more favorable; but the frequent association of carcinoma and malignant sarcoma with cystoma should render the prognosis guarded in any event.

Treatment.-The only treatment to be recommended is early removal; but in those rare cases where the non-maligancy is well demon strated, and where the patient has only one testis, one may follow the advice of Prof. Humphrey, of Cambridge, and carefully dissect out the diseased part only.

\section{DERMOID CYSTS OF THE TESTIS AND SCROTUM.}

Cysts similar to those in other parts of the body, and containing skin, hair, bones, teeth, cartilage, etc., and even the gray matter of the brain, have been found, sometimes in the testis and at others in the sac of the tunica vaginalis as well as in the scrotal walls. Dermoid cysts of the testis may be intimately incorporated with the gland, or they may only push it to one side, causing its atrophy from pressure, or they may prevent its development. The tumor may become connected with the testis during foetal life before its descent into the scrotum, while on the other hand it may develop in the scrotal wall and become subsequently connected with the testicle. The connection of the tumor with the testis before its descent may interfere with, delay, or prevent its transition.

The tumors are congenital, but may be small and attract no attention until the period of puberty, when the increased activity and growth 
generally bring them into prominence. The frequent'traumatisms to which they are subjected cause inflammation, which may result in softening at certain points, and even in the formation of the fistula through which the fotal structures composing them may be discharged.

Diagnosis.-The diagnosis is not, as a rule, difficult, though in some cases it may be impossible until an incision is made. The fact that the tumors are congenital is of the greatest importance. Puncture with a fine needle may discover bone or teeth, or these may be felt with a probe passed through a fistula, and the doubt cleared up.

Treatment. - The only treatment to be recommended is removal of the tumor. This should be done with as little interference with the testicle as possible. It is better to save even a part of the gland. There is no valid reason for removing a testicle because it is in a state of atrophy from pressure. It may develop under improved conditions. In case the tumor is intimately incorporated with the testis, or where the gland has been extensively invaded by repeated inflammations, its removal becomes necessary. In operating, the tumor may be exposed by an incision and dissected ont from its bed without opening its capsule; or this may be split open, the contents removed, and as much of the capsule taken away as possible.

\section{SOLID TUMORS OF THE TESTICLE.}

Enchondroma. - As a distinct disease enchondroma is rare, but cartilaginous growths in connection with other pathological states are tolerably frequent. The development of enchondroma testis is obscure and its growth is slow, requiring from four to six years.

The point of departure for the pathological process may be the interstitial connective tissue or the lymph-spaces of the gland. The rete testis is the most frequent site of the growth, though the epididymis may be primarily invaded. The size, shape, and character of the cartilaginous growths, as well as their appearance vary greatly, from the single round or oval nodule growing in the otherwise healthy gland-substance or epididymis to the most complex and varied forms. Occasionally fatty degeneration occurs in the new growth, followed by a cretaceous deposit in the intercellular substance.

It is often associated with other diseases, especially with the cystic, sarcomatous, and carcinomatous. When associated with fibro-cystic or carcinomatous growths, the tumor may attain a very great size. In medullary cancer the cartilaginous masses may be incorporated with the tissue, or they may be encapsulated. In cystic disease the nodules may be numerous, and scattered irregularly through the growth. Only when associated with other diseases does enchondroma appear to cause the testis to attain a very considerable size. Even when the disease appears in the 
form of a single nodule, some hydrocele fluid may be present which must be withdrawn to facilitate palpation, while, on the other hand, the disease may run its full course without hydropsy.

The tumor is oval in shape, heavy, and often has its surface studded with hard, cartilaginous nodules interspersed with fluctuating spots where softening has taken place. The cord is usually free of disease and the organ devoid of "testicular sensibility." There is absence of pain, the growth causing inconvenience only by its weight.

Diagnosis.-Diagnosis is beset with many difficulties. While one may-perhaps after tapping a hydrocele--detect a single hard and painless nodule, even in the substance of the gland, to determine its character would hardly be possible.

The slow progress of the growth, its weight, the absence of disease in the cord and of "testicular sensibility" in the gland when squeezed, are the chief points for differential diagnosis. The patient frequently refers the beginning to some injury to the organ, and urges the post hoc argument, as in sarcoma, cystoma, etc. Where the tumor continues to grow and to cause distress by dragging on the cord, the diagnosis remaining doubtful, an incision under strict antiseptic precautions, with the understanding that if found dangerously diseased the organ must be removed, would be justifiable. The tendency of these tumors to degenerate into other forms of disease capable of infecting the body-a fact long since called attention to by Rindfleisch and abundantly confirmed by experience-along with the inability of constitutional measures to cope with the disease, renders early removal the only treatment to be advised.

Fibrous Tumors of the Testis.-Diffuse syphilitic orchitis, when untreated, generally results in a fibroid degeneration of the gland which leaves only a lump the size of a small marble lying loosely at the bottom of the scrotal sac. This mass is composed chiefly of fibrous tissue, with here and there some wasted tubuli seminiferi in its meshes. The epididymis is generally included in the atrophy from pressure of the fibrous tissue. Interstitial, fibrous overgrowth is a not infrequent concomitant of various epididymal tumors. A hard fibrous knot at the tail of the epididymis following a gonorrhœal extension and blocking of the duct just where it begins to be a single tube, is quite common. When bilateral, these fibrous tumors are the efficient cause of an incurable impotentia generandi. A few cases are on record where very hard, bulky, and painless fibrous tumors have developed in these glands, the result of which has been enlargement instead of shrinkage; and this form has had no connection with syphilis as a cause. In all cases, however, the fibrous overgrowth seems to spring from the tunica albuginea and press inward at the expense of the secreting structures. This course seems to be pursued whether the organ increases or diminishes in size. In some few 
cases the fibrous growth is quite localized, resulting in the development, from the tunica albuginea, of a smaller fibrous mass imbedded in the secreting substance, and entirely indistinguishable by palpation from an enchondromatous lump.

In pure cases of fibrous tumor the cord and epididymis are left free of disease, and there is no hydrocele. "Testicular sensibility" is present or absent according to the extent of the glandular involvement. The slight tendency to become bilateral suggests a constitutional etiology, and raises the question whether syphilis, in some of its stages, may not have been overlooked in certain reported cases.

Treatment.-In doubtful cases the iodides may be tried in the beginning. Removal of the organ will rarely become necessary, and will generally depend on the size of the growth, the inconveniences it causes, the rate of its progress, and the amount of "testicular sensibility" as indicating the usefulness of the gland as a procreative organ.

Calcareous Masses, composed of phosphate and carbonate of lime and traces of magnesia mingled with animal matter, may form in the testis, epididymis, or cord, generally as a result of previous pathological changes. Fibro-cystic and cartilaginous growths sometimes undergo socalled chalky degenerations, but the great majority of these deposits are due to inflammatory processes in the chronic form. Tuberculosis stands at the head of the diseases producing this form of tumor. They are of stony hardness, variable in size and shape, and often mechanically irritate their surrounding capsules so as to produce inflammation and fistula. They are generally easily recognized, but in case of doubt a probe inserted through the fistula if present, or a needle through the tissues, will reveal their nature.

Treatment. - The treatment will depend upon the size and position of the concretion and the pain or inflammation it is causing. A small cretaceous mass encapsuled in the testis or epididymis, and causing no pain, may be left alone. In case mischief results in the way of abscess, fistula, etc., an incision should be made, the calcareous matter scooped out, and appropriate measures taken with a view of causing the cavity to granulate and fill up from the bottom. The actual cantery, after curetting, will be found to have a stimulating effect on the healing process. Small beadlike masses may form in the vas deferens and block the duct. They sometimes demand excision on account of the pain.

Carcinoma of the Testis occurs most frequently in middle life (thirty-five to forty-five), though cases have been observed in very young children as well as in the aged. The tendency to affect only one of the testes is a marked feature of the disease. Unlike almost all the other growths of the organ, the cancerous tissue is unmixed with other morbid products. It,is most easily confounded, in the earlier stages, with cystic 
sarcoma and syphilis, and is entirely indistinguishable from soft sarcoma, a fact of no great practical importance, since early removal of the diseased organ is demanded in both cases. The growth infects the body by way of the cord and the lymphatic channels passing upward to the groin and abdomen. Butlin places the duration of the tumors at seven to fortytwo months.

Two varieties of cancer of the testicle are distinguishable, scirrhous and encephaloid.

Scirrhous carcinoma of the testicle is very rare. It is of slow growth, and attains only a moderate size. The tumor is of stony hardness, irregular form, knotted, and rarely fungating. Sharp, lancinating pains accompany its advance, running up the cord to the loin. As the tumor develops, adhesions of the tunica vaginalis form, the glands above enlarge and press upon the veins, causing œdema of the leg, ascites, and gastric and intestinal disturbances, which, with the pain, render the patient cachectic.

Medullary, encephaloid, or soft cancer invades the body of the gland, cansing a swelling, which in the early stage disturbs the fibrous tunic so as to render the testicle quite hard, though still lacking the stony feel of scirrhus. Later on it involves the epididymis. The disease advancing rapidly causes thinning of the tunica albuginea in places, changing the heretofore smooth and even outline to a softly nodulated appearance and feel. The most prominent portions fluctuate or become more elastic. Where the tunica albuginea is most thinned the growth may burst through, causing adhesion and finally penetrating the skin, protruding as a sloughing, foul-smelling, and bleeding fungus hæmatodes. There is no pain in the early stages, and testicular sensibility is abolished only as the normal epidydimal structures are replaced by the growth. As the cord is attacked, sharp pains shoot up toward the loin. The tumor is at first globular in shape, with sides flattened by pressure, but later on as the cord is affected it becomes more pyramidal. The distinct pear shape is reached when a hydrocele appears. Irregularity is given by the uneven thinning of the tunica albuginea and the formation of blood-cysts due to the traumatisms to which the large mass is naturally subjected. The tumor grows to a large size in a few months, sometimes equaling that of a fœtal head, the scrotal skin becomes thin and dark-colored, and the veins enlarge. Cancerous masses form in the cord, and general infection goes on. Dyspepsia and diarrhœa appear, and assist in rapidly wasting the patient's energies.

Diagnosis.-The differential diagnosis is not easy in the earlier stages. The disease is most readily confounded with syphilitic orchitis and soft or cystic sarcoma. The fact that the carcinomatous is unmixed with other growths, aids in distinguishing it from cystic disease where 
cartilaginous masses may be present. The chief distinguishing features of soft cancer of the testis are, the rapid enlargement without inflammatory symptoms, the size attained by the growth, the enlargement of the scrotal veins, and the development of cancerous masses in the cord. The limit of size for a syphilitic testis is that of the closed fist, while cancer grows much larger. Cancer is always unilateral, while syphilis is sometimes double. In the early stages doubt may often be removed by a carefully conducted antisyphilitic treatment. It is not advisable to puncture a cancerous tumor unless castration has been decided upon, for the reason that even a needle puncture is apt to cause a bleeding fungus to sprout. In case this is done, however, a cancer would bleed without diminishing in size. Between cystoma, cysto-sarcoma, and carcinoma, differential diagnosis is not of great practical importance, since early removal is equally desirable in all cases.

Treatment.-Early removal is the only treatment to be considered, and the question of castration will be decided by the extent to which the disease has involved the cord and the tissues along the line of general infection. When undertaken in time, excision has been found to give the patient a tolerably good hope. The difficulty of determining in any case the question of secondary infection will render the prognosis necessarily a guarded one in any case.

Sarcoma of the Testis appears under two principal forms, the round and the spindle-celled. There is no great or practical clinical difference between the two. Indeed, it is to the pathological histologists that we chiefly owe the distinction between soft cancer and sarcoma, for neither in their clinical histories, their physical or naked-eye appearances, nor in their malignancy, do they present any striking or easily recognized differences. We may therefore content ourselves here with mentioning those few and slight variations in appearance and clinical evolution which will in certain instances assist in differentiating them. Nor is this differentiation of great practical importance to the surgeon. The gravity of both cancer and sarcoma is such as to demand early removal of the diseased organ; and in other forms of tumor of the testis the known intermingling of sarcomatous elements adds greatly to the urgency of the case as well as to the guardedness of the prognosis. It is not, therefore, so much a question of distinguishing between sarcoma and carcinoma as it is of separating these from those nonmalignant diseases, such as syphilis, which not only show no tendency to infect the body, but are amenable to constitutional treatment.

Diagnosis.-Histologically, carcinoma is simple, while with sarcoma are often associated different abnormal structures mingling in the same tumor. Sarcoma most resembles medullary carcinoma, and this mingling of other structures may serve to give a valuable hint, which will enable 
the surgeon to separate the former both from cancer and syphilis. Cartilage, mucous tissue, and unstriped muscle-cells may mingle with a sarcomatous tumor in such a way as to give it a feel on palpation quite unlike the other affections mentioned. Unlike cancer, which is unilateral, sarcoma, like syphilis, not unfrequently affects both testicles. The roundcelled variety is far more apt to be bilateral than the spindle-celled. In the matter of the ages of patients affected, Butlin noted only slight differences, the round-celled being most frequent before the age of ten months and between fifty and sixty years, while the spindle-celled variety was seen with equal frequency before the age of ten months, but appeared earlier in adults-that is, between thirty-five and forty-five. These differences so frequently overlap in the different forms as to render the observations of little value in given cases. The total duration of the spindlecelled variety was noted, by the same author, to be longer. The spindlecelled tumors seem to show a less active malignancy than the other, being more frequently combined with other morbid growths-cartilage, musclecells, etc.-less liable to affect both testes, and not so prone to involve the lymphatics in anatomical relationship. They also more rarely result in extensive generalization in distant organs. The lungs, among the distant organs, are liable to become involved.

The growth of the tumor without inflammatory symptoms, its shape, the size attained, and the appearance of the scrotal envelope so closely resemble those of carcinoma as to render differentiation difficult in all and impossible in the majority of cases. This resemblance is in all respects so close as to render it unnecessary to repeat here the method of distinguishing between cancer and syphilis.

Prognosis and Treatment.-Early removal of the diseased organ offers the only hope, but the prognosis should be guarded in any event. It is sometimes desirable, for the comfort of the patient and to minimize his sufferings and the task imposed upon his friends, to operate even after the cord and the lumbar glands are involved in the disease.

\section{EXCISION OF THE TESTIS.}

The influence of anæsthesia and antisepsis has been to entirely revolutionize this surgical procedure, enabling us to proceed step by step and at leisure, advantages denied our predecessors. Sir Astley Cooper's brilliant method of removing the gland with a part of the scrotum by one stroke of the catlin went out of vogue when anæsthesia came in, and the same may be said of the method of first tying the cord through a button-hole before exsection was begun. The preparation of the patient is now a matter of the first importance. The skin over the pubes, scrotum, perinæum, and inner aspect of the thighs is washed in soap and water, carefully shaved, and covered for an hour or so before operation with 
towels soaked in a bichloride solution 1 to 2,000 . The patient should be placed on a narrow table of convenient height and anæsthetized. The parts are again washed with bichloride solution, and the parts of the body immediately surrounding the field of operation shut off with the bichloride napkins. It is of importance to cleanse the perinæum and penis carefully, and have the latter kept away from the neighborhood of the wound by an antiseptic towel. The operator then seizes the tumor with his left hand passed beneath it, and puts the scrotum moderately on the stretch. The incision will vary according to the size and nature of the growth. In moderate-sized tumors there is no necessity to begin the incision at the external abdominal ring, but where the cord is also involved this may be advisable; but, begin where it may, it should be carried to the bottom of the sac to secure drainage. My own preference is to begin over the top of the tumor, going through the layers of the scrotum stroke by stroke with a very sharp knife. Thus one may decide whether it is necessary to divide the tunica vaginalis at all or to remove it entirely after separating it from the other coverings. The gland being exposed, it may be turned out, the mesorchium divided with scissors or knife, and then, the finger being thrust up the cord as a director, the incision may be prolonged as far as necessary. The cord, carefully separated from its coverings, may now be examined. It should be left to the last, lest the discovery be made that, owing to an error in diagnosis, the testis may be saved. Removal being decided on, a medium-sized silk ligature should be passed through its center, and the funis divided about a quarter of an inch below it. Thus retraction with bleeding, necessitating the opening of the inguinal canal, may be prevented. The vessels are now to be tied separately, the looped ligature being relaxed so as to favor their detection. The artery of the vas deferens should be sought out and tied whether it bleeds or not, otherwise secondary hæmorrhage may occur some hours after the patient has been put to bed. Some operators divide the cord with clamp and cautery, following the plan of Mr. Henry Smith, of London. The objection to the ligature of the cord en masse is the pain it gives and the difficulty with which the thread comes away.

All bleeding having been stopped, and as much of the scrotal sac having been removed as seems necessary, the ligature that has been passed through the cord and tied in a long loop is now brought out at the upper end of the incision, a decalcified bone drainage-tube (or one of rubber) is placed in the bottom of the sac, extending from the cut end of the cord and out of the bottom of the scrotum; the skin is now bronght accurately together over it by a continuous suture of catgut. Good drainage should be looked after, and care should be taken to prevent any turning out of the dartos between the stitches. These, if not corrected, sprout exuberant granulations and retard healing. The line of the incision may now 
be covered with a thin strip of cotton web in a 1-to-2,000 bichloride solution, the parts enveloped in the same material, and the whole neatly secured by bandages passed round the hips and between the thighs. This bandage adds to the comfort of the patient by permitting him to turn in bed, which he can not do if the sac is supported on a small pillow. In applying the bandage, care should be taken to leave the cotton covering the end of the drainage-tube free and loose, to prevent its being blocked by pressure when the cotton becomes wet. The bandage should be so applied as to permit the bowels to be moved without soiling it. Properly adjusting the pressure, it is possible to prevent much of the swelling of the loose scrotal tissue that takes place when the pillow is used. The dressing may remain for four days, and the anchor loop in the stump of the cord need not be disturbed until the change is made.

When, on account of its being adherent, diseased, or redundant, any part of the scrotum the surgeon desires to remove may be included between two semilunar incisions, which meet above and below. If an inguinal hernia complicates the disease, the opportunity may be taken advantage of to put into practice suitable methods of radical cure.

In case the testis is retained, an incision may be made over its site in the canal or even higher up. In either event it is necessary to open the peritonæum. Since the surgeon can never say whether peritoneal cavity must be invaded, all the steps of the operation should be antiseptically guarded.

\section{IRRITABLE TESTIS.}

Irritable Testis must be distinguished from neuralgia of that organ, a task not difficult to accomplish. The acute, darting, and paroxysmal, or the continuous dull and dragging pains of neuralgia, unrelieved by rest and recumbency, are quite unlike the simple but extreme sensitiveness of irritable testis.

In irritable testis the whole organ, or more frequently a particular spot, is exquisitely sensitive to the slightest touch, even contact of the clothing being in some cases unbearable. The patient may be robust and healthy. The disease occurs at any time between early puberty and the latter part of middle life, when the sexual powers decline. It is not met with in those who have flabby testes and relaxed scrotal sacs any more frequently than in those with tense sacs and plump glands. It is often bilateral, while neuralgia is almost always one-sided. The nervous, the anæmic, the neurasthenic, are not more apt to have it than the ordinarily healthy person. There does not seem to be any relationship of effect to melancholia, dyspepsia, oxaluria, or central disease of the nervous system, neither do disseminated tubercular nodules, nor localized syphilitic lesions, have any recognizable causative connection.

The etiology is in the highest degree obscure. The fact that it is 
often bilateral, that it belongs to the active period of puberty, that it occurs most frequently in cases of over-stimulated and ungratified sexual desire, and in those whose normal sexual habits have been suddenly interrupted, as well as the quite common involvement of only a small portion of the testis, all point to a perversion of nutrition of the peripheral nerves. This may well be so in an organ presenting the physiological peculiarities of the glands of reproduction. There are no observations of the histological changes in the diseased areas reported, and in the pure cases no anatomical alterations are discernible on physical examination.

Treatment.-Sexual hygiene, which means marriage, stands at the head of curative means. Fornication, to which no physician would give his consent, aggravates the disease. In one case that came under my observation the patient had broken off an engagement to marry on account of the disease, and in sheer desperation had flung himself into a life of sexual excess, with the result of greatly increasing his trouble. $\mathrm{He}$ recovered soon after being married to the woman of his choice. When marriage can not be advised, as in the case of persons who are too young, rest in bed, the protection of the organ by suitable apparatus from rough contact, and the application of an evaporating lotion, constitute our most effective means of relief. The following is an excellent lotion: R. Liq. ammon. acetatis., $\xi j$; spts. etheris nit., $\xi$ jss.; aquæ camphoræ, $\xi$ iijss. M. Sig. : Apply locally on thin muslin, kept constantly wetted. The ordinary antineuralgic remedies are not to be relied upon. Sulfonal, phenacetin, and antipyrine should be tried before giving opium or its alkaloids to relieve suffering. 



\section{HYDROCELE AND SPERMATOCELE.}

Bx JOHN A. WYETH, M. D., AND W. W. VAN ARSDALE, M. D.

Definition and Nomenclature. - The term hydrocele is employed to denote an accumulation of fluid in the serous sac of the testicle, the fluid being the natural secretion of serous membranes.

Since the serous sac of the testicle is a part of the pouched duplicature of the peritonæum, known as the vaginal process, not obliterated after the descent of the testicle, the term hydrocele may be applied, in conformity with the above definition, to any part of the process containing an unusual amount of serous fluid.

Generally, however, the term hydrocele, unless specially characterized, is used to designate the accumulation of an abnormal amount of serous fluid in the tunica vaginalis testis alone-the rest of the processus vaginalis being obliterated in the adult, in the normal state of development, in from ten to twenty days after birth.

Synonymous with the word hydrocele, when used in the latter, more special sense, are the terms periorchitis, orchidomeningitis, vaginalitis, which intimate that the condition in question is of inflammatory origin. As we shall show later on that inflammation does not always play a part in bringing about the affection, we use the term hydrocele in this article by preference.

'Hydrocele processus vaginalis peritonei may be variously classified, according to its extent and location. When hydrocele is congenital, the tunica vaginalis frequently communicates with the peritoneal cavity; or, in other words, the vaginal process remains open, leading into the peritoneal cavity. This form has been called hydrocele communicans.

In contradistinction to this variety of hydrocele, the usual form of effusion into the tunica vaginalis has been called incapsulated hydrocele of the testicle.

An accumulation of fluid about the spermatic cord is termed hydrocele funiculi spermatici, or perispermatitis. The effusion may be located in the sheath of the cord not obliterated after the descent of the testicle, in which case it is called incapsulated "hydrocele funiculi saccata." This form may communicate with the peritoneal cavity: hydrocele funiculi communicans. Or the effusion may not be confined to the serous 
sac representing the sheath of the cord, but may be located in the cellular tissue enveloping the cord and its sheath. This form has been termed diffuse hydrocele of the cord; it is exceedingly rare, and is of slight practical interest.

We must here mention two other variations in the location of accumulations about the testicle, for the sake of completeness, although but little practical interest attaches to them. An effusion of fluid may take place between the tunica vaginalis and the tunica albuginea testis. Again, we may have an accumulation of fluid between the fibrous layers of the tunica vaginalis communis, which, when present conjointly with hydrocele of the tunica vaginalis testis, represents one of the two forms of bilocular hydrocele.

The other form of bilocular hydrocele is the combination of hydrocele of the tunica vaginalis and one along the course of the cord, not communicating with the peritoneal cavity, but sometimes extending extra peritonceum toward the abdominal integument.

Both of these forms of bilocular hydrocele may be regarded as a simple hydrocele of the tunica vaginalis with a diverticulum, the direction only of the expansion of the diverticulum differing in the two cases. The two sacs in the bilocular hydrocele generally intercommunicate through a more or less well-defined aperture; so that the fluid may be forced from one sac to the other by pressure.

Multilocular hydrocele is a collection of encysted accumulations of fluid, not intercommunicating, lying most generally at the original site of the vaginal process of the peritonæum.

These various forms of hydrocele admit of combination and complications in great variety-complications with cysts, hydatic or embryonic, in the region of the head of the epididymis, and with hernial sacs, being the most frequent and important.

Clinically we must differentiate between acute and chronic hydrocele; such a distinction being important, not only in regard to the origin and pathology of the affection, but especially of practical value as influencing the treatment.

\section{ACUTE HYDROCELE.}

Pathogenesis.-In by far the greater majority of cases of acute inflammatory affections of the epididymis, and in many cases of those of the testicle, we find a concomitant hydrocele present. This is very frequently overlooked, and the more readily as no very great importance attaches to its presence. Pathologically these acute serous effusions into the tunica vaginalis are comparable to the secondary pleuritic effusions complicating pneumonia.

An acute hydrocele may further result from traumatisms of the scro- 
tum or its contents, or the urethra or bladder. Thus, contusions of the testicle or epididymis, septic infection of wounds of the scrotal coverings, the passing of instruments into the urethra or bladder, frequently produce acute hydrocele. Inflammations of the urethra, the prostate, the bladder, or the inflammation of other serous membranes of the body, also lead to the development of acute hydrocele.

Most frequently this affection is associated with epididymitis; and it may appear in pyæmia, variola, and spontaneously, in epidemics, secondary to epididymitis.

Etiology and Pathological Anatomy.-The acute inflammations of the tunica vaginalis are now generally considered to be most frequently due to organized infectious agents, although it must be conceded that any irritation capable of exciting inflammation, such as chemical agents, heat, etc., may cause acute hydrocele; any agent, in fact, capable of injuring the structure or the functions of the serous sac of the testicle.

These inflammations may vary in character, as in other parts of the body, the "serous" inflammation due to simple hyperæmia of the sac walls and hypersecretion being the most frequently observed; while the "adhesive" variety of inflammation, consisting in the formation of fibrinous masses in the cavity of the tunica vaginalis (periorchitis plastica), and the "suppurative" variety, generally attributed to the invasion of pyogenic micro-organisms, are of much rarer occurrence.

The serous membrane appears red and injected, the microscope showing the capillaries enormously distended and filled with blood. The surface is less smooth and glossy than normal, the epithelium having disappeared, and emigration of leucocytes having commenced. A soft, pinkish, elastic substance is sparsely distributed upon the portions of the surface of the membrane, and frequently forms connective filaments between portions of the serous membrane. Similar flaky masses are also to be found floating in the serous exudate inclosed in the sac.

Thus, hyperæmia and exudation are the two chief symptoms found in the first stages. Later on these inflammatory changes may develop new-formed connective tissue, as in the adhesive form of inflammation, or end in suppuration. At the commencement of the latter form of inflammation the serous surface appears coated with a milky-white membrane at a time when the serous effusion is still quite clear and transparent. At a later stage the fluid becomes turbulent through the entrance into it of pus-cells ; finally, it turns into pus.

Pathology.-The commencement of acute hydrocele is sudden and accompanied with fever, which, however, may be slight or not appreciable. Pain is frequent over the site of the swelling and along the cord. On pressure the pain increases, the caudal region of the epididy- 
mis being the point of greatest sensitiveness. Frequently the scrotal integument is reddened and œdematous.

The character of the swelling is that of chronic hydrocele. The form is oval or pear-shaped, and fluctuations can be detected, but the tenseness of the tumor is much more marked in the acute effiusions. In consequence of the greater pressure within the sac, the oval form of the tumor is apt to appear laterally flattened.

If the inflammation takes on a suppurative character, the clinical symptoms become somewhat changed-continuous fever, rising toward night, and more severe pain are present, and a more marked phlegmonous condition of the skin of the scrotum, ending in the formation of an abscess and perforation.

Course.-If, on the other hand, the inflammation does not go on to suppuration, the affection reaches its height on about the third or fifth day, when the fever abates and the effusion is gradually absorbed. In one or two weeks the patient has generally entirely recovered. Or the hydrocele may become chronic. In the adhesive variety of inflammation thickening of the walls may be noticed, and obliteration of the sac may be effected.

Diagnosis.-The diagnosis of acute hydrocele is not always easy. The most simple cases are those where we have induced acute inflammation in the sac after tapping for chronic hydrocele by injecting irritating substances, such as carbolic acid.

In other cases the differential diagnosis between hæmatocele, epididymitis, orchitis, and hernia must be made. As hæmatocele is frequently caused by traumatisms, this may easily be mistaken for acute hydrocele. The absence of 'fever, a lesser degree of translucency, and aseptic aspiration by means of a hypodermic needle, will determine the diagnosis in important cases, while in those cases where a hernia has suddenly appeared the continuation of the tumor into the inguinal canal and the abdominal cavity will point to hernia. Acute hydrocele is so frequently connected with epididymitis that the presence of the latter should always be suspected and sought for. The diagnosis of epididymitis is made by recognizing the long-shaped, hard, non-fluctuating body of the epididymis, behind the testicle, and continuous with the cord. Pressure of this body causes acute pain.

Orchitis may be distinguished from acute hydrocele by the absence both of fluctuation and of translucency, while the hardness and weight are more marked, and the symptoms of inflammation in the scrotal coverings are absent.

Prognosis. - The prognosis of acute hydrocele is generally favorable. Even when the suppurative form is observed, the proximity of the focus to the common integument is so great that there is little 
danger to the system, even when the abscess is not interfered with, especially if the scrotum is not subjected to pressure from without. In the other forms of the disease the greatest menace consists in the affection becoming chronic; this issue is, however, comparatively rare. Concomitant hydrocele, coexistent with epididymitis and inflammations of other organs, has the same prognosis as the primary disease.

Treatment.-Many cases of acute hydrocele recover without treatment; especially in the epidemic variety of disease, the patients frequently get well without being obliged to neglect their daily vocations. Rest in the house is, however, advisable in the first days during the febrile stage; elevation of the affected parts, application of cold, and judicious compression, are beneficial in less severe cases. General and local depletion, the application of leeches at the external ring of the canal, may be resorted to.

Aspiration of the exudate is best deferred until the febrile reaction is past, and is generally unnecessary. The exhibition of potassium-iodide solutions internally has been recommended.

In two or three days the patient may generally be allowed to go about with a well-fitting suspensory bandage.

In all cases of suppurative inflammation of the sac of the tunica vaginalis early incision with drainage should be insisted upon. This operation should be done under cocaine anæsthesia and with due antieptic precautions. The patient should remain in bed until the wounds is healed, and wear a suspensory bandage for some time afterward.

\section{CHRONIC HYDROCELE.}

When the term hydrocele is used without any additional explanatory epithet, chronic hydrocele is implied. This variety is most common, and very frequent, occurring at all times of life, and on either side with about equal frequency, and often simultaneously on both sides.

Heredity has no influence in the causation of hydrocele proper. Hydrocele was well known to the ancients, and was accurately described by Celsus. Chronic hydrocele generally develops in the course of two or three weeks. It shows no tendency to disappear spontaneously, but increases more or less rapidly and constantly in size.

Etiology.-The etiology of the affection is considered to be a discrepancy between the amount of fluid secreted by the tunica vaginalis and that absorbed. A priori we should expect to find hydrocele present as well in inflammatory conditions when a larger amount of exudation of serum takes place, as also in those cases where the absorbent mechanism is interfered with. This latter alternative is, however, excluded by clinical observation; for in those eases where dropsy of the serous cavities in other parts of the body is observed, as in hydrotherax, hydropericar- 
dium, and ascites, hydrocele is never present, and not even then, when œdema of the scrotal integument is very pronounced.

We must therefore have recourse to the inflammatory theories for an explanation of the etiology of hydrocele. But here we meet with another difficulty, for in many cases we are not only not able to explain the anatomical conditions present in hydrocele on any basis of inflammatory changes, but we frequently have occasion to observe the development of hydrocele without any concomitant symptoms of an inflammatory character.

Virchow, Klebs, and other authors in pathology have made a study of hydrocele, and have failed to find any inflammatory conditions present. The forms of congenital hydrocele exclude the idea of inflammatory changes as etiological factors in the causation of the affection.

There is also a variety of hydrocele known as senile hydrocele, where the disease develops spontaneously late in life, but without any inflammatory conditions whatever.

It is true there do exist cases where the cause of hydrocele may be traced properly to an inflammation-cases where the origin of the disease ean be traced to a blow upon the scrotum, or some other traumatism of the neighboring parts; and in many cases we can trace the development of hydrocele to inflammatory conditions existing in the epididymis, the testicle, the spermatic cord, the urethra, or the bladder. But in all these cases we recognize the conditions mentioned above as causing acute hydrocele; so that we may represent the actual facts more correctly by stating that acute hydrocele may become chronic.

We have mentioned above that in acute hydrocele exudations and fibrinous masses on the surface of the tunica vaginalis were not uncommon. It is not improbable that chronic hydrocele owes it existence to the presence of such proliferations and excrescences of the walls of the sac. Similar conditions are met with in other serous cavities. Moreover, small cysts, the perseverance and enlargement of the embryonic cyst, known as Morgagni's hydatid, are known to be a frequent cause of chronic hydrocele.

If we exclude inflammation as an important factor in the etiology of chronic hydrocele, we must also properly exclude from the nomenclature such terms as " periorchitis," designating an inflammatory condition.

Pathological Anatomy.-In chronic hydrocele the walls of the sac show the most important morbid changes; but they differ considerably in various conditions. In most cases the walls appear considerably thickened, and the more so, as a rule, the older the hydrocele is. The layers of the sac wall consist of sclerotic connective tissue, frequently indurated to the consistency of cartilage. Calcareous formations and even localized osseous deposit may here be found. 
The epithelium is but little impaired, and this condition is the chief characteristic point of interest in 'chronic hydrocele; it appears whitish, but the surface of the membrane retains its gloss.

The fluid contents of the hydrocele next engage our attention. The quantity formed varies with the size of the tumor; as much as twentysix quarts is said to have been evacuated at one time. The average in the cases met with in general practice ranges between eight ounces and a quart.

The color of this fluid is generally a pale straw or amber; but it may vary considerably, and present a greenishbrown or chocolate or opaque appearance. Most frequently it resembles serum, and is clear and transparent. Its consistency may be considerably greater than that of serum, however. Not infrequently it is gelatinous and ropy. If pus is present, in cases of beginning inflammation, the fluid is rendered opaque. Spermatozoa cause an opalescent appearance; blood even in small quantities is easily detected by the color. The fluid shows a neutral or alkaline reaction; the specific gravity generally ranges be-

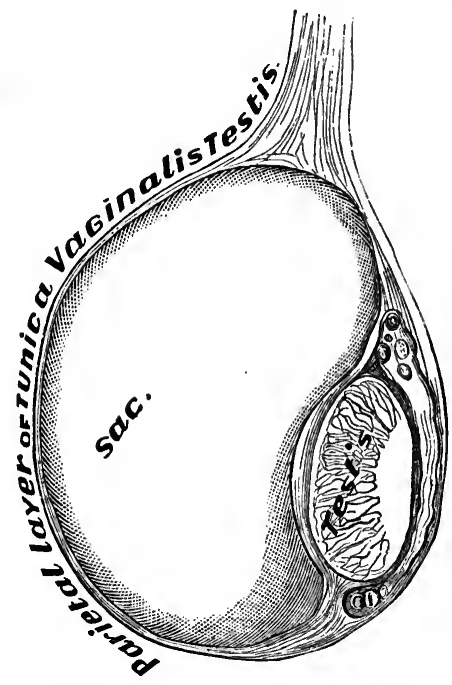

Fig. 1.-Usual form of hydrocele. (After Kocher.) tween 1,020 and 1,026. Chemically it contains large amounts of albumen, very frequently the addition of nitric acid causes the entire mass to coagulate.

The morbid anatomical conditions described thus far under this heading are those of hydrocele proper, or of chronic accumulation of fluid in the tunica raginalis.

Opposed to these conditions are those forms of chronic inflammation of the tunica vaginalis which may properly be termed chronic periorchitis. But we may, with advantage, consider these forms at this point for the sake of brevity, and especially since the clinical symptoms and the treatment of both classes of the chronic forms are practically the same.

We must, however, for the sake of greater clearness briefly refer to some theoretical points in the pathology of the subject. There are authors who insist that all chronic accumulations of fluid in the tunica vaginalis are inflammatory in origin (Rindfleish), and who regard hydrocele as analogous to ependymitis ("hydrops inflammatorius"). These authors have no difficulty in explaining the various forms of chronic 
hydrocele: they regard them simply as variations in degree as well as in quality of the inflammation.

Another class of writers, however, who hold, mainly from the reasons presented above, that hydrocele proper should not be classed among the inflammations, are put to the necessity of drawing a line which should separate chronic liydrocele from the less pronounced cases of periorchitis serosa chronica. There is no doubt but that these two forms gradually pass over into each other, and can not be definitely limited.

The salient point of difficulty in this question lies in the correct appreciation of the difference between hypertrophy and inflammation. Pathologists have ever insisted and agreed that a distinction should be made between these two conditions. But as our knowledge advances, the tendency to regard inflammatory conditions as reparative action in the tissues becomes more pronounced; and from this point of view the differentiation between hydrocle and periorchitis chronica appears tenable.

Periorchitis prolifera differs from hydrocele in the amount of thickening which the walls of the sac undergo. In well-marked cases large masses of newly formed fibrous tissue are found composing the sac walls, while the amount of fluid inclosed in the cavity is small, and the tumor is consequently not generally of large size.

In extreme cases there may be no fluid at all present, and the entire tumor may be due to the enormously increased and thickened tunica; in these cases the tumor resembles a chondroma more than aught else, and may be mistaken for a malignant neoplasm. In less well-marked cases, where there is more accumulation of fluid and less hyperplasia of the walls, the distinction can not be satisfactorily made between hydrocele proper and the inflammatory condition under discussion.

The surface of the tunica in periorchitis prolifera is usually found irregular; excrescences similar to synovial villi are found, which become harder with age and frequently undergo caleareous degeneration. Indeed, free bodies, consisting of sclerosed connective tissue of cartilaginous structure, sometimes ossified in the center, are found in the latter stages. The proliferation of connective tissue may not be restricted to the serous membrane alone, but may take place in the connective-tissue layers externally to it, and may grow about and through the epididymis, strangulating it and causing its atrophy. Indeed, the testicle itself may also be squeezed between two layers of proliferated connective tissue and be destroyed.

In periorchitis adhosiva the epithelium is destroyed, and the internal surface of the tunica vaginalis is covered with an organized exudate originating from the serous membrane, and varying in form and thickness. When both walls of the cavity are attacked, they beeome attached and may grow together. This generally occurs within limited areas, and 
bands and circumscribed adhesions approximate the opposite walls. In certain cases the entire cavity may be obliterated.

Another form of chronic inflammation has been described under the name of

Periorchitis Hcemorrhagica.-In this case the surface of the tunica vaginalis is covered with newly formed membranes of connective tissue which are highly vascularized, and which bleed spontaneously from time to time. The aspect of the effusion into the sac of the hydrocele is affected by these hæmorrhages, the color becoming darker and the consistency greater, and the tension within the sac may be considerable.

Kocher describes this form in connection with periorchitis prolifera.

It is this form of hydrocele which is generally described in the literature as chronic hæmatocele; the term acute hæmatocele being used to designate the hæmorrhage into the tunica by the rupture of a blood-vessel (" hæmatoma tunicæ vaginalis").

Suppurative inflammation is induced when the exudate from the serous membrane, instead of becoming organized, becomes infected by pyogenic micro-organisms and secretes large numbers of pus-cells, or when (according to the more recent theories) the coagulation of the inflammatory exndate is prevented from taking place by the presence of certain chemical agents or ptomaines. The internal aspect of the tunica vaginalis appears ulcerated, and large masses of the newly formed membrane are thrown off and undergo suppuration. The destruction of tissue is checked in certain places by the formation of a granulating surface ("pyogenic membrane"); in other places the inflammation progresses, by invasion of the infectious agents into the tissue, till the abscess is said to point externally, and spontaneous perforation of the skin ensues.

Pathology.-The commencement of chronic hydrocele is insidious and painless ; in those cases only where chronic hydrocele develops from acute hydrocele can it be said to be associated with acute pain; the sensation of weight is the symptom most generally complained of by the patients, and drawing pains in the spermatic cord may occasionally become associated with it, especially at such times when the hydrocele suddenly becomes enlarged. Such exacerbations are more acutely felt when the walls are thickened and present more resistance to the increasing fluid contents of the sac.

The development of hydrocele usually takes place from the lowest point of the scrotum upward. The testicle is generally dislodged toward the posterior aspect, where it may be recognized by means of the spermatic cord leading to it, and distinctly felt.

Symptoms. - When after the course of three or more weeks the hydrocele has reached its full development, it generally presents the following symptoms : 
Situated within the scrotum, generally unilaterally, is found a fluctuating, oval, pyramidal, or pear-shaped tumor, which usually bears a wellmarked and constant topic relation to the testicle. It is most frequently recognized in the front of the testicle and in the upper portion of the scrotum, just below the point where the skin of the scrotum joins that of the penis.

The fluctuation is most pronounced in the early stages of hydrocele; in old cases, where the walls are thick and where there is very great tension, as in cases of rapid development, it may be almost imperceptible. The shape is liable to undergo great changes. When it is pear-shaped the base is situated below and the apex above, pointing toward the inguinal canal. Very frequently, however, an elongated oblong form is found, or a perfectly round one. In cases where adhesions of the sac wall have occurred the shape may be most irregular and complex.

In children, hydrocele more frequently extends along the course of the spermatic cord. The surface of the tumor is smooth; no irregularities or roughness is encountered by passing the finger over it.

The consistency of the tumor is not the same in every part, the region where the testicle is located being uniformly harder to the touch than where the fluid lies nearer to the outer skin. The seat of the testicle, although generally at the posterior aspect of the tumor, as mentioned above, may vary with the following causes :

The developmental anomaly known as inversion of the testicle causes it to lie in the anterior region of the hydrocele. In this case the examination of the testicle on the sound side will indicate the anomaly and point out where to look for the testicle in the hydrocele.

Again, wherever adhesions have formed within the sac of the tunica vaginalis the testicle may be dislodged from its proper position and drawn to any portion of the tumor. In certain cases the epididymis with the vas deferens may be separated to a greater or less degree from the testicle, especially when the hydrocele is complicated with diffuse hydrocele of the spermatic cord, and give rise to errors in diagnosis, unless this condition is properly appreciated. In determining the position of the testicle in the hydrocele (which is important from a therapeutical point of view), it is advisable not to rely solely upon the sense of touch, but also to make use of the translucency test.

The testicle itself is generally in a sound condition. In the case of chronic inflammatory hydrocele mentioned above as periorchitis prolifera, the testicle may be compressed, anæmic, or atrophied; most generally the tail of the epididymis is affected, and the vas deferens may be compressed.

The most important symptom characteristic of hydrocele, and the one which enables us to differentiate it from other conditions simulating hydrocele, is its limitation in the inguinal canal or below it. 
The tumor is sharply defined above; so that the spermatic cord can be readily felt above the tumor, near the external ring, and moved laterally between the thumb and forefinger.

The weight of the scrotum in hydrocele is heavy to the feel, but not so much so as in some cases of orchitis.

Change of position of the patient, from the erect to the recumbent does not influence the usual form of hydrocele in any way. Only in case of communicating hydrocele, or when a branch of the sac extends far up into the inguinal canal, does posture affect the aspect of the tumor.

The general integument covering the hydrocele is attenuated, especially in large hydroceles. The veins frequently appear dilated and darkcolored. The skin is often drawn away from the surrounding parts by the growth of the tumor; and as a consequence of this the penis may appear shorter, or sometimes disappear from view altogether. The cremaster muscle is often found hypertrophied in cases of long standing. Edema of the skin of the scrotum is never present in uncomplicated cases of hydrocele. In cases of extreme tension of the skin in elderly patients, gangrene may occur.

Finally, the translucency of the tumor must be mentioned, a test which is not always reliable, but of considerable differential diagnostic importance. The test of transparency is best made by means of a threefourths-inch tube, or the tube of a straight stethoscope. The patient should be so placed that the scrotal tumor is situated between the observer and the light. Either the prone or standing posture may be employed, and a lighted candle does good service for illumination.

We here add the symptomatology of the other various forms of chronic hydrocele mentioned above as inflammatory in character, and more especially that of periorchitis prolifera.

In periorchitis prolifera the thickening of the walls may be so marked that no fluid can be felt through them, and consequently all the symptoms of a solid tumor may be present. In any case, however, the tumor is sharply defined above. Fluctuation may therefore be absent, or scarcely recognizable. The shape is oval or pyramidal, pear-like ; in wellmarked cases, irregular; the superficies being uneven or nodular. The irregularities may be so pronounced as to suggest the use of the synonymous term periorchitis deformans.

The consistency and weight of the tumor are considerable, and more pronounced in the older cases. The testicle may not be recognizable by the touch, being imbedded in the hard, fibrous tissue or destroyed by pressure. On the other hand, we may recognize one or more floating bodies within the tumor.

The skin of the scrotum is not altered; in exceptional cases, where the tumor has reached a large size, it appears stretched and attenuated. 
The tumor is not translucent.

When hydrocele goes on to suppuration from any cause, we encounter the usual symptoms of acute inflammation, pain, fever, redness and swelling of the skin, and local heat. The pain is auginented by pressure, and extends along the spermatic cord to the back. Fluctuation is well marked, the general consistency of the tumor varies in places, and is soft where the abscess points.

The tumor loses its translucency in the later stages, and the contents of the sac are puriform.

The general clinical picture of hydrocele is that of a benign tumor. The patients are usually in robust health; they have no pain, and are not prevented from attending to their daily vocations, unless it be by a sense of weight. This causes them, in well-developed cases, to move about more slowly.

The course of the disease is a chronic one, and unless surgical interference is employed the size of the tumor steadily but slowly increases, until some occasional violence causes its rupture. In certain cases the hydrocele may remain stationary.

Acute inflammation may attack hydroceles, induced either by external traumatisms or as an exacerbation of the initial causative process. Such intercurrent inflammatory crises cause enlargement of the tumor and thickening of the walls. Indeed, the growth of all hydroceles has been attributed entirely to these exacerbations by some authors. Very rarely does it happen that hydroceles go on to spontaneous suppuration, this course being limited to infection from neighboring wounds or that of the entire system with pyogenic material. Suppuration generally leads to the destruction of the sac, and frequently to that of testicle as well. Spontaneous recovery, on the other hand, is more frequent, the fluid being absorbed from unknown causes. The hydrocele may recur, however.

Rupture of the sac has been observed, after traumatisms, with effusion of the fluid into the cellular tissue surrounding the sac, and consequent absorption, and this accident is said to happen frequently to riders on horseback.

The occurrence of an acute epididymitis has also been observed to cause a hydrocele to disappear. And, lastly, confinement in bed and rest has in some instances had the same result.

Diagnosis. - The diagnosis of hydrocele can generally be made by palpation alone. We begin by examining the testicle on the sound side, in order to facilitate comparison with the affected side. The next step is to get satisfactory evidence of the limitation of the tumor in the scrotum. This is best achieved by grasping the spermatic cord between the thumb and forefinger at the point where it passes out from the tumor, and before it enters the inguinal canal. By means of this we can exclude herniæ and 
epididymitis; for in hernia, even when it is strangulated, the tumor is felt to be continuous into the abdominal cavity, while in epididymitis the cord is most generally swollen.

If we are able to prove the limitation of the tumor in the scrotum, the next step is to endeavor to establish fluctuation. If we find that the tumor fluctuates, the diagnosis lies between hydrocele, hæmatocele, and abscess.

Freedom from pain and absence of participation of the skin enable us to rule out abscess, and the test of translucency decides between hæmatocele and hydrocele. The only error in diagnosis now possible is the mistaking of some cystic tumor, other than hydrocele, for an effusion into the tunica vaginalis. In such rare cases the location of the testicle, as well as the history, must decide.

In case no fluctuation is established, we must bear in mind orchitis, solid tumors of the testicle, and those forms of periorchitis prolifera which possess very thick walls and little fluid under considerable tension.

Fever and rapid development point to orchitis, while tumors are readily recognized by their development from below upward-hydrocele developing in the opposite direction. Malignant tumors impinge upon the tissues of the skin of the scrotum at an early date.

Aseptic aspiration of the tumor with a hypodermic needle will decide the question in cases of difficult diagnosis. The history of syphilis in a patient with scrotal tumor may point to chronic orchitis as well as to periorchitis prolifera, while tuberculous diathesis is more indicative of epididymitis.

Thus far we have assumed the tumor to be well defined above, and not to extend toward the inguinal canal. If the tumor is found to extend into or through the canal, it becomes necessary to differentiate it from hernia, varicocele, and the various other forms of hydrocele. The reader is referred to page 288 for more detailed differentiation in these cases.

Prognosis. - The prognosis is favorable in hydrocele, both on account of the benign nature of the effusion and the relative freedom from danger of the surgical methods generally employed for its cure.

In cases where surgical interference is declined, the prognosis should be giren in accordance with the remarks made above concerning the course of the disease.

Special forms of chronic hydrocele, however, may alter the prognosis considerably. Periorchitis prolifera is apt to cause atrophy of the testicle from pressure, and can not be cured without graver surgical interference than that which is called for in hydrocele. Periorchitis adhesiva, on the other hand, is liable to result in spontaneous cure.

The prognosis of suppurative inflammation in the tunica vaginalis is identical with that of abscess in synovial membranes; if early incision and drainage are practiced, the outlook is favorable for a permanent cure. 
Treatment.-The treatment of hydrocele proper is based upon the recognition of two principles: firstly, the regulation of the existing balance between secretion and absorption in the sac of the tunica vaginalis; and, secondly, accomplishment of the adhesion of the walls of the sac by some surgical interference, resulting in its total or partial eradication.

The first of these methods was formerly more extensively practiced, and consisted in the application of collodion, tincture of iodine, or vesication externally to the scrotum, the use of leeches, of electricity, and the exhibition of digitalis or iodide of potassium internally. But, owing to the unsatisfactory results of these endeavors, surgical interference is the only principle which merits our attention.

The oldest method is that of puncturing the sac with the lancet, and should be unqualifiedly condemned. Any method which permits the fluid in the sac to escape into the loose cellular tissue underlying the skin of the scrotum is fraught with danger, owing to the tendency of the scrotal integument, when invaded by a traumatic œdema of this description, to become gangrenous. If not done antiseptically, moreover, this procedure most frequently leads to suppuration inside of the cavity, and may end in the destruction of the testicle.

The same objections hold good against the subcutaneous discision of the tunica vaginalis by means of a tenotome.

Acupuncture, electropuncture, and various other methods which flourished for a time, require no detailed mention here, as we have better and surer means at hand.

Simple puncture of the sac by means of a trocar under antiseptic precautions is only a palliative operation, and but rarely leads to a cure. It is indicated as a substitute for the operation to be immediately described in those cases only where the patient is in ill-health or infirm, and momentary relief is demanded.

Tapping, with consequent injection of some medicinal fluid into the sac, is an old method for curing hydrocele, and the most serviceable one for general use when carried out with all the modern improvements.

The fluids that have been used for such injections are many. Celsus is said to have used nitre, and others at different times have used sublimate, alcohol, wine, tincture of iodine, solutions of potassium iodide, of alum, of the sulphates of zinc, and of copper, of nitrate of silver, chloroform, ergotin, cold water, milk, hydrocele fluid, and many others, and even atmospheric air has been forced into the cavity. None of these have assured final success. Until comparatively recently the most favored of them has been iodine tincture, but on account of the violent and uncontrollable inflammatory reaction following the use of this drug, and the possibility of absorption to a dangerous degree, it has been largely superseded by pure carbolic acid. This method is known in this country as 
Levis's method. This being at the same time the safest and most effective method, and the only one which can be unqualifiedly and unconditionally recommended, we give it, as follows :

The skin of the scrotum is first shaved on the anterior aspect, and cleansed with soap and water, alcohol, and some antiseptic solution; about five minims of a four-per-cent cocaine solution are now injected immediately beneath the epidermis, so that the point at which puncture is to be made is rendered anæsthetic. The patient being placed in the recumbent position, with the thighs somewhat separated, the operator grasps the tumor from its posterior aspect, with his left hand rendering the skin of the anterior aspect tense, and forcing the liquid as much as possible toward the cocainized point. A medium-sized disinfected trocar, with the cannula in place, is now grasped firmly with the right hand, the thumb being placed on the cannula, to within an inch of the point of the instrument. This serves to prevent the trocar from entering the tumor too deeply. The cannula is now firmly introduced with a slight rotary motion through the anæsthetized zone, well within the cavity. As it enters the sac it is well to depress the handle of the instrument slightly, so as to direct the point away from the testicle and epididymis. Care must be taken not to withdraw the cannula again after the sac is once punctured, otherwise the fluid forces its way into the loose areolar tissue surrounding the tunica vaginalis, and thus may lead to gangrene. The stylet being withdrawn, the entire quantity of fluid empties itself through the cannula, the left hand making gentle pressure from behind upon the sac the while.

A small syringe charged with pure carbolic acid is now fitted to the cannula, and about twenty minims of the acid injected inside of the sac. Before withdrawing the cannula it is well to apply some aseptic salve to the skin about the point of puncture, to prevent the outer skin from being burned by the acid, a drop of which may follow the withdrawal of the tube. The sac should now be manipulated so as to distribute the injected fluid over the entire inner surface of the tunica vaginalis. This is very important. The operation is entirely free from pain in almost all cases. In some instances slight nausea or faintness is experienced before the anæsthetic effects of the carbolic acid have developed. The scrotum is then enveloped in cotton, and judicious pressure maintained.

"The patient should be kept quiet on the day of the operation, but with proper suspension of the scrotum he may be allowed to move about after twenty-four hours.

"On the day following, and for about a week afterward, the tumor swells up as if it were refilling, and is solid or doughy to the feel. After this it begins to decrease until the sac is obliterated and a permanent cure is effected." 
The trouble may recur again, however, at a later date, and in that case the operation may be repeated, a larger amount of carbolic acid being injected than before. This method may be adopted with propriety and excellent effect in the case of infants and young children. No cocaine need be used. A large-sized hypodermic needle serves for the trocar, and but one or two minims of carbolic acid should be injected.

The method known as Volkmann's operation consists in laying open the sac by a long incision under antiseptic precautions.

The patient having been anæsthetized and the scrotum and pubes shaved and properly cleansed, a longitudinal incision from two to four inches or more in length is made over the anterior aspect, and carried deeper until the sac is laid open to about the same extent. The fluid escapes. The edge of the parietal layer of the tunica vaginalis is now stitched to the edge of the wound in the skin by means of a good-sized catgut continuous suture. This makes an opening not unlike a buttonhole. The sac may now be irrigated with a 1-to-3,000 solution of sublimate, and two drainage-tubes are to be inserted into the sac, draining the lower and upper portions respectively. Iodoformized gauze may be used in addition to the tubes, being packed into the sac around them.

The dressing is done with sublimated gauze, laid on in sufficient quantity to absorb all secretions without necessitating a change for several days.

The penis should be well isolated from the dressings, so that they will not be soiled or infected during micturition. This may be achieved by passing the organ through openings of sufficient size in each successive layer of gauze applied to the scrotum. A sheet of protective tissue is finally added, adjusted in a manner to envelop the entire dressing and prevent contamination both by the fæces and the urine. The bandage is put on in a manner somewhat resembling a spica of both hips, but with additional turns, running from the pelvis of one side to the femur of the other, and crossing in the region of the perinæum. The patient is obliged to remain in bed for a period of two or three weeks after this operation.

The dressing is to be changed for the purpose of removing the drainage-tubes, or if the secretion moistens the edges of the dressing, so that decomposition of the secretions can take place, or if the temperature of the patient rises, indicating some retention or septic disturbance in the wound.

This latter operation (Volkmann's) is indicated in cases where the operation of injection of carbolic acid (Levis's method) has failed repeatedly. But it should not be forgotten that Volkmann's operation, if carried out with a strict attention to antiseptic details, may also fail and be followed by recurrence. In this case radical cure can only be attained by 
packing the cavity with iodoformized gauze, and leaving the latter in situ until suppuration ensues (ten days).

The operation is further indicated in cases where the sac walls are thickened to a great extent (periorchitis prolifera).

The only possible source of danger connected with this method, when it is antiseptically carried out, lies in insufficient drainage. Before antiseptic precautions were insisted upon (by Volkmann), extensive suppuration and pyæmia frequently followed the free opening of the sac.

The danger of retention is greatly averted by "button-holing" the incision.

More recently the excision of the sac of the hydrocele has been put forward as a radical cure, more especially in Germany. The method consists in making a longitudinal incision the entire length of the tumor, and then excising the serous membrane with the curved scissors and forceps, in a similar manner as is done with the synovial membrane of the joints. It is only necessary to remove the parietal layer of the tunica vaginalis, as the visceral portion in intimate connection with the testicle may be left untouched, and in fact is rarely affected by secondary changes. The operation should be done with the strictest antiseptic precautions.

We now turn to the description of the rarer forms and complications of hydrocele from a clinical point of view.

Hydrocele Communicans is an effusion in the tunica vaginalis propria testis which extends upward along the cord through the unobliterated processus vaginalis, and enters the peritoneal cavity. This form is always congenital, but it is comparatively rare.

There are other congenital forms of hydrocele, such as hydrocele proper (of the tunica vaginalis alone) and that of the cord, which are more frequent.

Etiology.-The etiology of hydrocele communicans is an embryonic inhibitory malformation, a failure of the vaginal process to become obliterated. The fluid in the sac in these cases is serum, and the same as is found between the coils of intestine in the abdominal cavity.

Symptoms. - The chief symptoms characteristic of hydrocele communicans is this, that, on compression of the tumor, the fluid disappears and the swelling becomes smaller. Considerable time may be required in order to displace all the fluid into the abdomen by pressure, especially when the opening leading into the peritoneal cavity is small. After the pressure has been removed the sac refills spontaneously, particularly when the parts are in a dependent position.

The shape of the tumor is more oblong or oval than that of hydrocele proper, and no limitation of the tumor below the inguinal canal can be made out.

In other cases, however, when the communicating canal is long and 
of very small caliber, there may be no difference in the shape and external aspect.

The other symptoms are the same as those of hydrocele proper-fluctuation, smoothness of surface, topic relation to the testicle, a normal state of the skin covering it, and translucency.

In the further course of bodily development, these congenital communicating hydroceles very frequently disappear spontaneously, probably in consequence of some traumatic inflammatory process; or they may lead to encysted forms of hydrocele, as that of the cord. In some cases, however, and especially when the communication is of a large caliber, they may persist, and have to be treated in the same manner as herniæ.

Diagnosis. - The diagnosis rests upon the possibility of emptying the tumor by manual pressure, and the symptom of its spontaneous decrease in size in the prone posture, and its increase in the erect one. Frequently a rippling sensation can be detected when pressure is made, caused by currents of the fluid at the abdominal opening, a sensation totally different from the murmur accompanying the return of coils of intestine during taxis.

Prognosis.-The prognosis in young infants is generally good, for the reason that in most cases cure is effected either spontaneously or by interference. In adults, or when the hydrocele communicans has persisted for a long time, spontaneous recovery is not to be expected; and surgical interference is indicated. If nothing is done, complications with hernia or the formation of encysted hydrocele of the cord must be expected.

Treatment. - The treatment of hydrocele communicans in the first days after birth is limited to judicious compression and cold applications. In cases where the trouble has persisted for some time and in adults, tapping or aspiration with a fine needle under antiseptic precautions is indicated, and subsequent compression of the neck of the sac by means of a truss or a pelotte and spica bandage. Tapping alone has frequently led to a permanent cure. Injections of irritants should be scrupulously avoided, as liable to set up inflammatory reaction in the peritoneal cavity.

Hydrocele of the Spermatic Cord may be either diffuse or encysted.

Encysted hydrocele of the cord (hydrocele funiculi saccata) is an accumulation of serous fluid in a closed sac, accompanying the spermatic cord, consisting of an nnobliterated portion of the processus vaginalis peritonei. It is pathologically entirely comparable to hydrocele of the tunica vaginalis. The acute form of encysted hydrocele of the cord is not known to exist; it is always chronic. There may, however, occur acute inflammatory processes in a chronic hydrocele of this kind, and these attacks we designate by the term perispermatitis acuta. Such attacks may 
result from traumatisms of the encysted hydrocele of the cord, through infectious agents, irritating injections, etc.

The character of the effusion may be serous, fibrinous, or purulent.

These acute exacerbations occurring in chronic hydrocele of the cord are clinically important principally for the reason that they may be readily mistaken for recent hernial incarcerations.

The diagnosis can be made by determining whether the tumor extends through the inguinal canal, in which case we have to deal with hernia. By invaginating the loose skin of the scrotum on the little finger and pushing it up into the canal, the limitation of the tumor in hydrocele can be readily recognized. Percussion gives a tympanitic note in the latter case, and translucency is absent. Aspiration with a fine needle will decide in difficult cases.

The treatment of acute perispermatitis is identical with that of acute vaginal hydrocele.

Chronic encysted hydrocele of the spermatic cord, or hydrocele funiculi proper, is quite common in infancy, but somewhat rare in later life. In young children it is frequently associated with vaginal hydrocele.

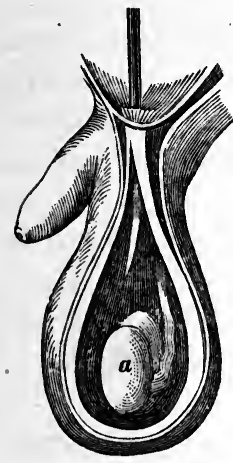

Fig. 2.-Hydrocele of the cord communicating with the tunica vaginalis testis. (After Linhart.)

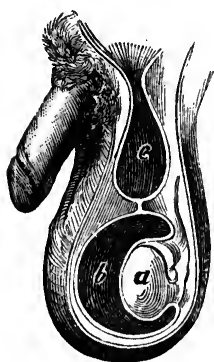

Fra. 3.-Hydrocele of the cord communi-
cating with the peritoneal cavity. (After Linhart.)

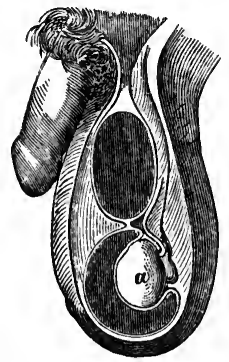

FIG. 4.-Encysted hydrocele of the cord. (After Linhart.)

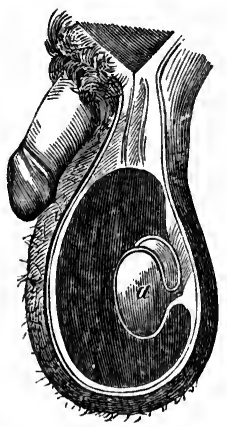

Fia. 5.-Hydrocele of the tunica vaginalis testis. (After Linhart.)

Any one part or the entire length of the abnormal serons sac accompanying the cord, from the peritoneal membrane down to the tunica vaginalis, may be affected, so that we may differentiate as to the site of the tumor between hydrocele funiculi intra-inguinalis, h. extra-inguinalis, or $\mathrm{h}$. totalis. The situation below the external ring is the most frequent. The effusion may take place at one point only (hydrocele partialis simplex), or at several points independently (hydrocele partialis multiplex).

Etiology.-The etiology of chronic encysted hydrocele of the cord is little understood; its origin has been attributed to repeated injuries 
connected with developmental abnormities. The pathological conditions of hydrocele of the cord differ little from those of the tunica vaginalis. The sac consists of fibrous tissue lined with a serous membrane covered with tessellated epithelium.

The effusion into this sac also resembles that in chronic hydrocele proper. The quantity may be as much as two ounces. It is of a paleyellow color, limpid, and contains much albumin.

Perispermatitis in rare cases may take on the conditions described under the heading Periorchitis, and we have consequently the various forms of hydrocele of the cord, characterized as perispermatitis prolifera, perispermatitis adhæsiva, perispermatitis hæmorrhagica, and perispermatitis suppurativa. For a more detailed description of these conditions, the reader is referred to the heading Periorchitis.

Symptoms. - The subjective disturbance caused by hydrocele of the cord is usually very slight, unless acute exacerbations in the growth of the tumor occur. In this latter case the patients may experience pain along the cord and show a rise of temperature.

The objective symptoms of hydrocele of the cord are the following:

Above the testicle and below the inguinal canal an oval tumor is to be seen and felt, which is hardly ever larger than a hen's egg, and which is covered by the normal skin of the scrotum, under which it is freely movable. The spermatic cord may be distinctly recognized immediately behind it, or situated internally to it. In rare cases the tumor may be found lying behind the cord.

Fluctuation is well marked, except in those cases where the sac is tense, owing to the presence of a comparatively large amount of fluid.

The tumor appears smooth to the touch, sharply defined, does not diminish on pressure or on change of posture, and is translucent, unless inflammatory changes have affected the walls.

Should suppuration ensue, the symptoms of acute abscess would supervene.

Diagnosis. - The diagnosis of hydrocele of the cord may be made when an oval tumor is recognized in the scrotum above the testicle, which is well defined above and below, is not painful on pressure, is movable under the skin, is soft and regular to the feel, although not fluctuating, and which is translucent. The testicle can be distinctly felt below it. The molility may be so great as to permit of the entire tumor being pushed through the canal into the abdomen; but in such a case it can not be retained there, and appears as before when pressure is released. There is no impulse felt on coughing when uncomplicated with inguinal hernia. Hydrocele funiculi may also be mistaken for spermatocele or a retained testicle.

In difficult cases aspiration under aseptic conditions with a fine 
hypodermic needle will lead to a correct appreciation of the conditions present.

The prognosis is the same as that of hydrocele vaginalis.

Treatment.-The treatment of hydrocele of the cord in adults consists in tapping the tumor with a moderate-sized needle under antiseptic precautions, and the subsequent injection of from five to ten minims of pure carbolic acid. If this treatment should fail to effect a permanent cure after repeating it once or twice, recourse should be had to the more radical operation of laying open the sac and suturing the sac walls to the skin so as to form a fistula large enough to admit two drainage-tubes. Antiseptic dressings are now to be applied, and the patient kept in bed until the cavity of the sac has become obliterated.

In certain cases the extirpation of the walls of the sac may be done, or at least part of the walls may be excised. In any case the trouble may return, however.

In children aspiration alone generally effects a cure. If after this treatment the fluid should again accumulate, the injection of one minim of pure carbolic acid may be resorted to.

Diffuse Hydrocele of the Cord.-This condition stands in the same relation to encysted hydrocele of the cord as does hydrocele of the tunica communis to that of the tunica vaginalis.

The conditions present in diffuse hydrocele of the cord were experimentally demonstrated by Kocher. By tying a cannula into the tissue surrounding the cord at the point of its egress from the inguinal canal and forcing plastic matter downward along the cord, he produced a sausage-shaped tumor, which inclosed the cord on every side and extended downward as far as the testicle. This newly formed tumor displaces the testicle, causing it to lie horizontally, and parting it from the cord. The mass forms a bell-like covering for the testicle which can be felt below, lying in the concavity of the tumor. The epididymis lies between the testicle and the tumor. If the injection is increased, the tumor expands outward toward the skin of the scrotum, until it is nearly round in shape.

By injecting upward instead of downward the mass can be made to extend up through the inguinal canal, and a tumor is produced lying in the iliac fossa, situated in the subserous cellular tissue and resting upon the iliac muscle.

From this description it is apparent that the conditions present in diffuse hydrocele of the spermatic cord may be characterized as acute œdema surrounding the cord. It is limited by the fascia Cooperi.

This condition is rare. It is said to occur in adults after the thirtieth year, and it may be caused by the rupture of cysts situated near the cord, or secondarily after inflammation of the testicle by an interference in the return circulation in the spermatic vein, or by unknown means. 
Etiology.-Probably the most frequent origin of the conditions described as diffuse hydrocele of the cord is to be found in the process of incapsulation and absorption of hæmorrhages in the regions surrounding the cord, caused by direct violence, or indeed by suddenly increased intra-abdominal pressure, resulting in the rupture of a vein. The hæmorrhages occurring after lesion of blood-vessels accompanying the funiculus spermaticus have the same anatomical location as the acute œdema described above, and hence when they become incapsulated, and serous fluid replaces the extravasation of blood, we encounter one or more cysts which correspond to the definition given above for diffuse hydrocele of the cord.

Symptoms and Diagnosis.-The symptoms of diffuse hydrocele of the cord much resemble those of an omental hernia; the tumor generally extends into the canal, the shape is pyriform, with the apex above; the surface is uneven or a little nodular to the touch; there is considerable resistance on pressure, and generally fluctuation in the lower portions. The size of the tumor decreases in the horizontal position of the patient, and increases again with the erect posture. The skin over the tumor appears normal.

In differentiation, however, the following facts must be brought out: The tumor can not be replaced inside of the abdominal cavity, but when pressure is made the fluid escapes into the subperitoneal or intermuscular tissues of the abdomen, and the tumor recurs as soon as such pressure is released. The configuration, in well-marked cases, is determinate, the bell-shaped formation surrounding the testicle from above being pathognomonic. Finally, diffuse hydrocele of the cord is translucent.

The prognosis of this condition should be guarded, on account of the liability of the affection to lead to gangrene of the parts affected through pressure.

The treatment of diffuse hydrocele of the cord varies with its age.

In the acute stage, incision under aseptic precautions is indicated; and in these cases special attention should be given to the tracing and checking of any hæmorrhage.

In chronic affections of the kind, aspiration with compression may be first undertaken, and if not successful, incision and drainage, or extirpation of the cyst walls, may be resorted to.

Bilocular Hydrocele. - This was first described by Dupuytren as hydrocele en bissac, and represents an hour-glass-shaped tumor, the lower portion of which corresponds to the distended tunica vaginalis, the upper portion to the greatly distended vaginal process of the peritonæum, not communicating with the peritoneal cavity, but obliterated at the point where the peritonæum is reached. Both these portions of the tumor connect by means of the narrow part, which is that portion of the vagi- 
nal process which lies in the inguinal canal. As the upper portion does not enter into the peritoneal cavity, and yet lies within the pelvis, it is necessary to describe its situation more precisely.

The upper sac lies in a subserous space close beneath the peritoneal membrane, and when it increases in size it dissects off the peritonæum and crowds it back.

In a hydrocele shaped in this manner the fluid can pass slowly from one enlargement of the sac to the other, through the narrow portion in the canal. When the patient therefore lies down, the scrotal tumor diminishes in size and the intra-abdominal one increases. This portion can be made out by bimanual palpation especially in narcosis, one finger being in the rectum. Pressure made here in the reclining posture will cause the scrotal portion of the tumor to enlarge.

This form of hydrocele is most frequent in adults ; it occurs, however, in children as well, but is rare. In some cases it has developed to an enormous size.

Etiology.-Etiologically the formation of bilocular hydrocele of this form is attributed to blows or other direct violence taking effect upon a hydrocele of the tunica vaginalis, and causing its sudden extension along the processus vaginalis upward into the abdomen.

Some writers view bilocular hydrocele simply as a diverticulum of the tunica vaginalis. And the term bilocular hydrocele is consequently applied to any diverticulum of the tunica vaginalis, irrespective of the fact whether this diverticulum extends upward into the abdominal cavity or in the scrotum, externally to the original sac, through the

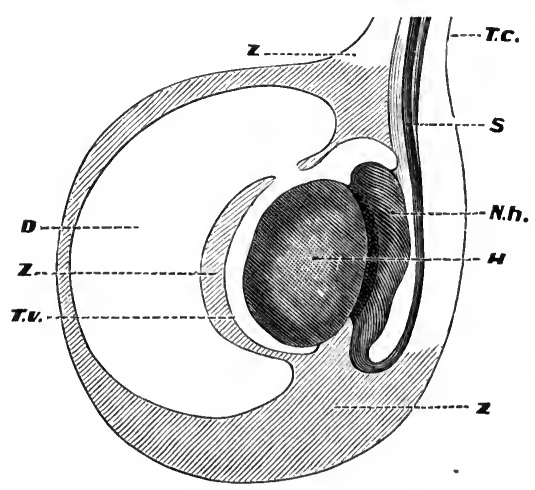

FIG. 6.-Bilocular hydrocele. fibrous layer of the tunica communis. This latter class of cases may be termed hydrocele bilocularis vaginalis. The tunica vaginalis is believed to expand at the point of least resistance. In any case, however, the two sacs of hydrocele bilocularis intercommunicate, while so-called multilocular hydrocele consists of entirely separate compartments.

Symptoms. - The symptoms of hydrocele bilocularis much resemble those of simple hydrocele at first sight. But upon placing the patient in a recumbent position, and exerting pressure upon the swelling in the scrotum, the fluid gradually disappears, frequently (if the communicating opening is small) with a rippling sensation. As soon as pressure is re- 
leased, however, the scrotal portion of the tumor refills; and in certain cases the abdominal portion of the tumor can be made out by palpation, combined, or in narcosis.

This expansion of the scrotal portion of the tumor also takes place when the patient coughs or exerts abdominal pressure.

The tumor is translucent, except in those cases where hæmorrhage has occurred after traumatisms, or where the sac is undergoing suppuration.

In other respects the symptoms are the same as in simple hydrocele, with the exception only as to the extent of the tumor.

The difference between diffuse hydrocele of the cord and bilocular abdominal hydrocele lies in the anatomical conditions. The fluid in both cases lies outside of the peritonæum. But in bilocular hydrocele this fluid is in an extension or diverticulum of the original processus vaginal peritonei, while in diffuse hydrocele of the cord, the fluid, when it has become encysted, lies externally to the vaginal process, and inclosed in a newly formed membrane, imbedded in the loose cellular tissue surrounding the cord.

The treatment is the same as that of simple hydrocele.

Multilocular Hydrocele.-This term is used to denote a multiplicity of cystic swellings accompanying the cord and extending from the tunica vaginalis toward the abdominal cavity, somewhat resembling a quantity of small hydroceles of the cord. This form is rare, and has been described by Kocher.

The pathology of this form is little understood.

The contents of these cystic tumors is not serous, as in true hydrocele of the cord, but watery or mucous. To the feel they present nodules, conglomerate, more or less tense, movable, transparent, and painless. The mass may extend through the inguinal canal and into the abdominal cavity.

The treatment is similar to that of simple hydrocele of the cord. Incision and extirpation will cure the most obstinate cases.

\section{COMPLICATIONS OF HYDROCELE.}

Simple hydrocele may be complicated with hernia, with a hydrocele of the cord, or with both; with a communicating hydrocele of the cord, with or without a hernia; with hydatic cysts, and with other affections of the epididymis and testicle. Communicating hydrocele may be complicated by a hernia.

The most frequent complication of hydrocele is that with hernia. If the hernia is reducible, there is little difficulty in forming an accurate conception of the size and variety of the hydrocele. If, however, the hernia is incarcerated, or is an adherent omental hernia, the appreciation of the conditions is rendered more difficult.

The history of the patient must be duly considered. The want of 
well-defined limitation of the tumor above will lead the practitioner to suspect a complication.

Fluctuation is well marked in the lower portion, but not in the upper one, where in simple hydrocele it is most pronounced. The consistency varies in the upper and lower portion of the tumor. In hernia of the omentum the feel is doughy; while in incarcerated intestinal hernia the percussion note is tympanitic. Translucency is present only in the lower portion containing the hydrocele. Aspiration with a fine hypodermic needle will establish the presence of the hydrocele, and, after tapping, the nature of the complicating tumor can be more readily appreciated.

If hydrocele, hydrocele of the cord, and hernia are all present together, we find three tumors, which present symptoms differentiated in the same manner as that just described. The configuration and the touch enable us to determine the presence of the hydrocele of the cord between the two other portions of the tumor; it is tense, diaphanous, painless, dull on percussion, and not movable. The aspirating needle reveals serum.

In some cases, however, the conditions present in these complications are much more difficult to appreciate. The descending hernial sac may impinge upon an existing hydrocele, either of the tunica vaginalis or of the cord, in such a manner that it drives in one pole of the hydrocele sac and causes it to ingulf it. The hernial sac is then surrounded on one or all sides by the hydrocele sac. These forms have been described by A. Cooper as encysted hernia. If the hernia can not be reduced, the main reliance in the diagnosis must be placed in hypodermic aspiration.

A communicating hydrocele in children may contain a loop of intestine if the opening at the abdominal ring is large enough to admit it.

In all these cases the hernia is generally readily replaced, and the hydrocele can then be treated as indicated above.

Hydatic cysts and other complications of hydrocele arising from an affection of the epididymis or testicle can not well be diagnosticated as long as the hydrocele is present. It is difficult in the extreme to appreciate whether or not the testicle is normal when the hydrocele is well marked; and if the tumor is tense and large, it may be impossible to do more than determine the seat of the testicle. In all cases where suspicion of affections of the testicle and its adnexes is entertained, the hydrocele should be tapped or its sac laid open.

Hydatic cysts are most frequently overlooked, and are not found until radical operation for the hydrocele is done. They should then be extirpated. These cysts are generally pedunculated, situated at the upper portion of the testicle alongside of Morgagni's body, or replacing it, and of a size no larger than a cherry. If the cysts are of larger size, inversion of the testicle sometimes follows. Such cysts may burst spontaneously, and are believed by some to cause hydrocele. 
Mention must be here made of a condition known as hydrocele hernialis, which is not, properly speaking, a hydrocele, but is described here for the sake of completeness.

This affection consists of a hernial sac, containing serous fluid identical with that of hydrocele, but not containing any coils of intestine or omentum or other abdominal organs.

The communication with the peritoneal cavity is generally maintained by means of a very small opening, which admits of our forcing the fluid into the abdomen; the tumor gradually refills, however, as soon as the patient assumes the erect posture and walks about.

These hernial hydroceles are not confined to the inguinal region and the scrotum alone, but occur wherever herniæ are met with, in the femoral and umbilical regions, and in the female as well-what is described as hydrocele in the female being a serous sac communicating through a small opening with the peritoneal cavity and lying in the canal of Nuck. In certain cases the opening communicating with the peritonæum may become obliterated. The tumor appears smooth and soft to the touch, fluctuating and translucent. It may generally be emptied by continuous pressure, but then it refills again. The testicle can be clearly defined beneath it, unless complications with hydrocele of the tunica vaginalis are present. The tumor, however, can not be defined above. The skin of the scrotum above the tumor is not affected. There is no pain on pressure, or it may be slight.

Hydrocele hernialis must be differentiated from omental incarcerated hernia, in which inflammatory exudation has taken place. In this latter case there is much pain, and complete reduction is not possible.

The treatment consists in extirpation of the sac, as for hernia.

Differential Diagnosis of the Various Forms of Hydrocele and their Complications.-Under the heading Diagnosis of Hydrocele the differentiation between hydrocele of the tunica vaginalis and other tumors of the scrotum was given. This differentiation applied only to such cases in which the limitation of the tumor in the scrotum was apparent.

If, now, by grasping the upper portion of the scrotum between the thumb and the forefinger we are able to feel that the tumor continues into the inguinal canal, we must next endeavor to determine whether the tumor is limited inside of the canal, or whether it extends into the abdominal cavity.

There are three varieties of tumor which extend into the abdominal cavity, and which may prove more difficult to differentiate-namely, hernia, hydrocele communicans, and bilocular hydrocele; for varicocele (which also extends into the abdominal cavity) is always easy of diagnosis, the peculiar feel, as of a number of earth-worms beneath the skin, the 
compressibility and the diminution of the tumor when the patient assumes the recumbent posture, being pathognononic.

Malignant tumors extending upward from the testicle through the inguinal canal are also manifest by means of their size, the history of their commencement, the involvement of the integument of the scrotum, their feel, etc.

Hernia, hydrocele communicans, and bilocular hydrocele present a number of symptoms in common. They can be entirely or at least almost entirely reduced, they decrease in size in the recumbent position of the patient, and they show an impulse when the patient coughs.

Attempted taxis, however, generally discloses the nature of these tumors. The reduction of herniæ is accompanied by a peculiar murmur, a bubbling of air in the intestine, and takes place suddenly, while the fluid in the hydrocele communicans recedes slowly and continuously, sometimes accompanied by a flowing sensation; and the fluid in the bilocular hydrocele, when reduced, exhibits an elastic tension, and a tendency to return at once, nor can it be entirely reduced.

Moreover, a hernia shows tympanitic resonance on percussion, while hydrocele communicans and bilocularis both appear translucent.

Differentiation between hydrocele communicans and bilocularis (en bissac) can be made by ascertaining whether the fluid when evacuated by pressure from the scrotal portion of the tumor is forced into a sac situated extraperitoneally, in the iliac fossa, or whether the fluid is forced into the peritoneal cavity itself (hydrocele communicans). In the first case the sac can generally be felt by combined methods of palpation; pressure upon it causes the scrotal portion to refill, and thus establishes the diagnosis of bilocular hydrocele. This symptom also establishes the differentiation between an omental hernia and bilocular hydrocele.

Simple hydrocele of the tunica vaginalis with a diverticulum reaching into the inguinal canal, on the other hand, can not be emptied by pressure; and by pulling down the tumor the limitation inside of the canal can generally be determined. The little finger introduced into the inguinal canal, invaginating a portion of the scrotal integument, is of much assistance in establishing this point.

In hydrocele of the cord we have not only this limitation inside of the canal, but another limitation below, above the testicle.

Diffuse hydrocele of the cord can not always be differentiated from bilocular hydrocele (en bissac). The history of its commencement and its development from a hæmorrhage may permit of a probable diagnosis on account of the subcutaneous discoloration attendant upon the causative injury. The symptoms of both conditions are otherwise identical, but for the shape. Hydrocele bilocularis being pyriform, diffuse hydro- 
cele of the cord is bell-shaped. The latter affection, however, is said to be so rare that little importance attaches to it.

The diagnosis of the nature of the fluid in various forms of hydrocele rests upon their translucency, a knowledge of the etiology and pathogenesis of the various forms, and the result of aspiration with a hypodermic needle.

The diagnosis of complications of the various forms of hydrocele may present considerable difficulties.

If simple vaginal hydrocele is complicated with simple hernia, we encounter two distinct tumors, the lower fluctuating, transparent; the upper one reducible, tympanitic. The hernia may be incarcerated, and contain no air (omentum); in this case the upper tumor is neither reducible nor tympanitic ; but it is not translucent, and presents an unmistakable direct impulse on coughing.

In case hydrocele of the cord is present combined with hernia, we have the same symptoms present as before, with this exception, that the testicle can be readily felt and appreciated in hydrocele of the cord, while in simple hydrocele of the tunica vaginalis it is not easily felt, being enveloped in the liquid.

Encysted hernia can not always be differentiated from hernia with much fluid, unless the herniæ can be reduced. In this case a tumor giving the symptoms of simple hydrocele, or of hydrocele of the cord, remains in the scrotum after reduction.

A hydrocele hernialis can not be differentiated from a communicating hydrocele of the cord, except by the history; the latter affection occurring more frequently in children.

\section{SPERMATOCELE.}

Definition.-Spermatocele is a cystic new growth containing spermatic elements situated between the testicle and the epididymis, near the head of the latter. It is comparatively rare.

Etiology.-As Kocher has shown experimentally, the vasa efferentia become dilated at their point of egress from the rete testis, and this is the most frequent origin of spermatocele. Dilatation of the efferent tubes at the point where they enter the head of the epididymis gives rise to the smaller subserous spermatic cysts frequently found in adults.

Rupture of a spermatic vessel by traumatism or disease (epididymitis) may also lead to spermatocele, if the extravasation becomes incapsulated. If hydrocele is present, communication may be established between the lumen of a spermatic duct and the cavity of the tunica vaginalis (hydrospermatocele).

Symptoms.-In conformity with the etiological data just given, spermatocele then begins-not before the age of puberty-as a small 
tumor situated above the testicle, between it and the epididymis. It gradually but slowly increases in size, until it becomes as large as an apple. Some have been known to contain a quart of fluid. The growth takes place upward toward the inguinal canal, and the tumor becomes larger in circumference as it ascends. In this manner a pear-shaped tumor is formed, with the base above and the apex pointing downward to the testicle. At this point the testicle can always readily be made out, both poles and both sides being accessible for palpation. The epididymis can be felt behind the tumor, the testicle being situated in front.

Diagnosis. - These symptoms serve to differentiate spermatocele from hydrocele of the tunica vaginalis and from that of the cord. In the former the testicle is enveloped in the fluid, and can not be distinctly felt. In hydrocele of the cord the testicle is in close proximity to the epididymis, with the tumor above, while in spermatocele the tumor appears inserted between the testicle and the epididymis.

From other affections spermatocele can be differentiated by the fluctuation and by the translucency present, and in doubtful cases (such as when other cystic tumors of the parts are suspected) aseptic exploratory aspiration with a hypodermic needle must decide the diagnosis, the microscope revealing spermatozoa floating in the aqueous contents of the cyst when spermatocele is present. If the spermatocele develops from the testicle proper instead of, as is usual, from the efferent vessels, the growth takes place within the cavity of the tunica vaginalis instead of extravaginally, and in such cases spermatocele can be differentiated from hydrocele only by aspiration.

Treatment.-The cure of smaller spermatoceles may be attempted by extirpation, or incision and drainage ("Volkmann's operation") may be done under antiseptic precautions. In some cases tapping alone is said to have led to a permanent cure. 



\title{
VARICOCELE.
}

\author{
By EDWARD L. KEYES, M. D.
}

Definition.-Varicocele is an unnatural, varicose enlargement of the pampiniform plexus and the veins of the spermatic cord. What extent of turgescence of these veins must exist in order that their enlargement may be elevated to the condition of a morbid affection is a matter of personal estimate. A very mild form of varicocele-that is, a slight enlargement of these veins-is so common, that, when it exists upon the left side at least, it may be considered normal; and even an abnormal enlargement in its mildest form is so common that it is hardly worthy of the name of a disease.

Frequency.-Common estimate places slight varicocele at a figure of at least ten per cent of all adult males. It habitually is confined to the left side, but has been seen by all writers also occasionally upon the right side alone, and, when it is very marked upon the left side, the right side may be involved to a slight extent. Breschet operated only once on the right side in one hundred and twenty recorded cases. I have never operated on the right side, although I have seen the veins considerably enlarged upon this side perhaps half a dozen times.

Etiology.--In young men, shortly after adolescence, budding erotic fancies and imperfect sexual hygiene often lead to eongestion of the testicle and over-distention of the veins of the cord, and when this is kept up too persistently, especially in subjects whose tissues are lax, or when the sexual faculties are taxed or teased, the foundation of varicocele is usually laid. The young bachelor is often unnecessarily alarmed by the presence of a cord slightly thicker upon one side than upon the other, especially if uneasy sensations exist in the testicle or groin, and he habitually distresses himself about this affection to an extent entirely disproportionate to its real significance. Commonly, marriage disperses both the slight turgescence of the cord and the unhappy fancies which have accompanied it; and Nature, by the aid of a well-regulated sexual life, disposes of the necessity for a surgeon. Old men rarely have varicocele, although the veins of their legs may be in a varicose condition, and the general tissues of their body more or less degenerated, and, when they do have it, it causes usually no symptoms, and they give it no attention. 
It is entirely appropriate that this malady should be looked upon generally rather as a sexual neurotic derangement, so far as its symptoms are concerned, than as an organic disease. It is accused by young men, by widowers, by individuals who have committed sexual excess, by oxaluric and sexually neurasthenic patients, as the cause of many symptoms with which it has no possible connection. I have been appealed to in many instances to operate upon a slight varicocele, the existence of which as a physical fact was quite obvious, the call for the operation being based upon a desire to be strengthened sexually; to be relieved of morbid sensations in the testicle, the perinæum, the groin, the back, the bladder or elsewhere, with the idea on the part of the patient that, if these veins were done away with, all his morbid symptoms would disappear. Experience has taught me that it is not wise to approach these cases with any promise of permanent relief in any of the directions which I have indicated. Truly, if the enlarged veins are obliterated and a proper circulation restored to the testicle, its functions and its sensations are likely to be improved; but in very many instances the enlarged veins are only a single symptom in the general tableaux of the disease, and, although the veins may be mechanically thrown out of function, still the other symptoms may persist unless removed by appropriate means. This fact can not be too prominently paraded before the generally diseased mind of the young patient who seeks relief for this very moderate malady.

Yet, that varicocele does cause neuralgia of the testicle and dragging pain in the groin, and more or less functional disability in a sexual way, is entirely true, and the relief of the malady, when it exists to any consicierable extent, is an entirely appropriate surgical procedure; and since it can be accomplished at the present day with so little distress and absolutely no risk or danger, what comfort it affords should not be denied the patient; but he must not be encouraged to expect a miracle as the result of a successful operation.

The left testicle, which is the common seat of varicocele, hangs habitually lower than the right. The larger veins only are furnished with valves, and on the left side the venous blood from the testicle empties into the left renal vein at a right angle, while upon the right side the blood flows at an acute angle into the ascending cava. This circumstance is believed to be the main predisposing cause of the more frequent occurrence of varicocele upon the left side. As an extra predisposing cause, the position of the sigmoid flexure of the colon on the left side, distended as it so often is in chronic constipation, must be noted. Yet, that the conditions upon the left side are more predisposing essentially to varicocele than on the right side, seems to be accentuated by the fact that in those cases of acute varicocele occurring after sudden effort, or sometimes after fatigue, local injury, or cold, or perhaps withont obvious exciting 
cause, the left side is the one habitually involved. These cases of acute varicocele are usually only important from the fact that they give rise to very decided local symptoms of pain, distress, and tension, and occasion considerable alarm. I have seen a number of them, but under my observation they have all disappeared within a few days, being mastered by rest, local suspension, cooling lotions, a moderate anodyne, and a few laxatives ; occasionally, slight permanent enlargement of the veins persists after an acute attack, but I have one individual in mind in whom the veins of the left cord were quite seriously enlarged by acute varicocele on two occasions, at an interval of several years, where mild treatment effected a positive and permanent cure, the veins returning absolutely to their normal condition.

Symptoms.-Leaving out the acute cases, whose consideration may terminate with the few remarks made above, varicocele usually comes on insidiously, and is discovered by accident, the patient's attention being attracted to the scrotum by some morbid sensations in this region, or a tired, dragged feeling in the groin and back, always worse after fatigue and prolonged standing. There may be an enormous varicocele with positively no symptoms whatsoever excepting the physical enlargement of the veins, while in other instances a very slight distention of the vessels seems to be the occasion of radiating pains up the groin, into the back, down the thigh, and more or less neuralgia of the testicle itself. These pains are aggravated by sexual excitement, especially if unrelieved, and by sexual excess; while in many instances they are temporarily relieved by the sexual act, but this relief is only of short duration.

In complete varicocele the vessels are elongated, their walls the seat of fatty atrophy, sometimes thickened, sometimes thinned; the valves broken down, the loops of veins within the scrotum feeling more like a bunch of earth-worms than anything else. Sometimes, when the veins are much degenerated, calcareous masses form in their walls, but this is exceedingly uncommon. In extreme cases, all the veins in and about the testicle and scrotum seem to be involved in the general varicose condition; even the veins between the tunica vaginalis and the tunica albuginea The scrotal veins are also enlarged. The scrotum itself becomes thin, the dartos atrophied, the texture of the scrotum so thin that the blue color of the blood in the veins becomes distinctly visible. In such extreme cases the integrity of the testicle itself is threatened in a physical way; the organ becomes reduced in size, soft, flabby, sensitive, and if the tone of the circulation be not improved by some operative measure, the function of the testicle necessarily becomes impaired and the organ itself goes on to partial atrophy. Yet, even in these extreme cases, the hypochondriacal and emotional side of the symptoms of the patient are often largely in excess of the actual physical signs of the disease. 
Diagnosis.-Simple, uncomplicated varicocele can not well be mistaken for anything else. The wormy feel of the tortuous veins is entirely a physical symptom sui generis. When the varicocele becomes complicated by hydrocele, tumor of the cord, or any of the various forms of hernia, difficulties in diagnosis may arise, but in such cases varicocele sinks into secondary significance, the complicating disorder being the one calling for attention and requiring treatment. As this treatment, if applied surgically, involves a cutting operation and open inspection of the diseased area, the enlarged veins become a matter of very secondary importance, and are treated or not in connection with the main disorder, according to the inspiration of the surgeon at the moment of the operative act.

A simple test, in common, uncomplicated varicocele, establishes the diagnosis. The patient is made to lie down, whereupon the blood flows back into the abdominal cavity, and gentle pressure immediately removes the slight deformity. The surgeon's fingers are now placed with moderate firmness upon the external abdominal ring and the patient told to rise. Hernia will remain reduced; not so varicocele, unless the pressure at the external abdominal ring be so firm as to occlude the arteries as well as the veins. If the veins only are compressed, the varicocele repeats itself, refilling from below. Hydrocele can be diagnosticated by the usual simple method, or in any case of doubt by capillary aspiration.

Treatment.-In those very common cases in which the neurotic symptoms preponderate, they will require treatment more than the distended veins, and for such cases palliative measures, such as cold douches, the regulation of intestinal evacuations, and a snugly fitting suspensory bandage, are usually better means in the surgeon's hands than a radical operation, which, although it may relieve the physical disease, might fail to satisfactorily influence the morbid symptoms which the patient has ascribed to it. Varicocele never compromises health in any serious way, and ought not to be raised to the rank of a serious disorder, but frequently the patient's mind is so concentrated upon this obvious physical irregularity that a radical operation is justified. Formerly, such a radical operation was a rather serious matter. The open, cutting operation is, in my opinion, unnecessarily severe, nor does it save the patient from the possibility of damage. I have seen atrophy of the testicle follow the open operation for varicocele at the hands of one of the most competent surgeons in New York. It is never unavoidable. I consider it obsolete, and shall not describe it. Curtailing the scrotum is a method devoid of risk, which has been advocated in many quarters; but this also, unless the scrotum be very long and flabby, seems to me to be an operation unnecessarily severe, as it confines the patient habitually ten days or thereabouts 
to his bed, calls for the use of an anæsthetic, and is liable to all the ordinary complications of surgical wounds.

The operation which I advocate and habitually perform is simplicity itself. I described it first in 1886 (New York Medical Record, Februery 20 and September 18, 1886, and November 26, 1887). Recently a country schoolmaster, aged forty-five, came to me complaining of various sexual neurasthenic symptoms; and while examining him I recognized that there was a ligature about the veins of his cord, and that the vessels were obliterated. I called his attention to the fact, and asked him when he had had his varicocele operated on, and who had done it, remarking that the silk ligature was still in place, and that the operation had been well done. He informed me that he had seen my description of the operation, had sent to town and purchased the operating needle, and had operated upon himself, and he had no knowledge whatsoever of medicine or surgery of any sort. On the other hand, I have personally operated a second time upon patients who had had their veins tied up by surgeons by the subcutaneous method with silk, and yet upon whom the operation had failed, the failure having certainly been due only to the fact that the first knot of the ligature had not been tied with sufficient firmness and force.

In my opinion, no minor operation upon the genito-urinary tract is so simple and so efficacious as that for the relief of varicocele, unless possibly it be the treatment of simple hydrocele by capillary aspiration and capillary injection of pure carbolic acid, without knife and without cannula. This is very simple and thoroughly effective, although there are modern surgeons who, preferring to cut, still resort to the excision of the tunica vaginalis and the laying open of simple cases of hydrocele.

The operation which clearly meets the indications in all cases of varicocele, with or without pendulous scrotum, be the veins ever so enlarged, numerous, and tortuous, is the subcutaneous application of aseptic silk. If the operation be properly performed, every case, in my opinion, can be radically and permanently cured. I have never seen a patient detained in bed more than ten days, and this occurred only in my earlier cases, and in patients unnecessarily hyperæsthetic. Usually, five days is enough. There is no cutting, no loss of blood, very moderate pain. One of my patients, within forty-eight hours after the operation, traveled by rail without distress from New York city to the central part of the State. No accident has ever happened more serious than the puncture of a vein on one occasion, in which case there was considerable subcutaneous hæmorrhage, but it was all reabsorbed without treatment, and the final cure was permanent. In my first published operations with this method I advocated the use of catgut, but I soon abandoned this substance; it never cures. The catgut unties, or is absorbed too rapidly to effect the 
obliteration of the veins, and the varicocele always reproduces itself. After I adopted silk as a ligature my success became permanent. I have never produced abscess about the ligature, although in two or three instances there has been a slight oozing of sero-pus superficially discharging itself at the point of puncture; and in three instances that I can recall, in a hundred cases or more, the ligature has worked out at a period varying from three to nine months. In all the others the ligature has remained upon the veins, and I have felt it at the end of two years and longer as a firm little ferule of silk about the cord, the veins being obliterated beneath. My experience now covers very many cases, and extends over eight years

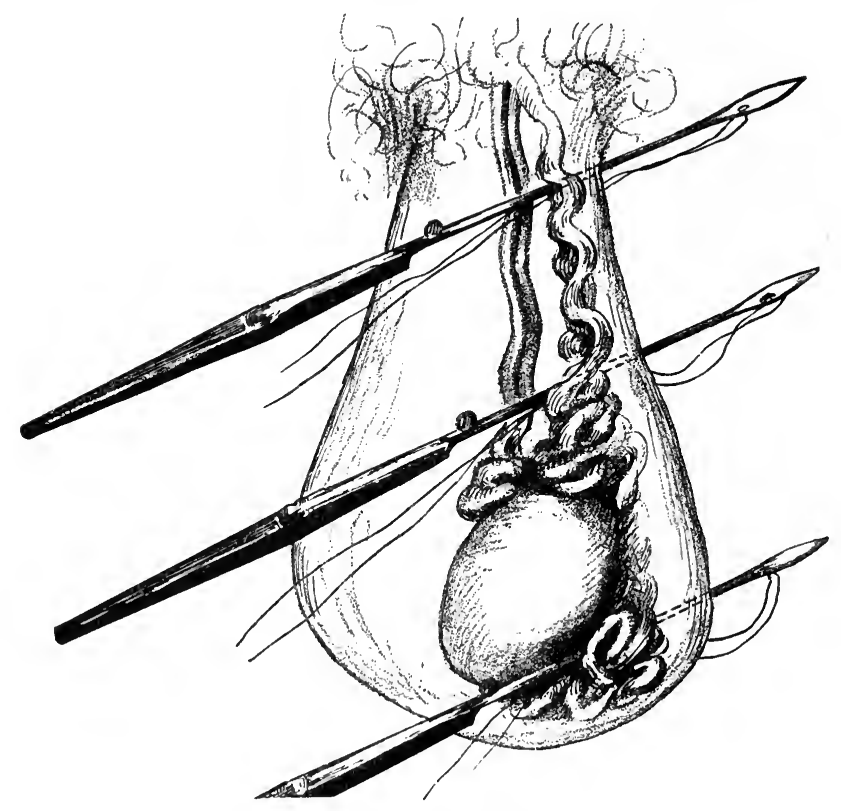

FIG. 1.

or thereabouts, and I have no hesitation in recommending this method for application in the radical cure of any material case of varicocele sufficiently severe to call for any operative interference.

I have in several instances tied the entire cord, transfixing first on the outer side of the vas deferens and tying the cord between it and the outer side of the scrotum in one ligature, and then have transfixed on the inner side of the same vas deferens and tied up the rest of the cord in another ligature on the inner side tightly, and in spite of this apparent jugulation of the circulation of the cord no atrophy of the testicle has followed in any case. At present, my method is to apply three ligatures if 
the varicocele be severe, two if it be moderate, the third ligature being placed below the globus minor. The veins that hang down below the globus minor are not enlarged in a certain minority of cases, but in almost all instances of severe varicocele, this bunch of veins is quite a prominent feature in the malady, and it should not be neglected in applying the ligatures; the cut (Fig. 1) shows the position in which the three ligatures are habitually placed-one, reasonably high up upon the cord on the outer side; the second, close down to the globus major on the outer side of the cord; the third, below the testicle, beneath the globus minor. Occasionally, when one or more large veins can be felt running down the inner side of the vas deferens, they may be also included in a fourth ligature, but I personally have never applied more than three upon any one case. In very young people, where the testicle is not developed and the vas deferens is exceedingly small and hard to feel with the thumb and finger beneath the tegumentary covering, I have applied my upper ligatures subcutaneously, and then, making a simple linear incision below the testicle, have exposed the veins hanging beneath the globus minor and applied a silk ligature upon them under the guidance of the eye, washing out the wound properly and immediately closing it with catgut. Such a combination of the open with the subcutaneous method does not militate against a prompt recovery. I operated in April, 1892, upon a boy of eleven years by this method, and he came to my office for inspection, and asked permission to go and run in the country at the end of one week.

The subcutaneous ligature of varicocele by silver wire or silk over an outside spring or button, or by elastic ligature, these foreign substances being allowed to cut their way out, has been practiced for many years. I did one operation by this method and was so disappointed in it as a surgical method that I renounced it at once. Suppuration is inevitable with these substances, and the open method, is far preferable. Exactly when silk was first used subcutaneously and left in place I do not know. Ogston (Annals of Surgery, August, 1886) mentions that he used silk in 1878, and Barker (London Lancet, September 30, 1882, page 521) details two cases in which he employed silk, passing it through a puncture made with a knife. I do not know whether in these instances the silk suppurated out or not. I employed silk for the first time in the spring of 1886, after catgut had failed me, knowing that silk, properly prepared, was perfectly capable of remaining permanently among the tissues without producing irritation, and not knowing at the time that any one else had ever used it in this manner, nor do I now know that it has been so used before. Since that date I have relied exclusively upon silk, and I find that it leaves nothing to be desired. The Chinese twisted silk of largest size, so strong that it can not be broken in tying a single knot 
without the use of very extreme force, is the most suitable size and material, even for children. It is prepared by simply boiling it in ordinary water for twenty minutes, fishing it out of its boiling medium with a clean tenaculum, and dropping it, cut into proper lengths, into a widemouthed bottle containing simple ninety-per-cent alcohol. It keeps indefinitely, and is ready for use without further attention. The needle (a result of six different modifications and efforts in the same direction), upon which I now rely entirely, is a modification of the Reverdin straight needle. Fig. 2 shows the instrument. The eye is kept permanently closed by a

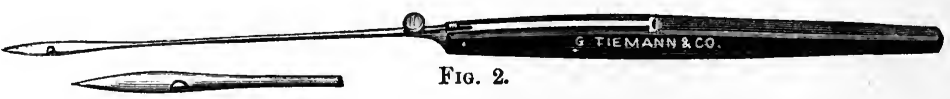

spiral spring concealed in the handle, and the instrument is a closed needle for all intents and purposes, unless the button is pressed backward. By this pressure the eye at the front end of the needle is opened. Reverdin's needle has no such spring, and requires constant attention on the part of the operator to keep the eye closed.

The Operation.-The patient should not take an anæsthetic. If he does so he must lie down, and in so doing the veins of the cord become empty and collapse, and some of them may escape, being inclosed in the ligature. If a patient be so sensitive that he insists upon the use of an anæsthetic, it may be given to him, provided the first and most important ligature be placed in its first position while he is in the erect posture; after this, with the needle in situ, he may be placed upon his back and an anæsthetic administered; because, after the first ligature is tied, the veins of the cord beneath become turgid and then they can be easily isolated, and the remaining ligatures placed under anæsthetic narcosis. Most patients prefer to have the entire operation done without the use of any anæsthetic. The pain of the puncture of the needle is very moderate, and although many patients faint perhaps to such an extent that they can not stand up, as they do in the case of many minor operations upon the genital apparatus, still it is a matter of no importance whether they faint or not, because, when two or three needles are placed in the first position, then the patient may lie down; and if he faints, it is all the better for him, becanse he is unconscious in that case of the only step in the operation which is really severely painful, namely, that in which the nerves of the cord are pinched in the first vigorous knotting of each ligature.

The patient stands upon a rubber cloth, well exposed to a strong light. The scrotum is thoroughly washed in a solution of the bichloride of mercury and water, 1 to 1,000. He should stand leaning against the side of the bed, so that the moment he becomes faint he may be placed upon his 
back, with his head low. He never does this until after the first puncture has been made, and this is the key to the entire operation. After this his fainting is unimportant. The distended veins are now separated by the thumb and finger of the left hand, grasping the scrotum from before backward, and the vas deferens, which may be easily distinguished by its whip-cord-like feel, separated from the package of veins and carried to the inner side. The Reverdin needle is now charged with a piece of silk fourteen inches long, and dipped into a solution of 1 to 2,000 bichloride of mercury, and the needle, so charged, with a vigorous thrust is made to transfix the scrotum from before backward, between the veins and the vas deferens, as high up upon the cord as it is convenient to operate. This needle is left in this its first position. Without delay the veins near the globus major are in a similar manner separated to the onter side of the vas deferens by the thumb and finger, which again grasp the scrotum from before backward, lower down than the first needle, and a second needle similarly charged with silk is with another vigorous thrust cansed to transfix the compressed tissues and emerge posteriorly. This needle is also left hanging, and the thumb and finger pass beneath the testicle, and separate the veins that hang down below the globus minor, and between these veins and the globus minor the third needle is made to transfix the scrotum from before backward. This is the most difficult step in the whole operation, for here a vein may be pierced. By this time the patient is generally pretty faint. He is lifted up and placed upon a rubber cloth on the bed, flat upon his back. The punctures, the scrotum, the needles, and the silk are thoroughly irrigated with a 1-to1,000 solution of bichloride of mercury, and, commencing at the upper needle, each ligature is placed about the veins in the following manner: With a tenaculum the loop of silk is seized posteriorly, and the button of the needle being pressed in the handle, its eye is opened and the loop of silk released. This loop is now pulled through posteriorly until its short end emerges, and then the position is that the scrotum is transfixed from before backward by a disengaged single thread of silk and an uncharged needle. The eye of the needle is now allowed to close, and the needle itself, by widening the scrotum, is pulled forward, but its point is not permitted to emerge at the anterior puncture, while the veins are all allowed to fall away into their natural, original position alongside the vas deferens. Then the point of the needle is again advanced under the dartos, and to the outer side of the veins, and cautiously brought up to the point of posterior puncture, out of which the strand of silk protrudes. Here care must be taken not by a diagonal puncture to include any considerable bit of dartos, or any cutaneous tissue, and not to transfix the silk, but the point of the needle must be brought ont as accurately as possible exactly at the point of original posterior puncture, alongside of the silk. 
The parts are again freely irrigated with bichloride solution, and the eye of the needle being opened by pressure of the button in the handle, the silk is inserted into the eye, enough slack being left upon the silk to allow the needle to be easily extracted, and by a sudden rapid motion the needle, recharged with the silk, is withdrawn through the anterior point of puncture. The same manœuvre is executed upon the other two needles, and after all the three silks have been thus placed, the scrotum is again thoroughly irrigated with the bichloride solution. The two strands of the upper silk are now to be grasped with one hand, and the scrotum, near the upper, posterior point of puncture, caught with the thumb and finger of the other hand, and, by a rapid and violent motion, any little piece of dartos caught in the posterior loop of silk is torn away, the tearing being distinctly appreciated by the fingers. Each loop of silk is treated in this manner. Now another irrigation, and then the few hairs growing about the points of puncture through which the loops protrude anteriorly are pulled away, so that they may not be included in the knot. Finally, a square knot is tied, the first motion of ligation being executed with very considerable force. The pain accompanying this first application of the knot is sometimes very intense. The completion of the knot, of course, causes no pain ; and I habitually apply a third knot, so that I may cut my ends close and not fear that the knot may become untied. The two ends of silk are now cut off very close to the knot, and then, by pulling on the scrotum, after another irrigation, the knot disappears beneath the surface, and a subcutaneous silk ligature has been applied about the veins. Each of the knots is made in this manner; the points of puncture, if they bleed, dusted with iodoform or any suitable powder; the scrotum enveloped in dry cotton and supported by a $\mathrm{T}$ bandage. A little anodyne, a slight laxative, and a mechanical support for the testicle, complete the treatment. Each point of ligation swells underneath the scrotum until it attains perhaps the size of a walnutsometimes larger. The testicle itself swells, and becomes quite tense and moderately painful on the second day. By the fourth day the pain becomes very moderate, and usually on the fifth or sixth day the patient may apply a suspensory bandage, and go about without much discomfort. The inflammatory effusion about the ligature persists for several weeks, but slowly subsides without suppuration, leaving the silken ligature in place, where it may be felt as a painless cord about the obliterated veins for a very long period-exactly how long I do not know. I have felt it after two years. Of course, the entire success of this operation depends upon its aseptic performance.

Ablation of the Scrotum.-When the scrotum itself is so redundant and flabby that the surgeon thinks it ought to be removed, it may be taken away by pulling down whatever portion seems excessive, and 
clamping it with a Cooper or a Henry clamp on either side of the raphé, cutting off with the scissors the redundant material. Then the clamp is removed, the parts irrigated with a mild bichloride solution, all bleeding points scraped with the nail and ligated with catgut, and the cut edges on either side united with a continuous catgut suture, a small drainagetube being inserted at the upper and lower angles of the wound. The dressing is according to the customary surgical methods, and union by first intention may be confidently expected if the operation has been performed in a cleanly manner. The patient should be kept in bed for about ten days, that union may become quite firm before he goes about. 



\section{DISEASES OF THE SEMINAL VESICLES.}

By PAUL THORNDIKE, M. D.,

Our knowledge of the vesiculæ seminales dates back to the early part of the sixteenth century, when we find the first descriptions of them. It was not, however, until toward the end of the seventeenth century that the first careful dissections were made and the first accurate descriptions of these organs written.

\section{ANATOMY.}

The vesiculæ seminales are two irregularly convoluted tubes lying one on each side of the under surface of the male bladder and separated from the rectum by a layer of fascia (recto-vesical). They vary greatly in size in different persons, and even on different sides of the same person, but average perhaps two inches in length as they lie on the bladder. If dissected off and straightened out they are found to be two or three times this length. These tubes terminate posteriorly, near the points where the ureters enter the bladder, in blind pouches, and converge anteriorly, ending in ducts which open at the back of the prostate, each into the common ejaculatory duct of the same side. As they lie on the surface of the bladder, to which they are but loosely connected and from which they can be easily dissected, the posterior halves of them are covered by peritonæum. The vasa deferentia pass down from the internal abdominal rings to the base of the bladder along the inner sides of the two vesiculæ, and unite at an acute angle with the ducts of the seminal vesicles to form the common (ejaculatory) ducts. Just before this union takes place each vas deferens widens ont into a tortnous sac or "ampulla" which closely resembles in structure the seminal vesicle itself.

The vesicles are made up of three coats-an onter fibrous covering derived from the recto-vesical fascia; a muscular coat having an external layer of longitudinal fibers; an inner circular layer ; and a mucous membrane lined with columnar epithelium, and containing very small tubular glands.

The walls of the vas deferentia are thicker than those of the vesicles, and their canals are much smaller. Their structure is essentially the same, but the lining membrane does not contain so many glands.

The ejaculatory ducts pass upward and inward through the substance 
of the prostate to open into the prostatic sinus on each side of the verumontanum by small, slit-like openings.

The walls of the ducts grow thinner and their canals smaller as they approach their urethral termination.

The arteries of the vesiculæ seminales are branches of the middle hæmorrhoidal and inferior vesical, and the veins and lymphatics accompany them. The nerves help to form the hypogastric plexus.

Many curious anomalies of development of the seminal vesicles are recorded, and Guelliot gives a very complete account of them. He men-

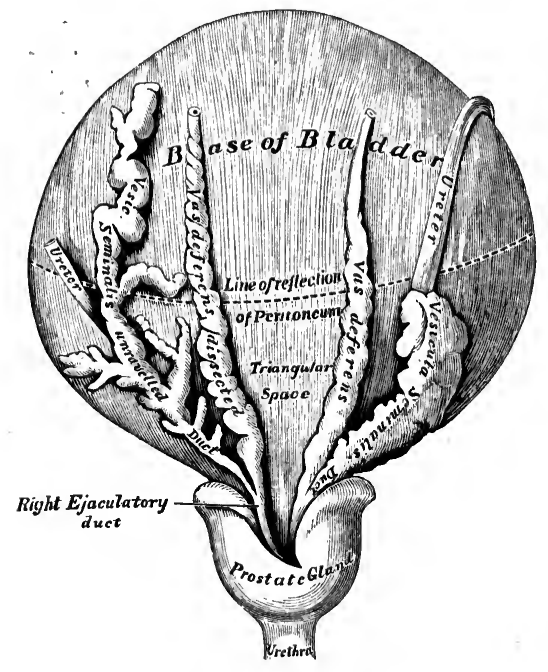

Fia. 1.-(From Gray.) tions nine cases where the two vesicles were not fully developed, two cases (both anorchids) where both vesicles were entirely wanting, and several cases where only one vesicle was present: In these last cases the corresponding testicle was normal, but the kidney was wanting, and the epididymis wholly or partly gone.

Two cases where the vesicles were fused into one are reported; but Guelliot thinks that both are open to question, as the dissections at the autopsies were not properly completed. Of 19 cases of anorchids collected by him, 12 had normal vesicles, while in the other 7 there was atrophy more or less marked, and in 6 the whole spermatic apparatus was wanting. Rott reports a case where the seminal vesicle was not connected with the vas deferens at all, but opened directly into the prostate, while the vas deferens opened into the ureter.

Weigert mentions an autopsy where the left ureter was found to empty directly into the left seminal vesicle.

The seminal fluid (semen) is a whitish fluid having a distinct odor of its own. It consists of a fluid vehicle (liquor seminis) which contains solid elements. This fluid is a clear, albuminous liquid, in which oilglobules and columnar epithelium may be seen. The solid elements of the semen are the spermatozoa and the seminal granules, the latter being small, round corpuscles always present in varying numbers, and of which further mention will be made. 


\section{PHYSIOLOGY.}

Of what use are the vesicles?

Ever since Fallopius wrote of these organs, nearly three hundred years ago; there has been endless discussion as to their function. Many writers have claimed that they are merely repositories for the spermatic fluid formed in the testes. Others say that they are something more than reservoirs-that they are secreting organs. true:

That the vesicles do secrete an albuminous fluid is undoubtedly

Their walls contain many albuminiparous glands, which secrete a fluid; and Guelliot mentions the case of a man without testicles, where the autopsy showed the vesicles to be full of such a fluid.

The fact that the vesicle does not atrophy after castration would seem to be additional evidence that it remains active in the absence of the testis. Atrophy does, however, occur in some cases after the removal of the testis, and Cruveilhier and Holmes both mention instances of such atrophy.

Carter thinks that the vesicular fluid serves as a vehicle for the testicular products. He says that at the time of ejaculation there is a vermicular action of the vas deferens and a contraction of the walls of the seminal vesicle, and that in this way the vesicular and testicular products are mixed together and poured into the urethra. This mingling of the two fluids is favored by the obliquity of union of the vas deferens and the duct of the vesicle.

That the vesicles are storehouses for their own secretions is true, but that they serve as receptacles for the semen formed in the testes is very doubtful. Kayser sums up the question very briefly and accurately. He had opportunities of examining the vesicular contents in seven cases after death, and found spermatozoa present in small but varying numbers in all cases. In concluding his thesis, he says that "the vesiculæ seminales are organs whose walls secrete an albuminous fluid; that they are recepta cles for their own secretions, whose physiological importance is not yet known; that in the contents of the vesicles we generally find some spermatozoa-sometimes a very few, sometimes a larger number-but that they are not so numerous or constant that one may consider the vesicles as receptacles for spermatozoa."

\section{INJURIES.}

The vesicles are so situated as to be very thoroughly protected from the effects of any external violence, and injuries to them, except as a result of surgical operations in their vicinity, are very rare, if not quite unknown. 
Lithotomy.-It is well known that perineal lithotomy, especially in young boys, may be followed by sterility due to injury of the vesicles or the ejaculatory ducts. It is for this reason that these operations are becoming less and less popular with the surgeons of to-day. Teevan reports four cases where the lateral operation for stone was followed by sterility, no emission taking place during connection. He says that the operation involves laceration of the floor of the prostatic urethra, and a consequent obliteration of the ejaculatory ducts, whose orifices are so close together that even a lateral operation is apt to injure both. $\mathrm{He}$ advocates very free perineal incisions, arguing that the laceration of tissue by the removal of the stone is thereby reduced to a minimum, and the risk of sterility greatly diminished.

Direct Violence.-As instances of injury from direct violence in this region are so rare, it seems proper to mention three cases spoken of by Holmes, as reported by Mr. Hilton. In all these the vas deferens was probably ruptured. The symptoms were: 1. A feeling of "sometling giving way" in the groin at the time of the injury. 2. Immediate swelling of the testis of the injured side. 3. Urine drawn from the bladder with a catheter perfectly clear, but that which flowed from the urethra was full of blood. 4. Recovery from gradual atrophy of testis. $\mathrm{Mr}$. Hilton thinks that in these cases the duct was torn across at a point between the internal abdominal ring and the place where the duct crosses the ureter. He mentions a case where at the autopsy he found an atrophied testis, and the vas deferens was torn across at the above point and the ends of it two inches apart.

Fistulæ.-Cases of pathological fistulæ involving the vesicles are almost never seen, but Lallemand mentions an instance where an abscess of the prostate involved the seminal vesicles and then broke into the rectum. The patient discharged his semen into the rectum at each connection, but at the end of two months a spontaneous cure took place.

\section{TUMORS.}

Malignant Disease.-Although the seminal vesicles are sometimes invaded by a cancerous process which started in some neighboring organ (generally the prostate), instances of primary malignant disease of these organs are very rare. Guelliot collected fourteen cases where the vesicles were the seat of cancer. Of these cases, seven were secondary to disease in the prostate, three started in the bladder, one started in the testicle, one started in the rectum, one was primary, one not accounted for.

This last case, the only one of primary cancer of the seminal vesicles on record, was reported by Méricamp (see Guelliot's thesis), and the autopsy showed a cancerous mass in the left vesicle, with nodules in the peritonæum. 
In May, 1891, Dr. G. W. Gay removed a seminal vesicle for malignant disease at the Boston City Hospital, and it is through his kindness that I am enabled to mention the case here.

The patient had suffered for several months with pain in the rectum and lower abdomen, had been troubled with constipation, and had lost considerable flesh and strength.

Rectal examination showed the prostate of normal size, or, if anything, rather smaller than normal, but the right seminal vesicle greatly enlarged, hard, with an irregular, roughened surface.

Under ether, an incision two inches long was made in the perinæum to the right of the median line and extending from the anus well forward to the scrotum. Careful dissection was made to the base of the bladder, and the growth was found to be not at all connected with the prostate, the latter being of normal size. The growth was firmly adherent, and could not be enucleated, but was removed as thoronghly as possible. A drainage-tube was left in the perineal wound. At the end of six weeks the wound was healed completely, but the symptoms were not much relieved. Since then the wound has broken open again, and now (November, 1891) there is a discharging sinus in the old scar, and rectal examination reveals a hard, finger-like mass in the situation of the right seminal vesicle. The microscope showed the growth to be of a cancerous nature, and as far as can be determined it was limited to the vesicle, and involved neither prostate nor bladder. The case is of especial interest, because (1) it was a case of cancer of the seminal vesicle (probably primary), and because (2) it is one of a very few cases where operation for the removal of a seminal vesicle has been attempted.

Guelliot believes that although so few cases of secondary cancer of the vesiculæ seminales are reported, the disease exists in many cases where it is not sought for or recognized, these organs being involved in many of the cases of a cancerous prostate.

Sarcoma.-Sarcomata of the seminal vesicles have been seen but once or twice. Zahn reports the only case of primary sarcoma of these organs. At the autopsy, which is most carefully detailed in his article, growths were also found in the kidney, heart, and mesentery; but Zahn believed the growth in the vesicle to be the primary one, because sarcomatous thrombi were found in the veins about the growth, and because no metastatic growth in the seminal vesical has ever been reported.

Coupland reports a case, with autopsy, of lympho-sarcoma of the prostate and seminal vesicles, with secondary nodules in the pancreas and suprarenal capsule.

Cysts.-Cysts of the vas deferens are occasionally seen, but cysts in the seminal vesicle have been noted but twice-by Ralfe and by Smith. 
Ralfe's case was unilocular cyst of the left vesicle, and reached nearly to the umbilicus.

Smith's case was equally as large, and was thought at first to be a distended bladder. The diagnosis was finally made by getting a fluctuation wave between the finger in the rectum and touching the tumor and the other hand on the abdominal wall. This cyst was tapped througli the rectum, and ten pints of fluid removed. A second tapping four wẹeks later resulted in a cure, as the cyst never refilled.

Several cases of hydatid cysts of the seminal vesicle were reported in the early part of this century by Lallemand and others, but their existence was by no means proved. Pinault, however, in 1826, reported a case of a large cyst between the bladder and rectum, which appeared to arise from the seminal vesicle to which it was attached.

\section{SPERMATIC COLIC.}

In 1874 Reliquet described a disease which he called colique spermatique, and reported a case which had come under his observation. This disease is caused by an obstruction more or less complete of the ejaculatory duct. It is rarely met with, but is of considerable clinical importance, as an accurate diagnosis may be easily made, and the cure may be promptly effected.

Symptoms. - The trouble begins with a sharp, cutting pain in the perinæum at the end of each sexual connection, or, if the patient be living a continent life, from time to time with each erection. Defecation is also painful. The pain is very severe, and of a similar character to the pain of hepatic or renal colic. If the stoppage of the duct is complete, there is of course no ejaculation at the end of coitus. With these symptoms are generally associated those pointing to irritation of the neck of the bladder and prostatic urethra. There may be blood in the urine. The passage of a sound into the bladder shows great hyperæsthesia of the prostatic urethra, and a finger in the rectum feels one vesicle much larger than the other, with a smooth surface and very sensitive to the touch. Fluctuation may be felt; there may also be felt a bean-like prominence on the side of the prostate corresponding to the obstruction in the duct.

Diagnosis. - The diagnosis, then, is to be made (1) from the characteristic pain, (2) absence of ejaculation and (3) symptoms of prostatic irritation, together with (4) rectal examination and (5) the examination of the urine. The examination of the urine will generally reveal the presence of numbers of small, whitish masses (the sympexions of Robin), which have been the cause of the obstruction in the duct. In Reliquet's case large numbers of these masses were seen in the urine, and Robin described them as opaline, transparent bodies, two millimetres in diameter, 
with edges more or less beveled. They are the spermatic granules of normal semen held together in a mucous vehicle.

Treatment. - In Reliquet's case the passage of a sound into the bladder was enough to dislodge these masses, and in this way to bring about an accidental cure. In a second article Reliquet describes a similar case, which was cured by crushing the obstructing masses between a sound in the urethra and a finger in the rectum, exerting pressure on the affected side. Two such treatments completed the cure.

Guelliot mentions two autopsies where these masses were found in the vesicles, and Bergh mentions another case.

Besides these typical cases caused by obstruction to the ejaculatory duct by these concretions, there occur cases of the same type but of less severity where there is no such complete obstruction. Here the trouble occurs in men who are living a continent life, and is probably due to a slight obstruction of the duct from inflammation in the prostatic urethra, affecting the ejaculatory ducts to a greater or less degree by inflammatory extension. The vesicle becomes distended with fluid, and the patient complains of the same colicky pain during and after defecation, and to a greater degree with some erections and nocturnal emissions. Regular sexual intercourse invariably effects a cure. Guelliot mentions such a case, and the writer has known of two others.

While speaking of these concretions formed in the seminal vesicles, it should be mentioned that true calculi similar to those found in the prostate have been found in the vesicles of old men at autopsies. Guelliot mentions eight such cases. These calculi are composed chiefly of phosphate and carbonate of chalk, are not very uncommon, and are of no clinical importance, as they give rise to no symptoms which can lead to a diagnosis.

\section{INFLAMMATION.}

Acute inflammation of the vesiculæ seminales (acute spermatocystitis) was recognized as a disease early in the present century, but Rapin's article (1859) gave us the first good description of it. The inflammatory process is the result of the direct extension of a posterior urethritis, and is therefore usually of gonorrhœal origin. That its existence is rarely demonstrated, is true; but it is undoubtedly often present where its existence is not suspected or looked for. Ricord says that in cases of gonorrhœal orchitis the seminal vesicle of the same side is almost invariably sensitive to the touch, and that inflammations of these organs would be much oftener found if oftener sought for. A mild form of the inflammation is doubtless the explanation of many of the cases of recurrent epididymitis and orchitis which are by no means uncommon; and the few autopsies which have been made upon patients who died during the course of an epididymitis confirm this idea. 
Etiology.-The inflammation, then, starts in most cases from a posterior clap, but may result from the extension of a retro-strictural inflammation, from inflammation due to urinary infiltration, or from a tubercular prostatitis. Jordan Lloyd says: "I have seen the vesicle inflame secondarily to urethritis set up by the passage of a bougie, by the presence of stricture, and after coitus with a leucorrheal woman. I have seen both vesicles suppurate in one case where their inflammation appeared to be primary; at least, there was no antecedent cause discoverable in the urethra."

Symptoms. - The symptoms are very like those of an acute prostatitis, and the trouble is often confounded with prostatic inflammation. There are present the pain and feeling of fullness in the perinæum, the frequent and painful micturitions, the painful defecations and erections.

There is usually a great frequency of seminal emissions, the vesicle discharging its contents at short intervals, as does the urinary bladder when it is acutely inflamed. These emissions are very painful, and the semen contains a greater or less quantity of blood. Rectal examination shows a vesicle larger and more sensitive than normal, the normal vesicle being scarcely appreciable to the examining finger in the rectum. These symptoms are often followed in a few days by an epididymitis on the same side, and this sequela is pathognomonic when present.

Diagnosis. - The diagnosis, then, must be made from the general history of the case, which will show, as a rule, a posterior urethritis as the cause of the trouble; from the painful and frequent emissions, with perhaps more or less blood in the seminal discharges; from the rectal examination; and, finally, from the epididymitis which so often follows the symptoms pointing to vesicular trouble.

If, however, the disease is of a mild type, and the symptoms which should localize the trouble are not marked in severity, one must rely largely upon the rectal examination, which, together with the general history of the case, will be enough to establish a diagnosis.

The inflammation may run its course, and subside without occasioning further trouble; may become chronic, and manifest itself only by an occasional exacerbation ; or may end in suppuration and abscess formation. The pus may point in the perinæum, or may break into the prostatic urethra, or even into the rectum.

The suppurating cases may result in a perivesiculitis, peritonitis and death, for it will be remembered that the vesicles are partially covered by peritonæum, and an inflammatory process in them may very readily extend to the peritoneal covering. Of such cases Gosselin reports one, Guyot one, Faucon one, Mitchell Henry one, Cock one, Velpean one, Lloyd two, and Sebeaux one. 
Purser speaks of a case where a purulent vesiculitis resulted in a general pyæmia, with abscesses in the heart, kidneys, bladder, etc.

Treatment. - The treatment of an inflamed vesicle should be palliative and symptomatic. Sitz-baths, poultices to the perinæum, with rest in bed and a bland diet, should always be enforced, and medication should be directed entirely to the relief of symptoms as they arise. If suppuration takes place, the pus should be evacuated as soon as found, either by puncture through the rectal wall, or preferably through a perineal incision, as recommended by Jordan Lloyd.

\section{TUBERCULOSIS.}

It is beyond doubt the belief of the medical world of to-day that tuberculosis is a general disease which shows itself, as a rule, by settling down in one organ or another, and giving rise to symptoms which point directly to that organ. The infecting material is evidently carried through the system in the circulation, and the disease manifests itself by symptoms which indicate a definite localization of lesions.

Genito-urinary tuberculosis is relatively a masculine disease, although it occurs in women oftener than is commonly supposed. It usually makes its appearance in middle life, when the sexual organs are in their most vigorous and active condition. The disease is thought by many writers to invade this region either from the kidney downward or the testicle upward; but Réclus always declared that the testis was never the seat of primary tuberculosis, but that the prostate and seminal vesicles were invariably invaded before it.

That the disease always marches onward in a definite line, involving one part after another, is certainly not true, for a study of cases shows no such uniformity of invasion. In fifty-nine cases collected by Guelliot the disease occurred in the seminal vesicles in one third of the cases when there was no disease of the prostate discoverable, and there are many recorded autopsies which show tubercular disease of urethra, prostate, and seminal vesicles with no invasion of bladder or testis. In these cases the primary focus was probably prostatic.

That certain parts of the uro-genital system are especially prone to infection in persons who are predisposed to tubercular troubles, is undoubtedly due to the fact that these localities are most often prepared for the invasion of the tubercle bacillus by an inflammatory process of longer or shorter duration.

Tuberculosis of the seminal vesicles is almost nerer seen except as secondary to the disease in neighboring organs. That it does occasionally originate in the vesicle is true; and Soloweitschik reports a most interesting case, with the autopsy in which he thought the primary disease was undoubtedly vesicular. 
At autopsies, tubercular disease of the vesicles is generally found in the form of caseous deposits which soften during life, and may break into the urethra, through the perinæum, or more rarely into the rectum. Occasionally one finds the walls of the vesicles studded with tubercular granulations, with little, if any, cheesy material.

In rare cases the patient may recover, the tubercular inflammation ending in a gradual fibrous degeneration and atrophy of the parts involved. More frequently, however, the softened products break through into the perinæum or prostatic urethra, forming sinuses which are constantly discharging.

Symptoms. - The invasion of the seminal vesicles by a tubercular process can be determined only by a rectal examination, combined with a general history of the case. The examining finger will find the affected vesicle larger and harder than the other; and, as the disease progresses, a tendency to soften will be noted at successive examinations before any fistulous traces are found.

Impotence and sterility almost always result in cases of long standing, but before these symptoms appear there is often a stage of great sexual excitability, with frequent seminal emissions, and a semen containing larger or smaller quantities of blood.

Diagnosis. - The general history of the case, the local symptoms, and the rectal examination will generally lead to a correct diagnosis, and a microscopic examination, showing tubercle bacilli in the semen, will confirm the diagnosis in some cases.

It must be remembered, however, that marked remissions, characterized by a subsidence of the local symptoms, and an improvement in the general condition of the patient are by no means uncommon in cases of tuberculosis in this region, and may prove very deceptive.

Treatment.-It should also be borne in mind that any instrumentation in cases of this kind is very apt to be followed by a marked increase in the severity of all the symptoms. This intolerance of any instrumental interference may give the surgeon his first suspicion as to the character of the disease with which he has to deal.

The treatment should, of course, be such as is followed out in tubercular disease of other organs. Change to a suitable climate, with proper care as to diet, fatty foods, etc., will accomplish a great deal in some cases. The question of operative interference in cases of genito-urinary tuberculosis with a hope of effecting a radical cure by the removal of the diseased areas is a very interesting one, but we are not yet in a position to advocate or condemn the procedure. The opinion of the conservative surgeons of to-day seems to be against any attempt of this sort. In a paper read before the American Association of Genito-Urinary Surgeons, in June, 1890 , Dr. Bryson, of St. Louis, sums up this question as follows, basing 
his conclusions on a study of eighty-four cases and twenty-three operations. He says: "That all the evidence points to the conclusion that we have to deal, even in its earliest recognizable stages, with a general disease which manifests itself by localizations; that present experience does not warrant the belief that operations, however extensive, undertaken with the view of excising localized lesions, and thereby radically curing the disease, will be successful; that our chief resource is still in general antitubercular treatment, reserving surgical interference for the palliation and relief of pain, bleeding, and wasting suppuration."

The results obtained from the use of Koch's fluid in cases of genitourinary tuberculosis have been for the most part either negative or distinctly unfavorable.

But one radical operation for the removal of a tubercular seminal vesicle has been recorded. Ullmann removed successfully the right seminal vesicle for tubercular disease, secondary to that in the epididymis. The

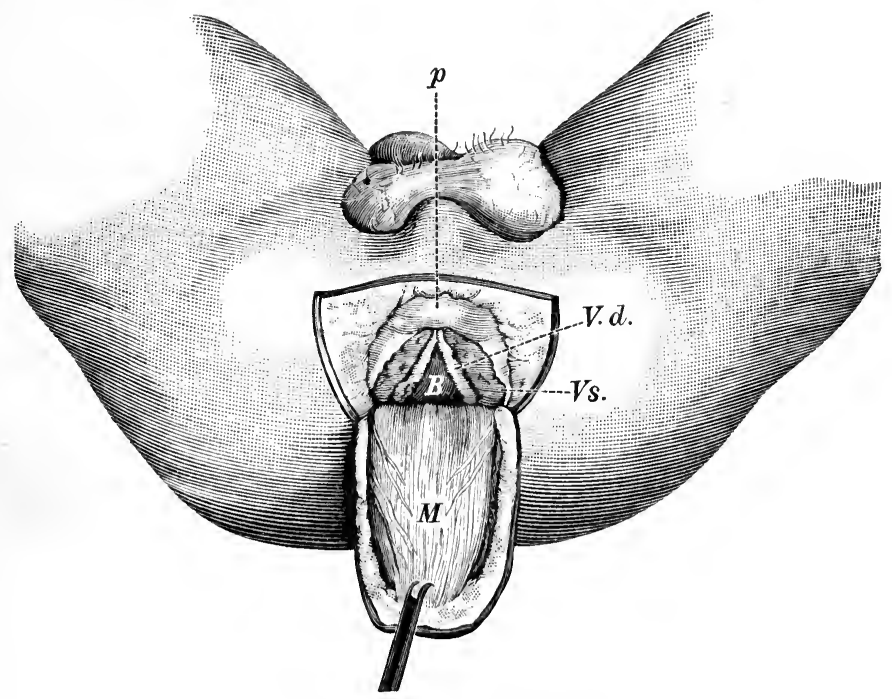

Fig. 2.-(From Zuckerkandl.)

$p$, prostate; $V . d$, vas deferens ; $V . s$, seminal vesicle $B$, bladder; $M$, rectum.

patient was a boy of seventeen years, with a right-sided tubercular epididymitis of several months' standing. Rectal examination showed the right seminal vesicle twice too large and very hard, while the left vesicle was apparently normal. On June 17 th the testicle was removed, and the vas deferens found pretty extensively infiltrated with tubercle; on July 27 th the seminal vesicle was removed by Zuckerkandl's method. Ullmann describes the operation as follows: "A semicircular incision is made in the perinæum, with its base toward the sacrum. Then, with an assistant's 
finger in the rectum as a guide, the parts are carefully dissected, the part of the levator ani which comes from the os pubis is divided and so the prostate is laid bare in the wound. Then the rectum is exposed a little more fully, and the base of the bladder brought more into prominence by means of a stone searcher introduced into it through the urethra. By this means the vasa deferentia and the seminal vesicles are brought well into view and may be dissected off.

"In Ullmann's case the right vesicle was found enlarged, cheesy at its upper part, and infiltrated below with tubercle. The vas deferens was also infiltrated. The left seminal vesicle was found somewhat enlarged and indurated. Both vesicles were therefore carefully dissected away, but the left vas deferens, which appeared healthy, was not removed. In the left upper corner of the prostate was a small abscess, and so this part of the prostate was also removed. The bleeding was readily controlled, a drainage-tube inserted, and the wound closed. There was some hæmorrhage that night, and so the wound was opened and plugged with iodoform gauze. During the convalescence some urine flowed through the wound, probably through the right ejaculatory duct, which was cut across with a knife during the operation. The convalescence was uninterrupted, and at the time the case was reported the patient was in excellent general condition, and the wound was all closed except one small fistula. Urine was passed entirely through the urethra, and the patient had had two erections since the operation. The microscopic examination verified the diagnosis of tuberculosis."

In commenting upon the operation, Ullmann says it should be performed-

1. In cases of primary tuberculosis of testis or epididymis at a time when no suspicious symptoms have appeared in the healthy side, but when the vesicle is involved on the diseased side. If both vesicles are involved, but one vas deferens is found healthy, the latter side should be left alone, both vesicles being removed.

2. In cases of primary tuberculosis of the seminal vesicle.

He says the resulting impotence should not militate against the operation, for in all reported cases of vesicular tuberculosis we find that impotence is sure to appear as a cardinal symptom of the disease.

\section{BIBLIOGRAPHY.}

A most accurate and complete bibliography of all the literature on this subject up to 1882 will be found in Guelliot's article on the diseases of the seminal vesicles.

Guelliot. Thèse de Paris, 1882.

Rott. Schmidt's Jahrbuch, 1879, vol. clxxxiii, p. 10.

Weigert. Virchow's Archiv, civ, 1, 1886, p. 10.

Cruveilhier. Anatomie, vol. ii, p. 375 . 
Holmes. System of Surgery, second edition, vol. ii, p. 739.

Carter. Glasgow Medical Journal, 188\%, vol. xxvii, p. 332.

Kayser. Untersuchungen über die Bedeutung der Samenblasen. Inaugural Dissertation, Berlin, 1889.

Teevan. British Medical Journal, 18\%4, p. 586.

Méricamp. Vide Guelliot.

Englisch. Medizinische Jahrbuch von Stricker, Wien, 1875, p. 127.

Gross. On the Urinary Organs, second edition, p. 721.

Chopart. Traité des Maladies des Voies urinaires, Paris, 1821.

Flemming. Dublin Hospital Gazette, 1858.

Heath. Transactions of Pathological Society, London, 1862, xiii.

Wyss. Archiv de Virchow, 1866, xxxv, 389.

p. 222 .

Berger. Cancer des vésicules séminales, etc. Bulletin Soc. Anat., 1871,

Zahn. Deutsche Zeitschrift für Chirurgie, 1885, v, 22.

Coupland. Transactions Pathological Society, London, 187\%, vol. xxviii.

Ralfe. Lancet, London, 1876, vol. ii, p. 782.

Smith. Lancet, London, 18\%2, vol. ii, p. 558.

Lallemand. Des pertes séminales involuntaires, Paris, 1836.

Pinault. Vide Guelliot.

Reliquet. Gazette des Hôpitaux, 1874; 1879, p. 891.

Bergh. Schmidt's Jahrbuch, 1879, vol. clxxxi, p. 35.

Rapin. Thèse de Strasbourg, 1859, No. 491.

Klebs. Handbuch der patholog. Anat, Berlin, 1863.

Kocher. Pitha u. Billroth, Bd. iii, Abth. ii, p. 463.

Horavitz. Wien. medizinisch Presse, 1889, xxx, 1313, 1349.

Finger. Wien. medizinisch Presse, 1885, Nos. 19-23.

Parkin. Lancet, London, October 17, 1891.

Humphrey. Holmes's System of Surgery, vol. v, p. 169.

Jordan Lloyd. Lancet, London, October 31, 1891.

Jordan Lloyd. British Medical Journal, April 20, 1889.

Gosselin. Clinique chirurgicale de la Charité, 18\%6, t. ii, 65\%.

Guyot. Vide Guelliot.

Faucon. Archives générales, October, $18 \% \%$.

Mitchell Henry. Med.-Chir. Transactions, London, vol. xxxiii, p. $30 \%$.

Cock. Article in Ashurst's Surgery.

Velpeau. Medico-Chirurgical Review, London, 185\%, vol. i, p. $25 \%$.

Sebeaux. Contractures du col de la vessie, etc. Thèse de Paris, 1876, No. 134.

Purser. Dublin Journal of Medical Science, 187\%, lxiv, 553.

Soloweitschik. Archiv für Dermat. u. Syph., 1870, p. 1.

Bryson. (Review) Journal of Cutaneous and Genito-Urinary Diseases, New York, July, 1890.

Ullmann. Centralblatt für Chirurgie, February 22, 1890. 



\section{FUNCTIONAL DISORDERS OF THE MALE SEXUAL ORGANS.}

Br PRiNCE A. MORROW, M. D.

UNDER the general class of functional disorders of the reproductive apparatus are comprehended the three affections which form the subject of this article: spermatorrhœa, impotence, and sterility. They are not, strictly speaking, functional diseases, as they may owe their origin to structural changes; nor do they always have a separate and independent existence, since they are oftentimes merely symptomatic of some local or constitutional state. Although they have many points in common in connection with their etiology, symptomatology, prognosis, and treatment, they are essentially distinct in their pathological import and significance.

Spermatorrhœa has reference to abnormal evacuations of the seminal fluid.

Impotence implies loss of virility or copulative power.

Sterility involves absence or pathological modifications of the semen.

All three morbid conditions may coexist in the same individual, or either may be present, independently of the others.

\section{SPERMATORRHEA.}

Definition. - Spermatorrhœe will be employed in this article as a generic term to denote all abnormal or pathological losses of the seminal fluid. As is well known, disorders of the genital function characterized by abnormal seminal losses exist in different forms and degrees of severity, and by most modern writers the application of the term spermatorrhœa has been restricted to those cases which represent the highest form, the superlative degree of seminal incontinence. While recognizing this distinction, it seems perfectly proper, since the greater includes the lesser, to group under a common heading different types of the same disorder.

Classification.-The several forms of seminal incontinence may be conveniently classified as follows:

1. Nocturnal pollutions. 2. Diurnal pollutions. 3. Spermatorrhœa, true and false. 
Nocturnal Pollutions.-By nocturnal pollutions is understood a more or less frequent evacuation of the seminal fluid which takes place at night, ordinarily under the influence of erotic dreams, attended with erection and specific sensations. In more aggravated cases the emissions occur without erection, without pleasurable sensations or sufficient excitement to awaken the sleeper, who may have only a confused recollection, or, indeed, no knowledge of the accident except that furnished by visible traces of the discharge upon his linen.

Diurnal Pollutions. - This term is used to designate seminal losses occurring during the day without sufficient mechanical cause, as coitus or masturbation. Diurnal pollutions take place under the influence of various slight causes, mechanical or psychical, and are attended with full or feeble erection and diminished sensation.

Spermatorrhœa.-In its strictest acceptation this term is applicable only to those cases in which loss of the seminal fluid takes place without erection or sensation. The semen, instead of being discharged in a forcible jet by the reflex contraction of the ejaculatory apparatus, escapes in a purely passive manner, and oftentimes without the knowledge of the individual.

False Spermatorrhœa.-By this term is understood the discharge of a clear, viscid fluid from the meatus which does not contain spermatozoa, with or withont erection or erotic excitement. It may appear at the meatus after straining at stool, the expulsion of the last drops of urine, or during exercise and certain movements. It is usually interpreted by the patient as an evidence that he is losing his seminal fluid, and not infrequently leads to a train of mental symptoms which are scarcely secondary in importance and gravity to those resulting from true seminal loss.

While the above division is convenient for purposes of description and study, it is evident that a strict line of demarcation can not always be established between the forms of seminal incontinence, since they represent different stages of the same morbid state, modified in degree according to the duration and progress of the case.

Between nocturnal and diurnal pollutions a clear line of distinction can be drawn, since the former always occur during sleep, while the latter take place when consciousness and volition are active ; but between diurnal pollutions and spermatorrhœa the distinction is not always possible, since from temporary causes erection and orgasm may at one time be present, at another absent. When the stage of spermatorrhœa is fully developed, pollutions usually cease, but not always; they may continue to recur at intervals, sometimes the symptoms of pollutions, at other times those of spermatorrhœa, predominating. The only constant basis of differentiation between the two forms is the element of consciousness. Un- 
conscious pollution would properly express the pathological condition present in spermatorrhœa.

Ultzmaun, who classes all abnormal seminal losses under Motor Neuroses of the Sexual System, defines pollution as a neurosis of the sexual apparatus with spasm of the muscular coat of the seminal vesicles, and spermatorrhœa as a similar neurosis with paresis of the ejaculatory ducts.

Pathological Significance.-Recognizing that the mechanism of the production of seminal losses depends essentially upon a disordered innervation of the motor apparatus concerned in the evacuation of the seminal fluid, there remains for us to inquire into their pathological significance-In what degree are they to be considered unhealthy or abnormal? Here we shall find it extremely difficult to differentiate between the physiological and the pathological. Occasional aberrations in the mode of function of an organ are not necessarily pathological, since they may be neither the cause nor the consequence of disease. The mere fact of the occurrence of seminal evacuation without normal coitus does not necessarily establish it as a pathological phenomenon.

Every healthy adult male whose organs are in a state of physiological activity, especially if he leads a continent life, is liable to have nocturnal pollutions. Occurring within certain limits of frequency, attended with strong erections and pleasurable sensations, and followed by a sense of relief, such emissions are essentially physiological. They may be interpreted as the expression of a compensatory effort on the part of Nature to relieve the seminal plethora accumulated by the ceaseless activity of the seminiferous organs. Such occasional overflows of the seminal fluid, due to the overdistention of the seminal ducts and vessels, should be recognized as an indication of the normal functional activity of the organs, and in no sense harmful; their frequent repetition and their associated constitutional effects constitute the sole evidences of a diseased condition.

Certain authorities state broadly that when pollutions occur once in ten or fourteen days they may be looked upon as physiological ; if they occur much oftener, they are pathological. The element of frequency alone can not, however, be accepted as a test of the physiological character of seminal emissions. It is difficult to generalize in a matter of this kind, or formulate a rule of universal application. Precisely as, in normal coitus, the frequency with which the act may be repeated within the limits of perfect health varies widely in different individuals, so also in the case of involuntary seminal emissions. The pathological influence of the absolute seminal loss is determined largely by the constitution of the individual : what might be excessive and harmful in one, may be supported by another with perfect impunity.

The effect of seminal loss upon the individual constitutes the surest test or measure of its normal or pathological character. Pollutions at- 
tended with that feeling which follows the gratification of a physical want, must be considered as healthful and sanative, irrespective of their frequency. On the other hand, when they are followed by languor, headache, backache, or other impairment of physical or mental vigor, they must be regarded as pathological, even though they occur quite infrequently and at long intervals.

Unfortunately, when nocturnal pollutions are fully established, under the influence of various causes, even from the force of habit alone, they have a tendency to become relatively more frequent, with the development of a train of mental and physical symptoms to be considered in connection with the symptomatology of seminal incontinence, and the patient passes from the boundaries of health into the confines of disease.

While it is impossible to draw a sharply defined line between normal and abnormal nocturnal pollutions, there can be no question concerning the pathological significance of diurnal pollutions. A healthy man may lose semen when asleep; a healthy man does not have an emission when awake, except as a result of coitus or some sufficient mechanical cause. Mere desire and venereal excitement, no matter how intense, are not sufficient in a state of health to provoke spontaneous ejaculation; there must be a peripheral impulse or excitation in the shape of mechanical friction. It may be positively affirmed that diurnal pollutions occurring during consciousness, when the will-power is active, except under the conditions just mentioned, always imply an abnormal excitability of the genital center or weakness of the motor apparatus, and are necessarily pathological.

As regards the pathological significance of seminal loss without erotic excitement, erection, or sensation, we must make a distinction between what may be regarded as a physiological accident and the symptom of a confirmed disease. The fact of a drop of semen appearing at the urethral orifice after defecation, especially after straining and the passage of a hard stool, is not of grave import. It is purely a mechanical phenomenon, due to the pressure of the hardened mass upon the seminal and prostatic ducts, and has no more significance than the milking of the seminal ducts for diagnostic purposes by the mechanical pressure of the finger introduced into the rectum. Men whose organs are in a perfectly healthy condition may have this experience. When, however, this seminal flow, instead of being an occasional accident, occurs habitually, with soft as well as hard fæces, without straining, and during the act of micturition, and is, in fact, more or less continuous, it constitutes the worst form of seminal incontinence.

Etiology.-Seminal incontinence has a most diverse etiology ; many constitutional as well as local factors are concerned in its causation. In certain cases it is merely a symptom of some constitutional state, or the expression of a nervous disorder, which later may manifest itself in the 
graver forms of epilepsy or insanity. Myelitis or other lesions of the cord or brain are not infrequently attended with abnormal seminal losses. In view of the sympathetic lines which unite the genital and nervous systems, it is not surprising that functional disorders of the former occur in connection with a great variety of nerve lesions, both peripheral and central. The morbid tendencies may be excited into action by either direct or reflex irritation of the most diverse character.

Disturbances of the nutrition, resulting in various forms of nerve exhaustion, act as predisposing causes by weakening the capacity of resistance and deranging the equilibrium of the reflex centers. Seminal losses occur in the course of many acute and chronic diseases as a result of anæmia and nervous debility. The proximate cause may be purely local, as seen in the pollutions which occur as the result of an abnormal sensitiveness of the prostatic urethra, induced by gonorrhœal inflammation or other forms of local irritation. Many other local disorders of the genital and perigenital regions, presently to be referred to, act as predisposing or proximate causes.

An inherent weakness and irritability of the genital passages and of the nerves which control the motor apparatus may act as a powerful predisposing cause. The important rôle which heredity plays in determining disorders of the genital function has not been recognized or sufficiently emphasized by writers upon this subject. A parent may transmit a weak, irritable sexual organization to his offspring, just as weak eyes, feeble digestion, weak lungs, or other organic defects may be inherited. The genital systems of different individuals differ as much in their constitution and proneness to derangement of function as do the digestive and other systems. The motor neurosis, which in childhood is characterized by incontinence of urine, may continue to be manifest after puberty by seminal incontinence; as pointed out by Trousseau, one morbid condition may succeed to and replace the other.

Of especial interest in connection with the etiology of spermatorrhœa is the undoubted influence of heredity in creating a disposition or inclination to the practice of masturbation. Unquestionably the tendency to this habit, as to other vicious habits, may be transmitted from parent to offspring.

Masturbation.-First in point of importance, as well as of frequency, is the practice of masturbation, or rather self-abuse, since the means employed for the production of the venereal orgasm upon one's self are not always such as are implied in the strict etymological sense of the word masturbation, or friction by the hand.

The sweeping statement made by certain authors that all male human beings at some time practice masturbation, is no doubt an exaggeration of the truth. Yet it can not be doubted that a large proportion of young 
men begin their sexual life as masturbators. The pernicious and ruinous effects of this solitary vice, not only in the production of spermatorrhcea but other functional disorders of the genital system, have been painted in the most lurid colors by ignorant pretenders who cultivate this field of quackery. On the other hand, the evil effects of "solitary pleasures" have been glossed over or minimized by text-book authorities, who claim that the seminal loss from masturbation is no more injurious than that of normal coitus. Sir James Paget asserts that the injurious effects of masturbation have been exaggerated, and that seminal loss through this practice entails no more ill effects upon the constitution than that through normal coitus exercised with the same frequency.

A priori, it might be assumed from analogies in the mechanism of masturbation and coitus, both involving a mechanical cause, that their effect upon the individual would be identical. This, however, is by no means the case; the nervous exhaustion and other reactionary effects upon the general system which follow the unnatural act are much more pronounced. In addition, there are other circumstances or conditions ordinarily connected with the practice of masturbation which have a tendency to aggravate or intensify its harmful effects.

In the first place, the habit is usually formed at an early age, often before puberty, when the sexual organs are immature and undeveloped, and entirely unfitted for this demand upon their functional activity. The injurious effects of this precocious and excessive use of rudimentary organs are oftentimes manifest in arrest of development, a retarded and stunted growth, so that long after the period of puberty the external genitals present an aspect of infantilism. Most masturbators who have begun the practice early and carried it to excess, have small and undersized organs. The effect of this early excess upon the composition of the semen and the functional vigor will be considered in connection with impotence and sterility.

In the next place, the very nature of the habit and the facility of its performance render its indulgence inordinately frequent-vastly more frequent than is possible with coitus, where not only consentment and co-operation of another party, but favoring time and opportunity, are requisite. To the devotee of this solitary vice the external factor as well as the opportunity are always present; there being no hindrance, no bounds are set to the indulgence. The first effect of this repeated indulgence remarked by the patient is a precipitation of the ejaculation, which he is apt to regard as an evidence of increased vigor, but which, rightly interpreted, is due to the congestion of the prostatic mucous membrane and an irritability of the reflex centers, so that abnormally slight causes are sufficient to bring about the spasmodic contraction of the seminal vesicles and ejaculatory ducts. When this pathological condition is established, in- 
voluntary emissions begin to appear, and continue with progressive frequency, it may be, long after the habit which occasioned them has been abandoned.

Sexual Excess.-After masturbation, sexual excess ranks as the most common cause of spermatorrhœa. The exercise of the sexual organs within certain bounds undoubtedly has the effect of strengthening, invigorating, and preserving them in their full integrity; their excessive exercise or abuse, on the contrary, engenders disorders of function, oftentimes associated with pathological changes in the organs involved. No absolute or even approximate rule can be formulated with regard to the frequency with which the sexual function may be exercised within physiological limits. A frequency of repetition which might constitute sexual excess in one individual may come within the physiological capacity of another more strongly sexed. The effect upon the system must be accepted as the sole test of its normal or abnormal character.

Since, as before premised, most men are initiated into sexual life by masturbation, the injurious effects of which continue to be manifest long after the habit has been relinquished, it is evident that in appreciating the pathological results which follow natural sexual excess the morbid impulse communicated by the former practice must act as a strong coefficient. Indeed, it is often impossible to disassociate these two pathogenic factors which determine analogous if not identical effects.

It is still a question whether the harmful result of sexual excess is due to the absolute loss of a highly elaborated "vital" fluid, or whether it is to be attributed to the intense nervous excitement engendered by the orgasm. If the seminal fluid ejaculated has been stored up in the seminal vesicles, and therefore stands in the relation of an excreted product to the general system, its mere evacuation should be a matter of slight or indifferent import. On the other hand, if the injurious effect of sexual excess were due solely to the explosion of nerve force attending ejaculation, women, in whom this excitement of the nervous system may be quite as intense, should also suffer bad effects. It is well known, however, that women may experience the "cynic spasm" in frequently repeated coitus without any sense of exhaustion. Then, again, in the advanced stage of spermatorrhoea, escape of the semen takes place without any nervous excitement, or, indeed, apparently without the active participation of the nervous system. In this class of cases it is fair to assume that the constant spermatic drain causes exhaustion of the nerve-centers, just as the saccharine drain of diabetes impairs the nutrition of the general system.

Sexual excess, whether by natural or unnatural practices, determines an irritable weakness of the genital organs. As a result of the frequently repeated erections and abnormal stimulation of the genital canals there are induced a hyperæmia and hyperæsthesia of the mucous membrane of the 
prostatic urethra. This constant congestion causes a chronic or subacute inflammation of the pars prostatica, analogous to the catarrhal inflammations of other mucosæ from long-continued irritation. The introduction of a sound shows the urethra to be inflamed and sensitive. The urine is often found loaded with shreds consisting of pus-corpuscles, epithelium, and occasional spermatozoa. Under endoscopic examination the mucous membrane appears swollen, reddened, and partly deprived of epithelium. The ejaculatory ducts also participate in these morbid changes; their walls become enlarged and relaxed and their orifices patulous, permitting a ready escape of their contents.

Associated with these morbid changes in the motor apparatus there is engendered, by frequently repeated stimulus, an irritable condition of the efferent nerves which convey sensations to the ejaculation center, conjoined with an abnormal excitability of the reflex cerebral and spinal genital centers, as a consequence of which the impulse to reflex discharge is set in motion by abnormally slight causes, hence pollutions and prema. ture ejaculations.

Continence.-The etiological significance of continence in relation to abnormal seminal losses is differently appreciated by different authors. The impulse of the genital organs to act is a law of Nature, and is expressed in physiological pollutions. In persons of nervous, excitable temperament, especially when the sexual instinct is stimulated and kept in unnatural excitement by lascivious thoughts and external impressions, pollutions not infrequently surpass the physiological limit and become pathological; a condition of mental masturbation is thus established. Many young men who lead continent lives and yet have involuntary emissions become possessed, from reading quack literature, of the most extravagant sexual ideas. They see in every emission an evidence of loss of virility. The constant concentration of mind upon the genital function, and the exaggerated fear of having pollutions, constitute a most efficient cause of their production. Sexual hypochondriasis is more frequently the cause than the effect of pollutions.

Continence as a provocative to masturbation may be considered indirectly a cause of abnormal seminal losses. Young men of virtuous instincts and studious tastes, theological students and others, often drift into the practice of masturbation, imagining that they preserve their purity while indulging in this vicious habit.

Local Causes. - Since abnormal seminal losses originate in congestive or inflammatory changes in the prostatic mucous membrane and ejaculatory ducts, it is evident that any condition which determines an irritation of this region will contribute to their production.

Phimosis may prove a source of reflex irritation from the pressure of a tight foreskin or the presence of retained smegma. Balano-posthitis, 
herpes, vegetations, etc., may also cause pollutions in persons predisposed thereto.

Various lesions of the urethral canal act as exciting causes. Gonorrhœal inflammation especially plays an important rôle in the genesis of pollutions, especially when it has penetrated the deep urethra. The inflammation creates a condition of increased irritability, by which spasmodic action of the expulsive apparatus is more readily excited. The common occurrence of pollutions in the course of urethritis, especially when complicated with posterior urethritis, prostatitis, epididymitis, inflammation of the vasa deferentia, etc., is constantly referred to in the article on. Urethritis. Acute prostatitis, or a chronic inflammatory state of the neck of the bladder from any cause, favors pollutions by inducing a morbid irritability of the prostatic ducts.

Stricture of the urethra at any point of the canal may be a cause of pollutions. The irritation may be set up by the pressure of decomposed urine retained behind the stricture. Congenital narrowing of the meatus, as well as spasmodic stricture, may act as exciting causes.

Inflammation of the seminal vesicles produces pollutions; in acute spermatocystitis the seminal discharge is usually painful, and mixed with blood and pus.

Contusions of the perinæum, followed by acute inflammation, may determine pollutions. Abnormal states of the rectum and anus, as hæmorrhoids, ascarides, eczema, fissures, pruritus, may cause sympathetic contractions of the seminal vesicles and expulsion of their contents. Any coincident inflammation of the pelvic organs attended with disorders of the nerves of that region may react upon the nerves of the genitalia and provoke pollutions.

Symptomatology.-The symptoms of spermatorrhœa are as diverse as are the causes which produce them; they vary according to the form of the disorder, its degree of development, and the constitutional peculiarities of the individual.

Course.-Spermatorrhœa is by no means typical in its course and mode of evolution. In the ordinary evolution of a spermatorrhœea which owes its origin to masturbation the beginning of the trouble is markèd by the occurrence of nocturnal pollutions; these may be succeeded by diurnal emissions, and finally the loss of seminal fluid takes place without erection or any form of erotic excitement. At first the nocturnal pollutions occur at comparatively rare intervals, and are attended with erection and normal sensation. After a time they are reproduced more frequently, until they may occur several times a week, or even more than once the same night. With this increased frequency of repetition there is a progressive enfeeblement of erection, and emission takes place from a flaccid organ without sufficient sensation to arouse conscionsness; the individual awakes and 
finds himself polluted. The normal sensation peculiar to the discharge of semen is not infrequently replaced by a disagreeable sense of heat and burning in the urethra.

When the disorder is more fully developed, involuntary emissions occur when the patient is awake. In an early stage the ejaculation may be attended with strong erections and specific sensations; in a more advanced stage it occur's with partial or incomplete erection, even when the organ is limp and flaccid, and is attended with perverted sensation.

A variety of slight causes may determine ejaculation. Any peripheral irritation, as friction of the glans from movements of the body, riding on horseback, or any slight mechanical irritation of the genital organs, may be sufficient to provoke it, or the impulse may come from a remote organ. Shampooing, shaving, or simply brushing the hair have been known to occasion an emission.

Purely psychical impulses, such as the desire excited by the presence or contact with women, the sight of obscene pictures, lascivious thoughts, the lewd images created by the imagination, may be sufficient to discharge the reflex act of ejaculation. In a more advanced stage, which marks the development of true spermatorrhœa, the loss of semen takes place without erection, sensation, or even without sexual thought or desire. While this loss is more apt to occur during the act of defecation or micturition, there may be an almost continuous escape of seminal fluid.

It is by no means to be assumed that the clinical history of all patients affected with abnormal seminal losses exhibits this progressive course; in the vast majority it ends with the stage of nocturnal pollutions, in a small minority it passes to the stage of diurnal pollutions, while in comparatively few does it culminate in true spermatorrhœa.

The pathological effects of abnormal seminal losses are ordinarily manifest in local inflammatory changes, and, secondarily, in affections of the nervous system or disturbances of the general nutrition.

Local Effects.-The local changes expressed in irritable weakness and derangement of the motor expulsive apparatus, and which are closely related to impotence, have already been referred to. No less significant are the changes in the character of the seminal fluid, which have an important bearing upon the production of sterility. As a result of the abnormal demand upon the secretory activity of the generative organs, the composition of the semen undergoes marked modifications. While the quantity may not be materially diminished, the quality is deteriorated. It becomes thinner in consistence, more watery, and less opaline, resembling lymph. The spermatozoa may be diminished in number, altered in quality, or entirely absent. They are small, immature, malformed, often motionless, and feebly charged or entirely deficient in fecundating power. 
The condition of the external genitals presents nothing absolutely characteristic. As a rule the organs are smaller, with less appearance of vigor. Many writers have spoken of the hardness of the corpora cavernosa as pathognomonic, but this is by no means a constant condition. The skin of the penis and the testicles is less sensitive to electrical stimulation; the urethra is hyperæsthetic, especially the pars prostatica, which explains the burning sensation often attending the emission of semen. In a more advanced stage of the malady, there is, coincident with a decline of the inflammatory condition, a gradual decrease of this disagreeable sensation in the prostatic and perineal region.

Constitutional Effects. - The pathological effects of abnormal seminal losses upon the general system present the greatest variations. The immediate effects of pollutions are certain subjective sensations-a sense of malaise, great fatigue, headache, backache, mental dullness, etc. In the early stage these sensations are ordinarily of short duration, and may entirely disappear in the intervals between the emissions.

The more profound disturbances of the general health of the individual which make up the clinical picture of spermatorrhœa depend largely upon constitutional peculiarities. In many cases the signs of the disorder are localized in the genital apparatus, the pollutions constitute the whole disease, while the general health is comparatively unaffected; in other cases emissions occur but rarely, and the entire train of morbid symptoms is expressed in nervous and nutritive disorders. Paradoxical as it may appear, the strongest and most robust individuals are often those in whom the local disturbances are the most severe. The etiological explanation is that their stronger physical organization permits of greater abuse, while in persons of feebler endowment the system remonstrates by symptoms that can not be disregarded before the abuse is carried to such harmful excess.

In an advanced stage of the malady the motor and sensory disturbances are more generalized. A condition of nervous irritability supervenes; the patient is restless, can not sit still or remain long in any position. This disposition to move, notwithstanding muscular fatigue, has been pointed out as pathognomonic by various observers. In more aggravated cases involuntary twitchings, trembling of the hands and limbs, and other choreic symptoms are developed; the gait is unsteady and vacillating.

Sensory disorders are also well marked. The subjective sensations which immediately follow pollutions become more constant. The patient is subject to various neuralgic pains which are exceedingly capricious, hyperæsthesia of one part succeeded by numbness, formication, hot and cold flashes, extremities cold and clammy, sensations of heat and heaviness in the occiput, tenderness of the scalp; in fine, a group of symptoms which 
are now recognized as characteristic of neurasthenia, and pointing to exhaustion of the brain and spinal cord.

The effects of abnormal seminal losses upon the higher intellectual centers are most characteristic; the essential mental faculties are weakened. The patient finds that his perceptive powers are less acute; he can not concentrate his mind upon any subject, and is therefore entirely incapacitated for intense or prolonged mental exertion. His memory is impaired, especially his memory for words; he constantly pauses in his speech for loss of a word, and his articulation becomes halting and stammering.

Mental disturbances are also manifest in the attitude and behavior. A morbid self-consciousness is almost pathognomonic of spermatorrhœea. The patient shuns society, likes to be alone, continually broods over his sexual trouble, imagines himself impotent, and that his humiliating secret may be read in his face; his glance is furtive; he rarely looks one straight in the eye. As a result of this morbid self-concentration, a fixed melancholy seizes upon him; this condition of sexual hypochondriasis is most deplorable, and forms the darkest shades in the clinical picture of spermatorrhœa.

A tendency to tabes, epilepsy, insanity, or other grave nervous disorders is not infrequently developed into active existence by the neuropathic influence of spermatorrhœea.

In addition to these purely nervous phenomena, disturbances of the general nutrition are seen in various disorders of the digestive, circulatory, and respiratory systems. Dyspeptic symptoms are common in the course of spermatorrhœa, characterized by inappetence, disgust for certain articles of food, acid eructations, epigastric cramps, intestinal pains, constipation, and sometimes by marked emaciation. The palpitation, oppression of breathing, shortness of breath, irregularity of pulse, anæmia, pallor of countenance, etc., are doubtless due to disorders of the vasomotor and nutritive systems. Morbid effects are also determined toward special organs; deafness, noises in the ear, muscæ volitantes, hemiopia, asthenopia, dilatation of the pupils, are well-attested symptoms.

Pathological Anatomy.-The anatomical changes induced by spermatorrhœa have not been investigated with sufficient care to furnish data of scientific value. Post-mortem examinations have shown the results of chronic inflammation of the mucous membrane of the prostatic urethra evidenced by induration and suppuration of the prostate, the ulceration extending toward the ejaculatory ducts and seminal vesicles. The latter have been found atrophied and sometimes notably enlarged and partially adherent to neighboring organs, the walls of the ejaculatory ducts are thinned, their lumina enlarged and their orifices dilated. No pathological alterations have been demonstrated in the lower portion of 
the cord connected with the genital organs. It is probable that in aggravated cases the nerve-cells concerned in the functions of the genital centers are exhausted, leading to paresis of the motor apparatus.

Diagnosis.-There can, of course, be no difficulty in diagnosticating pollutions; the subjective and objective signs of the disorders are sufficiently manifest to the patient. The question for the physician to determine is their pathological significance; of this their frequency of occurrence and systemic effects constitute the surest tests. Fortunately, in many cases the patient may be dismissed with the assurance that the symptoms af which he complains are physiological phenomena common to healthy persons, and are not at all indicative of disease.

The chief diagnostic difficulty lies in the differentiation of prostatic or other urethral discharges from spermatorrhœa. The physician is often consulted by patients suffering from prostatorrhcea or a gleety discharge, the persistence of which and the accompanying symptoms are interpreted by them as evidences of true seminal loss-they imagine themselves impotent, and become the prey of the most gloomy forebodings. This urethral hypochondriasis is quite common in young men of nervous, highly impressionable temperament.

While it is comparatively easy to identify a gleety discharge, the clinical similitude of spermatorrhœe and prostatorrhœa is so close that there may be considerable difficulty in distinguishing the two affections. They may both owe their origin to the same causes and be characterized by the same local symptoms-a more or less constant discharge of a clear viscous fluid which keeps the orifice of the urethra always moist or the lips agglutinated-an irritable weakness of the deep urethra, frequent micturition, sensation of weight or heaviness in the perineal region, pain and weakness in the loins, etc. Under the microscope the prostatic discharge is found to consist only of mucons corpuscles, epithelium-cells, débris from tubular glands, sometimes casts of follicles, with complete absence of spermatozoa.

No certain diagnosis of the existence of true spermatorrhœa can be made without microscopical examination of the fluid. The quantity of the discharge, its odor, color, consistence, or other physical characters do not furnish an absolute test of the presence or absence of spermatozoa. The absence of these bodies constitutes, it is true, negative evidence, but is insufficient, in view of the fact that the essential characters of the seminal fluid become altered in the course of the disease. In the early stage the semen shows no abnormal quality, but when the seminal losses become frequent and long continued the spermatozoa diminish materially in numbers, or they may be altogether absent. The prompt appearance of spermatic crystals in the evacuated fluid is, according to Ultzmann, a most characteristic and constant sign of the nonpresence of spermatozoa. 
Under these circumstances the history of the case and the associated symptoms will assist in the diagnosis. If there is a constant escape of fluid not only after defecation, but from urethral straining, or during each micturition, and the clinical history shows the patient to have been addicted to sexual excess and to have suffered from pollutions, the evidence is in favor of spermatorrhœa, although the result of the microscopic examination be negative.

Prognosis.- The prognosis of spermatorrhœa is by no means so unfavorable as many writers have maintained. Death from exhaustion rarely follows pollutions, and its termination in epilepsy, degeneration of the spinal cord, dementia paralytica, etc., must be ascribed to primordial derangements of the nervous system, rather than to the seminal loss. In certain affections of the brain and cord spermatorrhœa is merely an incidental symptom, and sinks into insignificance in comparison with the graver lesions of the primary disease.

The prognosis is materially influenced by the cause which produced the disorder. Occurring in the course of fevers or convalescence from acute diseases it spontaneously ceases with restoration of the general health. The prognostic significance of pollutions occurring in the course of phthisis and other chronic diseases is measured chiefly by their debilitating and exhausting effect upon the general system. When dependent upon phimosis, stricture, varicocele, lesions of the urethral canal, anal or rectal irritations, it usually disappears with the removal of the cause. Occurring as a result of gonorrhoeal inflammation of the genital tract, the prognosis is less immediately favorable, as the local causes of irritation are apt to persist for some time.

When the complaint is of recent date, and the emissions take place only at night and are unaccompanied by local structural changes or severe general symptoms, a favorable result may be confidently anticipated. Diurnal pollutions always carry with them a less farorable prognosis than nocturnal emissions.

In general it may be said that pollutions are amenable to treatment when the pathological changes are confined to hyperæsthesia or inflammatory lesions of the prostatic urethra and ejaculatory ducts. Where this inflammatory stage has been succeeded by an atony of the parts, with paresis of the motor apparatus, especially when associated with symptoms of general nerve exhaustion, the outlook is not nearly so favorable.

In regard to the age of the patient, it may be said that abnormal seminal loss occurring in early life as a result of masturbation has a more unfavorable prognosis than pollutions from sexual excess at a more mature age, when the activity of the organs is undergoing a physiological decline. On the other hand, the greater natural recuperative powers of youth constitute a favorable element of prognosis. 
The family history and constitutional peculiarities of the individual have an important bearing upon prognosis. In persons of nervous, excitable temperament, with hereditary tendencies to hypochondriasis, epilepsy, insanity, etc., the inherited neuropathic state constitutes an unfavorable element in the prognosis.

Treatment.-A most important part of treatment is prophylaxis. Since, in the large majority of cases, abnormal seminal losses owe their origin to masturbation and sexual excess, the correction of these abuses constitutes the most efficient prophylactic measure. Most young men grow up in absolute ignorance of the physiology of this most important function of their physical being and of the ruinous effects its abuse entails. With the advent of puberty they perceive themselves subject to certain phenomena, the nature and significance of which they have never been taught. The habit of masturbation is contracted often thoughtlessly and innocently; it is practiced in utter ignorance of its evil effects, and the eyes of the victim are only opened to the danger when it has engendered a train of local and general disorders which persist long after the habit is abandoned.

The only way to combat the evil is to instruct the young in a knowledge of the physiological laws which pertain to their sexual organization. They should be taught that the sexual organs can not be excited into abnormal activity without doing violence to their health; that selfabuse is destructive to every endowment of true manhood, an infraction of Nature's law, the penalty for which is debility and premature decay of power. They should recognize that continence is not incompatible with health, that incontinence is not justifiable on physiological grounds, and that harlotry is not a safe substitute for marriage.

How best to impart this knowledge is an important problem in the education of childhood and youth. Its solution requires, in addition to courage, tact, discretion, and good sense. Medical men should take the initiative in this matter. One can not too strongly inveigh against the false sentiment and attitude of the profession in relation to the reprodnctive apparatus. It is a forbidden topic; its discussion is rebuked as immodest and profane; it is scarcely considered a proper and legitimate subject for scientific inquiry. All knowledge relating to that function through which life is perpetuated, and with the preservation of which is bound up the mental, moral, and physical health and well-being of the individual, is a sealed book, which only unworthy quacks and pretenders venture to open and with erafty cunning pervert to their own selfish ends.

In the treatment of spermatorrhœa, the form of the disorder, the exciting cause, and the coexistent constitutional conditions, which may stand in the relation of cause or consequence, must be considered. 
Special Treatment.-When spermatorrhœa results from local organic canses, the removal of the cause usually suffices to bring about a cure more or less promptly. Phimosis and other irritable conditions of the glans should be relieved by circumcision; varicocele by palliative means or radical operation. Stricture and other lesions of the urethral canal should be treated by appropriate methods. When the disorder is dependent upon ascarides, piles, fissures, eczema, or other anal or rectal irritations, the removal of the local exciting cause is the first essential of cure.

In gonorrhœal prostatitis, epididymitis, and chronic inflammation of the neck of the bladder, which are so constantly accompanied by pollutions, the indications are to correct the material lesions which are the exciting cause of the seminal loss.

Spermatorrhœa, symptomatic of constitutional conditions, can be cured only by correction of the complicating cause. Obviously enough the cases dependent upon organic lesions of the cerebro-spinal axis are little amenable to treatment.

Methodic Treatment should be based upon a clear comprehension of the pathological condition present and adapted to the stage of the disorder. We have seen that in the large proportion of cases the exciting cause of the pollutions is a subacute or chronic inflammation of the prostatic mucous membrane and ejaculatory ducts with perverted innervation of the motor apparatus. The indications of treatment are to correct the hyperæsthesia and hyperæmia of the prostatic mucous membrane, to calm the irritability of the motor apparatus, and to diminish the excitability of the reflex spinal center. This can be most successfully accomplished by a combination of general and local measures. In a more advanced stage, when the local inflammatory condition has been succeeded by atony of the parts, a modification of treatment is necessary.

In addition to local structural changes, spermatorrhœa is characterized by nutritive and nervous disorders, hence a general tonic course of treatment is indicated. All hygienic and other measures calculated to build up the general health should be employed. The habits of life should be regulated, a substantial and nutritions diet ordered, highly seasoned articles of food should be proscribed; the last meal at night should always be light and simple. Alcohol is objectionable.

Any pathological modifications of the urine should be corrected, and the bladder thoroughly evacuated just before retiring. A full bladder favors erection, and as pollutions commonly occur toward morning, the patient should be directed to awaken and empty his bladder before morning. The expedient of using an alarm-clock renders this advice practicable. The patient should sleep on a hair mattress and pillow, with light covering, and always lie upon the side. In a dorsal position the distended 
bladder presses upon the return blood-vessels, thus increasing the congestion of the prostatic urethra.

Since pollutions occur from the excitation of dream impressions, which are often but the reflection of waking thoughts and desires, the patient should not allow his mind to be occupied during the day with sexual thoughts or imaginations; every form of erotic excitement should be avoided.

If the patient be married, abstinence from sexual intercourse should be enjoined for a period until the sexual organs regain their vigor by repose and proper treatment. Sexual intercourse, carefully regulated and indulged with moderate frequency, is beneficial in most cases, but excitement without gratification is always harmful.

Excessive and exhausting mental exercise should be avoided. Horseback riding is objectionable, on account of the tendency to cause local congestion and irritation of the sexual organs.

The tonic effect of hydrotherapeutic measures should be utilized; the patient should be directed to use saline douches night and morning to the genital parts and over the lower portion of the spine, followed by friction with a coarse towel until a healthy glow of the surface is established. In some cases a hip-batl, or hot douches alternated with cold, yield equally good results.

Drugs. - While no drug exercises a specific curative action upon spermatorrhcea, yet the use of certain remedies is undoubtedly beneficial when employed with discrimination and with an intelligent adaptation to the pathological condition present. In general, it may be said that sedatives are indicated in the early irritable stage of the disorder, while an essentially stimulating and tonic treatment is adapted to the more advanced, atonic stage.

Bromide of potassium exerts an undoubted calmative influence upon the abnormal excitability of the genital center. Camphor, lupulin, cannabis indica, and hyoscyamin, are also reputed to possess this property. In most cases I am accustomed to order the bromide, fifteen grains, in combination with tincture of the chloride of zinc, fifteen drops after each meal, with a double dose at bedtime. In an irritable, spasmodic condition of the ejaculatory apparatus, which, as before intimated, has certain analogies with irritability of the neck of the bladder attended with incontinence of urine, belladonna, or its alkaloid atropia, is one of the most efficient remedies. Gelsemium is another remedy of undoubted therapeutic worth, the value of which $\mathrm{I}$ have tested in numerous cases.

In the atonic stage, phosphorus, iron and strychnia, coca erythroxylon, quinine, and other nerve and nutritive tonics are indicated. They improve the tone of the nervous system, and relieve the neurasthenic symptoms which are so constant an accompaniment. Phosphorus may be 
given in the form of phosphide of zinc, phosphoric acid, or pure phosphorus, in combination with iron or strychnia, or both. Damiana, avena sativa, and other remedies of this class recommended for their aphrodisiac properties, I have used largely, without being able to convince myself that they possessed any special value. A most excellent remedy is a combination of a tincture of iron and cantharides in the proportion of twelve parts of the former to two of the latter, fifteen drops of which should be given after meals three times a day.

Local Treatment.-The seat of the pathological disorder and its easy accessibility suggest the use of direct topical applications.

The most effective means of relieving the irritability of the prostatic urethra is the systematic introduction of full-sized conical steel sounds, which overcome the hyperæsthesia and relieve congestion by distention and pressure and the contact of a cold surface. They should be used as large as the capacity of the urethra will permit, a congenitally small meatus being divided, if necessary, to admit the passage of an instrument 26 to 30 French scale. They should be introduced every other day, and allowed to remain from five to twenty minutes. The good effects of this procedure in relieving the abnormal sensitiveness of the urethra are as appreciable to the patient as to the surgeon.

The psychrophore or cold-water sound of Winternitz acts much in the same way, by combining the effect of mechanical pressure with the sedative influence of cold on the caput gallinginis and the prostatic urethra. By means of this appliance a current of any desired temperature may be kept continually flowing through the tube for fifteen to twenty minutes or longer at each sitting. It is desirable to begin with water of ordinary temperature, which may be gradually lowered.

A more indirect but sometimes equally efficient means of applying dry cold to the prostate is by the use of the rectal cold-water sound. It is especially indicated in cases where the existence of piles or inflammation of the seminal vesicles act as exciting or complicating causes of the seminal incontinence. The use of hot rectal douches is often followed by marked relief in inflammatory conditions of the prostate.

The application of astringents and caustics to the prostatic urethra constitutes a most efficient method of treatment, especially when the inflammation is circumscribed to a limited area involving the orifices of the ejaculatory ducts. The brilliant results of cauterization claimed by Lallemand and his followers have not always been substantiated by the experience of others. While it constitutes a most valuable resource in certain selected cases, the method has by no means the universality of application claimed by its originator.

Cauterization of the pars prostatica may be effected by the porte remède of Lallemand, by Langlebert's modification, which possesses cer- 
tain advantages, or by any of the numerous urethral applicators devised for this purpose. The caustic may be used combined with an ointment by means of cupped sounds or ointment bougies, or in the form of suppositories by Dittel's porte remède, or other appliances for introducing suppositories of any desired strength. In using Dittel's porte remède for the purpose of cauterization a small suppository containing 0.02 grammes of nitrate of silver in combination with cacao-butter is inserted into the opening of the catheter, previously introduced into the urethra, and is pushed down and deposited in the prostatic urethra. This method possesses the advantages of accuracy and precision; a given amount of the remedy may be deposited at any desired point. The effect of cauterization is manifest in a sharp, stinging pain, sometimes bleeding, with more or less vesical tenesmus, which may be relieved by rest in bed and cold compresses to the perinæum. Ordinarily a single cauterization is insufficient, and the procedure must be repeated two or three times. A modified cauterization may be effected by the use of liquid injections or instillations of nitrate of silver by means of Ultzmann's or Keyes's deep urethral syringe.

Equally good results are obtained in the majority of cases by the use of astringents which may be introduced in the form of suppositories or liquid injections carried directly into the prostatic urethra. Weak solutions of nitrate of silver, carbolic acid, sulphate of zinc, tannin, hydrastis, pinus canadensis may be employed for this purpose.

Electrical Treatment.-Electricity, when intelligently employed, is a most valuable addition to our therapentical resources, especially in an advanced stage of the disorder. In the generality of cases the use of the galvanic current yields the best results. The negative electrode should be introduced into the urethra or against the orifices of the ejaculatory ducts, and the positive pole be placed over the perinæum.

The current should be mild, rarely exceeding four milliampères, and the duration of its application at each sitting should be only a few minutes. In order to secure the best results from this treatment it should be employed at first daily, afterward two or three times a week, for two or three montlss. Its results in relieving the irritability of the urethra and arresting the discharges are often most gratifying.

Local faradization may be advantageously substituted for the constant current in cases characterized by diminished sensation or anæsthesia and deficient erectile power. When the faradic current is used it is better to introduce the cathode into the rectum, and apply the other electrode over the bulb of the urethra and external genitals. It should be used with caution, however, as its stimulating effect may be followed by an increase in the frequency of the pollutions.

Certain instrumental appliances in the shape of plugs or other devices 
for compressing the prostate, highly recommended by Trousseau and others, are of doubtful efficacy, besides being unpleasantly borne by the patient.

Castration is only mentioned to be condemned as an unscientific and barbarous practice.

Narriage as a remedy for abnormal seminal losses is often recklessly and injudiciously recommended. Unquestionably, moderate sexual intercourse constitutes a most efficient hygiene for the sexual organs, and in a majority of cases, when the pollutions have not induced actual impotence; it may, after a judicious course of treatment, be safely advised to complete the cure. But the view that a woman may be properly utilized as a therapentic agent can not be too strongly condemned, as degrading to the dignity of womanhood and subversive of the higher ends for which marriage is instituted. Marriage is a partnership formed for mutual advantage, enjoyment, and the propagation of the species. Each contracting party should contribute a sufficient amount of virile energy and health to make these objects realizable. But a man whose contribution to the capital stock is an emasculate manhood, an impaired virility, and a barren capacity, cheats and defrauds his partner. Especially would we protest against the admissibility to marriage of worn-out roués and libertines, who have exhausted their sexual forces by a long course of sensual excess, and who seek to rekindle upon the altar of marriage the fires of a wasted and burned-out passion.

\section{IMPOTENCE.}

Definition.-The power of copulation, or the ability to perform the sexual act, is the normal condition of every healthy adult during the physiological period of virility. The loss of this power constitutes impotence, and may be due either to organic causes, to incompleteness or absence of erection, or to a precipitancy of the reflex act of ejaculation before intromission of the penile organ and consummation of the act. The man who is impotent is practically sterile, although the semen may contain spermatozoa, for the mechanical reason that he is unable to deposit it in the generative tract of the female.

It is important to have a clear understanding of the distinction between impotence and sterility. While inability to accomplish the sexual act may be associated with loss of procreative power, yet one function may be preserved in entire integrity while the other is impaired or completely lost. There may be a complete abolition of the generative faculty in a man capable of consummating the sexual act normally and with full vigor. He is unable to procreate because his seminal fluid is absent or 
lacking in the essential elements of fecundation-the spermatozoa-or these bodies are altered, malformed, or deficient in vitality. As examples of the preservation of potency with loss of fecundating power may be mentioned a class of eunuchs whose testicles have been removed without other mutilation, and men whose vasa deferentia have been occluded by a donble epididymitis, in whom strong sexual desire, the power of erection and ejaculation, attended with normal specific sensations, may exist in full activity.

Impotence may be absolute or relative ; there may be a permanent absence of virility, or merely a temporary abeyance of this power. Since in its broadest sense impotence means inability to consummate marriage, it is evident that this definition embraces pathological conditions of a diverse character. It may depend upon abnormal physical conditions which act as mechanical obstacles to the introduction of the male organ in the vagina, or it may result from disorders of the nervous and muscular systems concerned in the mechanism of the complex act of erection and ejaculation.

It is a question whether the impotentia coeundi due to organic causes should properly be classed as true impotence. The subjects of this form of impotence are often endowed with strong desire and the mechanism of erection is normal; there is simply an inability to perform the sexual act under existing conditions. When these structural impediments are removed, as they often may be by surgical art, potency is found to be unimpaired.

Physiology of Erection. - Since erection is the essential condition of potentia coeundi, it is necessary to have a clear knowledge of the mechanism of normal erection in order to understand the pathological conditions which cause impotence. Erection is determined mechanically by the distention of the blood-vessels and erectile tissues of the penis with blood, causing an augmentation of its volume, with increased hardness, rigidity, and raising of the organ. This process is essentially under the control of the nervous system. Under the influence of the nervi erigentes which take their origin from the second, more rarely the third, sacral nerve, there is a relaxation of the muscular fibers of the cavernous tissue, permitting a free influx of arterial blood, entirely filling the short arterial branches anastomosing in the cavernous spaces. At the same time there is a compression of the efferent veins by the smooth muscular fibers of the corpora cavernosa, which hinders the reflux of the blood, and by its retention prolongs and strengthens the erection.

According to Landois, while the impulse to erection is obtained by increased supply of arterial blood, the full completion of the act is brought about by the activity of the following transversely striped muscles: 1 . The ischio cavernosi, by their tendinous union surrounding the penis; when 
they contract they compress the root of the penis from above, and laterally, so that the outflow of blood is hindered. 2. The deep transrersi perinei, perforated by the venæ profundæ coming from the corpora cavernosa, which in contracting compress these veins by their tense horizontal fibers. 3. The bulbo-cavernosus is concerned in the hardening of the urethral corpus spongiosum as it compresses the bulb of the urethra. All these muscles are partly under control of the will, whereby erection may be increased; normally, however, their contraction is excited reflex ly by stimulation of the sensory nerves of the penis.

Ejaculation takes place normally when the penis is in full erection. During erection the orifices of the ejaculatory ducts are directed forward and the passage from the urethra to the bladder is closed by the swelling of the caput gallinaginis. As a result, while the semen may be readily discharged during erection, a barrier is formed to the passage of urine. During the act of erection the urethral glands begin to secrete, and their secretion may appear, if the erection is prolonged before ejaculation, as a clear viscid fluid at the meatus. The act of ejaculation is brought about by the reflex stimulation of the ejaculatory center in the spinal cord. When the seminal fluid is poured out through the ejaculatory ducts it collects in the bulbous urethra, between the erectile tubercle behind and the compressor urethræ in front, until by mechanical distention of the canal it excites a reflex contraction of the bulbo-cavernosus muscle of a spasmodic character whereby it is forcibly expelled in a series of gushes from the urethra. Immediately preceding and coincident with ejaculation a sensation of voluptuous excitement is experienced.

It is probable that the spermatozoa ejaculated represent the accumulation stored in the seminal vesicles rather than the immediate secretion of the testicles.

Eckhard showed that erection can be excited by stimulation of the erigent nerves, but is impossible after their division. He also found that erection occurred from stimulation of the higher regions of the cord as well as the pons and crura cerebri, from which he concluded that the motor impressions were conveyed by fibers of the erigent nerves which pass down the cord. Goltz found that erection was much more powerful and promptly excited by irritation of the sensory nerves of the penis after division and separation of the lumbar segment of the cord; and from this he concluded that there was an independent center for erection in the lower portion of the cord ; also, that the brain exercised a more or less dominating, inhibitory action upon erection-a conclusion confirmed by the clinical manifestations of psychical impotence.

Irritation of the spinal cord of the most varied character from disease, concussion, or other injury, may occasion erection ; indeed, priapism is one of the first indications of incipient tabes. Stimulation of the 
medulla oblongata by asphyxiated blood or by muscarine excites erection.

Erection may be excited by peripheral or psychical impressions. As illustrations of the former, may be mentioned the morning fullness of the bladder, various local irritations of the urethra, bladder, and rectum, also the mechanical stimulation of the sensory nerves of the penis from friction of the glans in masturbation. As illustrations of the latter may be instanced lascivious or lustful thoughts, the sight or contact with women. While partly under the control of the will, erection may manifest itself independently of the will, or it may fail, notwithstanding the most pressing desire or the strongest effort of the will. Mere imagination may excite erection, and it may be arrested or neutralized by a sentiment of fear, disgust, or modesty.

The act of ejaculation is in a measure also controlled by the will ; after a certain degree of excitement, however, the ejaculative sensation becomes so exalted that the motor influences which complete the act are irrepressible. The inhibitory action of the will over the reflex center is exceptionally developed in certain individuals. This repressive influence of the will is not infrequently utilized by married men, who, in order to avoid impregnation, are accustomed to delay emission until after withdrawal of the penis from the vagina.

From these physiological facts it will appear that the normal evacuation of the seminal fluid is a complex motor act the mechanism of which involves the nervous, vascular, and muscular systems. In a perfectly healthy condition there is a harmonious and co-ordinate action of these elements.

In normal coitus erection and ejaculation usually result from the combined influence of central and peripheric stimuli. The central impulse awakened by desire or erotic excitement is quite sufficient to excite erection, but insufficient to arouse the ejaculation center which transmits the motor influence to the seminal vesicles and stimulates them to contraction and expulsion of their contents. The mechanical stimulus of the sensory nerves of the penis from contact with the rugæ of the vaginal walls determines ejaculation. In a healthy state this coefficient action of the mechanical stimulus is essential to the discharge of the reflex, and the transition into a morbid state may be measured by the progressive diminution of the peripheral impulse necessary to provoke ejaculation. In the large class of cases of impotence from irritable weakness there is an abnormal excitability of the motor apparatus with increased mobility of the reflex center, and ejaculation takes place without mechanical cause, during intromission, or even before actual contact.

It will thus be seen that in the large proportion of cases impotence is essentially an ataxia of the genital system characterized by incoordination of the expulsive and retentive muscles, and presenting certain analo- 
gies with tabes. This disordered action is due to a disturbance of the equilibrium of the motor influences emanating from the brain, the sensory nerves, and the reflex spinal center.

Classification.-Different divisions of the several forms of impotence have been made by different authors. The following classification appears to the writer to be the simplest and most scientific. According to the more prominent etiological element present in each case, the following forms may be distinguished:

1. Organic impotence. 2. Psychical impotence, including relative impotence. 3. Irritable impotence. 4. Paralytic impotence.

Organic Impotence.-Under this head are grouped various defects, mutilations, malformations, and vicious directions of the penile organ, which physical conditions preclude the possibility of successful sexual intercourse.

The cases of complete absence of the penis are of too rare occurrence to merit consideration. Small size of the penis is a relative cause of impotence. The penile organ may be abnormally short, or its available length may be reduced by the contiguous existence of a tumor, scrotal hernia, elephantiasis, or an overhanging or protuberant belly which prevents the close approach essential to copulation. Excessive size of the penis has been mentioned by certain anthorities as a cause of impotence. Cases are instanced in which stone in the bladder or strictures have determined a hypertrophy of the penis until it acquired such dimensions as to render intromission impossible. Extreme size of the penis involves only a relative incapacity for intercourse.

Hypospadias and epispadias when extensive, with or without extreme curvation, involve impotence as well as sterility simply from the vice of conformation which prevents the deposition of the semen in the genital canal of the female. A slight hypospadias does not necessarily involve impotence or even sterility. The semen may be deposited in the lower part of the vagina and impregnation follow. A short frænum may cause distortion of the penis and prevent intromission.

Distortions or deviations in the direction of the penis, due to inflammations or deposits of various kinds in the erectile tissue or fibrous sheaths of the corpora cavernosa, may be sufficiently pronounced to prevent intromission. Cicatricial induration, gummata, and calcifications of the corpora cavernosa have been assigned as causes.

The various diseases of the testicles have, as a rule, a closer causal connection with sterility, but they are not infrequently causes of impotence. Anæmia and atrophy of the testes, tubercular affections, syphilitic sarcocele, sarcomatous and cancerous infiltrations, by displacing or disorganizing the secretory structures of the organs, may be followed bv both sterility and impotence. 
Psychical Impotence.-No fact better establishes the controlling and dominating influence of the mind over the genital function than the existence of this neurosis. An individual may have all the appearances of virility, with strong sexual desire, yet when he attempts sexual intercourse he fails, from a nervous apprehension of his inability to perform the act. In consequence of the high degree of nervous excitement, the inhibitory action of the brain over the genito-spinal center is intensified, and the erection fails at the critical moment.

This form of impotence, which is of a purely psychical order, has been termed imaginary impotence, since the individual is impotent from the sole fact that he imagines himself to be impotent. It is most commonly met with in the young or newly married, and is often the expression of inexperience rather than of incapacity.

A young man contemplating matrimony, in order to test his virility or fitness for marriage, may attempt intercourse with a prostitute and fail, and in consequence imagine that he is impotent. He does not reflect that the conditions of sexual intercourse in married life and illicit cohabitation are altogether different. Indifference or disgust, inspired by some act or speech of the prostitute, shame, the fear of contracting disease, all tend to induce a state of mental perturbation which takes away the power of performance.

A newly married man, in the eagerness to consummate the marriage, may fail simply from the impetuosity which attends overwronght desire. The mortification which results from this failure in the marriage-bed is apt to beget a timidity and distrust of his powers which may render him really impotent. Fortunately, in most of these cases confidence is regained by repetitions of the act, and this abnormal condition spontaneously disappears under the corrective influence of the sexual hygiene of married life.

The influence of fear and dread on the production of erection may sometimes be studied, as suggested by Ultzmann, in the examination of such patients by sounds. "When they have unfastened their clothes and stretched themselves on the table the penis is seen gradually to shrink, grow smaller, and to move in a worm-like manner. The corpora cavernosa become smaller and harder. To the touch the penis feels cartilaginous ; the skin shows transverse folds due to the contraction of the corpora cavernosa. These appearances can only be explained by spasmodic contraction of the organic muscular fibers, which are imbedded in the trabeculæ of the corpora cavernosa. This constriction is a manifestation of the action of the inhibitory nerves of erection, stimulated by the fear and dread of catheterism. In exactly the same way, then, other purely psychical influences stimulate the action of the inhibitory nerves, and the patient is at such moments impotent." 
Among the causes of this form of impotence may be sexual indifference, sudden shock, violent emotions, as excessive grief, fright, intense and long-continued mental preoccupation, etc. As an instance of the latter may be mentioned the oft-quoted case of Grimaud de Caux, that of a celebrated mathematician, whose thoughts at the critical moment always went wandering toward the solution of some complicated problem. His wife finally succeeded in keeping him "on amorous thoughts intent" sufficiently long for an ejaculation, by the expedient of making him slightly intoxicated, so that he forgot his problems. Roubaud relates cases of impotence in previously healthy men following the shock of a railway accident, grief at the death of a beloved wife, etc. Ultzmann instances similar cases which have come under his observation, also impotence from the loss of money and property.

The sufferers from this form of impotence have in most cases been addicted to masturbation or other abuse of the generative organs and still have pollutions or spermatorrhœa, the existence of which they accept as an evidence, and at the same time as sufficient cause, for this fancied loss of virility.

Relative Impotence.-By this term is understood the inability to perform the sexual act with certain women, while with others it may be normally consummated. This form of impotence is not infrequently found in loveless marriages contracted from mercenary motives, where the parties are of incompatible temperament. Unquestionably the spiritual element of love, as well as affinity and mutual attraction, play an important rôle in exciting desire, which is an essential preliminary to the proper performance of the sexual act. Many men and most women are so constituted that this moral sentiment is a prerequisite or necessary condition of physical passion. Without it the sexual nature remains dormant and indifferent.

Apathy, coldness, or repugnance on the part of a wife often extinguishes sexual desire on the part of the man, and may be responsible for this relative impotence, which immediately disappears ontside the marriage-bed. A man may be impotent with a frigid and indifferent wife, while the opposite is the case with a passionate prostitute.

Irritable Impotence.-The impotence due to irritable weakness of the genital organs, with abnormal excitability of the refiex centers, represents by far the largest proportion of cases. Perfectly normal coitus involves not only desire and erection sufficiently powerful for penetration, but also sufficiently lasting to admit of a series of movements during a certain time, which act as a mechanical stimulus to the reflex discharge. The inability to have a powerful and lasting erection, and premature ejaculation, are the distinguishing features of this form of impotence, which varies in degree in different cases. There may be only 
feeble desire, the erection may be partial, irregular, or of such short duration that the ejaculation of semen takes place ante portas-before intromission. In another class of cases sexual desire and erection are strong and penetration possible, but ejaculation takes place prematurely, during or immediately after intromission, rendering satisfactory intercourse impossible.

While this form of impotence may be manifest in persons of a highly excitable temperament, simply from a peculiarity of nervous organization, the origin of the trouble may be most commonly traced to masturbation or other forms of sexual excess. As a result of this abnormal irritation of the genital passages there have been induced a hyperæmia and hyperæsthesia of the mucous membrane of the prostatic urethra, in consequence of which the nerves which excite the reflex act of ejaculation respond to an abnormally slight stimulus, either peripheral or central.

Such individuals will always be found to have a hypersensitive urethra. Upon the introduction of a sound the canal is found sensitive throughout. When it reaches the prostatic portion the hyperæsthesia is so pronounced that it occasions the most intense pain. The mucous membrane of this region seen under the endoscope is found to be hyperæmic, swollen, of a cherry-red or livid color, and bleeding easily. Associated with this morbid alteration in the mucous membrane, which renders it especially vulnerable and disposed to take offense at the slightest provocation, there is oftentimes an enlargement and patulousness of the ejaculatory ducts, permitting of a more ready escape of the contents of the seminal vesicles.

The impotence which not infrequently follows gonorrhœal inflammation, especially when complicated with posterior urethritis and epididymitis, stricture, etc., also certain affections of the prostate, is doubtless due to the prostatic inflammation, causing irritability of the nerve branches which transmit the reflexes. Impotence from this cause is usually of temporary duration, and spontaneously disappears with the restoration of the deep urethra to a normal condition.

Paraly tic Impotence.-By many writers, only those cases in which impotence is absolute and permanent are classed in this category. Obviously, however, this restriction is not admissible, since cases of the true paralytic variety of impotence are sometimes, though unfortunately very seldom, curable. In this form of impotence the nervous mechanism of the genital apparatus is so disturbed that normal erection rarely occurs. In the more advanced stage not only is there loss of power of erection, but sexual desire is completely absent.

In some cases partial or feeble erections may occasionally occur, sufficiently strong even to render possible the penetration of a capacious vagina, but the semen, instead of being discharged in a violent jet or gush, oozes or dribbles away in a sluggish, passive manner. This may be 
due to a paralysis of the bulbo-cavernosi muscles, by the contraction of which normally the semen is forcibly projected from the urethra. The secretory functions of the seminiferous organs, as well as the nerves and motor apparatus, are oftentimes in a state of organic weakness.

Upon examination, the genital organs are often found to be undersized, pale and cold, the penis flaccid, the testicles soft and atrophied, the scrotum relaxed, and the whole aspect of the external genitals weak, withered, and senile. The integument is, as a rule, insensitive to the action of the galvanic current. Upon examination with a full-sized sound the urethral mucous membrane is found to be deprived of its normal sensibility.

While this most aggravated and intractable form of impotence is often due to the exhaustion which follows masturbation and sexual excess, it may be the local expression of some constitutional state or dyscrasia, or profound lesion of the nervous system. Several years since I had under my care a medical man who had sustained a severe injury of the spine by being thrown from his carriage; as a result of which there followed a marked atrophy and wasting of the external genitals, with complete extinguishment of sexual desire and erection. Various writers have mentioned instances of atrophy of the testis and penis following injuries or concussions of the cord.

Diseases of the brain and cord which abolish or greatly diminish the reflex excitability of the nerves supplying the generative organs, softening of the brain, cerebral hæmorrhage, tumors of the brain, chronic myelitis, especially in an advanced stage, may be attended with complete anaphrodisia; also certain constitutional affections, as lepra, Bright's disease, diabetes, the cachexia and anæmia of syphilis, may depress or entirely abolish sexual desire and power.

In connection with the impotence symptomatic of conditions of the general system, the impotence due to drugs may be properly considered. The prolonged and excessive use of certain drugs, such as opium, cannabis indica, bromide and iodide of potassium, chloral, arsenic, ete., may seriously impair or entirely destroy sexual power. It is well known that Orientals not infrequently become impotent at an early age from the use of opium and hasheesh. Exposure to the fumes of antimony, lead, sulphide of carbon, arsenic, etc., in industrial occupations, may be followed by loss of virility.

The depressing effect of tobacco, alcohol, and other cerebral excitants upon the genital functions is a matter of common observation. It may be said that the anaphrodisiac effect of tobacco is not universal, being influenced by conditions of temperament or idiosynerasy. The excessive use of beer is said to diminish sexual power; the action of alcohol, in first stimulating, then blunting, nervous sensibility, is well-known; the im- 
mediate effect of drunkenness is to excite the sexual desire while taking away the power of performance. Old and confirmed alcoholics are often entirely impotent. The pathogenesis of this symptomatic impotence must be sought for in the depressant action of the drug upon the brain and spinal cord, associated with more or less profound alterations of the nervous structures.

Etiology.-The causes of organic impotence have been fully set forth in the description of the mechanical obstacles concerned in its production. The etiology of other forms of impotence is, in the majority of cases, identical with that of spermatorrhœa. It may be positively affirmed that the diminution or complete loss of the power of sexual intercourse can in most instances be traced to abuse of the sexual organs by natural or unnatural methods. Exception is, of course, made for certain cases of impotence symptomatic of general diseases-cachexiæ, cerebral diseases, or lesions of the spinal cord involving the centers of erection.

Symptomatology.-It is not surprising that two diseases which are united by such close etiological lines should have largely a common symptomatology. A description of the symptoms that characterize impotence would consist chiefly in a repetition of what has been detailed in this section under spermatorrhœa.

It may be said, however, that when the fear or the consciousness of impotence is ingrafted upon the train of mental symptoms engendered by spermatorrhoea their hypochondriacal character becomes more accentuated. The loss of virile power always carries with it a sense of personal degradation; and when a man becomes dominated by the fixed idea that he is deficient in the most essential attribute of manhood, he is apt to sink into a state of profound melancholia. Indeed, no mental state is more depressing in its influence than the dread of impotence. Whether the impotence be real or only fancied, its demoralizing effect upon the individual is none the less pronounced.

Prognosis.-The prognostic significance of the different forms of impotence varies according to the pathological condition present. In many cases organic impotence is absolute and permanent from the very nature of the physical causes which constitute the obstacle to successful intercourse; other cases, however, are remediable by surgical means. The prognosis of psychical impotence is almost uniformly favorable. The subjects of this form have ordinarily a normal genital apparatus, with strong desire and erection; the trouble lies in the fact that the nervous excitement is so exalted as to nullify the power of erection during the act of coitus. This disturbing element is usually of transient duration; after a single successful intercourse confidence in one's powers is regained as readily as it was lost. Scarcely less favorable is the prognosis in most cases of impotence from irritable weakness of the genital 
organs. Proper local and general treatment, supplemented by the sexual hygiene of married life, rarely fails to effect a cure.

The prognosis of the paralytic form of impotence is generally gloomy. In cases dependent upon structural lesions of the cerebro-spinal axis there is rarely hope of cure. In other cases due to prolonged and inordinate self-abuse, the tone of the sexual organs may often be restored by local and general tonic measures.

Treatment.-Many cases of impotence due to abnormities or lesions of the genito-urinary organs admit of surgical relief. Hernia, hydrocele, varicocele, etc., may be corrected by operative procedures; tumors of the penis, if nonmalignant, may be extirpated; the redundant integument of elephantiasis may be removed; hypospadias and epispadias may be cured by plastic operations, with re-establishment of the lost or impaired genital function. The deviations and curves of the penis from wounds of the urethra, indurations from cavernitis, ossification or calcification of the corpora cavernosa, are rarely remediable by surgical measures. On the contrary, when distortion of the penis arises from syphilitic infiltrations the outlook is favorable. Under the influence of specific treatment the indurations may melt away and the organ be restored to a normal condition. The reflex impotence which is consecutive to various lesions of the genital and perigenital regions, such as phimosis, herpes vegetations, granular patches or stricture of the urethra, diseases of the bladder and rectum, frequently disappears upon the correction of the cause.

The impotence which not infrequently follows gonorrhœa, prostatitis, vesiculitis, inflammation of the testicle and cord, is usually cured by the correction of the material lesion upon which it depends.

The large proportion of cases which come under the physician's observation are examples of the irritable or atonic form of impotence, characterized by a too precipitate ejacnlation. The patients are generally young men who have been addicted to the practice of masturbation and suffer from one of the forms of seminal incontinence. These cases are almost invariably characterized by marked hyperæsthesia of the prostatic mucous membrane, irritability of the ejaculatory ducts, and erethism of the genital nerve-centers.

The first indication is to correct the local irritation. This can be most effectively accomplished by topical applications. The methods of local treatment may be summed up as follows: (1) The daily passage of metallic sounds of sufficient size to distend the urethra; (2) the use of the coldwater sound or psycrophore; (3) the treatment of the pars prostatica by the direct application of caustics and astringents; (4) the use of elec tricity.

The application of these methods has been fully considered in the treatment of spermatorrhœa, and to avoid repetition the reader is referred 
to this section. Of especial service in irritable weakness is the cauterization of the caput gallinaginis.

The good effect of local treatment is not only manifest in the diminution of the morbid sensitiveness of the prostatic urethra, but in the production of frequent and more vigorous erections, the moral effect of which upon the patient is always most favorable.

As regards the use of electricity, it may be said that the constant current gives the best results in the generality of cases. As previously pointed out, the faradic current is more stimulating, and is therefore contraindicated in cases of seminal weakness characterized by pollutions, from its tendency to increase their frequency. Faradization is, however, especially useful in the paralytic form of impotence, when there is a paresis of the bulbo-cavernosi muscles and other structures concerned in the ejaculation of the semen. By its continued use the muscular apparatus concerned in erection and the expulsion of the semen is toned up and energized.

Cases of the purely psychical type are in many instances susceptible of psychological cure. The subjects of this form of impotence are, as a rule, nervous and highly excitable. It is necessary, first of all, to gain the patient's confidence, and impress him with the certainty of cure; very often, under the physician's confident assurance of his functional capability, his nervous apprehension will be allayed and the impotence disappear. In other cases it may be necessary to give for a few days a placebo in small doses, or some medicine of reputed aphrodisiac power. 'The faith of the patient in the virtue of the remedy will often suffice to assure the desired effect. An ingenious French writer has recommended the expedient, often successful, of giving the patient any indifferent remedy, with the positive prohibition of any attempt at sexual intercourse until a certain quantity has been taken. It succeeds so well that the patient rarely takes the prescribed quantity. A new emotion-a resolution to resist his desires-having replaced the fear of failure, the impotence vanishes with the cause which produced it. However paradoxical it may appear, the absolute prohibition of coitus is in many cases the surest way to render it possible.

It is important in all cases to study the temperament and peculiarities of each individual case and adapt the treatment accordingly. Some patients are more impressed by what acts upon the imagination, others by what appeals to the intelligence. The physician should not essay the róle of the Christian scientist-of assuring the patient that his trouble is merely fanciful, that it exists only in his imagination, and that, if he will only bring his mind to the conviction that his disease has no existence, it will immediately vanish. However much the patient may be impressed at the time by this logic, afterward the recollection of an experience which 
caused him so much humiliation is sure to beget a lively apprehension of its repetition under similar circumstances, and the dread of failure reasserts itself. Instead of endeavoring to convince the patient that his trouble is trivial and of no importance, it is better in these cases to respect his fears, and counsel a course of preparatory treatment before making a further test of his virility.

The constitutional remedies and general hygienic measures which are best adapted for the various forms of seminal weakness have been fully considered in connection with the treatment of spermatorrhœa.

In nervous, hypochondriacal patients, change of air, tonics, sea-bathing, with complete abstinence for a time from all attempts at coitus, are to be recommended. With the re-establishment of the general health there is frequently a spontaneous awakening of the genital sense, evidenced by more frequent and lasting erections, which the patient always interprets as a proof of virile power.

\section{STERILITY.}

The general subject of sterility has assumed a new phase in professional estimation within the last few years, from a recognition of the fact that sterile marriages, for which the woman was formerly held exclusively responsible, are not infrequently due to a lack of procreative power on the part of the husband. This opinion was based upon a confusion of copulative with procreative power. It was assumed that any man with a good development of his genital organs, and capable of consummating sexual intercourse in a normal manner, was possessed of the power of propagating his species. It is now recognized that a man may possess the potentia coeundi unimpaired, and even unusually developed, and yet be deficient in the potentia generandi.

The comparative frequency of sterility in the male can not be definitely determined, from the very nature of the case. When only two factors are involved, it might appear easy to identify one by eliminating the other; but the conditions which control generation are complex and obscure, and not always susceptible of analysis. Either or both parties to a sterile marriage may contract a new connection, and fruitful results may follow in either or both cases. Few, however, would subscribe to the opinion promulgated by the late Dr. Gross, that in more than sixteen per cent of sterile marriages the fault is to be ascribed to the man and not to the woman.

Definition.-Sterility on the part of the male is an inability to produce offspring. He may be possessed of virile power and have a discharge of seminal fluid, yet if this contains no spermatozoa, or at least no fertile spermatozoa, he is sterile. 
Physiological Characters of the Semen.-Since procreative power depends upon the quality of the semen, it is necessary to have an exact knowledge of the normal composition of the semen and the conditions of its secretion in order to elicit the significance in relation to sterility of the pathological modifications this fluid may undergo.

The semen is a composite substance made up of the secretions of the testicles, the seminal vesicles, and of the accessory glands-the prostate, Cowper's glands, and the glands of the urethral mucous membrane. Of these various secretions which collectively constitute the seminal fluid, that from the testicles alone contains the fecundating principle. The office of the accessory glands is to furnish secretions which dilute the semen, lubricate and prepare the urethra for its reception, and facilitate its discharge.

The semen when ejaculated is an opalescent mass of varying consistency, which coagulates upon exposure to the air and then becomes more fluid. It possesses an alkaline reaction, and a characteristic odor which has been compared to that emitted by freshly sawed bone. This odor is derived from the secretions of the accessory glands, probably the prostate, since it is present in seminal fluid which does not contain the testicular secretion.

The semen consists of ninety per cent water with only ten per cent of solid matter; of this latter, six per cent consists of organic constituents, including the spermatozoa, three per cent of earthy phosphates, especially phosphate of lime, and one per cent of chloride of sodium. Of the organic constituents, semen contains spermatin, an albuminous body resembling casein in reaction. Besides spermatozoa, the microscope shows spermatic cells, epithelium from the prostate and urethra, and molecular detritus. The spermatic cells have been termed the breeding-places of the spermatozoa.

Spermatozoa presents the appearance of threadlike filaments, with an oval head, a middle portion tapering to a tail extremely fine at the other end, and are endowed with movement and activity. In fresh, healthy semen "they may be seen to move rapidly with a whiplike or wriggling motion across the microscopic field." They are also endowed with considerable vitality, which may be preserved from forty-eight to eighty-four hours after ejaculation. It is claimed that this vitality may be conserved for a period of eight days in a favorable medium, in the generative organs of the female. An alkaline medium favors their vitality, while an acid medium destroys them. Water checks their movements, "causing their tails to curl up in a loop." Solutions of salt, sugar, albumen, potash, and soda may revive motionless spermatozoa, while cold, and solutions of metallic salts and acids, arrest their movements (Fig. 1).

The amount of semen discharged at a single ejaculation varies from 
one half to one drachm or more, depending upon different conditions and circumstances. Frequent repetition of the sexual act diminishes not only the absolute quantity of the seminal fluid, but more notably the number of the contained spermatozoa. The greater proportion of the fluid comes from the accessory glands. It has been observed that in complete occlusion of the vasa deferentia, shutting off the testicular secretion, the amount was not materially diminished.

The secretion of the seminal fluid begins at an early age and continues for an indefinite period, depending upon the individual, his habits,

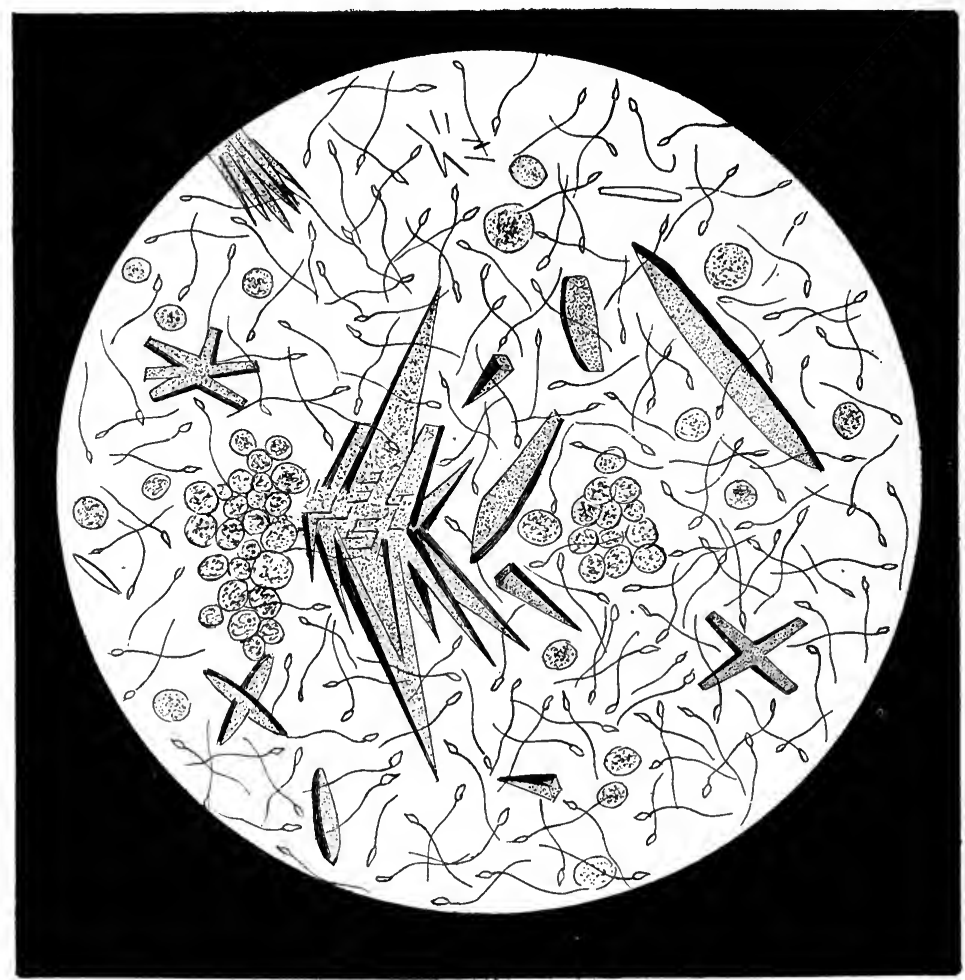

Fig. 1.-Spermatozoa in last drops of urine, with alkaline reaction. (Peyer.)

mode of life, etc. Spermatozoa begin to appear at the age of puberty, and may continue to appear, but with rapidly decreasing frequency, after the sixtieth year; they have been found as late as the eighty-sixth year. Before puberty and in old age no spermatozoa, only spermatic granules, are found. The period at which spermatozoa normally cease to appear may be anticipated by masturbation and other forms of sexual abuse.

The continuous secretory activity of the seminiferous organs during the 
entire period of virility renders the male human being apt for procreation at all times and seasons. This is in marked contradistinction to the sexual instinct and power in other animals, which are active only at certain seasons when the animal is "in heat," and dormant at others. Indeed, this ceaseless physiological activity of the reproductive organs in man constitutes a most marked anthropological differentiation. It must not be assumed, however, that the peculiar sexual organization of man demands for its health and preservation the full amount of exercise of which it is capable. The testicles afford examples of organs whose development and functional activity are not lost by disuse. A man may live to quite an advanced age in a state of continence, with comparative or complete inactivity of the sexual organs, without material impairment of their functional capacity.

Pathological Modifications. - The pathological modifications of the seminal fluid which are connected with sterility relate both to its quantity and quality as well as it color, and may be classified as follows : 1, Aspermia, absence of semen ; 2, oligospermia, diminished quantity of semen; 3 , oligozoöspermia, diminished number of spermatozoa; 4 , azoöspermia, absence of spermatozoa; 5 , chromospermia, colored semen.

It is evident that the first of these classes excludes all the others, since no seminal fluid whatever is ejaculated. The second and third conditions frequently coexist, and may owe their origin to the same pathological cause; the sterility resulting therefrom is relative rather than absolute.

Aspermia.-This term is applied to that form of sterility in which the act of coitus, though normal in other respects, does not culminate in the ejaculation of semen from the orifice of the urethra. The nonemission may be due to a failure of the reflex act of ejaculation in response to the mechanical stimulus of coition; it may be caused by alterations in the seminal passages which prevent its entrance into the urethra, or by some mechanical obstacle in the urethra which obstructs its outward flow.

Aspermatism may be absolute and permanent or relative and temporary. Absolute aspermia is characterized by an entire absence of semen, either in coitus or in the form of nocturnal pollutions. During coition, no matter how prolonged the act, no fluid is discharged, except a few drops of viscid fluid representing the secretion of the urethral glands. This form is usually congenital; it is not attended with any marked abnormity in the development of the external genitals, and can only be explained on the assumption of the nonexcitability of the reflex center of ejaculation.

The absolute aspermia caused by mechanical obstruction to the passage of the semen into the urethra may owe its origin to congenital defects or vices of conformation, such as occlusion, absence or vicious direction of the ejaculatory ducts. In the majority of cases, however, it is an acquired affection, and due to obliteration or occlusion of the 
ejaculatory ducts by various inflammatory affections of the prostate. Cicatricial occlusion of the duct may follow an abscess or any suppurative inflammation of the prostate. In tubercular affections of the prostate the infiltrations may disorganize the gland and by their pressure occlude the ejaculatory ducts. Division of these canals in perineal lithotomy, or from injury in the extraction of a calculus; may permanently destroy their function. According to Reliquet, the ducts may be blocked up by sympexions or concretions formed in the seminal vesicles.

The third variety of aspermia, which is relative rather than absolute, is caused by an obstruction situated between the pars prostatica and the meatus. Since semen is discharged into the urethra, there is in this class of cases a retention rather than an absence of seminal fluid, and it has therefore been properly termed false aspermia. Moreover, the condition is fortunately relievable by surgical treatment.

Stricture of the Urethra is the most common cause of the retention of semen within the urethral canal. A stricture which readily permits the passage of urine may, under the changed vascular conditions which attend venereal excitement and erection, have its caliber so encroached upon as to prove impervious to the denser seminal fluid. Under these circumstances the ejaculated semen regurgitates back into the bladder, to be passed with the urine when the erection subsides, or slowly escapes from the urethral orifice in a dribbling, intermittent manner. Fortunately, the sterility from stricture is amenable to surgical measures, and disappears with the removal of the obstacle that occasioned it.

A tight phimosis may prove an obstacle to the normal forcible discharge of the semen, and thus cause sterility. A number of cases are on record in which sterility from this cause was cured by the operation of circumcision.

Relative Aspermia.-This comparatively rare form is distinguished by the peculiarity that ejaculation of semen does not take place during coitus, but subsequently, as a pollution during sleep. While the most prolonged and exhansting efforts during coition or long-continued manipulation (masturbation) fail to provoke the seminal discharge, nocturnal pollutions under the influence of erotic dreams are not infrequent.

This form of aspermatism may be due to anæsthesia or a diminished excitability of the sensory nerves of the penis, which normally respond to the mechanical stimulus of coition by reflex contraction of the ejaculatory muscles. The absence of voluptuous sensations, which characterizes this condition, would confirm this view. Since in this class of cases emission never occurs when the patient is awake, the influence of the inhibitory nerves upon the center of ejaculation may be exaggerated or otherwise perverted. When this restraining action is suspended during sleep, pollutions take place. 
Relative aspermia presents certain analogies with the relative or psychical form of impotence, and, like it, is often of a temporary character; one morbid condition may replace the other, the patient being at one time aspermous, at another impotent; the same pathogenic cause may be invoked for both.

Temporary Aspermia.-In still another class of cases ejaculation takes place at certain times and under certain circumstances, while at other times it fails completely. Temporary aspermatism is found chiefly in persons who suffer from irritative debility of the genital organs. The majority of patients thus affected have been addicted to masturbation and venereal excesses, or have suffered from repeated attacks of gonorrhœa. The inflammatory and other changes in the prostate thus induced exert a paralyzing influence over the nervous apparatus, which controls ejaculation. With the improvement in the local condition the ejaculatory function may be restored in comparative or complete integrity.

Oligospermia.-By this term is understood a diminution in the quantity of the semen discharged by ejaculation. A variety of causes may contribute to the decrease or entire suppression of one or other of the secretions which collectively constitute normal semen. When the testicular secretion is diminished, the associated condition known as oligozoöspermia is present. Both are normal conditions of advanced age, when the physiological activity of the seminiferous organs have begun to decline.

Oligospermia may be congenital from feebleness, or inherent debility, or nondevelopment of the sexual organs. It is more often acquired, and occurs in the course of exhausting diseases, or as one of the manifold manifestations of neurasthenia. As a result of malnutrition or faulty innervation, the secretory activity of the sexual organs is impaired. A marked diminution of the seminal fluid is found in cases where the prostatic secretion is arrested from inflammatory or suppurative disease of this organ, or where the seminal ducts are occluded.

Oligozoöspermia may be defined as the diminution of the number of spermatozoa. As is well known, the semen of different individuals varies not only in the amount discharged at each ejaculation, but also in the relative number and proportion of contained spermatozoa. Ultzmann has endeavored to fix as a standard of comparison the number of spermatozoa which shonld appear in the field of vision of a given objective. He asserts" that "normal healthy semen should show in the microscopic field, with a Hartnack objective 7 and ocular 3, about one hundred living spermatozoa."

The spermatozoa vary not only in number, but in their period of development, activity, and other physical qualities, and consequently in fecundating power. In oligozoöspermia the spermatozoa are not only 
diminished in number but often altered in quality; when small and feebly vitalized they involve a diminution, when motionless or dead an entire loss, of procreative power.

This condition is a common result of masturbation and the frequent seminal losses induced by this habit. The testicles can not respond to the inordinate demands made upon their functional capacity by the production of healthy and well-formed spermatozoa. The injurious effects of natural sexual excess are no less pronounced. Nature requires a certain period of repose for the development of fully formed spermatozoa. It has been observed that when coitus takes place quite frequently the semen becomes more watery and scanty, and the spermatozoa are fewer and smaller, until after a certain number of repetitions of the act they may entirely disappear from the ejaculated fluid, which consists merely of the secretions from the accessory glands.

The more pronounced and permanent forms of oligozoöspermia result from various affections of the testes, epididymis, and vasa deferentia. Partial occlusion of the efferent vessels of the testes from unilateral epididymitis, monorchidism, etc., may cause a diminution of the number of spermatozoa. Pressure upon or disorganization of the testicular glandtissue from the presence of tubercular or syphilitic neoplasms may cause a gradually increasing diminution in the number of spermatozoa, until they finally cease to appear. In this case there is a transition of this condition into azoöspermia.

Azoöspermia.-This condition implies an entire absence of spermatozoa from the semen (Fig. 2). Although the seminal fluid deprived of the testicular secretion is fatally impaired in its fecundating capacity, there is no notable diminution of its quantity or obvious macroscopical change in its physical characters. It is perhaps more fluid, but the property of coagulation and the color and odor peculiar to normal semen remain unchanged. When placed in a test-tube and allowed to settle, it will be found that the whitish sediment is less dense and abundant in proportion to the liquor seminis than in normal semen. This sediment, according to Ultzmann, consists mostly of epithelium from the seminal ducts and urethra, seminal granules, and very often also of numerous well-formed spermatic crystals. The so-called spermatic or Böttcher crystals are larger, more numerous, and appear more quickly than in normal semen.

Azoöspermia not infrequently results from sexual abuse or excess. The sterility thus induced is usually of a temporary character; with rest and time, healthy and fruitful spermatozoa are again reproduced. It is a matter of common observation that a newly married pair who indulge excessively in sexual intercourse may remain sterile for a long period, unless impregnation has taken place at the first embrace or soon thereafter, before the quality of the semen has become deteriorated. If, now, they 
are separated for a period until the spermatozoa have time to mature and fully develop, impregnation takes place when they come together again.

General diseases, especially diathetic conditions, may cause an absence of spermatozoa by weakening the organism. The emasculating influence of diabetes is generally recognized. Spermatozoids are seldom found in the genital passages of persons who have died from tuberculosis.

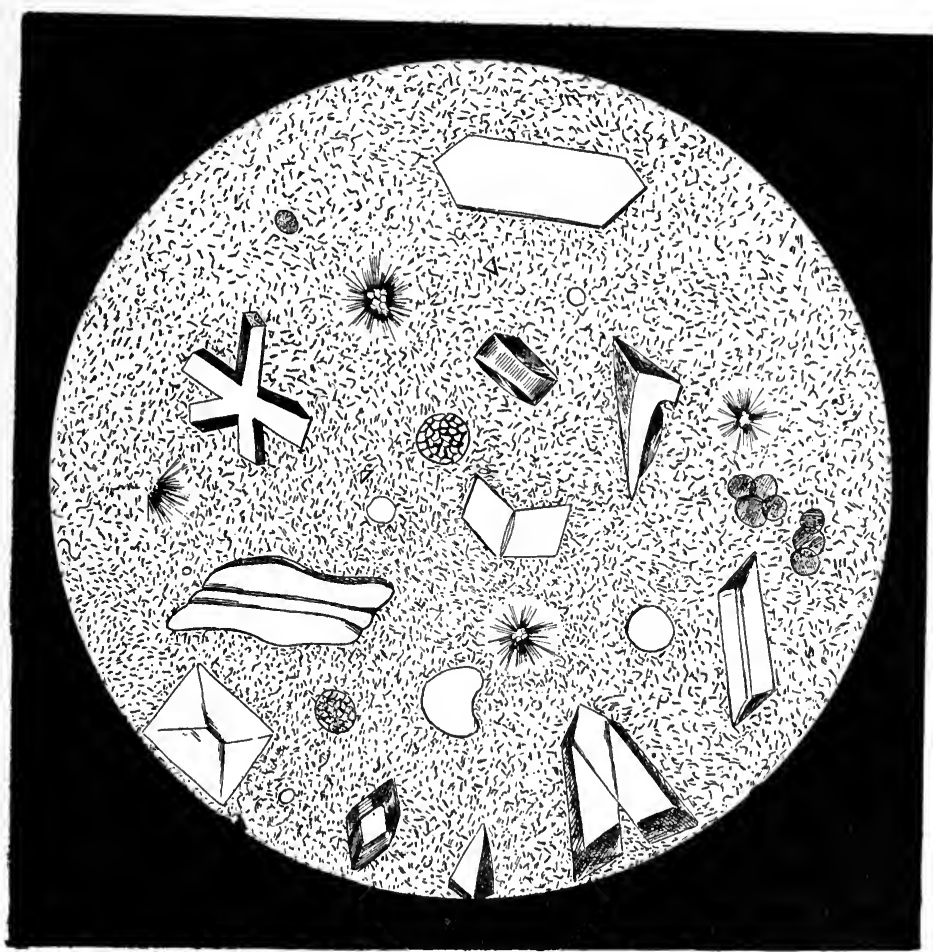

Fır. 2.-Spermatic fluid in azoöspermia. (Peyer.)

From the nature of its etiology azoöspermia is usually permanent. It may be congenital or acquired. Permanent absence of spermatozoa is occasionally, though very rarely, an idiopathic affection.

The congenital anomalies or vices of conformation which cause azoöspermia are absence of the testes, failure of the testes to descend (cryptorchidists are usually but not invariably sterile), and congenital deficiency of the vasa deferentia.

The most common cause of the acquired form is gonorrhœal inflammation of the testes and spermatic cord. If the epididymitis is bilateral and results in occlusion of both vasa deferentia, the sterility is absolute. 
Tubercular epididymitis, syphilitic sarcocele, cystic or solid tumors of the testicle, or parenchymatous orchitis from whatever cause, may interfere with the secretory structures of the organ to such a degree as to render the patient azoöspermous.

Chromospermia. - This term I have coined to express the different modifications in color which the semen presents under certain conditions, the exact pathological significance of which, especially in relation to sterility, has not been carefully studied. Some of these color changes in the seminal fluid present certain analogies with similar modifications in the secretion of the sweat-glands. It is probable that they occur much oftener than is generally supposed, but escape observation for obvious reasons, the conditions under which the semen is discharged presenting few opportunities for examination-mostly, in fact, in masturbators, who are not disposed to confess their practices, much less to place en évidence visible and palpable proofs.

The following varieties of chromospermia have been observed in diseases of the genital apparatus:

1. Red or Reddish-brown Semen, due to an admixture of blood, and which may properly be designated as homatospermia. The coloring material may arise from hyperæmia or a congested condition of the prostatic urethra, induced by gonorrhœal inflammation. It may come from the seminal vesicles, as a result of acute inflammation (spermatocystitis) or tubercular and cancerous disease. Ultzmann endeavors to differentiate the source of the blood in bloody pollutions, by the character of the stains. When from the pars prostatica "the dried stains on the linen appear irregularly colored, on the rusty-brown stains are usually small bloodspecks, while in the second case the stains appear evenly colored, which indicates an intimate mixing of the blood with the semen, as might happen in the seminal vesicles. When the stains are of a brownish-yellow color there is usually an admixture of pus with the blood."

Hæmatospermia may also result from the intense congestion of the mucous membrane of the prostatic region, which follows masturbation and sexual excess.

Yellow or Greenish-yellow Semen.-A greenish-yellow color is imparted to semen by the admixture of pus. The purulent discharge may come from the prostatic neck in the course of an acute gonorrhœa, or from epididymitis followed by a catarrhal inflammation of the vasa efferentia, the result of gonorrhœa. Terillon has collected a number of cases of bilateral epididymitis, in which the ejaculated fluid was of a yellowish tint verging on green. While it contained abundant pus-corpuscles and a few large granular corpuscles, spermatozoa were nearly always absent. The same condition may result from inflammation of the seminal vesicles. Ultzmann states that, when the pus comes from the 
neck of the bladder, "the stains have not an even yellow color, but the pus appears on the dried seminal stain in smaller or larger patches, irregularly distributed; when from the seminal vesicles, the stains appear evenly colored."

Wine-colored Semen.-The nature of this stain excited considerable comment until it was discovered to be due to indigo ; under the microscope the presence of crystalline indigo is readily demonstrated, and serves to differentiate it from bloody semen, to which it bears a macroscopic resemblance. The intimate admixture of the stain gives it a more even coloring than is usually seen in hæmatospermia.

Grass-green Semen.-This color is said to be due to the admixture of pus with strong indigo-colored semen.

Blue Semen is mentioned by Ultzmann as a probably rare modification of the grayish-white color of normal semen. Its occurrence is problematical.

As before intimated, the pathogenic influence of these color modifications upon the vitality of the semen has not been carefully studied. From the fact that greenish-yellow semen is almost always characterized by the absence of the essential anatomical elements, it would seem evident that pus is destructive to the evolution and life of the spermatozoa. Heitzmann's observations on spermato-cystitis would indicate that there may be a transformation of spermatozoa into pus-corpuscles.

The etiology and symptomatology of sterility have been indicated in the foregoing description.

Prognosis.-The prognosis of sterility will depend upon the cause and nature of the pathological condition present; in general, it may be said that it is not favorable.

When due to a mechanical impediment in the urethra to the passage of semen, which may be removed by surgical art, the prognosis is favorable. When the obstacle is situated more deeply in the ejaculatory ducts, the vasa deferentia, or involving the secretory structures of the testicle, their inaccessibility precludes the possibility of direct treatment, and the prognosis is correspondingly unfavorable. Nevertheless, we often find that the azoöspermia from gonorrhœeal inflammation and that attending syphilitic infiltrations is of temporary duration. With the subsidence of the epididymitis, the inflammatory engorgement occluding the efferent vessels may be resorbed and their lumen be restored; likewise, syphilitic infiltrations in the body of the testicle may undergo involution either spontaneously or as the result of treatment. In eighty-three cases of bilateral epididymitis due to gonorrhœea, collected by Liégeois, spermatozoa reappeared in the semen of only eight of these patients. The prognosis in cases of syphilitic origin is always more favorable.

Onanismal azöospermia, or the sterility from sexual excess, is usually 
of temporary duration, and disappears under the corrective influence of time and repose.

Treatment.-The treatment of male sterility does not, as a rule, yield favorable results. The exceedingly rare cases in which phimosis causes sterility may be cured by circumcision. The sterility resulting from coarctation of the urethra, or other impediment situated between the orifices of the ejaculatory ducts and the meatus, may be cured by removal of the cause. Absolute aspermia presents no available point for treatment.

In the partial stenosis of the vasa efferentia from gonorrhoeal inflammation, and in obstruction of the ejaculatory ducts, faradization of the testicles has been recommended with the view of exciting their secretory activity. It is claimed that the vis a tergo of the increased seminal flow serves to dilate the partially occluded vessels.

In occlusion of the vasa efferentia from bilateral epididymitis little hope of successful treatment can be offered, especially if the imperviousness is complete and has existed for some time. Under the influence of iodide of potassium internally and the local use of resolvent ointments, occasional cures of chronic cases have been reported. The very hopelessness of these cases emphasizes the necessity of prophylaxis. In all cases of bilateral epididymitis prompt and vigorous antiphlogistic treatment should be instituted and kept up until the indurations have undergone complete involution.

In advancing atrophy of the testicles good results may sometimes be obtained by galvanization. Where the tubular structures of the testicle are pressed upon or blocked up by diffuse or circumscribed syphilitic infiltrations, specific treatment-iodide of potassium and mercurials, either in the form of inunctions or plasters-should be energetically employed.

Ultzmann reports favorable results in the treatment of chromospermia when the blood or pus comes from the prostatic urethra from cauterization of the caput gallinaginis. When the blood and pus come from the seminal vesicles, cauterization does not accomplish much. 


\title{
GONORRHEA IN THE FEMALE.
}

\author{
By ANDREW F. CURRIER, M. D.
}

History.-The term gonorrhoea, like so many others which have been retained in medical terminology, is based upon a misconception. It is attributed to Galen, who used the word yovóṕpoua, from yov' or yóvos, semen, and $\hat{\rho} \hat{\epsilon} \iota \nu$, to flow (De Locis Affectiones, vi, 6 and xiv, 10). He considered it an affection of the vessels which are designed for the excretion of semen, and not of the genital organs proper. The term blennorrhoea, which is in common use with many writers, especially French writers, and derived ultimately from $\beta \lambda \lambda^{\prime} \nu o s$, mucus, and $\hat{\rho} \hat{\epsilon} \iota \nu$, to flow, is more nearly expressive of the true condition of affairs, especially in the gleety stage, while pyorrhoea, which signifies a discharge of pus, would be still more correct. All these terms are inaccurate, however, for they do not indicate whether the discharge is from the urethra, the conjunctiva, or any other tissue which is capable of secreting pus or mucus.

But the term rovóṕóna is older than Galen, for it may be found in the writings of Hippocrates and Herodotus, and their observations were probably of phenomena similar to those which were observed by Galen. In the writings of Paulus Eginetus, Actuarius, and Trallianus, gonorrhœa is described, but only as it occurs in males. Aretæus observed that women who suffered with gonorrhœa experienced gnawing pains in the sexual organs, and desire for coitus, while such sensations were not experienced by male sufferers. In all the ancient writers who have made any reference to gonorrhœa in women, this condition seems to have been confused with leucorrhœa and abnormalities of menstruation. The contagiousness of the disease was clearly recognized by them, whether it occurred in males or females. The ancients also recognized the presence of condylomata which are so common an accompaniment of gonorrhœa in women, descriptions of them being given by Atins, Aretæus, Paulus, Aginetus, and Archigenes. Cedrenus observed that condylomata when ulcerated cause infection during coitus (Synopsis, Paris, 1647, page 266), and a similar observation was made by Palladius (Vita patrum, cap. 148). Condylomata were also recognized by Galen and Hippocrates, the latter recommending their destruction with knife or caustic (De Natur. Mulierum, vol. ii). 
The most ancient description of gonorrhœa is said to be found in a Chinese manuscript written by the Emperor Hoang-ty in the year 2637 в. с. It is referred to by Verrier in an article entitled De la blennorrhagie chez les peuples de race jaune (Le Médecin, Paris, October 10, 1886). In this ancient document gonorrhœa is described as an inflammation of the urethra and vagina, and often of the bladder, which is accompanied with a white or red discharge. The symptoms in the female are said to be similar to those in the male, namely, pruritus ani, heat and swelling of the vagina, burning pain in micturition if the urethra is involved, a discharge of green or yellow pus, and pain extending from the hypogastrium to the perinæum. Such a description is a fairly satisfactory one, but one is inclined to be skeptical as to the authenticity of the record.

A Japanese manuscript of the ninth century A. D. is also said to contain descriptions of the various forms of venereal disease, including gonorrhoea (Scheube, Virchow's Archiv, March, 1883, page 448). Again, in the fifteenth chapter of Leviticus Moses refers to a condition which made Israelites possessing it ceremonially unclean, and this has been interpreted as signifying gonorrhœea. Josephus states that the Israelites contracted venereal disease from the Moabites while on their journey through the wilderness to Canaan. Many other facts in connection with the history of the nations of antiquity concur in leaving little doubt that venereal diseases were about as rife with them as with us, and it is safe to assume that gonorrhoea was one of them.*

The absence of checks and restraints upon the appetites and passions among savage and barbarous people would suggest the prevalence of venereal disease among them, and such seems to be the fact. Accurate information in regard to the condition of women among such people is extremely difficult to obtain. I have made extended inquiries, through physicians in the employ of the War and Interior Departments, concerning venereal disease among the women of our native Indians, but have failed in getting as definite information as could be desired. It is easily ascertained that syphilis is exceedingly common among them, for that is evident, without interrogation. The medical officers also speak, with scarcely an exception, of the almost utter absence of virtue among Indian women, more particularly the young ones, but to obtain definite data as to the amount of suffering which they experience from venereal disease seems impossible, both on account of their ignorance and their unwillingness to submit to the necessary examination and treatment. $\dagger$

What the influence of religion in the form of Nature-worship, which

* Some of the foregoing data, and inany other curious and interesting ones, have been collated by Dupouy in a work entitled La prostitution dans l'antiquité, Paris, 1887.

+ See the author's paper on a study relative to the functions of the reproductive apparatus in American Indian women, Trans. Amer. Gyn. Soc., vol. xv. 
with its licentious rites and orgies was practiced by so many of the nations of antiquity, may have been in the way of producing this disease which is so intimately associated with excesses and filth, we can only conjecture.

Etiology.-The statement of Fournier that the causes of gonorrhœa nay be reduced to contagion and excessive irritation of the urethra, though applicable to the male sex, is equally applicable to the female when slightly modified.

The concept of the relationship of the disease to germs and fungi, however ancient, was impossible of verification until the development of modern physiological chemistry and the improved microscope, hence our knowledge of the subject in this direction is of very recent date. Donne's spore trichomonas vaginalis was discovered in discharges from the vagina in 1844 ; Salisbury's crypta gonorrhoica in 1868 ; Jousseaume's algue genitale in 1872; Hallier's konotecium gonorrhoicum in 1872.

These discoveries marked a new era in the study of gonorrhœea, and were prophetic of the revolution which was soon to take place in the investigation of infectious disease. The new science of bacteriology was beginning to assume definite proportions. But it is not within the province of the present article to discuss the question of etiology except in a very general way. The article on the Etiology of Urethritis should be consulted for such information.

Another important chapter in the evolution of our present knowledge concerning gonorrhœa in the female was furnished by the investigations of Nöggerath. In 1872 appeared his first paper on this subject (Die latente Gonorrhöe im weiblichen Geschlecht), and in 1876 his second (Latent Gonorrhcea, especially with regard to its Influence on Fertility in Women). The conclusions of Nöggerath were accepted by Angus Macdonald, Amann, and Hennig, but not by gynæcologists in general. He maintained that gonorrhœa was not only extremely common, but that it often remained latent for years; that newly wedded women were very often infected by husbands who had long since ceased to show any active symptoms of gonorrhœa-sterility, dysmenorrhœa, perimetritis, salpingitis, and ovaritis resulting; and that gonorrhœa, either in the male or the female, was practically incurable. Nöggerath barely missed the discovery of the gonococcus, for he says (Trans. Am. Gyn. Soc., vol. ii, page 268): "In my work published four years ago I expressed the hope that the key to solve the question might be found in the presence of a fungus peculiar to the secretion of women affected with latent gonorrhœa, of which my researches up to that time had given much encouragement. I have, however, not followed them up for several reasons, principally owing to the fact that I found the same fungus in discharges from very young children, in cases where I was unable to trace all the points in the etiology 
of the case that would be necessary to establish the value attributable to the presence of this growth." Subsequent investigations, both clinical and microscopical, have fully demonstrated the value and importance of Nöggerath's observations.

In 1879 Neisser, of Breslau, announced the discovery of the gonococcus, a microbe which he claimed was characteristic of gonorrhcea (Ueber ein der Harnröhrentrippers eigenthümliche Mikrokokkenart, Centralbl. für die med. Wissen., 1879, page 28). He asserted its constant presence in gonorrhœal pus, and its identity with a microbe which is found in purulent ophthalmia neonatorum. It was usually found in groups upon the surface of pus-corpuscles, being first round in shape, and then developing into the form of a long oval. Then there was a division in the cell with an interval between the two segments, and then elongation at right angles to the former plane of division. He also stated that this micrococcus differed morphologically and functionally from all other forms (Die Mikrokokkus der Gonorrhöe, Deutsche med. Wochen., 1882, No. 20). The investigations of Neisser were speedily supplemented by those of many others, who confirmed the statements which he had made. The list of such investigators is a long one, and includes such names as Weiss, Bokai, Brieger, Cheyne, Ehrlich, Rücker, Gaffky, Aufrecht, Bumm, and Oppenheimer.

Definition.-To define gonorrhœa in the female in a manner which shall be concise and at the same time comprehensive, is not an easy matter. The disease is, of course, identical with gonorrhoea in the male, so far as its pathological characteristics are concerned. It is an infectious disease, which primarily involves a larger or smaller area of the mucous membrane of the genital tract in the great majority of cases. It may extend within to the peitonæum, or withont to the skin. It may involve the mucous membrane which is not included in the genital tract, and other serous membranes besides the peritonæum. Its ultimate cause is probably the gonococcus Neisser, though other microbes are almost constantly found associated with this one, and the fact of mixed infection is generally admitted. It is frequently identical with purulent ophthalmia, especially in the newborn. Its phenomena are those which belong to inflammations of the mucous and serous membranes, but vary in intensity and in the area involved in different cases. The hypothesis of the microbial origin of the disease is a convenient one to explain many cases of the dormant or latent variety, in which a history of recent impure sexual intercourse is wanting.

But belief in the pathogenic power of the microbe gonococcus does not imply that its demonstration is possible in every case of gonorrhœea. The area over which or within which it may find lodgment is an extensive one, the number present at a given moment may be small, and the 
keenness of vision and skill in investigation of different.observers vary within wide limits. It has long been my opinion, in which I am supported by some of the ablest and most conscientious observers, that in most cases clinical phenomena will enable one to determine a diagnosis of gonorrhœe even though gonococci be not found. In cases to which medico-legal importance is attached their demonstration is of value, though even in such cases it is not always imperative. Recent opinions upon this subject vary. Kratter (Occidental Medical Times, June, 1891) states that without a bacteriological examination it can not be determined, in a case of suspected rape, whether a vaginal discharge is traumatic or not, and that the detection of gonococci is proof of the presence of gonorrhœa, which has probably been conveyed in the sexual act. But gonorrhoea can not be positively excluded if gonococci are not found. Vibert and Bordas (Jour. de Médecine de Paris, March 8, 1891) think it premature to give diagnostic value to micro-organisms found in vaginal and vulvar mucopurulent discharges. They made observations upon the discharges from the genitals of six little girls, upon each of whom a criminal assault had been perpetrated by men found to be free from gonorrhœa. In the vulvar discharge in each instance were diplococci, identical morphologically and bacteriologically with the gonococcus Neisser.

Boret (Provincial Medical Journal, Leicester, England, April, 1891) agrees with the opinion last mentioned, after investigation of cases of vulvitis in which there was no suspicion of impure contact or indecent assault.

The disease varies greatly in its intensity in different individuals, variations in susceptibility obtaining in this as in all other infectious diseases. The personal equation in such matters is one which can never be rigorously defined in advance. With some women the disease is sharp in its attack, severe in its course, and apparently over in a few weeks, especially if the treatment has been intelligently designed and carried out. With other women the symptoms are mild, of long duration, and recurrent at intervals, even when there is no suspicion of new infection. In other words, this disease, like other infectious diseases, may be severe or mild, and may continue a very long or only a short time.

Classification. - Little attempt was made to subdivide or classify gonorrhœa in women prior to the period which began with Nöggerath. By many of the older writers the disease was practically ignored, by others it was little understood, being briefly described as an inflammatory disease of the vagina, less often of the urethra or endometrium. In recent times it has been seriously questioned by more than one thoughtful writer whether gonorrhœa of the vagina ever exists as a primary condition.

In all descriptions of disease the simpler the classification the better and the less confusing. I think all clinical purposes may be satisfied by 
regarding gonorrhœa in women as acute and chronic, remembering at the same time that there may be repeated acute attacks, and that the disease may long remain dormant or latent. The difference between the acute and chronic conditions consists mainly in difference in the intensity of the symptoms. A number of writers speak of the diminished number of gonococci in the chronic state, and this is reasonable, since they are associated with pus-cells, and these are not abundant in the chronic condition.

Variety of Forms. - So great is the variety of opinion as to the frequency of different forms of gonorrhœa that I am persuaded its phenomena must vary with the locality, whether from difference of individual habits, varying effects of climate, or some more obscure cause. Bumm, whose writings and investigations upon this subject during the past ten years demand for him a respectful hearing, found as a result of examining and studying 53 cases from their very beginning during a period of five months or more (Franenarzt, 1891, vi, 345), that the endometrium of the vaginal portion of the cervix was the seat of gonorrhœa in seventyfive per cent of the cases, the endometrium of the corpus in fifteen per cent, and the mucous membrane of the tubes in 3.5 per cent. He formerly questioned the existence of a vaginal gonorrhœa in adults. He now believes that it seldom occurs in adults as an acute process, and never as a chronic one.

Steinschneider (Jahresb. der Schles. Gesellsch. für vaterl. Kult., 1887; Breslau, 1888, lxv, 49) has found the urethra the seat of the disease in the majority of acute cases, and the cervical endometrium in the majority of chronic cases.

Eraud (Cong. Internat. de Dermat. et de Syphilig., 1890, p. 642; Le bulletin méd., 1889, iii, 1080) found gonorrhœal urethritis and endometritis with equal frequency. The gonococcus was obtained from the endometrium by examination of the scrapings obtained with the curette. In only a few instances was he able to find the gonococcus in scrapings from the corporeal endometrium. He believes that the gonococcus does not live upon pavement epithelium, and hence, if found in the vagina, that it is there by accident, having migrated thither from the cervix uteri with the discharges from the cervical endometrium. In his opinion, therefore, gonorrhœal vaginitis is secondary to metritis.

Ingria (Gazetta degli Ospedali, 1887, viii, 805) thinks urethral gonorrhœa is far more frequent than vaginal, but the existence of the latter is not doubted.

In a total of 750 cases of gonorrhœa in women seen by Jullien in Paris hospitals there were 286 cases of acute or chronic urethritis.

Sänger (Annales de gynécologie et d'obstetrique, 1890, xxxiii, p. 111) finds gonorrhœa most frequently in the cervical endometrium; vaginitis is less frequently seen, the vagina receiving infectious matter without 
being always influenced by it. He has not found urethritis of very common occurrence.

Horand (Lyon médical, 1888, lix, p. 251) examined 5,090 prostitutes and 764 children, many of the latter the victims of rape, between the years 1882 and 1888. In the women he found that the urethra was most frequently the seat of gonorrhœa, while in the children it was the vagina. (Among 85 children he found vaginitis in 68 ; in 33 of the latter he found gonococci; and in 20 the vagina alone was diseased. He also found gonococci in 20 cases of vaginitis in women, but in 239 other cases he was unable to find them.) Welander (Le bulletin médical, 1889, iii, p. 3) found gonócocci more frequently in the urethra than elsewhere, especially among prostitutes.

Thus, though there has been strong opposition to the idea of the existence of primary gonorrhœal vaginitis, that opposition is becoming less pronounced. The principal objections that have been offered are that the gonococcus does not live upon the pavement epithelium of the vagina nor in the secretion of the vagina which is normally acid. If it happens to be found in the vagina, it is there by accident in connection with primary disease of the urethra or endometrium.

And yet the objectors to vaginal gonorrhœea admit its presence and frequency in young children, and its occasional occurrence in young women.

The case is, therefore, not conclusive against those who insist strongly upon the presence and frequency of vaginal gonorrhœea. Even Bumm has admitted that he had found the gonococcus in and under the deeper epithelial layers of the vagina (Neumann, Internationale klinische Rundschau, 1888, ii, p. 217), and his earlier statement that it was found only on cylindrical epithelium was denied by Jadassohn (Deutsche medicinische Wochenschrift, $1889, \mathrm{xv}$, p. 683). Welander (loc. cit.) admits the difficulty of finding the gonococcus in the vagina for the reasons given, and also on account of the great number of other microbes in the vagina with which the gonococcus might be confused, but he thinks that a negative result in this direction has not a sufficiently demonstrative value. Furthermore, he has seen several cases which he could only interpret as essential gonorrhœal vaginitis. Urethritis was also present; the vagina was the seat of an acute purulent catarrh, and so tender to the touch that a speculum could not be used. In nine such cases gonococci were found, though they were also found in the uterus. In six other cases of gonorrhoal vaginitis there was no involvement of the uterus. The differentiation which Welander would make between gonorrhœal vaginitis and ordinary vaginal catarrh consists in the abundance of pus-cells in the former, and in the diminution of the microbes which are usually present in the vagina. Zeissl has found vaginal gonorrhoea twenty times more frequent 
than urethral (quoted by Ingria, loc. cit.). Terillon (Le bulletin médical, 1890 , iv, p. 837) affirms that gonorrhœa involving the uterus is preceded by gonorrhœa of the vagina, the former developing from the latter, and the former frequently culminating, as Bernutz and Goupil have shown, in pelvic peritonitis. There is, then, great confusion among investigators concerning the frequency and significance of the various forms of gonorrhoea, also concerning the diagnostic value of the gonococcus, its presence in this or that locality, and upon or within this or that tissue, and whether it is this organism alone which causes disease, or a combination with other organisms. There is also great difficulty in deciding between the true gonococcus and the false with which it is morphologically identical (Bumm, Bockhardt). On the one hand, we are told it is always easy to find the microbe (Horand); on the other, that in fifty per cent of cases it can not be found (Schwarz). Equally puzzling are the statements that it is always a superficial disease (Bumm, Sänger), and that it may involve the tissues as well as the secretions (Schmitt, Wertheim, Wiener klinische Wochenschrift, 1890, iii, p. 476). My own experience with this disease, which has been in a field rich with opportunities during the past twelve years, has given me opinions which are decided and to my own mind convincing, though I confess that my faith in the influence of the gonococcus is less firm than it was a few years ago. If it is mixed infection which produces the severer phenomena of gonorrhœa, why may it not be possible to discard the influence of the gonococcus altogether? for it seems to be fairly determined that the presence of the staphylococcus and streptococcus is always injurious. Again, if the pseudo-gonococci which are so often associated with the gonococcus produce phenomena which resemble, if they are not identical, with those produced by the genuine microbes, a diagnosis dependent upon such a basis is always open to doubt.*

Therefore, until bacteriologists can reach something like settled conclusions in regard to the part played by microbes and with something like unanimity, I think, with Nöggerath, Sänger, and others, that we must depend mainly upon clinical phenomena in determining our diagnosis. I am still far from believing, with Welander, that the view that gonorrhceal vaginitis is a common or the most common form clinically, of the disease, is obsolete. Possibly the view of that author that it is a very common form among children and young women, especially young prostitutes, is correct; but it must also be remembered that the greater number

* Neumann (Internationale klinische Rundschau, 1888, ii, p. 217) states that Bumm has found several diplococci in the vagina, similar morphologically to Neisser's microbe, and differing very little in their cultures, but not causing gonorrhœe. Bockhart has produced pseudo-gonorrhœe, urethritis, and epididymitis from pure cultures of staphylococei and streptococci, not differing in appearance from ordinary gonorrhœa. 
of cases of gonorrhœa occur in just such individuals, the possible changes in the vaginal epithelium of those who have been long married, or a long time prostitutes, or of those who have borne children, together with the better opportunities for vaginal drainage in the latter furnishing them with a certain degree of protection against gonorrhœal vaginitis which the others referred to would not have. At any rate, in the cases of gonorrhœa in women which $I$ have seen the greater number have come with vaginitis as the most conspicuous symptom. They have been in most cases young prostitutes, or young married women, or young women who have complained that they were victims of seduction. With these the external manifestations have also been most frequent. Next in frequency were those who suffered with pelvic peritonitis of gonorrhœal origin, especially prostitutes and recently married women. Fewer in number were those who suffered with urethritis and cystitis, and they were not infrequently women in mature life. Next in frequency were those who suffered with abscesses, glandular inflammations, and endometritis. I do not say that in my opinion endometritis is the least frequent manifestation or variety of gonorrhœa, but in my experience it is the form which has caused least personal annoyance to the patient, and fewest acute symptoms upon which a diagnosis could be based.

The disease may have the following forms or varieties, which may appear singly or in combination, and with or without complications: 1 , vulvitis ; 2 , bartholinitis ; 3 , vaginitis; 4 , urethritis ; 5 , endometritis ; 6 , salpingitis; 7, ovaritis; 8 , peritonitis.

Gonorrhœa in a woman always implies a precedent gonorrhœa in some one else, which has been acquired by infectious contact, but not necessarily by the sexual act. It is quite conceivable that it may be communicated through the medium of towels, handkerchiefs, articles of furniture, or even water-closet seats. It never exists de novo either in man or woman, ex nitilo nitil fit. Whether all vaginal and endometrial discharges are or are not infectious is another matter. Considering the number and variety of microbes which constantly inhabit both the vagina and endometrium, it is quite possible that under favoring conditions these may be the cause, or a cause, of inflammation in the male urethra, as Bockhart has'shown, but that is quite a different thing from gonorrhœa, the disease par éminence of dissipation, sexual excess, and vice. As gonorrhœa is in the majority of cases a disease of youth in the male, it is equally so in the female, partly because the greater number of those who are likely to be exposed to it are young, and partly, as is supposed by some writers, because of changes in the epithelium as life advances which enable it to offer greater resistance to infecting media.

Period of Incubation. - The time which elapses from the reception of the infectious material to the appearance of the symptoms of gonor- 
rhcea is not usually a long one. Of course, the symptoms appear more quickly when the mucous membrane of the genital tract is hyperæmic, as during menstruation or pregnancy, than under ordinary conditions. In some cases it has seemed to me that influences from the nervous system, such as fear, anxiety, shame, and remorse, have hastened and heightened the symptoms. In the very young we should expect indications of the disease more quickly than in those of mature years. From two to five days may be regarded as the usual period of ineubation for this disease in women, and I have not observed that the duration of this period varied from one form or location of it more than for another.* Why one portion of the genito-urinary tract should first show evidence of infection rather than some other portion, may be due in part to greater susceptibility, but more to efficient exposure. Thus, the primary form of the disease in children is almost always vulvitis; in young women it is more frequently vaginitis; while in the mature, and those who have borne children, it is often urethritis or endometritis. The crypts and follicles with which the genito-urinary mucous membrane abounds also explain the difficulty of eradicating the disease, and the frequency of the recurrence of acute attacks, when favoring conditions cause renewed activity in secretions and germs which may have been long retained in a dormant or inactive condition. I have never agreed with Nöggerath's earlier views, that the disease was necessarily incurable. It is doubtless radically cured in many cases, but we must not forget that there are many others, perhaps many which are never diagnosticated, which terminate in fatal peritonitis.

I shall first describe the varions forms of gonorrhœa in the order mentioned, and then their complications :

1. Vulvitis.-This is especially an inflammation of childhood; it is common in young women, it is rare in the aged. It may be limited to the labia majora, or it may include the nymphæ and the mucous membrane of the vestibule and introitus vaginæ. It is a typical inflammation, for it includes heat, pain, swelling, and redness of the affected parts. If the mucous membrane becomes involved, there is a discharge of dirty, yellowish, or greenish pus, acrid and excoriating. Intertrigo becomes annoying; walking is difficult and painful. The disease is prone to extend to the ragina, and not infrequently to the urethra, especially in young

\footnotetext{
* I have seen cases in which the patient was conscious of the beginning of the disease - pain, or soreness, or swelling being present-within a few hours from coitus. I have recently seen a case of gonorrhœa of the rectum and anus, with extensive ulceration of the mucous membrane and contiguous skin, in a young woman about eighteen years of age, in which coitus per anum at night was followed by evidences of gonorrhœa in the morning. Of course, a portion of the symptoms was due to injury to the sphincter muscle and the anal mucous membrane, but the distinctive evidences of gonorrhoa quickly appeared in addition to the effects of trauma.
} 
children with whom the meatus urethræ is prominent. In those who have not reached puberty the underlying cellular tissue is seldom invaded.*

In those who have passed puberty the cellular tissue is frequently invaded, the skin becoming hard and dense, or œdematous and boggy. In the former case the condition is frequently suggestive of elephantiasis, the enlargement of the labia being sometimes very great; but the termination in all cases which I have seen has been resolution ad integrum, after a period of three or four weeks. In the other cases we have true phlegmonous inflammation, with the formation of abscess which is sometimes quite extensive, the pus burrowing into the cellular tissue of the pelvis. In the majority of cases which I have seen, however, the swelling is in the direction of the least resistance-that is, toward the other labium majus - the position being very favorable for its surgical treatment. Why the infectious medium should in one case cause a phlegmonous and in another a nonphlegmonous inflammation is not always apparent. Probably traumatic influences occasionally favor the former result, the tissues being bruised or contused by violence of some sort, and the infectious element entering through the broken skin or mucous membrane.

Differential Diagnosis.-The diagnosis of gonorrhœal vulvitis is sometimes easy and sometimes difficult. It is especially apt to be difficult in children, by whom it may be acquired from the parents, from contact with other children, boys or girls who are contaminated, and from criminal violence. The greatest caution and judicial impartiality of mind are necessary in making a diagnosis when rape has been alleged; for not only the liberty but in some States the life of the accused person depends upon the determination of the crime. In view of all the recent bacteriological developments in this field, a diagnosis of gonorrhœa should not rest mainly upon the presence or absence of the gonococcus. Vulvitis or vulvo-vaginitis in children is said to result from the migration of worms from the rectum to the vulva or vagina, but, considering the closed condition of the vagina in most children, I should be skeptical concerning such a cause unless the evidence were very conclusive. Differentiation from vulvitis associated with epidemic diseases in children must undoubtedly be made, especially the eruptive fevers. Contributions to this subject have been made by Von Dusch (Deutsche medicinische Wochenschrift, October 11, 1888), Ollivier (La semaine médicale, October 24, 1888), Suchard (Revue mensuelle des maladies de l'enfance, 1888, p. 265), Tait (Lectures on Gynæcology, 1888), and the writer of this article (Medical News, July 6, 1889). In addition to the differentiation from the catarrhal

* I can recall but one case, a child about ten years of age, seen in consultation in whom the inflammation in the right labium majus had terminated in an extensive ischiorectal abscess opening near the lower portion of the labium. 
form of vulvitis to which the foregoing remarks apply, one must be prepared to exclude the phlegmonous form, which, if it occurs in children, is more usually of traumatic than of infectious origin, and the diphtheritic which is not infrequent in epidemics in which diphtheritic inflammations are present. The differential diagnosis of vulvitis in those who have passed the age of puberty is not usually difficult. Enlargements and inflammations of the vulva from traumatism, puerperal accidents, and elephantiasis are easily excluded; syphilis in this location must be studied in connection with its other phenomena in the secondary stage, though the primary sore may sometimes be discovered in connection with the enlargement of the labia and inguinal glands. Cancer affecting the vulva is so pronounced in its symptoms, especially pain, corrosion, and swelling, that its exclusion can almost invariably be made without difficulty. The subject of complications and treatment will be discussed in a subsequent portion of this paper.

2. Bartholinitis.-Inflammation of the vulvo-vaginal glands, or glands of Bartholin or Duverney, is a not uncommon form of gonorrhœa. I have never seen it except in adults. It is sometimes found unassociated with other forms of the disease ; indeed, cases are recorded in which a single gland or even a single duct has been the seat of the disease, and for a prolonged period. I am disinclined to believe that the disease ever limits itself to so small an area. It is quite possible, however, that this portion of it might remain after others were cured. The symptoms are those of an abscess in any other tissue which is easily distensible. The abscess may be no larger than a chestnut, or it may be as large as a small egg. It may exist in connection with a vulvitis, and may be included in a phlegmonous inflammation of the labia majora. Destruction of the gland accompanies the disease. Differentiation must be made from simple catarrhal inflammation of the gland, from retention of glandular secretion, and from traumatic inflammation.

3. Vaginitis.-So far as symptoms are concerned, it is this form of gonorrhœa which has most frequently brought women to me for relief. The urethritis from which women suffer is not comparable, as to pain, with the urethritis of men, and the endometritis of gonorrhœa has few acute symptoms, in some cases not any. In those forms of the disease in which the peritonæum is involved the pain is so severe that individuals are not usually to be treated as out-patients. As has already been stated, I dissent entirely with those who say that gonorrhœal vaginitis is a rare condition, at least according to my experience. In children it is usually, perhaps always, associated with vulvitis. In young adults it is the most common form of the disease ; in the mature and aged it is comparatively rare. No one denies the possibility of development of the gonococcus upon the vaginal pavement epithelium in children: why should it not de- 
velop upon the same tissue in adults? It would be absurd to hold that it is always secondary to infection of the uterus in children; it is almost as absurd to maintain the same universal proposition in regard to adults. In young adults the os uteri is frequently almost impervious, and the vaginal portion of the cervix small and retracted. The urethra in such women is also less exposed to infectious media than the vagina, and the latter is more adapted to retaining fluids which may have been injected into it than the vagina in women who have been long married or have borne children. But whatever the theory may be, the fact with me is unquestionable that vaginitis is the commonest form of the disease in $\mathrm{my}$ experience, and probably for the reason that has been given, that children and young women include the greater number of sufferers with gonorrhœa. The symptoms are unmistakable in the great majority of cases, and in the present state of uncertainty concerning the specific influence of the gonococcus (see note, page 1046, concerning investigations of Bumm, Bockhart, and others), as the sole cause of the disease, and the liability to mistake the true gonococcus with one of the five varieties of the pseudoorganisms with which Bockhart says it is morphologically identical. I think we may eliminate the question of its presence, in the greater number of cases at least, and make our diagnosis on the basis of the clinical phenomena alone. It is only necessary to quote such names as Nöggerath, Sänger, and Welander in support of the statement that clinical facts are sufficient to determine a diagnosis. I have in a number of cases seen this form of the disease in young unmarried women whose habits and character have been irreproachable save for the lapse or two which was the cause of their misery. The symptoms in such cases have been very pronounced, and perhaps they will best serve as types of gonorrhœal vaginitis. Fear of discovery of their fault, retention of secretions within the vagina, and active condition of the vaginal epithelium, are all favorable conditions in such cases to the exuberant development of the disease. The period of incubation is short; it may not be more than twenty-four or forty-eight hours before pain is felt in the vagina, walking becomes difficult, the tissues are swollen and hot, and the color of the mucous membrane is a dark, dirty red, which I have compared to the color of wet hemlock sole-leather. The stage of congestion is followed by the stage of secretion, the secretion being purulent, the pus sometimes yellow and sometimes green. An examination even with a small speculum at such a time is exquisitely painful, and the slightest rudeness tears away the epithelium of the vagina or the vaginal portion of the cervix, leaving a bleeding surface. This acute stage may last one week, two weeks, three weeks, possibly longer if it is untreated, and almost always longer in young children, but I have never seen a case in which it was followed by cicatrization; so that the statement of Bumm and others that 
gonorrhœa is essentially a superficial disease is probably true, at least in so far as it applies to the vagina.

Differential Diagnosis.-The admission of sexual intercourse on the part of the patient, and the quick succession of the phenomena mentioned, make a diagnosis easy. Those conditions with which gonorrhœal vaginitis may be confused are usually to be eliminated without difficulty. They may be considered as catarrhal, diphtheritic, and phlegmonous vaginitis, also endometritis with profuse discharge. Catarrhal vaginitis may be an accompaniment of the eruptive fevers, constipation, rectal disease, and anæmia, but in none of these cases would gonorrhœa be suspected. In the pregnant or puerperal state a profuse vaginal discharge is not unusual, and it is quite necessary to be on the lookout for other symptoms of gonorrhœa, which not infrequently coexists with pregnancy, both in its acute and chronic or recurrent forms. The leucorrhœea which so often comes before and after menstruation must also be eyed with suspicion in suspected eases, and its relationship to infection carefully inquired about. The same remark will apply to the catarrhal vaginitis which is so common with women of uncleanly habits.

Diphtheritic vaginitis may be easily excluded, while the phlegmonous form is rare and should give a history of violence of some kind. Such a condition (phlegmonous vaginitis) might follow a severe labor, violent coitus, a blow or thrust; and in some cases gonorrhœa may coexist, and the diagnosis be obscured by the severity of other conditions. With reference to the misleading influence of acute endometritis, a careful examination will, of course, readily show that the symptoms do not proceed from vaginitis. That this form of gonorrhœa may long remain latent, is shown by the countless cases of gonorrhœa in men which are contracted from women who suffer with no acute symptoms, and in whom an examination reveals nothing conclusive of gonorrhœa. I have seen this fact demonstrated repeatedly.

Senile Vaginitis.-It would seem almost supererogatory to make a separate distinction for vaginitis under this nomenclature. This distinction has frequently been made, however, and with reasons which seem sufficient, for the conditions which are present in vaginitis in women who have passed the menopause differ from those which obtain in younger women. The tissues of the vagina and vulva are in a state of atrophy, the abundant fat of earlier years has been absorbed, the hyperæmia of pregnancy and menstruation no longer occurs, and the functional activity of glands and follicles is less marked. At this period of life a woman is less susceptible to the virulent action of gonorrhœa than in early life, and the influence of the disease, when contracted, is less intense and extensive. Vaginitis does occur, both gonorrhœal and nongonorrhœal, in the aged; and the former, in its latent form, may be carried over from the period of 
præ-menopause to that of post-menopause. It is characterized by an abundant muco-purulent discharge, the secretion being watery, of a dirtyyellow color; and though the epithelium is easily bruised, there is little or no swelling of the tissues, and the bleeding which follows bruising is not as free as in younger women. The mucous membrane has a dirty brown appearance, and under the mucous membrane of the vestibule one frequently sees slight effusions of blood. The discharge is irritating, excoriates the thighs and buttocks, and the consequent scratching and rubbing result in infiltration and parchmentlike hardness of the skin. Condylomata and inguinal enlargements rarely, if ever, occur. The condition is a most annoying one, is worse in cold than in warm weather, and may persist for many months, especially if the person is uncleanly in her habits. It is not easy to differentiate the gonorrhœal form from the nongonorrhœal, for the symptoms are quite similar. The history and surroundings of the patient must be of assistance in determining the diagnosis. In women who have had gonorrhœa just prior to the menopause, and whose lives are not above reproach, such a vaginitis as has been described is usually gonorrhœal. In other women, whose lives are exemplary and always have been, the vaginitis may be a concomitant of an irritated or torpid rectum; it may be associated with malignant disease, or it may be one of the expressions of anæmia.

4. Urethritis.-Gonorrhœal urethritis in women is not usually the serious disease that it is in men. It has its serious consequences, to be sure; it is very frequently accompanied or followed by cystitis; but the serious lesions of the ureters and kidneys, the destructive strictures of the urethra, and the disorganizing lesions of the bladder, which are matters of every-day occurrence as sequels of gonorrhœa in men, are rare indeed in women. Not that these may not occur in women; they may and do occasionally, but the topographical anatomy of the female renders them comparatively amenable to treatment. Gonorrhœa in the female has its serious consequences, but they occur rather when the disease progresses through the uterus and thence to the peritonæum, than when it follows the urinary channel. The greater frequency of urethritis compared with other forms of gonorrhœe, in the opinion of certain able writers, has been referred to. I have not found it thus frequent. Forty per cent would be a liberal estimate for those who have suffered with urethritis in my experience. It has not seemed to me a cause of great disturbance in children, though the situation of the urinary meatus in children is an exposed one. In married and mature women it is relatively frequent, in the aged it is rare. Its period of incubation is shorter than with men, and about the same as in vaginitis. Its invariable symptoms are burning and smarting in the urethra, frequent and painful micturition, and more or less swelling and sensitiveness of the urethral mucous membrane. The 
epithelium of the latter becomes eroded with the slightest touch, bleeding freely. Pus exudes from the meatus after the congestive stage has passed, but $I$ have never yet seen such intense inflammation and suppuration as commonly attend urethritis in the male. Were this the sum and substance of gonorrhœa in the female, it might usually be considered a trivial and easily cured disease. The glands and follicles by which the meatus and a portion of the lumen of the urethra are surrounded, furnish opportunity for ramification of the urethral disease, and may complicate it in a very troublesome manner, causing indefinite delay in the cure, unless the follicles are carefully investigated, one by one, and thoroughly treated. Eraud has described three varieties of gonorrhoal urethritis, based upon their differing histological characteristics. His paper may be consulted for this differentiation (Congres internationale de dermatologie et de syphiligraphie, 1890 , p. 642 ).

Sänger also describes a periurethritis, an interstitial urethritis, and a stricture of gonorrhœal origin, and states further that they are occasionally followed by suppurative catarrh of the bladder, ureters, and pelvis of the kidneys. The study of urethritis with the endoscope has not found as many workers as has endoscopy of the male urethra.

Janowski recommends for this purpose (Wien. med. Presse, 1890, xxxi, 1409) the straight endoscope of Grünfeld, the pan-electroscope of Leiter, the Nitze-Leiter instrument, or that of Skene. By means of the endoscope Janowski has demonstrated chronic papillary urethritis, and Janowski gives as varieties of gonorrhœal urethritis, from the same means of investigation, $U$. simplex chronica and $U$. granulosa. His paper (loc. cit.) may be consulted for an elaboration of the foregoing. The duration of urethritis is not usually so great as that of vaginitis. Plenty of cases are relieved of the troublesome symptoms in the urethra and bladder after two weeks of treatment.

5. Endometritis. - A gonorrhœal endometritis is likely to be a far more serious matter with a woman than a urethritis. In addition to the acute and chronic or recurrent varieties, we may distinguish an endometritis of the cervix, of the cervix plus the corpus, and of the cervix plus the corpus plus salpingitis, the gravity of the disease increasing with its extension. The extension is from cell to cell, but that does not imply that the entire endometrium becomes infected as the disease progresses. It may be involved in strips or islands, continuity being preserved, of course, at one or more points in order that progression from one cavity to another may be possible.*

\footnotetext{
* Bumm believes that extension may also be accomplished from cervix to corpus by the regurgitation of menstrual blood, but such a hypothesis should work both ways; and if a current setting back into the cavity of the corpus carried gonococci, which is very problematical, why may not the return current out of the uterus carry the offending organisms
} 
This fact shows the necessity for the development of uterine endoscopy ; for without an accurate knowledge of the areas of disease of the endometrium, which can be ascertained in some diseases at least only by sight, any method of treatment adopted, whether by applications to the endometrium or the use of the curette or other instruments, is crude and inexact, for it must include the entire mucons membrane, diseased and healthy alike. Steinschneider believes that all forms of gonorrhœal disease in women are secondary to metritis. Bumm believes all cases of gonorrhœal vaginitis are secondary to metritis. I am satisfied that there is a primary and a secondary endometritis, the former being the more common in married and parous women, the latter in the young and nulliparous.*

The symptoms with endometritis, if it exists alone, are usually few and unimportant. A dirty, muco-purulent, viscid discharge, and the fact that those who cohabit with her suffer with gonorrhœa, may be the only intimation a woman has that she is diseased. Such a history is repeatedly given by prostitutes. In a few cases the endometrial inflammation is intense, the vaginal portion of the cervix is swollen and very tender. In such cases gonorrhœa is certainly not a superficial inflammation, and these are probably instances of mixed infection. Erosion of the os uteri is also a very common symptom with endometritis. It occurs alike in the parous and the nulliparous. It is due partly to the bathing of the epithelium of the os in the discharge from the endometrium and partly to friction upon the floor of the vagina. It is not exclusively a symptom of gonorrhœa, for it accompanies abundant endometrial discharges which are not gonorrhœal in character. The os internum frequently serves as a barrier to the extension of the disease. The fact that in the young and nulliparous it is almost a sealed portal, and that infection does progress beyond it, is an argument in favor of the extension of the disease by the epithelium. If the disease advances to the corpus and pregnancy ensues, an abortion may result. This matter will be referred to again. Having reached the corpus, the disease may be arrested for some time, perhaps being intensified at the cornua before it advances to the tubes. $t$ The ex-

away He also thinks gonococci may be introduced into the uterus mechanically during coitus or intra-uterine instrumentation or medication.

* I have seen direct infection of the cervical endometrium in a hospital patient npon whom trachelorrhaphy had been performed. After the sutures were removed she was allowed to go home and spend Sunday. When she returned the cervical wound was gaping, intensely inflamed, and secreting pus abundantly. Her husband admitted that he had gonorrhœa.

f I recently saw such a case in a young prostitute. The uterine canal was extremely narrow, and in each cornu was an abscess about as large as a walnut, discharging constantly through the os uteri. In tying off the tubes the tissue broke down like wet paper. The tubes were congested, but scarcely pervious. The patient had suffered sereral weeks with the usual symptoms of acute peritonitis. 
tension of the disease to the tubes is a matter of common occurrence-in fact, the majority of women who suffer with disease of the uterine appendages probably suffer with gonorrhœa as a cause of it. The progress of this disease from one stage to another may be slow or rapid. In young newly married women the severe symptoms may culminate within a month, as was first shown by Nöggerath in his classical paper. Then a long period of invalidism may follow, with sterility and various other severe complications. The anatomical conditions of the endometrium are such that, if it has become infected, it is almost impossible to restore it to a healthy condition by any measure short of a severe surgical operation.*

It is often very difficult to prove the existence of gonorrhœal endometritis, especially in its latent condition. The presence of a purulent discharge from the uterus, development of gonorrhœa in those who come in contact with such a discharge, the latter individuals being free from the disease before such contact, promiscuous intercourse, or intercourse with one whose habits in regard to the sexual relation have been bad, are all suggestive factors in determining a diagnosis. The diagnosis is strengthened by the presence of gonococci in the discharge. Differentiation must be made from a simple endometritis with mucous or muco-purulent discharge; from malignant disease of the endometrium with profuse discharge, the corroding character of which soon becomes evident; and from endometritis due to benign tumors of the endometrium or the muscular tissue of the uterus.

6. Salpingitis. - It is hardly possible to conceive of gonorrhœal salpingitis as a primary disease. The same cause which produces it must previously have been at work upon the endometrial mucous membrane. (Saxinger is quoted by Sänger (loc. cit., p. 200) as reporting gonorrhœal salpingitis in virgins. This seems extraordinary, if true. Sänger explains it by the possibility of contracting gonorrhœa in other ways than by impure coitus.) This is eminently the disease of young adnlt life, and its destructive effects not only in sterilizing women by occlusion of the abdominal ends of the tubes, but in leading to lifelong misery and often death, make it one of the most important surgical diseases to which professional attention has ever been directed. Though it belongs to adult life, it is not unknown in childhood. Sänger has quoted a case of Welander's in which general peritonitis of gonorrhœal character (preceded, of course, by salpingitis) occurred in a child five years of age. Mercier observed as early as 1846 , in autopsies upon prostitutes, that salpingitis and ovaritis were very common among them. The epochmaking labors of Bernutz and Goupil in connection with pelvic peri-

* While the gonococcus is often found in the secretions of the cervical endometrium, it is rarely found in those of the corporeal endometrium. 
tonitis threw much light upon salpingitis, and incidentally upon the gonorrhœal variety. (Terillon (loc. cit.) quotes Bernutz and Goupil as reporting twenty-eight cases of pelvic peritonitis or salpingitis among ninety-nine cases of gonorrhœa.) Nöggerath clearly saw the destructive character of the disease, its especial relation to the child-bearing function, and its great frequency among newly married women whose husbands had at some time in their lives suffered with gonorrhœa. Tait, Sänger, and others have made important contributions to the knowledge of the subject, especially with reference to its destructive character, and the frequent necessity for its treatment by severe surgical measures. As already intimated, its course may be a rapid or a slow one. The entire mucous membrane of the tube may be found diseased, or only portions of it, and diseased parts may be encysted or walled off from parts which are less diseased or uninvolved. We may have tubal abscess or interstitial salpingitis. Terillon thinks the disease usually affects both tubes, and he accounts for the almost universal prevalence of sterility among prostitutes by the prevalence of salpingitis among them. (This can not be taken as a rule that is by any means universal, for there are countless cases in which prostitutes marry and have families, though they may have been sterile during the years in which they practiced prostitution.) Whatever the rôle of the gonococcus may be as an exciter of inflammation in other forms of gonorrhcea is probably exercised also in salpingitis. It is found not only in and on the pus-cells in suppurative salpingitis, but in and on the cylindrical epithelium of the mucous membrane of the tube (Bumm). Bumm's opinion that the gonococcus does not penetrate the deeper layers of tissue of the tube is opposed by Wertheim (Wiener klinische Wochenschrift, 1890, iii, 476), who found it in five cases of pyosalpinx in the pus of the tube, and in three of them in the epithelium and the connective tissue. Other observers who have found the gonococcus in connection with pyosalpinx are Westermark, Kelly, Martin, and Orthmann. To write the history and pathology of gonorrhœal salpingitis would be to cover the subject of salpingitis in general, and that, of course, would be more appropriate for a work on general pathology. This excuse may account for any apparent deficiency of detail in a subject which is so important as this. As in salpingitis from other causes, the gonorrhœal variety may be catarrhal or interstitial. In the former the tube may be only slightly enlarged, especially if the pus has free exit through the nterus. If there is obstruction to its exit, the tube may gradually become greatly distended, and its contents may be confined to one or several sacs. With catarrhal salpingitis of any severity we are almost certain to have pelvic peritonitis; and if the communication between the tube and ovary persists for any length of time after the establishment of the disease, the abdominal end of the tube remaining pervious, 
we will be almost certain to have ovaritis also. The disease is marked by frequent recurrences and exacerbations under the influence of exposure to cold, excesses of various kinds, the puerperal state, etc. Rupture of a pyosalpinx with fatal results is probably not uncommon. In the interstitial variety the development of the disease is gradual, there is great hypertrophy of tissue, and, while peritonitis is a common accompaniment, ovaritis is less common than with the catarrhal variety. Both of these forms of disease are attended with great pain and great danger to life, and, as a rule, the function of the organs is entirely destroyed. The surgical procedure by which these diseased organs are removed, in spite of its abuses, is a boon to humanity which can not be overestimated.

Ovaritis.-Althongh a gonorrhœal salpingitis may exist without a consequent ovaritis, a gonorrhoal ovaritis is impossible without an antecedent salpingitis. In the interstitial form of salpingitis, in which there is a slow and progressive hypertrophy of tubal tissue, the structure of the ovary may remain uninterfered with for a long time. Catarrhal salpingitis also may be localized, and the abdominal end of the tube occluded by an infratubal exudate which will prevent the passage of infectious material to the ovary. But if infectious material traverses the bridge which unites tube and ovary, or if peritonitis is excited in connection with an existing salpingitis, the inflammatory process will, as a rule, extend to the ovary also. The ovary may be simply dislocated and imbedded in a mass of exudate, more or less extensive and more or less dense, its nutrition and function being interfered with. In cases of slight intensity the tunica albuginea will bear the greater portion of the inflammatory process. If the process is severe and very virulent, abscess of the ovary will result, and the organ will usually be entirely disorganized. Like some forms of pyosalpinx, it may be so walled in by exudate that it is effectually shut off from the pelvic viscera, or, like others, its walls may be friable, and the possibility of rupture be a constant menace to the patient's life. The attachments which these inflamed and diseased tubes and ovaries make to the pelvic peritonæum and the pelvic and abdominal viscera, often render their removal among the most formidable and dangerous of surgical operations. The diagnosis of gonorrhoal ovaritis must be made in connection with other manifestations of the disease ; it is never primary. As to its duration, differentiation, prognosis, and treatment, the same remarks apply which are appropriate for salpingitis.

Peritonitis. - There are probably few cases of gonorrhœa which extend as far as the Fallopian tubes which do not excite a peritonitis. This may be of limited extent, including principally the peritonæum covering the tubes and the fundus uteri, it may extend to the peritonæum of the surrounding viscera and the pelvis, or it may become general. It may be 
of moderate intensity and with little or no appearance of exudate, or it may be manifested by the presence of serum, pus, and adhesions of greater or less extent and firmness. It is sometimes produced by the transmission of the infecting virus through the tubes to the peritonæum itself, and at others the infecting medium is probably carried by the lymphatics. All the phenomena which have been mentioned are perfectly familiar to every one who has had even a moderate experience with abdominal surgery. This is a disease which is particularly prone to occur before middle life. Sänger speaks of the period between the twentieth and thirtieth years, when the tissues and tissue changes are most active, as the period of greatest frequency.

Nöggerath's attention was directed to its frequency among newly married women, and I have seen it many times in young prostitutes under twenty-five years of age. One of its most marked features is its tendency to recur, especially in connection with the hyperæmia of the menstrual period. Nöggerath alludes to this fact under the term recurrent perimetritis. Recurrent attacks are almost certain to result in envelopment of the appendages in inflammatory exudate, both sides being usually implicated. One of the least serious of its results is the establishment of sterility, as the abdominal end of the tube becomes sealed. With it may be associated abscess of the pelvis apart from abscess of the tube or ovary, though dependent upon the extension of the disease from one or both of those organs. It is to be differentiated from traumatic noninfectious peritonitis, which may be caused by blows, by lesions accompanying a severe labor, or by rough manipulation of the genital organs, and from infectious peritonitis which is nongonorrhoal in character, such as the peritonitis of puerperal septicæmia, of the infectious fevers, or of severe disease of the rectum.*

\section{COMPLICATIONS.}

Condylomata.-Among the most common of the complications of this disease are the growths upon the skin of the external genitals which are known as condylomata. These are due to the infectious character of the secretions which are poured ont from the gonorrhœa-invaded tissues. They are highly infections, and the abraded surface of one of them when brought in contact with the contiguous uninjured skin (as in the opposing folds of the nates or labia) will cause a similar development. They are apt to be especially abundant around the fourchette of the vulva and the anus. They may be flat or acuminated, a lonse accumulation of epidermal scales, highly vascular, or nodes slightly raised from the surround-

* Bumm says that peritonitis associated with gonorrhœa is always due to mixed infection, the streptococcus and not the gonococcus being the efficient agent. 
ing surface. They may be dry, or abraded and secreting, the secretion being viscid and infectious. They may be isolated, or in groups or masses an inch or more in length. Isolated formations are prone to coalesce and conglomerate. They do not appear until the secreting stage of the gonorrhœe has been reached, and remain several weeks. They finally disappear without a scar. They are insensitive to the touch unless excoriated, and are most likely to occur with women of unclean habits. (The skin of some women seems to be insusceptible to this form of growth, or perhaps, in cases in which they are absent, the necessary virulence of the particular element which causes condylomata is wanting. Instead of condylomata, the discharge of gonorrhœa upon surfaces which are in apposition sometimes produces intertrigo, the epidermis being rubbed off and the parts becoming sore and painful, especially when the patient walks.)

Enlarged Inguinal Glands.-This complication is not uncommon with gonorrhcea, and very often results in abscess. Sänger thinks it always results from a mixed infection.

Gonorrhœa of Nose and Mouth.-These complications are possible, for the disease may occur on any mucous membrane. It is referred to by Sänger, but he quotes no case. I have never seen it in these locations.

Gonorrhœa of Anus and Rectum. - This form of the disease is not uncommon in women, and is the source of much pain and annoyance. In my experience it yields readily to treatment. Horand (loc. cit.) saw thirteen cases of this form of the disease among four hundred and eightythree cases of gonorrhœa in women.

Gonorrhœal Arthritis. - I have never seen but one case in women in which the gonorrhœea and the arthritis seemed definitely related. The intimacy of the two conditions has always seemed to me rather questionable. Jullien reports the discovery of gonococci in the blood, and if this is true they might be transmitted to the joints; or they might be carried in the lymph circulation, on the supposition that they are the sole cause of the disease. Welander says there may be a gonorrhoal arthritis from mixed infection. He doubts the presence of gonococci in the blood, and has not seen scientific evidence of their presence in the joints in a single case. This subject is discussed at greater length elsewhere.

Cystitis. - This complication is a very common one, but neither so severe nor so persistent as with gonorrhcea in men. It is quite amenable to treatment, and cases are rare in which it extends to the ureters and kidneys.

Syphilis and Chancroid.-These diseases may be associated with gonorrhoea, the symptoms of each disease being distinct and easily recognized. 
Gonorrhœal Ophthalmia.-This very important division of the subject is discussed elsewhere.

Relation of Gonorrhœa to Sterility.-Reciprocal influence of gonorrhœa with menstruation, the pregnant and puerperal states. Gonorrhœa being an inflammatory condition, it would naturally follow that if an acute attack were added to the hyperæmia of the menstrual epoch the result would be an unusual loss of blood, and possibly undue frequency of the menses. If the pelvis were the seat of new growths which implied an additional vascularity, the hæmorrhage night be sufficiently profuse to require active measures for checking it. Excessive menstruation or polymenorrhœa is therefore one of the symptoms which may be expected in connection with acute gonorrhœa. This statement has been verified by the observations of Welander and Sänger (loc. cit.). That gonorrhœa may be transmitted through the medium of the menstrual blood is extremely probable, the infectious elements being latent within the folds of the uterine or vaginal mucous membrane and carried out during coitus. The hyperæmic condition of the pregnant state, and the abundance of glandular secretion during that period, are also favorable to the reception and transmission of gonorrhœa. With young women who are thus infected the disease is often very severe, especially in the forms of vaginitis and endometritis. An exuberant crop of condylomata is also a very common phenomenon in such cases. That interruption of the pregnant state may be caused by gonorrhœa is a very reasonable supposition, and is vouched for by high authority. Nöggerath reports twelve cases of abortion in fifty-three cases of gonorrhœa in pregnant women (Archiv für Gynäkologie, 1888, xxxii, p. 322). Kroner (Archiv für Gynäkologie, xxxi, ii, p. 261) reports thirty-one cases of gonorrhcea in pregnant women, of which twenty-three went to term, five aborted, and in three premature labor occurred. Sänger thinks that both abortion and placenta prævia may be due to gonorrhœa, though why the last-mentioned accident should be a result I do not know. The influence of gonorrhœa during parturition and the puerperal state is a matter of great importance. The hosts of infants who lost their eyesight in years gone by, before this disease was properly appreciated, from an infected parturient tract, are witnesses of the truth of this statement. The lochial fluid, like the menstrual blood, may be the medium for the transmission of the disease, and conversely the puerperal state renders a woman very sensitive to the acquisition of the disease. A pre-existing gonorrhœa may become very active and intense during the puerperal period. Many of the severe and fatal cases of puerperal septicæmia are of gonorrhœal origin; while Sänger is authority for the statement that before Crédé's method of treating gonorrhœal ophthal$\mathrm{mia}$ in the newborn was introduced at the Leipsic maternity twenty-six per cent of the infants born there were sufferers from that disease. All 
the forms of gonorrhœa may be acquired during the puerperal state, or may be relighted into activity; and they may be independent of septic puerperal condition of other origin, or they may be associated with it.

The relation of gonorrhœa to sterility has been touched upon from time to time in this paper. It was one of the important features in Nöggerath's original article, and one which excited astonishment and unfavorable comment. Nöggerath's prediction, that time would prove the truth of his statements concerning the destructive influence of this disease upon the reproductive power, has been measurably verified. Sterility is the result of gonorrhœa, not in all cases, but in those in which the disease extends to the Fallopian tubes, excites intense inflammatory action in them, and seals their abdominal ends. Sterility in such cases is irremediable. Even if the function of the ovaries remained (and in most cases the same inflammation which has destroyed the functional power of the tube has destroyed that of the ovary also), the only avenue of approach for the ovum to the uterus is cut off; the spermatozoön is entirely out of reach. The efficient cause of sterility subsists, therefore, in the tube, and the tube being disorganized, conception becomes an impossibility. A more or less complete cure of the inflammatory condition is possible, but a restoration of the tube ad integrum is impossible.

Treatment.-The subject of treatment for gonorrhœa in women is a large one, for it embraces not only the management of the disease and its complications, but the consideration of precautionary measures in men who consort with infected women, and the adoption of preventive treatment in women. Gonorrhœa will probably exist as long as men and women are the slaves of sexual appetite. Far be it from me to defend the gratification of that appetite in any but the normal and proper waythat is, in the lawful relationship of husband and wife. The idea of licensing a vice by any system or plan yet devised-an expedient which has always proved a failure-ought to be abhorrent to good morals and good citizenship. But, whether we regard the moral side of the subject or not, we must consider the sanitary side, and accept the fact of susceptibility to gonorrhœa and its actual and widely spread existence. I shall not offer any theory as to the proper way to treat prostitution. I have discussed that question elsewhere (see author's paper on The Unrestricted Evil of Prostitution), and have seen no reason to change my views. With reference to prophylaxis, scrupulous cleanliness of the genital organs before and after coitus would prevent many cases of gonorrhœa. It is simply the application of the surgical gospel of asepsis, for gonorrhoeal virus could not successfully compete against the scrubbings and washings with which a careful surgeon prefaces his operations. But there are few who would be willing to use the brush, soap, and water with thoroughness before and after coitus. As a measure of comparative 
safety, an abundant vaginal irrigation with hot water or solution of carbolic acid, 1 to 40, or solution of creoline, 1 to 50, may be recommended. Sänger recommends that men instill into the urethra a few drops of a two-per-cent solution of nitrate of silver previous to coitus, and that prostitutes irrigate the genitals with a 1-to-20 solution of sublimate. The latter seems to me dangerous advice, and, in regard to prophylaxis in general, we must not forget that with very many individuals the gratification of the sexual appetite is a matter about which they are prone to reason and propose safeguards at any time rather than the time when they are to be put in practice. Equally important with prophylaxis in this matter is quarantine. All women suffering with gonorrhœa (and other venereal disease), who can not or will not be treated at their homes, should be placed under observation and treatment in hospitals. The great prevalence of the venereal diseases is largely due to the fact that many of those who suffer with them, both men and women, continue to indulge in sexual intercourse even when conscious that they are diseased, thus multiplying the disease indefinitely. It wonld be difficult in the present state of society to constitute the infecting of one person by another as a legal offense or misdemeanor, but it ought not to be difficult to secure the passage of a law compelling any one with this disease to have either public or private medical attention, as we compel those who have cholera, smallpox, and other infectious diseases which are very dangerous to the community. Another important question in this connection is, How long is the disease infectious? Certainly as long as any of its symptoms remain; when once a woman has been infected, her capacity for infecting others may remain indefinitely.

The treatment of gonorrhœa will vary according as the disease is in the acute or chronic stage. Above all things cleanliness is a prerequisite. The external genitals should be frequently sponged off with absorbent cotton moistened with a hot solution of sublimate or carbolic, salicylic, or boric acid of moderate concentration, the cotton being burned or otherwise effectively disposed of after use. Irrigation of the vagina with the same solution should also be practiced two or three times daily under the direction of a physician or a competent nurse; and a vulvar pad of absorbent cotton snugly secured by a T-bandage should be constantly worn as long as any discharge from the diseased parts continues. If the urethra or bladder is involved, alkalies and balsams may he used. In the beginning of the acute stage, when the vulva, vagina, urethra, or cervix uteri is engorged and very sensitive, it will usually be found inadvisable to make local applications on account of the intense pain caused by manipulation. When the engorgement and sensitiveness have in a measure subsided, a direct attack may be made upon the affected parts. The position which the patient assumes for treatment is immaterial, although it must be such as to enable one to 
remove all secretions with thoroughness. If the bladder and urethra are involved, irrigation should be practiced daily with warm boric-acid solution in moderate concentration. The balsams may be administered by the mouth, or, since they are eliminated by the kidneys, they may be applied in the form of bougies to the urethra and bladder. Severe manipulation of the urethra will not only cause intense pain, but will tend to increase the inflammatory condition and encourage the formation of stricture. If condylomata are present, the external genitals should be frequently washed with a two or three per cent solution of creoline, the hair upon the pubes should be removed, and applications may be made of nitrate-ofsilver solution, 1 to 8, fuming nitric acid, nitrate-of-mercury ointment, or the thermocautery. The latter method is excellent, especially if the growths are exuberant and pediculated. Of course, the operation requires an anæsthetic. Cysts and abscesses of the genitals are to be treated with the same deference to surgical principles that are observable elsewhere. In addition to the irrigation the vagina should be gently but thoroughly swabbed daily with a 1-to-8 solution of nitrate of silver and then tamponned with cotton soaked in a mixture of subnitrate of bismuth and glycerite of tannin, 1 to 8 . The cervical canal should be kept clean by frequent curettings with the dull curette, severity in scraping being avoided, after which the strong solution (1 to 8) of nitrate of silver, or a strong solution (thirty to forty per cent) of chloride of zinc, should be applied. Prochownick has recommended the use of the constant galvanic current for the treatment of acute gonorrhœa, and is satisfied with the results which he has obtained (Münchener medicinische Wochenschrift, 1890, vol. xxxvii, p. 467). If the disease invades the Fallopian tubes, the ovaries, and the peritonæum, there will usually be but one way of curing it, and that is by means of an abdominal section and the removal of the diseased appendages. If the disease goes no farther than the tubes, the abdominal ends of the latter becoming sealed, dilatation of the uterus and drainage with the gauze tampon may in some cases effect a cure, or at any rate a remission; but the persistency of gonorrhaœl elements is such that it would be assuming a great deal to say that a woman was cured of her pyosalpinx merely because it was efficiently drained. Even though the disease of the tubes were cured, their functional power would be destroyed. A woman who has once had gonorrhœeal salpingitis is in a condition of insecurity as long as the diseased organs are in her body. The condition is quite analogous with that of appendicitis, in which recurrences are almost as sure to come as in any inflammatory condition of which we have any knowledge. 


\section{N D E X.}

Ablation of scrotum, 980 .

Abscess of pelvis, 333 .

of penis, 56 .

perinephritic, 795.

periurethral, 103.

of prostate, 333 .

of scrotum, 876 .

of testicle, 902 .

of urethra, 103.

Adenitis, 181, 1060.

Albumin in urine, 396.

diagnostic significance of, 399 .

tests for, $\mathbf{3 9 7}$.

Angeioma of scrotum, 891.

Arthritis, gonorrhœal, in females, 1060.

Aspermia, 1031.

relative, 1032.

temporary, 1033.

Atresia of urethra, congenital, 75.

of deep urethra, 76.

of glandular urethra, 76 .

of meatus urinarius, 75 .

Azoöspermia, 1034.

Bacilli in urine, 416.

Balanitis, 67, 180.

secondary, 67.

simple, 67 .

specific, 68 .

treatment of, 68.

Balano-posthitis, 67 . chronic, 68.

diabetic, 69.

treatment of, 70.

Bartholinitis, gonorrhœal, 1050.

Bichloride of mercury in urethritis, 164.

Bladder, urinary, abnormities of, 541.

absence of, 532 .

anatomy of, 13.

atrophy of, 543.

bar at neck of, 544 .
Bladder, carcinoma of, 571.

in child, 18.

conditions favoring infection of, 502 .

congenital defects of, 537.

congestion of, 493.

contracted, with retention, 380 .

contusion of, 525 .

cystoscopic appearance of, 473.

examination of, 465.

cysts of, 571 .

development of, 21.

diseases of, 525 .

drainage of, 193, 323, 519, 613 .

exstrophy of, 533.

extirpation of, in female, 618 .

extra-peritoneal wounds of, 527 .

fissure of, 546.

ioreign bodies in, 528 .

gravel in, 378.

hernia of, 537.

hypertrophy of, 541 .

injuries of, 525 .

intra-peritoneal wounds of, 527 .

inversion of, 541.

malformations and malpositions of, 532 .

multiple, 533.

myoma of, 571.

myxoma of, 570.

neuralgia of neck of, 377 .

obstructive disease of, $\mathbf{5 4 5}$.

papilloma of, 567 .

physiology of, 35 .

puncture of, 547.

relations of, 16.

resection of $61 \%$.

rupture of, 553 .

sacculated, 20, 543.

sarcoma of, 618.

sensibility of, 493 .

stone in, 621 ,

suture of, $562,607,730$. 
Bladder, tumors of, 565 .

tuberculosis of, 864 .

wounds of, 526.

Bladder, neck of, bar at, 544 .

fissure at, 546 .

neuralgia of, 377 .

ulcer of, $37 \%$.

Blennorrhœa, neonatorum, 234.

Blood in urine, 413, 510.

Calcareous masses in testis, $\mathbf{9 3 3 .}$

Calcic oxalate in urine, 393.

Calculus (see Stone).

in bladder, 721.

in kidney, 764.

in prostate, 736.

in ureter, 740 .

in urethra, 737 .

Cancer of bladder, 571.

of kidney, 823.

of penis, 58.

of prostate, 358,380 .

of testis, 933.

of urethra, 111.

Casts in urine, 408.

Catheterization of ureter, 488.

Catheterism in urethral stricture, 294.

Chancre of urethra, 110.

Chromospermia, 1036.

treatment of, 1038.

Circumeision, 63.

methods of operating in, 63.

Colic, spermatic, 988.

Condylomata, 1059.

Conjunctivitis, gonorrhœal, 223.

Corpora cavernosa, 27.

bony and calcareous plates of, 74 .

diseases of, 73.

gummata of, 74 .

inflammation of acute, 73 .

chronic, 74.

Cowper's glands, 34 .

Cowperitis, 174.

Cryptorchidism, 897.

Cystine in urine, 394.

Cystitis, 491.

clinical causes of, 503.

complicating acute urethritis, 177 .

conditions which favor infection, 502.

diagnosis of, 512.

drainage in, 519.

instillations in, 518 .

interstitial, 496.
Cystitis, intravesical injections in, 523.

lesions of, 494.

microbes in, 499.

modes of infection in, 501.

pathology of, 497 .

productive, 497.

superficial, 495.

symptoms of, 507.

treatment of, $\mathbf{5 1 5}$.

Cystorraphy, 561 .

Cystoscopic appearance of healthy bladder, 470.

of diseased bladder, 473.

Cystoscopy, 435.

batteries employed in, 458 .

in bladder growths, 578 .

in catarrh of the bladder, 474.

cocaine in, 456.

the employment of, 455 .

instruments employed in, 438.

in kidney diseases, 483.

in tuberculosis of bladder, $\mathbf{8 6 7}$.

narcosis in, 458.

possible dangers of, 460 .

rules for performing, 463 .

in tuberculosis, 475.

Cystotomy, suprapubic, for removing bladder growths, 592.

for prostatic enlargement, 352 .

Cysts of bladder, 571.

dermoid, of scrotum, 930 .

dermoid, of testis, 930 .

of kidney, hydatid, $378,816$.

of prostate, 360 .

sebaceous, of scrotum, 883.

of seminal vesicles, 987 .

of testis, 929 .

urinary, of scrotum, 890 .

of vas deferens, $98 \%$.

Dermoid cyst of serotum, $\mathbf{9 3 0}$.

of testis, 930.

Diseases of bladder, 525 .

of glans penis, 67 .

of penis, 49.

of prepuce, 67 .

of prostate, 331.

of scrotum, 875 .

of seminal vesicles, 983 .

of testicle, 893, 899, 929 .

of urethra, 75 .

Disorders of micturition tabulated, 384 .

Diverticula of urethra, 79. 
Drainage of bladder, 191, 323, 519. for malignant growths, 613 .

Eczema of penis, 54 . of scrotum, 879.

Elephantiasis of penis, 58 . of scrotum, 41, 884.

Emphysema of scrotum, 878.

Enchondroma of scrotum, 891. of testicle, 931.

Endometritis, gonorrhœal, 1054.

Endoscopic appearance of normal urethra, 207. of diseased urethra, 213.

Endoscopy, 195. development of, 196. diagnosis with, 218. method of examination with, 205. treatment with, 189, 219.

Epididymis, canal of, 44. diseases of, 908. structure of, 43. syphilis of, 922.

Epididymitis, 169, 908. chronic, 920. symptoms of, 921 . treatment of, 922. complicating acute urethritis, 169. etiology of, 908. symptoms of, 910 . syphilitic, 922. treatment of, 914.

Epispadias, 30, 92. etiology of, 94. glandular, 92. of penis, 93. prognosis of, 97. symptoms of, 96. treatment of, 97 .

Epithelioma of penis, 59. of scrotum, 891.

Erection, physiology of, 1017.

Erosion of urethra, 105.

Erysipelas of scrotum, 883.

Evacuator for stone in bladder, 676.

Excision of stricture, 324. of testis, 936.

Fever, urinary (see Urethral Fever), 419. Fibro-adenoma of prostate, $33 \%$.

Fibroma of scrotum, 891.

Fibro-myoma, 337.

Filaria sanguinis hominis, 418, 886.
Fissure of neck of bladder, $37 \%$.

Fistula, perinephritic, 803 . renal, 803. of seminal vesicles, 986 . of urethra, 129. urethro-penile, 133. urethro-perineal, 132. urethro-rectal, 129.

Folliculitis, simple, 173.

Foreign body in urethra, 126.

Functional disorders of micturition, 362. of male sexual organs, 997.

Fungus of scrotum, 877 . of testis, benign, 926 .

Gangrene of kidney, 794. of penis, 57.

Genito-urinary organs, anatomy and physiology of, 1.

Glands, Cowper's, 34. of Littré, 35.

Glandulæ odoriferæ of penis, 30 .

Gleet (see Chronic Gonorrhora), 183. endoscopic treatment of, 189. etiology of, 184.

injections in, 186. treatment of, 185.

Gonocele, 243.

Gonococcus of Neisser, 138. diagnostic value of, 140,417 . staining of, 139.

Gonorrhœa. (See URETHRITIS.) acute, 145.

of axilla, 260 .

chronic, 183.

complications of, 166. in female, 1059.

of ear, 260.

etiology of, 137 .

in female, 1039.

forms of, 1044. of mouth, 258, 1060 .

of nose, 256,1060 .

ophthalmia, caused by, 223 .

pseudo-, 142.

of rectum, 250, 1060 .

rheumatism caused by, 237 .

treatment of, 154.

of umbilicus, 260 .

varieties of, 146.

Gonorrhœal ophthalmia, 224. incubation, 225.

symptoms of, 226 . 
Gonorrhæal ophthalmia, prognosis of, 228. treatment of, 228.

Gonorrhœal rheumatism, 23\%. acute monarticular, 243. chronic monarticular, 243. acute polyarticular, 244 . chronic polyarticular, 244. diagnosis of, 247. treatment of, 248.

Hæmatocele of spermatic cord, 894. of testicle, 893. of tunica vaginalis, 893 .

Hæmatoma of scrotum, 890.

Hæmaturia, with kidney wounds, 760 . with stone in bladder, 635 .

Hæmorrhage from bladder growths, 574 . from stone, 635 .

Hernia of bladder, 537.

Herpes progenitalis, 70. diagnosis of, 71 . etiology of, 71 . neuralgic, $\mathbf{7 0}$. recurrent, 71 . of scrotum, 881. simple, 70 .

treatment of, 72 . ulcerating, 70. varieties of, 70.

Hydatids of Morgagni, 43.

Hydrarthrosis, 243.

Hydrocele, 941. acute, 942 .

diagnosis of, 944.

treatment of, 945 .

bilocular, 942,962 .

chronic, 945.

diagnosis of. 952.

treatment of, 954 .

communicans, 941, 957.

complications of, 964 .

differential diagnosis of the various forms of hydrocele and their complications, 966.

diffuse, 942.

encysted, 958.

incapsulated, 941.

multilocular, 942, 964 .

of spermatic cord, 958 .

diffuse, 961.

Hydronephrosis, 806.

due to malformations of ureters, 13.

Hypertrophy of prostate, 337.

\section{Hypertrophy of prostate, complications of, 346.}

diagnosis of, 340 .

pathology of, 338.

symptoms of, 339.

treatment of, 342 .

operative, 348.

Hypertrophy of prostatic sphincter, $35 \%$.

Hypospadias, 30, 81.

balanic, 84 .

diagnosis of, 88 .

etiology of, 82 .

glandular, 84 .

penile, 85 .

perineal, 86.

prognosis of, 88.

symptoms of, 87.

treatment of, 88.

Impotence, 1016.

etiology of, 1025 .

irritable, 1022.

organic, 1020.

paralytic, 1023.

psychical, 1021.

relative, 1022.

symptoms of, 1025 .

treatment of, 1026.

Incontinence, of urine, 370 .

seminal, 997.

diurnal, 998.

nocturnal, 998.

spermatorrhœal, 998.

Indican in urine, 395.

Infectious urethritis, $13 \%$.

Inflammation, periurethral, 172.

Involuntary micturition, 373 .

Kidney, abnormities of, 11 .

abscess of, 795.

anatomy of, 1.

cysts of, $378,816$.

displacements of, 4,827 .

fistula of, 803.

floating, 827 .

gangrene of, 794 .

gravel of, 764 .

hydatids of, 820 .

laceration and rupture of, $75 \%$

lymphatics of, 10.

operations upon, 829.

physiology of, 11.

polycystic, 819 . 
Kidney, relations of, 4. stone in, 764.

surgical diseases of, 751 .

traumatisms of, 751.

tuberculosis of, 781,860 .

tumors of, solid, 823.

benign, 826 .

malignant, 823.

wounds of, 752.

Lacerations of urethra, 114, 126.

Lipoma of scrotum, 891.

Lithotomy, bilateral, 701, 719.

lateral, 700.

median, 701, 720.

medio-bilateral, 701.

perineal, 655, 699 .

suprapubic, 656, 723.

vaginal, 734.

Litholapaxy, 657, 674.

complications of, $693,697$. after-treatment, 698.

in woman, 733.

Lithotrite, 674.

Lithotrity, history of, 667. perineal, 721.

Lupus of scrotum, 882 .

Lymphangitis, 55, 181.

Male sexual organs, functional disorders of, $99 \%$.

Masturbation, 1001.

Meatotomy, 310.

Meatus urinarius, atresia of, 75.

stricture of, 77.

treatment of, 78.

Micturition, abnormal, 362.

disorders of, functional, 361.

force of, 381 .

involuntary, 373.

irrepressible, 366 .

obstructed, 370.

painful, causes of, 374, 576 .

physiology of, 361 .

Monorchidism, 897.

Morgagni, hydatids of, 43.

Myoma of bladder, 571 .

Myxoma of bladder, 570.

Neoplasms of urethra, 110.

Nephrectomy, 781, 833.

abdominal, 834.

lumbar, 833.
Nephritis, 781.

primary suppurative, 794.

Nephrolithiasis, 764.

Nephrolithotomy, 781, 831.

Nephropexy, 835.

Nephrorrhaphy, 835.

Nephrotomy, 831.

Neuralgia of neck of bladder, $3 \% 7,383$.

Nitrate of silver in cystitis, 524 .

in epididymitis, 916 .

in gonorrhœal ophthalmia, 231.

in urethritis, $\mathbf{1 5 9}$.

Edema of scrotum, 41, 877 .

Oligospermia, 1033.

Oligozoöspermia, 1033.

Ophthalmia, gonorrhœal, 223.

neonatorum, 234.

Orchitis, acute, 899.

symptoms of, 901 .

treatment of, 906.

chronic, $91 \%$.

symptoms of, 917 .

treatment of, 919 .

syphilitic, 923 .

Ovaritis, gonorrhœal, 1058.

Painful micturition, 374.

diagnostic significance of, 374, 509.

Pampiniform plexus, 45, 46.

Papilloma of bladder, $56 \%$. of prostate, 358 .

Paraphimosis, 65, 181. treatment of, 66.

Patent urachus, 53\%.

Pediculosis of scrotum, 881.

Pelvic abscess, 333.

Penis, abnormities of, 30, 49 .

abscess of, 56 .

absence of, 49 .

adenoma of, 58.

amputation of, 59 .

anatomy of, 26.

cellular inflammation of, 56 .

concealed, 50.

cutaneous affections of, 53 .

deformed, treatment of, 50 .

development of, 30 .

diseases of, 49.

dislocation of, 53 .

eczema of, 54.

elephantiasis of, 58.

epithelioma of, 59 . 
Penis, erysipelas of, 56.

fibroid tumors of, 57.

folliculitis of, 56 .

fracture of, 53 .

gangrene of, $5 \%$.

glandulæ odoriferæ of, 30 .

horny growths of, 58 .

imperfect, 50.

lupus of, 55 .

lymphatic affections of, 55 .

malignant tumors of, 58.

mucous-cystic tumors of, 57.

periurethral phlegmon of, 56 .

prepuce of, morbid conditions of, 60 .

rudimentary, 49.

sarcoma of, 60 .

scabies of, 54 .

strangulation of, 52 .

tuberculosis of, 55 .

tumors of, 57 .

mucous cystic, 57 .

multiple, 50.

vascular, 57.

wounds of, 52.

Penitis, 56.

Perineal section, 123, 319.

indications for, 322 .

urethrotomy, 351 .

Perinephritis, 795.

Periorchitis adhæsiva, 948.

hæmorrhagica, 949.

prolifera, 948.

Peritonitis, gonorrhœal, 1058.

Perinæum, male, anatomy of, 36.

Periurethral abscess, 103.

inflammation, 172.

phlegmon, 56.

Phimosis, 60, 181.

accidental, 61.

circumcision for, 63.

congenital, 60.

treatment of, expectant, 61 . operative, 61 .

Plexus of veins, pampiniform, 45, 46. spermatic, 46.

Polypus of urethra, 113.

Posterior urethritis, 166.

Prepuce, morbid conditions of, 60, 67.

Prostate, abscess of, 333.

anatomy of, 22.

calculi in, 736 .

cancer of, 358, 380 .

cysts of, 360 .

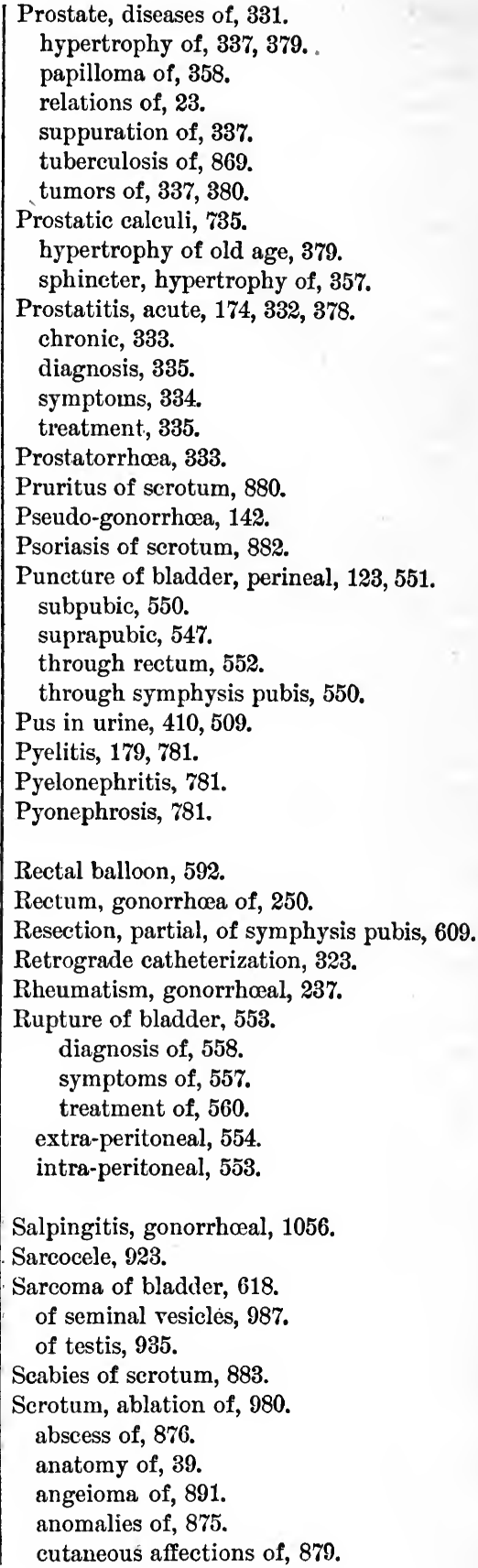


Scrotum, dermatitis of, 882 .

dermoid cyst of, 930 .

diseases of, 875 .

eczema of, 879 .

elephantiasis of, 41,884 .

emphysema of, 878 .

enchondroma of, 891.

epithelioma of, 891.

erysipelas of, 883.

fibroma of, 891.

fungus of, 877 .

hæmatoma of, 890 .

herpes of, 881.

hypertrophy of, 876 .

injuries of, 876 .

intertrigo of, 882.

lipoma of, 891.

lupus of, 882.

molluscum contagiosum of, 882

œdema of, $41,877$.

pediculosis of, 881 .

pigmentary affections of, 883 .

pityriasis versicolor of, 883 .

pruritus of, 880 .

psoriasis of, 882 .

scabies of, 883.

sebaceous cysts of, 883.

tuberculosis of, 892 .

tumors of, 889.

ulcer of, 883.

urinary cysts of, 890 .

Sebaceous cysts of scrotum, 883.

Semen, physiological characters of, 1029. pathological modifications of, 1031.

Seminal vesicles, acute inflammation of, 379.

anatomy of, 983 .

collic of, 988.

diseases of, 983.

fistula of, 986.

inflammation of, 989 .

injuries of, 985 .

malignant growth of, 986.

physiology of, 985.

sarcoma of, 987.

tuberculosis of, $869,991$.

tumors of, 986 .

Sexual excess, 1003.

Sexual organs, male, functional disorders of, 997 .

Spermatic cord, 45.

hæmatocele of, 894.

hydrocele of, 958 .

plexus of, 46 .
Spermatic cord, structures of, 46.

tuberculosis of, 872 .

Spermatocele, 968.

Spermatorrhœe, 997.

diagnosis of, 1009.

effects of, constitutional, $100 \%$.

local, 1006.

etiology of, 1000.

false, 998 .

pathological significance of, 999 .

symptoms of, 1005.

treatment of, 1011.

by drugs, 1013 .

electrical, 1015.

local, 1014.

methodic, 1012.

Sterility in male, 1028.

in female, relation of gonorrhœa to, 1061. treatment of, 1062.

Stone in bladder, 621.

chemical characteristics of, 621 .

consistence of, 628 .

cystic, 627 .

cystoscopic examination for, 479,642 .

diagnosis of, 636.

etiology of, 630.

exploration for, methods of, 642 .

in females, 732 .

operative treatment, 733 .

fracture of, spontaneous, 629 .

location of, 630 .

multiple, 628 .

oxalate of lime, 621 .

phosphatic, 621 .

physical characteristics of, 621 .

sounding for, 639.

symptoms of, 634 .

treatment of, 643 .

history of, $65 \%$.

local, 646.

operative, 651.

preventive, 643.

solvent, 647 .

uric acid, 621 .

Stone in kidney, 764 .

chemical composition, 766 .

diagnosis of, 775 .

etiology of, 768 .

symptoms of, 770 .

treatment of, $77 \%$.

Stone in prostate, 735.

in ureters, 740 .

in urethra, 737 . 
Stricture of urethra, acquired, 262.

catheterism in, 294.

cauterization of, 327 .

character of, 268.

congenital, 77, 261.

divulsion of, 326.

electrolysis in, 325 .

excision of, 324.

gradual dilatation in, 291.

in females, 328.

inflammatory, 262.

location of, 274 .

massage in, 327.

of large caliber, 270 .

organic, 263.

overdistention of, 326 .

perineal section in, 319.

prognosis of, 288.

results of, 286.

spasmodic, 262.

symptoms of, 278 .

traumatic, 266.

treatment of, 291.

Sugar in urine, 401.

Suprapubic operation in bladder growths, 592.

Suprapubic operation for stone, 728.

Suprapubic puncture, 547.

Suture of bladder, 562, 607, 730 .

Symphysis pubis, partial resection of, 609 .

Syphilis of epididymis, 922.

of testicle, 923.

Syphilitic urethritis, 143.

Testicle, abscess of, 902.

absence of, 895.

anatomy of, 42 .

anomalies of, 895 .

atrophy of, 896 .

calcareous masses of, 933.

carcinoma of, 933.

cystoma of, 929.

dermoid cysts of, 930 .

diseases of, 893, 899, 929.

enchondroma of, 931.

excision of, 936.

fibrous tumors of, 932 .

fungus of, benign, 926 .

gangrene of, 904.

hæmatocele of. (See HæMatocele.)

hydrocele of. (See Hydrocele.)

hypertrophy of, 896.

injuries of, 898.
Testicle, irritable, 938.

misplacement of, 897 .

pampiniform plexus of, $45,46$.

retraction of, 898 .

sarcoma of, 935 .

supernumerary, 895.

syphilis of, 923.

tuberculosis of, 872 .

tubuli seminiferi of, 44.

tumors, solid, of, 931.

undescended, 897.

wounds of, 898.

Tubercle bacillus, 417 .

Tuberculosis, uro-genitalis, 837.

avenues of infection, 839 .

of bladder, 864 .

cystoscopy in, 867 .

diagnosis of, 851.

differential diagnosis of, from renal stone, 861.

influence of heredity, 848 .

of prostate, 869 .

renal, primary, 781,860 .

of scrotum, 891.

of seminal vesicles, 869, 991 .

of spermatic cord, 872 .

of testis, 872 .

of urethra, 871 .

varieties of, 838.

Tumors of bladder, 565 .

hæmorrhage in, 574.

operative treatment of benign growths of 588.

in female, 610 .

cystic, 890.

of prostate, 337,380 .

of scrotum, 889.

of seminal vesicles, 986 .

of testicle, solid, 931.

ultimate results of, 611 .

Tunica albuginea, 44.

vaginalis, 40.

free bodies in, $\mathbf{8 9 5}$.

vasculosa, 44.

Ulcer of neck of bladder, 377 .

of scrotum, 883 .

of urethra, 105.

Urates, the, in urine, 393.

Ureters, abnormities of, 13.

anatomy of, 11.

catheterization of, 488.

course of, 12. 
Ureters, malformations of, causing hydronephrosis, 13.

physiology of, 13.

relations of, 12 .

stone in, 740.

diagnosis, 743.

symptomatology, 742.

treatment, 744.

Urethra, abscess of, 103.

absence of, 75 .

anatomy of, 31.

bulb of, 34 .

cancer of, 111.

chancre of, 110.

changes in, due to stricture, 275.

congenital atresia of, 75 .

direction of, 32 .

diseases and injuries of, 75 .

diverticula of, 79 .

erosion of, 105.

fistula of, 129 .

foreign bodies in, 126.

irrigation of, in gonorrhœe, 161.

lacerations of, 114, 126.

malformations of, 75 .

membranous portion of, 34 .

neoplasms of, 110.

obliteration of, 75.

physiology of, 35.

polypi of, 114.

prostatic portion of, 33 .

rupture of, 114.

spongy portion of, 34 .

stone in, 737.

strictures of, acquired, 262.

congenital, 77, 261.

syphilis of, 110, 143.

tuberculosis of, 143, 871 .

ulcer of, 105.

urinary pouches of, 79 .

vegetations of, 115.

wounds of, 114, 126.

Urethral calculus, 737.

cancer, 111.

chancres, 110.

fever (see Urinary Fever), 419.

fistula, 129.

neoplasms, 110.

polypi, 114.

synovitis. (See Gonorrhceal RheUmaTISM).

ulcers, 105.

vegetations, 115 .
Urethritis. (See Gonorrmas.)

acute, 145.

chronic, 183.

complications of, 166.

differential diagnosis of, 152.

etiology of, 137.

in females, 1053.

complications of, 1059.

history of, 145 .

incubation of, 149 .

infectious, 137.

noninfectious, 143.

pathology of, 151.

posterior, 166.

prognosis in, 153.

pseudo-, 142.

syphilitic, 143.

treatment of, 154.

expectant, 156.

internal, 156.

local, 159.

tuberculosa, 143.

varieties of, 146.

Urethro-penile fistulæ, 123.

Urethro-perineal fistulæ, 132.

Urethroplasty, operations of, 135.

Urethrotomy, combined external and internal, 317.

excessive, injuries caused by, 128.

external, 313.

internal, 304 .

in children, 312.

Uric acid in urine, 392.

Urinary fever, 419.

acute, 422 .

chronic, 424.

diagnosis of, 432.

etiology of, 426.

pathogenesis of, 428.

treatment of, 433.

Urination, physiology of, 361.

Urine, albumin in, 396, 511.

quantitative estimation of, 398.

significance of, 399 .

tests for, 396, 397.

ammoniacal fermentation of, $395,511,632$.

bacilli in, 416 .

bacilli, tubercle in, 417.

in bladder growths, diagnostic value of, 577.

blood in, significance of, $413,510,635$.

calcic oxalate of, 393.

casts in, 408. 
Urine, chemical reaction of, 387 . chemical constituents of, normal, 390. chemical decomposition of, products of, 395.

color of, 385 .

cystine in, significance of, 394. epithelia in, significance of, 404. general characteristics of, 385 . incontinence of, 370 . indican in, 395. modifications, pathological, of, $\mathbf{3 8 5}$. mucus in, significance of, 408. odor of, 386. organized sediments of, 404. overflow of, 366. parasites in, 418.

prostatic fluid in, significance of, 406 . pus in, significance of, $\mathbf{4 1 0}$. retention of, 364 . sediments, organized, of, 404. specific gravity of, 389 . seminal fluid in, significance of, 407 . sugar in, significance of, 401. quantitative estimation of, 403. Fehling's test for, 401.

fermentation test for, 402. transparency of, 386. urates of, 393. uric acid of, 392. volume of, 388.

Vaginitis, gonorrhœal, 1050. senile, 1052.

Varicocele, $\mathbf{9 7 1}$. etiology of, 972. operation for, 978. symptoms of, 973. treatment of, 974.

Vasa deferentia, 44, 983. cysts of, 987.

Vegetations of urethra, 113.

Verrucæ, 72. etiology of, 72. diagnosis of, 72. treatment of, 72 .

Vesical drainage in cystitis, 519. in gleet, 191. in prostatitis, 323.

Vesical growths, removal of, by suprapubic cystotomy, 598. through perineal opening, 589.

Vesiculitis, 176.

treatment of, $17 \%$.

Vulvitis, 1049.

Wounds of bladder, 526 . of kidney, 751. of penis, 52. of scrotum, 876 . of seminal vesicles, 985 . of urethra, 114, 126. 


\title{
A PRACTICAL TREATISE ON THE SURGICAL DISEASES OF THE GENITO-URINARY ORGANS, INCLUDING SYPHILIS.
}

\author{
DESIGNED AS A MANUAL FOR STUDENTS AND PRACTITIONERS.
}

\section{With Engravings.}

By E. L. KEYES, A. M., M. D.,

Professor of Genito-Urinary Surgery, Syphilology, and Dermatology
in Bellevue Hospital Medical College.

\author{
BEING A REVISION OF' A TREATISE, BEARING THE SAME TITLE, BY \\ VAN BUREN AND KEYES.
}

SECOND EDITION, THOROUGHLY REVISED, AND SOMEWHAT ENLARGED.

8vo. 688 pages. Cloth, $\$ 5.00 ;$ sheep, $\$ 6.00$.

\begin{abstract}
"The progress made in surgery during the last ten years, the changes of practice by the best surgeons with regard to sereral opcrative procedures, notably litholapaxy, suprapubic sary a thorough revision of the work published some years ago as the joint production of Drs. Van Buren and Keyes. Much of the work lias been rewritten entirely. There is a large amount of entirely new matter presented in this volume, to make room for which the reports of cases given in the former work are all omitted in this. The work in its present form stands fairly abreast of the latest advances in genito-urinary surgery. Dr. Keyes says of the book that it is an honest exhibit of his views upon all the subjects considered, and, in view of his wide experience and uuquestioned skill, we commend his book to the notice and study of all who work in this field." -St. Louis Courier of Medicine.

"We do not know of any one work in the English language, devoted to diseases, etc., of the genito-urinary organs, including the venereal diseases, that is so well adapted to the wants of the general practitioner. To the specialist this book is invaluable." - Virginia Medical Monthly.
\end{abstract}

"This handsome volume is not merely a new edition of the well-known work of Van Buren and Keyes, but a complete revision of that text-book. The original plan of the older work has been retained, and its scope remains the same; but it has been entirely recast, and in a large measure rewritten. This course has been made necessary by the vast progress which has marked the history of surgery during the last ten years, especially in the field of therapeutics and operative procedures. To bring the book up abreast of the times upon the new derice of litholapaxy, suprapubic cystotomy, the modern surgery of the kidncy, the treatment now followed in diseases of the tunica vaginalis, and the many minor changes which find expression in the use of new agents, Dr. Keyes was compelled to omit many things, to add considerable new matter, and largcly to modify much of the remainder. Some chapters are entirely new, and in order to make room for desired additions all the cases have becn dropped. As it now stands, it is a treatise which may safely be consulted, and which fairly and freely speaks of the most modern methods. Dr. Keyes is enthusiastic in his commenda tions of litholapaxy, and cordially indorses the high operation for stonc, while he decides that the time-honored and brilliant methods of reaching the bladder through the perinæum are only applicable in the cases of male children with stones of moderate size. Dr. Keves says the book 'is an loncst exhibit of ny views upon all the subjects considered'; and as his expcrience has been large, and his skill and prudence are undisputed, we have no hesitation in saying there is $\mathrm{n}$ () one in this country whose judgment is more worthy of confidence, or whose directions may be more safely followed."-American Journal of the Medical Sciences.

New York: D. APPLETON \& CO., 1, 3, \& 5 Bond Street. 


\section{A Practical TREatise on DisEASES OF THE SKIN.}

By HENRY G. PIFFARD, A. M., M. D.,

Clinical Professor of Dermatology, University of the City of New York; Surgeon in Charge of the New York Dispensary for Diseases of the Skin; Consulting Surgeon to Charity Hospital; Consulting Surgeon to the Bureau of Out-door Relief, Bellevue Hospital; Consulting Dermatologist to the Board of Health, ete.

\section{With Fifty full-page Original Plates and Thirty-three Illustra- tions in the Text.}

Folio form. Sold only by Subseription.

"The work beforo us is one of the best yet published for the general practitioner. The author's name is sufficient to give it authority on any subject with which it deals. . . . There are no theoretical or controversial discussions, which would serve only to perplex any one but a specialist, and what is necessary to be said is put in the most practical manner, and in the fewest possible words.

"The plan of the work and its execution are both indeed excellent. We have seen no work which we can, with so much confidence, recommend to our readers, who are in need of help in the diagnosis and treatment of this very important class of diseases. The plates and figures are really illustrative of the text, are beautifully executed, while the letterpress and binding are all that can be desired."-Canada Lancet.

". . . Beyond doubt the most important part of the book is the plates, and their excellence and number give it a unique character. Probably photography has never before been used so successfully to illustrate diseases of the skin, and the excellence of some of the plates in the book is so great as far to surpass anything of the kind that has as yet come under our observation.... Some of the plates are a revelation in so far as they show the extent to which photography may be used to depict the clinical appearances of some diseases of the skin.

“. . . Dr. Piffard has produced a work which testifies unmistakably to his power of observing accurately and of reproducing artistically those appearances on which the diagnosis and treatment of skin diseases chiefly depend, while his remarks on treatment give evidence of a wide experience in dermatological therapeutics."-British Medical Journal.

New York: D. APPLETON \& CO., Publishers, 1, 3, \& 5 Bond Street.

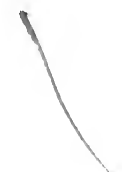




\section{MEDICAL}

ANI)

\section{HYIENIC WORKS}

PUBLISHED BY

D. APPLETON \& CO., 1, 3, \& 5 Bond Street, New York.

AULDE (JOHN). The Pocket Pharmacy, with Therapeutic Index. A résumé of the Clinical Applications of Remedies adapted to the Pocket-case, for the Treatment of Emergencies and Acute Diseases. 12mo. Cloth, $\$ 2.00$.

BARKER (FORDYCE). On Sea-Sickness. A Popular Treatise for Travelers and the General Reader. Small $12 \mathrm{mo}$. Cloth, 75 cents.

BARKER (FORDYCE). On Puerperal Disease. Clinical Lectures delivered at Bellevue Hospital. A Course of Lectures valuable alike to the Student and the Practitioner. Third edition. 8vo. Cloth, $\$ 5.00$; sheep, $\$ 6.00$.

BARTHOLOW (ROBERTS). A Treatise on Materia Medica and Therapeutics. Serenth edition. Revised, enlarged, and adapted to "The New Pharmacopœia." 8 vo. Cloth, $\$ 5.00$; sheep, $\$ 6.00$.

BARTHOLOW (ROBERTS). A Treatise on the Practice of Nedicine, for the Use of Students and Practitioners. Sixth edition, revised and enlarged. 8vo. Cloth, $\$ 5.00$; sheep, $\$ 6.00$.

BARTHOLOW (ROBERTS). On the Antagonism between Medicines and between Remedies and Diseases. Being the Cartwright Lectures for the Year 1880. 8vo. Oloth, $\$ 1.25$.

BILLINGS (F. S.). The Relation of Animal Diseases to the Public Health, and their Prevention. 8vo. Cloth, $\$ 4.00$.

BILLROTH (THEODOR). General Surgical Pathology and Therapeutics. A Text-Book for Students and Physicians. Translated from the tenth German edition, by special permission of the author, by Charles E. Hackley, M. D. Fifth American edition, revised and enlarged. 8vo. Cloth, $\$ 5.00$; sheep, $\$ 6.00$.

BOYCE (RUBERT). A Text-I3ook of Morbid Histology. For Students and Practitioners. With 1200 Colored Illustrations. Cloth, $\$ 7.50$.

BRAMWELL (BYROM). Diseases of the Heart and Thoracic Aorta. Illustrated with 226 Wood-Engravings and 68 Lithograph Plates-showing 91 Figures-in all 317 Illustrations. 8vo. Cloth, $\$ 8.00$; sheep, $\$ 9.00$.

BRYANT (JOSEPH D.). A Manual of Operative Surgery. New edition, revised and enlarged. 793 Illustrations. 8vo. Cloth, $\$ 5.00$; sheep, $\$ 6.00$

BUCK (GURDON). Contributions to Reparative Surgery, showing its Application to the Treatment of Deformities produced by Destructive Disease or Injury; Congenital Defects from Arrest or Excess of Development; and Cicatricial Contractions following Burns. Illustrated by Thirty Cases and fine Engravings. 8vo. Cloth, $\$ 3.00$. 
CAMPBELL (F. R.). The Language of Medicine. A Manual giving the Origin, Etymology, Pronunciation, and Meaning of the Technical Terms found in Medical Literature. 8vo. Cloth, $\$ 3.00$.

CASTRO (DUiIVEIRA). Elements of Therapeutics and Practice according to the Dosimetric System. 8vo. Cloth, $\$ 4.00$.

CHAUVEAU (A.) The Comparative Anatomy of the Domesticated Animals. By A. Chauveau, M.D., LL. D., Member of the Institute (Academy of Sciences); Inspector-General of Veterinary Schools in France; Professor at the Museum of Natural History, Paris. Revised and enlarged, with the co-operation of S. Arloing, Director of the Lyons Veterinary School ; Professor of Experimental and Comparative Medicine at the Lyons Faculty of Medicine. Second English edition. Translated and edited by George Fleming, C. B., LL. D., F. R.C. V.S., late Principal Veterinary Surgeon of the British Army; Foreign Corresponding Member of the Société Royale de Médecine, and of the Société Royale de Médicine Publique, of Belgium, etc. 8 vo, with 585 Illustrations. Cloth, $\$ 7.00$.

CORNING (J. L.). Brain Exhaustion, with some Preliminary Considerations on Cerebral Dynamics. Crown 8vo. Cloth, $\$ 2.00$.

CORNING (J. L.). Local Anæsthesia in General Medicine and Surgery. Being the Practical Application of the Author's Recent Discoveries. With Illustrations. Small 8vo. Cloth, $\$ 1.25$.

DAVIDSON (ANDREW). Geographical Pathology: An Inquiry into the Geographical Distribution of Infective and Climatic Diseases. 2 vols. 8vo. Cloth, $\$ 7.00$.

IEXTER (FRANKLIN). The Anatomy of the Peritonæum. 12mo. With 39 colored Illustrations. (In press.)

DOTY (ALVAH H.). A Manual of Instruction in the Principles of Prompt Aid to the Injured. Designed for Military and Civil Use. 96 Illustrations. 12mo. Cloth, $\$ 1.25$.

ELIIIOT (GEORGE T.). Obstetric Clinic: A Practical Contribution to the Study of Obstetrics and the Diseases of Women and Children. 8vo. Cloth, $\$ 4.50$.

EVANS (GEORGE A.). Hand-Book of Historical and Geographical Phthisiology. With Special Reference to the Distribution of Consumption in the Lnited States. 8vo. Cloth, $\$ 2.00$.

EW ALD (C. A.). Lectures on the Diseases of the Stomach. By Dr. O. A. Ewald, Professor of Pathology and Therapeutics in the University of Berlin, etc. Translated from the German by special permission of the author, by Morris Manges, A. M., M. D. Cloth, $\$ 5.00$; sheep, $\$ 5.00$.

FLINT (AUSTIN). Medical Ethics and Etiquette. Commentaries on the National Code of Ethics. 12mo. Cloth, 60 cents.

FLINT (AUSTIN). Medicine of the Future. An Address prepared for the Annual Meeting of the British Medical Association in 1886. With Portrait of Dr. Flint. $12 \mathrm{mo}$. Cloth, $\$ 1.00$.

FLIN'T (AUSTIN, JR.). Text-Book of Human Physiology; designed for the Use of Practitioners and Students of Medicine. Illustrated with three hundred and sixteen Woodcuts and Two Plates. Fonrth edition, revised. Imperial 8vo. Cloth, $\$ 6.00$; sheep, $\$ 7.00$.

FLINT (AUSTIN, JR.). The Physiological Effects of Severe and Protracted Mnscular Exercise; with Special Reference to its Influence upon the Excretion of Nitrogen. $12 \mathrm{mo}$. Cloth, $\$ 1.00$.

FLINT (AUSTIN, JR.). The Source of Muscular Power. Arguments and Conclusions drawn from Observation upon the Human Subject under Conditions of Rest and of Muscular Exercise. 12mo. Cloth, $\$ 1.00$. 


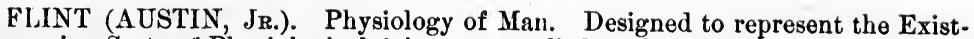
ing State of Physiological Science as applied to the Functions of the Human Body. Complete in 5 vols., 8 vo. Per vol., cloth, $\$ 4.50$; sheep, $\$ 5.50$.

** Vols. I and II can be had in cloth and sheep binding; Vol. III in sheep only. Vol. IV is at present out of print.

FLINT (AUSTIN, JR.). Manual of Chemical Examinations of the Urine in Disease; with Brief Directions for the Examination of the most Common Varieties of Urinary Calculi. Revised edition. 12mo. Cloth, $\$ 1.00$.

FOSTER (FRANK P.). Illustrated Encyclopædic Medical Dictionary, being a Dictionary of the Technical Terms used by Writers on Medicine and the Collateral Sciences in the Latin, English, French, and German Languages. This work will be completed in four volumes. (Sold only by subscription.) The work will consist of Four Volumes, and will be sold in Parts; Three Parts to a Volume. Nine Parts are now ready for delivery.

FOURNIER (ALFRED). Syphilis and Marriage. Translated by P. Albert Morrow, M. D. 8vo. Cloth, $\$ 2.00$; sheep, $\$ 3.00$.

FREY (HEINRICH). The Histology and Histochemistry of Man. A Treatise on the Elements of Composition and Structure of the Human Body. Translated from the fourth German edition by Arthur E. J. Barker, M. D., and revised by the author. With 608 Engravings on Wood. 8vo. Cloth, $\$ 5.00$; sheep, $\$ 6.00$.

FRIEDLANDER (CARL). The Use of the Microscope in Clinical and Pathological Examinations. Second edition, enlarged and improved, with a Chromo-lithograph Plate. Translated, with the permission of the author, by Henry C. Coe, M. D. 8vo. Cloth, $\$ 1.00$.

FUCHS (ERNEST). Text-Book of Ophthalmology. By Dr. Ernest Fuchs, Professor of Ophthalmology in the University of Vienna. With 178 Woodcuts. Authorized translation from the second enlarged and improved German edition, by A. Duane, M. D. Cloth, $\$ 5.00$; sheep, $\$ 6.00$.

GARMANY (JASPER J.). Operative Surgery on the Cadaver. With Two Colored Diagrams showing the Collateral Circulation after Ligatures of Arteries of Arm, Abdomen, and Lower Extremity. Small 8vo. Cloth, $\$ 2.00$.

GERSTER (ARPAD G.). The Rules of Aseptic and Antiseptic Surgery. A Practical Treatise for the Use of Students and the General Practitioner. Illustrated with over two hundred fine Engravings. 8vo. Cloth, $\$ 5.00$; sheep, $\$ 6.00$.

GIBSON-RUSSELL. Physical Diagnosis: A Guide to Methods of Clinical Investigation. By G. A. Gibson, M. D., and William Russell, M. D. With 101 Illustrations. 12mo. (Student's Series.) Cloth, $\$ 2.50$.

GOULEY (JOHN W. S.). Diseases of the Urinary Apparatus. Part I. Phlegmasic Affections. Being a Series of Twelve Lectures delivered during the autumn of 1891. With an Addendum on Retention of Urine from Prostatic Obstruction in Elderly Men. Cloth, $\$ 1.50$.

GRosS (SAMUEL W.). A Practical Treatise on Tumors of the Mammary Gland. Illustrated. 8vo. Cloth, $\$ 2.50$.

GRUBER (JOSEF). A Text-Book of the Diseases of the Ear. Translated from the second German edition by special permission of the author, and edited by Edward Law, M. D., and Coleman Jewell, M. D. With 150 Illustrations and 70 Colored Figures on Two Lithographic Plates. 8vo. Cloth. $\$ 5.00$.

GUTMANN (EDWARD). The Watering-Places and Mineral Springs of Germany, Austria, and Switzerland. Illustrated. $12 \mathrm{mo}$. Cloth, $\$ 2.50$. 
HAMMOND (W. A.). A Treatise on Diseases of the Nervous System. With the Collaboration of Graeme M. Hammond, M. D. With One Hundred and Eighteen Illustrations. Ninth edition, with corrections and additions. 8vo. Cloth, $\$ 5.00$; sheep, $\$ 6.00$.

HAMMOND (W. A.). A Treatise on Insanity, in its Medical Relations. 8vo Cloth, $\$ 5.00$; sheep, $\$ 6.00$.

HÁMMOND (W. A.). Clinical Lectures on Diseases of the Nervous Srstem. Delivered at Bellevue Hospital Medical College. Edited bv T. M. B. Cross, M. D. 8vo. Cloth, $\$ 3.50$.

HARVEY (A.). First Lines of Therapentics. 12mo. Cloth. $\$ 1.50$.

HOFFMANN-ULTZMANN. Analysis of the Urine, with Special Reference to Diseases of the Urinary Apparatus. By M. B. Hoffmann, Professor in the University of Gratz; and R. Ultzmann, Tutor in the University of Vienna. Third edition, revised and enlarged. 8vo. Cloth, $\$ 2.00$.

HIRT (LUDWlG). The Pathology and Therapenties of Nervous I liseases. For Physicians and Students. By Dr. Ludwig Hirt, Professor at the University of Breslau. Translated by Aug. Hoch, Mr. D., Assistant Physician to the Johns Hopkins Hospital. With an Introduction by William Osler, M. D., Professor of Medicine in the Johns Hopkins University, and Physician-inChief to the Johns IIopkins Hospital, Baltimore. With Illustrations. (In preparation.)

HOWE (JOSEPH W.). Emergencies, and how to treat them. Fourth edition, revised. 8vo. Cloth, $\$ 2.50$.

HOWE (JOSEPH W.). The Breath, and the Diseases which give it a Fetid Odor. With Directions for Treatment. Second edition, revised and corrected. 12mo. Cloth, $\$ 1.00$.

HUEPPE (FERDINAND). The Methods of Bacteriological Investigation. Written at the request of Dr. Robert Koch. Translated by Hermann M. Biggs, M. D. Illustrated. 8vo. Cloth, $\$ 2.50$.

JACCOUD (S.). The Curability and Treatment of Pulmonary Phthisis. Translated and edited by Montagu Lubbock, M. D. 8vo. Cloth, \$1.00.

JOHnstone (ALEX.). Botany : A Concise Mannal for Students of Medicine and Science. With 164 Illustrations and a Series of Floral Diagrams. 12 mo. (Student's Series.) Cloth, $\$ 1.75$.

JUNES (H. MACNAUGHTON). Practical Manual of Diseases of Women and Uterine Therapeutics. For Stndents and Practitioners. 188 Illustrations. $12 \mathrm{mo}$. Cloth, $\$ 3.00$.

JOURNAL OF CUTANEOUS AND GENITO-URINARY DISEASES. Published Monthly. Edited by John A. Fordyce, M. D. Terms, $\$ 2.50$ per annum.

KEYES (E L.). A Practical Treatise on Genito-Urinary Diseases, including Syphilis. Being a new edition of a work with the same title, by Van Buren and Keyes. Almost entirely rewritten. 8vo. With Illustrations. Cloth, $\$ 5.00$; sheep, $\$ 6.00$.

KEYES (E. L.). The Tonic Treatment of Syphilis, including Local Treatment of Lesions. 8vo. Cloth, $\$ 1.00$.

KINGSLEY (N. W.). A Treatise on Oral Deformities as a Branch of Mechanical Surgery. With over 350 Illustrations. 8vo. Cloth, $\$ 5.00$; sheep, $\$ 6.00$. 
LEGG (J. WICKHAM). On the Bile, Jaundice, and Blious Diseases. With Illustrations in Chromo-Lithogruphy. 8vo. Cloth, $\$ 6.00$; sheep, $\$ 7.00$.

LITTLE (W. J.). Medical and Surgical Aspects of In-Knee (Genu-Valgum, its Relation to Rickets, its Prevention, and its Treatment, with and without Surgical Operation. Illustrated by upward of Fifty Figures and Diagrams. 8vo. Cloth, $\$ 2.00$.

L.ORING (EDWARD G.). A Text-Book of Ophthalmoscopy.

Part I. The Normal Eye, Determination of Refraction, and Diseases of the Media. With 131 Illustrations, and 4 Chromo-Lithographs. 8vo. Buckram. $\$ 5.00$.

Part II. Diseases of the Retina, Optic Nerve, and Choroid: their Varieties and Complications. The manuscript of this volume, which the author finished just prior to his death, has been thoroughly edited and revised by F. B. Loring, M. D., of Washington, D. C., and is now issued in the same style as the first volume. Profusely illustrated. Part II, buckram, $\$ 5.00$. Two Parts, buckram, $\$ 10.00$.

LUSK (WILLIAM T.). The Sicience and Art of Midwifery. With 246 Illustrations. Fourth edition, revised and enlarged. 8vo. Cloth, $\$ 5.00$; sheep, $\$ 6.00$

MARCY (HENRY O.). The Anatomy and Surgical Treatment of Hernia. 4to, with about Sixty full-page Heliotype and Lithographic Reproductions from the Old Masters, and numerons Illustrations in the Text. (Sold only by subscription.)

MARKOE (T. M.). A Treatise on Diseases of the Bones. With Illustrations. 8vo. Cloth, $\$ 4.50$.

MATHEWS (JOSEPH M.). A Treatise on Diseases of the Rectum, Anus, and Sigmoid Flexure. 8vo. With Six Chromo-lithographs, and Illustrations in the text. (Sold only by subscription.)

MILLS (WESLEY). A Text-Book of Animal Pbysiology, with Introductory Chapters on General Biology and a full Treatment of Reproduction for Students of Human and Comparative Medicine. 8vo. With 505 Illustrations. Cloth, $\$ 5.00$; sheep, $\$ 6.00$.

MILLS (WESLEY). A Text-Book of Comparative Physiology. For Students and Practitioners of Veterinary Medicine. Small 8vo. Cloth, $\$ 3.00$.

THE NEW YORK MEDICAL JOURNAL (weekly). Edited by Frank P Foster, M. D. Terms, $\$ 5.00$ per annum.

Binding Cases, cloth, 50 cents each.

"Self-Binder" (this is used for temporary binding only), 90 cents.

General Index, from April, 1865, to June, 1876 (23 vols.) 8vo. Cloth, 75 cts.

NIEMEYER (FELIX VON). A Text-Book of Practical Medicine, with' particular reference to Physiology and Pathological Anatomy. Containing all the author's Additions and Revisions in the eighth and last German edition. Translated by George H. Humphreys, M. D., and Charles E. Hackley, M. D. 2 vols., 8vo. Cloth, $\$ 9.00$; sheep, $\$ 11.00$.

NIGHTINGALE'S (FLORENCE) Notes on Nursing. 12mo. Cloth, 75 cents.

OSLER (WILliaM). The Principles and Practice of Medicine. Designed for the Use of Practitioners and Students of Medicine. Solll only by subscription. Cloth, $\$ 5.50$; sheep, $\$ 6.50$; half morocco, $\$ 7.00$. 
PELLEW (O. E.). A Manual of Practical Medical Chemistry. 12mo. With Illustrations. (In press.)

PEREIRA'S (Dr.) Elements of Materia Medica and Therapeutics. Abridged and adapted for the Use of Medical and Pharmaceutical Practitioners and Students, and comprising all the Medicines of the British Pharmacopœia, with such others as are frequently ordered in Prescriptions, or required by the Physician. Edited by Robert Bentley and Theophilus Redwood. Royal 8vo. Cloth, $\$ 7.00$; sheep, $\$ 8.00$.

PEYER (ALEXANIER). An Atlas of Clinical Microscopy. Translated and edited by Alfred C. Girard, M.D. First American, from the manuscript of the second German edition, with Additions. Ninety Plates, with 105 Illustrations, Chromo-Lithographs. $\quad$ Square 8vo. Cloth, $\$ 6.00$.

PIFFARD (HENRY G.). A Practical Treatise on Diseases of the Skin. By Henry G. Piffard, A. M. M. D., assisted by Robert M. Fuller, M. D. With Fifty full-page Original Plates and Thirty-three Illustrations in the Text. 4to. (Sold only by subscription.)

POMEROY (OREN D.). The Diagnosis and Treatment of Diseases of the Ear. With One Hundred Illustrations. Second edition, revised and enlarged. 8vo. Cloth, $\$ 3.00$.

POORE (C. T.). Osteotomy and Osteoclasis, for the Correction of Deformities of the Lower Limbs. 50 Illustrations. 8vo. Cloth, $\$ 2.50$.

QUAIN (RICHARD). A Dictionary of Medicine, including General Pathology, General Therapeutics, Hygiene, and the Diseases peculiar to Women and Children. By Various Writers. Edited by Richard Quain, M. D., In one large 8vo volume, with complete Index, and 138 Illustrations. (Sold only by subscription.)

RANNEY (AMBROSE L.). Applied Anatomy of the Nervous System, being a Study of this Portion of the Human Body from a Standpoint of its General Interest and Practical Utility, designed for Use as a Text-Book and as a Work of Reference. Second edition, revised and enlarged. Profusely illustrated. 8vo. Cloth, $\$ 5.00$; sheep, $\$ 6.00$.

ROBINSON (A. R.). A Manual of Dermatology. Revised and corrected. 8vo. Cloth, $\$ 5.00$.

ROSCOE-SCHORLEMMER. Treatise on Chemistry.

Vol. 1. Non-Metallic Elements. 8vo. Cloth, $\$ 5.00$.

Vol. 2. Part I. Metals. 8vo. Cloth, $\$ 3.00$.

Vol. 2. Part II. Metals. 8vo. Cloth, $\$ 3.00$.

Vol. 3. Part I. The Chemistry of the Hydrocarbons and their Derivatives. 8vo. Cloth, $\$ 5.00$.

Vol. 3. Part II. The Chemistry of the Hydrocarbons and their Derivatives. 8vo. Cloth, $\$ 5.00$.

Vol. 3. Part IIl. The Chemistry of the Hydrocarbons and their Derivatives. 8vo. Cloth, $\$ 3.00$.

Vol. 3. Part IV. The Chemistry of the Hydrocarbons and their Derivatives. 8vo. Cloth, $\$ 3.00$.

Vol. 3. Part V. The Chemistry of the Hydrocarbons and their Derivatives. 8vo. Cloth, $\$ 3.00$.

ROSENTHAL (I.). General Physiology of Muscles and Nerves. With 75 Woodcuts. 12mo. Cloth, $\$ 1.50$. 
SAYRE (LEWIS A.). Practical Manual of the Treatment of Club-Foot. Fourtb edition, enlarged and corrected. $12 \mathrm{mo}$. Cloth, $\$ 1.25$.

SAYRE (LEWIS A.). Lectures on Orthopedic Surgery and Diseases of the Joints, delivered at Bellevue Hospital Medical College. New edition, illustrated with 224 Engravings on Wood. 8vo. Oloth, $\$ 5.00$; sheep, $\$ 6.00$.

SCHULTZE (B. S.). The Pathology and Treatment of Displacements of the Uterus. Translated from the German by Jameson J. Macan, M. A., etc.; and edited by Arthur V. Macan, M. B., etc. With One Hundred and Twenty Illustrations. 8 vo. Cloth, $\$ 3.50$.

SHIELD (A. MARMADUKE). Surgical Anatomy for Students. $12 \mathrm{mo}$. (Student's Series.) Cloth, \$1.75.

SHOEMAKER (JOHN V.). A Text-Book of Diseases of the Skin. Six Chromo-Lithographs and numerous Engravings. Second edition, revised and enlarged. 8vo. Cloth, $\$ 5.00 ;$ sheep, $\$ 6.00$.

SIMPSON (JAMES Y.). Selected Works: Anæsthesia, Diseases of Women. 3 vols., 8 vo. Per volume. Cloth, $\$ 3.00$; sheep, $\$ 4.00$.

SIMS (J. MARION). The Story of my Life. Edited by his Son, H. Marion Sims, M. D. With Portrait. 12mo. Cloth, $\$ 1.50$.

SKENE (ALEXANDER J. C.). A Text-Book on the Diseases of Women. Illustrated with two hundred and fifty-four Illustrations, of which one hundred and sixty-tive are original, and nine chromo-lithographs. Second edition. 8vo. (Sold only by subscription.)

STEINER (JOHANNES). Compendium of Children's Diseases: a Hand-Book for Practitioners and Students. Translated from the second German edition, by Lawson Tait. 8 vo. Cloth, $\$ 3.50$; sheep, $\$ 4.50$.

STEVENS (GEORGE T.) Functional Nerrous Diseases: their Oauses and their Treatment. Memoir for the Concourse of 1881-1883, A cadémie Royal de Médecine de Belgique. With a Supplement, on the Anomalies of Refraction and Accommodation of the Eye, and of the Ocular Muscles. Small 8 vo. With six Photographic Plates and twelve Illustrations. Cloth, $\$ 2.50$.

STONE (R. FRENCH). Elements of Modern Medicine, including Principles of Pathology and of Therapeutics, with many Useful Memoranda and Valuable Tables of Reference. Accompanied by Pocket Fever Charts. Designed for the Use of Students and Practitioners of Medicine. In wallet-book form, with pockets on each cover for Memoranda, Temperature Charts, etc. Roan, tuck, $\$ 2.50$.

STRECKER (ADOLPH). Short Text-Book of Organic Chemistry. By Dr. Johannes Wislicenus. Translated and edited, with Extensive Additions, by W. H. Hodgkinson and A. J. Greenaway. 8vo. Cloth, $\$ 5.00$.

STRÜMPELL (ADOLPH). A Text-Book of Medicine, for Students and Practitioners. With 111 Illustrations. 8vo. Cloth, $\$ 6.00$; sheep, $\$ 7.00$.

THOMAS (T. GAILLARD). Abortion and its Treatment, from the Standpoint of Practical Experience. A Special Course of Lectures delivered before the College of Physicians and Surgeons, New York, Session of 1889-'90. From Notes by P. Brynberg Porter, M.D. Revised by the Author. $12 \mathrm{mo}$. Cloth, $\$ 1.00$. 
THOMSON (J. ARTHUR). Outlines of Zoölogy. With Thirty-two full-page Illustrations. $12 \mathrm{mo}$. Cloth, $\$ 3.00$.

TRACY (ROGER S.). The Essentials of Anatomy, Physiology, and Hygiene. $12 \mathrm{mo}$. Cloth. $\$ 1.25$.

TRANSACTIONS OF THE NEW YORK STATE MEDICAI ASSOCIATION, VOLS. I and II. Being the Proceedings of the First Annual Meeting of the New York State Medical Association, held in New York, November 18,19 , and 20,1884 . Small 8 vo. Cloth, $\$ 5.00$.

TYNDALL (JOHN). Essays on the Floating Matter of the Air, in Relation to . Putrefaction and Infection. 12mo. Cloth. $\$ 1.50$.

ULTZMANN (ROBERT). Pyuria, or Pus in the Urine, and its Treatment. Translated by permission, by Dr. Walter B. Platt. $12 \mathrm{mo}$. Cloth, $\$ 1.00$.

VAN BUREN (W. H.). Lectures upon Diseases of the Rectum, and the Surgery of the Lower Bowel, delivered at Bellevue Hospital Medical College. Second edition, revised and enlarged. 8 vo. Cloth, $\$ 3.00$; sheep, $\$ 4.00$.

VAN BUREN (W. H.). Lectures on the Principles and Practice of Surgery. Delivered at Bellevue Hospital Medical College. Edited by Lewis A. Stimson, M. D. 8 vo. Cloth, $\$ 4.00$; sheep, $\$ 5.00$.

VOGEL (A.). A Practical Treatise on the Diseases of Children. Translated and editerl by H. Raphael, M.D. Third American from the eighth German edition, revised and enlarged. Illustrated by six Lithographic Plates. 8vo. Cloth, $\$ 4.50$; sheep, $\$ 5.50$.

VON ZEISSL (HERMANN). Outlines of the Pathology and Treatment of Syphilis and Allied Venereal Diseases. Second edition, revised by Maximil ian von Zeissl. Authorized edition. Translated, with Notes, by H. Raphael, M. D. 8 vo. Cloth, $\$ 4.00$; sheep, $\$ 5.00$.

WAGNER (RUDOLF). Hand-Book of Chemical Technologs. Translated and edited from the eighth German edition, with extensive Additions, by William Crookes. With 336 Illustrations. 8vo. Cloth, $\$ 5.00$.

WALTON (GEORGE E.). Mineral Springs of the United States and Canadas. Containing the latest Analyses, with full Description of Localities, Routes, etc. Second edition, revised and enlarged. $12 \mathrm{mo}$. Cloth, $\$ 2.00$.

WEBBER (S. G.). A Treatise on Nervous Diseases: Their Symptoms and Treatment. A Text-Book for Students and Practitioners. 8vo. Cloth, $\$ 3.00$.

WEEKS-SHA W (CLARA S.). A Text-Book of Nursing. For the Use of Training-Schools, Families, and Private Students. Second edition, revised and enlarged. 12mo. With Illustrations, Questions for Review and Examination, and Vocabulary of Medical Ternıs. 12mo. Cloth, $\$ 1.75$.

WELLS ('T. SPENCER). Diseases of the Ovaries. 8vo. Cloth, $\$ 4.50$.

WORCESTER (A.). Monthly Nursing. Second edition, revised. Cloth, \$1.25.

WYETH (JOHN A.). A Text-Book on Surgery: General, Operative, and Mechanical. Profusely illustrated, 8vo. (Sold only by subscription.)

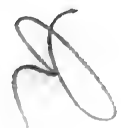





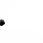





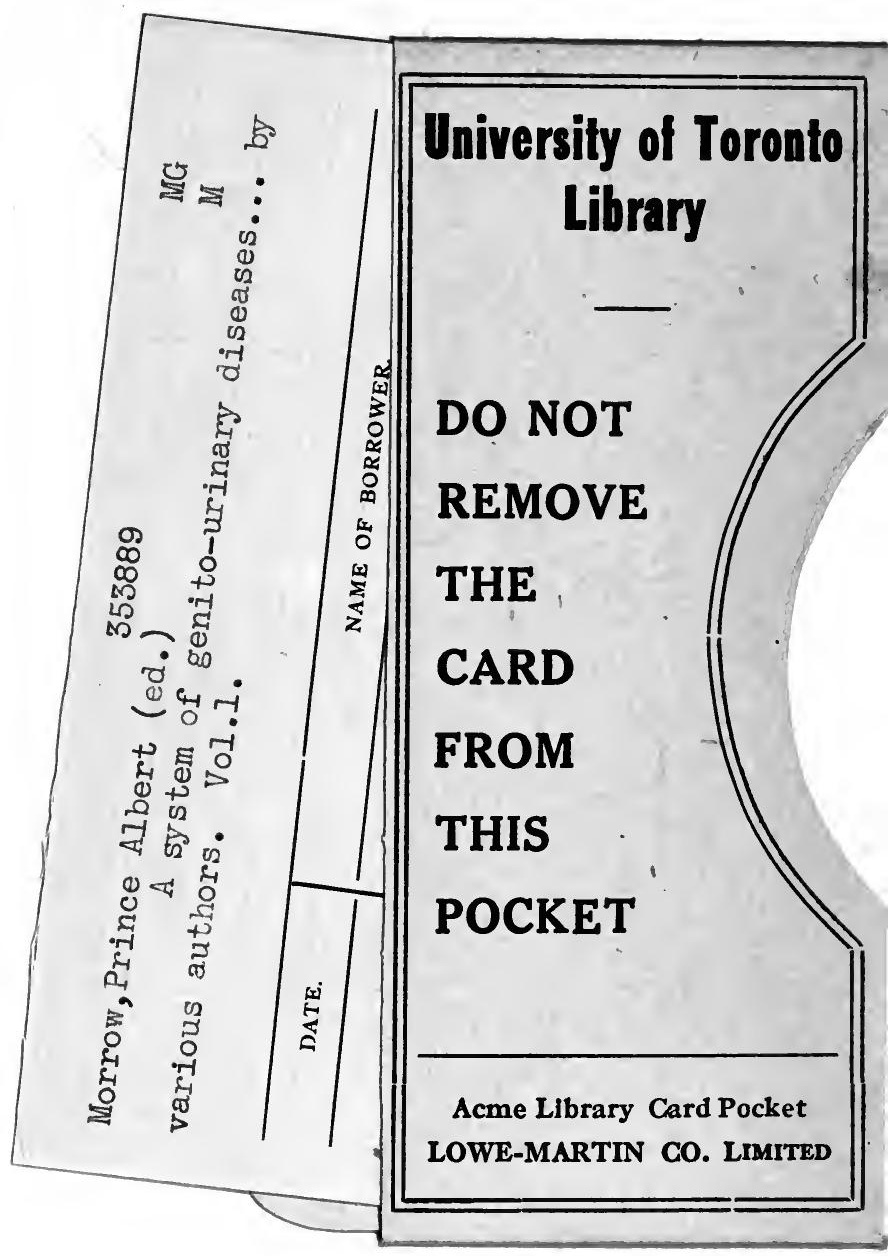


